\section{BEHAVIORAL SCIENCE AND HEALTH STUDIES} 1 AN APPROACH TO THE DEVELOPMENT OF LEVEL II PERINATAL EXTENDERS, Eugene W. Adcock, Frank H. Morriss, 0. Scott Hume, Joan W. Stoerner, Charles L. Paxson, and Susan E. Denson. (Spon. by R. R. Howe11). University of Texas Medical School at Houston, Departments of Pediatrics and $\mathrm{Ob}-\mathrm{Gyn}$, Houston.

Three Level III Regional Perinatal Centers (Level IIIs) combined efforts to develop Level II Perinatal Care Units (Level IIs) in rural and urban locations in a catchment area with 50,000 annual births. Continuing education programs included: surveys of facilities and education needs of community hospitals (CHs), lectures for Emergency Medical Technicians involved in transportation, a telephone "hotline" network, workshops and symposia for health professionals and consumers, specially prepared videotapes, and self-evaluation study guides. Pediatric nurse practitioners, nurse clinicians in neonatology and obstetrics, physicians, local citizens and pharmaceutical representatives worked as a team to increase awareness of the need for Level IIs and to provide education to consumers and professionals. Problems included: (1) assessment of immediate effectiveness; (2) personnel turnover in CHs; (3) coordination among Level IIIs; (4) long distance transportation; (5) patient care financing; and (6) $\mathrm{CHs}^{\prime}$ competition for recognition as Level IIs. Accomplishments after 18 months are: (1) enhanced utilization of facilities and patterns of referral; (2) development of 7 emerging ievel IIs; (3) 1,856 educational contacts; (4) concerted consumer effort in development of Level IIs; and (5) delayed but spontaneous interest among obstetricians following their recognition of a lag in obstetrical health care delivery.

2 PRIMARY CARE RESIDENCY. Joel J. Alpert, Alan S.

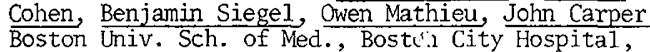
Boston Univ. Sch. of Med.
Ped. and Med., Boston, Ma.

Depts. of Ped. and Med., Boston, Ma.

A three year primary care residency (PC) for pediatricians and internists has functioned for two years and is one effort to modify pediatric training to meet primary care needs. The program retains much of usual training but differs by its multi disciplinary faculty, crossover rotations in Pediatrics and Medicine, social science and psychiatric content, and intensive ambulatory experience either in hospital or health center sites. A separate evaluation unit is studying program development and outcomes. Evaluation to date is principally descriptive due to the small size of the trainee cohort (16). Comparing the trainees to other BCH housestaff (80) showed no major differences in medical school performance, rank on match, and cognitive know1edge, (measured by pretests provided by the American Boards of Pediatrics and Medicine). PC pediatric trainees express greater interest in inner city practice, adolescents, behavior disorders, growth and development, and practice organization. Non PC pediatric trainees expressed greater interest in the medical subspecialties. Since one of the stated program goals is to attract physicians to inner city practice, one outcome measure will be how the residency reinforces or alters this goal. THE CORRELATION OF EDUCATIONAL LEVEL, ECONOMIC STATUS, AND MATERNAL-INFANT FEEDING METHODS IN AN URBAN POPULATION. Jay E. Berkelhamer, Ruth Whitham, Julia North and Janis Mendelsohn (spon. by Samuel Spector). Univ. of Chicago, Pritzker Sch
Illinois.

248 mothers of consecutively born infants at the Chicago Lying In Hospital were interviewed during their first postparturn day. Among the questions asked were: 1) whether breast feedings would be used, 2) the highest grade completed in school, and 3) the estimated family income per year.

37 mothers indicated that they planned to breast feed their infants. These included mothers that would use a combination of breast and bottle feedings. 188 mothers offered an income estimate when asked. Of the 152 with family incomes of less than $\$ 10,000$ per year, $19(12.5 \%)$ indicated they would breast feed. $11(30.5 \%)$ of the 36 with higher incomes planned to breast feed. The difference between the two groups is significant at the RK.02 level. All mothers volunteered their educational level. Of the 80 with a level of 10th grade or less $5(6.3 \%)$ planned to breast feed. $32(19 \%)$ of the remaining 168 with higher educational levels also planned to breast Eeed. This difference is significant at the P<.02 leve1.

Although the frequency of breast feeding is only $15 \%$ in the population studied, there is a direct correlation with increased income and educational levels. In parts of the world where breast feeding is nore conmon, increased income and educational status have been negatively correlated with breast feeding.
PRADER-WILLI SYNDROME: BEHAVIORAL TECHNIQUES RESULTING IN WEIGHT LOSS. Andrew S. Bondy, Kar1 I. Altman, and Joseph G. Hollowell (Spon. by Cheng Cho). Kansas University Medical Center, Childrens Rehabilitation Unit and Departments of Human Ecology and Pediatrics, Kansas City.

Behavioral techniques successful in wt. reduction with normal obese people have not had success with obese Prader-Willi children. This study of a Prader-Willi adolescent female demonstrated wt. loss which was maintained in a natural environment. She weighed $239.5 \mathrm{lbs}$, at the start of this program and had been gaining at $3.5 \mathrm{lbs}$./wk. The $8 \mathrm{wk}$. in-patient program to initiate and reward changes in eating patterns began by contracting with the client toward earning specific social rewards in exchange for self-monitoring of 1 ) food and cal. intake 2) wt. and 3) exercises. Hospital staff monitoring of the client's performance showed $91 \%$ agreement with the client's self-monitoring. Only after 2 wks. of self-monitoring were contracts for losing wt. introduced. At this time the client ate 200 cal./day over amt. prescribed. Each wt.-loss contract had rewards proportionate to amt. of wt. loss. The diet was maintained only when rewarded. Cal. intake was maintained at $1000 /$ day or less for the last 6 wks. The client lost 21.5 lbs. during the 8 wks. Measures of colateral behaviors (e.g., exercise, self-care, etc.) indicated that contracting was necessary for the behavioral changes. Contracting at home has resulted in continued wt. loss at $1 \mathrm{lb} . / \mathrm{wk}$. The results suggest that behavioral programs can alter eating behaviors of PraderWilli children.

5 PARENTAL EVALUATION OF VERY LOW BIRTHWEIGHT (VLBW) CHILDREN AT PRESCHOOL AGE. Michael H. Boyle, Airi E. Giffen, Pamela M. Fitzhardinge (SDon. by Paul $R$. Swyer). Research Inst., Hosp. for Sick Children, Univ. of Toronto, Toronto, Canada.

This study was done to determine whether the parents of VLBW infants feel their child is a burden at the preschool age. Parents of 75 infants horn between Jan I/70-July I/7I with birthweights under I5OI $g$ formed the study group. Parents of 55 infants born at the same time, normal at hirth with a weight over $2500 \mathrm{~g}$ served as controls. Home interviews were done when the children were 3-5 years old. Questions were divided into 7 major areas: development, health, play, toilet training, feeding, sleeping and discipline; the questions examined I) protlems reported by the parents 2) special care given to the child 3 ) parental reactions to the child's care and 4) parental comparison with a "normal" child. The study group showed more negative evaluations in four areas: development ( $p<0.0 I)$ health ( $p<0.01)$, play $(p<0.0 I)$ and training ( $p<0.05)$. Fiftytwo $(69 \%)$ of the VLBW children were free from C.N.S. defects and had I.Q. scores over 80 by 3 years. Parental evaluation of this subgroup of "normal" VLBW children were compared with the controls and gave more negative results in only one area: health $(p<0.05)$. The results indicate that health represents a common concern among parents of VLBW children at the preschool age, but only those children with signs of neurologic sequelae and/or low I.Q. continued to represent a hurden to their families.

6 LAWXER PRODUCED PAIN, DISEASE AND SUFFERING, Robert L. Brent, Jefferson Med. Coll., Pediat. Dept., Phila. $\mathrm{Pa}$.

Over the past 15 years I have received written or verbal communication concerning over 100 negligence lawsuits dealing with malformed infants and children. Many of the suits have been dropped, many have been settled out of court and approximately 25 have reached the courtroom. In $1967 \mathrm{I}$ indicated (Pediat. 71:288) that the number of lawsuits seemed to be increasing, not realizing that the increase was foretelling an epidemic. The reasons for this epidemic are due to attitudes and happenings in the medical, legal and lay sectors of our society. In this presentation I would like to focus on the contribution of the legal profession to the injured child and family in producing more pain and suffering and, in some instances, even disease by presenting case histories to exemplify this thesis. The case histories clearly indicate that when a family becomes orchestrated by a lawyer into the position of devoting a great deal of their energy to litigation, many high priority family responsibilities are ignored and important cultural standards are distorted. To win at all costs may be good for a football team, but it is obviously bad for a family. We must make drastic changes in the method of supporting the victims of disease and injury so that litigation is no longer necessary to compensate and support patients and families whether negligence is or is not a factor. The process of litigation rarely solves the patient's problems and frequentiy develops into a disease all its own

(Supported by NIH HD 630, HD 370, ES01121; (E(11-1) 3268) 
7 GENETIC KNOWLEDGE OF CONNECTICUT PEDIATRICIANS. K.K. Bucholz, Y.E.Hsia , S.P. Korper, S.S.Mick. Yale Univ. The genetic knowledge and attitudes of Connecticut pediatricians were surveyed by questionnaire, and compared with their training and their ranking of continuing educational material. Responses were received from 223 of 379 practitioners (59\%), and 52 of 117 house-staf $f(44 \%)$. Knowledge about neonatal screening for PKU( $99 \%$ correct)was better than that for galactosemia(64\% correct) Availability of prenatal testing for Down's syndrome was known by $95 \%$, but only $57 \%$ knew spina bifida was detectable, and just $54 \%$ knew PKU was not detectable. Comprehension of recurrence risks was fair for sickle cell anemia(75\%); weak for Fallot's tetralogy (53\%); and poor for the possibility of mutation causing a new case of Duchenne's muscular dystrophy (32\%)or achondroplasia( $24 \%$. Comparison with training variables showed significant correlations of correct responses with year of graduation. The 1960-1970 cohort scored higher than earlier or later graduates.

Most practitioners $(91 \%$ ) would inform their patients about prenatal genetic tests; $64 \%$ would explain recurrence risks to their families;but $70 \%$ rarely discussed problems with a geneticist.

For continuing education, practitioners rated journals highest, followed closely by rounds, specialty texts and consultations. Areas of genetics viewed as important were:inheritance patterns, congenital malformations, and genetic counseling.

This study has shown some weaknesses in pediatricians' knowledge of inheritance patterns and newer diagnostic tests.Continuing education in genetics should emphasize topics important to practitioners, using channels ranked high by them.

THE ORGANIZATION OF BEHAVIOR IN DOWN'S SYNDROME INFFANTS. Dante Cicchetti and Linda Mans. (Intr. by Amos S. Deinard, M.D.). Institute of Child Development, University of Minnesota, Minneapolis.

To date, most investigations of Down's syndrome (DS) children have paid perfunctory attention to developmental processes. Instead, they have been concerned with biochemical and cytogenic analyses, with the hope that these inquires would reduce the hiatus between the chromosomal anomalies and the ultimate biopsychological outcomes. Such studies reflect a defect model of retardation. In contradistinction, the developmental model stipulates that a comprehensive understanding of retarded children requires an integration of social and emotional factors with cognitive factors. In this position it is assumed that basically the developmental process is similar in retarded and normal children; that is, that there is a similar organization in the behavior and development of atypical children. 25 DS infants are participating in a longitudinal study examining the relationship between affective and cognitive development. It was found that the DS infants laughed to stimulus items in the same order as did the normal infants, although the process was delayed by several months. In addition, cognitive developmental status, assessed by the Bayley and Uzgiris-Hunt scales, paralleled the level of affective development. There was a striking individual consistency across these indices, suggesting the organized nature of retarded development. With all DS babies, attention to emotional growth must accompany the concern about cognitive development. For all babies development is an integrated process.

an aROUSAL MODEL OF AFFECTIVE EXPRESSion IN DOWN'S SYNDROME INFANTS. Dante Cicchetti and Dan A. Nichols. (Intr. by Amos S. Deinard, M.D.). Institute of Child Development, University of Minnesota, Minneapolis.

A recent review and previous research with normal infants has generated a model which suggests that tension fluctuation is central in affective expression and that such modulation of tension has functional significance for the infant. The salient features of this model are a period of affect-free orientation, which leads to a period of appraisal, and the subsequent development of tension. The resultant affective response is thus a function of the degree of stimulus intensity, stimulus incongruity, and pre-stimulus arousal leve1. 25 Down's syndrome (DS) infants have been participating in a longitudinal study of affective development. From 4 months until age 2 , each infant is administered a series of 30 items, found to elicit laughter in normal infants, twice per month in their own homes. Our data suggest that DS infants are less affectively responsive than normal infants, even after the effects of delayed cognitive development are removed. Concurrent laboratory psychophysiological studies, using the "Visual Cliff" and infants' responses to impending collision as indices of affect, yield similar results. Our data and that of others suggest that arousal plays a major role in the development of affect in DS infants. According to our model, affect and attention abnormalities may be correlates of a fundamental defect in DS infants' arousal systems. This basic arousal deficit may have important implications for cognitive intervention strategies with these infants.
10 MORAL REASONING, AITITUDES ABOUT TREATMENT OF CRITICALLY ILL PATIENTS, AND PERFORMANCE IN PEDIATRIC RESIDENTS: Charles D. Cook, SUNY, Brooklyn; Mark Bargen, $\frac{\text { T. Joseph Sheehan, Susan R. Husted, Gary Kasey, Univ. of }}{\text { Ct. Health Ctr., Farmington; Dan Candee, }}$ Using (1) a questionnaire devised by Diana Crane to assess their activism in treating critically ill, irreversibly damaged children and (2) the Defining Issues Test by James Rest to assess the developmental level of moral reasoning, complete data were obtained on 146 pediatric residents who were rated by their supervisors on overall performance and also on 18 sub-divisions considered important aspects of performance. The majority (117) of respondents were from two major university hospitals with 21 still residents at the time of the study and 96 former trainees who had left the programs 1-9 years before the study. An additional 17 were current residents from community hospitals with $46 \%$ FMG's and 12 were from an urban hospital with $95 \%$ FMG's. Striking differences were found between the various groups of residents in level of moral reasoning and activism: 47\% from the university hospitals, $71 \%$ from the community hospitals, and $91 \%$ from the urban hospital opted for active treatment. Among all current residents, there were significant associations between sensitivity to family attitude--socio-economic factors and moral reasoning** and rated performance**. Moral reasoning was significantly related to the following performance ratings: overall performance*, personal vs. impersonal patient care ${ }^{\star \star *}$, pediatric knowledge**, clinical judgment**, decision making ability*, compassion*, and relating well to patients*. (*p<.05, **p<.01, $* \star * p<.001)$.

11 ECOLOGICAL CHARACTERISTIICS OF A NEONATAL INTENSIVE CARE UNIT. Cecelia M. Daum, Katharine R. Lawson and Gerald C. Turkewitz, Depts. Of Pediatrics and Psy(Spon. by L. M. Gartner).

Intervention programs have assumed that there is a lack of adequate stimulation in intensive care nurseries which may be detrimental to the subsequent motor and cognitive development of hospitalized premature infants. This study was designed to determine the frequency and patterning of environmental stimulation normally present in a neonatal intensive care center. Acoustic events, illumination levels and handling were noted every 15 minutes during either a 24 or 72 hour period. All observations were made within each of 4 intensive care rooms. Speech stimuli were present during $82.2 \%$ of the observations; non-speech auditory stimuli were recorded during $96.3 \%$ of the observations. Total auditory stimuli ( 62 to $80 \mathrm{db}$ ) occurred during $95 \%$ of the the periods. Although there were illumination changes resulting from solar diurnal rhythmicity, infants were continuously exposed to moderate levels of illumination (mean:63.2 ft. candles; range 50.4-73.8 $\mathrm{ft}$. candles). Therefore, rather than being deprived of stimulation, hospitalized premature infants may be exposed to inappropriate and excessive stimulation which may provide few opportunities for intersensory integration. Intervention programs which attempt to provide even greater stimulation without increasing the possibilities for intersensory integration may be detrimental to subsequent neuro-behavioral development.
12 EFFECT OF MYLLODYSPLASTIC PARAPLEGIA ON ADOLESCENT ADJUSTMENT Sandra L. $\underline{H}$. Davenport, Patricia W. Hayden,

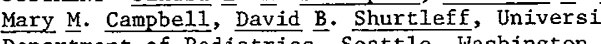

Washington, Department of Pediatrics, Seattle, Was

To test the clinical impression that adolescents with myelodysplasia mature early physically yet lag behind in emotional adjustment 20 myelodysplastic subjects age $10-18$ with average intelligence were compared to age-and sex-matched non-paraplegic controls using a structured interview of parent and child, physical examination and four standardized self-image, social adjustment scales. on Tanner criteria girls but not boys with myelodysplasia had earlier physical development than controls. The psychologic tests revealed significantly poorer adjustment in the myelodysplastic group particularly regarding self-esteem $(p=.003)$, sexual attitudes and body image $(p=.03)$ and social relationships $(p=.005)$. Although the two groups had comparable means of expressing feelings the physically handicapped were more defensive about these feelings in the test situation. Controls were able to express concerns about family relations whereas subjects tended to deny them. Subjects showed more concern about school and were less knowledgeable and realistic about future plans for family and career than their age-matched controls. Parents of both groups underestimated the sadness felt and teasing endured by the adolescent and overestimated the child's knowledge about sex and the likelihood of the child coming to them with problems of any type. This study confirms that paraplegia seriously impedes emotional maturation and indicates specific areas where communication and adaptive alternatives must be developed if these patients are to become functional adults. 
13 ASSESSMENT OF FANILY ADAPTATION TO CYSTIC FIBROSIS Carolyn R. Denning, Muriel M. Gluckson and Patricia cians \& Surgeons of Columbia Univ., Department of Pediatrics, NY

Parents of Cystic Fibrosis (CF) patients must simultaneously cope with the medical, psychosocial and hereditary aspects of htgh-risk, high-burden disease. This creates great stress. We have attempted to test the hypothesis that increasing parents' understanding of the genetic implications of CF would lessen stress within these families. A sample population of $104 \mathrm{fami-}$ lies vas interviewed to identify as many causes of marital stress as they felt applied, to determine their level of genetic comprehension, and to discuss the difficulties of living with a chronically ill child of uncertain life-span.

CAUSES OF STRESS NO. FAM. CAUSES OF STRESS NO. FAM.

Home treatment regimen $\quad 50$ Interference from relatives 14

\begin{tabular}{ll|l}
\hline liome treatment regimen & 50 & Interference from relatives 14 \\
Burden of care on one parent & 45 & Hereditary nature of $\mathrm{CF}$
\end{tabular}

Fear of patient dying 68 Fear of Pregnancy

Financial burden

39 Al coholism

The parents comprehension of genetics was tested in a series of questions. In this population where $73 \%$ of the mothers have a high school education, $91 \%$ could define the word "carrier" whereas on 1 y $60 \%$ understood that the recurrence risk was the same at each conception. The genetic questions distinguished between parents who memorized and those who made practical application of this information. In conclusion, the study clarified some causes of stress and provided data that became the basis for referrals and/or counseling.

14 AUTISM AND OCULOCUTANFOUS ALBBINISI TN THO UNRELATED BOYS. Steve J. Funderburk, Michae1 $\Lambda$. Rogawski, and Stephen D. Cederbaum (Spon. by Richard J. Schain), UCLA School of Medicine, Departments of Psychiatry and Pediatrics, Los Angeles.

Two boys with oculocutaneous albinism (one black and one white) manifested profound language retardation, delayed social and intellectual development, and bizarre, ritualistic behaviors consistent with early childhood autism. From birth both boys showed progressively darkening skin, hair, irides and pigmented nevi, and developed nystagmus, consistent with the tyrosinase-positive form of oculocutaneous albinism. Hair root incubation studies carried out on the black boy produced pigment formation with either tyrosine or dopa, verifying the presence of tyrosinase activity. Metabolic disorders in children such as phenylketonuria are known to produce retardation with autistic manifestations, whereas albinism has not previously been associated with behavioral disturbances in humans. Although the metabolic error in albinism is not known, its effect on the developing nervous system is evidenced by several reports consistently demonstrating disordered visual pathways in mammalian albinos including one albino human brain. It seems plausible that in the two boys reported here, there may be an etiologic relationship between the metabolic defect leading to oculocutaneous albinism and the autistic behavior syndrome.

15

THE ROLES OF BEHAVIOR AND ENVIRONMENT IN CHILDHOOD

LEAD ACCUMULATION. Maxybeth Gillette, Betty Jo McGrade, Martha L. Lepow, Univ. of CT Hlth. $\frac{\text { Ctr. }}{\text { H. }}$

$\mathrm{Sch}$. of Med., Dept. of Ped., Farmington, CT

Ten preschool children living in a high lead environment with blood lead levels consistently greater than 40 micrograms percen (Group I) matched with 10 neighborhood peers (Group II) with low lead levels and 10 children of similar age living in a lead-pain free housing development were studied in a doubleblind manner to determine whether differences could be demonstrated in available environmental lead, quality of parental and peer group interaction, availability of playthings and mouthing behavior.

The Home Inventory of Stimulation (STIM) was administered to all groups; environmental or hand dirt samples were tested for lead content.

Group II had significantly higher overall STIM scores than Group I as well as significantly greater numbers of appropriate playthings and greater parental and peer involvement. Group III was intermediate. Group I had greater mouthing behavior. No significant difference was found in environmental or hand dirt lead content between Groups I and II.

It is concluded that small differences in behavior and home environmental supports tip the balance toward low level intoxication among children living in a high lead environment. The STIM profile can be used at an early age to identify children needing preventive intervention.
16 PREDICTION AND PREVENTION OF CHILd ABUSE. Jane D. Gray, Christy A. Cutler, Janet Dean, C. Henry Kempe. eral Hospital, Department of Pediatrics, Denver, Colorado.

Utilizing an interview, a questionnaire, labor, delivery and post-partum observations, a sample of 100 mothers was identified as "high risk for abnormal parenting practices." These mothers were randomly divided into a "High Risk Intervene Group" ( $N=50)$ and a "High Risk Nonintervene Group" $(\mathrm{N}=50)$. In all 100 families our concern regarding the risk of abnormal parenting was shared with physicians and visiting nurses. The "Intervene" group received comprehensive pediatric follow-up by a single physician, a health visitor and/or public health nurse in the home. The "Nonintervene" group merely received routine care. A group of 50 mothers who also delivered during the same time period and were assessed as low risk in terms of abnormal parenting practices, served as "controls."

Results: 1. A high risk group was successfully identified on the basis of perinatal screening procedures; these children had significantly different parenting practices than the low risk ("control") group 2 Five children in the "High Risk Nonintervene" group required hospitalization for serious inflicted injuries as contrasted to no hospitalization in the "High Risk Intervene" and "Low Risk Control" groups. 3. Labor, delivery and nursery observations provide the most accurate predictive information; interview and questionnaire did not add significantly. Perinatal assessment and simple intervention with families "high risk for abnormal parenting practices" significantly improves the infants' chances of escaping serious physical injury.

17 PRE-SCHOOL SCREENING OF SPEECH, LANGUAGE, AND HEARING A MODEL PROGRAM OF EARLY IDENTIFICATION AND INTERVENTION. Gray, S.G. and Yamauchi, T. (Spon, by Robert H. Fiser). Harbor Gen. Hosp., UCLA Sch. of Med., and Ark. Child. Hosp., Depts. of Peds. and Speech Path., Torrance, CA. and Little Rock, AR.

Most communicative disorders are not uncovered until a child encounters difficulties in school. The purpose of this model screening program was to identify the pre-school child between the ages of 3 and 5 years with communicative disorders and initiate early treatment.

All children were selected from a supervised play area at Har bor General Hospital and had no known illnesses or abnormalities at the time of screening. The Fluharty Speech and Language Screening Test (FSLST), utilizing norms for both standard English and Black dialect, was used to assess phonological, semantic and syntactic components of language development. In addition, all children had standard audiometric screening procedures.

One hundred and eight children completed the screening program. Thirty-five (33\%) failed one or more sections of the test. Seventeen children (16\%) failed language and/or speech tests; and 3 (3\%) failed speech or language and hearing tests. All failures were referred for complete evaluation of communication function and habilitative treatment as indicated. Parenteral compliance was very favorable.

Utilization of the FSLST in combination with standard audiometric procedures identified a significant number of pre-school children with previously unrecognized communicative disorders.

Thomas A. Helmrath and Elaine M. Steinitz (spon. by A.F. Kohrman) Depts of Human Development, Psychiatry and Family Life Clinic, Michigan State University, East Lansing, Michigan.

In our society, birth is viewed as a normal happy event with most parents totally unprepared for the possibility of their infant's death. Beside initiating mourning in the parents, the baby's death also stresses their interpersonal relationship. Failure to resolve this crisis may explain the frequency of separation and divorce in these families. The purpose of this study was to determine the factors which facilitated or hindered the resolution of parental grief. Seven well educated, middle class couples with previously stable marriages were extensively interviewed following their baby's death. Each couple's inmediate family and friends were available but not supportive. Instead, they viewed the baby as replaceable, the grief as inappropriate, and discouraged any verbalization of feelings. Now isolated, parents had only each other for support. The men appeared to progress through the mourning process faster than the women, which produced frustration in both parents. At this time, serious but preventable problems in the relationship occurred. For these couples, sharing of feelings and mutual trust in each other were the key factors in resolving their grief. Five couples felt that their interpersonal relationship improved and viewed the infant's death as an opportunity for growth. Societal response to the death of a newborn infant is clearly different from the response to the death of an older child or adult. 
19

ENVIRONMENTAL CORRFIATFS OF PEDIATRIC SOCIAL ILLNESS J.Hyde, N.Morse, E. Newberger (Intr. by J.Richmond), Children's llospital Medical Center, Boston, Mass. A controlled prospective study of child abuse and neglect, failure to thrive, accidents and poisonings included 303 inpatients and 257 outpatients. Measurements of historical and contemporaneous familial, environmental, and child developmental realities were made in order to integrate into a more nearly etiologic (as opposed to manifestational) classification framework a set of hypotheses derived from clinical experience and the research literature. The objective was to form a more adequate rational base for clinical practise. Initial analysis of data demonstrated significant differences between cases and controls in summative indices of environmental stress,including housing, employment, and access to essential services. Subsequent analyses suggest associations with a postulated common causal underpinning of these illnesses which argue for a broadened,ecologic conceptualization of etiology and a wider range of intervention possibilities. A family advocacy program utilizing community based individuals addressed these problems for these families and was also offered to the comparision group. Indixect corroborration of the impact of environmental crisis is indicated by the prevalence of requests for this help in inpatient cases of abuse (388) and ingestions (38\%) vs. controls (14\%). Immediate, short-term work permitted solving problems in 838,548 , and $63 \%$ of these families respectively. The findings suggest that family advocacy, in the context of interdisciplinary, interagency program, is a valuable intervention mode where parental functioning is compromised by environmental crisis.

NEONATAL ASSESSMENT: IMPLICATION FOR INTERVENTION. J. Ivin, K. Massoud and D. Vidyasagar, 111 ino is InDisabilities and Abraham Lincoln School of Medicine, Univer. of 111 . Hosp., Dept. Pediat. Chicago, 11 .

The Brazelton Neonatal Behavior Assessment Scale has demonstrated its usefulness as a diagnostic tool with at-risk neonates. It has been used successfully to discriminate at-risk and control groups of infants and as a baseline measure for evaluation of intervention programs. We chose the Brazelton Scale as a screening tool for early intervention and sensori-motor areas. 50 at-risk infants have been examined in gestational age from 26-40 weeks and in birth weight from 850-2800 gms. Despite the fact that our sample is small and heterogeneous, thus far, and that there is no standardization of the Brazelton Scale for at risk infants, we are encouraged by the consistency between Brazelton profiles and later performance on the Bayley Scales. While it is generally agreed that infant tests yield poor predictive validity, our data indicates the Brazelton can be extremely useful in screening for specific, of ten subtle, sensorimotor liabilities. Results will be discussed in terms of specific developmental problems which were identified in the neonatal period. Examples will also be presented of infants who were considered medically at-risk as neonates but who required no special intervention, corroborating their Brazelton performance.

21

KWASHIORKOR IN WHITE INEANTS, NOT ASSOCIATED WITH POVERTY. T. Jacob John, Julie Blazovich, Elmer S. Lightner, Otto F. Sieber, Ronald Hansen and James J. Corrigan. University of Arizona Medical Center, Department of Pediatrics, Tucson, Arizona.

Within the past year we have diagnosed and treated 4 white infants with sugar-baby kwashiorkor. All of them were referred to the University Hospital as diagnostic problems, the diagnosis having been missed by several physicians at various levels. The presenting picture was that of failure to thrive and in addition dwarfism in one, skin rash in another, proximal muscle atrophy in the third and hepatomegaly in the fourth. All of them had the 4 constant features of kwashiorkor namely marked growth retardation, mental changes, muscle changes, and edema in association with hypoalbuminemia. A major problem in the nutritional rehabilitation of these infants was the extreme anorexia which had to be overcome by persistent attempts at feeding, sometimes hourly feeding and occasionally gavage feeding. All infants recovered by nutritional therapy alone, exhibiting accelerated growth response. These and other sporadic cases of kwashiorkor not caused by poverty are predominantly in infants under a year of age, mild in nature, sugar-baby type in appearance, and caused by severe protein deficiency without significant calorie deficiency, at least until anorexia occurs. Thus they are etiologically and epidemiologically different from cases widely prevalent in poor communities. Although marasmus is easily recognized, kwashiorkor may be missed by those who are unaccustomed to its clinical picture.
22 A DESCRIPTION AND EVALUATTON OF THE EXPANDED-ROLE NURSE IN HIGH-RISK NEWBORN CARE. Patricia J. Johnson and August L. Jung. (Spon. by John W. Reynolds). University of Utah College of Nursing and Department of Pediatrics, Salt Lake City.

The expanded-role nurse concept was implemented by one nurse in a newborn intensive care center for a period of seven weeks. During this role implementation, the expanded-role nurse was responsible for the primary care and management of fifteen sick newborns admitted to the center. A comparative evaluation was then conducted to describe the quality of primary care provided by the nurse to a representative group of ten high-risk newborns and compare it with the quality of primary care provided by pediatric interns to a similar group of ten newborns.

A chart audit was designed, involving ten neonatologists, to assess and rate the adequacy of problem identification, problem management, and documentation of patient care and progress in both groups. A simple statistical comparison was made of the mean numerical ratings between the two collective groups of infants. The quality of primary care provided by the nurse was consistent with the usual standard of primary care in this setting, thus demonstrating the viability and justifying the practical application of the expanded-role nurse concept in highrisk newborn care.

COMMUNITY HOSPITAL NEONATAL CARE: EVALUATION OF COGNITIVE PERFORMANCE OF NURSES. William P. Kanto, Jr. Joyce C. Maples, Gladys H. Goldberg, and Max D.

Miller (spon, by Alex F. Robertson). Medical College of Georgia, Department of Pediatrics, Augusta

RNs are primarily responsible for neonatal care in community hospitals and courses structured according to their deficiencies must be part of regional educational programs. Cognitive function of RNs was assessed by giving a standard test to 204 RNs from 28 community hospitals. The test was composed of 75 questions divided into 9 subtests. Performance on the test was negatively correlated with years' experience outside the nursery and time currently spent in areas other than the nursery. 84 RNs were employed in large hospitals (L), >1000 births/year, 35 in medium hospitals (M), 500-1000 births/year, and 85 in small hospitals (S), <500 births/year. Scores of $L$ on the test, $72.3 \pm$ 13.0 (mean \pm l S.D.) were significantly different from $M$ and $\bar{S}$, $60.8+15.7$ and $64.2+14.8$. L scored significantly better than $M$ and $S$ on subtests: Assessment, Sepsis, and Cardiorespiratory Distress. $L$ and $S$ exceeded $M$ on thermoregulation. Scores of 17 selected RNs (C) were used as a performance criterion. C mean, $79.4 \pm 10.2$, was not significantly different fxom L. 102 RNs (50\%) scored >1 S.D. below the C mean. C performed better than $L, M$, or $S$ on Thermoregulation and Resuscitation. RNs scoring $>1$ S.D. below $C$ mean on the subtests ranged from 40-117 (20\%-57\%), with $>50 \%$ falling below this level on Resuscitation and Thermoregulation. These data indicate characteristics of the nurses and critical deficiencies in her knowledge which must influence

\section{TTIMULANT-RELATED STATE-DEPENDENT LEARNING IN}

Marcel Kinsbourne

\section{Wendy Roberts}

\section{James Swanson}

Neuropsychology Research Department

The Hospital for Sick Children, Toronto, Ontario, Canada

Hyperactive and non-hyperactive children performed a learning task in two states -- while on stimulant medication

(methylphenidate) and while off medication -.. and were later tested for retention of the learned material either in the same learning state or in the alternate state. Symetrical state-dependent learning was demonstrated in the hyperactive group but not the non-hyperactive group; furthermore, the state-dependent effect was contingent upon the presence of drug facilitation during initial learning. This is the first report on record of state-dependent learning with a drug agent that facilitates rather than impairs performance of human subjects. 
25 BEHAVIORAL ANTECEDENTS OF COGNITIVE ABILITIES

Claire B. Kopp and Arthur H. Parmelee, Departments of Pediatrics and Psychiatry, UCLA, Los Angeles.

Investigation of relationships between infant behaviors and later cognitive skills yields knowledge about the meaning of ongoing responses, and can be particularly important in understanding the development of "at risk" groups. The present study focuses on relationships observed in the development of pre-term children. Previously, we found that 2 year olds who were successful problem-solvers had, at 8 months of age, demonstrated significantly better overall investigation of objects.

Recent addition of 2 year old subjects allowed more definitive analysis of earlier specific behaviors contributing to group differences, and of sex effects. 36 children equally divided by sex and problem solving ability comprised the sample. Male problem solvers showed they had significantly better investigating behavior at 8 mo. than did non-solvers (p.05); these differences were not observed for females. Analysis of male solvers and nonsolvers antecedent $8 \mathrm{mo}$, behaviors indicated significant differences in vigorous manipulative acts favoring the solvers (p.04) whereas the non-solvers showed significantly more random object movement (p.03). No differences were found in other visual and oral investigative object activities. Investigation of other antecedent behaviors, including performance on developmental examinations, did not yield group differences.

These data suggest that pre-term males' interactions with objects may be more predictive of later cognitive abilities than other behaviors, Evidence for this relationship has been found for full term males.

26 THP EFFECT OF MATERNAL METHADONE DOSE AND DURATION UPON NEWBORN SUCKING BEHAVIOR. Reuben E. Kron, Stuart L. Kaplan, Mitchell Litt, Marianne D. Phoenix, and Loretta P. Finnegan, (Spon.by Maria Delivoria-Papadopoulos), Phila. Gen.Hosp.,Depts.of Ped.and Psych., Univ. of Penna., Sch.of Med., Philadelphia, Pennsylvania.

The high incidence of disturbed states of CNS arousal reported among infants born to drug-dependent mothers has led us to use an objective method for measuring nutritive sucking to monitor effects upon newborn behavior resulting from maternal addiction and neonatal withdrawal. In prior studies, infants born to mothers on methadone maintenance were less effective in their sucking performance than infants born to street addicts on heroin. In this report, thirteen infants whose mothers were receiving daily methadone maintenance were studied. The length of time that the mother was in the methadone program correlated with maternal dose level $(r=0.50, p<0.05)$ indicating that those who were in the program longest tended to receive the highest doses of methadone. Measures of infant sucking varied inversely with maternal methadone dose level $(x=-0.44, p<0.05)$; these measures also varied inversely with duration of methadone intake during pregnancy ( $r=-0.70$, $p<0.005)$. These findings suggest that the length of time that a narcotic addict mother is enrolled in a methadone maintenance program, and, to a lesser degree, dosage level at delivery have a significant depressant effect upon adaptive behavior of her newborn.

27 PSYCHOSOCIAL RESEARCH IN CANCER. Shirley B. Lansky, Robert C. Trueworthy, and James T. Lowman. UniverA multidisciplinary team has investigated maladaptive pattern of behavior observed in families of children with malignancies. Accurate measurement of incidence has confirmed these observations and the use of psychological tools has allowed the further characterization and quantitation of these patterns which involve symbiotic child-parent relationships, school refusal, patient anxiety states, sibling relationships, and paxent marital relationships.

Symbiotic relationships were observed in twelve parent-child dyads. This severe regressive behavior is related to the patient's ordinal position in the family. A high incidence of school phobia was noted in this patient population, $10 \%$ vs. $1.7 \%$ in a normal group. School attendance differed (47\%vs.28\%) when compared with patients with comparable medical-care days(27vs.21). Anxiety was measured as a function of sex and age. Younger males were more anxious than younger females $(p<, 05)$, but older males were less anxious than older females ( $p<.05)$. Siblings' perception of the distress within the family was studied and compared to a normal population. The belief that there is a high divorce rate was not borne out by a statistical analysis of the marital status of 200 couples.

We have provided an effective treatment plan from the onset of the illness to counteract the psychological disabilities occurring in the families. The detailed results of these studies have applicability to all chronic disease.
28 AN EPIDEMIOLOG IC-DEVELOPMENTAL STUDY OF CHIIDREN WITII LEARNING DISORDERS. Craig B. Liden, Melvin D. Levine, Irving Hurwitz (Spon. by T. Berry Brazelton). Harvara Medical School, The Children's Hospital Medical Center, Department of Medicine, Boston.

Ninety-seven school age children referred with learning disorders were studied in a multidisciplinary program in order to demonstrate associations between their primary educational handicaps and a number of historical elements, developmental factors, and behavioral manifestations. A uniform data collection system was applied. This comprised: parent and school questionnaires; neurodevelopmental examination; osychoeducational testing; and psychiatric assessment. A taxonomy of primary handicaps was developed and included: visual spacial problem (13\%); temporal-sequential disorientation (9\%); receptive language deficit (7\%); communication or output handicap (78); attentional disorder (46\%); and emotional learning inhibition $(168)$. Significant variations between the primary handicap groups were seen with respect to sex, age at presentation, age at initial manifestation of dysfunction, family history, behavioral patterns, findings on a neurodevelonmental examination, and associated handicans. It was found that 628 of children with a strong history of perinatal stress fell into the attentional disorder category. This group also vielded the strongest association with infant temperamental dysfunction.

It is suggested that this investigation may provide a conceptual framework for the study of syndromes of learning dysfunction in a pediatric-educational diagnostic setting.

\footnotetext{
29 PREDICTIVE ASSESSMEN OF PRE-TERM INFANI RISK FACsity of California, Department of Pediatrics, Los
}

Angeles.

Developing a perinatal events scoring system has been difficult due to the number of possible occurrences and combinations. Efforts to date have been successful only in providing short term predictability of neonatol morbidity and mortality. We have devised a method of scoring obstetric, neonstal and infont biologic events using the optimal scoring technique of Prechtl. All forms were standardized on a pilot full term population; mean scores $(\bar{x})$ were fixed at 100 with a stondard devistion ( $s$. d.) of 20 .

113 preterm infants with a mean birth weight of 1903 gms. and gestational age of 33 weeks were assessed on these forms. All had Gesell tests at 9 months (Gesell 9) corrected age. Mean scores on the obstetric complications scele (OCS) were 80.4 , s.d. 18.5; on the postnatal factors scale (PNF) $\bar{x}$ was 94, s.d. 31.6; on the 4 month pediatric complication scale (PdCS) $\bar{x}$ was 107 , s.d. 20.4 ; on the 9 month PdCS, $\bar{x}$ was 114.7 , s.d. 22.5; Gesell 9 development quotient $\bar{x}$ was 100.1 , s.d. 9.0 .

Significent correlstions were: OCS-PNF, 0.31; PNF-PaCS 4 $0.24 ;$ PNF-PdCS 9, 0.25; PảCS 4 - PdCS 9, 0.3; PdCS 4 - Gesell 9, 0.25 ; PdCS 9 - Gesell 9, 0.31. By combining scores of medicsl events, the correlations to Gesell 9 were not improved.

OCS and PNF did predict pediatric events at 4 and 9 months. The PdCS 9 predicted Gesell 9. By cumulating scoring systems grester Gesell score predictability is not achieved.

CONSEQUENCES OF ELEVATED BLOOD LEAD LEVELS IN IN-

30 FANCY: A TWELVE-YEAR CONTROLLED FOLLOWUP STUDY. A. Harold Lubin, Malcolm Helper (Spon. by Bruce D. Graham) Ohio State Univ. College of Medicine, Children's Hospital, Department of Pediatrics, Columbus, Ohio.

Twenty-four children identified in 1961 with blood lead levels now appreciated to be moderately elevated, $\geqslant 40-60 \mathrm{ug} \%$, have been evaluated and compared with matched controls--children identified at the same time in the same area as having acceptable dicate that individuals in the experimental group still have a higher blood lead level $(\bar{X}=26 \pm 6$ ug\%) than those in the control group $(\bar{x}=16.5 \pm 5 \mathrm{ug} \%)$. Trends also reveal higher blood urea nitrogen levels, lower creatinines, and decreased amino levulinic acid dehydratase activity in the experimental group of subjects. Psychological scores on all children evaluated tend to be lower than those observed in individuals of a similar age and sex in the general population. Verbal I.Q. scores for black male high lead subjects are lower than those of their low-lead counterparts. The differences in the group of black females evaluated are less definitive. Speech, hearing, audiological, opthalmological, and neurological examinations on all of the children have failed to reveal any differences referable to prior elevated blood lead levels. In the absence of earlier clinical evidence of toxicity, a slight but sustained, increased, body burden of lead may be harmful to children in their future development, jut does not affect all individuals to a conclusive-
ly similar degree. Supported by State of Ohio Division of Mental Health. 
31 AN ATTEMPT bY A UNIVERSITY DEPARTMENT OF PEDIATRICS TO INFLUENCE THE IMPLEMENTATTON OF MEDTCAL ADVANCES IN ONE COMMUNITY. Thomas K. McInerny, Betty B. Satterwhite, Sydney A. Sutherland and Ivan B. Pless. Univ. of Rochester Sch. of Med. \& Dent., Dept, of Ped., Rochester, N.X. (Spon. by Gilbert Forbes)

The purpose of this study was to ascertain what influence a university medical center could have on private practitioners' implementation of medical advances. 71 practicing pediatricians and 12 family practitioners with clinical pediatric appointments at the UR Medical Center were surveyed regarding adoption of recommendations in two recently published articles, both before and after intervention by the Pediatric Dept. Intervention was of two kinds: 1) a letter from dept. chairman urging consideration of the findings of the enclosed reprints, and 2) a visit from a non-physician member of the dept. who brought and explained the findings of the reprints. Both groups were compared with a control group which received no intervention. Results were: 1) type of article and extent of exposure had a marked effect on reported adoption prior to intervention; 2) those physicians who were more highly integrated in the medical community were more apt to be prior adopters than those who were less integrated; and 3) the "visit" method yielded a higher subsequent adoption rate of the findings of both articles than did the "letter" method. The findings indicate that a university medical center can be effective in influencing practicing physicians to implement medical advances, especially if the physician is contacted personally.

\section{DOES TLAN ORGANIZATION ANI OUTREACI EFFECT OLTCOME?}

O. Mathieu, F. Charney, B. Pless, B. Satterwhite. Iniv. of Rochester, Rochester, $X, \bar{Y}$.

This study explores the extent to wilich teamwork and outreach influence outcomes of care. Three urban-based health centers were compared. They ranged from large, multidisciplinary colleague-oriented teams with maximal outreach, to sma11, phys ician-centered teams with minimal outreach. Tracer conditions were used as indicators of health care: (1) Immunization completion for infants of teen-aged mothers, (2) the successful resolution of anemia in $0-20$ montl old infants, and (3) the morbiality of chiluren with bronchial astima. Our hypothesis was that better outcomes would be seen in the center with the most outreach, but the hypotheses did not test out. There was a $40 \%$ immunization rate at the high outreach center, and a $47^{\circ}$. rate at the lowest, and $71 \%$ and $70 \%$, respectively, successful letection and resolution of anemia. If the asthma section sinowed any differences, they appeared to reflect the levels of in?ividual physician involvement with his patients rather than any methodology of care built in to the centers. The inconclusiveness of the results may reflect subtle patient differences in the populations that could not be measured, despite efforts to match the patient groups. This method of inquiry is a reasonable and useful instrument for cvaluating the quality of health care.

THE DEVELOPMENT OF THE CONCEPT OF PERSONAL ILLNESS Irwin Mohr and Carolyn R. Denning. College of Physicians and Surgeons of Columbia University, Department of Pediatrics, New York.

A chronic illness like Cystic Fibrosis (CF) lends itself to tha study of the ways this group of patients perceive illness. Interviews with $300 \mathrm{CF}$ patients, parents and siblings indicate that the development of the concept of personal illness evolves in 4 stages. Each stage is signified by aberrant behavior, a crisis and eventual resolution. The first stage occurs at about 5 years of age when the child grasps the duration of his disease and realIzes that it will not go away. Questions such as "When will I get we11?" increase markedly at this time. The crisis occurs with the parents as they agonize over appropriate responses to the child as well as how to cope with their own feelings of helplessness. The second stage occurs at about 8 years when the child realizes that he is different from his peers. The crisis involves the fear of being outcast and expresses itself in rejecting medical treatments and in underachievement in school. The third stage is reached at about 14 years when the adolescent contemplates his future and realizes the limitations he may face. The crists is the patient's realization that he is a sick person, and this leads to rejection of treatments and dental of illness. The fourth stage occurs at age 18 - the age of independence and adulthood. The crisis concerns his competence as an adult with an illness and how this will affect his future existence. The resolution of this crisis will have a major impact on the patient's future life-style. An awareness of the ways which these patients perceive chronic illness should be of help in thetr management.
STRESS FACTORS AND NURSING ATTITUDES IN NEONATAL INTENSIVE CARE. Robin Moore, Donald A. Kennedy an M. Jeffrey Maisels (Spon. by Nicholas M. Nelson). Penn State Univ. Co11. Med., M. S. Hershey Med. Ctr., Dept. of Ped. and Family and Community Med., Hershey, $\mathrm{Pa}$.

Thirty-one. nurses were interviewed to identify possible tressful situations during the performance of their duties in the Neonatal Intensive Care Unit. Eighty percent felt that their position placed them under tension. Situations which appeared to be particularly stressful (based on degree and frequency) were (1) Inadequate nurse staffing and accepting further admissions when the census was already high, (2) Inadequate communication between physicians and nurses and the feeling that some physicians did not trust the nurses' judgment, (3) Prolonged care of a severely ill infant with a poor prognosis. This was aggravated if the physicians were indecisive regarding their approach to therapy of such infants. (4) Being the charge nurse for the shift, (5) Difficulties dealing with other hospital departments (central supplies, pharmacy, lab, etc.), (6) Performing tasks that were not considered to be the responsibility of a nurse. The most rewarding aspects of the work were: (1) Seeing critically ill infants improve, (2) A feeling of the importance of the nurse's role in improved survival and subsequent development, (3) Work in emergency situations (also considered stressful), (4) Relationships with the families of infants and with coworkers, (5) Performance of technical skills. Working as a neonatal intensive care nurse is both stressful and rewarding. These observations suggest means of relieving some of the stress.

35 THE IMPACT OF NEONATAL INTENSIVE CARE ON THE FAMILY UNIT. Douglas Morgart, Anthony Cole, Zvi Friedman and M. Jeffrey Maisels (Spon. by Nicholas M. Nelson). Penn State Univ. Coll. Med., M. S. Hershey Med. Ctr., Dept. of Ped. and Family and Community Med., Hershey, $\mathrm{Pa}$.

Ten sets of parents whose infants were receiving care in the Neonatal Intensive Care Unit (NICU) and 19 whose infants had been discharged 4 weeks -6 months previously were questioned regarding their experience. $97 \%$ felt the experience was moderately or severely stressful. "High risk" factors which appeared to be related to an increase in stress were: (1) Baby born at referring hospital, (2) Pessimistic outlook by referring physician, (3) Parents <20 years old, (4) Parents living $>50$ miles from NICU, (5) First visit to unit, (6) Financial burden, (7) Discharge of the infant. Factors which appear to alleviate the stress were: (1) Level of care and expertise by the staff and facilities of the NICU, (2) Good communication with and reassurance by medical and nursing staff, (3) Handling infant by parents, (4) Feeling that nurses provided emotional care (mothering) as well as physical care to babies, (5) Rooming in with infant prior to discharge, (6) Verbalizing concerns to spouse, (7) Sibling visits to infant. Following discharge, $80 \%$ of parents said the subsequent relationship with their infan was unaffected although $60 \%$ felt they were "overprotective". Having the baby in the NICU had no effect on intra-family relationships in $55 \%$ and $42 \%$ felt the experience had brought the family closer together. Attention to the factors identified should help to alleviate some of the stresses which these parents experience. 


\section{PATHOGENETIC CONSIDERATIONS OF PICA IN Siegfried M. Pueschel, Susar \\ Rosanne B. Howard, and Marie M. Cullinane (Spon.} Leo Stern)

Brown University, Rhode Island Hospital, Department of Pediatrics, Providence, and Harvard Medical School, Children's Hospital Medical Center, Department of Medicine, Boston.

In search for the underlying mechanisms involved in the pathogenesis of pica in lead poisoning, information was obtained relative to the children's habits of eating nonfood substances, the parents' orality, and a variety of environmental, cultural, and social circumstances, including parent-child relationship, quality of mothering, and parental supervision in the home. Ninetytwo children with documented increased lead burden were included in the study group, and a control group of children living in the same area without lead poisoning were matched according to age, sex, and racial origin.

Inappropriate behavior management, undesirable feeding practices, failure of normal mother-child interaction, paternal deprivation, culturally dependent maternal oral interests, and significant stress factors in the home where abundant lead containing material is available were found significantly more of ten in the study group compared with the control group ( $<<0.01$ to $\mathrm{p}<0.001)$. These factors appear to be etiologically related to the development of pica in lead poisoning. Other pica associated factors observed were nutritional deficiencies and maladaptive behavior patterns in children from the study group.

\section{MOTHER'S SPEECH TO HER TWO-YEAR OLD, ITS EFFECT ON SPEECH AND LANGUAGE COMPREHENS:ON AT 5 YEARS. NOrma Ringler, Mary Anne Trause, Marshall Klaus, Case Western} Reserve University, Dept.of Ped.\& Speech Communication, Cleveland. As part of a continuing study designed to determine the eff
of early mother-infant contact on later maternal and child behavior, we studied the speech of ten mothers while addressing their 2-year olds and then assessed their children at 5 years using four standard tests. The 5 year olds' vocabulary comprehension $(p<.01)$ and ability to understand complex phrases with multiple critical elements $(p<.01)$ are significantly related to the number of words per proposition used by their mothers when children were 2. Mothers who used more adjectives have children who comprehend more complex syntactic structure $(0<.01)$. Mothers who used more elaborate end illustrative speech have chilidren with greater expressive ability $(p<.01)$. Mothers who asked more questions of their 2 year olds have children with somewhat higher $10 ' s$, comprehension, and expressive ability $(p<.10)$.

From previous studies, when their children were 2 years of age, mothers who had extended contact with their infant at birth used more adjectives, questions, words per proposition, and fewer content words and imperatives than mothers who did not. Significantly, the five year olds of the early contact mothers comprehended more complex phrases with 4 critical elements $(p<.005)$ indicating greater maturity in syntactic development than children of control mothers. Thus, the early linguistic environment of the young child, which may be altered by hospital practices at birth, appears to strongly affect the child's speech and language comprehension at 5 years of age.

\section{TEENAGE PREGNANCY - THE IMPACT OF A COMPREHENSIVE} PRENATAL CARE PROGRAM ON OUTCOME, LOIS M. Roeder, Isadore G. Ances and Felix P. Heald, Univ, of Maryland Sch. Med., University Hospital, Dept. of Ped., Baltimore. Pregnant adolescents at high risk for delivery of premature and low birth weight infants were provided comprehensive prenatal care. In 28 black 15-17 yr old primiparous teenagers, indices of nutritional status were obtained during pregnancy, and included levels of hemoglobin, albumin and transferrin, urinary urea, morphology of scalp hair and enzymatic activities of leukocytes. Fetal growth and development were evaluated at delivery. Outcome of pregnancy was markedly improved in adolescents enrolled in the program compared to a control non-program group matched for age, race and parity. Average birth weight was significantly higher ( $3213 \pm 90 \mathrm{vs} 2910 \pm 99 \mathrm{~g}, \mathrm{Mn} \pm \mathrm{SEM}$ ) for program infants. The distribution of infants according to gestational age was also altered, with fewer premature babies in the study group than controls. The difference in mean age at birth ( $40.5 \pm 0.3$ vs $38.2 \pm 0.5$ wks) was significant. Most indices of nutritional status in the study group of mothers were within normal ranges and unrelated to fetal growth. However, the activity of adenylate kinase in leukocytes at the 25 th week of pregnancy was positively and significantly related to birth weight of the infant and thus may be useful in predicting fetal growth. Multidisciplinary efforts to deliver comprehensive prenatal care to adolescent mothers has, therefore, significant benefits for the outcome of pregnancy. (Supp. in part by Grants - Mat.\&Ch1d.H1th. Svcs, \#MC-R-240200-01-0 and Nat, Fdn. 非-29.)

40 EFFECT OF THE ICU ENVIRONMENT ON HEARING. Carol Schulman-Galambos, Dept. of Neurosciences, Univ. of We have investigated auditory function in over 300 high-risk ICU survivors. 250 of these are being followed developmentally and any infant with delayed language is evaluated using the method described below (normal vocalizations on test and retest are presumed to indicate normal hearing). A series of 70 additional infants has been evaluated using Brain Stem Response Audiometry. This response, recorded from surface electrodes at the vertex and mastoid, is composed of a series of waves caused by the successive synchronous firing of the neurons of the brain stem auditory nuclei from the VIII nerve through the inferior colliculus. The incidence of auditory impairment in this group is 1:150 for significant loss having the $\mathrm{ICU}$ environment as the probable cause: 2 infants who were kernicteric on admission were profoundly hearingimpaired, and 1 infant of a congenitally deaf mother was found to have bilateral sensorineural loss. Only 2 infants had severe hearing loss unattributable to genetic or congenital causes. While both infants were small $(1000 \mathrm{~g}$.) and were in the ICU for long periods ( 80 and 84 days), they were neither the smallest nor the sickest in the sample. The incidence of risk of significant hearing impairment caused by the potentially ototoxic neonatal ICU environment, while statistically high, and therefore suggesting the need for careful followup, does not seem to be as serious as might be predicted.

4. DEVELOPMENTAL FOLLOWUP OF NEONATAL ICU SURVIVORS. Carol Schulman-Galambos, Morton Cohen, and Kristin Gist, Dept. of Neurosciences, Univ. of

We have followed all high-risk survivors discharged from our regional Neonatal ICU since July, 1972. Infants with diagnoses of asphyxia neonatorum, prematurity, RDS, or any combination of these were included ( $80 \%$ of the sample were retained for followup). The Bayley Scales of Infant Development were administered at 6 mos., 12 mos., and 2 years. Mean scores, median scores, and the distribution of scores approximate the normal curve for all groups including: 1) 1000g. ( $n=16) ; 2)$ 1500g. ( $n=53)$; 3) 1500-2000g. $(n=54) ; 4)$ asphyxia neonatorum $(n=82) ; 5)$ mild to moderate RDS $(n=36)$ and 6) severe RDS $(n=60)$. However, $2 \%$ (of Group 5$)$ to $10 \%$ (of Group 6) of the infants are impaired on the Mental Scale ( $2 \mathrm{SD}$ below the mean) and $8 \%$ (of Group 4) to $11 \%$ (of Group 6) are impaired on the Motor Scale. In addition, 7 infants have functional blindness at present from RLF and 2 additional infants are profoundly deaf. These infants seem to be developing normally otherwise. It. seems clear from this study that present methods for the care of the sick newborn have substantially improved the outcome for these infants. Whether the deficits present in the small but statistically significant group of profoundly impaired in each diagnostic category can be accounted for by treatment methods is now being investigated by discriminant analysis.

Sponsored by William Friedman 42 ADOLESCENT MEDICINE: SURVEY OF AGE LIMTITS CARE REMedicine Service, Tripler Army Medical Center, Honolulu, Hawaii and Richard G. McKenzie, M.D., Director, Division of Adolescent Medicine, Children's Hospital of Los Angeles, Calif. The American Academy of Pediatrics of ficially states that the responsibility of pediatrics may usually terminate by 21 years of age (Pediatrics: Vol. 49; No. 3; March 1972; p. 463). In this the majority of pediatric chairmen $(69 \%)$ agree with this policy. Expectedly, the majority of internal medical chairmen ( $89 \%)$ and chief residents $(90 \%)$ disagree with this AAP policy; but so do the majority of pediatric chief residents $(57 \%)$ who place the limit at 16-18. Responding pediatric chairmen (84), pediatric chief residents (70), internal medicine chief residents (59), internal medicine chairmen (55) and senior medical students (41), in general, reveal that outpatient departments and wards are more available to adolescents in internal medicine departments than in pediatrics, however, only a small percentage of internists $<20 \%$ feel they should be the primary physicians of adolescent patients. cine should be a subspecialty of pediatrics. Also, the majority believe pediatricians should be the primary physicians of adolescents. This discrepancy between the disposition of pediatricians to care for adolescents and present availability of facilities probably reflects the influence of AAP policy versus actual institutional experience, where the majority of pediatric departments tutional experience, where the majority of pediatric departments
presently limit the upper age limit to the early and mid teens.
It does suggest however, that pediatricians are oriented to assume the responsibility for care of older adolescents, and the majority of pediatric services need to modify their present age limit 
43 ECONOMICS OF CREATING A LEVEL III PERINATAL CENTER. C.Sia, A.Parekh, M.Sokal, S.Sokal, \& R.G.Harper, Depts.of Ped. \& Ob.-Gyn., North Shore Univ.Hosp.,Manhasset, NY, \& Depts.of Ped.\& Ob. Gyn.,Cornell Univ.Med.Col.,NY,NY. (Spon.by F.Lifshitz) While many hospitals may be attracted to the idea of upgrading a Level II neonatal unit to a Level III perinatal center, the financial outlay incurred in such a step may be prohibitive. A retrospective study was undertaken to evaluate the experience of the North Shore University Hospital in establishing a perinatal center whose services include a neonatal intensive care unit with an average daily census of 20 newborns, stetric service seeing an average of patients per week, and a mobile neonatal intensive care ambulance transferring approximately 100 newborns annually. The total direct expenditure in the initial 2-year period exceeded one million dollars. Of this sum, 20.08 was for fixed capital outlay and $80.0 \%$ for ongoing expenses; of the ongoing expenses $80.6 \%$ was for new personnel. Excluded were indirect costs and personnel costs from ancillary services. Finances were provided almost entirely from the hospital's operating budget.

Since economic considerations may mitigate against small hospitals undertaking an upgrading of their own perinatal services, regionalization of perinatal health care appears to offer a cost-effective alternative.

44. PARENTING BY NON-PARENTS. M.Sokal, C.Sia, S.Sokal, \& R.G.Harper, Depts.of Ped.\& Ob.Ped.\& Ob.-Gyn.,Cornell Univ.Med.Coll., NY, NY. Depts. of Ped.\& Ob.-Gyn.
(Spon. by F.Lifshitz)

To compare the ways in which non-parents and parents relate to infants in a newborn intensive care unit, 4 volunteer male college students and 2 sets of parents were filmed during their initial contacts with sick premature newborns. Observations were made of 15 specific attachment behaviors including fingertip and/or palm contact, stroking, cuddling, and en-face viewing. No significant differences were noted between parents and non-parents in their physical contact with these infants during the initial visit. During subsequent visits of non-parents to the same infant, an orderly progression of attachment behavior was noted: beginning with tentative fingertip contact on the extremities, the non-parents progressed to palm contact on the head, stroking, cuddling, encompassing, soothing, and playing behaviors. The non-parents assumed en-face positions early and frequently expressed interest and concern about the newborn's weight gain and progress, and were anxious to rock and feed the newborn as soon as possible. The progression of behaviors among nonparents parallelled closely that observed among parbehaviors previously analyzed as indicative of parenspecific behavior pattern with respect to newborns.

45 FACTORS DETERMINING PEDIATRICIAN'S ATTITUDES TO SURGERY IN DOWN'S SYNDROME AND MENLNGOMYELOCELE. I. David Todres, Diane Krane, Mary C. Howeil, Daniel C. Shannon. Harvard Medical Schoo1,

We elicited the attitudes of 406 Mass. pediatricians (Ped) toward ethical dilemmas in newborn care. Completed questionnaires were returned by $230 / 406$ (57\%). Dilemma \#1: Down's syndrome and duodenal atresia, parents refuse surgery. $46.3 \%$ (106) Ped advocated surgery, $50.7 \%$ (117) did not, $3 \%$ (7) gave no answer (NA). of those advocating surgery, 41/62 were Catholic, 25/50 Protestant $(p<.01), 22 / 57$ Jewish $(p<.01), 4 / 22$ Other, $14 / 39$ NA. $19.2 \%$ who advocated surgery would pursue a court order, $31.9 \%$ would not and $48.9 \% \mathrm{NA}$. Of those not advocating surgery, 66 recomnended custodial care, 6 intensive care, 2 hasten death, 43 NA. Dilemma "2: Large thoraco-lumbar meningomyelocele, microcephaly, paraplegia and incontinence. Of treatment options given, $33.3 \%$ (77/ 230) recommended surgery which related to: (a) religious activity$32 / 76$ active, $16 / 62$ fairly active $(p<.01), 17 / 62$ inactive $(p<.01)$ $12 / 30 \mathrm{NA}$; (b) age- $57 / 138$ were $<50 \mathrm{yr} ., 20 / 92>50 \mathrm{yr}$. $(\mathrm{p}<0.04)$; (c) $47 / 163$ were generalists, $30 / 67$ specialists ( $p<0.008$ ). $66.7 \%$ (152) recommended no surgery, $54 \%$ choosing custodial care, $11.7 \%$ skilled nursing. Of these 152 Ped, 117 would comply if the parents requested surgery. Of all red, $25.5 \%$ felt there was a moral difference between witholding surgery for Down's vs. meningomyelocele. The physician's religious persuasion and activity, age and degree of specialization were significant determining factors in advocating or witholding surgery.
46 THE IMPACT OF A CHILD'S CHRONIC ILLNESS ON HIS FAMILY WITH PARTICULAR REFERENCE TO SIBIINGS. John C. Vance, Betty B. Satterwhite, Louis E. Fazen, Michael F

Bryson, Ivan B, Pless. Depts, of Ped., Univ, of Rochester Sch, of Med., Rochester N.Y, and Johns Hopkins Med.Ctr., Baltimore, Md. Thirty-eight families of children with chronic nephrotic syndrome and 37 demographically similar families were studied to determine the impact of the nephrotic child on the family, 185 children - 95 siblings of ill children and 90 controls - were examined using parental questionnaires, behavior rating scales, psychological tests and teacher rating scales.

General questions on factors influencing family function surprisingly showed that control families were more affected by overtiredness, change in housing, and parental friction. The same questions, repeated to the nephrotic group alone referring specifically to the sick child, showed a marked increase in reporting of financial problems, parental tiredness, extra work and changes in household sleeping arrangements.

Ouestions about sibling interaction showed more fighting and anger in control families. Psychological testing showed social confidence to be lower in the nephrotic group and school affiliation higher. Results show that: 1) parents tend to deny family problems due to childhood illness unless specifically asked; 2) siblings of children with nephrotic syndrome are less aggressive; 3 ) decreased social confidence and a more positive view of school may be consequences of a chronically ill child in the home, This family and sibling profile may help in the management of families who have chronically ill children.

INFANT AND PRESCHOOL DEVELOPMENTAL TESTING AND LATER SCHOOL PERFORMANCE. William J. van Doorninck, Nathan P. Dick, William K. Frankenburg, Theresa N. Liddell, and John M. Lampe. University of Colorado School of Medicine, Department of Pediatrics, Denver.

The significance of early developmental assessments for later school problems has been challenged. Data presented in this study detail the accuracy and the errors in predicting school performance from results of the Denver Developmental Screening Test (DDST). In 1969-70, 2,715 children were screened with the DDST in the Denver Neighborhood Health Centers. Five to six years later, a random sample of 113 were followed-up. $\underline{S}$ s were representative of three age groups at screening (3-24, 24-48, and 48-72 months) and three DDST test results: Abnormal, Normal and Questionable. $96 \%$ of Ss with DDST Abnormal scores, $67 \%$ of Questionable scores, and $3 \overline{6} \%$ of Normal scores had substantial achievement and/or behavioral problems at school age. With prediction span held constant, accuracy of prediction improved when $\underline{S}$ s were screened at older ages. Accuracy was higher when Questínables and Abnormals were both treated as positives, regardless of age group of screening. Abnormals and Questionables who later had no apparent school problems had significantly less positive medical histories and neurological exams at follow-up. Normals who later developed school problems had significantly higher positive medical histories and neurologicals and significantly lower academic press from parents. Among these assessments, developmental testing provided the most accurate prediction in this sample.

48 SOME RELIABILITY DATA ON THE BRAZELTON NEONATAL BEHAVIORAL ASSESSMENT SCALE FROM SEVEN TO TEN DAYS OF LIFE. Brian E. Vaughn, Bonnie Taraldson, Amos Deinard and Byron Egeland, Inst. of Child Development and Dept. of Ped., Univ. of Minnesota, Minneapolis.

The Neonatal Behavioral Assessment Scale (Brazelton) was developed as an instrument which would be sensitive to subtle behavioral responses of the neonate. To date, a wide variety of studies have utilized this scale. As the scale's use expands, however, there will be an increasing need to have data available on the test-retest reliability on the various scoring techniques as well as on the individual items, themselves. The data reported here were obtained from 40 infants who were participating in a larger study of early infant development. They were all first born, ful1term infants whose mothers had participated in an extensive prenatal program. The Brazelton was administered to each infant twice during the first 10 days of life. On both occasions the exam was done in the infant's home. Test-retest reliabilities were assessed in three ways. Each individual item was studied across the whole sample, each infant was given a reliability score based on the two exams and a "clustering" profile, developed by the Brazelton group, was computed for both exams. Certain of the items were found to have significant variation, though the overall reliability of the exam for individual infants was quite satisfactory. The least day-to-day variation was found in the "cluster" profiles. Our results suggest factors which influence the variability of the exam from day-to-day; in addition, they provide confirming evidence concerning the sensitivity of the exam in reliably describing the behavior of the neonate. 
49

EVALUATION OF INFORMATION TRANSFER IN SICKLE CELI TRAIT (SCT) COUNSELING. Charles F. Whitten, Wayne State Univ. Sch. of Med, Dept. of Ped., Detroit. Trained laymen counseled using a 10 step protocol with 26 graphics. The counselors' adherence to the protocol for material related to 10 post test questions, and the counselees' narrative answers were rated. Sessions and pre and post tests were audiotaped. 236 tapes from 691 taped sessions held in a 6 mo. period were transcribed and analyzed. With two exceptions the frequency of satisfactory responses on each post test question and the counselees' age, sex, marital status, family size or education, were not significantly related $(p>.05)$. Adeq. responses were more frequently given by females on factors SCT couples might use in family planning decisions $(\mathrm{p}<.05)$ and by persons below 40 on the health status in SCT $(\mathrm{p}<.01)$. On 5 questions the frequency of adeq. counselor performance (CP) and counselee responses (CR) was high--94-99\% and $73-94 \%$ respectively. For 2 questions the $\mathrm{CP}$ was high (91\% and $92 \%$ ) but the $\mathrm{CR}$ was moderate $(48-54 \%)$ suggesting a protocol or evaluation related problem. For 2 questions $\mathrm{CP}$ and $\mathrm{CR}$ were $60-81 \%$ and $23-45 \%$ respectively. Part of the low $C R$ could be attributed to the $C P$, i.e. significantly more adeq. CR were associated with adeq. CP $(\mathrm{P}<.01)$. For one question with low $\mathrm{CR}(50 \%)$ and $\mathrm{CP}(58 \%)$ the $\mathrm{CP}$ was not a factor $(p>.05)$. The data suggest: 1 . laymen can transmit sickle information effectively; 2 . taping is acceptable (few refusals); 3. analysis of taped sessions is desirable in all genetic counseling to determine whether unsatisfactory counselee comprehension is related to the protocol, counselor, counselee or the evaluation.

\section{A CONTROLLED STUDY OF PRESCHOOL CHIIDREN OF HEROIN} ADDICTS - THEIR GROWTH, DEVELOPMENT AND BEHAVIOR. Geraldine S. Wilson and Richard McCreary (Intr. by Murdina M. Desmond). Baylor Col. of Med., Dept. of Ped. \& InterAgency Program for Multiple-Handicapped Children. Houston, Texas A previous study has shown disturbed somatic growth and behavior (hyperactivity and poor attention span) in $50 \%$ of infants 12 to 24 months of age whose mothers used heroin chronically during pregnancy. In anticipation of possible special educational needs 22 preschool children of addicts were evaluated at 3 year 1 month to 6 year 4 month. Evaluation included psychological instruments (ITPA, Columbia Mental Maturity Scale, \& McCarthy Scale), behavioral assessment by parent interview, direct observation measures, and biometric measures along with physical and neurologic examination. Control children comprised those who were not exposed to narcotics during pregnancy, but were reared in the drug culture, as well as a group of children of similar socioeconomic and high risk background. The somatic growth of the heroin-exposed group was found to be significantly less than that of control children. This group also showed poorer adjustment as measured by the Child Behavior Rating Scales. Psycholinguistic quotients of subjects and controls were below national test norms but did not differ between groups. Heroin-exposed children and controls differed significantly in performance on several subtests of the ITPA, and on the perceptual battery and other instruments. Tests on which heroin children performed more poorly than controls involved perceptual skills or short term memory, and may reflect an underlying difference in concentration or attention. Behavior differences reflect aggressiveness and poor impulse control.

51 ANALYSis OF NEONATAL HOSPital CHARGES. David D. Wirtschafter, Stephen R. Puckett and George Cassady. Decine, University of Alabama, Birmingham, Alabama.

During calendar 1974, there were 532 outborn admissions (304 $\leq 2500 \mathrm{gm})$ to our NICU。 Hospital charge data on 513 non-surgical admissions were collated by date of service. Grand total charges (GTC) were computed. The relationships between mortality and 1) GTC and 2) Length of stay (LOS) were (point biserial correlation) 1) GTC $r=-.0143, t=3.3, p<.01$ and 2) LOS $r=-.34, t=8.2$, $\mathrm{p}<{ }_{0} 01$. The hypothesis that GTC or LOS was directly related to mortality was rejected. Average daily charges (ADC), LOS, and survival status were plotted. The null hypothesis: Expired and lived infants are described by the identical form: $\mathrm{Y}=\mathrm{a}+\mathrm{b} \log$ $(A D C)+c(A D C)$ was tested by analysis of variance.

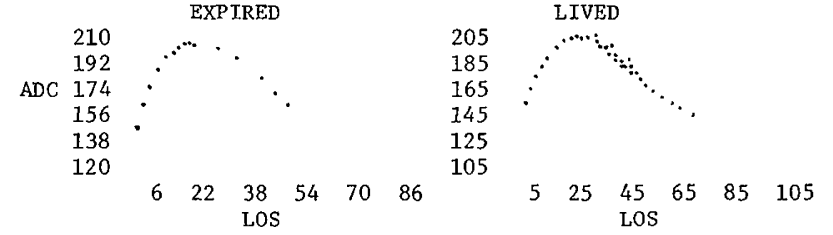

The hypothesis was rejected $(\mathrm{F}=56.97, \mathrm{df}=3,507, \mathrm{p}<.001)$. Use of both the ADC and LOS parameters results in a better fiscal index of neonatal mortality and may prove a more satisfactory fiscal index of neonatal morbidity.
52 COST-BENEFIT ANALYSIS OF AN X-LINKED CARDIOMUSCULAR vision of Medical Genetics, Department of Pediatrics, Emoxy University School of Medicine, Atlanta, Georgia.

A North Georgia kindred consisting of 212 members in 6 generations expresses cardiac and humeroperoneal muscular dystrophy with an $\mathrm{x}$-linked recessive pattern of inheritance (New Eng. J. Med. $293: 1017,1975)$. Private and societal costs and cost of prevention through genetic intexvention are calculated for the currently evolving generation (gen) VI. In hemizygous affected males, $I^{\circ}$ heart block appears in puberty and progresses to atrial paralysis requiring pacemakers. Slow twitch, Type I fibers of humeral and peroneal muscles degenerate. Disability is total by age 30, average retirement is at age 65 ; therefore, 35 wage earning years are lost. From clinical expression in gen IV and V, from pedigree analysis, and from an observed fecundity rate of $2.5,10$ affected males and 26 heterozygous females are anticipated for gen VI. Societal costs will be $\$ 1,765,050$ and private costs will be $\$ 3,829,200$, a combined cost of $\$ 5,594,250$ for these 10 affected males over 35 years. This cost is discounted to present value using the formula $\pi=C_{t} /(1+r)$ there $\pi$ is the net discounted cost in dollars today, $C_{t}$ the cost over time $(t)$ which is 35 years and $r$ is the net discount rate $(58)$. The discounted cost becomes $\$ 1,283,703$ in today's dollars. The cost of prevention through prenatal monitoring of all high risk pregnancies in gen VI is $\$ 21,750$. The cost-benefit ratio is, thus $5: 1$. We conclude that this unusual inherited disease constitutes a large public and private expense, can be prevented by expending $2^{\text {? }}$ of this expense, and emphasizes the need for public health programs in genetics.

\section{FATHER INFANT INTERACr'ION. Michael W. Yogman, Huzanne Dixon, Edward 'Ironick, Lauren Adamson,} sch. Dept. of Ped., Children's Hospital Medical Center, Boston. This study describes the interaction of infants with fathers and compares it to their interaction with mothers and strangers. Five, first-borm infants, their mothers and fathers were recruited in the newborm period and face to face interactions between infants and adults were videotaped biweekly for 6 months. Each session consisted of one 2 minute long interaction with each parent and an unfamiliar adult. By 6-8 weeks of age, infants acted differently with mothers, fathers and strangers. We documented these clinically apparent differences by analyzing the videotape second-by-second, and describing infant and adult gaze pattems, limb movements, facial expressions, vocalizations, body position and touching patterns. Infants frowned and cried with strangers 4 times as much as they did with either fathers or mothers (AINOVA, p<U.U1). They smiled more with mothers than with fathers and more with fathers than with strangers (ANOVA, p 6.01 ). Parents differed in the games they played with infants: mothers engaged in verbal play while fathers played rhythmic tapping games. These data suggest that infants have the capacity to differentiate fathers from mothers and strangers in the second month. By pointing out to fathers how inf ants can recognize them, pediatricians can foster early attachment between fathers and infants. By observing different patterns of infant facial expression and vocalization with different adults, and by noting games parents play with young infants, pediatricians can better assess early parent-inf ant interaction.

\section{CARDIOLOGY}

54 RESPONSES OF CARDIAC RATE AND RHYTHM TO ISOLATED CEREBRAL HYPOXIA IN NEWBORN PUPPIES. Robert A. Achtel, (Spon. by Nancy Holland). University of Kentucky College of Medicine, Department of Pediatrics, Lexington. The role of the central nervous system (CNS) in reflex chronotropic responses (CR) to hypoxia in newborns is unclear and was the subject of this investigation. Ten newborn mongrel puppies ranging in age from 6-12 hours of age were subjected to transient cerebral hypoxia by perfusing the cephalic portion of the animals with hypoxic blood. Cephalic pressure was maintained at systemic levels and flow monitored. Systemic and cephalic blood gases and $\mathrm{pH}$ were closely monitored. With combined systemic and cerebral hypoxia all animals showed a biphasic $\mathrm{CR}$, at first positive, $\mathrm{p}=<0.01$ (mean $\mathrm{pO}_{2}>45 \mathrm{mmHg}$ ) and then a negative $\mathrm{CR}$ as hypoxemia progressed. (mean $\mathrm{pO}_{2}<25 \mathrm{mmHg}$ ) Cerebral hypoxia alone witnessed either/or sinus or nodal bradycardia, A-V dissociation with intermittent capture, ventricular premature contractions, and ventricular fibrillation. These bradyarrhythmias were usually avoided by bilateral vagotomy or pretreatment with large doses of atropine, (1mg) and always eliminated by sectioning of Hering's Nerve. Beta adrenergic blockade with propanolol $0.01 \mathrm{mg} / \mathrm{kg}$, abolished the positive $\mathrm{CR}$ to combined systemic and cerebral hypoxia the negative $\mathrm{CR}$ response persisting despite vagotomy. It is concluded that the reflex negative CR associated with cerebral hypoxia is in part mediated through the carotid bodies. That the negative CR response persisted during combined systemic and cerebral hypoxia following vagotomy suggests either alternate parasympathetic pathways or direct myocardial depression. 
55 CARDIAC ARRHYTHMIAS IN NEWBORNS WITH CENTRAL NERVOUS SYSTEM DISEASE. Robert A. Achtel, M. Douglas Cunningham, Nirmala S. Desai, Carol J. Cottri11, and Jacqueline A. Noonan. University of Kentucky College of Medicine, Department of Pediatrics, lexington.

The reflex contribution of the central nervous system (CNS) to cardiac chronotropic responses has not been clearly established in newborns. In the past year continuous EKG monitoring revealed cardiac arrhythmias in the absence of associated heart disease in 5 term neonates with CNS pathology. (\#1Cerebral Mass, \#2Status Epilepticus, \#3Meningitis, \#4Arnold Chiari Malformation, \#5Cerebral Calcifications.) Infant "1 was without seizure activity, had an abnormal EEG, and developed a supraventricular tachycardia with aberrant conduction successfully treated with digitalis and propanolol and survived. Infants 非-5, demonstrated both local (LSA) and generalized (GSA) seizure activity in association with bradycardia, progressive $\mathrm{A}-\mathrm{V}$ dissociation and ventricular standstill. With GSA and apnea reflex vagal bradycardia may have resulted from CNS hypoxia. However, in $\$ 2-5$, arrhythmias persisted with LSA in the absence of metabolic abnormalities. Infants $\# 2 \& 3$ developed transient 3 rd degree $\mathrm{A}-\mathrm{V}$ block with LSA which continued despite right ventricular (RV) demand pacing. Both $\# 2 \& 3$ survived in normal sinus rhythm. Infants 非 $\& 5$ were treated with atropine and isoproterenol, were unresponsive, and expired. It is concluded that continuous EKG monitoring is a necessary adjunct in the management of infants with CNS pathology with/without LSA or GSA. Temporary RV demand pacing is effective in controlling bradyarrhythmias and should be considered when pharmocologic agents are ineffective.

\section{ECHOCARDIOGRAPHIC (ECHO) DEFINITION OF PROGRESSIV} Allen, Lyon.M. Taussig, Stanley J. Goldberg, David J. Sahn and John Gaines. Dept. of Peds., Ariz. Med. Ctr., Tucson.

Progressive cardiac changes with increasing lung disease in $C F$ have not been well defined. Thickening of the right ventricular anterior wall (RVAW) in CF has been directly assessed by ECHO but other cardiac and great vessel changes have not been reported. Thirty CF patients, age 3 months to 35 years with NYH clinical scores ranging from 34 to 99 were studied by ECHO, pulmonary function tests, and partial pressures of arterial oxygen ( $\left.\mathrm{PaO}_{2}\right)$. spective of clinical state. Patients with scores greater than 80 (mild) also had enlarged right ventricular cavities (RVC). Patients with scores of 60-80 (moderate disease) showed interventricular septal (IVS) and left ventricular posterior wall (LVPW) thickening, in addition to progressive RVAW and RVC changes. Those patients with the most severe lung disease (scores < 60) including those who died, showed progressive RVAW, IVS and LVPW thickening, RVC dilation, and, in addition, aortic root dilation (Ao) and abnormally small left ventricular cavities. Acute $\mathrm{PaO}_{2}$ measurements in 14 patients did not correlate with the long standing cardiac measurements. Measurements of large airway function correlated with RVAW $(x=-.7)$ and Ao $(x=-.7)$. No correlation between ECHO parameters and maximal flow in the low vital capacity range was found. Early cor pulmonale in CF involves the right side of the heart. As disease progresses, the septum, left ventricle, and aorta are also affected. This is best shown by echocardiography.

57 ETIOLOGY OF RIGHT BUNDLE BRANCH BLOCK (RBBB) PATTERN FOLLOWING SURGICAL REPAIR OF A-V CUSHION DEFECTS. Robert E. Antar, Ehud Krorigrad, Frederick 0 . Bowman Ir., James R. Malm. College of Physicians \& Surgeons of Columbia University, Department of Pediatrics, New York, N.Y. 10032. Injury to the right bundle branch(RBB)during surgical repair of various types of interventricular defects may occur throughout its entire course. In order to assess the possible effects of septal sutures on the genes is of RBBB, the electrocardiogram of 13 patients (pts) with complete A-V canal (AVC) and 26 pts with ostium primum( 1 ASD) were evaluated prior to and following open heart surgery. In all pts surgical repair was performed via a right atriotomy and no pt had a ventriculotomy or pulmonary infundibular resection. The operative age ranged from 3 months to 38 years (mean=7.5 years). Prior to surgery, 33 pts showed a counterclockwise superiorly oriented frontal plane QRS axis, 4 had indeterminate and 2 had normal axis. The mean QRS duration for both groups was $0.07 \mathrm{sec}$. Septal sutures were placed in all pts with AVC and 11 of these 13 pts developed RBBB. Septal sutures were utilized in 5 of the 26 pts with 1 ASD and all 5 developed postoperative RBBB. None of the pts with I ASD without septal sutures developed an RBBB pattern and none of the pts in this series developed complete heart block. The mean QRS duration postoperatively for pts with RBBB was $0.12 \mathrm{sec}$. Our data suggest:1) postoperative RBBB pattern following repair of various forms of A-V cushion defects is due to RBB injury by septal sutures,2) if possible. septal sutures should be avoided in pts with $1^{1} \mathrm{ASD}, 3$ ) increased efforts should be made for identification and preservation of the RBB during surgery.
58 REGULATION OF RESPIRATION IN INFANTS WITH CYANOTIC CCNGENITAL HEART DISEASE. Eduardo Bancalari, Tilo Gerharat (Intro. by W.w. Cleveland) University of Miami, School of Medicine, Dept. of Pediatrics, Miami, Florida. Infants with chronic hypoxemia due to CHD do not present with hyperventilation and hypocapnia in spite of their reduced $\mathrm{PaO}_{2}$. To determine to what degree the absence of hyperventilation is due to a lack of sensitivity of the peripheral chemoreceptors or to CNS depression secondary to hypoxia, we measured the ventilatory response to $100 \%$ inspired oxygen in 7 infants with $\mathrm{CHD}$ and severe chronic hypoxemia, (Mean $\mathrm{PaO}_{2} 27 \mathrm{mmHg}, \mathrm{PaCO}_{2} 39 \mathrm{mmHg}$ ). Minute ventilation $\left(\dot{V}_{E}\right)$ did not change during the first 30 seconds, but did increase $51 \%$ after 3 minutes of breathing $100 \%$ oxygen. The absence of an immediate decrease in $\dot{V}_{E}$ after exposure to $\mathrm{O}_{2}$ most likely reflects insensitivity of the peripheral chemoreceptors. On the other hand, this fall could be overridden by the subsequent increase in $\dot{V}_{E}$. The increase in $\dot{V}_{E}$ at 3 minutes $(51 \%)$ was significantly greater than the one observed in normal infants $(20 \%)$. This difference is more striking considering that the $\mathrm{PaO}_{2}$ in the cyanotic patients increased only from $2 ? \mathrm{mmHg}$ to $32 \mathrm{mmHg}$ when breathing $\mathrm{O}_{2}$. This marked increase in $\dot{\mathrm{V}}_{5}$ can be secondary to recovery from hypoxic CNS depression and or to increased $\mathrm{CO}_{2}$ production due to a higher metabolic rate secondary to improved oxygenation. The latter possibility is supported by the fact that the $\mathrm{PaCO}_{2}$ did not change in spite of the marked increase in ventilation.

REDUCED ADIPOSE CELLULARITY WITH CYANOTIC CONGENITAL

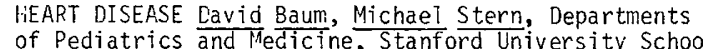
Stanford, $C A$

of Medicine Stanford, $C A$

Adjpose tissue mass, fat cell size and total body fat cell number were compared in two groups of children with congenital heart disease (CHD). Group I consisted of 16 children (age $6.9 \pm 1.1$ $\mathrm{yr}$; mean + SEM) with cyanotic CHD who had been hypoxic since infancy and who were short and underweight. Group II was composed of 16 normal sized children with acyanotic, asymptomatic CHD (age $6.7+.8$ yr). Age-adjusted adipose tissue mass was less in Group I than in Group II $(p<.05)$. This difference was not attributable to cell size which was not significantly different in the two groups $(p) .2)$. In contrast, age-adjusted cell number was markedly less in Group I $(p<.001)$.

The reduced fat cell number in children with cyanotic CHD (Group I) sungests their adipose tissue growth potential may be permanently reduced since ultimate fat cell number is largely determined in infancy and is little effected by later nutrition as is fat cell size. Since iean body mass was also less in Group I than in Group II $(p<.05)$, the cellular content of other organs may be altered similarly by chronic hypoxia. If true, these factors deserve consideration in timing surgical therapy.
60 hyperaldosteronism and aldosterone anatagonists in CONGESTIVE HEART FAILURE (CHF) AND COR PULMONALE (CP) IN INFANCY. Barry Baylen, Gregory Johnson, Reginald C. Tsang, Laxmi Srivastava, and Samuel Kaplan, University of Cincinnati, Children's Hospital, Dept. of Pediatrics. Cincinnati, Ohio

Infants with severe CHF from congenital heart disease (CHD) or bronchopulmonary dysplasia (BPD) with $\mathrm{CP}$ of ten fail to respond to conventional diuretic therapy. Secondary hyperaldosteronism may be a major contributing factor to this lack of response. We measured serum aldosterone (ald) by radioimmunoassay in 20 control infants, in 11 infants with $\mathrm{CHD}$ and $\mathrm{CHF}$ who received digoxin and intramuscular furosemide and in 4 with BPD and CP. Serum ald in controls was median (M) $19 \mathrm{ng} \%$, range $(R) .8-100.5$. Ald in infants with $\mathrm{CHD}$ and $\mathrm{CHF}$ was significantly increased $(M=169.8$; $R=3.4-597$, rank-t $p<.01)$. Ald was increased in 6 of $11 \quad(R=169-598)$ prior to, and 8 of $11 \quad(R=115-519)$ during therapy for CHF. Ald was increased in 1 of 4 with BPD prior to (146) and during therapy. Infants with normal ald responded with increased urinary output and $\mathrm{Na}: \mathrm{K}$ ratio $(\mathrm{UNa} / \mathrm{K})$. By contrast in 6 of 7 with increased Ald we observed decreased post furosemide urine volume, reversed $\mathrm{U} \mathrm{Na} / \mathrm{K}$, normal or low serum osmolarity and signs of persistent fluid retention. Addition of aldosterone anatagonist in these patients resulted in markedly improved diuresis and increased $U \mathrm{Na} / \mathrm{K}$.

Recognition and treatment of hyperaldosteronism is important for optimal medical management of CHF in infancy. 
61 CARDIOVASCULAR CHANGES DURING EXCHANGE TRANS. FUSION IN Rh DISEASE. Luis A. Cabal, Feizal Waffarn, Vellore G. Muraligopal, Bernardino Zanini, Joan E. Hodgman, Edward H. Hon Univ. of So. Calif. School of Medicine, Los Angeles County/USC Medical Center, Departments of $\mathrm{Ob} / \mathrm{Gyn}$ and Pediatrics, Los Angeles.

The acute hemodynamic changes during exchange transfusion(ET) were evaluated by continuous monitoring of instantaneous heart rate(HR), aortic blood pressure $(A B P)$ and instantaneous electromechanical interval(EMI) of the cardiac cycle measured from the onset of the $Q$ wave of the EKG to the onset of the ABP upstroke. Three sick neonates with severe $R h$ disease and respiratory distress had a total of six ETs with whole blood or packed cells during the first three days of life. The ETs were done with different aliquots $(5-15 \mathrm{ml})$ at varying rates through an umbilical venous catheter in the right atrium. No consistent changes were seen in HR and $A B P$. A consistent transient prolongation of the EMI during infusion was observed. At the same rate of infusion, larger aliquots were associated with greater prolon gation of the EMI $(p<0.02)$. With the same aliquots, the change in EMI was inversely related to the duration of infusion only in the range $1-10 \mathrm{sec} / \mathrm{ml}$. Short duration $(\leqslant 4 \mathrm{sec} / \mathrm{ml})$ was associated with greater prolongation of EMI(slope $=-4.86, p<0.01)$, intermediate duration $(4-10 \mathrm{sec} / \mathrm{ml}$ ) with less prolongation (slope $=-0.94, p<0.01)$. A consistent shortening of EMI was noted after infusion of calcium gluconate during ET. Ionotropic agents like Isuprel were observed to minimize or abolish the changes in EMI during infusion of blood. The EMI prolongation associated with larger aliquots of blood and higher rates of infusion may be explained by acute left ventricular overload and/or poor admixture of unphysiologic blood in the right atrium. The continuous evaluation of EMI provides a sensitive tool for monitoring cardiac performance during exchange transfusion. 62 PROGNOSIS OF INFANTS WITH COARCTATION OF AORTA, SuWilIman, (Spon. by Arthur E. McElfresh) St, Louis Univ。 Sch. of Med., Cardinal Glennon Tosp., Dept. of Ped., St. Louis, Mo Prognosis of infants with coarctation (coarco) is studied with regard to the medical or surgical management and the associated defects. 24 males and 12 females developed heart failure in tine first 6 months of life were followed from 14 months to $15 \mathrm{Jrs}$. (mean $4 \frac{1}{2}$ yrs.). There were 12 deaths, $(12 / 36,33.3 \%$ ), 10 in infancy, 2 at age $3 \mathrm{yrs}$ and $7 \mathrm{yx}$; 6 out of 23 operated patients died, $(26 \%), 6$ out of 13 non-operated pts. died, $(46 \%) .16$ pts. required surgery in infancy and $\delta$ of these died. None died when surgery was done above the ase of $1 \mathrm{yx}$. All had cardiac catheterization. Group (Gr.) I, 12 pts. had isolated coarc., Gr. II, 5 pts. had coarc. \& PDA, Gr. III, 9 pts. had coarc. \& VSD with or without PDA. Gr. IV, $10 \mathrm{pts}$, had coarc. and other lesion: 3 with TGV, 2 with A-V Canal and 3 with other defects. OnIy I in Gr. I required coarctectomy in infancy and 1 died at home after aspiration. In $\mathrm{Gr}$. II, 3 were operated in infancy and survived, 1 died without surgery. In $G r$. III, 5 pts. had coarctectomy and PA banding with 3 deaths ( 1 early, 2 late deaths), 1 died without surgery, 1 had surgery after infancy and 2 have not had surgery. In Gr. IV 7 pis. were operated on in infancy and 3 died, 3 nonoperated pts. died. Among the 7 non-operated surviving pts. 6 are below age $3 \mathrm{yrs}$. and well, $I$ is $12 \mathrm{yrs}$. with mild coarc. In 5 of the $9 \mathrm{Gr}$. III pts. tho had coarc. \& VSD, the VSD had either closed spontaneously (1) or became smaller (4), this suggests that PA banding at the time of coarctectomy should be discouraged.

63 MEDICAL MANAGEMENT OF SYMPTOMATIC DUCTUS SHUNTS IN SMALL PRETERM INFANTS. Cotton, R., Kovar, I., Catterton, Z., Selstam, U., Cordell, D., Graham, T.,Stahlman, M. Vanderbilt University School of Medicine, Vanderbilt University Medical Center, Department of Pediatrics, Nashville, Tennessee.

To evaluate the effectiveness of medical management of symptomatic $\mathrm{L} \rightarrow \mathrm{R}$ ductal shunting (DS) in small preterm infants, the course of all infants with $\mathrm{BW} \leq 1200 \mathrm{~g}$ admitted to NICU during 1975 was studied: $32(48 \%)$ of 66 infants (482-1200 g, mean 952 g) developed evidence of DS during the first 7 days of life. DS occurred in $31(65 \%)$ of 48 infants who lived $\geq 4$ days after birth. Evidence of DS included typical murmur, elevated basal HR, hyperdynamic precordium and pulses, gallop, rales, and radiologic features of cardiomegaly and pulmonary plethora. 28 infants (BW 709-1191 g) with DS were managed medically without ductal ligation. Cardiac failure was treated aggressively with digitalis, diuresis, and fluid restriction. Pulmonary edema was controlled with CDAP via nasal prongs or endotracheal tube when mechanical ventilation was required. Transfusions were given to maintain the hematocrit $>40 \%$ and adequate perfusion. Early adequate caloric intake was attempted via total parenteral nutrition or jejunal feeding. Hemodynamic course was assessed by echo and impedance cardiography. With this regimen $16(57 \%)$ of the 28 infants survived including $3(38 \%)$ of 8 infants with hyaline membrane disease. These results indicate the effectiveness of medical management. To evaluate surgical management, a program of randomised treatment assignment is in progress.

\section{ATYPICAL PRESENTATION OF HYPOPLASTIC LEFT HEART SYNDROME. Delores A. Danilowitz, Monika R. Rutkowski and Eugenie F. Doyle.}

New York University Medical Center, Department of Pediatrics, New York, N.Y.

The infant with hypoplastic left heart syndrome (HLHS) usually presents with symptoms of severe congestive heart failure or with a shock like picture. However, the primary manifestation may vary and can be quite misleading.

Out of a group of fourteen infants with HLHS demonstrated by cardiac catheterization and/or autopsy 8 presented with atypical manifestations. One presented with intrauterine arrhythmia; one with bounding pulses and high output failure associated with a huge patent ductus arteriosus; one with severe hypoglycemia; one with irritability and hypocalcemia; two with jaundice and tachypnea and two with respiratory distress syndrome.

In view of the high frequency of misleading initial manifestations routine screening of all sick neonates with echocardiography should be helpful in identifying HLHS.

\section{EARLY LIGATION OF THE DUCTUS ARTERIOSUS AS AN ADJUNCT TO VENTILATORY SUPPORT IN
INFANTS WITH RESPIRATORY DISTRESS SYN-}

DROME. Robert A.delemos, Lawrence C. Franklin, Donald $M$ Null, Jr., Howard H. Johnson, Jr., \& Paul A. Dumas (Spon. by M. J. Sweeney). Wilford Hall USAF Medical Center, Dept of Pediatrics, San Antonio, Tx.

To define the relationship between a left to right shunt across a PDA and the need for prolonged ventilatory support in infants with IRDS, aortograms were performed on 40 consecutive babies under 1300 grams who either failed to improve or clinically deteriorated within 72 hours of intervention. All were receiving CPAP and assisted ventilation (IMV). None of the infants had cardiomegaly or signs of heart failure. Pulse,pressure and EKG were normal. Eight had murmurs audible when IMV was discontinued; LA/root of aorta ratios were elevated in 5 . Left to right shunts were demonstrated angiographically in 28 infants, 27 of whom underwent immediate surgical ligation. There were no surgical deaths. Lung compliance fell post operatively in $4 / 4$ patients studied. Alveolar ventilation and $\mathrm{V}_{\mathrm{T}}$ increased significantly in 22 infants within 6 hours of surgery. All of these survived; 16 were extubated within 3 days. The five deaths were caused by intraventricular hemorrhage. These findings suggest that a ductal left to right shunt may adversely affect lung compli ance prior to development of heart failure. Elimination of the cardiac shunt may improve lung function and hasten extubation.

\section{THE MECHANISM OF DYSRHYTHMIAS AFTER MUSTARD'S}

Desmond F. Duff, Paul C. Gillette, Charles E. Mullins,

\& Dan G. McNamara, Dept. of Pediatrics, Section of Cardiology, Baylor College of Medicine and Texas Children's Hospital, Houston

Dysrhythmias are a frequent serious late complication after Mustard's operation (M) for transposition of the great arteries. Eighty-seven $(68 \%)$ of 128 patients who survived more than one week after $M$ developed at least one dysrhythmia during followup (1-8 years). The dysrhythmias included sinus and junctional bradycardia, atrial flutter, and paroxysmal supraventricular tachycardia. Six $(7 \%)$ of 87 patients with dysrhythmias died suddenly, apparently of dysrhythmias. Forty-two (33\%) patients have had postoperative electrophysiologic studies. All had normal internodal, atrioventricular nodal, and His Purkinje system conduction as evaluated by intracardiac conduction intervals(35), atrial pacing (34), and refractory periods(7). Sinus node function (SNF) was abnormal as measured by overdrive suppression in 22 patients $(65 \%)$ Eighty-two percent of patients with abnormal SNF developed dysrhythmias VS $24 \%$ of those with normal SNF. Eight (19\%) of patients developed severe sinus bradycardia during electrophysiologic study while $6(14 \%)$ developed tachydysrhythmias, 2 atrial flutter, 3 sinus node reentry tachycardia, 1 undetermined mechanism.

Based on the findings indicating that sinus node damage was responsible for post-Mustard dysrhythmias, the technique of the procedure was modified to attempt to protect the sinus node. After the modifications the incidence of dysrhythmias was decreased $27 \%$ from $82 \%$ to $55 \%$ during 3 years of followup. 
67

EFFECT OF ISOPROTERENOL ON REGIONAL CORONARY FLOW FOLLOWING RIGHT VENTRICULOTOMY. David E. Fixler, James $M$. Wheeler, James $T$. Willerson, and John $T$. Watson, University of Texas Health Science

We infused isoproterenol (ISO) $0.05-0.1 \mathrm{mcg} / \mathrm{kg} / \mathrm{min}$., into 8 anesthetized dogs after right ventriculotomy to determine if in creases in contractility were accompanied by increases in coronary flow. A $3.5 \mathrm{~cm}$ vertical incision was made in the RV free wall. Coronary flow was measured with 9 micron radioactive microspheres during control state, $45 \mathrm{~min}$. after ventriculotomy, and then during infusion of 150 . Flow was measured near the incision (myocardium $1 \mathrm{~cm}$ from the incision) and in distal areas of RV myocardium. After ventricułotomy, ISO did not alter RV pressures or cardiac output significantly, but did increase heart rate from $166 \pm 7(S E)$ to $178 \pm 4(p<0.05)$ and increased RV peak $\mathrm{dP} / \mathrm{dt}$ by $\overline{3} 0 \%(\mathrm{p}<0.01)$. Coronary flow $(\mathrm{ml} / \mathrm{min} / 100 \mathrm{~g})$ to the distal RV area did not change with ventriculotomy, but then increased from $64 \pm 8$ to $89 \pm 11$ ( $p<0.05$ ) with IS0. In contrast, flow to the peri-incisional area fell from $90+7$ to $30+$ $6(p<0.05)$ with ventriculotomy and failed to increase significantly with iso being $34+8$. These data indicate ventriculotomy reduces coronary flow to nearby myocardium. Although iso increased coronary flow to distal regions, it failed to increase flow to the peri-incisional region. Following ventriculotomy the ISO induced changes in $\mathrm{dP} / \mathrm{dt}$ and heart rates could potentially increase myocardial injury if increased oxygen needs are not accompanied by increased oxygen supply.

\section{VASCULAR INSUFFICIENCY RESULTING FROM}

BLALOCK-TAUSSIG SHUNT; SUBSEQUENT SUCCESSFUL REVASCULARIZATION OF THE ARM. Henry Frey, O. Wayne Isom, Eugenie F. Doyle, New York University Medical Center, Departments of Pediatrics and Surgery, New York, N.Y.

Vascular insufficiency following Blalock-Taussig anastomosis is rare. Three previously reported cases resulted in either death or amputation.

An 8-year-old mongoloid boy, severely incapacitated and profoundly cyanotic from $\mathrm{A}-\mathrm{V}$ canal and pulmonic stenosis underwent an end-to-side right subclavian-pulmonary artery anastomosis performed through a right anterolateral thoracotomy. The right arm became cool with absent radial pulse. Mild weakness of the right arm 24 hours later progressed to flaccid paralysis and complete sensory loss within 72 hours. The incision became necrotic. a line of demarcation developed over the shoulder. A saphenous vein autograft anastomosis of $4 \mathrm{~mm}$ diameter was made between the right common carotid artery and the distal right subclavian stump. Immediate return of the radial pulse and good perfusion of the arm followed. After one year sensation has returned and arm and hand motor function is almost normal.

Possible causes for the vascular insufficiency include anomolous subclavian artery branching, thrombosis, and the incision employed.

69 PHARMACOlOGic Closure of PATENT DUCTUS in THE PREMATURE. William F. Friedman, Michael J. Hirschklau, Paul T. Pitlick, and Stanley E. Kirkpatrick, Dept. of Peds., Peds. Card., Univ. of Calif., San Diego.

Patent ductus arteriosus (PDA) is a common complication of prematurity and may critically influence the morbidity and mortality of infants with respiratory distress syndrome (RDS). Prostaglandins (PG) have been shown recently to alter the contractile response of ductus smooth muscle in animals and man. Thus, PGE and $E_{2}$ has been shown to dilate the ductus and increase pulmonary blood flow in infants with pulmonary atresia. In animals, inhibition of PG synthesis may close the PDA in utero. At UCSD we have employed Indomethacin $(\mathrm{I})$, a potent inhibitor of PG synthesis, to close the PDA promptly in two premature infants 1360 and 2020 gms with RDS. Evidence for ductal closure after I consisted of disappearance of physical findings (cardiomegaly, heart murmur, bounding pulses) and most importantly of serial echocardiographic studies. The latter analyzed the change in the dimension ratio of left atrium/aorta (LA/Ao), an index shown previously by us to accurately detect acute alterations in the magnitude of left to right shunting due to changes in ductal caliber. In these infants the LA/Ao fell $32 \%$ and $36 \%$ to within the normal range by 24 hours after a single PO $(2.5$ $\mathrm{mg} / \mathrm{Kg}$ ) or rectal dose $(5 \mathrm{mg} / \mathrm{Kg})$ of $\mathrm{I}$. These provocative findings suggest that pharmacological techniques to close the PDA may assume progressive importance in the care of the preterm infant and support the need to analyze in depth the role of PG synthesis inhibition in the management of infants with PDA and RDS.
70 WLECTROPHYSIOLOGIC STUDIES IN MAN PauI C. Gillette and Dan G. MCNamara, Baylor College of Medicine and Texas Children's Hospital, Houston, Texas

Anomalous atrioventricular conduction pathways known as Kent (K) bundles are present in more than $50 \%$ of children with supraventricular tachycardia. Medical and surgical management of these patients is enhanced by knowing the function of these pathways. This study examined the antegrade and retrograde conduction properties of $\mathrm{K}$.

Nine subjects ages 5 days to 19 years were studied by recording intracardiac electrograms, and by premature atrial and ventricular stimulation and pacing. Six subjects had manifest WolffParkinson-White syndrome (WPW). In 3 others the $K$ was shown to participate in the reentry circuit during SVT but could not conduct antegrade correlating with lack of PR interval shortening and QRS aberration on their electrocardiograms. In the 5 patients with both WPW and SVT, the $K$ was part of the reentry circuit. In the one subject without SVT, reentry through the $K$ was impossible as it could not conduct retrograde. The effective and functional refractory periods of the $K$ during antegrade conduction were within the same range as normal children's AV node refractory periods. Ventricular rate during atrial fibrillation or flutter, SVT rate and maximum paced rates with maintenance of $1: 1$ conduction were also in the normal range compared to children without $K$.

The functional properties of $K$ are similar to those of the normal conduction system in children. Some $K$ may be detected only by intracardiac electrophysiologic study.

ECHOCARDIOGRAPHIC DETERMINATION OF VENTRICULAR DIMENSIONS: USE IN AOR TIC STENOSIS. Sidney Glanz, William E. Hellenbrand, Michael A. Berman, Norman S. Talner.* Yale Univ. Sch. Med., Yale-New Haven Hosp., Dept. Ped., New Haven.

The magnitude of ventricular hypertrophy in response to afterloading is determined by wall stress with wall thickness increas ing in proportion to ventricular load until systolic wall stress is normalized. Using echocardiographic measurements of end-systolic wall thickness (Ws) and cavity transverse dimension (Ds), the ratio (c) of peak systolic pressure $(P)$ to relative wall thickness (Ws/Ds) was calculated in 16 normal patients, thus, $c=$ $\mathrm{P} \times \mathrm{Ds} / \mathrm{Ws}$. The mean value for $\mathrm{c}$ was $225+6.7$. From this value left ventricular pressure was estimated in 13 patients (4-17 years) with aortic stenosis using the formula $P=c x W s / D s$. No subject had evidence of cardiac failure. Peak systolic aortic pressure difference $(\Delta P)$ was calculated by subtracting cuff measured brachial artery peak systolic pressure from the estimated left ventricular pressure. Excellent correlation was obtained between the estimated $\triangle F$ and that found at catheterization $(x=0.892$, $\mathrm{p}<.0005)$. In 3 patients, echocardiographic data predicted a significant obstruction in the presence of normal ECG, VCG, and $\mathrm{XYZ}$ tracings. Echocardiography offers a noninvasive method for estimating the severity of aortic stenosis that appears to be more sensitive than other currently employed techniques.

7 CARDIOVASCULAR ADRENERGIC RESPONSES IN NEWBORN PIGS. Norman Gootman, Nancy M.Buckley, Phyllis M.Gootman, Isaac D.Frasier. Long Island Jewish-Hillside Medical Center and SUNY at Stony Brook, Albert Einstein College of Medicine and SUNY Downstate Medical Center; Depts. Of Pediatrics and Physiology. New Hyde Park and New York, New York.

Adrenergic receptor function was tested in piglets aged 1-4days $(N=16), 1$ week $(N=13)$ and 2 weeks $(N=12)$ and in sexually mature Miniswine $(N=6)$, with norepinephrine (NE) or isoproterenol (ISP) before and after receptor blockade. All animals were lightly anesthetized with Na-pentobarbital, paralyzed with decamethonium, and ventilated to maintain normal arterial $\mathrm{pH}, \mathrm{pCO}_{2}$ and $\mathrm{pO}_{2}$. Cardiovascular function was evaluated from simultaneously recorded ECG, aortic pressure (AoP), right ventricular pressure and its $\mathrm{dP} / \mathrm{dt}$; and femoral, renal and carotid flows registered by electromagnetic transducers. Vascular resistances (R) were calculated as mean AOP/ mean flow. The pressor effect of $0.5 \mathrm{ug} \mathrm{NE} / \mathrm{kg}$ was smaller in piglets than in mature swine. $\alpha$-adrenergic blockade with phentolamine $(0.25 \mathrm{mg} / \mathrm{kg})$ produced hypotension and blocked NE action on AoP and renal $R$. The depressor effect of $0.1 \mathrm{\mu g} I S P / \mathrm{kg}$ was observed only in 2 week old piglets and mature swine, although cardiac inotropic and chronotropic responses and decreased femoral $R$ occurred at all ages. B-adrenergic blockade with propranolol $(0.1 \mathrm{mg} / \mathrm{kg})$ produced cardiac depression in all animals, increased femoral $R$ in 2 week old piglets and mature swine, and blocked all ISP actions at all ages. These results suggest the differential postnatal development of $\alpha$ and $\beta$ adrenergic mechanisms in the piglet. 
73 EFFECT OF INHALATION OF CARBON MONOXIDE(CO) ON THE REGIONAL DISTRIBUTION OF BLOOD FLOW IN LAMBS. Kathryn Grumbach, Robext J. Hollister, Louis Lipsitz, Luis Blascoe and Maria Delivoria-Papadopoulos. University of Pennsylvania, School of Medicine, Philadelphia, Pennsylvania, 19104. We have investigated the redistribution of blood flow to the various organs following an acute increase $(20 \%)$ of blood $\mathrm{HbCO}$ following inhalation of $\mathrm{CO}$ by the microsphere technique. Catheters were placed chronically in the right heart, left ventricle, and femoral artery of 2-6 week old lambs. Following surgical recovery all lambs were injected with microspheres $15 \mu$ in diameter labelled either ${ }^{141} \mathrm{Ce}$ or ${ }^{85} \mathrm{Sr}$ for determination of baseline organ blood flows. Hb saturation, blood gases, $\mathrm{SaO}_{2}$, and Hct were determined before and 2 hours after $\mathrm{CO}$ administration. After $\mathrm{HbCO}$ had been increased, microspheres labelled with the other isotope were injected. In the 7 experimental animals regional blood flow in $\mathrm{m} 1 / \mathrm{min} / 100 \mathrm{~g}$ tissue to the heart increased from mean values $\pm S D$ of $147 \pm 51$ to $238 \pm 92(p<0.05)$, to the brain from $67 \pm 9$ to $106 \pm 36(p<0.05)$ Blood flow to the adrenals, kidneys and lungs did not change significantly. Arterial-venous oxygen difference decreased from 5.2 to $4.3 \mathrm{ml} / 100 \mathrm{ml}$ blood, $\mathrm{PVO}_{2}$ from 39 to $23 \mathrm{mmHg} ; \mathrm{PaO}_{2}$ and Hct remained unchanged. Cardiac output increased from $157 \pm 42$ to $195 \pm 41$ $(p<0.07)$. In the 6 control animals all these parameters remained unchanged. These data indicate that $\mathrm{O}_{2}$ delivery to the brain and heart is maintained by an increase in blood flow to compensate for a decrease in venous $0_{2}$ tension reflecting a decrease in $0_{2}$ content as well as an increase in oxygen-hemoglobin affinity.

\section{LEFT VENTRICULAR FUNCTION IN CHILDREN WITH CONGESTIVE}

Howard P. Gutgese11, Marc Paquet, Desmond F. Duff, and Dan G. McNamara, Department of Pediatrics, Baylor College of Medicine and Texas Children's Hospital, Houston, Texas

Echocardiography was used to evaluate left ventricular (LV) function in 16 children with congestive cardiomyopathy. Measurements included LV end-diastolic diameter (EDD), percent change in $L V$ diameter with systole $(\% \Delta L V D)$, mean velocity of circumferential fiber shortening $\left(V_{c} f\right)$, and, from high speed recording of aortic valve motion, the ratio of the LV pre-ejection period to ejection time (PEP/LVET). The results were compared to similar data from 65 normal children.

The EDD was increased in 14 of 16 patients. The $: \triangle L V D$ was reduced in all, and averaged $15 \%$ compared to $36 \%$ in normals $(p<0.01)$. Mean Vcf was likewise lower than predicted in all patients with cardiomyopathy. PEP/LVET was increased in 12 of 14 patients in which it could be determined (normal $0.29 \pm 0.03 \mathrm{SD}$, cardiomyopathy $0.51 \pm 0.16$ ), and was useful in 2 patients in whom paradoxical septal motion precluded calculation of $\% \Delta L V D$ or $V c f$.

The $: \triangle L V D$ is easily determined and clearly separated patients with cardiomyopathy from normal. Increased EDD and PEP/LVET and reduced Vcf helped confirm LV dysfunction.

\section{Dilatation of the Ductus Arteriosus in Infants with} Rudolph, University of California San Francisco,

Department of Pediatrics, San Francisco.

Maintenance of ductus arteriosus (DA) patency is crucial to post-natal survival of infants with pulmonic atresia. Despite severe hypoxemia, the DA constricts within hours or days thus reducing pulmonary blood flow. Since Prostaglandin $\mathrm{E}_{1}\left(\mathrm{PGE}_{1}\right)$ dilates the DA experimentally, Elliott el al infused $\mathrm{PGE}_{1}$ into 2 infants with cyanotic heart disease and demonstrated an increase in arterial $\mathrm{O}_{2}$ saturation; however they did not examine the DA. We studied the effects on arterial blood gases and the $\mathrm{DA}$ of $\mathrm{PGE}_{1}$ infusion in 4 infants with pulmonic atresia. The DA was examined by cineaortography before and after $\mathrm{PGE}_{1}$ infusion into the thoracic aorta at a rate of 0.1 micrograms $/ \mathrm{kg} / \mathrm{min}$. Arterial PO rose from a range of 15-29 (mean 24) torr to 3847 (mean 459 torr 30 min after the infusion was started, an average increase of $108 \%$. The diameter of the DA at its narrowest point ranged from 2.4-2.94 (mean 2.75) mm and dilated to $4.4-5.53$ (mean 5.18 ) $\mathrm{mm}$, an average increase of $89 \%$. The minimal cross sectional area, which reflects conductance, increased from a range of 4.52-6.79 (mean 5.96) $\mathrm{mm}^{2}$ to 15.21 24.02 (mean 21.26 ) $\mathrm{mm}^{2}$, an average increase of $257 \%$. Arterial blood pressure and heart rate were unaltered throughout the infusion which was continued for periods of 3-5 hours until a palliative surgical aortopulmonary connection was completed. $\mathrm{PGE}_{1}$ is an effective dilator of the DA in infants with pulmonic atresia; it dramatically improves systemic arterial $\mathrm{PO}_{2}$ due to a marked increase in pulmonary blood flow. 76 PRE AND POST-OPERATIVE EXERCISE STUDIES IN CHILDREN Frederick W. James, Samuel Kapian and David C. Schwartz, University of Cincinnati College of Medicine, Childrens Hospital Medical Center, Dept. of Pediatrics, Cincinnati, Ohio Non-invasive assessment of the degree of left ventricular outflow tract (LVOT) obstruction due to aortic stenosis is difficult especially in the late post-operative period. Thirteen children underwent a continuous graded bicycle exercise test (GBET) before and 6 to 24 months after aortic vaivotomy or resection of discrete subvalvar obstruction. Preoperatively (preop) syncope and/or chest pain were present in $7 \mathrm{pts}$, and the routine electrocardiogram (ECG) revealed left ventricular hypertrophy in $12 \mathrm{pts}$. The gradients ranged from 50 to $235 \mathrm{~mm} \mathrm{Hg}$ preop and were $<40 \mathrm{~mm} \mathrm{Hg}$ in 12 pts after operation (postop). The end points of the GBET were development of symptoms, exhaustion, pallor, ST depression (ST) $\geq 3 \mathrm{~mm}$, ventricular arrhythmia or fall in exercise systolic pressure below resting levels. The results of the exercise ECG (in $\mathrm{mm}$ ) and working capacity (WC) (Kg-meters/minute) were:

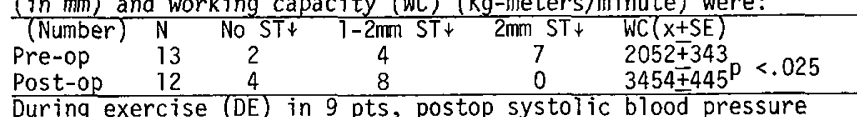

During exercise (DE) in 9 pts, postop systolic blood pressure preop SP increase of 4 to $41 \%$. We demonstrated improvement in preop SP increase of 4 to $41 \%$. We demonstrated improvement in ischemia DE postop. We suggest that this non-invasive method is useful in assessment of pre and postop LVOT gradients.
THE EFFECT OF HEMODYNAMTC VARTABLES ON ALBUMIN TRANSPORT ACROSS THE INTIMA OF CORONARY ARTERIES. Jay M.
Jarmakani, M.D. UCLA Medical Center, Department of Pediatrics, Los Angeles, California

The effect of pressure (tensile force) and flow (sheer force) on the transport of T-1824 dye-tagged albumin across the intimal surface of the systemic and coronary arteries was determined in 34 awake dogs which were divided into three groups: I-control (coronary flow $=1.0$ to $1.5 \mathrm{ml} / \mathrm{kg} / \mathrm{min} ; \mathrm{n}=10$ ), II- high pressure (peak aortic pressure $=250-300 \mathrm{mmHg}$; coronary flow $=250-300 \%$ of control; $n=13$ ) induced by infusing phenylephrine; III- high flow (normal aortic pressure; aortic flow $200 \%$ of control, coronary flow $=200-300 \%$ of control; $n=11$ ) induced by isoproterenal infusion. $\mathrm{T}-1824$ dye $(2 \mathrm{mg} / \mathrm{kg}$ ) was given intravenously. Drugs were infus $\epsilon d$ over a 3 hour period. The animals were sacrificed; then the aorta, coronary arteries and control artery obtained before the procedure were dissected, opened and pinned on white background with Kodak color strip then photographed under water. The amount of albumin entering the intima was semiquantitated by measuring the blue color density on a scale from 0 (control vesse1) to 100 (Kodak blue color). At the aortic branching sites, color intensity in both the pressure and flow groups averaged 32,22 respectively and was significantly $(P<0.01)$ higher than control $(<4)$. In the coronary bed the staining in the high pressure group averaged 20 (range 10 to 35 ) and was significantly higher than both high flow $(<8)$ or control $(<2)$ group. The data suggests that pressure is a determinant in the pathogenesis in coronary athrosclerosis and both flow and pressure contribute significantly to athrosclerosis in the systemic arteries.

\section{RIGHT VENTRICULAR ISOVOLUMIC CONTRACTION PERIOD IN CONGENITAL HEART DISEASE} Gregory L, Johnson, Richard A. Meyer, Joan Korfhagen and Samuel Kaplan, University of Cincinnati, Children's Hospitat, Dept. of Pediatrics, Cincinnati, Ohio

It has been difficult to non-invasively assess right ventricular (RV) function utilizing the parameters commonly applied to the left ventricle. We have attempted to evaluate RV function by echocardiographic measurement of the time interval between tricuspid closure (TC) and pulmonic valve opening ( $\mathrm{PO}^{\circ}$ ) in 50 children, aged 2-20 years. Twenty-two patients without evidence of cardiac disease demonstrated RV isovolumic contraction period, as defined by the Tc-Po interval, between 0 and $20 \mathrm{msec}$ (mean $11 \mathrm{msec}$ ). Eight patients with proven pulmonary hypertension secondary to left-to-right shunting demonstrated markedly prolonged TC-Po intervals (mean $42 \mathrm{msec}$, range $28-60 \mathrm{msec}$ ). Twelve patients with secundum type atrial septal defect and normal pulmonary artery pressure but with enlarged RV end-diastolic dimension demonstrated negative TC-Po intervals (mean -12 , range -22 to -2 ). Eight patients with pulmonic stenosis were also evaluated and were found to have Tc-Po intervals overlapping all the other three groups. values obtained were all highly reproducible. We believe that echographic evaluation of the TC-Po interval may provide valuable information concerning right ventricular performance in various congenital heart defects. 


\section{9}

MEDICAL \& SURGICAL MANAGEMENT OF CONGENITAL HEART DISEASE IN CHILDREN WITH DOWN'S SYNDROME Mark R. Katlic, Edward B。Clark, J。Alex Haller, Jr。 and Catherine A. Neill; Johns Hopkins University, Baltimore, Md。 Children with Down's syndrome and congenital heart disease (CHD) have been thought at increased risk when undergoing cardiac surgery. A recent survey suggests wide variations in clinical management by cardiologists. The records of 173 patients seen from 1953 to 1975 were analyzed: the clinical course of 60 who underwent cardiac surgery was compared with 77 followed medically for at least one year. Clinical severity was graded from 0 (asymptomatic) to 4 (severe cyanosis or uncontrolled failure) at first and last evaluation and number of hospitalizations were noted. Mean follow-up was 4.2 years.

Two sub-groups of surgical patients were identified by operative risk. In the low risk group (PDA, secundum and primum ASD, VSD) operative mortality was $10 \%$ while $77 \%$ showed improvement in severity grading: in 43 similar patients followed medically, mortality was $30 \%$ and only $19 \%$ improved. Among 30 high risk patients (AV canal I PS, tetralogy, tricuspid atresia) surgical mortality was $50 \%$ and $50 \%$ were improved: in 34 similar patients followed medically, mortality was $32 \%$ while $12 \%$ improved. The number of hospital admissions in surgical patients averaged 3.0 preoperatively, 0.6 postoperatively: the average in patients followed medically was 2.1 .

These data show contrary to prevalent opinion that selected lesions can be repaired with acceptable mortality and impressive postoperative improvement. This series forms a basis for a logical and aggressive approach to such patients.

Q8 RIGHT VENTRICULAR MASS ESTIMATION IN CHILDREN Lucille Lester, Rajamma Mathew, Peter Sodt, ot to Thilenius and Rene A. Arcilla; University of Chicago, Wyler Children's Hospital, Department of Pediatrics, Chicago, Illinois

Estimation of RV mass (M) has not been previously done. A method was derived from 30 in situlleart specimens/casts using parallelepiped approach:

$M=1.050\left[\left(\frac{A_{f}}{L_{1}}+2 T\right)\left(\frac{A_{1}}{L_{1}}+T\right)\left(L_{1}+T\right) 0.795-1.12\right]$ $-0.65\left(\frac{\mathrm{A}_{\mathrm{f}} \mathrm{A}_{1}}{\mathrm{~L}_{1}}\right)$ where: $\mathrm{A}_{\mathrm{f}}=$ area frontal; $\mathrm{A}_{1}=$ area lateral; $L_{1}=$ apex base length; $T=w a l l$ thickness. True mass (Mt) vs. estimated mass (Me) revealed: $M t=0.997 \mathrm{Me}-$ $0.11, r=0.97, \mathrm{SEE}=4.7 \mathrm{gm}$. C I inical application using angiograms for RV volume, and RV wall echo for $T$ was done on 36 children with normal right heart (N), 13 with RV pressure load (P), and 10 with RV volume load (V). Data from iv, 1 to 19 years, showed excellent correlation with BSA $\left(M^{2}\right): M e(g m)=45.9 B S A-0.77, r=$ 0.94 . Mean Me $\left(\mathrm{gm} / \mathrm{M}^{2}\right)$ was $44.6 \pm 1.1 \mathrm{SE}$ for $\mathrm{N}, 74.4 \pm$ 7.9 SE for $P(p<0.001)$, and $71.8 \pm 5.0 \mathrm{SE}$ for $V$ $(p<0.001)$. Mass/end-diastolic volume ratio $\left(\mathrm{gm} / \mathrm{cm}^{3}\right)$ was $0.53 \pm 0.01 \mathrm{SE}$ in $\mathrm{N}, 0.98 \pm 0.1 \mathrm{SE}$ in $\mathrm{P}(\mathrm{p}<0.001)$ and $0.54 \pm 0.02 \mathrm{SE}$ in $\mathrm{V}(\mathrm{p}=\mathrm{n} . \mathrm{s}$.$) . LV mass in 30 \mathrm{~N}$ children were $78.1 \mathrm{gm} / \mathrm{M}^{2} \pm 1.89 \mathrm{SE}$ and $1.26 \mathrm{gm} / \mathrm{cm}^{3} \pm 0.04 \mathrm{SE}$.

81 THE EFFECT OF CUFF SIZE ON THE ACCURACY OF BLOOD PRESSURE MEASUREMENTS IN NEONATES.

Lawrence G. Lum and M. Douglas Jones, Jr. (SPON. by L. Joseph Butterfield), Department of Perinatology, The Children's Hospital and Division of Perinatal Medicine, University of Colorado Medical Center, Denver

The relationship of the size of the extremity to the size of the blood pressure cuff is known to be important in BP determinations in chilaren. However, there are no studies available that explore this relationship in neonates.

This study examines the effects of cuff width, using $3.5,5.0$, and $6.0 \mathrm{~cm}$. cuffs, on systolic BP's utilizing the Doppler method. Twenty-four neonates with arm and thigh circumferences from 5.4 to $16.0 \mathrm{~cm}$., with weights from 0.72 to $4.2 \mathrm{kgs}$. , and with gestational ages from 24 to 42 weeks were studied. Circumferences were measured at the midpoints of the arm and thigh. Doppler systolic BP'S (SBP) were compared with simultaneous aortic systolic BP's ( $\mathrm{SBP}^{D}$ ) measured by pressure transducer.

The relationship between the cuff width and the extremity cixcumference was found to be critical to the accuracy of the SBP measurements. For example, using a $5.0 \mathrm{~cm}$. cuff width, the difference (SBP $-\mathrm{SBP}_{\mathrm{m}}$ ) can vary from -20 to $+15 \mathrm{~mm} \mathrm{Hg}$ depending upon the extremity circumfexence.

Using the information obtained from this study, we were able to define a range of extremity circumference associated with a $\mathrm{SBP}_{\mathrm{D}}-\mathrm{SBP}_{\mathrm{T}}= \pm 5 \mathrm{~mm} \mathrm{Hg}$ for each cuff width. We also found that arm and thigh circumferences could be accurately predicted from the body weight within the first week of post-natal life.
82 PROSPECTIVE NATURAL HISTORY OF PDA IN PREMATURE INWay, Michael A. Simmons, Robert R. Wolfe, James J. Nora, univ. of Colorado Med. Sch., Dept. of Pediatrics, Denver

35 prospectively studied premature infants developed heart failure (CHF) associated with PDA. All patients met specific diagnostic requirements assessed by clinical exam, chest $x$-ray, ECG and daily echocardiographic measurements of the LA/Ao ratio. The protocol treatment included digoxin, diuretics and fluid and salt restriction in addition to routine care. $8 / 35$ had little to no lung disease; 7 of these 8 had clinical evidence of spontaneous closure of the PDA at a mean post natal age of 3 months. 4/35 died from causes unrelated to PDA/CHF. 21/35 had moderate/severe hyaline membrane disease (HMD) and required mechanical ventilation; 13 of these 21 required ligation of PDA for failure to improve on medical management. One died 3 days after surgery from severe BPD. 8 of the 21 with severe HMD improved with. medical management alone. One died from severe bronchopulmonary dysplasia with no evidence of PDA. The 13 patients requiring surgery differed as a group from the 8 who improved on medical management in that they were gestationally younger ( $30 \mathrm{vs} 33 \mathrm{wks}$ ) and smallex (1369 vs 1628 gins). Mean age of onset of CHF/PDA was the same in both groups ( 3.6 days). Using the criteria of $\mathrm{LA} / \mathrm{Ao}, \mathrm{F}_{\mathrm{T}} \mathrm{O}_{2}$, cardiothoracic (C.T.) ratio, and ventilator requirements, the medical and surgical groups were identical at onset of CHF. By day 3 of therapy the medical group had improved significantly $(p<.025)$ by the same criteria but the surgical group was unchanged.

83 MEDICAL MANAGEMENT OF VERY SMALL NEONATES WITH CONGESTIVE HEART FAILURE. Brenda L. Marino and William P. Kanto, Jr. (Spon. by Alex F. Robertson)

College of Georgia,

Management of the small neonate with patent ductus arteriosus (PDA) and congestive heart failure (CHF) remains controversial. We have added nasal continuous positive airway pressure (NCPAP) to our medical regimen and treated 6 very small neonates $(B W<1$ $\mathrm{kgm})$ with PDA and CHF in this manner. The average gestational age and birth weight were 28.2 weeks (range 17-32) and $862 \mathrm{gms}$. (780-920). Three infants had RDS and 3 required a mechanical ventilator (MV) prior to diagnosis of PDA. PDA was diagnosed at a mean age of 6 days (2-10 days) with onset of CHF at 12 days (5-23). INCPAP was applied on day $16(6-35)$ and was used for 11.3 days $(1-34)$. Three infants were weaned from MV to NCPAP. Two had apnea within 2 days, were returned to MV and subsequently died. Three were breathing spontaneously at time of diagnosis of $\mathrm{CHF}$, were treated medically with NCPAP and all suxvived. During the first 12 hours of medical management with NCPAP in this latter group the following mean changes were observed: $\mathrm{PaCO}_{2}+1.8 \mathrm{mmHg}, \mathrm{pH}+.08$, systolic blood pressure $+6.9 \mathrm{mmHg}$, respiratory rate $-9.2 / \mathrm{min}$, and heart rate $-11.4 / \mathrm{min}$. From 12-36 hours when digitalization was complete the mean change in $\mathrm{PaCO}_{2}$ was $-9.7 \mathrm{mmHg}$ and respiratory rate $-6 / \mathrm{min}$. There were no further changes in $\mathrm{pH}$, blood pressure, or heart rate. The survival of 4 infants suggests that NCPAP may be a valuable adjunct in the medical management of small infants with PDA and CHF.

\section{ECHOCARDIOGRAPHIC CHANGES DURING DIGITALIZATION IN} INFANCY William J. Marvin, Jr.; James L. Spratt: Richard vi. Schieken Sponsored by Ronald M. Lauer. University of Iov'a Hospitals, Department of Pediatrics, Iowa City, IA The correlation between echocardiographic left ventricular volume and function with serum digoxin level was assessed during dicitalization in infants. Four infants with left to right shunts 1 with congenital mitral requrgitation presented with the syndrome of conoestive leart failure (Clif ). All infant's improved following digitalization. Each had an echocardiogram (echo) initially, and again 2 hours after each digitalizing dose and after the 48 hour maintenance dose. Digoxin levels for radioimmunoassay vere trawn at the same time as the echo. The echo mean velocity of circumferential fiber shortening ( $\mathrm{VCF}$ ), \% shortening of LV minor axis (\%) and LV diastolic dimension (LVDd) were measured. All infants, despite severe CHF, had increased initial VCF $1.98+$ .11 (SE) $(n 1=1.34+.04)$ and increased \% 37.2+2 $(n 1=34)$. The LVD was increased $2.3 \bar{\sigma}+.58$. No significant change was found retween initial and post digitalization measurements: $Y_{C F} 1.99+.16$, \% $40.8+1.9$; and $L^{2} D_{\mathrm{d}} 2.04+.47$. Mean serum digoxin mainteñance leve1 was $\overline{2} .65+.81 \mathrm{ng} / \mathrm{mi}$. No correlation between V/CF $(r=0.06)$ or LVDd $(r=.01)$ with serum digoxin levels was found. CHF in infants with ventricular volume overload is associated with increased myocardial contractility. Clinical improvement in these infants following digitalization was not associated with further increase in myocardial contractility nor with significant lowering of LV diastolic volume. This suggests another mechanism must be present to account for the clinical response of the infants to digoxin. 


\section{5}

PARADOXICAL SPLITTING OF SECOND HEART SOUND IN TETRALOGY OF FALLOT

Kareem Minhas, Francisco Elbl, T.P. Ly, Allan J. Rees, Robert Solinger and Robert $W$. Hodge. (Sponsored by Billy $F$. Andrews). University of Louisville School of Medicine,

Department of Pediatrics, Louisville

This study was designed to investigate the nature and timing of pulmonic closure (PC) in Tetralogy of Fallot (TOF). of 125 consecutive cases, 92 were suitable for analysis. Procedures included cardiac catheterization, selective angiocardiography and simultaneous external phonocardiography with intracardiac pressure tracings in root of aorta and main pulmonary artery (MPA). A single second heart sound (S2) was revealed in 49 cases, 39 had a delayed and attenuated $\mathrm{Pc}, 3$ had normal splitting, and one had close splitting. In the 4 cases with a single S2, 12 showed one or all of the following: (a) P2 before A2; (b) MPA dicrotic notch prior to $\mathrm{A} 2$; (c) two distinct systolic murmurs at upper left sternal border. Only 9 of these 12 patients had MPS tracings which were almost identical; sharp upstroke, low early dicrotic notch and secondary wave before A2. No significant gradient was found across the pulmonary valve in these 9 cases. Hence, total or subtotal occlusion of right ventricle outflow tract during systole may cause premature closure of the pulmonary valve resulting in paradoxical splitting of $\mathrm{S} 2$ in TOF.

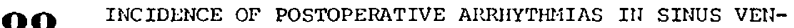
OSUS AND SECUINDUM ATRIAL SLPTAL DEFECTS, Marlene Rabinovitch, Macdonald Dick, William F. Bernhard, Aldo 12. Castaneda (Spon. by Alexander S. IJadas) Harvard Medical school, Children's Hospital ledical Center, Cardioloqy Denartment, Boston.

To answer the cruestion "Do sinus venosus(SV)atrial sental defects(ASD) have a higher incidence of rostoperative cardiac arrhythrias than secundum(II) ASD's?", we reviewed our surgical exnerience at the Children's llospital Medical Center of Boston witlin the last 15 years (yrs) $(1960-7(j)$. One hundred thirty five patients (pts)with ASD II, age range $1-51$ yrs (median=10 yrs) and 18 pts with sinus venosus defects, aqe range $5-46$ yrs, (median=12 yrs), were subjected to corrective surcery under cardionulmonary bypass. $\lambda$ mong the sinus venosus groun $50 z$ (9 out of 13) had transient supraventricular dysrhytumias, all disappearing before discharqe. In comparison, only 4 out of 40 pts(108 with ASD II matched to the sinus venosus groun for age and year of surgery had similar txansient postoperative dysrhythmias $(n<0.002)$. Eight of the 13 pts in the sinus venosus qroup were followed for $1-6$ yrs (median $=2$ yrs) postoperatively. Wone showed dysrhythmias during the follow-up period. By contrast, among the $70 / 134$ pts with ASU II followed postoperatively for a median period of 2 yrs (range 1-15 yrs) there were $4(5.78)$ with persistent dysrhythmias. ( 2 junctional rhythms, 1 sinus bradycardia, 1 sick sinus syndrome): none recfuired treatment. Thus although early transient postoperative arrhytlumias seem to be more common among the sinus venosus group, late nostoperative arrhythmias were only noted among the ASD II group.

89 NONINVASIVE ESTIMATION OF LEFT-TO-RIGHT SHUNT IN ISOLATED VENTRICULAR SEPTAL DEFECTS. Allan H. Rees, Judy J. Rigby, P. Syamasundar Rao and William B. Strong. Medical College of Georgia, Dept. of Pediatrics, Augusta, Georgia.

Cardiac catheterization (CC), an invasive technique, has traditionally been used to estimate the pulmonary to systemic flow ratio (Qp/QS) in infants and children with ventricular septal defects (VSD). Echocardiography (ECHO), a non-invasive technique, is used in this study to estimate CC determined Qp/Qs with ECHO dimensions. Ninteen patients, ages 6 wks. to 8 yrs., with isolated VSDs had ECHO within 24 hours of CC, and 19 children ages 3 to 11 yrs. served as controls. The left atrium to aorta ratio in the control group was $1.08 \pm 0.14$ and in VSD group, $1.37 \pm$ $0.13(\mathrm{p}<0.01)$. The left atrial internal dimension (LAID) and left ventricular internal dimension in diastole (LVIDd) were also determined and compared with the magnitude of $Q p / Q s$. The $r$ values for these respectively were 0.57 and 0.41 .

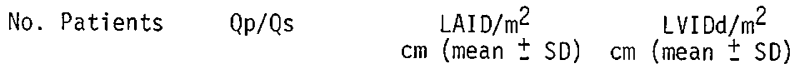
1
7
11
$<1.5$
1.5 to 2.0
$4.9^{2} \dot{+}^{3}+1.02$
$6.4 \pm 2.02$
$7.1^{4}+0$
$7.1 \pm 2.5$
$9.04 \pm 2.7$

propionate did not increase acetoacetate oxidation in hearts
taken from vitamin $B_{12}$ deficient rats.

These results indicate that propionate may be therapeutic in ketones.

\section{INDIRECT FETAL VECTORCARDIOGRAPHY-John T. Oldenburg,} Reserve University, Dept. Biomedical Engineering, Cleveland, Ohio.

Fetal electrocardiograms (FECG) recorded from the maternal abdomen have not been very useful for the study of the fetal heart for several reasons: 1) It has been difficult to record leads, other than midline, which are sufficiently noise-free for waveform analysis. 2) The orientation of the fetus in utero at the time of FECG recording was difficult to assess. 3)An orthogonal vectorcardiographic (VCG) lead system to accommodate the variety of fetal positions was not available. 4) Controversial "preferred conduction pathways" (low resistance connections between fetus and maternal abdomen) have been postulated to exist which for the fetal case would invalidate the volume conductor hypothesis upon which vectorcardiography is based. A study was done to evaluate and eliminate these problems.

A low-noise recording system incorporating "active electrodes" was designed to record four simultaneous FECG leads. B-mode ultrasound laminography was employed to determine orientation of the fetus in utero. A computer system was assembled for group averaging of fetal waveforms and for display of fetal QRS vector loops, both in the maternal VCG reference frame and by lead resolution in the fetal VCG reference frame.

Results from more than 50 normal subjects have shown that a change from volume to preferred FECG conduction apparently occurs over a period of 3 to 5 weeks between 25 and 35 weeks gestation and that indirect vectorcardiography is only possible in most subjects prior to 30 weeks gestation.
This study suggests that ECHO is a useful non-invasive technique in estimating $Q p / O S$ in patients with VSD.

PATHOGENESIS OF PARADOXICAL HYPERTEISION AFTFR COARCTATION RESECTION. Albert P. RocChini, Amnon Rosenthal A. Clifford Barqer, Aldo R. Castaneda, Alexander S. Ladas, harvard Medical School, Children's llospital Medical Center Cardiology Department, Boston, Massachusetts.

The pathoqenesis of paradoxical hypertension after resection of coarctation of the aorta(coarc) was investigated by comparing the course of 7 patients (pts)aged 7 to 14 yrs underqoing renair of coarc with 5 acyanotic pts 4 to 17 yrs undergoing othex types of elective cardiovascular surgery. Blood pressure(BP), plasma renin activity(PRA), cold pressor test(CPT), $24 \mathrm{hr}$ urinary sodium(INA) and potassium excretion, plasma volume and fluid balance were determined both preoperatively and daily postoperatively. During the first 24 hrs after surqery all coarc pts demonstrated a rise in systolic (sys) BP $(35 \pm 15.5 \mathrm{mmHq}$; mean $\pm S \mathrm{~S} ; \mathrm{p}<.001)$ a depression in $\mathrm{CPT}$ (sys $13.7 \pm 2.1 \mathrm{mmlig}$ and diastolic $8.2 \pm 1.4)(p<.001$ ) and onlv a slight elevation in PRA $(2.5 \pm 2.9 \mathrm{mq} / \mathrm{ml} / \mathrm{hr})(\mathrm{n}<.05)$. In the next 24$72 \mathrm{hrs}$ coarc pts developed a rise in diastolic BP $(26.8 \pm 10.6 \mathrm{mmHq}$; $\mathrm{D}<.001), \operatorname{PRA}(22.9 \pm 10.2 \mathrm{mg} / \mathrm{ml} / \mathrm{hr} ; \mathrm{p}<.001)$ and fluid retention with a decrease urinary $\mathrm{NA}$ excretion $65.1 \pm 8.2 \mathrm{mEq} / \mathrm{L}(\mathrm{p}<.001$ ). By contrast control pts had no significant postoperative chanqes in BP, CPT, PRA and only exhibited mild NA retention lasting for 2 days. Abdominal pain occured in 5 of 7 coarc pts during the period of maximal PRA. The data suggests that the sympathetic nervous system (i.e. depressed CPT and oublished reports of increased urinary catecholamines) mav be resnonsible for the initial nhase of sys hypertension after coarc resection and that the renin anaiotensin system plays a major role in the second nhase of paradoxical hypertension and in the nathogenesis of mesenteric arteritis. 
91

SUBAORTIC OBSTRUCTION AFTER THE USE OF AN INTRACARIDIAC BAFFLE TO TUNNEL THE: LEFT VENTRICLE TO THE AORTA. Albert P. Rocchini, Mmon Rosenthal, Aldo R. Castaneda, Alexander S. Nadas. (Spon. by Alexander S. Nadas). Harvard Medical School, Children's Hospital Medical Center, Cardiology Department, Boston, Massachusetts.

Postoperative hemodynamic studies in 5 patients(pts)document subaortic obstruction after surgical repair utilizing an intracardiac baffle to establish continuity between the left ventricle (LV) and the aorta(AO). Four of the pts had a Rastelli procedure for D-transposition of the great arteries (DTGA) with a ventricular septal defect(VSD) and pulmonary stenosis(PS)and one pt had repair of double outlet right ventricle (DOIV) with a VSD and PS. The pts aqe at surgery ranged from 8 to $26 \mathrm{yrs}$. All pts were thought to have a nonrestrictive VSD at preoperative catheterization and at surgery. Postoperative catheterization was performed 5 days to 8 yrs after repair. The LV outflow was shown to be a lonq narrow tunnel by anqiography in 4 of 5 pts and by echocardiography in 1 pt. Resting LV and no peak systolic pressure gradient ranged from 6 to 42 mmllq (mean 24) with a simultaneous cardiac index of 2.8 to $5.9 \mathrm{~L} / \mathrm{min} / \mathrm{N}^{2}$ (mean 4.2 ). The obstruction was localized to the proximal end of the LV to Ao tunnel(i.e. site of VSD) in 5 pts. One pt with an LV to Ao qradient of 42 mulla has angina and decreased exercise tolerance. Reoperation is planned with enlargement of the VSV and revision of the patch. Subaortic obstruction is a newly described sequela after the Rastelli procedure for DTGA or revair of DORV. The obstruction may be hemodvnamically siqnificant and should be searched for at postoperative cardiac catheterization.

92

INHOMOGENEOUS RECOVERY OF THE VENTRICLE: A PROPOSED MECHANISM OF SUDDEN DEATH IN THE LONG Q-T SYNDROME. J-Michel Roland, H. Leon Greene, P. Jacob Varghese, Johns Hopkins Hospital, Department of Pediatrics, Baltimore, MD. The mechanism of ventricular fibrillation (VF) in the long Q-T syndrome remains unclear. A patient ( $\mathrm{pt}$ ) without congenital deafness, who had spontaneous VF and long Q-T was evaluated by intracardiac electrocardiograms and stimulation studies. Five control pts with congenital heart disease and normal Q-T were also studied by the same methods.

Sinus node function was determined by overdrive suppression. AV node function was evaluated by pacing the atrium at a constant rate and introducing progressively premature atrial beats.

Effective refractory period (ERP) of the ventricle was assessed by introducing progressively premature ventricular beats synchronized to the sinus beat of the pt. The ERP was determined in 3 sites within the right ventricle.

Sinus node and AV node functions were normal in the pt with long $\mathrm{Q}-\mathrm{T}$ and the control pts. In the pt with long Q-T the ERP varied from 290 to $370 \mathrm{msec}$ within the right ventricle and the Q-T/ERP was greater than 1 . In the control pts the ERP ranged from 220 to $260 \mathrm{msec}$ and the Q-T/ERP was less than or equal to 1 . The data demonstrate a wide range in the recovery of the ventricular muscle in this pt with long Q-T interval whereas the recovery is homogeneous in the control group. This non-homogeneity of the ventricle is an ideal situation for re-entry and VF. This may be the mechanism of sudden death in the long Q-T syndrome. 93 THE UTILITY OF REAL TIME CROSS-SECTIONAL ECHOCARDIOTRICLE (SV). David J. Sahn, Stanley J. Goldberg, Hugh D. Allen and William F. Friedman, Dept. of Peds., Ped. Card. Univ. of Ariz. Med. Ctr., Tucson and Univ, of Calif., San Diego.

Multiple crystal cross-sectional echocardiograms were obtained of 10 patients (aged 1 day to 7 years) with a hemodynamic and angiographic diagnosis of $\mathrm{SV}$. In 4 patients, questions regarding great vessel orientation and atrio-ventricular (AV) valve structure remained even after angiographic study. The determination of complex spatial relationships of great vessels and intracardiac anatomy was substantially facilitated by this method compared to standard single crystal techniques. Dextrocardia in 3 of the 10 patients did not preclude reliable echo evaluation. Seven of the 10 could be shown echocardiographically to have transposition of the great vessels $(4 \mathrm{~d} ; 31)$. Five had pulmonary stenosis. A small outflow chamber unrelated to an $A V$ valve was demonstrated in 8 patients. Two $A V$ valves were demonstrated in 8 patients; in 3 , however, there was a gross discrepancy in the size of the two valves. Two patients had a single grossly abnormal AV valve. of 100 children with various forms of congenital heart disease examined subsequently, 2 patients with "corrected" transposition and ventricular malposition could not be distinguished from SV anatomy because of gross distortion of septal and $A V$ valve spatial orientation. Real time cross-sectional echocardiography is of significant utility in the noninvasive evaluation of this complex abnormality for delineation of intraventricular anatomy and great vessel relationships.
94 InCOMPlete CONGENITAL ATRIO-VENTRICULAR BLOCK IN M. Donner. $\frac{\text { David W. Sapire, Jitendra J. Shah }}{\text { (Spon. by A.B. Gruskin). Temple Univer- }}$ M. Donner. (Spon. by A.B. Gruskin). Temple Univer sity Department Philadelphia, Pennsylvania.

Five children with no obvious reason for prolonged atrio-ventricular (AV) conduction were studied by His Bundle Electrograms (HBE) and atrial pacing (AP). Three of the patients had normal cardiac anatomy, one had a patent ductus arteriosus and one had endocardial cushion defect. All had P-R intervals of $200 \mathrm{msec}$. or greater on Scalar electrocardiograms (ECG) and 2 patients demonstrated Wenckebach periodicity. HBE recorded at rest revealed $\mathrm{A}-\mathrm{H}$ intervals $1 / 3$ to twice normal values for age in 4 out of 5 cases and in one out of 4 there was also intra-atrial conduction delay, the P-A interval being $80 \%$ greater than normal for age. $\mathrm{AP}$ at rates only slightly higher than their normal basic rate rapidly produced prolongation of the $\mathrm{A}-\mathrm{H}$ interval in all patients. Wenckebach periodicity was seen at a pacing rate $50-75 \%$ lower than the expected rate for age. $\mathrm{H}-\mathrm{V}$ intervals were all normal. Intravenous Isuprel at a rate of $1 \mathrm{microgram} / \mathrm{min}$. resulted in normalization of the A-H interval in 3 patients and reduction of the $\mathrm{A}-\mathrm{H}$ interval in 2 , and rapid $\mathrm{AP}$ was achieved at rates $40-150 \%$ higher than resting rates. Atropine in a dose of $0.4 \mathrm{mgm}$. intravenously produced similar results.

These studies suggest that there is a form of congenital incomplete AV block possibly due to autonomic mechanisms that can be spontaneously overridden by endogenous catecholamines in response to exercise or anxiety and that the block is above the
Bundle of His.

95 ANGIOGRAPHY OF THE MITRAL VALVE IN CHILDREN WITH SECUNDUM ATRIAL SEPTAL DEFECT. Jon P. Shematek, Lilliam Valdes-Cruz, Glenn C. Rosenquist, Robert 1. White, Jr. The Johns Hopkins Hospital, Baltimore, Maryland.

The association of mitral valve abnormalities in children with ostium secundum atrial septal defect is ill-defined. Clarification of this relationship is of importance for long-term management of these patients. The purpose of this study was to correlate angiographic evaluations of the mitral valve leaflets with clinical data. Right anterior oblique left ventriculograms performed in 22 children (aged 1-11 years) with precatheterization diagnoses of uncomplicated ostium secundum defect were examined for scallops (distinct "bulges" in the posteromedial, anterolateral and/or medial portions of the posterior leaflet), prolapse (projection of any scallop beyond the plane of the annulus) and regurgitation (contrast entering left atrium only during systole, without premature ventricular contractions). Normal scalloping of the mitral valve was present in 15 of 22 children $(68 \%)$, mild prolapse in 5 $(23 \%)$ and regurgitation in none. Auscultatory and phonocardiographic examinations suggested mitral valve disease in 3 , none showing angiographic abnormalities. ECG showed superior QRS axes in 3 , all with normal mitral valves on ventriculograms. None of the 5 children with prolapse on angiography had clinical signs of mitral valve disease. In conclusion, until the results of longterm follow-up are available and the natural history of patients with subclinical mitral valve prolapse is clarified, children with secundum defects undergoing catheterization should have left ventriculography. Those with evidence of prolapse should be periodically evaluated.

\section{ECHOCARDIOGRAPHIC EVALUATION OF THE SEVERITY OF} VALVAR AORTYC STENOSIS IN CHILDREN. Norman $H$. Silverman, James W. French, Joanne Wallington, Richard $L$. Popp. Stanford University, California. (Spon.by M.A. Heymann). Echocardiography (ECHO) was used to predict the severity of valvar aortic stenosis(AS) in 15 children, aged 3 weeks to 18 years. We measured left ventricular(LV)dimensions and wall thickness, aortic root diameter and aortic leaflet motion. LV ejection time in msec(LVET)was measured between the points of separation and coaption of the aortic leaflet echoes; the pre-ejection period(PEP) was derived by subtracting the LVET from the interval between the onset of the QRS and the point of coaption of the aortic root echo signal. LVET was corrected for the heart rate (LVETC) from the formula LVETc (msec) $=1.6 \mathrm{x}_{t}$ heart rate + LVET. 15 matched controls had a mean LVETC of $389-21$ (S.D.), which was less than the LVETC of patients with AS 434 $27, \mathrm{p}<0.01$. ECHO results were compared to the peak systolic pressure difference across the aortic valve in $\mathrm{mm} \mathrm{Hg}$ (PSD). LVETc exhibited a close correlation with the PSD with PSD $=0.90 \mathrm{x}$ LVETc $-341, \mathrm{r}=.95$. In 5 children who underwent aortic valvotomy LVETc diminished from a mean of $457 \pm 28$ to $417 \pm 14 \mathrm{msec}, \mathrm{p}<0.05$. The PEP/LVET ratio, LV cavity dimensions and mean velocity of circumferential fiber shortening did not correlate with the severity of AS. While suggesting AS, aortic leaflet motion, multiple diastolic or eccentric lines within the aortic root, and increased LV wall thickness did not correlate we11 with severity. We conclude that LVETc measured by $\mathrm{ECHO}$ is a useful non-invasive guide to judge the severity and follow the progress of AS in childhood. 


\begin{abstract}
ECHOCARDTOGRAPHIC FEATURES OF TOTAL ANOMALOUS PULMONARY VENOUS DRAINAGE IN THE NEONATE

Robert Solinger, Francisco E1b1, Lily George, T.P. Ly, Madapura S. Satyanarayana, Kareem Minhas. (Sponsored by Billy F. Andrews). University of Louisville School of Medicine, Department of Pediatrics, Louisville.

Echocardiographic features of right ventricular diastolic volume overload (increased right ventricular dimension + abnormal septal motion) and decreased left sided (left atrial, left ventricular, and aortic root) dimensions have been described in older infants with total anomalous pulmonary venous drainage (TAPVD). However, little data is available for the newborn. We have studied three newborn infants with pulmonary vein atresia and one infant with TAPVD into the portal vein within the first 5 days of life. In addition, we studied one infant with TAPVD into the coronary sinus at ten days of age. All five infants had normal right sided dimensions and septal motion. Four had normal left sided dimensions. One of the infants with pulmonary vein atresia had decreased left sided dimensions. These findings are consistent with the type of quantitative chamber and great artery dimensions anticipated as a result of known fetal circulation and suggest that TAPVD cannot be ruled out by a normal echocardiographic study in the early neonatal period. Further, they imply that a finite amount of extra-uterine time is required to develop the findings of right ventricular diastolic volume overload, and in most cases, of a small left side.
\end{abstract}

98 THE NATURAL HISTORY OF DOUBLE OUTLET RIGHT VENTRICLE. Children, Toronto Henry M. Sondheimer, Robert M. Freedom, and Peter M. lley (Intr. by R.D. Rowe), Hospital for Sick

We have performed a retrospective study of all children with double outlet right ventricle born from 1956 through 1975. There have been 87 such patients, 43 of whom are currently alive, 35 dead $(40 \%)$, and nine lost to follow-up. Autopsies were performed on 31 of the 35 that died. Patients were placed in four groups; those with subpulmonic VSD, subaortic VSD, subaortic VSD and pulmonic stenosis, and those with uncommitted VSD.

Patients with subpulmonic VSD presented earliest (median six weeks) and had the poorest prognosis. There were 26 such patients; 16 died $(62 \%)$, two have pulmonary vascular disease, five are palliated, and only one is "corrected". Two are lost. of 17 with subaortic VSD, eight died (47\%), three are "corrected", three are palliated, and one is awaiting surgery. Two are lost. The 28 patients with subaortic VSD and pulmonic stenosis presented at a median age of four months; only seven died (25\%), seven are "corrected", 12 palliated, one unoperated, one lost.

of the 35 deaths, nine were related to abnormalities of the mitral valve - six atresias, two severe mitral stenosis, and one straddling mitral valve which was replaced at the time of intracardiac repair. Of eight patients with aortic coarctation, only two have survived and they were palliated in the last two years. We conclude that the prognosis in double outlet right ventricle depends on the specific anatomy present and that particular attention must be paid to the mitral valve and aortic arch.

\section{9}

SARCOMERE LENGTH IN THE LEFT VENTRICLE OF THE PUPPY William D. Spotnitz, Henry M. Spotnitz, Nestor J. Truccone, Thomas S. Cottrell, Weiton Gersony, James R. Malm, and Edmund H. Sonnenblick, Columbia Univ. College of Phys-

icians and Surgeons, Depts. of Pediatries and Surgery, N.Y., N.Y. Although the relation of sarcomere length (SL) to diastolic pressure ( $P$ ) and volume (VOL) in the left ventricle (LV) of the adult dog have been defined, data have not been detailed for the immature animal. Accordingly, in $18 \mathrm{chloralose-anesthetized} \mathrm{mon-}$ grel puppies (1 $\mathrm{Kg}$ average weight) hearts were arrested with potassium chloride. After reproducible curves relating $P$ and VoL were obtained, each LV was fixed by glutaraldehyde coronary perfusion, while $P$ was maintained at a predetermined value between 0 and $30 \mathrm{~mm} \mathrm{Hg}$. Midwall SL in the free wall of the LV was related to fixation $P$ and VOL (silastic casts). Results were compared with adult data from other studies. Averages are tabulated:

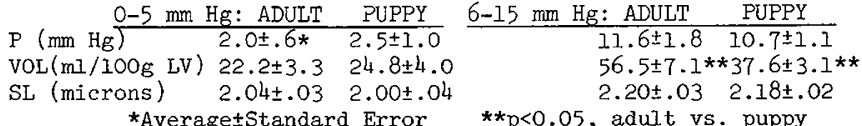

In the puppy, the relation of SL and $P$ is similar to the adult $\mathrm{LV}$ at physiologic $\mathrm{P}$. However, compliance is reduced, and sarcomeres resist stretch above $20 \mathrm{~mm} \mathrm{Hg}$ P. Variation in SL and cell size appears increased. Electron microscopy shows many immature sarcomeres under assembly from free myofilaments. While the ultrastructural limits on performance are similar in the adult and puppy LV, the puppy appears better protected against damage from volume overloads.
100

FLUID ADMINISTRATION IN THE ASSOCIATION OF PATENT DUCTUS ARTERIOSUS COMPLICATING RESPIRATORY DISTRESS SYNDROME (PDA/IRDS). James G. Stevenson (Spon. by william F. Fxiedman) Dept. Pediatrics, Div. Pediatric Cardiology, Naval Regional Medical Center, San Diego, Calif 92134

To determine the effect of fluid administration in PDA/IRDS, the nursery courses of 21 infants with IRDS and birth weights less than 2000 grams were reviewed. In 11, there was significant left to right shunting through PDA manifest by murmur, cardiomegaly, elevation of echocardiographic left atrial to aortic ratio, and need for increased respiratory support. In 9 of 11 , there was unusual difficulty in weaning from respiratory support, and 2 required PDA ligation. 10 of the 21 infants with IRDS did not develop PDA and served as controls. There was no significant difference between groups in terms of weight $(1.5 \mathrm{Kg})$, gestational age (33wk), postnatal age at time of PDA(5days), Hematocrit $(42.6)$, use of radiant warmers, phototherapy or antibiotics. There was a significant difference in mean daily fluid volumes administered, the PDA group receiving $222 \pm 33.2 \mathrm{cc} / \mathrm{kg} /$ day, and the control group, $147+26.1 \mathrm{cc} / \mathrm{Kg} /$ day; $\mathrm{F}=<.001$. The timing of increase in fluid administration was closely related to appearance of PDA, and diuresis was associated with improvement in, or resolution of, signs of PDA. In 4, later fluid excesses led to return of PDA. While the association of PDA/IRDS must have several contributing factors, these results suggest that excessive fluid administration to premature infants with IRDS may be casually associated with PDA complicating IRDS.

\section{CARDIAC INVOLVEMENT IN JUVENILE RHEUMATOID ARTHRITIS \\ (JRA)}

Aluizio Stopa, Richard A. Meyer, Joseph Levinson, Joan Korfhagen and Samuel Kaplan, University of Cincinnati, Children's Hospital, Dept. of Pediatrics, Cincinnati, Ohio

This study was undertaken to define the extent of cardiac involvement in JRA and to demonstrate the incidence of pericardial effusion. We performed 74 echocardiograms in 67 patients (ages 2-22 years) with JRA diagnosed by clinical and laboratory findings. Patients with other Collagen disorders and doubtful diagnoses were excluded. Most were examined as outpatients and were grouped at the time of the examination as Active-43 (Systemic-3, Polyarticular-20, Pauciarticular-13, Monoarticular-7) Remission-22 and Undecided-2. Pericardial fluid was detected in only one patient who had systemic disease and an acute illness. Pericardial thickening was observed in one patient in remission. Left ventricular function assessed by measuring shortening fraction (SF) revealed that only 3 of 54 patients had SF less than $28 \%$ while 8 exceeded $40 \%$, as compared to a normal value of $35 \%(+5 \%)$. However, the right ventricular dimension exceeded norma $\bar{T}$ values in $30 \%$ of patients. All chest radiographs were norma 1. The electrocardiogram showed right ventricular hypertrophy in 3, first degree heart block in 3 , and the rest were normal. We concluded that (1) right ventricular enlargement of unknown cause occurred in about $1 / 3$ of patients, (2) left ventricular function is usually normal and (3) pericardial effusion is rare.

\section{SYSTOLIC BLOOD PRESSURE IN BLACK CHILDREN: AT REST} AND IN RESPONSE TO ISOMETRIC AND DYNAMIC EXERCISE:
Michael R. Striplin, William B. Strong, Max D. Miller, Mansoor Salehbhai; Medical College

epartment of Pediatrics, Augusta.

One hundred and seventy normal black children were studied to determine their resting blood pressure (BPrest) and their systolic blood pressure response to isometric exercise ( $\mathrm{BP}_{\text {iso }}$ ) and dynamic exercise at a heart rate of 170 ( $B P 170)$ and maximum ( $B P_{\max }$ ). Resting blood pressure increased with age and body surface area $(B S A)$, no differences were noted between males and females at any age or $B S A(p<0.02)$. BP response to isometric exercise (BPiso) increased with age (males(M) $r=.37$, females $(F) r=.40$ ) and BSA $M$, $r=.42, F, r=.37$ and no differences were noted at any BSA for $M$ or $F$. $B P$ response to dynamic exercise at a heart rate of 170 and maximum correlated similar to the isometric for age and BSA with no difference between $M$ and $F$. There was an inverse relation of $B P$ rest and absolute change in $B P_{i s o}$ and $B P 170$ and $B P_{\max }$ (i.e. the higher $B$ Prest the smaller the absolute change in response to exercise). The peak values observed for $\mathrm{BP}_{\mathrm{iso}}, \mathrm{BP} 170$ and $\mathrm{BP}_{\max }$ were 160,180 and 200 respectively. BP is proportional to the cardiac output (CO) times the systemic vascular resistance (SVR). The variable of $\mathrm{CO}$ can be minimized by producing an elevated $\mathrm{CO}$ by exercise and thus making SVR the major variable of BP response to exercise. It is concluded that blood pressure response to exercise exceeding $2 S D$ above the mean is suggestive of increased systemic vascular resistance and may be a risk factor for the development of hypertension later in life. 
103 THE PHYSICAL WORK CAPACITY OF NORMAL BLACK CHILDREN: William B. Strong, Dennis Spencer, Max D. Miller, Talmadge Hospital, Department of Pediatrics, Augusta.

The purpose of this study was to design a standardized exercise protocol and to evaluate the protocol by determining the physical work capacity (PWC) of normal black children. A continuous exercise protocol using a mechanically braked bicycle ergometer was designed. Measurements included (1) work performed (in $\mathrm{KgM} / \mathrm{min}$ ) as either a submaximal (PWC at a heart rate of 170--PWC 170) or maximal load (total work load--TWL), (2) the response of the electrocardiogram to stress (J-point deviation and ST segment slope quantitation), (3) blood pressure response to isometric and dynamic exercise. The protocol is simple to perform in the hospital or in the field with normal children or children with heart or pulmonary disease. The instrumentation is reasonably inexpensive requiring minimal maintenance. The adoption of a "standard" protocol for children would enable better comparison of information from various centers. One hundred and seventy normal b]ack boys and girls seven to fourteen years of age were evaluated by means of the above protocol. Maximum heart rates achieved varied from 170 to 210 . Generally, the absolute values (PWC170 and TWL) were greater for boys than for girls. However, for a given BSA, boys and girls less than ten years of age had similar PWC 170. There was a good correlation between PWC 170 and total work performed for boys $(r=0.81)$ and girls $(r=0.69)$. The results of the PWC 170 , a submaximal workload, are comparable to values previously obtained from California children.

ECHOCARDIOGRAPHIC ASSESSMENT OF CHILDREN WITH HOMOZY GOUS SICKLE CELL DISEASE (SS): Allan Rees, Priscilla Gilman, Judy Rigby, Judith McFarlane, Max D. Miller William B. Strong; Medical CoTle ge of Ga, Dept of Peds, Augusta. By means of the following echocardiographic measurements: ve1ocity of circumferential fiber shortening (Vcf), ejection fraction (EF), left ventricular posterior wall thickness (LVPW) and left ventricular diastolic internal dimension (LVIDd), 44 SS patients (pts) and 27 normal black children were evaluated. On the basis of cardiovascular examination and x-ray findings, the pts were classified according to disease severity: mild (GP I), moderate (Gp II), severe (Gp III)

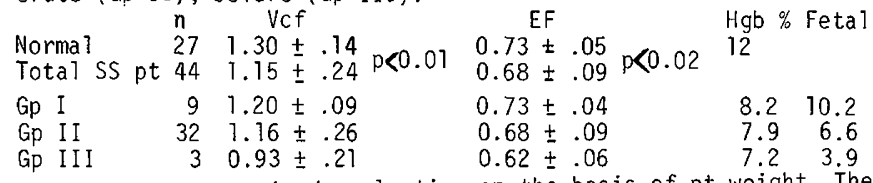
LVPW and LVIDd required evaluation on the basis of pt weight. The mean LVPW of the total SS pts group was greater than the mean mean LP the normals. The mean value for LVIDd for total SS pts was also greater than the control group LVIDd. This study demonstrates that LV performance is impaired in children with SS anemia. The degree of impairment correlates with clinical severity but not with \% fetal Hgb or Hgb level between groups. Echoity but not with measure by which clinical severity and effects of therapeutic interventions can be assessed on these pts.

\section{5}

NON-INVASIVE DETECTION OF LEFT-TO-RIGHT SHUNTS BY INHALATION OF $\mathrm{Cl}^{15} \mathrm{O}_{2}$. Dolores M. Tamer, Dennis R. Watson, Warren R. Janowitz, Peter J. Kenny, Albert J. Gilson, and Henry Gelband. Univ. of Miami, Dept. of Ped, and Nuc. Med. Dept. of Nuc. Med., Mt. Sinai Hosp., Miami Beach, Fla.

Cardiac catheterization (CC), an invasive procedure is the definitive method employed to document the presence and magnitude of a left to right $(L \rightarrow R)$ intracardiac shunt. Oxygen 15 labelled carbon dioxide $\left(\mathrm{C}^{15} \mathrm{O}_{2}\right)$, which has a half 1 ife of 2 min., was used to develop a totally non-invasive method to detect $L \rightarrow R$ shunts. $A$ bolus of inhaled $\mathrm{C}^{15} \mathrm{O}_{2}$ is delivered to the pulmonary venous blood via the lungs. Radioactivity counted over a peripheral artery is displayed as an indicator-dilution curve and analyzed similarly. A prospective study was undertaken in 39 patients to define the value of $\mathrm{C}^{15} \mathrm{O}_{2}$ inhalation in the detection and quantification of $L \rightarrow R$ shunts in children age $2-16$ years admitted for elective CC. 17 patients had no evidence of $a \mathrm{~L} \rightarrow \mathrm{R}$ shunt at the time of $\mathrm{CC}$ by oximetry or angiography. None of these patients had a positive $\mathrm{C}^{15} \mathrm{O}_{2}$ study; 20 of $22 \mathrm{CC}$ documented shunts were detected by the $\mathrm{C}^{15} \mathrm{O}_{2}$ inhalation method. Two false negatives occurred in shunts of $<23 \%$ of pulmonary blood flow. In 20 cases where the shunt ratio by oximetry was $>1.3 / 1$, there was a good correlation $(\mathrm{r}=.78)$ with the $\mathrm{C}^{15} \mathrm{O}_{2}$ method. This technique is non-invasive, repeatable, safe, and correlates well with oximetry values obtained at CC. Thus it is a valuable new method for the detection, confirmation and quantification of $L \rightarrow R$ intracardiac shunts.

106

ACCELERATED OR DELAYED "FUNCTIONAL" CLOSURE OF DUCTUS ARTERIOSUS (DA) AND LUNG MATURATION IN PRETERM INFANTS Donald W. Thibeault, George C. Emmanouilides, Michael E. Dodge, and Ralph Lachman, UCLA School of Medicine, Harbor General Hospita1, Department of Pediatrics, Torrance, California.

It is established that accelerated alveolar surfactant maturation in the fetus can occur. However, it is not known if a concomitant ductai maturation occurs. The clinical course of 143 preterm infants (<34 weeks gestation) was analyzed for the presence of the respiratory distress syndrome (RDS) and patent ductus arteriosus (PDA). The patency of the $D A$ was established clinically or by aortography, RDS by clinical criteria and alveolar surfactant maturation by the Foam Stability Test (FST).

Infants with closed ducti had intermediate or positive FST and did not have severe RDS. However, $50 \%$ of those infants with open ducti had severe RDS and large left to right ductal shunts after 24 hours. No RDS occurred in infants born after premature rupture of membranes (PROM) $>48$ hours. However, PDA was present in $50 \%$ of infants with PROM 48-72 hours, but rarely after 72 hours.

Fifteen infants 35-37 weeks gestation with severe RDS were also studied. In 5 of these, the FST was measured and it was negative. All had a PDA and the aortograms (in contrast to the younger infants with RDS) in the majority of them (10/15) suggested persistence of high pulmonary vascular resistance for several days.

These observations suggest that when there is accelerated alveolar surfactant maturation, there is accelerated ductal maturation as well; and when alveolar surfactant maturation is delayed, ductal maturation is also delayed.

\section{7}

COARCTATION OF THE AORTA IN RABBITS: A VECTORCARDIOGRAPHIC AND :1ORPHOLOGIC STUDY, JOn B. Tinqelstad and Jack L. Haar. (Spon. by H. M. Mauxer). Depts. of Pediatrics and Anatomy, Medical Collece of Virqinia, Richmond. Coarctation of the abdominal aorta was produced in 8 young rabbits (594 - $824 \mathrm{gm}$ ) to determine the effect on the Frank vectorcardiogram (VCG) and on the myocardium as demonstrated by light and electron microscony (Group 1). Seven rabbits (368 $856 \mathrm{~cm}$ ) served as controls (Groun 2). VCG's were obtained at the beqinning and end of study periods ranging from 51 - 84 days. Five in Group 2 showed the expected increase in left ventricular oreponderance. Only 3 in Groun 1 demonstrated increasing left ventricular voltage, whereas 5 showed right ventriculax preponderance or hypertronhy althouah there was no demonstrable cause for or gross evidence of right ventricular hynextronhy (RVH). Mean pressure differences across the coarctations in 6 rabbits ranged from 1 to $31 \mathrm{mmil}$ (mean $15 \mathrm{mmlla}$ ). At necronsy Group 1 hearts were increased in size, $2.32 \mathrm{~cm} / \mathrm{Kq}$ of body weight, comnared to $1.9 \mathrm{~cm} / \mathrm{Kg}$ for Group 2. Measurements of ventricular wall thickness showed significant increase in the left ventricular base in Groun 1 specimens $(p<0.05)$. Linht and electron microscopy demonstrated changes of hypertrophy, namely, increases in intercellular snace, connective tissue, numbers and diameter of blood vessels, numbers of mitochondria with multi-lobulated configurations, and sarcomere lenath, limited nrimarily to the base of the left ventricle or interventricular septum. These observations suqqest that hypertronhy of the base of the left ventricle oroduces VCG changes which have previously been read as RVH.

108

HYPERLIPIDEMIA (HL) IN CHILDREN (Ch.) FROM HIGH RISK FAMILIES. Chandra M. Tiwary (Spon. by C. Angle)Dept. of Ped., Univ. of Nebr. Med. Cent., Omaha, NE Hyperlipidemia is a major risk factor associated with the development of premature atherosclerosis; therefore, detection of $\mathrm{HL}$ in $\mathrm{Ch}$. would be a major initial step in its control. As mass screening for HL is too expensive, Ch. from 63 high risk families (in which one or both parents had clinical and EKG features of coronary heart disease (CHD), or had a stroke or were known to have HL) were screened for hypercholesterolemia and hypertriglyceridemia using age related normal values established by Friedman and Goldberg and by Frederickson et. al. The Ch. were on a regular diet. Venous blood was obtained after a 12-14 hr. fast. In 4 families there was a history of stroke; in 51 families a history of CHD; and in 8 families, HL. The 63 families had 205 Ch.; lipid analysis was performed in $162 \mathrm{Ch}$. The age range was $1 \frac{1}{2}-32$ yrs.; $3 / 4$ of the offspring were 21 years old or less. Of the 162 offspring, $39(24 \%)$ had HL (32 were type II and 7 were type IV). Of the 120 offspring of 51 families with a parental history of CHD, $30(25 \%) \mathrm{Ch}$. had HL. These $30 \mathrm{Ch}$. came from 19 families. Of 8 families screened because of parental HL, 5 had Ch. with HL. In 13 additional families, a parent had HL and also CHD, 4 of these families had Ch. with HL. None of the parents with a history of a stroke had Ch. with HL. Since $24(38.9 \%)$ families out of 63 had Ch. with HL it is recommended that serum cholesterol and triglyceride be determined in all offspring from high risk families. It is expected that $>1 / 3$ of such families would have offspring with HL. The study was supported by a grant from the Nebraska. Regional Medical Program. 
109

COMBINED HEMODYNAMIC EFFECTS OF PAIRED VENTRICULAR

PACING (PVP) AND ISOPROTERENOL (I) IN DOGS. Nestor J. Truccone and Ehud Krongrad. College of Physicians Surgeons, Columbia U. Department of Pediatrics, N.Y.C. 10032. Increased tachycardia with I infusion may constitute a contraindication for its use in some patients with low cardiac output $(\mathrm{LCO})$. The present study was undertaken in order to evaluate the hemodynamic effects of simultaneous PVP and I infusion and its potential use for the treatment of LCO. In each of 8 dogs, simultaneous ECG, cardiac index (CI), left ventricular (LV) systolic (LVSP), end diastolic (LVEDP) and aortic (AOP) pressures and LV $\mathrm{dp} / \mathrm{dt} / \mathrm{p}$ were measure and LV stroke work index (LVSWI) calculated in 4 states: ) concrol state (2) PVP at $150 / \mathrm{min}$. (3) I infusion $(0.04 \mu / \mathrm{kg} / \mathrm{min})(4)$ simultaneous PVP $(150 / \mathrm{min})$ and I $(0.04 / \mathrm{\mu} / \mathrm{kg} /$ min). Our results show the following:

\begin{tabular}{|c|c|c|c|c|c|c|}
\hline \multirow[t]{2}{*}{ HR } & $\mathrm{CI}$ & LVSP & LVEDP & $\mathrm{AOP}$ & $\mathrm{LV} d p / d t / p$ & LVSWI \\
\hline & $\mathrm{L} / \mathrm{m} / \mathrm{M}^{2}$ & $\mathrm{~mm} \mathrm{Hg}$. & $\mathrm{mm} \mathrm{Hg}$. & $\mathrm{mm} \mathrm{Hg}$. & sec-1 & $\mathrm{g} / \mathrm{m} / \mathrm{M}^{2}$ \\
\hline & $\frac{1.98}{2}$ & 150 & 7.2 & 127 & 53.9 & 29.0 \\
\hline 2) 150 & 4.05 & 154 & 6.8 & 125 & 75.2 & 47.9 \\
\hline 3) 222 & 6.61 & 152 & 4.1 & 117 & 101.2 & 46.3 \\
\hline 4) 150 & 6.53 & 148 & 5.6 & 112 & 104.9 & 61.9 \\
\hline
\end{tabular}
I resulted in significant increase in heart rate, CI and LV $\mathrm{dp} / \mathrm{dt} / \mathrm{p}$ when compared to control state and PVP. Simultaneous PVP at $150 /$ min with I resulted in similar increase in cardiac hemodynamics with marked increase in LVSWI. Our findings suggest that simultaneous PVP and I infusion may be a useful method for the treatment of patients with LCO with tachycardia.

\section{1 eFfect of Partial ganglionic blockage upon RENAL AND} CARDIOVASCULAR FUNCTION IN BURNED DOGS. Richard Turner, Hugo F. Carvajal and Daniel L. Traber, Spon. by Luther B. Travis, Univ. of Texas Med. Br. and Shriners Burns Inst., Depts. of Anesth. and Ped., Galveston, Texas.

Studies to test the effects of partial ganglionic blockade on renal and cardiovascular function were carried out in 16 mongrel dogs which under chloralose anesthesia had been subjected to ful thickness flame burn to approximately $25 \%$ of their body surface. A11 animals received intravenous fluid replacement according to the same resuscitation program we use in burned children (SBI). Forty minutes after the burn half of the animals received 0.3 $\mathrm{mg} / \mathrm{kg}$ of chlorisondamine hydrochloride; the remaining 8 dogs received on1y the vehicle. Among the variables monitored before burning and before and after blockade were glomerular filtration rate, renal plasma flow, water and osmolar clearances, sodium and potassium excretion, cardiac output, mean arterial, right atrial, and left ventricular end diastolic pressures, peripheral resistance, peak $\mathrm{dP} / \mathrm{dt} / \mathrm{P}$ and $\mathrm{Ph}$ and blood gases. Analys is of the data has revealed that pharmacologic blockade of the sympathetic system during the immediate post burn period results in a marked improvement in cardiac output and moderate improvement in kidney function. The changes were so dramatic that such therapy should be seriously considered in severe burn victims.
112

LIGATION OF PATENT DUCTUS ARTER IOSUS (PDA) IN HYALINE MEMBRANE DISEASE (HMD): A CONTROLLED STUDY. D. Vidyasagar, T.N.K. Raju, E. Fisher, A. Hastreiter, S. Levitsky, E. Bennett, Abraham Lincoln School of Medicine, U. of 11 Various studies suggest that ligation of PDA in infants with HMO increases survival. We report the results of a controlled study of infants with HMD and PDA requiring assisted ventilation (Asst. Vent.) who were randomized for ligation of POA. Infants with significant PDA ( 2 of the 4 set criteria: (1) bounding pulses (2) hyperactive precardium (3) third heart sound (4) cardiac murmur) were digitalized. If there was no improvement after $24 \mathrm{hrs}$ they were randomly assigned to 1 . Surgical or 11 . Medical treatment. Group I infants were treated with surgical ligation (early surgery). Group II infants were continued on digitalis until recovery or 10 days. If the infant did not recover by 10 days PDA was ligated (late surgery). 27 infants entered the study overall $9 / 14$ surgically treated, $9 / 13$ medically treated infants survived. Birth weight, gestational age, Apgar scores and initial acid base status between the two groups were not significantly different. Survival of infants with early and late surgery was not significantly different $(3 / 6,6 / 8)$. The duration of Asst. Vent. was shortest for medical group $(9.5 \pm 1.8$ days) and was not different between early and late surgical groups $(33.33+5.7$ and $31.6+6.4$ days). Duration of Asst. Vent. after digitalization in medical group was significantly shorter than after PDA ligation in early surgery group $(5.0+1.66$ and $26.6+6.06$ days, $p<0.001)$. The results suggest that 1 igation of PDA in HMD did not alter the outcome or course of the disease.

13 PULMOIIARY VENOUS WELGE PRESSURE IN PULMOHARY ARTFRY MYPERTEINST ON. J. Deane Waldman, Michael A. LaCorte, (spol, by Alexander S. Nadas). Harvard lledical School, Children's Hospital iledical Center, Department of Cardiology, Boston.

pulmonary venous wedge pressure (PVWP) was compared to pulmonary artery pressure (PAP) in patients with significant pulmonary artery hypertension (PAH)-systolic (syst) PAP $\geqq 50 \mathrm{mmila}$. Forty-seven of 49 patients $(96 \%)$ with such PAIl had a mean PVIT $\geqq 20 \mathrm{mmHg}$.

\begin{tabular}{|c|c|c|c|c|c|c|}
\hline \multicolumn{4}{|c|}{ TOHAI, GROUP ( $n=49)$} & \multicolumn{3}{|c|}{ IPSL GROUP $(n=20)$} \\
\hline & $\overline{\mathrm{PVHP}} \star$ & $\overrightarrow{\mathrm{PAP}}$ & Correlation & PVWP* & $P \wedge P^{*}$ & Correlation \\
\hline sy & $\overline{17-61}$ & $50-110$ & IIS & $\overline{27-53}$ & $\overline{50-96}$ & ITS \\
\hline dias & $10-40$ & $10-61$ & $r=0.33$, reco.05 & $10-40$ & $12-45$ & $r=0.75, p<0.001$ \\
\hline , & $17-44$ & $30-77$ & $x=0.34, x<0.05$ & $17-44$ & $32-59$ & $r=0.71,0<0.001$ \\
\hline
\end{tabular}

When diastolic(diast) and mean PVWP and PAP were compared (Table) weal but significant $(p<0.05)$ correlations were found. Comparison of mean branch PAP and ipsilateral(Ipsl)mean PVWP yielded a strong correlation: $r=0.71$, with $\mathrm{SE}=6 \mathrm{mmlig}$. It is concluded that carefully measured mean PVIT is a reasonably reliable indicator of mean branch $P \Lambda P$ up to $60 \mathrm{mmHq}$ mean $P A P$, and may be helpful in identifying those at high risk for pulmonary vascular disease (when PAP cannot be obtained directly).
111 PATHOLOGICAL ANALYSIS OF THE MITRAL APPARATUS IN OSTIUM SECUNDUM ATRIAL SEPTAL DEFECT. Lilliam ValdesCruz, Jon P. Shematek, Glenn C. Rosenquist, Hugh A. McAllister. The Armed Forces Institute of Pathology, Washington, D.C.
Recent awareness of clinically silent mitral valve anomalies Recent awareness of clinically silent mitral valve anomalies
isolated ostium secundum atrial septal defect (OSD) prompted this study of 35 specimens (16 infants $<1$ year, 9 children, 10 adults) with OSD (absent or fenestrated valve of fossa ovalis). Ratio of circumference of tricuspid and mitral valve annuli (TVC: MVC), number and prominence of scallops (semicircular segments of posterior leaflet) and abnormalities of leaflets, papillary muscles or chordae were compared to 32 age-matched normal hearts. In OSD the average TVC:MVC $=1.43(1.05-2.06)$, normal (N) TVC:MVC $=$ $1.09(.98-1.22)$. In hearts with OSD 3 distinct scallops were noted in $62 \%$ of infants $(\mathrm{N}=64 \%), 77 \%$ of children $(\mathrm{N}=74 \%)$ and $80 \%$ of adults $(\mathrm{N}=100 \%)$; ill-defined scallops in $38 \%$ of infants $(\mathrm{N}=27 \%), 11 \%$ of children $(\mathrm{N}=21 \%)$ and $10 \%$ of adults $(\mathrm{N}=0 \%)$; supernumerary scallops in no infants $(\mathrm{N}=9 \%), 11 \%$ of children $(\mathrm{N}=5 \%)$ and $10 \%$ of adults $(\mathrm{N}=0 \%)$. No mitral valve anomalies were noted in normal specimens. Abnormalities in OSD hearts included prominent scallops ( 1 infant, 2 children and 2 adults); thickened leaflets ( 1 child, 4 adults); accessory papillary muscle supporting a restricted anterior leaflet (1 adult); forme fruste arcade of anterior leaflet ( 1 child); and abnormal lengths of chordae (4 children, 2 adults). In conclusion, with increasing age, a significant percentage of hearts with OSD have mitral valve anomalies, demonstrating the need for greater clinical attention to this combination of defects.
11 ECHOCAR̈DIOGRAPHIC LEFT VENTRICULAR FUNCTION IN INFANTS NITH CONGESTIVE FAILURE. Robert D. White, Lidward B. Clark, P. Jacob Vargnese, Daniel R. Pieroni, and Paul . Lietman. The Johns Hopkins Hospital, Baltimore, Maryland.

Congestive heart failure (CHF) is thought to represent impaired myocardial contractility, al though this relationship is not clear in infants. Left ventricular (LV) function was evaluated by echocardiogran in 33 infants under 6 months age with CHF 13 myocarditis, 5 LV obstructive lesions, 25 left to right shunts). Velocity of circumferential fiber shortening $\left(V_{c f}\right)$, ejection frac tion $(\mathrm{EF})$, and percent change in IV diameter witn systole $(\% \Delta D)$ were calculated.

Results (graph) show that infants with myocarditis or obstructive lesions nad low Vcf, while the Vcf in infants with shunts was normal or increased. Graphs of $E F$ and $B \triangle D$ were similar. Irifants with shunts and increased Vcf usually responded poorly to anticongestive therapy and required early surgery.

These data suggest that factors other than impaired myocardial contractility are responsible for the signs and symptoms of CHF in infants with shunts; understanding of these factors may improve the success of medical management of $\mathrm{CHF}$ in this group of infants. 
115

THE EFFECT OF CARDIAC GLYCOSIDES ON MECHANICAL

FUNCTION IN THE HYPOXIC MYOCARDIUM. Steven M. Yabek, Jay M. Jarmakani, Makoto Nakazawa, UCLA School of Medicine, Department of Pediat rics, Los Angeles.

The inotropic effects of acetylstrophanthidin (ACS) on hypoxic myocardium were studied in 17 arterially perfused rabbit interventricular septa. The results were compared to a control group of 23 septa subjected to hypoxia alone. Hypoxia was introduced for 30 minutes by perfusate equilibrated with $95 \% \mathrm{~N}_{2}$ and $5 \% \mathrm{CO}_{2}$ following one hour of perfusion with $95 \% \mathrm{O}_{2}$ and $5 \%$ $\mathrm{CO}_{2}$ to achieve a steady state. Muscles were stimulated at 60 beats/min. and maintained at $26^{\circ} \mathrm{C}$. Ten minutes of $1 \times 10^{-6} \mathrm{M}$ ACS in 5 non-hypoxic muscles resulted in a positive inotropic effect with increases in maximum $+d P / d t ~(12 \%)$ and maximum $-d P / d t$ (7\%). ACS administered during the initial 10 minutes of hypoxia was not associated with positive inotropism, but rather a significantly greater degeneration $(p<0.05)$ in developed tension (DT) and $+\mathrm{dP} / \mathrm{dt}$ compared to that produced by hypoxia alone. The $-\mathrm{dP} / \mathrm{dt}$, however, was reduced less than by hypoxia alone. The rise in resting tension produced by hypoxia was significantly increased $(p<0.05)$ and the recovery of mechanical function following one hour of reoxygenation was significantly decreased ( $p<0.001$ ) when ACS was given during the hypoxia. ACS given for 10 minutes prior to hypoxia provided no protection against the hypoxia induced deterioration of mechanical function. These results indicate that in rabbit myocardium, cardiac glycosides do not improve mechanical function during hypoxia but actually enhance the detremental effects of hypoxia on myocardial function.

\section{DEVELOPMENTAL BIOLOGY}

116 Developmental changes in erythrocyte enzymes OF LAMBS DURING THE FIRST EIGHT WEEKS OF LIFE. Cruz Alvarade, Susan F. Iravis, Elizabeth Cannen and Maria Delivoria-Papadopoulos, Univ of Penna. and Thom. Jeff. Univ., Philadelphia, Pa.

The present studies were designed to provide base line data in determining the postnatal aye that red cell enzymes of newborn lambs reach normal adult levels. Sequential measurements of lactic dehydrogenase (LDH), glutathione reductase(GR), g1ucose-6-phosphate dehydrogenase (G-6-PD) and hexokinase(HK) were made in 7 term lambs at birth and weekly thereafter. The mean value of LDH activity was $101.9 \pm 16.6$ units/gm Hb on day l and approached adult values ( 33.6 units) by 38 days $(48.9 \pm 16.7$ units/gm Hb; $<<0.005)$ while GR activity was similar to that observed in adults. Mean G-6-PD activity was $4.39-1.24$ units/gm Hb on day $l$ as compared to 0.76 units/gm Hb in the adult sheep, and declined progressively to $2.78-0.65$ units by 38 days $(p<0,002)$ and slowly thereafter. Mean HK activity was $1.19-0.33$ units/gm Hb on day 1 as compared to adults ( 0.58 units) and progressively declined during the first 60 days of life. The reticulocyte count was elevated at birth and was zero at 38 days in lambs and in adult sheep. It appears from these studies that HK, LDH and G-6-PD are age dependent enzymes in lambs and that maturity is approached at approximately 38 days, a 1 though enzyme levels remain slightly elevated until 60 days of 1 ife.

\section{EVIDENCE FOR IMMATURITY OF THE HYPOTHALAMIC-PITUITARY} AXIS (HPA) IN MCCUNE-ALBRIGHT SYNDROME (IIAS). K. Arulanantham, and L. E. Seyler, Jr., Newington Child. Hosp., and Depts. of Ped. and Med., Univ. Of CT School of Med., Farmington. (Spon. by Martha L. Lepow)

To determine if the sexuaT precocity (SP) of MAS is due to pre mature HPA activation or to peripheral gland hyperactivity, the maturity of the HPA was evaluated in an 11.0-yr.-old girl with fibrous dysplasia, café-au-lait pigmentation, SP of 9 yrs. duration; thyromegaly, and hyperthyroidism of 6 yrs. duration. As is often seen in MAS, the sequence of sexuial development was atypi = cal, the first manifestation being irregular vaginal bleeding at age 2, followed by thelarche at age 4. Plasma and urinary estrogens were in the pubertal range (plasma $E_{2} 220 \mathrm{pg} . / \mathrm{ml}$., 24-hr. urinary estrogen $38 \mathrm{mcg}$.) but hLH by RIA was undetectable in ic plasma samples drawn between 9:30-11 A.M. Sleep-induced augmentation of $\mathrm{hL} \cdot \mathrm{H}$ secretion was absent in 10 samples drawn during sleep between 3-4:30 A.M. hLH response to I.V. injection of $100 \mu \mathrm{g}$. LRH (Ayerst Labs) revealed a prepubertal pattern (baseline undetectable, maximal $\mathrm{hLH}$ response $60 \mathrm{ng}$. LER $907 / \mathrm{ml}$.). Thyroid function tests revealed high normal $\mathrm{T}_{4}, \mathrm{~T}_{3} \mathrm{U}$ and elevated I 137 uptake. TSH and LATS were undetectable. Tc $99 \mathrm{~m}$ pertechnetate scan showed enlarged thyroid with nonhomogenous distribution of activity.

These data are indicative of a prepubertal HPA. Peripheral organ hyperfunction in the absence of HPA maturation or hyperfunction best explains the findings in this child. This may represent an unusual sensitivity to low levels of trophic hormones or be a manifestation of peripheral aland autonomy.
18 THE ADAPTIVE REgULATION OF RED CELL OXYGEN (P50) IN UTERO : THE LIMITATION OF 2,3 DIPHOSPHOGLYCERATE LEVELS (DPG). H. Bard, A. Cornet, J.-C. Fouron, J. Robillard. Centre de Recherche Pédiatrique, Université de Montréal, Montréal, Canada.

The higher $P_{50}$ of fetal blood encourages placental $\mathrm{O}_{2}$ transfer. Large decreases in red blood cell $\mathrm{P}_{50}$ in utero could jeopardize the fetal supply of $\mathrm{O}_{2}$. In order to evaluate the physiological modulation of fetal $\mathrm{P}_{50}$, a study was carried out using 16 different fetal sheep whose gestational age (GA) varied from 70-148 days. Catheters were inserted into the fetal carotid artery when GA was > 110 days and exteriorized on the pregnant ewe. For fetal blood samples at < days, a hysterotomy was performed. The following analyses were done and correlated with $\mathrm{GA}$; the \% of adult hemoglobin ( $\mathrm{Hb}$ ) by column chromatography, DPG levels by method of Kiett and $P_{50}$ by gas mixing tonometry. $\mathrm{P}_{50}$ remained constant from 70 to 120 days of $\mathrm{GA}$ (mean $\pm \mathrm{SD}=14 . \mathrm{I}$ $\pm 0.1 \mathrm{mmHg} ; \mathrm{n}=6$ ), before any adult (Hb) was detected. Once adult $\mathrm{Hb}$ appeared near 120 days of $\mathrm{GA}$, there was a decrease in $\mathrm{P}_{50}$ which correlated with the increase in adult $\mathrm{Hb}(\mathrm{r}=0.70$, $\mathrm{p}<0.025, \mathrm{n}=10)$. $\mathrm{P}_{50}$ decreased in relation to GA only after 120 days of gestation ; $\mathrm{P}_{50}$ rose from 14 to $18.5 \mathrm{mmHg}(r=0.62$, $\mathrm{P}<0.025, \mathrm{n}=11$ ). The leve1 of DPG remained constant throughout gestation (mean $\pm \mathrm{SD}=3.5 \pm 1.5 \mu$ moles $/ \mathrm{gHb}$ ). When sheep erythropoietin (200 units) or hydrocortisone (180 mg) were injected into the fetus it resulted in a reticulocyte response without any effect on DPG levels or $\mathrm{P}_{50}$. The high $\mathrm{P}_{50}$ of fetal red cell may be controlled by an unknown regulatory mechanism which limits the lexel of DPG in utero.

\section{9} GLUCONEOGENESIS IN THE SUCKLING RAT: Marc-A.Beaudry, J.H.Exton, J.L.Chiasson, depts Physiol.\&Med., Vanderbilt U., Nashville Tn.(spon.by-Ian Burr) Gluconeogenesis from $\left[U-{ }^{14} \mathrm{C}\right]$ Lactate was studied in perfused livers and isolated liver cells from 10- to 21-day old suckling rats. In perfused livers of fed sucklings, gluconeogenesis from saturating lactate accounted for $50 \%$ of total glucose production compared with $28 \%$ in fed adult rats. Gluconeogenic rates were $125^{+} 7,106^{+} 6,73^{t_{9}}$ umol/100 g B.W./hr in 10-,15-and 21 day old rats $v s$. $49 t^{t} 2$ for adult rats. Fasting did not increase gluconeogenesis in sucklings, but caused a two-fold increase in adults. Isolated liver cells from fed sucklings showed a 2-fold increase of gluconeogenic rate compared with adult liver cells. The concentration of lactate for half-maximum gluconeogenesis was similar for suckling and adult liver cells. Gluconeogenesis in cells from suckling rats was stimulated by glucagon concentrations as low as $5 \times 10^{-10} \mathrm{M}$. However, the maximum increase with glucagon in cells from sucklings was only $23 \%$ compared with about $200 \%$ in cells from aduzts. Peripheral venous glucagon levels were markedly elevated in sucklings: $322^{t-29}, 366^{t}-39$, $117^{t_{7}} \mathrm{pg} / \mathrm{ml}$ in fed $12-$ day, $23-$ day and adult rats, respectively. It is suggested that the high gluconeogenic activity of livers of suckling rats is due in part to hyperglucagonemia.

Supported by NIH grants AM07462 and AM 17026.

120 CORD SERUM THYROXINE, THYROID BINDING GLOBULIN,

120 WEIGHT AND PONDEROSITY OF HEALTHY NEWBORN INFANTS. Betty Bernard, Omar A. Ghazzawi, Thomas H. Oddie and Delbert A. Fisher, LAC-USC Med. Ctr., LOS AngeTes and UCLA-Harbor General Hospital, Torrance, Cal if., Depts. of Pediatrics.

The weight $(W)$, ponderosity (P) and cord serum thyroxine (T4) concentrations were determined for 1992 healthy newborn infants at 30-46 weeks of gestational age (GA) born at the LAC-USC Medical Center. The group included 78.3\% Latin American (LA), 14.2\% Caucasian (C), 6.7\% Black (B) and $0.8 \%$ Oriental infants. The mean value of each T4 radioimmunoassay (RIA) run was normalized to the mean of the first 20 runs to correct for between-assay variance. Analysis showed that cord T4 concentrations were statistically independent of sex and race, but rose significantly with GA $(p<.001)$ froin a mean value of $9.3 \mu \mathrm{g} / \mathrm{dl}$ at 30 weeks to $11.3 \mu \mathrm{g} / \mathrm{dl}$ at 45 weeks. Serum T4 binding globul in (TBG) concentrations determined by specific RIA in a random sample of 200 newborns also showed significant correlations with GA. Weight analysis showed that males were $4 \%$ heavier than females, that there was no statistical difference between $C$ and $L A$ infants, and that $C$ and LA babieswere 1.043 times the weight of $B$ babies. Pooling our data and those of Lubchenco with correction for the greater average weight of Californian babies, regression equations were derived for the variations of $T 4, W$, and $P$ each with GA. There was a significant correlation of each with GA. The correlation of T4 with P could be accounted for on the basis of GA. The results indicate significant variation in T4 and TBG with GA, and establish normal values in the healthy newborn population. These standards will be usefut in assessment of thyroid function. 
121 HIBERNATING GROUND SOUIRRELS: A MODEL FOR THE RECAPITULATION OF FETAL LUNG DEVELOPMENT. Will R. Blackburn Jan De1li-Bovi, Phylis A. Logsdon and John Alexander. University of South Alabama College of Medicine, Mobile.

Certain biochemical events during lung development are unique and do not recur after birth (Glycogen synthesis-utilization; Surfactant synthesis surge-steady state synthesis). These events appear to be hormonally controlled and initiated by the fetal hypothalamus. Hibernation in ground squirrels is regulated by the hypothalamus, secondary hormones and cyclic AMP. Such animals accumulate large quantities of lipid prior to hibernation and then, during hibernation, undergo extreme physiological changes: hypothermia $\left(2-7^{\circ} \mathrm{C}\right)$, bradycardia $(2-3 / \mathrm{min})$, bradypnea $(0.5-1 / \mathrm{min})$, and acidosis (pH 6.01-6.23). We have studied the biochemical and structural aspects of GS-lung during "activity" and hibernation, noting changes which resemble those of fetal lung development in non-hibernating rodents. The following values $=\mathrm{mg} / \mathrm{lung}$ :

\begin{tabular}{|c|c|c|c|c|c|}
\hline Period & DNA/lung & Total Lipid & P-lipid & Lecithin & Glycog \\
\hline Activity & 17.44 & 43.90 & 19.75 & 6.91 & 0.52 \\
\hline Hibernation & 18.08 & 48.87 & 17.46 & 3.93 & 1.32 \\
\hline $\mathrm{t}$-Test & $P=0.2$ & $P>0.2$ & $\mathrm{P}>0.2$ & $P<.01$ & $P<.01$ \\
\hline
\end{tabular}

These observations suggest that during hibernation the lung
reverts to a condition resembling that of the fetus. Once the

"wake up" phase is initiated, the lung rapidly synthesizes surfactant, glycogen disappears, respirations resume at a rapid rate, and acidosis disappears. Hibernating animals may therefore pro vide a powerful model for the study of factors controlling certain unique biochemical events normally occurring only during the intrauterine period in human fetuses. Support: USPHS NIH HL-17328-03
124

WATER CONTENTS AND DISTRIBUTION IN HUMAN MUSCLES IN RELATION TO FETAL GROWTH. Y. Brans, P. Ortega, and P. Bailey. Univ. of Chicago, Dept. Pediatrics,

Chicago.

Triceps or quadriceps samples were collected at autopsies of 21 normally-grown (NG) and 15 intrauterine growth retarded (IGR) neonates. Birthweights ranged from 425 to $3750 \mathrm{~g}$, gestational ages ranged from 21 to 44 weeks and postnatal ages at death ranged from 0 to 29 days. Half the neonates in each group died within 24 hours of birth. Contents in total water (TW) were estimated by desiccation, in extracellular water (ECW) as the corrected chloride space and in intracellular water (ICW) as the difference between the former. Results were expressed in 1 per $100 \mathrm{~g}$ of tissue.

Although muscle samples from IGR and NG neonates had similar TW (mean \pm S.D.: $86.5 \pm 3.71$ vs. $85.8 \pm 2.52 \%$ ), the former contained smāler proportions of ECW $(20.2 \pm 6.36$ vs. $30.3 \pm 7.37 \%$, $p<0.001)$ and larger proportions of ICW $(66.3+9.31$ vs. $55.6+$ $8.73 \%, p<0.005)$. A considerably larger proportion of TW was inside the muscle cells of IGR neonates (ICW/TW: $0.76+0.078$ vs. $0.65 \pm 0.094, \mathrm{p}<0.001$ ). Birthweight, gestational age, postnatal age at death and duration of the period between death and autopsy did not affect these trends.

These data suggest that intrauterine growth retardation may significantly and durably affect intracellular water balance of muscle cells. They also support the assertion that the higher total body water contents of IGR neonates are unevenly distributed throughout body tissues. 125 PAH TRANSPORT AND ACETATE METABOLTSM BY MATURE AND Barbara R. Cole, Linda Peterson and Alan M. Robson. Wash. Univ. Sch. of Med. Dept. of Ped. St. Louis, Mo.

This study investigated the mechanisms responsible for the well-described reduction in organic acid transport by the proximal tubules of developing rabbit kidneys. Mean para-amino-hippuric acid (PAH) slice to medium ratios (S/M) for renal cortical slices from mature and fetal rabbits, incubated one hour in an acetatefree medium were, respectively, 8.1 and 3.7 . The addition of 10 or $30 \mathrm{mM}$ acetate, known to stimulate $\mathrm{PAH}$ uptake in mature kidney slices, resulted in an $S / M$ ratio of 16.3 in adult tissue. However, it rose only to 5.6 in fetal tissue. To investigate the cause for this difference, both the rates of ${ }^{14} \mathrm{C}$-acetate entry into cells of kidney cortical slices and its metabolic rate were measured. Acetate uptake and final tissue levels after $l$ hour of incubation were similar in slices from fetal and adult animals. In contrast, $\mathrm{CO}_{2}$ production from $1-14 \mathrm{C}$-acetate in fetal tissue was on $1 \mathrm{y} 60.7 \%$ of the adult rate and that from $2-{ }^{14} \mathrm{C}$-acetate was only $68.2 \%$.

Thus, the inability of acetate to stimulate S/M PAH significantly in the renal cortex of developing animals was not due to the failure of acetate to enter the cells but was associated with reduced metabolic rate of acetate. Thus, the studies suggest there may be an additional mechanism by which acetate augments PAH transport other than that proposed by Schachter et al. (AJP 182, $537,1955)$. In addition, the findings are consistent with the the sis that fewer available transport sites for PAH contribute to the decreased organic acid uptake by immature renal cortex.

126 THE EFFECT OF WEANING AND DIET ON PAH TRANSPORT BY THE KIDNEYS OF DEVELOPING ANIMALS. J.Trevor Brocklebank, Brenda Murray, and Alan M. Robson. Wash. Univ. Sch. of Med. Dept. of Ped. St. Louis, Mo.

This study investigated the effect of weaning on the maturation of organic acid transport by kidneys of developing rabbits. Slice to medium ratios (S/M) of para-amino-hippuric acid (PAH) in renal cortical slices from fetal animals incubated for 1 hour averaged 5.0. In animals reared by their mothers the equivalent $S / M$ ratios for slices obtained from 2 and 4 wk. old animals increased significantly to 9.7 and $14.0(p<.001)$, the latter value being similar to that of adult kidney slices. As the increasing ability to concentrate PAH occurs during weaning when the animal's diet is changing markedly, the effect of diet on the matuxation of organic acid transport was studied. Two wk. old animals were removed from their mothers and fed either a low protein diet $(4.5 \mathrm{~g} \%$ protein) or a high protein diet similar to that recommended for weaning $(22.5 \mathrm{~g}$ $\%$ protein). Diets were otherwise identical and were isocaloric. At age $4 \mathrm{wks} S / M$ PAH for the animals fed a low protein diet was 9.9, a value similar to that seen in normal 2 wk. old animals. In contrast, litter-mates given a high protein intake showed the normal ability to concentrate PAH at age 4 wk. (S/M ratio 15.0). The difference between the two groups was highly significant $(p<.001)$. There were no differences in other cell functions studied - cell size, water content or acetate metabolism. These studies demonstrate that a low protein diet can arrest the development of organic acid transport by the kidneys of immature animals and suggest that the we11-described pattern of maturation in PAH transport is dependent upon an adequate dietary protein intake. 
127

CARNITINE PALIMITOYL TRANSFERASE ACTIVITY IN NORMAL AND PREMATURE NEWBORNS AND ADULTS. Maria Buch, Paul B. Wieser, Milan Novak. Department of Pediatrics, University of Miami School of Medicine, Miami, Florida.

Palmityl-CoA:Carnitine Palmitoyl Transferase (CPT) activity in subcutaneous adipose tissue was measured in three groups of individuals: Infants less than $12 \mathrm{~h}$ old, infants greater than $12 \mathrm{~h}$ old and adults. Two methods for the assay of CPT were used. Method A measured spectrophotometrically the release of CoASH from palmityl-CoA by complexing with DTNB and method B measured conversion of ${ }^{14} \mathrm{C}$ labeled carnitine to palmitylcarnitine. There was no significant difference in the results between methods A and B. Normal infants under $12 \mathrm{~h}$ had CPT activity of $8.2+1.4 \mathrm{nmoles} / \mathrm{mg}$ protein $/ \mathrm{min}$. Infants over $12 \mathrm{~h} 58.3 \pm 7.5$ nmoles $/ \mathrm{mg} / \mathrm{min}$. Adults $10.0+3.6$ nmoles $/ \mathrm{mg} / \mathrm{min}$. $P$ for infants $<12 \mathrm{~h}$ was $<0.001 . \quad P<0.025$ for infants $>12 \mathrm{~h}$ old as compared to adults. CPT activity was also measured in premature infants of 32-36 weeks gestational age. These were compared to infants in the same age group ( 16 to $76 \mathrm{~h}$ postpartum). CPT activity was $26.2+8.5 \mathrm{nmoles} / \mathrm{mg} / \mathrm{min}$ in the normal group and $9.2+3.0 \mathrm{nmoles} / \mathrm{mg} / \mathrm{min}$ in the premature group $\mathrm{P}<0.05$. The data suggest that in very young and especially in premature infants that there is a decreased capacity to utilize free fatty acids. - - Supported by NIH grant HD 04946.

128

THYMIDYLATE SYNTHETASE ACTIVITY DURING CEREBELLAR DEVELOPMENT: EFFECT OF THYROXINE. Brian R. Clark and Morton E. Weichse T, Jr., UCLA Sch. Of Med., Harbor Gen. Hosp., Depts. of Psychiatry and Pediatrics, Torrance, Cal if. Thymidylate synthetase (TS), an enzyme of the pathway that converts uridylate (an RNA precursor) to thymidylate (a DNA precursor), and thymidine kinase (TK), a pyrimidine salvage enzyme, are elevated in cells undergoing replicative DNA synthesis. In rat cerebellum, postnatal replicative DNA synthesis and the associated rise in TK activity have been shown previousiy to be accelerated by daily subcutaneous injections of thyroxine $\left(T_{4}\right)$.

We measured TS activity, not previously investigated in developing cerebellum, as well as DNA content and TK activity in cerebella of norma 1 and $T_{4}$-treated neonatal rats. Daily $T_{4}$ injections significantly increased cerebellar TS and TK activities over control values on days 2-7 and 2-6, respectively, and significantly decreased both activities below control values on days 9-15. Whereas TS activity was maximal on day 6 in treated rats and day 7 in controls. TK activity was maximal between days 5 and 6 for both groups. $T_{4}$ injections increased the rate of cerebellar DNA accumulation (derived by differentiation of an equation obtained by using the method of least squares to fit a sigmoid curve to the variation of cerebellar DNA content with time) over control values on days 1-5 and decreased the rate below control values on days 7-12; maxima 1 rate occurred on day 6 in treated rats and day 7 in controls. Thus, $T_{4}$ accelerated replicative DNA synthesis and the associated rises in TS and TK activities. Thymidylate formation appears to be a critical factor in the control of cell replication rate in developing rat cerebellum.

129

DETECTION OF INTRAUTERINE GROWTH RETARDATION WITH ULTRASOUND. L:RRY COOK, JOHN T. QUEENAN, SANDRA KUBARYCH, BILLY F. ANDREWS. University of LnUisville

School of Medicine Affiliated Hospitals, Depts. of ped.. or/ry, Louisville.

The increased perinatal morbidity and mortality of intrauterine growth retardation is well documented. Serial biparietal diameter determinations(BPD) were explored as a non-invasive means of detecting retarded fetal growth.

800 fetal BPD determinations in 475 normal pregnancies between the 16 and 43 weeks of gestation were done. The mean BPD \pm 2 SD were determined and a BPD growth curve constructed which revealed a straight line. The $\triangle \mathrm{BPD}$ representing fetal BPD growth in normal pregnancies was determined to be $0.26 \pm 0.005 \mathrm{~cm} / \mathrm{wk}$. from 18 to 38 weeks.

100 random high risk pregnancies were screened for IUGR. Neonates were evaluated with clinical estimates of gestational age and anthropometric data plotted on Colorado growth grids. Two patterns of suspected IUGR are observed: 1) BPD's falling below 2 SD on our normal growth curve and 2) serial values falling within the normal growth curve range but showing an arrest in the $\triangle$ BPD. 18 total infants were predicted with a confirmation rate of $50 \%$. 7 neonates were identified postnatally as having IUGR who could not be detected in utero with a single BPD.

The study is ongoing in selected high risk groups, i.e., Rh disease, chronic hypertension, pre-eclampsia and others.
130 TRANSFER AND METABOL.ISM OF CORTISOL BY PERFUSED HUMAN PLACENTA。 Joseph Dancis, Valerie Jansen, Will iam Rosner and Mortimer Levitz. Departments of Pediatrics and Obstetrics \& Gynecology, New York University School of Medicine, and Department of Medicine, Columbia University College of Physicians \& Surgeons, New York City.

The effect of protein-binding on the placental transfer and metabolism of cortisol was investigated in an in vitro perfusion system of human placenta. The transfer rate with 50\% bound to cortisol-binding protein (CBG) was the same as or slightly less than that from buffered salt solution, and greater than when bound $50 \%$ to albumin, eventhough the strength of interaction of cortisol with CBG is approximately one thousand fold greater than with albumin.

Following one circuit through the placenta, about one-third of maternal cortisol is converted to cortisone and over $80 \%$ of steroid appearing in the fetal circulation is also cortisone. When the placenta is perfused with cortisone, there is negligible conversion to cortisol. Equilibrium dialys is revealed more effective binding of cortisone by albumin than by CBG. The possibility must be considered that the mechanism of release of cortisol from CBG involves conversion to cortisone.

Maternal cortisol is presented to the fetus primarily as cortisone.

ROLE OF CARBOHYDRATE METABOLISM IN MATURATION OF FETAL

131 RABBIT LUNGS BY HYDROCORTISONE. Carl Gilden, Alex Sevanian, Donald F. Tierney, Solomon A. Kaplan, and Cynthia T. Barrett, UCLA School of Med

The mechanism by which hydrocortisone (HC) regulates fetal lun maturation is associated with an alteration in carbohydrate metabolism. On days 24,25 and 26 of gestation 12 does were given HC subcutaneous $1 \mathrm{y}, 2 \mathrm{mg} / \mathrm{Kg} /$ day divided TID, a dose which did not cause fetal wastage. Controls received saline and were studied simultaneously. On day 27 fetal lung slices were prepared and incubated with glucose-6- ${ }^{14} \mathrm{C}$ in Krebs-Ringer bicarbonate for $90 \mathrm{~min}$ Glycogen and the major lipid fractions were then studied quantitatively and for ${ }^{14} \mathrm{C}$ incorporation. Before incubation the level of glycogen in the fetal lungs of the HC treated group was lower than in the control group $(2.42$ vs $3.81 \mathrm{mg} / \mathrm{Gm}$ of lung, $\mathrm{P}<.05)$. After incubation the glycogen levels were similar. Along with this was a significant increase in the incorporation of glucose- $6-{ }^{14} \mathrm{C}$ into glycogen in the $\mathrm{HC}$ group (.116 vs $145 \mathrm{uM} / \mathrm{Gm}$ of lung, $\mathrm{P}<.05$ ). Glucose oxidation as reflected by ${ }^{14} \mathrm{CO}_{2}$ production was not changed by HC. Incorporation of glucose- $6-{ }^{14} \mathrm{C}$ into phosphatidyl choline (PC) increased from $038 \mathrm{uM} / \mathrm{Gm}$ of lung in the controls to .057 $\mathrm{uM} / \mathrm{Gm}$ of lung in the HC group $(\mathrm{P}<.05)$, in pool sizes that were not significantly different (HC 4.70 and control $4.45 \mathrm{mg} / \mathrm{Gm}$ of lung). Results for other neutral phospholipids were similar. These data demonstrate a depletion and increased turnover of glycogen in fetal lung exposed to HC. Part of the mechanism of action of $\mathrm{HC}$ in inducing fetal lung maturation may be through an increased utilization of glucose.

SYNTHESIS OF SULFATED GLYCOSAMINOGLYCANS (GAGS) IN THE

132 Francis J. Manasek. (Spon. by Alexander S. Nadas). HarFrancis J. Manasek. (Spon. by Alexander S. Nadas) Harren's Hospital Medical Center, Department of Cardiology, Boston and University of Chicago, Anatomy Department, Chicago.

The synthetic patterns of the sulfated GAGs of embryonic chick hearts age 3 days to hatching were studied employing in vitro and in vivo incorporation of $\mathrm{Na}_{2}{ }^{35} \mathrm{sO} 4$. Hearts dissected into atria (A), ventricles (V) and great vessels(G), at days $3.5,5,7,10,14$ and 19 were inculated with precursor for 5 hours, digested with papain and liberated GAG carbohydrate initially senarated on a DENE-cellulose column eluted with a linear qradient of 0 to I M lithium chloride. Individual labeled fractions were chaxacterized further on the basis of their sensitivity to a variety of agents and analyzed on DE cellulose and Sephadex G50 columns. Sulfate was incorporated into 3 GAGs: (1) a major component, chondroitin sulfate(CS) which was identified by coelution with known CS, sensitivity to testicular hyaluronidase (TH) and resistance to HNO2, (2) small amounts of heparan sulfate identified by $\mathrm{TH}$ resistance and $\mathrm{HNO}_{2}$ sensitivity and (3) small amounts of dermatan sulfate which was resistant to both $\mathrm{TH}$ and $\mathrm{HilO}_{2}$. Controls were labeled in ovo. Incorporation (expressed as counts/minute ner gram in wet wt. )of sulfate into $\mathrm{CS}$ by $\mathrm{V}$ diminished in an exponential fashion with increasing age while incorporation by $A$ and $G$ was similar but varied at the older ages. GAGs synthetic patterns do not appear to relate directly to specific cardiac embryonic events but sulfate incorporation does appear to be most intense during earlier periods of orgarogenesis when the basic organ is developing. 
132A

CAMP STIMULATES PHOSPHOLIPID SYNTHESIS BY FETAL RAT

LUNG IN ORGAN CULTURE.

Ian Gross and Seamus A. Rooney (Spon。by Joseph B. Warshaw) Yale University School of Medicine, Departments of Pediatrics, OB/Gyn and Lung Research Center, New Haven, Conn.

Studies in intact animals have indicated that pulmonary phosphatidylcholine (PC) synthesis is stimulated by the administration of aminophylline. We have investigated the direct effect of dibutyryl cAMP on the incorporation of choline into phospholipids by 18 day fetal rat lung explants in organ culture. The explants were incubated in F12 medium with or without added cAMP for 48 hours. [MethyI - 30] choline was then added to the medium for 6 hours and incorporation into PC and sphingomyelin determined. Activity is expressed as pmoles choline incorporated per ing protein per hour.

$\begin{array}{lcccc} & \text { Control } & \text { cAMP } & \text { Stimulation } & \text { P } \\ \text { PC } & 359.8 & 486.6 & 35.2 \% & <0.005 \\ \text { Sphingomyelin } & 14.3 & 17.4 & 21.7 \% & \text { NS (0.06) } \\ \text { PC/sphingomyelin } & & & & \\ \text { ratio } & 25.9 & 29.3 & 13.1 \% & \text { NS }\end{array}$
Statistical analysis was by paired $t$ test.

These data indicate that cAMP acts directly on lung tissue to stimulate the incorporation of choline into $\mathrm{PC}$. There is also a tendency towards increased incorporation into sphingomyelin. cAMP may act by non-specifically stimulating pulmonary phospholipid synthesis.

133 STUDIES OF THE HYPOTHALAMIC-PITUITARY AXIS IN FETAL AND NEWBORN SHEEP. Harvey Guyda and Robert Williams. McGil1 University-Montreal Children's Hospital Research Institute, Department of Pediatrics, Montreal, Canada. Chronic fetal lamb preparations (110-140 days gest.) had catheters placed in the fetal carotid artery, amniotic cavity and a maternal leg vein to enable continuous sanpling 'in utero'. In addition electrodes were inserted in the fetal hypothalamus. Antisera to growth hormone (OCH), prolactin (OPRL), bTSH and porcine insulin (IRI) were generated for specific RIA of these hormones Immunochemical TSH was a gift from Dr. J. Pierce, standards for oTSH and oGH from NIH and oPRL from Sigma. A11 samples were assayed at $50 \mu l$ in duplicate. Basal fetal serum oGH levels were higher than maternal levels whereas maternal levels of oPRL and oTSH were similar or higher than fetal levels. Basal fetal IRI levels were low. Hypothalamic electrical stimulation 'in situ' of 3 lambs near term did not produce a consistent change in basal fetal levels of oGH $(18-33 \mathrm{ng} / \mathrm{ml})$, OPRL $(10-100 \mathrm{ng} / \mathrm{ml})$ and OTSH $(14-18 \mu \mathrm{U} / \mathrm{m} 1)$. Somatostatin $(200-300 \mu \mathrm{g}$ by IV bolus) decreased fetal oGH $30 \%$ in 1 of 3 experiments only. Maternal levels of all hormones were not altered by these stimuli, and amniotic levels remained low. TRH ( $100 \mu \mathrm{g}$ IV) given to the newborn lamb at 1 week and 2 weeks of age increased serum oPRL (peaks of $190 \& 470 \mathrm{ng} / \mathrm{ml}$ ) serum oTSH (peaks of $42 \& 59 \mu \mathrm{U} / \mathrm{ml}$ ), and did not change serum oGH Our preliminary studies suggest fetal pituitary unresponsiveness 'in utero' to hypothalamic electrical stimulation and the bolus injection of somatostatin. The feasibility of this model for the study of perinatal hypothalamic-pituitary regulation has been

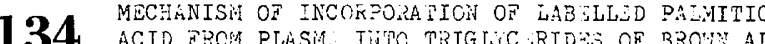
ACID FROM PLASIA. IHTC TRIGLIC ARIDES OF BROYN ADIPOS: TISSU;, Tijor Heim, Herwart Schenk, Śnjor Molnár, Hans Hąnar, Christa Kabus. /Intr. by J.B. Narshaw/. Dept. Ped. Univ. Mod. Sch. Pécs, Hungary, Inst. Päth. Physiol. Univ. Jena, GDK.

In vitro studies demonstrated two triclyceride/TG/ pools in white adipose tissue /iAT/, but no data were available for brown adipose tissue /BAT/. The function of the two compartments and their role during calorigenesis remained also unknown, since in vivo observations were lacking. After i.v. injection of 14-C-palmitic acid into newborn rabbits the compartmentation of newly esterifiad TG was calculatad from the time response curve using an analos computer proeramme. At the neutril amoient temperature $/ \mathrm{T}_{2} /$ of $35^{\circ} \mathrm{C}$ the TG of BAT were distributed between two separate compartments with different turnover timos. Turnover time in the smaller compartment was $1,3-2,9 \mathrm{~min}$. and the incorporation rate of plasma FFA was $0,9-$ 2, I Mmol/min. loo g body weight/bwt/. The influx of plasma FFA into the large TG pool, called slow pool, was found to be $0,367 \pm 0,11 \mu \mathrm{mol} / \mathrm{min}$. $100 \mathrm{~g}$ bwt $/ \mathrm{M}+\mathrm{SE} /$ under the same circurastancess. During exposure to cold $7 \mathrm{~T} 20^{\circ} \mathrm{C} /$ the smaller compartment with rapiz turnover disappeared and the TG of BAT constituted only one compartment with a turnover time longer than $45 \mathrm{~min}$. The inflow rate of plasma FFA into this slow pool was about $0,5 \mathrm{\mu mol} / \mathrm{min}$. $100 \mathrm{~g}$ bwt. These results are the first in vivo demonstration of two TG pools in BAT and show that the distribution of TG-FA between the two pools varies with $T_{a}$.
125 EFFECTS OF TOTAL PARENTERAL NUTRITION ON BRATN GROWTH OF BEAGLE PUPPIES. W.C. Heird, M.A. Bieber, J. Bassi, A.R. Pulito and J.A. Brasel. Columbia University College of Physicians and Inst. of Human Nutr. N.Y.

Brain weight (BW), DNA and protein content, plus major lipid classes and fatty acid patterns of ethanol phosphoglyceride (EPG) were determined in beagle puppies at birth $(n=9)$ and after 10 days of either normal feeding (C), total parenteral nutrition (TPN), or $10 \%$ glucose IV $(G)$. BW of $C(n=8)$ and TPN $(n=7)$ increased $103 \%$ and $89 \%$ respectively (NS), but BW of $G(n=5)$ increased only $68 \%$ ( $p<0.005$ compared to $C$ ), Cerebral DNA of $C$ increased on $1 y$ $40 \%$ in 10 days and neither cerebral DNA nor protein content of TPN and $G$ differed from $C$. Cerebellar DNA increased approximately $400 \%$ in $C$ and TPN, but only $260 \%$ in $G(p<0.005)$. Cerebellar protein content of $G$ was less than that of $C$ $(\mathrm{p}<0.01)$ but not TPN. Total lipid (TL) content of both $\mathrm{C}$ and TPN doubled in 10 days but increased only $44 \%$ in $G(p<0.001$ ). Gangliosides, reflected by sialic acid content, were higher in TPN and $G(p<0.005)$. Other 1ipid classes were distributed similarly in all groups. Linoleic and arachidonic acid of both cerebral and cerebellar EPG were reduced in TPN and $G$ while eicosatrienoic acid was increased; these changes were more marked in TPN. The data show that TPN, as opposed to glucose alone, supports normal growth in mass and cell number of the puppy brain. However, the lipid which accumulates in the absence of exogenous fat, especially during rapid growth, has an abnormal fatty acid pattern.

136 STIMULATION OF HUMAN FIBROBLAST GROWTH - A COMPARISON OF INSULIN, FIBROBLAST GROWTH FACTOR AND SERUM. Kenneth L. Jones and Judith Addison. Departmen

ornia, San Diego.

We have studied the mechanisms of human fibroblast growth and the factors controlling this growth. We have compared the effects of bovine insulin and fibroblast growth factor (FGF) isolated from bovine pituitary tissue. DNA synthesis was arrested by serum starvation. FGF produced stimulation of incorporation of $\left[{ }^{3} \mathrm{H}\right]$-thymidine at concentrations as low as $5 \mathrm{ng} / \mathrm{ml}$ and maximum stimulation at $100 \mathrm{ng} / \mathrm{ml}$. FGF was more active than was insulin at 4 logs greater concentration. Serum and FGF produced a complimentary stimulation of DNA synthesis with FGF producing up to a 6-fold increase in counts incorporated over that seen with serum alone. Though insulin, FGF and serum all stimulated DNA synthesis, only serum was capable of sustaining cell growth. The addition of FGF to serum decreased the population doubling time from 29 hours to 24 hours. In contrast to the effect in chondrocytes and $3 \mathrm{~T} 3 \mathrm{cells}$, glucocorticoids inhibited rather than enhanced FGF stimulation. Fibroblasts from patients with hypopituitarism showed an identical response to cells from normal subjects. Bovine FGF affects growth in human cells and provides a valuable tool for studying the mechanisms of growth.

137 ARginine is an ESSENTIAL AMINO ACID IN A BOY WITH DEFICIENCY OF CARBAMYL PHOSPHATE SYNTHETASE (CPS). Jeffrey Kline, George Hug, Ted Brown, William Schubert, Edgar Ballard and Helen Berry, Children's Hospital Research Foundation, Cincinnati, Ohio.

A 6 mo old boy had seizures, developmental delay and persistent hyperammonemia of $300-600 \mu \mathrm{g} / 100 \mathrm{ml}(\mathrm{N}:<130)$. Activity of CPS in liver was less than $5 \%$; that of the other 4 urea cycle enzymes was norma1. He was fed $2 \mathrm{~g} / \mathrm{kg} / \mathrm{day}$ of an arginine free mixture of 10 essential amino acids (AA) supplemented by fat and glucose for a daily intake of $310 \mathrm{cal} / \mathrm{kg}$. After seven days of this diet, he had stopped growing and had developed a severe, exudative dermatitis. When the diet was changed to $2 \mathrm{~g} / \mathrm{kg} /$ day of milk protein, growth resumed and his skin healed within 3 days. Reinstitution of the initial mixture of essential $A A$ again led to growth cessation and desquamative dermatitis that worsened during the next 7 days. The AA mixture was then fortified with $80 \mathrm{mg} / \mathrm{kg} /$ day of arginine. No other changes were made, nor was the dermatitis treated in any other way. Growth resumed and the dermatitis resolved completely within 3 days. The effect of arginine was documented photographically and by light and electronmicroscopy of skin biopsy specimens. Posttreatment specimen was normal. Histology of the pretreatment skin lesion indicated superficial dermal perivascular inflammation and moderate spongiotic acanthosis without vesicles. We conclude that arginine is an essential amino acid in children with CPS deficiency and perhaps also in children with defects of other urea cycle enzymes. 
138

STIMULATION OF SWISS 3T3 CELL GROWTH BY EPIDERMAL GROWTH FACTOR (EGF), FIBROBLASTIC GROWTH FACTOR (FGF) AND DEXAMETHASONE (DEX). Roger L. Ladda and Lia S. LaPiana (Spon. by Nicholas M. Nelson). Penn State Univ.

Cells in culture require serum for the stimulation and maintenance of optimal growth. EGF and FGF are small polypeptides with growth-promoting effects which may account for at least part of the serum effect. We have studied initiation of DNA synthesis and growth of quiescent Swiss $3 \mathrm{~T} 3$ cells in culture in the presence of different combinations of EGF, FGF and Dex in comparison to the effects of serum excess. W None of these growth factors (GF) alone or in combination sustained growth in the absence of permissive amounts of serum (PAS) in the culture medium. PAS is that minimum serum concentration that allows cells to survive without dividing; cells are capable of maximal growth response when exposed to excess serum or GF. EGF and FGF in combination with PAS induced a $1-2$ fold greater response than PAS alone $(0.25 \%$ serum). EGF or FGF with PAS and Dex induced a 2-3 fold greater response than $1 \%$ serum and was about $60-70 \%$ as effective as $4 \%$ serum. EGF, FGF, PAS and Dex together were about $80-85 \%$ as effective as $4 \%$ serum. EGF in combination with PAS and Dex showed a $25 \%$ greater effect on DNA stimulation than FGF in similar combinations. Maximum growth stimulation required daily replenishment of GF. Serum growth effect appears to result from the synergistic action of its numerous macromolecular components which presumably have different mechanisms of action. (Supported by NIH Grant \#1 R01 HD 09232-01).

139 MORPHOLOGIC DEVELOPMENT OF THE PULMONARY VASCULAR BED IN EXPERIMENTAL FETAL HYPERTENSION. Daniel Levin,Allen Hyman, Michael Heynann, Abraham Rudolph. Univ. of Calif. San Francisco,Dept. of Pediatrics and Cardiovascular Research Inst. Persistent pulnonary hypertension is common in many newborns after acute or chronic intrauterine stress. Although hypoxia is known to increase pulmonary arterial smooth muscle,little is known about the effects of chronic fetal hypertension, a major response to stress, on development of pulmonary vasculature. Chronic hypertension was created in 4 fetal lambs by unilateral renal artery constriction; catheters were placed in fetal upper and lower limb vessels. Hypertension began within 3 days and rose to a peak systemic mean pressure of 70-100 $\mathrm{mm} \mathrm{Hg}$ (normal $+50-55 \mathrm{~mm} \mathrm{Hg}$ ). Blood gases were normal with $\mathrm{PaO}_{2}$ 's consistently $>20 \mathrm{~mm} \mathrm{Hg}$. Animals were killed 8-15 days post operatively (126-139 d.gestation). Serial lung sections were prepared and 5 th generation (resistance)vessels identified as previously described. Mean medial width/external dimeter ratio in 6 normal fetuses $(85-140 \mathrm{~d}$.) was $0.16(\mathrm{~N}=529)$. Ratios in the hypertensive animals were:126d.0.18 $(\mathrm{N}=50) ; 129 \mathrm{~d} 0.18(\mathrm{~N}=28)$; 136d.0.19 $(\mathrm{N}=34)$; and 139d.0.20 $(\mathrm{N}=50) . \quad(\mathrm{p}<0.05)$.

Since fetal pulmonary and systemic arterial pressures are equal, pulmonary vessels are exposed to elevated pressure. This pulmonary hypertension is a stimulus to increased pulmonary vascular smooth muscle development in utero. In chronically stressed fetuses, hypertension as well as hypoxia can induce hypertrophy or hyperplasia of medial stoooth muscle in the resistance vessels. This may explain persistent pulmonary hypertension in newborn infants subjected to chronic stress in utero. USPHS Grant HL06285 and NHLI Grant HD00397.

140 IRON ABSORPTION AND GROWTH IN WEANLING RATS George L. McElroy and Calvin W. Woodruff. Univer

The hypothesis that the rate of growth is a major determinant of the efficiency of iron absorption has been tested in weanling rats 24 days of age. They were fed $3 \mathrm{milk}$ based infant formulas ad libitum for 3 weeks and contained $1.5,2.4$ and $3.3 \%$ protein, each formula containing both 1.4 and $12 \mathrm{mg}$ iron per quart. Iron absorption was measuref in a whole body counter after the administration of $\mu .2 \mathrm{Ci}{ }^{2} \mathrm{Fe}$ by gavage in $2 \mathrm{mI}$ formula and calculated as corrected counts at 7 days as percentage of counts 4 hrs after administration. Weight gains were 21,45 and 75 gms in 22 days with increasing protein intake in the formulas supplemented with iron. Only at the higher protein intake did the lower iron intake suppress growth or produce anemia. Iron absorption:

\begin{tabular}{|c|c|c|c|}
\hline protein & $1.5 \%$ & $2.4 \%$ & $3.3 \%$ \\
\hline low iron & $31.1 \pm 1.4 \%$ & $40 \overline{.7 \pm 1.6}$ & $76 . \overline{9 \pm 2.3}$ (SEM) \\
\hline high iron & $22.4 \pm 1.3$ & $27.4 \pm 1.4$ & $40.2 \pm 2.0$ \\
\hline
\end{tabular}
Differences among all 6 groups are statistically significant $(p<.01)$ and were independent of the type of formula in which the iron was given. The weanling rat absorbs iron in response to his needs for growth as well as his needs for hemoglobin synthesis. These findings support the hypothesis of Gorten et al. (J. Pediat. 63:1063,1963) that the same response is found in the human infant. The weanling rat is a suitable model for studying the mechanisms controlling iron absorption by the intestinal mucosa. Milk proteins do not interfere with iron absorption in this model. Supported by USPHS HD 04776.
141 ONTOGENY OF INSULIN ACTION ON DEVELOPING LIVER, William M. Maniscalco, Sherry Loo, Ian Gross, Joseph B. Warshaw, Yale University School of Medicine Departments of Pediatrics, OB/Gyn, New Haven, Connecticut

Infants of diabetic mothers become macrosomic and develop large amounts of adipose tissue after 28 weeks of gestation. This suggests that fetal tissues become more sensitive to insulin during the latter part of pregnancy. We have examined the effect of insulin on hepatic fatty acid synthesis in fetal rat liver explants by measuring $\mathrm{C}^{14}$-acetate incorporation into long chain fatty acids. The addition of insulin $(0.1 \mathrm{U} / \mathrm{ml})$ to the culture media $(F-12)$ of explants from 21 day fetuses and 2 day postnatal animals resulted in a $50 \%$ increase in acetate incorporation into fatty acids. Stimulation was not observed in explants obtained from 19 day fetuses. Insulin binding to isolated rat liver membrane as a function of developmental age was also studied. Specific binding increased from $1.6 \%$ at 16 days gestation to $3.0 \%$ at 21 days gestation and $5.8 \%$ at 2 days postnatal age. Since there was also an increase in $5^{\prime}$ nucleotidase activity (a membrane marker) during development, it is not possible at this time to distinguish whether the change in binding is due to an increase in receptor per unit membrane or to an absolute increase in total membrane isolated. These results suggest that a stimulatory effect of insulin on hepatic fatty acid synthesis is seen only after a critical developmental stage and may further provide an explanation for the increased growth and fat deposition seen in infants of diabetic mothers after the 28 th week of gestation.

142 MATERNAL CELL METABOLISM RELATED TO FETAL GROWTH Metcoff, Jack; Mameesh, Mostafa; Jacobson, Gai 7 Crosby, Warren; McClain, Phil; Costiloe, Paul: Sandstead, Harold; Hernandez, Omar; Rosado, Adolfo. U.0kla. Health Sci.Ctr. OKC., Agric.Res.Div.,USDA, N.Dak \& MD, \& Centro Medico Nacional, IMSS, Mexico, D.F.

Maternal nutritional factors which regulate fetal cell growth also must modulate metabolism of rapidly replicating maternal cells such as circulating leukocytes. Cell replication \& growth is thought to be impaired in babies born with fetal malnutrition. The metabolism of circulating leukocytes has been examined in mothers of FM babies. Kinetic analyses of the leukocyte enzyme pyruvate kinase indicated allosteric inhibition by L-alanine with activation by fructose 1,6-diphosphate (FDP). In mothers of FM babies the enzyme was more inhibited by alanine \& less responsive to activation by FDP. Protein synthesis ( ${ }^{3} \mathrm{H}$-leucine incorp.) in maternal leukocytes was correlated with wt/gest age of the baby. In a prospective study of 56 women for whom the outcome of pregnancy was known multivariate analyses showed significant correlations between sets of leukocyte \& nutrient data at midpregnancy \& the ultimate size attained by the fetus \& the placenta at term. Among the significant variables were protein/DNA,energy charge \& protein synthesis (leukocytes), diet protein,plasma zinc, $\mathrm{Fe}$, \& wt gain (nutrients). Arginine+ornithine/isoleucine ratio of plasma at midpregnancy also correlated with baby size. These data suggest that some nutrients, amino acid substrates, protein synthesis \& enzyme regulation may be altered in cells of mothers having undergrown babies. (Supported by HD-06915 NICHD and WHO)

142 THE ROLE OF "LIPID BOUND CARNITINE" ON THE RESPIRATION OF HUMAN NEWBORN AND ADULT WHITE ADIPOSE TISSUE MITOCHONDRIA, Milan Novak, Pau1 B. Wieser, Maria Buch. Department of Pediatrics, University of Miami School of Medicine, Miami, Florida.

The mitochondria isolated from subcutaneous white adipose tissue (WAT) of newborns exhibit increased oxygen consumption in the presence of carnitine ( 16 nmoles $\mathrm{O}_{2} / \mathrm{mg}$ protein $/ \mathrm{min}$ in the absence and 30 nmoles $\mathrm{O}_{2} / \mathrm{mg} / \mathrm{min}$ with carnitine). The respixatory rate will decrease if carnitine acyl transfer is inhibited by deoxycarnitine ( 5 nmoles $0_{2} / \mathrm{mg} / \mathrm{min}$ ). This effect is not seen in mitochondria isolated from adult WAT. However, mitochondria from adult WAT have a higher respiratory rate than mitochondria from newborn WAT ( 16 nmoles $\mathrm{O}_{2} / \mathrm{mg} / \mathrm{min}$ for newborns compared to 290 nmoles $\mathrm{O}_{2} / \mathrm{mg} / \mathrm{min}$ for adults). Carnitine palmitoyl transferase activity (CPT) per mitochondrial protein is lower in adult WAT mitochondria. The increase of lipid bound carnitine after birth in WAT and the high levels that we observed in adult WAT mitochondria are consistent with the increased fatty acid oxidation measured in adult mitochondria and implies that lipid bound carnitine is part of another transport system which operates in adults to accomplish acyl transfer into mitochondria. Attempts to chemically identify lipid bound carnitine indicate that these compounds could be composed of palmitoyl-carnitine or phosphatidyl-carnitine.

Supported by NIH grant HD 04946 . 
14 EFFECT OF THYROXINE $\left(T_{4}\right)$ ON RAT SERUM CORTICOSTERONE (CS) DURING EARLY DEVELOPMENT. Russell E. Poland and Morton E. Weichsel, Jr., UCLA Sch. of Med., Harbor

Gen. Hosp., Depts. of Psychiatry and Pediatrics, Torrance, Cal if Experimental hyperthyroidism and hyperglucocorticoidism in the rat have been shown to cause profound morphological and biochemical effects on early brain development. In order to define the effect of experimental hyperthyroidism on serum glucocorticoids, we measured levels of serum CS in stressed and nonstressed $\mathrm{T}_{4}$ treated $(0.4 \mu \mathrm{g} / \mathrm{gram}$ body weight) and saline injected control rat pups from ages $0-18$ days. The normal postnatal developmental pattern and levels for CS in stressed (stress induced by handling) and non-stressed control animals were similar to results previously reported. In $T_{4}$ treated stressed animals, levels of CS were significantly elevated by day 4 of treatment compared with controls $(2,3$, and 5 times control on days 4,12 , and 18 respectively). The day 4 level of $\mathrm{CS}$ in $\mathrm{T}_{4}$ treated stressed animals was not reached until day 15 in the stressed controls. However, $T_{4}$ treatment produced no changes in the circulating level of CS in non-stressed animals. Results show that $\mathrm{T}_{4}$ alters the developmental pattern of stress responsiveness while not affecting basal circulating levels of CS. Thus, interhormonal relationships appear important in mediating the effects of perinatal endocrinopathies on brain development.

147

CARNITINE CONTENT OF HUMAN ADIPOSE TISSUE DURING EARLY DEVELOPMENT. Eberhard Schmidt-Sommerfeld*, Paul B. Wieser, Maria Buch, Milan Novak, Department of Pediatrics, University of Miami School of Medicine, Miami, Florida Carnitine was shown to enhance lipolysis and fatty acid oxidation as well as fatty acid reesterification in human newborn white adipose tissue (WAT). To elucidate its role in energy metabolism during early development, carnitine was measured in WAT of normal newborns. The acid soluble (free) carnitine as well as acid insoluble (bound) carnitine was significantly lower during the first 30 hours of life than after 30 hours (free carnitine 48 nmoles/g tissue compared to 127 nmoles/g tissue and bound carnitine 31 nmoles/g compared to 56 nmoles/g). The free carnitine level of adult WAT was comparable to the level in the post 30 hour newborns. However, acid insoluble carnitine was two fold higher in adults than in newborns when the data was expressed on a protein basis. Comparative measurements in brown adipose tissue (BAT) of neonatal rabbits showed a four times higher level of acid soluble carnitine than in human newborn WAT whereas acid insoluble carnitine was in the same range. The amount of carnitine in rabbit milk was ten times higher than in human breast milk. Commercial formulas with a soybean base had little or no carnitine ( 0 to 11 nmoles/m1) while other formulas with a milk base had higher levels (55 to 210 nmoles $/ \mathrm{m} 1$ ). Sufficient carnitine intake during the first days of life may be important for energy metabolism of the newborn.

*Deutsche Forschungsgemeinschaft Fellow.

Supported in part by NIH grant HD 04946.

145 INTESTINAL DISACCHARIDASES (D) IN HUMAN, SHEEP AND RABBIT AMNIOTIC FLUID (AF) DURING GESTATION. M. Potier ${ }^{1}$, P. Guay ${ }^{2}$, P. Lamothe $^{2}$, D. Bousquet $^{2}$

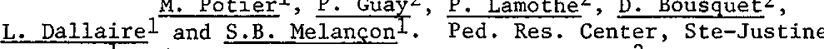
Hospital ${ }^{1}$ and Res. Center on Animal Reproduction ${ }^{2}$, Faculty of Veterinary Med., U. of Montreal, Quebec, Canada.

The fetal intestine has been implicated as the source of amniotic fluid D (maltase, sucrase, trehalase, turanase, cellobiase and lactase) in the human on the basis of very similar kinetic and physical properties between intestinal and AF D as well as a high degree of correlation between levels of intestinal and $\mathrm{AF} \mathrm{D}$ activities. The time-course of $\mathrm{D}$ release into the amniotic cavity has been studied in human, sheep and rabbit. D activities in the human and sheep AF fall rapidly at 22 weeks and 85-90 days gestation respectively, and thereafter remain low. In the human and sheep, these periods of gestation correspond to the rapid accumulation of large amounts of meconium in the fetal large intestine. No such drop of $D$ activities was observed in the rabbit which is much less mature at birth than the human and sheep and does not accumulate meconium in the large intestine. $D$ activities are apparently released into the $A F$ by fetal defecation. The fall of $\mathrm{D}$ activities in $\mathrm{AF}$ suggests that the human and sheep fetuses stop defecating or that rapid changes in the permeability of the intestinal mucosa to $D$, which would affect extrusion of $\mathrm{D}$, occurs at these periods of gestation. Supported by the Medical Research Council of Canada (grant MA-5163).

14.6 SELENTUM LEVELS IN MATERNAL AND CORD BLOOD: CORRELATION WITH GLUTATHIONE PEROXIDASE ACTIVITY. Nathan Rudolph (Spon. by Charles D. Cook) Downstate Med.Ctr, S.U.N.Y., Dept. of Ped., Bkiyn., N.Y.

Selenium has been recognized as an essential trace metal in animal nutrition. Though its biological and metabolic functions are not fully understood, Se appears to interact with Vit. E and sulfur amino acids in protecting tissues from oxidative damage. Recent animal studies demonstrated that Se is an integral part of the enzyme glutathione peroxidase (GSH-Per), and that the activity of the latter is a function of dietary Se. GSH-Per activity is known to be reduced in newborn RBC's. To evaluate whether any correlation exists in the human between Se levels and GSH-Per activity, 19 pairs of maternal and cord blood samples were assayed for Se, with the following results:

$$
\text { Mean Plasma } \mathrm{Se}(\mu \mathrm{g} / \mathrm{ml}) \pm \text { S.D. }
$$

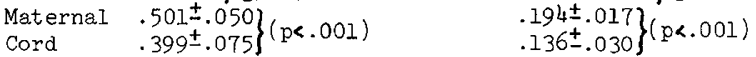

With the exception of one RBC sample, each mother's RBC and plasma Se levels were consistently higher than respective values in her offspring. RBC GSH-Per activity was also consistently higher in the mother as compared to her offspring. A close correlation ( $r=0.86)$ was found between GSH-Per activity and Se levels, confirming animal data. It is uncertain whether the low Se levels in cord plasma and red cells represent inadequate placental transport of the metal, or the correlate of a developmental phase of low GSH-Per activity adapted to a low intrauterine oxygen environment.
148 FETAL METABOLISM IN FASTING SHEEP. Richard L。 Schreiner, Laurence I. Burd, M. Douglas Jones, Jr. James A. Lemons, Roger E. Sheldon, Michael A. Simmons, Frederick C. Battaglia, and Giacomo Meschia. University of Colorado Medical Center, Division of Perinatal Medicine, Denver.

Maternal and fetal arterial concentrations and umbilical venousarterial concentration differences of glucose, fructose, lactate, pyruvate, acetoacetate (AcAc), $\beta$-hydroxybutyrate $(\beta \mathrm{OH})$, free fatty acids, and oxygen content, as well as maternal and fetal $\mathrm{pH}, \mathrm{pCO}_{2}, \mathrm{pO}_{2}$, urea, hematocrit, and protein were measured in 11 chronic sheep preparations remote from surgical stress. After two days of stable data in the fed state, maternal fasting was initiated. Fasting induced a decrease in maternal and fetal concentrations of plasma glucose, umbilical glucose/oxygen quotient (.64 fed; . 30 fasting), and fetal fructose $(88 \pm 6 \mathrm{mg} / 100 \mathrm{ml}$ fed; $24.8 \pm 1.0 \mathrm{mg} / 100 \mathrm{ml}$ fasting). Although there was a significant uptake of lactate by the fetus (Iactate/ $\mathrm{O}_{2}$ quotient . 19 fed; . 15 fasting), there were no significant changes during fasting. Maternal $\mathrm{AcAc}$ and $\beta \mathrm{OH}$ concentrations increased during fasting to $.99 \pm .28 \mathrm{mM}$ and $2.41 \pm .70 \mathrm{mM}$, respectively, on the 7th day. The changes in fetal AcAc $(.23 \pm .006 \mathrm{mM}$ fed; $.38 \pm .004 \mathrm{mM}$ fasting) and $\beta \mathrm{OH}(.11 \pm .01 \mathrm{mM}$ fed; $.18 \pm .1 \mathrm{mM}$ fasting) were much smaller。 There was no significant fetal uptake of $\beta \mathrm{OH}$ and AcAc during fasting. The fetal-maternal arterial concentration differences of urea increased with fasting, indicating a higher rate of amino acid catabolism by the fetus.

\section{A DEVELOPMENT OF ORGAN BLOOD FLOWS IN THE} Douglas Jones, Jr. Edgar L. Makowski, Frederick C. Battaglia, and Giacomo Meschia. Division of Perinatal Medicine, University of Colorado Medical Center, Denver.

While there is considerable knowledge about organ blood lows in adults, the changes in organ flows have not been studied during different stages of development.

Blood flow to individual organs was measured using nuclide-labeled microspheres in 16 fetuses and 11 lambs aged -12 to 226 days. Percentage of left ventricular output (\%LVO) and oxygen delivery were calculated for each organ. The development of cerebral, renal and coronary blood flows was very different: blood flow per gram of tissue declined in brain to half its fetal value, while in kidney, it rose to 3-4. times fetal levels. The brain grew to twice its fetal weight; thus its absolute flow remained constant. The kidneys grew to $\sim 6$ times fetal weight, and absolute blood flow increased nearly twentyfold. Oxygen delivery to brain (per gram of tissue) was constant at all ages, while that to kidney showed a fourfold rise. Brain \%LVO fell from $\sim 4$ to $\sim 1 \%$, while kidney \%LVO rose from $\sim 5$ to $\sim 15 \%$.

The development of coronary blood flow produced a decline in flow per gram to $\sim$ half the fetal value. This decline is the same as that seen in brain, but in contrast, the heart grew to 9 times its fetal size, and thus its absolute blood flow increased nearly fourfold. Oxygen delivery showed no significant change over time. 
149

PERTURBATION OF THE RENIN-ANGIOTENSIN-ALDOSTERONE SYSTEM IN THE NEWBORN LAMB. Sharon R. Siegel and Delbert A. Fisher, UCLA-Harbor General Hospital, Department of Pediatrics, Torrance, California.

Plasma renin activity (PRA) and aldosterone concentrations are high during the neonatal period but little is known about the physiology of the renin-angiotensin-aldosterone system in early extrauterine life. The purpose of this study is to determine in the newborn lamb whether the system can respond to stimulation.

The system was perturbed with the natriuretic drug, Furosemide, $2 \mathrm{mg} / \mathrm{kg}$, infused at 24-72 hrs. of age I.V. Five lambs were studied; blood samples were drawn at $8,20,35,65$, and 125 min. post infusion. PRA (measured as angiotensin I generated) and aldosterone were measured by RIA.

The baseline PRA values increased from a mean (and SEM) value of $5.2 \pm 1.5$ to $10.8 \pm 2.4 \mathrm{ng} / \mathrm{ml} / \mathrm{hr} .,(\mathrm{p}<.02)$ at $8 \mathrm{~min}$. post infusion, and to $20.3 \pm 4.6 \mathrm{ng} / \mathrm{ml} / \mathrm{hr}$. at $15 \mathrm{~min}$. ( $\mathrm{p}<.05$ ). PRA remained elevated through $120 \mathrm{~min}$. Plasma aldosterone levels increased by $30 \mathrm{~min}$. and plateaued through $125 \mathrm{~min}$. There was no change in plasma sodium or osmolality. Sodium diuresis was not observed until after $30 \mathrm{~min}$.

The results indicate that the renin-angiotens in system responds promptly to stimulation and angiotens in evokes prompt aldosterone secretion in early extrauterine life in the lamb. PRA levels increased $8 \mathrm{~min}$. post infusion without change in plasma sodium or osmolality and before sodium diuresis was observed. Thus, it is possible that Furosemide may directly stimulate renal renin release.

150 REgULATION OF ALDOSTERONE SECRETION IN THE NEONATE. Richard L. Siegler, Ronald $\mathrm{H}$. Crouch, Theodore $\mathrm{N}_{\text {。 }}$ Hackett, Jr., Margaret W. Miller, William Jubiz

(Spon. by Lowell A. Glasgow). Dept. of Ped. and Med., Univ of Utah Col. of Med., Univ. and VA Hosp., Salt Lake City.

In adults aldosterone secretion is regulated primarily by the renin-angiotensin system (RAS), and there is good correlation between plasma renin activity (PRA) and serum aldosterone levels.

Concurrent PRA, and serum aldosterone levels were measured by radioimmunoassay in cord blood $(n=14)$, at $2-3$ days of age $(n=10)$, and at 3-12 months of age $(n=10)$. The following values (mean + SD) and correlation coefficients were obtained.

\begin{tabular}{|c|c|c|c|c|}
\hline & $\begin{array}{l}\text { Cord } \\
\text { Blood }\end{array}$ & $\begin{array}{c}2-3 \\
\text { Days age }\end{array}$ & $\begin{array}{c}3-12 \\
\text { Months age }\end{array}$ & $\begin{array}{l}\text { Norma } 1 \\
\text { Adults }\end{array}$ \\
\hline $\begin{array}{c}\text { Aldosterone } \\
(\mathrm{ng} / \mathrm{d} 1)\end{array}$ & $95.6 \pm 38.8$ & $105.9 \pm 51.2$ & $59.8+34.6$ & $14.2+4.0$ \\
\hline $\begin{array}{l}\text { PRA } \\
\quad(\mathrm{ng} / \mathrm{m} 1 / \mathrm{hr})\end{array}$ & $10.1 \pm 4.7$ & $11.7 \pm 5.5$ & $6.3 \pm 3.9$ & $1.1 \pm 0.5$ \\
\hline $\begin{array}{c}\text { Aldosterone } \\
\text { vs. PRA }\end{array}$ & $r=.03$ & $r=-.01$ & $\mathrm{r}=.81, \mathrm{p} .01$ & \\
\hline
\end{tabular}

Although mean neonatal PRA and serum aldosterone levels were both significantly higher $(\mathrm{p}<.01)$ than adult values, there was no correlation between concurrent samples in individual infants until 3-12 months of age. There was also no significant correlation between neonatal aldosterone and serum potassium concentrations. By 3-12 months, however, there was strong correlation $(r=.81, p<.01)$. These observations suggest, that contrary to older infants and adults, neonatal aldosterone secretion does not appear to be regulated primarily by the RAS.

\section{HEAT PRODUCING EFFECT OF CARNITINE IN} 151. NEWBORNS. Uwe Stave, Milan Novak, Paul B. Wieser, Maria Buch. Department of Pediatrics, University of Miami School of Medicine, Miami, Florida.

Carnitine enhances free fatty acid (FFA) oxidation by facilitating FFA transport across mitochondrial membranes and in addition, it activates ATP generation. Administration of L-carnitine to adult animals increased their $\mathrm{O}_{2}$ consumption and body temperature only if they were pretreated with catecholamines. In newborns, however, this conditioning is not necessary as we have demonstrated in $24 \mathrm{~h}$ old rabbits $(n=15)$ kept in thermoneutral environment. $20 \mathrm{~min}$. after $50 \mathrm{mg} / \mathrm{kg}$ carnitine i.p. $(n=14)$, we recorded a marked increase $\left(1.5^{\circ} \mathrm{C}\right.$ ) of the skin temp. over the intrascapular fat pads, and a lesser increase of deep rectal temp. Blood glycerol levels increased from $0.11 \mu \mathrm{moles} / \mathrm{ml}$ in saline injected controls to a mean of $0.17 \mu$ moles $/ \mathrm{ml}$ in carnitine treated newborns ( $p<0.001$ ); simultaneously the FFA blood levels decreased to half of the control values. 12 newborns were kept in cold environment $\left(20^{\circ} \mathrm{C}\right)$ and after the same i.p. dose of carnitine their skin temp. showed similar increases; relative changes of blood glycerol and FFA were even more significant than in the normothermic group. The ability of the newborn mammal to tolerate cold stress seems to be dependent on exogenous carnitine (mother's milk) until his own snythesis suffices to meet the need. - -Supported in part by NIH grant HD 04946.
7 COMPARISON OF GLUCOSE-6-PHOSPHATE DEHYDROGENASE (G6PD) STEROID DEHYDROGENASE (SD), AND MALATE DEHYDROGENASE (MD) IN HUMAN PLACENTAS OF DIFFERENT GESTA TIONAL AGES. D.P. Walker, D. Nabich, P.R. Williams. Wm. Beaumont Hosp, Dept.of Peds., Royal Oak, Mi, and Wayne State Univ. Sch.of Med., Dept. of Biochem., Detroit, Mi. (Introduced by R.L. Poland.) Initially regarded as a modified semi-permeable membrane, the placenta is now considered capable of many biosynthetic processes. This study was done to establish a method of evaluating marker enzymes for steroidogenesis, the hexose monophosphate shunt (HMP) and Kreb's cycle in partially purified homogenates derived from placental tissue ( $8-40$ weeks gestation). Samples were assayed for G6PD, SD, and MD. The specific activity of SD was significantly weeks gestation $(0.015+0.012 *, \mathrm{p}<0.05)$, indicating increased steroidogenesis. Mitochondrial MD activity increased with gestational age $(1.49 \pm 0.294 *$ at term) compared to $0.232 \pm 0.221 \%$, $(\mathrm{p}<.01)$ in the immature placenta, suggesting a mechanism of providing reducing equivalents for intramitochondrial processes. In contrast, cytoplasmic MD and G6PD did not appear to increase with gestational age. Cytoplasmic $M D$ is important in the maintenance of cytoplasmic NAD via the malate-oxaloacetate shuttle. It probably operates at maximal activity throughout gestation and is not rate-limiting. The failure of G6PD to increase at term suggests: 1) either the enzyme does not reflect the activity of the HMP; or, 2) the demand for products of the shunt changes with gestational age.

*Change in optical density $(340 \mathrm{~nm}) / \mathrm{min} / \mathrm{mg}$ protein.

\section{FETAL UPTAKE OF AMINO ACIDS AND BRAIN ENERGY METABOLISM IN EXPERIMENTAL HYPERPHENYLALA- NINEMIA. Raul A. Wapnir, Susan A. Moak and Fima} Lifshitz. Dept. of Peds., North Shore Univ. Hosp., Manhasset, NY and Dept. of Peds., Cornell Univ. Med. Col., New York, NY.

The effects of experimental hyperphenylalaninemia (HyPhe) on transplacental transport of amino acids and brain energy metabolism were assessed on pregnant rats made HyPhe by a diet with 7\% excess phenylalanine (Phe) during the last week of gestation, plus an oral load of 500 $\mathrm{mg} / \mathrm{kg}$ of Phe $1 \mathrm{hr}$ prior to the procedure, carried out one or two days before delivery. Controls $(\mathrm{C}+\mathrm{l})$ were fed a lab chow ad lib. Placental transport was estimated by measuring radioactivity of maternal and fetal blood, and placenta at $15,30,60,90$ and $120 \mathrm{~min}$ after a $4 \mu \mathrm{Ci} / 100 \mathrm{~g} \mathrm{~b} . \mathrm{w}$. pulse of ${ }^{3} \mathrm{H}-\mathrm{L}$ tryptophan (Trp) or ${ }^{3} \mathrm{H}-\mathrm{L}$-tyrosine (Tyr). HyPhe caused a marked reduction in fetal blood Trp levels at all times. The mean $\mathrm{dpm} / \mathrm{ml}$ in this fluid for HyPhe vs $\mathrm{CHI}$ were $\left(\times 10^{-3}\right)$, at $15^{\prime}: 28.5$ vs $50.9 ; 30^{\prime}$ : 26.6 vs $43.8 ; 60^{\prime}: 22.0$ vs $37.5 ; 90^{\prime}: 21.4$ vs 34.4 and $120^{\prime}: 19.6$ vs 31.6 ( $p<.07$ or less in all cases). Placental uptake, however, was unchanged. in contrast, Tyr uptake by placenta and transport to the fetus was not impaired. HyPhe also inhibited fetal brain pyruvate kinase (means \& SEM, HyPhe: .248 $\pm .021 ; \mathrm{CHl}: .401 \pm .015 \mathrm{U} / \mathrm{mg}$ protein, $p<.01)$. Fetal brain hexokinase was unaltered. The fetal: placental weight ratio was identical in both groups. These data suggest that intrauterine damage in HyPhe can be associated with impaired placental transport of certain essential amino acids and interference with brain metabolism.

154 REGULATION OF GROWTH IN CULTURED HEART CELLS, Joseph B. Warshaw, Brian J. Coyne, Lynn Barrett, Yale Univ. School of Medicine, Departments of Pediatrics and OB/Gyn, New Haven, Connecticut

Growth of chicken embryonic heart cells in culture declines as cells reach confluency. The decline in rate of growth has been associated with a reduction in $\mathrm{pH}$ of the culture media and a decreased capacity for glucose oxidation by the pentose phosphate pathway. We have carried out experiments to further define the role of the pentose phosphate pathway in regulation of cell growth. An increase in the $\mathrm{pH}$ of the culture medium by $0.3 \mathrm{pH}$ units with $14 \mathrm{mil}$ bicarbonate or a mixture of organic buffers resulted in a $40 \%$ enhancement of cell proliferation. This was preceded by a significant increase in pentose phosphate pathway activity assessed by measuring the ratio of $14 \mathrm{CO}_{2}$ production from glucose-1-14 C and glucose-6-14 C by cultured cells. Total glucose uptake by $\mathrm{pH}$-enhanced cells was increased by $70 \%$ as compared with controls. Stimulation of growth and increased uptake of glucose was always associated with increased activity of the pentose phosphate pathway. These data provide further evidence for a possible controlling relationship between activity of the pentose phosphate pathway and cell growth. 
155 INFLUENCE OF GLUCAGON AND INSULIN ON ALANINE RELEASE FROM CULTURED CHICK SKELETAL MUSCLE, Joseph B. Warshaw, Robert Taylor, Yale University School of Medicine, Departments of Pediatrics and OB/Gyn, New Haven, Conn.

Control of muscle catabolism in response to endocrine and nutritional influences remains a major question。 We have utilized monolayer cultures of chick embryonic skeletal muscle to examine influences of glucagon and insulin on alanine release from cultured muscle cells. Chick embryonic myoblasts were isolated from thigh muscle of 14 day chick embryos and grown to confluency in minimal essential medium (MEM) supplemented with $10 \%$ horse serum and $5 \%$ chick embryo extract. The cultures containing myofibrils were carefully washed with Krebs-Ringer phosphate buffer (KRP) and incubated for four hours in KRP containing 0.1 unit insulin or 10 um glucagon. After four hours of incubation at $37^{\circ}$, the media was retained for assay of alanine content. MEM contains no alanine so that all alanine produced derives from the cells themselves. Addition of glucagon decreased alanine release into the assay medium by $40-60 \%$. Incubation with insulin also resulted in a $40-50 \%$ decline. Addition of $2 \mathrm{mM}$ glucose to the assay medium in addition to glucagon or insulin resulted in an increased alanine release compared with control cultures. The data suggest that when precursors are absent, alanine release is decreased and both insulin and glucagon have a protein conserving effect. However, if glucose is available, alanine release from cultured skeletal muscle can take place even in the presence of glucagon and insulin. This provides evidence for the first time for a direct effect of glucagon on gluconeogenic substrate availability at the
level of muscle.

\section{6}

THYROXINE EFFECT UPON URIDINE KINASE IN DEVELOPING CEREBELLUM. Morton E. Weichsel, Jr., UCLA School of Med., Harbor Gen. Hosp., Dept. of Peds., Torrance, Ca Activity of uridine kinase (UK), a salvage (reutilization) pathway enzyme for pyrimidine nucleotide biosynthesis, has been shown previously to peak at 12 days of age in developing rat cerebellum and to fall only slightly thereafter. Because of the known shift to an earlier age in the developmental curve for cerebellar DNA synthesis and thymidine kinase (TK) activity in rats treated with thyroxine $\left(\mathrm{T}_{4}\right)$, we studied the activity of UK during cerebellar development in $T_{4}$ treated rat pups and controls.

UK activity was found to be increased significantly above control values at ages $2,4,6$, and 9 days (maximum $119 \%$ of control at age 4 days) forlowing which activity fell significantly below control values by 12 days of age. UK activity from both treated and control animals fell only moderately after the peak activity at 12 to 15 days of age. By day 18 , UK values in cerebella of control and treated animals differed by only $9 \%$. These data show a less pronounced early stimulation of UK by $T_{4}$ compared with previously reported $\mathrm{T}_{4}$ enhancement of TK activity. However, both enzymes are affected by $\mathrm{T}_{4}$ throughout early cerebellar ontogenesis. Developmental curves for cerebellar UK under control and hyperthyroid conditions thus differ from those established under similar conditions for other enzymes involved in pyrimidine biosynthesis. Whereas TK is felt to be related to DNA biosynthesis, these data support the hypothesis that UK is associated with the biosynthesis of RNA.

\section{DE NOVO PYRIMIDINE BIOSYNTHESIS DURING CEREBELLAR} DEVELOPMENT: EFFECT OF THYROXINE. Morton E. Weichsel, Jr., UCLA School of Medicine, Harbor General Hosp., Dept. of Pediatrics, Torrance, Calif.

Thyroxine $\left(T_{4}\right)$ administered from birth has been shown to accelerate cerebellar DNA synthesis in the rat from 2-6 days of age and to decrease DNA synthesis thereafter. This hormone-induced developmental acceleration has led to studies of the relationships between $T_{4}$, DNA, and activities of de novo and salvage pathway enzymes involving pyrimidine nucleotide biosynthesis during cerebellar development. The activity of rat cerebellar aspartate transcarbamylase (ATC), the second enzyme in the de novo pathway, has been shown to peak around 9 days and to decline thereafter. Cerebellar ATC activity in animals injected daily with $T_{4}$ was found to be similar to that in controls through age 6 days; subsequently, the activity in treated animals fell significantly below control values at ages $8,10,14$ and 19 days. These results show that $T_{4}$ affects cerebeliar ATC activity during the period of decreasing rate of cerebellar DNA synthesis, with the maximum deficit being $81 \%$ of control at age 14 days. Unlike enzymes studied thus far from salvage and interconversion pathways, ATC activity is not significantly affected during early cerebellar development when DNA synthesis is accelerated by thyroxine. This unique effect of $T_{4}$ on the de novo pathway enzyme ATC suggests that hormones may affect each pathway for pyrimidine nucleotide biosynthesis in a different manner at each age of early development and that specific contributions of given metabolic pathways to both DNA and RNA biosynthesis during different stages of development must be further elucidated.
158 VARIATIONS IN AMNIOTIC FLUID OSMOLALITY WITH GESTATIONAL AGE AND MATERNAL DISEASE, AND ITS RELATION
L/S RATIO, CREATININE, URINARY ESTRIOL LEVELS AND OXYTOCIN CHALLENGE TEST. Paul Y.K. Wu, Uwe Goebelsmann, Sze K. Ho, Omar Ghazzawi. Univ. of Southern California Sch of Med. LAC-USC Medical Center, Depts. of Pediatrics and OB/GYN, LoS Angeles.

Both volume and composition of amniotic fluid is known to vary with cestational age. The current studies involved amniotic filuid (AF) specimens obtained from 700 pregnant mothers at varying lengths of gestation. 0smolality was found to decrease from $290 \mathrm{~m} 0 \mathrm{sm} / \mathrm{L}$ at 28 weeks' gestation to values ranging from 195 to $258 \mathrm{mOsm} / \mathrm{L}$ at term. The fail in osmolality was correlated with a rise in $L / S$ ratio and creatinine in AF. In pregnancies complicated with diabetes, chronic hypertension and pre-eclampsia, osmola? ity was found to be higher than values obtained from uncomplicated pregnancies during corresponding weeks, and sequential specimens indicate a rise in osmolality w1th increasing gestational age. This rise was associated with a fall in urinary estriol levels, but had no relation to L/S ratio or creatinine levels in AF. In postmaturity, an increase in AF osmolality was observed to be correlated with SGA infants rather than to increasing gestational age. In placenta previa and Rh sensitization the change in osmolality in AF was variable depending on the fetal condition. No relation was found between amniotic fluid osmolality and response to oxytocin challenge test.

\section{DEVELOPMENTAL PHARMACOLOGY}

159 LEFT VENTRICULAR CONTRACTILITY IN NEWBORNS OF SMOKING MOTHERS. Robert A. Achte1, and John L. Erickson, (Spon. by Charlton C. Mabry). University of Kentucky College of Medicine, Department of Pediatrics, Lexington. Left ventricular contractility, (LVC) in newborns exposed to nicotine throughout gestation has hitherto not been considered, and was the object of this investigation. Immediately after breeding, 12 ewes were the subject of infusions of nicotine, $400 \mu \mathrm{g} /$ day, $\mathrm{n}=6$, and, normal saline, $\mathrm{n}=6$, into previously placed sialastic jugular venous reservoirs. LVC was studied in 17 (nicotine $=8$, normal saline $=9$ ) closed chest reflexic newborn lambs subjected to transient systemic hypoxia and infusions of isoproterenol $(0.1 \mu \mathrm{g} / \mathrm{kg})$. LVC was assessed by changes in Vce (dp/dt/developed pressure $40 \mathrm{mmHg}$ ) at a constant heart rate. Arterial blood gases and $\mathrm{pH}$ were closely monitored. In response to hypoxia, all animals demonstrated an increase in LVC $(p<0.001)$, a greater increase being seen in the nicotine group $(p<0.05)$. Isoproterenol infusions elicited a comparable increase in LVC in both groups. Beta adrenergic blockade abolished an increase in LVC to either hypoxia or isoproterenol in both nicotine and control animals. It is concluded that hypoxia evokes a greater increase in LVC in newborns exposed to nicotine throughout gestation. That a similar increase in LVC in all animals was elicited by the infusion of isoproterenol supports previous findings that endogenous myocardial norepinephrine synthesis is increased with chronic exposure to nicotine.

160 PHARMACODYNAMIC ASPECTS OF CYCLOPENTOLATE AND PHENYLEPHRINE OPHTHALMIC DROPS IN TPE NEWBORN. J.V. Aranda and A. Fuchs. (Intro. by E.Colle), Dept. Newborn Med. Montreal Children's Hosp. \& Dept. of Pharmacol.\& Thera. (Roche Dev. Pharmacol. Unit). Mcfill Univ., Montreal, Canada.

Cyclopentolate (Ly) and phenylephrine (F) are used routinely to produce cycloplegia and mydriasis for ophthalmoscopic examination (CEX) in preterm infants during or after oxygen therapy. The pharmacodynamic properties of these drugs have been studied in 9 nreterm for a 24 hour period during a routine OEx. Mean birth weights ( \pm S.E.) was $1855.9 \pm 162.9 \mathrm{gm}$ and weights during $\mathrm{OEx}$ was $2143.3 \pm 156.7 \mathrm{gm}$. Gestational age was $31.7 \pm 0.8$ weeks and age Oi: OEx was $26.4 \pm 6.4$ days. After base line measurements were chtained, one drop of Cy $(0.5 \%)$ and one drop of $\mathrm{P}(2.5 \%)$ per eye were administered to 4 neonates. One drop of $1 \% \mathrm{Cy}$ alone per eye was sj.milarily administered to 5 neonates. Relative to baseïine pupillary size $(1.6 \pm 0.1 \mathrm{~mm})$ significant increase in pupillary size was noted 5 min post-administration of either Cy\&P or of $\mathrm{Cy}$ alone. Time to maximal pupillary dilatation $(5.2 \pm 0.2 \mathrm{~mm})$ was 30 min with Cy\&P and 20 min with $C y$ alone $(4.8 \pm 0.2 \mathrm{~mm})$. Duration of pupillary dilatation was $6 \mathrm{~h}$ with Cy\&p and $9 \mathrm{hrs}$ with Cy. Light reflex was lost between 5-10 $\mathrm{min}$, with Cy\&P and less than $5 \mathrm{~min}$ in $\mathrm{Cy}$ alone. Loss of light reflex lasted for $12 \mathrm{~h}$. Transient increase in systolic blood pressure $(\triangle \mathrm{BP}=12 \mathrm{mmHg})$, was noted only with Cy\&P at $60 \mathrm{~min}$. No change in heart rate with both dose regimens. The data suggest that $\mathrm{OEx}$ should be done 20-30 min after $C y \pm P$ and the newborn should be protected from intense light exposure at least $12 \mathrm{~h}$ following administration of Cyt $\mathrm{P}$. 
161 PHARMACOKINETICS OF TOBRAMYCIN SULFATE IN NEWBORNS. Allan M. Arbeter, Marta Blesa, Michelle Danish, Brian Rosenfeld, George Peckham, Sumner Yaffe and Stanley A. Plotkin; Children's Hosp., Philadelphia, Pa. 19104

The pharmacokinetics of tobramycin sulfate (TS) were studied in 47 newborns with suspected or proven sepsis. Gestational ages varied from 28 to 43 wks. with birth wt. of 0.9 to $4.4 \mathrm{~kg}$. TS was given IV at $1.7 \mathrm{mgm} / \mathrm{Kg} /$ dose $g 8 \mathrm{hr}$. in combination with ampicillin or carbenicillin. TS levels were determined by an agar diffusion method against $B$. subtilis. Maltiple serun levels of TS were determined on each pt. The mean TS level at 60 to 90 minutes after completion of a dose was $5.4 \mu \mathrm{gm} / \mathrm{ml}$ (range= 2.5 to 24 ); at 7 to $8 \mathrm{hr}$ after a dose the mean was $1.6 \mu \mathrm{igm} / \mathrm{ml}$ (range= 3.8 to $<1$ )。 Mean serum half life was $5.6 \mathrm{hrs}$ (range 5 to $10 \mathrm{hr}$ ); inversely related to the gestational and extrauterine age. Nine newborns were studied in greater detail incluzing $2+\mathrm{hr}$. urine excretion, as well as matched urine-serun pairs, to yeild data on volume distribution, renal clearance, and the relationship of drug levels to creatinine clearance. It was impossible to predict levels on any given newborn by age or serum creatinine. All newborns were monitored for changes in blood chemistries, hematologic indices, and urinary sediment with no evidence of toxicity daring therapy.

\section{PHARMACOKINETICS OF MEPERIDINE IN INFANTS}

Gerald F. Atwood, Michael A. Evans, and Raymond D. Harbison (Sponsored by Thomas P. Graham, Jr.)

Departments of Pediatrics and Pharmacology, Vanderbilt University Medical Center, Nashville, Tennessee 37232

Meperidine (Demerol) is commonly used in infants and children as premedication undergoing various procedures. Clinical experience in infants has suggested that the duration of action and response of meperidine may be reduced in this age group when compared to adults. Nine infants ranging from 3 to 18 months of age undergoing cardiac catheterization procedures were given an intravenous bolus of meperidine in doses ranging from 0.65 to 1.1 $\mathrm{mg} / \mathrm{Kg}$ during the procedure. Blood samples were withdrawn and plasma meperidine was analyzed by gas-1iquid chromatography. Binding was also determined in plasma samples from both infant and normal adult subjects using (n-methy $1-C-14$ ) meperidine HCL.

The $\mathrm{Tl} / 2$ (B) in the infants was $2.3 \pm 0.4 \mathrm{hrs}$ with a steady state volume of distribution [Vd (ss)] of 51 iters $/ \mathrm{Kg}$. Previous studies from this institution demonstrated that in normal adults the $\mathrm{Tl} / 2$ (B) was $3.3 \pm 0.9 \mathrm{hrs}$ and mean $\mathrm{Vd}(\mathrm{ss})$ was $4.17 \mathrm{liters} / \mathrm{Kg}$. Plasma binding of $\mathrm{C}-14$ meperidine in adults was $64 \%$. Infants, however, had an increased plasma binding value up to $85 \%$.

It appears that the decreased $T] / 2$ (B) of meperidine in infants correlates with the clinical impression of a diminished duration of action. The increased plasma binding in infants associated with the lower Tl/2 $B$ and essentially similar Vd(ss) would suggest either increased hepatic blood flow in the younger age group or greater availability of the drug for hepatic metabolism because of higher plasma binding.

\section{3}

METABOLISM OF PROSTAGLANDINS A AND E $\left(\mathrm{PGA}_{1}\right.$ \& PGE $\left.\mathrm{PG}_{1}\right)$ BY THE PER FUSED RABBIT LUNG: DEMONSTRATION OF AGE DEPENDENCE. Michael A. Berman, K. Baker, C.N. Gillis, Yale Univ.Sch, Med., Depts. Ped, \& Anesth., New Haven。 (Intr. by Norman S. Talner)

Removal and metabolism of $\mathrm{PGA}_{1}$ and $\mathrm{PGE}_{1}$ was measured in isolated perfused rabbit lung preparations at ages $1,2,14,35,42$, $56,84,112,140$ days. Lungs were perfused through individual right and left pulmonary artery cannulae with $100 \mathrm{ng} / \mathrm{ml}$ of tritiated $P G A_{1}$ and $P G E_{1}$ at $2 \mathrm{ml} / \mathrm{min}$. Pulmonary venous effluents were acidified and extracted with ethyl acetate. PG compounds and their extractable metabolites were identified by thin layer chromatography. Representative data for the 1 and 56 day groups are listed.

$\%$ Nonlipid Sol-

\begin{tabular}{|c|c|c|c|c|c|c|c|c|}
\hline e & ubl & & $\% \mathrm{U}$ & & $\% \mathrm{Me}$ & ab. I & $\mathrm{Me}$ & II \\
\hline & $\overline{\mathrm{PGA}_{1}}$ & $\mathrm{PGE}_{1}$ & $\mathrm{PGA}_{1}$ & $\overline{\mathrm{PGE}_{1}}$ & $\overline{\mathrm{PGA}_{1}}$ & $\overline{P G E}_{1}$ & $\mathrm{PGA}_{1}$ & $\overline{\mathrm{PGE}}_{1}$ \\
\hline $1 d$ & 17.3 & 1.5 & 66.8 & 62.9 & $30.5^{\circ}$ & 43.1 & 10.7 & 9. \\
\hline & \pm 3.3 & +.2 & +8.1 & +10.0 & \pm 5.7 & \pm 6.3 & \pm 1.7 & \pm .8 \\
\hline & 38.3 & 5.2 & 8.3 & 26.7 & 43.5 & 60.1 & 18.2 & 11.0 \\
\hline & +1.0 & \pm .5 & \pm 2.4 & \pm 3.1 & +3.0 & \pm 4.0 & \pm 5.2 & th. \\
\hline
\end{tabular}
PGA the table reveals that PGA is significantly metabolized by the perfused rabbit lung. Moreover, changes in uptake and metabolites of $\mathrm{PGA}_{1}$ and $\mathrm{PGE}_{1}$ with age are demonstrated.

164 MATERNAL-FETAL TRANSFER OF CEFATRIZINE。 Betty Bernard, Salvador Garcia-Cázares, Omax Ghazzawi, Pau1 Thielen and Charles A. Ballard. (Spon. by Paul $F$. Wehrle). Univ. of Southern California, IAC-USC Med. Ctr., Depts. of Pediatrics and Obstetrics \& Gynecology.

To investigate the maternal-fetal transfer and fetal pharmacology of a new semi-synthetic cephalosporin antibiotic, 12 healthy gravidas received a single $1000 \mathrm{mg}$ oral dose of cefatrizine (BL-S640) 3 to 20 hours prior to therapeutic abortion and sterilization by hysterectomy. BL-\$640 concentration was assayed microbiologically in maternal serum (at $1,2,4,8$ hours and at delivery), myometrium, fetal tissues (placenta, brain, kidney, liver, lung) and fluids (amniotic, CSF, urine and serum).

Maternal serum half-life $\left(T-\frac{1}{2}\right)$ was 1.5 hours and the peak serum concentration was $18 \mathrm{\mu g} / \mathrm{ml}$ at 4 hours. Antimicrobial activity was not detected in myometrium, fetal liver or brain. Placental concentrations of cefatrizine reached $1.6 \mu \mathrm{g} / \mathrm{gm}$ and plateaued between 5 and 14 hours. BL-S640 was found in the CSF of $<16$ weeks' gestation fetus and $<0.2 \mathrm{\mu g} / \mathrm{mI}$ for 14 hours. Fetal lung and kidney tissue concentrations were <lug/gm by 6 hours and fetal urine concentrations reached $2 \mu \mathrm{g} / \mathrm{mI}$ by 5 hours following maternal drug administration. Ammiotic fluid peaked at $0.2 \mu \mathrm{g} / \mathrm{mI}$ at 6 hours and disappeared by 14 hours. These data indicate very little maternal-fetal transfer of cefatrizine and no specific tissue accumulation.

165 EFFECT OF PROPRANOLOL IN THE TREATMENT OF 165

Robert C. Boerth (Sponsored by Thomas P. Graham, Jr.)

Vanderbilt University Medical Center, Departments of Pediatrics and Pharmacology, Nashville, Tennessee

Propranolo1 (P) is used to treat some types of hypertension (HBP) in adults, but no information is available on effective doses or plasma levels of $P$ when $P$ is used as an antihypertensive agent in children and adolescents. This study reports the results from 11 hypertensive pediatric pts (ages 7 mos to $16 \mathrm{yrs}$ ) in whom plasma levels of $P$ were measured at the end of dosage intervals. The HBP in 6 pts (ages 7 mos to 11 yrs) was secondary to chronic renal parenchymal disease or hypoplastic abdominal aorta (Group 1), and 5 pts (ages 14 to 16 yrs) had primary HBP (Group 2). Four pts had high peripheral renins and 7 pts had normal renins. Pretreatment BP averaged 144/98 in Group 1 and $143 / 104$ in Group 2. $P$ was the only pharmacologic agent in 5 $\mathrm{pts}$, and $6 \mathrm{pts}$ received $\mathrm{P}$ plus diuretics. A dose of $\mathrm{P}$ was considered to be effective when diastolic BP was reduced to $\leq 90 \mathrm{mmH}$ $\mathrm{P}$ reduced $\mathrm{BP}$ in all 11 pts. Effective doses of $\mathrm{P}$ ranged from 2.5 to $16 \mathrm{mg} / \mathrm{kg} /$ day in Group $1 \mathrm{pts}$, and effective plasma levels averaged $140 \mathrm{ng} / \mathrm{ml}$ (range $=40$ to $226 \mathrm{ng} / \mathrm{ml}$ ). In Group 2 the effective dose of $P$ ranged from 1.8 to $704 \mathrm{mg} / \mathrm{kg} / \mathrm{day}$, and effective plasma levels ranged from 54 to $310 \mathrm{ng} / \mathrm{ml}$ (mean=11l ng/ml). There were no observed side effects in any of the pts. It is concluded that 1) P may be effective for treating HBP of various etiologies in pediatric pts, 2) there is wide variation in dosage requirements of $P$ between hypertensive pts and 3) $P$ has no effect on HBP until plasma levels $>40$ to $50 \mathrm{ng} / \mathrm{ml}$ are reached.

PROGNOSIS OF INFANTS BORN TO DRUG DEPENDENT MOTHERS: 166 ITS RELATION TO THE SEVERITY OF WITHDRAWAL DURING THE $\frac{\text { Ir. Milton E. Strauss, Joan C. Stryker, Wayne State Univ., Hut- }}{\text { zei Hosp. \& Children's Hosp. of Mich., Depts. of Peds., Psychol. }}$ \& OB, Detroit, Michigan (Spon. by Sanford N. Cohen) A systematic follow-up of 103 infants of drug dependent mothers
(IDDM) was conducted to determine their growh \& mental \& psycho-
motor developments. Based on specific criteria (Int'1. J. Addict. motor developments. Based on specific criteria (Int'1. J. Addict.
Dis. 2:187, 1975 ), the severity of the withdrawal was assessed as Dis. $2: 187,1975$ ), the severity of the withdrawal was assessed as
minor to mi1d (MM) in 79 infants (77\%) \& moderate to severe (MS) in 24 (23\%). Six (27\%) of the infants in the MS group receiv RESULTS: During the first 8 weeks of follow-up, a significant $1 \mathrm{y}$

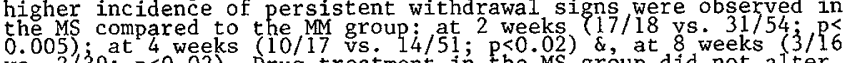

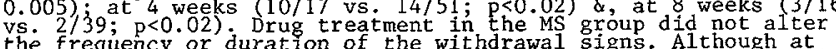

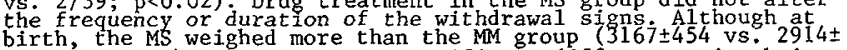
birth, the MS weighed more than the MM group (3167士454 vs. $2914 \pm$

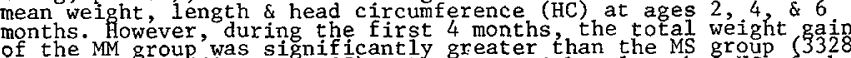

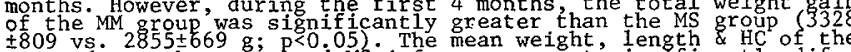
\pm 809 vs. $285 \pm 569$ g; p<0.05). The mean weight, length \& HC of the
treated vs. the unteated MS infants were not significantly dif-
ferent. Mental \& psychomotor development scores did not signififerent. Mental \& psychomotor development scores did not signifi-
cantly differ in the MM \& MS group when examined at $4 \& 6$ months.

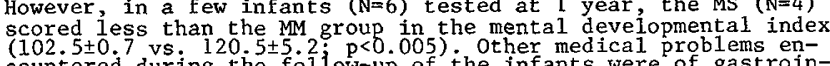
countered during the fol ow wp of the inf ants were of gastroin-
testinal, skin \& respiratory origin. The occurrence of these problems in both groups was essentially. Thimilar. However more infants in the MS group were hospitalized (5/19 vs. $6 / 65 ; p<0.025)$. Two infants in the series died, bot
sudden infant death syndromes. 
167 RESPONSE OF THE LAMB FETUS TO EXOGENOUS VASOof Perinatolog. S. James, Coll. of Phys. \& Surg., Columbia Univ.,Div. Extremely high levels of vasopressin have been found in fetal blood both at delivery and under hypoxic conditions. Experiments have been undertaken in 8 fetal lambs intact in utero and in 8 newborn lambs in order to study the renal response to $5.0-10.0 \mathrm{mU} / \mathrm{kg}$ vasopressin $1 . \mathrm{V}$. Vasopressin caused an increase in urine output from 0.17 to a maximum of $0.58 \mathrm{ml} / \mathrm{kg}$ min and in osmolality from 149 to $310 \mathrm{mOsm} / \mathrm{kg}$ at the end of one hour . This response was accompanied by an increase in the concentration of both sodium and chloride in the urine. In the newborn vasopressin also caused a rise in urine output, but the rise was transient and much smaller, from 0.09 to $0.21 \mathrm{ml} / \mathrm{kg} \mathrm{min}$ after $15 \mathrm{~min}$ while urine osmolality rose from 330 to $510 \mathrm{mOsm} / \mathrm{kg}$ and remained elevated for two hours. In both the fetus and the neonate, vasopressin caused a fall in free water clearance which lasted for approximately $30 \mathrm{~min}$ in the fetus and one hour in the newborn. Vasopressin caused a rise in mean arterial blood pressure of $10-20 \mathrm{mmHg}$ within 15 seconds. Blood pressure began to decline after 5 $\mathrm{min}$ and returned to control level by $15-30 \mathrm{~min}$. These studies indicate that in the well hydrated healthy fetus, vasopressin, in a dose that produces antidiuresis in the adult, has a predominantly natriuretic action which cannot be explained entirely by its pressor effect. This response provides an explanation for the natriuresis that occurs following partial or complete occlusion of the umbilical cord in the fetal lamb.

\section{EFFECT OF RITODRINE INFUSION ON UTERO-PLACENTAL}

CIRCULATION IN PREGNANT SHEEP. Richard A. Ehrenkranz Adrian M. Walker, Gary K. Oakes, Margaret ${ }^{-}$K. McLaughl in and Ronald A. Chez, (Spon. By Joseph D. Sch

We studied the response of the utero-placental circulation in chronically instrumented Dorset sheep to ritodrine ( $R$ ), a $B-$ adrenergic agonist effective in arresting premature labor in humans. At 122-132 d gestation, electromagnetic flow probes were applied to the main uterine artery of the pregnant horn and the common intra-abdominal umbilical vein; catheters were placed in maternal and fetal vessels and the amniotic cavity. At 2-13 d postoperation, R was infused IV to the ewe for $120 \mathrm{~min}$; uterine contractions were not present. During $R$ infusions of incrementa 1 doses from 100 to $800 \mu \mathrm{g} / \mathrm{min}(n=5)$, uterine blood flow (UtBF) fell progressively to $43 \pm 6 \%$ (mean $\pm S E, p<.01$ ) below control levels, and mean maternal arterial pressure (MMAP) declined 20 $6 \%(p<.05)$. During constant infusions of $100 \quad(n=5)^{\prime}, 400 \quad(n=6)$ or $800 \mu \mathrm{g} / \mathrm{min}(n=4)$, UtBF decreased $10 \pm 4 \%(p<.05), 37 \pm 5 \%(p<.001)$ and $3 T \pm 7 \%(p<.05)$ respectively, and MMAP decreased $6 \pm 2 \%(p<.05)$, $20 \pm 5 \%(p<.02)$ and $25 \pm 9 \%(p<.05)$ respectively. Dose-related elevations of maternal heart rate ranging from $24-100 \%(p<.01)$ occurred with both regimens. During all of these infusions, umbilical blood flow (UmBF), mean fetal arterial pressure, and fetal heart rate did not change significantly, and maternat and fetal arterial $\mathrm{pH}, \mathrm{PCO}_{2}$ and $\mathrm{PO}_{2}$ remained normal. Thus, $\mathrm{R}$ infusions in this sheep preparation decrease UtBF significantly without impairing UmBF or fetal acid-base status.

169 MORTALITY IN INFANTS OF DRUG-DEPENDENT MOTHERS.Loretta P.Finnegan,M.D., Dian Reeser, B.A., Jacob Schut, M.D. (Spon. by Maria Delivoria-Papadopoulos, M.D.), Phila.Gen.Hosp. (PGH),Depts. of Ped.\& Psych., Univ. of Penna.Sch.of Med.,Phila.,Penna.

With the increasing improvement in recognition and treatment of neonatal illness, the mortality rate $(34 \%-96 \%)$ in infants of drugdependent mothers (IDDM) has been reduced to $3-20 \%$ in various centers. Neonatal mortality in control infants born at PGH was compared to that in IDDM. The drug-dependent mothers were of 3 types: Group A $(\mathrm{N}=65)$ heroin dependent, no prenatal care; Group B ( $\mathrm{N}=98$ ) methadone dependent, aver. 1.8 prenatal visits; Group $\mathrm{C}$ $(\mathrm{N}=166)$ methadone dependent, aver. 8.2 prenatal visits. In 1586 controls, $15.2 \%$ were low birth weight (LBW), $<2500$ gms. The overall mortality was $1.6 \%$ and in LBW infants $10.0 \%$. In 329 IDDM, $27.9 \%$ were LBW; the overall mortality was $4.9 \%$ ( 3 times that of controls) and in LBW infants it was $13.0 \%$. The incidence of LBW in Groups $\mathrm{A}, \mathrm{B}$, and $\mathrm{C}$ was $47.6 \%, 37.7 \%$, and $16.2 \%$ and mortality rate was as follows:

$$
\begin{array}{lcr}
\text { Group A }(\mathrm{N}=65) & \text { Overall \% } & \text { LBW\% } \\
\text { Group B }(\mathrm{N}=98) & 8.6 & 9.7 \\
\text { Group C }(\mathrm{N}=166) & 2.4 & 23.5 \\
& & 3.7
\end{array}
$$

The most striking mortality rates are in Group $B$ where total

births and LBW infants are affected. This suggests that methadone given in an uncontrolled manner in pregnant women may be more harmful than illicit heroin. In contrast, methadone maintained mothers with adequate prenatal and addictive care have a somewhat increased overall mortality but decreased mortality in LBW infants in comparison to the control population.
170 REVERSAL OF NARCOTIC RESPIRATORY DEPRESSION IN THE NEWBORN. Tilo Gerhardt, Eduardo Bancalari, Harry Cohen, Luis Fernandez Rocha, Kathy Holsinger, (Intro. by W.W. Cleveland) University of Miami, School of Medicine, Dept. of Pediatrics, Miami, Florida.

This study was done to evaluate the effectiveness of Naloxone when administered to depressed infants at birth. Minute ventilation $\left(\dot{\mathrm{V}}_{\mathrm{E}}\right)$ end tidal $\mathrm{PCO}_{2}\left(\mathrm{P}_{\mathrm{A}} \mathrm{CO}_{2}\right)$ and ventilatory response to $\mathrm{CO}_{2}$ were measured in 20 infants born to mothers who received $I$ to 1.5 $\mathrm{mg} / \mathrm{kg}$ of Meperidine IV within $2 \mathrm{hrs}$. prior to a normal vaginal delivery. These determinations were done 20-30 minutes after birth

\begin{tabular}{|c|c|c|c|c|c|}
\hline$\stackrel{D}{V}_{E}(m)$ & g) & $\mathrm{P}_{\mathrm{A} \mathrm{CO}_{2}}(\mathrm{~m}$ & $\mathrm{nHg})$ & $\mathrm{CO}_{2}$ resp. & $\begin{array}{l}(\mathrm{mI} / \mathrm{kg} \times \mathrm{minx} \\
\left.\mathrm{mmHgP} \mathrm{CO}_{2}\right)\end{array}$ \\
\hline before & afte & befor & after & befo & after \\
\hline \pm+3 & $388+4$ & $42 \cdot 5+20$ & $40.7+1.6$ & $16.4+2.0$ & $31.4+2.4$ \\
\hline $301+37$ & $314+45$ & $43.6+25$ & $41.6+2.6$ & $20.1 \overline{+3} .7$ & $16.6+2.6$ \\
\hline
\end{tabular}
and repeated 10 minutes after the infant received either Naloxone $0.01 \mathrm{mg} / \mathrm{kg} I M(n=I 2)$ or placebo $(n=8)$.

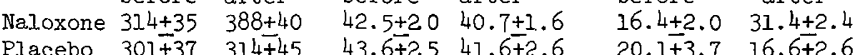
$\begin{array}{llllll}\text { Placebo } 301 \pm 37 & 314+45 & 43.6+25 & 41.6+2.6 & 20.1 \pm 3.7 & 16.6+2.6\end{array}$ fested mainly by a decreased $\mathrm{CO}_{2}$ response. After medication, $\mathrm{CO}_{2}$ response and $\dot{V}_{E}$ increased significantly in the Naloxone group $(P<0.05)$ and this increase was larger in the more depressed infants. There was a significant negative correlation between this improvement and the initial $\mathrm{CO}_{2}$ response. $(\mathrm{r}=0.88 \mathrm{P}<0.001)$ In the neonates who received placebo no significant change occurred in the $\mathrm{CO}_{2}$ response or $\dot{V}_{\mathrm{E}}$. In conclusion, Naloxone effectively reversed neonatal respiratory depression secondary to Meperidine given to mothers. No depressant agonistic effect was observed in infants who were not depressed and received this medication.

EFFECT OF MEPERIDINE ON RESPIRATION IN THE NEWBORN, Tilo Gerhardt, Eduardo Bancalari, Paul Tocci, Harry Cohen, Luis Fernandez Rocha, Kathy Holsinger, Intro. duced by $w$. W. Cleveland) University of Miami, School of Medicine Department of Pediatrics, Miami, Florida.

The purpose of this work was to determine the effects of Meperidine given to the mother during labor on the respiration of the newborn. We studied 24 full term infants born to mothers with normal pregnancy who received only Meperidine $1.0-1.5 \mathrm{mg} / \mathrm{kg}$ IV within 2 hours prior to an uncomplicated delivery. Apgar Score was recorded at 1 and 5 minutes and respiratory rate (RR), tidal volume $\left(V_{T}\right)$, minute ventilation $\left(\dot{V}_{F}\right)$, end tidal $\mathrm{CO}_{2}\left(\mathrm{P}_{A} \mathrm{CO}_{2}\right)$ and ventilatory response to $\mathrm{CO}_{2}$ were determined 20-30 minutes after delivery. Meperidine levels were measured by GLC in maternal and cord blood. The following results were all within the normal range: Apgar Score 7/9, RR 5l+4, $\mathrm{V}_{\mathrm{T}} 21.4 \pm 2.1 \mathrm{ml}, \dot{\mathrm{V}}_{\mathrm{E}} 339 \pm 24 \mathrm{ml} /$ minxkg, $\mathrm{P} C O$ 40.8+1.3 mmHg. The mean ventilatory response to $\mathrm{CO}_{2}$ of $21.8+2.7 \mathrm{ml} / \mathrm{kg}$ xminxmmHg $\mathrm{PCO}_{2}$ was decreased but showed a large individual variation from $4.1^{2}$ to 46.2 . No correlation was found between the response to $\mathrm{CO}_{2}$ and the other determinations, or between Meperidine levels in mother or infant and any of the respiratory measurements. This supports the hypothesis that Meperidine metabolites are in part responsible for the observed res piratory depression. In conclusion, Meperidine used in the described dose causes mild respiratory depression which can only be detected by the ventilatory response to $\mathrm{CO}_{2}$, but not by the Apgar Score, $\dot{\mathrm{V}}_{\mathrm{F}}$ or $\mathrm{P}_{\mathrm{CO}}$. A low Apgar Score at firth is not likely to be solely caused by Meperidine given to the mother and suggests additional causes such as fetal asphyxia.

772 CALCIUM BINDING BY HOMOGENATES OF HUMAN PLACENTAL VILLI. Wallace A. Gieason, Jr. (Introduced by A. E. McElfresh). Department of Pediatrics, St. Louis University School of Medicine, St. Louis, Missouri

In examining the relationship of calcium binding proteins (CaBP) to calcium transport by human epithelial tissues, human placental villi were investigated. Epithelial surfaces of human placenta were dissected free of connective tissue, rinsed in saline and homogenized in 3 vols. $15 \mathrm{mM}$ Tris-HCl buffer. After centrifugation $\left(10^{6} \mathrm{xg}\right)$ the supernate had $31 \%$ more calcium binding activity than similar renal homogenates. $(35 \% / \mathrm{mg}$ protein vs. $24 \% / \mathrm{mg}$ ). Sephadex G-100 chromatography revealed 3 peaks of $\mathrm{Ca}^{++}$binding activity. The first was felt to represent non-specific binding, being associated with excluded proteins. The second was eluted in the range of $50 \times 10^{3} \mathrm{M} . \mathrm{W}$. The third peak, eluted in the area of small M.W. proteins, had the highest specific calcium binding activity. Polyacryamide gel electrophoresis revealed a rapidly moving band in the original supernate and the third peak which had mobility similar to the CaBP of human kidney and gut. This is preliminary evidence of the existence of a CaBP in human placenta which may be related to placental calcium transport. 
173 ELEVATED FREE ERYTHROCYTE PORPHYRIN (FEP) IN THE NEWBORN. Michael A. Gottuso, Barbara F. Osk1 and Frank A. Oski. Department of Pediatrics, State University of New York, Upstate Medical Center, Syracuse, New York.

The measurement of FEP is now widely employed for the screening of lead poisoning. Studies were conducted in newborns in an attempt to determine the utility of the FEP as an index of intrauterine lead exposure. In 100 term infants, cord blood FEP was measured. The mean FEP of $94 \mu \mathrm{gm} / 100 \mathrm{ml} \mathrm{RBC}^{\prime} \mathrm{s}$ (range 45-199) was found to be significantly higher than that observed in healthy childxen ages $1-6$ years $(50+20$; range $17-72)$. Seventyfive per cent of the values exceeded $70 \mu \mathrm{gm} / 100 \mathrm{ml} \mathrm{RBC}$ 's. Blood lead determinations were performed in 12 infants with FEP values of 125 or greater and contrasted with those of 13 infants with FEP leve1s of less than 65 . All 25 infants had blood lead levels of less than $15 \mu \mathrm{gm} / \mathrm{dl}$. Cord blood FEP was not related to cord blood hematocrit. FEP levels were also elevated in infants weighing less than 2500 grams at birth. Administration of Imferon did not produce a fall in FEP during the first 4 weeks of life in 7 premature infants, while FEP did not increase over intital values in 12 infants fed a non-iron fortified formula over the first 4 weeks of life.

The elevated FEP in the newborn does not appear to be a consequence of elther increased body lead burden or iron deficiency. This elevation may reflect a transient maturational abnormality in heme synthesis.

174 OUTPATIENT USF OF A SIX HOUR LFAD MOBILIZATION TEST IN CHELATION THERAPY. John V. Graef, Children's Hospital Medical Center, Boston, Massachusetts

The $24 \mathrm{hr}$. lead mobilization test has been used to confirm increased lead burden when the ratio of, umoles lead excreted in $24 \mathrm{hrs} / \mathrm{m}$ mole EDTA injected is $>1.0$. To modify this test for outpatient use, children suspected of asymptomatic increased lead burden (elevated blood lead, "lead lines on $x$-ray", microcytic, hypochromic anemia, or severe oica, or other) were administered $50 \mathrm{mgm} / \mathrm{kg}$ EDTA $\mathrm{IM}$ in a single dose. Urine was collected for 6-8 hours in an outpatient setting and an excretion ratio was computed.

Children were divided into 5 grouns by blood lead concen-

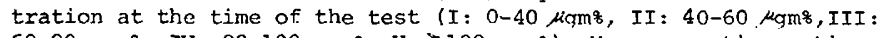
$60-80 \mu \mathrm{gm} \%$, IV : $30-100, \mu \mathrm{qm} \%, \mathrm{~V}:>100 \mu \mathrm{gm} z)$. Mean excretion ratios for each aroup were: Group I: $0.4 \pm 0.21$ (7 patients), II: $0.59 \pm$ 0.34 (43 patients), III: $0.72 \pm 0.32$ (21 patients), IV: $0.67 \pm$ 0.27 ( 8 patients), V: $2.8 \pm 1.1$ (5 patients). Correlation of excretion ratio $>0.5$ with increased metaphyseal densities on $x$-ray was 958 .

These data sugrest that the 6 hour lead mobilization test can be used effectively on an outpatient basis. In this setting an excretion ratio $>0.5$ over a $6-8$ hour collection period is correlated well with other indicators of increased lead burden. This test may be particularly useful as a guide to the notential vield of chelation therany in borderline cases or in previously chelated children in whom further chelation is contemplated.

\section{STANDARDIZATION OF ERYTHROCYTE PROTOPORPHYRIN} MEASUREMENTS. David A. Hart and Sergio Piomelii, NYU School of Medicine, Dept. of Pediatrics, New York The concentration of zinc protoporphyrin ( $\mathrm{ZnPP}$ ) in RBCs is increased in lead intoxication and in iron deficiency anemia, while protoporphyrin IX (PPIX) free base is elevated in erythropoietic protoporphyria. Protoporphyrins fluoresce and this fact is the basis for screening tests for these diseases. Nationwide agreement and correlation in screening programs depends on accurate standardization. Quantitation may be accomplished by direct fluorescence of RBCs or by fluorescence of extracted PPIX in $\mathrm{HCl}$. Concentration is measured by comparison with a standard in the same diluent. The accurate analysis of PP standards is determined spectrophotometrically based on absorbance of sample, diluent used, and on the millimolar extinction coefficient ( $\varepsilon \mathrm{mM})$. Recently it has been stated that the $\varepsilon \mathrm{mM}$ of PPIX in HCI which is accepted in the literature is not accurate since a lower value is obtained when highly purified ZnPP in pyridine solution is diluted with $\mathrm{HCl}$. We have, however, reconfirmed the literature value of 262 as the correct value for $\varepsilon \mathrm{mM}$ of PPIX in $\mathrm{HCl}$. We have accomplished this by using a highly purified PPIX dimethyl ester as well as one prepared from the same stock as the above $\mathrm{ZnPP}$. We have al so shown that the addition of pyridine-solubilized ZnPP to acid solution does not result in a pure PPIX solution, thus this method of solubilization should not be used in standardization. The literature reported PPIX concentrations in RBCs based on the $262 \varepsilon \mathrm{m}^{\mathrm{M}} 1$ for extracted PPIX are therefore valid.
176 NEONATAL WITHDRAWAL SEIZURES. Robert A. Herzlinger, $\frac{\text { Stephen R. Kandal1 }}{\text { Pediatrics and Neurology, Albert Einstein Coll. of }}$ Medicine, Bronx, New York. (Spon. by L. M. Gartner)

Neonatal seizures associated with maternal narcotic addiction is well known, but detailed clinical and EEG data on these infants has not been previously reported. Of 261 infants born to addicted mothers in a $41 / 2$ year period, $17(6.5 \%)$ were identified as having withdrawal seizures. Other possible seizure etiologies were ruled out by appropriate studies.

Nine of the 17 mothers had used primarily methadone (M) during the pregnancy, one had used heroin $(\mathrm{H})$, and 7 had mixed patterns of abuse. The incidence of seizures in the $M$ group was $9 \%$ and $1.4 \%$ in the $\mathrm{H}$ group $(p<0.05)$. In the total group the mean day of seizure onset was $9.5+7.8$ days. Eighty-eight percent of the infants were on withdrawal medication at the time of the seizure; in $29 \%$ the medication was being tapered when the seizure occurred Seizures were equally divided between "generalized" and "nongeneralized." The "generalized" seizure group had a significant increase in the number of days of seizure activity $(p<0.05)$.

Comparisons were made to 16 random1y selected, seizure-free infants of addicted mothers. The seizure group had a significant increase in the incidence of withdrawal symptoms $(p<0.05)$, and the need for symptomatic treatment $(p<0.05)$. Seizures were more frequent in infants whose withdrawal symptoms were treated with valium compared to paregoric $(p<0.05)$. The electroencephalographic data of all available study infants were analyzed.

177 URINARY EXCRETION OF PHENOBARBITAL BY THE 17 NEONATE. Reba M. Hill, Linda M. Tennyson
Baylor Col. of Med., Dept. of Ped., Inst. for Lipid Research, St. Luke's Episcopal Hospital, Newborn Research Center, Houston, Texas 77025

Utilizing methods of gas chromatography-mass spectrometry computer systems, the urinary excretion of phenobarbital (Pbs) was monitored in newborn infants. Group I consisted of 7 infants who were prenatally exposed to Pbs. Range of maternal dose was 30 to 120 mgs daily for 2 to 120 days prior to delivery. Group II consisted of 5 newborn infants treated parentally with Pbs (4-10 mg/kilo/24 hrs) for seizures.

In Group I two types of Pbs excretion was observed. Infants whose mothers received short term therapy with $\mathrm{Pbs}$ had lower initial levels ( $M=648 \mathrm{ng} / \mathrm{m} 1)$ which remained relatively constant with a gradual trail off. Excretion of $\mathrm{Pbs}$ was demonstrated for 17 days (47 $\mathrm{ng} /$ m1) post delivery. The second type of excretion occurred in infants whose mothers received $\mathrm{Pbs}$ for a longer period ( $>5$ weeks). Higher initial levels ( $M=$ $5.7 \mu \mathrm{g} / \mathrm{m} 1$ ) were present with a rapid decline after $5-$ 7 days. Levels $(97 \mathrm{ng} / \mathrm{ml})$ were still present for 18 days post delivery.

The urinary half-life of $\mathrm{Pbs}$ in $\mu \mathrm{g} / \mathrm{hr}$ in Group II patients was found to be 36-44 hours. Excretion of $P b s$ was present for greater than 26 days after the drug was discontinued. (Supported. by.NIH Grant GM 16216-08)

\section{TIE EFFECT OF INSULIN ON AVIAN SALT GLAND NaK-ATPase. Thomas J. Hougen, Barry E. Hopkins, Thomas W. Smith. Sppon. by Alexander S. Nadas, Harvard Medical School,
Departments of Pediatrics and Medicine, Children's} Hospital Medical Center,

Bxigham Hospital, Boston.

Sodium and potassium-activated adenosine triphosphatase (NaKATPase) is involved in the active transport of monovalent cation across cell membranes and is specifically inhibited by cardiac brane phenomena we studied the effects of insulin on NaK-ATPase. Duck salt gland contains a high concentration of NaK-ATPase which : has shown functional and structural homology with mammalian NaK-

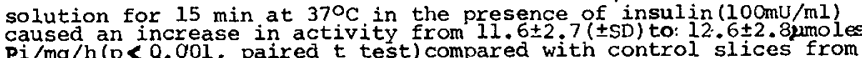
$\mathrm{pi} / \mathrm{mg} / \mathrm{h}$ (p< 0 .001, paired $t$ test) compared with control slices from the same gland incubated without insulin. When 2-deoxy-2-glucose
was substituted for glucose the change was from $12.5 \pm 1.7$ to $14.9 \pm$ was substituted for glucose the change was from $12.5 \pm 1.7$ to $14.9 \pm$
$2.6(p<0.05$ ) indicating that changes in intracellular metabolizable substrate were not responsible for the observed increase in activity. Broken cells exposed to insulin showed no change in activity. When homogenates of control and insulin-exposed slices were to maximally activate NaK-ATPase, activities of detexgent-treated control and insulin-exposed slices were no longer different. Thus with insulin exposure; this effect requires intact cells but not giucose. After detergent activation, insulin enhancement of activity was not evident suggesting that insulin acts to increase the catalytic activity of existing NaK-ATPase units. 
179

EFFECT OF LUCAL TOPICAL BACITRACIN AND DRY SKIN CARE ON STAPHYLOCOCCAL COLONIZATION AND INFECTION IN THE NEWBORN INFANT. John D. Johnson, Philip Sunshine, Natalie Malachowski and Kenneth L. Vosti. Stanford University School of Med., Depts. of Pediatrics and Medicine, Stanford, Ca.

We have previously shown that the application of bacitracin ointment to the umbilicus is an effective method for maintaining low rates of colonization and infection with coagulase-positive staphylococci in our well-baby nursery. We have recently compared the use of local topical bacitracin ointment with "dry skin care", the technique currently recommended by the American Academy of Pediatrics. Cultures of the umbilicus and nares were obtained from all infants discharged on one day of each week. Follow-up data for the entire population were obtained via postcards from examining physicians indicating whether infection had been found in the infant following discharge from the nursery. The colonization rate (C.R.) and infection rate (I.R.) for each time period is shown in the Table.

$\begin{array}{llrr}\frac{\text { Dates }}{775-09 / 75} & \frac{\text { Regimen }}{\text { Bacitracin }} & \text { C.R. } & \text { I.R. } \\ 10 / 75-11 / 75 & \text { Dry skin care } & 85.2 \% & \text { 1.1\% } \\ 12 / 75- & \text { Bacitracin } & 6.7 \% & -0 \%\end{array}$

Dry skin care was ineffective in controlling staphylococcal colonization and infection in our well-baby nursery. We have not found bacitracin-resistant strains of staphylococci and have not detected bacitracin in serum of infants treated with topical bacitracin. Local application of bacitracin to the umbilical area appears to be a safe and effective method of controlling endemic staphylococcal colonization and infection in the nursery.

180 BIOAVAIIAABILITY AND EFFICACY OF NAPROXEN SUSPENSION IN CHILDREN WITH POSTOPERATIVE PAIN. Ralph E. Kauffman, Rolf Habersang, Joseph Oren, and Jerry $\frac{D}{\text { D. }}$. Johnson (Intr. by C. Cho), Depts. Ped. and Pharmacol., Univ. Kans. Sch. Med., Kans. City, Kans. and Syntex Research, Palo Alto, Calif.

The analgesic efficacy of naproxen oral suspension was compared to that of codeine phosphate oral suspension in 16 children ages 5-13 years suffering from postoperative pain. Naproxen $10 \mathrm{mg} / \mathrm{Kg}$ or codeine $0.5 \mathrm{mg} / \mathrm{Kg}$ were administered in identicall. flavored suspensions. Patients were randomly assigned to naproxen and codeine treatments under double-blind conditions. Pain severity was evaluated by the same observer at $30 \mathrm{~min}$. intervals the first two hours and hourly for 8 hours after dosing. The degree of pain relief was scored by two independent rating scales. Blood was obtained at 30 min., $1,2,4,8,12,24$, and 48 hours for naproxen assay. There was no statistically significant difference in pain scores between the patients receiving naproxen and those receiving codeine. All patients in both groups experienced significant pain relief, with maximum relief occurring at $2-3$ hours post dose. The time course of pain relief was virtually identical in both groups. Naproxen was readily absorbed with peak serum levels $(49+14 \mathrm{~S} . \mathrm{D}$. $\mathrm{\mu g} / \mathrm{ml}$ ) occurring $2-4$ hours post dosing, except in two patients who had peak levels at 8 hours. Serum halflives ranged from 10.4 to 17.4 hours with a mean of $14.1+2.5$ S.D. hours. This is comparable to halflives in adults receiving naproxen in capsule or tablet form. Naproxen appears to be well absorbed when administered in suspension and provides analgesia comparable to orally administered codeine.

\section{1}

PLACENTAL TRANSFER OF SALICYLAZOSULFAPYRIDINE. RaIph E. Kauffman and Paul A. Hensleigh (Spon. by H.C Mill.er), Depts. Ped., OB-Gyn., and Pharmacol., Univ. Kans. Sch. Med., Kans, City, Kans.

The placental transfer of salicylazosulfapyridine (SASP) and its metabolites was studied in a full term male infant delivered while the mother was receiving SASP $2 \mathrm{Gm}$ daily for ulcerative colitis. Concentrations of SASP, sulfapyridine (SP), N-acetyl sulfapyridine (AcSP), sulfapyridine glucuronide (SP-GIuc), and Nacetyl sulfapyridine glucuronide (AcSP-Gluc) were determined using the methods of Sandberg and Hansson (1973) in maternal serum, maternal urine, cord blood, and amniotic fluid obtained at delivery. The concentrations of SASP, SP, AcSP, and ACSP-Gluc in maternal (M) and cord (C) serum were: $M-S A S P=2.6 \mu \mathrm{g} / \mathrm{ml}, C-S A S P=$ $2.9 \mu \mathrm{g} / \mathrm{ml} ; \mathrm{M}-\mathrm{SP}=11.0 \mu \mathrm{g} / \mathrm{ml}, \mathrm{C}-\mathrm{SP}=10.0 \mu \mathrm{g} / \mathrm{ml} ; \mathrm{M}-\mathrm{ACSP}=4.1 \mu \mathrm{g} / \mathrm{ml}$, $\mathrm{C}-\mathrm{AcSP}=4.0 \mu \mathrm{gg} / \mathrm{ml} ; \mathrm{M}-\mathrm{AcSP}-\mathrm{Gl} u c=1.0 \mu \mathrm{g} / \mathrm{ml}, \mathrm{C}-\mathrm{AcSP}-\mathrm{Gluc}=0.9 \mu \mathrm{g} / \mathrm{ml}$. Maternal SP-GIuc=0.3 $\mu \mathrm{g} / \mathrm{ml}$ while no SP-Gluc was detectable in cord serum. Concentrations in amniotjc fluid were $S A S P=0.9 \mu \mathrm{g} / \mathrm{ml}$, $\mathrm{SP}=12.4 \mu \mathrm{g} / \mathrm{ml}, \mathrm{AcSP}=6.3 \mu \mathrm{g} / \mathrm{ml}, \mathrm{SP}-\mathrm{Gluc}=1.5 \mu \mathrm{g} / \mathrm{ml}$, and AcSP-Gluc= $1.4 \mathrm{\mu g} / \mathrm{ml}$. SASP and metabolites, excepting SP-Gluc, readily cros the placenta and exist in equal concentrations in mother and fetus. The presence of SP-Gluc in amniotic fluid indicates either placental passage of SP-Gluc or glucuronidation of SP by fetal liver and subsequent fetal urinary excretion. Excessive concentrations of SP could theoretically interfere with bilirubin binding in the neonate thereby increasing the risk of kernicterus. Use of SASP in women nearing parturition should be avoided.
182 EFFECT OF SMOKING IN PREGNANT WOMEN AND THEIR OFFBodenbender, Daniel Couri, Barbara Bayer, Leandro Cordero and Frederick Zuspan. Ohio State Univ. Col1. of Med. Depts, of Ped., OB-Gyn., and Pharmac. and Children's Hospital Research Foundation, Columbus.

Respiratory distress, lethargy, poor feeding and seizures have been described in neonates with high hematocrits (hct). The cause of this "pathologic hyperviscosity" is not known. The possibility of a relationship between smoking in the mother and polycythemia in the infant was raised by studies which have shown higher values for hct. and red cell mass in young aduit male smokers compared to non smokers, due to chronic hypoxia caused by elevated carbon monoxide levels. The relation of maternal smoking to pathologic polycythemia in infancy was explored in the present study. Hemoglobin (hgb), hct., viscosity (visc) and carboxyhemoglobin ( $\mathrm{COHb}$ ) concentration were determined in 55 smoking and non smoking mothers. Cord blood was studied in 46 babies and venous blood in 16 newborn infants. The resulting data shows: 1) The birth weights of infants born to mothers who smoked were lower than the birth weights of infants born to non smoking mothers $(p<0.004)$. 2) CoHb concentrations were higher in maternal blood and the infants cord blood in the smoking mothers compared to non smoking mothers $(p<.001) .3)$ Hgb., hct. and blood visc. in the cord blood of the babies of smoking mothers were higher $(p<.003)$ than the values of the cord bloods of infants of non smoking mothers. 4) Hgb., hct. and visc. values did not differ significantly between smoking and non smoking mothers and in 16 of their infants who
were studied although 2 of these had abnormally high values.

189 DEPRESSANT EFFECTS UPON NEWBORN FEEDING BEHAVIOR WITH THE USE OF DIAZEPAM FOR NEONATAL ABSTINENCE.Reuben $\mathrm{E}$. Kron, Marianne D.Phoenix and Loretta P. Finnegan, (Spon. by Maria Delivoria-Papadopoulos), Phila.Gen.Hosp., Depts.of' Ped. \& Psych., Univ. of Penna. Sch. of Med., Philadelphia, Pennsylvania.

The neonatal abstinence syndrome seen in infants born to narcotic dependent women is usually treated by pharmacotherapeutic means which include opiates, sedatives, and minor tranquilizers. In order to test the relative usefulness of these agents in ameliorating narcotic withdrawal symptoms in the newborn, four groups of infants were compared: 10 infants receiving phenobarbital, 5 infants receiving paregoric, and 6 infants on diazepam,along with 10 control infants born to non-drug dependent mothers. A major effect of neonatal narcotic abstinence is disruption in the organization and effectiveness of nutritive sucking behavior. The infants' feeding activities were evaluated using an objective method for recording nutritive sucking. Normal controls averaged $39.6 \pm 4.6$ sucks/minute, paregoric treated infants averaged $30.5 \mp 3.0$ sucks/minute, phenobarbital treated infants averaged $19.4 \pm 2.3$ sucks/minute and diazepam treated infants averaged $13.4 \mp 7.2$ sucks/minute. These data show that opiate treated infants approach normal control levels. Five diazepam treated babies hardly sucked at all, although the high sucking scores of the sixth infant increased the group mean. These results raise questions about the safety and efficacy of current pharmacotherapeutic approaches for the passively addicted newborn and specifically, the powerful depressant effect of diazepam on nutritive sucking behavior.

\section{METABOLISM AND TOXICITY OF ACETAMINOPHEN DURING} DEVELOPMENT IN MICE. George H. Lambert and Snorri S. Thorgeiixsson (Spon. by Joseph Schulman). Nation Institutes of Health, Bethesda, Maryland.

Large doses of acetaminophen (Tylenol ${ }^{(R)}$ ) cause fulminant hepatic necrosis in both man and experimental animals (Lancet 1 : 519 [1971]); (J.Pharmaco1.Exp.Ther. 187:185 [1973]). Hepatic necrosis caused by acetaminophen parallels the formation of a toxic metabolite(s) that binds covalently to liver proteins ( $\mathrm{J}$. Pharmacol.Exp.Ther. 187:195 [1973]) and glutathione (J.Pharmacol. Exp. Ther. 187:21] [1973]) is very important in protecting the livex against the toxic metabolite(s). We have examined various developmental aspects of acetaminophen metabolism in mice. The glutathione content in the liver reached adult levels at 10 days of age. The rate of cytochrome $P_{1} 450$-dependent metabolism of acetaminophen, as measured by percent glutathione depletion, was slow until day 10 , then rapidly reached adult levels by day 15 . An increase in covalently bound acetaminophen metabolite(s) was found to be coincident with the development of cytochrome $\mathrm{P}_{1} 450-$ dependent metabolic pathways. Our results suggest that the glutathione-dependent detoxification pathway matures prior to the maturation of the drug-metabolizing enzymes which conver acetaminophen into the toxic metabolite(s) in mice. These developmental aspects of acetaminophen metabolism and detoxification are now under investigation in humans. 
185

INDITCTION OF FETAL RAT ENTTEROKINASE(ENTEPOPFPTIDASE E C 3.4.4.8) IN UTERO BY CORTICOSTFROIDS AND ACTINOMYCris D. Emanuel Lebenthal, Harvard Medical School, Children's Hospital Medical Center, (Introduced by llarry Shwachman) Enterokinase is a alycoprotein enzyme that initiates the activation of trypsinogen into active trupsin. We found that enterokinase activity first appeared in the small intestine in utero at the 20th day of gestation. Pregnant Riverside rats 14 to 18 days of gestation were injected intraperitoneally dajly for 3 days with hydrocortison $50 \mu \mathrm{g} / \mathrm{gm}$, Actinomycin $\mathrm{D} 0.25 \mu \mathrm{q} / \mathrm{gm}$, Cvclohexamide $7 \mu \mathrm{d} / \mathrm{gm}$, alone and in various combinations. Preqnant rats injected daily with hydrocortison from the 16 th day of gestation and killed 3 days later, revealed de novo synthesis in the fetuses of enterokinase activity $(60.0 \pm 7.5 \mu \mathrm{M} / \mathrm{gP} / \mathrm{min})$. After injection of hydrocortison and Actinomycin $D$ there was a synergism evidenced by a marked increase in the precocious activity of enterokinase $(255.0 \pm 25.1$ units). No effect was elicited when Actinomycin $D$ and cyclohexamide was given alone.

Small intestinal sucrase activity is not detected before the 14 th day of Iife. There is an increasing effect with aqe in nost natal life on intestinal sucrase following the administration of hydrocortison $(1.7 \pm 0.4$ units in 3 days vs $7.2 \pm 1.3$ units in 16 days of age). The addition of Actinomycin $D$ to hydrocortison causes a slight reduction in the sucrase activity. No induction of fetal appearance of sucrase activity was noted by hydrocortison alone or with Actinomycin D. In conclusion, intestinal enzymes respond differently to corticosteroids at different ages and the superinduction caused by hydrocortison and Actinomycin $D$ appear with enterokinase, but not with sucrase activity.

\section{PHARMACOKINETICS OF CO-TRIMOXAZOLE IN} Sharon Chiumento, Joyce Colaiace and Jerome o. Klein. Univ. of Roch., Sch. of Med., Dept. of Ped. and Boston City Hosp., Dept. of Ped., Bos., Mass. (Spon. by: R.H. Schwartz).

The pharmacokinetics and toxicity of trimethoprim (TMP) - sulfamethoxazole (SMZ) was studied in 33 infants and children. Three dosage regimens were employed: $I=$ $150 \mathrm{mg} / \mathrm{m}^{2}(7.3 \mathrm{mg} / \mathrm{kg})$ q $12 \mathrm{~h}\left(\overline{\mathrm{age}}=13 \mathrm{mos}, \quad \mathrm{SA}=.37 \mathrm{~m}^{2}\right) ;$ I I $=150$ $\mathrm{mg} / \mathrm{m}^{2}(5.2 \mathrm{mg} / \mathrm{kg})$ q $24 \mathrm{~h} \quad\left(\overline{\mathrm{age}}=4.7 \mathrm{yrs}, \mathrm{SA}=.68 \mathrm{~m}^{2}\right) ;$ I II $=92.5$ $\mathrm{mg} / \mathrm{m}^{2} \quad(4.0 \mathrm{mg} / \mathrm{kg})$ q $12 \mathrm{~h} \quad\left(\overline{\mathrm{age}}=3.9 \mathrm{yrs}, \quad \mathrm{SA}=.44 \mathrm{~m}^{2}\right)$. Regimen

$$
\mu \mathrm{g} / \mathrm{ml} \text { (hrs after lst dose) }
$$

\section{$T \frac{1}{2}(h r s)$}

\begin{tabular}{llrrrrrrr} 
TMP & I & .6 & 1.7 & 2.6 & 2.5 & 1.9 & 1.1 & 7.6 \\
TMP & I I & 1.1 & 1.6 & 2.2 & 2.0 & 1.1 & 1.1 & 5.5 \\
TMP & I I I & .9 & 1.6 & 1.8 & 1.5 & .9 & .8 & 8.4 \\
\hline SMZ & I & 27.8 & 36.0 & 57.7 & 69.4 & 49.4 & 46.5 & 14 \\
SMZ & I I & 38.7 & 42.7 & 72.0 & 60.3 & 53.7 & 38.7 & 10 \\
SMZ & I I I & 31.3 & 43.6 & 46.2 & 41.6 & 34.0 & 24.4 & 14
\end{tabular}

SMZ III $31.3 \quad 43.6 \quad 46.2 \quad 41.6 \quad 34.0 \quad 24.4 \quad 14$

Accumulation of both TMP and SMZ was demonstrated in blood obtained 1 and 12 hrs after the 5 th dose for regimen I but for only SMz in regimen III. Serum SMz/ TMP ratios varied from $1: 22-1: 72$. Mean urinary $T / S$ ratio=1:2.1. Pharmacokinetic data suggested enterohepatic recirculation of TMP. Moderate depression in absolute PMN count was frequently observed but, because of intercurrent (viral) illness, could not be ascribed with certainty to the drug.

\section{7}

EQUENTIAL PULMONARY FUNCTION STUDIES OF DRUG DEPENDENT INFANTS SINCE BIRTH. Tsun-Hsin Lin, Loretta P. Finnegan, Dian S. Reeser Philadelphia General Hospital and Univ. of Pennsylvania School of Medicine, Philadelphia, Pa.

Previous studies of infants of drug dependent mothers (IDDM) showed alterations in pulmonary function during narcotic withdrawal. In the present study sequential measurement of lung compliance( $\left.C_{L}\right)$, tidal volume (TV), respiratory resistance ( $\left.R_{L}\right)$, respiratory rate(RR) and minute volume $\left(\dot{V}_{E}\right)$ were made in 18 infants born to drug dependent mothers and 13 term babies from birth to 3 days old. Mean ISE control group values, $C=1.82 \pm$ $0.19 \mathrm{ml} / \mathrm{cm} \mathrm{H} O / \mathrm{kg}, \mathrm{TV}=7.23-0.51 \mathrm{ml} / \mathrm{kg}, \mathrm{R}_{2}=46.8-5.1 \mathrm{~cm}$ $\mathrm{H}, 0 / \mathrm{L} / \mathrm{sec}, \mathrm{RK}=63-3 \mathrm{br} / \mathrm{min}$ and $\hat{V}_{\mathrm{T}}=1372-67 \mathrm{~L} 1 / \mathrm{min} \mathrm{did}$ not significantly vary during the study period. In $\mathrm{IDDM}, \mathrm{C}=1.5 \mathrm{l}-0.3 \mathrm{ml} / \mathrm{cm} \mathrm{H} \mathrm{H}_{2} \mathrm{O} / \mathrm{kg}$ and $\mathrm{TV}=5.86-0.42 \mathrm{mI} / \mathrm{kg}$ were less than control data $(p<0.06)$ on day 1 ; however. by day $3 \mathrm{C}_{\mathrm{T}}$ and $\mathrm{TV}$ increased $(\mathrm{p}<0.0 \mathrm{l})$, to respective con trol levels. $R R=76-5 \mathrm{br} / \mathrm{min}$ did not vary significantly from day 1 , to 3 but was always greater $(\mathrm{p}<0.005)$ than controls. $\dot{V}_{E}$ increased $(p<0.001)$ from contro1 leve 1 on day 1 to greater $(p<0.05)$ than control level on day 3 $\left(V_{E}=1760-135 \mathrm{~m} 1 / \mathrm{min}\right)$. No significant alterations in $R_{I}$ were observed. The mechanism by which $C_{\text {and }}$ TV improved in IDDM by 3 days of age is not Eotally understood; however, the persistent tachypnea and increased $\dot{V}_{E}$ is consistent with CNS hyperirxitability.
188

DOSE-RESPONSE RELATION OF DIAZOXIDE IN CHILDREN WITH HYPERTENSION

William R. Long and Robert C. Boerth (Sponsored by Thomas P. Graham, Jr.), Vanderbilt University Medical Center, Departments of Pediatrics and Pharmacology, Nashville, Tennessee

Although diazoxide (D) has been shown to rapidly lower diastolic blood pressure (DBP) in hypertensive patients (pts), no data are available on the effect of dosage of $D$ on this response in children. Doses of rapidly administered intravenous D (2 to 7.5 $\mathrm{mg} / \mathrm{Kg}$ ) and the reduction in DBP 15 to 60 minutes after $\mathrm{D}$ were obtained from an analysis of 16 severely hypertensive pts (ages 10 months to 13 years). Hypertension in 14 of the pts was secondary to renal parenchymal disease. Admission BP in the 16 pts was $179 \pm 8 / 130 \pm 5 \mathrm{mmHg}$. DBP just prior to $\mathrm{D}$ administration was not significantly different between the various doses of D. A significant, linear log dose-response relation was obtained which showed that a $3 \mathrm{mg} / \mathrm{Kg}$ dose of $\mathrm{D}$ lowered $\mathrm{DBP}$ by an average of $30 \mathrm{mmHg}$ and that doubling the dose of $\mathrm{D}$ produced a mean $12 \mathrm{mmHg}$ greater reduction in DBP. Five pts' initial exposure to $D$ was as 2 or 3 small injections at 10 to 15 min intervals (cumulative dose). The fall in DBP at the end of the cumulative dose was no different than the response after the same dose of $D$ given as a single injection in these same pts. It is concluded that 1) this is the first evidence that $D$ has a significant dose-response relation in hypertensive pediatric pts such that larger doses of $D$ produce a greater reduction in DBP, 2) most hypertensive children will have significant lowering of DBP by doses of D which are smaller than those usually recommended, and 3) lowering of DBP in hypertensive pts can be titrated using repeated small injections of $D$.

DRUG EFFECT ON PERIPHERAL LYMPHOCYTE POPULATIONS OF ATOPICS: Steve McGeady, Yildiz Saraclar and Herbert Mansmann

Effect of drugs on peripheral lymphocyte populations was examined in 51 atopic children aged 2-15 years and 30 age matched non-atopic controls. Acopics were divided into Group I treated with antihistamines, catecholamines, and methylxanthines and Group II which received these agents plus systemic corticosteroid; while controls received no drugs. Since lymphocyte populations are age dependent, subjects and controls were divided into those 10 years of age and under and those over 10 years. Peripheral lymphocytes were identified as thymus derived ( $\mathrm{T}$-cells) if they formed spontaneous rosettes with sheep red cells (E-rosettes), or as bone marrow derived ( $B$-cells) if they formed rosettes with human red cells coated with rabbit antibody and mouse complement (HEACrosettes); they are reported as absolute Iymphocyte numbers.

Results: $\quad G P$ I GP II Control P Values vs. Control

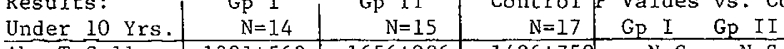
\begin{tabular}{l|r|r|r|ll}
\hline Abs T Cells & $1391+563$ & $1656+986$ & $1426+759$ & N.S. & N.S. \\
Abs B Cells & $551+225$ & $725+304$ & $564+437$ & N.S. & p .05
\end{tabular}

\begin{tabular}{l|r|r|r|rr} 
Over lo Yrs. & \multicolumn{1}{|c|}{$\mathrm{N}=10$} & $\mathrm{~N}=12$ & $\mathrm{~N}=13$ & & \\
\hline Abs T Cells & $1308+590$ & $1447+908$ & $1350+576$ & $\mathrm{~N} . S$. & N.S. \\
Abs B Cells & $501+228$ & $488+266$ & $448+174$ & N.S. & N.S.
\end{tabular}

The Groups differed in the finding of significantly increased $B$ cells in the younger steroid treated atopics. These findings differ from published reports describing a steroid induced 1ymphopenia. Inherent differences in the atopic individual, age related response, or the effect of steroid dosage or duration of
administration may account for these findings.

190 CLINICAL STUDIES OF ALTERNATIVE REGIMENS OF NEONATAL SKIN CARE. Frances H. McLean, Allen H. Neims, Robert H. Usher. McGill Univ., Royal Victoria Hospital, Department of Pediatrics, Montreal, Quebec.

Neonatal skin care involved whole body (WB) bathing with $3 \%$ hexachlorophene (HCP) until 1972, when an iodophor was introduced. Within 2 months, Staphylococcus aureus infection rates led to reinstitution of HCP. Subsequent trials in 3640 fullterm infants were designed to compare the efficacy and blood levels associated with various treatment regimens. $\mathrm{HCP}, 3 \%$ and $0.3 \%$ both applied to WB, were first compared. A subsequent study compared 4 regimens: WB bathing with castille soap or $0.03 \% \mathrm{HCP}$, and umbilical applications (U) of $3 \%$ HCP or Triple Dye.

\begin{tabular}{|c|c|c|c|}
\hline 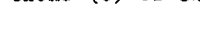 & $\mathrm{Col}$ & tion & Mean Blood HCP Conc \\
\hline Regimen & Umb. & Ant. Nares & $\mathrm{ng} / \mathrm{ml}$ \\
\hline Iodophor (WB) & 75 & - & - \\
\hline $3 \%$ HCP (WB) & 18 & - & 101 \\
\hline $0.3 \% \mathrm{HCP}$ (WB) & 32 & - & 67 \\
\hline Soap (WB) & 46 & 34 & 35 \\
\hline $0.03 \%$ HCP (WB) & 50 & 32 & 45 \\
\hline $3 \%$ HCP (U) & 25 . & 27 & 86 \\
\hline Triple Dye (U) & 10 & 18 & - \\
\hline
\end{tabular}

$3 \%$ HCP (U)

Premature infants wB-bathed with $0.3 \% \mathrm{HCP}$ had a higher mean bloo HCP concentration ( $172 \mathrm{ng} / \mathrm{ml})$ than fullterm infants. Acceptable alternatives to $3 \% \mathrm{HCP}$ (WB) may be $0.3 \% \mathrm{HCP}$ (WB) or $3 \% \mathrm{HCP}$ (U); each results in a moderate decrease in blood concentration, but also a moderate decrease in efficacy. Cord care with Triple Dye is efficacious, but toxicologic studies are needed. 
191

PLASMA RENIN ACTIVITY IN HEALTHY AND SICK NEWBORNS Richard Marshall, Cheryl Bartlett, Michael Sheehan, Michael Maurer, Diane E1lenberger,

Philip Needleman (Intr. by Rniv. Sch. Med,, St. Louis, Missouri. Kotchen has reported elevations of plasma renin activity (PRA) in normal term newborns at birth and 3-6 days. We have determined PRA in normal term newborns and sick newborns by the radioimmunoassay (Squibb kit) and expressed as ng angiotensin I formed/ml/ $\mathrm{plasma} / \mathrm{hr}$.

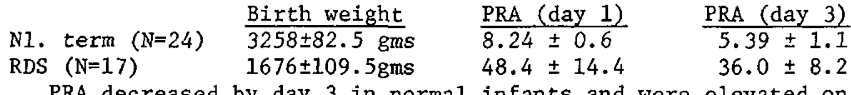

PRA decreased by day 3 in normal infants and were elevated on days 1 and 3 in patients with respiratory distress syndrome(RDS). There is good correlation between the PRA and the severity of the patients' RDS. Six RDS patients had initial elevations of PRA that declined as they improved $(41.9 \pm 14.5$ to $8.1 \pm 2.4)$. Four patients whose RDS did not improve had initial elevations of PRA that remained high. $(86.5 \pm 26.3$ to $90.0 \pm 39.5)$. Two patients had initial low PRA that increased as their iclinical condition deteriorated $(5.3,14$ to $16.5,46)$. Two patients with RDS had no elevations of PRA $(3.2,5.1$ to $2.1,6.6)$. Preliminary data on PRA in healthy, low birth weight prematures shows elevation in some, normal values in others. Six patients without RDS had elevated PRA's. Their diagnoses included hypertension, transposition of great vessels, persistence of the fetal circulation, shock, subarachnoid hemorrhage, and erythroblastosis. Thus, it is clear that multiple disorders can be associated with elevation of PRA.

\section{EFFECTS OF HYPOXIA ON AMIKACIN PHARMACOKINETICS,} Najwa Mirhij, Michael $D_{0}$ Reeves, Robert J. Roberts, and Martin $G$. Myers, The Universi ty of Iowa College of Medicine, University of Iowa Hospitals and Clinics, Departments of Pediatrics and Pharmacology, Iowa City, IA.

Aminoglycoside pharmacokinetics are variable between patients even in the absence of renal impairment. However, peak serum levels and half-lives are relatively reproducible over time in most patients. Four of 6 newborns with hypoxia $\left(\mathrm{PaO}_{2}<50 \mathrm{~mm} \mathrm{Hg}\right)$ were observed to have prolonged Amikacin half-lives ( $>8$ hours). In 2 infants who had serial measurements, shorter half-lives were noted on subsequent determinations after improvement of hypoxia. The mean serum half-life for the 6 hypoxic infants was 8.0 hours (range 3.8 to 12.0 hours) and for normoxic infants was 4.3 hours (26 determinations, including those from previously hypoxic children; range 1.0 to 9.0 hours). In 19 infants without hypox$i a$, only $?$ had a serum ha?f-life in excess of 6.5 hours. Because of these observations, the effect of hypoxia on Arjikacin pharmacokinetics was examined in experimental animals. Sprague-Dawley rats (790 to $250 \mathrm{gm}$ ) maintained in an environment of 0.075 to $0.095 \mathrm{FiO}_{2}$ had significantly longer Amikacin half-lives (80 minutes) after a $15 \mathrm{mg} / \mathrm{kg}$ intramuscular dose than rats maintained normoxic (45 minutes) $(p<0.05)$. Peak serum level $(13.4 \pm 1.7$ and $13.3 \pm 1.4 \mathrm{ugm} / \mathrm{ml}$ ) and volume of distribution were not statistically different for the two treatment groups. An effect of hypoxia on aminogiycoside pharmacokinetics is suggested.

\section{3}

AMIKACIN PHARMACOKINETICS IN NEWBORNS, Martin G。 Myers, Najwa Mirhij, Michael D. Reeves, and Robert J. Roberts, The University of Iowa College of Medicine, University of lowa Hospitais and Clinics, Departments of Pediatrics and Pharmacology, Iowa City, IA.

Because of the recent emergence of gentamicin-resistant Enterobacteriaceae in our nursery, the pharmacokinetics of Amikacin in newborns was studied. Amikacin ( $7.5 \mathrm{mg} / \mathrm{kg} /$ dose) was administered intramuscularly every 12 hours to 22 infants less than 10 days of age with normal renal function. Serial serum Amikacin levels were determined by an enzymatic analysis technique. Peak serum levels occurred at 30 to 60 minutes after administration. Mean peak serum 7 evels of $18.7 \mathrm{ugm} / \mathrm{ml}$ ( 37 determinations in 22 infants) were obtained with a range from 10 to $36 \mathrm{ugm} / \mathrm{ml}$. The mean serum halflife was 4.8 hours (30 determinations in 22 infants) with a range from 1.0 to 12.0 hours. Three infants also received Amikacin as a timed 30 minute intravenous infusion. The mean peak serum leve? in these infants was $20.4 \mathrm{ugm} / \mathrm{ml}$ (range 19.0 to $21.3 \mathrm{ugm} / \mathrm{ml}$ ) and the mean serum half-life was 6.1 hours (range 2.5 to 10.5 hours). As with other aminoglycosides, the pharcokinetics of Amikacin were quite variable between patients but appeared to be independent of hemoglobin and protein concentrations. In 7 newborns who had Arikacin pharmacokinetic studies on more than 1 occasion, the peak serum levels and half-lives were relatively reproducible. However, 2 infants with severe respiratory disease had prolonged serum half-lives in association with hypoxia which subsequently became shorter with resolution of the hypoxic condition. No nephrotoxicity or ototoxicity due to Amikacin administration was detected in the course of these studies.
19 THE EFFECT OF LITHIUM (Li) ADMINISTRATION ON Li CON194 CENTRATION IN MATERNAL BLOOD, BREAST MILK, AND NEONATAL BLOOD IN SUBHUMAN PRIMATES. Bruce R. Parks,

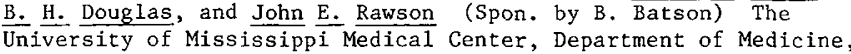
Jackson

Pregnant Rhesus monkeys weighing approximately $5 \mathrm{~kg}$ initially were given $\mathrm{Li}(25 \mathrm{mg} / \mathrm{kg} / \mathrm{day})$ orally beginning 10 to 21 days prior to delivery and for one week following delivery. This dose elevated the maternal blood level of $\mathrm{Li}$ to $0.5 \mathrm{mEq} / 1$ or above. In one animal the $25 \mathrm{mg} / \mathrm{kg} / \mathrm{day}$ dose raised the maternal blood level above $1.25 \mathrm{mEq} / 1$ and had to be reduced to $6.25 \mathrm{mg} / \mathrm{kg} / \mathrm{day}$ to produce the desired maternal blood level. After delivery the maternal blood level of $\mathrm{L} i$ decreased by $50 \%$ even though the mothers continued to receive the Li. Neonatal blood levels of $\mathrm{Li}$ were approximately $0.75 \mathrm{mEq} / 1$ on the day of birth and ranged from 0.25 to $0.75 \mathrm{mEq} / 1$ during the time the mothers continued to receive Li. Breast milk levels of $\mathrm{Li}$ averaged approximately 0.25 $\mathrm{mEq} / \mathrm{l}$. Within two days following withdrawal of the Li treatment the maternal blood level, neonatal blood level, and breast milk level of Li dropped to below $0.25 \mathrm{mEq} / \mathrm{l}$. These data indicate that in the subhuman primate, maternal therapeutic blood levels of $\mathrm{Li}$ will produce elevated blood levels of $\mathrm{Li}$ in newborns which persists while they are on breast milk.

195 ELIMINATION OF TRANSPLACENTALLY ACQUIRED CAFFEINE IN FULITERM NEONATES. William D. Parsons, Jacob V. Aranda, Allen H. Neims. McGill Univ., Depts. Pharmacol. \& Ped., Montreal Children's Hosp. \& Lachine Gen. Hosp., Montreal. During recent studies in the treatment of apnea in premature infants $(760-1985 \mathrm{~g})$, remarkably slow elimination of caffeine (C) and theophylline was noted ( $t \frac{1}{2} \mathrm{C}, 36-144 \mathrm{hr} ; t^{\frac{1}{2}}$ theophy1line, 20-67

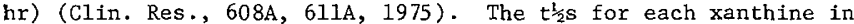
adults is 3-5 hr. Although prematurity per se might have contributed to this delayed elimination, the finding of Horning et al of a relative decrease of demethylated metabolites of $\mathrm{C}$ in urine of term infants implied that the immaturity might apply to term infants as well. The elimination of transplacentally acquired plasma $C$ in 15 healthy term non-breastfed infants was measured by radioimmunoassay supplied by C.E. Cook, Research Triangle Park. C was measured in cord plasma and 4-5 days later in conjunction with PKU screening. Cord plasma $\mathrm{C}$ values ranged from 0.02 to 5.50 $\mathrm{mg} / \mathrm{l}$, mean 1.4. The $t^{\frac{1}{2}}$ of $\mathrm{C}$ in these term neonates $(80 \pm 26 \mathrm{hr})$, as in prematures, was remarkably longer than that in adults $(3.5 \mathrm{hr})$. Certain other drugs that are oxidized also exhibit slow elimination in neonates, but the 23-fold prolongation of $\mathrm{C} \mathrm{t}^{\frac{1}{2}}$ is a substantially greater difference than that seen with other drugs. We have observed (Fed. Proc., Apr. '76) that C elimination in rats is increased 4-fold by pretreatment with 3-methylcholanthrene, suggesting possible participation of cytochrome P-448 monooxygenase activity in $\mathrm{C}$ metabolism. Maternal smoking did not enhance $\mathrm{C}$ elimination in these term infants. These data confirm the presence of considerable $\mathrm{C}$ at birth, and indicate that $\mathrm{C} \mathrm{t}^{\frac{2}{2}}$ is remarkably prolonged in term, as well as preterm, infants.

196 SERUM DIGOXIN LEVELS IN PREMATURE INFANTS Departments $\frac{\text { McNamara, Sections of Cardiology and Neonatology, }}{\text { of Pediatrics and Nuclear Medicine, Baylor College of }}$ Medicine and Texas Children's Hospital, Houston, Texas

The appropriate dose of digoxin for premature infants has not been clearly established. In 19 premature infants receiving digoxin we evaluated the influence of birth weight (BW) on the serum digoxin level (DL). The BW ranged from 870 to 3170 grams (mean= $1372 \mathrm{gms}$ ). The age range at time of digitalization was 2 to 45 days (mean 10 days). Both the total digitalizing dose (TDD) and maintenance dose (MD) were administered parenterally. The mean TDD was $31 \mu \mathrm{g} / \mathrm{kg}$ (SD $\pm 4.2 \mu \mathrm{g} / \mathrm{kg}$ ) and the mean $\mathrm{MD}$ was $8.2 \mu \mathrm{g} / \mathrm{kg}$ / $24 \mathrm{hrs}$ divided into 2 doses ( $\mathrm{SD} \pm 2.6 \mu \mathrm{g} / \mathrm{kg} / 24 \mathrm{hrs}$ ). The DL was determined 12 hours after the preceeding MD. Patients (pts) whose $\mathrm{BW}$ was $<1000 \mathrm{gms}$, the mean $\mathrm{DL}=6.1 \mathrm{ng} / \mathrm{ml} ; \mathrm{BW} 1000-1250 \mathrm{gms}$, $4 \mathrm{pts}$ $\mathrm{DL}=4.4 \mathrm{ng} / \mathrm{ml} ; \mathrm{BW} 1250-1500$, 3pts, $\mathrm{DL}=3.5 \mathrm{ng} / \mathrm{ml} ; \mathrm{BW} 1500-2000 \mathrm{gms}$ $2 \mathrm{pts}, \mathrm{DL}=2.1 \mathrm{ng} / \mathrm{ml}$; BW 2000-2500 gms, $4 \mathrm{pts}, \mathrm{DL}=2.05 \mathrm{ng} / \mathrm{ml}$; and $\mathrm{BW} 2500 \mathrm{gms}, 1 \mathrm{pt}, \mathrm{DL}=1.4 \mathrm{ng} / \mathrm{ml}$. Comparison of $\mathrm{DL}$ in pts above and below $B W$ of 1250 gms is significant to $p<.005$. It is known that premature infants have a less efficient renal function, hence a decreased clearance of digoxin. Because of toxic effects of higher serum levels in premature babies, such as displacing bilirubin from albumin binding sites, we conclude that smaller premature infants should be given a smaller total digitalizing dose and maintenance dose of digoxin. 
197

VASOPRESSIN IN HUMAN UMBILICAL CORD BLOOD. R.A.POlin, A. Frantz, K. Hussain, L.S. James. Coll. of P\&S, Columbia U., Div. of Perinatology \& Endocrinology, NYC

There is accumulating evidence of an increase in the activity of fetal endocrine systems at or about the time of birth, suggesting participation by the fetus in the onset \& control of labor. Arginine vasopressin (AVP), primarily an antidiuretic hormone, has the potential of influencing the onset \& progress of labor by vir tue of its vasoconstrictor effect on uterine blood vesse1s. To evaluate the role of asphyxial stress in AVP release, we determined umbilical artery \& vein AVP concentrations in 4 infant populations (a) 12 infants 48-72 hrs. old with no apparent stress; (b) 10 infants 24-48 hrs. old who were stressed with respiratory distress syndrome; (c) 24 infants delivered vaginally without the use of oxytocic agents; (d) 14 infants delivered by elective cesarean section. In general, AVP levels in the nonstressed neonate were barely detectable, \& did not exceed $5 \mathrm{pg} / \mathrm{ml}$. In infants stressed postnatally with respiratory distress syndrome, the AVP levels ranged from $2.5 \mathrm{pg} / \mathrm{m} 1-25 \mathrm{pg} / \mathrm{ml}$ (mean $6.4 \mathrm{pg} / \mathrm{ml}$ ). Among infants delivered vaginally, all but 2 values exceeded $25 \mathrm{pg} / \mathrm{ml}$ (mean 453.5 $\mathrm{pg} / \mathrm{ml}$ ) \& in those delivered by elective cesarean section, all but 1 value was less than $25 \mathrm{pg} / \mathrm{ml}$ (mean $17.7 \mathrm{pg} / \mathrm{ml}$ ). The concentration of AVP in the umbilical artery was greater than that in the umbilical vein. There was no correlation between AVP concentrations in umbilical cord blood \& the severity of fetal asphyxia as assessed by $\mathrm{pH}$, base excess or one minute Apgar score. The increased concentration of AVP in infants delivered vaginally suggests mechanisms other than stress for its release \& a possible role for the fetus in the initiation of labor.

198 THE MEASUREMENT OF FREE ERYTHROCYTE PORPHYRIN (FEP) AS AN INDEX OF BODY LEAD BURDEN. Ernest Post, Thelma Schneider, Barbara F. Oski, Michael L. Weltzman, and Frank A. Oski. Department of Pediatrics, State University of New York, Upstate Medical Center, Syracuse, N.Y.

Screening for lead exposure employing the FEP is now widely practiced. Although the FEP has been found to correlate with the blood lead values, very little information is available relating the FEP to the EDTA-chelatable body lead burden. Our experfence with 113 children ( 11.5 months -14 years) who underwent initial $8 \mathrm{hr}$. EDTA mobilization tests was reviewed in an attempt to determine the sensitivity and the specificity of the whole blood FEP (Piomelli technique) as an index of the body lead burden. Results were as follows:

\begin{tabular}{|c|c|c|c|c|}
\hline$\frac{\text { FEP }}{(\mu g m / d 1)}$ & $\frac{\text { Blood Lead }}{(\mu \mathrm{gm} / \mathrm{d} 1)}$ & $\%$ with & $\frac{\text { Increased Lead Excretion }}{(0.5 \mu \mathrm{gm} / \mathrm{ml} \text { EDTA })}$ & Patients \\
\hline $0-29$ & $0-39$ & & 0.0 & 15 \\
\hline $30-237$ & $0-39$ & & 0.0 & 32 \\
\hline $0-29$ & $40-82$ & & 12.5 & 8 \\
\hline $30-237$ & $40-82$ & & 43.8 & 48 \\
\hline
\end{tabular}

Employing an FEP of $30 \mu \mathrm{gm} / \mathrm{d} 1$ as a screening level has a $95 \%$ sensitivity and a $23 \%$ specificity for detecting patients with a urinary lead excretion of greater than $0.5 \mu \mathrm{gm} / \mathrm{mg}$ EDTA. Results also suggest that variations exist in the lead sensitivity of the host in terms of biochemical alterations produced and that older patients demonstrate less FEP elevations at any blood lead value. When used appropriately, it is a valuable screening procedure.

SUDDEN INFANT DEATH (SIDS) IN INFANTS OF NARCOTIC199 ADDICTED MOTHERS. B.K. Rajegowda, Stephen R. Kandall and Horacio Falciglia. Dept. of Pediatrics, Lincoln Hospital and BMHC, Albert Einstein College of Medicine, Bronx, New York. (Spon. by L. M. Gartner).

The frequency of sudden infant death (SIDS) between 1-12 months of age in the general population is reported to be $2.5-3$ per 1000 live births. From 1972-1974 we cared for 383 infants born to mothers with histories of active narcotic usage; 182 used primarily methadone (M), 95 heroin (H), and 106 both drugs combined (HM). Eight of their infants experienced sudden unexpected death; clinical and autopsy data ruled out other causes of death.

Five of the 7 mothers were in registered M programs; the remaining two admitted to abuse of $\mathrm{H}$ and $\mathrm{M}$ during the pregnancy. Two of the 8 patients were sibs. The incidence of SIDS in our study population was 8-9 times that in the general population; in those in whom $M$ use was confirmed, the incidence was 13 times the expected number. These figures are minimal incidences, since not all patients are followed at the two hospitals.

Mean birth weight of the infants was 2736 grams; 6 of the 8 were male. Peak age of SIDS in both the study and general population was $21 / 2-3$ months, suggesting that the drug group might provide a model for the general study of SIDS. Persistent neurobehavioral abnormalities have been recognized in addicted infants for up to 6 months, suggesting that autonomic control of respiration during sleep may be related to the increased incidence of SIDS.
200 DISPLACEMENT OF BILIRUBIN FROM ALBUMIN BY DIURETICS. L. Fraser Rasmussen and Richard Wennberg. Department Medicine, Davis, California.

The demonstration that furosemide displaces bilirubin(B) from albumin(A) has led to an increased use of alternative diuretics in $\mathrm{j}$ aundiced infants. We have studied the effect of furosemide, ethacrynic acid and chlorothiazide on bilimbin binding in adult human plasma using the peroxidase assay, red cell uptake and Sephadex G-25 gel filtration. Enzymatic oxidation demonstrated a marked increase in unbound bilirubin in the presence of all three drugs (drug concentration 500umol/1; B/A molar ratio 0.8 ). Calculated apparent drug binding constants $\left(K_{d}\right)$ at the primary bilirubin binding site were equivalent to, or higher than, sulfisoxazole. At the primary binding site ethacrynic acid was a stronger displacer than either furosemide or chlorothiazide.

\begin{tabular}{|c|c|c|}
\hline Drug & Unbound Bilirubin & $\mathrm{K}_{\mathrm{f}}$ (drug) \\
\hline Control (0.8 MR) & $10.5 \mathrm{nmol} / \mathrm{I}$ & \\
\hline Ethacrynic acid & $\mathrm{nmol} / 1$ & $3.4 \times 10_{4}^{4} 1 / \mathrm{mol}$ \\
\hline Furosemide & $\mathrm{nmol} / \mathrm{l}$ & $2.7 \times 10_{4}^{4} 1 / \mathrm{mol}$ \\
\hline Sulfisoxazole & nmol/1 & $2.8 \times 10_{4}^{4} 1 / \mathrm{mol}$ \\
\hline Chlorothiazide & $\mathrm{nmol} / \mathrm{l}$ & $2.6 \times 10^{4} 1 / \mathrm{mol}$ \\
\hline
\end{tabular}

These results were confirmed using erythrocyte uptake and gel filtration. Bilirubin displacement was observed at both primary $(B / A M R<1.0)$ and secondary $(B / A M P 1.0)$ binding sites.

Although these diuretics are structurally dissimilar, they appear to have a common high affinity for plasma albumin that may increase the risk of kernicterus in a sick jaundiced infant.

201 PHENOBARBITAL DISTRIBUTION IN NEWBORN PUPPIES WITH ACUTE AND CHRONIC ACIDOSIS. John E. Rawson, Bruce The University of Mississippi Medical Center, Departments of Pediatrics and Pharmacology, Jackson

Asphyxiated infants are more sedated for a given dose of phenobarbital than non-asphyxiated infants. The asphyxiated infant may be more sensitive because of his acidosis. In this study the distribution of phenobarbital in various tissues was studied in newborn puppies made acidotic acutely or chronically.

In the acute acidosis study mongrel puppies were given $\mathrm{NH} 4 \mathrm{Cl}$ $(100 \mathrm{mg} / \mathrm{kg}$ ) beginning at 6 days old. Thirty minutes later phenobarbital $(8 \mathrm{mg} / \mathrm{kg})$ was given. The animals were sacrificed 8 hours after phenobarbital. In the chronic acidosis study puppies were given $\mathrm{NH} 4 \mathrm{Cl}(25 \mathrm{mg} / \mathrm{kg})$ every 6 hours $\mathrm{X} 4$ days. Sacrifice occurred 6 hours after the last dose of $\mathrm{NH} 4 \mathrm{Cl}$ and 14-16 hours after the administration of phenobarbital.

Despite no significant change in serum concentration between control and acutely acidiotic animals, the brain, lung, and kidney showed increased concentrations of phenobarbital. With acute acidosis the liver showed a reduction in concentration while a slight increase was seen in the chronically acidotic animals.

This study shows that acidosis results in a increased brain concentration of phenobarbital and that this may be part of the explanation of the increased sedation of asphyxiated infants treated with phenobarbital.

FETAL GROWTH INHIBITION: RELATIVE EFFECTS OF METHA202 DONE AND MORPHINE. John R. Raye, Joseph W. Dubin, and Jack $\underline{N}$. Blechner (Intr. by Arnold J. Altman) Univ. of CT Hlth. Ctr., Depts. of Ped. and Ob/Gyn, Farmington, CT

Methadone is widely used in clinical practice as an alternative narcotic for pregnant drug addicts. We have previously shown that chronic maternal morphine administration results in significant growth inhibition in the fetal rabbit (Ped. Res. 9:279,1975). This study attempts to define the effects of maternal methadone administration on fetal growth in the rabbit and to compare the relative effects on growth of these two narcotics. Does treated with methadone ( $15 \mathrm{mg} / \mathrm{kg} / \mathrm{d}$ s.c.) 1 week prior to conception and throughout gestation were pair fed with weight matched controls. At $29 \mathrm{~d}$ litters were delivered surgically and measurements made of fetal weight, length, organ weight, protein and DNA.

The litters of methadone treated does showed a significant increase in later intrauterine deaths. Significant $(\mathrm{p}<0.001)$ reductions were seen in fetal weight, length, and in brain and lung weights in the methadone group. This growth inhibition associated with methadone administration $(15 \mathrm{mg} / \mathrm{kg} / \mathrm{d}$ ) was comparable to that resulting from the administration of $100 \mathrm{mg} / \mathrm{kg} / \mathrm{d}$ morphine and was greater than that seen with $50 \mathrm{mg} / \mathrm{kg} / \mathrm{d}$ morphine. In the methadone group, however, the inhibition of brain growth was significantly increased ( $p<0.001$ ) over that seen in the comparable morphine group. This localization of methadone effect was of particular interest because of the concomitant appearance of CNS anomalies restricted to the methadone group. The growth inhibition noted in the lung and brain seemed to be the result of a reduction in cell number (Supported in part by funds from SOADAP, NIMH \#DA00633-01). 


\section{3}

LEAD-203(203Pb)AND EDTA: INTERACTIONS WITH PARATHYROT HORMONE (PTH, CALCITONIN (CT)AND I, 25-DIHYDROXYV ITAMIN Albert Einstein Col1.Med., Kontefiore Hosp. \& Med.

Ctr, Department of Pediatrics, Bronx, New York.

Since bone is the reservoir for the majority of the body burden of $\mathrm{Pb}$ and the main source of $\mathrm{Pb}$ mobilized during $\mathrm{CaNa}_{2}$ EDTA (EDTA) therapy, this study was undertaken to define further interactions between EDTA and hormonal regulators of bone metabolism. Pregnant rats on day 1318 of pregnancy were injected IV with $500 \mu \mathrm{Ci}$ of ${ }^{20} \mathrm{~Pb}$ On day \#19, paired fetal bones were placed on grids in B.GJ medium (M) that resembled the ionic concentration of the extracellular fluid (ECF). After 3-5 days of culture at different concentrations of EDTA, ${ }^{20} \mathrm{~Pb}$ released from bones into the experimental medium(EM) was compared to that released from bones into the appropriate control medium (CM). The effects of adding hormones to the EM were expressed as CPM EM/CM ratios. The results were:
$M+\operatorname{EDTA}(.001 \mathrm{mM})$
2) $+\mathrm{PTH}$
3) $+1,25-(\mathrm{OH})_{2} \mathrm{D}_{3}$ $\mathrm{EM} / \mathrm{CM}$ Ratio
$4.67 \pm .11 *$
$5.04 \pm .16 *$
$5.04 \pm .16^{*}$
$.58 \pm .10^{*}$
$*=p<.01$ different from 1.00

Increasing medium levels of calcium and phosphate reduced the ef-

flux of $203 \mathrm{~Pb}$ in groups 1,2 and 3.

These data represent the first demonstration in vitro that EDTA chelation of $203 \mathrm{~Pb}$ from a rapidly exchangeable skeletal compartment is increased by PTH and $1,25-(\mathrm{OH}){ }_{2} \mathrm{D}_{3}$, and decreased by $\mathrm{CT}$ and ionic changes in the ECF. Future studies in this system are likely to define the hormonal and ionic environment in which chelating agents are the most effective.

2 CLINICAL MANAGEMENT OF HYPERTENSION WITH MINOXIDIL: A 204 CONTROLLED TRIAL IN CHILDREN. Alan Sinaiko and Bernard L. Mirkin, Univ. of Minn. Health Sciences Center Div. of Clin. Pharmacology, Depts. of Ped. and Pharm., Minneapolis, Mn.

The therapeutic efficacy of 3 antihypertensive regimens has been evaluated in a series of 9 children age 1 mo. -16 yrs. with unsatisfactory regulation of their blood pressure (BP). In this group 7 children were treated with Minoxidil (M) and Propranalol (P), P alone and Methyldopa (MD) for 6 week study periods in a randomized cross-over fashion. 2 children received only M\&P and P. Hydrochlorothiazide was included with each of the therapeutic regimens and was administered as the sole antihypertensive agent for 4 weeks prior to the start of the study and for a 2 week washout period between each of the 6 week study periods. The following average blood pressures were noted for each regimen: (each value represents the mean for each group)

$\begin{array}{lccc} & \text { systolic } & \text { diastolic } & \text { mean BP } \\ \text { control } & 147 & 98 & 115 \\ \text { M\&P } & 129 & 79 & 96 \\ \text { MD } & 138 & 87 & 104 \\ \text { P } & 137 & 85 & 103\end{array}$

The diastolic BP was significantly reduced from control values with each regimen studied; however, the same was seen for systolic BP on Iy with M\&P. M\&P decreased diastolic BP to a significantly greater extent than either $P$ or $M D$, but systolic $B P$ was not significantly lowered. The only major adverse effect noted a profound hypertrichosis.

Supported by USPHS grant \#HD 08580-01.

\section{BACTERIAL COLONIZATION IN THE NEWBORN -} EFFECT OF CORD CARE. William T. Speck, John $\mathrm{M}$. Driscoll, Ir., Richard A. Polin and Herbert S. Rosenkranz. (Spon by Jo Anne Brasel). Col. of Physicians and Surgeons, Columbia University, Babies Hosp., Department of Pediatrics, New York, New York 10032

A randomized controlled and blinded study to compare the effectiveness of three regimens (dry cord.care (D.C.), triple dye (T.D.) and silver sulfadiazine (S.S.)) in controlling neonatal bacterial colonization was conducted. Two hundred and eighty-four infants participated in a 12 -month study. Cultures of the anterior nares and umbilical area were obtained on Days 3, 14 and 42. Staphylococcal colonization was inhibited by both T.D. and S.S., however, T.D. was significantly more effective $(p=<.001)$. Group B Streptococcal colonization was most significantly inhibited by S.S. while T.D. appeared to promote the growth of this microorganism。 D.C. treated infants had less Streptococcal colonization than T.D. infants $(p=<。 01)$. There were no significant differences in gram negative colonization between D.C. and T.D., however, S.S. infants had significantly less gram negative colonization than infants treated with the other regimens $(p=<.005)$. The study demonstrated that treatment of the umbilicus with S.S. is a safe and effective method for inhibiting neonatal bacterial colonization with gram positive and gram negative organisms.
206 PHARMACOKINETIC STUDIES OF HEXACHLOROPHENE IN THE NEWBORN. Eileen E. Tyrala, W. Edwin Dodson, Laura S. Hillman, and Richard E. Hillman, Washington Univ. Sch. Of Missouri.

To help control a staphylococcal epidemic, bathing with a soap containing $3 \%$ hexachlorophene (HCP) was instituted during 2,12 day periods in a regional neonatal intensive care unit. $H C P$ in $100 \mu 1$ whole blood samples obtained by heel stick was measured by GLC using dichlorophene as an internal standard and a ${ }^{63} \mathrm{Ni}$ electron capture detector. More than 200 measurements of HCP concentrations (HCP) were made on 49 infants. Among 27 infants bathed 3 or more times, the median weight was $1490 \mathrm{gms}(740-3340 \mathrm{gms})$, the median gestational age at birth was 30 weeks (25-40 wks), and the median age was 15 days ( 3 days -5 months). No overt clinical toxicity was recognized. In these infants $\mathrm{HCP}$ twenty-four hours after a bath ranged from $150-4350 \mathrm{ng} / \mathrm{ml}$. All eight infants weighing less than $1100 \mathrm{gms}$ had HCP greater than $1000 \mathrm{ng} / \mathrm{ml}$. There was great variation in HCP in larger infants. The HCP of 4350 was measured in a $1700 \mathrm{gm}$ infant. No correlation could be shown between HICP and age, gestational age, or the number of baths $(>3)$. The elimination of HCP followed 1st order kinetics. The T $\frac{1}{2}$ varied from $13-78 \mathrm{hrs}$ among 8 patients and did not correlate with age or weight. Absorption was complex and followed no simple kinetic pattern. These data indicate that although accumulation of HCP is significant it is not possible to predict HCP in any given infant. Studies of the toxicology of HCP must be correlated with HCP in blood and tissue rather than administered dose.

\section{DEMONSTRATION OF SATURATION KINETICS FOR THEOPHYLIINE} DISPOSITION. Miles M. Weinberger, Elliot Ginchansky, (Spon. by M.K. Younoszai) University of Iowa, U. of Iowa kiospitals, Dept. of Pediatrics and Pharmacology, Iowa City. Previous studies of theophylline pharmacokinetics have assumed first order metabolism. The current study examined the disposition of theophylline in 19 asthmatic children by infusing theophylline intravenously using an IVAC constant infusion pump at 2 infusion rates on sequential days until the serum concentration, as measured by a rapid specific assay utilizing high pressure cation exchange chromatography, reached steady-state at each infusion rate. The clearance was then calculated at each steady-state serum concentration by dividing the rate of metabolism (the rate of infusion at steady-state) by the steady-state serum concentration. At the lower infusion rate of $.73 \pm .17 \mathrm{mg} /$ $\mathrm{kg} / \mathrm{hr}$ (mean $\pm \mathrm{S} . \mathrm{D}_{.}$), steady state theophylline concentrations averaged $9.2 \pm 2.1 \mu \mathrm{g} / \mathrm{ml}$ and clearance rates were $1.39 \pm .41 \mathrm{ml}$ $\mathrm{kg} / \mathrm{min}$. The higher infusion rates averaged $1.44 \pm .39 \mathrm{mg} / \mathrm{kg} / \mathrm{hr}$ and resulted in steady-state serum concentrations of $20.0 \pm 4.4$ $\mu \mathrm{g} / \mathrm{ml}$ with clearances of $1.20 \pm .28 \mathrm{ml} / \mathrm{kg} / \mathrm{min}$, a significant reduction $(p<.025)$. Fifteen of the 19 patients had clearances reduced to some degree at higher serum concentrations. Four of these patients had clearances reduced by $>20 \%$ with $2>40 \%$. These data indicate saturation kinetics for theophylline disposition and suggest that disproportionate changes in serum theophylline concentrations may occur as a result of dosage changes at the upper end of the $10-20 \mu \mathrm{g} / \mathrm{ml}$ therapeutic range.

\section{ENDROCRINOLOGY}

20 CLINICAL AND BIOCHEMICAL CORRELATIONS OF TRIIODOTHY C Val Abbassi and Carolyn Aldige. (Intr.by P.L.Calcagno)

Georgetown Univ. Sch. of Med., G.U. Hosp., Dept. of Ped.

Washington, D.C.

Radioimmunoassay (RIA) of $\mathrm{T}_{3}$ has become a routine test of thyroid function. Although normal levels have been established the behavior of $\mathrm{T}_{3}$ in various thyroid disorders has not been evaluated. $\mathrm{T}_{3}$ by RIA was determined in 40 children with thyroid disease ( 6 toxics, 7 hypothyroids, 14 chronic lymphocytic thyroiditis [CLT], 10 euthyroid goiters and 3 thyroid adenomas). The results were correllated with clinical presentations and with $\mathrm{T}_{4}$ and TSH concentrations determined by $\mathrm{CBP}$ and RIA respectively. In toxjc group $T_{3}$ was increased $(366 \pm 48 \mathrm{ng} / \mathrm{DL}$ ) and in hypothyroid group decreased $\left(18.5^{ \pm} 6\right)$. In remaining groups, $\mathrm{T}_{3}$ concentrations were normal. The CLT group could be subdivided into 4 groups; $\uparrow \mathrm{TSH}, \mathrm{nl} \mathrm{T} \mathrm{T}_{4}$ (2): $4 \mathrm{TSH}$, J.ow $\mathrm{T}_{4}$ (2); $\mathrm{nl} \mathrm{TSH,} 4 \mathrm{~T}_{4}$ (3) and $\mathrm{n} 1 \mathrm{TSH}$, $\mathrm{n} 1 \mathrm{~T}_{4}(6) .{ }^{4} \mathrm{~T}_{4}$ in the last subgroup with CLT $(7.4 \pm 0.4 \mathrm{ug} / \mathrm{DL})$ and in pts. with euthyroid goiter $(6.9 \pm 5)$ was significantly different from controls $\left(T_{4}=9.4^{ \pm}\right) \quad p<0.01$. Mean $T_{3}$ in these two groups $(146 \pm 7$ and $130 \pm 9)$ was normal and did not significantly differ from the control group $\left(135^{ \pm 4}\right), p>0.2$. Nine euthyroid pts. had abnormal $\mathrm{T}_{4}(3 \uparrow, 6 \downarrow)$ but normal $\mathrm{T}_{3}$. There was a significant correllation between $\mathrm{T}_{3}$ and $\mathrm{T}_{4}(\mathrm{r}=.89)$ in all pts. but not between $T_{3}$ and TSH. These observations are indicative of the excellent clinical correlation of the $\mathrm{T}_{3}$ RIA, however, with $\mathrm{T}_{3}$ determination alone abnormalities of thyroid function in certain disorders may not be detected. Such information is useful in interpretation and clinical application of the T3 assay. 
209

CYPROHEPTADINE MODIFICATION OF GLUCAGON-INDUCED GROWTH HORMONE RELEASE:POSSIBLE HYPOTHALAMIC SITE OF GLUCAGON ACTION. Theodore W. AvRuskin, Vinod R. Lala, Kutsi Onur, and Christinas. Juan. New York Univ. Sch. Med. The Broo

Glucagon(GL)stimulation of growth hormone (GH)has been well documented but its mechanism is unclear. Cyproheptadine(C), an anti-serotoninergic agent inhibits GH release after insulin-hypoglycemia. To investigate $C$ effects on GL-GH release, 12 genetically short children ( 8 males, 4 females) and 8 children with diabetes mellitus ( 4 males, 4 females)had paired IV GL tests before and after 8 doses of $4 \mathrm{mg} \mathrm{C}$. Tests were done after overnight fasts; serial samples for sugar (BS, mg/dl), insulin(IRI, $\mu \mathrm{U} / \mathrm{ml}$ ), and growth hormone (IRGH, ng/m 1 ) were obtatned to 3 hrs. In short stature: Pre-and post-C BS were similar Post-C IRI values were higher $(p<0.01-0.001)$ than pre-C, except at 15 and $30 \mathrm{~min}$. Post-C $\triangle I R G H$ values in all 12 patients were lower but significantly lower $(p<0.05)$ in the 8 males. In diabetes:Post-C mean max. IRGH and $\Delta$ IRGH were higher than pre-C values ( $\triangle I R G H, p r e-C: 9.8 \pm 0.9$, post-C:14.8 5.8$)$ and were significant $(p<0.002)$ on paired " $t$ " tests. Post-C $\triangle I R G H$ values were higher $(p<0.01)$ in all males but lower $(p<0.001)$ in $3 / 4$ females. Conclusions These data suggest that GL-GH secretion may involve hypothalamic release of serotonin and that $C$ modifies this GH release in short stature but has paradoxic effects in diabetes mellitus. 210 A CHARCOAL ASSAY FOR TRANSCORTIN-BOUND AND UNBOUND
CORTISOL IN PLASMA. Philip $L$. Ballard and Janet $P$. Carter. Spec. Center of Res. in Pulm. Dis., Cardiovos. Res. Inst. and Dept. of Ped., Univ. of Calif. San Francisco 94743. We have developed a simple charcoal assay for determination of the fractions of plasma cortisol unbound and bound to transcortin (CBG). $0.1 \mathrm{ml}$ of plasma is incubated with a tracer amount of $3 \mathrm{H}$ cortisol $(0.4 \mathrm{ng})$ for $60 \mathrm{~min}$ at $25^{\circ} \mathrm{C}$ and then for $30 \mathrm{~min}$ at $2^{\circ} \mathrm{C}$. $0.025 \mathrm{ml}$ of activated charcoal suspension $(170 \mathrm{mg} / \mathrm{ml})$ is added, the mixture is agitated, and then centrifuged 3 min later for 5 min at $5000 \times \mathrm{g}$. The radioactivity remaining in the supernatant represents cortisol bound to $C B G$, and steroid absorbed to charcoal includes that which was unbound and bound to albumin. Unbound cortisol is calculated from the albumin concentration. The procedure is reproducible (coefficient of variation $5 \%$ ) and values for unbound cortisol in both umbilical cord and adult plasma correlate well with those obtained by equilibrium dialysis at $37^{\circ} \mathrm{C}$. CBG capacity determined by this method was $10.8+1.1$ (S.E.) $\mu \mathrm{g} / 100 \mathrm{ml}$ in 7 cord bloods and $20.3+1.7 \mu \mathrm{g} / 100 \mathrm{~m} 7$ in 10 adult specimens, and the $K_{a}$ in 4 adult samples determined by Scatchard analys is ranged from 1.9 to $4.1 \times 107 \mathrm{M}$. Similar values have been reported by gel filtration, uitrafiltration and equil ibrium dialysis. Compared with these procedures, the charcoal method is faster and requires a smaller volume of plasma. Used in conjunction with an assay of total plasma cortisol, this procedure allows routine determination of the unbound (apparently physiologically active) cortisol concentration in plasma.
211. L-DOPA AS A SCREENING TEST OF HYPOTHALAMO-PITUITARY ADRENAL \& PANCREATIC FUNCTION IN CHTLDREN. Dorothy J. Becker, Salvador Villalpando, \& Allan L. Drash Univ. of Pittsburgh Sch. of Med., Dept. of Peds., Pittsburgh.

L-Dopa (250-750 $\mathrm{mg})$ was given to $17 \mathrm{children}$ with constitutional short stature (5), partial or complete growth hormone (GH) deficiency(7), organic intracranial lesions(3) or Turners syndrome(2). Blood samples vere collected basally $\times 2$ \& at intervals for 3 hrs. Al1 patients (pts) also had an arginine-insulin tolerance test (AITT). In all but 1 case there was a corresponding $\mathrm{CH}$ response during the 2 tests, the peak being 30-60 min. after the L-Dopa. L-Dopa induced a significant increase of plasma cortisol(F) in all the pts who had a similar rise during the AITT. The rise of $\mathrm{F}$ occurred simultaneously with that of $\mathrm{GH}$, peaking usually at 45$60 \mathrm{~min}$. There was a suppression of prolactin during all but $1 \mathrm{~L}-$ Dopa test. In al1 of 15 pts plasma glucagon rose from a mean basal level of $135 \mathrm{pg} / \mathrm{ml}$ to a mean peak of $333 \mathrm{pg} / \mathrm{m} 1$. Thirteen pts had an initial pe ak at 30-60 min \& 8 showed a second, usuall y smaller peak at 90-180 min. The rise in glucagon always preceded that of $\mathrm{F}$. An insulin rise from $9.6 \mu \mathrm{U} / \mathrm{ml}$ to a mean peak of 19.0 $\mathrm{\mu U} / \mathrm{m} 1$ occurred in 13 of $15 \mathrm{pts}$, usua11y at $30-45 \mathrm{~min}$. There was no clear relationship between changes of insulin \& glucagon. Blood glucose rose concomitantly with glucagon. Increases of the pancreatic hormones were independent of the presence or absence of a $\mathrm{GH}$ response. The changes of all the hormones occurred with or $w /$ out nausea due to L-Dopa administration \& therefore appear to be mediated by dopaminergic or adrenergic receptor stimulation, rather than stress-induced.
COMPARISON BETWEEN 1-DEAMINO-8-D-ARGININE VASOPRESSIN (DDAVP), CLOFIBRATE (CE), CARBAMEZEPINE (Cb) AND PITRESSIN (P) IN THE TREATMENT OF CENTRAL DIABETES INSIPIDUS (DI) IN CIILDHOOD, Dorothy J. Becker \& Thomas P. Foley, Ir., Univ of Pittsburgh, Sch of Med., Dept.of Per., Fittsburgh.

Thirteen cilildren aged $1-1 / 2$ to 16 years with $D I$ due to histiocytosis $x$ (3), septo-optic dysplasia (2), pinealoma (1) or idiopathic (7) have been treated with $P-i n-o i l$ or $C f(1-2 \mathrm{gms} /$ day)with or without $\mathrm{Cb}(200-400 \mathrm{mg} /$ day). Therapy with $\mathrm{CF} \& \mathrm{Cb}$ in pts with partial DI in whom $P$ had heen withdrawn or never begun, resulted in a reduction of daily urine volumes from a pre-treat ment mean of $4.7 \mathrm{~L}$ to $2.1 \mathrm{~L}$, with excellent symptomatic control.Cf and $\mathrm{Cb}$ had no antidiuretic effect in 5 pts with complete DI and did not decrease their $P$ requirements. In these pts $P$ decreased urine volumes from $6.3 \mathrm{~L}$ to $0.9 \mathrm{~L} /$ day. Ten of the pts (youngest 1 $1 / 2$ yrs) are now receiving intranasal DDAVP, requiring 2 daily doses of $5-15 \mathrm{mg}$ at \pm 8 am and $2.5-7.5 \mathrm{mg}$ at $\pm 8 \mathrm{pm}$. Those with partial DI require smaller doses and the duration of action is longer. The effectiveness of each dose lasts 8-20 hrs. Urine volumes decreased from 5.5 ro $1.5 \mathrm{~L} /$ day. All pts who had received therapy with $\mathrm{P}$ have developed growth hormone (GH) antibodies (abs), but continued to grow normally unless there was pre-existing GH deficiency. There was no evidence to suggest the presence of prolactin, TSH or antithyrold abs. GH abs were still present up to 9 months after the withdrawal of $P$. DDAVP did not induce acute changes of serum osmolality, $G$ or prolactin, nor did it influence nocturnal patterns of secretion of the hormones. No side effects were experienced. Although partial DI was effectively, con-
trolled with Cf \& Cb, results are more satisfactory using obAvp.

213 IN UTERO THYROID TRANSPLANTATION UITH PREVENTION OF ATHYROTIC CRETINISM. Robert P. BeT in, Dorothy $R$.

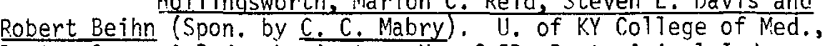
Depts. Surg. Ped., Lexington; U. of ID, Dept. Animal Ind., Moscow; and V.A. Hospital, Lexington, KY.

Thyroid aland transplantation in utero is technically possibie. A.t 82-93 days gestation, total fetal thyroidectomy was performed and autologous thyroid tissue transplanted to fetal thigh in 6 lambs. Serum thyroxine iodine $[T 4(C)]$, triiodothyronine (T3RIA), thyroid stimulating hormone (OTSH), $13 i$ I uptake and scan, and pituitary response to thyroid releasing hormone (TRH) were followed in twins to age 6 months. Results: Two ewes aborted post operatively and 2 delivered stilloorn cretin lambs at term. By $131 \mathrm{I}$ scan, I lamb had a small surviving thigh graft, but died with partial cretinism. The 6 th lamb, with twin unoperated control, has survived for 6 months with normal development and thyroid function (thigh only by scan), but markedly elevated levels of OTSH. Even with high OTSH levels, the transplanted lamb can further increase its OTSH output after TRH stimulation.

\begin{tabular}{lrrlrrrr} 
Day 1 & T4(C) & T3RIA & OTSH & OTSH $\overline{\mathrm{p}}$ TRH $(24$ wk) & \multicolumn{2}{c}{$\% 131$ I uptake } \\
& ug/dT & nq/d1 & $n g / m 1$ & Omin & 30 min & Neck & Thigh \\
Norma & 4.1 & 344 & 13.7 & $<0.8$ & 6.0 & 9.5 & 0 \\
Transplant & 3.2 & $>400$ & $>100$ & 44 & 88 & 0 & 10.2
\end{tabular}

Conclusion: This successful thyroid transplantation in an in utero cretin suggests a new approach for treating congenital hypothyroidism.

214 INTRACRANIAL CHORIONIC GONADOTROPIN (HCG) SECRETING TUMOR IN A PREPUBERTAL GIRL. Hans H. Bode, Barry B. Bercu, Inese 2 . Beitins, Bruce D. Weintraub, Harvard Med. Sch., Massachusetts Gen. Hosp., Dept of Ped, Boston and NIH.

The syndrome of ectopic HCG production has been confined to males and is accompanied by sexual precocity. We described an 8 y o gir1 referred with diabetes insipidus, poor growth, malnutrition and no evidence of sexual development. Growth hormone secretion, adrenal and thyroid function were normal. Two randon plasma samples contained undetectable FSH, but high $\mathrm{LH}$ (40 and $56 \mathrm{mIU} / \mathrm{ml}, 2^{\text {nd }} \mathrm{IRP}$ ). In a specific radioimmunoassay (RIA) for LH basal levels of 1.9 and $2.2 \mathrm{mIU} / \mathrm{ml}$ (LER 907) rose to $4.1 \mathrm{mIU}$ after LHRH stimulation; FSH remained undetectable. Neither HCG nor its $\beta$-subunit could be identified in specific RIA's. Extensive evaluation failed to reveal evidence of tumor.

Four months later the patient was referred moribund with increased intracranial pressure. A vascular hypothalamic tumor was diagnosed on biopsy as germinoma. Laboratory findings: Serum LH $31.4 \mathrm{mIU} / \mathrm{ml}$ LER 907 (no increase after LHRH), FSH undetectable HCG $220 \mathrm{ng} / \mathrm{ml}$ (CR 117), $\not \beta-H C G 389 \mathrm{ng} / \mathrm{ml}$ (CR 116), LHRH $20 \mathrm{pg} / \mathrm{ml}$, urine Gravindex positive, urinary HCG $138 \mathrm{ug} / 12 \mathrm{~h}$ by RIA and $110 \mathrm{ug} / 12 \mathrm{~h}$ by receptor assay. The tumor contained HCG and secreted HCG in tissue culture (no increase in HCG secretion or cell growth by addition of LHRH). In tumor tissue membranes, LHRH did not stimulate adenylate cyclase. After radiation and chemotherapy, serum HCG and $\boldsymbol{B}$-HCG became undetectable while LH was 0.4 and FSH $1.1 \mathrm{mIU} / \mathrm{ml}$ (LER 907). Ectopic HCG production in girls has previously escaped detection because $i$ in the absence of FSH, sexual precocity does not occur. 
215 MALE TO MALE INHERTTANCE AND LHRH RESPONSE 'IN KALLMANN'S SYNDROME. Hans H. Bode, George R. Merriam, and Inese Z. Beitins, Harvard Med. Sch., Massachusetts Gen. Hosp., Dept. of Ped., Boston.

Hypogonadotropic hypogonadism and anosmia, usually considered to be inherited as $\mathrm{X}$-1inked recessive, was found in a father and son who presented with cryptorchidism, hypogonadism, and hyposmia. The father's fertility had been induced with chorionic gonadotropin, leading to the birth of 3 children, for whom paternity was verified by blood group analysis. The mother had normal olfaction and no family history of infertility. The father was studied five weeks after the last testosterone therapy.

Study results (LH/FSH in MIU/ml LER 907 , testosterone(T) in $\mathrm{ng} / \mathrm{dl}$ ):

Father Son(9yxs) Son(7yrs) Daughter(12 y)

Olfaction

$\downarrow$
1
$1.5 / 2$

LH/FSH, basal

$1.5 / 2.4$

$0.3 / 0.6$

$\mathrm{N}$

Daugh

$0.3 / 0.4$
$1.2 / 2.1$

$0.3 / 2.8$

$180^{\circ}$ p. LHRH

$13.3 / 3.9$
$7.2 / 4.2$

$0.7 / 1.6$

$5.2 / 8.3$

Testo., basal

205
475
4.76

$0.4 / 0.1$

$0.4 / 2.8$

$3.6 / 13.2$

$90^{\prime}$ p. LHRH

48

16

$--$

40

0.14

11.88

0.46

0.50

--

$-$

Basal serum gonadotropins and plasma testosterone in prepubertal children prove of little value for the diagnosis of Kallmann's syndrome. LHRH stimulation is helpful in differentiating patients with Kallmann's syndrome from those with primary gonadotropin deficiency. Like other genetic midline defects, Kallmann's syndrome is autosomal dominantly inherited with incomplete expression in the female.

\section{6}

MUSCULAR HYPERTROPHY VERSUS SUPRACLAVICULAR "FAT PADS" IN CONGENITAL HYPOTHYROIDISM, JOSE Cara, S.U.N.Y., Brooklyn, Maimon
Dept. Pediatrics

Supraclavicular fat pads were described as a sign of hypothyroidism more than 100 years ago, and since then it has been repeated in almost every publication dealing with congenital hypothyroidism. Our observations, however, indicate that supraclavicular "fat pad" is a misnomer. The bulging in the supraclavicular area is the result of hypertrophy of the muscles of the neck, particularly the trapezius. A fold of skin fails to demonstrate any accumulation of fat or mixedematous material in the amount necessary to account for the bulging in that area. Palpation however reveals enlarged hard muscles during contraction. The muscular hypertrophy is more easily observed in the supraclavicular area because the infant is able to move his head against gravity after the first day of life. However muscular hypertrophy can be demonstrated in the hypothyroid infant during the contraction of other muscular groups. If the infant is held from the axillae allowing him to support his weight, one can palpate enlarged hard muscles in the gastrocnemic area. When the hypothyroid infant cries, contracting the abdominal muscles, it is sometimes possible to see the enlarged muscles bulging between the tendinous intersections. The consistency of the abdominal wall may be board like, making palpation of the abdomen difficult. The gluteal and the paravertebral areas may present the same phenomena. We also consider that the large tongue of the hypothyroid infant is the result of muscular hypertrophy of that movable organ rather than mixedematous infiltration.

\section{FAMILIAL DIABETES MELLITUS. E. Colle and M. M.}

There have been recent reports of a familial, dominantly inherited type of diabetes mellitus. Detection of these

individuals often occurs in childhood. We present extended longitudinal studies on one such family with one affected member in the first generation, four members in the second generation, and six members in the third. These studies reveal (1) inappropriately low insulin levels in the fasting state and following an oral glucose challenge, (2) an increase in insulin levels following intravenous tolbutamide with a delayed drop in plasma glucose levels (nadir 40-90 minutes), (3) mildly elevated fasting glucagon levels which fall after oral glucose, (4) absence of ketonuria. The age at diagnosis depends on the performance of a routine urinalysis which reveals glucosuria since symptoms are minimal or absent. Longitudinal studies indicate that the most abnormal oral glucose tolerance tests occur during puberty.

After mature height is established glucose values more closely approximating norms for age are found. Growth rates and sexual development are normal. Anti islet cell antibodies are not present in the sera of affected individuals.

It is important to recognize this varient of diabetes mellitus since the inclusion of these individuals in the total group may confuse studies on the etiology of the more common form(s) of juvenile diabetes and erroneously attribute treatment effects where none exist.
218 INTERACTION OF hGH AND bGH WITH AMPHIPHILES. G. w. Conner, P. J. Collipp and V. T. Maddaiah, Dept. of Ped., Nassau County Med. Ctr., E. Meadow, N.Y. 1I554. Although bGH has about $60 \%$ homologous sequences with hGH it is not active in humans. We have reported some tertiary structural differences around the single tryptophane $(T)$. It is likely that the hormones may undergo different structural transitions when bound to receptors in vivo. As an in vitro model we have studied fluorescence $(F)$ changes when monomeric forms of hormones interact with amphiphiles like cetyl trimethylammoniumbromide (C), sodiumdodecylsulfate (S), deoxycholate (D), anilinonaphthalenesulfonate (A) and lysolecithin (L). F (350 nm) increased till the critical micellar concentration of amphiphiles was reached. The $\%$ change of maximum $F$ (Table), except with $A$ where $F$ was measured at $475 \mathrm{~nm}$, would be a measure of structural transition around $\mathrm{T}$.

hGH

\begin{tabular}{|c|c|c|c|c|}
\hline & mum & & & \\
\hline & S & D & $\mathrm{A}$ & L \\
\hline 100 & $10-25$ & 0 & 3000 & 0 \\
\hline
\end{tabular}

Anionic $S$ and $D$ did not show significant interaction. Neither ionic strength nor $\mathrm{pH}$, between 3.5 and 9.5 significantly influenced $F$ change by $C$. These results suggest the following: 1 . hormones show selectivity in interaction with cationic amphiphiles, 2. binding which may involve mostly hydrophobic interaction shields the molecules from the effects of $\mathrm{pH}, 3$. bGH interaction is stronger than hGH probably due to greater hydrophobicity around $\mathrm{T}$.

219 ABNORMAL CORD THYROID HORMONE LEVELS IN NEONATAL RESPIRATORY DISTRESS SYNDROME (RDS). Raul A. Cuestas, Arnold Lindall, and Rolf R. Engel, University of Minnesota Medical School, Minneapolis.

The finding of low cord thyroxine (T4) levels in infants who develop RDS was confirmed and extended by also assaying triiodothyronine (T3), thyroid stimulating hormone (TSH) and T3 uptake ratio (T3UR) in the cord serum from 120 premature and 39 term newborns. The incidence of RDS correlated inversely with both cord T3 $(p<0.01)$ and cord T4 $(p<0.01)$ levels; directly with cord TSH $(p<0.05)$ levels; and not at all with the T3UR results. The table lists the mean +SEM for a subgroup (gestational age 33-37 weeks) of 37 control and 18 RDS prematures matched for mean gestational age and body length, after excluding all infants with Apgar score below 5, delivery by Cesarean section or maternal diabetes.

\begin{tabular}{llllll}
\hline & T3 ng/dl & T4 $\mu$ g/d1 & T3UR & TSH $\mu \mathrm{U} / \mathrm{ml}$ \\
\hline \hline RDS & $10.8 \pm 2.1^{*}$ & $6.3 \pm 1.0^{*}$ & $0.92 \pm 0.04$ & $18.6 \pm 3.6^{*}$ \\
Control & $20.6 \pm 2.9$ & $7.5 \pm 0.3$ & $0.88 \pm 0.02$ & $12.7 \pm 1.0$ \\
\hline
\end{tabular}

$\widehat{*_{\mathrm{p}}<0.05}$

The compensatory increase in cord TSH levels of prematures with RDS suggests that the decrease in cord T4 and the proportionately larger decrement in the more active hormone, T3, reflect a biologically significant limitation in thyroid hormone activity which could contribute to delayed lung maturation.

ABSENCE OF YLACENTAL TRANSFER OF L-TRITODOTHYRONINE (T) IN THE RAT. Jean D. Dubois, Alain Cloutier, Peter Walker, and Jean H. Dussault (Spon. by Delbert A. Fisher). C.H.U.L., Dept. of Endocrinology, Quebec.

To detect placental transfer of L-Triiodothyronine (T3) in pregnant rats, we have injected $1 \mu \mathrm{Ci}{ }^{125} \mathrm{I}_{-} \mathrm{T}_{3}$ at the $16 \mathrm{th}$, 18th and 20th day of gestation. Three hours after the injection, which corresponds to the equilibrium time determined by a method of constant infusion, the pregnant rats and their fetuses were sacrificed. The $125 \mathrm{I}_{3} \mathrm{~T}_{3}$ was extracted from the serum or the homogenate by butanol extractions and alkaline washes. The transfer rate was calculated from the quantity of $125 \mathrm{I}-\mathrm{T} 3$ in the serum of the fetuses after 3 hours, and the maternal metabolic clearance rate $(8.19 \pm 0.449 \mathrm{ml} / \mathrm{hr} / 100 \mathrm{~g}$ body weight; Mean \pm S.E.M.). At the 16th day of gestation, the placental transfer of $\mathrm{T}_{3}$ was $0.824 \pm 0.105 \%$ of the total maternal clearance rate/1itter weight; it was $1.053 \pm 0.245 \%$ at the 18 th day of gestation and $0.578 \pm 0.101 \%$ at the 20 th day gestation. There were no significant differences between these results. The maternal $\mathrm{T}_{3}$ concentration was $68.27 \pm 20.6 \mathrm{ng} / 100 \mathrm{ml}$ and its production rate was $5.57 \pm 0.305 \mathrm{ng} / \mathrm{hr} / 100 \mathrm{~g}$; with these data we calculated a maternal-fetal T3 transfer of $0.046 \pm .0063 \mathrm{ng} / \mathrm{hr}$. Nor was there any $\mathrm{T}_{3}$ transfer observed when the mother received $1.9 \mu \mathrm{g}$ unlabelled $\mathrm{T}_{3}$ which led to a significant rise in maternal $\mathrm{T}_{3}$ concentration $(68.27 \pm 20.6 \mathrm{ng}$ to $102.23 \pm 7.41 \mathrm{ng} / 100 \mathrm{~m} 1 ; \mathrm{p}<$ $0.01)$; there was no detectable T3 in the fetal serum. From these results we conclude that there is no placental transfer of $\mathrm{T}_{3}$ in the rat and that the hypothalamo-pituitary-thyroid axis of
the fetus develnos autonomously. 
221 THYROID FUNCTION IN NEONATAL HYPOTHYROIDISM. Jean H.

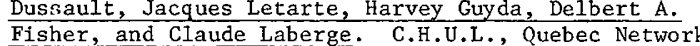
for Genetic Medicine, Quebec, and U.C.L.A., Dept. of Pediatrics, California.

We have measured various thyroid hormone parameters in 26 cases of neonatal hypothyroidism detected through the Quebec screening program for neonatal hypothyroidism. In all instances the filter paper spot $\mathrm{T}_{4}$ was below 2 S.D. of the mean of the day, averaging $0.35 \mathrm{ng} / 40 \lambda \pm 0.05$ (Mean \pm S. F.M.) of eluted blood. Filter paper spot $\mathrm{T}_{4}$ on a second sample averaged $0.21 \pm .04 \mathrm{ng} /$ $40 \lambda$ of eluted blood a value significantly lower than the first value, indicating a progressive disappearance of thyroid hormone reserve. Serum T 4 averaged $1.9 \pm 0.4 \mu \mathrm{g} / 100 \mathrm{ml}$ whereas the serum $\mathrm{T}_{3}$ ranged from 0 to $21 \mathrm{I} \mathrm{ng} / 100 \mathrm{ml}$. An Il31 uptake was performed in 8 infants and averaged $10.3 \pm 5.2 \%$. The mean TSH concentration was $365 \pm 124 \mu \mathrm{U} / \mathrm{ml}$ but 2 infants were hypothyroid with a normal TSH. One infant had a subnormal response to TRH thus diagnosed as hypothalamic hypothyroidism, the other presenting a small goiter with a normal $\mathrm{T}_{3}$ indicating probably a compensated dyshormonogenesis. Reverse $\mathrm{T}_{3}$ (RT3) was measured in 8 hypothyroid infants and the mean value of $45 \pm 9.6 \mathrm{ng} / 100$ $\mathrm{ml}$ was well below the normal mean of that age $(178 \pm 12 \mathrm{ng} / 100$ $\mathrm{ml}$ ). From these results it would appear that filter paper spot $\mathrm{T}_{4}$, serum $\mathrm{T}_{4}$, and possibly $\mathrm{R}_{\mathrm{T} 3}$ are the best indicators of thyroid function in the newborn for the detection of hypothyroidism.

222 EVALUATION OF THE GROWTH HORMONE (GH) EXERCISE TEST. Evelyn Eisenstein, Leslie P. Plotnick, Peter A. Lee, Claude J. Migeon, and A. Avinoam Kowarski, Department of Pediatrics, Johns Hopkins University, School of Medicine, Baltimore, Maryland.

Two hundred children with short stature have been evaluated for growth hormone status by a standard exercise test (Pediatr. 50:760, 1972).

A diagnosis of hypopituitarism was made in 18 patients based on an inadequate response to intravenous arginine and insulin $(\mathrm{GH}<10 \mathrm{ng} / \mathrm{ml})$ and an abnormal growth rate. The maximum $\mathrm{GH}$ value in the exercise test for these patients was $5.1+3.4 \mathrm{ng} / \mathrm{ml}$ (mean + S. D.). Only one of them had a value $>10 \mathrm{ng} / \mathrm{ml}(14)$ which on repeat study was $4.5 \mathrm{ng} / \mathrm{ml}$.

One hundred seventy-six children were not growth hormone deficient based on diagnostic criteria other than the exercise test. The GH levels from before, immediately after and 20 minutes after exercise were $8.8+9.4,28.1+15.1$, and $11.9+9.9 \mathrm{ng} / \mathrm{ml}$, respectively. The mean maximum level in any of the 3 samples was $29.6 \pm 14.3 \mathrm{ng} / \mathrm{ml}$. Thirteen of the non-GH deficient children had a maximum GH value of $<\mathrm{I} 0 \mathrm{ng} / \mathrm{ml}$. All of these $13 \mathrm{had}$ a response to arginine and insulin stimulation of $>10 \mathrm{ng} / \mathrm{ml}$. Thus only $7 \%$ of the non-GH deficient patients had an abnormal response to the exercise test.

Six patients who had maximum GH responses $<10 \mathrm{ng} / \mathrm{ml}$ are not yet included in the study because further information is being obtained for final diagnosis. 223 W.Finkelstein, Robert M. Boyar, Richard HK Wu, Jack Katz and Leon Hellman, Albert Einstein Coll. Med., Montefiore Hosp. \& Med. Ctr.,Inst. for Steroid Rsch., Departments of Oncology, Pediatrics, Psychiatry, Bronx, New York.

Nine patients, ages 17-25 years had findings typical of anorexia nervosa. They all showed avoidance of food with bizarre dietary habits resulting in severe weight 1 oss of from $24-40 \%$ of their ideal weights. No organic abnormality could be found to explain this weight loss. All patients were studied prior to any treatment. A 20 minute, 24-hour blood sampling study was performed to evaluate the spontaneous secretion of hGH, which was measured by radioimmunoassay. All studies from each patient were run in the same assay. During the initial studies all patients had normal patterns of growth hormone release with episodic releases occurring during both waking and sleeping periods but always with greater release occurring during the sleep periods. The mean maximum concentration of hGH reached during sleep was 16.2 (range $5.0-46.0) \mathrm{ng} / \mathrm{ml}$. The mean 24 -hour concentration was 3.5 (range.44-16.7) ng/ml. These results are within the normal range for a matched control group. Four patients were restudied one to 16 months after varying degrees of weight gain. All patients had no significant change in hGH secretion during recovery. All patients had ammenorrhea and all showed regression of $\mathrm{LH}$ secretion from the normal adult pattern to a pre-pubertal or adolescent pattern. The normal growth hormone secretion suggests that the factors responsible for regression in gonadotrophin secretion do not affect the release of hGH which is normal in this disorder.
224 CORTISOL (F) SECRETION \& METABOLISM IN CHILDREN AND ADOLESCENTS WITH SHORT STATURE. Jordan $W$. Finkelstein, David K. Fukushima, H. Leon Bradlow, Robert M. Boyar, Richard Hk Wu and Leon Hellman, Albert Einstein Coll. Med., Montefiore Hosp. \& Med. Ctr., Inst. for Steroid Research, Depts. of Oncology and Pediatrics, Bronx, New York.

Cortisol (F) secretion was measured in a group of children with idiopathic short stature by 1) Isotope dilution $(N=25)$, 2) By a formula derived by summation of 3 metabolites of $F(N=$ $23)$, 3) By calculation from the metabolic clearance rate (MCR) and the 24-hour mean plasma $F(N=9)$. MCR was determined after a pulse dose of labelled $F$ and mean plasma $F$ during the course of a 24-hour, 20-minute sampling study. F secretion $\mathrm{rate}\left(\mathrm{mg} / \mathrm{m}^{2} /\right.$ day) was $11.8( \pm 3.3 \mathrm{SD})$ by isotope dilution, $11.4( \pm 2.9 \mathrm{SD})$ by 3 metabolite method, $11.1( \pm 1.7 \mathrm{SD})$ by the MCR method. There was no difference between secretion rate between prepubertal children and adolescents. However, in 4 prepubertal children MCR $\left(\mathrm{L} / \mathrm{m}^{2} / \mathrm{d}\right.$ ) was 159 (range 153-181) and volume of distribution (VD) $\left(\mathrm{L} / \mathrm{m}^{2}\right.$ ) was 7.4 (range $\left.7 \cdot 0-8,0\right)$. F half time was 47 minutes (range 44-50). In 6 adolescents MCR was 254 (range 244-300), VD 14.2 (range 12.7-17.5) and $F$ half time 50 (range 40-56). MCR \& VD in adolescents are significantly greater than in prepubertals but are the same as in adults. Half time is shorter in all children than in adults. These data indicate a change in the metabolism of $F$ with increasing age or sexual maturation in children with short stature.

225

TSH METABOLISM IN THE NEWBORN LAMB. Delbert A. Fisher, Joseph Sack, Robert W. Lam, and Che-Ching Wang, UCLAHarbor Gen. Hosp., Dept. Ped., Torrance, California.

We have reported that serum $\mathrm{T} 3$ in the lamb increases rapidly in the newborn period and that this increase is provoked by cutting the umbilical cord (CCT). To further clarify the role of TSH in this T3 surge we studied serum OTSH levels and the OTSH response to exogenous TRH. In controls serum TSH levels increased modestly to peak levels at $30 \mathrm{~min}$. A similar 30-45 min. peak occurred in newborns in which CCT was delayed $60 \mathrm{~min}$. but in these animals, in spite of a normal TSH surge, the T3 response was delayed until after CCT. Warming the newborn at $39^{\circ} \mathrm{C}$ (water bath) prevented the TSH surge. The response to TRH $(250 \mu \mathrm{g})$ was studied in 8 lambs; 4 were $<6 \mathrm{hr}$. of age and 4 were $7-18 \mathrm{hr}$. Blood was sampled serially for measurements of TSH, T4 and T3. There was a rapid rise in serum TSH during the first $15 \mathrm{~min}$., from a mean of $7.3 \mu \mathrm{U} / \mathrm{ml}$ to $14.2 \mu \mathrm{U} / \mathrm{ml}$. A second peak was observed at $120 \mathrm{~min}$, (TSH $24.6 \mu \mathrm{U} / \mathrm{ml}$ ), suggesting new synthesis as well as release of hormone. T4 began to rise between 60 and $120 \mathrm{~min}$. and continued to rise through $6 \mathrm{hr}$. T3 increased by $30 \mathrm{~min}$. with levels gradually rising thereafter; SRIF ( $125 \mu \mathrm{g}$ over $45 \mathrm{~min}$.) blocked the TRH response in two animals over $45 \mathrm{~min}$., and when discontinued T4 and $T 3$ levels increased in response to a small increase in TSH. These data indicate 1) that the TSH surge in the newborn lamb is less marked than in the newborn infant 2) the TSH and T3 responses can be dissociated by CCT 3) the TSH surge may be due to newborn cooling 4) the T3 response is not entirely due to TSH 5) the newborn lamb responds to TRH with a rapid increase in TSH and 6) SRIF inhibits the TRH effect.

226 NEURAL PROGRAMMING OF SACCHARIN PREFERENCE BY ANDROGENS IN THE RAT. Allen S. Goldman, ent of Experimental Pathology, Children's Hospital of Philadelphia, Phila, Pa. 19104

The present studies were performed to determine whether gonadal hormones may program in the rat a sexually dimorphic, non-reproductive brain function, namely preference for sweet solutions. The selection of saccharin solutions relative to water was compared among normal males, females, genetically androgen insensitive male pseudohermaphrodites (PS), and male pseudohermaphrodites made androgen insensitive in fetal and neonatal 1 ife (CypAc) by cyproterone acetate. Ps displayed the characteristically female pattern of increased preference for saccharin solutions while CypAc expressed typically masculine preference. Treatment with estrogen and progesterone and/or castration of mature CypAc did not induce the female pattern. These results suggest that androgen action is required for programming male saccharin preference centers in the brain. However, since blockade of androgen action by cyproterone acetate is only blocked until two weeks of age in CypAc, the programming of the saccharin preference center must occur after the first week, the time of imprinting by androgens of the centers controlling reproductive functions. 
227 THE DETECTION OF THE HETEROZYGOTE CARRIER FOR CONGENITAL ADRENAI HYPERPLASIA
(CAH): STUDIES ON PARENTS AND SIBLINGS OF CHILDREN WITH CAH. James P. Gutai, Peter A. Lee, Avinoam A. Kowarski, Claude J. Migeon, Department of Pediatrics, Johns Hopkins University, School of Medicine, Baltimore. The response of plasma progesterone, 17 -hydroxyprogesterone (17-OHP) and cortisol to intravenous ACTH (1.0 mg Cortrosyn) was determined in 17 control subjects, 7 sets of parents and 11 siblings of children with CAH. There was no difference in baseline or post stimulation concentrations of progesterone and cortisol of parents and controls. Only after ACTH stimulation was the concentration of $17-O H P$ in parents significantly higher than in the control group. Rates of increase of progesterone and 17-OHP were significantly greater in the parents when compared to control subjects, all 14 individual parents having a combined rate of increase of progesterone and 17-OHP that was greater than the mean $+1 S 。 D$ o of the control group.

The eleven siblings studied were from 3 families in which there are 5 affected children. The mean +2 S. D. of the combined rate of increase of the control group was considered to be the upper limit of normal. Using these criteria, 7 of the 11 siblings would be predicted to be heterozygotes while 4 would be unaffected homozygotes. These results ( $31 \%$ affected, $44 \%$ heterozygotes and $25 \%$ normal) are in good agreement with an autosomal recessive mode of inheritance.

This work was supported by U.S.P.H. S. Grants AM-00180, HD06284, T1-AM-05219 and 5-K06-AM-21855.

228

Pituitary Regulation of Leydig Cel1 Function. R.L.Hauger, Y.I.Chen, A.H.Payne, \& R.P.Ke $1 \mathrm{ch}$, Rep. Endo. Program, U. of Mich., Ann Arbor.

To examine whether the pituitary regulates testicular LH (hCG) receptors, binding of 125 I-hCG to $20,000 \times g$ membrane fraction and serum testosterone( $T$ ) were measured in intact and hypophysectomized(HPX) mature rats. Highly purified hCG (40\% biologically active) with a SA of $5 \times 10^{4} \mathrm{cpm} / \mathrm{ng}$ was used to determine specific binding. Leydig cell response was assessed by measuring serum $T$ $2 \mathrm{~h}$ after i.p. administration of $25 \mathrm{ug}$ LH(NIH-LH-S19). Serum $\mathrm{T}$ decreased from $2.7 \pm 0.2 \mathrm{ng} / \mathrm{ml}$ to $0.34 \pm 0.04 \mathrm{ng} / \mathrm{ml}$ 1 day after HPX, while intratesticular $T$ was $1 / 100$ less by day 3. Following HPX, Leydig cell response was biphasic and did not decrease as much or as rapidly as did hCG binding.

$\begin{array}{lrrrr}\text { ng T/ml } & \text { Intact } & \text { HPX Day } 1 & \text { HPX Day } 3 & \text { HPX Day6 } \\ \text { cpmXI0-5/testis } & 22.1 \pm 0.9 & 24.5 \pm 1.9 & 6.2 \pm 0.4 & 3.4 \pm 0.2 \\ \text { cpm/ug protein } & 35.4 \pm 1.5 & 44.1 \pm 3.4 & 12.1 \pm 0.6 & 6.5 \pm 0.6\end{array}$

Administration of $\mathrm{T}, \mathrm{DHT}, \mathrm{T}+\mathrm{FSH}, \mathrm{T}+\mathrm{LH}, \mathrm{FSH}$, or $17 \mathrm{~B}-$ estradiol did not prevent decline in hCG binding. A highly specific antiserum for GnRH(LRF) decreased serum $T$ in intact rats by $60 \%$, but had no effect on $L H$ receptors. Although it has been proposed that protein hormones regulate their own receptors, we suggest that pituitary factors other than gonadotropins may be necessary for maintenance of the testicular LH receptor.

\%29 SOMATOMEDIN IN CHILDPEN WITH PROTEIN CALORIE MALNUTRITION. Raymond L. Hintz, Robert Suskind, Kosin Amatayakul and Rohert 01sen, Department of Pediatrics Stanford University Medical School, and Anemia and Malnutrition Research Center, Chiang Mai, Thailand.

This study was undertaken to define the interrelationships of somatomedin (SM), growth hormone (HGH) and an inhibitor of SM in both marasmus and kwashiorkor. The patients, 8-60 months of age, were hospitalized 50 days. A total of 27 patients were studied, 13 with maramus $(M), 11$ with maramus-kwashiorkor $(M-K)$, and 3 witr: kwashiorkor (K). 21 well-nourished Thai children acted as contro] SM, HGH and the presence of an inhibitor were assessed.

\begin{tabular}{|c|c|c|c|c|}
\hline )ay & 8 & 2.9 & 50 & Control \\
\hline $\begin{array}{ll} & 0.22 \pm 0.02 \\
1 / \mathrm{ml} & (\mathrm{n}=18)\end{array}$ & $\begin{array}{c}0.23 \pm 0.02 \\
(n=21)\end{array}$ & $\begin{aligned} 0.43 & \pm 0.03 \\
(n & =26)\end{aligned}$ & $\begin{array}{c}0.63 \pm 0.09 \\
(n=25)\end{array}$ & $\begin{array}{c}0.48 \pm 0.07 \\
(\mathrm{n}=21)\end{array}$ \\
\hline
\end{tabular}

\pm S.E.

SM was significantly depressed at days 2 and $8 \quad(p<0.01)$ but were not different from controls at day 29 and 50 . There were no differences between the $M, M-K$, and $K$ groups, and no correlations with plasma albumin. When SM values were compared to simultaneous HGH levels, there was an inverse relationship observed. Patients with $\mathrm{SM}>0.35 \mathrm{u} / \mathrm{ml}$ had normal $\mathrm{HGH}$ values. 16 out of 30 patients with $\mathrm{SM}<0.35$ had HGH levels $>20 \mathrm{ng} / \mathrm{ml}$. 9 out of 27 -day 2 samples and 6 out of 27-day 8 samples showed the presence of an inhibitor These data show that SM is reduced in children with $M$ as we11 as $M-K$ and $K$. These levels did not rise until after a full intake of calories and protein. The low SM levels in the face of markedly elevated HGH levels in many of our patients suggest functional block in the synthesis and/or release of SM.
ANTITHYROGLOBULIN ANTIBODIES BY RADIOIMMUNOASSAY IN CHILDREN AND ADOLESCENTS WITH THYROID DISORDERS N.J. Hopwood, T.P. Foley, Jr., R. Rabin, and R.L. Peake. Dept. of Ped., Univ. of Michigan Med. Ctr., Ann Arbor, MI, Dept. of Ped. and Path., Univ. of Pgh Sch. of Med., Pittsburgh, PA, and Dept. of Med., Univ. of Tex. Med. Br. Hosp, Galveston, TX

A comparative study employing 3 methods for determining thyroid antibodies--double antibody radioimmunoassay (RATA), antithyroglobulin (ATA) and antimicrosomal (AMA) by hemagglutination, was performed in 125 children with thyroid disorders and in 53 short normal children (controls). RATA was assayed at $1: 100$ dilution $(+=>99 \%$ confidence limits for normal adult population).

\begin{tabular}{|c|c|c|c|c|c|c|}
\hline & Pt. & Sample & RATA $1: 1$ & & $\mathrm{ATA} \geqq 1: 4$ & AMA \\
\hline Patients & 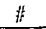 & 非 & $\bar{x} \%$ Bound & $8+$ & $\%+$ & $\%+$ \\
\hline $\begin{array}{l}\text { Controls } \\
\text { Diffuse Goiter }\end{array}$ & 53 & 53 & 6.3 & 11.3 & 13.2 & 1.9 \\
\hline Euthyroid & 34 & 55 & 23.0 & 78.2 & 21.2 & 1.8 \\
\hline Hypothyroid & 20 & 34 & 38.3 & 97.0 & 14.7 & 8.8 \\
\hline Enzymatic & 6 & 6 & 6.5 & 16.6 & 0 & 0 \\
\hline Hyperthyroid & 23 & 41 & 25.7 & 85.3 & 24.4 & 4.9 \\
\hline Cong. Hypothyroid & 21 & 21 & 5.6 & 9.5 & 0 & 0 \\
\hline Acquired Non-Goitr. & & & & & & \\
\hline Hypothyroid & 13 & 22 & 12.0 & 54.4 & 4.5 & 9.0 \\
\hline Thyroid tumor & 8 & 8 & 14.3 & 25.0 & 12.5 & 12.5 \\
\hline
\end{tabular}

The RATA is simple and quantitative with sensitivity and specificity exceeding present tests for circulating antithyroglobulin antibodies.

\section{1}

CONGENITAL TOTAL LIPODYSTROPHY (CTL): AN ENDOCRINE STUDY IN 3 SIBLINGS. Caro1 A. Huseman, Ann J. Johanson, Madan M. Varma, $\frac{\text { Elsa P. Paulsen, Robert } M}{\text { Blizzard. }}$ Virginia, Charlottesville, Virginia 22901.

Three siblings ( 1 male, 2 female) age $(6 / 12-128 / 12)$ years with congenital total lipodystrophy were evaluated for endocrine and metabolic disturbances.

Marked virilization in the $12 \mathrm{y} / \mathrm{OXX}$ female was associated with increased testosterone (T) 300-500 $\mathrm{ng} \%$, and serum hLH to $33.6 \mathrm{mIu} / \mathrm{ml}$. HCG (7000u I.M.) but not ACTH $\left(25 \mathrm{u} / \mathrm{m}^{2} \mathrm{I} . \mathrm{V}.\right)$ produced $a$ rise in $\mathrm{T}$. $\mathrm{E}_{2}$ ( $0.18 \mathrm{mg} \mathrm{q} 8 \mathrm{~h} \times 7$ days) suppressed $\mathrm{T}$ but Decadron (1.5mg q $8 \mathrm{~h} \times 14$ days) did not. Polycystic ovary disease was confirmed by laporotomy in this patient. $T$ and hLH levels decreased to normal levels after bilateral ovarian wedge resection. However, $T$ gradually returned to preoperative levels 4 months after surgery

Marked insulin resistance $(0.3 \mathrm{u} / \mathrm{kg} \mathrm{I.V.)}$ associated with diabetic OGTT's, hyperinsulinism, and hyperglucagonemia, were found in all three siblings. Hyperglucagonemia may explain the insulin resistance.

Cholesterol, triglycerides, and lipoproteins were normal in contrast to values reported in the literature. Elevated liver gly cogen $(6.5 \%$ vs $\mathrm{n} 1<5 \%)$ was found, as well as fatty metamorphosis and periportal fibrosis in the liver biopsy of the $12 \mathrm{y} / \mathrm{o}$ patient.

Accelerated height age and bone age were present in all 3 patients but no explanation is apparent except in the $12 \mathrm{y} / \mathrm{o}$ virilized patient. Growth hormone responses to arginine, L-DOPA, and glucagon were normal in all 3 children.

\section{MICROVASCULAR CHANGES IN CHILDREN WITH DIABETES.} $\frac{\text { Robert L. Jackson, Richard A. Guthrie, James A. }}{\text { Esterly. Ahmet N. Bilginturan, Ronald C. James, and }}$ Jeanne F. Saathoff, Columbia, Mo.

Clinical, anatomical, and biochemical evidence is accumulating which indicates that the microvascular changes which occur in diabetics are related to the degree of regulation of their disease. Using Williamson's method, we have measured muscle capillary basement membrane thickness (CBMT) in children with diabetes in varying degrees of control. All measurements were done as unknowns and diabetic control was rated prior to knowledge of CBMT. Most diabetics were observed sequentially from onset. The subjects were 54 boys (age 7 to 24 ) and 43 girls (age 5 to 21). Patients were classified according to (a) age (prepubescent, pubescent and postpubescent), (b) duration of the disease $(0$ to 5 , 5 to 10,10 to 15 , and over 15 years), (c) degree of control (good, fair, fair to poor, and poor to fair). Increase in CBMT was segmental, preceded retinopathy and was not observed before CHOH intolerance. CBMT was increased in some young adults with late chemical diabetes. Diabetics near time of onset had normal or high normal values ( 5 boys $\bar{c}$ high values at onset maintained in good control had decreased CBMT in $<18$ months). Thirty-two diabetics maintained in good control up to $15 \mathrm{yrs}$. after onset had normal CBMT; 26 of 29 patients in fair control up to 19 yrs. had normal CBMT; 13 patients in fair to poor control for 5-11 yrs. and 10 adolescent girls in poor control for $<10$ yrs. had increased CBMT. During maturation, $6 \mathrm{girls}$ in poor control had increased CBMT in $<3$ yrs. Additional studies including serial observations are in progress. 
233 MUSCLE CAPILLARY BASEMENT MEMBRANE THICKNESS IN NORMAL CHILDREN AND SIBLINGS OF CHILDREN WITH OVERT DIABETES. Robert L. Jackson, Richard A. Guthrie, James A. hoff, and Diana W. Guthrie, Columbia, Mo.

Measuring the thickness of capillary basement membranes is an objective method for early detection of microvascular changes in patients with diabetes. Using Williamson's method, measurements of muscle capillary basement membrane thickness (CBMT) were made from electron photomicrographs of 49 normal children (age 9 to 18 , 24 boys and $25 \mathrm{girls}$ ) with no family history of diabetes and in 39 heal thy siblings of diabetics (age 13 to $21 ; 17$ boys and 22 girls) with one or more normal glucose tolerance tests (OGTT's) including both blood sugar and serum insulin determinations. Our data confirm Williamson and Kilo's observations that physiological thickening increases with age. We found that the rate of thickness accelerates during the pubertal growth spurt in both groups. No differences in CBMT were found between normal children and the siblings of children with overt diabetes with normal OGTT's. Reliable norms for boys and girls are necessary as CBMT is not only related to chronological age and sex but also to maturational age. Esterly, Ahmet N. Bilginturan, Larry W. Romang, Jeanne F. Saat-

SEXUAL PRECOCITY AND HYPOTHYROIDISM-DIMINTSHED ANDROGEN SYNTHESIS AND ABSENCE OF PUBARCHE. Bruce S. Keenan, Rebecca T. Kirkland, Ronald M. McNeel, Robert A. Boyd, and George W. Clayton. Baylor College of Medicine, Dept. of Pediatrics, Houston.

A $9 \frac{1}{2}$ year old girl with menorrhagia was found to be severely myxedematous. Breast development of 18 months duration was Stage IV but pubic and axillary hair were absent. Skull x-ray showed an enlarged sella turcia and bone age was $6 \frac{1}{2}$ years. Plasma testosterone (T) and dehydroisoandrosterone (DHA) concentrations were appropriate for a prepubertal child of less than 9 years. Luteinizing hormone ( $\mathrm{LH})$, follicle-stimulating hormone (FSH), and 178estradiol $\left(E_{2}\right)$ were in the adult female range. Although response of the symptoms of hypothyroidism was prompt, return of gonadotropin secretion to the prepubertal state was gradual, as were cessation of menstruation and the regression of secondary sexual characteristics. Plasma androgen concentrations remained in the prepubertal range. The data suggest that a selective maturational change in the hypothalamic-pituitary-gondal axis occurs in primaxy hypothyroidism which regresses gradually. Adrenarche did not appear to be activated despite gonadotropin secretion.

\begin{tabular}{|c|c|c|c|c|c|c|c|}
\hline THERAPY & $\begin{array}{l}\mathrm{LH} \\
\mathrm{mI}\end{array}$ & $\begin{array}{l}\text { FSH } \\
\mathrm{mI}\end{array}$ & $\mathrm{E}_{2}$ & $\begin{array}{c}\mathrm{T} \\
10\end{array}$ & $\begin{array}{l}\mathrm{DHA} \\
\mathrm{ml}\end{array}$ & $\begin{array}{c}\mathrm{T}_{4} \\
\mathrm{\mu g} / 100 \mathrm{ml}\end{array}$ & $\begin{array}{r}\mathrm{TSH} \\
\mu \mathrm{U} / \mathrm{m} 1 \\
\end{array}$ \\
\hline Before & 20 & 14 & 10.3 & $<25$ & 38 & $<1$ & $>200$ \\
\hline $\mathrm{T}_{4}+$ Provera 1 mo. & 5.8 & 6.2 & 0.9 & $<25$ & 30 & 8.9 & \\
\hline $\mathrm{T}_{4}^{4}$ alone $2 \mathrm{mo}$. & 4.4 & 10.1 & 28.0 & $<25$ & 47 & 9.4 & 2.2 \\
\hline $\mathrm{r}_{4}$ alone $5 \mathrm{mo}$. & 3.9 & 6.5 & & $<25$ & 75 & & \\
\hline $\mathrm{T}_{4}$ alone $9 \mathrm{mo}$. & 4.4 & 3.9 & & $<25$ & & 8.2 & \\
\hline
\end{tabular}

234 EFFECT OF GROWIH HORMOIE (GH) ON RAT HEART MITOCHONDRIA (HM). D. Katkocin, G. Gwosdow, P. J. Collipp and V.T. Naddaiah, Dept. of Ped., Nassau County Ihed. Ctr., SUNY, Stony Brook, East ileadow, N. Y. 11554.

We have already shown that $\mathrm{GH}$ is important for the maintenance of a normal population of properly functioning liver mitochondria. Now we find that hypophysectomy (Hy) decreased heart weight to $100 \mathrm{~g}$ body weight ratio $(0.38 \pm 0.02$ to $0.27 \pm 0.02 \mathrm{p}<0.01)$. Hy also decreased HM protein content which is in contrast to an increase in liver. (9.81 \pm 2.10 to $6.70 \pm 2.14$ per $g$ tissue.) HM protein synthesis was decreased $(p<0.001)$ as measured by in vitro and in vivo incorporation (cpm/mg protein, Table) of labeled leucine. GH treatment of Hy rats restored these levels.

$\begin{array}{llrl} & \text { In Vitro } & \text { In Vivo } \\ \text { Normal } & \frac{1637 \pm 227}{1637} & \text { (6) } \\ \text { Hy } & 1022 \pm 289 \pm(6) & 751 \pm 32 & (5)\end{array}$

$\begin{array}{lllll}\mathrm{Hy} & 1022 \pm 289 & (6) & 751 \pm 32 & (6) \\ \mathrm{Hy}+\mathrm{bGH} & 1570 \pm 338 & (6) & 1100 \pm 111 & (5)\end{array}$

There was no change in mitochondrial ATPase levels and only a slight decrease in respiratory rate after Hy. Hy increased liver mitochondrial proteins and particularly high molecular weight matrix proteins. Some of these proteins are enzymes of gluconeogenesis which is known to be increased after Hy. Since heart lacks gluconeogenic activity Hy reduced HM protein content. Therefore, the effects of $\mathrm{Hy}$ and $\mathrm{GH}$ on mitochondria of different tissues depend on the overall metabolic role of the tissue in normal growth and development. (Supported by Nassau Heart Association and NIH AM 16649.)

\section{HETEROGENEITY OF COMPLETE TESTICULAR FEMINIZATION}

235 (CTF) REVEALED BY DIFFERENT LEVELS OF $5 \alpha$-DIHYDROTESTOSTERONE (DHT) BINDING IN CULTURED SKIN FIBROBLASTS. Morris Kaufman, Cipia Straisfeld, and Leonard Pinsky. Ce11 Genet. Lab., Lady Davis Inst., Jewish Gen. Hosp., Dept. of Ped., McGill Univ., Montreal.

Specific DHT-binding activity $\left(B_{S}\right)$ has been measured in intact confluent skin fibroblast monolayers derived from controls and patients with CTF. Nine labium majus (LM) strains from adult females and 9 preputial strains from males of all ages had a mean $B_{S}$ three times greater than that of 13 nongenital (NG) strains from adults of both sexes [33 (15-53) v.s. 11 (5 23) fmol per mg cell protein]. The variation was not correlated with sex, chronologic age of skin donors, or in vitro age of cultures. Notably, the $B_{S}$ of $3 N G$ strains from control infants was $\leq 5$. Seven of 8 NG strains from patients fulfilling the gonadal and clinicogenetic criteria for CTF had $B_{S}<2$; the value for the eighth was 5. The lower limit of $B_{S}$ for normal LM strains was 15 . Three of five LM strains from unrelated patients with CTF had $B_{S}<3$; the other two fell between 10 and 15. Conclusions: (1) genital skin fibroblasts preserve their differentiative status in serial culture; (2) the gap between the $B_{S}$ in NG fibroblasts from controls and from most patients with CTF is small; (3) fibroblasts from some patients with CTF have levels of $B_{S}$ close to or within the normal range; (4) LM fibroblasts appear able to reveal types of CTF associated with partial DHT-binding deficits; (5) CTF with normal or near-normal. $B_{S}$ may be due to primary defects in the nuclear events of target cell responsiveness to androgen.
237 EVIDENCE FOR SECRETION OF LUTEINIZING HORMONE RELEASATED WITH PRECOCIOUS PUBERTY, Howard Kulin, David Judge, Richard Santen, and Robert Page, The Pa. State Univ., M.S. Hershey Med. Ctr., Depts。 of Ped.,Path.,Med。\& Surg。, Hershey, Pa. dynamic control of gonadotropin secretion before and after neurosurgery were investigated in a 21 -month old boy with precocious puberty. Electron microscopy of the tissue showed glial and neural elements and many dense, membrane-bound granules of approxiUsing an indirect immunofluorescent antibody technique, LRF was demonstrated in sections of the hamartoma with 2 different highly specific anti-LRF antibodies. Specificity of the antibody reaction was demonstrated by the addition of excess synthetic LRF and resultant elimination of fluorescence. Since these neuropathologic studies suggested LRF within the hamartoma, 3 test substances nad and the hypothalamic-pituitary axis in the patient. Clomid was administered for 7-10 days on 2 occasions and produced no significant changes in the elevated levels of blood and urinary FSH and LH. Aldactone, an inhibitor of testosterone synthesis, caused a decrease in testosterone production rate and a consequent rise in gonadotropins. Medroxyprogesterone acetate, in a dose of 250$350 \mathrm{mg}$ I.M. every 2 weeks, caused only negligible gonadotropin suppression. These studies suggested partial autonomy of the hypothalamic-pituitary unit induced by tumor. Conclusion: Precocious puberty associated with an hypothalamic hamartoma may be due to direct secretion of LRF by the abnormally placed neural tissue.

LOW SOMATOMEDIN ACTIVITY (SM) AND LACK OF RESPONSE TO 238 IGH IN GLYCOGEN STORAGE DISEASE. L.S.Levine, E. Jniv.Med.col., New York, VA Hosp., Bronx, New York

To evaluate possible factors in the growth retardation of glycogen storage disease the acute metabolic response to human growth hormone ( $\mathrm{hGH}$ ) was determined in 2 sibs with Type VI glycogenosis, phosphorylase deficiency. Clinical data were as follows:

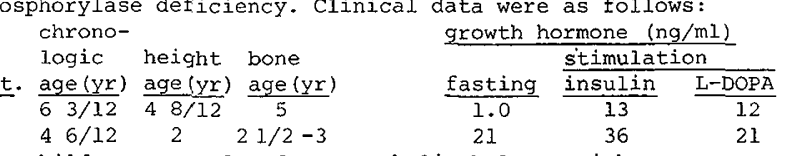

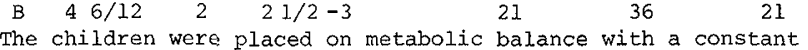
daily diet and daily $24 \mathrm{~h}$ urine collections. Following a 5 day pretreatment period, hGH (5 IU IM daily) was given for 5 days. serum somatomedin activity (SM) was in the hypopituitary range $(<0.5 \mathrm{U} / \mathrm{ml})$ in both patients in the pretreatment period and did not rise with growth hormone administration. In addition, there was no change in fasting blood sugar, insulin sensitivity, blood uxea nitrogen, sexum insulin, serum fibrinogen or urinary excretion of nitrogen, calcium, phosphorus, sodium or potassium.

The findings of low SM with no increase in response to growth hormone administration are similar to those in alcoholic liver cirrhosis. These findings as well as the absence of other metabolic response to hGH administration suggest growth hormone unresponsiveness may be a factor in the growth retardation of glycogen storage disease. The hormonal content of a partially excised hamartoma and the mately $100 \mathrm{~nm}$ in diameter, suggestive of neuro-secretory material. were used to characterize feedback control mechanisms between go- 
239

PITUITARY ADENOMA IN MCCUNE-ALBRIGHT SYNDROME: A RESULT OF ABNORMAL HYPOTHALAMIC STTMULATION? E.S. Lightner and S.S.C. Yen, (intr. by Grant Morrow, III) Dept. of Ped., Univ. of Arizona Medical Center, Tucson, Az. and Dept. of Ob. and Gyn., Univ. of California, San Diego School of Medicine, La Jolla California.

Previously reported studies in a 5-5/12 year old male with McCune-Albright syndrome and giantism suggested hypothalamic dysfunction as a cause of both his precocious puberty and rapid growth. At age 6-3/12 years, evaluation of pituitary function with hypothalamic releasing factors, with and without simultaneous infusion of somatostatin, indicated multiple abnormalities of hormonal function, possibly mediated through both abnormal hypothalamic input to, and abnormal hormonal interaction within, the pituitary gland. At 7-4/12 years, $2.5 \mathrm{mg}$. of ergocxyptine resulted in a prompt and significant fall in the patient's elevated levels of growth hormone, which previously were non-suppressible with glucose and chlorpromozine. Sequential roentgenograms for sella size, neurological and visual field examinations were normal from diagnosis until age $7-4 / 12$ years. At $7-8 / 12$ years, repeat examination revealed bilateral optic atrophy and visual field changes. Tomograms of the optic foramen revealed them to be almost obliterated by bony changes, with probable optic nerve compression. A pneumoencephalogram revealed a $1.5 \mathrm{~cm}$. suprasellar mass, which following surgical removal was diagnosed as a pituitary eosinophilic adenoma. The results of our studies, and the clinical course, suggest that prolonged abnormal hypothalamic stimulation of the pituitary may be an etiologic factor in the development of this patient's pituitary tumor.

240

THYROTROPIN RELEASING FACTOR (TRF) IN EVALUATION OF THYROID HORMONE REPLACEMENT THERAPY. Anne W. Lucky Elihu N. Goren, and Roger E. Johnsonbaugh, NIH and Naval Med. Center, Bethesda, Md. (Spon. by Joseph D. Schulman). Assuming that optimal replacement with l-thyroxine $\left(1 \mathrm{~T}_{4}\right)$ will reduce basal TSH to normal but will not suppress TSH responsiveness to exogenous TRF, we have used the TRF test to detect subtle manifestations of overtreatment in primary hypothyroidism. Nine tests were performed on 8 children, ages 6 to 16, who were clinically euthyroid and had normal serum levels of T,D and TSH on therapy; 2 had normal, 2 had partial, and 4 had absent responses to 500 ug of TRF given intravenously 24 hours after the last dose of $1 \mathrm{~T}_{4}$. One non-responder was retested after 6 weeks on a reduced dosage of $1 \mathrm{~T}_{4}$ and had a partial response. Responders had mean Igwer $\mathrm{T}_{4} \mathrm{D}$ and were on smaller average doses of $1 \mathrm{~T}_{4}$ ( $\mu \mathrm{g}$ per $\mathrm{kg}$. or $\mathrm{m}^{2}$ ) than non-responders (table). Although our data are preliminary and based on a single provocative test per patient, we have demonstrated pituitary suppression of TSH despite "normal" $T_{4}$ D and TSH in 6 of 8 patients studied. We do not recommend TRF tests on all children being treated for primary hypothyroidism, but suggest that TRF may be a useful tool to better define the range of physiologic replacement doses of thyroid hormone.

\begin{tabular}{|c|c|c|c|c|c|}
\hline $\begin{array}{c}\text { TRF RESPONSE } \\
\text { (Max. TSH in } \mathrm{uU} / \mathrm{ml} \text { ) }\end{array}$ & $\begin{array}{l}\text { NO. } \\
\text { TESTS }\end{array}$ & $\begin{array}{l}\text { BASAL TSH** } \\
(<10 \mathrm{UU} / \mathrm{ml}) \dagger\end{array}$ & $\begin{array}{r}T_{2} D^{* *}{ }^{A V G} . \\
(4.6-10.7 u g \%)+\end{array}$ & $\begin{array}{c}\mathrm{DOSE}_{\mathrm{Hg}} \mathrm{g} \\
\mathrm{kg}\end{array}$ & $:{ }_{\mathrm{m}}^{{ }^{1 \mathrm{~T}}}$ \\
\hline Normal $(>14)$ & 2 & $3.0 \pm 0.4$ & $7.5 \pm 0.2$ & 3.5 & 110 \\
\hline$a I(<4)$ & 3 & $1.2 \pm 0.2$ & $8.1 \pm 1.1$ & 4.5 & 142 \\
\hline Absent $(<1)$ & 4 & -7 & $10.2+0.2$ & 6.2 & 175 \\
\hline
\end{tabular}

ENDOCRINE FUNCTION IN CYSTINOSIS. Anne W. Lucky

241 Klara Megyesi, Peter M. Howley and Joseph D. Schulman, N.I.H., Bethesda, Maryland.

The severe growth failure long preceding renal insufficiency in cystinosis remains largely unexplained. Hypothyroidism has been noted in some cases. We have studied endocrine function in 4 patients ages 2 to 9 and reviewed autopsies of 4 cases ages 3-13 with classical nephropathic cystinosis. All subjects manifest severe growth retardation. The patients had normal peripheral thyroid indices $\left(\mathrm{T}_{3} \mathrm{RIA}, \mathrm{T}_{4} \mathrm{D}\right.$, and $\left.\mathrm{FT}_{4}\right)$, but 2 of them had elevated serum TSH. In one, an exaggerated response to thyrotropin releasing hormone, from a basal TSH of $33 \mathrm{uU} / \mathrm{ml}$ to a maximum of $103 \mathrm{uU} / \mathrm{ml}$, was documented as additional evidence of compensated thyroid failure. Examination of thyroid glands at autopsy showed cystine crystals in follicular epithelium and interstitium. Despite numerous cystine crystals in the adrenal cortices of the autopsy cases, the 4 patients had normal a.m. plasma cortisols, normal 24 hour excretions of urinary $170 \mathrm{H}$ and I7KS, and appropriate increases in plasma cortisol upon stimulation with intravenous ACTH. Growth hormone responses to sleep, exercise or l-DOPA were normal, >IOng/ml, in all patients. Preliminary studies of serum NSILA-s, a somatomedin-like substance, showed overlap with age-matched controls. We conclude that in cystinosis: (1) compensated primary thyrojd failure may occur early and be diagnosed by measurements of serum TSH, (2) despite numerous cystine crystals, adrenal cortical function is well preserved, and (3) growth failure cannot be attributed to lack of growth hormone.
242

GROWTH: HORMONE AND SOMATOMEDIN DURING FAILURE OF CATCH-UP GROIITH AFTER EXPERIMENTAL HYPOTHYROIDISH II RATS. H. David Mosier, Jr., Regina A. Jansons, and Rosemary Rae Hill. Department of Pediatrics, University of CaTi. fornia, Irvine.

After experimental hypothyroidism in rats recovery may be accompanied by incomplete catch-up growth (Pediatr Res 5:51, 1971). This study, was carried out to determine whether prolonged disturbance of pituitary GH and/or somatomedin (S!I) accounts for the incomplete catch-up. Imnunoassayable GH in the pituitary and serum and SII activity (porcine cartilage bioassay) in serum vere measured at the end of 17 days of PTU-feeding, starting at 57 days of age, and for 14 days after resumption of normal diet. Serum. immunoassayable thyroxine concentration dropped significantly during PTU-feeding but was normal by 7 days of recovery. Grow'th rates decreased during PTU feeding and increased during recovery; but cathc-up growth did not occur with tody weight and only partially occurred with tail length. Pituitary GH content and concentration dropped during PTU-feeding; by recovery day 14 there was significant but not complete repletion of the gland. Serum GH rose slightly during PTU-feeding and was more variable than in controls during recovery. Serum SM activity dropped significantly during PTU-feeding but returned to normal by recovery day 14 . Thus, while GH and SH functions were disturbed during hypothyroidism, prompt return to normal function occurred in recovery. Return to normal GH and SM function does not assure catch-up growth acceleration. Other factors, including alterations of chondrocytes and cartilage matrix, may be responsible for the failure of catchup growth.

24 PROPAGATION OF HUMAN GROWTH HORMONE PRODUCINC

243 PITUITARY CELls. Mandayam J. Narasimhan and John A. Anderson, Dept. of Pediatrics, School of Medicine, University of Minnesota, Minneapolis, Minnesota.

Utilizing an improved culture medium and a modified culture procedure human anterior pituitary growth hormone producing cells were propagated serially in suspended cell cultures and continued to produce large amounts of immune reacting human growth hormone, $\mathrm{hGH}$, for a prolonged period. An initial tissue inoculum (about $1 / 3$ of an anterior pituitary gland) was grown in serial culture with $1 / 2$ volume transfers every 8 days for 32 days. The following average concentrations of hGH in $\mathrm{ng} / \mathrm{ml}$ found in the medium were: at 8 days 21,166 ; at 16 days 18,666 ; at 24 days 21,750 ; and at 32 days 20,000 . Each culture flask contained $30 \mathrm{ml}$ and hence because of one-half dilutions and volume reconstitution at sub-culture times, the total amount of hGH produced at each subculture time was as follows: 8 days $5.01 \mathrm{mg}$ in 6 flasks; at 16 days $6.7 \mathrm{mg}$ in 12 flasks; at 24 days $15.6 \mathrm{mg}$ in 24 flasks; and, by 32 days $28 \mathrm{mg}$ in 48 flasks. Cultures have now been grown for two months and additional hGH analysis of medium concentrations are in progress. Preliminary characterization studies utilizing a sephadex gel filtration procedure indicate that $80-90 \%$ of the immune reacting hGH is a monomer. Ce1ls were epithelial type, 10-14 u in diameter, contained chromatin granules and a large highly chromatic nucleus. Mitosis was present. The procedure offers a possibility for the production of large amounts of hGH from small amounts of human pituitary gland.

LOW RENIN HYPERTENSION AND HYPOKALEMIA IN A 3-YEAR-OLD 24.4 GIRL WITH DEFICIENCY OF ALL KNOWN STEROIDS. M.I.NeW, P.Saenger, M.Novogroder, L.S.Levine, and S.Ulick, Cornell Univ.Med.Col., NewYork, V A Hosp. Bronx, N.Y.

Low renin hypertension and hypokalemic alkalosis was observed in a 3-year-old girl whose secretion of all known steroidal hormones including aldosterone and cortisol was low or absent. The patient survived salmonella sepsis without glucocorticoid treatment. Both plasma renin activity and ACTH levels were suppressed and inappropriately low for the deficient secretion of aldo and cortisol. Renin was not stimulated by salt depletion. ACTH administration raised blood pressure and aggravated the hypokalemia. Cortisol secretion increased sluggishly to prolonged ACTH administration but the resulting urinary steroid pattern was normal as judged by gas chromatographic/mass spectroscopic analysis. Metabolism of tracer doses of hormones was normal. Prolonged metyrapone administration which raised $A C T H$ level was associated with normalization of blood pressure, indicating inhibition of a steroid which was causing hypertension and suppressing ACTH. These findings suggest the secretion of an unknown life-sustaining adrenocortical factor stimulable by ACTH which is both a glucocorticoid and mineralocorticoid, suppressing both $\mathrm{ACTH}$ and renin. A biologically active factor with potent salt xetaining activity was localized to the urinary sulfate-conjugated steroid faction. This factor is being purified. It is not $15 \beta$-hydroxydehydroisoandrosterone, a steroid that has been implicated in adult low renin hypertension. Structural characterization of the ACTH stimulabje factor may have significance in the general problem of human hypertension. 
- DEXAMETHASONE BLOOD LEVELS IN PREGNANT AND NONPREGNANT ADULTS AND NEWBORN INFANTS. Rapin Osathanondh, Dan Tulchinsky, Homa Kamali, Montserrat deM. Fencl, and $\mathrm{H}$. William Taeusch, Jr., Depts. of Pediatrics and Obstetrics and Gynecology, Harvard Medical School, and the Boston Hospital for Women, Boston, Massachusetts 02115 .

Plasma dexamethasone (DX) concentration was measured by radioimmunoassay (RIA) following paper chromatography (Steroids 22: 193, 1973). Recovery averaged $70 \pm 10$ (SE)\%. The intra-assay coefficient of variation was less than $15 \%$ and the lower limit of sensitivity was $0.2 \mu \mathrm{g} / 100 \mathrm{ml}$ of plasma. Highest blood levels $(5.3 \pm 3[\mathrm{SE}] \mu \mathrm{g} / 100 \mathrm{ml})$ were observed 30 minutes after an intramuscular administration of $4 \mathrm{mg}$ DX phosphate to 4 normal adults and a $40 \%$ fall of DX levels was observed in the next 90 minutes. Eight to eleven hours following an oral administration of $8 \mathrm{mg}$ DX to 5 normal term pregnant women, DX blood levels at elective repeat cesarean section were $2.2 \pm 0.7,2.9 \pm 0.7$, and $2.6 \pm 0.8 \mathrm{\mu g} /$ $100 \mathrm{ml}$ in maternal peripheral vein, umbilical vein, and umbilical artery, respectively. Umbilical artery cortisol levels of patients receiving DX were less than $10 \%$ of untreated patients, indicating complete fetal adrenal suppression. The data demonstrate that this dose of DX provides levels that result in fetal glucocorticoid activity well above physiologic levels.
248 LUTEINIZING HORMONE (LH) AND FOLLICLE STIMULATING HORMONE (FSH) EXCRETION IN TIMED OVERNIGHT URINE COLLECTIONS: NORMAL MALE CHILDREN AND ADULTS. Robert Penny, Irwin P. Goldstein, S. Douglas Frasier. Univ. So. Calif. Sch. Med., LA County-USC Medical Ctr., Dept. Pediatr., Los Angeles.

Utilizing acetone precipitates of timed overnight urine collections extrapolated to 24 hours and radioimmunoassay, LH and FSH urinary excretion was determined in 147 males $3.0-16.9$ yrs and 20 normal adult males. LH and FSH progressively increased with age from $2.1 \pm 1.2$ (LH) and $1.8 \pm 1.1$ (FSH) IU/24 hrs (2nd IRP-HMG) for the $3.0-4.9 \mathrm{yr}$ age group to $18.8 \pm 8.0(\mathrm{LH})$ and $8.6+3.2$ (FSH) IU/24 hrs for the 15.0-16.9 yr age group. Mean LH and FSH excretion for succeeding age groups $(3.0-4.9,5.1$ $6.8,7.0-8.8,9.0-10.8,11.2-12.9,13.0-14.9$ and $15.0-16.9$ yrs) increased significantly $(p<0.01)$ for the $11.2-12.9,13.0-14.9$ and 15.0-16.9 yr age groups. Mean FSH $(9.3 \pm 3.4 \mathrm{IU} / 24 \mathrm{hrs})$ excretion of adults was not significantly $(p>0.3)$ different from that of the 15.0-16.9 yr age group. In contrast, mean LH $(14.5+5.7$ IU/24 hrs) excretion of adults was significantly $(p<0 . \overline{0} 5)$ less than that of the 15.0-16.9 yr age group. LH and FSH excretion increased progressively with stage of sexual development.

The results are comparable to those reported for 24 hour urines in normal males. Mean LH excretion greater in 15.0 $16.9 \mathrm{yr}$ males than in adult males is consistent with the reported increase in gonadotropin secretion during sleep that occurs prior to achieving the adult state of sexual maturation.

\section{THE EFFECTS OF ADRENAL SEX STEROIDS ON FEEDBACK 246 CONTROL OF LH AND FSH SECRETION IN PATIENTS WITH} CONGENITAL VIRILIZING ADRENAL HYPERPLASIA (CVAH). Songja Pang, Julane Hotchkiss, Thomas Foley, Allan Drash, David Archer, and Frederic M. Kenny. Univ. of Pittsburgh, Sch. of Med., Depts. of Ped., Physiology and Ob-Gyn. Pittsburgh, PA.

The negative(-) and positive(+) feedback control of gonadotropin(GTP) secretion was examined in two $16-1 / 2$ yr old females with CVAH. Pt 1 was an untreated subject ovariectomized at 12 yrs; Pt 2 was diagnosed at birth, poorly controlled and amenorrheic. Serum progesterone(P), estradiol(E), testosterone(T), FSH and LH were measured by RIA $q$ 30-120 min for $24 \mathrm{hrs}$ and at 0800 and 2000 hrs during the study. After a 2-day control period, LH and FSH response to synthetic LHRH was examined on day 3. To determine the presence of a functional - and + GTP feedback system, estradiol benzoate (EB, $12 \mathrm{ug} / \mathrm{kg} / \mathrm{d} \mathrm{im)} \mathrm{was} \mathrm{given} \mathrm{on} \mathrm{days} 4-6$. In both pts $\mathrm{LH}$ and FSH increased in response to LHRH. The steroid levels in $\frac{\mathrm{Pt} 17 \mathrm{KS}(\mathrm{mg} / \mathrm{d}) \mathrm{E}(\mathrm{pg} / \mathrm{ml}) \mathrm{P}(\mathrm{ng} / \mathrm{ml}) \mathrm{T}(\mathrm{ng} / \mathrm{ml}) \mathrm{LH}(\mathrm{ng} / \mathrm{ml}) \text { basal EB } \mathrm{rx} 24 \mathrm{~h} / 72 \mathrm{~h}}{\mathrm{P}}$

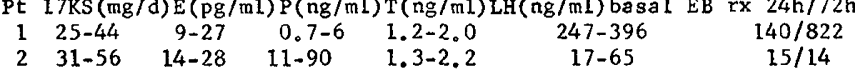
Norma $15-15 \quad 30-150 \quad 1-10 \quad<.6 \quad 25-167$ these pts are similar except for the high levels of $\mathrm{P}$ in pt $2 . \mathrm{Ba}-$ sal LH levels in pt 1 are lower than those of agematched pts with sal LH levels in pt 1 are lower than those of agematched pts with in midpubertal subjects. EB $r x$ induced a -feedback suppression of LH in both pts and a subsequent surge in pt 1 only. CoNCLUSIONS: 1) Excess androgen will partially suppress LH secretion but does not prevent the maturation of the + feedback mechanism;

2) Excessive $P$ may inhibit the + feedback response of $\mathrm{LH}$ to $\mathrm{E}$.

LUTEINIZING HORMONE (LH) AND FOLLICLE STIMULATING 247 HORMONE (FSH) EXCRETION IN TIMED OVERNIGHT URINE COLLECTIONS: NORMAL FEMALE CHILDREN AND ADULTS Robert Penny, Irwin P. Goldstein, S. Douglas Frasier. Univ. So. Calif. Sch. Med., LA County-USC Med. Ctr., Dept. Pediatrics, Los Angeles.

Utilizing acetone precipitates of timed overnight urine collections extrapolated to 24 hours and radioimmunoassay, $\mathrm{LH}$ and FSH urinary excretion was determined in 140 normal females $3.0-$ 16.9 yrs. Timed overnight urines were collected throughout a menstrual cycle from 3 normal adult females. LH and FSH progressively increased with age from $2.0 \pm 0.4$ (LH) and $2.0 \pm$ 0.5 (FSH) IU/24 hrs (2nd IRP-HMG) for the 3.0-4.9 yr age group to $11.5 \pm 3.8(\mathrm{LH})$ and $8.1 \pm 1.8$ (FSH) IU/24 hrs for the 15.3$16.9 \mathrm{yr}$ age group. Mean LH and FSH excretion for succeeding age groups $(3.0-4.9,5.0-6.9,7.1-8.7,9.0-10.9,11.0-12.9$, $13.0-14.9$ and $15.3-16.9$ yrs) increased significantly ( $p<0.01$; $p<0.025$ ) for the $5.0-6.9,9.0-10.9$ and $11.0-12.9$ yr age groups. Mean LH but not FSH excretion increased significantly $(p<0.025)$ for the 13.0-14.9 yr age group. LH and FSH excretion increased progressively with stage of sexual development. In the 3 adult menstrual cycles, LH (173.7 $\pm 7.8 \mathrm{IU} / 24 \mathrm{hrs})$ and FSH $(43.6 \pm$ $5.0 \mathrm{IU} / 24 \mathrm{hrs}$ ) peaks occurred on the same day. Luteal phase excretion of LH and FSH was lower than follicular phase excretion. Timed overnight urine collection allows integration of several hours of LH and FSH secretion. It provides a noninvasive method for the evaluation of changing gonadotropin levels associated with sexual maturation. 249 CORRELATION OF LUTEINIZING HORMONE (LH) AND FOLLICLE AND BODY FAT (BF). Robert Penny, Irwin P. Goldstein,
Frasier. Univ. So. Calif. Sch. Med., LA County-USC S. Douglas Frasier. Univ. So. Calif.

The urinary excretion of LH and FSH was determined in 140 normal females (3.6-16.9 yrs) and 142 normal males (3.0-16.9 yrs) utilizing acetone precipitates of timed overnight urine collections extrapolated to $24 \mathrm{hrs}$ and radioimmunoassay. TBW and BF were calculated using the formulas of Mellits and Cheek. An increase in $\mathrm{LH}(4.8 \pm 2.8$ vs $3.0 \pm 1.0 \mathrm{IU} / 24 \mathrm{hrs} ; \mathrm{p}<0.01)$ and FSH $(4.0 \pm 1.9$ vs $2.9 \pm 1.0 \mathrm{IU} / 24 \mathrm{hrs} ; \mathrm{p}<0.025)$ excretion occurred in $10.0 \pm 0.6 \mathrm{yr}$ females compared to $7.9 \pm 0.5 \mathrm{yr}$ females. A decrease in mean TBW $(55.2 \pm 7.4$ vs $59.3 \pm 7.6 ; \mathrm{p}<0.05)$ and an increase in mean BF $(23.2 \pm 10.7$ vs $17.4 \pm 4.0 ; p<0.01)$ as $\%$ of body weight occurred in females at $12.0 \pm 0.6$ yrs compared to $10.0 \pm 0.6$ yrs. At the time of initial testicular enlargement in males $(11.7 \pm 1.3 \mathrm{yrs}) \mathrm{LH}(7.3 \pm 2.9 \mathrm{vs} 2.9 \pm 1.7 \mathrm{IU} / 24 \mathrm{hrs}$; $p<0.01)$ and FSH $(4.1 \pm 1.9$ vs $2.3 \pm 1.0$ IU/24 hrs; $p<0.01)$ excretion was higher, $\mathrm{BF}(12.2 \pm 2.3$ vs $16.2 \pm 4.6 ; \mathrm{p}<0.005)$ as $\%$ body weight was lower and TBW $(63.3 \pm 10.1$ vs $60.2 \pm 10.4)$ as $\%$ body weight was unchanged compared to prepubertal boys. Significant $(p<0.005)$ correlation coefficients (LH, females $R=0.78$; males $R=0.83$. FSH, females $R=0.75$; males, $R=0.77$ ) were found for the plot of the log of LH and FSH urinary excretion against TBW. The data is compatible with an inter-relationship between changes in hormone secretion and changes in body composition during sexual maturation.

INTEGRATED CONCENTRATIONS OF GROWTH HORMONE: 250 A PHYSIOLOGIC MEASUREMENT FOR THE DIAGNOSIS O HYPOPITUITARISM AND PARTIA L HYPOPITUITARISM. Leslie P. Plotnick, Robert J. Winter, Peter A. Lee, $\frac{\text { Claude J. Migeon, }}{\text { and A Avinoam Kowarski Department of Pediatrics }}$ University, School of Medicine, Baltimore, Maryland.

Integrated concentrations of growth hormone (ICGH) have previously been determined on consecutive 30 -min plasma samples collected with a constant withdrawal pump over 24 -hrs in 8 normal children (JCEM 40: 240, 1975). Eight patients with evidence of hypopituitarism have been studied using the same technique and compared with normals. Three of these patients had low mean ICGH $(2.2-3.6 \mathrm{ng} / \mathrm{ml})$. The pattern was characterized by flatness and no peak was above 7.5. These patients had subnormal responses to arginine-insulin tolerance tests (AITT) and A ITT with epinephrine and propranolol (A ITT-EP) and a good growth rate response to exogenous GH administration. Only one patient had organic hypopituitarism (post-op craniopharyngioma). His mean integrated concentration was 6.2 with individual values falling between 4.3 and 8.9. He had a very subnormal response to AITT and AITT-EP.

The other four patients had ICGH patterns and levels different than those of the previous four patients. Three of these patients had 2 peaks $>10$ and IC from 4.8 to 6.4 . The fourth had 5 peaks $>10$ and an IC of 11.9 . While all four had borderline responses to AITT and AITT-EP, the fourth patient had the greatest response. Based on ICGH, the fourth patient is indistinguishable from normal children.

Hence, the ICGH mean values and peak patterms give more precise information than AITT or AITT-EP. Because it reflects actual physiologic parameters, it may be a more refined index for diagnosing hypopituitarism and selecting those who might benefit from exogenous GH. 
251 E:!DOGEFIOUS LH SUPPRESSION ANID BILATERAL TESTICULAR TIMORS IN CONGEUITAL ADRENAL HYPERPLASIA (CAH) llezam Padfar, Frederic C. Bartter and Richard J. Sherins (Spon. by PauT $\lambda$. di Sant'Aanese), MIH, Bethesda, Md. 20014

A 19 year old male with CAN and bilateral testicular tumors present for one year was evaluated durino a basal period when he received cortisol (F), $25 \mathrm{ma} /$ day and during a neriod when he also received Dexamethasone, $0.5 \mathrm{ma} \mathrm{a} 6 \mathrm{hr}$. Blood was obtained at $30-$ min. intervals for 20 hrs before and after ACTH suppression.

Durina basal studies mean $17 \mathrm{nH}$ nronesterone (170HP) was 30fold normal, while plasma $F$, testosterone $(T)$, Estradiol $\left(E_{2}\right)$ were normal. Pulsatile fluctuations of plasma $T, E$ and $770 H P$ occurred at 90 min. intervals in AM and PM. By contrast. periodic pulses of LH were present in AM but absent in PM. Durinn ACTH suppression mean plasma $170 \mathrm{HP}$ fell to $2 \mathrm{ng} / \mathrm{ml}$. Mean PM plasma $T$ and $E_{2}$ decreased $25 \%$ while mean $L H$ levels increased 4-fold and periodic LII release returned. Testis size decreased durina $A C T H$ suppression and re-enlarqed after ACTH administration. Spermatic vein $F(14.3 \mu \mathrm{n} / \mathrm{d} 1)$ was 3 -fold the simultaneous peripheral value $(5.2)$. Microscopically the tumor cells resembled "Leydia cells" but had no Reinke crystals. The normal testicular tissue revealed maturation arrest of aerm cells and Leydia cells contained Reinke crystals.

These data suagest that in CAH: 1) "Leydig cell" tumors may secrete cortisol and be ACTH-dependent; 2) endonenous suppression of LH may occur if ACTH suppression is inadequate.

25 EFFECT OF CONSTANT INFUSION OF GONADUTROPIN RELEASING 252 HORMONE (Gn-RH) UPON EXCRETION OF LH AND FSH IN CHILDREN. E. Reiter, G. Duckett, and A. Root, Univ. So. Fla., All Children's Hosp., Dept. Ped., St. Petersburg, Florida. The pituitary gonadotropin secretory capacity has been examined by measuring the excretion of immunoreactive LH and FSH in $3 \mathrm{hr}$ urine specimens collected prior to, during, and after the constant infusion of $100 \mathrm{\mu g}$ synthetic Gn-RH to 9 normal prepubertal (PRE), 12 pubertal (PUB) and 5 hyposomatotropic (HS) children:

\begin{tabular}{lllll} 
& & \multicolumn{1}{c}{ Control } & Infusion & Post-infusion \\
\cline { 3 - 5 } Prepubertal & LH* & $0.11 \pm 0.02(\mathrm{SE})$ & $0.62 \pm 0.16$ & $0.68 \pm 0.31$ \\
Pubertal & & $0.82 \pm 0.14$ & $2.91 \pm 0.61$ & $5.08 \pm 1.38$ \\
Prepubertal & \multirow{2}{*}{ FSH* } & $0.41 \pm 0.15$ & $3.79 \pm 0.54$ & $2.25 \pm 0.56$ \\
Pubertal & & $1.81 \pm 0.15$ & $2.39 \pm 0.45$ & $4.87 \pm 0.50$
\end{tabular}

* (IU, IRP-2-hMG/3 hr)

The peak urinary LH in PUB $(5.7 \pm 1.3)$ was greater $(P<0.005)$ than in PRE $(1.0 \pm 0.4)$. There was no difference in maximal FSH excretion between PUB and PRE. In HS peak LH was $0.42 \pm 0.17$; peak FSH was $0.61 \pm 0.15$. The mean maximal percentage increment in LH was: PRE $(796 \%)$, PUB $(718 \%)$, HS $(61 \%)$; and in FSH: PRE $(2712 \%)$, PUB $(328 \%)$, HS $(67 \%)$. Conclusions: 1) There is a maturityrelated increase in Gn-RH-evoked LH but not FSH release as quantified by urinary gonadotropin excretion. 2) Comparable mean percentage rises of $\mathrm{LH}$ in PRE and PUB suggest that gonadotrope sensitivity, though not capacity, may be similar; 3) The increment in urinary $\mathrm{LH}$ after $\mathrm{Gn}-\mathrm{RH}$ in $\mathrm{PRE}$ is greater than in serum (9X vs. $3 \mathrm{X}$ ) ; 4) Determination of releasable pituitary LH by measurement of urinary $\mathrm{LH}$ excretion may permit separation of prepubertal from hypogonadotropic subjects.

POSTNATAL CHANGES IN SERUM TOTAL ESTRIOL AND SERUM

253 DEHYDROEPIANDROSTERONE SULFATE (DHAS) IN ABNORMAL NEWBORN INFANTS. John $W$. Reynolds, Karen Bentley and Marvin R. Turnipseed. University of Minnesota Medical School, Department of Pediatrics, Minneapolis.

Serum total estriol and DHAS were measured by radioimmunoassay in 131 newborn infants. Serial sampling was carried out in 40 infants. The infants were in an NICU for prematurity, RDS, intrauterine growth retardation (IUGR) and postmaturity. Mean DHAS values on day 1 of life were similar in all gestational age groups, ranging from 4486-6461 ng/m1. Mean DHAS levels did not decline over the first $3 \mathrm{wk}$, and individual infants showed increases associated with severe clinical stress. IUGR and postmature infants $>35$ wk gestation had significantly lower ( $p<.001$ ) DHAS levels on day 1 than normal controls. Mean total estriol levels on day 1 of life were similar in all gestational groups, ranging from $996-1071 \mathrm{ng} / \mathrm{ml}$. The postnatal $t 1 / 2$ of total estriol in 21 infants ranged from 0.6-3.6 days and was negatively correlated with gestational age. Four small premature infants, all with severe intestinal hypomotility and later necrotizing enterocolitis, had prolonged high total estriol levels during the first 2 wk.

High postnatal DHAS levels indicate persistance of fetal pattern of prominent $\triangle 5-3 \beta-0 H$ steroid secretion, and lower levels in IUGR infants suggests that the fetal zone of the adrenal is the major source. Pexsistent high total estriol levels in small prematures with intestinal hypomotility suggests that enterohepatic circulation of the estriol in meconium may contribute to circulating leve1s.
DELAYED ONSET OF HYPOPITUITARISM: SEQUELA OF THERAPEUTIC IRRADIATION TO TUMORS OF THE EYE, MIDDLE EAR, AND CENTRAL NERVOUS SYSTEM. Gail E. Richards, William M. Wara, Melvin M. Grumbach, Selna L. Kaplan, Glenn E. She line Califormia San Francisco, San Francisco, California 94143.

Seven children who received irradiation to the head in conventional doses for tumors not involving the hypothalamus or pituitary had clinical and laboratory evidence of hormonal deficiencies several years after treatment; 6 had significant height retardation ( -1.7 to -4.8 S.D.). One patient who was evaluated 1 year after irradiation was at the mean height for age. Growth hormone deficiency was documented in all by lack of response to insulin hypoglycemia, arginine, and/or L-dopa. ACTH function was evaluated by plasma cortisol response to insulin hypoglycemia in 6;4 had subnormal responses. Plasma gonadotropins were measured after LRF in 6 patients; only 1 had an abnormal response for age and stage of sexual maturation. Of 5 patients tested with TRF, 4 had a normal rise in plasma TSH and prolactin. The fifth patient, whose thyroid was included in the irradiation field, had an elevated piasma TSH and exaggerated TSH response to TRF, suggesting primary hypothyroidism in spite of normal plasma thyroxine and normal prolactin response to TRF. Since hypothalamic-pituitary deficiencies may occur insidiously over many years, careful follow up of patients who have received irradiation to the head is necessary. Periodic tests of hypothalamic-pituitary function should be performed in children who have had irradiation to the head in order to detect and treat hormonal deficiencies before growth and development are seriously compromised.

1,25-DIHYDROXYVITAMIN $\mathrm{D}_{3}\left\{1,25-(\mathrm{OH}) \mathrm{D}_{3}\right\}$ : ITS USE IN THE

255 LONG-TERM MANAGEMENT OF IDIOPATHIC HYPOPARATHYROIDISM (IHP) IN CHILDREN. John F. Rosen, Alan R. Fleischman, Laurence Finberg, Hector DeLuca, A1bert Einstein College of Medicin Montefiore Hospital \& Medical Center, Dept. Ped., New York; and Univ. of Wisconsin, Dept. of Biochem., Madison, Wisconsin.

Since parathyroid hormone(PTH)plays a central role in regulating the synthesis of $1,25-(\mathrm{OH})_{2} \mathrm{D}_{3}$, the production of $1,25-(\mathrm{OH})_{2} \mathrm{D}_{3}$ is reduced in the absence of PTH. By managing IHP with subphysiologic doses of $1,25-(\mathrm{OH})_{2} \mathrm{D}_{3}$ given orally, one can increase the intestinal absorption of calcium(Ca) and restore serum levels of $\mathrm{Ca}$ to normal. Three children with previously untreated and undiagnosed IHP have been managed in this manner for 1050 treatment days.

Two 4-year-old children achieved and maintained normal serum levels of total and ionized $\mathrm{Ca}$ after $1-4$ days on $0.03 \mu \mathrm{g} / \mathrm{kg} / \mathrm{d}$ of $1,25-(\mathrm{OH})_{2} \mathrm{D}_{3}$. While the hormone was continued, each child was placed on a low $\mathrm{Ca}$ diet: the total serum $\mathrm{Ca}$ fell by 0.75 to $1.0 \mathrm{mg} / \mathrm{dl}$ within 2 days.During 800 treatment days, both children have remained normocalcemic on a regular diet. The third child needed phenobarbital before and during treatment, responded rapidly, but required $0.16 \mu \mathrm{g} / \mathrm{Kg} / \mathrm{d}$ of $1,25-(\mathrm{OH})_{2} \mathrm{D}_{3}$. During 250 days of therapy, 4 brief episodes of hypercalcemia (total serum $\mathrm{Ca}: 10.5-11.7 \mathrm{mg} / \mathrm{d} 1$ ) occurred. On each occasion, $1,25-(\mathrm{OH})_{2} \mathrm{D}_{3}$ was discontinued for 2 days, with prompt return to normocalcemia, and the dose was decreased by $.03 \mu \mathrm{g}$ $\mathrm{Kg} / \mathrm{d}$. This infant is now normocalcemic on $0.04 \mu \mathrm{g} / \mathrm{Kg} / \mathrm{d}$ of the hormone.

This approach avoids the use of large doses of long-acting vitamin $D$ analogues. Subphysiologic doses of $1,25-(\mathrm{OH})_{2} \mathrm{D}_{3}$, given ora1ly, present considerable advantages in the long-term management of IHP.

256 NEW DATA DESCRIBING TISSUE TESTOSTERONE $5 \alpha$-REDUCTASE DEFICIENCY AND DIHYDROTESTOSTERONE BINDING IN MALE PSEUDOHERMAPHRODITES (MPH) . P.Saenger, A.S.Goldman, L.S.Levine, S. Korth-Schutz, Y.Doberne, M. Katsumata,M. I. New, Corne 11 Univ.Med.Col., New York, Childrens Hosp. Phila.

The diagnosis of $5 \alpha$-reductase deficiency was proven in two prepubertal MPH of Italian descent. Both had 46XY karyotype and were reared as females; one child had been castrated in infancy. Clitoromegaly,urogenital sinus and a short vaginal pouch were present in both, inguinal gonads were palpable in one.Endocrine findings supporting the diagnosis were: (a) testosterone/dihydrotestosterone (T/DHT) ratio in excess of 35 after HCG stimulation, (b) ratio of $5 \alpha /$ $5 \mathrm{~B}$ metabolites of testosterone in urine decreased to 0.12 and 0.17 (nI for age $>2$ ), (c) conversion of $T$ to $D H T$ during constant $3 \mathrm{H}-\mathrm{T}$ infusion decreased to 0.3 and 0.48 ( $\mathrm{nl} 2.7-10 \%$ ), (d) conversion of ${ }^{14} \mathrm{C}-\mathrm{T}$ to 5 areduced metabolites by skin fibroblasts from nongenital skin decreased to 58 ( $\mathrm{nl}$ 80-908), (e) incubation of slices of sex skin and testes with ${ }^{14} \mathrm{C}-\mathrm{T}$ demonstrated for the first time a deficiency of 5oreductase; conversion of $T$ to DHT was reduced to 0.28 , as compared to 218 in a nonaffected child, (f) despite deficient tissue DHT formation binding of $3 \mathrm{H}-\mathrm{DHT}$ to cytosol of skin fibroblasts in one patient was $4.2 \mathrm{dpm} / \mathrm{ug}$ DNA (nl 3.7) and higher than that observed in 2 patients with testicular feminization (TF) $(0.7$ and $1.0 \mathrm{dpm} / \mathrm{ug} \mathrm{DNA})$.

In conclusion these studies show that this form of MPH is due to deficient tissue 5areductase activity and DHT formation. DHT binding is normal in contrast to TF. Since peripheral metabolism is defective, this disorder can be documented in the castrate. 
27 KLINEFELTER'S SYNDROME (47XXY) WITH ABSENCE OF GROWTH HORMONE. Annemarie Sommer and Carolyn Romshe. Sponsored by Stella B. Kontras. Ohio State University College of Med., Children's Hospital, Dept. of Peds., Columbus.

Klinefelter's syndrome (47XXY) is characterized by males with incomplete development of secondary sexual characteristics, eunuchoid body proportions, gynecomastia, small testes and increased incidence of antisocial behavior. Childhood features of value in clinical detection are dull mentality, small testicular size, small phallus, body habitus of long legs with decreased upper-tolower segment ratio and slim build. Although an increased incidence of diabetes mellitus, mild hyperlipidemia and mild hypercholesterolemia have been found in males with Klinefelter's, cases associated with growth hormone deficiency have not been reported. A 12 year old boy was evaluated because of short stature. Diagnosis of Klinefelter's syndrome was made at 3 years because of signs of hypogonadism. Because of the diagnosis, he received only routine care and his short stature was not evaluated until the patient voiced his concerns. When first seen, this boy was an immature young man with a high pitched voice. He was $112 \mathrm{~cm}$ tall and weighed $17.2 \mathrm{Kg}$. Intelligence was normal. The cytogenetic study showed a 47XXY karyotype. Endocrinological evaluation revealed complete absence of growth hormone with no response to arginine and L-Dopa. The bone age was that of a 3-4 year old male. Growth hormone therapy is planned. The association of Klinefe1ter's syndrome with absence of growth hormone has not previously been reported. Although cytogenetic diagnosis was made early, the second diagnosis was delayed until growth parameters very differ-
ent from those expected became apparent.

258 GROWTH SUPPRESSTON IN TRFATED CONGENTTAL ADRENAL HYPERPLASIA (CAH). Dennis M. Styne, Selna L. Kaplan, and Melvin M. Grumbach. Dept. Pediat., University of California San Francisco, San Francisco, California 94143. Glucocorticoid replacement therapy can suppress linear growth in children with $\mathrm{CAH}$ even if used in doses recommended for control of virilization. Previous reports suggest discrepancies in comparative potency between steroid growth effect (GE) and antiinflammatory effect (AIE). We determined the dose-response of cortisone acetate $(E)$, hydrocortisone $(F)$, prednisone $(P), 6 x_{-}$ methylprednisolone (MP), and dexamethasone (D) on growth in patients with 21-OH deficiency. Height velocities of 39 children treated for a mean period of 11.8 years were correlated with dose and type of steroid. The estimated optimal dose (EOD) associated with normal growth was determined for each steroid. Relative $\mathrm{GE}$ and AID using the EOD of $\mathrm{E}$ as a basis with a value of 1 were calculated. While there were striking individual differences, average doses were:

DRUG

\begin{tabular}{lccc} 
DRUG & EOD $\left(\mathrm{mg} / \mathrm{m}^{2} / \mathrm{d}\right)$ & Rel. GE & Rel. AIE \\
\hline Oral cortisone acetate (E) & 22 & 1.6 & 1 \\
IM cortisone acetate (E) & 14 & 1.6 & 1.9 \\
Hydrocortisone (F) & 18.4 & 1.2 & 1.2 \\
Prednisone (P) & 3.7 & 6 & 4.7 \\
6x-Methylprednisolone (MP) & 2.4 & 9 & 6.6 \\
Dexamethasone (D) & .23 & 96 & 33
\end{tabular}

Dexamethasone (D)

96

The growth effects of $P$ and MP were 1.5 and of D 3 times greater than their anti-inflammatory effects. An appreciation of these relationships is crucial in treatment of $\mathrm{CAH}$ if optimal growth is to be ensured while controling virilization.

CUSHING'S SYNDROME (CD) IN INFANCY: CLINICAL 259 VARIABILITY AND THERAPY INCLUDING ADRENAL AUTOTRANSPLANTATION (AA): Shiu-Ching Tang, $\frac{\text { Kadan Sau, and Theodore W. AvRuskin. New York Univ. }}{\text { Sch. Med., The Brookdale Hosp. Med. Ctr., Dept. of Ped. }}$ Sch. Med., The
Brooklyn, N.Y.

Cushing's Syndrome(CS) is rare in infants and therapy is difficult. A 2-month old girl had hypertension, acne, c11toromegaly, advanced bone maturation and osteoporosis Diurnal cortisols $(F, \mu g / 100 \mathrm{ml})$ were elevated:8AM, $31.1 \pm 3$, $M \pm S E$ (controls: $9.1 \pm 2.6, \mathrm{p}<0.001) ; 4 \mathrm{PM}, 30.2 \pm 4.8$ (controls : $10.3 \pm 1.8, \mathrm{p}<0.002)$. IM ACTH gel $\left(20 U^{2}\right.$ bid): Control F 35.6 ; peak F 236; IM Cortrosyn(0.125 and $0.25 \mathrm{mg}$ respectively) Contro1 F 23.2,20.6; peak F:54,54.9; two metyrapone test decreased $F: 6.0$ to $5.5,12.0$ to 2.2 ; and Increased $S: 3.6$ to $6.8,5.9$ to 9.9 . Dexamethasone ( $2 \mathrm{mg} /$ day) suppressed $\mathrm{F}$ once $(35.6$ to 18.0$)$ but not subsequent1y. At surgery, the adrenals were minimally enlarged and had normal light microscopy. Hemiadrenal slices were implanted beneath the rectus abdominis muscles. Progressive hypertension and $F$ increase (65.2) were noted on postop day 20. Gradual steroid withdrawal lowered $B P$, and $F$ to 10.2 and 4.0. Patient was well for 4 days without therapy but explred following Salmonella enteritis. AA histology revealed outer cortical preservation, fetal zone swelling, inner zone degeneration, but some growth into adjacent tissue. Conclusions: I) Infantile CS may present with variable adrenal tests, 2) serial $F$ best documents functional defects, 3) AA is of limited therapeutic value
260 SOLITARY MAXILLARY CENTRAL INCISOR ASSOCIATED WITH SHORT STATURE AND GROWTH HORMONE DEFICIENCY. Robext A. U1strom, Robert J. Gorlin, Elizabeth B. Rappaport Anne w. Lucky, Eleanor $\frac{\text { Colle, and James Miser. Depts. Of Pedia- }}{\text { trics and Oral }}$ Pathology, University of Minnesota, Minneapolis, Depts. of Pediatrics, McGill University, Montreal, and University of Washington, Seattle, and the Reproduction Research Branch, NIH, Bethesda.

Three male children have been studied and all have unresponsiveness of serum growth hormone levels to stimulation with Ldopa, argenine, and insulin, as well as short stature (<-3 S.D.) and a single upper central incisor. Four females with short stature (<-3 S.D.) and the same dental anomaly were also tested for stimulated growth hormone response. Two responded normally and two did not.

The female normal responders were a 44 year old who also had treated Graves' disease, and a one year old infant. No cause for their shoxt stature was found.

All other patients were tested between $1 \frac{1}{2}$ and 6 years of age. A male grew $10.2 \mathrm{~cm} / 13 \mathrm{mo}$. during HGH therapy. Others are yet untreated.

All patients except one male have normal intelligence. No other functional abnormality of pituitary-hypothalamus was demonstrated. Each of the single maxillary central incisors was increased in all dimensions and present in both deciduous and permanent dentition of all patients. A wide nasal tip and rostrally tilted nares was also present in 6 of 7 . No other midline defects were found. No familial cases were found.

MECHANISM OF GLUCAGON INDUCED GR

261 SECRETION. H. Lawrence Vallet, Maureen E. Day, Ehud Ben-Galim (spon. by R. Pickering). Birth Defects Inst., N.Y.S. Dept. of Health and Albany Med. Col., Dept. Ped., NY $A$ rat pituitary (P) and hypothalamus (H) organ culture was developed to determine if glucagon (G) induced growth hormone (GH) release results from a direct action on components of the $\mathrm{H}-\mathrm{P}$ axis and independent of variation in blood glucose. 20 day o1d $P$ were explanted, hemisected, cultured independently and with $\mathrm{H}$ in co-culture; also with $\mathrm{G}$ at concentrations of $1.0,10.0$, and $100.0 \mathrm{ng} / \mathrm{m} 1$ in Medium $199-1 \mathrm{X}$ with $95 \%$ air and $5 \% \mathrm{CO}_{2}$ at $37^{\circ} \mathrm{C}$. Media changes at $15^{\prime}$ intervals were assayed by radioimmunoassay and $\mathrm{rCH}$ levels were determined with NIAMD reagents using NIAMDRat-GH-RP-1 as standard.

\begin{tabular}{|c|c|c|c|c|c|c|c|c|}
\hline & $P$ & & $\mathrm{P}+\mathrm{G}$ & & $\mathrm{P}+\mathrm{H}$ & & $+\mathrm{H}$ & G \\
\hline G CONCENTRATION & & 1. & 10. & 100. & & 1. & 10. & 100. \\
\hline $\mathrm{ng} / 1.0_{\mathrm{X}} \mathrm{mg} \mathrm{P} / \mathrm{min}$. & 4.46 & 8.46 & 7.86 & 10.53 & 9.21 & 7.11 & 15.9 & 39 \\
\hline ISEM & .53 & 2.2 & 2.1 & 2.6 & 1.6 & 1.1 & 6.7 & 0 \\
\hline
\end{tabular}

G at $100 \mathrm{ng}$ directly stimulates $\mathrm{P}$ secretion of $\mathrm{rGH}$ to $2.3 \mathrm{x}$ $\mathrm{G}$ at $100 \mathrm{ng}$ in the absence of $\mathrm{H}(\mathrm{p}=.05)^{*}$. H stimulates $\mathrm{p}$ to baseline values in the absence of $H(p=.05) \%$. H stimulates $P$ to
$2.1 \times$ baseline $(p=.02)$ but $G$ is equally effective $(p>.50)$. G does not improve $\mathrm{P}+\mathrm{H}$ secretion rates at 1 . and $100 . \mathrm{ng} / \mathrm{ml}$ ( $\mathrm{P}>$ $.50)$ but possibly does so at 10 . This demonstrates that $G$ directly stimulates $\mathrm{P}$ to secrete $\mathrm{GH}$ independent of an $\mathrm{H}$ effect and obviously independent of variations in blood glucose. Studies with other agents and adrenergic blockers $_{t}$ test in progress.

PERINATAL FACTORS IN RELATION TO CORD BLOOD TSH AND Reginald C. Tsang. Univ. of Cincinnati Med. Center t. of Pediatrics and the Radioisotope Laboratory, Cincinnati. Radioimmunoassay (RIA) of thyrotropin (TSH) and thyroxine ( $\mathrm{T}_{4}$ ) in the neonate have been used in the early identification of congenital hypothyroidism. However, little information is available on the possible compounding influence of perinatal factors on these hormones. TSH and $T_{4}$ were measured by RIA in the cord blood serum of 147 full term neonates. Mean $T_{4}$ was $13.1 \mu \mathrm{g} / \mathrm{dl} \pm 2.8$ (1SD) and mean TSH was $8.7 \mu \mathrm{u} / \mathrm{ml}$ with a range of 1.5 to 34.5 . $\mathrm{T}_{4}$ correlated only with maternal age $(r=.194 ; p<.05)$ and meperidine administration during labor: with meperidine $\mathrm{T}_{4}$ was lower (12.7 $\mathrm{\mu g} /$ dl \pm .3 , mean \pm SEM) than without $(13.8 \pm .4 ; t=2.4 ; p<.02)$. TSH correlated weakly with length of membrane rupture $(x=.20 ; p<.05)$, was lower with Cesarean section $(3.8 \mu \mathrm{u} / \mathrm{ml} \pm .9)$ than with vaginal delivery $(8.9 \pm .6 ; t=2.0 ; p<.05)$, and lower with post term delivery $\geq 42$ weeks $(4.9 \pm .68)$ than term $(9.1 \pm .65 ; t=2.18 ; p<.05)$; TSH was high-

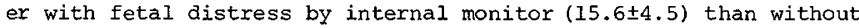
distress $(8.3 \pm 0.57 ; t=2.72 ; p<.01)$. Neither $T_{4}$ nor TSH was correlated with sex, race, birth weight, presentation, Apgar scores, length of labor, or maternal parity, anesthesia, bleeding, toxemia or diabetes. Infants of appropriate weight for gestation had similax $\mathrm{T}_{4}$ and TSH to those large or small for gestation. Thus perinatal clinical factors appear to have only a slight effect on cord blood T4; TSH values may be affected by Cesarean section, post term delivery and fetal distress. These findings may be useful in evaluating future screening programs for congenital hypothyroidism using cord blood. 
263 EVIDENCE FOR DEFECTIVE ADENYLATE CYCLASE ACTIVITY IN SEVERAL TISSUES IN PSEUDOHYPOPARATHYROIDISM (PHP) J.I. Wolfsdorf, R. L. Rosenfield, R. Kobayashi, A.K. Razdan, M. H. Kim, V.S. Fang. University of Chicago Pritzker Sch. of Med., Dept. of Ped., Med., Obstet. and Gynec., Chicago. Gonadotropin resistance in a is $y / 0$ patient with typical Type (Albright) PHP suggested that adenylate cyclase deficiency may be more widespread than previously suspected. The effectiveness of glucagon, glucose, TRH, ACTH and ADH were determined since cyclic adenosine-3', $5^{\prime}$-monophosphate (CAMP) is known to mediate the actions of these substances, as it does for PTH, LH and FSH.
Ovarian insufficiency was indicated by ol igomenorrhea and hypoestrogenism (e.g., 3-5\% vaginal cornification) with modest elevation of basal LH and FSH $(272 \pm 84$, S.D. , \& $593 \pm 83 \mathrm{ng} / \mathrm{ml}$, resp; norm $? 20$ and $<400$, resp.). Ovarian histology demonstrated poor stimulation and karyotype was normal. The hypoestrogenism was overcome by the administration of pharmacologic doses of menopausal gonadotropins, a response indicating resistance to endogenous LH \& FSH Two glucagon infusion tests produced subnormal increments in plasma cAMP (90-123nM/1) compared to 4 normal controls (156-294 $\mathrm{nM} / 1$ ); however, the glycemic response was normal. Intravenous glucose tolerance test elicited a delayed peak in insul in secretion; however. the glucose disappearance rate was normal. The TSH and thyroxine response to TRH, cortisol reserve and the cAMP response to $A D H$ were normal.

n conclusion we have adduced evidence indicative of deficient activity of adenylate cyclase in several tissues in PHP. This to the adenylate cyclase of the PTH receptor system. are compatible with the possibility that certain adenylate cyclases consist of a family of related isozymes.

(Supported by USPHS grants RR-305,HD-7052, HD-07110, \& HD-06308)

\section{EPIDEMIOLOGY}

264 ENVIRONMENTAL CONTROLS AND THE DECLINE OF BLOOD LEAD. Carol R. Angle and Matilda S. McIntire, Univ. of Neb. Coll. of Med., Dept. of Ped., Omaha. A comprehensive environmental study, 1970-1975, in Omaha, for the correlation of lead from multiple environmental sources with the blood lead of children shows a marked decrease in lead in the air and dustfall, parallelling the antipollution measures enacted by the EPA. Lead in both urban and suburban air fell to neglible amounts in 1973, the time of introduction of unleaded gasoline. Lead as measured in 30 day collections of dustfall also decreased dramatically. Environmental measurements at a school adjacent to a battery plant before and after antipollution measures showed a greater decrease in the air lead (particles below $1 \mathrm{u}$ ) than in the large particle dustfall lead. Since industrial filters are maximally effective for large particles, this phenomenon is attributed to the recirculation of previously contaminated soil.

During this same time interval, 1972-1973, blood lead of both urban and suburban children decreased, although the levels of the urban children were consistently higher. Comparable measures (same season, same technique, same age) at an urban junior high school showed a significant $(p \cdot 05)$ decrease in mean blood lead from $25.1 \mathrm{ug} / \mathrm{dl}$ in 1971 to 21.0 in 1973, to 16.1 in 1975 . This longitudinal study in Omaha conclusively demonstrates that environmental controls do reduce blood lead.

65. THE VIETNAMESE ORPHAN: THE MEDICAL IMPACT UPON THE

265 FAMILY. Roger M. Barkin and Thomas M. Vernon (Spon. by Kennech McIncosh) Univ. of Colo. Sch. Of Med., vepi. of Ped., and the Colorado Department of Health, Preventive Medicine Services Division, Denver, Colo.

The medical impact of Vietnamese orphans upon their adopting families was assessed by a retrospective questionnaire which was recurned by $186(87.7 \%)$ families adopiting orphans through one adoption agency in Apri1, 1975. Similar questionnaires were recurned by $275(84.6 \%)$ families on the adoption agency's waiting lisit and by $54(49.7 \%)$ birch certificate matched families, these 2 groups serving as controls. Demographic data were not significantily different among the groups investigated.

Vieinamese orphans had more medical problems than their American counterparis during the comparable two month siudy period. The Vietnamese orphans were reporied to be more symptomatic than matched birth certificate children in all symptom categories investigated. Twenty-three (11.8\%) Vietnamese orphans required hospitalization subsequent to their adoption, while none of the control children received such care. Adopiting families and conirol families experienced the same degree of medical problems with the exception of an increased incidence of runny noses, skin infections, and chicken pox in the adopting families.

The immediate medical impact of the Vietnamese orphan on the family is minimal, but continued surveillance for nonparenteral transmission of hepatitis $B$ anitigen $\left(\mathrm{HB}_{\mathrm{S}} \mathrm{Ag}\right)$ is necessary. Aitention must also be focused upon the psychological and developmental aspects of the acculturation of these children.
LOWER RESPIRATORY TRACT INFECTIONS (LRI) IN CHILDREN WITH LEFT-TO-RIGHT INTRACARDIAC SHUNTS. H.A. Baskin, L.M. Taussig, L.A. Platt, H.D. Allen, S. Goldberg and D. Sahn, Univ. of Arizona, Dept. of Pediatrics, Tucson, Arizona.

Actual statistics for the prevalence of LRI (pneumonia, bronchiolitis) in children with left-to-right shunts $(\mathrm{L} \rightarrow \mathrm{R})$ have not been published. Medical records of 341 infants and children with $\mathrm{L} \rightarrow \mathrm{R}$ shunts were reviewed to study the frequency of LRI. 701 children without congenital heart disease from a SCOR epidemiological study served as controls. LRI was determined by detailed history and/or examination.

\begin{tabular}{|c|c|c|c|}
\hline & of Patients & LRI & LRI \\
\hline All patients with $L \rightarrow R$ shunt & 341 & 34 & 10.0 \\
\hline Ventricular septal defect & 177 & 13 & 7.3 \\
\hline Atrial septal defect & 55 & 7 & 12.7 \\
\hline ent ductus arteriosus & 63 & 1 & 1.6 \\
\hline Complex lesions - Down's & 17 & 6 & $35.3 *$ \\
\hline Complex lesions - Non-Down's & 29 & 7 & $24.1 *$ \\
\hline Control group & 701 & 100 & 14.3 \\
\hline
\end{tabular}
*Above upper $95 \%$ confidence limits. Complex lesions were defined as having $\mathrm{L} \rightarrow \mathrm{R}$ at more than one 1evel. No hemodynamic differences in amount of $\mathrm{L} \rightarrow \mathrm{R}$ or pulmonary artery pressures existed between children with and without LRI for each lesion. More than one episode of LRI occurred in 13 patients; 6 of these had the Down Syndrome and 8 had complex lesions. The postulated increase of LRI in children with simple $L \rightarrow R$ shunts is not confirmed by this study.

TRACHEAL, BACTERIOLOGY OF NEWBORNS WITH RESPIRATORY 267 DISTRESS SYNDROME AND MECONIUM ASPIRATION. I. BroOK \& w.J. Martin (Spon. by P.F. Wehrle); University of California, Los Angeles School of Medicine, Departments of Pediatrics and Clinical Microbiology, Los Angeles, Çalifornia, 90024.

Tracheal cultures were obtained from 21 newborns after intubation and daily while intubated. Fifteen had respiratory distress syndrome, 4 had meconium aspiration and 4 had congenital heart disease; there were 11 deaths. Four received antibiotics prior to first culture. Negative cultures were obtained on the day of intubation in 4 of the 21 newborns, isolation of non-pathogenic organisms (Staphylococcus epidermidis, diphtheroids, bacillus, and propionibacterium species) in 5 , and 11 had pathogenic organisms recovered on the first day. Viridans group streptococci was isolated in 7 cases, 6 of which expired later. One or more of the following organisms was isolated in the remaining cases: Staphylococcus aureus with one of the following gram negative rods: Escherchia coli, Klebsiella pneumoniae, Pseudomonas aeruginosa, Hemophilus vaginalis and Hemophilus influenzae. Anaerobic organisms isolated were: Clostridium perfringens (1), Peptococcus species (2) and Propionibacterium acnes (6). Only in one case did a newborn with neyative cultures become positive following continuous intubation and antibiotics and grew Pseudomonas aeruginosa. Cultures from the 4 patients with meconium aspiration grew one of the following: H. influenzae, E. coli and the anaerobes: C. perfringens and Peptococcus variabilis.

THE PECOVERY OT ANAEROBIC BACTERIA FROA PEDIATRIC PA268 TIENTS, A ONE YEAR EXPERIENCF. Itzak Brook, William Pediatrics and Pathology, i.C.L.A., Los inseles, Ca.

During 1975127 specimens from pediatric ward and nursery patients were submitted to the clinical laboratory for anaerobic culture. In most instances specimens were collected in anaerobic transport tubes containing peptone yeast extract and glucose. In the laboratory, samples were processed by the holding jar Gas Pak method ('lartin, W.J., Anpl. Nicrobiol. 22:1168, 1971) Seventyfour percent of the specimens vere positive viclding, 119 isolates. Bacterioides sp. were the most common isolates (37 nositive cultures, 21 of which were speciated): these were recovered from 11 wounds, 7 gastric aspirations in newborns, 5 abscesses, 4 external ear canals of newborns, 4 amniotic fluids, 2 cerebrospinal fluids, 2 bloods, and 2 peritoneal fluids. nther isolates included: 24 anaerobic cocci (5 of which vere Deptostreptococcus sp.), 24 Propionibacterium sn., 9 Veillone1la sp., 9 Clostridium sp., (3 of which were $C$. perfringens and 1 C. paraputrificum), 8 Fusobacterium sp., 6 Bifidobacterium sp., and 3 Eubacterium sp. In 51 patients the anaerobic isolates (14 wounds, 10 amniotic fluids, 9 abscesses, 9 cerebrospinal fluids, 6 bloods and 3 eyes) were felt to be significantly related to a disease process. nur findings suggest that anaerobic infections are more common in children than generally realized. Anaerobic cultures should be performed on all specimens collected from body fluids as well as wounds and abscesses. 
269 FEVER UNDER THREE MONTHS OF AGE. Frederic W. Bruhn and Kennech McIntosh. Univ. of Colo. Sch. of t. d., Denver General Hosp., Depc. of Ped., Denver, Colo. Few systematic investigations of fever in very young infancs have been performed. Accordingly, we conducted a prospective siudy of fever in infants under 3 months of age who presented at Uenver General Hospital from 1 Oct. 1974 through 30 Sept. 1975. Criteria for inclusion were rectal iemperacure over 38, no DPT immunization within $48 \mathrm{hrs}$ and no prior antibiotics. Bacterial (blood), and viral (NP, throat, rectal) cultures, acute and convalescent sera, CBC, ESR, and urinalys is were obtained from all patients. Additional specimens (CSF, myringotomy fluid, other bacterial culcures) were obtained when indicated.

66 episodes of fever occurred in 65 infanis ( 35 males). 3 infanis had significant bacteremia (iN. meningitidis, N. gonorrheae, S. aureus). Bacterial pachogens were isolated from 9 additionat patients. Of the 12 patients with bacterial infection 9 had concomicant viral infection. All 3 infants in whom myringocomy fluid grew bacierial pathogens had simultaneous viral infection. There were 28 additional patients with virus infection. Respiratory syncyitial and echo viruses were the mosi frequeni pachogens. In 6 infanis encerovirus was recovered from the CSF: 3 of these CSF specimens were otherwise entirely normal for age. In 26 patients no microbial pachogen was isolaced.

Historical and clinical findings, degree of fever, WBC and differential and ESR were of absolutely no value in distinguishing bacterial from viral etiologies or in dececting combined bacterial-viral infections.

EPIDEMIOLOGIC STUDIES OF $\underline{S}$. PNEUMONIAE: LONGITUDINAL

270 CARR IAGE OF PNEUMOCOCCAL SEROTYPES IN YOUNG INFANTS. George M. Converse, Hugh C. Dillon, University of A Tabama in Birmingham, School of Medicine, Dept. of Pediatrics, Birmingham, Alabama

79 healthy term infants were followed from birth up to 12 mos. of age. The acquisition and carriage of given serotypes of Str. pneumoniae, as determined by serial monthly cultures, is now reported.

Paired nasopharyngeal and throat swabs, obtained at each visit, were planted on blood agar medium containing gentamycin, which facilitates isolation of pneumococci. 211/460, or $45 \%$ of all cultures, were positive, yielding 223 strains. 222/223 pneumococcal strains were typable with Danısh reference antisera. 55/79 $(70 \%)$ of the infants harbored pneumococci, most for four or more months duration. Mean age of first acquisition was 14 weeks; acquisition by age 4 weeks was rare. Disappearance of a given serotype was frequentiy followed by acquisition of a new serotype. Spontaneous eradication (no antibiotic therapy) occurred only twice. Carriage of multiple serotypes was observed 14 times. 20 pneumococcal serotypes were identified, the 8 most common ones being types $6,19,23,3,15,11,8$ and 9 in that order, and accounting for $72 \%$ of the total. The first 4 are known to be a frequent cause of otitis media in infants. Among carrier infants, persistent carriage was not uniformly limited to a single serotype. I11nesses were recorded to determine advantages or disadvantages to the healthy host of harboring pneumococcí.

These epidemiologic data provide further information on the natural history of pneumococcal infection in infancy.

THE SIGNIFICANCE OF STAPHYLOCOCCAL COLONIZATION OF THE

271 NEWBORN. Gerald W. DeWitt and J.O. Hendley. U. Va. Sch. Med. Dept. of Pediatrics, Charlottesville.

Since discontinuation of hexachlorophene for routine newborn bathing, the significance of newborn colonization with non-epidemic strains of Staphylococcus aureus (SA) has become critical. In this study the risk of development of SA disease (pustulosis, abscess, omphalitis, conjunctivitis) in newborns followed longitudinally for the first month of life was correlated with nursery colonization and later acquisition of SA.

227 ful1-term newborns were examined and had cord and nose cultures at 2-3 days and 2 and/or 4 wks. of age. A1l SA isolated were phage-typed.

\begin{tabular}{|l|c|c|c||c|c|c|}
\hline & COLONIED IN NURSERY & \multicolumn{3}{|c|}{ NOT COLONIZED IN NURSERY } \\
\hline Time & No. & Culture + & Disease & No. & Culture + & Disease \\
\hline 2 wk. & 91 & $67(74 \%)$ & $31(34 \%)$ & 114 & $54(47 \%)$ & $20(18 \%)$ \\
\hline 4 wk. & 64 & $35(55 \%)$ & $4(6 \%)$ & 89 & $41(46 \%)$ & $5(6 \%)$ \\
\hline
\end{tabular}

$43 \%$ of babies were colonized with SA prior to discharge. At 2 wks. $74 \%$ of this group still had SA while $47 \%$ of the babies not colonized in the nursery had acquired SA. Carxiage rates at 4 wks. were similar in both groups. $25 \%$ of the babies developed presumed SA disease by 2 wks.; $80 \%$ of the disease was pustulosis. $1 / 3$ of babies colonized in hospital but only $1 / 6$ of those not colonized developed disease. Of the culture proven SA disease in the colonized group $55 \%$ was caused by a phage-type different from that acquired in the nursery.

Babies colonized with non-epidemic SA in the nursery had twice the risk of developing disease as noncolonized babies, but only $1 / 2$ of the disease was caused by the nursery acquired SA.
DISACCHARIDE INTOLERANCE AMONG CANADIAN INDIANS AND

272 ESKIMOS. Judith J. Ellestad-Sayed and J.C. Haworth Univ. Mani toba, Dept. Pediatrics, Winnipeg, Canada.

In view of relatively recent dietary changes, Canadian Indians and Eskimos were screened for lactose and sucrose intolerance. A $15 \%$ solution of $2 \mathrm{~g}$ disaccharide $/ \mathrm{kg}$ (max. $50 \mathrm{~g}$ ) was drunk by subjects, aged 2-90 years. Intolerance was defined as failure of blood glucose, measured by glucose oxidase, to rise $20 \mathrm{mg} \%$ or more above fasting. A dietary history was taken, and the number and severity of symptoms following the test were scored (4-16).

The numbers intolerant and tolerant to lactose and sucrose were:

\begin{tabular}{|c|c|c|c|c|c|c|c|c|}
\hline \multirow[b]{3}{*}{ Age (yrs.) } & \multicolumn{4}{|c|}{ Indi an } & \multicolumn{4}{|c|}{ Eskimo } \\
\hline & \multicolumn{2}{|c|}{ Lactose } & \multicolumn{2}{|c|}{ Sucrose } & \multicolumn{2}{|c|}{ Lactose } & \multicolumn{2}{|c|}{ Sucrose } \\
\hline & Intol & Tol. & Tntol. & Tol. & Intol & Tol. & Intol. & Tol \\
\hline $0-3$ & 3 & 5 & $T$ & 7 & 2 & 7 & $\overline{0}$ & 7 \\
\hline $4-7$ & 1 & 6 & 0 & 7 & 10 & 1 & 1 & 8 \\
\hline $8-11$ & 4 & 4 & 1 & 7 & 8 & 1 & J & 8 \\
\hline $12-15$ & 6 & 5 & 0 & 10 & 7 & 4 & 0 & 12 \\
\hline $16-19$ & 10 & 1 & 0 & 11 & 7 & 0 & 1 & 6 \\
\hline $20-$ & 12 & 3 & 0 & 11 & 9 & 3 & 1 & 11 \\
\hline
\end{tabular}

The prevalence of lactose intolerance was $60 \%$ and $73 \%$ among the Indians and Eskimos. Of the 6 who were sucrose intolerant, 5 were also intolerant to lactose. Symptom scores were significantly higher $(p<0.01)$ among the lactose intolerant subjects. The consumption of free milik at school resulted in gastrointestinal disturbances in 13 children. School milk programs for Canadian native children should therefore be reconsidered.

A COMPARISON OF A SHORT COURSE OF AMPICILLIN AND SUL273 FAMETHOXAZOLE-TRIMETHOPRIM FOR BACTERIURIA IN GIRLS WLTH RECURRENT URINARY TRACT TNFECTIONS, Robert $S$. Fenne11, III, Eduardo H. Garin, Norman D. Pryor, Carl D. Sorgen, R. Dixon Walker, and George A. Richard (Spon. by Elia M. Ayoub) Univ. of Fla. College of Med., Dept. of Pediatrics, Gainesville.

Twenty-five girls ages four through twelve with active urinary tract infections were treated for ten days with either ampicillin or sulfamethoxazole-trimethoprim. Criteria for inclusion in the study were a recent history of recurrent bacteriuria and no history of allergy to either penicillin or sulfa. Patients were randomly assigned to either therapy group. Bacteriuria was de$\mathrm{f}$ ined as $>100,000$ colonies per $\mathrm{ml}$ on two separate urine specimens or growth on a suprapubic aspiration. Therapeutic success was defined as elimination of the original organism and freedom from reinfection for three months following the initial ten-day course of therapy.

E. coli was the initial organism in nine out of thirteen girls treated with ampicillin and eight out of twelve treated with sulfamethoxazole-trimethoprim. Only two out of thirteen girls (15\%) treated with ampicillin had a therapeutic success, whereas seven out of twelve $(58 \%)$ treated with sulfamethoxazole-

trimethoprim had a successful therapeutic course. When a recurrence was observed, the average time after the initiation of therapy for ampicillin was 14.2 days and for sulfamethoxazoletrimethoprim, 43.6 days. No major side effects from either drug were noted.

TIME SERIES ANALYSIS IN PREDICTING UTILIZATTION OF 274 NEONATAL (EMERGENCY) TRANSPORT SERVICE IN NYC. Angelo Ferrara, Rajagopalan Indra, (Spon.by Joseph Dancis) NYU Sch.Med. -Bellevue Hosp.Ctr., Dept. Ped.

In a 45 mo. period $(4 / 71-12 / 74)$ the \# of calls to an emergency transport service averaged $86 / \mathrm{mo} .(2.8 /$ day $)$ with a range of 61-114 per mo. To predict the monthly \# of calls, 2 time series models were used: 1 based on the \# of monthly calls in time period studied (corrected for season \& mo.) and the other based on the monthly $\%$ of neonates transported from the live borns in NYC. The resulting trend line equations are in Fig.1. Results: In estimating the \# of calls for the first 6 mo. of 1975, the first model had a $\%$ error (coefficient of variation) of $400 \%$ and a success prediction of $50 \%$ with 1 S.D. while the 2 nd model had less $\%$ error (84\%) with $100 \%$ successful prediction but the estimate came too late (\# of births in any I mo. not known for several months) to be practical. Time series analysis is not a valid indicator of actual service.

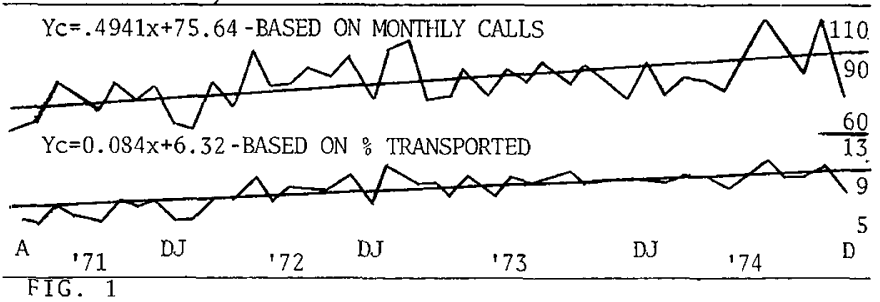



Asa E. Seeds, and Lewis W. Wannamaker. Univ. of Minnesota Medical School, Dept. of Pediatrics, Minneapolis, Minn. A prospective study was carried out to determine prevalence of genital tract carriage of group B streptococci (GBS) in pregnant women and acquisition of GBS by their newborn infants. Cervical and vaginal cultures were obtained at approximately 36 weeks of pregnancy and vaginal cultures at time of labor. Infants had cultures of the nares (N), ear canal, and umbilicus (U) at bixth and of $\mathrm{N}$ and $\mathrm{U}$ at discharge. A low carriage, 5.6\%, was found during the third trimester of pregnancy, increasing to $8.3 \%$ in labor. At birth, $3.4 \%$ of infants were culture positive $(+)$ for GBS; an additional $2.1 \%$ were $(+)$ at discharge. Maternal site(s) of carriage did not influence the frequency of GBS acquisition by the infants. GBS carriage during labor was common in women previously $(+)$ during the third trimester. Of women culture (t) in labor, however, $31.7 \%$ had converted from a culture negative (-) state. The opposite event--conversion from $(+)$ to $(-)$--was also seen. Nearly $50 \%$ of babies born to women $(+)$ in labor acquired GBS at birth. GBS contamination at multiple sites was common but $94 \%$ of culture $(+)$ infants had $(t)$ ear canal cultures. Three infants had asymptomatic bacteremia after birth with no morbidity. The distribution of GBS serotypes recovered was similar among mothers and infants with types Ic, II and III predominating.

Persistence of GBS carriage was common in parturients as was conversion of culture status; the latter event may hamper identification of the "at risk" mother-infant pair.

276 ETIOLOGICAL FACTORS IN HEMORRHAGIC CYSTITIS (HC) Bernard G. Gauthier, Hooshang Mirlohi, M. Ejaz Qureshi, and Donald 1. Moel. (Spon. by Philip Lanzkowsky). Department of Pediatrics, Long $|s|$ and Jewish-Hil|side Medical Center, New Hyde Park, N.Y. and SUNY, Downstate Medical Center, Brooklyn, N. Y.

Sixty-five children with HC (gross hematuria with one or more of: dysuria, frequency, suprapubic pain) were compared with 134 children with urinary tract infections (UTI) with reference to sex distribution, prevalence of significant bacilluria (SB;single arganism, more than $105 / \mathrm{ml}$., grown on one or more occasions), and radiological abnormalities (RA). $38 \%$ of the boys and $69 \%$ of the girls with HC had SB. This sex difference was significant $(p<$ $0.01)$. 13\% of the boys with $\mathrm{HC}$ and $44 \%$ of the boys with UTI had RA. This difference was significant $(p<0.01), 26 \%$ of the girls with HC and $36 \%$ of the girls with UTI had RA (not significant). Among the children with RA, all of those with $\mathrm{HC}$ and $85 \%$ of those with UTI had vesicoureteric reflux or pyelonephritic scarring. The data suggest that the incidence of nonbacilluric HC is significantly higher in males. The prevalence of $R A$ and of $S B$ in $H C$ suggests that, in about $1 / 3$ of males and in about $2 / 3$ of females, $\mathrm{HC}$ is caused by a bacterial infection. The table below summarizes the data.

$\begin{array}{llccc} & & \text { Patients } & \text { Bacilluria } & \text { Anomalies } \\ \text { HC } & \text { Boys } & 29 & 38 \% & 13 \% \\ & \text { Girls } & 36 & 69 \% & 26 \% \\ \text { UTI Boys } & 58 & 100 \% & 44 \% \\ & \text { Giris } & 76 & 100 \% & 36 \%\end{array}$

\section{USAGE OF ANTIBIOTICS IN TWO NEWBORN NURSERIES}

\section{7 herion}

rics, Harvard Medical School, Boston University School of Medicine Tufts University School of Nedicine, Boston, Wass.

Usage of antibiotics $(\mathrm{AB})$ in neonates delivered at two hospitals was studied prospectively. Hospital A (A) serves a community that is mainly midole class and Caucasian, whereas Hospital B (B) serves a community of black and hispanic urban poor. During November-December 1975, 563 infants were delivered at A and 284 at $B$. The percentage of infants with birth weight $\leq 2500$ gm was $3.7 \%$ at $\mathrm{A}$ and $7.7 \%$ at $\mathrm{B}$. Antibiotics were administered prior to delivery to $0.9 \%$ of mothers at $A$ and $3.8 \%$ of those at $B$. Almost all infants had cultures of blood ( $B C)$, urine and CSF prior to treatment. Most received two $\mathrm{Ab}$, an aminoglycoside and a penicillin.

Intants cultured

Infants treated with $\mathrm{Ab}$

Median duration of Ab usage (days)

No. positive cultures

The organism isolated at $A$ was $\alpha$ hemolytic streptococcus, nongroup $D$; that isolated at $B$ was Salmonella group $E$. The results indicated differences in usage of $A b$ in the two hospitals.

The ratio of infants treated with $A b$ to those with documented bacterial infection was $\cong 20: 1$. These data reaffirm the need to establish the safety of $\mathrm{Ab}$ used in neonates since many without bacterial diseases are treated.
WHERE CAN PERINATAL MORTALITY BF REDUCFD?

278 R.G.Harper, S.Sokal, M.Sokal,V.F.Mastrota, \& J. Davis,Depts. of Ped. \& Ob.-Gyn.,North Shore Univ.Hosp. ,Manhasset,NY, \& Depts.of Ped.\& Ob.-ryn., Cornell Univ.Med.Coll.,NY,NY. (Spon. by F.Lifshitz)

To develop a strategy for reducing perinatal mortality, a panel of perinatologists reviewed the medical, obstetric, and pediatric factors involved in perinatal care among the 315 perinatal deaths in Nassau County during 1973. Analysis indicated that 248 of the 135 stillborns and $27 \%$ of the 180 neonatal deaths were preventable. Intrapartum deaths accounted for $30 \%$ of the fetal deaths; 408 of the intrapartum deaths were greater than 2000 gms and $50 \%$ greater than 36 weeks' gestation. Postmature deliveries, infants surviving the first 48 hours, and transfers were disproportionately over-represented among preventable deaths. Physician error, the factor which accounted for 6.38 of all deaths, accounted for $24 \%$ of the preventable deaths. Major reductions in the Countywide perinatal death rate of $27.4 / 1000$ livebirths could be accomplished if fetal monitoring were routinely used during all labors, if postmature pregnancies were vigorously monitored, if pediatric management of infants surviving more than 48 hours were upgraded, and if pre-delivery transfer of mothers with compromised fetuses were routinely employed. Further, physician reduce perinatal mortality.

CERVICAL CYTOLOGIC SCREENING IN THE ADOLESCENT.

279 K. Hein, K.Schreiber, A.J. Sheehy, M.I. Cohen, Albert Dept. Ped. and Path., Bronx, New York.

The Papanicolaou smear will detect cervical carcinoma ten years before symptoms become apparent in the adult female. A study was conducted to investigate the usefulness of cervical cytologic screening in the adolescent. Pelvic examinations were performed in 574 fentales aged 12-15 years, over a 48 week period of time. The girls were predominantly minority group members and $99 \%$ were sexually active. A detailed history of contraceptive use, venereal disease and pregnancy was obtained from 307 who had pap smears. The pap smears revealed trichomonas, monilia or other infections in 111 samples. Cytologic abnormalities in 307 pap smears included inflammation (20\%), inflammatory atypia (10\%), atypia (5\%) and dys plasia-intraepithelial carcinoma (3\%). Thus a significantly abnormal pap smear, as defined by dysplasia or atypia without inflammation, was found in $8 \%$ of girls tested. The mean age of the 11 patients with dysplasia was 14.8 years with menarche at 11.6 years and sexual activity present for 30 months. Follow-up studies to date included colposcopy with multiple biopsies of the cervix in 3 of these 11 girls. Biopsies confirmed dysplasia or intraepithelial carcinoma and cryosurgery or conization was performed. The presence of such pathology correlated with sexual activity, venereal disease, contraception and/or pregnancy. These data show that pap smears performed on adolescent females yield important information regarding the appropriate management of cervical cytologic abnormalities.

STOCHASTIC RESPONSE (POISSON DISTRIBUTION) OF

280 EMERGENCY URBAN INFANT TRANSPORT CALLS AFFECTINC STAFFING PATTERNS. Rajagopalan Indra, Angelo Ferrara (Spon. by Joseph Dancis) NYU Sch.Med.-Bellevue Hosp.Ctr.Dept.Ped. A $12 \%$ random sample of 56 months (4/71-11/75 with 4885 transports) of a neonatal transport service showed the frequency distribution ( $(\%)$ of emergency calls by work shifts as follows: 8 Am-4 PM 45\%, 4PM-Midnight 35\%, Midnight-8AM $20 \%$. Though the daily average \# of calls was 2.8 , empiric observations revealed no calls on an average of 2 days a month while on an additional 2 days the transport staff was active moving $>6$ calls in 24 hours. For more precise resource allocation of nursing personnel, a distribution of calls by days was done on an additional $18 \%$ randomization of months (181 days). The actual \# of days with varying \# of calls (0-6) was predicted by a Poisson distribution. Results: If nursing schedules were prepared weekly or biweekly, a more rational manpower allocation could be predicted by the poisson distribution, but month1y scheduling patterns could not improve allocation by this predictive model.

\begin{tabular}{l|r|r|r|r|r|r|r} 
FIGURE 1 & $x^{2}=\langle .1$ & \& & $\mathrm{P}>.7$ & - No significant difference \\
\hline \# OF CALLS & 0 & 1 & 2 & 3 & 4 & 5 & $\geq 6$ \\
\hline $\begin{array}{l}\text { ACTUAL \# } \\
\text { OF DAYS }\end{array}$ & 11 & 33 & 43 & 36 & 32 & 14 & 12 \\
\hline $\begin{array}{l}\text { PREDICTED } \\
\text { (POISSON) }\end{array}$ & & & & & & & \\
\# OF DAYS & 11 & 31 & 43 & 40 & 28 & 16 & 11 \\
\hline
\end{tabular}




\section{1 \\ SIBLING AGGREGATION OF PLASMA CHOLESTEROI \\ Mary J. Jesse, Barbara E. Klein, Charles H. Hennekens, Janet E. Gourley, Sidney Blumenthal.}

Plasma cholesterol levels were analyzed for 41 sibling groups containing 75 children and 41 of their infant-siblings. Plasma levels were obtained from the 41 infants during the neonatal period and at one month of age.

Cholesterol values were converted to geometric mean values, and were expressed in standard deviation units to adjust for age. Analysis of variance was performed for the 75 siolings. There was less variance within than between the sibling groups ( $F$ ratio of $2.14, p=.013)$. The intraclass correlation coefficient $\left(r_{r}\right)$ was .39. To determine the earliest age at which this tendency could be demonstrated, data from the newborn were then included. The $\mathrm{F}$ ratio was $1.47, \mathrm{p}=.08 ; \mathrm{r}_{\mathrm{T}}=.14$. When the value for the infant at one month was substituted for the newborn value, the $F$ ratio rose to $2.45, \mathrm{p}=.0004 ; \mathrm{r}_{\mathrm{I}}=.34$.

These findings demonstrate significant aggregation of cholesterol, and suggest that the sibling similarity may be detected by one month of age. Etiologic studies of this tendency should be geared to the prenatal and early postnatal period.
284 NATIONAL IMPACT OF IMPROVED PERINATAL CARE: ANALYSIS OF U.S. NEONATAL MORTALITY STATISTICS OF $1968-1973$. Robert Manniello and Philip Farrell, NIH, Bethesda, MD Advances in perinatology have affected neonatal mortality rates in the 1968-1973 period studied by us. Analysis of computerlogged data from all states and D.C. confirms a rate decline from 16.1 to $12.9 / 1000$ live births. Percent contribution of the 7 most commonly reported causes of neonatal death demonstrated changing trends as shown. While (2), (3), and (5) declined in frequency, the HMD \& RDS category has increased $6 \%$ during the 6 year period.

\begin{tabular}{|c|c|c|c|c|c|}
\hline$\frac{1968}{35}$ & $\frac{1969}{36}$ & $\frac{1970}{37}$ & $\frac{1971}{3.5}$ & $\frac{1972}{32}$ & $\frac{1973}{3}$ \\
\hline $\begin{array}{l}\text { Live births } \times 10^{-3} \\
\text { Total neonatal deaths } \times 10^{-3} \frac{3.5}{56}\end{array}$ & $\begin{array}{l}3.6 \\
56\end{array}$ & $\begin{array}{l}3.7 \\
56\end{array}$ & $\begin{array}{l}3.5 \\
50\end{array}$ & $\begin{array}{l}3.2 \\
44^{2}\end{array}$ & $\begin{array}{l}3.1 \\
40\end{array}$ \\
\hline
\end{tabular}
$\%$ Deaths due to:

(I) HMD \& RDS

(2) Asphyxia

(3) Immaturity

(4) Congential anomalies 13

(5) Pregnancy complications 12

(6) Placental conditions

(7) Birth injury

Further analysis revealed that $65 \%$ of all neonatal deaths occur within the first 48 hours of life with male infants predominating in most categories. A $10 \%$ decrease in total live births has occured with the percent of low birth weight infants $(<2.5 \mathrm{~kg})$ falling from 8.2 to 7.5 . Although emphasis on comprehensive perinatal care has produced a significant decline in overall U.S. neonatal mortality, prematurity and its consequences must remain of foremost concern if further improvements are to be realized.
282 RELATIVE IMPORTANCE OF ENTEROTOXIGENIC E. COLI AND UNCLASSIFIED FECAL VIRUS II (UFV II) AS CAUSES OF INFANTILE GASTROENTERITIS. Benny Kerzner, Manuel Buchwald and Richard Hamilton, Res. Inst., Hosp. for Sick Children, Dept. of Paed., Univ. of Tor onto, Ontario.

We examined stools from 54 children, 44 attending hospital with acute "non-bacterial" diarrhea and 10 healthy controls, for UFV II (previously named orbi, reo-like, duo or rota virus) and Enterotoxigenic E.coli between Dec. 1974 and Sept. 1975. Children under age 3 years, with diarrhea less than 4 days and no known bacterial pathogen were studied, 34 during a winter epidemic, 10 sporadic cases in the summer. UFV II was found by direct electron microscopy in 30 of 34 winter cases, none of summer cases, none of controls. E.coli was grown from all patients except 9, positive for UFV II; 5 colonies from each child plus 6 known positive controls were serotyped and analysed for enterotoxin production. No stable toxin (ST) producing colonies were found in control or diarrhea patients using the infant mouse assay. Heat labile toxin (LT) producing colonies were found in one colony from a control patient, one from a UFV II positive case and in 7 colonies from 2 summer cases. "Enteropathogenic" serotypes were found in 2 diarrhea patients, one producing LT. In our community, UFV II is the major cause of infantile gastroenteritis in winter; our identifications of enterotoxin producing $E_{0} c o l i$ were confined to LT producers in a small proportion of cases in the summer.
EPIDEMIOLOGY OF HIGH TENSION ELECTRICAL INJURIES IN

283 CHILDREN, Elizabeth McLoughlin, Michael P. Joseph, John D. Crawford, Harvard Med. School, Shriners Burns Institute, Mass. Gen. Hosp., Dept. of Pediatrics, Boston.

Twenty-seven children whose injuries resulted from contact with high-voltage electric current have been admitted as acute patients to this burn unit in its 7 years of operation. All patients were male age 7 to 16 . Pole climbing (9), trespassing (9), tree climbing (7), and target shooting (2), were the activities which led to the accidents. The vast majority happened between May and October, on weekends between 4 and $8 \mathrm{p.m.}$, to boys for whom acknowledged out-door danger zones become a proving ground for testing physical prowess and daredeviltry. 14 sustained electric current injuries; 8 only clothing ignition burns, and 5 boys sustained both electric and ignition injuries. 13 suffered amputations; 2 boys died.

The high precision with which one can define the circumstances and individuals at risk of these frequent and mutilating accidents invites an exploration of stratagems for prevention. Preventive education directed to this high-risk population should illustrate properties of high-voltage electricity, effects on the body and how to cope with peer pressures in unstructured time. We have followed the suggestion of Janis (1968) that a moderately fear-provoking message, combined with positive recommendations on how to avoid such dire consequences, is an effective approach. Burn victims and families collaborate with Institute staff in the presentation of programs.
285 EPIDEMIC SPREAD OF H. INFLUENZAE TYPE B (HIB) IN A 285 DAY CARE CENTER. Marian E. Melish, Alvin J. Nelson, Tigi E. Martin, Carl W. Norden. (Spon. by James D. Connor). Dept. of Pediatrics, Univ. Cal., San Diego and Dept. Med., Univ. Pittsburgh.

HIB is generally considered to cause endemic but not epidemic disease. However, during a 5 week period, 4 cases of systemic HIB infection occurred within a group of 48 normal children aged 5 months to 5 years attending a preschool/day care center. This attack rate is $-400 x$ the general incidence for HIB meningitis. 3 children $(3.5$ yrs., 2.3 yrs., and 10 mos.) developed HIB meningitis; 1 child (6 mos.) developed HIB pneumonia. 3 weeks after onset of Case \#1, the center children were surveyed for HIB nasopharyngeal (NP) carriage and for HIB serum antibody (ab) (Survey \#1). 50\% carried HIB. 20 days later, 7 days after onset of illness in Case $\# 4,30 \%$ had HIB NP carriage. At this point, chemoprophylaxis with sulfamethoxazoletrimethoprim for 4 days was instituted. HIB carriage was eradicated from all children when surveyed 10 days later.

At Survey \#1, 36\% had "significant" HIB ab (RIA $>0.8 \mu \mathrm{g} / \mathrm{ml}$ )。30 days later, $60 \%$ had HIB ab while $40 \%$ had low ab levels. Nasopharyngeal HIB carriage occurred mainly in children with HIB ab and persisted despite HIB $a b$.

The HIB carriage rate in this population is $10 x$ that reported for an open population of children. The potential for epidemic spread of HIB disease exists when a group of susceptible children are exposed to high carriage rates of HIB as in a day care center. Chemoprophylaxis for HIB may be warranted in special exposure situations.

DRIED UMBILICAL CORDS IN THE STUDY OF POLLUTANTS.

286 Robert W. Miller. Clinical Epidemiology Branch, NCI. Bethesda, Maryland.

Ingeniously, Japanese investigators have used dried umbilical cords from up to 48 years ago to show peaks in methylmercury content in the 1950's when an epidemic of Minamata disease (a neurologic disorder) is known to have occurred -- and 20 years before that, when a similar episode went unrecorded (Jpn. J. Hyg. 27:115, 1972; Nature 258:324, 1975). In Japan $5 \mathrm{cms}$. of dried cords are traditionally kept for possible use as a folk-medicine. The report suggests a wider use of dried cords, which can be easily obtained and stored, for evaluation of pollutants anywhere in the world. In I raq, where about 7,000 people were poisoned with methylmercury from using fungicide-coated grain for baking bread, dried cords could have been obtained for later study under optimal conditions. In Japan study could be made of cadmium content in relation to "ltai-itai disease" or of polychlorinated biphenyls (PCB's) in contaminated cooking oil which caused an epidemic of cola-colored babies and chloracne. Study could be made in Michigan of extensive contamination from polybrominated biphenyls (PBB's) or in Virginia from kepone. In Glasgow or El Paso, with respect to lead pollution of water or air, one could study high vs. low I.Q. scores in the future as related to lead content of umbilical cords obtained and stored now. Systematic collection and storage of dried cords might be used as a biologic dosimeter for changes in levels of pollutants over time, or possibly in relation to etiologically puzzling diseases of childhood. 
$\mathbf{2 8 7}$ PEDIATRIC INTENSIVE DRUG SURVEILLANCE,

Allen A. Mitchell, Peter Goldman, Samuel Shapiro, Victor siskind Dennis slone (Spon. by Mary Ellen Avery). Harvard Medical School, Departments of Pediatrics and Pharmacology; Children's Hospital Medical Center, Clinical Pharmacology Unit; Boston University. Medical Center, Drug Epidemiology Unit; Boston.

Rational drug therapy in pediatrics requires information on the risks and benefits of drugs as they are used in clinical practice. To provide this information, a prospective, intensive drug surveillance program, in which nurse-monitors collect data on consecutively admitted patients, has been established at the Children's Hospital Medical Center. The goals of this program in clude the detection of previously unknown adverse drug reactions, the quantitation of known reactions, and the identification of risk factors which may alter a patient's response to drugs.

By December 1975, records of 716 patients were on computer file; mean values for this population were: age, 7.9 years; days in hospital, 7.9; drugs per patient, 7.0. Forty-two $(5.9 \%$ ) of these patients were hospitalized as a result of adverse reactions to drugs taken prior to admission. Eighty-three patients (11.6\%) suffered 118 adverse reactions to drugs administered in the hospital. Medication orders were modified because of the reaction in 45 instances (38\% of adverse reactions), and one or more additional drugs were used in the treatment of an adverse reaction in 30 instances (25\% of adverse reactions). Life-threatening reactions occurred in five patients, and included coma, gram-negative sepsis, bone-marrow depression, and fluid overload (two patients).
COMPARISON OF MINOCYCLINE, CEPHALEXIN, AND SULFISOXA-

290 ZOLE IN THE TREATMENT OF BACTERIURIA IN COLLEGE COEDS. Norman D. Pryor, Car1 D. Sorgen, Robert S. Fennell, III, Eduardo H. Garin, R. Dixon Walker, and George A. Richard, (Spon. by Elia M. Ayoub), University of Florida College of Med., Department of Pediatrics, Gainesville.

377 coeds with dysuria were interviewed for a past history of bacteriuria, sexual activities, and use of oral contraceptives. A complete genitourinary exam was done and culture obtained. Significant bacteriuria was demonstrated in $232(61.5 \%)$ and dysuria without bacteriuria in $145(38.5 \%)$. The most common organisms isolated were E. coli $(67.0 \%)$, proteus $(15.0 \%)$, and coagulase negative staphylococcus $(12.5 \%)$. Patients with significant bacteriuria were treated with Minocycline ( $\underline{M})$, Cephalexin ( $\underline{C})$, or Sulfisoxazole (S) for $3,7,14$, or 21 days. Urine cultures were obtained monthly for 6 months. Recurrence rates one month post therapy in $M(5 / 79$ or $6.3 \%)$ and $C(11 / 77$ or $14.3 \%)$ were signif $i-$ cantly greater than in group $\underline{S}(\overline{2} / 58$ or $3.4 \%)$. The combined treatment group recurrence rate at 6 months was $27.7 \%$. Side effects occurred more frequent $1 y$ in the patients on $\underline{M}$ and $\underline{C}$, e.g., monilia vaginitis and dizziness $(M)$. A history of sexual activity within 24 hours prior to symptoms was obtained in $77 \%$ of dysuric coeds with bacteriuria and in $70 \%$ of those without bacteriuria. The 145 dysuric coeds without bacteriuria demonstrated no etiology $(69.7 \%)$, monilia vaginitis $(16.6 \%)$, trichomonas $(4.1 \%)$, herpes genitalia $(2.7 \%)$, nonspecific vaginitis $(5.5 \%)$, and gonorrhea $(1.4 \%)$. One of 232 dysuric patients with bacteriuria had associated gonorrhea.
288

ONE YEAR'S EXPERIENCE WITH AN OUTBREAK OF KANAMYCIN CENTAMICIN RESISTANT KLEBSIELLA BACTEREMIA IN A REGIONAL PERINATAL CENTER. E1len F. Monkus, D. Stewart MacIntyre, and Joan Turner (Spon. by William $\overline{\mathrm{W}}$. Cleveland), Univ. of Miami Sch. of Med., Dep. of Ped. and Med., Miami, Florida.

Kanamycin-Gentamicin resistant Klebsiella became epidemic in the premature nurseries of a Regional Perinatal Center in the Fall of 1974 . E. coli resistant to Kanamycin and Gentamicin emerged in December, 1974, possibly by episomal transfer. Usual control measures were ineffective and attempts to cohort were incomplete due to staff and space limitations, particularly in the Newborn Intensive Care Unit. Restrictions in antibiotic use were strictly enforced, with stronger indications for antibiotic therapy, elimination of prophylactic antibiotics and shorter presumptive treatment with negative cultures. Patient-days of antibiotic therapy and average days of treatment with negative cultures were significantly decreased. Periodic colonization prevalance surveys (rectal swab cultures) showed a marked decrease in resistant Gram negative bacilli in the nursery, with continued high prevalance elsewhere in the hospital.

1974-75 SEP OCT NOV DEC JAN FEB MAR APR MAY JUN JUL SEP OCT NOV \begin{tabular}{lrrrrrrrrrrrrr}
$1974-75$ & Coloniz & 75 & 70 & 50 & 11 & 66 & & & 59 & 50 & 7 & 3 \\
\hline Disease & 2 & 1 & 2 & 2 & 1 & 5 & 2 & 3 & 3 & 1 & $=15$ & $\mathrm{K1} . ; 7$ & E.coli
\end{tabular} $\begin{array}{lllllllllll}\text { Deaths } & 0 & 0 & 2 & 1 & 1 & 2 & 0 & 2 & 0 & 1\end{array}=9$ Deaths $(41 \%)$

From July through December, 1975, no new cases of disease due to Kanamycin-Gentamicin resistant Klebsiella or E. coli occurred. Control of antibiotic usage appears to have been an important factor in containing this epidemic of resistant Gram negative bacteremia in a Regional Perinatal Center.

289 SINGLE-DOSE AITIBIOTIC THERAPY FOR GONORRHEA IN PREPUBERTAL CHILDREN. The Pre-pubertal Gonorrhea Cooperative Group, presented by John D. NeIson. Univ. of Texas H. Sci. Center at Dallas, Dept. of Ped, Dallas.

A controlled study of gonorrhea treatment has not previously been done. 18 institutions participated in a study of singledose treatment comparing procaine penicill in $\mathrm{G}(100,000 \mathrm{u} / \mathrm{kg}$ I.M.) and amoxicilitin $(50 \mathrm{mg} / \mathrm{kg}$ P.O.). Probenecid $(25 \mathrm{mg} / \mathrm{kg}$ 1.M.) and aiven simultaneously with both antibiotics. There were P.0. was given simut taneousfirmed gonorrhea in 100 children. 108 episodes of culture-confirmed gonorrhea in 14 mos. and 14 yrs. of There were 15 boys and 85 girls between 14 mos. and 14 yrs. of age. Multiple episodes, presumably due to re-exposure, occurred
in 6 girls. Both regimens provided prompt bacteriological and in 6 girls. Both regime treatment and 5 days and 12 days later. (The only treatment treatment and 5 days and 12 days later. (Thl treated with spectinomyfailure was in a penicillin-allergic girl treated with spectino cin. Tetracycline was in all of 47 costa Rican children but cultures were negative in allofen. Anal cultures yielded gonocommon cocci in $52 \%$ of girls and $25 \%$ of boys and oratances rectal culitive in $18 \%$ and $13 \%$, respectively. In 3 instances rectal cultures confirmed the diagnosis when vaginal cultures were regative. Gonorrhea is not rare in pre-pubertal children and must be condose penicillin or amoxicillin treatment (with probenecid) is uniformly curative for uncomplicated gonorrhea.
DIETARY SERVICES DURING PREGNANCY, AND BIRTHWEIGHT:

291 A RETROSPECTIVE MATCHED PAIR ANALYSIS. David Rush, Agnes C. Higgins, Mark D. Sadow, \& Susan Margol1s, Columbia Univ., Div. Epidemlology \& Dept. Pediatrics, N.Y., N.Y. An aggressive program of dietary counselling and supplementatreal Diet Dispensary (M.D.D.), among non-systematically chosen women registered for prenatal care at the Royal Victoria Hospital (R.V.H.), Montreal. There were no concurrent controls. Of 1544 (R.V.H.), Montreal. There were no concurrent controls. Of 1544 singleton pregnancles delivered from $1962-1970,1341$ of white
Catholfc or Protestant women were studied; 1182 could be retrospectively matched to other R.V.H. public patients for year of delivery, parity, pregravid weight, trimester of registration for prenatal care, and religion. Propositi and controls were similar for such unmatched variables as marital status, age, and past pregnancy loss. For these 1182 pairs, perinatal mortality was lower among those receiving dietary services (16.9/1000 vs. $24.5 / 1000, .10<\mathrm{p} 6.25$, n.s.). The difference was 1 imited to deaths prior to labor (8 M.D.D. births, 17 among controls).

Infants of M.D.D. mothers had significantly higher birthweight than controls (48 gms, p 4.05); the difference was $87 \mathrm{gms}$ for first, $41 \mathrm{gms}$ for second, and $20 \mathrm{gms}$ for third trimester registrants; also, differences were greater for 11ghter women, and those of lower parity. The M.D.D. group gained slightly more weight in pregnancy (.53 1bs, n.s.) and had minimally longer gestations $(0.66 \mathrm{~d}$, n.s.)

This is the first reported controlled study demonstrating a significant increase in birthwelght following dietary services in pregnancy in a western, industrialized population.

292 COLLEGE STUDENTS? M.R. Seashore, C. Austein, S.S. Mick. Yale Univ. Sch. Med., Depts. Human Genet. an Ped., New haven, CT (Spon. by L.E. Rosenberg)

A questionnaire examined knowledge and attitudes about genetics and Tay-Sachs Disease (TSD) in a sample of single, Jewish indergraduates; 219 of 348 questionnaires mailed were returned $(63 \%)$. The student's knowledge varied: $78 \%$ answered all general genetics questions correctly but only $19 \%$ were as knowledgeable about TSD; $37 \%$ knew little about TSD. The major TSD knowledge source was news media and only $0.9 \%$ had learned about TSD from a physician. They were interested: $77 \%$ would attend a TSD screening $>80 \%$ would consult a physician upon learning they were a TSD carrier. Most $(55 \%)$ felt that TSD screening should be done in high school or college and would tell a prospective mate of carrier status early $(71 \%)$.

Most who felt that knowledge of carrier status was important would attend a screening ( $85 \%$ ) and use the results in family planning $(96 \%)$. Erroneously, $2 \%$ of these would forego children if one spouse were a carrier. Those feeling such knowledge of little importance would neither at tend screenings nor consider carrier status in family planning.

The predicted compliance $(77 \%)$ and interest indicate that screening single college students for TSD carrier state may be advisable. If this is to be effective, the news media and physicians must be accurately informed and properly educated as community knowledge sources. 
293 URINARY TRACT INFECTIONS DURING PREGNANCY-MATERNAL AND PEDIATRIC FINDINGS. John L. Sever, Jonas H. E1lenberg, Mary R. Gilkeson, Dorothy M. Edmonds and Helen M. Krebs. National Institute of Neurological and

Urinary tract infections (UTI) during pregnancy have been reported by several investigators to be associated with maternal anemia, prematurity and perinatal mortality. Not all studies have supported these observations.

As part of the Collaborative Perinatal Project we have investigated maternal and pediatric findings associated with maternal UTI in approximately 56,000 pregnant women. There were 1,933 women with symptomatic UTI $(3.4 \%)$. A11 women were treated and approximately half received sulfa drugs. Low socioeconomic level was significantly associated with UTT. Because of this finding, for all subsequent analysis, matching of controls included this variable. Detailed analysis was possible for 1,513 cases and an equal number of matched controls. Matching of controls included age, race, last menstrual period and birth date. There were 312 women with temperatures of $\geq 38^{\circ} \mathrm{C}$ and UTI. Clinical findings significantIy increased among women with UTI included anemia, thrombosis a/o phlebitis, abortions of siblings and delivery by cesarean section.

For the children, there was a significant association with low birth weight, shortened gestation, stillbirths and deaths in the neonatal period. Most effects were found for UTI during the second or third trimester.

90 WHO HAS THEIR BLOOD PRESSURE MEASURED? John T. Sladky, Bennett A. Shaywitz, Norman J. Siegel, Dept. of Ped., Yale Univ. School of Med., New Haven, Conn.

Over the past several years increasing emphasis has been placed on the routine measurement of blood pressure (BP) in children. To determine which children were having their BP measured during hospitalization, 184 consecutive admissions to the pediatric service over a 2 -month period were reviewed. A BP was recorded on admission by either a nurse or physician in 111 cases $(60 \%)$ and at some time during the hospitalization in an additiona1 19 cases $(11 \%)$. Consequently, in 54 cases $(29 \%)$ no blood pressure was determined: 53 of these 54 patients were $<6$ yrs of age at the time of hospitalization and comprise $45 \%$ of the total admissions (118) in that age group.

Specific indications for $\mathrm{BP}$ measurement on history, P.E., or laboratory data were found in $109(60 \%)$ of the admissions: 89 ( $82 \%)$ had had a BP recorded; and of the 20 (18\%) who had no BP taken, 19 were $<6$ yrs of age. However, $47(71 \%)$ of the 66 patients $<6$ yrs of age with an indication of BP measurement had had a BP recorded. Importantly, al1 9 children in this age group who were hypertensive (diastolic >2 S.D. for age) had a specific indication for $B P$ measurement whereas on $1 y$ 7/10 hypertensive pts $>6$ yrs of age had an indication for BP measurement.

These data suggest: 1) virtually all children $>6$ yrs of age had a BP recorded duxing hospitalization; 2 ) on 1 y $55 \%$ of pts $<6$ yrs of age had a BP taken; 3) BP was recorded more frequently (71\%) in this younger age group when there was a specific indication; and 4) the majority of hypertensive pts had one of the specific indications which suggested the need for BP measurement.

205 CLINICAL, LABORATORY, EPIDEMIOLOGICAL FEATURES OF A

295 RECENTLY RECOGNIZED VIRAL ENTERITIS. Susan Tallett, Benny Kerzner, Carrie MacKenzie, Peter Middleton and Richard Hamilton (Spon. by H.W. Bain), Res. Inst., Hosp. for Sick Children, Depts. of Ped. and Microbiol, , Univ. of Toronto, Canada. We studied 28 children under age 3 , hospitalized with acute diarrhea caused by unclassified fecal virus II (UFV II) (previously termed orbi, reo-like, duo or rota virus). In a11, stools contained UFV II virus, but no enterotoxin-producing E,coli. All vomited, 24 were febrile, 13 dehydrated, 10 had apparent URI. Stools contained $\mathrm{Na} 27.9 \pm 3.6, \mathrm{~K} 43.8 \pm 8.1$ and $\mathrm{Cl} 16.4 \pm 2.8 \mathrm{mEq} / \mathrm{l}$ (m \pm s.e.), no blood, no excess fat, sugar concentration exceeded normal $(0.5 \mathrm{~g} / 100 \mathrm{ml})$ in 4 infants. A compensated metabolic acidosis was noted in 25 cases, serum $\mathrm{Na}$ was normal in all but 6,5 of whom were hypernatremic. In hospital, vomiting, fever settled in one day, diarrhea in most by 4 and all were home by 9 th day. symptoms recurred in 4 but by 3 weeks all were well, stools then contained $\mathrm{Na} 10.0 \pm 1.3, \mathrm{~K} 34.1 \pm 4.0$ and $\mathrm{C} 14.6 \pm 0.7 \mathrm{mEq} / \mathrm{I}$ but no UFV II, serum antibody titre to UFV II had risen in 20 of 23 . In 59 contacts, adults and children from 19 households, we found seroconversion in 19 , G.I. symptoms in 13 , UFV II virus in stools of 6,3 of whom were asymptomatic. This newly diagnosable disease is the major cause of acute diarrhea in many communities, causing up to $80 \%$ of hospitalized gastroenteritis in Toronto in winter. Our study has shown a consistent disease pattern and high infectivity. By defining the clinical features and functional defects caused by UFV II our data should aid practitioners in preventing, diagnosing and actively treating the problem.
WHO WILL ACCEPT GENETIC SCREENING? - COOPERATION IN AN ALPHA 1 ANTITRYPSIN RESEARCH SCREENING STUDY WAS UNRELATED TO RESPIRATORY DISEASE. John C. Vance, Klaus J. Roghmann, Robert H. Schwartz. Univ, of Rochester Sch.of Med, and Dent., Dept. of Pediatrics, Rochester, N.Y.

Genetic screening has achieved considerable importance because of increased availability of tests and potential preventive aspects such as amniocentesis and available abortion. Yet little is known about the acceptability of such screening to patients.

At the end of a household interview for a community health survey (random sample of 1104 families), the genetic deficiency of alpha 1 antitxypsin and its relationship to chronic lung disease was explained and genetic screening was offered. $57.1 \% \quad(n=630)$ of eligible families agreed to a separate visit by a nurse during which $10 \mathrm{cc}$ of blood would be drawn from each parent, a questionnaire administered and lung function studies performed.

We found significant relationship between positive response and being white $(p<0.05)$, living in the suburbs $(p<0.01)$, the father having a high education $(p<0.02)$, and a high family income $(p-0.001)$. However respiratory illness, such as asthma, in the responding mothers or their children was not related to positive response.

In this study, agreement to participate in a research screening program was related to socioeconomic factors rather than to illness. For planning research and service screening programs, our findings point to the danger of including only the healthy middle class motivated to participate and missing the sick and lower class person possibly in greatest need of screening.

\section{7} VIREMTA IN CHILDREN WITH HIGH TEMPERATURE. Yamauchi, T., Gammon, J.A., Imagawa, D., and Nishita, M.Y. Sch. of Med., and Ark. Child. Hosp., Dept. Of Peds., Torrance, CA. and Little Rock, AR.

Forty-one childxen between the ages of 7 months and 15 years were studied because of $103^{\circ}$ or greater temperatures and no obvious source of infection. No child had received antibiotics for at least two weeks prior to this evaluation. Every child had bacterial cultures of blood, throat, cerebrospinal fluid (CSF), and urine. In addition, viral cultures of throat, stool and buffy coat were a1so obtained. Thirty-eight children had their CSF cultured for viruses.

Viruses were recovered from 14 children (34\%). Eight children had viruses isolated from their throats, 7 from feces, 2 from $\mathrm{CSF}$ and 6 from the blood. Several children had viruses cultivated from multiple sites. Paramyxovirus was recovered from the blood and pharynx of 2 siblings. One of these children developed parotitis 5 days after culturing. The only symptom in the other child was the initial febrile episode. Picornaviruses were retrieved from cultivation of buffy coats in 4 additional children. One child was carrying the picornavirus in his throat and stool as well as blood.

The recovery of paramyxovirus or picornavirus in the blood of children with high temperatures is unique. The possibility that temperature elevation secondary to viremia is similar to that seen with bacteremia and must be considered in the evaluation of high temperatures in children.

298 AN OUTBREAK OF MEASLES AMONG ADOLESCENTS. Leonard Weiner, Robert Corwin and Phillip Nieburg (Intro. by H.A. Feldman). SUNY, Upstate Med. Ctr., Depts. of Peds. and Prev. Med., Syracuse, N.Y.
In a May 1975 outbreak 147 adolescents, ages $12-16$ yrs., were dentified as having rubeola by a physician or school nurse. A junior high school (JHS) with an enrollment of 1,122 had 131 cases. Among the 147 students, 54 were seen by physicians. Their immunization data were obtained from physicians' records; $19 / 54$ $(35 \%)$ had received live measles virus vaccine (L) without measles immune globulin (MIG), after the age of $1 \mathrm{yr}$. Fourteen of the 19 recelved immunization more than 8 yrs. previously; the others in 1968 (1), 1969 (2) and 1971 (2). The remaining 35 students received: killed virus vaccine $(K)$ or $K+L(5), L+M I G$ (4), L at <1 yr. of age (4), L+(?) MIG (4), only serum immune globulin (SIG) for exposure (6), no vaccine but previous history of rubeola (9); uncertain in 3 . These 54 were immunized at various ages in a variety of geographic locations. Paired hemaglutination-inhibition antibody (HIA) titers confirmed the diagnosis of rubeola in 14. Duration of illness was 7-25 days; 2 students with iliness $>20$ days required hospitalization for atypical measles. Both had received KKL series and had the highest convalescent HIA titers $(>512)$. No index case(s) was identified and no secondary cases occurred within the families of the 54. Three sibling pairs, similar in age (13-15 yrs.) and attending the same JHS, had measles concurrently. This measles outbreak in immunized adolescents ratses questions about the duration of protection. 


\section{GASTROENTEROLOGY AND NUTRITION}

299

TOTAL PARENTERAL NUTRITION IN ANURIC CHILDREN. CarOlyn Abitbol and Malcolm A. Holliday, University of California, San Francisco, Department of Pediatrics.

Children who are uxemic and acutely sick often are unable to eat and may be body protein depleted. The acute illness often induces body protein catabolism and an increase in urea production (UrP). Since they are uremic, this increases the degree of uremia. Therapy is designed a) to spare body protein, b) to suppress UrP, and $c$ ) to increase body protein nitrogen balance using essential amino acid nitrogen (EAAN). Body protein nitrogen balance, with a correction, is derived as the difference between EAAN intake and UrP. We report results in 6 anuric malnourished children given glucose alone and glucose + EAAN successively. Daily UrP was derived from changes in urea pool during each day of study and nitrogen was measured from intake of solutions containing EAAN which were given under strict supervision. Glucose alone, providing from $20-70 \mathrm{kcal} / \mathrm{kg} /$ day, progressively reduced UrP, i.e., the more glucose given, the greater the protein sparing effect (kcal vs. UrP; $x=-.81)$. Glucose + EAAN, which provided from 60-100 mg nitrogen/ $\mathrm{kg} /$ day, suppressed UXP frequently to zero. SUN increased $3 \mathrm{mg} / \mathrm{dl} /$ day when amino acids were given compared with an increase of $15 \mathrm{mg} /$ dl/day when only glucose was given. Nitrogen balance was positive (+ $38 \mathrm{mg}$ nitrogen $/ \mathrm{kg} /$ day) in children given EAAN. The study suggests aggressive parenteral nutritional therapy is useful in acute anuria in suppressing urea production in children. Adding essential amino acids further suppresses urea production and results in a positive protein nitrogen balance.

INEFFICACY OF POSTOPERATIVE INTRAVENOUS HYPERONCOTIC

300 ALBUMIN THERAPY (IV AIb) ON PERTANASTOMOTIC BOWEL EDEMA. Eugene W. Adcock, Gabrie1 G. Haddad, John M. Daly, Frank H. Morriss, and Stanley J. Dudrick. (Spon. by R. R. ments of Pediatrics and Surgery, Houston.

Wound edema following bowel anastomosis may delay healing and cause poor motility and absorption. The use of IV Alb to decrease such edema is unproven, although it is often administered and may have untoward side effects. This study was conducted to assess the efficacy of IV Alb.

Eighteen Sprague-Dawley rats had standardized small bowel anastomoses performed. At 36-48 hours following surgery, 9 (Group I) received IV $0.5 \mathrm{gm}$ albumin/kg body weight and the other 9 (Group II) received an identical volume of $5 \%$ glucose in water IV. Following infusion of either solution for one hour, segments of healthy (control) and perianastomotic bowel were removed from each rat and the percentage of extracellular fluid (\%ECF) was measured using inulin-carboxyl- ${ }^{14} \mathrm{C}$.

$\begin{array}{lcccc}\text { ResuIts: } & \text { Control Tissue \%ECF* } & \frac{\text { Perianastomotic }}{\text { Tissue \%ECF* }} & \text { P** } \\ \text { Group I } & 34.38 \pm 1.73 & \frac{P}{37.23 \pm 2.14} & <0.005 \\ \text { Group II } & 28.68 \pm 3.20 & 36.21 \pm 2.78 & <0.025\end{array}$
In each group there was significant perianastomotic edema. How ever, between Groups I and II there is no significant difference in either the control or perianastomotic tissue \%ECF. No difference in hematocrit, serum albumin, or osmolality was found between groups. Our study demonstrates that this regimen of IV Alb does not reduce bowel edema. *Mean \pm S.E.M. $* \star$ Paired $t$ test

CONTROLLED TRIAL OF INTRAVENOUS GLUCOSE VS GLUCOSE

301 AND AMINO ACIDS IN PREMATURE INFANTS. T.L. Anderson, J.F. Nicholson and W.C. Heird. Columbia University College of Physicians and Surgeons, Dept. of Peds., N.Y.

To test the efficacy of adding amino acids to conventional intravenous therapy for sick premature infants, 2 groups (BW 1000-2000 $\mathrm{gm}$ ) were randomly assigned to nutritional regimens delivering $60 \mathrm{cal} / \mathrm{kg} / \mathrm{d}$. Regimen $A(n=7)$ delivered $15 \mathrm{gm} / \mathrm{kg} / \mathrm{d}$ of glucose. Regimen B $(n=7)$ delivered $12.5 \mathrm{gm} / \mathrm{kg} / \mathrm{d}$ of glucose $\mathrm{plus}$ $2.5 \mathrm{gm} / \mathrm{kg} / \mathrm{d}$ of crystalline amino acids. Electrolytes, minerals and vitamins were identical. Weight gain, nitrogen balance, plasma essential fatty acid (EFA) status and aminograms were compared after 5 days. Infants receiving A lost $10.5 \mathrm{gm} / \mathrm{kg} / \mathrm{d}$ whereas those receiving $B$ gained $8.7 \mathrm{gm} / \mathrm{kg} / \mathrm{d}(\mathrm{p}<0.005)$. Nitrogen balance was $-0.12 \mathrm{gm} / \mathrm{kg} / \mathrm{d}$ with $A$ and $+0.20 \mathrm{gm} / \mathrm{kg} / \mathrm{d}$ with $B$ $(p<0.005)$. Blood acid-base status and plasma electrolytes were similar in the 2 groups. Blood urea was higher in infants receiving $B$ ( $13 \mathrm{mg} / 100 \mathrm{ml}$ vs $7 \mathrm{mg} / 100 \mathrm{ml}$ ). EFA deficiency developed in both groups, but more rapidly in B. Plasma amino acid levels were higher in infants receiving $B$; threonine, methionine serine, glycine and alanine consistently exceeded normal fasting or post-prandial levels. In infants receiving $A$, plasma valine, leucine, isoleucine, lysine and arginine were of ten below normal fasting values. Plasma cystine was low in both groups These data demonstrate that provision of as little as 60 $\mathrm{cal} / \mathrm{kg} / \mathrm{d}$ with amino acids results in weight gain and positive nitrogen balance and indicate that classical nutritional requirements of premature infants should be re-evaluated.

\section{2}

HYPERLIPOPROTEINEMIA IN THE NEWBORN. Billy F. AndrewS, Larry N. Cook, Sophia Franco, Philip Kalayil, Univ.

Louisville.

Advances in diagnostic techniques and reports in the medical and 1 ay press regarding atherosclerosis, heart disease and more recently the specific hyperlipoprotenemias have generated interest in early recognition of hyperlipoprotenemias because of possible treatment benefits. To determine the incidence of hyperlipoprotenemias, the time of sampling was made concomitantly with that required for blood sampling for phenylalanine on the day of departure from the newborn nursery on a three to four hour fasting sample. Microtechniques for triglycerides, cholesterol and lipoprotein electrophoresis with densitometric analysis have been performed on 1928 infants. Elevated lipids were surprisingly detected in 31 infants; 28 with Type IV; 2 with Type III and one with Type $V$. Early investigations of parents, siblings, and other family members has revealed early death and currently major related diseases in many. Should dietary and/or accepted adult treatment be initiated prophylactical $\dot{y}$ or therapeutically? What should be the role of the pediatrician who diagnoses the first family member?

DCCHARIDASE ACTIVITIES IN PANCREATIC INSUFFICIENC

303 AND REAPPRAISAL OF LACTASE ACTIVITY IN CYSTIC FIBROSIS Irena Antonowicz, Emanuel Lebenthal, Harry Shwachman Harvard Medical School, Children's Ilospital Medical Center, Boston

In a recent study Alpers and Tedesco $(B B A, 401,28,1975)$ found elevated disaccharidase activities in pancreatetomized rats. In light of this observation we studied the activity of disaccharidases in 63 patients with cystic fibrosis(CF) and pancreatic insufficiency. We found significantly higher disaccharidase activities in patients with $C F$ as compared with control patients (177) of the same age, the same ethnic group and with normal morphological appearance of the intestinal mucosa. For patients with CF lactase (L) : $45.7 \pm 27.0$, sucrase $(S): 91.6 \pm 38.6$, maltase (M) : $285.4 \pm 131.2$, palatinase $(\mathrm{P}): 24.2 \pm 13.3 \mathrm{uM} / \mathrm{min} / \mathrm{gProte}$ in, as compared with controls $L: 34.2 \pm 11.3, S: 61.4 \pm 19.3, M: 201.3 \pm 62.4$ and $\mathrm{P}: 16.5 \pm 5.5 \mathrm{uM} / \mathrm{min} / \mathrm{gProtein}$. In patients with $\mathrm{CF}$ under 5 years of age and with normal intestinal mucosa no low lactase activity was found. In those with $\mathrm{CF}$ over 5 years of age, two groups emerge one with high and one with low lactase activity as occurs in healthy Caucasian controls. In 7 patients with $C F$ with grade 2 atrophy of the intestinal mucosa when compared to 14 patients with chronic diarrhea and the same degree of mucosal injury, the decrease in the activity of all disaccharidases followed the same pattern. This study showed that in humans with pancreatic insufficiency the activity of disaccharidases is elevated and that low lactase activity in patients with CF is a normal phenomenon not related to the disease entity.

EFFECT OF KAOLIN-PECTIN ABSORBENT ON STOOL CONTENT IN 304 LACTOSE-INDUCED DIARRHEA. Robert D. Beck, H. Juhling McClung, Priscilla Powers (Spon. by Bruce D. Graham), The Ohio State Univ., Columbus Children's Hospital, Dept. Ped.

Kaolin-pectin absorbents are currently in widespread use for treatment of "non-specific" diarrhea. Although these compounds are said to be absorbents, no reports have appeared on the effect of kaolin-pectin mixtures on stool electrolyte and fat losses.

Diarrhea was induced with a $40 \%$ lactose diet in Sprague-Dawley male rats who served as their own controls during a four way cross over study. Stool electrolyte and fat content were determined for each rat while on the following diets (A) control, (B) control plus kaolin $20 \%$-pectin $1 \%$, (C) $40 \%$ lactose, diarrhea producing diet, (D) $40 \%$ lactose plus kaolin $20 \%$-pectin $1 \%$.

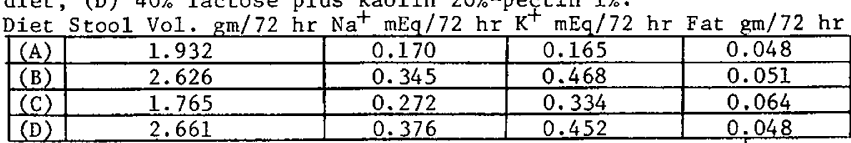

Lactose-induced diarrhea increased stool losses of $\mathrm{Na}$ $(p<0.005), \mathrm{K}^{+}(p<0.001)$, and fat $(p<0.025)$. When kaolin-pectin absorbent was added to the diarrhea diet, stool fat malabsorption was corrected to control levels, stool volume and $\mathrm{K}^{+}$losses were slightly increased and stool $\mathrm{Na}^{+}$losses were significantly increased $(p<0.025)$. The addition of kaolin-pectin absorbent appeared to complicate the electrolyte management in this diarrheal model and suggests that increased caution is required when kaolin-pectin absorbent is used in severe diarrheal states. 
305

ANALYSIS OF THE IMMUNOCOMPETENCE OF HIIMAN MILK

LYMPHOCYTES. Alan E. Beer \& Michael J. Parmely (Spon. by Philip Sunshine). Department of Ceil Biology, The University of Texas Health Science Center at Dallas.

Immunological transactions between a mother and her offspring may continue beyond birth. Our recent studies in rats and mice have documented that during suckling the perinate receives a dowry of maternal immunities in the form of viable lymphocytes in the mammary exosecretion. Human colostrum and milk contain large numbers of macrophages and lymphocytes, $1.3 \times 10^{6}$ and $1.45 \times 10^{5} / \mathrm{ml}$ respectively。 Purified human milk lymphocytes (ML) were cultured in microtiter plates with appropriate mitogens or antigens and were labelled with tritiated thymidine to assess the extent of DNA synthesis. Peripheral blood lymphocytes (PBL) from the same patient were cultured under identical conditions in order to compare the reactivity of both cell populations. It was found that: I) ML are as viable and long-lived as PBL in culture; 2) The total thymidine incorporated by ML following PHA stimulation is less than the uptake by PBL; 3) Mixed lymphocyte reactive lymphocytes (MLC) reside in milk; however, their reactivity is somewhat muted when compared with MLC-reactive PBL; 4) ML from tetanus or Candida positive patients are usually unresponsive in culture; however, the reverse applies for responses to the $k 1$ antigen of E.coli; 5) This selective reactivity of ML is not due to suppressive factors, antigen specific populations of suppressor cells, or lack of adherent cells in milk. The data suggest that milk may lack certain antigen-reactive lymphocytes in pexipheral blood and be a source of specific clones of reactive maternal lymphocytes. Supported by NIH grants N33314 and N30160.

306 EFFECTS OF ACUTE HYPOTHERMIA ON INTESTINAL FUNCTION. Jon Blumenfeld, Fima Lifshitz, Raul A. Wapnir and Saul Teichberg, Dept. of Peds., North Shore Univ. Hosp., Manhasset, NY and Dept. of Peds., Cornell Univ. Med. Col., NY, NY. To discriminate the effects of hypothermia from those of other metabolic stresses on intestinal function; cell turnover, enzymes, histologyand ultrastructure were assessed in four groups of 60-80 gms. rats. Shaven and unshaven rats were exposed to a cold stress at $4^{\circ} \mathrm{C}$ for $4-6 \mathrm{hrs}$. (SE and UE). Control animals remained at room temperature $\left(26^{\circ} \mathrm{C}\right)(\mathrm{SC}$ and UC). Hypothermia was induced in group $S E$, with deep rectal temperature (T) of 230 $\pm 2^{\circ} \mathrm{C}$. UE animals had $\mathrm{T}=35^{\circ} \pm .5^{\circ} \mathrm{C}$. Groups SC and UC were normothermic $\left(T=36^{\circ} \pm 1^{\circ} \mathrm{C}\right)$. All rats had similar arterial $\mathrm{pO} 2$ and hepatic tryptophan oxygenase levels. Acute hypothermia induced a sloughing of superficial cells from the villus into the lumen of the gut, as indicated by a decrease in intestinal mucosal/luminal cell number ratios (means + SD; $\mathrm{SE}=.76 \pm .29 ; \mathrm{SC}=1.76 \pm .45, \mathrm{p}<.05) .{ }^{3} \mathrm{H}$ thymidine incorporated into DNA in intestinal mucosal cells and luminal washes was similar in all groups (mucosal: $\mathrm{SE}=.018 \pm .010, \mathrm{SC}=.014 \pm .004, \mathrm{UE}=.014 \pm .010, \mathrm{UC}=$ $.020 \pm .010 ;$ luminal: $\mathrm{SE}=.135 \pm .110, \mathrm{UE}=.139 \pm .050, \mathrm{UC}=.095 \pm$ $.059 \mathrm{dpm} / \mathrm{mg}$ DNA. In addition, intestinal disaccharidases (lactase, sucrase, maltase) and alkaline phosphatase were not altered. There were no histological and ultrastructural abnormalities caused by acute hypothermia. Therefore, intest inal cellular alterations reported earlier in hypothermia may have been caused by associated factors other than a decreased body temperature.

\section{7}

AGAROSE-GEL LIPOPROTEIN ELECTROPHORESIS IN REYE'S SYNDROME (RS) Robert C. Bobo, William K. Schubert, John C. Partin, Jacqueline S. Partin, Cathy McGraw Children's Hospital Research Fdn., Cincinnati, Ohio

Defective formation of very low density lipoprotein-like particles(VLDL) by the liver of patients with severe RS has been reported. This report describes the results of plasma lipoprotein electrophoresis(LPE) in 41 patients with biopsy or autopsy proven RS. LPE at the time of diagnosis showed that 29 patients with severe RS (Grades III, IV,V) had absent pre-beta-lipoprotein (PBL) and 4 had trace amounts. Of 8 patients with mild to moderate RS (Grades I,II) 4 had absent PBL, 2 had trace and 2 had normal amounts. The PBL returned to normal in al1 32 survivors except 2 during the recovery phase and at followup. Alpha-lipoprotein was decreased or absent in 20 patients with severe RS and 2 with mild to moderate RS. Beta-lipoprotein was reduced in 15 patients with severe RS and in 4 with mild to moderate RS. Electron microscopy (EM) of the liver of patients with severe RS showed flat and empty golgi saccules (site of storage of VLDL) with reaccumulation of VLDL during the recovery phase and at followup. Eight severely ill children clinically suspected of having RS because of encephalopathy and elevated liver enzymes had PBL present in their plasma. They were subsequently proven by liver biopsy, with light and EM, not to have RS although 7 had mild to severe fatty change in their liver. Thus a predictable pattern of blocked hepatic triglyceride secretion is consistently seen early in RS which relates well to the severity of the hepatic injury and to hepatic recovery.

Support: NIH \#IF-22-AM-03204-01 and CRC Grant \#RR-00123
308 LATIONSHIP TO NECROTIZING ENTEROCOLITIS (NEC) Edwin G. Brown, Eugene Ainbender and Avron ‥ Sweet. Mount Sinai School of Medicine, Department of Pediatrics New York, New York.

In our case material, $89 \%$ of infants with NEC had been fed formula. Of these, $70 \%$ developed symptoms within 72 hours after the first feed and most of the remainder were symptomatic before 28 days from onset of feeding. Since symptoms of NEC are common to endotoxic shock and because absorption of endotoxin from the gut has been shown in animals, it is possible that endotoxin may be the cause of many symptoms of NEC in human infants.

We have measured endotoxin and counted the number of bacteri in fresh stool of formula fed and breast fed term infants as well as formula fed low-birth-weight infants. Regardless of the kind of milk given, $75 \%$ of infants had at least $10 \mu \mathrm{g}$ endotoxin and $5 \times 10^{8}$ Gram-negative bacteria/g wet stool within 72 hours after starting feeds. The remainder attained the same values within 14 days after initiation of feeds. Low-birth-weight and term infants had $0.1 \mu \mathrm{g}$ or less endotoxin/g wet stool until feedings were begun. These findings indicate that the presence of endotoxin and Gram-negative bacteria in the stool of newborn infants results primarily from the intake of milk and is not influenced by the source of milk used. It is of interest that the appearance of large numbers of Gram-negative organisms and endotoxin in stool and the first symtoms of NEC frequently occur within 72 hours after the intiation of feedings.

309 LIVER IN REYE'S SYIDDOME (RS): NORMAL $\mathrm{K}_{\mathrm{m}}$ OF MITOCHONDRIAL UREA CYCLE ENZYMES (CPS, OTC) AND FATLURE OF 4PENTENOIC ACID (PA) TO ACCOUNT IN VITRO FOR THE TRANSIENT REDUCTION OF ACTIVITY OF THE TWO ENZYMES IN VIVO. Ted Brown, George Hug, Lester Lansky, Kevin Bove and William Schubert, Departments of Pediatrics, Universities of Cincinnati and Kansas, Cincinnati, OH and Kansas City, KS.

Our finding of reduced carbamy1 phosphate synthetase(CPS) and ornithine transcarbamylase(OTC) in RS liver has now been confirmed by others. They proposed increased $\mathrm{K}_{\mathrm{m}}$ of OTC or in vitro inhibition by PA of CPS and OTC as genetic and/or acquired basis of RS We studied liver of 31 "normal" and 13 RS patients some biopsied repeatedly for: urea cycle enzymes(in $\mathrm{nm}$ citrulline for CPS, OTC $\mathrm{nm}$ urea for ASS, ASL, arginase/mg prot/min); $\mathrm{K}_{\mathrm{m}}$ of CPS and OTC(in $\mathrm{mM}) ;$ effects on CPS or OTC of $30 \mathrm{~min}$ incubation at $37^{\circ} \mathrm{C}$. with PA $10^{-3}$ or $10^{-2} \mathrm{M}$ [norma1s in brackets]: CPS $6.27 \pm 2.45$ [11.54 \pm 4.24 ]; OTC $139.19 \pm 41.83[307.49 \pm 94.15]$; ASS $16.19 \pm 3.78[16.75 \pm 6.37]$; ASL $16.10 \pm 3.36[15.77 \pm 4.68]$; arginase $746.68 \pm 160.30[713.17 \pm 258.01] ; \mathrm{K}_{\mathrm{m}}$ CPS for $\mathrm{NH}_{4}^{+} 38.68 \pm 19.62[52.31 \pm 22.67] ; \mathrm{K}_{\mathrm{m}}$ CPS for ATP $4.42 \pm 2.84$ $[4.21 \pm 1.93] ; \mathrm{K}_{\mathrm{m}}$ OTC for ornithine $0.24 \pm 0.12[0.23 \pm 0.11] ; \mathrm{K}_{\mathrm{m}}$ OTC for carbamyl phosphate $0.80 \pm 0.61[0,81 \pm 0.45]$. Since PA did not reduce CPS or OTC in vitro, the significant $(\mathrm{p}<0.005)$ in vivo reduction by 46 and $56 \%$ has not been linked to PA. This reduction is greatest at onset of RS and then disappears as shown in late autopsy and repeat biopsy specimens. Normal $\mathrm{K}_{\mathrm{m}}$ did not indicate a genetic basis of RS 。CPS and OTC reduction may be typical for RS; it did not occur in liver with fatty metamorphosis of aspirin intoxication or argininosuccinic synthetase deficiency.

310 COMPARED TO CONTROLS. Dennis L. Christie, Marvin E. Ament. UCLA School of Medicine, Department of Pediatrics, Los Angeles, California, 90024.

Fifty-eight children and adolescents between 5 and 20 years of age had fasting serum gastrin determined by radioimmunoassay. Nine children between 11 and 15 years of age with duodenal ulcer (DU) diagnosed by evidence of an ulcer crater on upper gastrointestinal radiographs or by gastroduodenoscopy also had fasting serum gastrin levels determined during an active phase of their disease.

The mean fasting serum gastrin for the duodenal ulcer children was $39.4 \pm 8.0 \mathrm{pg} / \mathrm{ml}$; the 58 controls had a level of $39.9 \pm 6.2$ $\mathrm{pg} / \mathrm{ml}(\mathrm{P}>0.05)$. Statistically there was no significant difference in fasting gastrin among the control children when age was considered. (Table I).

These results demonstrate that fasting serum gastrin in children with duodenal ulcer is similar to levels in a control population.

\begin{tabular}{|c|c|c|c|}
\hline \multicolumn{4}{|c|}{$\begin{array}{l}\text { FASTING SERUM GASTRIN LEVELS IN CONTROL } \\
\text { AND DUODENAL ULCER CHILDREN }\end{array}$} \\
\hline & Age (years) & No. Patients & Mean Gastrin \\
\hline Normals & $5-10$ & 21 & $28.6 \pm 3.3$ \\
\hline & $11-15$ & 22 & $45.4 \pm 7.8$ \\
\hline & $16-20$ & 15 & $41.4 \pm 14.6$ \\
\hline
\end{tabular}

${ }^{*}(\mathrm{pg} / \mathrm{ml} \pm 1$ S.E. $)$ 
311 GASTRIN RESPONSE TO A PROTEIN MEAL IN CHILDREN WITH DUODENAL ULCER. Dennis L. Christie, Marvin E. Ament. UCLA School of Medicine, Department of Pediatrics, Los Angeles, California, 90024.

Gastrin release after a protein meal was evaluated in 9 normal children (N) and 8 with duodenal ulcer (DU). Each child ate 2 hard boiled eggs, 2 pieces of buttered toast and $8 \mathrm{oz}$. of skim milk. Venous blood was withdrawn just prior to eating and 15,30, 45 and 60 minutes after eating. Serum gastrin determinations were done by radioimmunoassay. Fasting serum gastrin was similar in DU and $\mathrm{N}$. Both groups showed a statistically significant rise in gastrin levels after eating. Mean serum gastrin was higher at each time period for DU when compared to $\mathrm{N}$ but was significantly different only at 15 minutes $(P<0.025)$.

(P values represent change of gastrin over fasting level)

\begin{tabular}{|c|c|c|c|c|c|}
\hline & Fasting & $15^{\prime}$ & $30^{\prime}$ & $45^{\prime}$ & $60^{\circ}$ \\
\hline DU & 42.5 & $9 5 \longdiv { . 0 }$ & 76.9 & 79.5 & 72.6 \\
\hline & 8.4 & \pm 18.5 & \pm 15.1 & \pm 18.9 & \pm 16.8 \\
\hline${ }_{P}$ & & $<0.025$ & $<0.025$ & $<0.05$ & $<0.05$ \\
\hline $\mathrm{N}$ & 32.3 & 43.8 & 51.7 & 48.0 & 51.6 \\
\hline $\mathrm{P}$ & \pm 6.3 & $\begin{array}{l} \pm 8.9 \\
\text { NS }\end{array}$ & $\begin{array}{r} \pm 11.3 \\
<0.05\end{array}$ & $\begin{array}{r} \pm 9.4 \\
<0.01\end{array}$ & $\begin{array}{l} \pm 7.8 \\
<0.001\end{array}$ \\
\hline
\end{tabular}
Conclusions: 1) After a protein stimulus $\mathrm{N}$ and DU children have a gastrin response similar to adults. 2) DU children have a higher gastrin rise after eating which peaks earlier than in N. children (15 minutes post-stimulus $(\mathrm{P}<0.025)$.

CARISON OF ARM PATCH (APS) AND WHOLF. BODY SWFAT

312 (WBS) IN CYSTIC FIBROSIS (CF) PATIENTS ANN CONTROLS. Lawrence F. Cohen, Philip M. Farrell, David W. Lundgren and Paul A. di Sant'Agnese. NIH, Bethesda, Md.

A comparison of sweat electrolytes from single site (ADS by pilocarpine iontophoresis) and whole body (WBS, rinsed after thermal stimulation) was done in $19 \mathrm{CF}$ and 5 control young adults. For all subjects, the two methods correlated significantly $(\mathrm{p}<.001)$ for sweat $\mathrm{Cl}(\mathrm{r}=.830)$ and $\mathrm{Na}(\mathrm{r}=.842)$ concentration. Sweat $\mathrm{Na}$ and $\mathrm{Cl}$ were significantlv higher $(\mathrm{p}<.001)$ for CF by each method while the range of sweat rates was similar.

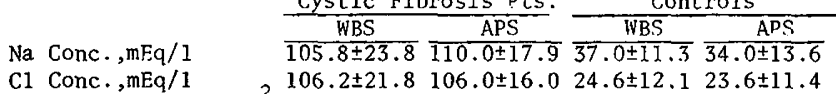

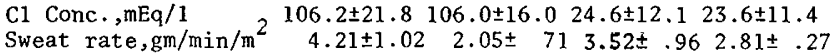

By each method, Na excretion rate $\left(\mathrm{mEq} / \mathrm{min} / \mathrm{m}^{2}\right)$ was a linear function of sweat rate for CF subjects (slope $.125 \mathrm{mFq} / \mathrm{gm}$ for APS and $.116 \mathrm{mEq} / \mathrm{gm}$ for WBS) but not controls, for the range of sweat rates induced. Sweat rates were greater by thermal stimulation than pilocarpine iontophoresis, but final Na concentration was not affected because $\mathrm{Na}$ excretion rate varied with CF sweat rate. These data support recent studies suggesting that the CF sweat defect is due to a Na reabsorption inhibitory factor.

This is the first comparison of APS and WBS in CF subjects. Since the electrolyte concentration of WBS reflects single-site collection, whole body sweat may be useful in studying chemical and other properties of sweat.

DELAYED SMALL BOWEL TRANSTT IN ACUT'E AND CHRONIC

313 DIARRHEAL SYMDROMES. J.L. Cupe110, J.T. Rodriguez; ment of Pediatrics, Baylor College of Medicine, Houston, Texas 77025. *Unidad de Estudios Clinicos, Roosevelt Hospital, Guatemala City, Guatemala.

Although it is widely recognized that mouth to anus transit time is accelerated in diarrhea, little information is available concerning transit in the small bowel. Twenty-four children between the ages of 6 months and 6 years with acute or chronic diarrheal syndromes were investigated for small bowel transit time (SBTT). Using an application of the Fick principle, all subjects had a double lumen catheter positioned in the small intestine with ports located 1 meter apart. A bolus of 4\% PEG was delivered into the stomach and the rate of appearance was monitored at the two collecting ports. Four normal children had an SBT'T of $60 \mathrm{~min} /$ meter. One subject with shigella was $30 \mathrm{~min} / \mathrm{m}$. Twelve children with acute non-specific diarrhea had a mean SBTT of $90 \mathrm{~min} / \mathrm{m}$ with a range of 75 to $120 \mathrm{~min} / \mathrm{m}$. Seven subjects with sub-acute nonspecific diarrhea had a mean of $100 \mathrm{~min} / \mathrm{m}$ with a range of 90 to 150. In each case, the increase in SBTT was associated with an increase in luminal free bile acids.

This investigation points out that transit in the small bowel may actually be delayed in certain kinds of diarrhea and suggests that the deamidation of luminal bile acids results from relative stasis in the small bowel in non-specific diarrheal syndromes.

Research support is acknowledged from NIH Training Grant USPH 5 ROI AM-05721, USPH Research Grant RR-00188 and Universidad del Zulia, Maracaibo, Venezuela.
314 BACTERIAL FLORA OF HUMAN MILK. Arthur L. Eidelman, George Szilagyi and Shirley Eahie. (Intr. by: Lawrence Gartner) Depts. Of Peds \& Lab. Med., Hosp. of the Albert Einstein College of Medicine, Bronx, N.Y.

To assess the potential bacteriologic risk of utilizing fresh unsterilized human milk for feeding of high risk infants, milk was obtained from 44 healthy mothers between the ist and 3 rd day of life utilizing a sterile Egnell breast pump after cleansing of the breast with sterile water. Individual cultures were obtained from the nipple and milk at the time of collection.

All cultures were positive. In $37(80 \%)$ mothers the same organisms were recovered from both the nipple and milk. Culture of the nipple revealed: coagulase neg. staph (CNS) 43 (38\%), strep viridans $3(20 \%)$, coagulase pos. staph (CPS) $6(14 \%)$ alpha strep $3(7 \%)$, enterococcus $2(5 \%)$ and Klebsiella $1(2 \%)$ From the milk CNS was cultured in $43(98 \%)$, CPS $3(20 \%)$, strep viridans $9(20 \%)$, alpha strep $3(7 \%)$, enterococcus $2(5 \%)$, Klebsiella, E. Coli and Herellia $1(2 \%)$ each. Colony counts in the milk exceeded $100,000 / m l$ in two samples. In the remainder the mean colony count was $15,700 \pm 20,000$ (SD) (range 900-90,00:/ $\mathrm{ml}$ ).

The data demonstrates that cleanly collected unsterilized milk is contaminated by the bacterial flora present on the nipple. The predominant organisms are non-pathogenic staph and strep, in individual samples colony counts exceeding $10,000 / \mathrm{ml}$ of coagulase positive staph and pathogenic gram negative organism were recovered.

\section{DIETARY EVALUATION OF CHILDREN UNDERGOING CHRONTC}

315 HEMODIALYSIS. Demetrius Ellis, Inge Mauger, Gladys $H$. by Wellington Hung.) Department of Nephrology, Children's Hospital National Medical Center and George Washington University, Washington, D.C.

The severe growth retardation in 7 of 8 children undergoing maintenance hemodialysis (HD) at our center prompted this study into their calorie, protein and mineral intake. The mean age was $13.5(8-20)$ years with a mean duration of HD of $15.3(5-24)$ months. The diet diary was recorded by the mother after detailed instructions by the research dietitian. Data from 2 to 6 weekend periods were remarkably similar permitting accurate quantitation and comparison with the recommended dietary intake as shown below:

$\begin{array}{lcc} & \text { ACTUAL } & \text { RECOMMENDED } \\ \text { Calories } / \mathrm{m}^{2} / \mathrm{d} & 833+253 & 2400 \\ \text { Protein } \mathrm{g} / \mathrm{kg} / \mathrm{d} & 1.3 \mp 0.5 & 2 \\ \text { Sodium } \mathrm{mEq} / \mathrm{m}^{2} / \mathrm{d} & 33+17 & 40 \\ \text { Potassium } \mathrm{mEq} / \mathrm{m}^{2} / \mathrm{d} & 22+9 & 30\end{array}$

The one patient who maintained normal growth was found to consume substantially higher quantities of calories and protein and is not included.in the above calculations.

These data indicate that on 1 y $35 \%$ of the recommended amount of calories and $64 \%$ of the desired amount of protein were ingested. This deficiency in calorie and protein intake may limit nitrogen turnover and contribute to increased frequency of $\mathrm{HD}$ and decreased growth.

316 DECREASED LOWER ESOPHAGEAL SPHINCTER PRESSURES (LESP) IN GASTROESOPHAGEAL REFLUX OF CHILDHOOD. Arthur $R$. Euler, Marvin E. Ament.* UCLA School of Medicine, Department of Pediatrics, Los Angeles, California, 90024.

The study of esophageal motility with measurement of resting LESP by a constant perfusion $(0.8 \mathrm{cc} / \mathrm{min})$ catheter system and rapid pull through technique is a well established method for evaluation of gastroesophageal reflux (GER) in adults but has not been widely used in pediatrics. Fifteen infants and ten children (age $=4.5-18$ yrs.) with symptoms of GER were studied to determine if this examination could predict which patients would require surgical correction. All infants and one child were sedated with meperidine, chlorpromazine and promethazine. GER was present on the esophagram of five infants and three children. Al1 infants received a medical regimen (thickened feedings every 2-3 hours and were kept in an upright position 24 hours a day) for 3 weeks. Six infants did not cease vomiting and/or gain weight; they had Nissen fundoplications. These six infants had a mean LESP of $12.7 \mathrm{~mm}$. Hg. (Range $=11.3-15.0$ ) while the remaining nine had a mean LESP of $19.6 \mathrm{~mm}$. $\mathrm{Hg}$. (Range $=15.0-25.0$ ). Four children had endoscopic or histologic proven esophagitis and required surgery. Their mean LESP was $8.25 \mathrm{~mm}$. Hg. (Range $=6.5-$ 10.0). The remaining six children did not have esophagitis and had a LESP of $16.6 \mathrm{~mm}$. Hg. (Range $=10.0-20.0$ ). The mean LESP is significantly lower in patients who required surgery regardless of age (Infants $=\mathrm{P}<0.001 ;$ Children $=\mathrm{P}<0.005$ ). Esophagea 1 motility studies should be done in all children with GER symptoms to help determine which patients will require surgical correction. 

317 INTRALIPID (TPN-IL). Frank A. Franklin, Jr., John B. Watkins, Lesley Heafitz, Alexander W. Clowes and Jan L. Breslow, (spon. by H. William Taeusch,Jr.) Harvard !fedical School, Child. Hosp. Med. Ctx., Dept. of Pediatrics, Boston.

14 neonates appropriate for gestational age (wgt 709-1l20gms) without surgical disease, received TPN-IL daily with glucose (12 gms $/ \mathrm{kg}$ ) amino acids (3 gms $/ \mathrm{kg}$ ) and Intralipid 10\% (IL) $(30 \mathrm{cc} / \mathrm{kg}$ ) by peripheral vein at day $10 \pm 1$ (Avg \pm S.E.) for $18 \pm 3$ days. Growth in 14/14 infants equalled intrauterine rates while on TPNIL at $90-100 \mathrm{cal} / \mathrm{kg} /$ day, with survival in $11 / 14$. The disappearance rate of plasma lactescence after an IL bolus $(3.5 \mathrm{cc} / \mathrm{kg})$ was 3.1 $\pm 0.3 \% / \mathrm{min}$ with no change during TPN-IL. Lactesence and/or elevated serum triglycerides (TG) developed during TPN-IL in only $1 / 14$ infants. However, all 14 developed elevated serum cholesterol (CH) to peak levels $(216 \pm 14 \mathrm{mg} \%)$ within $1-3$ weeks, which returned to pre TPN-IL levels (111 $\pm 6 \mathrm{mg} \%$ ) by 1 week after TPN-IL and adequate oral intake. This increase was as free, unesterified $\mathrm{CH}$ and was accompanied by a prominent $\beta$ band on electrophoresis. Similar changes developed in adult rats by 1 week on TPN-IL: (baseline $\mathrm{CH} 78 \pm 7 \mathrm{mg} \%$ to $420 \pm 28 \mathrm{mg} \%$ with normal $\mathrm{TG}$ ). The low density lipoprotein fraction of rat plasma had $\beta$ migration and was markedly enriched in phospholipid and free $\mathrm{CH}$. Similar lipoproteins occur in biliary obstruction and LCAT deficiency. Conclusion: Small neonates were capable of intrauterine rates of growth on TPN-IL. However, all developed elevated $\mathrm{CH}$ as free, unesterified $\mathrm{CH}$. An experimental model has been developed to define the basis and significance of this a1tered $\mathrm{CH}$ metabolism.

SSENTIAL FATTY ACID DEFICIENCY, PLATELET AGGREGATION

318 AND THE MAJOR URINARY METABOLITES OF THE E PROSTAGLANDINS (PGE-M) IN THE NEWBORN. Zvi Friedman, Edward Lamberth, Hans Seyberth and John A. Oates (Spon. by Nicholas M. Nelson). Penn State Univ. Coll. Med., M. S. Hershey Med. Ctr., Dept. of Ped., Hershey, Pa. and Vanderbilt Med. Ctr., Depts. of Pharm. and Med., Nashville, Tenn.

Six newborn infants (five prematures) receiving fat free parenteral nutrition, developed biochemical evidence of essential fatty acid (EFA) deficiency. These changes were manifested by a decrease in arachidonic and linoleic acid, an increase in 5,8,11 eicosatrienoic acid, palmitoleic and oleic acids and a trienoic/ tetraenoic ratio of more than 0.4 . At the time of EFA deficiency, platelet challenge with $A D P$ revealed impaired aggregation and marked disaggregation when compared with controls (18\% vs $43 \%$ at $2.5 \mu \mathrm{M}$ ADP and $34 \%$ vs $52 \%$ at $5.0 \mu \mathrm{M} A D P$ ). Platelet function returned to normal upon recovery from the EFA deficient state. PGE-M, 7 $\alpha$-hydroxy-5, 11-diketotetranorprostane-1, 16-dionic acid was analyzed in the urine of EFA deficient infants and compared with six controls. Significant differences $(p<0.05)$ were found in the excretion of PGE-M between the two groups $(0.53$ 0.11 vs $0.92 \pm 0.14 \mathrm{\mu g} / 24 \mathrm{hrs})$. It is concluded that the EFA deficiency state is associated with decreased prostaglandin production and may explain the impaired platelet function.
320

CHOLESTYRAMINE THERAPY IN CHILDREN WITH CHRONIC DIARRHEA: EVIDENCE FOR THE INHIBITION OF AMINO ACID ABSORPTION BY BILE SALTS. Adolfo D. Garnica, * Owen M. Rennert, and Bradley M. Rodgers. University of Florida College of Medicine, Division of Genetics, Endocrinology, and Metabolism, Department of Pediatrics, Gainesville.

Five children, ranging in age from 5 weeks to 4 years, were treated for chronic diarrhea with oral cholestyramine, 3-12 gm/ day, in 4-6 equally divided doses. Treatment with cholestyramine resulted in a marked decrease in 24-hour stool output, and of total stool sodium, amino acids, protein, nitrogen and bile acids. The concentrations in stool of sodium, amino acids, and bile acids also decreased. In vivo, water, electrolyte and hexose absorption has been shown to be inhibited by bile acids. In vitro, the inhibition of amino acid absorption in rat ileum by glycocholate, taurocholate, and deoxycholate has recently been demonstrated. Bile acids are known to be bound by cholestyramine. Our in vitro studies, however, indicate that there is a negligible binding of amino acids by cholestyramine at pH 5, 6 and 7 .

This data verifies the observation that some patients with chronic diarrhea might be effectively treated with oral cholestyramine. It suggests, moreover, that the beneficial effect of cholestyramine apparently results from its bile acid binding capacity, and a consequent improvement in water, electrolyte, hexose and amino acid absorption. These observations provide, for the first time, in vivo evidence for the inhibition of amino acid absorption by bile acids.
A JISTERAL-ENRILHED FORMULA FOR THE <1500-GM

321 INFANT. Judith Gihbs, David Sands, and Robert Usher. Mciill unj.v., hoyal Victoria Hospital, Depts. of Obs. Uyn. and Ped., Montreal.

To attain calculated intra-uterine (IV) accretion rates, Similac (SIM) was enriched by increasing protein $14 \%, \mathrm{Na}^{+} 52 \%$, vä $140 \%$ all $\mathrm{P}_{i} 62 \%$ (Ë̈P); EXP was red to 15 infants until $2-K_{g}$ weight, with 15 controls.

liesults: GI tolerance, fluid retention, tyrosinemia, fat ahsorption ( $87 \%)$, and growth parameters were simiJare letabolic acidnsis and bone demineralization were less frequent and BUN was higher on EXP. 3-day balance studies tone at 1.5 and $2 . t 2 \mathrm{Kg}$ showed almost normal IU accretion rates for $\mathrm{va}^{\text {th }}$ and $\mathrm{P}_{i}$ in infants fad EXP, and much lower values for SIM.

Conclusion: The mineral-enriched formula is welltoleraced, and entiances vä and $p_{i}$ retention and bone mineralization.

Balance-study data: retentions/ $\mathrm{kg} / \mathrm{day}$ :

\begin{tabular}{|c|c|c|c|c|c|}
\hline & & $\mathrm{K}_{\mathrm{m}}^{+}$ & $\mathrm{CH}_{\mathrm{B}}^{+4}$ & $\operatorname{mig}_{\mathrm{m}}$ & Nig \\
\hline $\mathrm{EXP}$ at $1.5 \mathrm{Kg}$ wt. & $2.4 \%$ & 1.1 & $8.2^{4}$ & $75.6^{3 \%}$ & 405 \\
\hline Exp at $2 \mathrm{Kg} w x$. & 1. 7 * & 0.6 & $6.4 *$ & $83.0 \%$ & 361 \\
\hline$S I M$ at $1.5 \mathrm{Kg} w t$ & $1.4^{*}$ & 1.8 & $2.7 *$ & $52.2 *$ & $3+4$ \\
\hline SIY at 2 KE wt. & $1.3 *$ & 1.3 & $3.0 *$ & $49.7 \%$ & 336 \\
\hline CALU. IU & 1.5 & 0.8 & 10.8 & 88.3 & 374 \\
\hline AUURETICN & 1.5 & 0.5 & 6.4 & 85.4 & 368 \\
\hline
\end{tabular}

( $*$ signif. diff. between EXP and SIM)
CORRECTION OF ESSENTTAL FATTY ACID (EFA) DEFICIENCY

319 IN NEWBORNS BY CUTANEOUS APPLICATION OF SUNFLOWERSEED (SFS) OIL. Zvi Friedman, Stephen J. Shochat, Edward Lamberth, Keith H. Marks and M. Jeffrey Maise1s (Spon. by Nicholas M. Nelson). Penn State Univ. Coll. Med., M. S. Hershey Med. Ctr., Dept. Ped., Hershey, Pa. and Vanderbilt Med. Ctr., Dept. Med., Nashville, Tenn.

Two newborn infants receiving long-term, fat-free parenteral nutrition developed biochemical evidence (one also developed clinical manifestations) of EFA deficiency. Biochemical evidence of EFA deficiency was documented first in plasma and then in red blood cells and adipose tissue and included a decrease in arachidonic and linoleic acids, an increase in 5,8,11-eicosatrienoic acid, palmitoleic and oleic acids and a trienoic/ tetraenoic ratio of more than 0.4 . Cutaneous application of SFS oil, a source rich in the essential fat linoleic acid, reversed the clinical and biochemical manifestations of EFA deficiency.

Plasma EFA Values (\% Total Fatty Acids in Plasma Phospholipids):
Linoleic Acid

Arachidonic Acid

Eicosatrienoic Acid

\begin{tabular}{cr} 
Before SFS Oil & After SFS Oil \\
\cline { 1 - 2 } $2.6 \pm 0.2$ & $6.5 \pm 0.2$ \\
$3.4 \pm 0.1$ & $16.2 \pm 0.6$ \\
$10.9 \pm 0.2$ & $2.6 \pm 0.1$
\end{tabular}

$16.2 \pm 0.6$
$2.6 \pm 0.1$
It is concluded that cutaneous application of oil rich in linoleate is an efficient route for supplementation of EFA and may correct the development of EFA deficiency in sick infants.
SITE OF BILE ACID DEAMIDATION IN CHILDREN WITH SUB-

322 ACUTE DIARRHEAL SYNDROME (SDS). G. S. Gopalakrishna, J. T. Rodriguez*, J. Alvarado*, C.T. L. Huang and B. L. Nichols. Department of Pediatrics, Baylor College of Medicine, Houston, Texas 77025. *Unidad de Estudios Clinicos, Roosevelt Hospital, Guatemala City, Guatemala.

The presence of increased free bile acios (FBA) in the upper small bowel of children with SDS was previously reported by our group. Purpose of the current investigation was to determine the site of deamidation of conjugated bile acids (BA).

Seven children, 1 - 3 years of age, with subacute diarrhea of over two weeks were studied. All had. negative cultures for conventional pathogens (bacterial and parasitic).

The upper small bowel was intubated using a triple lumen tube. 1\% PEG was infused through the proximal port at a constant rate. After giving $3 \mathrm{cc}$ of corn oil orally, luminal fluid was sampled from the two distal ports located $20 \mathrm{~cm}$ apart. At a steady state, 45 minutes following gall bladder stimulation, luminal FBAs increased from $4.2+2.1 \%$ to $10.3 \pm 3.2 \%$ of total BAs $(p<.05)$ (normal $3.2+1.1 \%(\bar{M} \pm S . D$.$) ). When luminal fluid was subjected$ to an in vitro assay of deamidase using $14 \mathrm{C}$ labeled taurocholic acid, no measurable activity was noted.

These data indicate that deamidation occurs within the upper small bowel of children with SDS and suggests that the enzyme is fixed to the mucosal surface. The possibility that the liver might secrete FBAs in SDS is excluded. Research support is acknowledged from NIH Training Grant USPH 5 ROl AM-0572l, Children's Clinical Research Center NIH 2-MOl-RR 00188 and Ross Laboratories. 
LIPID ABNORMALITIES IN PSYCHOSOCTAL DWARFISM (PSD):

323 A POSSIBLE SCREENING TEST. Nancy J. Hopwood, Dorothy $\mathrm{J}$. Becker, Fay $\mathrm{H}$. Hengstenberg, \& A $\frac{\text { Hlan }}{\mathrm{L}}$. Drash. Depts. of Peds. Univ. of Mich.

A distinctive pattern of changing blood lipids has been observed in the first week of hospitalization of 8 children, age 1.9$10.7 \mathrm{yrs}$, with growth retardation later proven to have PSD. On day 1 (morning after admission) the mean $(\vec{x})$ fasting serum tri glyceride leve1 (TG) was $66 \mathrm{mg} \%$ \& cholesterol (C) $180 \mathrm{mg} \%$. A11 fasting sera were turbid without clearing after standing 48 hrs. On day 2 there was an often dramatic rise of TG ( $\bar{x} 106 \mathrm{mg} \%$ ) which decreased to normal by day 7 ( $\bar{x} 59 \mathrm{mg} \%$ ) without significant change of $\mathrm{C}$, but with total disappearance of serum turbidity. Paper 1ipoprotein electrophoresis of $12 \mathrm{hr}$ postprandial plasma showed a light chyle spectrum characteristic of a non-fasting sample. Fat tolerance test showed delayed clearing of the absorbed lipid from the serum of 1 case \& normal clearing in another whose fasting serum was already clear. Recurrence of turbidity followed parental visits to the hospital \& cleared again with parental-child separation. By contrast, 5 children with growth retardation due to protein-calorie malnutrition, age 1.2-3.9 yrs \& 12 children, age 3-10.4 yrs, with isolated growth hormone deficiency had clear fasting sera on day 1. Thus fasting serum turbidity in children with growth retardation may suggest the diagnosis of PSD \& differentiate these children from those with non-functional hypopituitarism or malnutrition.

DEFICIENCY OF ARGININOSUCCINIC ACID SYNTHETASE (ASS):

324 HEPATOCYTES WXTH FATTY METAMORPHOSIS AND GLYCOGEN DEPOSITED AS BETA-PARTICLES, NOT ALPHA-ROSETTES. George Hug, Linda Book, Ted Brown, John Herbst, Kevin Bove and James Wu, Departments of Pediatrics, Universities of Cincinnati and Utah, Cincinnati, $\mathrm{OH}$ and Salt Lake City, UT

A $4 \mathrm{~d}$ old boy whose sister had died of acidosis at $4 \mathrm{~d}$ of age had hypotension, hemoglobinuria, generalized aminoaciduria, prothrombin time $100 \mathrm{sec}$, SGOT 209,BS $8-25 \mathrm{mg} \%$, art.pH 7.09 , in serum: lactate 28.5 , pyruvate $0.25(\mathrm{~N}<1.3,0.07 \mathrm{mM})$, no methylmalonic, propionic or isovaleric acid, no citrulline. He died at $6 \mathrm{~d}$ in metabolic acidosis of encephalopathy and liver failure. Liver urea cycle enzymes were - in nm citrulline for CPS, OTC; nm urea for ASS, ASL, arginase/mg prot/min - (normals in parentheses): carbamyl phosphate synthetase $9.6(11.5 \pm 4.2)$, ornithine transcarbamylase 193.1(307.5 \pm 94.2$)$, argininosuccinic synthetase $0(15.2 \pm$

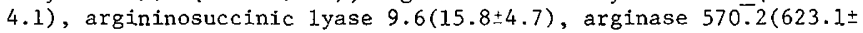
286.3). On light microscopy, fatty metamorphosis of hepatocytes was similar to that in Reye's syndrome in paraffin embedded, but not in plastic embedded perimortal tissue. On electronmicroscopy, hepatocytes had normal mitochondria, many lipid vacuoles some dwarfing nuclei, and glycogen in the form of B-particles of $20 \mathrm{~m} \mu$ diameter, and none as $\propto$-particles of $200 \mathrm{~m} \mu$ diameter that are characteristic of glycogen in human hepatocytes. We do not know of any previous instance of exclusively $\beta$-glycogen in liver parenchymal cells, how to explain this abnormal configuration, or why the baby's blood sugar was not sustained in the presence of abundant liver glycogen.

ENHANCED DEGRADATION OF INTESTINAL SURFACE GLYCOPRO-

325 TEIN IN BACTERIAL OVERGROWTH. Anita C. Jonas, Gordon G.Forstner (Spon. by J.R. Hami 1ton). Hospital for Sick Children, Dept. of Pediatrics, Toronto, Canada.

Bacterial contamination of the intestine is a well recognized cause of disaccharidase deficiency. Since disaccharidases constitute part of the surface glycoprotein coat, we explored the possibility that low activity was associated with increased degradation of brush border giycoproteins. Rat brush border membranes (BB) were isolated from self filling blind loops (SFBL) 8 weeks after construction and compared with $\mathrm{BB}$ from self emptying $\mathrm{BL}$ and midgut from nonoperated rats (controls). Maltase, sucrase and lactase were assayed in the whole mucosa and BB. Relative degradation rates $\left({ }^{3} \mathrm{H} /{ }^{14} \mathrm{C}\right.$ ratios) of glycoprotein were determined by a double isotope method. 10 uc $\left({ }^{4} \mathrm{C}\right) \mathrm{D}-\mathrm{glucosamine}$ was injected IP $19 \mathrm{~h}$, and $25 \mathrm{uc}(6-3 \mathrm{H}) \mathrm{D}$-glucosamine $3 \mathrm{~h}$ prior to sacrifice. Disaccharidase activity (maltase, shown in table) was redyced to the same extent in mucosa and $\mathrm{BB}$ from SFBL. Glycoprotein $\mathrm{H} /{ }^{14} \mathrm{C}$ ratios were increased in mucosa and BB from SFBL (table). These results indicate that bacteria reduce the number of disaccharidase molecules per unit of membrane probably as part of a general destruction of the surface glycoprotein coat.

\begin{tabular}{|c|c|c|c|c|}
\hline & \multicolumn{2}{|c|}{ Maltase $\mathrm{U} / \mathrm{mg}$ protein } & \multicolumn{2}{|c|}{${ }_{\mathrm{H} /} /^{14} \mathrm{C}$} \\
\hline & Mucosa & $\overline{\mathrm{BB}}$ & Mucosa & BB \\
\hline D. & $0.41 \pm 0.10$ & $8.1 \pm 1.7$ & $8.6 \pm 1.8$ & $7.8 \pm 2.0$ \\
\hline 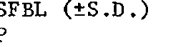 & $\begin{array}{l}0.10 \pm 0.02 \\
<.01\end{array}$ & $\begin{array}{l}4.0 \pm 0.72 \\
<.01\end{array}$ & $\begin{array}{l}11.2 \pm 0.6 \\
<.05\end{array}$ & $\begin{array}{l}28.4 \pm 15.4 \\
<.05\end{array}$ \\
\hline
\end{tabular}

$<.05$
ELFVATION OF THE SINGLE BREATH DIFFUSION CAPACITY IN

326 YSTIC FIBROSTS. T G Keens, H Levison N. Zame1, Research Institute, Hospital for Sick Children, and University of Toronto, Department of Medicine, Toronto, Ontario. Many have observed an elevation of the Single Breath Diffusion Capacity (Dco) in Asthmatics. If this is related to the degree of airways obstruction, one might expect and elevation of the Single Breath Dco in Cystic Fibrosis patients also. In 173 patients with Cystic Fibrosis, the single breath Dco was markedly elevated $(122+26 \%$ predicted, $P<0.00 \mathrm{I})$. They all showed moderate airways obstruction with a Maximum Mid-Expiratory Flow Rate (MME) of $62 \pm 22 \%$ predicted. Patients who had the most obstruction, as manifest by a decreased MMEF, also had the greatest elevation of Single Breath Dco above normal $(\mathrm{P}<0.01)$. 17 Cystic Fibrosis patients were tested before and after bronchodilator aerosol. No change was seen in Single Breath Dco or the degree of airways obstruction. The elevation of the Single Breath Dco may relate to the degree of obstruction. Increased inspiratory resistance causes the patient to draw more negative intrathoracic pressure during the rapid inspiration required for the test. This negative intrathoracic pressure wil increase capillary blood volume and therefore increase Dco. We have caused an increase in the Single Breath Dco in normal subjects by imposing an inspiratory resistance. Thus the increased Single Breath Dco in Cystic Fibrosis probably reflects an artifact due to rapid inspiration, and not a chronic physiologic change in the lungs.
327 ELEVATED RATES OF PROTEIN SYNTHESIS IN CHILDREN AFTER 32 THERMAL INJURY. Craig L. Kien, Dennis K. Rohrbaugh, Bode). Harvard Med. Sch., Shriners Burns Inst, Depts. of Ped. and Surgery, Boston, and Mass. Inst. of Technology, Dept. of Nutrition and Food Science, Cambridge, Mass.

The effects of stress after burn trauma on total body protein synthesis was studied in nine children aged 4 mos. to 12 yrs. in whom the percentage of total body surface area burned ranged from 13 to 83 . Using the method of Picou and Taylor-Roberts (Clin. Sci. 36:283, 1969) which involves the continuous administration of $15 \mathrm{~N}$-glycine for $48-72$ hours, whole body nitrogen flux was estimated and, in combination with nitrogen balance data, rates of whole body protein synthesis were determined. In five of the patients two studies were performed during the second and again during the fourth week post burn. The rate of whole body protein synthesis was positively correlated $(P<0.05)$ with percent initial third degree burn $(r=.68)$, energy intake $(r=.81)$, and protein intake $(r=.88)$. There was no significant change in protein synthesis with time after injury. Since protein synthesis normally accounts for a significant proportion of the basal ATP utilization, it is considered that elevated rates of protein synthesis, documented in these studies, may contribute to the increased daily energy expenditure and basal oxygen consumption found in burned patients. Further analytical data allowing also calculations of protein catabolic rate should provide more insight into the metabolic effects of burn injury. (Supported by NIH grant GM21700). $<.01$
TREATMENT OF AN INFANT BOY OF CARBAMYL PHOSPHATE SYN328 THETHASE (CPS) DEFICIENCY WITH ALPHA KETO ANALOGUES (KA) OF ESSENTIAL AMINO ACIDS. Jeffrey J.KIine, George Hug, William K. Schubert, Ted Brown, and Helen K. Berry, Cincinnati Childrens Hospital, Cincinnati, Ohio.

A 6 month old male infant was evaluated for seizures, developmental delay, and hyperammonemia. He was subsequently found to have hyperammonemia Type 1 , with a level of CPS less than $5 \%$ of control in liver tissue. Attempts at controlling the high amonia were unsuccessful and a trial with KA was instituted. The child was placed on a control diet for 14 days consisting of $2 \mathrm{gr} / \mathrm{kg} /$ day of the ten essential amino acids, plus arginine supplementation. Each essential amino acid was given in approximately four times the daily requirement as described in Foman, Infant Nutrition. Two KA were then substituted, on a gram for grambasis, for their respective amino acids (methionine and phenylalanine). Amonia fell from an average of 480 to $215 \mathrm{ug} / 100 \mathrm{ml}$. Three more KA were substituted on day four for their respective amino acids (isoleucine, leucine, valine), with resultant amonia levels dropping to an average of $110 \mathrm{ug} / 100 \mathrm{ml}$. Amino acid imbalance became apparent necessating a three day return to amino acids, and then a retrial with $11 / 2$ times the previous amounts of $K A$. It was again difficult to maintain amino acid balance and $K A$ were discontinued. Later comparison to a low protein diet with arginine supplementation showed both diets substantially controlled amonia levels, but only the arginine supplemented amino acid diet maintained linear growth. 
329

THE EFFECTS OF INTESTINAL SOLUTE LOAD ON WATER SECRETION IN ACQUIRED MONOSACCHARIDE INTOLERANCE (AMI). W.

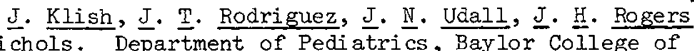
and $\mathrm{B}$. L. Nichols. Departint 77025 .

Infants with AMI have defective glucose absorption which correlates with reduced mucosal surface. The osmotic effect of unabsorbed glucose on intestinal secretion was investigated by jejunal perfusion in 8 infants ranging from 2 - 10 months of age. All infants had or were recovering from AMI Isocaloric solutions of glucose and glucose polymer (Polycose ${ }^{(R)}$ ) containing 1\% PEG as a non-absorbable marker were tested. Absorption and water flux were determined by the methods of Fordtran. When 10\% solutions were compared, no significant change was noted in carbohydrate absorption (M 27.1 vs $30.6 \mathrm{mg} / \mathrm{min} / 30 \mathrm{~cm}$ jejunum $(p>.1$ )) but water secretion decreased from $.43 \mathrm{ml} / \mathrm{min}$ to $0.05 \mathrm{ml} / \mathrm{min}$ with Polycose $(p<.01)$. 5\% solutions revealed similar results ( $a b-$ sorption of 33.6 and $33.0 \mathrm{mg} / \mathrm{min} / 20 \mathrm{~cm}$ jejunum) for glucose and Polycose $(p>.6)$ and a mean water secretion of .53 and $.06 \mathrm{ml} /$ min respectively $(p<.05)$.

The results indicate that the increased intestinal secretion in AMI is a function of osmolar load in the presence of a reduced absorptive capacity. The use of an isocaloric glucose polymer with a larger molecular weight resulted in a significant reduction in jejunal secretion. The rate of glucose absorption from the polymer was equivalent to that from glucose in 7 subjects. In one infant reduced amylase production was associated with reduced digestion of the polymer. Research support from NIH AM-05721, Ross Laboratories and NIH RR-00188.

330

SKIN FOLD LENGTH, A MEASURE OF ACUTE WEIGHT LOSS.

Detroit.

The weight deficit in malnutrition may develop because of weight loss or chronic failure to thrive. Studies of 19 and 27 infants, respectively, showed that the two types of malnutrition differ with regard to BMR and plasma Rivase. The group difference is not expressed by standard anthropometric measures, like skin fold thickness (ST) or weight deficit for age and height. An objective measure of skin fold redundancy was therefore developed. We designed an addition to a standard caliper which measures in one step ST and maximal skin fold length (SL). During a second step circumference (C) of an extremity (arm) is measured which excludes the volume of the extruded skin fold and is an improved measure of muscle mass. The method was tested in 233 hospital infants, age 1 to 26 months. Height was normal in 105, below the 10 th $\%$ ile in 100 , and above the 90 th \%ile in 28 infants; weightfor-height was variable. 81 infants were normal for both height and weight. This group showed no sex difference for ST, SL, and $\mathrm{C}$ and was used to calculate regressions on age. Only $\mathrm{C}$ and SL were related to age. Multiple regressions on age, height and $\%$ weight-for-height were calculated for the total population. Regressions for $S T$ and $C$ on weight-for-height were hichly significant. As expected, there was no relation between SL and weightfor-height; but patients with known acute weight loss had a high SL. Acute weight loss can be expressed in + S. D of the normal SI for age.

DELAYED NERVE CONDUCTION VELOCITIES IN CHILDREN WITH 331 PROTEIN-CALORIE MALNUTRITION (PCM). Ashir Kumar, Om P. Ghai and Naunihal Singh. (Spon. by Donald N. Medearis, Jr. Dept. at Clevel and Metropolitan General Hosp., Cleveland, Ohio \& All India Inst. of Med. Sciences, New Delhi, INDIA.

The effects of PCM on the developing nervous system were eval uated by measuring the conduction velocities in 93 children ( 38 Marasmus, 13 Kwashiorkor (Kwash) \& 42 Control) in ulnar, median, peroneal \& post. tibial nerves. Mean conduction velocities in meters/sec ( \pm S.D.), grouped according to age, are listed below: $\frac{\text { GROUPS (Gr) }}{\text { I } 6-12 \text { Mo. }}$ ULNAR MEDIAN PERONEAL POST. TIBIAL

Control (15) $43.3 \pm 5.5 \quad 42.4 \pm 4.2 \quad 40.4 \pm 4.0 \quad 40.3 \pm 4.7$ Marasmus (15) $\quad 36.7 \pm 4.1 \quad 37.7 \pm 4.4 \quad 32.7 \pm 9.0 \quad 35.3 \pm 4.3$ II $13-24$ Mo. Marasmus (14) $39.7 \pm 3.7 \quad 40.0 \pm 4.0 \quad 38.8 \pm 6.2$ Kwash (5) $43.1 \pm 8.2 \quad 40.7 \pm 1.3 \quad 38.4 \pm 1.9 \quad 40.3 \pm 6.2$

III 25-48 Mo. $50.1 \pm 5.9 \quad 49.9 \pm 7.7 \quad 45.3 \pm 6.2 \quad 48.4 \pm 6.9$ $\begin{array}{llllllll}\text { Control (11) } & 50.1 \pm 5.9 & 49.9 \pm 7.7 & 45.3 \pm 6.2 & 48.4 \pm 6.9 \\ \text { Marasmus (9) } & 43.5 \pm 4.5 & 44.1 \pm 8.0 & 37.5 \pm 4.9 & 39.5 \pm 6.1\end{array}$ Kwash (8) $45.4 \pm 4.942 .4 \pm 5.3 \quad 38.6 \pm 6.3 \quad 42.5 \pm 4.5$ All Marasmus pts, had significantly reduced velocities. In Kwash reduced velocities were found in all nerves in Gr III and only in lower limb nerves in Gr II. Since PCM in oldex age groups does not have any effect on conduction velocities, these data indicate that PCM, when it occurs during the development of the nervous system, effects myelination of the peripheral nerves.
332 NITROGEN EXCRETION IN THE LOW-BIRTH-WEIGHT INFANT. James A. Lemons, Gilberto Pereira, and Michael A. Simmons (Spon。 by Frederick C. Battaglia). University of Colorado Medical Center and The Children's Hospital, Division of Perinatal Medicine, Denver.

To document the relationship of protein catabolism to total caloric and protein intake in the small infant, 13 preterm AGA infants (weights 980-1600 grams) were studied over the first 35 days of life. Timed 8hour urine samples were collected every 3 days and urine urea (UN), ammonia (AN) and total nitrogen (TN) excretion determined. Daily weights and protein, carbohydrate, fat and caloric intakes were recorded. During the first 5 days of life, the mean excretion of TN was $132 \pm 17(\mathrm{SEM}) \mathrm{mg} / \mathrm{kg} / 24 \mathrm{hr}$, and of UN $92 \pm 14 \mathrm{mg} / \mathrm{kg} / 24 \mathrm{hr}$. After day 5 the urine TN and UN excretion decreased to $79 \pm 5$ and $41 \pm 3 \mathrm{mg} / \mathrm{kg} /$ $24 \mathrm{hr}$ and did not change significantly from days 6 through 35 . Excretions of TN and UN before and after day 5 were significantly different $(P<.005)$. AN excretion remained constant during the study period $(8.7 \pm 0.5 \mathrm{mg} / \mathrm{kg} / 24 \mathrm{hr})$. Mean caloric intake prior to day 5 was $59.7 \pm$ $4.9 \mathrm{Cal} / \mathrm{kg} / 24 \mathrm{hr}$, increasing during days $6-8$ to $83.7 \pm 4.5 \mathrm{Cal} / \mathrm{kg} / 24 \mathrm{hr}$ $(P<.001)$. Increased protein catabolism occurs during the first days of life in the small premature infant in association with a caloric intake of $<75 \mathrm{Cal} / \mathrm{kg} / 24 \mathrm{hr}$. Protein catabolism then decreases in association with a caloric intake of $>75 \mathrm{Cal} / \mathrm{kg} / 24 \mathrm{hr}$ and remains at this low level during subsequent growth (days 13-35)。 This relatively greater TN and UN excretion during the first days of life is consistent with previous findings of high nitrogen intake and nitrogen excretion by the fetus.

SUREY OF VITAMIN E STATUS IN CHILDREN. Sharon L.

$333 \frac{L e v i n e}{\text { Philip M. Farrell, NICHD, }}, \frac{\text { M. Diane Murphey, Bethesda, MD }}{\text { NNMC }}$ Vitamin $E$ is a biological antioxidant which is necessary for stabilization of lipid membranes. Normal values of plasma vit. E have been established for adults but not for children. This study is designed to determine the range of vit. E or tocopherol levels present in random populations of normal children. Plasma tocopherol, peroxide-induced hemolysis (an index of red cell tocopherol), and total plasma lipids were determined in 17 children, 2-10 years of age (mean $=5.4$ years) and compared with values in 21 normal adults. Mean plasma tocopherol in these children was $0.53 \pm .13 \mathrm{mg} / \mathrm{dl}$ and differed significantly $(p<.001)$ from the adult mean of $0.79 \pm .20$. Vit. E levels in $47 \%$ of the children fell below $0.5 \mathrm{mg} / \mathrm{dl}$, the previously accepted lower limit of normal. Despite this, peroxide hemolysis tests in all subjects were normal, suggesting that in chizdren plasma tocopherol levels $<0.5$ do not necessarily represent biological vit. E deficiency. Horwitt et al. have proposed that tocopherol: total lipid ratios are better indicators of $\mathrm{E}$ adequacy than plasma tocopherol levels alone and that 0.8 represents the minimum normal ratio. Graphic comparison of plasma $E$ with total lipids revealed good correlation for children $(r=.80)$ and adults $(r=.75)$. However, the mean $E$ : lipid ratio in children of $1.08 \pm .17$ was significantly lower $(p<.001)$ than that of adults $(1.43 \pm .22)$. Nonetheless, $94 \%$ of the E:lipid ratios in children were greater than 0.8 . These data therefore indicate that new vit. E norms are needed in children and that tocopherol: lipid ratios in plasma are better predictors of vit. E status than plasma tocopherol levels alone.

HYPOXIA (HY) EFFECTS ON CARBOHYDRATE (CHO) TRANS334 PORT. Fima Lifshitz, Raul A. Wapnir, Robert Pergolizzi and Saul Teichberg, Dept. of Peds., North Shore Univ. Hosp., Manhasset, NY and Dept. of Peds., Cornell Univ. Med. Col., NY, NY.

The effects of $\mathrm{HY}$ on jejunal transport were assessed. Weanling rats were exposed to $78 \% \mathrm{~N}_{2}$ controls $(\mathrm{CH})$ or $93 \% \mathrm{~N}_{2} \mathrm{HY}$, for 4 to $72 \mathrm{hr}$ in acrylic chambers. A $20 \mathrm{~cm}$ jejunal segment was sampled for mucosal enzymes and ultrastructural studies, or perfused in vivowith Krebs-Henseleit bicarbonate buffers containing 4 to $40 \mathrm{mM} \mathrm{CHO}$ and $1 \mathrm{mM} \mathrm{L-aminoacids}$ with tracer amounts of ${ }^{3} \mathrm{H}$-labelled solutes and $600 \mathrm{mg} \%$ polyethylene glycol. $\mathrm{HY}$ rats had lower blood $\mathrm{pH}, \mathrm{PaO}_{2}$ and $\mathrm{HCO}_{3}^{-}$as well as higher concentrations of liver tryptophan oxygenase as compared to $\mathrm{Ctl}$. All $\mathrm{HY}$ rats had a decrease in $\left(\mathrm{Na}^{+}-\mathrm{K}^{+}\right)$ATPase activity in the jejunum. HY of more than $4 \mathrm{hr}$ duration reduced glucose (Glc) transport at 4 and $10 \mathrm{mM}$ concentrations. After $48 \mathrm{hr} \mathrm{HY}$, Glc transport at $4 \mathrm{mM}$ was $14.0 \pm 1.7$ vs $20.4 \pm$ 1.3 and at $10 \mathrm{mM}$ was $36.8 \pm 4.1$ vs $58.4 \pm 2.6 \mathrm{nmole} / \mathrm{min} / \mathrm{cm}, \mathrm{p} 0.01)$. The transport of both $4 \mathrm{mM}$ galactose and 3-0-methyl-Glc was also impaired: after $48 \mathrm{hr}$ of HY it was $11.0 \pm .5$ vs $13.7 \pm .5$ and $6.3 \pm .7$ vs $9.5 \pm$ $.6, \mathrm{nmoles} / \mathrm{min} / \mathrm{cm}$, respectively $(p .05)$. The transport of other actively transported solutes was also reduced, i.e. sodium and aminoacids. However, the transport of fructose by HY rats was similar to that of $\mathrm{CHI}$ and there were no alterations in intestinal disaccharidases, alkaline phosphatase, histology and ultrastructure. These studies suggest that HY rapidly induces a loss of intestinal transport capacity of $\mathrm{CHO}$-sodium dependent mechanisms without affecting intestinal permeability and ultrastructure. 
PROSTAGLANDIN E AND ENTEROCOLITIS. John D.

335 Lloyd-Still and Laurence Demers (Intr. by H. Nadler). Northwestern Univ. Med. Sch,, Children's Mem. Hosp. Dept. of Ped., Chicago and Dept. of Path. The Penn. State Univ. Col. of Med., Hershey, Pa.

The etiology of the enterocolitis of Hirschsprung's disease remains unclear. Prostaglandin $\mathrm{E}$ (PGE) causes secretion of water and electrolytes from the jejunum and an increase in transit time through the small intestine and colon. Recently a trial of cholestyramine has been recommended for infants with protracted diarrhea. We investigated an infant with Hirschsprung's enterocolitis characterized by a fulminant secretory diarrhea unresponsive to all therapy for 28 days until cholestyramine was administered. Fecal volume decreased from $460 \mathrm{ml} /$ day to $50 \mathrm{ml} /$ day. A twelve fold decrease in PGE concentration in the colostomy fluid was documented in response to cholestyramine (pre 128.0 $\mathrm{ng} /$ day, post $10.5 \mathrm{ng} /$ day). We a1so estimated fecal PGE concentration from a premature infant following extensive intestinal resection for necrotizing enterocolitis (NE) and demonstrated a 60 fold increase in PGE concentration $(2,666 \mathrm{ng} /$ day) in the colostomy fluid compared to a control ( $40 \mathrm{ng} /$ day). It is postulated that increased PGE concentration may be involved in the enterocolitis of Hirschsprung's disease as well as NE of prematurity. The possibility that bile acids exert their effect on the colon through a mediator such as increased PGE concentration must also be considered. In infants with secretory diarrhea that does not respond to conventional measures, increased prostaglandin activity should be considered. Therapy with cholestyramine may be curative.

336 EFFECT OF DIETARY PROTEIN LEVEL ON FAT ABSORPTION. Will iam C. MacLean, Jr., Guillermo Lopez de Romaña (Ped。), Sch. Hygiene \& Public Health (Internat. Hl th.), Baltimore Dietary protein adequacy affects wt. gain of man and animals. Previous data suggested that it influenced fat absorption. To test this, 9 heaithy children of 3.5-18 mo. were given a diet in which fat (soy-cottonseed oils $80: 20$ ) provided $50 \%$ and prote in (casein) $6.4 \%$ of $125-150 \mathrm{kcal} / \mathrm{kg} /$ day. Fecal fat was compared with that while consuming the same diet with protein doubled or omitted. Successive 3-day dietary periods were sequenced 6.4$12.5-0-6.4 \%$ protein calories ( 4 children) or $6.4-0-12.5-6.4 \%(5$ children). Results (tSD).

$$
\begin{array}{lccccc}
\text { Protein Calories } & \frac{6.4 \%}{2.2 \pm 4.3} & \frac{12.5 \%}{5.3 \pm 2.6} & \frac{0 \%}{11.7 \pm 7.7} & \frac{6.4 \%}{5.9 \pm 4.2} \\
\begin{array}{llll}
\text { Fecal fat: g/day } \\
\% \text { of intake }
\end{array} & 14.5 \pm 6.0 & 10.0 \pm 2.6 & 23.9 \pm 7.7 & 11.7 \pm 5.0
\end{array}
$$

Fecal fat from the protein-free diet was greater than from $6.4 \%$ $(P<0.01)$ or $12.5 \%(P<0.001)$ diets (by paired "t"l). Difference be tween 6.4 and $12.5 \%$ periods was not significant $(P<0.1)$. Stool wet wt. was higher $(P<0.01)$ during 0 than during $12.5 \%$ diet. Stool dry wts. were similar in all periods. The presence of protein appears to favor fat absorption. The promptness of the effect suggests a physico-chemical rather than a nutritional cause. This may be one way in which high or low protein intakes at extremes of the social scale affect energy balance. (USPHS Fellowship F22 AM-03252-02; AID Research Contract csd-2946.)

IN VITRO STUDIES OF SECRETORY CELL FUNCTION IN CYSTIC

\section{FIBROSIS (CF): J.A. Mangos, Dept. of Pediatrics,} Univ. of Florida ColTege of Medicine, Gainesville. Florida.

A secretory cell dysfunction has been assumed to be the pathogenetic basis of CF. In this in vitro study, isolated parotid acinar cells from cadavers of 5 patients with CF (P) and 5 organdonor controls (C) and from 3 surgical specimens ( $C-S$ ) were used. The morphologically and functionally intact parotid acinar cells were isolated and studied using methods previously developed in this laboratory (Am. J. Physiol. 229:560, 1975). The following. were observed: i) $\bar{P}$ cells had significantly higher concentrations of $\mathrm{Na}, \mathrm{Ca}$ and amylase and lower concentrations of $\mathrm{K}$ than $\mathrm{C}$ and C-S cells; 2) Activation of beta adrenergic receptors by isoproterenol or epinephrine resulted in significantly higher rates of secretion of amylase and synthes is of CAMP by $P$ cells than by $C$ or C-S cells; 3) Activation of alpha adrenergic receptors by epinephrine and Ách-chol inergic receptors by acetylcholine or carbamyl choline resulted in significantly lower cellular responses ( $K$ efflux) in $\mathrm{P}$ cells than in $\mathrm{C}$ or $\mathrm{C}-\mathrm{S}$ cells; 4) Following activation of cholinergic receptors by equimolar concentrations of carbamy? choline, the intracellular levels of cGMP were significantly lower in $\mathrm{P}$ cells than in $\mathrm{C}$ and $\mathrm{C}-\mathrm{S}$ cells; 5 ) The rates of transmembrane $\mathrm{Na}$ fluxes in $P$ cells $(6.9 \pm 1.2 \mathrm{mEg} \mathrm{Na} / \mathrm{min}$. L of cells were lower than the same fluxes in $C$ or $C-S$ cells $(11.7+1.9$ mEq/min.L). It is concluded that isolated parotid acinar cells from patients with CF show physiological and biochemical differences from control cells.
A PROTEIN SPARING MODIFIED FAST IN THE OBESE ADOLESCENT PATIENT. Russell Merritt, Bruce R. Bistrian. Robert M. Suskind (Spon. by Richard J. Grand). Deptof Nutrition and Food Science, MIT, Children's Hospital Medical Center, Division of Gastroenterology and Nutrition, Boston

At present, there is no recognized method for achieving rapid weight loss in the obese adolescent without negative nitrogen balance and growth retardation. Previous studies have shown that fasting modified by continued dietary protein intake is a safe and efficacious method of weight control in adult obese patients. We have applied this program to a group of adolescent patients, ages $13-16,4$ males and 1 female. Our data suggests that approx. 1.5 grams of protein/ $\mathrm{kg}$ ideal body weight is sufficient to achieve positive nitrogen balance in a state of controlled ketosis. Patients were followed with anthropometric measurements total body $\mathrm{K} 40$ counting and determination of metabolic substrates. There has been no evidence of abnormal organ function and all patients have been hunger free. Our first patient has achieved a greater than $25 \mathrm{~kg}$ weight loss without a decrease in total body $\mathrm{K}$ Three out of the 5 initial patients have been able to continue the modified fast as outpatients with continued weight loss and evidence of decreased adipose tissue. Studies of these patients growth are continuing. A model is being developed and will be discussed for eventual application of a multidisciplinary program directed at the obese adolescent outpatient.
339 AMINO ACID RESPONSES TO CONTROLLED NITROGEN INFUSION IN NEONATES. Charles E. Mize, Gerald R. Faloona, Zaven $\underline{H}$. Chakmakjian, and Constance Richards. Uni versity of Texas Health Science

30 patients requiring total parenteral nutrition received on of 3 randomized inputs of L-Amino acids (AA) (FreAmine ${ }^{B}$ ), viz. $1.5,3.0,4.5 \mathrm{gm} / \mathrm{kg} / \mathrm{d}$, during incremental caloric input adjusted with glucose $(60,90,120,150 \mathrm{cal} / \mathrm{kg} / \mathrm{d})$. Plasma/urinary AA were determined, coincident with $\geqq 24 \mathrm{hr}$ stabilization at each stepwise substrate level (glucose) change, on a Beckman 119-P Analyzer. While plasma AA levels, generally low at $t=0$ compared to normal infants, appear to increase as the highest carbohydrate infusion is reached, there is significant variation among individual AA. Several AA (Phe, Leu, Pro, Cys, Met) are abnormally low at $\geqq 90 \mathrm{cal} / \mathrm{kg} / \mathrm{d}$, in contrast to infants receiving casein or beef fibrin hydrolysate (J. Ped. $78: 595,1971$ ). In $24-\mathrm{hr}$ urines, 1 -and 3 -methylhistidine excretion is significantly below normal infant excretion. Urinary AA such as Lys, Gln, and Ala increase ( $\mu \mathrm{gm}$ $\mathrm{AA} / \mathrm{mg}$ creatinine) as a function of increasing caloric input to approximately $90 \mathrm{cal} / \mathrm{kg} / \mathrm{d}$, appearing to decline thereafter at $120-150 \mathrm{cal} / \mathrm{kg} / \mathrm{d}$, an almost identical pattern to that for total urea nitrogen excretion. The latter data suggest a point of significant changeover in control of nitrogen utilization in these infants. (Supported by USPHS 5ROl HD07707). 
341 INTRACTABLE DIARRIEA (AND PROTEIN ENERGY MALNUTRITION) P. Pencharz \& E. Colle. Montreal Children's Hospital. A series of 34 patients with intractable diarrhea of infancy treated with TPN over a 2 year period is reported. Twenty-five of the patients were Eskimos and they differed from the 9 Caucasian infants in having a higher incidence of premature births, a higher percentage of male infants and a higher mean age on admission. Specific diagnoses were established in 7/9 Caucasian infants, but once the severe protein energy malnutrition was corrected all but 2 of the Eskimo infants appeared to have no underlying disease. TPN was provided as an protein-hydrolysate, (Amigen(R)) lipid emulsion (Intralipid $(R)$ ) mixture by peripheral veins or central venous catheters. During 34 periods of peripheral TPN the mean duration of treatment was 29 days (5-50 days) the mean caloric intake was $93 \mathrm{cal} / \mathrm{kg} /$ day $(67-121 \mathrm{cal} / \mathrm{kg})$, and the mean protein intake was $2.9 \mathrm{gm} / \mathrm{kg} /$ day. During 11 periods of central TPN the mean duration of treatment was 13.4 days $(4-20)$, mean calories $116 \mathrm{cal} / \mathrm{kg} /$ day (93-137) and mean protein 3.35 (2$4 \mathrm{gm} / \mathrm{kg} /$ day). There were 2 deaths, both Eskimos, both in the central TPN group. Elevation of liver enzymes and eosinophilia were common. Liver biopsies on 7 patients developing elevation of enzymes showed eosinophilia, increased Kupffer cell pigmentation and slight portal lymphocytosis. Liver enzymes returned to normal before discharge. Frequent intercurrent viral and bacterial infections occurred often preceded by a short period of lipid intolerance and/or neutropenia. Tolerance to lipid returned following the infectious episode.

ANTITRYPSIN ACTIVITY IN SOY FORMULAS. Arlynn Mulne, 342 H. Juhling McClung, Penelope Tokarski (Spon. by B.D. Graham). Ohio State Univ. Coll. of Med., Cols. Children's Hosp., Dept. of Pediatrics, Columbus, Ohio.

soy based formulas are used in children who cannot tolerate milk formulas. Since supplementation, soy formulas provide adequate nutrition for heal thy infants. These formulas have not proven successful in premature, malnourished or cystic fibrotic infants. Veterinary experience has shown that the presence of antitryps in substances in soybean protein causes growth retardation in non-ruminant animals. Heat processing of soy protein eliminates most of the antitrypsin activity. The question remains whether commercially available formulas contain excess antitrypsin substances. This study compares antitryps in activity in soy based formutas and milk based formulas. Formulas investigated were $5 \mathrm{milk}$ based, 5 soy, homo. milk, and 4 other formulas containing neither soy nor milk protein. Assays were performed by a modification of the gelatin tube dilution method of Schwachman. Standard trypsin diTutions showed liquefaction of gelatin to a level of $1 \mathrm{mg} \%$. Against the standard trypsin dilution, processed milk based formulas showed complete neutralization of trypsin at a level of $2.5 \mathrm{mg} \%$. Meat base, protein hydroTysate, banana base and amino acid formulas neutralized trypsin at levels of $2.5 \mathrm{mg} \%$. Homo. milk neutralized at levels of $10 \mathrm{mg} \%$ trypsin. Soy based formulas showed complete neutralization between levels of 10 to $25 \mathrm{mg} \%$ trypsin. The persistance of antitrypsin activity in commercially processed soy formulas could be an important factor in the frequent failure of soy formulas in

THE EFFECT OF ORAL ZINC SUPPLEMENTATION ON PLASMA

343 LEVELS OF VITAMIN A AND RETINOL-BINDING PROTEIN IN CYSTIC FIBROSIS. H. Dawn Palin, Barbara A. Underwood and Carolyn R. Denning. Pennsylvania State University, Nutrition Program, University Park, Pa. and College of Physicians and Surgeons of Columbia University, Department of Pediatrics, New York.

Low levels of zinc as well as a defect in the transport of vitamin $A$ have been reported in patients with Cystic Fibrosis (C F). Because of growing evidence of an interrelationship between zinc and vitamin $A, 36$ patients with $C F$ and 17 age-matched controls were given either $100 \mathrm{mg}$ of zinc sulfate or a lactose placebo dally for eight weeks. Plasma samples collected four times during the study period were analyzed for vitamin A, retinolbinding protein (RBP), zinc, total protein, albumin and liver enzyme content. Taste acuity was assessed in all subjects. The zinc content of hair samples taken at the beginning and end of the study period was measured in all subjects. Zinc supplementation did not af fect either the plasma vitamin A or RBP, both of wh1ch were found to be lower in the patients than in the controls. Plasma zinc levels were normal in both $C F$ patients and controls prior to supplementation with zinc and increased only slightly after recelving it. All patients and controls had normal taste sensitivities which were not altered by zinc supplementation. No correlation was found between taste acuity and plasma levels of vitamin $A$ or zinc. In conclusion, CF patients supplemented with $100 \mathrm{mg}$ of zinc sulfate daily for 8 weeks showed no significant change in plasma vitamin $A$ and RBP nor in any other biochemical or clinical parameter measured.
SUPPLEMENTAL PARENTERAL NUTRITION A PEDIATRIC CRYSTALLINE AMINO ACID SOLUTION IN NEWBORNS. Bruce R. Parks, John E. Rawson, and M. Don Turner (Spon. by B. Batson) The University of Mississippi Medical Center, Departments of Pediatrics and Surgery, Jackson

A crystalline amino acid solution designed for use in pediatric patients was utilized in the formulation of a parenteral nutrition infusion used as a supplement in low birth-weight infants. In its final composition the fluid contained in each $100 \mathrm{ml}$., crystalline amino acids $2.5 \mathrm{Gm}$ and dextrose 12.5 to $22.5 \mathrm{Gm}$. Electrolytes and vitamins were added as indicated.

The infants, all less than $1,000 \mathrm{Gm}$, were maintained on the nutrient solution for periods ranging from 7 to 11 days. Following the initiation of oral feedings, the supplement was decreased as tolerance to the oral intake increased.

The daily protein intake averaged $2.80 \mathrm{Gm} / \mathrm{Kg}$ with a mean total caloric intake of $96.4 \mathrm{Cal} . / \mathrm{Kg}$. This provided a total calorie $/ \mathrm{Gm}$ protein ratio of 34.5 . The average weight gain was $17.7 \mathrm{Gm} /$ day. Nitrogen balance studies, performed on two consecutive days, showed a mean positive balance of $301.9 \mathrm{mg} / \mathrm{Kg}$.

These data indicate that steady weight gain with a positive nitrogen balance can be achieved with a pediatric crystalline amino acid solution and dextrose until such time as oral intake becomes adequate.

345SENTIAL FATTYY ACID DEFICIENCY DURING TOTAL PAREN-

345 TERAL NUTRITIAN. Bruce R. Parks, Harold B. White, John E. Rawson, and W. Henry Wooldridge. (Spon. by B. Batson) The University of Mississippi Medical Center, Departments of Pediatrics and Biochemistry, Jackson

Total Parenteral Nutrition (TPN) with protein hydrolysate or amino acids and sugar furnishes no essential unsaturated fatty acid (EFA) to the body. Previous work has shown an EFA deficiency state in both human infants and dogs on TPN. Administration of human plasma or whole blood does not correct this deficiency.

Four pre-term infants had serum assayed for EFA and 20:3w9 for one week while on TPN alone and assayed the following week while receiving wheat germ oil $(0.5 \mathrm{~m} 1 /$ day $)$ per gastric tube. An increase in $20: 3 \omega 9$ has been shown to be the most sensitive indicator of EFA deficiency state.

All infants showed a decrease in EFA and an increase in 20:369 with TPN. 20:3w9 progressively increased to more than fivefold the baseline values in 7 days. The administration of wheat germ oil caused drops in the concentration of $20: 3 \omega 9$ in two of the four infants, but the values in these infants at the end of seven days were still twice the baseline values.

The data demonstrates that all four infants on TPN manifested an EFA deficiency. This was not corrected to baseline values by administration of wheat germ oil in the amount of $0.5 \mathrm{ml} /$ day.

COMparative STUdy bETWEen tRAnS-Pyloric (NASO-JEJUNAL)

346 AND INTERMITTENT GAVAGE FEEDING IN SMALL PRE-TERM INFANTS. Gilberto R. Pereira, James Lemons (Intro. by Joseph Butterfield) Dep. of Perinatology, Children's Hospital, Division of Neonatology, University of Colo., Denver, Colo.

Several recent reports have indicated an advantage of transpyloric (T.P.) feeding over intermittent gavage feeding for small pre-term infants. Other studies have questioned the efficacy and safety of trans-pyloric feeding. This prospective controlled study was designed to evaluate these 2 methods of feeding. Fiftythree patients less than 32 weeks of gestation and appropriate for gestational age were randomly assigned into 2 groups. Mean birth weight + S.D. in the T.P. group $1.14 \pm 0.17 \mathrm{~kg}$ vs $1.13 \pm$ $0.20 \mathrm{~kg}$ for the gavage group. Infants were studied from admission until discharge (mean hospitalization 7.8 weeks range of 6 to 12 weeks). Babies with congenital anomalies were not included. Feedings were started and advanced the same way in both groups, according to degree of tolerance. The first week of life infants fed by gavage had higher caloric intake (gavage mean \pm S.D. $96 \neq$ $21.7 \mathrm{cal} / \mathrm{kg} / \mathrm{day}$ vs trans-pyloric mean $72 \pm 21.0 \mathrm{~S}$.D. cal $/ \mathrm{kg} / \mathrm{day}$ ) $(P<0.05)$. No significant difference was observed in caloric intake after the first week of life, growth parameters (weight, length, head circumference), serum total protein levels, feeding related complications and length of hospitalization. No cases of intestinal perforation or difficulty in removing the feeding tubes was observed in the T.P. group. Based on this study trans-pyloric feeding appeared to be a safe method of feeding pre-term infants but not more efficacious than conventional gavage feedings. 
IDENTIFICATION OF IMMUNOGLOBULIN BEARING LYMPHOCYTES IN FRESH HUMAN BREAST MILK. WiIl iam Pittard, Stephen Polmar, Michael Fanger, Avroy Fanaroff.Case western Reserve University, Depts. of Microbiol. \& Peds. Cleveland.

Fresh human breast milk contains significant quantities of immunocompetent $B$ \& T lymphocytes but only IgA synthesis has been demonstrated. To determine whether selective IgA synthesis was due to restriction of the breast milk B-cell population or regulation of B-lymphocyte differentiation, surface immunoglobulin-bearing Iymphocytes from human breast milk were studied. Fresh human breast milk was collected from ten healthy breast feeding mothers on the 4th to 10th post partum day. The cellular components were isolated using centrifugation and the iymphocytes separated from the phagocytic mononuclear cells (macrophages) by incubation with carbonyl iron particles followed by differential centrifugation on a ficoll-hypaque gradient. This process resulted in a lymphocyte suspension with fewer than $5 \%$ phagocytic cells as determined by myeloperoxidase staining. The lymphocytes were incubated with specific fluorescein conjugated goat antisera to human IgA, IgG, \& IgM. The percentage of cells bearing each class of immunoglobulin on their surface was determined. Surface immunoglobulins were detected on 19.1-29.0\% (mean 22.8\%) of the breast milk lymphocytes. I gA was present on 7.6-12.9\%(mean 9.0\%), IgM on 8.0-10.5\%(mean 9.5 $\%$ ) and $\mathrm{IgG}$ on $1.7-6.4 \%$ (mean $3.7 \%$ ). These data indicate that there is not restriction of the breast milk B-cell population. Therefore, IgA synthesis by breast milk Iymphoid celis may result from either selective enhancement of IgA B-cell differentiation or suppression of other classes of B-cell differentiation.
NATURE OF WEIGHT GAIN DURING TOTAL PARENTERAL

348 NUTRITION (TPN). A.R. Pulito, J.P. Nicholson and and Surgeons, Dept. of Peds., N.Y.

Body weight $(\mathrm{BW})$, individual organ weights, eviscerated carcass weight (CW) and composition (CC) as well as in vivo THO and ${ }^{36} \mathrm{Cl}$ spaces of beagle puppies were determined at birth (0-day) and after 10 days of normal feeding (C), TPN (200 caI/ $/ \mathrm{kg} / \mathrm{d})$, or $10 \%$ glucose IV (S). At 10 days, BW of $C(n=9)$ and TPN $(n=7)$ had increased $67 \pm 5 \%$ and $66 \pm 4 \%$ respectively (N.S.), while BW of S $(n=5)$ had decreased $3 \pm 2 \%$. Liver weight was larger in TPN than $C$ $(p<0.01)$. Weights of other organs, as percentages of BW, were similar in all groups. CW at birth and after 10 days of each nutritional regimen accounted for $69.7-74.8 \%$ of BW (N.S.). As determined from wet, dry and fat-free dxy weights, CC of TPN and $S$ contained more water $(p<0.01)$ and less fat $(p<0.01)$ than $c$. Except for slightly more fat in TPN, CC of both TPN and $S$ resembled that of 0-day animals. Fat-free dry solids of all groups contained $65-70 \%$ protein (N.S.) and represented similar percentages of $\mathrm{CW}$. In vivo measurements of THO space agreed with carcass composition data--i.e., grea̧ter in 0-day, TPN and $S$ than $C$ $(p<0.005)$. Compared to $C,{ }^{36} \mathrm{Cl}$ space was greater in 0 -day $(p<0.01)$, TPN and $S(p<0.005)$ but represented similar percentages of THO space in all groups. The data demonstrate that the tissue laid down during TPN contains more water and less fat than observed in normally fed animals.

349 CONIINUOUS DRIP NASOGASTRIC (NG) FEEDINGS: A CONRosita S. Pildes: Cook County Hosp. Depts. of Pediat, Univ. of 111 . and Chgo. Med. Sch., Chgo.

The need to supply optimum nutrition for premature infants has led to a continuing search for a safe and simple method by which adequate calories can be provided. The purpose of this randomized controlled study was to evaluate the efficacy of NG feeding by continuous drip as compared to nasojejunal (NJ) feedings. Ninestandard 20cal/oz formula within the first 30 hrs of pos tnatal age. I.V. fluids, 5\%DE. 2NS were supplemented as needed. Both groups were started on 5 imilar amounts of formula depending on birth wt. Birth wt. and gestational age in the NG group $\left\{1.3^{+}\right.$ $.18 \mathrm{Kg}$ (Mean+SD) and $30.1+2.2$ wks were similar to those in the $\mathrm{NJ}$ group, $(1.2 \mp .23 \mathrm{Kg}$ and $30.1+2.0 \mathrm{wks})$. All were appropriate for gestation. Four NG newborns and I NJ had severe RDS; 5 NG and 2 $\mathrm{NJ}$ needed assisted ventilation. Birth wt. was regained in $14.4 \pm$ $6.5 \mathrm{~d}$. in NG and $15.9+5.5 \mathrm{~d}$. in NJ groups. At $21 \mathrm{~d}$., there was no significant difference in wt. between NG and NJ $(1.4+.26$ vs $1.3+.32 \mathrm{Kg})$ groups. During days $1-7$, mean $\mathrm{cal} / \mathrm{Kg} /$ day were similar in both groups; at $7 \mathrm{~d}$., NG was given $90+15$ vs $73+30 \mathrm{cal} / \mathrm{Kg}$ for $N J$ group. From 8-14 d., NG tolerated significantly more ( $p<.05)$ $\mathrm{cal} / \mathrm{Kg} /$ day than did the NJ group; NG intake at $14 \mathrm{~d}$. was $120+24$ vs $96+21 \mathrm{cal} / \mathrm{Kg}$ in $\mathrm{NJ}$. These differences were no longer present by day 21 when many infants were taking feedings by nipple. No aspiration or other significant complications were seen in either group. Thus, continuous NG feeding provides an efficient, practical and safe technique for provision of cal. to small infants.
BACTERIAL COLONIZATION OF HUMAN MILK, by Griffith E.

350 Andrews. University of Louisville, Norton-Children's Hospitals, Department of Pediatrics, Louisville, Kentucky.

An increased incidence of necrotizing enterocolitis stimulated animal studies into the etiology and prevention of this disease. Early reports indicated a possible preventive role for breast milk. The utilization of unprocessed breast milk was begun at the University of Louisville in March, 1975. To date, $178 \mathrm{high}$ risk infants have been nourished, wholly or partially with human milk. As a quality control measure, cultures were taken from 129 random samples of milk that were currently in use in the nursery. Bacterial colonization of milk occurred in $93 \%$ of samples; $7 \%$ were sterile. Sixty-five percent of samples were colonized with gram positive organisms on $1 y, 25.6 \%$ with both gram positive and gram negative organisms, and $2.3 \%$ with gram negative organisms alone. Organisms observed with greatest frequency, as either pure isolates or mixed cultures were: S. epidermidis, $90.6 \%$; Klebsiella sp., $17.8 \%$; Enterobacter sp., $13.1 \%$; S. aureus, $12.4 \%$; other organisms totaled $5.1 \%$. No adverse effects were noted in infants attributable to colonization of milk. The bacterial growth kinetics in milk as utilized in this nursery are under continuing investigation. These data suggest: 1) an improved collection technique is needed to achieve lower levels of bacterial colonization, 2) fresh, unprocessed human milk collected under currently available techniques can be safely utilized in the neonate. Data on the efficacy of human milk in the prevention or amelioration of necrotizing enterocolitis remains to be
evaluated.

351 J.T. Rodriguez, M.D., J. Abdo E., M.D., N. Flores S. M.D., T.L. Fuang, Ph.D.*, J. Alvarado, M.D., B.L. Nichols, M.D.*. Unidad de Estudios Clinicos, Department of Pediatrics, Roosevelt Hospital, Guatemala City. *Section of Nutrition \& Gastroenterology, Baylor College of Medicine, Houston, Texas.

Primary bile acids (BA) and some BA metabolites are known to produce celiular damage to the intestinal mucosa and inflammation when injected in muscle. Under the hypothesis that skin irritation in the dianer area observed in children is due to altered fecal BA, 60 children were admitted to Roosevelt Hospital for study. They were divided into 3 groups of 20 each and treated ouring a 5 day period in a double blind fashion with any of the following medications: 1) Desitin ointment; 2) Cholestyramine ointment; and 3) Placebo. The results are indicated as follows:

\begin{tabular}{lcccc}
\hline & COMPLETE & OBVIOUS & PARTIAL & \\
& CURE & IMPROVEMENT & IMPROVEMENT & FAILURE \\
\hline Desitin & $30 \%$ & $35 \%$ & $25 \%$ & $10 \%$ \\
Cholestyramine & $65 \%$ & $30 \%$ & $5 \%$ & $0 \%$
\end{tabular}

Cholestyr

$30 \% \quad 20 \%$

$45 \%$

$5 \%$

Treatment of diaper rash with a $20 \%$ cholestyramine ointment in a water soluble base showed a statistically significant difference from the groups treated with Desitin and Placebo. We postulate that skin irritation in the diaper area is due to organic acids found in the stools. Since cholestyramine bas a great affinity for binding BA, it is possible that they may play a role in the genesis of diaper rash. Partial support for this study is acknowledged from NIH Training Grant AM05721-02.

352 VERY LOW BIRTH WEIGHT (VLBW) INFANTS. Neil Roy, Robert Pollnitz, Richard Hamilton and Graham Chance, Research Institute, Hospital for Sick Children, Department of Paediatrics, University of Toronto, Ontario, Canada.

We studied, prospectively, intestinal absorption of a standard $80 \mathrm{cal}$ per dl infant formula in 18 healthy VLBW infants assigned randomly to one of 2 feeding groups. The one group was fed continuously by the NJ route, the other in 2 hourly bolus feeds by the nasogastric (NG) route. The two groups did not differ significantly with respect to mean gestational age, (NJ $30.3 \mathrm{vs.}$ NG 31.0 weeks $)$, bixth weight $(1280 \mathrm{~g}$ vs. $1285 \mathrm{~g})$ and body weight at age 8 days when the study began $(1150 \mathrm{~g}$ vs. $1166 \mathrm{~g})$. Between 8 and 15 days of age NJ fed infants did not differ from NG infants in their growth, blood sugar, $\mathrm{Na}, \mathrm{K}, \mathrm{Ca}$, urea $\mathrm{N}$ or amino acid pattern. However, during the same period stool frequency, fat and potassium excretion were significantly greater in the NJ fed group.

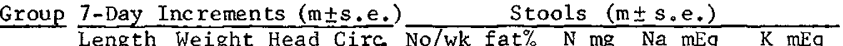

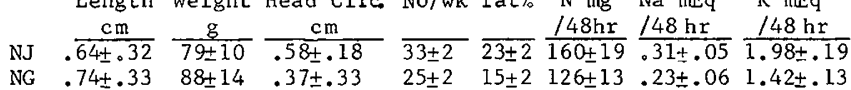
We conclude that a conventional formula is absorbed less efficiently when fed by NJ tube than when fed by NG tube to young VLBW infants. 
HYPERURICOSURIA DUE TO PANCREATIC ENZYME THERAPY,

353 Bruder F. Stapleton, James Kennedy, Sanda N. ArvaniDepts. of Pediatrics and Medicine, Univ. of Kansas Medical Center Kansas City, Kansas.

Metabolic complications of excessive amounts of pancreatic enzymes in patients with cystic fibrosis have not been reported. Recently we observed a 4 year old boy with cystic fibrosis who presented with dysuria, uric acid crystalluria and hyperuricosuria shortly after an enzyme dosage increase. Known causes of hyperuricosuria were eliminated. On a controlled diet, hyperuricosuria disappeared when enzyme dosage was greatly reduced, though no gastrointestinal symptoms developed. Symptomatic hyperuricosuria reappeared when enzyme doses were raised to initial levels. Two additional patients, on different enzyme preparations, were similarly found to be hyperuricosuric due to pancreatic extract. In these 3 patients, a close correlation was found between pancreatic enzyme dosage and 24 hour urinary uric acid excretion. When daily enzyme purine content was reduced by a mean of $227 \mathrm{mg}$, urinary uric acid excretion decreased by a mean of $214 \mathrm{mg}$ ( $p<.02$ ) Our chemical analysis of the purine content in commercial enzyme preparations revealed: Preparation

Cherry-flavored pancrealipase powder Pancrealipase capsule Pancreatine tablet

\section{Purine content} $23.6 \mathrm{mg} /$ pack $3.7 \mathrm{mg} /$ capsule cause symptomatic hyperuricosuria from intestinal therapy can purines contained in these preparations.

\section{TER}

354 EXTRACELLULAR VOLUME. James Sumners, Sherry Burris and Howard Arkans. (Spon. by George Cassady) University of Alabama, Department of Pediatrics, Division of Perinatal Medicine, Birmingham, Alabama.

Timed measurement of skinfold thickness (SFT) with simultaneous estimation of extracellular water volume by corrected bromide space (CBS) was done on 26 occasions in 17 infants. Postnatal age at study varied from 2 to 47 days. Study weight ranged from 1040 to 4280 grams with only one baby weighing more than 2500 grams. SFT was obtained at 4 sites bilaterally (midtriceps, subscapular, flank and quadriceps). SFT decreased with time after application of the Harpenden calipers and the values at 5 and 60 seconds ( $\mathrm{SFT}_{5}$ and $\mathrm{SFT}_{6} 6$ were used to express SFT compressibility as rate of change $\left(\triangle \mathrm{SFT}=\mathrm{SFT} 5-\mathrm{SFT}_{6} \partial\right.$ and as percent of the SFT which was compressible $\left[\% \Delta \mathrm{SFT}=\left(\mathrm{SFT}_{5}-\mathrm{SFT}_{6} \partial /\right.\right.$ $\mathrm{SFT}_{5} \times 100$ ] $\mathrm{CBS}$ was evaluated in relation to body weight $(\mathrm{CBS} / \mathrm{kg})$ and crown-heel length ( $\mathrm{CBS} / \mathrm{CH}$ ).

SFT compressibility correlated with extracellular water volume. This was true both in the relationship between $\% \Delta S F T$ and $\mathrm{CBS} / \mathrm{kg} \quad(\mathrm{r}=.41 ; \mathrm{p}<.05)$ and that between $\triangle \mathrm{SFT}$ and $\mathrm{CBS} / \mathrm{CH}$ $(\mathrm{r}=.54 ; \mathrm{p}<.005)$. Intravenous therapy during the day prior to study was associated with larger $\mathrm{CBS} / \mathrm{kg}(t=2.09 ; \mathrm{p}<.05)$ and larger \% $\triangle \mathrm{SFT} \quad(\mathrm{t}=5.84 ; \mathrm{p}<.001)$.

Implications of these data are 1) that SFT compressibility may be used in assessment of quality of weight gain and 2) that these measurements may be useful in the clinical evaluation of hydration of sick infants.

JAMAICAN VOMITING SICKNESS : BIOCHEMICAL INVESTIGATION

355 OF TWO CASES, K. Tanaka, E. A. Kean and B. Johnson, Yale univ. Sch. of Med., Dept. of Human Genet., New Haven; Dept. of Biochem., Univ. of West Indies and Children's Hosp., Kingston, Jamaica. (Spon. by L. E. Rosenberg)

The interest in Jamaican vomiting sickness has been rejoined recently due to its similarities to Reye's syndrome. It is clinically characterized by acute onset, vomiting, and coma which are accompanied with severe hypoglycemia and acidosis. It occurs most frequently in children below ten and is of ten fatal. Diffuse fatty infiltration of liver is observed in autopsy cases. The ingestion of unripe ackee fruit which contains a toxin, hypoglycin, has been implicated as its cause. We analyzed the urine from two siblings who developed the typical symptoms by gas chromatograph-mass spectroscopy. We identified methylenecyclopropylacetic acid, the toxic metabolite of hypoglycin, in significant amounts ( 16 and $8 \mu \mathrm{g} / \mathrm{mg}$ creat.). We also detected large amounts (50 to 1000 times normal) of glutaric, adipic, suberic, sebacic, octenedioic and decenedioic acids which we have previously identified in urine of hypoglycin treated rats (J. Biol. Chem., 247, 7465, 1972). This evidence conclusively links hypoglycin to the cause of this disease for the first time. In addition, ethylmalonic, methylsuccinic and several short chain fatty acids are also identified in large quantities. In contrast, these unusual urinary metabolites were not significantly increased in five patients with Reye's syndrome although in one case slightly increased amounts of adipic and sebacic acids were excreted. These results indicate that biochemical mechanisms of these two clinically similar diseases are basically different.
GASTROINTESTINAL ENDOSCOPY IN CHILDREN. Annie B. Terry, Harrison J. Shull, Jr., and Harry L. Greene. Vanderbilt University, Department of Pediatrics,

\section{Nashville, Tennessee.}

The development of smaller fiberoptic endoscopes, permitting direct visualization of the UGI tract has provided an improved method of evaluating gastrointestinal problems in children. Endoscopies were performed in 38 patients, ages 6 months - 17 years, with no complications. Indications were UGI hemorrhage, chronic abdominal pain, protracted vomiting, or equivocal radiographic studies. The site of bleeding was identified in 6 of 7 children with hematemesis and 1 of 3 with melena. Twenty-two patients were examined because of chronic abdominal pain and possible ulcer disease: lesions of the esophagus, stomach, or duodenum were found in ten, 7 of whom had normal x-ray examinations.

Three of 5 patients endoscoped for chronic vomiting were found to have ulcer disease, including an infant with protracted vomiting, and 2 children with "cyclic vomiting." These preliminary results demonstratee the usefulness of endoscopy and suggest that: 1) uicer disease in children may be difficult to diagnose clinically in that symptoms are often atypical and may include protracted or cyclic vomiting, 2) gastric ulcers are more common under 5 years of age, and 3) radiologic studies may fail to demonstrate ulcers which can be visualized through endoscopy.
BILIRUBIN(B) METABOLISM AND TISSUE DISTRIBUTION IN

357 JAUNDICED RATS TREATED WITH INTRALIPID(I). M. Michael Thaler, University of California, San Francisco, CA
cts of infused । (10\% fat emulsion) on the kinetics

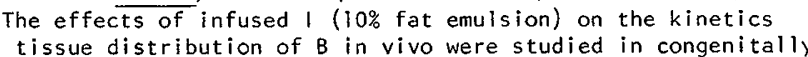
and tissue distribution of $B$ in vivo were studied in congenitally miscible $B$ pool had been uniformly labeled with $\mathrm{B}-14 \mathrm{C}$ for at least $30 \mathrm{hrs}$. I was infused $(100 \mathrm{mg} / \mathrm{hr}$.) for 6 or $2 / \mathrm{hrs}$., or pulse injected $(100 \mathrm{mg})$. Control rats received equivalent volumes of saline or $5 \%$ dextrose. Blood was sampled at intervals, animals were sacrificed $6 \mathrm{hrs}$. after injection or at the end of infusion, and tissue $B$ determined isotopically and chemically. Plasma B concentrations remained unchanged during $I$ treatment of rats with moderate $(7-9 \mathrm{mg} \%)$ and severe $(>15 \mathrm{mg} \%)$ hyperbilirubinemia. Plasma $B$ specific activity curves were identical before and after 1 infusion, indicating that $B$ production, fractional turnover, and biological half-life were not affected. The total $B$ pool and plasma $B$ pool remained constant through the $24-\mathrm{hr}$. I infusion period. The plasma B pool represented $13.3 \%$ of the total B pool in 1 -infused rats, and $12.7 \%$ in controls. Tissue $B$ concentrations were not significantly different in 1 -treated and saline-infused rats, and were higher in liver and kidney than in plasma. In contrast, brain, muscle and fat contained relatively little $B$. Thus, $B$ concentration in brain of 1 -treated and control rats averaged $13 \%$ and $12 \%$ of plasma $B$ concentrations, respectively. These results indicate that 1 infused in concentrations equal to or higher than those utilized clinically has no detectable effects on $B$ transport or tissue distribution in jaundiced rats.

THE NEONATAL FORM OF CITRULLINEMIA:SURVIVAL 358 IN A PATIENT TREATED WITH KETOACID ANALOGUES OF ESSENTIAL AMINO ACIDS. JesS G. Thoene, Mark Batshaw, Elaine B. Spector, Stanko Kulovich, Saul W. Brusilow, Mackenzie Walser, and William L. Nyhan. University of California, San Diego and Johns Hopkins University, Baltimore, Maryland.

Type I citrullinemia results in seizures, coma, and death by age 7 days. Absence of argininosuccinic acid synthetase (E.C.6.3.4.5.) and extremely high levels of ammonia and citrulline in the blood are characteristic. Success in treating hyperammonemia with a-keto acid analogues of essential amino acids led us to a trial of this therapy in a patient with type I citrullinemia. A male infant presented with seizures, coma, and a blood ammonia of $550 \mu \mathrm{M}$. The plasma citrulline at 20 days of age was $3.86 \mathrm{mM}$ (normal<0.03mM), and a diagnosis of neonatal citrullinemia was made. Intravenous injection of the o-keto analogues of leucine, isoleucine, valine, phenylalanine and methionine produced marked (2 to 25 fold) rises in the plasma concentrations of the corresponding amino acids. Skin fibroblast argininosuccinic acid synthetase activity was $<0.01$ of normal. Oral therapy with the keto acid mixture produced a dramatic decline in both blood ammonia and plasma citrulline (to $50 \mu \mathrm{M}$ and $0.5 \mathrm{mM}$, respectively) with a concomitant linear increase in weight. At 7 months the patient had milestones of 5 months. An episode of hypernatremic dehydration was fatal at age 8 months. 
PHE PHYSICAL BASIS OF INTESTINAL WATFR SECRETION IN 359 CHOLERA. J. N. Udall,$\frac{\text { L. A. Alvarez }}{\text { and B. L. Nichols. D. Hazlewood }}$ College of Medicine, Houston, Texas 77025.

An ordered intracellular protein-water system within normal intestinal mucosa has been proposed to prevent the extravasation of fluid and solutes into the lumen. In addition, cholera entero toxin has been postulated to alter the configuration of the cellular proteins, thereby increasing mucosal permeability to water. Using nuclear magnetic resonance spectroscopy to determine the mobility of the water in the intestinal tissue, this hypothesis was tested. Small intestinal loops in the rat were injecteo with $0.5 \mathrm{ml}$ of either Schwarz/Mann cholera enterotoxin ( $40 \mu \mathrm{gm} / \mathrm{cc}$ saline solution) or normal saline. Normal intestinal tissue in the rat contained $79.5 \pm 2.7 \%$ water $(\overline{\mathrm{X}} \pm \mathrm{S} . \mathrm{D}$.$) . \mathrm{T}_{1}$ (spin-lattice) relaxation time was $521 \pm 70 \mathrm{msec}$ and $\mathrm{T}_{2}$ (spin-spin) $62 \pm 10 \mathrm{msec}$. In intestinal tissue exposed to cholera enterotoxin, \% hydration increased to $84.5+2.0$. T relaxation time was $668+119 \mathrm{msec}$ and $\mathrm{T}_{2}$ was $80 \pm 21 \mathrm{msec}$. Increased relaxation times are related to increased motional freedom of intracellular water. The water in the tissue exposed to enterotoxin had greater motional freedom and hence could be interpreted to be more like liquid water. These changes in relaxation times support the concept that cholera enterotoxin alters the intracellular protein-water system in such a manner as to enhance fluid loss.

Research supported in part by NIH Training Grant AM-05721, Office of Naval Research Contract N0004-76-C-0100, USPH Research Grants GM-20154 and RR-00188 and Robert A. Welch Foundation.
FURTHER EXPERIENCE WITH JOYBEAN ENUULSION AND CASEIN HYDRULYSATE (SECH) IN. PARENTERAL NUT-

360 CASEIN HYDRULYSATE (SECH) IN PARENTER

Robert H. Dhital, Depts. of Ped. Obs.' and Syn., Kontreal

The use of $3 \mathrm{GH}$ in parenteral nutrition of $\angle 1500 \mathrm{~g}$ infants has been reported in 19 survivors (Ped. 56:8, 1975). This propram has now been used in a further weighing 501-1500g, almost all Caucasian, with excellent survival rates:

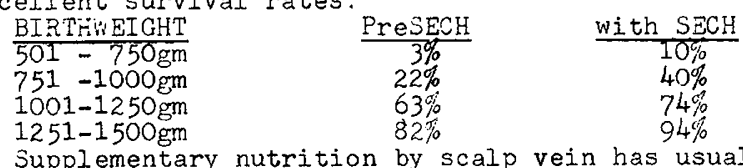

Supplementary nutrition by scalp vein has usually been required for 2-6 weeks until milk feeds were adequate. Inadequate metabolism or excretion of fat, protein, carbohydrate, or water led to reduced intakes in some of the smallest, sickest infants. Good growth occurred on 93 calories $/ \mathrm{kg} / \mathrm{d}$, anc infants were discharged 2-4 weeks before their F.D.C. Kernicterus, coagulopathies, infection, necrotising enterocolitis, apneic spells and acidosis have not increased.

SECH is a useful adjunct to nutrition of the small premature. Further studies of dose, metabolism and follow-up are needed.

THE IMMUNOLOGIC CONTROL MECHANISM FOR TOXIGENIC 361 MrARRHEA. W. Allan Walker and Ai-Lien Wu. Harvard Boston.

The mechanism of antibody protection against toxigenic diarrhea is not completely understood; however, one possible role of local antibodies is to prevent the binding of toxin to specific receptors on the intestinal microvillous membrane, thereby inhibiting secretion (diarrhea). To study this possibility, 125 Icholera toxin (CT) (20 ug) was injected into the small intestine from immunized and control rats; after $10 \mathrm{~min}$. intestine was removèd and microvillous membranes prepared. To maximize the local intestinal antibody response, immunized rats were given repeated intraperitoneal injections of crude CT (2 mgCT in adjuvant) and boosted orally ( $100 \mathrm{mgCT}$ ) 10 days before study. Compared to negligible binding of 125 I-bovine serum albumin used as a control, measurable quantities of $125 \mathrm{I}-\mathrm{CT}$ were recovered from the microvillous fractions suggesting binding of $C T$ to a specific receptor site. In ten experiments, an average \pm SEM of $45 \pm 8.5 \mathrm{pg}$ CT per ug membrane protein was bound by microvillous membranes from control animals compared to $20+2.8 \mathrm{pg}$ bound/ug protein by immunized rats $(p<0.02)$. Furthermore, after $8 \mathrm{hr}$. incubation with CT (1 ug) $10 \mathrm{~cm}$ loop) a highly significant $(\mathrm{p}<001)$ decrease in fluid accumulation occurred in ileal loops from immunized versus control rats. Furthermore, CT-binding and decreased fluid accumulation in ileal loops were directly related to levels of intestina1 CT-antibody. These studies strongly suggest that intestinal antibodies protect against enterotoxigenic diarrhea by interfering with binding of toxin to the intestinal surface.
DIETARY SOY PROTEIN INTOLERANCE IN INFANTS. Peter F. 362 Whitington and Richard C. Gibson, (Spon. by James N. Ette1dorf), University of Tennessee Center for the Health.Sciences, Department of Pediatrics, Memphis, Tennessee.

An infrequently encountered and poorly understood infantile disorder is gastrointestinal soy protein intolerance (SPI). Four patients with intractable diarrhea of infancy and failure to thrive were tested by per oral challenge with soy protein isolate and found to have SPI. All four exhibited concomitant sensitivity to cow's milk protein. The response to challenge with soy protein included diarrhea, vomiting, hypotension, lethargy and fever. These symptoms were immediate, of short duration and occurred with each subsequent challenge dose. Although prominent in the families, no patient exhibited cutaneous, pulmonary or hematologic evidence of allergy. A diet devoid of intact soy and cow's milk protein allowed symptomatic recovery and rapid weight gain. Oral disodium chromoglycate therapy was ineffective in one trial. We conclude that SPI should be suspected in infants with intractable diarrhea while being fed a soy based formula since specific therapy is available and effective. No pathogenic mechanism can be concluded from our data.
TOOL BACTERIA IN LOW BIRTH WEIGHT (LBW) INFANTS :

363 CHANGES WITH MILK FORMULA. Brenda Wilkinson, Keith Nicholas M. Nelson). King's College Hospital, Dept. Child Health, London.

The effects of the composition and preparation of milk on aerobic and anaerobic fecal bacteria were studied in 'normal' LBW infants. The groups studied: (A) 6 infants ( $\bar{x} 1.6 \mathrm{~kg}, 33$ wks) fed pooled expressed breast milk which had been boiled, dispensed aseptically and stored (up to $I \mathrm{yr}$ ) at $-20^{\circ} \mathrm{C}$, (B) 16 infants (र $1.9 \mathrm{~kg}, 34$ wks) fed "humanized" milk formulas, (C) $6 \mathrm{full-}$ term breast fed infants. At 1 week feces were examined using standard aerobic and anaerobic bacteriological techniques. Colony counts per gram of stool: Mean \pm SD

$\begin{array}{lrr} & \text { E. coli }+ \text { coliforms } & \text { Lactobacillustbifidobacteria } \\ \text { Group A } & 19 \times 10^{9} \pm 36 \times 10^{9} & 2.0 \times 10^{9} \pm 1.7 \times 10^{9} \\ \text { Group B } & 6.1 \times 10^{9} \pm 7.9 \times 10^{9} & .19 \times 10^{9} \pm .37 \times 10^{9} \\ \text { Group C } & .88 \times 10^{9} \pm .54 \times 10^{9} & 5.4 \times 10^{9} \pm 3.0 \times 10^{9}\end{array}$

Coliform predominance over bifidus bacteria in Groups A or B is a reversal of the pattern found in normal breast fed infants, although in Group $C$ the predominance of bifidus bacteria is not as marked as expected. Colonies of staphylococcus and bacteroides species were cultured only in Groups A or B. The results indicate that (1) human milk, if boiled before storage, loses its efficacy in maintaining a predominant 'bifidus' fecal flora and supports the growth of coliforms, (2) LBW infants fed 'humanized' cows' milk formula develop a mixed fecal flora.
364 IRON NUTRITION IN THE BREAST-FED INFANT. Calvin W.

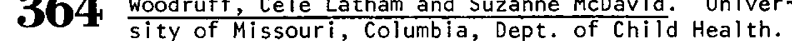
Nineteen infants whose only milk intake was their mother's milk were compared with 31 infants fed a prepared formula not supplemented with iron for the first 9 mos. of 1 ife. All infants weighed over $3 \mathrm{~kg}$ at birth and their growth rates were comparable. No other food was given during the first 3 mos. Cereal fortified with sodium iron pyrophosphate, fruits and vegetables were fed at 3 mos of age. Between 3 and 6 mos the breast-fed infants ate less iron than the formula-fed group. Iron intakes between 6 and 9 mos of age were $6.52 \pm 1.16$ (SEM) mg/day for the breast-fed infants and $7.22 \pm 0.21 \mathrm{mg} /$ day for the formula fed infants. The difference was not significant. No significant differences in $\mathrm{Hgb}$, HCt, RBC, MCV, serum iron or transferrin saturation were found at 3,6 or 9 mos. Total iron binding capacity was significantly higher in the formula-fed infants at 3,6 and 9 mos. Hgb concentration between 10 and $11 \mathrm{gm} / \mathrm{dl}$ with transferrin saturation less than $16 \%$ at 9 mos occured in 1 of 8 breast-fed and 4 of 31 formula-fed infants. Although the breast-fed infants had a lower iron intake between 3 and 6 mos and lower total iron binding capacity at 3, 6 and 9 mos, there was no difference in the incidence of $i$ ron deficiency as measured by transferrin saturation, microcytosis or $\mathrm{Hgb}$ concentration less than $11 \mathrm{gm} / \mathrm{dl}$. Much of the iron intake was in a poorly absorbed form. The iron requirement of both groups was similar but could not be determined quantitatively under the conditions of this study. Supported by Ross Laboratories. 
365

OSMOLAR AND FREE WATER CLEARANCE $\left(\mathrm{C}_{\mathrm{OSM}}, \mathrm{C}_{\mathrm{H}} \mathrm{O}\right)$ IN PRETERM INFANTS ON "HIGH" AND "LOW" SOLUTE FORMULA.
Paul Y.K. Wu and Sze K. Ho. University of Southern California Sch. of Med., Los Angeles County-USC Medical Center. Dept. of Pediatrics.

The neonate's ability to conserve or excrete excess water under varying intakes is less efficient than in adults. Maximum and minimum urine volumes are dependent on the amount of solutes that require excretion by the kidneys. Net loss or gain of water to the body may be calculated from simultaneous values of the $\mathrm{C}_{\mathrm{osm}}$ and urine flow. Twenty-four well, preterm infants, were divided into 2 groups of 12 infants each. Group $A$ was fed a "High Solute" diet (Similac 24). Group B was fed a "Low Solute" diet (Similac $60 / 40,24 \mathrm{Cal} / \mathrm{oz}$ ). The volume of milk consumed was comparable in both groups. Infants were studied at weekly intervals during the first 3 weeks of 1 ife. Each study period consisted of 24 hours. RESULTS: (Mean values)

\begin{tabular}{|c|c|c|c|c|c|c|}
\hline \multirow[b]{2}{*}{$\begin{array}{l}\text { Age } \\
\text { (Wk) }\end{array}$} & \multicolumn{3}{|c|}{ "High Solute" } & \multicolumn{3}{|c|}{ "Low Solute" } \\
\hline & $\mathrm{C}_{\text {osm }}$ & $\mathrm{C}_{2} \mathrm{O}$ & $\begin{array}{l}\text { Urine } \\
\mathrm{ml} / \mathrm{kg} / \mathrm{h}\end{array}$ & $C_{\text {osm }}$ & $\mathrm{C}_{\mathrm{H}_{2} \mathrm{O}}$ & $\begin{array}{l}\text { Urthe } \\
\mathrm{ml} / \mathrm{Kg} / \mathrm{h}\end{array}$ \\
\hline 1 & 0.044 & 0.041 & 3.22 & 0.046 & 0.052 & 3.67 \\
\hline $\begin{array}{l}2 \\
3\end{array}$ & $\begin{array}{l}0.056 * \\
0.059 *\end{array}$ & $\begin{array}{l}0.044 \\
0.044\end{array}$ & $\begin{array}{l}3.51 \\
3.29\end{array}$ & $\begin{array}{l}0.037 * \\
0.036^{\star}\end{array}$ & $\begin{array}{l}0.051 \\
0.055\end{array}$ & $\begin{array}{l}4.08 \\
3.52\end{array}$ \\
\hline
\end{tabular}

$\mathrm{p}<0.05$

There were no significant differences in serum water, osmolarity and electrolytes between the two groups. Data suggest that preterm infants can maintain homeostasis with both diets.

INPIBITION OF ORIITHINE TRANSCARBANTLASE (OTC) ACTI-

366 VITY IN MORIAL DAT AND HUMAY LIVER BY T, IVER IIOMO GENATES FROM PATLEITS UITII REYE'S SYNDRNM. (RS). Takashi Yoshida, Frank Sinatra and Philip Sunshine. Stanford University School of Medicine, Dept. of Pediatrics, Stanford, California.

Decreased OTC activity has recently been observed in liver from patients with RS. Two natients with the clinical and histologic diagrosis of RS died within 72 hours following the onset of encephalopathy. Liver was obtained within 1 hour of the time of death and was frozen at -700 . I Iomogenates were prepared and allowed to pre-incubate from 1 to 24 hours in the presence of an equal volume of either the homogenizing solution, normal rat liver homogenate, or normal human liver homogenate. Radiochemical determination of OTC activity revealed a $57-70 \%$ reduction in rat liver ITC at 1 hour and 94\% reduction at 24 hours. l.uman liver OTC was inhibited by only $10 \%$ at 1 hour but was reduced by $68 \%$ at 24 hours. Hixing of normal human liver with both rat liver and other control human liver caused no reduction in predicted OTC activity. Liver from a third patient with RS obtained 15 days following the onset of encephalopathy produced only a $14 \%$ reduction in the predicted OTC activity of rat Iiver. At the time tissue was obtained in this patient liver function studies, including serum ammonia, had returned to normal. These observations suggest the possible existence of a substance within the liver during the early stages of RS which may be capable of producing acquired refects in urea biosynthesis.
LIPOPROTEIN-X IN OBSTRUCTIVE JAUNDICE IN INFANCY.

367 Barry S. Yoss and Philip J. Lipsitz. Dept. Of Peds. Jewish-Hillside Medical Center, New Hyde Park, N.Y.

Measurement of serum Lipoprotein-X $(L P-X)$ has been proposed as a reliable test that will distinguish between biliary atresia (BA) and neonatal hepatitis (NH). We studied 6 infants under 3 months of age presenting with obstructive jaundice. Metabolic, hematologic, and infectious diseases were excluded. One of these had alpha1 antitrypsin deficiency (AATD). Rose Bengal excretion after cholestyramine and alpha fetoprotein were measured in all patients, but in only 2 did both tests correctly predict the final diagnosis. All had $L P-X$ measurements before and after cholestyramine. Decreasing LP-X correctly predicted NH in 3 of 3 cases. LP-X increased in one patient, correctly predicting $B A$. One infant with a choledochal cyst (CC) had LP-X values suggesting both $\mathrm{BA}$ and $\mathrm{NH}$, but this diagnosis might have been made by sonography or GI series. We conclude that increasing LP-X in an infant with normal alpha, antitrypsin is an indication for open biopsy and cholangiogram. Decreasing LP-X, after exclusion of choledochal cyst, confirms the presence of a patent biliary tree and obviates the need for surgery. This test should be part of the routine evaluation of infants presenting with obstructive jaundice in the first three months of life.

\begin{tabular}{|c|c|c|c|c|c|c|c|}
\hline CASE \# & & 1 & 2 & 3 & 4 & 5 & 6 \\
\hline$L P-X \quad(m g \%)$ & $\begin{array}{l}\text { before } \\
\text { after }\end{array}$ & $\begin{array}{l}83.5 \\
62.0\end{array}$ & $\begin{array}{l}62.5 \\
30.0\end{array}$ & $\begin{array}{r}154.4 \\
83.0\end{array}$ & $\begin{array}{l}709.0 \\
790.0\end{array}$ & $\begin{array}{r}62.5 \\
104 / 0\end{array}$ & $\begin{array}{l}359.0 \\
396.0\end{array}$ \\
\hline OIAGNOSIS & & NH & $\mathrm{NH}$ & $\mathrm{NH}$ & $B A$ & $C C$ & AATD \\
\hline
\end{tabular}

IN VIVO BICARBONATE (HCOB) TRANSPORT IN THE

368 SMAll intestine OF SuCKLing(S) And ADULT(A) RATS. M. K. Younoszai and Jean Robillard. University of Iowa, College of Medicine, Department of Pediatrics, Iowa City, Iowa.

Using a one pass perfusion technique we measured net absorption(NA) and lumen-to-mucosa(LM) and mucosa-tolumen (ML) fluxes of $\mathrm{HCO}^{\overline{3}}$ in segments of the proximal(P) and distal(D) intestine. The perfusion solutions con tained, in moles/litex, NaCL $115 ; \mathrm{KCL} 5 ; \mathrm{NaCHO}_{3} 25$; glucose 17 and/or mannitol 17 or 35 and phenol red (20 mg/liter). $\mathrm{HCO}_{3}^{-} \mathrm{NA}(\mu \xi \mathrm{q} / \mathrm{hr}$ per $\mathrm{g}$ wet wt, ) was not different in corresponding segments of $S$ and $A$ rats

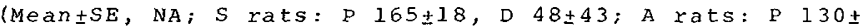
10 , D $-17+18)$. With the exception of the ML flux in the distal segments, $L M$ and $M L$ fluxes in segments of $S$ were significantly greater than corresponding values in $A$ rats $(p<0.05)$ (LM flux; S rats: P 524 51 , D $316 \pm 55$; A rats: P $240 \pm 20$, D $149 \pm 30$. ML flux; S rats: $P 359 \pm 39$, D 268 288 ; A rats: P $109 \pm 18$, D $166 \pm 35$ ). Glucose did not affect $\mathrm{HCO}_{3}^{-} \mathrm{NA}$ or fluxes. In our previous study where tygon tubing was used for the perfusions, $\mathrm{HCO}_{3}^{-} \mathrm{NA}$ was significantly different in segments of $S$ and $A$ rats (Ped. Res. 9:308, 1975). In this study $\mathrm{CO}_{2}$ leak through the tubing was prevented by using glass tubing. The difference in tubing may explain the discrepancy. The present results suggest that rate of transport of $\mathrm{HCO}_{3}$ across the mucosal membrane specially in the proximal segment is greate $x$ in $S$ than in $A$ rats.

\section{GENETICS}

60 THYMIC ABNORMALITIES IN A FAMILY WITH CANDIDIASIS 369 ENDOCR INOPATHY SYNDROME(CES) K. Arulanantham, M. Cene1, $\mathrm{Sch}_{\mathrm{c}}$ of Medo, New Haven, Ct. Depts. of Pediat, and Med, Yale Univ.

An immunologic basis for the C-E syndrome has of ten been suggested but studies in family pedigrees reported to date are remarkable only for their inconsistency. Extensive immunologic \& endocrinologic studies were performed in the parents and 7 children ( 3 with CES) of an afflicted family. Clinical manifestations were hypoparathyroidism $(2 / 3)$, adrenal insufficiency $(2 / 3)$, mucocutaneous candidiasis $(3 / 3)$, autoimmune hepatitis $(1 / 3)$, alopecia totalis $(1 / 3)$, and malabsorption(1/3). Parents and 5 of the progeny showed $\mathrm{T}$-cell dysfunction in vitro with impaired lymphocyte replication in response to Con- $\bar{A}, P H A$ or candida stimulation. In contrast, skin testing with these antigens was positive in all but 1 and MIF production normal in the 2 children tested. Variable abnormalities in humoral immune function were found with absent IgA (2);increased $\operatorname{IgE}(2)$ and positive organ-specific antibodies ( $R \circ M$, Blizzard and A.P.Forbes) : thyroid (2), gastric(2), adrenal(1), smooth muscle(1), parietal cell(1), and DNA(1) one child, who died with autoimmune hepatitis, had the total spectrum of abnormalities with negative skin testing, low T-cel1 number and defective function by in vitro testing, absent IgA, elevated IgE, and positive autoantibodies. The thymus was not detected at autopsy。

While each of these immunologic abnormalities has been separately described in association with $\mathrm{T}$-cell dysfunction, the clustering in this pedigree suggests a more basic underlying defect of T-cell differentiation and/or regulation with variable expression in individual affected members. (Supported by NIH grant RR-125)

HUMAN B-GLUCURONIDASE DEFICIENCY MUCOPOLY-

370 SACCHARIDOSIS: IDENTIFICATION OF CROSS-REACT ING ANTIGEN IN DEFICIENT PATIENTS BY PRIMARY ENZYME IMMUNOASSAY. Elliott Bell, Jr., William S. Sly, Frederick E. Brot, and Daniel Achord, Washington Univ. Sch. of Med., Depts. of Ped., Path., and Med., St. Louis Children's and Barnes Hospitals, St. Louis, Missouri.

An important variable in the potential for enzyme replacement therapy in lysosomal storage diseases is the presence or absence of antigenically cross-reactive material (CRM) in the patient. CRM-negative mutants are more likely than CRM-positive mutants to develop antibodies to infused enzyme that would limit the effectiveness of further enzyme administration. Since the primary enzyme immunoassay (PEIA) reported by Neuwelt et al (Immunochem. 10:767, 1973) has advantages for detecting CRM, we developed a similar PEIA for human $\beta$-glucuronidase. In this assay, goat antibody to purified placental $\beta$-glucuronidase (immobilized on Sepharose) is exposed to test material, washed, and the binding sites occupied by CRM are estimated from the residual binding capacity of the antibody for standard enzyme. Results with this assay indicated: 1) Antibody to human placental $\beta$-glucuronidase did not recognize differences in normal enzyme from human placenta, fibroblasts, liver, or blood platelets. 2) All four of the unrelated B-glucuronidase deficient patients studied contained CRM in their fibroblasts. 3) Titration patterns indicated genetic heterogeneity among the four mutant proteins. 4) Fibroblast enzyme from two obligate heterozygotes was distinguishable immunologically from normal enzyme. 5) Liver enzyme from other mammalian species is cross-reactive, but distinguishable from enzyme from human liver. 
371 MUTATION IN HUMAN LYMPHOCYTE LINES. Soja P. Bennett, Arthur D. Bloom, and Frank T. Nakamura. College of partment of Pediatrics, New York.

Biochemically mutant human lymphoid lines, whether derived from patients with inborn metabolic errors or derived by incubation of wild type cells in selective medium, provide loci (hpt, specifying hypoxanthine-guanine phosphoribosyl transferase activity; and as, specifying argininosuccinic acid synthetase activity) for the determination of spontaneous and induced mutation rates (forward and reverse at $\mathrm{hpt}$, reverse at as) and for study of mechanisms of mutation at these loci. Using preparations of Epstein-Barr virus, we have established lymphocyte lines from citrullinemic and Lesch-Nyhan patients and have derived, by incubation of normal cells in $10 \mu \mathrm{g} / \mathrm{ml}$ of 6 -thioguanine (6TG), lines and clones which are resistant to $6 \mathrm{TG}^{-}\left(6 \mathrm{TG}^{\mathrm{r}}\right)$. While the specific HGPRT activity of the natural HGPRT cell population (UM-10) was $1.3 \%$ of the control line (UM-42), the line (UM-1-6 $\mathrm{TG}^{\mathrm{r}}$ ) derived from incubation in $6 \mathrm{TG}$ had $5.6 \%$ of the activity. Our determinations (with S. Streeter) of the spontaneous forward mutation rate, from $6 \mathrm{TG}^{s} \rightarrow 6 \mathrm{TG}^{\mathrm{r}}$ (HGPRT ${ }^{+} \longrightarrow \mathrm{HGPRT}^{-}$) for UM-61, a diploid $1 \mathrm{ym}-$ phoid line of normal donor origin, were $0.9-6.4 \times 10^{-7} / \mathrm{cell} / \mathrm{s}$ generation. Further, a line (UM-21-5-6 TG ${ }^{r}$ ) has been derived which is mutant at both the hpt and as 1oci, by incubation of $\mathrm{AS}^{-}$citrullinemic cells in $6 \mathrm{TG}$, with the resulting line having $4.6 \%$ of the HGPRT of the control and being unable to grow in citrulline. This double mutant will allow estimates of mutation rates at 2 loci in the same cell population.

$\mathbf{3 7 2}$ RAPID DIAGNOSIS OF PYRUVATE DEHYDROGENASE DEFICIENCY

372 IN PLATELETS FROM TWO SIBLINGS. John P. Blass and Stephen D. Cederbaum (Spon. by Solomon A. Kaplan).

UCLA School of Med., Depts. of Biol. Chem., Psych., and Ped.

The accurate diagnosis of pyruvate dehydrogenase (PDH) deficiency has up to now required the study of disrupted cultured fibroblasts (which requires at least 6 weeks from the time of the skin biopsy) or of biopsied liver or muscle. We now report the rapid demonstration of PDH deficiency in 2 sibs using blood platelets.

Platelets were isolated from $5 \mathrm{ml}$ or more of blood by differential centrifugation, washed, and homogenized in $40 \%$ glycerol. Activity of PDH was measured from the conversion of [1-14 C ]pyruvate to $14 \mathrm{CO}_{2}$ at $\mathrm{pH}$ 7.4. Activity showed the expected dependence on added $\mathrm{COA}, \mathrm{NAD}^{+}$, and thiamin pyrophosphate; it was not increased by treatment which activates (dephosphorylates) PDH. Control experiments indicated that activity was stable for at least $48 \mathrm{hr}$ at $-20^{\circ} \mathrm{C}$; did not vary with age, sex, activity, or time of day; and did not depend on diet (control vs high fat vs high carbohydrate, fasting vs $2 \mathrm{hr} p \mathrm{pp}$ ). The activity in 23 controls was $162+17$ pmoles/min/mg protein (mean+SEM). In 2 sibs previously described with PDH deficiency, it was $19+6$ and $11+1$ (for each sib, $n=4$ and $p<0.001$ ).

Recent studies have indicated that children with PDH deficiencies can improve on a ketogenic diet (Falk et al., Ped. Res. 2: 350, 1975 and Pediatrics, in press; Benke \& Tocci, Clin. Res. 24 : tate the diagnosis of these patients.

H-Y(MALE) ANTIGEN DETERMINA TION IN DELINEA TION OF SEX

373 CHROMOSOME ABNORMALITIES. W. Roy Breg, Myron Gene1, Yale Univ., Depts. of Hum. Genet. and Ped., New Haven; Mem.-S loan Kettering Cancer Ctr., N.Y.C.; Depts. of Hum. Genet. \& Dev. and Obs. \& Gyn., N.Y.C.; Southbury Training School, Southbury.

Antibodies produced in female mice by the injection of cells from males of the same inbred strain react with $\mathrm{H}-\mathrm{Y}$ antigen (H-Ya) of leukocytes of human males (Wachtel et al, P.N.A.S. 71, 1974). That the gene controlling the expression of $\mathrm{H}-\mathrm{Ya}$ is $\mathrm{Y}-$ linked in humans is indicated by excess amounts in XYY males (Wachtel et a1, N.E.J. Med. 293, 1975). Study of $\mathrm{H}$-Ya expression of patients with structura11y abnormal sex chromosomes not delineated by banding technics suggests this method may be useful in deciding whether male-determining $Y$ chromosome material is present. Some of the cases studied and $\mathrm{H}-\mathrm{Ya}$ results axe:

\begin{tabular}{|c|c|c|c|}
\hline Males & $\underline{\mathrm{H}}-\mathrm{Ya}$ & Females & $\mathrm{H}-\mathrm{Ya}$ \\
\hline $45, \mathrm{X} / 46, \mathrm{XY} *$ & + & $45, \mathrm{X} / 46, \mathrm{XY*}$ & + \\
\hline $45, x / 45, x, 22 q+$ & + & $45, X / 46, X, r(? X \circ \circ Y) * *$ & + \\
\hline $45, \mathrm{x} / 46, \mathrm{x}, ? 1(\mathrm{YP})$ & 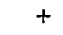 & $45, X / 46, X, r($ ?XorY $)$ & \pm \\
\hline $46, x, x p+$ & + & $45, X / 46, X, r(? X \circ r Y)$ & \\
\hline
\end{tabular}

$* Y=a v$, length, lacks intense $Q F ; * *$ dysgenetic gonads $w /$ tubules The males lacking a norma $1 \mathrm{Y}$ chromosome but containing possible Y material are $\mathrm{H}-\mathrm{Ya}$ positive. Some females are also positive; mosaicism with 45 , $X$ cells may have modified the male-determining effect of H-Ya in these cases. H-Ya determination may prove useful in recognizing those females with possible $Y$ material who are likely to develop neoplasia in dysgenetic gonads.
POLYAMINE BIOSYNTHETIC PATHWAYS IN CYSTIC FIBROSIS AND 374 CONTROL FIBROBLASTS. Bruce A. Buehler, * Reba Wright, * and Owen M. Rennert, University of Florida College of Medicine, Division of Genetics, Endocrinology, and Metabolism, Department of Pediatrics, Gainesville.

Abnormal levels of polyamines have been found in the blood of patients with neoplastic diseases, psoriasis, and cystic fibrosis. Ornithine decarboxylase (ODC) and S-adenosyl methionine decarboxylase (SAM decarboxylase) are two key enzymes in the polyamine biosynthetic pathways. Ornithine decarboxylase and Sadenosyl-methionine decarboxylase were studied in cultured fibroblasts from patients with cystic fibrosis and controls. ODC and ornithine keto aminotransferase activity decreased as fibroblast proliferation diminished, whereas SAM decarboxylase and adenylate cyclase activity increased as the cells approached confluence. older cells (higher passage numbers) showed decreased SAM decarboxylase activity when compared to younger (low passage number) cells. The activities of ODC and SAM decarboxylase were indistinguishable in control and cystic fibrosis fibroblasts; all fibroblasts responded equally to putrescine. SAM decarboxylase activity was enhanced, and ODC activity was inhibited by equal concentrations of putrescine.

\begin{tabular}{lccc}
\hline FIBROBLASTS & $\begin{array}{c}\text { SAM } \\
\text { DECARBOXYLASE }\end{array}$ & $\begin{array}{c}\text { SAM DECARBOXYLASE } \\
\text { with } 2.5 \text { mM Putrescine }\end{array}$ & ODC \\
\hline $\begin{array}{l}\text { Contro1 Mean } \\
\text { Activities }\end{array}$ & $22.7 *$ & $568 *$ & $33.4 *$ \\
$\begin{array}{c}\text { Cystic Fibrosis } \\
\text { Mean Activities }\end{array}$ & $21.7 *$ & $463 *$ & $38.2 *$ \\
& & &
\end{tabular}

*pMoles/mg Protein $/ \mathrm{hr}$

TREATMENT OF CYSTINOTIC FIBROBLASTS WITH LIPOSOME-

375 ENCLOSED AGEITS. Jean Butler, Francoise Pellefigue, Bethesda, Ma.

Cystine can be removed from cystinotic fibroblasts by cysteamine (MEA), dithiothreitol (DTT) or ascorbic acid (vit.c) added to the culture medium. MEA and DTT are toxic compounds. The feasibility of targetting potentially therapeutic agents at the lysosomally compartmentalized cystine was examined by attempting to enclose selected compounds in phospholipid vesicles (liposomes) and presenting them to cystinotic cells in culture. MEA, cystamine, vit.C, L-amino acid oxidase and glutathione were retained within negatively-charged liposomes while $\mathrm{N}$-acetyl cysteine, BAL, lipoic acid, penicillamine, DTT, and sodium sulfite were poorly retained. After 2 hours exposure, cystine content of cystinotic cells was reduced approximately twice as much by liposome-enclosed MEA as by MEA dissolved at the same dose $(0.1 \mathrm{mM})$ in normal or protein-free medium. Other compounds produced slight or no reduction of intracellular cystine over that caused by non-liposomeenclosed drug action. 35s-Cysteamine in solution or enclosed in liposomes was injected intravenously into mice and uptake of label by various tissues was determined after 3 hours. There were significantly increased radioactivity ratios in kidney, liver, spleen, and lung compared to brain when the compound was administered in liposomes in contrast to administration in solution $(p<.005)$. This preferential distribution of encapsulated MEA in tissues where high concentrations of cystine accumulate in cystinotic patients may have future therapeutic implications.
376 HYPERARGININEMIA. Stephen D. Cederbaum, Kenneth N.F. 376 Shaw, at Los Angeles Depts. of Psych. \& Ped., \& Childrens Hosp. \& Univ. of Southern Cal. Dept. of Ped., Los Angeles. Male \& female siblings aged $6 \& 12$ years developed near normal1 y for 3 years, then gradually deteriorated intellectually \& phys ically. The sister walked \& was bilingual at 6 , but at 12 could not stand or speak ( $\mathrm{I}<20)$. The brother is regressing likewise. Clinical observations include increasing spasticity, hyperreflexia, seizures \& abnormal EEG, but no other neurological abnormalities. A single self-limited episode of coma \& hepatomegaly occurred in the boy, with liver biopsy findings showing increased fibrosis. Both patients show a marked hyperargininuria (up to 370 $X$ norma1) \& a generalized aminoaciduria. In plasma, arginine is $10-17 \mathrm{mg} / \mathrm{dl}$ ( $4-7 \mathrm{X}$ normal) but other amino acids are not increased. In CSF, arginine is $3-5 \mathrm{X}$ normal, \& numerous other amino acids are also high. Plasma urea $\mathrm{N}$ is low normal $(7-10 \mathrm{mg} / \mathrm{d} 1)$, \& CSF urea $\mathrm{N}$ is normal $(9-10 \mathrm{mg} / \mathrm{d} 1)$. Plasma $\mathrm{NH}_{3}$ is generally normal, but $\mathrm{x}$ ises to $0.25 \mathrm{mg} / \mathrm{d} 1$ ( $5 \mathrm{X}$ norma1) following increased protein intake. The erythrocyte arginase level is $<0.5 \%$ normal in the patients. The mother has heterozygote arginase levels; 2 healthy sisters show normal levels. The father is dead. Five other patients with hyperargininemia have been described with the same downhill course. The increased levels of numerous amino acids in CSF \& urine of the present patients contrast with our previous patient \& those of Terheggen et al. in whom only arginine ( $\&$ sometimes other dibasic amino acids) is abnormal. A distinctive aminoaciduria is a Iiagnostic clue for some patients, but others show normal urinary amino acids. 
377

MULTIPLE ENZYME DEFECTS IN FAMILIAL HYPERLYSINEMIA. Joseph Dancis, Joel Hutzler, Norman C. Woody, and Rody B. Cox. New York University School of Medicine \& Tulane University School of Medicine, Depts. of Pediatrics \& Medicine. New York City \& New Orleans.

Lysine-ketoglutarate reductase deficiency in skin fibroblasts has been previously reported in patients with familial hyperlysinemia providing an adequate explanation for the biochemical derangements noted clinically. In the present study, analysis of liver obtained at autopsy from a patient with familial hyperlysinemia confirmed the lysine-ketoglutarate reductase deficiency but, unexpectedly also revealed an absence of saccharopine dehydrogenase and saccharopine oxidoreductase activity. Skin fibroblasts from 2 siblings with the disease and a third patient from an unrelated family were also deficient in all three enzymes (lysine-ketoglutarate reductase, average $9 \%$; saccharopine dehydrogenase, average $4 \%$; saccharopine oxidoreductase, $10 \%$ of normal). The possibility that saccharopine dehydrogenase is a substrate inducible enzyme was investigated by maintaining normal skin fibroblasts in a medium with minimal lysine concentration, and hyperlysinemia fibroblasts were exposed to elevated saccharopine concentrations. There was no significant modification in saccharopine dehydrogenase activity.

Familial hyperlysinemia represents another inborn error of metabolism affecting more than one enzyme.

PRENATAL DIAGNOSIS OF CAT CRY SYNDROME. Karen

378 David, Sara Kaffe, Andrea Serotkin, Lillian Y. F. Ped. and Path., Mt. Sinai School of Medicine, New York City.

We wish to report the prenatal diagnosis of cat cry syndrome. The indication for prenatal cytogenetic diagnosis was advanced maternal age (39 yrs.) previous pregnancies resulted in three normal children and one first trimester spontaneous abortion. Amniocentesis was performed at $161 / 2$ weeks gestation. Sonogram revealed a normal fetal head. At 20 weeks gestation, harvest of two culture flasks showed a $46, \mathrm{XY}$ karyotype with a suspicious very small deletion of the short arm of one of the $B$ group chromosomes in some cells. Using both $G$ and $Q$-banding, the partially deleted chromosome was identified as $5 \mathrm{p}-$. The banding patterns of all other chromosomes were normal. Chromosome analysis with banding of the peripheral leukocytes of both parents was normal. This deletion was a de novo event, either an interstitial deletion involving $5 \mathrm{p} 13$ and $5 \mathrm{p} 14$ or the result of an unbalanced reciprocal translocation with the break point in 5p13. The parents were told of these findings and of the likelinood of the cat cry syndrome. They elected to terminate the pregnancy by saline abortion at 23 weeks gestation. The amniotic fluid obtained at the time of abortion was cultured and again demonstrated the $5 p-$. Autopsy revealed a male fetus with stigmata suggestive of the cat cry syndrome including slight facial asymmetry, hypertelorism, small palpebral fissures, antimongoloid slant, high arched palate, micrognathia, low set ears, preauricular tag and ASD. Microcephaly was not present.
PHENOTYPIC VARIATION IN THE 3q TRISOMY SYNDROME.

380 Robert M. Fineman, Ronald C. Ablow, Rufus O. Howard, Ped., Radiol., Ophthal., New Haven; Southbury Training Sch., Southbury, CT.

We are reporting observations on 2 families with individuals who are trisomic for different amounts of the distal end of the long arm of chromosome 3. Our data, plus those published by Allderdice, et al. (Am. J. Hum. Genet. 27:699, 1975) describing individuals from a third family, support the hypothesis that the severity of the phenotypic expression in a trisomic syndrome is proportional to the amount of extra chromosomal material present: Trisomic Segment Mental Retardation

Physical Growth

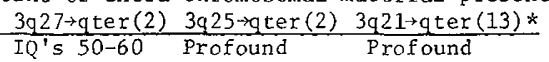
Cardiovascular Anom. None Gastrointestinal Anom. None Spine Deformity None Limb Deformity < 3\% Moderate Severe None 7 of 13 1 of $2 \quad 7$ of 13

1 of $2 \quad 5$ of 13
* from Allderdice; $(\quad)=$ number of cases; Anon, = anomalies The phenotypic expression of the $3 q$ trisomy syndrome within these families is quite similar. In spite of the variability in expression among the families, the facies of all of the affected individuals bear a striking resemblance suggesting that these patients delineate a new chromosomal syndrome. In addition, all 4 of our patients have prominent frontal and parietal digital impressions on skull x-ray.

AN ASSOCIATION OF DOIN 'S SYNDROME (DS) WITH ADVANCED 381 MATERNAL AGE, AND ALPHA-1 ANTITRYPSIN (ATT) VARIANTS, Robert M. Fineman, A. Myron Johnson, Kenneth K. Kidd, W. Roy Breg. Yale Lniv. Sch. Med., Depts. of Human Genet, and Ped. New Haven; Univ. of N.C., Dept. Ped., Chape 1 Hill; Southbury Training School, Southbury, CT.

In attempting to find an association of etiologic significance in DS, we have performed AAT phenotyping on 63 patients with DS and 116 matched, chromosomally normal controls. We divided our subjects prior to testing into 2 groups according to the maternal age (MA) at the time of the individual's birth:

AAT Phenotype

Mi homozygote

$M /$ variant heterozygote

Total

\begin{tabular}{|c|c|c|c|}
\hline \multicolumn{2}{|c|}{ Controls } & \multicolumn{2}{|c|}{ DS } \\
\hline$M A<\overline{30}$ & $\overline{\mathrm{MA}}>35$ & $M A<30$ & $M A>35$ \\
\hline 50 & 53 & 30 & 21 \\
\hline 7 & 6 & 1 & 11 \\
\hline 57 & $\overline{59}$ & $\overline{31}$ & $\overline{32}$ \\
\hline .12 & .10 & .03 & .34 \\
\hline
\end{tabular}

These 4 groups are significantly heterogenous $(p \approx .002)$, primarily due to the increased frequency of AAT variants in the last group. In addition, the DS group with a MA>35 is significantly different from the control group with a $M A>35,(p \simeq .012)$.

These data suggest that decreased AAT activity, as found in heterozygous AAT variants, in conjunction with advanced maternal age, may be a factor in the production of trisomy 21 . Further studies may make it possible to identify those individuals who have a significantly increased risk of having of spring with chromosomal abnormalities; this high risk group could be readily dentified by AAT phenotyping.
2703 EFFECT OF CYSTIC, FIBROSIS (CF) AND NORMAL SFRIM ON 379 H-L-FUCOSE INCORPORATION IN FIRROBIASTS. Pamela B.

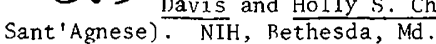

A previous report (Ped. Res. $9: 312,1975$ ) suggests that incorporation of $3 \mathrm{H}$-L-fucose into TCA-precipitable material by cultured human fibroblasts is stimulated by normal but not CF serum.

We find that fibroblasts incubated in $0.01 \mathrm{M}$ sodium phosphate buffer, $\mathrm{pH} 7.0-0.15 \mathrm{M} \mathrm{Na} \mathrm{Cl-0.5 \%}$ bovine serum albumin (Buffer $A$ ) with $5 \mu \mathrm{Ci} 3 \mathrm{H}$-L-fucose for $3 \mathrm{~h}$ at $37^{\circ}$, incorporate $1234 \mathrm{dpm} / 10^{6}$ cells into TCA precipitate. This is not stimulated by either normal ( $1264 \mathrm{dpm} / 10^{6}$ cells) or CF ( $1101 \mathrm{dpm} / 10^{6}$ cells) serum. If Eagle's minimal essential medium (MEM) is substituted for Buffer A under the same conditions, incorporation is $2080 \mathrm{dpm} / 10^{6}$ cells and is stimulated by normal ( $3707 \mathrm{dpm} / 10^{6}$ ce11s) or CF (3419 $\mathrm{dpm} / 10^{6}$ cel1s) serum. No significant difference between normal and $C F$ serum in effects on $3 \mathrm{H}-\mathrm{L}$-fucose incorporation was found under any test conditions, including: storage of serum at $-20^{\circ}$ storage at $4^{\circ}$ for $18 \mathrm{~h}$, dialysis at $4^{\circ}$ for $18 \mathrm{~h}$ against Buffer $\mathrm{A}$, use of fresh sexum; or use of normal or $\mathrm{CF}$ fibroblasts, or harvesting by scraping or trypsinization. There was no significant difference in $3 \mathrm{H}-\mathrm{L}$-fucose incorporation between normal and $\mathrm{CF}$ fibroblasts when expressed per $10^{6}$ cells (2080 vs. $2074 \mathrm{dpm}$ in MEM, 1234 vs. $1390 \mathrm{dpm}$ in Buffer A) but CF fibroblasts have $2 / 3$

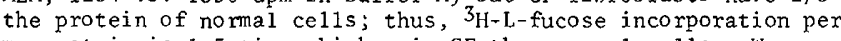
$\mathrm{mg}$ protein is 1.5 times higher in $\mathrm{CF}$ than normal cells. We cannot confirm the previous study which differentiates CF from normal serum by effect on fucose uptake by fibroblasts.
TRISOMY 8p RESULTING FROM A PATERNAL RECIPROCAL TRANS382 LOCATION. Steve J. Funderburk, Helga M. Muller, and School of Medicine, Depts, of Psychiat, and Ped., los Angeles.

A 3.7 kilogram male died neonatally with the hypoplastic left heart syndrome. Clinical examination including necropsy revealed the Dandy-Walker syndrome as well as agenesis of the corpus callosum, hypoplasia of the hippocampus and micropolygyria, the Pierre Robin anomaly, agenesis of the gallbladder, inguinal hernias, a single umbilical artery, low set and malformed ears and deep plantar furrows. Trypsin-Giemsa banding analysis in the infant and both parents revealed trisomy for the short arms of chromosome no. 8 in the infant, due to a reciprocal translocation between the short arms of a chromosome no. 8 and no. 15 in the father. This infant appeared distinct from other reported cases of $8 \mathrm{p}$ trisomy, who have survived with mental retardation, short stature and only minor anomalies. Similarly this infant was quite distinct from the mosaic trisomy 8 syndrome, supporting the suggestion that the phenotypic features of the latter may be the result of trisony for only a small segment of the long arm of chromosome 8. For accurate diagnosis and genetic counseling, banding chronosome analysis is needed in all critically 111 newborns whose multiple malformations may be fully evident oniy after careful clinical and postmortem examination. Additionally, the chromosome abnormality in this infant with a posterior U-shaped palatal cleft, micrognathia and relative glossoptosts illustrates the varied etiologies for the Pierre Robin anomaly. 
ORNITHINE KETOACID TRANSAMINASE DEFICIENCY ASSOCIATED 383 WITH HYPERAMMONEMIA AND ORNITHINEMIA. Ado1fo D. Gar-
nica, * Owen M. Rennert, and W. Y. Chan,
Kniversity of Florida College of Medicine, Division of Genetics, Endocrinology, and Metabolism, Department of Pediatrics, Gainesville.

A 4-year-old male with psychomotor retardation and hypexkinesis was evaluateu because of refractory seizures and intermittent ataxia, the sudden onset of which was at age 5 months with myoclonic and major motor seizures. On physical examination, his height and weight were at the 25 th percentile for age and his head circumference at the 50th percentile. Quantitation of serum amino acids demonstrated normal levels of acidic and neutral amino acids but elevated levels of the basic amino acids ornithine, asparagine and glutamine. Plasma ammonia was $190 \mathrm{mcg} / 100 \mathrm{ml}$ (normal $17-60$ $\mathrm{mcg} / 100 \mathrm{ml})$. After protein restriction $(0.5-1.0 \mathrm{gm} / \mathrm{kg}$ body weight/day), there was a marked reduction in seizure activity; plasma ammonia decreased to a range of $18-68 \mathrm{mcg} / 100 \mathrm{mI}$ and serum ornithine to $13 \mu$ moles/100 m1. Quantitation of urinary amino acids both before and after protein restriction revealed excessive excretion of glutamine and asparagine only. Ornithine ketoacid transaminase (OKT) and ornithine decarboxylase (ODC) activities were measured in fibroblasts cultured from patient and normal skin biopsies: patient OKT levels were low, as compared with nor$\mathrm{mals}(0.011 \mathrm{mM} / \mathrm{mg}$ protein $/ \mathrm{hr}$ vs normal $0.134 \pm 0.01 \mathrm{mM} / \mathrm{mg}$ protein/ $\mathrm{hr}$ ); patient ODC activities during exponential growth and in confluent cultures were comparable to normals. This case possibly represents a variant of the ornithinemia with mental retardation, without hyperammonemia, reported by Bickel. Family studies, including parents and two normal siblings, will be presented.

ABNORMAL COPPER METABOLISM IN FIBROBLASTS DEVELOPED Ped., Univ. $\frac{\text { Patrick M. Hefferan, and R. Rodney Howel }}{\text { of Texas Medical Schoo1, Houston, Texas. }}$

We have recently reported the discovery of an in vitro genetic marker for Menkes disease (Clin Res 23, 262A; Proc Nat Acad Sci, in press). This marker is expressed as elevated copper content in cultured skin fibroblasts developed from individuals with this genetic anomaly. To further study this genetic expression, we
utilized radiocopper- $64\left({ }^{64} \mathrm{Cu}\right)$ from 0 to 10 hours to evaluate copper uptake. Both Menkes and control cultures were incubated in media containing various concentrations of ${ }^{64} \mathrm{Cu}$. A plot of the ${ }^{64} \mathrm{Cu} \mathrm{DPM} / \mathrm{mg}$ protein versus time revealed a rectangular hyperbola shaped curve for both Menkes and control cultures. Cultures from 6 patients with Menkes disease however, consistently accumulated more ${ }^{64} \mathrm{Cu}$ than did control cultures, and the initial rate of ${ }^{64} \mathrm{Cu}$ uptake was greater in Menkes cultures.

INITIAL RATE OF ${ }^{64} \mathrm{Cu}$ UPTAKE (DPM/mg PROTEIN/hr)

\begin{tabular}{ccc}
${ }^{64} \mathrm{Cu}$ Concentration (Media) & $\frac{\text { Control (2) }}{359}$ & $\frac{\text { Menkes (2) }}{1530}$ \\
\hline $1 \mu \mathrm{Ci} / \mathrm{ml}$ & 1269 & 4208
\end{tabular}

These quantitative differences in the uptake of copper between Menkes and control cultures are consistent with the idea that the abnormality involves the cellular transport or handling of copper, resulting in an elevated copper content.

CARRIER DETECTION IN ORNITHINE TRANSCARBAMYLAS

385 DEFICIENCY. Jerold T. Hokanson, William E. O'Brien, Irwin A. Schafer Case Western Reserve University School of Medicine, Cleveland Metropolitan General Hospital, Department of Pediatrics, Cleveland, Ohio.

Ornithine transcarbamylase (OTCase) deficiency is an $\mathrm{X}$-1inked genetic defect in urea cycle metabolism which produces lethal hyperammonemia in males and varying degrees of illness in females. Affected individuals excrete increased amounts of orotic acid in the urine. Goldstein et al (Ped. Res. 8:5, 1974) suggested that carriers could be detected by monitoring urinary orotic acid after protein loading. We have confirmed this observation, by studies carried out in a large kindred with at least three obligate heterozygotes. The diagnosis of OTC deficiency was established in this family by enzyme studies of liver obtained post mortem in an infant male and his female cousin. The three obligate heterozygotes (III-6, IV-4, IV-13) showed urinary orotic acid concentrations that differed by more than three standard deviations from controls $(29 ; 208 ; 123$ vs. controls, $6 \pm 3 \mathrm{ug} / \mathrm{mg}$ creatinine). Three hours after protein feeding venous blood ammonia concentrations in the same three individuals also differed by three standard deviations from the controls ( $82 ; 149$; 92 vs. control $50 \pm 8 \mathrm{ug} / 100 \mathrm{cc}$ ). OTCase activity in leukocytes did not differ from control values. In pedigrees in which an enzymatic diagnosis of OTCase deficiency is established, protein loading with serial measurements of urinary orotic acid and blood ammonia may be as effective as enzyme assays on biopsied THIRD COMPONENT OF COMPLENEIT (C3) IN CYSTIC FIBROSIS

386 Robert $\frac{J}{\text { Schwart }}$. Holzhauer. Univ. of Rochester. Rochester. New York.

Serum $\mathrm{C} 3$ has been reported to be elevated in cystic fibrosis (CF) and in CF parents (obligate heterozygotes). Human C3 exhibits genetic polymorphism with 15 predicted phenotypes of which 3 are common (SS,FS,FF). The possibility that elevated $C 3$ in $C F$ and $C F$ parents depends upon $\mathrm{C} 3$ phenotype was investigated in $61 \mathrm{CF}, 88$ CF parents, 64 CF siblings and 39 healthy adult controls.C3 serum levels were measured by radial immunodiffusion (Hyland plates). C3 typing was done by high voltage agarose gel electrophoresis.

Nean $C 3$ levels were elevated in $C F$, parent and sibling groups

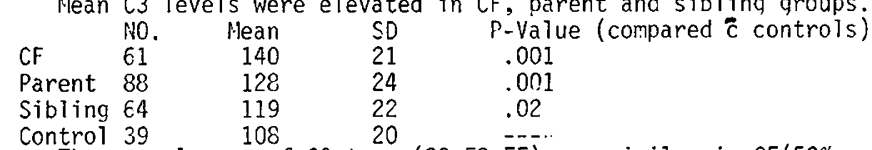

The prevalences of C3 types(SS.FS, FF) were similar in CF( $59 \%$, $39 \%, 2 \%) ; C F$ parents $(66 \%, 27 \%, 7 \%)$ :CF siblings $(63 \%, 34 \%, 3 \%)$; and control $(57 \%, 39 \%, 4 \%)$ groups. CF C3 levels were elevated when compared to a 11 controls and those matched for sex and $C 3$ types ( $p<.001$ to< $.005)$. Those with moderate and severe disease had higher levels than the healthier group $(p<.005)$.SS and FS mothers had higher levels than female controls $(p<.02$ to<.05).SS and FS fathers, however, were not significantly elevated compared to SS and FS male controls. Al though the C3 mean level was elevated for the sib
group, this was due to elevation in the female SS siblings.

Important variables affecting $\mathrm{C} 3$ levels are severity of disease for CF and C3 type and sex for parents and siblings. ren, Research Institute, Toronto, Canada.

A 10 month old French Canadian girl who presented with developmental delay, seizures and autistic behaviour was found to consistently excrete large quantities of benzoate (B) in the urine Her developmental level was 4 months but physical findings, including head size were norma 1 . Electroencephalograms (EEG) showed frequent seizure activity. No bacteriuria was present. On a normal diet for age baseline $24 \mathrm{hr}$. urinary excretion of $B$ was $34.7+5.3$ mgs and hippurate $(H) 85.2+9.5 \mathrm{mgs}$. Removal of benzoate from the diet produced no change in $\bar{B}$, nor did dietary exclusion of phenylalanine and tyrosine. L-Glycine $1 \mathrm{gm} / \mathrm{Kg}$ was added to the diet with a fall in $B$ to $2.1 \pm 1.1 \mathrm{mg}$. and a rise of $\mathrm{H}$ to $181.0 \pm 15.8 \mathrm{mg}$. Over 4 weeks this diet allowed development to progress to the 6 7 month leve1, and a decrease in autistic behaviour. EEG showed much less seizure activity. Removal of dietary glycine produced behaviour deterioration, overt seizures, and a return of benzoate in the urine. Hepatic glycine $\mathrm{N}^{\prime}$ acylase, and benzoyl CoA synthetase activities were normal in vitro. An oral load of phenylalanine $0.1 \mathrm{gm} / \mathrm{kg}$ did not increase $B$ excretion and Neomycin $225 \mathrm{mg}$ twice daily for 7 days to reduce bowel flora had little effect on $B$ or $H$ in the urine. Return to a high glycine intake produced an improvement in alertness, affect \& appetite and a cessation in overt seizures. B is known to be neurotoxic in excess. The data presented suggests that this girl may have a previously undescribed defect in $B$ metabolism, associated with brain dysfunction but improved by stimulation of glycine $-\mathrm{N}$ acylase activity.
388 ASE (PCC) BY AN UNIDENTIFIED FACTOR. Y. Edward Hsia and Katherine J, Scully, Depts. Human Genetics and Pediat., Yale Univ. Sch. Med., New Haven, CT

Cultured fibroblasts from patients with propionicacidemia have markedly reduced but variable PCC activity. The variability is due at least in part to decreased thermostability of the mutant enzyme. In highly concentrated extracts (c $0.8 \mathrm{mg}$ protein $/ 0.1 \mathrm{mI}$ incubation mix) mutant cell PCC activity was up to $50 \%$ of normal. When diluted $1 / 10$, PCC activities of those mutant extracts was only about $3 \%$ of normal, but could be stimulated more than 10fold by preincubation with a small dialysable heat-stable factor. Assay Conditions Fibroblast Lines This factor was (all include $\mathrm{K}+$ ) Control Mutant 1 Mutant 2 present in both

\begin{tabular}{|c|c|c|c|c|}
\hline Concentrated & $1.17 *$ & 0.58 & 0.35 & cultured cells, \\
\hline Diluted $1 / 10$ & 1.04 & 0.03 & 0.02 & normal human liver \\
\hline$"+$ Factor & 1.06 & 0.35 & 0.32 & rat liver, and in \\
\hline
\end{tabular}
$*_{\mathrm{n} m o l e} / \mathrm{min} / \mathrm{mg} /$ protein pure mitochondrial preparations from rat liver. It is water-soluble, sparingly soluble in methanol:chloxoform ( $1: 1$ vol:vol), stable to boiling, and resistant to mild acid or alkali treatment. It has a molecular size of $<300$ dalton, and is positively charged. The normal enzyme contains biotin and is activated by potassium, but the factor's effect on mutant enzyme was not reproduced by either increased potassium or added biotin. The factor was resistant to avidin; it enhanced mutant PCC activity only after preincubation; and it did not protect the mutant enzyme from heat inactivation. 
ANTENATAL TESTING FOR PROPIONIC ACIDEMIA. Y.E. HSIa,

389 M.J. Mahoney \& K.J. Scully. Depts, Human Genetics Pediatrics, Yale Univ. Sch. Med., New Haven, Conn.

Two pregnancies at risk for propionic acidemia have been monitored by assaying for propionyl-Co A carboxylase (PCC) activity in cultured amniotic cells. Both families had previous affected probands with deficient, thermolabile PCC in cultured fibroblasts. Control amniotic cell PCC activity was found to be 3 times that of control fibroblasts. Amniotic cells from each fetus at risk had only $20 \%$ of control amniotic cell activity. In each case, enzyme thermostability was normal. Whole cell oxidation of propionate was also normal. The pregnancies were allowed to go to term.

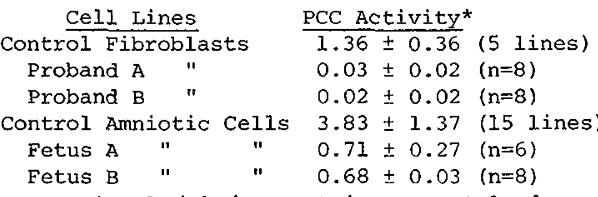
Cord blood leucocyte PCC activity from infant A confirmed that she was unaffected. She is tolerating milk protein well. without any metabolic disturbance.

Infant $B$ is also clinically well. He had no elevation of blood or urine propionate while taking milkon the fifth day of life. The predictions that these two fetuses were unaffected have been verified, helping to establish the PCC assay for antenatal diagnosis of propionic acidemia. The reduced PCC activity in these amniotic cells suggests heterozygosity. Cultured cells from these infants may clarify that possibility.

PRENATAL DIAGNOSIS OF $45, \mathrm{X} / 46, \mathrm{XY}$ MOSAICISM WITH POSTNATAL CONFIRMATION IN A GROSSLY NORMAL MALE INFANT. Lillian Y.F. Hsu, Hyon J. Kim, Richard Hausknecht, and Kurt Hirschhorn, Dept. Peds., Mount Sinai School of Med., NYC.

Prenatal detection of chromosome mosaicism has always been a diagnostic dilemma. Thus far, only one case of trisomy 13 mosaicism (A. Bloom et al) has been confirmed in an abortus. We report a case of $\mathrm{XO} / \mathrm{XY}$ mosaicism detected prenatally and confirmed postnatally in a grossly normal male infant. The mother, 38 years old, had amniocentesis at 19. weeks of pregnancy. A diagnosis of $\mathrm{XO} / \mathrm{XY}$ mosaicism was made at $22 \frac{1}{2}$ weeks of pregnancy from 2 separate culture flasks with $45, X$ cells predominating $(86.4 \%$ ). The $Y$ chromosome was of normal size but carried no fluorescent band. The parents were counseled and were advised that the phenotype of $\mathrm{xO} / \mathrm{XY}$ mosaicism can range from relative normality to sexual maldevelopment. They decided to continue this pregnancy. The infant was born at term by C-section and was a grossly normal male with normal penis and descended normal sized testes. The B.W. was $3170 \mathrm{gm}$ and length $51 \mathrm{~cm}$. Dermatoglyphics showed an elevated total finger ridge count of about 190. Leukocyte culture from the cord blood and a skin fibroblast culture from the foreskin confirmed the mosaicism of XO/XY (Blood:XO-26\%,XY-74\%; Skin: $\mathrm{XO}-65 \%, \mathrm{XY}-35 \%$ ). The $\mathrm{Y}$ chromosome was again shown to be nonfluorescent and non-heterochromatic in nature. The father's $\mathrm{x}$ chromosome was of identical size and carried a small fluorescent band. By comparing Q-banding polymorphisms, the paternity of this father was essentially confirmed. We have therefore confirmed a prenatal diagnosis of mosaicism in this case resulting
in a grossly normal infant.

\section{PARTIAL TRISOMY 1q IN MARROW CELLS OF PATIENTS. WITH}

39] MYELOFIBROSIS AND POLYCYTHEMIA VERA. Lillian Y.F.HSU Dona Pinchiaroli, Harriet Gilbert, Neal Weinreb, and Kurt Hixschhorn, Depts. Ped. \& Med., Mt. Sinai Sch. Med., N.Y.C. The new banding techniques have allowed recognition of nonrandom chromosomal abnormalities in hematologic disorders. Trisomy 8, trisomy 9 and monosomy 7 are the three most common chromosomal aberrations. We have identified partial trisomy la in the marrow cells of two patients with different hematologic disorders. The first was a 55 year old female with myelosclerosis and myeloid metaplasia diagnosed at age 38 years presenting with anemia, fatigue, bruising, fever and splenomegaly. At age 49, she developed pancytopenia and was treated with Halotest in and prednisone. At age 52, she had a splenectomy. Since 1973, she has had 50-958 myeloblast cells and 95-100 nucleated rbc precursor per 100 wbc. Chromosome analysis of unstimulated leucocytes with $Q-$ and G-banding showed $46, \mathrm{XX},-6,+\mathrm{t}(1 \mathrm{q} ; 6 \mathrm{q})$ in all metaphases. In vitro incorporation of $\mathrm{Fe}^{55}$ was demonstrated in all metaphase cells by autoradiography. This indicates the erythroid nature of the abnormal cells. Skin fibroblasts showed a normal karyotype. The second patient, a 49 year old male, was diagnosed as polycythemia vera at age 30 . He has since developed hepatosplenomegaly. He has had various forms of therapy. Chromosome analysis from a direct bone marrow preparation at age 44 and 45 showed grossly normal karyotypes. At age 49 , his marrow. by $Q-$ and G-banding, showed 1008 cells with $46, X Y,-13,+t(1 q ; 13 q)$. It is possible that trisomy lq may represent another non-random chromosomal abnormality in hematologic disorders.
TWO TYPES OF GLYCOGENOSIS (GSD) IN THE SAME GIRL: COMBINED DEFICIENCY OF PHOSPHORYLASE KINASE IN LIVER (GSD IX) AND DEBRANCHER IN LIVER AND MUSCLE (GSD III). George Hug, David Harris and Jerome A. Grunt, Children's Hospital, Cincinnati, OH and Children's Mercy Hospital, Kansas City, MO.

A 5 mo old girl had hepatomegaly, vomiting, doll face, body length $<3$ rd\%tile, SGOT $490(\mathrm{~N}<30)$, CPK $160(\mathrm{~N}<50)$, biventricular hypertrophy on EKG but normal heart clinically and by $x$-ray, no symptoms of muscle disease or hypoglycemia but BS between 20-35 $\mathrm{mg} \%$ on fasting glucagon test. Biopsy specimens (normals in parentheses):

: Liver Muscle phosphorylase kinase $\mu \mathrm{mP} / \mathrm{g} / \mathrm{min}^{2}$ phosphorylase $\mu \mathrm{mP} / \mathrm{g} / \mathrm{min} \quad * 4.8(25.1) \quad 50.8(60)$ glycogen conc. $\% \quad 7.1(<6) \quad 3.8(<1.5)$ $\begin{array}{lll}\text { amylo-l.6-glucosidase } \mathrm{cpm} / \mathrm{g} / \mathrm{hr} & 44(1800) & \text { 43(6770) }\end{array}$ $\begin{array}{ll}\text { glucose-6-phosphatase } \mu \mathrm{mP} / \mathrm{g} / \mathrm{min} & 2.9(4.7) \\ \text { electron microscopy consistent with GSD } & \text { IX \& III III, not IX }\end{array}$ electron microscopy consistent with GSD IX
$*$ Exogenous kinase restored normal activity The findings indicate that the girl had GSD IX (liver phosphorylase kinase deficiency) as well as GSD III (amylo-1.6-glucosidase deficiency). Children with isolated GSD IX do not have abnormal EKG, hypoglycemia, flat BS curve after glucagon or elevation of $\mathrm{CPK}$ and muscle glycogen; these findings in our patient are indicative of GSD III as is the fact that the girl died suddenly at home, perhaps of hypoglycemia or cardiac insufficiency. Coexistence of GSD IX and III is of importance prognostically and as an explanation of reports by others (at variance with our experience) of muscle involvement in GSD IX.

STIMULATION OF MONOCLONAL IgG FOLLOWING PIASMA TRANS-

393 FUSIONS IN A CHILD WITH COMBINED IMMUNODEFICIENCY (CID-ADAt). John J. Tacuone, Beatrice C. Lampkin, Sheldon A. Horowitz, Kevin E. Bove, Dept. of Pediatrics and Pathology, College of Med., Univ. of Cincinnati, and Dept. of Pediatrics, Div. of Immunology, Univ. of Wisconsin, Madison

J.B., the second male sibling with CID-ADAt, had no E and EAC rosette forming lymphocytes, decreased lymphocyte stimulation by PHA and absent serum and surface immunoglobulins ( $\mathrm{Ig}$ ) until $3 \frac{3}{2}$ months of age when he received deep frozen plasma prophylactical$1 y$. Subsequently, normal numbers of $E$ rosette forming lymphocytes were present transiently but marked elevation of serum Ig developed and persisted until he died at 1 year of age of P. carinii pneumonia. Serial immunoelectrophoresis showed restricted heterogeneity of IgG and the development of a monoclonal IgM after plasma transfusion. Gm subtyping of parental and patient's serum Ig showed expression of paternal haplotype only. Absence of graft is host disease on lymph node and skin biopsy after plasma transfusion and the presence of $\mathrm{Ig}$ with paternal haplotype suggest that the elevated IgG and monoclonal IgM were produced by the patient's own B cells. Autopsy findings were consistent with incomplete development of the B cell system (widespread plasmacytosis but absent follicular development in lymph nodes, spleen, gut and tonsils). The thymus was dysplastic and lacked Hassel1's bodies. The results suggest that some factor in the transfused plasma triggered the incomplete development of a $B$ cell system. Such a finding may indicate that in some patients with CID-ADAt the potential for immune competency exists and that plasma factors may play an inductive or depressive role.

REMOGLOBLN SYNTHESIS IN CULTURED HUMAN

394 RABBTT ERYTHROBLAST HETEROKARYONS. Patricia Jargiello, Sch. of Med., Child. Hosp. of Pittsburgh, Dept. of Ped., Pa.

Erythroblasts from the femora of phenylhydrazine treated male rabbits were purified by centrifugation on a BSA gradient; and then fused with cultured human lung fibroblasts using inactivated Sendai virus. The cell mixtures (10-33\% heterokaryons)were next incubated with radioactive amino acids and harvested at timed intervals. Soluble cell proteins were separated by iso-electric focusing ( $\mathrm{pH} \mathrm{6-8)}$ on polyacrylamide gel with cold carrier hemoglobins. De novo synthesized hemoglobin was identified by elution of hemoglobin bands from the gel and reaction of the eluate with specific antisera followed by autoradiography. The heterokaryon mixtures produced up to 10 times the amount of rabbit hemoglobin as rabbit erythroblasts (with or without Sendai virus)alone with a peak at 3-5 hrs. post cell fusion. After 5 hrs.rabbit hemoglobin synthesis in the heterokaryons declined but remained above that of rabbit erythroblasts alone for an additional 5 hrs. By $24 \mathrm{hrs}$. rabbit hemoglobin synthesis in the heterokaryon mixtures was similar to that of rabbit erythroblasts alone. Rabbit erythroblasts mixed with cultured human fibroblasts without Sendai virus formed no apparent heterokaryons and always made the same amount of rabbit hemoglobin as rabbit erythroblasts alone. There was never any apparent synthesis of human hemoglobin by any cells. Apparently there are factors in cultured human fibroblasts which temporarily allow an increased level of heterologous hemoglobin mRNA translation. 
395

ORIGIN OF CHROMOSOMAL ABNORMALITIES: EVIDENCE FOR DELAYED FERTILIZATION IN MEIOTIC NONDISJUNCTION. Richard C. Juberg and John B. Mailhes, Dept. of Pediatrics, L.S.J. School of Medicine in Shreveport, Louisiana.

We sought to record events around the time of conception of couples with aneuploid progeny and compare with controls to evaluate factors in the etiology of meiotic nondisjunction.

We identified 33 aneuploid patients, 16 with $47, X Y,+G, 14$ with $47, X X,+G$, and 3 with $45, X$, referred for diagnosis or parental counseling. For comparison we chose 33 couples similarly referred because of an abnormal outcome of pregnancy; most progeny had congenital defects and all were euploid. We matched parents of the control group (CG) with parents of the study group (SG) by their age at conception of the abnormal offspring. We confidentially interviewed each couple about their habits and exposures.

Mean ages were 27 and 32 for the mothers and fathers of the SG and 27 and 31 for the CG. Mean sibship size was 2.5 in the SG and 2.0 in the $\mathrm{CG} ; 14$ of the $\mathrm{SG}$ and 19 of the $\mathrm{CG}$ were first births. Parents of 8 SG and of 3 CG subjects were unmarried at the conception. Among environmental exposures, parents in neither group ever had infectious hepatitis, and I SG mother and 3 CG parents recalled drug ingestion. There had been radiation exposure to 8 SG parents and 2 CG parents. Thyroid disease occurred in 2 SG families and I CG family. Difference between the two groups lay in their sexual histories; evidence for delayed fertilization (contraceptive failure, infrequent intercourse, or premarital conception occurred in 24 of the SG but in only 8 of the CG.

Thus, sexual histories may give evidence of delayed fertilization and so explain the origin of some meiotic nondisjunctions.

906 PRenatal diagnosis of MeCKel syndrome. Sara Kaffe,

396 Lynn Godmilow, Barbara Walker, Thomas D. Kerenyi, Nicholas G. Beratis, Eugene Ainbender, Judith Rose, and Kurt Hirschhorn, Department of Pediatrics, Mount Sinai School of Medicine, New York City.

Meckel syndrome is an autosomal recessive disorder manifested by encephalocele, microcephaly, microphthalmia, polycystic kidneys and, occasionally, polydactyly, cleft lip and palate, and small external genitalia. We have studied a fetus at risk for Meckel syndrome in a couple who previously had two children with this disorder. Monitoring of the third pregnancy included serial ultrasonography scans, in order to determine the presence of amniotic fluid and the size of the trunk and head. If amniotic fluid were obtainable we planned to assay for alpha-fetoprotein and neutral alpha-glucosidase which is produced from the fetal kidney. Three sonograms were done between 16 and 22 weeks gestation and no amniotic fluid was present. However, the sonogram demonstrated a large trunk suggestive of polycystic kidneys. The fourth sonogram at 25 weeks gestation revealed a significant increase in head circumference and a small area of fluid behind the skull. Analysis of the aspirated fluid showed no cells, and protein levels ( $I_{G}$ and alpha-fetoprotein) consistent with cerebrospinal fluid. No neutral alpha-glucosidase was detectable. On X-ray following injection of hypaque, an encephalocele was well documented. The family opted to abort this pregnancy and the anatomical findings confirmed the diagnosis of Meckel syndrome.

PRENATAL DIAGNOSIS OF HEREDITARY HEMOGLOBIN DISORDERS

397 CURRENT DEVELOPMENTS. Yuet W. Kan, Mitchell s. Golbus Moses Grossman)

University of California, San Francisco. (Spon. by

Methods for prenatal diagnosis of major hemoglobin disorders are being developed. This is a report of our progress to date. In $\alpha$ thalassemia (thal), since the genetic defect results from deletions of the aglobin structural genes, the diagnosis can be made by analysis of amniotic fluid fibroblast DNA by molecular hybridization with radioactive DNA complementary to globin mRNA sequences. We have used this method in a pregnancy at risk; the prenatal diagnosis of $\alpha$ thal trait was made and confirmed after birth. This technique is not applicable to $\beta$ globin abnormalities as the $\beta$ globin structural genes are present. Hence, prenatal diagnosis of $\beta$ thal and sickle cell anemia (SS) depends on fetal blood acquisition. Fetal blood was obtained by placental aspiration under ultrasound guidance and globin chain synthesis was measured. Of 11 pregnancies tested for $\beta$ globin chain abnormalities, adequate fetal blood samples for accurate diagnosis were obtained in 9 cases. The results include 3 fetuses homozygous for $B$ thal and 1 homozygous for SS. Two complications (infection and fetal bleeding) resulting in fetal death occurred, and methods are being developed for their prevention. We conclude that prenatal diagnosis of $\alpha$ thal is now possible using molecular hybridization on fibroblast DNA obtained by established, safe techniques. For $B$ chain abnormalities, although prenatal diagno$s$ is is feasible, the methods used need to be improved to increase the success rate and to avoid complications.
A SELECTIVE SCREENING METHOD FOR HYPERAMMONEMIA.

398 Ingeborg Krieger \& Qamar Taqi. Wayne State Univ. Sch. of Med. \& Children's Hosp. of Mich, , Detroit. screening for hyperammonemia of infants hospitalized for convulsions led to the diagnosis of 2 cases with urea cycle enzyme deficiency which would have been unrecognized if screened for aminoacidopathy by paper chromatography. Detection at the onset of symptoms would have prevented mental retardation in 1 case This experience emphasized the need to test infants who ccme to the ER and OPD because of vomiting, lethargy and/or convulsions preferably while they are symptomatic. We therefore developed a method which allows overnight sample storage. This method is pre ferable over plasma storage because it utilizes blood in smaller amounts, and obviates centrifugation and freezing. It is a modification of an ion exchange column method (Clin. Chim. Acta 42:141, 72 ? 0.25 or $0.5 \mathrm{ml}$ of blood is added to screw-topped bottles $3 / 4$ filled with resin (Dowex 50 ) in 0.24 M sucrose, sealed, shaken by repeated inversion, and refrigerated until further processing within 24 hours. The mean of random samples of 17 aaults was $36.0 \mu \mathrm{g} \%$ (range 25.9-53.1) compared to 38.2 $\mu$ g\% (range 21.1-55.1) by the standard method. The mean after overnight storage of resin and blood was $37.4 \mu \mathrm{g} \%$ (range 25.2-50.3); the mean difference between stored vs fresh samples was $12.1 \%$ (range $-27.1 \%$ to +39.5 ) Occasional large differences ( $>15 \%$ in 4 tests) are due to the well known vagaries of ammonia determination rather than storage, because the following comparisons gave similar results: difference between duplicates; between 0.25 and $0.5 \mathrm{ml}$ blood; fresh vs washed resin storage; and fresh vs frozen plasma storage
390 and GALACTOSE METABOLIC ABNORMALITY. Harvey L. Levy Public Health, Harvard Med. Sch., and Mass. Gen. Hosp., Neuro. Serv., Boston.

All known disorders of galactose (gal) metabolism are characterized by deficient activity of a gal metabolizing enzyme demonstrable in erythrocytes (rbc). These include galactosemia (gal]-phos uridyltransferase deficiency), galactokinase deficiency, and UDPGal-4-epimerase deficiency. We have been studying an infant who consistently accumulates gal in blood (10-12 mg/dl) and urine $(>50 \mathrm{mg} / \mathrm{dl})$ when ingesting milk. The gal tolerance curve following milk loading revealed gal accumulations that peaked at 30 minutes $(10 \mathrm{mg} / \mathrm{d} 1)$ and plateaued between 1 and 3 hours (6-8 $\mathrm{mg} / \mathrm{dl})$, intermediate between that of the normal infant and the infant with galactosemia or the Rennes-like variant. No gal-1-phos could be detected. Below are enzyme activities:

\begin{tabular}{|c|c|c|c|c|}
\hline & Gal-Kin & Gal-1-P UT & Epim & ${ }^{14} \mathrm{CO}_{2}$ \\
\hline $\mathrm{Rbc}$ & $0.0473,0.0232$ & $\begin{array}{r}11.0,19.5 \\
21\end{array}$ & $0.194,0.346$ & $\begin{array}{l}\text { (cpiii) } \\
2,673\end{array}$ \\
\hline Normal & $0.0297+0.0049$ & .5 & $0.137+0.026$ & $2,000-10,00$ \\
\hline kin Fibr & roblasts - & & $=$ & \\
\hline Norma & - & $38.1+11.1$ & - & 350 \\
\hline
\end{tabular}
Normal
Rbc UDPG pyrophosphorylase activity and gal uptake were also normal. Thus, it would appear that gal can be normally transported and metabolized in rbc and probably normaliy metabolized in cultured skin fibroblasts. This infant may have a defect in ga? metabolism expressed in liver but not in rbc or cultured skin fibroblasts.
100 EVALUATION OF DUARTE:GALACTOSEMIA GENETIC COMPOIND. Harvey L. Levy, Stephen J. Sepe and David S. Walton. Med. Sch., Mass. Gen. Hosp. and Mass. Eye and Ear Inf., repts. of Neuro. Serv. and Opthal., Boston.

Galactosemia is characterized by lack of oalactose-1-phosphate uridyltransferase (Gal-1-P (IT) activity. Other low activity transferase variants have been described. Among these is the genetic compound involving both the Duarte variant enzyme and, presumably, enzyme for the "classical" galactosemia mutation. It has been suggested that such genetic compound individuals, when untreated, are at risk for the clinical complications of calacto'semia. We have encountered by routine screening four individuals ages 4-30 years, having this genetic compound. None has received any dietary restrictions or other therapy. Erythrocyte aal-1-P
UT activity has been $3.7-8.6 \mathrm{El} / \mathrm{om} \mathrm{Hb} / \mathrm{hr}$ (16-37\% of norma 1$)$. In all irstances an enzyme with the electrophoretic mobility of the Duarte variant was demonstrable in erythrocytes. N'o clinical abnormalities have been detectable. This includes the absence of any signs of cataracts as determined by slit-lamp examination, absence of liver disease as determined by examination and norma SGOT, and normal intelligence. In addition, we are following six younger children with the genetic compound who up to the age of 3 years have not developed any clinical complications. It is likely that this genetic compound of transferase is benian and does not warrant treatment. 
NORMALIZATION OF HEPATIC PHOSPHORYLASE KINASE ACTIVITY AND GLYCOGEN CONCENTRATION IN GLYCOGEN STORAGE DISEASE TYPE IX (GSD IX) DURING TREATMENT WITH SODIUM DEXTROTHYROXIN. Derrick Lonsdale and George Hug, Cleveland Clinic Fndn., Cleveland and Children's Hospital, Cincinnati, Ohio.

In 1960 , treatment with $2 \mathrm{mg} /$ day of sodium $\mathrm{D}-3,5,3^{\prime}, 5^{\prime}$ tetraiodothyroxin (S.D.) in a 5 mo old boy with GSD I (G-6-P' tase deficiency) produced acidosis. In 1968, $4 \mathrm{mg} /$ day S D. in 4 children with low liver phosphorylase activity the cause of which was not determined produced decrease of liver size and serum triglyceride and increase of body growth but not phosphorylase activity. In $1974,4 \mathrm{mg} /$ day of S.D. for 15 months in a 2 y old girl with GSD IX (i.e. phosphorylase kinase deficiency in liver but not in muscle) produced disappearance of hepatomegaly, drop of serum triglycerides from 528 to $96 \mathrm{mg} \%(\mathrm{~N}=35-135)$, normalization of hepatic light and electronmicroscopy and bioptic liver tissue changes:

\begin{tabular}{lccl} 
& \multicolumn{2}{c}{ Patient } & Normal values \\
& Before Rx & After $\mathrm{Rx}$ & \\
phosphorylyase kinase & 0.1 & 1.4 & $1-3 \mu \mathrm{mP} / \mathrm{g} / \mathrm{min}^{2}$ \\
phosphory lase & $* 4.0$ & 32.5 & $25.1 \pm 6.5 \mu \mathrm{mP} / \mathrm{g} / \mathrm{min}$ \\
gly cogen concentration & 8.1 & 3.8 & $1 \mathrm{ess} \mathrm{than} 6 \%$ \\
glucose-6-phosphatase & 5.3 & 5.1 & $4.7 \pm 1.9 \mu \mathrm{mP} / \mathrm{g} / \mathrm{min}$
\end{tabular}

Exogenous kirase restored normal activity

Normalization of phosphorylase activity was secondary to that of phosphorylase kinase. The latter, and the reversal of clinical, biochemical and morphologic abnormalities of GSD IX did not seem the result of nonspecific stimulation of enzymatic activities since microsomal G-6-P'tase remained unchanged.

402

METABUL IC ABNDRYALITIES IN LOWE SYINDROME FIBROBLASTS.

Roderick R. McImnes, Vivian E. Shih, Richard W. Erbe. Harvard Ked.Sch. Mass.Gen.Hosp.(Genetics), Boston. The biochemical basis of the $X-1$ inked oculocerebrorenal symdrome of Lowe (OCPS) is unknown. Our studies of skin fibroblasts from 2 patients suggest a disturbance in glutamate (Glu) metabolism. OCRS cells grown to high density often died if not refed for 7-10 days, while nomal cells survive over 3 weeks. Intracellular [Glu] was $2.1 \mathrm{X}$ \&[Asp] 3.3X normal in unrefed cells, with an intra- to extracellular [Glu] $5.7 \mathrm{X}$ noma1, whereas all other amino acids (aa) in the media \& cells were comparable. Further, Glu-1$14 \mathrm{C} \& \mathrm{Glu}-5-14 \mathrm{C}$ oxidation to $14 \mathrm{CO} 2$ were only $1 / 3$ of normal over wicle ranges of [Glu] \& time. Only L-Glu of 21 aa $(2 \mathrm{~m} / 1)$ differentially inhibited cell growth, with 2 doublings/ 5 days by OCRS cells vs. 4 by nomals. Increased cel1[Gilu] could enchance pyruvate to Ala transmination $\xi$, according ly pyruvate-1-14C decarboxylation was $30 \%$ normal at $0.01 \mathrm{~m}^{\mathrm{M}}$ pyruvate $\& 65 \%$ at $1.0 \mathrm{~m}^{\mathrm{\prime}} \mathrm{s}$. In contrast, Ala-1-14 C \& Ala-i-14C oxidation were even more reduced (6-15\% of nomial). The oxidation of succinate-1,4-14C was normal, suggesting integrity of the citric acid cycle (CAC). Thus the anaplerotic conversion of Glu to the CAC intermediate $\alpha$-ketoglutarate, and of $\mathrm{Al}$ a to pyruvate, is reduced. Defective aa uptake is unlikely since, for comparable cell densities, incorporation of $\mathrm{Ala}$ \& Leu into protein was normal. The increased cell [GIU] \& abnornal Glu metabol ism nay account for the OCRS phenotype, since Glu is (i) toxic to developing retina \& CNS, (ii) central to renal anmoniagenesis \& bioenergetics $\xi_{\text {f }}$ (iii) especially concentrated by nomal eye, brain \& kidney. (Supported by USPHS grant 1 DD06356 \& a Canadian MRC Fellowship to RRM.)

COBALAMIN (VITAMIN B 12 ) TOXICITY IN METHYLMALONIC-

ACIDEMIA. M.J. Mahoney, J.F. Nicholson, A.C. Hart, ACIDEMIA. M.J. Mahoney, J.F. Nicholson,
L.E. Rosenberg \& R. Challop. Depts Hum. Genet. \& Ped. Yale Univ., New Haven; Dept. Ped., Columbia Univ., New York.

After failing to respond to $1 \mathrm{mg} / \mathrm{d}$ cyanocobalamin for $24 \mathrm{~d}$, an infant with methylmalonicacidemia due to a defect in adenosylcobalamin (AdoCb1) synthesis was given hydroxocobalamin ( $\mathrm{OH}-\mathrm{Cbl}$ ), $2 \mathrm{mg} / \mathrm{d}$. This was followed by clinical improvement and a fall in urinary methylmalonate from $3.2 \mathrm{~g} / \mathrm{d}$ to $<0.3 \mathrm{~g} / \mathrm{d}$. Clinical improvement was not sustained, however, and urinary methylmalonate actually rose despite continued $\mathrm{Cbl}$ therapy. Other observations also suggested adverse effects of treatment. He twice developed acidosis within hours of receiving $\mathrm{OH}-\mathrm{Cbl}$. Cessation of $\mathrm{Cbl}$ therapy then led to temporary improvement. Efforts to define optimum treatment failed and he died at 13 mo. During the month before he died, serum $\mathrm{Cbl}$ concentrations, measured by radioactive assay, were greater than $100 \mathrm{ng} / \mathrm{ml}$ (normal $<1$ ).

Cultured fibroblast extracts were used to investigate possible inhibitory effects of $\mathrm{OH}-\mathrm{Cbl}$ upon methylmalonyl- $\mathrm{COA}$ mutase activity. In control extracts $\mathrm{AdoCbl}$, the required mutase coenzyme, had a $\mathrm{K}_{\mathrm{m}}$ of $3 \times 10^{-8} \mathrm{M}$ whereas $\mathrm{OH}-\mathrm{CbI}$ was found to be a competitive inhibitor with a $\mathrm{K}_{i}$ of $6 \times 10^{-6} \mathrm{M}$. Fibroblasts from the affected infant gave apparent $\mathrm{K}_{\mathrm{m}}{ }^{\prime} \mathrm{s}$ for AdoCbl and methylmalonyl- $\mathrm{COA}$ and a $\mathrm{K}$. for $\mathrm{OH}-\mathrm{Cbl}$ which were similar to controls. Some mutant lines increase mutase activity when $\mathrm{OH}-\mathrm{Cbl}$ is added to culture medium cells from our patient showed no response. This severe block in AdoCbl synthesis plus the presumed exceedingly high intracellular concentration of $\mathrm{OH}-\mathrm{Cbl}$ probably combined to reduce even further the mutase activity in the child and render $\mathrm{OH}-\mathrm{Cbl}$ toxic.
Clonal Evolution and Leukemic Transformation in 404 Fanconi's Anemia. L. Meisner, A. Taher, and N. T. Shahidi, University of Wisconsin, Madison.

It is well known that patients with Fanconi's anemia have a high incidence of acute leukemia. This has been attributed to increase chromosome breakage leading to clonal changes with malignant potential. The nature of this relationship, however, has not been clarified. Recently a 15 year old boy with classical Fanconi's anemia developed acute erythroleukemia, characterized by a high number of nucleated erythroids $(140,000 / \mathrm{cu} . \mathrm{mm})$ and a large number of myeloblasts in the peripheral blood and bone marrow. Cytogenetic studies from a direct marrow specimen revealed a modal karyotype characterized by loss of a $\mathrm{C}$ chromosome $(45, \mathrm{XY},-\mathrm{C})$, although 5 years ago his marrow karyotype had been norma1. Subsequently similar chromosome studies were performed on the patient's 19 year old sister, also with Fanconi's anemia, who had remained in hematologic remission for the past 6 years. Although her previous marrow studies demonstrated normal karyotype the current cytogenetic studies on her bone marrow revealed four distinct clones, all with 46 chromosomes. One clone had an apparently normal female karyotype, one had an extra no. 1 chromosome associated with a missing $\mathrm{C}$, another had an extra no. 2 chromosome and a missing $\mathrm{C}$, and the fourth clone exhibited an extra B-like chromosome and a missing C. Despite these findings, the patient remains in good hematologic remission without any evidence of leukemia. This hematologic discrepancy between the two siblings may be the result of a selective advantage of the single abnormal marrow clone as compared to four different clones without any proliferative advantage.

405 TETRAHYDROCANNABINOL AND OLIVETOL. Akira Morishima, Richard T. Henrich, and Gabriel G. Nahas, Departments of Pediatrics and Anesthesiology, College of Physicians and Surgeons, Columbia University, New York, New York.

Increased frequency of metaphase nuclei containing less than 30 chromosomes ("hypoploid") in lymphocytes obtained from marihuana smokers and induction of this phenomenon by addition of olivetol or $\Delta^{9}$-tetrahydrocannabinol (THC) to culture medium of normal human lymphocytes were reported previously. It was speculated that segregational errors of chromosomes may account for the underlying mechanism of this observation.

In the present study, partially purified T-lymphocytes obtained from 11 healthy adult males were cultured for 3 days in the presence of olivetol $\left(5 \times 10^{-5} \mathrm{M}\right)$ or THC $\left(3.2 \times 10^{-6} \mathrm{M}\right)$. Cells were harvested for microscopic examination, without exposure to colchicine or hypotonic solution. All cell divisions under the field of observation were subjected to photomicrography and analyzed by 3 independent observers in a double blind fashion. In cells exposed to THC, $10.96 \%$ were judged to be abnormal, while only $2.45 \%$ of cells in the control group exhibited abnormalities. In cells exposed to olivetol, $6.2 \%$ were abnormal compared with $0.95 \%$ in the controls. Abnormal cell divisions in cells exposed to THC included anaphase bridges, anaphase lagging, multipolar anaphases and telophases and other distinctly abnormal patterns of chromosome segregation. It appears that THC and olivetol may affect the formation of microtubules resulting in exrors of chromosome segregation.

\section{6} DEFICIENT GLUCOCEREBROSIDASE ACTIVITY IN GAUCHER'S

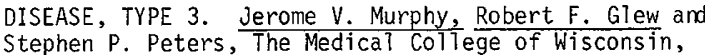
'Milwaukee Children's Hospital, Department of Neurology, Milwaukee WI 53233, University of Pittsburgh School of Medicine, Department of Biochemistry, Pittsburgh, PA 15213.

Glucocerebrosidase is deficient in Gaucher's disease, Types 1 and 2, but the enzymology of Type 3, juvenile Gaucher's, has not been reported. Our patient ceased acquiring new developmental skills at age 1 year, and Gaucher cells were demonstrated in her bone marrow and spleen at age 3 years. Presently she is nine years old, nonambulatory, and her only vocalization is crying or laughing. Seizures are intermittently frequent.

Glucocerebrosidase activities were determined in white blood cells from the patient, her mother, and her maternal grandmother utilizing ${ }^{14} C$-glucocerebroside and:-4-methylumbel 7 ifery $1-B-D-$ glucopyranoside. The patient's enzyme activities were $12 \%$ and $23 \%$ of controls respectively, utilizing the natural and artificial substrates. The enzyme activity of her mother's white blood cells were about $50 \%$ of control values. We believe these are the first demonstrations of a reduced white blood cell glucocerebrosidase activity in Type 3 Gaucher's disease. They do not explain the difference in the clinical presentations for this type of Gaucher's disease. 
407

LONG TERM LIVER CELL CULTURE. H. Negishi and P. Benke (Intr. by R. Stempfel, Jr.) University of Miami Mailman Center for Child Development, Miami, Fla.

It has not yet been possible to obtain liver-specific cells in long term tissue culture because of the accompanying overgrowth of fibroblast-like cells. Reported here is the successful longterm growth of human and rat hepatic epithelial cells (ec) with liver specific properties. Hepatic biopsies were treated with $0.05 \%$ chymotrypsin and $0.1 \%$ collagenase in Mg+ - Ca+t free Hanks salt solution and plated sequentially in three flasks. Successful culture of hepatic ec depends on 1)primary cloning from the mixed cultures 2) use of dilute (0.05\%) trypsin solutions $\mathrm{pH} 7.2-7.4$ for subculturing 3) use of non-toxic fetal bovine serums. Hydrocortisone ( $5 \mathrm{mg} \%$ ) has a strong and insulin a weak stimulating effect on ec growth. Tripeptide (glycyl-histidyl-1ysine) and dipyridamole were without effect. D-valine substituted for L-valine in the media selects for ec growth. Cultured hepatic ec secrete albumin and contain liver specific induceable enzymes;

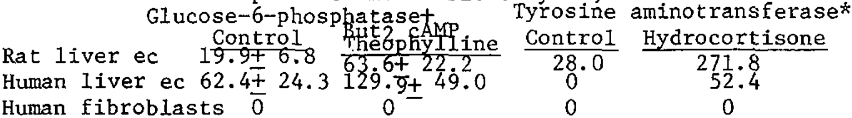
Human fibroblasts $0 \quad 00$

(+nanomoles $\mathrm{Pi} / \mathrm{mg}$ protein/hr. *nmoles $\mathrm{HPP} / \mathrm{mg}$ protein/hr.) Human and rat liver ec have been maintained in culture up to 5 months before slowing of growth. Cell strains examined to date do not have some constitutional liver enzymes (e.g., ornithine transcarbamylase). Nevertheless, it should be possible to examine cultured hepatic cells for some metabolic processes not found in skin
fibroblasts.

\section{A CASE OF PARTIAI 13q TRISOMY WITH A BALANCED}

408 t(Cp+;13q-) CARRIER FATHER. Richard $L$. Neu and

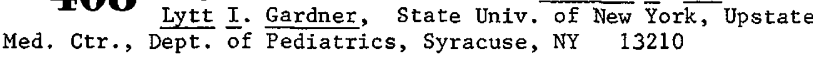

Paxtial trisomy for the distal one-third to distal two-thirds of the long arm of chromosome 13 has recently been recognized as a distinct clinical entity. The case presented here is that of an eleven month old female referred to us for evaluation because of multiple congenital malformations. She was the product of the first pregnancy from a 25 year old mother and 23 year old father. Examination revealed an infant with severe psychomotor retardation, cleft lip and palate, low set ears, sloping forehead, strabismus, long curved eyelashes, micrognathia, short neck, polydactyly of both hands and feet, and a dimple on the lateral surface of the elbow. Other tests are underway to determine if deafness or any congenital heart defects are present. A chromosome analysis from the proposita revealed a $46, \mathrm{XX}, \mathrm{Cp}+$ karyotype. The mother's karyotype was normal; the father had a

$46, \mathrm{XY}, \mathrm{t}(\mathrm{Cp}+; \mathrm{Dq}-)$ complement. G-banding studies demonstrated the Dq- chromosome to be a number 13; further banding studies ar underway to determine which $\mathrm{C}$-group chromosome is involved.

409 TRANSLOCATION CARRIERS. Richard $\mathrm{L}$. Neu and Lytt I. Gardner, State Univ. of New York, Upstate Med. Ctr. Dept. of Pediatrics, Syracuse, NY 13210

To our knowledge, no translocations between the short arm 0 i a number 3 and the long arm of a number 15 chromosome have been reported. The case presented here is that of a family with a $t(3 ; 15)(p 27 ; q 21)$ translocation. The index case was a three year old female referred for evaluation because of delayed speech and language acquisition. Examination revealed retarded psychomotor development and a physical growth rate in the third percentile. Several facial abnormalities were present including dolicocephaly microphthalmia, hypertelorism, epicanthal folds, narrow palpebral fissures and low set posteriorly rotated ears. A hearing loss in both ears of a conductive nature was demonstrated. The mother's first three pregnancies ended in spontaneous abortions in the first trimester; the proposita has two younger male siblings. A chromosome analysis and G-banding study from the proposita revealed a $46, \mathrm{XX}, \mathrm{t}(3 ; 15)(\mathrm{p} 27 ; \mathrm{q} 21)$ karyotype. Her father and one brother were found to be carriers of the same translocation; phenotypically they are not remarkable. The father has difficulty in reading and writing, and the brother is deficient in the area of language acquisition. Both are being tested further to determine the extent of any retardation. The variation observed between the three translocation carriers may be explained using the aneusomy by recombination model.
410 A $t(5 ; 21)(p 13 ; q 13)$ IN A FAMILY WITH DOWN'S SYNDROME. Syracuse, NY 13210 .

The proposita was a 57 year old woman who sought genetic counseling because both she and an older sister had given birth to infants with Down's syndrome. The proposita's oldest daughter was expecting her first child and the family wanted to know if they might be translocation carriers. Chromosome analysis performed on the proposita revealed a $46, \mathrm{XX}, \mathrm{t}(5 ; 21)(\mathrm{p} 13 ; \mathrm{q} 13)$ karyotype; one of her three daughters was also a balanced carrier of the same translocation. The components of the translocation were identified by G-banding. The two children in the family with Down's syndrome and the proposita's sister had died and were unavailable for karyotyping. The carriers of such translocations as described here, involving chromosome 21 , may increase the risk of producing a child with trisomy 21 . Such structural rearrangements may affect pairing and segregation during meiosis, resulting in an increased frequency of nondisjunction.
411 John $\mathrm{F}$. Nicholson, pablo Rubinstein, and Nicole Suciu-Foca, Columbia University, Collere of Physicians and Surgeons, and The New York Blood Center, New York, N.Y.

Studies of famillal incidence of diabetes mellitus have been inconclusive as to the mode of inheritance of the predisposition for diabetes. Recently an association between certain HI, $-A$ antigens and fuvenile diabetes mellitus has been shown in some populations. The suggestion has also been made that genes in the $\mathrm{HL}-\mathrm{A}$ region may not be randomly assorted in fuventle diabetics. We have studied 20 genetically determined erythrocyte and plasma characters and HL-A A, B, C, and D in 11 familles laving 1 or more children with fuvenile diabetes mellitus. The inheritance of erythrocyte and plasma characters proved the reliabllity of the studies and demonstrated random assortment. However, studies of the HL-A region showed clearly both an increased number of crossovers and non-random assortment of haplotypes, establishing ( $P<0.001$ ) a relationshin hetween the chromosome carrying HL-A genes and fuvenile diabetes mellitus. The frequency of HL-A identity among affected siblings suggests that (a) gene(s) in the HL-A region may predispose to juvenile diabetes. In view of the non-l'endelian sepregation rates of HL-A in families with J.D.M. it is therefore possible that studies of the incidence of the disease in families cannot define the mode of inheritance of the predisposition.
412 FTROSIS Ronald D. Pearson, A harold IIC

rold Lubin (Spon, by Bruce D. Graham) Ohio State Univ., College of Medicine, Children's Hosp., Dept. of Pediatrics, Columbus, 0. The hypertriglyceridemia in children with Cystic Fibrosis has been hypothesized to be due to a known decrease in post heparin lipolytic activity. Serum from individuals with Cystic Fibrosis produces a dense, granular precipitate when diluted in a heparincontaining buffer unlike the focculant precipitate observed with serum of adults and children unaffected by Cystic Fibrosis. The ability of heparin to reverse the ciliostatic character of serum and salivary secretions of fibrocystic individuals and to bind to many different proteins present in blood suggests that an abnormally high affinity for heparin of one or more serum components in Cystic Fibrosis affected individuals may cause the formation of the unusual precipitate and influence in vivo stimulation of lipolytic activity by heparin. Measurements of protein precipitated by heparin from fibrocystic serum in a buffered solution indicate increased amounts of protein compared with normal subjects, (mg protein/ml serum: Normals, $\overline{\mathrm{X}}=13.03 \pm 1.86$ ;CF: $\overline{\mathrm{x}}=17.14 \pm 2.4)$. Heparin co-precipitated with protein is proportionately increased in amount compared with normal subjects, (mg heparin/m1 serum: Normals, $\overline{\mathrm{X}}=0.873 \pm 0.09 ; \mathrm{CF}, \overline{\mathrm{X}}=1.287$ $\pm 0.22)$. Results are significant at a level of $p<.001$. This data suggests that an increased capacity to bind heparin in serum of fibrocystic individuals affected the previously noted low post-heparin lipolytic activities. Supported by CHRF GRS Grant \#81-779\& Women's Wing of Children's Hospital \& Mask IV. 
13 AMINO ACID TRANSPORT IN GLUTATHIONURIA DUE TO GAMMAGLUTAMYL TRANSPEPTIDASE (GGTP) DEFICIENCY. Francoise Pellefigue, Stephen I. Goodman, Stephen P. Spielberg, Jean Butler, and Joseph D. Schulman, NIH, Bethesda, and Univ. Colorado Med. Center, Denver.

We report studies of amino acid transport (AATx) in a patient (BBRC $65: 68,1975)$ with gross tissue deficiency of GGTP $(<0.5 \%$ normal activity in fibroblasts). GGTP has been considered a significant mediator of mammalian AATX (Science 180:33, 1973).

Baseline plasma AA concentrations and urinary excretions were normal. Individual AAs were infused I.V. Plasma methionine and alanine levels rose 90 and 6 fold respectively without exceeding capacjty for full renal resorption. Tm lysine was $70 \mathrm{u} \mathrm{mol} / \mathrm{min} /$ $1.73 \mathrm{~m}^{2}$ (published nl. 32-86). Tm proline was a moderately reduced 77 ( $\mathrm{nl}$. 180-300). The patient's cultured fibroblasts had normal intracellular AA concentrations. Apparent uptake kinetics of four of the best AA glutamyl acceptors into normal and mutant fibroblasts, as determined with radioactive AAs, are summarized (table). No significant differences were observed (all p>.20). The data do not support the view that GGTP plays a significant role in transport of AAs in the human kidney or fibroblast.

\begin{tabular}{|c|c|c|c|c|}
\hline Amino Acia & \multicolumn{2}{|c|}{$\mathrm{V}^{*} \max \left( \pm \mathrm{S} \cdot \mathrm{E} \cdot \mathrm{M}_{.}\right)$} & \multicolumn{2}{|c|}{$\mathrm{KmH}$ ( \pm S.E.M.) } \\
\hline & Mutant & Controls & Mutant & Controls \\
\hline Cystine & $.268(.047)$ &.$\overline{334(.050)}$ & $.107(.042)$ &.$\overline{116}(.028)$ \\
\hline Methionine & $.193(.039)$ & $.226(.025)$ & $.055(.009)$ & $.051(.010)$ \\
\hline Glutamine & $.412(.027)$ & $.352(.039)$ & $.011(.003)$ & $.011(.002)$ \\
\hline Alanine & $.442(.135)$ & $.472(.099)$ & $.044(.014)$ & $.055(.012)$ \\
\hline
\end{tabular}

\section{ABNORMAL GENITALIA AS PRESENTING SIGN IN MALE}

4.14 INFANTS WITH HYDANTOIN EMBRYOPATHY SYNDROME.

Waltex Pinto, Jr., Lytt I. Gardnex and Paula Rosenbaum. Genetic and Endocrine Unit, Dept. Peds., Upstate Med. Ctr. State Univ. N.Y., Syracuse.

Abnormal genitalia as a primary clinical finding is described in two unrelated $46, \mathrm{XY}$ male infants with the hydantoin embryopathy syndrome. Both their mothers had received diphenylhydantoin (Dilantin) $300 \mathrm{mg} \cdot /$ day throughout pregnancy. The infants also exhibited hypoplastic nails of fingers and toes, hypertelorism, a flat nasal bridge and bifid scrotum. In one case the scrotal skin was extremely redundant; this together with hypothe foregoing findings need to be differentiated from those with the Aarskog syndrome and from males with the Noonan syndrome. It is possible that diphenylhydantoin interferes with the action of fetal testicular hormones on the male genital primordia at critical stages of embryonic development. Whether this interference is via the fetal testosterone-dihydrotestosterone system, or via the feto-protein known as Miillerian-inhibiting hormone (or both) is not known. The available evidence strongly suggests that mothers receiving diphenylhydantoin who have given birth to one affected infant should be placed on a different anticonvulsant for future pregnancies.
416 TRISOMY 10 MOSAICISM IN A FEMALE INFANT. Qutub Qazi, Akiko Masakawa, Cecily Madahar, and Robert Ehrlich State Univ. of New York, Downstate Med. Ctr.,Brook 1 yn The infant was born after 36 weeks of gestation weighing 2300 grams. Parents were in their mid-twenties. Parents and two older siblings were phenotypically normal and healthy.

The major clinical and roentgenographic manifestations in the proband included mongoloid slanted eyes, microphthalmia, blepharophimosis, low-set malformed ears, narrow high arched palate, retromicrognathia, absent fifth sacral segment, right pelvic kidney, congenital heart disease and failure to thrive. The head size was normal at birth but showed very little growth in subsequent three months. On the left hand $b$ triradius was missing and the axial triradius was in intermediate position. The chromosome studies on cultured lymphocytes and skin fibroblasts showed 33 per cent cells with 47 chromosomes, incliting an extra $C$ group chromosome which was identified as number 10 by the G-banding technique. The parents' karyotypes were normal.

To our knowledge, no patient with complete trisomy 10 has been reported. Nakagome et al (Jap. J. Hum. Genet. 18:216, 1972) have described the only known case of mosaic trisomy 10 in a five year old boy. Clinically their patient differed from ours in having normal physical growth and head size, spacious forehead, long trunk, narrow pelvis and deep plantar furrows, the features more commonly associated with trisomy of chromosome 8 . Their patient, in addition, lacked microphtha1mia, blepharophimosis and congenital heart disease. spadias gave a truly ambiguous appearance. Male patients with

\section{CONGENITAL HEART DISEASE IN DOWN'S SYNDROME - ITS \\ 417 GENOTYPE DISTRIBUTION. P. Syamasundar Rao and Mansoor Salehbhai. Medical College of Georgia, Dept. of Pediatrics, Augusta, Georgia.}

The association of congenital heart disease (CHD) with Down's syndrome (DS) and the specific types of CHD in DS have been well documented. However, the incidence and type of CHD in different genotypes of DS nameiy nondisjunction, translocation or mosaic have not been studied, which this study investigates. The records of 420 patients with diagnoses of DS seen at Medical College of Georgia Institutions were reviewed for the presence and type of CHD and type of chromosomal abnormality. Both clinical and chromosomal data were available in 276 patients of whom $80(29 \%)$ had CHD. The genotype distribution was nondisjunction $-90 \%$, translocation $-7 \%$ and mosaic - $3 \%$. The incidence of CHD in these respective genotypes was $23 \%, 29 \%$ and $50 \%$. The types of CHD in all cases of DS is similar to those reported by others: Endocardial cushion defect (ECD) $-35 / 80$, ventricular septal defect (VSD) $-25 / 80$, tetralogy of Fallot $-6 / 80$ and other CHD $-14 / 80$. The type of CHD in nondisjunction and mosaic DS is similar to that described above. But, in translocation DS, 3 of 4 patients had ECD and the fourth, patent ductus arteriosus (PDA). Being a persistent fetal structure, PDA may be ignored. The presence of only ECD in translocation DS by contrast to the usual ECD, VSD and other CHD combination in nondisjunction DS (although could be due to small numbers of translocation DS cases studied)may have some etiologic significance in CHD. These findings may call for chromosomal studies, especially with the newly developed techniques, in ECD patients in order to evaluate minor chromosomal etiology of CHD.

415 ACTIVATION OF N-ACETYL- $\beta$-HEXOSAMINIDASE (HEX) S heXosamines (H). M. Potier, S.B. Melançon and
L. Dallaire. Sect. Med. Genet., Ped. Res. Center, Hospital, U. of Montreal, Montreal, Quebec, Canada. Ste-Justine Hospital, dosis are deficient in both isoenzymes $A$ and $B$ of HEX but contain a small residual activity (less than $10 \%$ normal). Most of this activity is represented by an isoenzyme, HEX S, which has a higher isoelectric point than HEX A but was, otherwise, very similar to HEX A. Both isoenzymes were thermolabile and showed similar pH optimun, apparent $\mathrm{Km}$, molecular weights and immunological properties. The two isoenzymes were further compared by studying the effect of various known inhibitors of HEX A (N-acetylhexosamines, $\mathrm{H}, \mathrm{p}$-chloromercuriphenylsufonic acid and acetate) on an extract and on purified HEX S $\left(1,600\right.$-fold by $\left(\mathrm{NH}_{4}\right)_{2} \mathrm{SO}_{4}$ precipitation, DEAE-Sephadex A-25, SP-Sephadex C-25 and Sepharose $6 \mathrm{~B}$ chromatographies) from the liver of a patient deceased of type 0 GM2 gangliosidosis. All substances were found inhibitory except for $\mathrm{H}$ which activated the liver extract as well as purified HEX S (135-143\% of control activity between 50 and $125 \mathrm{mM} \mathrm{H}$ ). Since $H$ are competitive inhibitors of HEX A, it appears that there exists a binding site for $\mathrm{H}$ different from the catalytic site on the HEX S molecule. The HEX S polymer is thought to be formed from HEX A subunits ( $\alpha$ and $\beta$ ) but to contain a higher proportion of $\alpha^{\prime \prime}$. It is not known whether this rearrangement of subunits could induce conformationnal changes in the $\alpha$ and $\beta$ subunits which would explain $\mathrm{H}$ activation of HEX $\mathrm{S}$ or if HEX $\mathrm{S}$ is formed from at least one different subunit type. Supported bv the Medical Research Council of Canada (grant MA-5163)
418 A PROSPECTIVE STUDY OF BOYS WITH 47, XXY AND 47, XYY

418 KARYOTYPES. Arthur Robinson, Mary H. Puck, Katherine and National Jewish Hospital and Research Center, Denver. A survey of consecutive newborns between 1970 and 1975 has identified the following karyotypes in males: 47, XXY (12); $47, X Y Y(4) ; 46, X Y / X X Y$ (1). These have been studied developmentally since birth. Half of the group did not deviate from normal in physical growth, development, or psychosocial adjustment. The remainder of the $47, X X Y$ group, but none of the boys with $47, X Y Y$, had poor motor coordination, stiff hip joints, slow tempo, and placid dispositions during the first two years of life. Two of the boys with 47, XYY karyotype, unlike the other two, were we11coordinated, but during the second year of 1 ife were unusually impulsive and showed a low frustration tolerance. Language usage was mildly delayed in both groups. One child with poor emotiona development had a deprived environment. The developmental risk suggested by the excess of adults with these karyotypes in mental We are impressed by finding only one of these 17 boys with notable evidence of the traits associated with the XXY karyotype in adults. In half the group there were no distinguishing characteristics to set them apart from children with normat karyotypes. and penal institutions may be elucidated by this ongoing study. 
B-METHYL GLUTACONIC ACTDURIA: A NEW DISORDER OF LEUCINE METABOLISM. Brian H. Robinson, W. Geoffrey Sherwood, Maxwe11 Lampty, and J. Alexander Lowden, The Hospital for Sick Children, Research Institute, Genetic Metabolic Programme, Toronto, Canada.

A 3-year old girl presented with severe progressive neurological deterioration, having developed normally both physically and mentally to the age of 9 months. After this age, her head circumference, weight, and length ceased to increase. She exhibited hypotonia, bizarre posturing of the right arm, self mutilation of the left hand, and seizures on E.E.G. At presentation, she functioned at a 4-month level. There was no family history of mental retardation. There was neither metabolic acidosis nor hypoglycemia Gas liquid chromatography and mass spectrometry (GLC-MS) showed that the urine contained B-Methylglutaconic acid (BMGCA) a product of leucine (L) catabolism. Plasma L was normal. Daily urinary excretion of BMGCA was approx. $300 \mathrm{mg}$. This fell to $<10 \mathrm{mg}$ within 5 days of commencing a diet containing $<1.5 \mathrm{~g}$ protein $/ \mathrm{kg} /$ day. Following this, $75 \mathrm{mg} \mathrm{L} / \mathrm{kg}$ induced reappearance of BMGCA in the urine. No change in neurological status occurred during these studies. BMGCA hydratase activity was measured in muscle biopsy specimen as nmoles substrate utilized $/ \mathrm{min} / \mathrm{g}$ muscle. Compared to controls, the activities were $59.2 \pm 5$ (SE) vs. $18.4 \pm 2.5$, and $49.9 \pm$ 7.4 vs. $21.6+2$ in the dehydration and hydration directions respectively. This disorder cannot be accounted for by absent enzyme activity in muscle. Thus, it may be manifest only in nervous tissue or the accumulation of BMGCA is secondary to a different enzyme defect. GLC-MS combined with enzymatic analysis is useful in the delineation of developmental disorders.
IMMUNOLOGICAL EVIDENCE FOR ENZYMATICALLY INACTIVE 420 ARGININE ESTERASE (AEase) IN CYSTIC FIBROSIS (CF). Emmanuel Shapira, G.J.S. Rao, Hans U. Wessel, and Henry L. Nadler. iJorthestern University Medical School, Chi Íden's Memorial Hospital, Department of Pediatrics, Chicago.

Previous work in our laboratory has demonstrated a deficiency of proteolytic activity towards arginine esters (AEase) in plasma of patients with CF. This activity could be separated into 6 bands by isoelectric focusing on polyacrylamide gel. To further define the nature of the deficient AEase activity, the enzymes inhibited by soybean trypsin inhibitor (STI) were purified by affinity chromatography using STI bound to Sepharose 4B. This purified protein fraction was resolved by preparative isoelectric focusing. The fraction between $\mathrm{pH} 4.7$ and 6.4 was used to immunize rabbits in an attempt to develop an immunoassay for AEase. This fraction was chosen as previous studies demonstrated a deficient or possibly absent AEase in plasma of patients with CF. When normal human plasma was examined by immunoelectrophoresis with the antiserum, 5 precipitin arcs were observed of which 3 demonstrated AEase activity. When plasma from patients with CF was examined, similar precipitin arcs were demonstrated, however, one was found to be inactive in contrast to the normal. Samples from 15 normal controls and 15 patients with CF were also examined, in a double blind study, by electrophoresis in agarose gel containing the antibodies (Laurell technique). 13 of 15 normals revealed 3 enzymatically active "rockets". In contrast, 14 of $15 \mathrm{CF}$ samples revealed only 2 corresponding rockets with enzymatic activity. In all 14, the same rocket was deficient. These data provide additional support for the concept of an inactive AEase in patients with CF.

INCREASED MITOTIC RATE OF AMNIOTIC FLUID CELLS

421 TREATED WITH HOMOLOGUS MATERNAL SERUM. L.R. Shapiro, Z.R. Graves, and P.L. Wilmot, N.Y. Med. College, Depts. Ped. \& Path., Valhalla, N.Y. and Letchworth Village D.C., Cytogenetics Lab., Thiells, N.Y. (Intr. W.M. Gersony).

Since the serum of pregnant women contains elevated levels of many hormones and substances at physiologic levels, it can be postulated that the use of homologus maternal serum in amniotic fluid cell culture media would provide a more favorable environment and result in more efficient cell growth.

At the time of amniocentesis, serum was obtained from the mother and substituted for a portion of fetal calf serum usually employed in the media. The cells to which the homologus maternal serum was added were harvested on the tenth day as was a control from the original specimen which had the usual amount of fetal calf serum and no maternal serum. The slides were coded and evaluated blindly for mitotic rate per thousand as well as for usable mitotic rate (i.e., those mitoses suitable for cytogenetic analysis). Both the mitotic rate and usable mitotic rate were significantly increased with homologus maternal serum as compared to the controls.

Thus, homologus maternal serum has a beneficial effect on cultured amniotic fluid cells, and it is quite possible that this effect may be able to be translated into a consistent and significantly shorter culture time for prenatal cytogenetic diagnosis.
FABRY'S DISEASE: DETECTION OF HEMTZYGOUS AND HETERO-

422 ZYGOUS STATE BY $\mathcal{C}$-GAIACTOSIDASE IN SERUM AND LEUKOCYTES AND NERVE CONDUCTION. Kumudchandra J. Sheth, Thomas A. Good, John P. Thomas, Jr., Herbert M. Swick, and Jerome V. Murphy. Medical College of Wisconsin, Milwaukee Children's Hospital, Departments of Pediatrics and Neurology, Milwaukee, WI.

49 family members from 3 kindreds - 1 Mexican; 1 black; aid 1 white - with Fabry's Disease were studied by physical examination, EKG, peripheral nerve conduction (PNC) studies and biochemical estimation of the enzyme $\alpha$-galactosidase $(\alpha-G)$ in serum and WBC. The clinical expression in 2 propositus hemizygous males (ages 26 \& $42 \mathrm{yr}$ ) was severe with angiokeratomas, renal involvement, and abnormal EKGs. 7 other affected hemizygous males (age 4-13 $\mathrm{yx}$ ) had variable clinical expressions: 5 of these had abnormal EKGs and 6 had abnormal PNCs. A propositus heterozygous female (age $25 \mathrm{yr}$ ) had a severe clinical expression with episodic and severe pain in her extremities, arthralgia, liver involvement and palmar angiectasias. 16 heterozygous females (age 3-40 yx) had variable clinical expression but no angiectasia. Five of 11 heterozygous females had abnormal EKGs while 5 of 10 had abnormal PNCs. Both serum and WBC $\alpha-G$ were useful in detection of the hemizygous disease state. Serum $\alpha-G$ was consistent in separating the hemizygous males and hetexozygous females from normals. Leukocyte $\alpha$-G identified hemizygous males but overlap was seen between heterozygous females and normals. Interfamilial variation in EKGs and PNCs was evident.

423

SULFITE OXIDASE DEFICIENCY. Vivian E. Shih, Israel $F$. Abroms, Roseann Mandell, Miriam Th. Carney, Jean L. Johnson, K.V. Rajagopatan, Neurot. Services, Mass. Gen Hosp. and children's Flosp. Med. Center, Boston; Dept. of Biochem., Duke Univ., Durham.

Sulfite oxidase deficiency (SOD) has been described only once by Mudd et al (1967) in a severe neurologically defective child. We recently studied a natient, who at ace 2 suddenly developed ataxia, myoclonic seizures and right hemiparesis following a bout of vomiting and diarrhea. At 3 , he had spastic quadriparesis, oross choreoathetoid movements and severe truncal ataxia, diannosed and treated as cerebral arteritis. Bilateral dislocated lenses were discovered at $41 / 12$. A diaqnosis of $S 00$ was then made by finding sulfite and S-sulfocysteine (SSC) in the urine, blood and CSF, and thiosulfate in the urine. These comoounds markedly increased after oral L-cysteine and L-methionine loadina. SO activity was demonstrated in normal skin fibroblasts rut not measurable $(<5 \%)$ in the patient's fibroblasts. A aood biochemical response to restricted intake of sulfur amino acids was obtained:

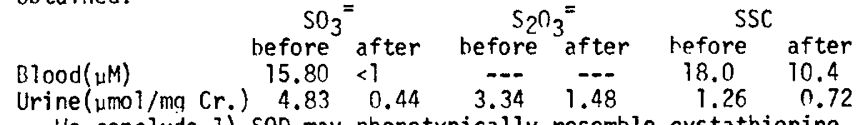

We conclude 1) SOD may phenotypically resemble cystathionine synthase deficiency, 2) early dietary treatment may nrevent clinical symptoms, 3) prenatal diaanos is is potentially possible, 4) the rarity of SOD may in part be due to technical difficulties in diagnosis.

\section{ING. Vivian E. Shih, J. Thomas Coulombe, Miriam M. Carney, Phyllis M. Madigan and Arline M. Erickson.} Neurol. Service, Mass. Gen. Hosn. and Harvard Med. Sch., State Lab. Inst., Mass. Dept. Public Health, Boston.

Argininosuccinic aciduria (ASAciduria) is a rare urea cycle disorder. Most reported patients have shown protein intolerance and mental retardation. We have studied 6 children detected by routine newborn screening. The initial laboratorv findinas are: $\begin{array}{lllllll}\text { Patient } & 1 & 2 & 3 & 4 & 5 & 6 \\ \text { Urine ASA (mq/ma Creat.) } & 22.2 & 7.0 & 6.1 & 2.1 & 3.7 & 0.26\end{array}$ $\begin{array}{lllllll}\text { Urine ASA (mq/mg Creat.) } & 22.2 & 7.0 & 6.1 & 2.1 & 3.7 & 0.26 \\ \text { Bl. } \mathrm{NH}_{3}(\mathrm{~N}:<110 \mu \mathrm{g} / \mathrm{dl}) & 150 & 137 & 133 & 220 & 150 & 100\end{array}$

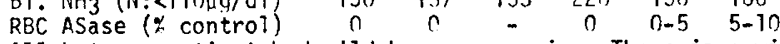
All but one patient had mild hynerammonemia. There is a wide range of urinary ASA excretion, the amounts in Patients 1 and 6 being 100 fold different. Patient 6 , with the least amount of urinary $A S A$, had a small residual activity of $\mathrm{RBC}$. ASase. A1l patients were normal at the last follow-up $(4 / 6>3$ years of age). Four natients have received a restricted protein diet supplemented with arginine. One affected older sib, al though untreated, was normal at $4-1 / 2$ years of age. The biochemical data suquest heterogeneity among these patients. ASAciduria may be asymotomatic in certain instances.

ASAciduria is, thus far, the only urea cvcle disorder that has been detected by routine newborn screening. The incidence is 1 in 70,000 in Massachusetts. 
BIOCHEMICAL INVESTIGATION OF A SYNDROME OF PERIODIC MUSCLE WEAKNESS, CARDIAC ARRHYTHMIA AND HYPERTAURNURIA Alf E. Slonim, William H. Olson, Ian M. Burr, Peggy Borum, William R. Long. Vander

Four members of a family of six manifest a syndrome of periodic muscle weakness, periodic cardiac arrhythmia, icthiosis, lordosis, arthralgia, and growth retardation. Intermittent increase in taurine and decrease in carnitine appears to be associated with the intermittent muscie weakness and cardiac arrhythmia. Skeletal muscle biopsies demonstrated fissuring of Type I muscle fibers and ultrastructural examination revealed subsarcolemmal tubular aggregates with lipid droplets within the aggregates, endoplasmic reticulum was abnormally dilated. Oral glucose load induced abnormal insul in response, muscle weakness, ventricular extrasystoles, increased taurine and decreased carnitine in the urine, while the serum $\mathrm{K}, \mathrm{Ca}, \mathrm{Na}$ all remained with normal limits. The significance of these biochemical abnormalities and their possible relevance to the clinical findings will be discussed.

428

ELEVATED CYSTINE LEVELS IN I-CELL FIBROBLASTS. Frank

Tietze. NIH, Bethesda. (Spon. by Joseph D. Schulman) Cultured fibroblasts from patients with I-cell disease (mucolipidosis II) exhibit prominent intralysosomal accumulations due to a deficiency of multiple lysosomal enzyme activities. We have examined such cells for other possible manifestations of the underlying derangement. Fibroblasts from 6 patients with I-cell disease were examined for intracellular cystine content. Three of the 6 mutant lines showed distinctly elevated levels of intracellular cystine (means: $2.2,4.5$, and 5.6 umoles $1 / 2$ cystine/gm cell protein) approaching the cystinotic range $(10-30)$. Normal cells have cystine values of $<0.5$. No such increase was observed for several other amino acids. A fourth I-cell line showed marginal elevation (0.85) while two of the mutant lines were within the normal range. Intracellular cystine contents of two cell lines from patients with the biochemically related but less severe mucolipidosis III, as well as one variant line of uncertain classification, were within normal limits. In addition to its recognition by ion-exchange chromatography, excess free cystine in I-cells was confirmed by highvoltage paper electrophoresis of extracts from cells incubated in the presence of ${ }^{35} \mathrm{~S}$-cystine and by a binding protein assay ( $\mathrm{J}$. Biol. Chem., 249: 6033 (1974)). In one I-cell line studied in detail cell fractionation indicated that a large proportion of intracellular cystine was associated with the granular fraction. These studies confirm biochenical heterogeneity in I-cell disease and may have implications for understanding the pathogenesis of cystinosis. GLUTATHIONE METABOLISM IN NORMAL AND MUTANT HUMAN

426 CELLS. S.P. Spielberg, $\frac{\text { S.I. Goodman }}{\text { Butler, F. Tietze, }}, \frac{\text { J. }}{\text { Mramer and J.D. Schulman, }}$ Bethesda; Univ. Colorado Med. Ctr. Denver; Dept. Ped., SUNY, Buffalo; and Dept. Ped., Georgetown Med. Sch., D.C.

We are investigating the regulation of glutathione (GSH) metabolism in normal cells and in the known mutations of GSH synthesis including the first U.S. case of 5-oxoprolinuria (50P), reported here. Two patients (NEJM 286:557,'72) with <10\% normal red cell (nl.RBC) $\gamma$-glutamylcysteine synthetase (GC-S) activity and GSH have $>30 \% \mathrm{~nJ}$. GC-S and GSH in fibroblasts, but nl. fibroblast GSH synthetase (GSH-S) activity. Fibroblasts and RBCs from our patient with $50 \mathrm{P}$ both have normal to slightly increased GC-S, $5 \% \mathrm{nl}$. GSH $-S$, and $\sim 10 \% \mathrm{nl}$. GSH content, while fibroblasts from an unrelated patient with $50 \mathrm{P}$ have $-10 \% \mathrm{nl}$. GSH-S and $\sim 25 \% \mathrm{nl}$. GSH. GSH-S in fresh extracts of both $50 \mathrm{P}$ fibroblast lines is $~ 50 \%$ inhibited by $50 \mathrm{mM}$ Tris, but $\mathrm{nl}$. fibroblast GSH-S is not. Fibroblasts from a patient with undetectable RBC GSH-S without 5OP (NEJM 283:1253, 170) have $40 \% \mathrm{nl}$. GSH-S activity but GSH levels in $\mathrm{nl}$. range. Unlike the $\mathrm{nl}$. enzyme, GSH-S in these cells is $>95 \%$ inactivated by $16 \mathrm{hr}$. dialysis $\left(4^{\circ} \mathrm{C}\right.$.), or by heating ( $1 \mathrm{hr}$, $50^{\circ} \mathrm{C}$ ), without added GSH, but like nl. GSH-S is not inhibited by $50 \mathrm{mM}$ Tris. This disorder is thus associated with unstable GSH-S and is enzymatically distinct from GSH-S deficiency with 50P. Furthermore, partial heat inactivation $\left(1 \mathrm{hr} ., 50^{\circ} \mathrm{C}\right.$.) of $\mathrm{nl}$. fibroblast GSh-S is potentiated by $30 \mathrm{mM}$ GSH while the effect of heat on $\mathrm{nl}$. RBC GSH-S is inhibited by GSH. These results, confirmed by mixing experiments, suggest molecular differences between GSH-S in $\mathrm{nl}$. RBCs and fibroblasts.

47 ABNORMAL AMINO ACID COMPOSITION OF LENS FROM

427 MICE WITH A DOMINANTLY INHERITED CATARACT, CAT F. Linda J. Stirk, Reynold J.M. Gold and $F$. Clarke Eraser (Spon. by Charles R. Scriver) Medical Research Council Genetics Group, Montreal Children's Hospital, McGill University, Department of Biology, Montreal, Quebec, Canada.

Water-soluble crystallins and urea-soluble albuminoids were extracted from normal murine lenses, and from lenses in which a dominantly inherited cataract, Cat $/ t$, was present. The amino acid composition of the protein hydrolysates was determined. The amino acid composition of the mutant albuminoid fraction was shown to be significantly different from that of the normal albuminoids. The composition of the mutant crystallins was found to be much more variable than that of their normal counterpart. Upon examination of the crystallin subclasses $(\alpha, \beta$, and $\gamma)$, it was found that mutant $\beta$ and $\gamma$ crystallins were significantly different in amino acia composition from these proteins in the normal lens. The resultant of all these changes is that the crystallins are less uniform in composition in the mutant lens, and that the albuminoid fraction is less markedly different from the crystallin fraction in the mutant than in the normal lens.
CHROMOSOME POLYMORPHISM AND TWIN ZYGOSITY. D.L. Van

429 Hosp., Detroit; Indiana University, Indianapolis: Med. Coll. of Virginia, Richmond (Spon, by Lester Weiss).

To demonstrate the heritability of chromosome variants and to assess the value of chromosome studies in twin zygosity evaluation, Q- and G-banded metaphases were examined from $23 \mathrm{MZ}$ and 17 like-sexed DZ twin sets, plus a set of male quadruplets. A11 karyotypic examinations were performed without prior knowledge of true zygosity. Variation in acrocentric short arm morphology (G-banding) was much greater between $D Z$ than between MZ twins, and therefore in part genetically determined, but was of limited utility in differentiating twin zygosity because of non-genetic factors which influenced the appearance of satellites in the acrocentric short arm regions.

Better results were obtained by scoring Q-banding variants, and centromeric heterochromatin variants on chromosomes 1 , 9, and 16 after G-banding. Most of the DZ twins were identifiable as DZ solely on the basis of their discordant variant scores, whereas the $M Z$ twins were completely concordant. In agreement with the genotyping data, the quads were shown to be assymetric dizygotic.

The conclusion that variants seen at polymorphic chromosome regions are heritable is reasonable, since differences were found between $D Z$ twins only, with complete concordance between MZ twins. The usefulness of karyotypic examination in the evaluation of zygosity can be extended to the comparison of maternal and fetal tissues obtained for antenatal diagnostic studies, and other work which requires differentiation among genetic individuals.

\section{GLYCOSAMINOGLYCAN FORMATION IN NORMAL AND CYSTIC FI- Rennert, $R$ Mishael Roberts, University of} College of Medicine, Department of Biochemistry, Gainesville, Fla There have been several conflicting accounts of abnormalities in the accumulation and secretion of glycosaminoglycans (GAG) by cultured skin fibroblasts from patients with cystic fibrosis (CF). Here we report more detailed studies of complex carbohydrate metabolism in normal (N), heterozygous (HZ) and CF cells. The major GAG produced by skin fibroblasts in vitro was hyaluronic acid, with smaller amounts of heparan sulfates, and chondrotin sulfates. In experiments with $10 \mathrm{CF}, 2 \mathrm{HZ}$ and $10 \mathrm{~N}$ cell lines double label techniques were used employing radioactive D-glucosamine to compare the extracellular products of the different cultured cells. Radioactive materials associated with the cell surfaces and secreted into the medium were analyzed by ion exchange chromatography and by electrophoresis. No consistent differences could be observed between $\mathrm{CF}, \mathrm{N}$, or $\mathrm{Hz}$ cells with regard to the relative proportions of GAG and labeled glycopeptide materials they produced. In addition, experiments in which $\mathrm{CF}, \mathrm{N}$ and $\mathrm{Hz}$ cells were grown in $\log$ phase for up to 5 days in the presence of $\left[{ }^{35} \mathrm{~S}\right]$ sulfate and $\left[{ }^{3} \mathrm{H}\right]$ glucosamine revealed that CF fibroblasts neither accumulate nor secrete complex carbohydrate in abnormal amounts. Considerable variation was observed between individual cell lines even when great care was taken to carry out the experiments under identical growth conditions and passage number. Because of such variability, reasonable comparisons can only be made if relatively large numbers of cell lines are analyzed. The CF fibroblast is not abnormal with regard to its metabolism of GAG and other complex saccharides. 
DETECTION AND CHARACTERIZATION OF PROPIONATE-METHYIMALONATE DISORDERS IN CELI CULTURE: A SIMPLE METHOD. H.F. Willard, L.M. Ambani, M.J. Mahoney, and L.E. Ros enberg. Yale Sch. Med., Depts. Human Genet. and Ped., New Haven. To detect inherited disorders of propionate, methylmalonate, and cobalamin ( $\mathrm{Cbl}$ ) coenzyme metabolism, cells are incubated for $10 \mathrm{hr}$ with ${ }^{14} \mathrm{C}$-propionate $\left(10^{-4} \mathrm{M}\right)$ in medium containing $50 \mathrm{pg}$ $\mathrm{Cbl} / \mathrm{ml}$. Four normal fibroblast lines incorporated $79 \pm 24 \mathrm{cpm} / \mathrm{\mu g}$ protein ( $\mathrm{X} \pm \mathrm{sd} ; \mathrm{n}=18$ ) into 5\% TCA-precipitable material. Incorporation by 12 mutant lines was markedly deficient:

$\frac{\text { Mutant lines (no.) }}{\text { ionyl-CoA carboxylase (1) }} \frac{1{ }^{14} \mathrm{C} \text { incorporation (cpm/ } / \mathrm{gg} \text { protein) }}{2}$ $\begin{array}{ll}\text { propionyl-COA carboxylase (1) } & 2 \\ \text { methylmalony } 1-C O A \text { mutase (2) } & 2,3\end{array}$ $\mathrm{cbl} A$ (4) $3-9$

$\mathrm{cbl} B(2) \quad 3,14$

cbl C (3) $2-5$

When hydroxocobalamin $(\mathrm{OH}-\mathrm{Cbl})$ is added to the growth medium at 1-100 $\mathrm{ng} / \mathrm{ml}$, preliminary discrimination among these mutant classes is possible. OH-Cbl supplementation had no significant effect on ${ }^{14} \mathrm{C}$ incorporation in control lines or in carboxylase or mutase apoenzyme mutants, but restored incorporation in each cbl $\mathrm{C}$ mutant to normal. $\mathrm{Cbl} \mathrm{A}$ and $\mathrm{B}$ mutants were heterogencous; in some, $\mathrm{OH}-\mathrm{Cbl}$ supplementation increased incorporation 3-4 fold; in others, no effect was observed. This method has also successfully identified a fetus at risk for mutase apoenzyme deficiency. Three control amniotic cell lines incorporated $30-73 \mathrm{cpm} / \mu \mathrm{g}$ protein, cells from the affected fetus only $1 \mathrm{cpm} / \mu \mathrm{g}$. This technique constitutes a convenient, reliable prototype for prenatal and postnatal detection of inborn errors in cultured cells.

THE RELATION BETWEEN HETEROZYGOSITY AND IQ IN PHENYL-

432 KETONURIA. Malcolm Williamson, Richard Koch, Samuel School of Medicine, Department of Pharmacology, Los Angeles.

Twenty-five mothers of positively identified phenylketonuric individuals and 48 of their non-phenylketonuric progeny were administered $100 \mathrm{mg} \mathrm{L}$-phe/ $\mathrm{kg}$ bwt mixed in a single dose of orange juice. Serum phenylalanine and tyrosine were determined for blood samples drawn at $0,1,2,3$ and 4 hours post administration. The progeny were classified by various procedures into normal and heterozygote groups for the phenylalanine hydroxylase enzyme. These groups were then compared for the significance of the difference in IQ's. The findings suggest that normal individuals have significantly higher IQ's than do their heterozygote siblings. These results extend the evidence of Ford and Berman who also found that diminished ability of heterozygous siblings of PKU homozygotes to convert phenylalanine to tyrosine is associated with relatively lower performance on IO tests. The justification theory of mental retardation related the inability of heterozygotes to synthesize a non-essential amino acid to relative dietary deprivation as an important cause of mental retardation. Further study confirming these findings in the light of the above theory would mean that a small dietary supplement of tyrosine to all pregnant mothers could greatly reduce or even eliminate a large percentage of cases of mental retardation now called non-specific and dietary in origin.

433

POLYAMINES AND ADENYLATE CYCLASE IN NORMAL AND CYSTIC FIBROSIS (CF) CULTURED FIBROBLASTS. Reba Wright, * Bruce A. Buehler, * and Owen M. Rennert, University of Florida, college of Medicine, Division of Genetics, Endocrinology and Metabolism, Department of Pediatrics, Gainesville.

During cellular mitosis, entrance into the " $\mathrm{S}$ " phase of the cell cycle is associated with the induction of ornithine decarboxylase (rate limiting for polyamine synthesis); polyamines have been shown to act as cell growth factors. Since cAMP and adenylate cyclase modulate proliferative activity, we examined the relation of this pathway to spermidine and spermine fluxes. Adeny1ate cyclase activity was assayed in cultured fibroblast membranes in the presence of $1 \mu \mathrm{M}$ to $2 \mathrm{mM}$ spermine. There was inhibition of adenylate cyclase activity; 50\% inhibition occurring at $1 \times 10^{-6 \mathrm{M}}$. This concentration is within the physiologic range for mamalian tissue. We postulate a second messenger function of polyamines on cell surfaces.

\section{ADENYLATE CYCLASE ACTIVITY (pMoles/mg $10 \mathrm{~min} \pm \mathrm{SEM}$ )}

$\frac{\text { Basa1 }}{36.4 \pm 4.1} \frac{\text { NaF-Stimulated }}{102.5 \pm 12.9} \frac{\text { Spermine }}{\frac{10^{-4 M}}{24.5 \pm 3.4} \frac{10^{-5 M}}{16.9 \pm 2.3} \frac{10^{-6 M}}{18.7 \pm 0.7}} \frac{\mathrm{NaF}+\mathrm{SPM}}{67.9 \pm 13.9}$
In view of the role of cyclic nucleotides in exocrine secretor processes, Buchwald (Am. J. Hum. Genet. 27, 22A, 1975) reported abnormal cAMP levels in CF fibroblasts. In our studies, CF fibroblasts had adenylate cyclase activity indistinguishable from controls at basal conditions, NaF- and isoproterenol-stimulated, and modulation by spermine and spermidine. Adenylate cyclase activity and CAMP levels vary significantly as cell cultures transit from exponential growth towards confluence.

\section{HEMATOLOGY}

POLYVALENT PNEUMOCOCCAL POLYSACCHARIDE IMMUNIZATION in PATIENTS WITH SICKLE CELL ANEMIA. Joseph Addiego, W. Bryon Smith, William C. Mentzer, Bertrand Lubin and Arthur J.Ammann.Childrens Hospital Medical Center, Oakland, Calif., San Francisco General Hospital and University of Calif. San Francisco, Departments of Pediatrics.

A pneumococcal polysaccharide (PPS) vaccine (types $1,3,6,7,14$, $18,19,23)$ was utilized to immunize 52 patients with sickle cell anemia (SS), 19 splenectomized patients (spherocytosis, Hodgkins, ITP, trauma) and 38 normals. Ages ranged from 2 to 18 years. $0.5 \mathrm{ml}$ of the vaccine containing 50 micrograms of each PPS was given s.c. Antibody titers were measured pre and 3 weeks postimmunization. Results were expressed as mean fold increase (F.I) (post minus-pre titer) and \% responders.

\begin{tabular}{cccccccccc}
\hline PPS type & 1 & 3 & 6 & 7 & 14 & 18 & 19 & 23 \\
\hline SS: F.I. & 1.4 & 68 & 9.3 & 14 & 10 & 5.4 & 1.5 & 4.9 \\
\hline$\%$ responders & 45 & 84 & 71 & 65 & 59 & 78 & 47 & 59 \\
\hline Splenectomy:F. I. & 3.9 & 72.4 & 4.9 & 3.4 & 14 & 7.5 & 1.2 & 1.7 \\
\hline$\%$ responders & 52 & 86 & 62 & 67 & 76 & 81 & 43 & 71 \\
\hline Normals: F. I. & 3.9 & 23.9 & 2.9 & 4.2 & 3.3 & 6.1 & 2.1 & 6.0 \\
\hline$\%$ responders & 66 & 95 & 44 & 79 & 69 & 79 & 50 & 61 \\
\hline
\end{tabular}

77 immunized patients ( $68 \mathrm{SS}, 7 \mathrm{~S}$-thalassemia, 2SD) were followed clinically among with 29 unimmunized SS patients. Three episodes of pneumococcal septicemia with 1 death (types 6 \& 23) occurred in unimmunized patients. Comparable antibody responses occurred in SS, splenectomy and normals. Results suggest that immunization with PPS vaccine results in a normal antibody response and may provide significant protection against pneumococcal infection.

AN IN-VITRO DEMONSTRATION OF THE ABILITY OF HUMAN

435 BONE MARROW STROMAL ELEMENTS TO SUSTAIN GRANULOCYTOHealth Center, Dept. of Pediatrics, Farmington, $C t$.

Granulocytopoiesis in-vitro requires committed granulocyte precursor cells and a source of colony stimulating activity (CSA) usually exogenously provided by "conditioned" medium, human serum, or a feeder layer of peripheral blood cells. In this study, the ability of human bone marrow stromal elements to independently sustain granulocytopoiesis was tested by culturing marrow particles at low concentration in the absence of an exogenous source of CSA. The particles (which contain a dense core of stromal elements surrounded by maturing hemic cells) were obtained by bone marrow aspiration, washed $\mathrm{x} 2$ in modified McCoy's $5 \mathrm{~A}$ medium containing $15 \%$ fetal calf serum, suspended at a concentration of 2-5 particles/cc in the same medium containing $1.6 \%$ methylcellulose, and incubated in $10 \% \mathrm{CO} 2$ in air at $37^{\circ} \mathrm{C}$. Evidence of granulocytopoiesis in this system included: 1) presence of mitotic figures in promyelocytes and myelocytes throughout the first 10 days, 2) disappearance of metamyelocytes, bands, and polymorphs after 48 hours followed by their reappearance in large quantities (200-several thousand per particle) after Day 4 in culture and their continued reappearance until Day 14, 3) presence of promyelocytes in cultures up to 10-14 days. Although no exogenous source of CSA was provided, a likely endogenous source was the particles' dense core of stromal elements; these particles with their high local concentrations of CSA bathing responsive granulocyte precursor cells may serve as the functional units of the granulocytopoietic microenvironment in-vivo.

436 THE METABOLIC BASIS FOR HYPERPHAGOCYTOSIS IN CHRONIC GRANULOMATOUS DISEASE (CGD) POLYMORPHONUCLEAR LEUKOCYTES (PMN). Robert L. Baehner, Laurence A. Boxer, and Jacqueline Davis, Indiana University School of Medicine, J.W. Riley Hospital for Children, Department of Pediatrics, Indlanapolis, Indiana.

CGD PMN and normal PMN deprived of oxygen have been observed to be hyperphagocytic while failing to reduce oxygen to superoxide anion $\left(0^{-}\right)$and to hydrogen peroxide $\left(\mathrm{H}_{2} \mathrm{O}_{2}\right)$. In order to explain these observations, we studied the influence of $\mathrm{O}_{\overline{2}}, \mathrm{H}_{2} \mathrm{O}_{2}$, and myeloperoxidase on the phagocytic rate utilizing $C_{3}$ coated lipopolysaccharide-paraffin ofl droplets. CGD PMN and normal PMN in anaerobic chambers ingested (respectively) .071, .075 and .057 $+.007 \mathrm{mg} / 10^{7} \mathrm{PMN} / \mathrm{minute}$ compared to normal PMN in air ingesting $.038+.010 \mathrm{mg} / 10^{7} \mathrm{PMN} / \mathrm{minute}$ PMN were exposed to one or more of the following enzymes in the extracellular medium: 1) superoxide dismutase (SOD) to deplete cells of $0 ;$; 2) horseradish peroxidase to enhance peroxidation; and 3) catalase to deplete cells of $\mathrm{H}_{2} \mathrm{O}_{2}$. The respective rates were $.034 \pm .006 ; .036+.005$; and .045t $.007 \mathrm{mg} / 10^{7} \mathrm{PMN} / \mathrm{minute.} \mathrm{Since} \mathrm{only} \mathrm{catalase} \mathrm{potentiated} \mathrm{inges-}$ tion, the effect of $\mathrm{H}_{2} \mathrm{O}_{2}$ on normal PMN ingestion was examined. $\mathrm{H}_{2} \mathrm{O}_{2}$ at both $1 \times 10^{-6}$ and $1 \times 10^{-3} \mathrm{M}$ was placed in the extracellular media and the phagocytic rates were .035 and $.032 \mathrm{mg} / 10^{7}$ $\mathrm{PMN} / \mathrm{minute.} \mathrm{Addition} \mathrm{of} \mathrm{catalase} \mathrm{again} \mathrm{increased} \mathrm{the} \mathrm{ingestion}$ rate to $.046+.006 \mathrm{mg} / 10^{7} \mathrm{PMN} / \mathrm{minute}$. The metabolic basis for hyperphagocytosis in CGD PMN and normal PMN in anaerobiosis is due solely to the absence of $\mathrm{H}_{2} \mathrm{O}_{2}$ generation from the PMN during ingestion. 
17 EVALUATION OF MICRHOGAM IN THE RH NEGATIVE NEONATE。 B. Bernard, M. Presley, G. Rodriguez, J. Gruenhagen o. Ghazzawi and J. McGregor (Spon. by P.Y.K. Wu) Univ. of Southern California, LAC-USC Med. Ctr., Dept. of Pediat.

The first born infant may have a high risk of erythroblastos is fetalis if the maternal grandmother is $\mathrm{Rh}$ positive ( $\mathrm{Rh}+$ ) and had sensitized her $R h$ negative (Rh - ) daughter at birth. In order to determine both incidence of $\mathrm{Rh}$ sensitization at parturition and the role of human $\mathrm{Rh}_{\mathrm{o}}$ (D) immune globulin in the prevention of this sensitization, $174 \mathrm{Rh}$ - infants $\left(900^{\circ}, 849\right)$ born of $\mathrm{Rh}+$ mothers have been studied. Seventy females received 50ug of MICRhoGAM (MRG) in the first 72 hours of life and 104 infants served as controls. To date, 50 controls and 31 MRG babies have been followed by 6 mos. Screening for antibody by room-temperature saline, albumin and indirect anti-globulin techniques revealed that none of the $174 \mathrm{Rh}$ infants demonstrated Rh sensitization at birth nor did 50 of the controls at followup. Of 31 infants receiving MRG, 5 had weak $1 y$ positive indirect antiglobulin tests. This is probably due to MRC being detected as RhoGAM may be detected 6 mos a after injection. By an enzyme technique, Bowen \& Renfield have reported a $12 \%$ incidence of sensitization by 6 mos. in the Rh- baby of an Rh+ mother Our data indicate that either $\mathrm{Rh}$ sensitization is not occurring in the control infants or that it may not be detected by the laboratory methods being utilized. Thus, more sensitive methods must be performed to identify the Rh- baby sensitized at birth. Duplicate samples are being stored for further testing by more sensitive methods. MRG has produced no ill-effects when administered to the newly born Rh- infant and long term followup will decide its ultimate immuno-prophylactic role.

438 APLASTIC ANEMIA WITH EVIDENCE OF PROLIFERATION OF A TRUE "FETAL" RED CELL CLONE FOLLOWING ANDROGEN THERAPY. Brown, Audrey K., Rao, A.N. Raghavendra, \& Rieder, Ronald F. S.U.N.Y., Downstate Medical Center, Department of Pediatrics, Brooklyn, New York.

A $6 \frac{1}{2}$ year old girl with acquired aplastic anemia treated with Halotestin and Prednisone showed a gradual rise in hemoglobin (Ho) from $4.5 \mathrm{~g} \%$ to $16.8 \mathrm{~g} \%$. Concomitantly, there was a remarkable rise in the absolute concentration of $\mathrm{Hb} F$ from $0.85 \mathrm{~g} \%$ to $9.6 \mathrm{~g} \%$. Discontinuation of therapy was followed by a fall in both total $\mathrm{Hb}$ and $\mathrm{Hb} \mathrm{F}$ to $7 \mathrm{~g} \%$ and $3.3 \mathrm{~g} \%$ respectively. On reinstitution of Halotestin the $\mathrm{Hb}$ again rose to $10 \mathrm{~g} \% ; 5 \mathrm{~g} \%$ was $\mathrm{Hb} F$. The $\mathrm{Hb} F$ was distributed heterogeneously on Betke stain. Globin chain synthesis by reticulocytes was studied using radioactive leucine $\left(\mathrm{H}^{3}\right)$. The alpha to beta plus gamma chain synthetic ratio was $1: 1$. Analysis of the amnioacid composition of the gamma chains revealed only glycine at the 136th position, a phenomenon not previously described in aplastic anemia. In addition, several "fetal" characteristics of the red cells were documented, including enhanced amount of $i$ antigen decreased $I$ antigen, as well as decreased red cell carbonic anhydrase and catalase. $\mathrm{Hb} \cdot \mathrm{A}_{2}$ was decreased to $0.4 \%$ (DEAE Cellulose). These findings suggest that the red cells which appeared during androgen therapy in this subject may have been produced by activation of a true "fetal" clone.

PLATEIET FUNCTION IN THE CHEDIAK-HIGASHI SYNDROME

439 (CHS). George R. Buchanan and Robert I. Handin, (Spon. by David G. Nathan), Harvard Medical School, Children's Hospital Medical Center and Peter Bent Brigham Hospital Boston. The CHS, a generalized disorder of membrane bound granules, is characterized by albinism, neutropenia and frequent pyogenic infections. Studies of platelet (P) function in human patients with the CHS have not been reported despite the common occurrence of bleeding symptoms, even when P count is normal. Two patients with the CHS, a $5 \frac{1}{2}$ year old child in the "accelerated phase" and an adult with a clinically more mild course were studied. In both, P counts and size were normal, template bleeding times were over $20 \mathrm{~min}$, and $\mathrm{P}$ aggregation to collagen and epinephrine was impaired. After $\mathrm{P}$ were labeled in vitro with ${ }^{14} \mathrm{C}-$ serotonin, there was decreased maximal uptake and excessive spontaneous leakage of the label. Total P ADP was reduced in both patients $\left(0.45\right.$ and 0.85 umoles $/ 10^{11} \mathrm{P}$ vs. normal value of $2.73+$ 0.97 ) and ATP/ADP ratio was increased ( 12.2 and 6.76 vs. normal $2.65+0.80)$. Specific radioactivity of $\mathrm{P}$ adenine nucleotides after ${ }^{3} \mathrm{H}$-adenine labeling was increased. These findings are consistent with a storage-pool deficiency (SPD) of nucleotides and serotonin in CHS P and extend earlier findings in several animal analogues of the CHS. SPD, one of two general mechanisms of P dysfunction causing abnormal secondary aggregation also occurs in some albinos without the CHS and may reflect a generalized defect in packaging of secretory granules.
THE EFFECT OF ASPIRIN, GLYCEROL GUTACOLATE, TRIPROLIDINE-PSEUDOEPHEDRINE, AND DIPHENHYDRAMTNE ON PLATELET FUNCTION. Lorraine A.A. Champion, Ruth E. Luddy, Susan Schindler, and Allen D. Schwartz. University of Maryland School of Medicine, Department of Pediatrics, Baltimore.

A number of commonly used drugs have been reported to interfere with platelet function, leading many physicians to avoid their use in patients with hemostatic defects or organic lesions with the potential to bleed. Because of our clinical impression that some of these drugs do not influence the incidence or the severity of hemorrhage in hemophilic or thrombocytopenic patients we studied the effects of a number of them on platelet function. Each of 21 healthy adult volunteers was given one of the following drugs: 1) aspirin, 10 grains, 2) diphenhydramine (Benadry1) $50 \mathrm{mg}$ every 6 hours for 3 doses, 3) triprolidine $2.5 \mathrm{mg}$ combined with pseudoephedrine $60 \mathrm{mg}$ (Actifed) every 6 hours for 3 doses, or 4) glyceryl guiacolate, $200 \mathrm{mg}$. Ivy bleeding times and platelet aggregation studies were performed immediately before, 1 hour after and 24 hours after ingestion of the first dose of each drug. 4 of 5 subjects who received aspirin had prolonged bleeding times 1 and 24 hours after drug ingestion, and al1 5 failed to have normal platelet aggregation with ADP, epinephrine or collagen. There were no significant alterations in the bleeding times of the subjects receiving diphenhydraminine, triprolidine-pseudoephedrine, or glyceryl guiacolate, and platelet aggregation remained normal after ingestion of these drugs. These three drugs do not affect platelet function in the dosages used and, in contrast to aspirin, probably can be safely administered to the patient with a predisposition to hemorrhage.

441 Robert R. Chilcote, Dept. Peds., Univ. Chicago at Michael Reese Med. Ctr., Chicago, Robert M. Weetman, Jay L. Grosfeld, Robert L. Baehner, Dept. Peds., Indiana Univ. School of Med., James Whitcomb Riley Hospital for Children, Indianapolis

Splenectomy alleviates hemolysis in hereditary spherocytosis, though the spleen's role in rbc injury is incompletely understood. The splenic environment may exacerbate membrane ion leak resulting. in osmotic lysis associated with increased mean cell volume (MCV). Alternatively, as suggested by in vitro studies, rbc membrane fragmentation associated with decreased surface area/volume may occur with no change in MCV. We studied five patients with HS by sampling splenic arterial and venous blood at the time of splenectomy. When venous was compared to arterial samples, MCV was identical while osmotic fragility increased and red cell deformability, as measured by time required to traverse $3 \mu$ nucleopores, decreased. Histograms of red cell volume distributions were superimposable and did not show any new peaks. Phase contrast and scanning electron microscopy of rapidly fixed splenic tissue illustrated $\mathrm{rbc}$ fragmentation and spherocyte trapping. Increased osmotic fragility without swelling indicates that membrane fragmentation is the most important injury in vivo. Geometric considerations dictate that decreased surface area is the result of the loss of small but numerous membrane fragments. Our observations support the hypothesis that the spleen in HS injures rbc's primarily by accelerating fragmentation, hastening ultimate sequestration and destruction by the reticuloendothelial system.

442

CONGENITAL LEUKEMIA, MYELOCYTIC OR LYMPHOCYTIC? JenO'Connor and Paul Byrne. (Introduced by Arthur E. St. Louis University School of Medicine, Cardinal Glennon Memorial Hospital for Children, Departments of Pediat rics and Pathology, St. Louis, Missouri.

A newborn male infant with fetal distress had a peripheral blood count of $337,000 / \mathrm{mm}^{3}$ WBC with $90 \%$ blast cells. Exchange transfusion was performed because of this high count and evidence of intravascular coagulopathy. Chemotherapy with vincris tine and hydrocortisone was started after exchange but he died at 32 hours of age. Autopsy showed hemorrhage into many organs and extensive leukemic infiltration of liver, lungs, pleura, heart, esophagus, testes, kidneys, leptomeninges, and brain. Cytogenetic study revealed normal chromosomes. Morphology of blast cells by Wright stain could not distinguish myelocytic from lymphocytic leukemia nor did cytochemical stains for PAS, alkaline phosphatase, myeloperoxidase, and chloracetate esterase. Tests for $\mathrm{B}-$ and $\mathrm{T}$ - cells indicated the absence of $\mathrm{B}$ - and $\mathrm{T}$ cel1 markers. DNA synthes is of isolated WBC culture was inhibited rather than stimulated by phytohemagglutinin. Soft agar culture of both peripheral blood and bone marrow revealed no definite colony formation, but many small clusters (about 100 clusters per $4 \times 10^{4}$ nucleated cells plated, $<40$ cells each cluster). Cells from clusters were bizzare myeloid cells. In conclusion, we believed the patient had myelocytic leukemia. Soft agar culture techniques may be helpful in resolving the controversy as to whether congenital leukemia is myelocytic or 1 ymphocytic. 
443

FANCONI'S APLASTIC ANEMIA: DEFECT OF BOTH STEM CELL AND MICROENVIRONMENT? Jen-Yih Chu, Judith Ho, Patricia Monteleone, Virginia Herrmann and Arthur E. McElfresh. St. Louis University School of Medicine, Cardinal Glennon Memorial Hospital for Children, Department of Pediatrics, St. Louis, Missouri.

Twin brother and sister developed anemia at 14 years of age. Both had pancytopenia, macrocytic erythrocytes, reticulocytos is, high fetal hemoglobin and a horseshoe or ectopic kidney. Chromosome study revealed an increase in breakage. Bone marrow aspirate was slightly hypocellular. However, soft agar culture technique revealed marked decrease in colony forming unit (CFU-C) in bone marrow $\left(1-3 / 2 \times 10^{5}\right.$ vs. $40-200 / 2 \times 10^{5}$ nucleated cells plated in "control"). CFU-C of peripheral blood was also decreased. Colony stimulation activity (CSA) of peripheral white cells was also reduced to $1-20 \%$ that of control. No plasma inhibitor was observed when tested with "control" cells. Mother had normal CFU-C in peripheral blood. Bone marrow scan using $99^{\text {mTc sulfur - }}$ colloid revealed increased marrow space with skip areas. There was absence or diminution of activity in some areas but increase or normal activity in adjacent areas. In conclusion, soft agar culture techniques and bone marrow scan are useful in diagnosis of Fanconi's aplastic anemia. Defect in stem cell is evident by decrease in CFU-C and CAS producing cells. The scan probably indicates bone marrow microenvironmental abnormality. Whether this microenvironmental abnormality may interfere with successful engraftment of bone marrow transplantation needs further evaluation.

COAGULOPATHY ASSOCIATED WITH VITAMIN E. James J.

4.4.4 Corrigan,Jr. and Truda Mikel. Dept. of Peds. Univ. of Ariz. Med. Center, Tucson, Arizona.

Vitamin $\mathrm{E}$ ( $\alpha$ tocopherol) is reputedly non-toxic to humans. However, we have reported the development of an acquired coagulopathy in a vitamin $K$ deficient patient taking megadoses of vitamin $E$. The purpose of this investigation was to develop an experimental model of vitamin $E$ induced coagulopathy in order to determine where the vitamin interacts in the coagulation mechanism. Dogs were given vitamin E (800 IU/day), warfarin, or both orally. platelet function tests and detailed coagulation assays were performed serially on each dog. Warfarin was given in two different amounts; one which did not prolong the prothrombin time but reduced the levels of the vitamin $K$ dependent coagulation factors $50 \%$, and a dose which would prolong the prothrombin time. A11 animals were fed the same diet. No significant changes occurred in the platelet or coagulation mechanisms in normal dogs on vitamin $E$ alone, and no changes were evident in platlet function in dogs given warfarin or warfarin plus vitamin E. Dogs given vitamin $\mathrm{E}$ after high or low dose warfarin regularly developed profound reduction in coagulation factors II, VII, X and IX but not in factors $I, V$, or VIII. Within 7 days of removing vitamin $E$ but continuing warfarin the depressed procoagulants returned to previtamin treatments levels. These data suggest that the use of vitamin $E$ in animals with reduced vitamin $K$ dependent factor causes further reduction in these factors leading to a coagulopathy. The data also suggest that humans with vitamin $\mathrm{K}$ deficiency may be at risk for developing a hemorrhagic state if vitamin $\mathrm{E}$ is
administered.

445 HAGEMAN FACTOR AND DISSEMINATED INTRAVASCLLAR COAGULATION (DIC) IN NEWBORNS AND RABBITS. James J. Corrigan,Jr. and Cheryl Pagel. Dept of Peds. Univ. of Arizona Medical Center, Tucson, Arizona.

Hageman factor (coagulation factor XII) activation is importan in initiating the intrinsic coagulation mechanism. In septicemia, experimental and human data suggest that activation of factor XII may be instrumental in activating the coagulation mechanism to produce DIC. Pretreatment of rabbits with lysozyme (which inactivates factor XII) reportably protects the animals from developing liquoid induced DIC. Normal human newborns are known to have low levels of factor XII. The purposes of this study were: to investigate the role of factor XII in sick neonates with associated coagulopathies and to study the effect of factor XII inhibition on endotoxin induced DIC in rabbits. Of the 98 sick infants studied $75 \%$ had RDS. There was no statistical difference in the levels of factor XII between the sick infants and normal age matched controls although the levels in both groups were lower than older children. Detailed coagulation studies on 27 sick infants revealed 11 to have DIC (thrombocytopenia with reduced fibrinogen, factor II, $V$, and VIII levels). In those with DIC the mean Hageman factor level was $20 \%$, and in those without DIC $25 \%(\mathrm{p}>0.05)$. Rabbits given a constant infusion of lysozyme $(20 \mathrm{mg} / \mathrm{kg} / \mathrm{min}$.) showed laboratory evidence of factor XII inhibition. However, endotoxin induced DIC could not be prevented in the lysozyme treated animals. These data suggest that neither reduced factor XIX levels nor Hageman factor inhibition provide protection from DIC. The data also suggest that another coagulation pathway must be involved
in order to elicit the DIC. (SAL). James J.Corrigan, Jr., Charles L. Witte, Marlys H. Witte, and Robert E. O'Mara. Depts. of Peds., Surg. and Radiology. Univ. of Ariz. Med. Center, Tucson, Ariz.

Splenectomy has been the traditional approach to the management of the patient with hypersplenism. Asplenic individuals, especially children, are subject to an increased risk of fulminant and often lethal sepsis. Also, there are clinical conditions in which the spleen cannot be removed for technical reasons. Previous studies from our lab have shown that rats made hypersplenic with methylcellulose became hematologically normal following ligation of the splenic artery. SAL markedly reduced splenic mass although some splenic tissue persisted. The purpose of this study was to investigate the effect of SAL in a patient with hypersplenism. A 4 year old girl with congenital hemolytic anemia (pyruvate kinase deficiency) required frequent transfusions since birth. For the previous 6 months the need for RBC transfusions increased and she developed leukopenia, thromboctopenia, with massive splenomegaly. RBC survival study using $\mathrm{Cr}^{51}$ tagged normal compatible RBC's was 14 days. She underwent SAL and had an uneventful post-operative course. Dynamic and static splenic scans using technetium sulfur-colloid $99 \mathrm{mTc}$ before and after SAL demonstrated marked reduction in flow rate and functional splenic mass. The leukopenia and thrombocytopenia disappeared and the reticulocyte count increased from $4-7 \%$ to $11-26 \%$. The RBC survival increased to 21 days and 19 days, 8 and 16 months post-operatively. The data suggest that reduction in the splenic mass by SAL offers an alternative to splenectomy for the treatment of hypersplenism.
4.47 "LYMPHOBLASTIC CONVERSION". William M. Crist, Abdel H. Ragab, Raphael Ducos, Alexander R. Lawton, William $\mathrm{T}$. Shearer, Washington Univ. School of Medicine,St. Louis Children's Hosp..Dept.of Peds., St. Louis, Mo. and the Univ. of Alabama School of Medicine, Children's Hosp., Dept. of Peds., Birmingham, Alabama.

An 11 year old boy presented with $\mathrm{Phl}^{\mathrm{l}}$ positive acute leukemia without a preceeding symptomatic chronic phase of his disease. At presentation his blast population resembled lymphoblasts. Remission was induced and while not effectively maintained typical $\mathrm{CML}\left(\mathrm{Ph}^{1+}\right)$ developed. During the initial and/or subsequent "blast crisis"cyto chemical stains (PAS, Sudan black and myeloperoxidase) were negative and marked elevation of peripheral blood terminyl transferas (patient $59.5 \mathrm{u} / 10^{8} \mathrm{cells}$ with normal being $<1 \mathrm{u} / 10^{8}$ cells were found. Soft agar growth parameters of peripheral blood revealed
marked increase in colony forming cells (CFU) (mean 65.5 col $1 / 4 \times 10^{5}$ marked increase in colony forming cells (CFU) (mean 65.5 col
cells). The colonies were very large and consisted of myelocytes, metamyelocytes, polys, and monocytes. These studies suggested lymphocytic origin of the acute leukemia. "T" and " $B$ " lymphocyte marker studies (FC receptors, surface immunoglobulins. "E"roset tes and anti"T" antisera)performed on blast cells revealed no markers. We feel that the above data supports the concept that CML is a pluripotential stem cell disease involving lymphoid as well as
other hematopoietic cell lines. Previously reported cases of $\mathrm{Ph}^{1}$ chromosome positive AML or ALL may in fact have presented as did this patient and been misdiagnosed. If this is true the specificity of the $\mathrm{Ph}^{1}$ chromosome abnormality for chronic myeloproliferative disorders is reaffirmed. Lack of 1ymphoblast surface markers may reflect their lack of maturation.
48 GRANULOPOESIS IN PHILADELPHIA CHROMOSOME POSITIVE CHILDHOOD CHRONIC MYELOGENOUS LEUKEMIA. William M.
Crist,
ra, The Abdel H. Ragab, Hernan Moreno, John C. Foster
Univ. of Alabama School of Medicine, Dept. of Fabio Pereira, The Univ. of Alabama School of Medicine, Dept. of Ped., Children's Hosicine, St. Louis Children's Hospital, Dept. of Ped., St. Louis, Missouri. Intro. by Benton, J. W.

Three children with chronic myelogenous leukemia (CML) ( $\mathrm{Ph}+$ ) have been studied using the in vitro soft agar culture technique to examine peripheral blood ( $P B$ ) and bone marrow (BM) granulopoeisis. In addition patient sera and $P B$ nucleated cells were assayed for colony stimulating activity using a modification of the soft agar technique.

One child, studied in "blast crisis", had increased colony forming units (CFU) in his PB at that time (mean $65.5 \mathrm{col} / 4 \times 10^{5}$ cells plated)and after successful remission induction he had $32 \mathrm{col} / 4 \times 10^{5}$ cells plated. BM CFU were normal during remission (mean $50 \mathrm{col} / 10^{5}$ nucleated cells plated). A 2nd child had inc. PB CFC (mean 37.5 col/5 $10^{5}$ cells) and normal BM CFC at diagnosis (mean of $40.25 / 10^{5}$ cells plated.) The 3 rd child had dec. CFC from BM (mean $4.5 \mathrm{col} /$ $10^{5}$ cells) and high normal CFC from $P B$ (mean of $5.0 \mathrm{col} / 10^{5}$ cells plated). Patient sera mixed with normal BM cells showed patterns ranging
from inhibition to stimulation of myelopoeisis in different patients. PB cells in all patients supported colony growth poorly when used in a "feeder layer" under control BM cells. colonies contained myelocytes, neutrophils and monocytes. These results are similar to those reported in adult CML. Growth patternsduring "blast crisis" are helpful in distinguishing lymphoid from myeloid conversion of CML. 
49 STUGIES ON THE ANEMiA OF UREMIA. Melvin $H$. Freedman and $E$. Fred Saunders, Dept. of Pediatrics, Hospital for Sick Children, Univ. of Toronto, Toronto, Canada.

Factors affecting red cell proliferation in chronic rena? failure were studied by assessing marrow erythroid colony growth in tissue culture. Each colony represents an erythroid stem cell (CFU-E) that has differentiated to its progeny in response to added erythropoietin (EPO). Serum from 5 of 6 anemic uremic children produced a mean decrease in CFU-E of $32 \%$ using control human marrow, compared to human $A B$ or fetal calf serum (FCS). When the 6 sera were tested with mouse marrow, CFU-E growth was profoundly inhitited in all experiments compared to control studies with human serum. Marrow from 1 uremic patient yielded a normal EPO dose-response with autologous serum or FCS with a peak of $124 \mathrm{CFU}-E / 10^{5}$ cells plated (mean of 30 controls 158 CFL-E/ $10^{5}$, range $31-317$ ). The patient's CFU-E increased to $262 / 10^{5}$ when $A B$ serum was substituted. Nandrol one decanoate, $10^{-8} \mathrm{M}$, produced an $8 \%$ increase in the patient's CFU-E when added to the cultures containing autologous serum, and a $30 \%$ increase in cultures with FCS. We conclude that in uremia: erythroid stem cells are present in adequate numbers; they are responsive to EPO and androgen stimulation; uremic serum contains inhibitory factors whose site of action is early in erythropoiesis and impairs red cell proliferation and differentiation.

A SIMPLE TEST FOR DETECTION OF HB $S$ IN THE NEONATAL PERIOD。 Shlomo Friedman, Bruce Back, Maria DelivoriaPapadopoulos, Jean Atwater, Toshio Asakura, and Elias Schwartz, Univ. of Pennsylvania Sch. of Med., The Children's Hosp. of Philadelphia and the Hosp. of the Univ. of Pennsylvania, and Jefferson Med. Coll., Cardeza Foundation for Hematologic Research, Philadelphia, Pennsylvania.

The detection of sickling syndromes in the newborn period is of importance for the early entry of children with sickle cell disease into a comprehensive care system. The ease of denaturation by shaking of oxy $\mathrm{Hb} \mathrm{S}$ forms the basis for a simple and reliable test for confirmation or rapid detection of $\mathrm{Hb} \mathrm{S}$ in children and adults (JAMA 233:156, 1975). In the present study we applied this test to 187 capillary blood samples obtained from black neonates at 2-4 days of life and compared the results with those from electrophoresis on agar gel and starch gel. Capillary bloods were washed and hemolyzed, and hemolysate was added to a vial containing $10 \mathrm{mM}$ sodium phosphate buffer, $\mathrm{pH} 8.0$. Optical densities were measured at $577 \mathrm{~nm}$ before and after shaking for 5 minutes, and precipitation was calculated. The precipitate range.d from 0 to $4.7 \%$ of the total hemoglobin in 177 neonates. of these, 171 showed $\mathrm{Hb}$ FA on agar and starch gels. The other six neonates had $\mathrm{Hb} F A C$. In ten neonates the precipitation ranged from 5.3 to $12.0 \%$. Nine showed $\mathrm{Hb}$ FAS and one had $\mathrm{Hb}$ FSC on agar ge1. Hb $S$ was not detected in 3 of these infants by starch gel, which is therefore not suitable for detection of $\mathrm{Hb} S$ in the newborn. These results indicate that the shaking test may be used to detect $\mathrm{Hb} \mathrm{S}$ in the newborn period reliably, inexpensively and rapidly before discharge of the neonate from the hospital.

PATHOPHYSTOLOGY OF GROWTH FAILURE IN THALASSEMIA 451 MAJOR. Myron Genel, Howard Pearson, Richard O'Brien, Raymond Hintz, Depts. of Pediat, Yale UnivoSch. o Med., New Haven,Ct., and Stanford Univ.Sch, of Med。, Stanford,Ca。 Growth failure is a recognized consequence of thalassemia major, but of inexplicable origin. Improvement of growth noted with chronic hypertransfusion regimens has suggested a causal relationship to chronic anemia. In order to define the factors af fecting growth in thalassemia, endocrinologic and hematologic studies were performed on 18 patients, ages 2-31 years, 11 on a chronic hypertransfusion program and 7 of intermediate severity not receiving regular transfusions.

In non-transfused patients and in patients prior to hypertransfusion, impaired growth was noted with advancing age.Growth hormone secretion was assessed via standard provocative tests (insulin hypoglycemia, arginine, or L-dopa) in 11 patients with normal to exaggerated responses in all, and high basal unstimulated hGH in 4 . Somatomedin levels determined by porcine bioassay were normal in all 14 patients testèd $(\bar{M}=0.74 \pm 0.18 \mathrm{U} / \mathrm{ml} 1 . \mathrm{vs}$. control of $0.65 \pm 0.16$ ). Hematologic studies in non-transfused patients demonstrated low whole blood p50 $\left(22.9 \pm 2.1 \mathrm{~mm} . \mathrm{Hg}_{\circ}\right)$ and inappropriately low $2,3 \mathrm{DPG}(5943 \pm 811 \mathrm{nmo} 1 / \mathrm{ml}$. RBC) for the degree of anemia(Hgb.7.5 $\pm 1.5 \mathrm{gm} . \%)$. These findings could not be explained entirely by levels of \%Hgb.F, serum $\mathrm{PO}_{4}$ or blood $\mathrm{pH}$.

These data suggest that growth failure in severe thalassemia may reflect "end-organ" unresponsiveness,possibly induced by peripheral hypoxia related to the chronic anemia, as well as to altered 0 dissociation and inappropriately low levels of 2,3 DFG.
452 Natural History(NHx) of Sickle Cell Anemia:Reevaluation of a 15 Year Cord Blood Testing Program.Priscilla A. Gilman, Judith M. McFarlane. Titus H.J.Hulsman, Med.Col.of Ga. (MCG), Dept.of Ped. and Cell and Mo1.Biol., Augusta. (Spons by A.F.Robertson)

True incidence and $\mathrm{NHx}$ can not be obtained from hospital,clinic, or death records. From 1-1 61 to 6-30-75, 15,679 black infants born at MCG were tested by starch gel electrophoresis for hemoglobin(Hgb)type. Followup (F/U) of 73 cases with suspected sickle cell anemia(SS) or sickle C disease(SC)revealed:SS-22,SC-8,sickle tha1assemia(Stha1)-4, CC-1, AS-17, AC-11, AA-3, 1ost-5(1 possible SS). At $F / U$ the diagnosis(dx)was unknown to 3 SS and 4 Sthal patients(pts). Seven of 22 sS pts $(31 \%)$ died: mean age 2 years. SS was not on the death certificate in 3. Five of 7 died with acute sequestration crisis (ASC) : 3 were dead on arrival (2 were well $2-4 \mathrm{hrs}$ prior to death; 2 were febrile and died within $4 \mathrm{hrs}$ ). Two ASC pts never had symptoms and the dx was unknown. One pt died with pneumococcal meningitis. One died at home "with infection". No correlation existed with prior severity of disease and age at or cause of death. In 15 survivors(1-14 yrs)a score for clinical severity correlates with \% fetal $\mathrm{Hgb}$ but not with age or Hgb. Complications:meningitis-1; os teomyelitis-2;pneumonia-2pts 7 times;hospitalizations-10 pts 41 times; transfusions7 pts 21 times. Two $(9$ and 12 yrs)had no symptoms and were unknown to any physician. Six were older and acutely ill when diagnosed.

INFECTIOUS MONONUCLEOSIS IN CHILDHOOD Depart. Of Pedi., University of Texas Health Science Center, Dallas and Univ. of Pennsyivania, Philadelphia. (Spon. by G. H. McCracken)

Primary EBV infections in childhood have commonly been thought to remain either silent or to be accompanied by mild, non-characteristic illnesses. Little information is available on the type of clinical illness associated with the primary infection. Sera from 32 children ranging in age from $1-13$ yrs. With characteristic clinical signs and symptoms of acute infectious mononucleosis and positive mono spot tests were tested for IgM and/or IgG antibodies to EBV specific antigens; i.e., the viral capsid antigen (VCA), the diffuse (D) component of the early antigen complex and the EBV associated nuclear antigen (EBNA). On the basis of the serologic results all 32 children had current or recent EBV infection. The mean age was 7.4 yrs, with $28 \%$ of the patients being 5 years of age or less. Physical and laboratory features of the disease were virtually identical to those commonly found in adults except for a greater frequency of exudative pharyngitis and periorbital edema. Sixteen percent had platelet counts below 100,000 . In addition, eleven children with non-specific aggiutination on their mono spot tests showed no serologic evidence of recent or current EBV infection. The clinical and laboratory features of these patients were indistinguishable from those with evidence of current infection. There was no serologic evidence of
other viral diseases associated with the mono syndrome in these pt.

THE PROCOAGULANT ACTIVITY (PCA) OF CEREBROSPINAI 154 Marie J. Stuart (Spon. by F.A. Oski). SUNY, Upstate Med. Ctr., Dept. of Peds., Syracuse, N.Y.

Thromboplastic activity has been measured in human brain, and its liberation may be implicated in DIC following cerebral trauma. We have developed a method for the measurement of PCA of CSF using a modification of the plasma recalcification time (RT), PCA is being defined for the purpose of our study as the activity contained in $0.1 \mathrm{ml}$ CSF that will shorten the RT of $0.1 \mathrm{ml}$ pooled normal plasma as compared to saline control values of $155^{\prime \prime}-175^{\prime \prime}$. Activity is then calculated as \% shortening. The presence of up to $200,000 \mathrm{RBC} / \mathrm{mm}^{3}$, secondary to traumatic lumbar puncture, did not influence the PCA activity. CSF from normals contained a mean activity of $8.2 \% \pm 13.3$ (2SD). In 17 children with acute lymphatic leukemia without evidence of CNS disease, who have received prophyla :ic intrathecal methotrexate \pm cranial irradiation within the past 3 years, the mean activity was $34.5 \% \pm 20.0$ (2SD). In 10 infants tapped to $R / 0$ sepsis with normal CSF values and negative cultures the mean activity was $35.2 \% \pm 35.8$ (2SD). One neonate with $\mathrm{E}$. Coli meningitis had $90 \%$ activity. The activity measured significantly shortened the recalcification time of VIII deficient plasma from $>750 \mathrm{sec}$. to a mean of $236 \mathrm{sec}$. and, therefore, appears to be tissue factor related. These studies indicate that PCA is present in spinal fluid of some patients. The usefulness of this test as an indication of cerebral injury requires further evaluation. 
THE USE OF INTRAMUSCULAR VITAMIN E IN THE PREMATURE INFANT. OPTIMUM DOSE AND IRON INTERACTION. Janet E. Graeber, Linda A. Butler, Margaret L. Williams and Frank A. Osk1. Dept. of Peds., SUNY, Upstate Med. Ctr., Syracuse.

Premature infants are all born vitamin $E$ deficient. Prolonged vitamin $E$ deficlency may produce hemolytic anemia if the infant's diet is rich in polyunsaturated fatty acids and is supplemented with iron. Studies were performed to determine if the early administration of intramuscular vitamin $E$ (IME) could produce $E$ sufficlency during the 1st. 5-6 wks. of life in infants weighing less than 1500 gms. at birth. The dose of IME (Hoffmann-LaRoche) varied from 50-150 untts/Kg and was given to groups of infants receiving iron fortified formulas and to infants recelving additional (100 mg) intramuscular iron.

Infants receiving 100 to 150 units $/ \mathrm{Kg}$ of vitamin $\mathrm{E}$ during the 1st. wk. of life had serum tocopherol levels that averaged from 1.6 to $1.9 \mathrm{mg} / \mathrm{dl}$ at $35-40$ days of life. Infants who recelved intramuscular iron with 125 units $/ \mathrm{Kg}$ of IME had mean tocopherol levels of $1.9 \mathrm{mg} / \mathrm{dl}$; in contrast, infants who recelved intramuscular iron without additional $E$ had mean tocopherol levels of $0.44 \mathrm{mg} / \mathrm{dl}$. In 17 infants on the iron fortified formula alone, the mean tocopherol level was $1.2 \mathrm{mg} \%$. With IME the $\mathrm{H}_{2} \mathrm{O}_{2}$ hemolysis test was normalized $(<10 \%)$ within the 1st. wk. of 1 ife and remained normal during the 1st. 6 wks. of life in $17 / 18$ infants receiving 125 units $/ \mathrm{Kg}$ or more of IME. In contrast $\mathrm{H}_{2} \mathrm{O}_{2}$ tests remained elevated in $2 / 3$ of infants not receiving this dose of additional $\mathrm{E}$. These studies indicate that the administration of $125 \mathrm{U} / \mathrm{Kg}$ of $\mathrm{E}$ to premature infants is a safe, effective means of producing prompt and persistent $\mathrm{E}$ sufficiency.

DECREASED RED CELI FILTERABILITY IN THE PREMATURE 456 INFANT. Steven J. Gross, Rosemarie A. Cittadino, Stephen A. Landaw, Patricia L. O'Neal and Frank A. oski. Departments of Pediatrics and Medicine, S.U. N. Y., Upstate Medical Center, Syracuse, New York.

Red cell filterability -- a characteristic believed to be an Important determinant of red cell lifespan -- was measured in normal term and premature infants. Filterability was measured in conjunction with blood carboxyhemoglobin ( $\mathrm{COHb}$ ) -- an index of hemolysis. For this study, a micromethod was developed that allowed filterability measurements to be performed on capillary blood samples. Studies employed cells at a $1 \%$ hematocrit and a filter with a su pore size. The mean filtration time (FT) in 40 normal adults was $13.0+1.2 \mathrm{sec} . / \mathrm{ml}$. In $40 \mathrm{term}$ infants, the FT was significantly longer averaging $15.5+1.7$. The FT in 20 premature infants was significantly longer than in both other groups and averaged $18.4 \mathrm{sec} / \mathrm{ml}$. In 28 patients with a variety of hematologic disorders, FT's ranged from 14 to $180 \mathrm{sec} . / \mathrm{m} 1$. Mean COHb levels, measured on the third day of Iife, were higher in both term $(0.56+0.11 \%)$ and premature infants $(1.08+0.20 \%)$ than in normal adults $(0.34+0.10 \%)$. Within the groups of normal term and premature infants there was no correlation between FT's and COHb. This study confirms the previous report that red cell filterability is reduced in term infants and demonstrates an exaggeration of this phenomenon in the premature infant. The technique provides a simple means of detecting abnormalities of the erythrocyte.

THE INCIDENCE OF LIVER DYSFUNCTION IN HEMOPHILIA A,B

457 AND VON WILLEBRAND'S DISEASE. Margaret W. Hilgartner and Patricia Giardina (Spon. by Denis R. Miller). Cornel1 U.Med.C0i1. New York \& Beth Srael Hosj. Dept. of Ped. New York Patients in the Hemophilia clinic were reviewed to evaluate liver function \& susceptibility to serum hepatitis. They received either episodic \&/or prophylactic replacement factor conc. therapy between 1973-75. Liver function studies included SGOT,SGPT; HBSAG and $H B_{S} A B$ were measured by CEP and RIA. 80 Patients had hemophilia A with $<1 \%$ F.VIII, 4 had vW. disease with $7-4 \%$ F.VIII and 9 had hemophilia $B$ with F.IX 1-4\%. 40 F.VIII Deficiencies were treated by episodic transfusion therapy; $19 / 34$ required average $506 u F$. VIII conc./week. 32\% (13/40) had elevated SGOT \& SGPT; $2 \%(1 / 40)$ were $\mathrm{HB}_{S} \mathrm{AG}+\& 60 \%(67 / 80)$ were $\mathrm{HB}_{S} \mathrm{AB}+$. The average amount of F.VIII conc. used per week by those patients with elevated liver enzymes (480u/week) was not significantly different from those with nomal liver enzymes. 40 Hemophiliacs on both episodic and prophylactic transfusion therapy required average $1166 \mathrm{u}$ F.VIII conc./week; $42 \%$ $(17 / 40)$ had elevations of both SGOT \& SGPT; $2 \%(1 / 40)$ were HB AGt. The average amount of F.VIII conc. used per week for those patients with elevated liver enzymes was $1524 \mathrm{u}$ whereas those with normal liver enzymes used $809 u$. 9 Patients with F. IX deficiency were treated with episodic prophylactic therapy. $7 / 9(77 \%)$ had elevated SGOT/SGPT; 2 were $\mathrm{HB}_{\mathrm{S}} \mathrm{AG}+\& 6$ were $\mathrm{HB}_{\mathrm{S}} \mathrm{AB}+$. Both $\mathrm{HB}_{\mathrm{S}} \mathrm{AG}+$ patients have cirrhosis by liver biopsy and one is on steroid therapy. The incidence of asymptomatic liver dysfunction as demonstrated by elevation of SGOT \& SGPT chemistries is $38 \%$ in the F.VIII patients $\& 7 \%$ in F.IX patients. There is no remarkable difference between the 2 F.VIII therapy regimens.
HLA IN FAMILIAL STEROID RESPONSIVE THROMBOCYTOPENIA AND NEUTROPENIA. Dorothy Horns, Jon Gerrard, Edmond Yunis, and William Krivit. Univ. of Minn., Dept. of Peds., Minneapolis, Minn.

Thrombocytopenia and neutropenia beginning at age two in daughter (propositus) and at age thirty in her mother were associated with increased megakaryocytes and normal granulocyte precursors in the marrow. The daughter responded on several occasions to steroids and later to splenectomy. Myasthenia gravis, scleroderma, polycythemia vera, hypothyroidism, and adrenal hypoplasia have been noted in the maternal family.

The HLA histocompatible antigen type of the propositus was $1-8 / 1-8$, and $1-8$ occurs frequently in the family. For 10 years in the propositus, abnormalities of the humoral immune system (abnormally low immunoglobulins, failure of IgG stimulation) have been noted. "B" cells were within lower limits of normal. Anergy to Candida, streptokinase, and Trichophyton antigens and gradual deterioration of response to mitogens (PHA, Pokeweed) has occurred despite normal numbers of "T" cells. Spleen tissue was normal, but nodular lymphoid hyperplasia of immunoblastic origin was noted in the liver. Nonspecific granulomas were present in the bone marrow.

We report the present family because of the unique combination of autoimmune thrombocytopenia with neutropenia in a mother and daughter and to document a family which represents an example of the association of HLA 1 and 8 with autoimmunity. We hypothesize that this may be related to an abnormality of suppressor " $\mathrm{T}$ " cells. LYTIC AlNEMIA. T. Jacob John, Carla Lohman, Robert C. Strunk and James J. Corrigan, Jr. University of Arizona Medical Center, Department of Pediatrics, Tucson, Arizona. The adsorption of 3 or more human RBC's on a human lymphocyte is called an autorosette. Only $1-2 \%$ of normal 1 ymphocytes form autorosettes with normal RBC's. While evaluating the immunohematology of an infant with severe autoimmune hemolytic anemia with warm (IgG) antibodies it was found that her $R B C^{\prime} s$ readily formed rosettes with her own lymphocytes, as well as with those from control subjects. Average results of 4 tests showed that at $4^{\circ} \mathrm{C}$ and $37^{\circ} \mathrm{C}, 47 \%$ and $25 \%$ of lymphocytes from control subjects rosetted with patient's RBC's. Patient's lymphocytes did not rosette with RBC's from control subjects. Thus the abnormality was located on the patient's RBC's. However, when RBC's from control subjects were incubated in patient's serum or plasma, they acquired this property of enhanced autorosetting. Heating patient's serum at $56^{\circ} \mathrm{C}$ for 30 minutes did not reduce significantly its ability to modify normal RBC's. Three samples of patient's serum collected at different times were treated with $40 \%$ ammonium sulfate and the precipitated immunoglobulins and supernatents were tested for their ability to modify $R B C^{\prime} s$. RBC's incubated in untreated serum, immunoglobulin fraction and supernatent formed rosettes with $23 \%, 23 \%$ and $3 \%$ of lymphocytes, respectively. These results suggest that the basis of enhanced autorosetting was the coating of RBC's by patient's immunoglobulins, presumably the anti-red-cell antibodies. This is a previously unrecognized phenomenon, and is probably related to the pathogenesis of hemolysis. AbduT J. Khan, Chuck K. Lee, James A. Wolff, Parvin Khan and Hugh E. Evans, Dept. of Ped., Jewish Hosp. and Med. Ctr., State Univ. of N.Y., Brooklyn and College of Phys. and Surgeons, New York, N.Y.

An abnormality of neutrophil (PMNS) specifically elevated NBT reduction in thallasemia major (TM) (J. Ped. 82:352, 1973) prompt ed us to study other PMN functions in 11 noninfected children with 11 controls (C). Chemotactic indices (CI), random migration (RM) NBT tests and chemotactic activity of patient's sera (PS) were investigated. The CIs were determined utilizing a modified Boyden's technique. A chemotactic stimulus was generated in the lower chamber by Endotoxin activation of 1) normal $A B$ serum (ABS) and then 2) PS while the cells were piaced in the upper chamber on a $3 \mathrm{~m}$ micropore fitter. RM represented CIs in the absence of any chemotactic stimulus. The results represent mean and (standard deviation). CI with ABS CI with PS RM NBT

$\begin{array}{lllll}\text { Patients } & 230(123) & 239(115) & 60(19) & 8.4(3.8) \\ \text { Controls } & 418(128) & 357(118) & 91(42) & 3.8(1.5)\end{array}$
Controls

$P$ value $<\begin{array}{ll}418 \\ 0.01\end{array}$

$357(118)$

$91(42) \quad 3.8(1.5)$

The $C I$ and RM were significantly decreased and resting NBT scores higher in TM compared with C. PS generated activity comparable to ABS. No correlation was found between NBT scores, CIs RMs and immunoglobul in (IgA, IgM and IgG) concentrations. These results suggest a defect in PMNs which may be correlated with a predisposition to infection sometimes observed in this disease. 
USE OF RADIONUCLIDE BONE IMAGING TO DIFFERENTIATE OSTEOMYELITIS FROM BONE INFARCTION IN S HEMOGLOBINOPATHIES, Elizabeth M. Kurczynski and Letty G. Lutzker, Albert Einstein College of Medicine, Bronx Municipal Center, Sponsored by C.M. Edelmann, Jr .

In patients with sickle cell anemia, it is often difficult to differentiate bone infection from infarction since the symptoms and signs can be similar. Bone imaging with $99 \mathrm{mTc}$-labelled phosphates was performed on a number of patients in an attempt to distinguish between these entities. Nine patients with sickle hemoglobinopathies and severe local pain in one or more extremities were examined 3-14 days after the onset of symptoms. All had normal radiographs of involved areas. In six patients, at least one focal area of decreased radioactivity was seen in the affected bone, substantiating the clinical impression of infarction. Two patients had normat studies. One demonstrated patchy areas of mildly increased activity but none showed the intensely increased activity typical of osteomyelitis. Seven patients recovered quickly after treatment with hydration and analgesi cs. Two received long-term intravenous courses of antibiotics, but no organism was isolated in either case .

The optimal time of imaging appears to be between 3 and 8 days after the onset of symptoms. This technique is less helpful later in the course, since reactive bone in an infarcted area becomes "hot" and may resemble infection. Early bone imaging has allowed significant shortening of hospital stay plus decreased diagnostic procedures in cases where osteomyelitis is a definite clinical possibility.

462 VITAMIN E DEFICIENCY INDUCED ABNORMAL PLATELET FUNCDept. of Peds., SUNY, Upstate Med. Ctr., Syracuse. In rats made $E$ deficient, Machlin has demonstrated increased platelet aggregation concommitant with increased $\mathrm{PGE}_{2}$ synthesis. Platelet function studies in $\mathrm{E}$ deficient humans have not been reported to date. A 17 month old infant with a decreased Vitamin $E$ level of $0.2 \mathrm{mgm} \%$ was studied during the period of $\mathrm{E}$ deficiency and 2 weeks following intramuscular and oral E administration with a normal $\mathrm{E}$ level of $0.6 \mathrm{mg} \%$.

\begin{tabular}{lrrrrr} 
Group & Plt. Count & $\begin{array}{c}\text { Bleeding } \\
\text { Time }\end{array}$ & $\begin{array}{c}\text { Least ADP } \\
\text { Conc. Causing } \\
\text { Biph. Aggreg. }\end{array}$ & $\begin{array}{c}\text { Least Collagen } \\
\text { Dilution } \\
\text { Causing } \\
\text { Aggreg. }\end{array}$ \\
\hline Control & $150-450,000$ & $1.5^{\prime}$ to6' & $1.6 \mathrm{um}$ & $1: 16$ \\
PTT (pre) & 440,000 & $4^{\prime} 30^{\prime \prime}$ & $0.2 \mathrm{uM}$ & $1: 64$ \\
PTT (post) & 268,000 & $5^{\prime} 00^{\prime \prime}$ & $1.6 \mathrm{uM}$ & $1: 8$
\end{tabular}

Platelet sizing revealed a significant shift in platelet populations to megathrombocytes during the period of $\mathrm{E}$ deficiency. Following $\mathrm{E}$ administration platelet populations returned to normal, suggesting increased platelet turnover during the E deficiency. This data demonstrates a state of platelet hyperaggregability in humans similar to that observed in the $\mathrm{E}$ deficient rat. This is rapidly reversible with $\mathrm{E}$ therapy.

PHYSIOLOGICAL SIGNIFICANCE OF ERYTHROCYTE PROTOPOR-

463 PHYRIN (EP) IN MODERATE BODY LEAD BURDEN. Donald S. Levi, Anita S. Curran, Sheryll D. Alexander, Bernard Davidow, Sergio Plomellit. (Spon. by

Screening for body $\mathrm{Pb}$ burden tests absorption (blood $\mathrm{Pb}-\mathrm{BPb}$, in $\mu \mathrm{g} / \mathrm{dl}$ blood) or metabolic interference (EP, in $\mu \mathrm{g} / \mathrm{dl}$ blood; $\mathrm{EP}+\geq 60, \mathrm{EP}-<60)$. All children tested with $\mathrm{BPb} \geq 55$ are $\mathrm{EP}+$, but at lower levels a minority are EP-. This study investigated how EP reflects $\mathrm{Pb}$ absorption in this lower level group. In 1971-72 $\mathrm{EP}$ was measured in venous samples from 406 children with $\mathrm{BPb} 40$ 49 ; $122(30 \%)$ were EP-. As at the time EP results were not reported, management of individual children was not affected. Search of the computerized NYC records revealed 149 of these children with repeated $\mathrm{BPb}$ within a year; 44 (29\%) had been EPThe initial $\mathrm{BPb}$ (index $\mathrm{BPb}$ ) was compared to the highest recorded $\mathrm{BPb}$ (follow-up $\mathrm{BPb}$ ). Mean index $\mathrm{BPb}$ was 43.9 in the $\mathrm{EP}+$ and 43.1 in the EP- group; mean follow-up $\mathrm{BPb}$ was 44.7 and 40.5 respectively. Of $105 \mathrm{EP}+$ children 12 had follow-up $\mathrm{BPb} \geq 55$, while of 44 EP- only one did. This boy was 6 yrs old at testing, with a 3 yr history of slowly declining $\mathrm{BPb}$ (mean $\mathrm{BPb} 56.0$ in 1970-71 and 51.7 in 1972-73). These data indicate that among children with moderately elevated $\mathrm{BPb}$, those $\mathrm{EP}+$ are at greater risk of achieving a markedly elevated $\mathrm{BPb}$ within a year, while in those $\mathrm{EP}-$ the elevated $\mathrm{BPb}$ is either spurious or declining. Thus EP screening predicts the trend of $\mathrm{Pb}$ absorption hetter than $\mathrm{BPb}$ itself. EP appears the screening test of choice as it is not only simple, economical, reproducible and unaffected by environmental contamination, but also more physiologically significant,
SERUM INHIBITOR TO GRANULOPOI ESIS IN THALASSEMIA (THAL) 464 MAJOR. Naomi L.C.Luban and Denis R. Miller, Cornell Univ.

Children with Thal major frequently exhibit marked variability in white blood cell counts when corrected for nucleated red blood cells, unrelated to underlying infection or clinical symptomatology. Utilizing semisolidagar culture, peripheral blood (pb) of 30 children was evaluated for colony stimulating activity (CSA)utilizing normal pb feeder layers as a source of colony stimulating factor (CSF). The addition of $2 \times 10^{5}$ nucleated Thal pb cells per plate produced $1.45 \pm 0.35$ colony forming cells (CFU-C) while $2 \times 10^{6} \mathrm{pb}$ cells produced $1.0 \pm 0.35$ CFUs These results compare with $2.25 \pm 0.25 \mathrm{CFU}-\mathrm{Cs}$ at $2 \times 10^{5}$ cells and 4.2 \pm 0.99 a $2 \times 10^{6}$ cells in normals $(N=13)$. The addition of Thalassemic serum to normal bone marrow (BM) cells, to a final concentration of $15 \%$ significantly inhibited CFU-C in 6 out of 6 experiments. The serum CF inhibitor is nondialyzable, precipitates upon dialys is and can be recovered from the precipitate. Cellular and noncellular sources of CSF were prepared using Ficoll-Hypaque separated mononuclear feeders and human spleen conditioned media respectively; the inhibitor was still manifest with increasing concentration of the precipitated fraction using $2 \times 10^{5}$ normal $\mathrm{BM}$. Thal BM exhibited a lower CF capacity (4.0 CFU-CS $N=2)$ than other hereditary hemolytic anemias $(33.4 \pm 11.3 \mathrm{~N}=22)$ or acute leukemics in remission $(27.4 \pm 4.63 \mathrm{~N}=10)$. Thus Thals exhibit a nondialyzable serum factor which is either directly inhibitory to CSF or interacts with a cell, probably a monocyte, resulting in the inhibition of CSF release; this then results in depressed CF.

\section{5} NORMAL PLATELEI SURVIVAL AND ULTRASTRUCTURE IN HOMOCYSTINURIA (CYSTATHIONINE SYNTHASE DEFICIENCY). Anne W. Lucky, Edward R. Uhlemann, John H. TenPas, N. Raphael Shulman, S. Harvey Mudd, and Joseph D. Schulman. NIH and NIMH, Bethesda, Maryland.

A recent study (NEJM 29l:537, 174) described shortened platelet survival in vivo in 4 of 4 patients with homocystinuria, a disorder associated with greatly increased risk of serious thromboembolism. Abnormal platelet ultrastructure with increased vacuolization has been reported in another investigation ( $\mathrm{z}$. Kinderheilk 112:309, ' 72 ). We studied the survival in vivo of autologous $5 \overline{\mathrm{I}-\mathrm{Cr}}$ labelled platelets in 5 pyridoxine non-responsive homocystinuric patients ages 6 to 25, including one who had a previous retinal artery occlusion. All patients had normal platelet counts and had not recently received antithrombotic agents considered to affect platelet function. Based on at least 8 days of study after the administration of $51-C r$ platelets, the half-time of platelet survival was 4.75-5.60 days, not shortened from the 4.0-5.5 day normal range found in our laboratory. Platelets from 4 homocystinuric patients, 3 obligate heterozygotes, and 6 controls were examined by electron microscopy. Coded photomicrographs were scored by several observers. No ultrastructural features were found which could distinguish the homocystinuric platelets. Our inability to confirm reduced platelet survival in vivo or a morphologic abnormality of platelets in homocystinuria does not exclude the usefulness of antithrombotic medications such as dipyridamole or aspirin for prophylaxis of thromboembolism if supported by clinical observations. 


\section{7}

CHRONIC ACTIVE LIVER DISEASE (CALD): PREVELANCE IN HEMOPHILIA

J.R。 Moran, A.B. Terry, L.L. McKenzie, and H.L

ville,

Management of hemophilia has been dramatically improved with the use of blood concentrates rich in clotting factors. However, current methods of preparing concentrate do not remove hepatitis virus and therefore patients with hemophilia are at greater risk from hepatitis virus infection than the general population. Records of 21 children (ages 3-16 years) from 1972-1976 were reviewed with respect to development of clinical hepatitis, elevated serum transaminase (SGOT) and presence of hepatitis $B$ antigen $\left(\mathrm{HB}_{\mathrm{S}} \mathrm{Ag}\right)$. Patients were treated with cryoprecipitate and AHF concentrate, Systematic evaluation for the presence of liver disease was not begun until 1974. Signs and symptoms of acute hepatitis were present in 7 patients. Elevation of SGOT $>500$ IU occurred in 11 patients and persistent elevation, $>100$ IU has been present in 4 patients for more than 12 months and 2 patients for more than 5 months. $\mathrm{HB}_{\mathrm{S}} \mathrm{Ag}$ became positive in 5 patients and remained persistently positive in 2. Biopsy evidence of CALD (disruption of limiting plate, bridging and piece meal necrosis) was present in 3 patients ( $1 \mathrm{HB}_{\mathrm{S}} \mathrm{Ag}$ carrier) and benign persistent hepatitis was present in 1 ( $\mathrm{HB}_{\mathrm{S}} \mathrm{Ag}_{\mathrm{g}}$ carrier). These findings support the contention that CALD results from exposure to hepatitis virus and suggests that patients with hemophilia are at greater risk to CALD than the general population because of repeated exposure to blood products.
6 MEMBRANE PERMEABILITY AND ACTIVE CATION TRANSPORT IN NEONATAL RED BLOOD CELLS (RBC's). Aixa Muller, Bonnie Watts Ramsey and Bertil E. Glader, Dept. Pediatrics Harvard Medical School, Children's hosp. Med. Ctr., Boston, Mass. Neonatal RBC survival is shorter than normal. Numerous biochemical differences exist between infant and adult RBC's, but which of these is related to decreased RBC lifespan is not known. In this study we examined the functional significance of decreased Na-K ATPase activity in newborn RBC's. Active Na and $K$ trans port were measured after pretreatment of RBC's with amphotericin in order to elevate $\mathrm{RBC} \mathrm{Na}$ content (up to $60 \mathrm{meq} / 10 \mathrm{l0} \mathrm{RBC}$ ) and therefore stimulate Na-K activated ATPase. At all RBC Na concentrations studied, ouabain-inhibitable $\mathrm{Na}$ efflux and $\mathrm{K}$-influx were identical in neonatal and adult RBC's. Passive $K$ efflux from newborn RBC's reportedly is increased when net fluxes are measured with radioactive $K$. Since we now know that $K-K$ exchange can interfexe with isotopically measured cation flux determinations, we reassessed this abnomality in a nonisotopic study. No increased $\mathrm{K}$ leak was observed in metabolically replete neonatal RBC's. Furthermore, separation of young and old neonatal RBC's revealed an identical age related $\mathrm{K}$ loss (15meq/10 10 $\mathrm{RBC}$ ) in adult and infant RBC's. In summary these data suggest that (1) decreased Na-K ATPase activity is an interesting developmental marker, but it has little effect on active cation transport in neonatal RBC's and (2) accelerated $\mathrm{K}$ loss leading to cellular dehydration is not a major factor responsible for decreased $R B C$ survival in the neonate.
470 HYPOFOLICACIDEMIA AND GROWTH RETARDATION IN SICKLE CELI ANEMIA (SSA) Olu O. Ogunye, Anne L. Knase1, Corazon G. Uy, and Darry1 A. Morton (Spon. by Roland B. Scott) from the Dept, of Pediatrics and Child Health and the Ctr. for Sickle Ce11 Disease, Howard Univ. Col. of Med., Washington, D.C

To evaluate the possible role of basal serum folic acid levels in the pathogenesis of growth retardation in SSA patients and the therapeutic effect of folic acid supplementation, serum folate levels were determined in 20 children with SSA and 25 age and sex-matched controls. The SSA patients were then randomly divided into two groups, one group was treated with folic acid $5 \mathrm{mg}$ for an average duration of 8 months with measurement of body weight, height, hemogram, and red cell indices.

Basal serum folic acid level in SSA patients was lower $5.03 \pm$ $1.77 \mathrm{ng} / \mathrm{m1}$ ( \pm S.D.) than but not statistically different from those of normal controls $8.8 \pm 3.0 \mathrm{ng} / \mathrm{mI}(\mathrm{p}>0.05)$. The incidence of decreased serum folate values was significantly higher in SSA children than control subjects $(\mathrm{p}<0.01)$. There was high correlation between serutn folate level and age-adjusted weight and height in SSA patients, and normal levels of serum folate in this group was associated with a "mild" form of the disease. However, $5 \mathrm{mg} /$ day of folic acid did not accelerate weight or height gain in SSA patients.

We conclude from the preliminary results that folate deficiency can be one of the factors contributing to the pathogenesis of growth retardation in patients with Sickle Cell Disease. Lon term follow-up is necessary to determine whether retarda
somatic growth can be reversed by folic acid therapy.

471

HYPOTRANSFERRINEMIA (HTf), ANEMIA AND INCREASEO SUSCEPTIBILITY TO INFECTIONS IN CHILDHOOD NEPHROTIC SYNDROME (NS). Lawrence Opas, Jack Lazerson, Stuart Siegel and Ellin Lieberman. USC Sch. Med, Dept. Pediatrics, Childrens Hosp. of Los Angeles, Divisions of Nephrology and Hematology.

Transferrin(Tf) enhances host resistance to infection by withholding iron necessary for microbial growth and plays a major role in red cell production. NS in childhood is characterized by increased incidence of infection, but not anemia. 5 patients (aged 2-15 years) with corticosteroid-resistant, non-minimal change, NS with serum creat inine levels from $0.7-1.9 \mathrm{mg} \%$ had a normochromic normocytic anemia ( $\mathrm{Hgb} 7-10 \mathrm{~g} / \mathrm{dl}$ ). 3 of 5 had documented episodes of bacterial sepsis. Serum Tf levels in these 5 patients were $17-50 \mathrm{mg} / \mathrm{dl}$ as compared with normal $(120-200 \mathrm{mg} / \mathrm{dl})$. Serum iron levels were depressed $(20-47 \mathrm{ug} / \mathrm{dl})$ and percent saturation was increased $(44-92)$. Urinary losses of Tf (mean $36 \mathrm{mg} / \mathrm{dl}$ ) might account for $20 \%$ of the low serum Tf. Erythropoiet in levels by bioassay were appropriately increased for the degree of anemia. Stainable iron was decreased in the bone marrow.

These data suggest a causal relationship between HTf and sepsis as well as a partial etiologic role for HTf and hypoferremia in the development of the anemia. NS patients at higher risk for sepsis may be detectible by monitoring $\mathrm{Hgb}$, serum iron serum Tf and percent saturation. Tf infusions may have a role in the therapy of septic anemic patients with NS.
469

HEMATOLOGIC AND IMMUNOLOGIC STUDIES OF TOXIC ERYTHEMA OF THE NEWBORN. William J. Oetgen, Richard Evans, Richard D. Landes. (Spon.by James W. Bass). Walter Reed Army Medical Center, Department of Pediatrics, Washington, D.C. Toxic erythema of the newborn (TEN) is a benign dermatitis of the first week of Iife. The characteristic pustules contain numerous eosinophils, and undocumented accounts suggest that a circulating eosinophilia also occurs. The cause of TEN is unknown; however, because of the association of eosinophils and immunologic diseases, an allergic etiology has been proposed. This study attempted to verify the postulated eosinophilia in TEN and to determine if differences in immunoglobul in and C3 levels exis ted in infants with and without TEN. On the third day of Iife blood specimens were obtained from 33 consecutively born infants. TEN was diagnosed by physical examination and was confirmed by showing eosinophils in the vesicular fluid of seven of the infants. No difference was found between TEN and non-TEN groups in hematocrit and white blood cell counts. Absolute counts of neutrophils, bands, monocytes, lymphocytes, and basophils also showed no differences. The mean absolute eosinophil count for infants with TEN was $618 \pm 237 \mathrm{cells} / \mathrm{mm}^{3}$ and the mean for non-TEN in fants was $249 \pm 142$ ( $p<.001)$. Quantitation of total serum IgG and IgM by immunodiffusion and of total IgE by radioimunoassay showed no difference between TEN and non-TEN infants. The mean serum C3(immunodiffusion) level was $60 \pm 10 \mathrm{mg} / \mathrm{dl}$ in control infants and $69 \pm 6 \mathrm{mg} / \mathrm{dl}$ in TEN infants $(p<.025)$. These observations confirm the presence of an absolute circulating eosinophilia in infants with TEN and suggest that elevations of the third component of complement may be a previously unrecognized finding in TEN.
772 A BENEFICIAL EFFECT OF THE IN SITU KIDNEY ON IN VITRO

472 MARROW ERYTHROPOIESIS IN CHRONIC RENAL DISEASE. Jorge $\mathrm{A}_{\circ}$ Ortega, Mohammed $\mathrm{H}_{\circ}$ Malekzadeh, Peter P. Dukes, Richard N.Fine, Andrew Ma, and Nomie AoShore. USC School of Medicine, and Childrens Hospital of Los Angeles, Department of Pediatrics, Los Angeles.

The effect of erythropoietin (Epo) and prostaglandin $\mathrm{E}_{2}\left(\mathrm{PGE}_{2}\right)$ on cultures of marrow obtained from anemic patients with chronic renal disease (CRD) on a hemodialysis program was investigated. The study was a double-blind for selection of patients with 7 of the 14 patients being anephric. Marrow was incubated in normal $A B$ positive serum, the patient's pre-dialysis serum, and the patient's post-dialysis serum. Either exogenous Epo, PGE 2 or nothing was added to the 3 different culture systems. 59 Fe incorporation into heme was determined as an index of erythroid activity. Marrow from patients with CRD responded to Epo in normal serum. (Table: Fraction indicates responders/total patients):

\begin{tabular}{|c|c|c|c|c|c|c|}
\hline & Epo & 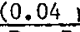 & (m1) & $\mathrm{PGE}_{2} \quad(3$ & $10^{-7} \mathrm{M}$ & $r 3 \times 10^{-8}$ \\
\hline & $A B \overline{+}$ & Pre-D & Post-D & $\overline{A B+}$ & Pre-D & Post-D \\
\hline & $\begin{array}{l}\text { serum } \\
6 / 7\end{array}$ & $\begin{array}{l}\text { Serumm } \\
5 / 7\end{array}$ & Serum & Serum & Serum & $\begin{array}{l}\text { Serum } \\
5 / 7\end{array}$ \\
\hline tep & $5 / 7$ & $1 / 7$ & $2 / 7$ & $3 / 6$ & $3 / 6$ & $3 / 6$ \\
\hline
\end{tabular}
PGE2 was stimulatory under all conditions but in fewer patients. Incubation in post-dialysis serum did not improve response to either Epo or $\mathrm{PGE}_{2}$. Only a few responses to Epo were obtained in pre- and post-dialysis sera from anephric patients. However, the same sera did not affect the response to $\mathrm{PGE}_{2}$. The data suggest that an in situ kidney favorably modified the patient's serum to allow a response to Epo. 
473 IMPROVEMENT OF OXYGEN DISSOCIATION AND TISSUE OXYGENATION WITH ANDROGENS IN THALASSEMIA INTERMEDIA. Howard A. Pearson, Myron Gene1, Yale Univ. Sch. of Med., Dept. of Pediatrics, New Haven, Conn.

Patients with severe thalassemia have failure of intraerythrocytic adaptive mechanisms resulting in functional anemia more severe than predicted from hemoglobin levels. A 15 year old male with homozygous $\beta$ thalassemia of intermediate severity had weakness, growth failure, lack of puberty and priapism. Pituitaryend organ endocrine function was intact with normal $\mathrm{HGH}$, thyroid and adrenal responses to provocative testing. Serum gonadotropins were detectable with a positive testosterone response to HCG stimulation. He was anemic and had inappropriately low levels of RBC 2,3 DPG. Whole blood P50 was shifted to the left of normal. Testosterone, in doses for delayed puberty, produced growth and improvement of weakness and increased exercise tolerance. Priapism ceased.

$$
\text { Hgb 2,3 DPG }
$$

(4 $\mathrm{mol} / \mathrm{m}$ l

5,020

p50

$$
23.1
$$

Hgb $\mathrm{F}$

7.6 5,700

23.1

$(\%)$

$9 / 16 / 75$

$$
7.9
$$$$
\text { 5,700 - wks. I.M. }
$$$$
\text { Testosterone enanthate } 200 \mathrm{mg} \mathrm{q} 4 \text { wks. I.M. }
$$

$1 / 6 / 76$ 8.7 6,400

26.4

33.0

Increases in p50 should enhance tissue unloading of oxygen and

contribute to symptomatic improvement. In selected patients with thalassemia intermedia, administration of androgens in tolerably low doses may be of hematological and physiological benefit. 474 GROWTH-RETARDED FETUSES. Loren R. Pickart, Robert K. Departments of Pediatrics and Obstetrics, San Francisco, Calif. The force required to maintain pulsatile blood flow in the microcirculation (yield shear stress) must overcome red cell aggregates formed in capillaries during the static phase. Formation of aggregates is a function of plasma fibrinogen concentration ( $F$ ) and hematocrit $(H)$. The relationship between $F$ and $H$ was examined in 25 fullterm newborns at $4 \mathrm{hrs}$ of age. F averaged $173 \pm 1 \mathrm{r}$ $\mathrm{mg} \%$ in 12 babies with $\mathrm{H}<55$, and $306 \pm 16^{*} \mathrm{mg} \%$ in 13 babies with $H>55$. The difference in $F$ between low and high $H$ groups was highly significant $(p<0.005)$. The effects of chronic arterial hypoxemia and polycythemia on fetal $F$ and albumin were measured in sheep fetuses in whom $\mathrm{PO}_{2}$ was reduced $15 \%$ to $25 \%$ by embolization of the maternal uteroplacental vascular bed between 110 and 140 days of gestation. Blood was drawn from catheters implanted in femoral arteries of 19 embolized and 24 non-embolized (control) fetuses. In comparison with control fetuses, embolized fetuses were growth-retarded $(p<0.05)$ and polycythemic $(p<0.05)$. F was unchanged in controls between 110 and 140 days of gestation. In growth-retarded polycythemic fetuses, $F$ increased to $195 \%$ of initial values by 125 days $(131 \pm 24 * \mathrm{mg} \%$ vs $247 \pm 89 * \mathrm{mg} \% ;<0.025)$. This difference was magnified during the last 10 days $(p<0.0005)$. in contrast, plasma albumin increased linearly and showed no difference between the groups. The findings indicate that $F$ may be dangerously elevated in polycythemia, and may contribute to inadequate tissue perfusion in growth-retarded or hypoxic newborns. *S.E.M.

475 CONSTANT SUBBCUTANEOUS (SC) INFUSTON OF DESFLRRIOXANINE (DF) IN TRNNSFUSED BETA THIALSSSEMIA PATIIENTS (THALS). R. Propper, S. Shurin and D. Nathan. (hildren's Hosp.

Nedical Center and Harvard Medical School, Boston, Mass.

Iron (Fe) overload is the major cause of death in tha1s. DF has a high affinity for $\mathrm{Fe}$, and ferrioxamine, the Fe-chelator com plex, is nearly quantitatively excreted in the urine. But a single $\mathrm{IM}$ dose of $0.75 \mathrm{~g} \mathrm{DF}$ may be painful and induced only 14-26 $\mathrm{mg}$ Fe excretion in 6 tha1s. To devise a more effective approach, 2 patients, who excreted 13 and $15 \mathrm{mg} / \mathrm{d}$ Fe after IM DF, received constant IV infusions of IF at a dose range of 0.75 to $16 \mathrm{~g} / \mathrm{d}$. Urinary Fe excretion was $74-180 \mathrm{mg} / \mathrm{d}$. Quantitative conversion of DF to ferrioxamine occurred at doses less than $1.5 \mathrm{~g} / \mathrm{d}$. After 10 days of constant IV treatment Fe losses were $\sim 1000 \mathrm{mg} \mathrm{Fe}$, a rate of elimination equal to effects of phlebotomy in hemochromatosis. In 1 thal severe arrhythmias markedly improved. The marked augmentation in net le excretion seen with constant intravenous administration of DF compared to a single $\mathrm{MM}_{\text {injection of equal }}$ dose is probably due to lengthening of the time of exposure of DF to the "labile Fe pool". Therefore, we devised a simple method for constant infusion on an ambulatory basis. Two thals wear a 14 $o z$ battery operated syringe type intermittent infusion pump on their belts. The pump infuses $16 \mu \mathrm{DF}$ at $250 \mathrm{mg} / \mathrm{ml}$ SC every 8 minutes for a total daily dose of $0.75 \mathrm{~g}$. Fe excretion was 21-60 $\mathrm{mg} / \mathrm{d}$, a $2-4$ fold increase over IM injection. The syringes and SC needles are changed daily by patients. There has been neither pain, psychological hazard nor allergic response in these early trials. Constant SC infusion of DF is a promising approach to cheiation therapy for thals with iron overload.
476 Utilization OF a FUnCTIONAL PHAGOCYTIC ASSAY SYSTEM IN THE EVALUATION OF OPSONIC ACTIVITY IN SERA FROM PATIENTS WITH SICKLE CELl ANEMIA (SCA). Arthur J. Provisor, John M. Allen, and Robert L. Baehner, Indiana Univerment of Pediatrics, Indianapolis, Indiana.

The susceptibility of children with SCA to pneumococcal sepsis is well recognized. Others have postulated a defect in the alternate pathway of complement activation and have questioned the role of autosplenectomy in depressing synthesis of alternate pathway factors. Alternate pathway opsonic activity was measured by the uptake of ${ }^{14} \mathrm{C}$ Streptococcus pneumoniae type II by neutrophils (PMN) with control (C) and SCA sera. The bacteria to PMN ratio was $10: 1$. Sera were treated with $0.1 \mathrm{M}$ EGTA and $0.1 \mathrm{M} \mathrm{MgCl}_{2}$ chelating $\mathrm{Ca}^{+}+$required by the classical complement pathway, but leaving $\mathrm{Mg}^{++}$for alternate pathway function. The sensitivity of this system was demonstrated by heat inactivating normal sera and combining it with varying amounts of $\mathrm{C}$ sera chelated with EGTA and $\mathrm{MgCl}_{2}$. Type specific antibody was added to maximally activate the alternate pathway without affecting the direct pathway. Twenty-eight patients with SCA, ranging in age from 3 months to 16 years, were studied. There was no difference in uptake of $14 \mathrm{C}$ s. pneumoniae between $C$ and SCA sera when treated with EGTA and $\mathrm{MgCl}_{2}$. Two of these sera were obtained from SCA patients during an episode of fatal pneumococcal sepsis. Rather than pointing to a defect in alternate pathway function, our results indicate that a generalized malfunction of the reticuloendothelial system may be responsible for the increased incidence of pneumococcal sepsis in patients with SCA.

477 CATION PERMEABILITY AND HYDRATION. BOnnie Watts Ramsey and Bertil E. Glader, Dept. Pediatrics,

Medical School, Children's Hosp. Med. Ctr., Boston, Mass.

Increased $x$ loss and depletion of RBC cation content leads to cellulax dehydration and osmotically resistant RBC's. This is a normal event of $R B C$ aging which is magnified in many hemolytic anemias. RBC's containing Heinz bodies (HB) are unique in that $K$ and cation depletion produces osmotically fragile cells. In an attempt to resolve this paradox, we generated $A B$ 's in normal RBC's by pretreatment with acetylphenylhydrazine. Cation measurement of $\mathrm{HB}-\mathrm{RBC}^{\prime} \mathrm{s}$ washed in $\mathrm{MgCl}_{2}$ (standard procedure) revealed a normal $\mathrm{Na}$ while the $\mathrm{K}$ and total cation content was markedly reduced (40\%). Measurement of $\mathrm{Na}$ and $\mathrm{K}$ in unwashed HB-RBC's (calculated from cation difference in RBC suspension and medium) indicated an increased $\mathrm{Na}$, an equivalent $\mathrm{K}$ loss and no change in total cation. The MCV (index of cell $\mathrm{H}_{2} \mathrm{O}$ content) of the unwashed HB-RBC's was increased and there was only minimal MCV reduction in the sevexely cation depleted $\left(\mathrm{MgCl}_{2}\right)$ washed cells. This discordance between cation and $\mathrm{H}_{2} \mathrm{O}$ content is due to an increased intracellular osmotic activity unaccounted for by $\mathrm{Na}$ and $\mathrm{K}$. In support of this concept, the chloride ratio $\left(\mathrm{Cl}_{\text {in }}: \mathrm{Cl}_{\text {out }}\right.$ ) is increased in HB-RBC's. Thus these studies indicate that the membrane lesion of HB-RBC's equally augments $\mathrm{Na}$ and $\mathrm{K}$ permeability. Apparent $\mathrm{K}$ loss in excess of $\mathrm{Na}$ gain is an artifact of washing injured cells in a $\mathrm{K}$-free medium $\left(\mathrm{MgCl}_{2}\right)$. $\mathrm{HB}-\mathrm{RBC}$ 's are not dehydrated but in fact are $\mathrm{H}_{2} \mathrm{O}$ loaded and osmotically fragile. We believe this is due to increased osmotic activity as monovalent $\mathrm{Cl}^{-}$replaces the denatured polyvalent hemoglobin anion.

478 ACTIVITY. Kenneth C. Rich, Robert I. Lehrer, UCLA, Pachman. Depts. of Ped. and Med., Los Angeles, (Spon. by L.M.

Leukocytes (WBC) contain tissue thromboplastin activity and contribute to fibrin deposition in inflammation. To identify the responsible WBC type(s), we separated human WBC into fractions enriched in neutrophils (PMN), lymphocytes (LY), and monocytes (MN) by density centrifugation and differential adherence. MN were distinguished from LY histochemically by their peroxidase content. WBC procoagulant activity was assayed by a one-stage recalcification time. In a representative experiment, the clotting time (CT) of $2.5 \times 10^{6} \mathrm{MN}$ was $25.8 \mathrm{sec}$ (control CT, 133.6 $\mathrm{sec}$ ). LY had little activity beyond that attributable to MN contamination. PMN lacked activity. MN homogenates (in $0.34 \mathrm{M}$ sucrose) exerted twice the procoagulant activity of equivalent numbers of intact MN. The procoagulant activity of MN homogenates was completely sedimentable at $27,000 \times \mathrm{g}$ for $20 \mathrm{~min}$, and was inactivated by heating $\left(80^{\circ} \mathrm{C}, 30 \mathrm{~min}\right)$. Comparable fractions of PMN lacked activity. The procoagulant activity of intact MN was greatly augmented by permitting the cells to adhere to a surface for $60 \mathrm{~min}$. MN exerted procoagulant activity in native and Factor VIII-deficient plasma, but were inactive in Factor VII-deficient plasma. These data show MN to be the major source of WBC procoagulant activity in man, and to act via the extrinsic limb of coagulation.

Sponsored in part by NIH Fellowship 1 FO3 HD57101-01 
479 MEMBRANE-ASSOCIATED HB S IN SICKLE CELL DISEASE. Marie $\frac{0 \text {. Russe11 }}{\text { Asakura, and }}$ John McSweeney, $\frac{\text { Shlomo Friedman, }}{\text { Elias Schwartz }}, \frac{\text { Toshio }}{\text { Univ. of Pennsylvania }}$ Asakura, and Elias Schwartz, Univ. of Pennsylvania

$\mathrm{Sch}$. of Med., and The abnormalities are common in sickle cell disease. We have studied the association of newly-synthesized th $S$ with RBC membranes by incubating red cells from 4 patients with $14 \mathrm{C}$-leucine and subsequently preparing membranes by repeated washes with 5mM phosphate buffer, pH 7.4. The washing elutes the initial red color, leaving brown membranes. The spectra of membranes after successive washes show removal of oxy $\mathrm{Hb}$, leaving hemichrome. The radioactive $\alpha$ and $\beta^{S}$ chains associated with the membranes were $0.12-0.32 \%$ of the total globin, with $\beta^{S} / \alpha$ of $0.96-$ 1.24. Globin comprised $89.9 \%$ of total radioactivity in the hemolysate, while it was only $25.5 \%$ of membrane radioactivity, indicating mainly other radioactive proteins in stroma. These data conflict with results of a previous study which showed $9-15 \%$ of total $\beta^{S}$ bound to stroma and stromal $\beta^{S} / \alpha$ of 2.4 when using $1 \mathrm{mM}$ EDTA, pH 7.2, for lysis (Bank et al, J.C.I. 54:805, 1974). With the same EDTA lysis we found oxy $\mathrm{Hb}$ on pink membranes and 2.6$6.8 \%$ of the total radioactive globin associated with membranes, with $\beta S / \alpha$ of only $1.03-1.33$. Globin comprised $68.1 \%$ of total radioactivity associated with these membranes, showing incomplete removal of $\mathrm{Hb} \mathrm{S}$. The phosphate lysis results show only a small fraction of newly-synthesized globin chains associated with the membranes. The 14-50 fold excess globin with preferential accumulation of $\beta^{S}$ in studies by the EDTA method appears to be an artifact of washing.

$4 \mathbf{8 0}$ Rate of precipitation of Deoxyhemoglobin s. H. Sabio* and D. Komp, Univ. of Virginia, Dept. of Ped.,

A method utilizing turbidometry(Chronolog Aggregometer) to quantitate the rate of precipitation of deoxyhemoglobin $S$ in a concentrated phosphate buffer was evaluated. A $2 \%$ dithionite-phosphate $\left(\mathrm{KH}_{2} \mathrm{PO}_{4} 1.18 \mathrm{M} ; \mathrm{K}_{2} \mathrm{HPO}_{4} 1.61 \mathrm{M}\right)$ solution was used as the reducing agent. Addition of the dithionite-phosphate solution to the hemolysate produced a deflection in the turbidometer $\left(\mathrm{T}_{1}\right)$ directly proportional to the solubility of the hemoglobins in the hemolysate, and indirectly proportional to the concentration of hemoglobin. Precipitation of hemoglobin was continuously recorded to a point of maximal turbidity $\left(\mathrm{T}_{2}\right)$. $\mathrm{T}_{1}-\mathrm{T}_{2}$ is a measurement of hemoglobin precipitation. The rate of precipitation during the first 30 seconds of the reaction(expressed \% of $\mathrm{T}_{1}-\mathrm{T}_{2}$ ) was determined for hemolysates containing $\mathrm{Hb}^{\prime} \mathrm{s} \mathrm{A}, \mathrm{S}, \mathrm{C}$, and $\mathrm{F}$ in various combinations. Results paralleled the "sickling tendency" observed in vivo and also demonstrated an inhibitory effect of $\mathrm{Hb}-\mathrm{F}$ on the precipitation of $\mathrm{Hb}-\mathrm{S}$. The method is rapid and provides good distinction between $\mathrm{Hb} \mathrm{A} / \mathrm{S}$ and $\mathrm{Hb} \mathrm{S} / \mathrm{S}$ on the basis of solubility.
Hb A A - $98 \%$

Hb A $-20 \%$; Hb F $-80 \%$

Hb A $-55 \%$; Hb S $-45 \%$

$\mathrm{Hb} \mathrm{F}-50 \% ; \mathrm{Hb} \mathrm{S}-50 \%$

$\mathrm{Hb} F-25 \%$; $\mathrm{Hb} \mathrm{S}-75 \%$

Hb F $-6 \%$; Hb S - $90 \%$

Hb C $-50 \%$; Hb S - $50 \%$
$\%$ PRECIPITATED IN 30 SECONDS

0
0
$2.6-7.3$
0.06
14.2
70.1
$55.3-58.1$

482

TRANSIENT ACQUIRED RED BLOOD CELL (RBC) APLASIA IN CHILDREN WITHOUT HEMATOLOGIC DISEASE. Narayan R, Shah, James A. Wolff, Anneliese Sitarz, Babies Hosp., NYC, Acquired RBC aplasia is a rare disease in children. In the last 8 years, 5 cases ( 3 males and 2 females) have been seen. The age of onset ranged from 22 to 36 months. Three patients were seen because of upper respiratory infections, at which time they were noted to be pale. Two patients were noted to be pale on routine examination. No causative agents could be elicited by history. Physical examination was negative, except for pallor in all cases. Hemoglobin varied from $4.4 \mathrm{gms} \%-7.5 \mathrm{gms} \%$ on admission. White blood cell, differential count and platelet counts were normal. Reticulocyte count at admission ranged from $0.1 \%-3.6 \%$. Haptoglobin was absent in 1 patient and normal in the others. Fetal hemoglobin ranged from $2.5 \%-7.2 \%$ at admission. Bone marrow aspiration at admission showed very decreased erythropoiesis in 2 of 5 patients. Autologous RBC survival done in 3 patients was normal. Chest xray was negative in all patients. Serum iron and iron-binding capacity findings in 3 patients at diagnosis were $19 / 216$ (reticulocytes $3.6 \%$ ); $58 / 338$ (retics $1.7 \%$ ); and $186 / 286$ (retics $0.1 \%$ ). One patient was treated with prednisone because of continued aplasia for 18 days, with subsequent response. The second patient was treated with prednisone before referral and recovered well. Three patients recovered spontaneously. Patients have been followed for 2 to 96 months (median 24 months), none of whom relapsed. Temporary red cell aplasia with secondary anemia may occur in children who do not have underlying hemolytic disease. Smith, Sue H. Fujimura, Bertram H. Lubin, Thomas B. Bradley. Children's Hospital Medical Center, Oakland, V.A. Hospital, San Francisco (Spon. by Peter Dallman).

Unstable hemoglobins exhibit early precipitation in $17 \%$ isopropanol (ISP) in $0.1 \mathrm{M}$ Tris, $\mathrm{pH} 7.4$ at $37^{\circ} \mathrm{C}$. Fresh hemolysates of cord blood ( $60 \pm 4 \%$ fetal hemoglobin) developed a definite precipitate within 5 minutes in ISP while adult hemolysates remained clear for 20 minutes. Our studies to date suggest that this finding is due to the propensity of fetal hemoglobin to convert to methemoglobin and to a greater instability of fetal methemoglobin. Although the whole blood methemoglobin levels in fresh cord blood were identical to adult samples, immediately after preparation of hemolysates in $\mathrm{CCl}_{4}$, the methemoglobin content of cord blood was $9.0 \pm 3.9 \%$ compared to $2.0 \pm 0.3 \%$ in adults. Furthermore, the rate of conversion of fetal hemoglobin to methemoglobin by $\mathrm{K}_{3} \mathrm{Fe}(\mathrm{CN})_{6}$ was twice that observed in the adult samples. Following the conversion of both samples to methemoglobin, the rate of precipitation in ISP was still greater in the fetal samples. When methemoglobin was stabilized by the addition of KCN to the hemolysates, the precipitation of fetal hemoglobin was inhibited. In mixtures of cord and adult hemolysates, precipitation was observed when the concentration of fetal hemoglobin was greater than $6 \%$. There was preferential precipitation of fetal hemoglobin from these mixtures. These studies indicate that a positive ISP test should be considered with caution in the presence of fetal hemoglobin. The reported observation of Heinz bodies in newborn red cells suggests that insta-
bility of fetal hemoglobin may occur in vivo.

EFFECTS OF PREGNANCY ON HEMOGLOBIN A IC IN NORMAL, 481 GESTATIONALLY DIABE'TIC AND DIABETIC WOMEN. Herbert C. Schwartz, Katherine C. King, Alan L, Schwartz, Diana Edmunds and Robert Schwartz. Stanford University School of Medicine, Department of Pediatrics, Stanford; Case Western Reserve University, Departments of Pediatrics and Pharmacology, Cleveland Metropolitan General Hospital, Cleveland; and Brown University, Rhode Island Hospital, Department of Pediatrics, Providence. The etiology of the erythrocytosis observed in infants of diabetic mothers is unknown, but might be secondary to fetal hypoxia. Hemoglobin $A_{I_{c}}\left(\mathrm{HOA}_{I c}\right)$, a normal minor $\mathrm{Hb}$ with linked hexose $(s)$, has an increased affinity for oxygen; thus in creases of this glycohemoglobin could limit materno-fetal oxygen transport. Since increased levels of $\mathrm{HbA}_{\mathrm{A}}$ have been reported in insulin dependent diabetics, $\mathrm{HbA}_{\mathrm{B}}$ levels were determined in sixty pregnant(or non) and diabetic(or non) subjects. HbA Ic $_{c}$ was separated from other $\mathrm{Hb}$ 's on IRC-50 columns, and expressed as per cent of total Ho after correction for $\mathrm{HbF}$. Compared to normal females $(m=5.68 \%)$, $\mathrm{HbA}$. was elevated in pregnant normals $(\mathrm{m}=6.97 \%$ $\mathrm{p}<0.001)$, and in obese non diabetic pregnant $(\mathrm{m}=6.89 \% \mathrm{p} \times 0.001)$, in gestationally diabetic $(m=8.77 \% \mathrm{p}<0.001)$, and pregnant diabetics $(\mathrm{m}=$ $8.46 \%$ p $(0.001)$. The latter was a significantly lower level compared to non pregnant insulin dependent diabetics $(m=12.8 \%$ p $<0.001)$ After 30 weeks of pregnancy, gestationally diabetic levels were higher than normal pregnant values $(p<0.001)$ observed and could be disadvantageous to the fetus. The relatively lower levels in insulin dependent pregnant women may reflect either a better state of diabetic control and/or a compensatory mechanism to protect
OXIDANT DAMAGE AND NEWBORN RED BLOOD CELLS: THE ROLE

484 OF SUPEROXIDE DISMUTASE (S.0.D.). James A. Stockman III (Spon. by Frank A. Oski), Dept. of Pediatrics, S.U.N.Y., Upstate Medical Center, Syracuse, New York. ble to oxidant injury. Normal values for newborn red cell S.0.D. have not been determined, nor has the role of this enzyme in relation to oxidant induced damage been examjned. To study this possible relationship, fresh cord blood was obtained from 28 full term infants and examined immediately for Heinz Body formation induced by acetylphenylhydrazine (APH). Superoxide dismutase activity was assayed by the method of Winterbourn, et. a). ( $J$. Lab Clin Med. 85:337, 1975). As expected, Heinz Bodies formed more readily in the presence of APH in newborns as compared to modult controls (3.05 Heinz Bodies/RBC vs 0.3 Heinz Bodies/RBC). S.0.D. activity did not vary significantly between newborn $R B C$ 's and adult controls (newborn: $2126+304$ units/gm Hb, adult 2183 +291 units/gm $\mathrm{Hb}$ ). Variations existed in the degree of Heinz Body formation, with RBC's with higher S.O.D. activity demonstrating greater Heinz Body formation $(R=0.40, p<0.05)$ implying that the end products of the S.O.D. mediated reaction ( $\mathrm{H}_{2} \mathrm{O} 2$ + hydroxyl free radical) are defectiveiy detoxified in newborn cells and may contribute to the production of Heinz Bodies. These data demonstrate that S.0.D. in newborn RBC's is quantitatively similar to adult RBC's. The proclivity to oxidant injury in newborn RBC's is not due to S.0.D. deficiency and may in fact be accentuated by increased amounts of intracellular S.O.D.
Newborn red biood cells (RBC's) are known to be more suscepta- 


\section{5}

THE ANEMIA OF PREMATURITY. FACTORS GOVERNING THE ERYTHROPOIET IN RESPONSE. James A. Stockman, III Joseph F. Garcia and Frank A. Oski. Department of Pediatrics, S.U.N.Y., Upstate Medical Center, Syracuse, and the Lawrence Berkeley Lab., Univ. of California, Berkeley, Cal. Factors governing the role of erythropoletin in response to anemia in premature infants have not been elucidated. It has been a clintcal impression that variability exists in the infant's response to any given hemoglobin $(\mathrm{Hb})$ level. To define the factors determining this response, sequential studies were performed in 30 infants (birth weights $<1500 \mathrm{gm}$ ) for periods up to 12 wks. of age. $\mathrm{Hb}$, red cell 2,3-DPG, fetal hemoglobin, reticulocytes and plasma erythropoiet in (EP) were measured. EP was determined by radioimmunoassay. Employing the 2,3-DPG and fetal hemoglobin concentration, $P_{50}$ values were determined and calculations made regarding the infant's $0_{2}$ unloading capacity. Although the relationsh1p between absolute th level and plasma EP was signiflcant, much individual variability was present. The most striking relationship was observed between the plasma EP and the infant's oxygen releasing capacity (ml $02 / 100 \mathrm{ml}$ blood) at a venous $0_{2}$ tension of $40 \mathrm{~mm} \mathrm{Hg}(x=.50 ; \mathrm{p}<.001)$. When oxygen delivery was less than $2.0 \mathrm{ml} / 100 \mathrm{ml}$ blood, 29/30 EP values were increased. These studies demonstrate that response to anemia in infants is governed by oxygen supply and they respond appropriately. Oxygen supply is determined not by $\mathrm{Hb}$ alone but by factors modulating $\mathrm{Hb}^{\prime}$ 's affinity for oxygen (\% fetal hemoglobin and 2,3-DPG). Recognition of these factors will result in better decisions regarding transfusions in low birth weight infants.

486

IgG IMMUNE HEMOLYTIC ANEMIA: EVALUATION BY A NEW C1 FIXATION AND TRANSFER TEST. Robert C. Strunk, James J. Corrigan, Jr, and T. Jacob John. U. of Az. Coll. of Medicine, Arizona Medical Center, Dept. Pediatrics, Tucson.

The fixation and transfer of the first component of complement (CIFT) had been used to quantitate the number of IgM-C1 fixing sites in cold agglutinin disease. We have used a new method for C1FT in evaluation of a patient with severe warm ( $\lg G$ ) autoimmune hemolytic anemia. She did not respond to splenectomy, thymectomy and immunosuppressants, and died of sepsis at 12 months. Her blood collected in $\mathrm{NaF}$ or normal RBC sensitized with her NaF plasma were washed with Veronal buffered saline (VBS)-sucrose buffer, $\mathrm{u}=0.065$, at $0^{\circ} \mathrm{C}$. The $\mathrm{Cl}$ was titrated after transfer to EAC4 in VBS, $u=0.15$. Sequential evaluations of the patient's cells over a 6 week period showed that the number of $\mathrm{C} 1$ molecules/cell varied from 12 to 50 . Using a comparable NaF plasma, passively sensitized RBCs showed 4-37 C1/cell. Control blood collected in $\mathrm{NaF}$ or normal RBC incubated with normal NaF plasma had no C1. Comparative tests have shown that the number of $\mathrm{Cl}$ fixing sites were underestimated by $60-80 \%$ and by $30-40 \%$ with the standard ClFT, using partially purified guinea pig $\mathrm{Cl}$ and human $\mathrm{Cl}$. The severity of immune hemolytic anemia is probably related to the number of antibody molecules on the RBC surface. Since there is fixing sites, we believe that ClFT had clinical application in predicting the severity of hemolysis and evaluating therapy. In addition this modified C1FT may be a more sensitive indicator of the presence of $\mathrm{Cl}$ fixing sites in IgG immune hemolytic anemia than the standard ClFT.

THE EFFECT OF HEMOFIL (HF) ON PLATELET FUNCTION IN 487 CLASSICAL HEMOPHILIA (CH). Marie J. Stuart, Gerald vladimer and Joel A. Wolk (Intro, by W.H. Berg

Dept. of Peds., SUNY, Upstate Med. Ctr., Syracuse, N.Y.
Infusions of cryoprecipitate (cryo) have been demonstrated to Infusions of cryoprecipitate (cryo) have been demonstrated to
use abnormalities in platelet ( 1 lt) function. (Hathaway, Transf $13: 6^{\circ} 73$ ). The effect of hemofil (HF) a glycine precipitate VIII conc. was studied in 2 grps, of pts, with $\mathrm{CH}$. Grp.I comprised 11 $\mathrm{CH}$ (4-14 yrs.) with VIII AHF $<1 \%$, VIII AGN (>50\%) without VIII inhibitors. During a non-bleeding period (no VIII for 10 days prior) this grp. was administered a single $\mathrm{HF}$ infusion to raise VIII $\mathrm{AHF}$ to $60 \%$. Studies pre $\& 1 \mathrm{hr}$, post $\mathrm{HF}$ included PTT, TT, fibrinogen, FSP, salzman retention (SR), Ivy template bleeding time (BT) and p1t. aggregation studies (PA). The most significant finding was the presence of prolonged baseline BTs. Mean BT pre $H F$ was $1.3^{\prime} \pm 7.3^{\prime}$ (ISD), with a significant shortening post HF to $8.4^{\prime} \pm 2.9^{\prime}$. (Mean Control BT being $3.6^{\prime} \pm 1.1^{\prime}$ ). No differences were observed in SR, FSP, or PA pre vs. post HF. When compared to controls, hyperaggregability to collagen was observed in $\mathrm{CH}$. PTTs $\downarrow$ into the normal range post HF with modest $\uparrow$ in fibrinogen. Grp. II consisted of 3 post-op pts. with severe $\mathrm{CH}$ who were given daily infusions of $\mathrm{HF}$ for 7-14 days, daily dosages being calculated to raise VIII AHF $60 \%$. Similar studies were performed pre HF and on 2-4 occasions during the course of daily HF administration. Findings similar to Grp.I were observed. HF unlike cryo does not appear to cause plt. functional abnormalities \& therefore should be used when daily VIII administration becomes necessary in $\mathrm{CH}$. The abnormal BTs in our pts. with severe $\mathrm{CH}$ and its partial correction by $\mathrm{HF}$ has not been previously documented.
488 THE ASPIRIN TOLERANCE TEST IN THE DIAGNOSIS OF BLEEDMNG DISORDERS. Marie J. Stuart, Joel A. WoIk, Oski). Depts. of Ped. and Path., SUNY, Upstate Med. Ctr., Syr. To determine the usefulness of the aspirin (ASA) tolerance test, 20 "controls" with negative bieeding histories were evalua platelet aggregations including Ristocetin (R), and modified IVy bleeding times (BT's) pre and $2 \mathrm{hrs}$. post 600 mgms ASA. $2 / 20$ "controls" (without bleeding post tonsillectomies and teeth extractions) were diagnosed as VWD (VIII AHF and AGN $<40 \%, \downarrow S R$ ). In $18 / 20$ normal controls the mean pre ASA BT was $3.6^{\prime} \pm 2.2^{\prime}$ (2SD). 2 hrs. post ASA, the BT was $6.9^{\prime} \pm 2.4^{\prime}$ (2SD). Both "controls" with VWD had normal pre ASA BT's but post ASA values of $17^{\prime}$ and $11^{\prime}$. Evaluations for hemostatic abnormalities were performed in a referral population with 11 diagnosed as having VWD. $5 / 11$ had classical findings of $\downarrow$ VIII AHF and AGN, $\downarrow S R$ and prolonged pre ASA BT's. $6 / 11$ had $\downarrow$ VIII AHF and AGN $\pm \downarrow$ SR and $R$ aggregations but normal BT's pre ASA. All 6 demonstrated abnormal post ASA BT's as did 2 pts. with platelet dysfunction states. In addition, 5 pts. with bleeding histories, normal aggregations and borderline VIII AHF and AGN (50-60\%) with $\downarrow$ or normal SR had prolonged post ASA BT's $\left(11^{\prime}-20^{\prime}\right) ; 2$ of these have siblings with documented VWD. VWD appears the most common symptomatic/asymptomatic bleeding disorder. It is mandatory to test all "controls" used in determining "normal" values for this entity. The post ASA BT appears to identify all pts. With VWD and may be the most sensitive parameter in pts. with borderline VIII and significant bleeding histories.

489 Erythropoeitic Islands in Autonomous Erythropoeisis Associated with Hypotransferrenemia. A. Taher an whereby iron is transferred to erythroid cells for hemoglobin synthesis. Bessis has proposed a second mechanism namely direct transfer of iron from the reticulum cells to the developing erythroids. In this process several erythroblasts surround a large reticulum cell to form a distinct anatomical unit referred to as exythroblastic island. These islands are seldom seen in the stained films of bone marrow aspirates and their role in iron transfer to erythroids and the extent of such a transfer in normal and pathological conditions remains unsettled. Recently we observed a unique association of an acute and autonomous erythropoiesis, as evidenced by $14 \times 104 / \mathrm{cmm}$ nucleated erythroids in the peripheral blood, with a marked reduction in plasma transferrin (TiBC $74 \mathrm{ug} \%$ ), in a 15 year old boy with Fanconi's anemia. A bone marrow aspiration revealed numerous erythroblastic islands ( $3 / \mathrm{Hp}$ field). All reticulum cells surrounded by erythroblasts contained hemosiderin. No iron granules could be seen in the erythroid surrounded by the reticulum cells. The patients bone marrow specimen obtained prior to the onset of erythroleukemia and hypotransferrenemia contained no erythroblastic islands. Despite adequate bone marrow iron stores the erythropoeisis remained hypochromic (MCHC 25\%). In agreement with Bessis hypothesis the occurrence of large numbers of erythroblastic islands in the bone marrow of our patient is probably in response to increased demand for iron caused by neoplastic erythropoiesis in the absence of adequate amounts of transferrin.

\section{INVESTIGATIONS OF CYCLIC NEUTROPENIA. Kamran Tebbi, and Samuel Gross. Case Western Reserve University,} Department of Pediatrics, Cleveland, Ohio

Cyclic neutropenia is characterized by periodic variation in the percentage of polymorphonuclear leukocytes and monocytes. At its lowest level it is accompanied by fever, malaise, oral pharyn. geal ulcers and mild infections. To elaborate the etiology of cycles in this disease, bone marrow culture as well as production of colony stimulating activity (CSA) in various stages of the cycle were carried out in two siblings with this disorder. At the height of neutrophile count, the marrow contained decreased numbers of colony forming units in culture (CFU-C). Colony stimulating activity from these patients at the nadir of neutropenia and from normal individuals restored the CFU-C to normal values. CSA isolated from peripheral blood at various stages of the neutrophile cycles was measured using non-adherent bone marrow cells. This proved to have a cyclic variation with an inverse relation to the neutrophile cycle, i.e. the nadir of neutrophile count coincided with the height of CSA values. The frequency and amplitude of CSA cycle was directly related to absolute monocyte levels. These observations indicate defects both in feedback mechanisms and CSA production in patients with cyclic neutropenia. Furthermore, this study supports data indicating that monocytes are the major CSA producing factors and as such play a prominent role in granulocytic proliferation and differentiation. Attempts are being made to harvest exogenous CSA in an effort to treat this disorder. 

CHARACTERISTICS OF ERYTHROCYTES FROM NORMAL TERM INFANTS DURING THE FIRST YEAR OF LIFE. Susan F. Travis, Bakulesh Patel, Elizabeth Cannon and Maria Deliveria-Papadopoulos. Thom Jeff. Univ. and Univ. of Pennsylvania, Philadelphia, Pa.

The activities of red cell phosphofructokinase(PFK), phosphoglycerate kinase(PGK) and enolase were measured sequentially the first year of life in 10 term infants and contrasted with the activities of pyruvate kinase $(\mathrm{PK})$, hexokinase(HK), aldolase and fetal hemoglobin ( $\mathrm{HbF}$ ) to establish whether developmental patterns were related to cell age or the postnatal decline of $\mathrm{HbF}$. Red cell glycolytic intermediates were studied at the same time intervals, as were intra- and extra-cellular $\mathrm{pH}$ and plasma inorganic phosphorus concentration(Pi) to determine the effects of environmental (extra-cellular) factors. Maturation changes in the activities of PFK, $P G K$ and enolase correlated best with the decline in HbF ( $p<0.001$ ), not red cell age. Red cell PK, HK, G-6-PD and aldolase activities were elevated the first year of life, consistent with a young red cell population. Glucose-6-phosphate(G-6-P) and fructose-6-phosphate (F-6-P) were significantly elevated and correlated with $\mathrm{Pi}(\mathrm{p}<0.001)$ not the decline of $\mathrm{HbF}(\mathrm{p}>1.0)$. These data suggest that changes in $P F K$, enolase and $P G K$ follow a normal developmental pattern that coincides with the decline in HbF and that alterations in the concentration of G-6-P and F-6-P are modulated by $P i$.

492 RED CELL MEMBRANE PROTEIN PHOSPHORYLATION IN HEREDITARY SPHEROCYTOSIS (HS). Lawrence C. Wolfe and Samuel E. Lux, Harvard Medical School, Children's Hospital Medical Center, Department of Pediatrics, Boston, MA It is unclear whether phosphorylation of spectrin and (PK) is membrane proteins by endogenous membrane proteln HS. Avruch and Fairbanks recently delineated 2 distinct PK activities in RBC ghosts: one stimulated by monovalent cations and unresponsive to CAMP (high salt PK), the other inhibited by salts and stimulated by CAMP (low salt PK). We measured the activity of these 2 PKs toward membrane protein substrates in ghosts from 7 HS patients and 6 controls (C). We also measured membrane phosphorylation in intact RBC of 3 HS and $3 \mathrm{C}$ by monitoring $32_{\mathrm{P}}$ uptake and incorporation into ATP and specific membrane proteins. Membrane protein composition (SDS gels) of HS and C ghosts were identical. Phosphorylations of spectrin (includes Band 2.1) and Band 3, the major endogenous membrane substrates, are shown below.

HIGH SALT PK LOW SALT PK LOW SALT PK INTACT RBC

$$
\text { - CAMP }
$$$$
+C A M P
$$

\begin{tabular}{|c|c|c|c|c|c|}
\hline \multirow{3}{*}{ SPECTRIN } & \multirow{3}{*}{ HS } & (pM $P$ in & cporated/mg & and protein & $/ \min )$ \\
\hline & & 6.7 & $5.3 \pm 1.0$ & $13.8 \pm 6.7$ & $6.3 \pm 1.6$ \\
\hline & & 20.0 & $6.7 \pm 1.3$ & $13.3 \pm 6.5$ & $5.8 \pm 0.8$ \\
\hline BAND 3 & HS & $3.9 \pm 0.8$ & $10.6 \pm 2.8$ & $13.3 \pm 5.0$ & $4.6 \pm 0.8$ \\
\hline & & 3.2 & $16.0 \pm 2.7$ & $16.0 \pm 2.6$ & $4.2 \pm$ \\
\hline
\end{tabular}

The values for HS and C PK activities are statistically identical.

We conclude that RBC membrane protein phosphorylation in our HS patients is normal. The intact cell studies, in particular, argue agalnst any physiologically significant phosphorylation defect.

\section{IMMUNOLOGY}

493 TRANSFER FACTOR EFFECT ON IgE ANTIBODY. Ziad Alqusus and Photini S. Papageorgiou.

$T$ and $B-1 y m p h o c y t e$ interactions are instrumental in the modulation of antibody production. Transfer factor (TF) from healthy adults was administered to a 9 year old boy with congenital agammaglobulinemia, who had been unsuccessfully treated with gamma globulin. Immunological evaluation prior to TF (14 days post IgG) revealed $140 \mathrm{mg} \% \mathrm{IgG}$, undetectable serum and secretory IgA, $35 \mathrm{mg} \% \mathrm{IgM},<10 \mathrm{u} / \mathrm{ml} \mathrm{IgE}$ (undetectable). B-cell markers were $4.6 \%$ by EAC rosette and $7 \%(a, \gamma, \mu$, chain $)$ by immunofluorescent staining. Pokeweed mitogen response of the lymphocytes ${ }_{8}$ was normal. Following an initial course of 3 doses of TF $\left(2 \times 10^{8}\right.$ lymphocytes per dose) within 6 weeks, patient receives bimonthly doses, depending upon the status of his cutaneous reactivity to antigens to which the donor responded. During the first 9 months of TF therapy, patient was completely free of infections. Since then, he has had two episodes of pneumonia. Eighteen months post $\mathrm{TF}$, he developed copious amounts of clear mucous into the upper respiratory tract relieved temporarily by antihistamines and repeated episodes of generalized hives. His IgE rose to $30 \mathrm{u} / \mathrm{ml}$ and the RAST test showed specific IgE antibody to a variety of allergens. Serum IgG and IgM have remained low with some initial fluctuations. Two months after discontinuation of $\mathbf{T F}$, the IgE is undetectable and the episodes of hives have subsided. This coincided with the loss of patient's transferred cutaneous reactivity to monilia to which the donor was positive. These data suggest that $\mathrm{T}$-cells play a regulatory role in IgE producdata suggest that $\mathrm{T}-\mathrm{cells}$ play a regulatory role in lge produc-
ANTIBODY RESPONSE OF INFANTS TO THE CAPSULAR POLYRI-

494. BOPHOSPHATE (PRP) OF HEMOPHILUS INFLUENZAE b (HIB).

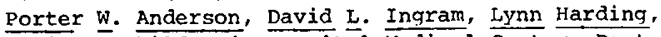
and David ${ }_{-}$. Smith*, Children's Hospital Medical Center, Dept.of Med., Boston, Mass.

Previous work indicated that $A b$ to $P R P$ protects vs. HIB invasive disease and that purified PRP is immunogenic in adults and children. Preliminary data, however, suggested a weaker response in infancy. In the present studies 303 infants under 1 year of age were immunized and $\mathrm{Ab}$ responses determined by radio-labelled antigen binding assay. Single or multiple injections of 1-10uq generally failed to raise titers measured $\overline{c a} 2$ mo. later; many titers fell. Interpretation is complicated because titers of non-immunized infants fall during the first $6 \mathrm{mo}$. of life. Agespecific titers of further groups of immunized and of control infants were therefore compared through age 2 yrs. PRP $(5 \mu \mathrm{g})$ was given in 3 injections combined with, simultaneous with, or alternated with $\mathrm{AlPO}_{4}$-ppted DPT beginning at $\overline{\mathrm{Ca}} 2 \mathrm{mo}$; or at $\overline{\mathrm{ca}} 8$ mo. (after the DPT course). Titers in the 4 immunized groups were slightly, but not significantly greater, than those of nonPRP-immunized infants. Tolerization was not observed. Thus, injection of purified PRP does not siqnificantly affect specific $\mathrm{Ab}$ activity during the ages of greatest susceptibility.

We are now studying a newly developed preparation in which the native PRP-protein-lipopolysaccharide complex is conserved. This complex induces high, boostable titers of anti-PRP $\mathrm{Ab}$ and bactericidal $\mathrm{Ab}$ to other surface antigens in young rabbits (in which pure PRP is not immunoqenic) and in adult humans.
495 DEFECTIVE NEUTROPHIL AND MONOCYTE CHEMOTAXIS IN DOWN SYNDROME. Rogex M. Barkin, William L. Weston, James by Charles s. August). Univ. Colo. School of Medicine, Depts. of Pediatrics, Medicine, and Biophysics/Genetics, Denver.

Defects in host defenses may account in part for the increased incidence of infection in Down syndrome (DS) patients. Evaluation of cellular and humoral chemotaxis in neutrophils (PMNL's) and monocytes (MNL's) was done to explore these aspects of phagocytic function. Fourteen children with DS and 15 matched institutionalized controls (IC) were studied and compared with 16 normal adults (NA). Modified Boyden chambers with double filters were employed. An E. coli filtrate was used to evaluate cellular PMNL chemotaxis while endotoxin-activated serum served as attractant in the other assays. Results are expressed as percentage of $\mathrm{mi}$ grating cells $(\bar{x} \pm S E M)$ (number of subjects in parentheses)

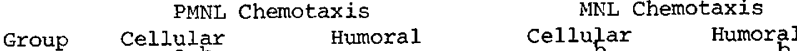
DS $\quad 1.2+0.3^{\mathrm{a}, \mathrm{b}}(14) \quad 8.9 \pm 1.0(14) \quad 5.0 \pm 1.1^{\mathrm{b}}(12) \quad 4.2 \pm 0.4^{\mathrm{b}}(14)$ IC $3.3 \pm 0.5^{\mathrm{C}}$ (15) $11.4 \pm 1.0(15) \quad 7.2 \pm 1.4$ (14) $7.3 \pm 0.4$ (15) $\mathrm{NA} \quad 6.0+0.7 \quad(16) \quad 11.3+1.6(8) \quad 10.0+0.6$ (13) $8.9+0.3$ (7) $p<0.05:$ a DS vs IC; $\bar{b} \overline{D S}$ vS NA; ${ }^{C}$ IC $\overrightarrow{v S}$ NA.

PMNL chemotaxis of DS patients was significantly impaired. MNL chemotaxis in IC was lower than the NA group. DS patients also failed to generate adequate humoral attractant for normal MNL's. These defective aspects of cellular and humoral chemotaxis in DS may be partly responsible for the frequent infections observed in that condition. Institutionalization probably accounts for part of the observed defects. 496 CELLS ON BOUYANT DENSITY GRADIENTS. W. Gary Becker Medicine, Department of Pediatrics, Durham, North Carolina. Most reports of mononuclear (MN) cell yields using FicollHypaque (FH) gradients have not exceeded $90 \%$. The relative recovery of Ig bearing B lymphocytes to spontaneous $E$ rosetting $T$ lymphocytes compared with that expected on the basis of $100 \%$ recovery was determined for various \% recoveries and found to be 1.6 at $75 \%$ and 3.0 at $40 \%$, indicating relatively greater losses of $T$ cells with diminishing cell yields. Optimal conditions for FH separations were then investigated in normal adults. Highest yields of MN cells were obtained when heparinized blood was diluted $7: 4$ with a diluent of $325 \mathrm{mosm} / \mathrm{L}$, placed on $\mathrm{FH}$ of a minimum depth of $2 \mathrm{~cm}$, and centrifuged for $30^{\prime}$ at $400 \mathrm{~g}$. Yields diminished with changes in diluent osmolarity. Saline $(0.95 \%)$ gave higher yields $(94.0 \pm 4.1 \%)$ than RPMI $1640(73.7 \pm 12.9 \%)$ or Hank's balanced salt solution (HBSS; $79.0 \pm 3.4 \%$ ). Human or fetal calf serum added to the diluent did not increase yields, but increased polymorphonuclear contamination at concentrations greater than $5 \%$ In contrast, wash solutions gave best yields when containing small amounts of serum $(2 \%)$ and when adjusted to $300 \mathrm{mosm} / \mathrm{L}$. Phosphate buffered saline gave higher yields $(94.0 \pm 4.1 \%)$ than RPMI 1640 $(78.6 \pm 7.8 \%)$ or HBSS $(80.3 \pm 11.7 \%)$. Yields were equal in siliconized glass and plastic tubes. Total mononuclear cell yields were $98.2 \pm 1.8$ at $5^{\circ} \mathrm{C}$ and $94.0 \pm 4.1 \%$ at $25^{\circ} \mathrm{C}$. Myeloperoxidase stains showed that losses occurred primarily in the monocyte fraction. $4.8 \%$ at $25^{\circ} \mathrm{C}$. Viability of separated cells was $>99 \%$ at 10 hours. 


\section{7}

COMPONENTS OF CELLULAR IMMUNITY IN MIDDLE EAR EFFUSIONS: POSSIBLE ROZE IN THE PATHOGENESIS OF OTITIS MEDIA. J.M. Bernstein, C. Sysmanski, B. Albini, and Buffalo

The distribution of $B$ and $T$ lymphocytes and macrophages in the middle ear fluid (MEF) was studied in groups of children with otitis media associated with the presence of serous, seromucinous or mucoid effusions in the middle ear. The subpopulations of $T$ and $B$ lymphocytes and macrophages were identified in the MEF by employing the techniques of membrane immunofluorescence and erythrocyte, erythrocyte-amboceptor-complement and erythrocyteamboceptor rosette formation respectively. A significant number of $B$ and $T$ lymphocytes or macrophages were detected in all specimens. However, certain differences were observed in their relative distribution in different types of effusions. The serous MEF was characterized by a cellular response associated predominantly with macrophages, although $\mathrm{T}-1$ ymphocytes were also seen frequently. No B-Iymphocytes were seen in such exudates. The seromucinous MEF contained $B$ and $T$ lymphocytes and macrophages in approximately similar proportions. Significantly, however, the mucoid effusions consisted mainly of $B$ lymphocytes. Only few macrophages and no $T$ cells were observed in these effusions. The cytologic findings reported here may be helpful in a more definitive classification of middle ear effusions. These data suggest a possible role of the different components of cellular immunity in the development of otitis media. It is possible that the various types of effusion seen in otitis media may represent progressive stages of a single inflammatory process in the middle ear.

PROGNOSIS IN JUVENILE RHEUMATOTD ARTHRITIS (JRA): A

498 15-YEAR FOLLOW-UP. John J. Calabro, Harry L. Staley, \& Stephen L. Burnstein (Intr. by James Bo Hanshaw). University of Massachusetts Medical School, Worcester City Hospital, Division of Rheumatology, Worcester, Massachusetts.

Having completed our prospective 15-year follow-up of 100 patients with JRA, we can now report that 64 are in remission, 33 are active, and 3 have died. These impressions have been gained from observations of $62 \mathrm{girls}$ and 38 boys; their current mean age is 21 years, the youngest is 15 , the oldest 33 . The onset, disease course, and outcome of our 100 patients are shown below. No. Modes of Onset No. Disease Course Active Incap. Ixido.

10 Polyarthritis

48 Polyarticular 48 polyarthritis 24 oligoarthritis

$\overline{100}$

$\begin{array}{rrr}1 & 0 & 1 \\ 3 & 3 & 0 \\ 19 & 11 & 1 \\ 4 & 0 & 1 \\ \frac{6}{33} & \frac{0}{14} & \frac{6}{9}\end{array}$

Remissions occurred most often $(80 \%)$ with the most severe systemic involvement initially (acute febrile onset). This surprised us, as did the disclosure that asymptomatic iridocyclitis developed most often (25\%) with the mildest form of disease (oligoarthritis). Only 14 patients are incapacitated (Functional Classes III-IV), all but 2 of these have had progressive polyarthritis and hip involvement. Of 9 with iridocyclitis, 2 are blind in 1 eye when first referred to us. Since then, blindness has been successfully prevented because of early detection made possible by routine slit-lamp examinations.

SEPARATION AND FUNCTIONAL CHARACTERISTICS OF 499 LYMPHOCYTE SUBPOPULATIONS IN PERIPHERAL BLOOD R.K. Chandra, Fac. of Med., Memorial Univ. of Newfoundland, St. John's, Newfoundland, Canada.

Lymphocyte preparations from peripheral blood were seperated into populations enriched with one subset by formation of spontaneous rosettes with sheep red blood cells (SRBC), immune rosettes with SRBC, anti-immunologlobul in coated nylon wool column filtration, and buoyant density centrifugation. C3- and Fc- receptor bearing lymphocytes exhibited antibody-dependent cytotoxicity. T-cell enriched populations showed a high response to mitogen stimulation. B-7ymphocyte enriched preparations and the "null" cells showed a relatively lower response but axhibited cell-mediated cytotoxicity against xenogeneic target cells. Mitogen-treated "null" cell preparations depleted of $T$ and $B$ Iymphocytes were able to suppress mitogen-stimulated DNA synthesis by 7 ymphocytes of healthy donors. The relative increase in the proportion of null cells and B-lymphocytes may be of pathophysiologic significance in diseases characterized by impaired delayed hypersensitivity, eg. primary immunodeficiency, malnutrition, lymphoreticular malignancy, lepromatous leprosy, etc. Supported by a grant from the World Health Organization, Geneva.
500 IMMUNOREGULATORY ROLE OF ALPHA-FETOPROTEIN. R.K. Newfoundland, Canada. Med. Memorial Univ. of Newfoundland, St. John's,

The ontogenetic development of embryo-specific alpha-fetoprotein (AFP) was studied. The peak serum levels were reached at 14-15 weeks of gestation and the main site of AFP synthes is was the liver. Serum AFP concentration was elevated in patients suffering from ataxia-telengiectasia, Indian childhood cirrhosis, infants with perinatal malnutrition, and in pregnancy, conditions with a common denominator of depressed cell-mediated immunity. AFP purified by affinity chromatography and polyacrylamide gei electrophoresis was injected intravenousiy into 8-week old mice immunized simultaneously by intraperitoneal administration of sheep red blood cells (SRBC). Spleen cells forming antibody to SRBC were significantly reduced compared with findings in control cnimals. A similar immunosuppressive effect of AFP was demonstrated in vitro. It is suggested that AFP has an important immunoregulatory role in mammalian biology. Supported by a grant from the World Health Organization, Geneva
501 ABNORMALITIES OF CELLULAR TMMUNE RESPONSES IN ARTHRITIS INDUCED BY RUBELLA VACCINATION. Y. Chiba, J.L. Dzierba and P.L. Ogra. Depts. of Peds. and Microbiol. State Univ. of N.Y, at Buffalo.

Employing hemagglutination inhibition, immunodiffusion, immunoelectrophoresis, E-rosette formation and, in-vitro lymphocyte transformation (LTF) in response to phytohemagglutinin (PHA) and rubella virus, the levels of immunoglobulins, complement components and rubella specific antibody activity in the serum, and the number of T-cells and the nature of cell-mediated immune (CMI) response in circulating lymphocytes was studied in a group of children who manifested arthralgia or arthritis after parenteral immunization with HPV $-77 \mathrm{DE} / 5$ or $\mathrm{RA} 27 / 3$ live attenuated rubella virus vaccines. Immunized subjects who developed no post-vaccine complications were included as controls. The immunoglobulin and complement levels were similar in all subjects, although rubella antibody levels were somewhat elevated in subjects with arthritis. The cellular immune function in subjects with arthritis was characterized by a slightly decreased number of E-rosette forming lymphocytes and appreciable LTF activity to PHA. Although significant rubella specific CMI was detectable in most controls, little or no LTF activity specific for rubella virus was observed in arthritis subjects. These findings suggest a selective depression of CMI particularly for rubella virus, in subjects with rubella arthritis. Such immunosuppression may represent prolonged rubella infection or antigenemia and may be an important factor in the pathogenesis of joint symptoms associated with rubella vaccination and possibly with other viral infections.

THE FIRST THYMIC TRANSPLANT: A PROGRESS REPORT.

502 William W. Cleveland and Philip R. Glade. University of Miami School of Medicine, Department of Pediatrics, Miami

Florida 33152 .

In 1967 the first successful transplant of fetal thymic tissue was performed in a 9 mos. old infant with the DiGeorge Syndrome. Since that time the child has shown no clinical evidence of immunologic deficiency. He is developmentally retarded for undetermined reasons. Hypoparathyroidism persists and is reasonably well controlled. No tissue has ever been palpable at the site of the transplant and no biopsy of this area has been performed. Periodic studies of immunologic function have shown no gross abnormalities but the total lymphocyte count, although usually normal, has been as low as 1250, suggesting decreased numbers of T cells; in vitro response to PHA has remained adequate. He continues to manifest a negative Schick test as well as positive cutaneous hypersensitivity to DNCB and to SK-SD; he did not however respond to candida and trichophyton antigens when recently tested at age $8-1 / 2$. Evaluation at this age also showed the following: IgG 1,450; IgA 29l; IgM 62; $\beta$ cell immunofluorescence Ig G $9.9 \%$ (normal $7.9 \%$ ); IgM $17.5 \%(5.6 \%) ; \lg A 4.3 \%$ (3.5\%); T-cells (rosette forming) were $27 \%$ (normal $40-50 \%$ ); normal in vitro response to pokeweed mitogen, PHA; and mixed lymphocyte stimulation.

These studies indicate that the child has grossly adequate immunologic status although there is a suggestion of mild abnormalities in both $T$ and $B$ cell function. If serial measurements confirm these observations consideration will be given to other approaches to improving immunologic capability. 
FAMILIAL DYSGAMMAGLOBULINEMIA IN IDTOPATHIC NEPHROTIC 503 SYNDROME.B.R. Cole, L.Peterson, and A.M.Robson, Wash. Univ. Sch. of Med., Dept. of Ped., St. Louis

That idiopathic nephrotic syndrome of childhood (INS) may be a manifestation of an altered immunologic state has been suggested by the findings of low IgG, IgA and high IgM levels in our patients (NEJM 293:8, 1975). These changes were present acutely and persisted even after long-term remission. To determine whether this immunologic abnormality might be inherited, serum immunoglobulin levels were determined by radial immunodiffusion in the 117 parents and siblings of 26 patients with INS, each of whom was screened for recent illnesses, medications and urinary abnormalities. Expressed as \% of normal for age, the following levels were found

Patients in remission

Fathers

Mothers

Brothers

Sisters

*" $t$ " test: $p<.05$

The finding of elevated $\mathrm{IgM}$ levels in parents and siblings of patients with INS suggests there may be an inherited factor which predisposes to INS. This could explain the reported familial incidence of INS. The parents did not show the decreased IgG and IgA levels characteristic of INS patients. This may indicate the incomplete penetrance of an inherited defect fuliy manifested in patients with INS. Alternately the etiology of INS may be multifactorial with as yet unknown causes precipitating the clinical syndrome only when the immunologic abnormality is present.
504 IgG as a serum Vector of MUCOCILIARY disturbances in 504 ALLERGIC ASTHMA. James H. Conover, Michael Kornfield, and Elaine J. Conod. (Son. by Emstein College of Medicine, Bronx, N.Y.

Disturbances in mucociliary function of cultured rabbit tracheal explants is consistently noted after exposure to sera from either cystic fibrosis (CF) patients or their carrier parents. This serum-borne ciliary dyskinesia factor (CDF), characteristic of the CF disorder, is not demonstrable in the majority of normal controls. We have found, however, in pediatric populations of allergic asthmatic individuals, a serum CDF indistinguishable in response from that of CF sera. The incidence of a serum CDF in asthmatics is too high to consider all these patients to be CF carriers. In addition, sera from a variety of other pediatric lung disorders tested (except croup) were seen to demonstrate a CF-like CDF response.

In CF sera, the IgG fraction has been shown to possess the CDF activity. We have separated IgG from the sera of each of six asthmatics with differing allergic sensitivities, by DEAEcellulose chromatography. In every instance, the purified IgG fractions gave rise to a CDF response by bioassay. Thus, it would seem that asthmatic IgG mediates ciliary dyskinesia or that some IgG-associated substance plays a role in evoking the CDF response. These data support the view that respiratory disorders have serum vectors which can be monitored by a respiratory test system.

505 CHRYSOTHERAPY IN JUVENILE RHEUMATOID ARTHRITIS. Clifford DeBenedetti (Spon. by James J. Corrigan, Jr) Dept. of Peds., Univ. of Arizona Medical Ctr., Tucson Drug therapy in children with Juvenile Rheumatoid Arthritis (JRA) who have chronic polyarthritis unresponsive to acetylsalicylic acid (ASA) is very limited. Gold therapy has been shown to be effective in suppressing inflammation of rheumatoid arthritis. However, chrysotherapy has been said to be more toxic, especially to young children. We reviewed the course of 14 children with JRA who were treated with gold. Following 2 test doses the patients were treated with $1 \mathrm{mg} / \mathrm{kgm} /$ week IM of gold thioglucose (Solganol) or gold thiomalate (Myochrysine). After an adequate trial of weekly injections the schedule was adjusted to every 2 weeks and then once a month. Complete blood counts were done every second or fourth injection and urinalysis every week. The patients were followed over a prolonged period (mean duration: 143 weeks; mean number of injections: 93) and received significant doses of gold (mean: $3300 \mathrm{mg}$ ). During the course of therapy all patients were maintained on therapeutic doses of salicylates and 7 patients were on Prednisone. Three patients developed skin rash; gold was subsequently re-instituted in 2 of these without recurrence of rash. There was no evidence of bone marrow depression. Renal toxicity was limited to 3 patients who had transient $2+$ proteinuria on 2 occasions during the course of treatment. Six of the patients responded with decrease in rheumatic signs and symptoms, 6 patients had ESR return to normal levels, and in 3 of the 7 patients on steroids, prednisone could be discontinued. These data suggest that chrysotherapy is a well tolexated,
effective adjunct in the treatment of ASA unresponsive IRA
16 DEFECTIVE ANTIVIRAL CELL-MEDIATED IMMUNITY IN A. CHILD WITH RECURRENT VIRAL INFECTIONS. Ibrahim I. Elaraby, William E. Rawls, Peter B. Dent, Max Chernesky. (Spon. by John C. Sinclair) McMaster Univ., Depts. Pediatrics and Pathology, Hamilton, Ont.

$J P$ is a 3 year old boy who has had monthly severe oral and ocular Herpes simplex (HSV-I) infections for $1 \frac{1}{2}$ years as well as persistent molluscum contagiosum lesions. His 6 year old brother (SP) has recurrent (6/year) oral herpes infections of mild to moderate severity. Assessment of general cellular and humoral immune competence of the 2 boys showed no abnormalities and both had normal levels of anti-HSV antibody. We therefore measured the cell-mediated response to HSV by calculating the ratio (SI) of 3H-thymidine uptake by lymphocytes in the presence of antigen derived from HSV-infected BHK 21 cells compared to the uptale in presence of antigen from uninfected cells. SI ( \pm SD) of 8 seronegative subjects was $1.0 \pm 0.3$, and 20 seropositive individuals were $9.5 \pm 7.5$. In the latter group the SI was inversely proportional to the frequency of labial herpes infections. The SI was not correlated with the level of anti-HSV antibody. The SI of JP was 1.1 and of SP 1.4, and that of the parents, who were both seropositive but not subject to recurrent herpes infections, was 2.0 and 1.9. Thus we have uncovered an impairment of HSVinduced lymphocyte stimulation in this fanily with severe recurrent herpes infections. As these individuals have no generalized defect in cellular immunity and all possess normal levels of anti-HSV antibodies, further qualitative evaluation of specific humoral and cellular antiviral immunity will be required to elucidate the mechanism of hyporesponsiveness.

507 ALTERED LYMPHOCYTE METABOLISM IN IMMUNE DYSFUNCTION. S.A. Feig, M. Das, and E.R. Stiehm. UCLA School of

The activities of certain lymphocyte ATP-producing enzymes have been measured in normal adults, term newborns, premature newborns and patients with malnutrition, in an attempt to correlate metabolic capacity with immunologic function. Pyruvate Kinase (PK) and Phosphoglycerate Kinase (PGK) activities were reduced in the lymphocytes of term newborns compared to adults (see Table). These deficiencies were more severe and accompanied by a deficiency of Adenylate Kinase (AK) in the lymphocytes of premature neonates. Reduced activity of $P K$ and PGK was also observed in the lymphocytes of all subjects with malnutrition while AK activity was variable. The severity of PK deficiency was directly correlated with the percentage of circulating thymus-derived lymphocytes ( $\mathrm{T}$-cells) in malnourished subjects. This correlation did not pertain to the level of PGK activity. Severe reduction of circulating $T$-cells was accompanied by normal $\mathrm{AK}$ activity while mild reduction of circulating $T$-cells correlated with marked ele vation of AK activity. There are developmental abnormalities in ATP-producing enzymes in lymphocytes that are reflected in patients with malnutrition. The data suggest a metabolic correlate to immunologic dysfunction and a potential alternate method for the identification and improved understanding of these conditions.

\begin{tabular}{|c|c|c|c|c|}
\hline \multirow{3}{*}{ Adults } & \multicolumn{4}{|c|}{$\mathrm{mi}$} \\
\hline & $\mathrm{N}$ & $\mathrm{PK}$ & PGK & $\mathrm{AK}$ \\
\hline & 50 & $\overline{22+7}$ & $415+95$ & $\overline{34+13}$ \\
\hline Ter & 60 & $15 \overline{+7}$ & $194 \mp 67$ & $35 \pm 17$ \\
\hline ture & 8 & $5+1$ & $71 \overline{+} 33$ & $15 \pm 5$ \\
\hline
\end{tabular}

NEUTROPHIL CHEMOTAXIS IN JUVENILE RHEUMATOID ARTHRI

508 TIS. Senih M. Fikrig, Juana C. Phillipp, Kamala Suntharalingam. Downstate Medical Center, $\frac{\text { Department }}{\text { Ded }}$ of Pediatrics, Brooklyn, New York.

Impaired polymorphonuclear (PMN) chemotaxis in adult rheumatoid arthritis have been reported. The present study was undertaken to study similar PMN function in juvenile rheumatoid arthritis (JRA). Chemotactic activity was generated from fresh serum by incubating with zymosan and chemotaxis was measured in modified Boyden's chambers. Final evaluation was made by taking the average measurements in five random fields a) of the distance traveled from the top of the filter to the furthest plane by at least two cells and b) of the number of neutrophils found on the lower surface of the filter. Similar measurements were made in filters obtained from control chambers without chemotactic factors. The difference between the two values was reported as final chemotaxis. The results demonstrated no difference in chemotaxis between the PMN of juvenile rheumatoid arthritic patients and proper controls.

\section{Chemotaxis}

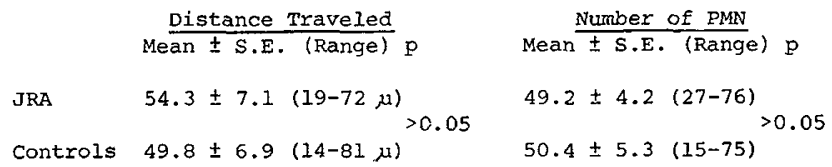


509 LEVAMISOLE: A SYNTHETIC AGENT WITH THYMOSIN-LIKE

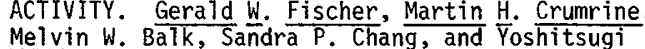
Hokama. (Spon. by James W. Bass) Trippler Army Medical Center, Department of Pediatrics, Honolulu.

Thymosin appears to have an essential role in the development and maturation of normal cell mediated immunity. Thymosin as presently available is a thymus extract and, therefore, variable in its activity and limited in its availability. These studies suggest that the synthetic agent levamisole (LMS) has thymosinlike activity by enhancing the maturation of cellular immunity. Tritiated thymidine studies demonstated that suckling rat spleen cells incubated with LMS have increased DNA uptake through a wide range of LMS concentrations varying from 0.5 to $25 \mu \mathrm{g} / \mathrm{ml}$. This effect of LMS is not observed in 2 to 3-week-old animals. Viral studies with Herpes simplex type 2 also demonstrated that protective cell mediated immunity was not present until the second to third week of life and that LMS was capable of accelerating this maturation process. Levamisole was not effective in further augmentation once immunity was functionally normal. New Zealand black (NZB) mice were also studied to determine if LMS could aiter the lymphocytes from these animals that are thymosin deficient. The administration of LMS in vivo to adult NZB mice significantly increased tritiated thymidine uptake of spleen cells cultured in vitro. These studies demonstrate that LMS has thymosin-like activity by enhancing maturation of cellular immunity in normal animals and in increasing immunologic activity in a thymosin deficient model.

\section{0}

DEPRESSED SPECIFIC CE LL-MEDIATED IMMUNITY TO CYTOMEGA LOVIRUS IN PREGNANCY. Lawrence D. Frenkel, David A. Fuccillo, Monroe M. Vincent, Joseph A. Bellanti. Georgetown University School of Medicine, Washington, D. C.; NIH; Microbiol. Assoc. Inc., Bethesda, Md.

The present studies were performed to study changes in specific cellmediated immunity (CMI) to cytomegalovirus (CMV) during pregnancy employing a specific lymphocyte-mediated ${ }^{51} \mathrm{Cr}$-release cytotoxicity microassay. The specific immune release (SIR) mean + SEM in 7 seropositive pregnant women was $13.7 \pm 4.3 \%$ as compared to $27.4 \pm 3.5 \%$ in 10 seropositive nonpregnant women $(p<0.05)$; in 2 seronegative pregnant and 10 seronegative nompregnant controi subjects no significant differences in SIR were observed. The SIR value in 6 nonpregnant CMV excretors was $23.0+5.7 \%$ which was not significantly different from the seropositive control values. The mean indirect hemagglutination inhibition (IHA) antibody titer to CMV was $1: 34$ in the 7 pregnant and $1: 30$ in the nonpregnant seropositive subjects $(p>0.05)$ and $1: 147$ in the 6 excretors $(\mathrm{p}<0.005)$. In 3 subjects studied $2-6$ months postpartum, 2 seropositive mothers demonstrated a rise in SIR from 8.8 to $22.2 \%$ and from 5.2 to $24 \%$, respectively; no postpartum changes were detected in the seronegative mother. These findings are consistent with the transient depression of CMI to rubella virus, which we have described previously, and provide further evidence for the greater susceptibility of the pregnant female for certain viral infections which pose risk to the fetus.

511

IMMUNODEFICIENCY AND AUTOTMMUNITY: CONTROLLED CLINICAL AND IMMUNOLOGICAL FAMILY STUDIES by Jan M. Friedman, Philip J. Flalkow, Starkey D. Davis, Hans D. Ochs and Ralph $\mathrm{J}$. Wedgwood (University of Washington, Departments of Medicine, Genetics and Pediatrics, and VA Hospital, Medical Service, Seattle).

In an attempt to define an hereditary factor important to the development of variable immune deficiency (VID), medical histories were obtained on 338 members of 12 families of patients with VID and 387 members of 12 control families. In addition, 143 VID relatives and 113 control relatives were tested for autoantibodies to thyroglobulin, thyroid microsomal antigen, and gastric parfetal cells, for rheumatold factor and antinuclear antibodies, and for serum IgG, IgM and IgA concentrations. A family history of immunodefictency was obtained in 4 cases, Including a set of affected 1dentical twins. The frequency of malignancy or clinical autoimmune disease did not differ between the VID and control families. Antibodies to thyrofd microsomal antigen and gastric parietal cells were significantly more frequent in the relatives of VID patients than in the controls. Abnormal serum 1mmunoglobuin levels were no more common in the VID famflies than in their controls. These data suggest that hereditary, as well as environmental, factors are important in the development of VID. No differences in the frequenctes of the autoantibodies tested or of abnormal levels of serum immunoglobulins were observed between the families of patients with $X-1$ inked infantile agammaglobulinemia and their controls. Relatives of patients with ataxia-telanglectasia showed a pattern of serological abnormalities similar to
512 HLA-D LOCUS TYPING: THREE EXCEPTIONS TO THE MEMPEL HYPOTHESIS. Richard A. Gatti, Wolfgang Leibold and Erik A.J. Svedmyr (intr. by Benjamin M. Kagan) Cedars logie der Tierärztlichen Hochschule, Hannover, West Germany; Karolinska Institute, Dept. of Tumor Biology, Stockholm, Sweden.

The Mempel hypothesis suggests that Tymphoid cells homozygous at the HLA-D locus (typing cells) will not stimulate lymphocytes vigorously in mixed lymphocyte cultures (MLC) if the latter are from a donor sharing at least one allele with the typing cell. While this hypothesis has proved generally true and has served as a basis for most D-locus typing until recently, we are aware of three situations which appear to violate this rule. First, while comparing the stimulatory effects of peripheral blood lymphocytes to those of a lymphoblastoid cell line from the same typing donor, results suggested that in the peripheral blood more than one antigenic system was responsible for stimulation, allowing apparentiy "false" positive responses to occur in four persons. Second, discordant responses were observed to lymphoblastoid typing 7 ines (TLCL) between three HLA/MLC identical siblings; false positive MLC responses were observed in the sibling with aplastic anemia. Third, one healthy random donor gives "typing responses" to TLCL cells representing at least four HLA-D alleles. A lacunar deficiency appears responsible for these false negative responses. Taken together, these observations suggest that it may be unwise to rely heavily upon lymphocyte responsiveness of the host to characterize a genetic locus influencing the immune response. Alternative approaches will be suggested.

513 HOST RESPONSE IN OTITIS PRONE CHILDREN, G. ScOt Giebink, Elaine L. Mills, John S. Huff, S.K. Juhn Department of Pediatrics \& Otolaryngology, Minneapolis, MN.

Forty-four children with persistent middle ear effusions (MEE) and/or recurrent acute otitis media were studied at the time of myringotomy and ventilation tube placement. Mean age was 4.5 years ( 6 mos. -10 years). The majority ( $53 \%$ ) of these children had their first episode of otitis prior to age 1 year; $72 \%$ prior to 2 years. Hearing loss ( $\geq 15 \mathrm{db}$ ) was detected in $97 \%$ of children prior to surgery. MEE was obtained from 54 of 62 ears: 16 samples were serous, 38 samples were mucoid. Aerobic cultures were positive in $41 \%$ of the effusions. $H$. influenzae were isolated from $38 \%$ of serous and $8 \%$ of mucoid effusions. Coagulasenegative staphylococci were found in $21 \%$ of mucoid MEE. M. pneumoniae, anaerobic bacteria and virus were absent in all MEE samples cultured for these organisms. Peripheral neutrophils showed normal phagocytosis and killing of bacteria in all 29 children studied. Neutrophil chemotaxis was abnormal (< $30 \%$ of the simultaneous control) in $12(29 \%)$ of 42 patients. Increased chemotaxis ( $>150 \%$ ) was found in 9 patients, 6 of whom had culture positive MEE.

The MEE/serum ratio of IgA was higher in mucoid (6.5:1) than serous (2.1:1) MEE $(p<.025)$. Children with high IgA ratios tended to have sterile MEE $(p<.10)$. IgG and IgM ratios were not different in mucoid versus serous samples. Serum immunoglobulins (IgG, IgA, IgM and IgE) were normal in all children.

514 VIETNAMESE INFANTS WITH PNEUMOCYSTIS PNEUMONIA. Wallace A. Gleason, Jr. and Stanford $T$. Roodman. (Introduced by A. E. MCE 1fresh). Departments of Pediatrics and Pathology, St. Louis University School of Medicine, St. Louis, Missouri.

To study the relationship between malnutrition and immunodeficiency in three Vietnamese infants with pneumocyst is pneumonia, thymus derived $(T)$ and bone marrow derived (B) 1 ymphocytes were quantitated. Lymphocytes obtained two months and eight months after recovery, were purified and studied by the erythrocyte rosette test and by immunofluorescence for surface immunoglobulins. When first tested, all patients were below the third percentile for height and weight. At that time, the patients had $39 \pm 4 \% \mathrm{~T}$ and $15 \pm 5 \% \mathrm{~B}$ cells, compared to $59 \pm 8 \%$ and $16.5 \% \mathrm{~B}$ for controls $(\bar{n}=19)$. When restudied, all patients were between the 5 th and 20th percentile for height and weight. $T$ cells increased to $59 \pm 6 \%$ and $B$ cells were $8 \pm 2 \%$. T cell deficiency may thus be a reversible factor in the immunodeficiency of malnutrition. 
515 ABNORMAL ADENINE METABOLISM IN SEVERE COMBINED DEAMINASE(ADA)ACTIVITY . Randall M.Goldblum, Frank C. Schmalstieg, Armond S.Goldman, J. Arly Nelson and Thomas M.Monahan. Depts.Pediatrics \& Human Biol.Chem .and Genefics, Univ.Texas Medical Branch, Galveston, Tex. \& Southern Research Inst „, Birmingham, Ala.

Some patients with SCID have a deficiency of ADA. Recent evidence suggests that this metabolic defect is causally related to the immune deficiency. Because of similar immunologic abnormalities in SCID patients with normal $A D A$, we have examined adenine metabolism in such patients,

A 10-month-old male with deficiencies in T and B lymphocyte functions had normal activities of nucleoside phosphorylase and 5 other erythrocyte enzymes in this pathway. Neutrophil $5^{2}$-nucleotidase was normal . Adenine, adenosine and adenine nucleotides were measured in plasma, urine or erythrocytes using ion exchange chromatography and a fluorescence assay. Lymphocyte nucleotides and CAMP were examined by high pressure liquid chromatography and protein binding assays. While adenosine levels were normal ,plasma adenine was elevated.Adenine nucleotides were increased in erythrocytes and lymphocytes as was CAMP in lymphocytes.

With the exception of normal adenosine levels, these values were similar to those we have found in SCID with ADA deficiency. This suggests that some patients with SCID and normal ADA have an undetermined enzymatic defect in purine metabolism which leads to an accumulation of adenine nucleotides and which may ultimately mediate their immune deficiency.

PHAGOCYTIC FUNCTION IN IMMUNE DEFICIENCY. E.R.Grimes,

516 S.G.Pahwa, R N Pahwa S A Schwartz, J o' Reilily, R.A. cer Center. Department of Pediatrics, NYC,N.Y. Sequential analysis of phagocytic cells was done in 5 immune deficient children: 4 with severe combined immunodeficiency (SCID) and 1 with Di George syndrome(DGS). All patients were in laminar flow isolation and were studied during transplantation procedures and in stages of de- and recontamination. Two were chronically infected, K.M. with M. avium and J.C. with cytomegalovirus.

Neutrophils (PMN) and monocytes (M) were assayed for chemotactic and bactericidal capacities, the results of which are summarized below as number abnormal/number tested.

\begin{tabular}{|c|c|c|c|c|}
\hline \multirow[b]{2}{*}{ NT } & \multicolumn{2}{|c|}{ CHEMOTAXIS } & \multicolumn{2}{|c|}{ BACTERICIDAL } \\
\hline & PYN & $M$ & PMN & \\
\hline M.R. & $6 / 8$ & $7 / 9$ & $6 / 7$ & \\
\hline J.C. ADA deficient & & $1 / 2$ & $0 / 2$ & \\
\hline K.M. B cell + & $4 / 7(3 \uparrow)$ & $1 / 6(1 \uparrow)$ & $0 / 5$ & \\
\hline M.W. B cell + & $0 / 3$ & $2 / 3$ & $0 / 3$ & \\
\hline 7 & $2 / 4$ & $2 / 3$ & $0 / 3$ & \\
\hline
\end{tabular}

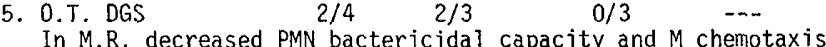
occurred prior to and during a period of aplasia. Abnormal PMN chemotaxis was present before and persisted during graft-vs-host disease, aplasia and peripheral eosinophilia. Impairment of chemotaxis was of a lesser degree in the 2 SCID's with detectable $B$ cells. Although defects were found in varying therapeutic and clinical situations, the findings suggest a primary abnormality in phagocytic cell function in some patients with SCID.

517 THE PROPHYLAXIS OF ALLERGY IN INFANTS DEBATE. Robert N.Hamburger and H.Alice Orgel. Univ. of Calif., San Diego, School of Medicine,Pediatric Department, Pediatric Immunology and Allergy Division. Since 1935 it has been claimed that the onset of allergic disease in early childhood could be delayed or prevented by providing a hypoallergenic environment before birth and during the first year of life in selected infants. Confirmations of this concept and refutations have appeared in the literature during the subsequent 40 years.

The discovery of the genetic control of serum IgE levels suggested that early-onset or infant allergy would not be amenable to environmental control. However, in a single blind study our preliminary data suggest that rigorous environmental controls including breast feeding, delay of DPm immunization and provision of a hypolilergenic environment do not alter the expected serum IgE levels but do alter the phenotypic expression, with a much lower number of infants with manifest allergic disease than expected.

In the first fourteen patients observed, the number of allergic infants expected (based upon serum IgE level and our previous study, J. Allergy Clin. Immunol.56: $296,1975)$ was five. To date only one baby has been diagnosed as having an allergic disease. If these proportions are maintained in the entire study group we may provide a definitive answer to the debate over the value of prophylaxis in childhood allergy.
SERUM IgD IN PREMATURE INFANTS. Douglas C. Heiner, 518 Kouji Tateno, Louis Kuehn, William Oh, and Bascom $F$. Anthony. UCLA School of Medicine, Harbor General

Hospital, Department of Pediatrics, Torrance, California.

IgD has recently been shown to be an important surface immunoglobulin of B-cells where it has no relationship to serum IgD levels. The function and amount of serum IgD have not been determined in small infants because of the limit of sensitivity of available techniques $(0.1 \mathrm{mg} / \mathrm{m} 7)$. A radioimmunoassay with 4- $\log$ greater sensitivity has permitted measurement of IgD in virtually all individuals. Adults tend to maintain consistent levels. Prospective study of 62 serums from 13 premature infants revealed no relation between cord serum $I g D$ and birth weight. Serum IgD decreased during the first month of life followed by a steady increase thereafter. Two infants developed bacteremia during the first week of life. These were the only ones showing a transient rise in IgD, indicating involvement of serum IgD in immune responses early in life.

\begin{tabular}{lccccccc} 
Age (Days): & $0-3$ & $4-14$ & $15-28$ & $29-56$ & $57-112$ & $113-181$ \\
\hline Non-infected \# & 9 & 9 & 8 & 11 & 8 & 9 \\
IgD (ng/ml): & 58 & 37 & 47 & 61 & 80 & 307 \\
\hline Bacteremic \# & 2 & 2 & 7 & 7 & 1 & \\
IgD (ng/ml): & 44 & 84 & 38 & 80 & 132 &
\end{tabular}

Preliminary studies have shown that it is feasible to measure

low levels of total serum IgD plus specific antibodies of the IgD variety by radioimunoassay on $0.1 \mathrm{ml}$ of serum using $125 \mathrm{I}$ anti-IgD $(F C)$. This will permit new insight into its immunobiological role.

STIMULATION OF PROIEIN SYNTHESIS (SPS) IN VITRO OF

519 LYMPHOCYTES FROM INFANTS IMMUNIZED WITH PNEUMOCOCCAI CAPSUIAR POLYSACCHARIDES (PCP) . V.M. Howie, J.L. Slover, Jr., and J.H. Ploussard. Huntsville Hosp. and Univ. of Alabama, SPMC Huntsville, Ala.

Employing the use of C14 leucine, SPS was measured in peripheral blood Iymphocytes (PBL) from infants immunized with PCP to prevent acute otitis media due to pneumococcus. PBL were cultured for 36 hours individually with PHA, PWM, ConA, or each of eight PCP. Group I had 22 patients receiving I.M. 25 ug of each PCP at 2-5 months. A booster was given 18 months later. Group II had 22 patients immunized at 18-24 months. Group III had 15 patients who received a saline placebo. SPS revealed no significant differences between groups for PHA, PWM, ConA thus indicating immmological similarity between groups. The mean stimulation index however for PCP induced synthesis was:

\begin{tabular}{rcc} 
& Pre-Immune & Post-Inmune \\
\hline I & 1.45 & 1.59 \\
II & 1.16 & 1.47 \\
III & 1.34 & 1.31 \\
\hline
\end{tabular}

The data suggest that administration of PCP in the first six months of life does not result in a decrease of specific lymphocyte reactivity, but rather in an increase of such activity. Hence the use of such vaccines to attempt to prevent meningitis, pneumonia, or otitis media should not be hampered by threats of inducing immune tolerance.

WEUTROPHIL AND MONOCYTE PHAGOCYTIC FUNCTION IN DOWN

520 SYNDROME. James R. Humbert, Roger M. Barkin, William L. Weston, Patricia A. DeArmey, Roberta Dustin. (Spon. by Charles S. August), Univ. Colo. School of Medicine, Depts. of Pediatrics, Medicine, and Biophysics/Genetics, Denver.

Increased susceptibility to infections is frequently noted in Down syndrome (DS). Host defense adequacy was thus investigated in 14 institutionalized DS children with regard to the phagocytic function of neutrophils (PMN'S) and monocytes (MC's). Controls consisted of 15 matched institutionalized patients and of 16 normal adults. MC and PMN phagocytosis and intracellular bactericidal activity towards staphylococcus aureus were simultaneously studied in an assay using lysostaphin to eliminate extracellular bacteria. Early phagocytosis by MC's was studied with the paraffin oil red o technique. Nitroblue tetrazolium (NBT) reduction by MC's and PMN's was determined quantitatively and histochemically. The only abnorma $M C$ function in DS was the paraffin oil red o phagocytosis. This dysfunction was also observed in institutionalized controls. PMN intracellular bactericidal activity was significantly impaired in both institutionalized groups, but this defect was not physiologically important. Cellular PMN phagocytosis was depressed in DS only. Opsonization of staphylococcus by the subjects' serum and PMN NBT reduction were identical in the 3 groups. These results suggest that defective $M C$ and PMN phagocytosis might contribute to the increased susceptibility to infections of some DS patients. Whether institutionalization alone causes these dysfunctions is open to question. 
521 COMPLEMENT ABNORMALITIES IN LIPODYSTROPHY. MOShe M. Ipp, Joe 0. Minta and Erwin W. Gelfand. Department of of Pathology, University of Toronto, Toronto, Canada.

Partial lipodystrophy (PLD) is frequent1y accompanied by hypocomplementemia. The serum of some of these patients contains an ill-defined anticomplementary substance which seems to have properties similar to the $\mathrm{C} 3$ nephritic factor (C3NeF) described in patients with membranoproliferative glomerulonephritis (MPGN). of seven patients with PLD studied, one had MPGN. Six had low C3 levels and impaired hemolytic activity by classical and alternative pathway assays. The remaining patient with PLD and three patients with total lipodystrophy had normal C3 levels. All ten patients had normal $\mathrm{C} 2, \mathrm{C} 4, \mathrm{C} 5$ and factor $\mathrm{B}$ levels. Five of the six hypocomplementemic sera contained a factor capable of cleaving $C 3$ in normal human serum. Partial purification of this factor showed it to be a heat stable $6.8 \mathrm{~S}$ pseudoglobulin which was eluted together with IgG following 1on exchange chromatography. Activity of this factor did not require $\mathrm{C2}, \mathrm{C4}$, or $\mathrm{CaH}$, but did require factor $\mathrm{B}$ and $\mathrm{MgH}$, indicating alternative pathway dependence. Properdin was not required for its activity. It was concluded that (a) only the partial form of lipodystrophy is associated with hypocomplementemia (low C3), (b) not all patients with PLD have a low $\mathrm{C} 3$, (c) the low $\mathrm{C} 3$ may not be associated with a circulating anticomplementary factor and (d) paralle1 studies using serum from a patient with MPGN showed that the anticomplementary factor in PLD has similar immunochemical and physicochemical properties to the C3NeF of MPGN.

Supported by the MRC of Canada, MA-4875 and MA-5063.

CLINICAL, IMMUNOLOGIC AND GENETIC FINDINGS IN C4

522 DEFICIENCY. Charles G. Iackson, Hans D. Ochs, Jane Univ. of Wash. Schaller, Bruce C. Gillliland, Ralph J. Wedgwood.

A 5 -year-old boy presented with a scaly erythematous rash, recurrent fevers, transient arthritis, anemia, hepatosplenomegaly, malaise and weight loss. Following a Salmonella B enteritis he developed diffuse proliferative glomerulonephritis. Antinuclear antibodies and DNA binding were elevated and serum $C 3$ was temporarily depressed. Evaluation of the complement (C) system consistently demonstrated complete absence of $\mathrm{C} 4$, both immunochemically and functionally, and lack of hemolytic C activity. The alternate pathway was intact. Reduced opsonization of zymosan by the patient's serum was corrected by adding $C 4$. Antibody response to bacteriophage $\emptyset x 174$ was impaired, immunologic memory was absent and specific IgG could not be detected. However, antibody titers to Salmonella 0 antigen, a polysaccharide, were adequate. A decreased percentage of peripheral blood lymphocytes formed Erosettes, and in vitro lymphocyte transformation to mitogens and allogeneic cells was depressed. Incubation of the patient's serum with endotoxin did not generate chemotactic factor. Eight family members including both parents are heterozygous for $\mathrm{CA}$ deficiency with half normal C4 levels. Linkage of the C4 deficient. gene to the major histocompatibility loci, $\mathrm{PGM}_{3}$, properdin factor $B$ and glyoxalase-I could be demonstrated further strengthening the importance of chromosome 6 in immune regulation. Abnormal

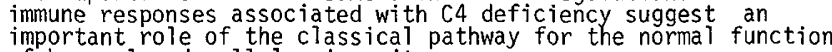
of humoral and cellular immunity.

523 CHEMOTACTIC MIGRATION OF NEUTROPHILS UNDER AGAROSE: EFFECT OF VARIOUS FACTORS. T. Jacob John and Otto F. Sieber, Jr. (Spon, by Vincent A. Fulginiti) University of Arizona Medical Center, Department of Pediatrics, Tucson, Arizona.

Human peripheral blood neutrophil leukocyte chemotaxis was tested in a new and simple technic. The cells and chemotactic attractant were placed in wells cut $3 \mathrm{~mm}$ apart in an agarose medium. The cells moved rapidly towards the attractant, but not towards control substances such as bovine albumin and tissue culture medium. The speed of migration was calculated from the mean distance travelled by the 10 fastest cells at time intervals such as $2,4,6$ and 8 hours. The speed of migration was unaffected by 1) the presence or absence of mononuclear cells or non-excessive numbers of red blood cells in the neutrophil cell suspension; 2) cell concentrations between $1 \times 10^{6}$ and $1 \times 10^{8}$ per $\left.0.1 \mathrm{ml} ; 3\right)$ a delay of upto 24 hours between collection of blood and testing; and 4) small variations of the distance between the wells. Freezing and thawing fresh-frozen human serum upto 5 times did not diminish its ability to attract neutrophils. The addition of endotoxin to serum was not found to enhance the speed of migration. Factors that retarded migration wexe 1) agarose medium older than one day; 2) cell concentrations below 1 x 106/0.1 m1; 3) cells older than one day; and 4) distance between the wells of over $4.5 \mathrm{~mm}$. This test is simple and reproducible, requires no special equipment or training and may be done on 2-3 ml of blood if neutrophil counts are not too low. Thus it is eminently suited for routine clinical application in evaluating patients with increased susceptibility to infection.
THE EFFECT OF STEROIDS ON THE IMMUNE FUNCTIONS IN

524 RHESUS MONKEYS. T. Jacob John, Marlene Stahl, Carla Medical Center, Department of Pediatrics, Tucson, Arizona

The effect of cortisone acetate and dexamethasone on the nonspecific and specific imnune functions especially those against vaccinia virus infection, was investigated in groups of young adult rhesus monkeys. Cortisone, in doses of $50-125 \mathrm{mg} / \mathrm{Kg} / \mathrm{day}$ produced marked neutrophilic leukocytosis. However, dexamethasone, in doses of $5-15 \mathrm{mg} / \mathrm{Kg} /$ day did not produce neutrophilic leukocytosis. Among the various analogs of cortisone, this appears to be the only agent which does not cause neutrophilic leukocytosis. Both drugs reduced the proportions of neutrophils reducing nitroblue tetrazolium dye, the numbers of circulating lymphocytes and the thymidine incorporation of lymphocytes stimulated with phytohemagglutinin, pokeweed mitogen and vaccinia virus antigen. When vaccination was performed while monkeys were on dexamethasone, their cutaneous reactivity and the thymidine incorporation of their lymphocytes in response to stimulation with vaccinia virus antigen, were both suppressed. Moreover vaccinia virus neutralizing antibody production was delayed in them and their peak antibody levels were significantly lower than in other vaccinated animals not treated with steroids. These results indicate that glucocorticoids may have profound effects on several non-specific and specific immune functions, including the numbers and functions of neutrophils and lymphocytes, cell mediated immunity and antibody production.

525 MODIFICATION OF NEUTROPHIL CHEMOTAXIS BY AMNIOTIC FLUID. Abdu? J. Khan, Leonard Glass, Chia Chang, Parvin Khan, Ethan Levy and Hugh E. Evans, Dept. of Pediatrics, Jewish Hospital and Medical Center of Brooklyn, N.Y. The immunologic properties of amniotic fluid (AF) are not weit understood. We have assessed the effect of 8 term AFs on chemotactic indices (CI) of neutrophils (PMNs) obtained from 8 nonpregnant adults. CIs were determined utilizing a modified Boyden's technique in which PMNs were placed on a 3 Mmicropore filter in the upper chamber containing Hank's solution $(H)$, and a chemotactic mixture of endotoxin (E), serum $(S)$, and $H$ in the lower chamber. CIs with $\mathrm{H}$ alone in both chambers represented random migration (RM). The effect of AF was evaluated by 1 ) adding $A F$ to the upper chamber 2) adding $A F$ instead of $E$ to the chemotactic mixture 3) placing only $H$ and $A F$ in the lower chamber. The results represent means and (standard deviations).

$\begin{array}{llllll}\text { Upper } & H & H+A F & H & H & H \\ \text { Lower } & S+E+H & S+E+H & S+A F+H & H+A F & H \\ \text { CIs } & 340(115) & 219(102) & 172(50) & 81(30) & 60(27)\end{array}$

CIs $\quad 340(115) 219(102) \quad 172(50) \quad 81(30) \quad 60(27)$ chamber $(p=0.03)$. When added to $S$, they generated chemotactic activity which was less $(p<0.01)$ than that generated by $E$ but higher $(p<0.07)$ than AF and $\mathrm{H}$ or $\mathrm{H}$ alone in the lower chamber. The activity of $\mathrm{AF}+\mathrm{H}$ was similar to RM. It may be speculated that AF in association with serum chemoattracts PMNs at the onset of amniotic infection syndrome, and later on keeps them at the site by inhibition of chemotaxis.
526 FTIOTPEXATE IN TIE: TREAT TENT OE STEROTI-PESTSTANT CHILDHOON DERATn: ryostTIS. Robert B. Klein, Gary S. $\frac{\text { nachelefsky, Thoinas J. Fischer, liarold E. Paulus, }}{\text { Stiehm. UCT.A School of fiedicine, nent. of Pediatrics, }}$ L. Nichard Stiehm. UCT.A

: iethotrexate (ITX) has been used successfully in adults vith steroid-resistant dermatomyositis $(n i)$. Te used imry in 5 children with $D W$ who vere either resistant to steroids after 3 months of therapy or who developed unacceptable side effects. Niagnosis of $\mathrm{D}$ : was documented by clinical course, physical exam, serum muscle enzyme elevations, muscle hiopsies and electromyograms.

Two girls, ages 12 and 4 , had minimal resnonse to daily nrednisone $(2 \mathrm{mg} / \mathrm{kg})$ given for 6 months. Toth developed marked cushingoid changes with striae; the former also develoned hypertensive encephalopathy and seizures. A 12 year old male improved on steroids but developed bilateral cataracts; decreasing steroids caused relapse of $D^{\prime}$. A 6 year old male developed respiratory failure while on steroids and a 14 year old male had persistent muscle weakness despite steroid therapy for over 3 years.

All patients were treated with iTx (1 mg/kg) given intravenously at weekly intervals; these intervals were progressively lengthened as improvement ensued. :larked clinjcal and laloratory improvement occurred within 4 weeks; by 3 months 2 of 5 patients were free of symptoms while the others were markedly improved. Prednisone was then tapered to $15-20 \mathrm{mg}$ q.o.d. The only side effect of ITX was transient nausea. Two patients are now off ITX after 1 year and remain well. itis. 
$\mathbf{5 2 7}$

CRYOGLOBULINEMIA AND AUTOIMMUNE HEMOLYTIC ANEMIA IN A $2 \frac{1}{2}$ YEAR OLD CHILD. Roberto R. Kretschmer, Armando R. Orlina, Phyllis J. Unger, Ameeta Bamzai and Samue1 P. Gotoff. Div. Immunology, Dept. Peds., and Blood Res.Lab. Michael Reese Med. Ctr., Pritzker Sch. Med., Univ. of Chicago.

A $2 \frac{1}{2}$ year old black male with autoimmune hemolytic anemia, diffuse hypergammaglobulinemia, rheumatoid factor and antinuclear antibody activity, hypocomplementemia and depressed cell mediated immunity was studied. Anti-e, anti-i and anti-d1 antibodies were found in the serum. Anti-e antibodies of the IgG class were also eluted from the red blood cells.

The serum contained $15 \mathrm{mg} / \mathrm{d} l$ of cryoglobulin, an unusual finding in this age group, which upon isolation and fractionation on Sephadex G-200 revealed polyclonal IgG and monoclonal (lambda) IgM. The former had anti-e antibody activity, but no anti-i or anti-d1 antibody activity. The monoclonal IgM of the cryoprecipitate was a rheumatoid factor. The rheumatoid factor remaining in the serum after cryoprecipitation reacted against a panel of human anti-e IgG antibodies, and against Rh-Null cells coated with one source of anti-dl antibodies. It did not react against anti-D IgG antibodies. Thus a rheumatoid factor of limited specificity (anti-e and anti-d1) was demonstrated, some of which cryoprecipitated (anti-e)

The patient showed signs suggestive of immune complex disease while the cryoglobulin was present in his serum. His clinical condition, the cryoglobulin and most of the immunohematological abnormalities disappeared following high dose steroid therapy.

528 CHEMOTAXIS OF HUMAN CORD BLOOD MONOCYTES. Roberto R. Kretschmer, Prudence Stewardson, Cynthia Papierniak and Samuel P. Gotoff. Div. Immunology, Dept. Peds., Michael Reese Med. Ctr., Pritzker Sch. Med., Univ. of Chicago. Chemotaxis of human cord blood monocytes and production of chemotactic factors by cord blood lymphocytes (LDCF) were evaluated in this study. Monocytes and lymphocytes were obtained by Ficoll-Hypaque gradient separation. Chemotaxis was measured with a modified Boyden's chamber technique using supernatant fluid from phytohaemagglutinin stimulated 1ymphocytes as the chemotactic attractant.

Random mobility of cord monocytes was comparable to that of adult monocytes. Unstimulated lymphocytes, particularly cord lymphocytes, produced some LDCF as evidenced by the brisk chemotaxis of adult monocytes towards supernatants of unstimulated cord lymphocytes. Yet, when cord monocytes were exposed to supernatants of unstimulated cord lymphocytes, inhibition of cell migration was observed.

Chemotaxis of cord or adult monocytes towards adult LDCF was normal, and chemotaxis of adult monocytes was also normal with cord LDCF as the attractant. In contrast, chemotaxis of cord monocytes towards cord LDCF was significantly diminished when compared to that of adult monocytes attracted by adult LDCF.

These results suggest that abnormal cord monocyte chemotaxis results from the production of inhibitory factors by cord lymphocytes, for which only cord monocytes have appropriate receptors. These inhibitory factors may contribute to the slow establishment of delayed hypersensitivity reactions in newborn
infants.

529 ERAFT VS HOST REACTION (GVH) AFTER TRANSPLANTATION OF LIVER CELLS TO A PATIENT WITH SEVERE COMBINED IMMUNODEFICIENCY DISEASE (SCID). James $\mathrm{V}$. Lustig, Christian H.L. Rieger and Richard M. Rothberg. University of Chicago Hospitals, Dept. of Peds., Chicago, I1. 60637

A $5 \frac{1}{2}$ month old boy with SCID had no mixed leucocyte reaction (MLR) compatible bone marrow donor. He was therefore given an intraperitoneal injection of $3.7 \times 10^{6}$ hepatic lymphoid cells from a $1.7 \mathrm{~cm}$ long embryo (4-5 weeks gestation). Prior to trans plantation, T-rosettes, MLR and 1 ymphocyte response to PHA were absent. B-cells, absent at birth, increased to $50 \%$ of circulating lymphocytes but there was no significant immunoglobul in production prior to transplantation. Red cell adenosine deaminase was normal. Three weeks after transplantation, the patient developed fevers to $39^{\circ} \mathrm{C}$, diarrhea, eosinophilia $\left(9500 / \mathrm{mm}^{3}\right)$, alopecia, edema and a maculopapular rash. There was no hepatosplenomegaly or alteration of liver enzymes. Diarrhea and edema resolved without treatment. The rash, alopecia and eosinophilia improved but have fluctuated since. A skin biopsy during one of these episodes demonstrated vacuolization and spongiosis of the epidermal basal layer with a perivascular lymphocytic infiltrate consistent with $\mathrm{GvH}$. With the onset of the $\mathrm{GvH}$, T-rosettes appeared and eventually rose to $18 \%$. By one year after transplantation, the patient had developed a normal MLR and lymphocyte response to candida, and a significant increase in responsiveness to PHA and pokeweed mitogen. There is no evidence of B-cell function. This data suggests that even the most immature lymphoid cells technically obtainable are capable of participating in a $\mathrm{GvH}$ reaction.
SECRETORY ANTIBODY FOLLOWING RESPIRATORY SYNCYTIAL

530 VIRUS INFECTION IN INFANTS. Kennech McIntosh, Helen Masters, Inara Orr, Ru K. Chao and Roger M. Barkin.

Univ. of Colo. Sch. of Med., Dept. of Ped., Denver, Colo.

Recurrent respiratory syncytial virus (RSV) infections are common, and this lack of natural immunity is likely to impede vaccine efficacy. The immunologic events following natural infection are therefore of interest. In particular, the role of secretory antibody is noi defined. We therefore obtained serial virus cultures and nasal secretions in 51 children under 6 months old hospitalized with RSV infections. Secretory neutralizing (neut) activity response ( $23 x$ measured by plaque reduction) was seen in only $9(18 \%)$, whereas a fluorescent-staining $(f-s)$ antiRSV IgA response $(>4 x)$ was found in $31(61 \%)$. There was no correlation in individual secretions between neur activity and $f-s$ anci-RSV IgA level. Neut activity was often $(44 \%)$, and $f-s$ anit IgA rarely $(2 \%)$ found in acute specimens.

Among 30 children where cessation of virus shedding was documented and a secretory specimen available 1-5 days laier, 21 $(70 \%)$ developed secrevory $f-s$ anti-RSV IgA. Age was not a significant factor in the incidence or speed of this response.

In infants under 6 monichs secretory IgA antibody to RSV commonly appears during or shortly after infection. This antibody is, however, usually non-neutralizing. Conversely, secrecory neutralizing activity does not appear to be IgA ancibody. The non-functional (i.e. non-neutralizing) nature of this antibody may be related to the relative lack of postinfection immunity in young infancs.

\section{1} SURVEY OF CHILDREN FOR OCCULT RHEUMATOID FACTORS John J. Miller, III, Lynne 01ds-Arroyo, Toru Akasaka, and John H. Vaughan. Children's Hospital at Stanford, Department of Pediatrics, Stanford University, Stanford, CA., and Scripps Clinic and Research Foundation, La Jolla, CA.

occult antibodies to IgG (anti-IgGs, rheumatoid factors) have been reported in juvenile rheumatoid arthritis (JRA). We have studied the incidence and diagnostic usefulness of occult antiIgGs with 2 procedures. Glutaraldehyde-aggregated Cohn fraction II globulin (FII) adsorbed equal amounts of globulin from sera from normal and JRA children and only distinguished sera from latex fixation (LF) positive ( + ) adults. Sepharose-bound FII in columns was used to study sera from 52 children with JRA, 5 normal children, 5 adults with RA and 2 normal adults. Acid eluates of the columns produced LF $(+)$ slide tests with sera from all adults, from 2 of 5 normal children, and from 27 of 52 children with JRA. This activity was labile, and tube LF assays were (+) in only 10 of 44 neutralized and concentrated eluates. Protein was adsorbed to Sepharose-bound FII from all sera, but 11 children with JRA had increased amounts, similar to those from LF ( + ) adults ( $>0.2 \%$ of serum protein), and greater than those from other children. These were from 1 of 3 with active systemic JRA; 8 of 26 with active polyarticular JRA, 2 of 8 with inactive polyarticular JRA, 0 of 2 with inactive systemic, and 0 of 13 with pauciarticular JRA. Of the 11,7 were already known to have LF $(+)$ serum. Increased levels of occult anti-IgGs may define a subgroup, but they distinguish only a small proportion of children with JRA from normal children and are not useful for diagnosis.

\section{DISORDERS OF HUMAN NEUTROPHIL MOVEMENT.M.E. Miller Pediatrics. Los Ang. Myers. Harbor Ge}

Primary defects of the cell membrane are thought to underlie some of the intrinsic abnormalities of human polymorphonuclear leukocyte(PMN) movement.Confirmation of this hypothesis has, however, been limited by available methodology. The studies now reported utilize cell elastimetxy in demonstrating the first evidence for abnormal membrane function in a number of intrinsic abnormalities of PMN movement. Two groups of studies were performed. In the first group, deformability of PMNS from subjects with a variety of recognized defects of PMN movement were measured.Results were expressed in $\mathrm{cmHg}$ negative pressures required to aspirate PMNS into 3-5u micropipettes. Simultaneous measurements were also made of chemotaxis(Boyden chamber) and random mobility (capillary tube). Subjects studied included 2 children with the "lazy leukocyte syndrome", 2 brothers with a familial movement defect, a child with an isolated defect of chemotaxis but normal random mobility, and patients with diabetes mellitus, both on and of insulin. In all cases,PMNS which were deficient in movement also showed markedly decreased deformability-i.e. had significantly more rigid membranes than normal PMNS $(p<.001)$. In the second group of studies, normal PMNS were measured in each of the three assays under a variety of conditions known to alter membrane deformability.Membrane deformability is $\mathrm{Ca}^{2+}$ dependent and decreased by glycolytic inhibitors. PMNS so treated were also found to have decreased chemotaxis. The data, thus, correlate membrane deformability with cell movement. 
533 DECREASED OPSONIC AND CHEMOTACTIC ACTIVITIES IN SERA OF POST-BURN PATIENTS AND PARTIAT OPSONIC RESTORATION WITH PURIFIED PROPERDIN AND PROPERDIN CONVERTASE.M.E. Miller,G. Nathenson and R.E. Spitzer.Harbor General Hospital, Montefiore Hospital and Upstate Medical Center;Departments of Pediatrics.Los Angeles, New York City, and Syracuse.

Bacterial infections remain the major cause of death in burn injury. Indirect evidence has supported an etiologic role for the alternate complement pathway. We have studied, over the past three years, serially collected sera from 12 patients with $1^{\circ}$ to $3^{\circ}$ burns involving from $20 \%$ to $85 \%$ of body surface. Mean opsonic (towards baker's yeast) and chemotactic activities were significantly decreased ( $p<.001)$ in all patients, for periods as long as 6 weeks post burn. Serially collected samples from three patients were assayed for a number of complement parameters, including $\mathrm{C} 3, \mathrm{C} 4$, C3-9 hemolytic activity, Factor B, properdin(P) and properdin convertase $(\underline{\mathrm{PC}})$. All factors were decreased for the first 3-4 days post burn. C3, C4, C3-9 hemolysis and Factor B then returned to normal, however, while $\mathrm{P}$ and $\mathrm{PC}$ remained at levels less than $50 \%$ of normal ( $P<.001$ ) for up to $4-6$ weeks. The addition of either purified $P$ or purified PC to opsonically deficient sera resulted in significant ( $p$ r.001), but not total restoration of yeast opsonic activity. We conclude: 1)Patients with severe thermal injury have significant and persistent decreases in serum opsonic and chemotactic activities;2) The duration of these abnormalities correlates with persistent decreases of $P$ and PC; 3) Since the addition of $\mathrm{P}$ or PC partially reconstitutes opsonic activities, a significant defect of the alternate pathway is suggested. PURPURA (TP). Nasser Movassaghi, Sanford L. Leikin. Geo. Wash. Univ. Schl. Med., Dept. Child
latren's Hosp. Nat. Med. Ctr., Wash., D.C.

Develop.; Children's Hosp. Nat. Med. Ctr., Wash., D.C.

Childhood TP is due to shortened platelet survival of unknown cause. The serotonin release test (SR) has been shown to detect platelet antibody. 31 children with TP were studied using SR and platelets from a panel of jonors with different HLA types (P) and autologous platelets after recovery (A). The test was done with sera obtained in the thrombocytopenic phase in all patients, in partial recovery in 4 and after complete recovery (CR) in 13. The thrombocytopenic sera were positive in 52 \% of the patients with $P$, in $72 \%$ with $A$ and in $89 \%$ with $P$ or $A$. Although many of the positive SR's were with platelets from panel donors which shared one or more HLA antigens with the patients, a definite pattern in relation to the HLA system could not be established. $\mathrm{CR}$ occurred in $87 \%$ of patients whose sera were $\mathrm{P}$ positive at diagnosis. Post-CR sexa from all patients were negative with $P$ and $15 \%$ were positive with $A$. Of 4 patients in partial recovery one remained negative and 3 positive with either $P$ or $A$. The results of this study indicate that childhood TP is an autoimmune disorder caused by the appearance of platelet antibody, its presence at diagnosis does not have prognostic significance and the antibody usually disappears after CR. Since some autoimmune disorders are associated with an increased frequency of cextain HLA antigens, the HLA type of 21 patients was obtained. The frequency of $\mathrm{w}-27$ was greater than expected $(0.24$ vs. 0.08 , $\mathrm{p}<.05)$; however, when correlated for the number of specifici$p<.05)$; however, when correlated for the number of
ties examined these differences lacked significance.

DELAYED CUTANEOUS HYPERSENSITIVITY IN CONTRO

535 CHILDREN. Aurea I. Munoz and David Limbert. (Intr. by Antonio Ortiz). Dept. of Ped., San Juan City Hosp., P. R. Med. Center

Tests for delayed cutaneous hypersensitivity were done on a group of patients, excluding those with conditions known to impair cellular immunity, in order to assess the normal pattern of reactivity at various ages. One hundred-thirty infants $0-11$ mos old (Group IX and $164 \mathrm{pts} 1-5$ yrs old (Group II) were skin-tested with Candida (C) $1: 100$, Streptokinase (S) (100 U in $0.1 \mathrm{cc}$ ) and $\mathrm{c} 1: 10$ in various combinations. There was a significantly greater frequency of induration (I) reactions $5 \mathrm{~mm}$ or more in diameter, and erythema reactions $10 \mathrm{~mm}$ or more, in Group II than in Group I. The frequency of such reactions in the entire group was greatest with $\mathrm{C} 1: 10$ and comparable with $\mathrm{C} 1: 100$ and $S$. Induration reactions less than $5 \mathrm{~mm}$ decreased sharply in fxequency after age 2 , but still occurred in $31.1 \%$ of $3-5$ yr old pts tested with $\mathrm{C} 1: 100$ and $25.8 \%$ of those tested with $\mathrm{S}$. In the entire group, a similar proportion of pts reacted with less than $5 \mathrm{~mm}$ I to both $\mathrm{C} 1: 100$ and $\mathrm{S}(30.2 \%)$ as with $5 \mathrm{~mm} \mathrm{I}$ or greater to both antigens $(27.9 \%)$. Delayed cutaneous hypersensitivity is a function of age; an appreciable number of normal infants and young children react to commonly employed antigens with less than $5 \mathrm{~mm} \mathrm{I}$, the numbex decreasing progressively with age.
FOUR YEAR SURVIVAL AFTER THYMUS TRANSPLANT IN COMBINED

536 IMMUNODEFICIENCY (CID). Stephen Murphey, James Nagel, Michael Facktor, David Gitlin and Philip Fireman. Univ. of Pittsburgh Sch. of Med., Dept. of Ped., Pittsburgh, Pa. Thymic dependent lymphocyte function in CID has been restored with a fetal thymus transplant but humoral antibody function has not. An 18 month old male presented with recurrent pulmonary infections, impaired growth, profound lymphopenia, deficient serum IgG and IgA and elevated IgM. Delayed hypersensitivity skin tests to Candida, tetanus and SK-SD were negative as were in vitro lymphocyte responses to mitogens. Lymph node biopsy was compat ible with severe CID. At 23 months of age a 16 week male fetal thymus was transplanted into the rectus abdominis muscle. Delayed skin tests to Candida became positive at 2 weeks post transplant and to tetanus at 3 months, but lymphopenia and in vitro responses to mitogens were variable. Repeated at tempts at DNFB sensitization have been unsuccessful. Now, 4 years post transplant, delayed hypersensitivity skin tests were positive. Spontaneous T-rosette formation was $45.7 \%$. In vitro lymphocyte responses to PHA, pokeweed and MLC were normaI. Serum inimunoglobulins have shown a gradual increase of $\operatorname{IgG}(750 \mathrm{mg} \%)$ and IgA $(187 \mathrm{mg} \%)$ and reduction of IgM $(37 \mathrm{mg} \%$ ). Isohemagglutinins were absent. Specific antibody to a variety of antigens including diphtheria, tetanus, typhoid, octavalent pneumococcal polysaccharide and influenza remain deficient and exogenous gamma globulin therapy has been continued. These data indicate that reconstitution of thymic dependent immunological function in CID can be achieved with a fetal thymus transplant. Survival of this
patient, however, is still dependent upon exogenous IgG therapy.

537 DEVELOPMENT OF TETANUS AND DIPHTHERIA IGE ANTIBODIES AFTER IMRUNIZATION. James Nage1, Dianna Svec, Tomi Waters and Philip Fireman. Univ. of Pittsburgh Sch. of Med., Dept. of Ped. Pittsburgh, $\mathrm{Pa}$.

Tetanus and diphtheria IgE and IgG antibodies were measured by radioallergosorbent test (RAST) and sheep cell hemagglutination respectively in 39 selected individuals (9 to 57 years) 10-21 days after booster immunization with alum precipitated tetanusdiphtheria toxoids. These individuals had no severe adverse reactions to this or prior immunizations. Tetanus IgG increased from a geometric mean antibody titer of $8 \times 10^{-2}$ to $8 \times 10^{-4}$ following immunization. Tetanus IgE increased from a mean $\%$ bound radioactivity of $2.0+0.2$ (pre) to $3.1+0.6$ (post): this increase was not statistically significant $(P>\overline{0} .1)$ but 2 of 17 atopic subjects increased tetanus IgE greater than 3 standard deviations (S.D.) above the mean. Diphtheria IgG antibody increased from a geometric mean antibody titer of $2 \times 10^{-2}$ to $1.3 \times 10^{-3}$ following immunization. Mean diphtheria IgE increased significantly ( $P>0.01$ ) from $1.3+0.1 \%$ activity (pre) to $2.9+0.7 \%$ (post): one non-atopic and two atopic subjects had increases greater than 3 S.D, above the mean. There was no correlation of these tetanus and diphtheria IgE responses to elevated total serum IgE greater than $200 \mathrm{ng} \%(\mathrm{P}>0.1)$ or pre-existing ragweed IgE antibody $(P>0.1)$. Mean diphtheria IgE antibody rise was greatex in atopics than non-atopics $(P>0.05)$ but tetanus $\operatorname{IgE}$ responses were not $(P>0.1)$. These data document the development of diphtheria and tetanus $\operatorname{IgE}$ antibodies after normal immunization and has provided an investigative model to study the synthesis of IgE antibodies in certain atopic and non-atopic individuals.

538 DIPHTHERIA TOXOIDS. Gerald Nathenson, $\frac{\text { Steven Litwin, }}{\text { Med., Montefiore }}$ Ctr.,Dept. Ped.; Cornell Med. Coll., Dept. Med., New York, New York In response to certain antigens, antibodies of restricted specificity have been identified in the human, as for example,the kappa (light chain) $\gamma \mathrm{G}_{2}$ (heavy chain) homogeneous population induced by levan. Our analysis of antibodies reactive to the toxoids of tetanus and diphtheria suggests a restricted response in antibody production to each of these antigens. Purified antibodies from specimens with elevated hemagglutination titers to tetanus (8) and diphtheria (4) were prepared by precipitation with the appropriate toxoid, then antibody separation with an acid glycine buffer. The purified specimens yielded only IgG proteins in concentrations of 160 to $500 \mathrm{\mu g} / \mathrm{ml}$. Though some of the tetanus specimens were derived from cord sera, in none of the total group were antibodies found in the IgA and IgM classes. Utilizing immunoelectrophoresis against heavy chain subclasses, genetic markers and kappa and lambda quantitations, specificity to the kappa $\gamma G_{1}$ antibody was established for both tetanus and diphtheria, since lambda light chains and other heavy chains were absent. Control experiments with three normal sera reacted directly with acid glycine buffer at $Y$ globulin concentrations similar to those of the purified tetanus and diphtheria preparations demonstrated persistence of all $\gamma G$ subclasses, both light chains, as well as IgA. IgM globulin was not found however; thus it is not possible to ascribe significance to the absence of IgM in the purified antibody preparations. The data tend to support the selective production of tetanus and diphtheria on the kappa $\gamma \mathrm{Gl}$ molecule. 
539

NON-SPECIFIC LYMPHOCYTE EFFECTOR FUNCTION IN IMMUNODEFICIENCY PATIENTS: MITOGEN-INDUCED CELLULAR CYTOTOXICITY (MICC) AND ANTIBODY-DEPENDENT CELLULAR CYTOTOXICITY (ADCC). David L. Nelson, Warren Strober, Andrew V. Muchmore, and R. Michael Blaese. NCI, NIH, Bethesda.

We have recently shown that T-cells mediate phytohemagglutinin (PHA)-induced MICC while sIg negative lymphocytes with receptors for IgG-FC (K-ce11s) mediate ADCC. In patients with hypogammaglobulinemia $(a \gamma)$ and ataxia-telangiectasia, MICC was normal in spite of profound functional $\mathrm{T}$-cell defects in other in vivo and in vitro (including PHA-induced lymphocyte proliferation) assays. $\overrightarrow{\mathrm{ADC}} \overline{\mathrm{C}}$ was variable and even increased in some patients; the latter a sub-class of ay patients with normal percentages of $F \mathrm{C}$ receptorbearing but absent $\mathrm{slg}$ bearing lymphocytes. Patients with the Wiskott-Aldrich syndrome and selective IgA deficiency had generally normal MICC and ADCC. A patient with intestinal lymphangiectasia had enhanced MICC and diminished ADCC by chylous effusion lymphocytes relative to peripheral blood lymphocytes suggesting that MICC effector cells are selectively lost in this disease. In general, MICC correlated poorly with other in vitro and in vivo tests of $\mathrm{T}$-cell function but was related to the percentage of 1 ymphocytes rosetting with sheep RBC (T-cells). Hence the capability of mediating MICC is a function unaffected by perturbations of lymphocytes resulting in $\mathrm{T}$-cell deficiencies identified by other techniques. Our finding of variable ADCC effector cell function demonstrates that $\mathrm{K}$-cells are also subject to abnormalities in immunodeficiency patients. Analysis of immunodeficiency patients with tests of non-specific cytotoxic effector function provide unique insights into defects of lymphocyte function in man.

540 JÖGREN'S SYNDROME WITH COLLAGEN VASCULAR DISEASE IN CHILDREN. Michael E. Norman, Balu H. Athreya, Nabih H. Abdou, and Mary Ann South. Departments of Medicine and Pediatrics, University of Pennsylvania School of Medicine, and The Children's Hospital of Philadelphia.

sjögren's syndrome has rarely been described in children, but we report 2 cases recently seen in girls. Collagen vascular disease has been associated with Sjögren's syndrome but glomerulonephritis (GN) is rare; our patients both have collagen vascular disease and GN. The diagnosis was established by the presence of sicca complex, schirmer test, sequential parotid scintigraphy, and lip biopsy in both patients, and sialography and parotid biopsy in 1 patient. Onset of symptoms was at 7 and 11 years. Both patients had increased immunoglobulins, positive ANA, DNA antibodies, LE cells, and cryoglobulinemia. One girl had normal urine, renal function, and complement profile, but mesangial GN with $\mathrm{C}_{3} / \mathrm{IgM}$ deposits on renal biopsy. The other girl had RBC casts, proteinuria, renal insufficiency, hypocomplementemia, and diffuse proliferative $G N$ on biopsy. Immunologic studies on the first patient showed normal peripheral blood lymphocyte proliferative responses to phytohemagglutinin and pokeweed mitogen, decreased $E$ rosettes, but increased $B$ cells (EAC rosettes and surface lg-bearing cells). Co-culture of $T$ and $B$ cells revealed enhanced $\mathrm{Ig}$ synthesis of normal and hypogammaglobulinemic B cells when cultured with patient but not normal $\mathrm{T}$ cells, suggesting a lack of suppressor Tcells to explain hyperactivity of the humoral immune system in Sjögren's syndrome.

Veterans Administration Grants and Public Health Service Grant RR-00240.

ARE IDIOPATHIC SYSTEMTC LUPUS ERYTHEMATOSUS (SLE) AND 541 ANTICOINULSANP ASSOCIATED SIE (A-SLE) DIFFEREIT DISEASES? James D. Northway, Sudhir K. Anand, Stevart P. Smith, (Spon. by Ira Ko Brandt) Ini Univ Sch Med, Dept of Peds, Indianapolis.

Several anticonvulsants have been implicated in the etiology of a clinical syndrome resembling SLE. 13 children with SLE have been analyzei in regards to clinical disease, serological abnormalities and renal involvement. 5 children with $A-S i$ were compared to 8 rith SIE. Both groups vere similar in age of onset. 2 of 5 A-SLE and 1 of 8 STE were male. Clinical and laboratory findings vere similar in both groups, except for arthritis which was more prevalent in the SLE group. 4 of 5 vith A-SLE had overt renal disease; 3 diffuse membranoproliferative (MP) glomerulonephritis (GN) and one focal GN. 8 of 8 with SLE had renal involvement. $2 / 3$ had focal $\mathrm{GN}, 5$ had MPGN and 1 membranous nephropathy. Antinuclear antibody titers were the same for both groups. Only $l$ in the SLE group and none in the A-SLE group had a titer of $1 / 80$. With 1 exception, all in both groups had antibodies to both native and denatured DNA. The clinical course in both groups has been variable. All of the patients have been treated with Prednisone and/or immunosuppressives. Serological abnormalities have persisted in both groups despite Prednisone therapy and removal of the anticonvulsant. None of the patients in either group have died. $3 / 4 \mathrm{~A}-\mathrm{SLE}$ and $3 / 8 \mathrm{SLE}$ have proteinvria. None are uremic.

A-SLE does not appear to be significantly different from SLE.
IMMUNE RESPONSES IN INFANTILE CORTICAL HYPEROSTOSIS

542 (I.C.H.) Olu 0. Ogunye and Robert C. Farmer (Spon. by Melvin E. Jenkins) from the Dept. of Pediatrics and Child Health, Howard Univ. Col. of Med., Washington, D.C.

Recent reports of I.C.H. occurring in two unrelated male infants with the Wiskott-Aldrich Syndrome and another case of I.C.H. with hypergammaglobulinemia and thrombocytosis have raised the possibility of an immunological defect playing an etiological role in Caffey's disease. We have studied humoral and cell mediated immune responses in an infant with I.C.H.

Percentage of rosette forming peripheral lymphocytes was $65 \%$ (n1. 60-80) with an absolute T-cell count of $3,412 / \mathrm{mm}^{2}$; the percentage of immunoglobulin bearing Iymphocytes detected by immunoflorescence microscopy was $22 \%$ (n1. 10-25). In vitro 1ymphocyte proliferative responses were $170 \%$ and $190 \%$ of simultaneously performed control values for the non-specific mitogens Phytohemagglutinin and Pokeweed Mitogen respectively. Delayed cutaneous sensitivity reaction to Keyhole Limpet Hemocyanine could not be demonstrated 48 hours after the challenge dose and specific antibody response to $\mathrm{KLH}$ and diphtheria and tetanus toxoids was less than normal.

We conclude from these results that the efferent limb of the immune system in I.C.H. is intact. The possible deduction of a defective afferent limb may be due to the disease or to the normal state of functional immaturity of neonatal macrophages.

HOMOZYGOUS C3 DEFICIENCY PRESENTING WITH FEVER, SKI 543 RASH AND ARTHRALGIAS AND RESPONDING TO WHOLE BLOOD TRANSFUSION. Stephen G.Osofsky, Barry H.Thompson, Thomas F. Lint, Henry Gewurz. Rush Medical College, Dept.of Immunology, Chicago, I11. and USAF Medical Center, Dept. of Pediatrics, Keesler AFB, Miss A previously well 34 month old male (SA) presenting with fevers skin rash and arthralgias was found to lack C3 by immunochemical (undetectable) and hemolytic ( $1 \%$ normal) assays. No infectious agent was demonstrated.Protein levels of $\mathrm{Cla}, \mathrm{C} 4, \mathrm{C} 5$, properdin and $\mathrm{C} 3 \mathrm{~b}-\mathrm{INA}$ were normal and complement (C) components $\mathrm{Cl}-9$ except $\mathrm{C} 3$ were normal by hemolytic assay. Factor $B$ was 702 (normal 175-275) ug/m1. The $\mathrm{CH}_{50}$ (13\% normal) was reconstituted by addition of purified C3. Immune adherence was normal. SA serum did not support phagocytosis of C.albicans or E.coli, generate significant neutrophil chemotactic

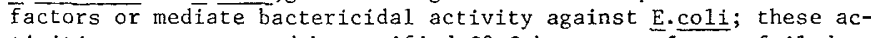
tivities were restored by purified C3. Cobra venom factor failed to cleave factor $B$ to $\bar{B}$. Transfusion of $320 \mathrm{ml} 1$ hour-old normal whot blood on the 52nd day, resulting in transient elevation of C3 level to $25 \mathrm{mg} \%$ to levels undetectable by 69 hours, was followed by disappearance of fever, arthralgias and rash as well as return of previously elevated CRP levels to normal. A defect in the synthesis of C3 was supported by absence of $\mathrm{C} 3$ antigens;genetic studies in 24 family members showing autosomal codominant inheritance;lack of anti-C 3 activity when normal serum or purified $\mathrm{C} 3$ were preincubated with SA serum; and a normal rate of catabolism of transfused C3. SA thus represents a unique individual with homozygous C3 deficiency whose clinical signs and symptoms were abated by whole blood transfusion and demonstrates the value of $\mathrm{C}$ analyses in the evaluation of patients with similar unexplained symptoms.

544 MORAL ASPECTS. Savita G. Pahwa, Rajendra N. Pahwa, Sloan-Kettering Cancer Center, Dept. Ped., NYC, N.Y.

Chemotaxis (CTX) of leukocytes from cord blood of heal thy term infants was compared to that of adult controls. The aim was to study the monocyte, a cell important in host defense but little analyze $f$ in the human newborn. The assay was done in modified Boyden chambers using as stimuli endotoxin-activated serum (EAS) and lymphocyte-derived chemotactic factor (LDCF) for monocytes (M) and EAS for neutrophils (PMN). Results were expressed as \% of control. CTX of cord blood was normal - increased: $126 \%$ with LOCF $(p<0.0025)$ and $115 \%$ with EAS $(p<0.05)$ whereas PMN response was decreased, $80 \%(p<0.005)$. Random migration of cord $M$ was also increased, $170 \%$. Generation of CTX factors for PMN from activated cord sera was $55 \%$ of that generated by normal serum. This is best explained on the basis of hypocomplementemia as no inhibitors or inactivators of CTX factors were detected in cord sera. When tested for effects on cells, cord sera inhibjted M CTX by $26 \%$ whereas adult sera did so by $51 \%$. There was minimal inhibition of PMN CTX by either sera. This lack of inhibitory activity for M in cord sera may represent a developmental delay.

The increased random mobility of cord $M$ suggests that these cells are activated in their resting state which could result from an undefined maternal stimulus or from the stress of delivery. The dissociation of chemotactic responses of the 2 different phagocytic cells may represent a protective mechanism whereby one cell can compensate for a defect in the response of the other. 
CHEMOTACTIC DEFECTS IN SEVERE COMBINED IMMUNE DEFI545 CIENCY(SCID). S. G.Pahwa, E.M.Smithwick, R.J.0'Reilly R.A.Good. Memorial Hospital, Dept. Ped., NYC, N.Y. Cellular and humoral components of monocyte(M) and neutrophil (PIIN) chemotaxis (CTX) were investigated as parameters of phagocytic function in a male child with SCID. Since admission to laminar flow germfree isolation at 5 mo., he has had 6 bone marrow transplants (BMT) from an unrelated MLC-matched female donor. With evidence of T-cell take after BMT\#5 (19 mo.), he was recontaminated with non-pathogenic organisms. Because he developed aplastic anemia (24 mo.), he was argain decontaminated and given BMT\#6 at 27 mo Now at 30 mo. he has hematopoietic and T-cell reconstitution. Test results at various times were: (EAS=endotoxin-activated serum)

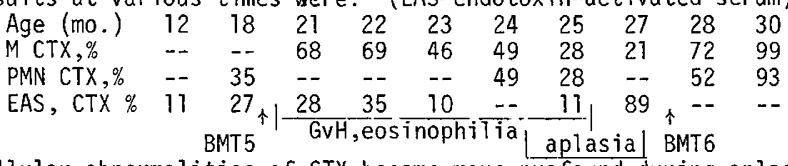

Cellular abnormalities of CTX became more profound during aplasia and are normal now after successful engraftment. Serum generation of CTX factor was markedly abnormal except once post-platelet transfusion $(89 \%)$. No evidence was found in his serum for excess amounts of CTX factor inactivator or a cell-directed inhibitor of CTX. Defective generation of CTX factor was found in 3 other SCID's which did not conrelate with $\mathrm{CH}_{50}$, Cla or $\mathrm{C} 3$ and may reflect either a defect in the alternate pathway or a lack of some other CTX component. The cellular defects may indicate a primary abnormality or relate in some way to BMT.

\section{CLINICAL TRIALS OF IMMUNIZATION WITH LIVE} HUMAN CYTOMEGALOVIRUS (TOWNE STRAIN). Stanley A. Plotkin, Max Just and John Farquhar,

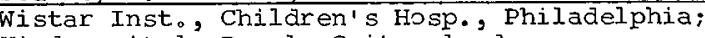
Kinderspital, Basel, Switzerland.

The Towne strain of Human cytomegalovirus (HCMV) passaged 129 times in human ribroblasts has been tested in 64 adult volunteers. Thirty-three volunteers negative for HCMV antibodies were inoculated subcutaneously. All developed antibodies within 2-3 weeks. The only clinical reaction observed was local erythema and induration at the site of the inoculation 10-12 days after inoculation, which disappeared. Biopsy of the skin lesions revealed mild inflammation. Hematologic values such as WBC and differential, and liver chemistries including serum transaminases remained normal throughout an 8 week study period. Attempts to recover HCMV from blood cells, throat swabs, urines, and semen specimens were uniformly negative. Antibodies have persisted for at least two years post-inoculation. Towne strain in amounts as large as 104.7PFU given intranasally to 19 seronegative and 4 seropositive volunteers failed to elicit antibody responses or virus excretion, suggesting that the Towne strain does not infect by the presumed natural route. Two instances of apparent reinfection of naturally immune subjects by Towne given subcutaneously were studied.

DEMONSTRATION OF A REQUIREMENT FOR ANTIBODY IN THE 547 ALTERNATIVE COMPLEMENT PATHWAY (ACP). Rutherford B Polhill, Jr., Kenneth M. Pruitt, and Richard B Johnston, Jr. Univ. of Ala. Med. Ctr., Depts. of Ped. and Mol. Bio1., Birmingham.

It has been shown that unsensitized rabbit erythrocytes (RaE) activate the ACP in nonimmune human and animal sera. This activation occurs when the classical complement pathway is blocked by chelation of calcium and in sera deficient in C2 or C4. Results in other assay systems suggest that antibody may be required for activation of this pathway. We have examined this possibility using a turbidometric assay that continuously monitors the lysis of RaE. Sera from 7 of 10 agammaglobulinemic (AY) patients collected prior to $\gamma$-globulin injection showed abnormal ACP activity. $\gamma$-globulin injections restored activity in the one patient studied. The addition to these sera of purified nonimmune human IgG at $250 \mathrm{\mu g} / \mathrm{ml}$ increased activity by a mean of $59 \%$ (range, $30-95 \%$ ) compared to $19 \%$ increase for standard normal human serum. IgM was also restorative. AY chicken sera were deficient and could be corrected with IgG. Absorption at $0^{\circ} \mathrm{C}$ with $\operatorname{RaE}$ but not sheep erythrocytes or zymosan removed the restorative capacity of IgG or serum. IgG eluted from $\mathrm{RaE}$ but not sheep erythrocytes restored activity to Ar sera. These findings suggest that antibody may be an essential part of both "natural" and specific immunity and that the inability to activate complement efficiently through either the alternative or classical pathways may be responsible for the frequency and character of infections in A $\gamma$ patients.
ENZYME REPLACEMENT THERAPY FOR ADENOSINE DEAMINASE

548 DEFICIENCY AND SEVERE COMBINED IMMUNODEFICIENCY DISEASE by Stephen H. Polmar, Robert C. Stern, Alan L. Schwartz, and Rochelle Hirschhorn, Case Western Reserve University Departments of Pediatrics and Pharmacology, Cleveland, Ohio, and New York University, Department of Medicine, New York, New York.

Deficiency of adenosine deaminase (ADA) is associated with an autosomal recessive form of severe combined immunodeficiency disease (SCID). Addition of ADA in vitro to lymphocytes from an ADA deficient SCID patient (ADA-SCID) was shown to restore their proliferative response to lymphocyte mitogens (Polmar et al. Lancet $2: 745-746,1975)$. These observations suggested the possi. bility of enzyme replacement therapy for ADA-SCID. Frozen, irradiated red blood cells (FIRBC) are a safe and readily available source of human ADA. Transfusion with FIRBC with and without fresh frozen plasma (FFP) was evaluated as therapy for ADA-SCID. Transfusion therapy resulted in restoration of in vitro lymphocyte responses to mitogens and allogeneic cells as well as the appearance of increased numbers of lymphocytes in the peripheral blood and the development of a thymus shadow. Therapy with FIRBC and FFP was more effective than FIRBC alone. FFP alone was ineffective. Following transfusion therapy, immunoglobulin levels rose and the patient responded to immunization with diphtheria and tetanus toxo1ds. Transfusion therapy resulted in reduction of plasma adenosine and intralymphocyte ATP levels, but there was no increase in lymphocyte ADA levels. The patient has remained free of infection for the eight months since the initiation of therpy.

DEFECTIVE MONOCYTE CYTOTOXIC EFFECTOR FUNCTION IN PATIENTS WITH IMMUNODEFICIENCY DISEASE. David G. Poplack, E. Clinton Lawrence, B. J. Holiman, Charles H. Kirkpatrick, and R. Michael Blaese. NIH, NCI, Bethesda, Md. Monocytes present in the mononuclear peripheral blood leucocyte fraction of a Ficoll Hypaque whole blood separation were found to mediate the 1ysis of $51_{C R}$ labelled human red blood cells coated with anti blood group antibody. We confirmed this cytotoxicity as an exclusive function of monocytes and applied this monocytemediated antibody dependent cellular cytotoxicity (MMADCC) assay as a clinical test of monocyte function. Cytotoxicity in this system correlates significantly $(p<.01)$ with monocyte number, thus monocytopenic aplastic anemia patients fail to exhibit MMADCC activity. Patients with the Wiskott-Aldrich Syndrome were found to have a profound defect in monocyte killer function despite normal numbers of monocytes. Interestingly, their monocytes possess normal concentrations of $F_{C}$ receptors on their cell surfaces with normal affinity for antibody coated red blood cells. Some individuals with chronic mucocutaneous candidiasis and a number of patients with a variety of 1ymphoreticular malignancies, including an individual with Iymphohistiocytosis, have also demonstrated defective monocyte cytotoxicity in this assay. MMADCC is a useful quantitative and qualitative assay which may help define the contribution of monocyte-macrophage effector function in patients with impaired host defenses.

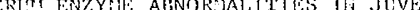
550 ARTHRITIS. Gary S. Rachelefsky, Nirmal A. Kar, Anne Coulson, Harold E. Paulus, F. Richard Stiehrl. liCL $\Lambda$ School of Medicine, Department of Pediatrics, Los Angeles, Cal. Although elevated serun transaminases ( $\mathrm{SGOT}$, SFPT) have been described in children with juvenile rhematoid arthritis (JPA), the frequency and the cause of the elevations are not well established. The posstbility that elevated SGOT and SGPT are secondary to muscle involvement in IJ $A$ was studied by assessing creatine phosphokinase (CPK), a specific enzyme for muscle lamage, along with SGOT, SGPT and alkaline phosphatase (AP). These were correlated with aspirin (ASA) therapy.

Sixteen male and 21 female (mean age 10 years) JRA patients were evaluated; 20 were on ASA continuously, 6 were on ASA intermittently and 11 were on no therapy. Tight had monarticular, 23 had polyarticular arthritis; 6 had Still's disease. Thirtyfive healthy age-matched control children were also studied,

In the healthy controls the SGNT was $22 \pm 7$ (mean $\pm 1 \mathrm{sn}$ ), SGPT $(9+7)$, CPK $(45+12)$ and $A P(200 \pm 4 \overline{6})$. In the 11 untreated JMA patients the mean SFOT $(39+3 \mathrm{SE})$ and SGPT $(19+1)$ were higher ( $p$.001) than the normal controls while levels of CPK $(45+5)$ and AP were not; in these 11 patients ST,OT vas elevated in 8 , SGPT in 6 and CPK in 4 . In the 20 ASN-treated JRA patients, mean SFOT $(43+5$ SE) and SGPT $(24+3)$ vere higher ( $p$.001) than normal while CPK was normal. In these 20 patients $S G O T$ was elevated in $11, S G P T$ in 7 and CPK in 9. In the 7 patients on intermittent $\Lambda S \Lambda$, the serum enzymes varied.

Thus, abnormalities of serum enzymes in JPA patients are related both to intrinsic disease and to the adrinistration of $\Lambda \mathrm{S} \Lambda$. 
PARTIAL CHARACTERIZATION OF SPONTANEOUS EOSINOPHIL CHEMOTACTIC ACTIVITY AND ITS ANALOGY TO C5a.Lawrence D. Robinson, Jr., Sandra K. Wooten and Michael E. Miller. Charles R. Drew and U.C.L.A. Schools of Medicine, Dept. of Pediatrics, Los Angeles, California.

Spontaneous eosinophil chemotactic activity (SECA) can mediate the directed movement of human eosinophils and neutrophils (Robinson and Miller, J. All. \& Clin. Imm., Oct., 1975). SECA is primarily found in the heat inactivated serum of patients with immune complex diseases. Preliminary characterization of SECA has been carried out. SECA is non-dialyzable and heat stable $\left(56^{\circ}, 30^{\circ}\right)$. Chromatography on Sephadex 675 demonstrated that SECA had elutional and functional properties similar to C5a (prepared from endotoxin activated normal sera). Polyacrylamide gel electrophoresis (PAGE) using 15\% Bis-acrylamide gels of 1yophilized, chemotactically active column fractions demonstrated a single protein band of identical electrophoretic mobility from either SECA or C5a preparations. Enzymatic hydrolysis with carboxypeptidase $B$, a known inhibitor of $\mathrm{C5}$ a activity, significantly decreased chemotactic activities of C5a and SECA. The addition of purified anti-C5 to either SECA or C5a significantly inhibited chemotactic activity. We conclude: SECA is a heat stable, nondialyzable material with elutional properties similar to $\mathrm{C} 5 \mathrm{a}$. Results obtained with PAGE, enzymatic hydrolysis and anti-C5 indicate that SECA is identical or very similar to $\mathrm{C} 5 \mathrm{a}$.
CLINICAL AND ANTIBODY RESPONSES FOLLONING IXRUNIZA-

554 TION WITH RA27/3 PUBFLLA VACCINE ANALYZFD BY HL-A TYPE. Sary J. Spencer, James D. Cherry, Paul I. Terasaki, Keith R. Powell, Ciro V. Sumaya, and S. Michael Jarcy intrics and Surgery,

aiser Foundation Hosp., Panorama City, $\mathrm{Ca}$.

HL-A typing was performed on 232 rubella seronegative (HAI titer $(8)$ children immunized with $P A 27 / 3$ vaccine. This vaccine is prepared in human diploid tissue culture (WI.38) which has the following HL-A phenotype 2,-,-,- (C. Brautbar, et al, Exp Cell Res $78: 367,1973)$. Of the 6 children in whom seroconversion did not occur HL-A type 2 was present in 4 and HL-A types 12 and 17 were present in 3 . The highest geometric mean convalescent-phase titer vas noted in children with HL-A types $\mathrm{N} 14$ and W22 (G!TT 152). This titer is significantly greater $(p<0.01)$ than the combined titer (GMT 88) of all vaccinees without HL-A types $W 14$ and $1: 22$. of 10 children with rubella titers $2512,40 \%$ had $H L-\Lambda$ type $V 28$; in contrast on $1 \mathrm{y} 8 \%$ of the total group had $\mathrm{W} 28 \mathrm{HL}-\mathrm{A}$ type $\left(X^{2}=\right.$ 12.06; $p=0.0005 \times 26=0.01)$. Of the 37 sibling pairs in the study only 4 pairs $(10 \%)$ had $\geq 4$-fold differences in titers. In contrast $39 \%$ of 71 randomly matched pairs of nonsiblings had titers that were different by 24 -fold $(p<0.005)$. Although 8 children had arthralgia and/or arthritis no correlation could be found with any specific HL-A type. There was also no association between vaccine related 1ymphadenopathy and $F L-A$ type. In a previous study with a live intranasal influenza $A$ vaccine we noted that subjects with !L-A type $\$ 16$ had poor antibodv responses; this trend was not observed in the present study following RA27/3 rubella vaccine.

555 HYPOPLASIA: IN VITRO RESPONSE TO THYMOSIN AND THYMUS RECONSTITUTION. Russell W. Steele, Howard A. Britton, Claude $\mathrm{T}$. Anderson and William T. Kniker. Dept. of Ped, Brooke Army Med. Ctr., Santa Rosa Med. Ctr., Univ. of TX Health Sci. Ctr. San Antonio, TX.

A 3 mo. old male infant with cartilage-hair hypoplasia also had severe combined immunodeficiency. Quantitative immunoglobulins were markedly depressed (IgG:141, IgA:0, IgM:24 mg\%) as were percent lymphocytes bearing surface immunoglobulins; $54 \%$ of peripheral lymphocytes formed EAC (B) rosettes (control 21\%). Lymphocyte responses to mitogens yielded a blastogenic index (BI) for PHA of 3.5 (normal 57-125), while the BI for pokeweed and Con A were $<2$ as were responses to monilia and tetanus toxoid. Recall antigen skin tests were negative and a lymph node biopsy showed depleted lymphocyte stores in both cortical and paracortical regions. Spontaneous (E) rosettes were $21 \%$ (contro1 $65 \%$ ). Erythrocyte adenine deaminase (ADA) activity was normal. In vitro assays in the presence of thymosin, fraction 5 (supplied by $\mathrm{Dr}$. A. Goldstein) were as follows: $25 \mathrm{\mu g}$ thymosin - $36 \% \mathrm{E}$ rosettes, 50 $\mu \mathrm{g}-45 \%, 100 \mathrm{Mg}-45 \%, 250 \mathrm{mg}-49 \%, 500 \mathrm{mg}-56 \%$ and $1 \mathrm{mg}-26 \%$. The BI to PHA also increased to 11.7 in the presence of thymosin, but responses to pokeweed and Con A didn't change. Transplantation of $24 \mathrm{wk}$ fetal thymus in millipore diffusion chambers and subsequently $18 \mathrm{wk}$ fetal thymus by intraperitoneal injection resulted in increased $\mathrm{E}$ rosettes to $32-41 \%$ although severe lymphopenia persisted. Responses to PHA, pokeweed and Con A also increased to 5-8 times previous values but still well below control ranges. Patient expired 5 months after transplantation.

IgE ANTIBODIES TO WHEAT (W) RICE (R), AND BUCK556 WHEAT(B). Kouji Tateno, Wai-Ying Lee, Tatsuo Pediatrics, Gunma University, Japan and UCLA School of Medicine. Torrance, California.

Subjects with proved celiac disease, other clinical sensitivities to W, R, B, or non cereal foods, and controls were tested by the radioallergosorbent test (RAST) for IgE antibodies. Subjects with antibodies to $W$ were likely to have antibodies to $R(r=.97)$ and to $B(r=0.72)$. There was also a relationship between antibodies to $R$ and $B(r=.76)$. Some subjects with only clinical $W$ sensitivity had elevated antibodies to $R$ and $B$. Celiac subjects had antibodies to wheat (which most were not ingesting) and to rice (which most were ingesting wi thout recognized symptoms). Several heal thy adults had IgE antibodies to wheat which they ate wi thout symptoms. The data suggest multiple antigens in each of the 3 cereals, some of which are shared. Relative levels of specific IgE, other antibodies, cellular immunity and specific organ sensitivity probably decide symptom production. Mean RAST counts were:

Diagnosis

Celiac Disease

Misc W. Sensitivity

$R$ and $B$ Sensitivity

$\bar{B}$ and $R$ Sensitivity

other Food Sensit.

Control Children

Heal thy Adults

\begin{tabular}{rrrr}
$\#$ & \multicolumn{1}{c}{ W } & \multicolumn{1}{c}{$R$} & \multicolumn{1}{c}{$B$} \\
\hline 10 & 5,327 & 5,479 & $T, 532$ \\
10 & 30,753 & 47,793 & 15,958 \\
4 & 30,536 & 74,189 & 33,544 \\
3 & 16,288 & 102,211 & 90,166 \\
3 & 5,575 & 3,589 & 1,981 \\
5 & 2,745 & 2,536 & 145 \\
13 & 10,936 & $<100$ & $<100$
\end{tabular}


557 ALLERGY TO PANGREATIC ENZYMES IN PARENTS OF PATIENTS WITH CYSTIC FIBROSIS. Frank J. Twarog, Steven F. Harvey R. Colten. Harvard Medical School, Children's Hospital Medical Center, Department of Medicine, Boston, Mass. 02115. Immediate hypersensitivity reactions to inhaled pancreatic extracts have been reported in parents of patients with cystic fibrosis (C.F.). To define the mechanism of these reactions patients with C.F. and their parents were studied. Eleven of 29 parents but none of the 13 patients had a history of asthmatic symptoms or rhinitis on exposure to the extracts. All symptomatic but only $10 \%(3 / 31)$ of asymptomatic individuals had positive immediate skin reactivity to one or more pancreatic enzymes used for testing. None had precipitating antibodies. Antigen mediated histamine release and provocative challenge studies were performed in six symptomatic and 2 asymptomatic parents. Specific leukocyte histamine release was induced by inactivated porcine trypsin (PT-I) only in symptomatic persons. Inhalation challenge with PT-I caused a significant and immediate decrease in lung function as judged by spirometry and flow volume curves in 5 persons with a history of bronchospasm but not in one person with rhinitis or in 2 asymptomatic persons. Passive transfer studies suggested that the response to pancreatic enzymes is mediated by $\mathrm{IgE}$ antibodies. Atopic, IgE mediated sensitization to trypsin is thus a significant factor in the allergic reaction induced by pancreatic extracts. There are no data suggesting IgG mediated hypersensitivity.

559 THE IN VITRO AND IN VIVO EFFECT OF THYMOSIN IN PRIMARY TMMUNODEFTCIENCY DISEASE. Diane W. Wara and Arthur J. Ammann. Univ. of Calif., San Francisco,

Department of Pediatrics

$\%$ T cell rosettes (TCR) in patients with certain immunodeficiency disorders increased following in vitro incubation with thymosin: \% increase in 4 patients with Nezelof's syndrome ranged from $86 \%$ to $430 \%$; patients with Wiskott-Aldrich syndrome, ataxiatelangiectasia and $\mathrm{Di}$ feorge syndrome had increases ranging from $29 \%$ to $88 \%$. Four patients with severe combined immunodeficiency disease had less than 5\% TCR which did not increase following thymosin incubation; presumably, a stem cell population is necessary for thymosin effect in vitro.

Five patients whose TCR increased in vitro following thymosin incubation have now received thymosin therapy for from 1 mo. to 2 yrs. Four of 5 patients improved clinically having an increase in TCR to normal within one month following initiation of therapy. Total numbers of lymphocytes increased and delayed hypersensitivity skin test(s) became positive in 3 of the 4 . Within 6 mos. $\bar{T}-$ lymphocyte function as assayed by in vitro lymphocyte response to phytohemagglutinin and/or allogeneic cells increased. One patient had alteration of immunoglobulin levels following 6 mos. of therapy; IgG increased from $380 \mathrm{mg} / \mathrm{dl}$ to $1200 \mathrm{mg} / \mathrm{dl}$.

Patients whose TCR increase following thymosin incubation in vitro may respond to therapy in vivo. In certain patients with primary immunodeficiency disease and abnormal thymic function, thymosin therapy may first expand the $T$ cell population and then improve $T$ cell function. Enhanced $T-B$ cell cooperation may then permit improved $B$ cell function.

559

SYNDROME OF CELLULAR IMMUNODEFICIENCY WITH IMMUNOGLOBULINS ASSOCIATED WITH ADENOSINE DEAMINASE DEFICIENCY J.W. Wolf, J.A. Anderson, R.H. Reid, L. Weiss, Henry Ford Hospital, Depts. of Int. Med. and Ped., J.J. Lightbody, wSU Medica1 School, Dept. of Biochemistry, Detroit.

A 30-month-old $B / M$ with failure to thrive, multiple pneumonias and oral/skin moniliasis was lymphopenic $(<200$ cells) but had normal immunoglobulins (IgG $1052 \mathrm{mg} . \%$, IgM $99 \mathrm{mg} . \%$, IgA $50 \mathrm{mg} . \%$ ), antibodies and $B-c e l l s$ (EAC rosettes $50 \%$ ). Cellular functions were depressed: D.H. skin tests, neg.; DNCB sensitization, unreactive; and T-cells, low (E-rosettes $6 \%$ ); PHA stimulated lymphocyte cultures were normal ( $60 \mathrm{~s} / \mathrm{C}$ ratio), mixed lymphocyte cultures were normal ( $10 \mathrm{~S} / \mathrm{C}$ ratio), and antigen stimulation of lymphocytes was not possible. Lymph node biopsy showed germinal centers but absent 1ymphocytes in the thymic-dependent areas. Adenosine deaminase (ADA) was $1 \%$ of normal in the red cells and $0-10 \%$ of normal in the lymphocyte/monocyte/platelet mixture ( $R$. Hirschhorn). Purified thymosin (A.L. Goldstein) increased in vitro rosettes from $6 \%$ to $60 \%$. After administration of thymosin, moniliasis cleared ( 1 wk.), E-rosettes increased to $60 \%$ ( 1 wk.), and the child developed a positive DT skin test ( 3 wks.); however, severe lymphopenia and ADA deficiency remained ( $4 \mathrm{wks}$.). At that time, normal red cells and plasma were given as ADA replacement therapy. This case shows the variable pattern found in severe combined immunodeficiency disease associated with ADA deficiency by presenting as a case of cellular immunodeficiency with immunoglobulins. Partial immune reconstitution was achieved with thymosin therapy. Complete reconstitution is probably dependent upon alteration of the ADA deficiency.
560 CHRONIC LYMPHOCYTIC THYROIDITIS: IDENTIFICATION OF RUBELLA VIRUS ANTIGEN IN THE THYROID
OF A CHILD WITH CONGENITAL RUBELLA. Philip R. Ziring, Sayenna A. Uduman, Gloria Gallo, Milton Finegold, Cyril Abrams, Elena Klein, Pearay Ogra and Louis Z. Cooper. College of Physicians and Surgeons, Columbia University and The Roosevelt Hospital, Department of Pediatrics, N.Y., N.Y.

A 5-3/12 year old boy with congenital rubella developed a goiter in association with symptoms of hypothvroidism. PBI was $1.6 \mathrm{mcq} . \%$, T4 (as jodine) was $2.5 \mathrm{mcq}$. $\%$ T4 (as thyroxine was $3.8 \mathrm{mcg} . \%$, antithyroglobulin titer was 1:2048, thyroid microsomal CF antibody was l:64, TSH (by radioimmunoassay) was $83 \mathrm{microunits} / \mathrm{ml}$. Biopsy of the thyroid revealed Hashimoto's Thyroiditis by light microscopy. No viral particles were seen on electron microscopy. Rubella virus was not isolated in tissue culture after inoculation of a suspension of biopsy material. Fluorescein labeled rubella specific antiserum revealed the presence of rubella virus antigen in the lymphoid follicles. It is now apparent that endocrine disorders (diabetes mellitus, thyroid disorders, adrenal disease) may be a delayed manifestation of congenital rubella. Congenital viral infection should be considered at least one of the possible triggering events in the development of Hashimoto's Thyroiditis. Its role in other autoimmune diseases and in carcinogenesis "deserves further exploration.

\section{INFECTIOUS DISEASE}

561 DETECTION OF ENDOTOXEMTA IN BACTEREMIC AND ABACTEREMIC NEONATAL SEPSIS. Allan M. Arbeter, Ann Stark, Stanley Watson, William Fox and Stanley A. Plotkin, Children's Hosp.

The Limulus lysate technique for detection of endotoxin was modified to detect changes in optical density (O.D.) of diluted test plasma when reacted with the Iysate. This modification eliminated the subjective observations intrinsic to the gelation method. Our method was sensitive to 10 picograms (pcg) of endotoxin. The O.D. was linear between 10 and $100 \mathrm{pcg}$, but the absolute values varied from day to day requiring standardization each time the test was performed. Typical $0 . D_{0}$ values for $0,10,50$ and 100 $\mathrm{pcg} / \mathrm{mI}$ of endotoxin were $0.084,0.116,0.155$, and 0.382 , respectively. The plasmas of 20 neonates with clinical sepsis and seven neonates with respiratory distress syndrome without clinical sepsis were assayed for endotoxin. A positive result for endotoxin was considered to be those samples with greater than $50 \mathrm{pcg}$. Endotoxin was detected in the plasma of $7 / 10$ neonates with bacteremic sepsis, 6/10 with abacteremic sepsis and $1 / 7$ without sepsis. Since the clinical picture of sepsis is not always accompanied by positive blood cultures, these data have pathogenetic and diagnostic implications.

562 Mohamad S. Badri, Sam Zawaneh, Amelia Cruz, Herman Pediatrics and Obstetrics, Univ. of Florida, Gainesville, Florida. Ano-rectal carriage as a possible primary source of vaginal colonization by Group B streptococci was investigated. The study was carried out during two different periods - April to June 1974 and October to December 1975. It included 789 pregnant women and 422 neonates. Vaginal and rectal cultures were obtained on all women; ear canal, nose, umbilical and rectal cultures were obtained on all infants. Culture swabs were plated onto a selective medium containing $8 \mu \mathrm{g}$ of gentamicin sulfate and $15 \mu \mathrm{g}$ of nalidixic acid per ml. Serological identification of Group B streptococci was performed by the Lancefield technique. Cultures positive for Group B streptococci were obtained from 162 of 789 women $(20.5 \%)$. Vaginal cultures were positive in $81(10.2 \%)$, while rectal cultures were positive in 142 (17.9\%). Of the 142 women with positive rectal cultures only 61 had positive vaginal cultures. Among the 422 neonates cultured, $42(11.8 \%)$ yielded positive cultures. Rectal cultures were positive in $36(8.5 \%)$ while umbilical, throat and ear cultures accounted for about 15 each $(3.5 \%)$. The rates of isolation of Group B streptococci from various sites were similar during both study periods. Preliminary studies in adult males suggest that rectal carriage rate for Group $B$ streptococci is as high as in females. These findings suggest that the gastrointestinal tract acts as a resevoir for Group $B$ streptococci and that vaginal colonization may be secondary to contamination from this source. 
THE ROLE OF ANTTBODY IN NEONATAL GROUP B STREPTOCOC-

563 CAL INFECTION, TYPE III. Carol J. Baker, Dennis L. Kasper, Abel Paredes, and William C. McCormack (Intr. by Martha D. Yow). Baylor College of Medicine, Department of Pediatrics, Houston, Texas, and Channing Laboratory, Boston City Hospital, and Harvard Medical School, Boston, Massachusetts.

Numerous epidemiologic studies have proved the significant association of type III strains with the majority of neonatal and infant group B streptococcal infections, particularly meningitis. A1though this observation may be related to enhanced virulence of the sialic acid-containing type III capsular polysaccharide, the role of antibody has not yet been determined. A radioactive antigen-binding assay which utilizes purified antigen from a type III strain was developed for the detection of antibody in human sera. Seventeen infants with invasive, type III infections were studied. None of these babies and only one of their mothers had antibody detected in acute sera. In contrast, 22 of $29(76 \%)$ type III vaginal carriers delivering babies who failed to develop symptomatic group B streptococcal infection had antibody demonstrated in sera collected during pregnancy. A11 infants with bone or joint infection (4) and only $29 \%$ of those surviving sepsis or meningitis developed antibody during convalescence. These data suggest that maternal antibody deficiency is a risk determinant for the development of symptomatic type III, group B streptococcal infections.
566

ROLE OF ADENOVIRUS IN PERTUSSIS SYNDROME. L.J. Baraff, J. Wilkins, G.D. Overturf, P.F. Wehrle. Los Angeles County/University of Southern California Medical Center, Department of Pediatrics, Los Angeles, Ca. 90033.

Forty-four patients with clinical pertussis were cultured for adenovirus and Bordetella pertussis. The criteria for the diagnosis of clinical pertussis was cough for $>1$ week, with either whoop, vomiting, or cyanosis. Thixty-five/44 had positive nasopharyngeal cultures for B. pertussis. Four $/ 44$ patients had positive throat cultures for adenovixus; all 4 were also culture posi tive for $B$. pertussis. In 33 of the 44 patients, viral cultures were repeated 2-3 weeks after the original culture to determine if activation of latent adenoviral infection occurred. Only 2 of the 4 adenovirus positive patients were recultured; both were neg ative. One repeat viral culture was positive for adenovirus in a patient previously negative. Twenty-nine patients had both acute and convalescent sera available for adenoviral complement fixation and B. pertussis agglutinin titers. In those tested, no patient had a significant rise in adenoviral titers. Thirteen/29 patients had a rise in pertussis agglutinins; all had a positive B. pertussis culture. Four $/ 5$ patients with positive Bordetella cultures had negative acute and convalescent agglutinin titers.

Clinical pertussis was not associated with adenoviral infection alone; those 5 patients with adenovirus isolated also had B. pertussis present in their nasopharynx, and none had a significant rise in adenoviral titer. The pertussis agglutinin test was not reliable to exclude the diagnosis of pertussis.
564

ANTIFUNGAL AND IMMUNOLOGIC ACTIVITY OF MICONAZOLE. *Melvin W. Balk, Martin H. Crumrine, and Gerald W. Fischer. (Spon. by James $w$. Bass) Tripler Army Medical Center, Department of Pediatrics, Honolulu.

Systemic candidiasis occurs most commonly in patients with impaired or suppressed immune systems. Eradication of invading organisms is, therefore, often difficult. Miconazole, a new broad spectrum antimycotic agent, was studied in a rat model of disseminated candidiasis. AduTt Wistar rats were given an intravenous injection of $10^{6}$ Candida albicans. Death in untreated animals general7y occurred between 4 and 8 days postchallenge. The animals developed progressive lesions in the kidneys, heart, and brain. Survival was $17 / 50(34 \%)$ in control animals compared to $62 / 6 \%(90 \%)$ in treated rats $(P<.005)$. Histopathologic examination of treated rats demonstrated splenic lymphoid hyperplasia observed as increased lymphocytes around central arteries. Addition of miconazole to mitogen stimulated human lymphocyte cultures suggests that miconazole may alter the activity of certain cell populations. In this model, miconazole is an effective antifungal agent and, in addition, appears to al ter celluiar immunity. This activity may make it uniquely effective in certain patients with immunologic impairment and severe mycotic infections.
PNEUMONIA IN INFANTS INFECTED WITH CHLAMYDIA

567 TRACHOMATIS. Marc O. Beem, Evelyn A. Saxon. U. of Chicago, Wyler Children's Hosp., Dept. of Peds. (Introduced by Albert Dorfman)

A rather distinctive pneumonia may be seen in infants infected with Chlamydia trachomatis (Ct), with: gradual onset at age 4 to 6 wks. , absence of fever and malaise, increased nasopharyngeal (NP) secretions, tachypnea, repetitive staccato coughing, lungs with diffuse interstitial infiltrates plus localized areas of alveolar involvement on $x$-ray, (often) follicular conjunctivitis and secretory otitis media, occasional eosinophilia, and usually elevation of immunoglobulins A, G and M. Symptoms may last weeks; signs and $x$-ray changes, months. NP \&/or tracheal secretion cultures of 12 such infants yielded $\mathrm{Ct}$ from all, various bacterial species, and viruses from 7 (CMV 4, picorna 2, adeno 1). Seven cultured for $M$. pneumoniae were negative. Lung biopsies of 2 showed interstitial pneumonia and negative cultures. Microimmunofluorescent (MIF) IgG antibodies vs LGV I were at sustained, high titer. Compared with 8 infants of like age w:th Co coril wilinitis only, pneumonia infants yielded NP Ct more consistently $(12 / 12$ vs $5 / 8)$, and at higher titer ( $>68$ inclusions/cover slip, $6 / 12$ vs $1 / 8$ ); mean MIF titers were significantly higher $(1 / 28,000$ vs $1 / 1,600)$. These findings indicate $\mathrm{Ct}$ infections of early infancy involve the upper respiratory tract more often than generally appreciated and suggest but do not establish, an etiologic role for $\mathrm{Ct}$ in certain pneumonias.

\section{CPSONOPHAGOCYTIC ANTIBODY AND DRAUNITY TO TYPE III}

565 STRAINS OF GiROUP B STREPTOCOCCI. Robert S. Baltimore, (Spon. by Jennis L. Kasper, Carol J. Baker and Di.ana K, Goroff Hospital, Channing Laboratory, Departments of Pediatrics and Hedicine, Boston, N'ass.

Previous epiderliologic studies have shown that type III strains of group B streptococci are responsible for the majority or neonatal infections aue to this organism. Biologically relevant tests of immunity are needed. An opsonophagocytic test has been developed which detects antibody to type III strains. In this systern huran polymorphonuclear leukocytes, complement, and inmune serum are all required for phagocytosis. Significant antibody levels vere defined as $>1$ log reduction in colony forming units in one hour when the mixture was rotated at $37^{\circ} \mathrm{C}$.

Irrmune raubit sera prepared to viable type III strains opsonized this organism at titers of $>1: 1,000$, while non-imnune serum did not opsonize and there was no killing. The recent isolation of a purified large molecular weight capsular type III polysaccharide allows study of the specificity of the opsonophagocytic antibody in irmune sera. Deficiency of maternal antibody to this antigen has previously been correlated with neonatal susceptibjlity to type III disease using a radioactive antigen bindine assay. This antigen inhibits the opsonopnagocytic activity of imune sera $(\ll \mathrm{ug} / \mathrm{ml})$. This inhibition demonstrates that the rajor determinant of immunity is antibody to this capsular antigen.
CLINICAL EVALUATION OF NEURAMINIDASE SPECIFIC INFLU-

568 ENZA A2 vaCCINE. K.R. Beutner, T. Chow, C. Rizzone, E. Rubi and P.L. Ogra. Depts. of Peds. and Microbiol. State Univ, of N.Y. at Buffalo.

In order to learn whether the induction of neuraminidase specific immunity will provide better protection against influenza $A_{2}$ infection, 1000 school children were immunized with, an inactivated recombinant influenza vaccine specific for the neuraminidase antigen of Port Chalmer (Ch) influenza A2 virus ( $/ \mathrm{Eq} / \mathrm{N} 2 / \mathrm{Ch}$ ), a commercial $\mathrm{Ch}$ strain of influenza vaccine $(\mathrm{H} 3 / \mathrm{Ch} / \mathrm{N} 2 / \mathrm{Ch})$, or a placebo, before the onset of natural Ch influenza infection. Immunization with either vaccine was found to be safe and without any major adverse effects. Vaccination with $\mathrm{H} 3 / \mathrm{Ch} / \mathrm{N} 2 / \mathrm{Ch}$ regularly induced seroconversion for antibody to the hemagglutin (HAI) and frequently for antibody to neuraminidase (ANAB). Immunization with $\mathrm{H} / \mathrm{Eq} / \mathrm{N}_{2} / \mathrm{Ch}$ resulted in no $\mathrm{HAI}$ response al though specific ANAB was observed in over $90 \%$, and the ANAB titers were 5-6 fold higher than in $\mathrm{H} / \mathrm{Ch} / \mathrm{N}_{2} / \mathrm{Ch}$ vaccinees. No serologic response was observed in placebo group. Subsequent natural outbreak of $\mathrm{Ch}$ influenza virus resulted in serologically (HAI) proven infection in $26 \%$ of $\mathrm{H}_{3} / \mathrm{Ch} / \mathrm{N}_{2} / \mathrm{Ch}, 47 \%$ of $\mathrm{H} / \mathrm{Eq} / \mathrm{N}_{2} / \mathrm{Ch}$ and $44 \%$ of $\mathrm{placebo}$ vaccinees. How ever, the incidence of clinical illness in infected subjects was $80 \%$ in the placebo, and $34-36 \%$ in the influenza vaccine groups. All vaccinees infected with clinical illness, were found to have very Iow HAI and ANAB titers, and vaccinees infected without any illness were found to have $4-8$ fold higher ANAB titers prior to infection, than those who were infected with illness. These observations provide tentative support to the use of neuraminidase specific vaccines in protection against influenza $A 2$ infections. 
OPSONTC REQUIREMENTS AND GRANULOCYTE RESPONSE TO

569 VARIOUS STRains of E. COLI. Bengt H. Bjorksten, Elaine L. Mills and Paul G. Quie, University of Minnesota Med. School, Dept. of Pediatrics, Minneapolis, MN.

Ten freshly isolated strains of E. coli were compared with respect to opsonic requirements and ability to stimulate granulocyte chemiluminescence. Six of these E. coli strains were isolated from stools of healthy newborns and four were recovered from the blood of septicemic infants. Three of the six strains from healthy newborns were spontaneously agglutinating "rough" strains and were serum-sensitive. These were not opsonized in serum chelated with MgEGTA, i.e. did not activate the properdin complement pathway. Three of the strains were "smooth" and serum-resistant and were opsonized in serum via the properdin pathway of complement activation.

The four strains of E. coli recovered from septicemic infants differed in serum sensitivity and three were opsonized via the properdin pathway. The strain opsonized only by the classical pathway had the $\mathrm{K}-\mathrm{I}$ antigen. The chemiluminescence response in normal granulocytes stimulated by the four E. coli strains from septic infants was markedly depressed when compared with the response of five strains from healthy newborns $(p<.005)$. This was not due to difference in opsonization.

These differences in opsonic requirements of, and chemiluminescence response to $\mathrm{E}$. coli strains suggests that factors which affect complement activation and granulocyte oxidative metabolism may contribute to the pathogenicity of certain strains.

\section{0} IMPROVEMENT OF CHEDIAK-HIGASHI (C-H) LEUKOCYTE (PMN) FUNCTION BY CXCLIC GUANOSINE MONOPHOSPHATE (GMP). Laurence A. Boxer, Manfred Rister, John M. Allen, Robert L. Baehner, Dept. of Ped., Indiana Univ. Sch. of Med. James Whitcomb Rilev Hosn. for Children, Indianapolis, Ind. $\mathrm{C}-\mathrm{H}$ sundrome is a disorder characterized bv recurrent infection and glant granules in PMN and monocvtes, and these cells are associated with compromised bactericidal capacity and degranulation. Since cvclic GMP regulates granular movement, we studied the effect of this compound in leukocytes of an infant with $\mathrm{C}-\mathrm{H}$ syndrome. The $\mathrm{C}-\mathrm{H} \mathrm{PMN}^{\prime} \mathrm{s}$ contained $17+5 \%$ myeloneroxidase and $58+10 \%$ B-glucuronidase of control PMN. C-H PMiN's and mixed leukocytes when rendered secretory by cvtochalasin $B$ and unon exposure to opsonized zymozan manifested impaired release of $B-$ glucuronidase. During degranulation $\mathrm{C}-\mathrm{H}$ PMN's and mixed leukocytes released $21+1.6 \%$ and $10.5+1 \%$ B-glucuronidase comnared to $27+1 \%$ and $22+2 \%$ of control resnectivelv. Following exposure to $10^{-2} \mathrm{M}$ GMP but not $10^{-5} \mathrm{M}$ for $30 \mathrm{~min}$, the $\mathrm{C}-\mathrm{H}$ leukocytes increased their enzvme release to normal values of $33+3 \%$ without changing their absolute amount of total enzyme activity. There was no bactericidal activity by $40 \mathrm{~m} / \mathrm{n}$. In PMN's from $\mathrm{C}-\mathrm{H}$ but by $90 \mathrm{~min}$. there were $5.19 \times 10^{6}$ viable bacteria remaining comnared to control of $1.13 \times 10^{6}$. Pre-exposure of C-H PMN's to $10^{-2} \mathrm{M}$ GMP for $10 \mathrm{~min}$. markedlv improved the kinetics of bacterial killing to values within normal. The addition of cyclic GMP corrected the impaired degranulation and bactericidal canacity suggesting faultv cyclic GMP activity in C-H leukocytes.

SPINAI FUUID IACTIC ACID, A DIAGNOSTIC AID IN BACTERIAL MENINGITIS. I. Brook, K.S. Bricknel1, G. Overturf, S.M. Finegold (Spon. by P.F. Wehrle). Veteran's Administration Hospital, Wadsworth, Infectious Disease Section, Los Angeles, Ca., 90024, \& Los Angeles County/University of South ern California Medical Center, Department of Pediatrics, Los Angeles, California, 90033.

Cerebrospinal fluid (CSF) lactic acid (LA) levels were determined by gas chromatography on serial specimens obtained from adults and children with CNS infections and control patients. Initial CSF LA levels were consistently $>35 \mathrm{mg} / 100 \mathrm{ml}$ in 30 patients with bacterial meningitis; patients with partially treated meningitis also had elevated levels of LA. Concentration of LA gradually decreased to normal levels ( $<25 \mathrm{mg} / 100 \mathrm{ml}$ ) during therapy and all treated patients had normal levels by 7 days. A late rise in CSF LA was demonstrated in 2 patients with recurrent meningitis and $I$ patient with brain abscess had a markedly elevated $(720 \mathrm{mg} / 100 \mathrm{ml})$ LA level.

CSF LA levels in 26 patients with aseptic meningitis were consistently $<35 \mathrm{mg} / 100 \mathrm{ml}$ (average, $21 \mathrm{mg} / 100 \mathrm{ml}$ ). Twenty-four control patients without meningitis (normal CSF) had average IA levels of $14 \mathrm{mg} / \mathrm{ml}$. Differences between the various groups were highly significant statistically. Concomitantly drawn serum LAA levels were consistently less than CSF levels; serum levels did not correlate with the type or absence of meningitis. Determination of CSF LA levels is a potentially useful aid in the diagnosis and therapy of CNS infections, particularly in patients who have received partial treatment.
572 BIOASSAY FOR ARA-A, HUMAN PLASMA. Yvonne J. Bryson, Lawrence Sweetman, James D. Connox. Dept.

Adenine arabinoside $(a r a-A)$ is an antiviral of low toxicity and clinical potential, currently in blinded clinical trials. At low intravenous doses, ara-A is metabolized rapidly through deamination to hypoxanthine arabinoside (ara-HI) which has 1/50 the antiviral activity (AVA) of ara-A. Previous assays for ara-A quantitate $50.1 \mathrm{\mu g} / \mathrm{ml}$ using high pressure liquid chromatography (HPLC) and UV absorbance. However, ara-A levels on present doses frequently are $<0.1 \mu \mathrm{g} / \mathrm{ml}$ in plasma collected with adenosine deaminase inhibitor. We have developed a bioassay for ara-A and ara-Hx using small plasma samples $(0.1-0.2 \mathrm{ml})$ which has $30 \mathrm{x}$ greater sensitivity than HPLC. This method combines the prior separation of these nucleosides by thin layer chromatography (TLC) on silica gel plates as described by Drach and Novack (Anal. Biochem.52:633,1973) with estimation of AVA of separated TLC eluates in adenosine deaminase deficient equine fibroblastic microcultures against vaccinia virus (VV). Using doubling dilutions of eluates prepared from plasma samples, standard replicate activity curves were prepared, finding that $\overline{5} 0.003 \mu \mathrm{g} / \mathrm{ml}$ of ara-A and $>0.3 \mu \mathrm{g} / \mathrm{ml}$ ara-Hx prevented $100 \%$ cytopathic effect from developing at $48 \mathrm{hr}$ post infection with VV. With HPLC, 20 of 99 plasmas contained $0.02-0.35 \mu \mathrm{g} / \mathrm{ml}$ ara-A and ratios ara-A:ara-Hx of 1.13 $1: 189$. With the bioassay, a patient's plasma had AVA equal to 2.5 $\mathrm{\mu g} / \mathrm{ml}$ ara- $\mathrm{Hx}$ and $0.012 \mu \mathrm{g} / \mathrm{ml}$ ara-A during infusion with no AVA prior to infusion or $12 \mathrm{hr}$ afterward. These methods should be useful in further pharmacologic studies of axa-A and the monophosphate salt, ara-AMP.

573 HERPESVIRUS HOMINIS. C.T. Cho and K.K. Feng, Depts of Ped. \& Microb., University of Kansas Medical Center, Kansas City, Kansas.

Experimental $\mathrm{H}$. hominis encephalitis in mice was uti7ized to evaluate the combined effects of antiviral agents (adenine arabinoside "ara-A", or phosphonoacetic acid "PAA") and humoral antibodies (HIG) directed against H. hominis. Treatment with ara-A or HIG reduced the \% mortality of infected mice that show no gross thymic defects (3-week-old Swiss mice, and phenotypically normal littermates "nu/t" of a strain of nude mice). However, the protective effects of ara-A or HIG were not observed in athymic nude (nu/nu) mice. On the other hand, combined administration of HIG with ara-A in immunologically normal (Swiss or nude "nu/+") or abnormal (nude "nu/nu") hosts resulted in an enhanced protection (see table), a decreased viral concentration of the infected tissues, and diminished tissue injury.

\begin{tabular}{|c|c|c|c|c|c|}
\hline \multirow[b]{2}{*}{ Treatment } & \multicolumn{5}{|c|}{ Mortality $(\%)$} \\
\hline & Swiss & Nude & $(n u / n u)$ & Nude & $(n u /+)$ \\
\hline irus control & $40 / 40(100 \%)$ & $15 / 15$ & $(100 \%)$ & $74 / 14$ & $(100 \%)$ \\
\hline HIC & $31 / 40$ & $15 / 15$ & $(100 \%)$ & $7 / 14$ & $50 \%)$ \\
\hline Ara & $29 / 40$ & $15 / 75$ & $(100 \%)$ & $7 / 14$ & $50 \%)$ \\
\hline IG & $42 \%)$ & $10 / 75$ & $67 \%)$ & $0 / 14$ & $0 \%)$ \\
\hline
\end{tabular}

our data suggest that 1) intact host defense mechanisms are necessary for the enhanced protection afforded by antiviral agent(s) or humoral antibodies, and 2) chemo immunotherapy may provide another approach to the control of severe viral

infections, particularly in host with abnormal defense mechanisms.

THE ROLE OF HUMORAL ANTIBODIES IN HOST RESISTANCE TO

574 EXPERIMENTAL HERPESVIRUS HOMINIS INFECTION. C.T.Cho, K.K. Feng, and J.A. Pittenger. Depts. of Ped. \& Microb., University of Kansas Medical Center, Kansas City, Kansas

Experimental $\mathrm{H}$. hominis infection in newborn mice was used to examine the role of humoral antibodies (HIG) directed against $H$. hominis. Exogenous administration of HIG to pregnant mice resulted in an enhanced resistance to intranasal challence of $H$. hominis in the offsprings. Postnatal administration of HIG to the newborn mice, before or soon after virus inoculation, also enhanced host recovery from the infection (see Table). The protective effects were reproducible and consistent up to a point (approximately 24 to 48 hours) in the infection cycle.

\begin{tabular}{l} 
HIG treatment \\
\hline Control \\
$-1 \mathrm{~h}$ \\
$+1 \mathrm{~h}$ \\
$+24 \mathrm{~h}$ \\
$+48 \mathrm{~h}$ \\
$+72 \mathrm{~h}$
\end{tabular}

\begin{tabular}{cc}
\multicolumn{3}{c}{ Mortality (\%) } \\
\hline H. hominis -1 & H. hominis -2 \\
\hline $55 / 65(85 \%)$ & $32 / 53(61 \%)$ \\
$13 / 50(26 \%)$ & $7 / 43(16 \%)$ \\
$19 / 53(36 \%)$ & $15 / 40(38 \%)$ \\
$18 / 53(34 \%)$ & $11 / 43(25 \%)$ \\
$50 / 53(95 \%)$ & $10 / 43(23 \%)$ \\
$50 / 55(91 \%)$ & $32 / 46(69 \%)$
\end{tabular}

Simultaneous administration of HIG and adenine arabinoside (ara-A) resulted in an augmentation of the antiviral action of ara-A.

The findings indicate that humoral antibodies 1) play a significant role in host recovery from severe $\mathrm{H}$. hominis infection, and 2) enhance the protective effect of antiviral agent. 
575 CONGENITAL CYTOMEGALOVIRUS INFECTION: A GUINEA PIG MODEL. W. S. Connor and K. P. Johnson (Intr. by M.M. University of California School of Medicine, San Francisco.

Human congenital cytomegalovirus (CMV) infection is the mos frequently recognized viral cause of congenital malformation and mental retardation. An animal model might yield valuable information on pathogenesis and perhaps prophylaxis and therapy. Efforts to obtain congenital infection with mouse CMV have been unsuccessful. But studies with guinea pig CMV (GPCMV) indicate this virus causes congenital infection.

Two strains of GPCMV, one more virulent than the other, have been used. 14 guinea pigs were inoculated 6 subcutaneously (sc) at various stages of pregnancy with either $10^{6}$ or $5 \times 10^{6} \mathrm{TCID}$ ( of the less virulent strain. Fetuses from 3 mothers inoculated at midgestation, 2 with low dose and 1 with high dose, 3 to 4 weeks before sacrifice, showed GPCMV in various tissues including brain by virus isolation but without histologic change. The high dose mother also showed leukocyte associated viremia 3 weeks after inoculation. Abortions or maternal death occurred only after high dose inoculations. Eosinophilic intranuclear inclusions (EII) in fetal viscera were found after direct fetal inoculation in utero.

The more virulent strain was first isolated from a young animal with EII in lung, kidney, spleen, and liver. Frequent abortions or maternal deaths occurred about 10 days after sc inoculation of $2 \times 10^{5}$ TCID $_{50}$. EII were found in trophoblastic cells of placenta. Efforts to document fetal infection after sc inoculation with this strain are continuing. Study of GPCMV in pregnant guinea pigs may aid understanding of features of congenital human CMV infection.

\section{6}

LIMULUS TEST IN GRAM-NEGATIVE (GN) MENINGITIS Michael S. Cooperstock and Linda L. Riegle (spons. by Calvin $W$. Woodruff) University of Missouri, Columbia, Department of Child Health.

The limulus test for endotoxin-like activity (ELA) was used to test $158 \mathrm{CSF}$ samples from 83 infants and children and $l$ adult. Prior centrifugation and heat extraction of CSF was needed for very proteinaceous or bloocy fluids. The test sensitivity was 10-100 $\mathrm{pg} / \mathrm{ml}$ 026B6 E.coli Boivin endotoxin. Quantitation was by serial dilution in parallel with this standard. 59 samples from 21 patients had ELA. 14 patients had I ng to $100 \mathrm{lg} / \mathrm{ml}$ ELA; 13 of these had proven GN meningitis; 1 had partially treated meningitis with petechiae. 7 additional patients had $<1 \mathrm{ng} / \mathrm{ml} \mathrm{ELA}$ and no evidence of $\mathrm{GN}$ meningitis.

21 of the 59 positive samples were from patients with GN meningitis whose CSF had been sterilized by previous therapy. Increasing ELA levels were associated with a poor prognosis in 2 newborns.

We conclude: (1) the limulus test can rapidly and correctly identify patients with $\mathrm{GN}$ meningitis whose CSF had been sterilized by prior treatment; and it may be useful in diagnosis and follow-up of these patients, (2) sensitivity must be rigidly controlled to avoid false positives, (3) serial quantitation of ELA can reveal trends not readily recognized by other means.

577 OXPERIMENTAI SEPTICEMIA: EVALUATION OF IYSATE TEST FOR ENDOTOXIN.James J.Corrigan, Jr. and James $\mathrm{F}$. Kiernat. Dept. of Peds. Univ. of Ariz. Med. Ctr.Tusson

Bacterial endotoxin causes gelation of a liquid material (lysate) derived from the amebocyte of the horseshoe crab (Limulus polyphemus). This phenomenon has been used as a test for endotoxin in various body fluids. A controversy exists with regard to its usefulness in detecting blood endotoxin in humans with gramnegative sepsis. The purpose of this study was to evaluate the effectiveness of the lysate test in a animal model for gram-negative septicemia. Fifty rabbits were given live Pasteurella multocida organisms intraperitoneally. Blood was obtained at 0,2 $4,8,12$ and 24 hours. The animals became bacteremic within 2-4 hours and were clinically ill by 8 hours. Quantitative colony counts (CFU $\log 10 / \mathrm{ml}$ blood) and lysate tests were performed on all blood samples. No rabbit had a positive lysate test and concommitant negative blood culture. By 4 hours $95 \%$ of the animals had positive blood cultures and $63 \%$ positive lysate tests. At 12 hours both were positive in $100 \%$ of the rabbits. The number of positive lysate tests was related to the CFU such that $<10^{3}$ org/ $\mathrm{ml}$ gave neg lysate tests, $10^{3}$ to $10^{5} 25$ to $60 \%$ were positive and $>10^{5} 100 \%$ were positive. Quantitative endotoxin levels revealed a range between 0.04 to 500 micrograms per $\mathrm{ml}$ of plasma which correlated with the CFUs. The data indicate that the lysate test can be used to detect and quantitate endotoxin activity in blood. The data suggest that there is a direct relationship between the number of infecting organisms per $\mathrm{ml}$ blood and quantity of endotoxin which, therefore, will influence the qualitative lysate test.
578 EXPERIMENTAL HEMOPHILUS INFLUENZAE, TYPE B TION. Francis J. Crosson, Jr., E. Richard Moxon, Jerry A. Winkelstein. Johns Hopkins Univ. Sch. of Med., Dept, of Pediatrics, Baltimore. (Spon. by David $\mathrm{H}$. Carver)

The mechanisms by which the non-immune infant controls infection by Hemophilus influenzae, type B, are only partially understood. Using the infant-rat meningitis model, we have attempted to determine if the complement system plays an important role in the host defense against this organism.

Ten-day old rats were injected with cobra-venom factor (CoVF), an agent known to selectively deplete C3-9; C3 activity in the treated animals remained <5\% of initial values for five days. These animals and controls were then inoculated intranasally with $10^{7} \mathrm{H}$. influenzae, type B. Bacteremia occurred in $95 \%$ of the CoVF-treated rats and $70 \%$ of the controls (P<.05). High level bacteremia ( $5 \times 10^{5} \mathrm{org} . / \mathrm{ml}$.) developed in 95\% of the CoVF-treated and $10 \%$ of the control animals ( $\mathrm{P}<.001)$. The mortality rate was $70 \%$ among the CoVF-treated rats and $0 \%$ among the controls ( $<<.001$ ). Both changes in the CSF indicative of meningitis and mortality were directly related to the level of bacteremia reached in each animal. Thus, in this model, complement appears to contribute significantly to the defense mechanisms of the non-immune infant rat against $H$. influenzae, type $B$.
579 IMMUNODEFICIENCY AND CYTOMEGALOVIRUS (CMV) INFECTIUN Audra Deveikis, Anthony J. Strelkauskas, $\frac{\text { Victoria }}{\text { Schauf (Spon. by Ira Rosenthal), Abraham Lincoln }}$ Schauf (Spon. by Ira Rosenthal), Abraham Lincoln School of Medicine and School of Basic Medical Sciences, Univer-
sity of 1 llinois at the Medical Center, Departments of Pediatrics sity of Illinois at the Medi
and Microbiology, Chicago

Immunodeficiency is known in congenital rubella, but has not been described in congenital CMV infection. Frequent infections occurred in an infant with CMV viruria. The number and proportion of peripheral leucocytes, $T$ and $B$ lymphocytes, and the blastogenic response to phytohemagglutinin (PHA) and pokeweed mitogen (PWM) were determined in this infant and in 2 infants with congenital CMV infection without recurrent infections. T cells were measured as $E$ rosettes and by an indirect rosette technique in which lymphocytes were sensitized with rabbit ant $i$-human $T$ cell 1 antibody $(A b)$ and then rosetted with erythrocytes coated with $A b$ to rabbit immunoglobulin ( $\mathrm{g}$ ) light chains. B cellswere measured by direct rosetting with erythrocytes coated with $A b$ to human Ig light chains. Replicate lymphocyte cultures were incubated with PHA, PWM, or no mitogen; labeled with $3 \mathrm{H}$-thymidine and harvested automatically. The infants with CMV infection had significantly decreased percentages of $\mathrm{T}$ cells $(13 \%, 29 \%$, and $40 \%)$ compared to age-matched controls $(61 \pm 2 \%)$. Compensatory increases in $B$ cells and in cells lacking $B$ and T markers occurred. Transient granulocytopenia occurred in 2 patients. Lymphocyte reactivity to PHA and PWM was decreased from $34-57 \%$ of control values in these 2 patients. These immunologic abnormalities may result from CMV $i_{1}-$ fection of lymphocytes and may be responsible for prolonged viruria and, in some cases, recurrent infections.
580 RELAPSE DURING PENICILLIN TREATMENT OF GROUP B STREPTOCOCCAL MENINGITIS. Rodney D. Dorand and Garrett Louisville School of Medicine Department of Pediatrics, Louisville A $3534 \mathrm{~g}$ term female infant with Group B streptococcal (GBS) meningitis responded initially to recommended doses of penicillin but relapsed on the 8 th day. To our knowledge this is the first reported case of such relapse. CSF examination at $24 \mathrm{hrs}$. of age revealed: WBC $2,170 / \mathrm{cu} \mathrm{mm}$, Gram positive diplococci on smear and normal glucose and protein. GBS sensitive to penicillin grew out $48 \mathrm{hrs}$. later, at which time penicillin G $100,000 \mu / \mathrm{kg} / 24 \mathrm{hrs}$. IV in 2 doses was substituted for the initial therapy of ampicillin $200 \mathrm{mg} / \mathrm{kg} / 24 \mathrm{hrs}$. IV in 2 doses and gentamicin $5 \mathrm{mg} / \mathrm{kg} / 24 \mathrm{hrs}$. IM in 2 doses. CSF then showed WBC $1,620 / \mathrm{cu} \mathrm{mm}$, and no organisms on smear or culture. On the 8 th day of therapy clinical and bacteriologic relapse occurred. GBS type III was cultured from the CSF, ventricular fluid and blood. Penicillin G $300,000 \mu / \mathrm{kg} / 24 \mathrm{hrs}$. IV in 3 doses was then given for 21 days and she recovered without apparent sequelae.

Dosage and administration errors were ruled out. Laboratory identification of the organism and penicillin susceptibility wer validated. Tube dilution susceptibility testing showed the MIC to penicillin to be $0.12 \mu \mathrm{g} / \mathrm{ml}$, relatively high for GBS. This treatment failure as well as recent penicillin pharmacokinetic studies suggest that to avoid prolonged periods of low penicillin levels in the CSF and serum, current penicillin dosage recommendations for the newborn should be increased to at least 150,000 $\mu / \mathrm{kg} / 24 \mathrm{hrs}$. in 3 doses for the first 3 days of life and then to $200,000 \mu / \mathrm{kg} / 24 \mathrm{hrs}$. in 4 doses. 
FALSE POSITIVE RUBELLA HEMAGGLUTINATION-INHIBITION (HAI) TITERS IN NEONATES AND CHILDREN WITH DIRECT HYPERBILIRUB INEMIA. S.C. Elliot t*, F. Kleinberg, T.F. Smith, R.D. Ellefson. (Spon. by G.S。Gilchrist) Mayo Clinic, Department of Pediatrics, Rochester, Minnesota.

Two neonates, one with extrahepatic biliary atresia and one with cystic fibrosis, and a nine year old with atresia of the common bile duct all had direct hyperbilirubinemia and elevated rubella HAI titers when examined by our routine testing method using kaolin pretreatment of serum. A $\beta-1$ ipoprotein fraction of the serum that is frequently found in association with bile obstruction was shown to be the source of our rubella HAI inhibitor. This $\beta$-lipoprotein was not removed by standard kaolin treatment of serum but was removed completely by dextran sulfatecalcium chloride ( $\mathrm{DS}-\mathrm{CaCl}_{2}$ ) treatment. The $\mathrm{DS}-\mathrm{CaCl}_{2}$ treatment was shown not to be removing specific rubella $\mathrm{Ab}$ but only the inhibitor. The $\beta$-lipoprotein molecule responsible for these false-positive results is an abnormal lipoprotein complex (LP-X) or a similar abnormal lipoprotein complex which has been shown to be present in the serum of children with bile obstruction. In the presence of direct hyperbilirubinemia, routine kaolin pretreatment of serum is not recommended as false-positive rubella HAI results are consistently obtained and may confuse the clinical diagnosis.

DECREASED VIRULANCE IN AMPICILLIN RESISTANT HAEMOPHILUS INFLUENZAE. Gerald W. Fischer Martin $\frac{H}{\text { Spon }}$. Crumrine, Melvin W. Balk , and Wili iam H. Topper. ( Spon. by James W. Bass) Tr Recently there have been numerous reports of ampicillin (AMP) resistant Haemophilus influenzae isolated from patients with meningitis and sepsis. ATthough these organisms are resistant in vitro, the clinical response to AMP therapy is often favorable. A recent case of neonatal $\mathrm{H}$. influenzae seps is was treated effectively with AMP. SubsequentTy, this organism was shown to be resistant to AMP, further emphasizing the difference between in vitro resistance and in vivo response. A test of virulance was therefore established in neonatal rats to study this dichotomy. Split litters of 1 to 2 -day-old rats were injected IP with $0.1 \mathrm{ml}$ of 48 -hour broth cultures of the test organisms (adjusted to equalize both number of organisms and volume). Animals were observed 7 days to determine survival. All animals challenged with the most resistant organisms survived without apparent disease $(14 / 14)$, while $(11 / 18-61 \%)$ animals challenged
with AMP sensitive $H$. influenzae died $\left(P<0.005, x^{2}\right)$. The AMP sensitivity of the organisms ranged from $0.39 \mu \mathrm{g} / \mathrm{ml}$ up to $32 \mu \mathrm{g} / \mathrm{ml}$ and 711 resistant bacteria produced beta-lactamase. As organisms increased their resistance to AMP, their growth rates declined. These studies demonstrate that the acquisition of AMP resistance alters the normal growth rate and may decrease the virulance of $\underline{H}$. influenzae.

RFLATTONSHIP OF CYTOMEGALOVIRIS AND HFPATITIS $B$

582 VIRUS TO HFPATITIS IN A MULTITTRANSFUSFD POPUIATTION, Robert F. Fnck, Robert F. Betts, Marilyn R. Brown, Gerald Miller, University of Rochester School of Medicine and Dentistry and Hemophilia Center-Rochester Region, Inc., Rochester, New York.

Hepatitis is a common problem in treated patients with hereditary hemorrhagic disorders. This study evaluated the role of cytomegalovirus (CMV) and hepatitis B virus (HBV) in the development of hepatitis in this population. The study population was divided into two groups: group l, consisting of 32 frequently transfused ( $>12$ times/vear) patients and group 2, consisting of 29 less frequently transfused (K1? times/vear) patients. The occurrence of IgG antibody to CMV and antibody of ad or ay subtype to hepatitis B virus was significantly higher in group I than group 2. Of group I patients with abnormal liver function tests (IFT), 100\% had antihody to HBV, while of group 2 patients with abnormal LFTs, $56 \%$ had antibody to KBV. Antibodies to CMV and HBV frequently overlapped, and the presence of antibody to CMV was not directly related to abnormal JJFTs. The higher incidence of antibody to CMV in the multitransfused group was striking in the younger age group and was not acquired passivelv by transfusion. The direct association of antibody to HBV with abnormal IFTS in the multitransfused group implies a role for HBV in the development of hepatitis. However, the occurrence of antibody to IIBV in $56 \%$ of the less frequently transfused group with abnormal LFTs suggests the possibility of other viruses not detected hy the current methods.

583

CLEARANCE OF BACTERIA AND BACTERIAL ANTIGEN FROM CEREBROSPINAL FLUID OF PATIENTS WITH BACTERIAL MENINGITIS. William E. Feldman (Intr. by John D. Nelson) Univ. of Texas H. Sci. Center at Dallas, Dept. of Ped., Dallas.

Concentrations of bacteria in cerebrospinal fluid (CSF) of 16 patients with bacterial meningitis ranged from $1.5 \times 10^{3}$ to $7.1 \times$ $10^{8}$ colony-forming units (CFU)/ml before antibiotic therapy (mean $\left.8.4 \times 10^{6} \mathrm{CFU} / \mathrm{ml}\right)$. Levels of bacterial antigen in pre-treatment CSF, measured by countercurrent immunoelectrophores is (CIE), ranged from 0.2 to $12.8 \mu \mathrm{g} / \mathrm{ml}$ (mean $3.6 \mu \mathrm{g} / \mathrm{ml}$ ). By polynomial regression analysis, concentrations of bacterial antigen and of viable bacteria were significantly related $(p<0.017)$

Bacterial growth was detected in 5 of $15 \mathrm{CSF}$ samples (range 5 to $50 \mathrm{CFU} / \mathrm{ml}$; mean $25 \mathrm{CFU} / \mathrm{ml}$ ) obtained after 17.5 to 35 hours (mean $22 \mathrm{hr}$.) of therapy with penicillin and chloramphenicol. The concentrations of bacterial antigen ranged from 0 to $4 \mu \mathrm{g} / \mathrm{ml}$ (mean $1.2 \mu \mathrm{g} / \mathrm{ml}$ ).

These data show that pre-treatment concentrations of bacterial antigen in CSF are related to the numbers of bacteria.

This relationship was not observed after antibiotic therapy probably because the increased clearance of bacteria releases enough antigen to maintain relatively high concentrations as the numbers of viable bacteria decreased.

Chloramphenicol therapy reduced the concentrations of bacteria in CSF rapidly but positive cultures persisted in one-third of samples on the second day of treatment.
BACTEREMIA AMONG NEONATES IN A REFLRRAL

585 CENTER. Alfred L. Florman, Lois L. Neumann, Robert S. Holzman, and Beatrice Toharsky. NXU Sch. of Med., Dept. of Pediatrics, New York. Increasing numbers of sick newborns are being sent to regional centers. Hence the usual experience with neonatal infections may have been altered. Taking bacteremia as a parameter of infection, we compared for 1974 the incidence and agents causing bacteremia during the lst 30 days of life among term and premature infants born at Bellevue with that among infants transported there soon after birth. There were 13 significant bacteremias - 3 among 1097 Bellevue born term babies (3/1000 live births), 2 among 134 Bellevue born prematures $(15 / 1000)$ and 8 among 129 transported neonates $(62 / 1000)$. Eleven isolates were gram positive and 2 were gram negative. Five were streptococcus Group $B$ (4 detected in the first 2 days of life) and 4 were $\mathrm{S}$. aureus (all detected after day 6 ). There was 1 Pneumococcus, 1 Enterococcus, $1 \mathrm{H}$. influenza type $\mathrm{B}$ and $1 \mathrm{H}$. parainfluenza. The absence of enteric bacilli was striking and at variance with recent past experience. During the first 3 days of life the risk of bacteremia was the same both for locally born and for transpoxted prematures. However all 6 episodes occurring after day 3 were in transported babies.

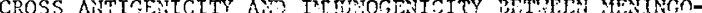

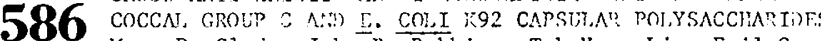
Mary P. Clocie, Jo:n B. Tohbins, Teh-Yuns Lin, Emi? C.

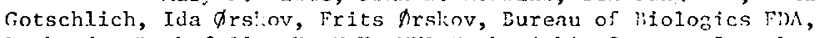
Bethosla, Rockefeller U. 'T.Y. 'iln Ischerichia Centre, Conenhagen

Cross-reactive bacteria vere sourint amons stnol, urine, blon and CSF cultures with the antisoram arar technique to identj.fy an antigenic stimulus $i$ addition to the homologous neranism to explain the age-relates ievelonment of meninsoncoccal froun $C$ polysaccharide antibodies in the US, if $2,0^{\circ} 3 \mathrm{~F}$. coll, $14 \mathrm{~K} 92$

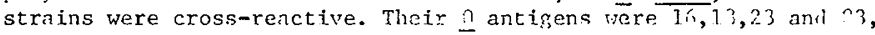
11 antigens were 4 and $3 / 4$, none produced enterntoxin, all were sensitive to most antihintics ani hat exnected formentations. Boti meningococcal Group $C$ and $E$. coli are acidic carsular nolvsaccharides and homonolymers of sialic acirl. The $K 92$ i.s $:$ but not 0 acetylated, alpha $2 / 8,2 / 9$ linke't, neuraninillase sensitive with only traces of protein, nucleic acir! and enciotoxin. 14 purified K92 polysaccharides were identica? by immunodiffusion, showed homogeneous peaks on Sepitarose 43, (Iid's 7. A to 0.5 ), precipitater about $60 \%$ of polysaccharide antihodies and remove: a nronortional amount of bactericidal antibndies ${ }^{2}$ rom meninsococcal froun $\mathrm{C}$ antisera. Intravenous iniection of formin-treated $502 \mathrm{E}$. coli yielded precipitating and bactericidal meningococcal Group $c$ polysaccharide antibodies. E. coli $\mathrm{k} 92$ strains may provide an alternate immunogen to meningncoccal froun $\mathrm{C}$ polvsaccharide in infants and young children, either as a source of a more immunozenic vaccine or as viable sacteria to colonize the ri tract. 
587 SERUM OPSONINS FOR GROOUP B STREPTOCOCCI VaT G. $\frac{\text { Hemming, Robert } T \text {. Hall and Harry R. Hij . University }}{\text { of Utah, Departments of Pediatrics and Pathology, }}$ Salt Lake City and Chi]dren's Mercy Hospital, University of Missouri, Kansas City, Missouri.

Little is known about immunity to Group B streptocncci because, to date, a reliable means for measuring functional opsonic activity for these organisms has not been developed. Neutrophils produce a chemiluminescence (CL) following phagocytosis of particulate matter and this event can be detected and quantitated in a liquid scintillation counter. We have adapted the CL procedure to detect opsonic activity for the 5 Group B streptococcal types in rabbit hyperimmune and human serum. Group B organisms opsonized in homologous but not heterologous rabbit serum produced a peak in $\mathrm{CL}$ when added to normal human neutrophils. Human adult serum, cord blood, and infant serum, similarly examined, also possessed opsonic activity for some of the Group B types. Examination of phanocytic mixtures showed a correlation in each instance between the uptake of bacteria, production of a peak in $\mathrm{CL}$ and the deposition of $\mathrm{Ig}_{2}$ and $\mathrm{C}_{3}$ on the bacterial surface. Paired maternal and infant sera from nine cases of early onset Group $B$ streptococcal sepsis were examined by this method. In each case opsonins directed against the organism recovered from the infant's blood were either absent or present in low titer. These studies suggest that the $\mathrm{CL}$ assay may provide valuable information regarding the role of serum opsonins in immunity to Group B streptococci.

588 THE ROLE OF REOVIRUS-LIKE AGENT AND HEAT-LABILE DIARRHEA. Mary T. Ho, Peter Echeverria, Neil R. Blacklow, James 01son (Spon. by David H. Smith). Naval Medical Research Unit No. 2, Taiwan, Harvard Medical School, University Hospital, Boston, Massachusetts.

In August-September 1975 a study was done in Taipei, Taiwan to determine the significance of reovirus-1ike agent and heatlabile enterotoxigenic $E$. coli (ent $+\mathrm{EC}$ ) and other enteric pathogens as causes of acute diarrhea. Stools from 85 children with diarrhea were screened for ent ${ }^{+} E C$ using the $Y-1$ adrenal cell morphological assay; acute and convalescent sera from 58 were tested for evidence of a recent reovirus-like infection by quantitating immunofluorescent-stainable antibody titers to the sero logically related Nebraska calf diarrhea virus. Stools were screened for other bacterial and parasitic pathogens. Specimens were inoculated into primary monkey kidney, MK-2 and Hep-2 tissue cultures. Forty-one percent of children tested showed evidence of a recent infection with a reovirus-like agent. Ent ${ }^{+}$EC were isolated from $7 \%$ of children who had not received antibiotics prior to culture and from $4 \%$ of well children $(p=0.49)$. Salmonella were isolated from $5 \%$ of children with diarrhea as compared to $3 \%$ of controls $(p=0.43)$. Entero, adeno, and herpes viruses were isolated more frequently from controls than from children with diarrhea, $27 \%$ vs $13 \%$. Other bacterial or parasitic pathogens were not found. This experience suggests that reovirus-like agent may be an etiology for a large proportion of pediatric diarrhea in Taipei, Taiwan and that ent ${ }^{+}$EC and Salmonella are responsible for only a small proportion of cases.

589 BY LEUKOCYTES (PMN) FROM PATIENTS WITH CHRONIC GRANULOMATOUS DISEASE(CGD). Philip C. Ho, Annette Lam, KamYin Wong \& W. Douglas Biggar (Spon.by J. Richard Hamilton) Depts. of Ped. \& Immuno1., Hospital. For Sick Children, Toronto, Canada. PMN from patients with CGD ingest bacteria normally but do not kill certain catalase-positive bacteria as vigorously as normal. PMN. Despite this PMN bactericidal abnormality, clinical improvement has been reported in CGD patients receiving long-term sulfonamide therapy. We have examined the effect of sulfisoxazole(SSX) and sulfamethoxazole(SMZ) on the capacity of CGD and normal PMN to kill staphylococci and E.coli in vitro. Although E.coli contain catalase, CGD PMN kill E,coli in vitro but less than normal PMN. SSX $(5-50 \mu \mathrm{gm} / \mathrm{ml})$, but not SMZ $(50 \mu \mathrm{gm} / \mathrm{ml})$ caused a small but significant increase in E.coli killing by both CGD and normal PMN. This observation was most likely due, in part, to an effect of SSX on E.coli. By contrast, no enhanced killing of staphylococci, an organism not killed by CGD cells, could be demonstrated in either normal or CGD PMN by the addition of SSX or SMZ. Rifampin, known to penetrate the PMN plasma membrane, was also examined. No enhanced killing of staphylococci by CGD or normal PMN could be demonstrated in the presence of Rifampin $(1 \mathrm{ugm} / \mathrm{m} 1)$. These results may indicate that although Rifampin can penetrate the PMN, the bactericidal concentrations for staphylococci may not be achieved within the phagosome of CGD PMN during the four hours of observation. Furthermore, some killing of staphylococci by 1ysates of CGD PMN was observed but this did not appear to be enhanced by preincubating PMN with Rifampin.

(Supported by the Medical Research Council of Canada, MA-5276).
CYTOMEGALOVIRUS IN SICK PRETERM INFANTS. K. Hufnagle, L. Orew and R. Ballard (Intr. by P. L. Ballard), Hospital and Med. Ctr., San Francisco, California

During a six-month period cytomegalovirus (CMV) was cultured from 8 sick preterm infants. Seven infants developed a recognizable symptom complex clinically suggestive of bacterial sepsis with marked hepatosplenomegaly. Other manifestations were listlessness, thrombocytopenia and a relative lymphocytosis with an increase in atypical lymphocytes. Seven had a worsening of their respiratory status, and, in five, a remarkable grey color was noted. Repeated bacterial cultures of blood, urine and CSF were negative. Viral cultures of urine grew CMV. Two of the infants died, and CMV was grown from multiple organs, including lung, brain, kidney and liver, and inclusions were found in lungs, pancreas and kidneys. The infants were all under 32 weeks' gestation, with an average birth weight of $1118 \mathrm{gm}$ ( $880-$ 1600). At birth, all had respiratory distress; six required mechanical ventilation. All of the infants had some degree of chronic oxygen dependency, in three severe enough to require prednisone. Prior to diagnosis of CMV, all received multiple blood transfusions (II to 30 units). Five of the eight had cultures for CMV at birth and were initially negative. We believe all of these infants acquired the disease while in the intensive care nursery and that it produced a significant change in their clinical course. Cytomegalovirus appears to be an important pathogen in the compromised premature host and should be considered when evaluating clinical deterioration in these infants.

COMPARISON OF TRIMETHOPRIM-SULFAMETHOXAZOLE 591 (TMP-SMZ) AND PENTAMIDINE (PNT) IN THE TREATMENT OF PNEUMOCYSTIS CARINII PNEUMONITIS (PCP). Walter T. Hughcs, Sandor Feldman, Subash Chaudhary, Michacl J. Ossi, and Shyamal K. Sanyal. St. Jude Children's Research Hospital, Infectious Discases and Cardiopulmonary Services, Memphis, TN.

PCP in immunosuppressed patients is fatal in 90 to $100 \%$ of cases if untreated. PNT is in current use for treatment of this infection but is associated with inordinate toxic and adverse side effects and is not commercially available in the United States. Recently we have shown that TMP-SMZ is effective in the treatment of PCP in both animals and humans. The present study was designed to determine the relative efficacies of PNT and TMP-SMZ for the treatment of unselected patients with PCP. All cancer patients with diffuse pneumonitis by rocntgenogram, tachypnea, hypoxia and $P$. curinii identified in specimens obtained by percutancous needle aspiration of the lung were randomized to receive either PNT or TMP-SMZ. If after 3 or morc days of treatment specified criteria indicated progression of disease, therapy was changed to the alternate drug.

Thirty-seven patients have been studied. Of the 18 treated with PNT, 11 recovered and one died receiving this drug alone; 6 others required crossover to TMP-SMZ and 3 recovered. Of the 19 treated with TMP-SMZ, 13 recovered recciving this drug alone; 6 required crossover to PNT and 2 recovered. Overall recovery rates were $78 \%$ for PNT and 79\% for TMP-SMZ groups.

Of the 12 patients treated with PNT alone, hypoglucosemia occurred in 5 , above-normal BUN values in 4 , and injection site reactions in 8 . None of these adverse effects occurred in the TMP-SMZ group. A transient rash occurred in 4 of the TMP-SMZ- and 2 of the PNT-treated patients.

Thesc data show that TMP-SMZ is as effective as PNT in the treatment of PCP and is associated with significantly fewer adverse effects. MUCOCUTANEOUS LYMPH NODE SYNDROME IN CONTINENTAL USA T. Jacob John, Clifford D. DeBenedetti and Maurice L. Arizona Medical Center and the Tucson Medical Center, Department of Pediatrics, Tucson, Arizona.

Mucocutaneous lymph node syndrome (MLNS) is a newly recognized acute illness of unknown etiology of children, widely prevalent in Japan, and also recognized in Korea, Hawaii and Greece. Recently a case has been reported from Texas and another from Canada. We present 4 cases of MLNS in white children in Tucson to illustrate the clinical features of this syndrome and to con firm its occurrence in the continental USA. They were 3 girls and 1 boy, between 3 and $4 \frac{1}{2}$ years of age. The principal signs and symptoms of MLNS are fever of over a week's duration, bilateral nonexudative conjunctivitis, changes of lips and oral cavity, indurative edema of hands and feet, pleomorphic nonvesicular exanthem and nonsuppurative cervical 1 ymph node enlargement. All of these features were found in each patient. other common features seen in them were arthralgia, arthritis, neutrophilic leukocytosis, elevated ESR, C reactive protein, drop in hernoglobin and hematocrit levels and peeling of skin of hands and feet during convalescense. The diagnoses of Stevens-Johnson syndrome, serum sickness, scarlet fever, infectious mononucleosis, atypical measles, and Rocky Mountain spotted fever were ruled out in them by appropriate clinical and laboratory criteria. A high titer of antihyaluronidase antibody was detected in one patient, but its significance is unknown. All children recovered without myocardial infarction of other sequelae. 
PERSISTENT CYTOMEGALOVIRUS (CMV) AND EPSTEIN-BARR VIRUS (EBV) GENOMES IN LYMPHOBLASTOID CELL LINES FROM AN INFANT, AFTER CONGENITAL INFECTION.

Jean H. Joncas, José Menezes and Eng S. Huang (Sponsored by J. Ducharme )

Armand Frappier Institute, Montreal; Ste Justine Hospital, Montreal; University of Montrea1, Montreal, Quebec; University of North Carolina, North Carolina, USA

Serial lymphoblastoid cell lines were established from an infant with congenital cytomegalovirus infection. By molecular hybridization, both EBV and CMV were found in the 2nd lymphoblastoid cell line (SD2) established at age 9 months. No detectable antigens of CMV but EBNA (nuclear antigen) and EA (early antigen) were expressed by EBV within that line. The EBV genome present in both the first and the second line shared only 65 to $70 \%$ homology with the HRIK-EBV suggesting that it could be a different or defective EBV strain. To our knowledge, this is the first time that the CMV genome is found unexpressed in a human lymphoblastoid cell line following infection. To date CMV could not be rescued from the 2nd lymphoblastoid cell line by cocultivation with human embryo fibroblasts known to allow replication of both CMV strain AD169 and the CMV strain from the infant's urine. A EBNA positive lymphoblastoid cell line of female karyotype was obtained by cocultivating lethally Xirradiated SD2 male cells with cord blood 1 ymphocytes from a female newborn. The latter experiment will be repeated using EBV positive CMV negative serum in an attempt to neutralize EBV and obtain a CMV transformed cord blood lymphocyte cell line.

\section{$\mathbf{5 9 4}$ INCIDENCE OF AMPICILLIN-RESISTANT STRAINS OF}

Waheed N. Khan, William J.Rodriguez, Gail L.Friedman, Guido A. Controni, Sydney Ross. (Sponsored by Robert H. Parrott). Research Foundation of Children's Hospital National Medical Center, 2125-13th. Street, N.W, Washington, D.C. 20009. During the past year, 500 infants and children, both sick and well, were surveyed for ampicillin-resistant strains of Haemophilus species in the Washington D.C. and its suburbs.

Haemophilus species were isolated from either nasopharyngeal or throat culture as follows: from both healthy children and children ill with respiratory infections, we found that $39 \%$ of children yielded Haemophilus influenzae and $35 \%$ Haemophilus parainfluenzae. Of considerable importance was the fact that among the H. Influenzae isolates, $9 \%$ were ampicillin-resistant. Similarly, -8 \% of H. parainfluenzae were also resistant to ampicillin.

These findings have disturbing clinical overtones and suggest that there may be an increasing incidence of ampicillinresistant strains of Haemophilus in the Washington area.

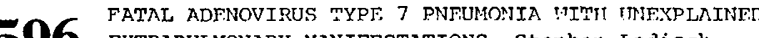
EXTRAPULMONARY MANIFESTATIOHS. Stenhan Ladisch, Michael N. Oxman, Frederick H. Lovejov, Gordon F. Vawter, Denise J. Strieder, Iarcje C. Hoore and Neil Finex Hospital Medical Center, Denartment of Medicine.

Three children 8-24 months of are were hosnitalized within a 3 week neriod with viral pneumonia. Their illness had herun with upner resniratorv sumntoms and prodressed to hronchopneumonia with extranulmonary manifestations involvina liver, skeletal muscle and central nexvous system. Blood ammonias were normal. In all three the clinical course ( 12 to 22 davs) had stahilized with satisfactory pulmonary status and improving parameters of muscle and liver function until the sudden onset of encenhalonathv with intractahle seizures and coma. All three died. Adenovirus tune 7 was isolated from bronchial tissue in one patient and from lung tissue obtained at autopsv from the other two. Brains showed toxic encenhalonathv and livers fatty infiltration with necrosis. Thouah histoloqical abnormalities were nresent there was no microscopic or viroloric evidence of adenovirus infection in orrans other than the lung. Fxtranulmonarv manifestations in certain adenovirus infections have been attrihuted to disseminated virus infection, but this is not the case in these natients. In fact, their disease was similar to Reve's sundrome, thourh differing in clinical course, in the absence of elevated hlood armonia and in histopatholorical detail. The presence in adenoviruses of cansid proteins known to he cytotoxic in vitro mav provide a possible mechanism for the production of disease in
organs distant from the site of virus multiplication.

597 CHRONIC GRANULOMATOUS DISEASE (CGD) : IN VITRO ENHANCEMENT OF BACTERICIDAL ACTIVITY OF CGD PHAGOCYTES BY RIFAMPICIN. Normand D. Lapointe (sponsored by G. Leboeuf). University of Montreal, Faculty of Medicine; Ste Justine Hospital, Department of Paediatrics; Montreal, Quebec, Canada.

Staphylococcus aureus is one of the most important pathogen responsable for infection in CGD. The extracellular action of antistaphylococcal antibiotics is a drawback in the treatment of CGD patients. The intracellular action of Rifampicin against staphylococcus aureus has been described (Mandell, 1972). In a serie of 29 experiments on CGD patients, we have studied the in vitro bactericidal capacity of normal and CGD phagocytes against Penicillin and Rifampicin sensitive staphylococcus aureus.

Different concentrations $(5,10,20 \mu \mathrm{gr})$ of a water soluble preparation of Rifampicin were used (Ciba-Geigy). The bactericidal capacity of phagocyte preparations was tested at different intervals during a 21 hours incubation. While CGD phagocytes bactericidal activity against staphylococcus aureus peaks after 90 minutes of incubation when Penicillin is used in the suspension, in contrast a significant enhancement of the bactericidal capacity is noted with Rifampicin at each concentration used. The enhancing, most likely non toxic, activity of Rifampicin is best noted after 21 hours of incubation.
IMMUNITY TO MEASLES 11 TO 15 YEARS AFTER

595 NATURAL INFECTION AND IMMUNIZATION. Saul Krugman, New York University School of Medicine, Department of Pediatrics, New York.

A prospective seroepidemiological study, in progress since 1960, has included the following groups: 1) 68 institutionalized children who had natural measles infection in 1960; and 2) 114 institutionalized and 220 home-dwelling children who received live further attenuated measles vaccine in 1962. Serial samples of blood obtained before infection or immunization, 1 month later and at yearly intervals were tested for measles $\mathrm{HI}$ antibody. Measles was eradicated in the institution in 1963 and since that time the children were not exposed to infection. Home-dwelling children had repeated exposures to measles in the community since 1963. The results of the 11 to 15 year follow-up were as follows: 1) No cases of measles in any group, 2) detectable levels of measles $\mathrm{HI}$ antibody in all immunized home-dwelling children and in all institutionalized children who had natural measles, and 3) measles $\mathrm{HI}$ antibody had declined to non detectable levels in $10 \%$ of immunized institutional children. The geometric mean $\mathrm{HI}$ antibody titers in the three groups at 11 years were as follows: 1) immunized homedwelling children - 1:50, 2) institutionalized children who had natural measles - 1:30, and 3) institutionalized immunized children - 1:10. These findings indicate that successful immunization with live further attenuated measles vaccine should provide lasting immunity.
RESPONSE OF YOUNG INFANTS TO POLYRIBO-

598 PHOSPHATE (PRP) VACCINE Edward B. Lewin, John Green and Sharon Chi umento. Univ. of Rochester, School of Med. and Dent., Strong Memorial Polyribophosphate (PRP) was administered IM to 40 infants to determine the immuno- and reactogenicity of the vaccine in this age group. Three groups were studied: GP I: PRP alone (N=20, $\overline{a g e}=4.6 \mathrm{mos})$, GP II: PRPDPT administered simultaneously at different sites $(\mathrm{N}=20, \overline{\mathrm{age}}=6.6 \mathrm{mos}), \mathrm{GP}$ III: DPT alone $(\mathrm{N}=4, \overline{\mathrm{age}}=4.3$ mos). Blood was obtained before, 3 wks and 3 mos after immunization. Antibody ( $a b)$ to PRP was measured by radioimmunoassay.

$A \geqslant 2$ fold $\uparrow$ in ab was observed over the 3 month period in only $3 / 20(15 \%), 1 / 20(5 \%)$ and $0 / 4$ in gPs I, II, III respectively. The mean ab rise found in Gp I responders was $45 \mathrm{ng} / \mathrm{ml}$. Ab returned to preimmunization levels by 3 mos in $3 / 4$ responders.

Reaction to the vaccine occurred as follows:

\begin{tabular}{llll} 
& GPI (PRP alone) & & GPII (PRP - DPT) \\
\cline { 2 - 4 } Temp 100F & $5 / 19(26 \%)$ & & $9 / 16(56 \%)$ \\
Erythema & $1 / 19(5 \%)$ & $2 / 16(13 \%)$ & $4 / 16(25 \%)$ \\
Swe11ing & $0 / 19$ & $0 / 16$ & $10 / 16(63 \%)$ \\
Tenderness & $0 / 19$ & $0 / 16$ & $6 / 16(38 \%)$
\end{tabular}

These data suggest that, while PRP in a $5 \mu \mathrm{g}$ dose is only mildy reactogenic, it produces few, low-level and transient ab responses in the age group at highest risk of $\underline{H}$, influenza b meningitis. 
COMMUNICABILITY OF H. INFLUENZAE B FROM

599 MENINGITIS Edward B. Lewin, Chinh $T$. Le and Sharon Chiumento. Univ, of Rochester, Sch. of Med. and Dent., Strong Memorial Hospital, Dept. of Ped., Rochester, N.Y. (Spon. by: R.H. Schwartz).

7 children, aged 4 to 13 mos ( $\overline{a g e}=8$ mos) were exposed to a 7 mos old with H. influenzae b (HFB) meningitis in a day care center. An epidemiologic study of HFB communicability as measured by bacterial acquisition and serologic response was performed in these children, their mothers and the day care staff. NP cultures and blood were obtained 5 days after hospitalization of the index case and repeated 5 wks later. Although $5 / 7$ children, $3 / 6$ of the siblings and $2 / 5$ of the staff members had symptoms of URI over the observation period, $\underline{H F B}$ was not recovered from any of the subjects. Anti-polyribophosphate (PRP) antibody was measured by radioimmunoassay. Only 1 infant ( 9 mos) had detectable anti-PRP ab in the initial sample. Convalescent sera were obtained in $5 / 7$ children. In $2 / 5$ (age= $8 \mathrm{mos}$ ) ab remained undetectable. One had no change in $a b$ titers. $2 / 5$ ( $\overrightarrow{a g e}=13 \mathrm{mos})$ had $a \geqslant 2$ fold rise in $a b$ titer (mean $\uparrow=105 \mathrm{ng} / \mathrm{m} 1$ ). $13 / 15$ adults had high initial titers (range $131-\geqslant 8,000 \mathrm{ng} / \mathrm{ml}$ ); none experienced a rise in titer. There were no secondary cases of meningitis or other invasive $\mathrm{HFB}$ disease. These limited serologic data suggest that, with meningitis as the source and under the epidemiologic conditions dessource and under the epidemio.
cribed, HFB is transmissible.

SEVERE NEONATAL RESPIRATORY DISTRESS AND FULMINANT 600 SEPSIS CAUSED BY GROUP B BETA HEMOLYTIC STREPTOCOCCA
INFECTION. Vichien Lorch, Marta S. De Ortiz, and Charles R. Bauer, (Intro. by W. W. Cleveland), University of Miami School of Medicine, Department of Pediatrics, Miami, Fl.

Currently Group B Beta Hemolytic Streptococcal Infection in the Newborn is classified into: Type I - an early sepsis with onset within the first 10 days of life. Type II - a late meningitis with onset after 10-14 days of life. This report describes a Type IA with severe respiratory distress at birth and fulminant sepsis within 12-24 hours of life. Twenty infants with culturaliy proven Group B Beta Hemolytic Streptococcal Infection were studied. The overall mortality was $60 \%$. Seventeen of the infants were premature; the had an increased mortality of $75 \%$. Membranes were ruptured for more than 24 hours in only 6 infants and 5 were born by Caesarean Section. All the infants presented with severe respiratory distress within four hours of birth. Initial chest radiographs were suggestive of pneumonia in only $30 \%$ of the infants. The most consistent and diagnostic findings distinguishing these infants from those with Hyaline Membrane Disease were persistent arterial hypotension and metabolic acidosis unrelieved by volume expansion. In sumary, we describe 20 infants, predominantly premature, with a distinct syndrome of Group B Beta Hemolytic Streptococcal Infection. This diagnosis must be suspected early if Pencillin therapy is to be effective. The difficulty in specifically diagnosing the cause of respiratory distress in the premature infant often leads to fatal delays in therapy. Intractable hypotension, in association with respiratory distress, should alert to the possibility of Streptococcal Disease.

ONTOGENY OF SERUM AND SECRETORY K1 ANTIBODIES

601 George H. McCracken, Larrie B. Sarff, John B. Robbins, Mary Glode, Bertil Kaiser and Lars Hanson. Dept. Ped.

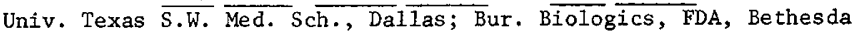
and Dept. Immunol., Univ. Gotebörg, Sweden.

Capsular $\mathrm{K} 1$ antigen is found in $80 \%$ of $\underline{E}$. coli strains causing neonatal meningitis; the amount and persistence of $\mathrm{Kl}$ in CSF and serum are correlated with outcome from disease. K1 strains colonize $20 \%$ to $40 \%$ of healthy subjects and the colonization to disease ratio in neonates is $\sim 200: 1$ suggesting that host immune factors are critical in the pathogenesis of $\underline{E}$. coli meningitis.

$\mathrm{K} 1$ antibodies (AB) in body fluids were measured by a specific enzyme-linked immunosorbent assay. $K 1$ AB was present in IgM and IgG fractions of most adult sera. IgG $\mathrm{K} 1$ was found in cord sera from $83 \%$ of neonates and $K 1$ AB titers in maternal and cord samples were comparable. IgM and IgA K1 AB were absent from cord sera. Of 13 women studied, IgA K1 AB was present in colostrum from 12 and in serum from only 4. Colostral $A B$ titers correlated with rectal carriage of $\mathrm{Kl}$ strains.

Serum IgM K1 AB first appeared in infants at 6 to 12 months of age and the levels of $\mathrm{KI} I \mathrm{IM} A \mathrm{AB}$ correlated with rectal colonization by $\mathrm{K} 1$ strains. The level of transplacentally acquired $A B$ decreased in the first months of 1 ife and IgG K1 AB production was noted at about 2 years of age. Women with recurrent $\mathrm{E}$. coli urinary tract infections had serum IgG and IgM KI levels that were 2 to 4-fold greater than those in healthy women. These data indicate that the presence of $\mathrm{K} 1 \mathrm{AB}$ is related to $\mathrm{E}$. coli $\mathrm{K} 1$ infectare currently under study.
602

QUANTITATIVE SPUTUM CULTURES IN PATIENTS WITH CYSTIC FIBROSIS. Elaine H. Mischlex, M. K. Younoszai, Martin G. Myers, The University of Iowa, College of Medicine, Department of Pediatrics, Iowa City, Iowa.

Quantitative sputum cultures (QSC) were obtained before and 10 days after intravenous antibiotics in 7 patients(pts.) with cystic fibrosis hospitalized because of lower respiratory infection. Probable pathogens isolated $\left(>10^{7}\right.$ organisms/ml) were $\mathrm{S}$. aureus, H. influenzae, pseudomonas species, Klebsiella species and str. pneumoniae, often in mixed culture. Preillness $Q S C$ were indicative of organisms present at the time of onset of illness, but differences in quantitation prior to illness did not correlate with symptomatology. After specific antibiotic therapy, the number of all organisms present initially was reduced by at least 1.00 fold in 5 pts. Six pts. with H. influenzaes 1 with Klebsiella and 1 with str. pneumoniae had <lo organisms/ml after therapy. Two of 3 with s. aureus and 3 of 5 with pseudomonas had a similar $\overline{\text { reduction in }}$ bacterial numbers. Two pts. had a reduction in H. influenzae without a concomitant decrease in either Pseudomonas or $\mathrm{S}$. aureus. One child acquired $10^{6}$ pseudomonas/ml during therapy. The quantitation of organisms in sputum was not helpful in evaluating the presence of acute infection; however, serial QSC were useful in the selection and assessment of specific antimicrobial therapy.

603 TIONSHIP OF BACTEREMIA TO CSF AND HISTOPATHOLOGIC FINDINGS. E. Richard Moxon, Peter T. Cstrow, Robert J. Kapko. Johns Hopkins Univ. Sch. of Med., Dept. of Pediatrics Baltimore. (Spon. by David H. Carver).

Infant (suckling) rats inoculated intranasally with type b Haemophilus influenzae develop bacteremia and meningitis. This model of bacterial meningitis simulates the human disease with regard to nasopharyngeal colonization, nematogenous spread, histological appearance of the inflamed meninges, and age-related susceptibility (J.Inf.Dis. 129:154, 1974).

A micropuncture technique (not previously described) was used to obtain CSF from the cisterna magna. Studies on 201 rats inoculated aged 5,10 or 20 days have defined the relationship of bacteremia to the occurrence of positive CSF cultures, CSF pleocytosis and the temporal relationship of those findings to histologic evaluation of the brain and meninges. It was found that (1) a critical concentration of blood-borne bacteria was necessary, but not always sufficient for the development of meningitis (See table), (2) Positive CSF cultures preceded the appearance of WBCs in CSF, (3) CSF pleocytosis was directly related to counts of viable bacteria in CSF, (4) Inflammation of the meninges, observed on histologic sections of brain, preceded the appearance of CSF pleocytosis, although CSF cultures were positive.

\begin{tabular}{|c|c|c|}
\hline & \multicolumn{2}{|c|}{ Meningitis } \\
\hline & present & absent \\
\hline Bacteremia $\geq 104$ & 73 & 18 \\
\hline Bacteremi $a<10^{4}$ & 3 & 25 \\
\hline
\end{tabular}

chi-square $p<0.001$

COEXISTENCE OF THYROIDITIS AND CONGENITAL RUBELLA

604 SYNDROME. Phillip $\frac{\text { I. Nieburg and Lytt }}{\text { S. }}$. Gardner, Ped., Syracuse, NY

Ziring et a1 (J. Ped. $87: 1002,1975$ ) have noted the coexistence of thyrotoxicosis and congenital rubella syndrome (CRS). Diabetes mellitus and other pancreatic dysfunctions have also been associated with CRS. Our study concerns a now $15 \frac{1}{2}$ yr. old boy initially referred for evaluation of a goiter. He was born in 1959 to a $28 \mathrm{yr}$. old woman whose full term pregnancy was complicated only by clinically diagnosed rubella early in the first trimester. He was small for dates $(1760 \mathrm{g.}$ ) and exhibited in the neonatal period: microcephaly, microphthalmia and cataract of the left eye, clinodactyly, interstitial pneumonia, lymphocytosis (WBC 36,600-65\% 1ymphocytes) and congenital heart disease. Later, growth retardation, deafness and mental retardation were noted. The goiter was noted for 3 wks. prior to our initial evaluation at age $11 \frac{1}{2}$. There was a diffusely and symmetrically enlarged thyroid. Laboratory data included thyroxine by column of $2.0 \mu \mathrm{g} / \mathrm{d} 1$, PBI of $6.1 \mu \mathrm{g} / \mathrm{d} 1$ and elevated antithyroid globulin titer (ATA) of $1: 128$. Therapy with thyroglobulin resulted in progressive diminution in size of the goiter. When last seen at age $14 \frac{1}{2}$ yrs., the thyroid was normal in size and consistency. ATA titer was $<1: 4$ (normal); thyroid microsomal antibody titer was $1: 16$ (elevated).

Patients with CRS may be at increased risk of "autoimmune" diseases. Periodic screening of thyroid and pancreatic function seems warranted in these patients. 
605

PROGNOSTIC/DIAGNOSTIC FACTORS IN GROUP B BETA HEMOLYTIC STREPTOCOCCAL (GBBS) AND OTHER NEONATAL SEPSES. Phillip I. Nieburg (Spon. by Margaret L. Williams), Upstate Med. Center, S.U.N.Y., Dept. of Peds., Syracuse, N.Y.

Absolute neutrophil (PMN) and band counts have been suggested as a diagnostic aid in neonatal bacterial infection. Our recent experience with GBBS and other neonatal sepses (NS) was reviewed to determine whether neutropenia (PMN + Band $\leq 1000 / \mathrm{mm}^{3}$ ) was a useful diagnostic sign and whether neutropenia or sex were prognostically important. Of 26 neonates (18 $\sigma^{\prime}$ and $8 \%$ ) with early onset (age $<2$ wks) GBBS NS, $11(42 \%)$ expired. Case fatality rates were $44 \%$ for $0^{7}$ and $37.5 \%$ for 9 . Of 21 neonates for whom WBC and differential count (diff) were known, 11 (52\%) had neutropenia. Eight of these (73\%) expired acutely while only 1 of $10(10 \%)$ non-neutropenic infants expired acutely $(p=.0017)$. One of 3 neonates with late onset GBBS NS had neutropenia; that child died. Thus, $9 / 10$ neonates with fatal GBBS NS had neutropenia.

Ten neonates had group A beta hemolytic strep. (3) or pneumococcal (7) NS; $5 / 80^{7}$ and $1 / 2$ q expired. WBC and diff were known in $7 / 10 ; 3 / 4$ with neutropenia expired. For NS caused by other Gram positives (coag. negative Staph-4; coag. positive Staph-8; enterococcus-3) 1 of $9 \sigma^{7}$ and 1 of $6 q$ expired. None of 12 had neutropenia.

These results indicate that GBBS sepsis is similar to other NS in its predilection for $\sigma^{x}$ al though the $q$ and $\sigma^{x}$ case fatality rates are similar. Neutropenia is often associated with GBBS and some other Gram positive NS. Although an ominous prognostic sign, its early recognition in potentially septic neonates may facilitate specific therapy with consequent improved prognosis.

606 COMPARISON OF CARBENICILLIN AND AMPICILLIN IN THE TREATMENT OF PURULENT MENINGITIS. Gary D. Overturf,
Jeanette Wilkins, P.F. Wehrle. University of SouthJeanette Wilkins, P.F. Wehrle. University of SouthAngeles, California.

Forty-five adult and pediatric patients with meningitis $(23$ H. influenzae, $13 \mathrm{~S}$. pneumoniae, $4 \mathrm{~N}$. meningitidis and 5 "purulent unknown") were randomly assigned treatment with ampicillin (AMP) or carbenicillin (CB); dosage varied from 200 to $400 \mathrm{mg} / \mathrm{kg} / \mathrm{day}$. Although patients treated with AMP suffered greater morbidity and mortality, AMP patients had more severe disease. All organisms were susceptible to $C B$ and AMP. Comparison of mean CSF concentrations of $C B$ and $A M P$ determined during the first 3 days of therapy $(200 \mathrm{mg} / \mathrm{kg})$ were equivalent $(4.20$ and $3.89 \mu \mathrm{g} / \mathrm{ml}$ for $\mathrm{AMP}$ and $\mathrm{CB}$ respectively); increased doses did not significantly increase CSF levels. CSF/serum ratios varied from 2-162\%; CSF concentrations ranged from $0.11-41 \mu \mathrm{g} / \mathrm{ml}$. High CSF levels were observed during earliex days of illness and with high protein concentrations.

However, 10 of 15 patients with $\mathrm{H}$. influenzae infections treated with $C B$ had a bacteriological failure as defined by positive CSF cultures on day one; only 1 of 8 patients treated with AMP had a similar failure. Methods to determine if $\mathrm{CB}$ is decarboxylated to benzyl penicillin failed to show a significant change of $\mathrm{CB}$; therefore, delay of CSF sterilization of $\mathrm{H}$. influenzae does not appear to be due to a failure to attain adequate C.SF concentrations of $\mathrm{CB}$. Although this delay does not affect the clinical course, $\mathrm{CB}$ may not be adequate treatment for meningitis due to H. influenzae.

VACCINE TRIAL IN MECRL ENEURG COUTTY, $\checkmark . C$. VITH

607 H. INFLUENZAE TYDe b (HITP) AND MENINGOCOCCAL GROUP $c$ (MGC) CAPSULAR POLYSACCHARIDES: PROGRESS REPORT J.C.

Parke, Jx. James Schlesselman, Rachel Schneerson, John B. Robbins Charlotte Memorial Hospital, N.C. Kational Institute of Child liealth and Human Development and Bureau of Biologics, Bethesda, Md.

Individuals, age 2 mon to 5 yr were injected with 25 ug of HITB or $10 \mathrm{ug}$ MGC s.c. with parental permission in a random, double-masked program from $3 / 74$ to-date. Approximately 15,000 doses were injected including, about $70 \%$ of $2 \mathrm{mon}$ to $2 \mathrm{yr}$ individuals. No adverse reactions including high fever were reported. Simultaneous antibody determinations of 300 vaccine recipients of all ages showed low response rate and average serum levels in infants increasing to adult values at about 18 mon for HITB and 2 yr for MCC. There were 4 HITB meningits cases in the HITr, group, 12 HI'iR cases in MGC group and 11 IITR cases in non-vaccinees. IITB septicemia without meningitis occurred in 2 HITB vaccinees, 3 in IGC vaccinees and 1 in non-injectees. Epiglottotis occurred in 2 non-injected children and $l$ adult. One IGC meningitis occurred in a IITTiB vaccinee. Meningococcal Group $B$ meningitis occurred in 2 HITB vaccinees, none in MCr vaccinees and 4 in non-vaccinees. There was no detectable change in the age distribution, overall attack rate or mortality in Mecklenburg County or adjacent areas of diseases caused by these or other encapsulated bacterfa.
EFFECT OF GLUCOSE CONCENTRATION ON WHITE BLOOD CELL

608 FUNCTION. Larry K. Pickering and Joan W. Stoerner.
(Spon. by R.R. HoweT?). Univ. of Texas Med. Schoo?, Depts. of Infectious Diseases and Pediatrics, Houston, Texas.

Phagocytosis and intracellular killing of bacteria are important functions of polymorphonuclear leukocytes (PMNL). Alterations of blood glucose concentrations often occur in neonates, diabetics and patients undergoing parenteral hyperalimentation. This study was undertaken to determine the effect of varying glucose concentrations on resting and stimulated hexose monophosphate shunt (HMPS) activity, phagocytosis and intracellular killing of bacteria by PMNL. PMNL obtained from normal volunteers were incubated in solutions containing glucose concentrations ranging from 0 to $600 \mathrm{mg} / \mathrm{dl}$. The cel ls in these solutions were evaluated for resting and stimulated HMPS activity and phagocytosis and intracellular killing of $\underline{S}$. aureus and $E$. coli. Resting HMPS activity of cells in all samples did not significantly differ from one another. Stimulated HMPS activity was significantly $(p<0.01)$ depressed at glucose concentrations of $0,20,40$, 400 and $600 \mathrm{mg} / \mathrm{dl}$ when compared to $100 \mathrm{mg} / \mathrm{dl}$. The optimal phagocytic activity and intracellular killing ability of PMNL occurred at glucose concentration of $100 \mathrm{mg} / \mathrm{dl}$. At glucose concentrations of $<40$ and $>200 \mathrm{mg} / \mathrm{dl}$ a significant $(p<0.05)$ decrease in PMNL function occurred during the first hour of incubation.

These results indicate that varying glucose concentrations effect bactericidal ability and oxidative metabolism of PMNL. These phenomena may provide a partial explanation for the weil known decrease in resistance to bacterial infections in patients
who have difficulty maintaining glucose homeostasis.

609 PRE AND POST-EXPOSURE USE OF NEW CELL CULTURE RABIES VACCINE. Stanley_A. Plotkin, Tadeusz Wiktor, Ernst Kuwert, T.T. Kramer and Howard Tint. Wistar Inst., Philadelphia, Pa. Inst. Med. Virol. Essen, W. Germany, Auburn Univ., Auburn, Ala., and Wyeth Labs., Philadelphia, Pa.

Presently available rabies vaccines are suboptimal owing to high rates of neurologic or allergic reactions and to poor immunogenicity. A rabies strain was adapted to hunan cell culture, and virus grown in vitro was concentrated and inactivated. The resulting material (HCCV) has been shown to be protective in animals, and has now been extensively studied in human clinical trials. A preexposure regimen of three $1 \mathrm{ml}$ intramuscular inoculation: at 0,7 , and ?I days provided $100 \%$ of volunteers with serum an'ibodies. Mean peak titers are about $1 / 500$ or 10 International Units, which is 10 times higher than $\exists$ fter duck embryo vaccine. Comparison with Semple nerve tissue vaccine was also favorable to $\mathrm{HCCV}$ in terms of faster antibody response. A six-dose post-exposure regimen $(0,3,7,14,30$, and 90 days) resulted in excellent an'ibody responses in humans exposed to proven rabid animals and thus far comblete protection from rabies. Reactions to the vaccine have been remarkably few, none serious, and the problem of sensitization seems to have been circumvented.

CLINICAL FEATURES OF HUMAN REOVIRUS-LIKE AGENT GASTRO610 ENTERITIS IN INFANTS AND CHILDREN. William J. Rodriquez, Hyun Wha Kim, Carl D. Brandt, Robert M. Chanock Albert 7. Kapikian, Richard Wate and Robert H. Parrott. Georqe Washinqton Universitv, Children's Hosnital
Washinqton, D. C., NIAID, NIH, Bethesda, Md.

Between January 1974 and June 1975 infection with a human reovirus-like agent HRVLA ("rotavirus", "duovirus") was shown by EM and serologic techninues in $47 \%$ of 152 infants and children with qastroenteritis. In ours and other studies it appears that this aqent is a major cause of acute diarrheal disease in infants and vouna children. Certain clinical and laboratorv findings appear to be helpful in distinquishing qastroenteritis due to HRVLA from other causes. Age: $67 \%$ of infants and children 6 to 12 months and $79 \%$ of those 1 to 2 vears of age had infection with the HRVLA whereas such infection was found in only $21 \%$ of infants under 6 months of aqe and 23\% of children 2 to 5 . Time of Vear: $60 \%$ of patients studied during the cooler months had HRVLA infection and such infection was not found from May to nctober: Frequency of Vomitina and Dehvdration: Twice as many patients infected with the HRVLA as those who were not had vomiting (92\%) and significant dehvdration, primarily isotonic $(83 \%)$. Since $16 \%$ of those infected with HRVLA had an excess of polvmorphonuclear cells in fecal material this parameter was not helpful in diagnosis. Recoverv was uneventfu? for patients with HRVLA infection, as compared with those without such infection, where seizures and meninqitis were observed. The patients with HRVLA infection were hospitalized an average of 4 davs as contrasted with 6 davs for 
ACCELERATED KILLING OF GROUP B STREPTOCOCCI BY AMPICILLIN AND GENTAMICIN. Victoria Schauf, Audra
Deveikis, Louise Riff (Spon. by Ira Rosenthal) Deveikis, Louise Riff (Spon. by Ira Rosenthal). Abraham Lincoln School of Medicine, University of 111 inois at the Medical Center, Departments of Pediatrics and Medicine, Chicago. Group B streptococci (strep) are susceptible to ampicillin, but despite its widespread use in the newborn, death rates vary from 14 to $70 \%$. Timed bactericidal studies were used to determine optimal conditions for eradication of these organisms. Nine strains isolated from blood or CSF and 5 reference strains of group B strep were studied, as was a clinical isolate of group A strep. Organisms were inoculated into broth containing either $1-2 \mathrm{mcg} / \mathrm{ml}$ ampicill in (Amp), $10 \mathrm{mcg} / \mathrm{ml}$ gentamicin (GM), or both. Quantitative colony counts were done at 1-4 hr intervals for 24 hrs. Growth rates of group $A$ and $B$ strep were similar. Sterilization of the group A strain by Amp required less than $4 \mathrm{hrs}$; $8-24$ hrs were needed for $99.99 \%$ killing of all group B strains. GM was not inhibitory for group B strains, but killing by Amp was accelerated when GM was present.

All strains showed a 1 to $4 \mathrm{log}$ greater reduction in colony count with Amp + GM than with Amp alone. Amp + GM produced $99.99 \%$ killing 2 to 21 hrs more rapidly than did Amp alone. Slow killing of group B strep by Amp may explain the pathogenicity of these organisms in nurseries where Amp is heavily used. Slow killing may also make eradication of organisms in vivo difficult. The current recommendation for use of Amp alone in treatment of group $B$ strep infection should be reevaluated.

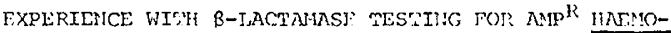

612 phIJIIS IHFLUFMZMI. David Scheifelc, Vassiliki Syriodren's Hosnital !icdical Certer, Dent, of Med. Hoston, Mass. We evaluated a rapid acidimetric test for $\beta$-lactamase production in ampicillin-resistant (Amp $\left.)^{R}\right)$ il. influenzae, hased on pll indicator color chance. $10^{7}$ Amp $R$ bacteria mixer with ampicillinphenol res solution hwdrolyse anysicillin to amicilloic acid producina a vellov color within 30 minutes at $37^{\circ} \mathrm{C}$. F'ortv $\mathrm{Amp} \mathrm{g}^{R}$ strains hydrolysed Pen. $f$, at a rate $>10 \mu q / \mathrm{min} / 10^{7}$ lacteria.

160 strains of $\underline{H}$. intiluenzae tyne $b$ (HIB) were tested blindly:

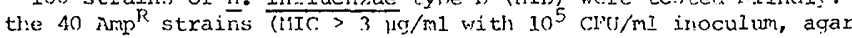
plate (lilution method) were correctly identified, as were the 120 Ans sensitive strains. Subsequent experience examining a total of 994 strains of defined Amn sensitivitv, includine 268 type $b$ $(42 \mathrm{Amp} R$ ) and 726 untypal) le strains (11 Amp $R$ ) yjelder no false negative, false positive or intermediate result?.

The B-lactamase test was used to screen recent isolates of Haemonhilus for Amp resistance. Among 100 consecutive nose and throat isolates of $\mathrm{H}$. influenzae fror patients at CHMC, $5 \mathrm{Amn}^{\mathrm{R}}$

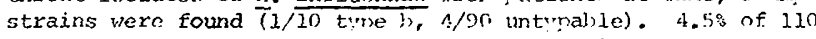
UIB strains isolated from blood or CSF of patients at ClimC in the past 36 months have been $\lambda m)^{R}$.

The B-1actamase test is a rapid, roliakis screening procedure for the detcction of amnicillin resistance in 1 . influenzae isolates and facilitates prompt revision of antib) $\overrightarrow{i o t i c}$ therary in patients with serious infection.
614 VS VS. H. INFLUENZAE, TYPE B, (HIB) IN YOUNG CHILDREN Vanderbilt University, Department of Pediatrics, Nashville, Tenn. Introduced by: David T. Karzon

Since serious infections from HIB tend to occur with greatest frequency in infants and young children, early immunization is desirable. However, it has been shown that young subjects respond poorly to bacterial polysaccharide antigens as a class and in particular to PRP derived from HIB.

This report concerns the effect of priming doses of purified PRP ( $5 \mathrm{mcg}$ ) administered, with or wi thout simul taneous DPT, to 38 infants at ages 2,3,4 and/or 10 months compared with 74 nonprimed children. All were challenged with PRP $(5 \mathrm{mcg})$ at ages 1624 months, with or without simultaneously administered DPT. 44 controls received no PRP at any time to determine background spontaneous antibody acquisition. Serum samples, taken before and 4-6 weeks after each injection-and matched control samples-were assayed against tritiated PRP by radioimmunoassay. Results indicated that:

1) Priming with PRP either in single or multiple doses, did not inhibit or enhance the response to the challenge dose of PRP. 2) Priming with PRP + DPT followed by challenge with PRP + DPT did not enhance or inhibit the response.

3) However, in non-primed children, response to PRP + DPT appeared to be enhanced.

4) Nasopharyngeal carriage of HIB was not prevented by priming with PRP in any combination.

\section{5} MEASLES IN PREVIOUSLY TMMUNIZED CHILDREN: VARIABIE Michigan, Department of Pediatrics, Detroit.

In November and December 1975, 21 cases of classical measles (two confirmed by HI antibody rise) occurred among school children in Northeastern Metropolitan Detroit where acceptance of measles immunization has been traditionally high. Twenty of the 21 cases were previously immunized. Analysis of immunization histories among children in a school where 9 cases occurred can be summarized as follows: The overall attack rate, $8 \%(9 / 115)$ ranged between 48 and 118 in the 4 classrooms involved. Imunization histories could be confirmed in 7 cases; 3 had been immunized at 8 and 9 months of age, the other 4 were older. The attack rate among those propexly immunized (3\%) gives a vaccine efficacy of $97 \%$.

However, vaccine efficacy among two sibships was found to be strikingly poor. In one family of 5 children, all had been immunized, all developed measles. One of these children was immunized at 8 months, but the others were at 12 months or older. In the other family, of 5 children, all had been immunized, three became ill. In two, immunization had been given at 8 and 10 months of. age in the other, at 5 years. Vaccine efficacy among properly immunized children in these two sibships was only $30 \%$.

Cases continue to occur and a prospective serologic investigation is underway to determine if vaccine induced antibody levels are low among this group of children and to determine if level of antibody can be used to predict "susceptible"
613

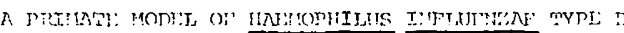

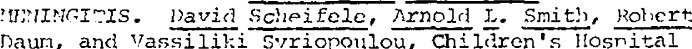
icdical Center, nept. of (1ed., Boston. (Intr. Wy navia !l. Smith) Ar infant primate model of 11 . infiuenzae meninoitis would? qreatly facilitate experimental studies of prevention, nathogenesis and tieray, Extrapolating from our model of meningitis in infant rats in which trpe " meningitis occurs following intranasal inoculation, five rliesus monlev's (lacacca mulatta), ared 3-14 davs, were inoculated intranasally vith $10^{7}$ or $10^{5}$ CFU HIB, strain $\mathrm{L}_{1}$. Inflammation and nersistent colonization of the nasa. mucosa $\left(10^{6} \mathrm{C} U / \mathrm{ml}\right.$ nasal vashincs), fever and bacteremia $\left(10^{3}\right.$. $10^{5}$ (.FU/mI blood) occurred vj.thin 24 hours of inoculation in all animals. Bacteremia and fever persisted over 5-10 davs of ohservation leading to splenomegaly and multifocal septic arthritis. leningitis develoned in 3 aninals. Bacteria were present in C.SF on initial lumisar puncture 48 hours after inoculation. Ilowever, a celiular response was not eviclent until 24 hours later. The classic sions of meningitis were variably present. Maximum hacterial and white cell counts observed in the CSF of each animal were: $10^{7} \mathrm{cFU} / \mathrm{mI}$ with $20,000 \mathrm{wBC} / \mathrm{mm}^{3}, 10^{5}$ with 2,500 cells and $10^{4}$ with 115 cells. Caysular polysaccharide antigen vas detected in CSF at 2 days, reaching a maximum at 3 days, and in serum at 2 days, with maximum of $8 \mathrm{~kg}$ at 4 days. Purulent leptomeningitis with focal vascular thromboses, similar to tirn lesions of HIB meningitis in children, were found on autopsy. This model allows measurement of cerehral hlood flow, detailer CSF ohservations and critical assessment of antibiotic therany.

\section{I6 TRANSFER FACTOR THERAPY OF ADOLESCENTS WITH CHRONIC}

616 AGGRESSIVE HEPATITIS: Stanford T. Shulman, Jack $H$. Florida, Gainesvilie.

Chronic aggressive hepatitis (CAH) is postulated to occur as a result of inadequate $T$-lymphocyte response to viral infection of the liver. The therapeutic potential of transfer factor (TF), the dialyzed lysate of peripheral lymphocytes which enhances T-lymphocyte function, was therefore evaluated in this disease. Four girls and two boys with CAH, aged 12-18 years, were treated with TF prepared from adults who recovered from acute hepatitis B or acute non-B hepatitis. The six teenagers included two who were ${ }_{H B_{s} A S} \oplus$ and four with pre-existing illnesses, including diabetes. hemophilia, as thma, and IgA deficiency. After withdrawal from immunosuppressants, and following reappearance of disease activity, subjects received three injections of TF, each representing $1-2 \times 109$ WBC, over a six-week period. Liver biopsies were performed at the beginning and at the end of the ten week study period in four patients. Chemical and immunologic parameters were followed in all patients. Three of six TF recipients showed histologic improvement and normalization of chemical values, especially SGOT and SGPT. These patients are now 34, 14, and 12 months post-TF therapy and lack evidence of disease activity. Two other patients showed equivocal responses, while the last patient had no evidence of response to TF. To date, only the latter patient has required re-institution of corticosteroid therapy. No patient deteriorated following administration of $T F$, and no adverse effects were encountered. These preliminary data indicate that further experience with immunoenhancement by TF in CAH is warranted. 
TYMPANOMETRY IN THE DIAGNOSIS OF OTITIS MEDIA, Paul A. Shurin, Stephen I. Pelton and Jerome 0 . Klein Harvard Medical School and Boston University School of Medicine, Boston City Hospital Department of Pediatrics, Boston.

Utitis media is comon in children, with highest rates in those less than two years old. Available non-invasive diagnostic methods are subjective and non-quantitative. Tympanometry provides an objective record of the acoustic properties of the tympanic membrane and middle ear. In this study, a quantitative method for the evaluation of tympanograms has been used to develop standards for the diagnosis of middle ear effusion.

Children 2 months to 12 years old were examined using a pneumatic otoscope. Ears were defined as normal on the basis of tympanic membrane mobility. Middle ear effusion was confirmed by needle aspiration. Susceptance tympanograms were obtained at $660 \mathrm{~Hz}$ over a pressure range of -300 to $+300 \mathrm{~mm} \mathrm{H}_{2} \mathrm{O}$, using a Grason-Stadler Model 1720 otoadmittance meter. The -300 and +300 $\mathrm{mm} \mathrm{H}_{2} \mathrm{O}$ intercepts of each curve were connected by a straight line and the area enclosed by the curve and intercept line was measured; positive values were assigned to traces above and negative values to those below the intercept line. The tympanograms of 124 normal ears had a mean area of $7.3 \pm 4.3$ (S.D.) $\mathrm{cm}^{2}$. Those of 75 ears with effusion had a mean area of $-0.79 \pm 2.6 \mathrm{~cm}^{2} \quad(t=14.6$, $\mathrm{p}<0.001)$. If an area of $<3.4 \mathrm{~cm}^{2}$ is set as a diagnostic standard for effusion, the test has a sensitivity of $98.7 \%$ and a specificity of $87.1 \%$.

Tympanometry is an accurate and objective means of detecting middle ear disease.

\section{8} PEDIATRIC PROTECTED ENVIRONMENTS. Stuart E. Siege1, Ronald Nachum, Jonathan Kellerman, Roger Uno and David Rigler. (Spon, by Harry T. Wright, Jr.) USC School of Medicine and Childrens Hospital of Los Angeles, Department of Pediatrics, Los Angeles.

A comprehensive protected environment ( $P E$ ) regimen adapted for the unique needs of the pediatric patient has been developed to determine if the risk of infectious complications can be reduced in children with compromised host defenses. The use of a modified semi-portable laminar airflow room has been combined with oral non-absorbable antibiotics, topical antimicrobials and a lowbacteria diet to reduce the exogenous and endogenous microbial load to levels at or below those previously obtained in similarly treated adults. Microbiological surveillance cultures of the PE revea1 $96 \%$ sterile air and $92 \%$ sterile surface samples. Results of weekly cultures of 6 children treated for 70-130 days in the $\mathrm{PE}$ include $89 \%$ sterile stoo $1,82 \%$ sterile skin and $38 \%$ sterile nasopharyngeal cultures. In contrast, organisms are regularly recovered from throat cultures. Mild diarrhea is occasionally noted during the first 10 days of oral decontamination. No other significant toxicity accompanied this treatment. Constant psychosocial evaluation and a continuing program of intervention is conducted by a team of individuals specifically assigned to this unit. Patient acceptance and adjustment to the PE has been excellent, and no serious or irreversible psychological disturbances have been noted. A microbiologically effective protected environment program for the pediatric patient has been developed without limiting toxicity or patient resistance.

619 CELLULAR IMMUNE RESPONSES TO HERPES GROUP VIRUSES DURING TREATMENT WITH ADENINE ARABINOSIDE. RUSSEII W. Steele and Ronald E. Keeney. Dept. of Pe $\overline{d .,}$ Brook Axmy Med. Ctr., San Antonio, TX and Dept. of Ped., Southern Illinois Univ. Sch. of Med., Springfield, IL. (Spon, by I. Canales Seventeen patients, including 5 neonates and 4 children with leukemia, were treated for severe herpes virus infection with IV adenine arabinoside (ara-A), and 3 others received placebo therapy. Blastogenic and cytotoxic responses specific for the virus infecting each patient were determined before, during and following therapy using methods previously described (steele, R. W. et. al. J. Infect. Dis. 131:528, 1975). In addition, blastogenic responses to the mitogens: phytohemagglutinin, pokeweed and concanavalin A were examined as well as viral antibody titers.

In vitro responses during and after treatment with ara-A were unchanged or often enhanced as compared to pretreatment values; therefore, unlike other antiviral chemotherapeutic agents, ara-A does not appear to depress the host's cellular immune responses which are vital for elimination of invading herpes group viruses.

Newborn infants, presumably infected at or shortly before birth, did not demonstrate cellular immune reactivity to the infecting virus until after 4 days of life suggesting that protective cellular immune responses are not functional during the first few days after infection. This delay in host resistance dictates the importance of early institution of antiviral therapy.

In the present series, prognosis appeared to be primarily determined by the cellular immune competence of the host at the time of infection, and in the case of neonates both the age and cellular responses to the virus at the time of diagnosis.
EFFECT OF TRIMETHOPRIM AND SULFAMETHOXAZOLE (T-S) IN

620 The therapy OF CHRONIC GRANUlomatous diSEASE (CGD). M. Akram Tamer and Kenneth R. Ratzin (Intr. by W.W. Cleveland). Univ. of Miami, Department of Pediatrics and Medicine, Mi.smi,Fla.

Clinical improvement in $\mathrm{CGD}$ has been reported following treatment with T-S, although the mechanism for this effect has not been satisfactorily defined; there have been contradictory data regarding its effect on leukocyte function. Granulocyte function was studied in two patients with CGD. One patient was recognized at four months of age when he developed Klebsiella osteomyelitis. The other boy was diagnosed at 12 years of age when he developed nocardiosis involving lung, pericardium and bone. There was remarkable clinical improvement in both patients after T-S treatment and they remained free of significant infection while on long-term therapy. In-vitro studies of granulocyte function were carried out after $\mathrm{T}-\mathrm{S}$ therapy and with addition of $\mathrm{T}, \mathrm{S}$ and $\mathrm{T}-\mathrm{S}$ to the leukocyte media. The granulocytes showed no change in quantitative nitroblue tetrazolium reduction, bactericidal activity against Staphylococcus epidermidis $(S-E)$, hexose monophosphate shunt activity, or iodinization of phagocytized heat-killed S-E. Our data do not support the findings that T-S acts by changing granulocyte function as currently measured. Nevertheless, we conclude that it is effective in the treatment and prophylaxis of infection in CGD, and recommend its empiric use.
621 SOCIO-ECONOMIC FACTORS IN THE EPIDEMIOLOGY OF HEMOPHILUS INFLUENZAE, type b MENINGITIS IN CHILDREN RHODE ISLAND 1970-74. Phillip I. Tarr and fearges Peter; Brown University Program in Medicine; Roger Will'iams Genera 1 Hospital, Department of Pediatrics; Providence, Rhode Island. (Sponsored by Leo Stern.)

Recent studies of Hemophilus influenzae, type b meningitis (H.I.) have not adequately separated race, income, education, and crowding in the epidemiology of this disease. To further investigate these factors, cases of bacterial meningitis in Rhode Island (RI) from 1970-74 were identified by review of data from the RI Department of Health, a private health care research organization which codes all hospital discharge diagnoses, hospital bacteriology laboratories, and death certificates. 116 of 169 cases of culture-proven bacterial meningitis and 75 of 81 cases of H.I. occurred in children less than 5 years old. A11 but 2 H.I. cases were reported to the Department of Health. The incidence of H. I. per 100,000 children less than 5 years of age per annum was 20.3 . Although blacks comprise $4 \%$ of the RI population under 5 years of age, $13.3 \%$ of H.T. cases were black. The disease incidence among black children ( $74.3 / 100,000 /$ annum) was significantly higher ( $\mathrm{p}<.001$ ) than that among white children (18.3) The incidence of H.I. in those census tracts (156 of 185) in which the population was greater than $99 \%$ white, was not related to family income, education, or number of household members. Race appears to be a critical and unexplained factor in the epidemiology of H.I., and should be an important consideration in future studies of Hemophilus influenzae vaccines.

\section{2} SIGNIFICANCE OF PNEUMOCOCCEMIA IN FEBRILE CHILDREN NOT INITIALLY ADMITTED TO HOSPITAL

David $W$. Teele, Loma Bratton, and Jerome 0. Klein. Departments of Pediatrics, Harvard Medical School, Boston University School of Medicine, and Boston City Hospital, Boston, Mass. Increasing use of cultures of blood (BC) in the evaluation of febrile ambulatory children has identified many with pneumococcemia. To determine the outcome of pneumococcemia in children not initially admitted to hospital we reviewed the records of 97 such children seen from $9 / 71$ to $11 / 75$ at Boston City Hospital. At the first visit antimicrobial agents were prescribed for 45 , most of whom had pneumonia or otitis media. No antimicrobial agents were prescribed for 52, most of whom had upper respiratory infection or fever without source. The status of all patients on recall to hospital is show below.

$\begin{array}{ccc}\text { Status at follow-up } & \text { Treated initially } & \text { Not treated initially } \\ \text { Improved, afebrile } & 25 & 15 \\ \text { BC negative } & 11 & 3 \\ \text { BC not done } & 6 & 18 \\ \text { Not improved, febrile } & 0 & 2 \\ \text { BC negative } & 1 & 12 \\ \text { BC not done } & 2 & 2 \\ \text { BC still positive } & 0\end{array}$

These data suggest that although pneumococcemia clears in many children, a significant number are at risk for persistent bacteremia or meningitis. 
623 INFLUENZA A INFECTIONS IN YOUNG, SERONEGATIVE CHILDREN Peter F. Wright, Katherine B. Ross, Juliette Thompson, David T. Karzon, Vanderbilt University, School of

Medicine, Department of Pediatrics, Nashville, Tennessee

Influenza A infections are poorly defined clinically, virologically and immunologically in small children. 147 children under 3 years of age in an experimental vaccine clinic were followed through an influenza A/Port Chalmers epidemic, Dec. '74 Feb. '75. Prior influenza experience defined 3 groups:1) 121 seronegative, 2) 10 seropositive, and 3) 16 successfully vaccinated with an experimental live vaccine, A/Hong Kong ts-1-[E] in the 6 months preceding the epidemic. Based upon age and lack of antibody to influenza A strains seronegative children were assumed to be undergoing primary exposure to influenza. Seroconversion (hemagglutination inhibition and antineuramindase) or virus shedding occurred in $49(40 \%)$ of seronegative children, $73 \%$ of whom were i11. No influenza infection occurred in seropositive children. Two of 16 vaccinees had an antibody rise, 1 of whom had otitis. 33 children with respiratory iliness were seen by a physician during the epidemic. Interval histories revealed 31 other respiratory illnesses, 12 of which were accompanied by seroconversion to influenza. of the 33 children examined 25 had influenza as judged by virus isolation (20) and/or seroconversion (24). Children with influenza had coryza,23; cough, 21; pharyngitis,20; irritability, 20; fever greater than $39.5 \mathrm{C}, 13$; anorexia, 13; otitis, 7 ; pneumonitis, 6 ; and croup, 1. Influenza causes extensive morbidity in otherwise heal thy children undergoing primary infection. A recently administered live Hong Kong influenza vaccine was protective against the related but nonidentical Port Chalmers strain.

\section{4}

FERTILIZATION AND PRENATAL MURINE CYTOMEGALOVIRUS (CMV) INFECTION FOLLOWING INSEMINATION WITH A SPERM AND VIRUS MIXTURE. James A. Young, Kwok-Sing Cheung, David J. Lang. Duke Univ. Med. Ctr.,Dept. of Peds., Durham, N. C. Previous attempts to induce congenital $\mathrm{CMV}$ infection in the mouse have been unsuccessful, although infection of pregnant mice with CMV has been associated with increased fetal wastage and resorption, and in some cases by infection of the maternal placenta. In no instance has infection of the fetal mouse been demonstrable. IIn the present studies mice were artifically inseminated employing sperm obtained from the vas deferens and epididymis of virusnegative males. Suspensions of sperm were mixed with medium (controls) or with murine CMV (infected), incubated and introduced into the vagina and uterus of female mice pretreated with gonadotropins. After 14 days of gestation the pregnant mice in both groups were exsanguinated, the embryos removed, washed, minced, and trypsinized to prepare embryonic cell cultures. Viral cytopathic effects (CPE) were not recognized in any primary cultures and after nine days these cells were trypsinized and passed. Typical CPE appeared after 3 days in the secondary cultures from infected embryos. The control cultures remained virus-negative. TCMV has been recovered from human semen and may persist in asymptomatic sexually active males. The effect in man of the presence of CMV in semen upon the induction of congenital infections is unknown. Our studies indicate that, in the mouse, the presence of CMV does not prevent fertilization and that congenital infection may result when this virus accompanies sperm at the time of insemination. This model may prove relevant to some instances of prenatal CMV infection in man.

\section{METABOLISM}

625 LIPID METABOLISM IN THE NEONATE: G. Andrew, * G. Chan* and D. Schiff, Dept. Pediatrics, Obstetrics \& Gynecology, University of Alberta, Edmonton, Alberta. To determine hormonal regulation and metabolism of lipid in the neonate, triglyceride (TG), free fatty acid (FFA), B-hydroxybutyrate $(B O B)$, glucose and insulin levels were measured during and after infusion of $1 \mathrm{gm} / \mathrm{kg}$ of Intralipid over 4 hours. Fifteen infants were studied in the first 48 hours of life in a fasting state: 9 were appropriate for gestational age (AGA) $(4<33$ and $5>33$ weeks gestation)and 6 were small for gestational age (SGA) from 33 to 41 weeks. Fasting levels of TG, FFA, BOB were similar in al1 infants. Maximal increases in these paranleters were reached at the 4 th hour of infusion in all infants but were greatest in SGA group. Table - The changes from 0 hours to 4 hours:

\begin{tabular}{|c|c|c|c|}
\hline roup & $\begin{array}{l}\triangle \mathrm{TG} \mathrm{mg} / \mathrm{d} 1 \\
\text { mean } \pm \text { SEMl }\end{array}$ & $\begin{array}{l}\triangle \mathrm{FFA} M \mathrm{MEq} / \mathrm{L} \\
\text { mean } \pm \mathrm{SEM}\end{array}$ & $\begin{array}{c}\triangle \mathrm{BOB} \mathrm{mMol} / \mathrm{L} \\
\text { mean } \pm \mathrm{SEM}\end{array}$ \\
\hline & & 30 & 15 \\
\hline & $.3 \mp 45.7$ & $1.04 \mp 0.33$ & $113 \mp 0$ \\
\hline & $650.8 \mp 121.2$ & $1.19 \mp 0.27$ & \\
\hline
\end{tabular}
At 2 hours post infusion, levels had returned to fasting in AGA but remained elevated in SGA infants. Fasting levels of glucose and insulin were similar in all infants with little change during or after Intralipid. Thus after TG was hydrolyzed to FFA, some FFA was rapidly metabolized to ketones in all infants. Increase in ketones for a given increase in FFA was greatest in SGA infants. The ketogenic effect of Intralipid was independent of changes in insul in concentration.
626

FAMILIAL PRIMARY HYPERALDOSTERONISM. LOTENZO C. Aschinberg, Conrado Samayoa, Neil A. Kurtzman, Petros M. Zeis. (Spon. by I.M. Rosenthal.) Abraham Lincoln School of Medicine, Univ. of Il1. Hosp., Depts. of Ped. and Med., Chicago, I11.

Primary hyperaldosteronism affecting more than one member of the same family is rare. Three members of a family, a male (T.W.) age 15, a female (A.W.) age 13 and their mother (J.W.) age 34, were found to have all of the principal features regarded as typical of primary hyperaldosteronism (hypertension, elevated serum aldosterone, hypokalemia and low plasma renin). Evidence of hypertensive vascular disease was present on ophthalmologic, roentgenographic and electrocardiographic examination. Expanded (E) and contracted (C) plasma renin activity, serum aldosterone (A) and differential aldosterone measurements after. percutaneous catheterization of the left $(L)$ and right $(R)$ adrenal vein were obtained:

$\begin{array}{lcccccc} & \text { B.P. } & \mathrm{E} & \mathrm{C} & \mathrm{A} & \mathrm{L} & \mathrm{R} \\ (\mathrm{mm} H) & (\mathrm{ng} / \mathrm{ml} / \mathrm{h}) & (\mathrm{ng} / \mathrm{ml} / \mathrm{h}) & (\mathrm{ng} / \mathrm{dl}) & (\mathrm{ng} / \mathrm{dl}) & (\mathrm{ng} / \mathrm{dl}) \\ \text { T.W. } & 160 / 130 & 0.1 & 0.2 & 58 & 28.5 & 1.5 \\ \text { A.W. } & 170 / 110 & 0 & 0.1 & 51 & 23.1 & 9.3 \\ \text { J.W. } & 150 / 120 & 0 & 0.1 & 42.8 & 50.6 & -\end{array}$

Administration of dexamethasone failed to produce a fall in serum aldosterone. Spironolactone $300 \mathrm{mg} /$ day resulted in normalization of blood pressure in all patients after 4-6 weeks. These results indicate the presence of a left aldosterone secreting adrenocortical adenoma responsible for the hypertension in each of these patients. After further observation surgical managementwa 11 be considered.

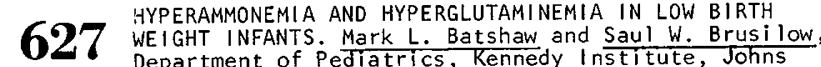
Hopkins Med. Inst., Baltimore, Maryland.

Plasma ammonium ( $\mathrm{NH}_{4+}+$, glutamine (gin), alanine (ala), and glutamate $(\mathrm{glu})$ were measured on capillary blood obtained from a group of neonates: full term $(\mathrm{N}=21)$, preterm appropriate for gestational age (AGA) $(N=12)$ and small for gestational age (SGA) ( $N=$ $8)$. These infants were followed from shortly after birth to 8 weeks of age. The $\mathrm{NH}_{4}+$ levels $(\mu M \pm S E M)$ at 3 days of age were as

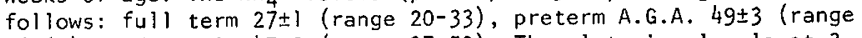

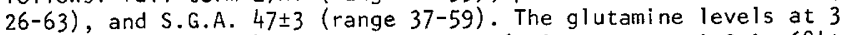
days of age ( $\mu M \pm S E M$ ) were: full term $560 \pm 17$, preterm A.G.A. $684 \pm$ 30 and S.G.A.651 26 . The elevation in $\mathrm{NH}_{4}+$ and $\mathrm{gln}$ in both preterm A.G.A. and S.G.A. groups was significant at $p<.01$. The elevated $\mathrm{NH}_{4}+$ levels persisted at $3-5$ weeks of age $(p<.01)$ : full term $26 \pm 3$, preterm A.G.A. $42 \pm 1$, S.G.A. 42 4 . Ala and glu levels were similar in all groups. There was no significant difference in $\mathrm{NH}_{4}+$ or $\mathrm{gln}$ levels comparing preterm A.G.A. to S.G.A. babies at either 3 days or 3-5 weeks of age. The degree of hyperammonemia was not associated with neurological abnormalities as measured by a Precht neurological exam. Protein intake $(\mathrm{g} / \mathrm{kg})$, sex and race bore no relationship to the elevated levels of $\mathrm{NH}_{4}+$ and gln. These abnormalities persisted past term ( 40 weeks gestational age) and after the babies had attained $3000 \mathrm{~g}$. We speculate that the hyperammonemia and hyperglutaminemia in low birth weight infants may be due to the immaturity of hepatic urea cycle enzymes. Although the hyperammonemia is not clinically symptomatic it may play a role in the late neurodevelopmental abnormalities associated wi th low birth weight.

GLUCOSE PRODUCTION RATES IN INFANCY AND CHILDHOOD.

628 Dennis M. Bier, Rosemary D. Leake, Kenneth J. Arnold, Morey Haymond, Larry D. Gruenke, Mark A. Sperling, and David M. Kipnis. University of California, San Francisco and Los Angeles, Harbor General Hospital Campus; and Washington Uni-
versity School of Medicine; Departments of Pediatrics and Medicine versity School of Medicine; Departments of

San Francisco, Los Angeles, and St. Louis. in children by continuous infusion of the non-radioactive, "irreversible" tracer, glucose-6,6-d 2 , and determination of deuterium isotopic enrichment in blood glucose by gas chromatographymass spectrometry. 41 patients, aged 25 weeks gestation to 14 years $(0.65-42 \mathrm{~kg})$ were studied. A 3 rd degree polynomial expression $(r=0.97)$ defined glucose production as slightly curvilinear to $25 \mathrm{~kg}$ when it reached $140 \mathrm{mg} / \mathrm{min}$, almost the adult value of $161 \mathrm{mg} / \mathrm{min}(2.1 \pm 0.2 \mathrm{mg} / \mathrm{kg} \cdot \mathrm{min}$, mean $\pm \mathrm{SE})$. Grouped by weight, production in prematures ranged from $3-13 \mathrm{mg} / \mathrm{min}(6.5 \pm 0.9 \mathrm{mg} / \mathrm{kg} \cdot \mathrm{min})$, in term infants from $17-31 \mathrm{mg} / \mathrm{min}(8.2 \pm 0.7 \mathrm{mg} / \mathrm{kg} \cdot \mathrm{min})$, in $5-24 \mathrm{~kg}$ children from $39-167 \mathrm{mg} / \mathrm{min}(7.2 \pm 0.3 \mathrm{mg} / \mathrm{kg} \cdot \mathrm{min})$, and in $25-42 \mathrm{~kg}$ children from $128-160 \mathrm{mg} / \mathrm{min}(4.6 \pm 0.3 \mathrm{mg} / \mathrm{kg} \cdot \mathrm{min})$. On the basis of estimated brain weight, however, glucose production was almost linear $(r=0.94)$ from premature to adult, with estimated cerebral glucose utilization for the child similar to that of the adult. These data, the first measurements of "new" glucose production in childhood, suggest that brain size may constitute a principal determinant of glucose production rates throughout $l i f e$. 
BURN EDEMA AND PROTEIN LEAKAGE IN THE RAT. I. RELA-

629 TIONSHIP TO TIME OF INJURY. Ben H. Brouhard, Hugo F Carvaja1, Hugo A. Linares, and Daniel L. Traber, Spon. by Luther B. Travis, Univ. of Texas Med. Br. and Shriners Burns Inst., Depts. of Ped., Path., and Anesth., Galveston, Texas Studies to determine the relationship between increased vascular permeability (edema formation and protein leakage) and time post injury, were performed in 108 sprague Dawley rats. In half of the animals three tiny deep second degree non-contact burns $(0.2 \%)$ were inflicted to the left of the spine. The remaining 54 animals served as sham controls. Six animals from each group were sacrificed at each of the following time intervals post injury: 30 min., 90 min., 3 h., 6 h., $12 \mathrm{h.}, 24 \mathrm{h.}, 36 \mathrm{~h}$. , and 48 h. RISA-I $131(200 \mathrm{mc} / \mathrm{kg})$ was given IV 30 minutes prior to sacrifice (decapitation). Segments of skin (burn and contralateral site), muscle, tail, liver, spleen, and kidney were obtained, weighed, counted (scintillation counting), dried $\left(60^{\circ} \mathrm{C}\right.$ for $96 \mathrm{~h}$.) and reweighed. In one third of the animals autoradiographic studies were also performed. Edema was maximal at $3 \mathrm{~h}$. post burn and was no longer demonstrable by $24 \mathrm{~h}$. Albumin leakage was maximal at $30 \mathrm{~min}$, and disappeared by $12 \mathrm{~h}$. post injury. In another set of experiments where RISA-I 131 was injected at the time of the burn, high tissue counts persisted for as long as $48 \mathrm{~h}$. These studies suggest that albumin leakage is not significant beyond $6 \mathrm{~h}$. post injury but that once in the interstitium albumin is trapped for up to $48 \mathrm{~h}$. Whether or not this response is similar for more extensive burns remains to be elucidated.

CALCIUM SUPPLEMENTATION IN NEONATES: EFFECTS ON 630 SERUM PTH, 25 HYDROXY D AND BTOCHEMICAL VALUES. David Cincinnati and Pittsburgh, Departments of Pediatrics.

In neonates at risk for hypocalcemia (prematurity, birth asphyxia) the effect of oral Ca supplement on serum parathyroid hormone (PTH), vitamin D (25 hydroxy D) and biochemical ( $\mathrm{Ca}$, ionized $\mathrm{Ca}, \mathrm{Mg}$ and $\mathrm{P}$ ) levels has not been studied. Sixteen infants were studied with carefully matched gestations $(\leqslant 33$ wks 1 pair, $34-35$ wks 3 pairs, $36-37$ wks 2 pairs, $\geqslant 38$ wks 2 pairs) and birth asphyxia (Apgar $\leqslant 6$ ). One of each pair was given oral $\mathrm{Ca}, 75 \mathrm{mg} / \mathrm{kg} /$ day from ages $12-72 \mathrm{hrs}$. PTH was determined by radioimmunoassay (antisera recognizing $\mathrm{N}$-terminal, normal undetectable to $180 \mu \mathrm{I}-\mathrm{Eq} / \mathrm{ml}, 89 \%$ of adults detectable), 25 hydroxy D by protein binding (modified Belsey's, normal 13-81 $\mathrm{ng} / \mathrm{ml}$, rickets evident at $<5 \mathrm{ng} / \mathrm{ml}$ ) and ionized Ca by Orion $99-20$ electrode. During supplementation, serum $\mathrm{Ca}$ was $9.5 \pm 0.4 \mathrm{mg} \%$ (mean \pm S.E.) ionized $\mathrm{Ca} 3.3 \pm 0.3 \mathrm{mg} \%$, vs $8.1 \pm 0.3$ (paired $t, p<0.001$ ) and $2.9 \pm$ $0.1 \mathrm{mg} \% \quad(p>0.10)$, respectively, in controls. Serum $\mathrm{P}$ and $\mathrm{Mg}$ were not different. Serum PTH levels were $89 \pm 20 \mu 1-\mathrm{Eq} / \mathrm{ml}$ pre and $107 \pm$ 27 with supplements, not different from $91 \pm 21$ and $97 \pm 22$ respectively, for controls. 25 hydroxy D levels were $47 \pm 6 \mathrm{ng} / \mathrm{ml}$ pre and $45 \pm 4$ with supplements, compared with $40 \pm 8$ and $48 \pm 6$, in controls. Clinically, there were no side effects (cardiorespiratory rates, apnea, weight change) except increased frequency of bowel movements $(4.7 \pm 0.5$ vs $3.0 \pm 0.5, \mathrm{p}<0.05)$. Oral Ca supplementation raises serum $\mathrm{Ca}$ in neonates at risk for hypocalcemia, has no discernible adverse effect on calcium regulatory hormonal or chemical factors, and has minimal side effects.

NEONATAL CYCLIC AMP (CAMP) AND PHOSPHATURIC RESPONSE

631 TO ENDOGENOUS PTH SECRETION: A STUDY MODEL UTILIZING EXCHANGE BLOOD TRANSFUSION. David R. Brown, Robert Johnson, I-Wen Chen, \& Reginald C. Tsang. Univs. of Cincinnati and Pittsburgh, Departments of Pediatrics, and Good Sam. Hosp.

Theoretical disadvantages in the use of exogenous parathyroid (PTH) extract have limited studies of target organ responsiveness to PTH in neonates. It has been suggested that the neonate has renal unresponsiveness to stimulation with PTH. In the present study, renal responsiveness to endogenous PTH was evaluated during exchange transfusions with citrated blood; citrate complexing of $\mathrm{Ca}$ has been shown to be a stimulus for PTH secretion. Seven neonates (30-41 wks gestation, weight $950-3380$ gms, ages $<5$ days) received exchange transfusions for hyperbilirubinemia. Timed voided urine and serial blood samples were obtained before, during, and after transfusion. Urinary cAMP was measured by radioimmunoassay (Steiner's), serum PTH by radioimmunoassay (antisera recognizing $\mathrm{N}$-terminal, normal undetectable to $180 \mu \mathrm{l}-\mathrm{Eq} / \mathrm{ml}$, $89 \%$ adults detectable). PTH levels were $58 \pm 4$ (mean \pm S.E.) before transfusion and $99 \pm 23$ during transfusion (paired $t=1.8$ ). Urinary cAMP increased from $822 \pm 218 \mathrm{pmol} / \mathrm{ml}$ before transfusion to a maximum of $1829 \pm 438$ after the start of the transfusion $(p<0.05)$, preceding a similar increase in urinary phosphate $(9.7 \pm 3.9 \mathrm{mg} / \mathrm{dl}$ to $34.9 \pm 13.9, \mathrm{p}<0.05)$. The increase in phosphaturia did not correlate with that for $\operatorname{cAMP}(r=-0.52)$. Five of 5 infants with gestation $\leqslant 35$ wks had phosphaturic responses, 4 of whom also had increases in CAMP excretion. In conclusion, urinaxy CAMP and phosphaturic responses to endogenous PTH can be demonstrated and evaluated in neonates during exchange blood transfusions.
CHOLESTEROL TURNOVER AND BALANCE STUDIES IN HOMO-

632 zYGOUS TYPE II CHILDREN. Guy A. Carter; William E. Lauer. Univ. of Iowa Hospitals, Dept. of Pediatrics, Iowa City IA

If the hypercholesterolemia in homozygous familial hyperlipoproteinemia type II is the result of deficient binding sites for low density lipoprotein, then increased cholesterol synthesis should occur as a result of decreased feedback inhibition of HMG CoA reductase. Isotopic cholesterol turnover and absorption, and sterol balance studies were done in two children with homozygous familial hyperlipoproteinemia type II. In the turnover the halflife of the first exponential ( 6.25 days) and the second exponential ( 69.5 days) were both prolonged (normal $=3.8 \pm 0.5$ and $51+14$ days respectively). The rate constant for the removal of cholesterol from pool A ( $\mathrm{K}_{\mathrm{AA}}$ ) was only 0.0652 (normal=0.1605+0.0236), and for excretion from the body $\left(\mathrm{K}_{\mathrm{A}}\right)$ only 0.0197 (normal $=0.0708 \pm$ $0.0221)$. The calculated metabolic clearance fraction was $2.5 \%$ (normal $=30 \%$ ), and the mean production rate was on $1 \mathrm{y} 7.5 \mathrm{mg} / \mathrm{kg} / \mathrm{d}$ (normal $=27+10 \mathrm{mg} / \mathrm{Kg} / \mathrm{d}$ ). Cholesterol absorption was norma1

$(57.8 \%)$. From the sterol balance studies their neutral sterol excretion was $5.22 \mathrm{mg} / \mathrm{Kg} / \mathrm{d}$ (normal $=7.39 \pm 1.07 \mathrm{mg} / \mathrm{Kg} / \mathrm{d}$ ), and bile acid excretion was $1.64 \mathrm{mg} / \mathrm{Kg} / \mathrm{d}$ (normal=3.94+0.94 mg/kg/d). This resulted in a total fecal cholesterol excretion of only $6.86 \mathrm{mg}$ / $\mathrm{Kg} / \mathrm{d}$ (norma1 $=11.33+2.01 \mathrm{mg} / \mathrm{Kg} / \mathrm{d}$ ). These studies indicate that the prominent defect is an inability to excrete cholesterol from the body rather than the oversynthesis predicted from the defect in the cell membrane binding sites.

\section{EFFECT OF PROLONGED CONTINUOUS GLUCOSE INFU-}

\section{SION (CGI) IN PRETERM INFANTS. O. Chaivorarat and}

H.S. Dweck (Spon. by M. Davidson). Albert Einstein Coll. of Med., Dept. of Ped., Bronx-Lebanon Hosp. Ctr., Bronx, N.Y.

Nineteen preterm infants $540 \mathrm{~g}-1840 \mathrm{~g} \mathrm{~b}$. wt. and $\leqslant 7 \mathrm{hr}$ old received CGI (4 hr@6 mg/kg/min +4hr@8 mg/kg/min). Baseline and hourly samples showed significant differences of plasma glucose $(G)$ and osmolality between babies $\leqslant 1 \mathrm{~kg}(n=7)$ and those $>1 \mathrm{~kg}(n=12)$.

Mean hourly G's were consistently higher in babies $\leqslant 1 \mathrm{~kg}$ after $2 \mathrm{hr}$ of CGI. Six of 7 babies $\leqslant 1 \mathrm{~kg}$ had osmolalities $>300 \mathrm{mOsm} / \mathrm{L}$ and persistent G> $200 \mathrm{mg} \%$ compared to only 1 of 12 babies $>1 \mathrm{~kg}(p<0.001)$. Twenty-eight of the $55 \mathrm{G}$ samples in babies $\leqslant 1 \mathrm{~kg}$ exceeded $200 \mathrm{mg} \%$ compared to only 1 of the 93 samples from babies $>1 \mathrm{~kg}(p<0.001)$. Osmolalify exceeded $300 \mathrm{mOsm} / \mathrm{L}$ in $15 / 53$ samples from babies $\leqslant 1 \mathrm{~kg}$ and in only $4 / 85$ samples from babies $>1 \mathrm{~kg}(p<0.001)$.

These data confirm our earlier demonstration that tiny babies are susceptible to hyperglycemia with parenteral glucose. They further demonstrate that significant hyperglycemia and hyperosmolality are likely to occur in tiny babies after prolonged $\mathrm{CGI}$ at current recommended glucose infusion rates.

THE RIBOFLAVIN STATUS OF DIABETIC CHILDREN,

634 Harold $S$. Cole, Rafael Lopez and Jack M. Cooperman, New York Medical College, Dept. of Pediatrics, New York, N. Y. 10029

Most diabetic children are placed on somewhat restricted diets, but with the normal amount of calories. Others subject themseives to fad diets. It is quite difficuit to maintain good metabolic control in juvenile diabetes, and consequently some may be vitamin deficient. The riboflavin status of 19 children with insulin-dependent diabetes mellitus and 103 normal children was studied. Riboflavin was selected because as the coenzymes flavin adenine dinucleotide (FAD) and riboflavin mononucleotide (FMN), it is intimately concerned with carbohydrate and fat metabolism. The chlldren in both groups ranged in age from 8-16 years and ali lived in a low socioeconomic area with similar dietary habits. None received supplemental vitamins. Erythrocyte glutathione reductase (EGR), a FAD-containing enzyme, was measured. The result is expressed as the activity coefficient (AC). Normal $A C$ values in children range from 0.9 to 7.2 . The decrease in saturation of EGR resuiting in AC vaiues above 1.2 is an early indicator of riboflavin deficiency.

Five of the 19 diabetics (26.3\%) were riboflavin deficient, with $A C$ values of $1.40-1.71$. Nine of 103 control children $(8.7 \%)$ were deficient and had $A C$ values of $1.37-2.12$. All deficient children in both groups were retested after 2 weeks of oral riboflavin supplementation, and all had normal $A C$ values. The exact mechanism for the increased incidence of ariboflavinosis in dfabetic children is unknown; however, it is advisable that they receive adequate vitamin intakes. 
635 HYPOCALCEMIA DUE TO LOSS OF PARATHYROID HORMONE (PTH) ACTIVITY ON RENAI FUNCTION: EVIDENCE FOR A DEFECTIVE ACTIVITY ON RENAL FUNCTION: EVIDENCE FOR A DEFEC
PTH. Natthew H. Connors, Julian J. Irias, Mahin Golabi, and Bagher M. Sheikholislam (Spon. by Eli Gold). School of Medicine, University of California, Davis, Department of Pediatrics, Davis, California.

Coexistence of biochemical evidence for hypoparathyroidism and radiological evidence for hyperparathyroidism has not received a satisfactory explanation. Studies of a 7 year old girl with seizures, laryngospasm, and tetany revealed hypocalcemia $(4.7 \mathrm{mg} /$ d1), hyperphosphatemia ( $11 \mathrm{mg} / \mathrm{dl}$ ), and normal serum magnesium. Plasma PTH was elevated, with values of $2063 \mathrm{pg} / \mathrm{ml}$ and $3693 \mathrm{pg} / \mathrm{ml}$ in radioimmunoassays for the amino-terminal region and for the carboxy-terminal region of PTH. Clinical features of pseudohypoparathyroidism were absent. Increased serum alkaline phosphatase activity $(335 \mathrm{mU} / \mathrm{ml})$ with roentgenographic evidence of subperiosteal bone resorption indicated PTH activity. Intramuscular administration of PTH extract caused a rise of serum $\mathrm{Ca}(9.6 \mathrm{mg} /$ dl) and a fall in serum $P(7.9 \mathrm{mg} / \mathrm{dl})$. The serum $\mathrm{Ca}, \mathrm{P}$, and alkaline phosphatase became normal during therapy with vitamin D2. PTH, as measured by both assay systems, decreased toward normal during therapy and evidence of subperiosteal bone resorption disappeared. While serum $\mathrm{Ca}$ and $\mathrm{P}$ were normal, a decrease in ionized $\mathrm{Ca}$ was induced by infusion of EDTA. This caused a marked rise in PTH levels without increased urinary cyclic AMP or decreased tubular reabsorption of phosphate.

The findings suggest that the patient's endogenous PTH affects bone resorption but not renal handling of $P$. Parathyroid function appears responsive to physiologic control by Ca concentration. From these observations we infer a defective endogenous PTH.

CARBOHYDRATE METABOLISM AND PANCREATIC ISLET CELL FUNCTION IN GONADAL DYSGENESIS (GD). G. Costin and M.D. Kogut, USC School of Medicine, Childrens tal of Los Angeles, Department of Pediatrics, Los Angeles. lasma glucose (G), immunoreactive insulin (IRI) and immunoreactive glucagon (IRG) levels were measured fasting and following oral (OGTT) and intravenous (IVGTT) glucose, and following I.V. tolbutamide ( $T$ ) and arginine (A) in 13 patients, 10-20 years old. The patients were divided into 2 groups, 6 non-obese (NOb) and 7 obese (Ob) (wt $>2$ SD for $h t$ ). In ob mean fasting $G$ was $>$ NOb but similar to norma1; fasting IRI and IRG were $>$ NOb $(p<0.005)$ and $>$ normal $(p<0.001)$. OGTT was normal in NOb and abnormal in $3 / 7$ Ob. During OGTT mean $G$ and IRI were $>$ normal in $0 b(p<0.05)$ and normal in NOb; IRG was suppressed by oral $\mathrm{G}$ only in NOb $(p<0.05)$. In both groups $\mathrm{Kg}$ was normal but IRG was not suppressed during IVGTT, Following $T$ the fall in $G$ below fasting was normal in NOb and < normal in Ob $(p<0.05)$; IRI was > normal $(p<0.05)$ in both groups. Following A mean IRI was normal in both groups; IRG increase above fasting was $>$ normal $(p<0.02)$ only in $0 b$.

Conclusions: Ob patients with GD have 1) elevated fasting IRI and IRG, 2) exaggerated IRG response to A, 3) failure of IRG suppression despite > normal G levels and 4) insufficient decrease of $G$ post T despite > normal IRI. In Nob, > normal IRI after T and failure of IRG to suppress with IVGTT were noted. Although hormonal alterations were noted in all patients, insulin resistance secondary to obesity may accelerate the occurrence of glucose intolerance in $O b$ patients with GD.

DOGENOUS GLUCOSE PRODUCTION DURING CONSTANT GLUCOSE

637 INFUSION IN THE NEWBORN LAMB. Richard M. Cowett, John B. Susa, William Oh and Robert Schwartz. Brown University Program in Medicine, Departments of Pediatrics, Women and Infants Hospital of Rhode Island and the Rhode Island Hospital, Providence. Fine control of hepatic glucose production is characteristic of the adult response to exogenous glucose. Since previous studies have assumed the suppression of endogenous glucose production during steady state infusion, we have tested this hypothesis in 11 unanesthetized term lambs(mean age 3.75 days) fasted initially for 7 hours. Basal plasma glucose was $107 \pm 8 \mathrm{mg} / \mathrm{dl}(\mathrm{m}+\mathrm{SEM})$ and insulin was $24 \pm 5 \mathrm{uU} / \mathrm{ml}$ following which control animals received $0.45 \% \mathrm{NaCl}$ and experimental animals received glucose at $5.63 \mathrm{mg} / \mathrm{kg} / \mathrm{min}$. After 6 hours of infusion, steady state glucose values of $91 \pm 7 \mathrm{mg} / \mathrm{dl}$ and insulin of $16 \pm 1 \mathrm{uU} / \mathrm{ml}$ in the control animals and $125 \pm 3 \mathrm{mg} / \mathrm{dl}$ and $22 \pm 3$ $\mathrm{uU} / \mathrm{ml}$ in the experimental animals were achieved. Glucose turnover was determined by the prime-constant infusion technique of steele using radio-labelled glucose, which gave rates of $5.25 \pm 0.6 \mathrm{mg} / \mathrm{kg} /$ min after 14 hours of fasting in the control animals compared to $9.69 \pm 0.86 \mathrm{mg} / \mathrm{kg} / \mathrm{min}$ in the experimental animals. The latter included endogenous hepatic glucose output of $4.06 \pm 0.89 \mathrm{mg} / \mathrm{kg} / \mathrm{min}$. The steady state hyperglycemia in the experimental animals was due to exogenous infusion plus persistent hepatic output. This developmental difference from the adult response may be due to (1) hormonal differences, (2) immature nervous system control, or (3) immaturity of intrinsic hepatocyte substrate-enzyme control.

SERUM TRIGLYCERIDE: CORRELATION BETWEEN 24 HOUR IN-

638 AND 2 PM SAMPLES. Mark M. Danney, John T. Hayford, Robert G. Thompson. Univ. of I I wa Col. of Med., University of Iowa Hospitals, Dept. of Pediatrics, Iowa City, Iowa 52242 The fasting triglyceride ( $T$ ) has been accepted for screening despite the known fluctuations of $T$ throughout the day. A total of 41 patients (26 normals (N1), 9 diabetics with normal fasting $T$ (DM), and 6 patients with hypertriglyceridemia (HT)) had 24 hour integrated concentrations (IC) of $T$ determined by means of a portable constant withdrawal pump. All subjects continued with their normal activities and consumed their normal diets during the study. Analysis by linear regression of fasting T vs IC, and mean fasting +2 hour post-prandial $T$ VS IC is as noted:

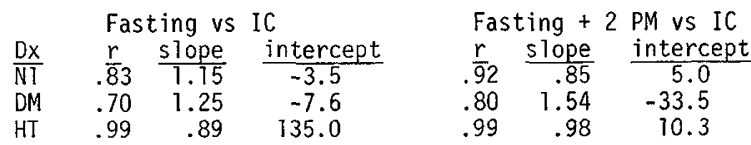

Assuming that IC represents the triglyceride milieu of the patient a single fasting sample is an accurate reflection of the IC of $T$ in normals and diabetics. However, the mean of a fasting and 2 PM sample more accurately reflects the IC of $T$ in these patients, since this mean includes a component of the dietary contribution to plasma triglycerides. In contrast, no increase in predictability of IC of $T$ in hypertriglyceridemic patients is achieved in the 2 sample vs fasting assessment of $T$.

GLUCONEOGENESIS IN THE LARGE FOR GESTATIONAL AGE IN-

639 FANT FOLLOWING ALANINE LOADING. Abdul Darki, Robert Medical Sciences Campus, University Hospital, Department of Pediatrics, Little Rock, Arkansas.

The ability of the large for gestational age (LGA) infant to initiate gluconeogenesis in response to alanine stimulation is unknown. We studied $30 \mathrm{LGA}$ infants all who received $500 \mathrm{mg} / \mathrm{kg}$ alanine in one-half normal saline by nasogastric tube and others received only normal saline at 2 to 5 hours of age. Ten infants were appropriate for gestational age (AGA) and of these 5 received alanine. Baseline 1,3 , and 6 hours determinations of blood glucose, insulin, alanine and glucagon were done.

Basal glucagon insulin and glucose levels were similar in the AGA vs LGA infants. In response to the alanine solution, glucagon levels were slightly increased in the LGA vs AGA. In contrast, a marked augmentation of glucose response was seen ( $38 \mathrm{mg} \%$ vs $5 \mathrm{mg} \% \mathrm{p}<.001)$. These levels remained slightly higher throughout the study period. Insulin levels in response to alanine were similar in both groups of infants.

These data document an accentuated glucose response to an amino acid stimulus in the large for gestational age infant. This increased glucose response in the face of minimal increases of glucagon suggests augmented gluconeogenesis and/or glycogenolysis or increased sensitivity to glucagon in the large for gestational age infant.

640 PARATHORMONE (PTH) AND CALCITONIN (CT) IN PREMATURES Walle, Philippe J. Chopard, Denis C. Grafmeyer (Spon. by Paul R. Swyer). Dept. Neonat., Hosp. E Herriot and INSERM U. 34, Lyon, France.

We have previously demonstrated in premature infants an increase in serum CT and PTH from birth to 48 hours of age. In order to evaluate the effect of Calcium infusion on hormonal regulation, we studied serum Calcium (Ca) Phosphorus (P) Magnesium $(\mathrm{Mg})$ PTH and CT in two groups of prematures, group I $(n=25)$ receiving $35 \mathrm{mg} / \mathrm{kg} / \mathrm{D}$ of Calcium, group II $(\mathrm{n}=15)$ without Calcium infusion. Blood samples were taken every $12 \mathrm{~h}$. from $1-3 \mathrm{~h}$. to $48 \mathrm{~h}$. Serum $\mathrm{Ca}, \mathrm{Mg}$ and $\mathrm{P}$ remained stable in group $\mathrm{I}$ while in group II, there was a decrease of serum Ca from $8.90 \pm 0.7 \mathrm{mg}$ $/ 100 \mathrm{ml}$ at time $\mathrm{l}-3 \mathrm{~h}$. to $6.57 \pm 1.1 \mathrm{mg}(\mathrm{P}<0.01)$ at $36 \overline{\mathrm{h}}$. and $\mathrm{Mg}$ from $1.64 \pm 0.13 \mathrm{mg} / 100 \mathrm{ml}$ at time $1-3 \mathrm{~h}$. to $1.45 \pm 0.13 \mathrm{mg}$ at $24 \mathrm{~h}$. $(\mathrm{P}<0.01)$. Serum $\mathrm{Ca}$ at time $12 \mathrm{~h}$. and $24 \mathrm{~h}$. and serum $\mathrm{Mg}$ at time 24-36 and $48 \mathrm{~h}$. were significantly lower in group II. Serum PTH and $\mathrm{Cr}$ changes were as follows without significant differences between the two groups:

Time

$1-3 \mathrm{~h}$.

Norma1

Groups (n) children I(25) II(14)

$\mathrm{PTH} \mu \mathrm{leq} / \mathrm{ml}<100$

CT $\mathrm{pg} / \mathrm{ml}<150$

$$
\text { (25) }
$$

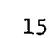

$\pm 90$

343

343
\pm 708
144
+116

567

$\pm 1005$
$24 \mathrm{~h}$.

$48 \mathrm{~h}$.

$\begin{array}{rrcc}I(19) & I I(13) & I(8) & I I(7) \\ 232 & 251 & 264.6 & 287.2 \\ \pm 11.2 & \pm 120 & \pm 112.4 & \pm 95.8 \\ 1272 & 1462 & 1590 & 535 \\ \pm 1000 & \pm 806 & \pm 1127 & \pm 578\end{array}$


EFFECT OF CITRULLTIN ON IYPFRAMMONEMIA IN REYE'S

64.1 SYNDROIE. G. Robert DeLong, Daniel C. Shannon, and setts General Iospital, Pediatric Neurology linit and Children's Service, Boston.

The effect of citrulline administration $(100 \mathrm{mg} / \mathrm{kg} / 6 \mathrm{hrs}$.) on arterial $\mathrm{Nil} 3$ was studied in 5 pts. with Reye's. Its use was suggested by 1.) absent citrulline in blood in Reye's 2.) presumed impairment of citrulline synthesis due to decreased ornithine transcarbamylase 3.) the role of citrulline in increasing incorporation of $\mathrm{NH}_{3}$ nitrogen into urea. Citrulline-treated pts. had initial mean arterial $N ?_{3} 985 \mu \mathrm{gg} / \mathrm{dl}$. (5 pts.); non-citrulline 852 (6 pts.). After 24 hrs., mean arterial $\mathrm{NH}_{3}$ was 107 (cit.) vs. 287 (non-cit.) $(\mathrm{p}=<.05)$. Effect on $\mathrm{NH}_{3}$ was expressed as $\mathrm{r}_{2}$ for decline of arterial $\mathrm{CH}_{3}$ after peak value. Citrullinetreated pts. had $\mathrm{T} \frac{1}{2}$ of $5.5 \mathrm{hrs}$. (at $12 \mathrm{hrs.}$ ) and $7.1 \mathrm{hrs}$. (at 24 hrs.) Non-citrulline patients, otherwise treated similarly, had $\mathrm{T}^{\frac{1}{2}} 8.5 \mathrm{hrs}$. (at $12 \mathrm{hrs.}$ ) and $25 \mathrm{hrs.} \mathrm{(at} 24 \mathrm{hrs.}$ ). Jata from literature corresponded to non-citrulline pts, ?wo pts. (init. NII 2300 and 660) received citrulline only for 12 !hrs.; Hll $_{3}$ declined wit $\mathrm{T}^{\frac{1}{2}} 5.0$ and $5.6 \mathrm{hrs}$.; subsequently, despite continuing peritoneal dialysis and exchange transfusions, $\mathrm{NH}_{3}$ rebounded sharply (to1250 and $550 \mu \mathrm{g} / \mathrm{dl}$ ). All five citrulline-treated pts. were decerebrate at nadir; four survived, one with moderate neurologic deficit. One pt., withnut neurologic function on admission, died. $T \frac{1}{2}$ for fall of blood $\mathrm{NH}_{3}$ affords a quantitative measure of $\mathrm{iNH}_{3}$ metabolism in Reye's, and the effect of treatment. Citrulline appears to hasten the fall of arterial $\mathrm{HH}_{3}$ probably by increasing incorporation into urea.

642

INTERRELATIONSHIP OF SERUM IMMUNOREACTIVE CALCITONIN (iCT) AND SERUM CALCIUM IN NEWBORN INFANTS. Hans $C$. Dirksen and Constantine S: Anast, Univ. of Mo. Medical School, Univ. of Mo. Med. Center and V.A. Hospital, Department of Child Health and Research Service, Columbia, Mo.

The purpose of the present study was to determine serum iCT levels in newborn infants and to relate these levels to the serum calcium concentration. The study group was divided by gestational age and by birth weight (from Lubchenco, L.0., et al., J. Pediatrics $81: 814,1972$ ). The serum iCT levels were significantly higher in newborn infants than in older children and adults; the highest values were found in the premature group. The serum iCT levels reached a peak during the first 24 hours of life and then decreased. The incidence of hypocalcemia was greater in both premature and term infants who had relatively high levels of iCT.

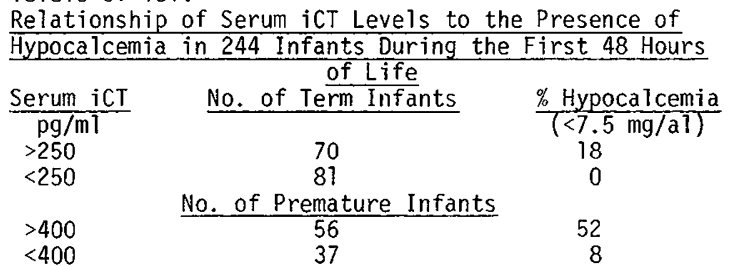

The results suggest that calcitonin may be of etiologic significance in early neonatal hypocalcemia. Work is underway to determine the relationship of serum iPTH with the above.

GLUCAGON SECRETORY RESPONSE IN CHILDHOOD OBESITY: RE-

643 SPONSE TO ALANINE AND WEIGHT LOSS, Robert H. Fiser, and George A. Bray, University of Arkansas Medical Sciences Campus and UCLA School of Medicine, University Hospital and Harbor General Hospital, Departments of Pediatrics and Medicine, Little Rock, Arkansas and Torrance, California.

Although the response of plasma glucagon to various stimuli has been variable in the obese adult, its role in producing the hyperinsulinemia in childhood obesity has not been elucidated.

To evaluate the role of glucagon in the obese state, we have measured the response of plasma glucagon, insulin and glucose to infusions of alanine in 8 obese children before and after weight loss.

Fasting levels of glucose and insulin were increased in the obese children while glucagon levels in the basal state were similar (216 vs $191 \mathrm{pg} / \mathrm{m} 1$ ). In contrast, alanine infusions markedly increased glucagon levels in the obese individuals, peak responses being 391 vs $212 \mathrm{pg} / \mathrm{ml}(\mathrm{p}<.001)$. After weight 1 oss, basal glucagon levels decreased from 216 to $154 \mathrm{pg} / \mathrm{ml}(\mathrm{p}<.001)$. Although the hyperglucagonemia in response to alanine stimulation diminished after weight loss, the response of plasma glucose increased. Glucose responses to alanine were minimal prior to weight loss but increased after weight Ioss $(p<.05)$.

These data document an augmented response of plasma glucagon in response to alanine infusions in the obese child. The hyperglucagonemia in response to alanine along with the diminished response after weight loss suggests altered hepatic response to glucagon stimulation of gluconeogenesis and/or glycogenolysis.
EVIDENCE FOR ALTERED ADRENERGIC CONTROL OF BASAI INSULIN LEVELS IN OBESITY. R. H. Fiser, Jr. and George A. Bray, University of Arkansas Medical Sciences Campus and UCLA School of Medicine, University Hospital and Harbor General Hospital, Department of Pediatrics and Medicine, Little Rock, Arkansas and Torrance, California.

The autonomic nervous system is important in the control of insulin secretion in normal man. Its possible role on the potentiation of the elevated basal insulin levels in obese individuals has not been defined.

To delineate the role of the autonomic nervous system on this phenomenon, we have measured plasma insulin and glucose concentrations in 8 obese and 5 lean individuals af ter alpha and beta blockade infusions. Phentolamine produced a slight increase in insulin levels in lean individuals while levels were decreased in the obese (no alpha stimulation). Propranolol infusions decreased insulin levels $\left(\Delta 35^{\%}\right)$ in both groups while glucose levels remained unchanged. Epinephrine infusions increased glucose levels ( $\triangle 80 \%$ vs $\Delta 40 \%) \quad p<.001$ in the lean subjects when compared with the obese. Although basal insulin levels were unchanged in the betablocked lean subjects, insulin levels were decreased $(\Delta 35 \%)$ in the obese $(\mathrm{p}<.001)$.

These data document altered adrenergic control of basal insulin levels in obesity. The failure of the obese individuals to increase plasma insulin levels with phentolamine and to decrease levels with epinephrine infusion in contrast to the lean individuals suggests that approximately $30 \%$ of the (elevated) basal insulin levels in the obese represent release from adrenergic inhibition.

645 Jeffrey Friedman, Frank P. Manginello, Peter A. M. Auld; Cornell University Medical College; The New York Hospital; Dept. of Pediatrics; New York, N.Y.

Glycosuria is observed as a frequent complication of fluid therapy in premature infants receiving intensive care. In a retrospective study of consecutive admissions weighing less than 2000 gms, more than $90 \%$ developed glycosuria at least once during the first week of life. A group of 14 infants uniformly distributed by weight from 730 to 1860 gms. was studied prospectively during the first two days of life to determine renal glucose thresholds (the serum glucose value at which glycosuria first occurs). This was done by infusion of dextrose solutions of increasing concentrations. There was no relationship between the measured threshold and weight or gestational age. However, the dose of glucose ( $\mathrm{gm} / \mathrm{kg} /$ day) required to reach the renal threshold was directly proportional to weight $(p<0.05)$. This dose ranged from $11 \mathrm{gm} / \mathrm{kg} /$ day in a $750 \mathrm{gm}$. infant to 15.5 $\mathrm{gm} / \mathrm{kg} /$ day for an infant of $1500 \mathrm{gms}$. In a second retrospective study, hourly doses of glucose in infants receiving conventional fluid therapy were determined. There was no evidence of renal damage in these infants. In greater than $90 \%$ of cases, glycosuria, when it occurred, was associated with an infusion rate in excess of the threshold mean for weight.

For patients without evidence of renal damage, glycosuria may be avoided by infusion of glucose-containing solutions at constant rates with the dose of sugar determined by the infant's weight.

646 (GRAND)PARENTAL DEPRIVATION AS TRIGGER FOR ONSET AND/OR RECURRENCE OF JUVENILE THYROTOXICOSIS. Lytt I. Gardner, Genetic and Endocrine Unit, Dept. of Ped., State Univ. of N.Y., Upstate Med. Ctr., Syracuse.

Case studies have been made on 3 kindreds affected with Graves' disease, all exhibiting an autosomal dominant pattern of inheritance. There appeared to be an association between actual or threatened loss of a grandparent or parent and the onset or recurrence of thyrotoxicosis. Proband 非 was a $9 \mathrm{yr}$. old boy who had lived in Alabama with his grandmother since age $2 \frac{1}{2}$. He was placed on a bus, alone, with a tag around his neck and "mailed" to his step-mother in Syracuse. He developed signs of hyperthyroidism in a few weeks and progressed rapidly to frank thyrotoxicosis and cardiac failure. Proband 非 was an $8 \mathrm{yr}$. old boy who suffered the death of a favorite grandmother. Shortly thereafter he presented with frequent blinking, soft stools, heat intolerance, muscle incoordination, weakness and a serm $\mathrm{PBI}$ of $18 \mu \mathrm{g} \%$. A grandfather died 16 mos. 1ater, at which time he developed a relapse with depression and denial of the death. Another relapse occurred 2 mos. later, when his father was hospitalized for a minor operation. Proband 33 was a $14 \frac{1}{2}$ year old girl whose onset of thyrotoxicosis was not associated with any known emotional event. However 9 mos. later a grandparent died, and her relapse was associated with serum T4 of $18.2 \mu \mathrm{g} \%$, higher than with the original diagnosis. One year later another grandparent died and once again she relapsed, with elevated serum T4, which had previously been controlled with PTU. 
ADIPOSE TISSUE CELLULARTTY IN THE PRADER-WILII SYNDROME Fredda Ginsberg-Fellner and Jerome L. Knittle, Mt. Sinai School of Medicine of C.U.N.Y., Dept. of Pediatrics, New York

Obesity in the Prader-Willi syndrome has been described as having its onset after the age of two. Thus, clinically it differs from most other extreme obesity where the onset is usually during infancy. Therefore it was of interest to compare adipose tissue cellularity and obesity in patients with Prader-Willi syndrome and in those with obesity with onset in infancy.

Eight patients, six girls and two boys with Prader-Wi11i syndrome, 7 ages $3 \frac{1}{2}$ to $11 \frac{1}{2}$ and one age 22 , were studied. Five of eight were less than the third percentile for height and the remaining three were between the third and tenth percentiles. Their percent idea 1 weights averaged $204 \pm 11 \%$ and all had norma 1 bone ages. Adipose cel1 size of tissue obtained by needle aspiration from the buttocks avexaged $0.79 \pm .04$ micrograms of lipid per cell, significantly greater than that found in other similarly aged grossly obese children. Total adipose cell numbers were only moderately elevated for age and degree of obesity. Weight reduction resulted in a rapid decrease in adipose cell size and was accompanied by return of glucose tolerance to near normal levels, decrease in serum hyperinsulinemia and in one case, loss of need for exogenous insulin therapy.

These findings differ from the hypercellularity seen in most cases of childhood obesity and are similar to those found in hypothalamic-lesioned animals. Thus, in this type of obesity excessive caloric intake after the age of two results primarily in increased cell size.

INSULIN, OBESITY AND TRIGLYCERIDE RELATIONSFIPS IN

64816 CHLDREN WITH FAMLLIAL HYPERTRIGLYCERIDENIA. C.J. Fallat, R.C. Tsang, S. Wacholder, C.R. Buncher. U.Cincinnati, Coll. Med., Gen. Clin. Res. Ctr., Cincinnati, Ohio.

In 16 consecutively ascertained children with familial hypertriglyceridemia (FTG), insulin (IRI), triglycerides (TG), and obesity $(O B)$ were studied to determine if FTG children had any distinctive IRI:TG relationships. Fat mass was $>97$ th percentile for age in 11/1.6 FTG children. Mean \pm SE TG for the 16 FTG children was $225+19 \mathrm{mg} / \mathrm{dl}$. When compared to 16 normal children (TG= 64+5) (matched for degree of OB) fasting IRI, IRI area (IA) and glucose areas (GA) during oral glucose tolerance tests, and IA/JA ratios were similar for normal and FTG, $\left(\bar{X}_{+}\right.$SE $)$:

$\begin{array}{lllllll}\text { Group } & n & \text { Age }(y x) & \text { IA } & \text { Fasting I } \overline{R I} & \text { GA } & \text { TA/GA } \\ \text { FTG } & 16 & 13+1 & 137+16 & 10+1 & 351+16 & .39+.05 \\ \text { TG } & 16 & 12+1 & 116+23 & 10+2 & 337+23 & .33+.05\end{array}$ Normal 16 1. $12+1 \quad 1.16+23 \quad 10+2 \quad 337+23 \quad .33+.05$ By simple correlation or multiple régression analysis, TG did not correlate significantly with ponderal index (PI) $(r=-.35)$, IA $(r=$ .30), GA $(r=-.01)$, or IA/GA $(r=.36)$ in FTG children, or in normals $(r=.27, .08, .27, .08)$. In FTG children, correlations between TG and IA/GA or IA $(r=.36, .30)$, were higher than in normals $(r=.08, .08)$, suggesting that IRI may play some role in control of TG metabolism. With minimal weight loss and adherence to the "Type IV" diet, TG ( $\overline{\mathrm{X}}+\mathrm{SE})$ fell from $238+24$ to $140+14 \mathrm{mg} / \mathrm{al}$ $(p<.02)$ in the 11 obese children with FTG, indicating utility of diet. Insulin does not appear to play a predominant role in FTG of chilaren.

FAMILIAL TYPE III HYPERLIPOPROTEINEMIA IN CHILDHOOD.

$649 \frac{\text { C.J.Glueck, R.W.Fallat, M.J.Mellies, P,M.Steiner, R.C. }}{\text { Tsang. University of Cincinnati, College of Medicine }}$ General Clinical Research Center, Cincinnati.

Familial type III hyperlipoproteinemia is usually first diagnosed in adults; the two 16 year old probands with type III represent only the 3 rd and 4 th children reported. Diagnosis of type III was based on the ratio of very low density lipoprotein cholesterol (C-VLDL) to triglyceride, with a ratio $\geq 0.30$ denoting type III. Type III was additionally identified by the presence of an abnormal low density lipoprotein (B-VLDL) floating in the ultracentrifugal density fraction $d<1.006$. Lipids and lipoproteins in $\mathrm{S} . \mathrm{E} .$, a I6 year old female and C.H., a 21 year old male (with palmar xanthomas at age 16), were as follows:

$\begin{array}{cccccc} & \text { Cholestero1 } & \text { C.VLDL } & \text { Triglyceride(TG) } & \text { C-VLDL/TG } & \text { B-VLDL } \\ \text { S.E. } & 177 & 52 & 167 & .31 & \text { present } \\ \text { C.H. } & 374 & 179 & 294 & .61 & \text { present }\end{array}$

Studies in the

brother $(C-V L D L / T G=.38), B$-VLDL and a type III pattern in a 57 year old mother and 78 year old grandmother, and type IV hyperlipoproteinemia in a 47 year old maternal uncle. Vertical transmission of type III through 3 generations was consistent with that of an autosomal dominant trait. C.H.'s father had type II hyperlipoproteinemia; a sister and paternal aunt had type IV hyperlipoproteinemia. S.E., obese at $5^{\prime} 9^{\prime \prime}$ ( 230 pounds), failed either to reduce or follow the type III diet with cholesterol 137, TG 182, and C-VLDL/TG . 39 on follow-up. On type III diet and Clofibrate, C. H. 's cholesterol fell to 210 , TG to 105 , but C-VLDL/TG remained characteristic of type III, .49 .
NEONATAL FAMTLIAL HYPOBETALIPOPROTEINEMTA.C.J.Glueck,

650 R.C.Tsang,M.J.Mellies, R.W.Fallat, P.M.Steiner.Univ. of Cincinnati, Coll.Med., Gen。Clin.Res.Ctr, Cincinnati.

Familial hypobetalipoproteinemia, FHB, characterized by low total cholesterol(C) and low density lipoprotein C(C-LDL), possible increased longevity, and decreased atherosclerotic event rates, provides a natural model for study of the role of C-LDL in atherosclerosis. Longitudinal study of infants with FHB allows the determination of any relationship between low C-LDL and development of nerve demyelination. Measurement of cord blood C-LDL in a propositus neonate led to identification of a kindred with 4 generation vertical transmission of FHB. Cord blood C and C-LDL were measured in a study of plasma lipids in 3000 live births. Cord blood C, high density $C(C-\mathrm{HDL})$, and triglyceride (TG) were normal, 53,37 , and $34 \mathrm{mg} /$ $\mathrm{dl} ; \mathrm{C}$-LDL $(9 \mathrm{mg} / \mathrm{dl})$ was $<2.5$ th percentile for C-LDL $(10 \mathrm{mg} / \mathrm{d} 1)$ in 60 normal neonates. At age 1.5 years on ad-1ib diet $(680 \mathrm{mg}$ cholesterol/ day), C and C-LDL $(85,19 \mathrm{mg} / \mathrm{d} 1)$ were $30 \%$ below lowest normal limits; C-HDL was normal $(57 \mathrm{mg} / \mathrm{dl})$. Relatives with FHB included:

\begin{tabular}{lcrrccc} 
Subject & Sex & Age & C & C-HDL & C-LDL & TG \\
\hline father & M & 23 & 117 & 74 & 39 & 10 \\
paternal uncle & M & 22 & 93 & 54 & 37 & 10 \\
paternal aunt & $\mathrm{F}$ & 10 & 108 & 66 & 37 & 32 \\
paternal grandmother & $\mathrm{F}$ & 44 & 132 & 70 & 50 & 34 \\
paternal great- " & $\mathrm{F}$ & 75 & 147 & 84 & 61 & 33 \\
paternal great-aunt & $\mathrm{F}$ & 44 & 132 & 70 & 50 & 34 \\
\hline
\end{tabular}
\begin{tabular}{ccccccc} 
paternal great-aunt & $F$ & 44 & 132 & 70 & 50 & 34 \\
\hline All FHB subjects had normal physical and neurological exams. Four
\end{tabular} generation vertical transmission of FHB was consistent with that of an autosomal dominant trait. Measurement of C-LDL, with family study, allows neonatal diagnosis of FHB.

\section{1} PORTAL VEIN DIVERSION (PVD): A MODEL FOR THE STUDY Ronald w. Gotlin, Charles W. Putnam, Thomas E. Starzl In the treatment of glycogen storage disease (GSD) and homo-
ygous familial hypercholesterolemia. (FH), PVD had been efficacious and has provided us with a human model with which to study the hepatic regulation of portal hormones. We have examined peripheral insulin (I), glucagon (G) [Unger's $30 \mathrm{~K}$ antiserum] glucose, free fatty acid (FFA), triglyceride (TG) and somatotrophin concentrations (GH) before and up to 8 months following PVD in 10 children with GSD or FH. Subjects were studied over a 24$\mathrm{hr}$. interval permitting meals, sleep and activity and during intravenous hyperalimentation (PHA) a $5-\mathrm{hr}$. oral glucose tolerance test and/or following intravenous arginine stimulation. The preoperative results reflect blunted or normal $I$ and $G$ changes in response to stimuli and a marked augmentation in I during PHA. Glucagon levels were elevated pre PVD in 2 of 3 subjects and only modest changes were observed after PVD. Consequently I:G molar ratios pre and post PVD reflect enhanced insulin delivery; however by the 4-5 month follow-up study peripheral I:G ratios were reduced. The pre PVD relative hyperinsulinemia is associated with appropriate changes in FFA and TGs and rebound hypoglycemia was noted in 2 of the younger subjects. GH levels were unchanged and neither enhanced $\mathrm{GH}$ nor somatomedin levels accompanied the enhanced growth velocities. These studies extend our knowledge of the hepatic influence on peripheral $I$ and $G$ levels in GSD and $F H$ and suggest that adaptive changes occur that can compensate for the loss of hepatic regulation.

\section{2} ESTROGEN EFFECT ON SERUM CALCIUM IN THE RAT. M.A. Wottuso, C.T. Nervez, R.D. Jacobs, R.J. Shott, M.L. Depts. of Peds. and Biochem., Syracuse, N.Y.

Estrogens inhibit mobilization of bone calcium by parathyroid hormone both in vivo and in vitro. Estradiol, estrone, and estriol $(0.2 \mathrm{ug} / \mathrm{gm} \mathrm{IP})$ were administered to newborn $(7 \mathrm{gm})$ rats and the following changes in plasma calcium were seen:

\begin{tabular}{llll} 
& \multicolumn{4}{c}{$\Delta \mathrm{Ca}, \mathrm{mg} / \mathrm{dl}$} \\
\cline { 2 - 4 } Time & Estradiol & Estrone & Estriol \\
\hline $2.5 \mathrm{hr}$ & $-2.2(23)^{*}$ & $-0.8(9) *$ & $-0.3(14)$ \\
$3.0 \mathrm{hr}$ & $-1.5(27)^{*}$ & & \\
$6.0 \mathrm{hr}$ & $-1.5(12)^{*}$ & ()$=\mathrm{n}$ & $*=\mathrm{p} \& 01$ \\
$16 \mathrm{hr}$ & $0(24)$ & ()
\end{tabular}

The hypocalcemic effect of estradiol was prevented by parathyroid extract and by the antiestrogenic compound ethamoxytriphetol. Intact adult rats showed no change in $\mathrm{Ca}$ after estradiol; following adrenal and parathyroid ablation Ca fell $1.2 \mathrm{mg} / \mathrm{d} 1$ at $3 \mathrm{hr}$. Offspring of dams fed $\mathrm{Na}$ barbital for 3 days (to induce hepatic steroid-inactivating enzymes) had no hypocalcemia after hepatic effect in immature rats at a time when hepatic and parathyroid function are known to be low. High plasma estrogens may contribute to hypocalcemia in human neonates as well. 
653 ADRENOCORTICAL FUNCTION IN SMALL FOR GESTATIONAL AGE (SGA) NEONATES - L. A. Grajwer, L. D. Lilien, R. L. Rosenfield, and R. S. Pildes: Cook County Hosp, Depts. of Pediat., Univ. of 111. and Univ. of Chicago, Chicago Plasma glucose and cortisol were measured during the first 2 days of age in 8 appropriate for gestational age premature (Group A), I3 normoglycemic SGA (Group B) and 6 SGA infants with hypogly cemia (Group C). Cortisol was assayed by competitive protein binding after separation by thin layer chromatography. Infants in group $C$ were significantly more mature than those in groups $B$ or $A$; no significant difference in birth weight was noted between the groups. Mean ( + SD) gestational age/birth weight (wks/kg) was $39.0+1.7 / 1.8+0.4$ in group $C, 36.6+2.0 / 1.7+0.3$ in group $B$ and $32.5+3.271 .6+0.5$ in group $A$ infants. Mean cord blood cortisol in group $B$ was not significantly different from that of group $A$ infants $(10.1+4.6$ vs. $6.9+4.1 \mu \mathrm{g} / \mathrm{dl})$. Cortisol values were not sig nificantly different in group $C(20+13 \mu \mathrm{g} / \mathrm{dl})$ at the time of hypoglycemia than in groups $B$ or $A(14+\pi)$ and $12+5.6 \mu \mathrm{g} / \mathrm{d}$ l respectively) at similar postnatal ages. Seven $S G \bar{A}$ in group $B$ and all group $C$ infants were tested with an $1 . V$. bolus of $.25 \mathrm{mg}$ synthetic ACTH between $18-44 \mathrm{hrs}$ of age. In the control group B, plasma cortisol rose $(\Delta) \quad 100$ to $500 \%$ and at least $13 \mu \mathrm{g} / \mathrm{dl}$. The response was subnormal in 3 of the 6 group $C$ infants. These data suggest that cortisol production is normal in normoglycemic SGA infants but may be blunted in some hypoglycemic SGA neonates and thereby contribute to a deficiency in gluconeogenesis. Adrenocor tical hypofunction may be secondary to intrauterine malnutrition of greater severity in some SGA infants.
656 INVESTIGATION OF NESIDIOBLASTOSIS USING SOMATOSTATIN. Harry J. Hirsch, Sherry Loo, Ilancy Evans, John F. Crigler, Jr., and Kenneth H. Gabbay. Dept. of Ped., Harvard Med. Sch., Boston, Mass.

A 2 mo. old gir] (b.w. $5.3 \mathrm{~kg}$ ) with hypoglycemia from birth due to nesidioblastosis (isiet dysgenesis) required IV glucose $(0.75 \mathrm{~g} / \mathrm{kg} / \mathrm{hr})$ to maintain blood glucose $(G)$ at $37 \pm 2 \mathrm{mg} / \mathrm{dl}+$ SEN with elevated insulin (IRI $9.8 \pm 1.2 \mathrm{uU} / \mathrm{ml}$ ), glücagon (IRG $339+13 \mathrm{pg} / \mathrm{ml}$ ) and growth hormone (GH $15.6+4.8 \mathrm{ng} / \mathrm{mi}$ ). Cessation of glucose infusion caused signs of hypoglycemia, a fall in $G$ to $<15 \mathrm{mg} / \mathrm{dl}$, a fall in IRI, and an increase in IRG, all within 15 minutes.

Infusions of synthetic cyclic somatostatin (120, 16.8, and 6 $\mathrm{ug} / \mathrm{hr}$ ) shoved a dose-dependent suppression of IRI and rises in $f_{3}$ $(325,215$, and $171 \mathrm{mg} / \mathrm{dl}$ respectively). Single s.c. injections of protamine-zinc somatostatin (PZS $50 \mathrm{ug}$ ) on four occasions raised $G$ to $83 \pm 3 \mathrm{mg} / \mathrm{dl}$ for 4 days each time, while peripheral IRI, IRf, and GH levels were unchanged. Cessation of qlucose infusion 2 days after PZS showed improvement, with a $G$ plateau of $55 \mathrm{mg} / \mathrm{dl}$ lasting $4 \frac{1}{2} \mathrm{hr}$. This was followed by an $3 \frac{1}{2} \mathrm{hr}$ G plateau of $30 \mathrm{mg} / \mathrm{dl}$ (unaffected by 4 -hourly feedings and elevated IRG of $394 \mathrm{pg} / \mathrm{ml}$ ) which rose to $103 \mathrm{mg} / \mathrm{dl} 30 \mathrm{~min}$. after glucagon, $30 \mathrm{ug} / \mathrm{kg} \mathrm{I} . \mathrm{M}$.

At surgery, the pancreas showed nesidioblastosis and the liver showed marked glycogen and fat deposition. These data are consistent with a defect in islet function with abnormal reguiation of insul in and glucagon secretion leading to abnormal hepatic glucose regulation. Somatostatin ameliorates the defective hormona] regulation in this disorder.
654

RELATION BETWEEN MATERNAL GLUCOSE TOLERANCE AND GLUCOSE UTILIZATION IN INFANTS OF DIABETIC MOTHERS. Robert T. Hall, Philip G. Rhodes, Fernando Fernandez, and Jerome Grunt, Kansas City, Missouri.

Glucose utilization $\left(\mathrm{K}_{\mathrm{T}}\right)$ was determined in 3 rd trimester pregnant women by means of an intravenous glucose tolerance test (IVGTT) and compared with the $\mathrm{K}_{\mathrm{T}}$ of their newborn infants. Insulin production (IRI) was assayed in both maternal and neonatal blood in mother-infant pairs not receiving insulin. The normal maternal control population had $\mathrm{K}_{\mathrm{T}}$ greater than 1.13 , gestational diabetics had $\mathrm{K}_{\mathrm{T}}$ less than 1.13 which returned to normal postpartum and diabetics had $K_{T}$ less than 1.13 which remained abnormal after delivery. Results of these studies appear in the table. Mothers

No. KT $\triangle$ Area IRI KT $\triangle$ Area IRI

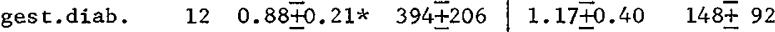
\begin{tabular}{lrll|ll} 
diab.total & 8 & $0.77 \mp 0.26 *$ & - & $1.19 \mp 0.59$ & \\
diab.no insulin 4 & $0.79+0.16 *$ & $77+73 *$ & $1.03 \mp 0.41$ & $341+407 *$
\end{tabular} * paired T-Test $\mathrm{p}<0.001$ Compared with controls

Although insulin production was significantly lower in diabetic mothers and significantly higher in their infants when compared with controls, there was no relationship between maternal and neonatal glucose utilization. These data indicate that factors other than maternal glucose utilization and insulin production are operative in determining neonatal glucose utilization.

657 HYPOPHOSPHATEMIC VITAMIN D RFSISTENT RICKETS (VDRR): MINERAL BALANCE STUDIES UNDER SHORT AND LONG TERM VITAMIN D PLUS INORGANIC PHOSPHATE (Pi) SUPPIEMENTATION. Gladys H Hirschman, Thomas H Coleman, David D Rao, Hooshang Hodjati and James CM Chan. (Intr by Robert H Parrott) George Wash Univ, Children's Hosp Nat Med Ctr, Dept Nephrology, Washington DC

The reversal of growth failure in VDRR under the influence of vitamin $D$ and inorganic phosphorus ( $\mathrm{Pi}$ ) supplementation (Glorieux et al, NEJM 287:481, 1972) is tested in our patient JH, who was followed for 19 mos. of therapy. She was 23 mos. old and in $3 \mathrm{rd}$ percentile height when therapy (vitamin $\mathrm{D}_{3} 10,000 \mathrm{IU}$ and $\mathrm{P}_{1} 1.8 \mathrm{~g}$ daily) was started.

Twenty-one days mineral balance studies prior to staxting treatment with this regimen showed the following data:

\begin{tabular}{lccc} 
& PRE & IN & POST \\
\hline Pi balance mg/day & $-5.33+287$ & $3554+804$ & $603+961$ \\
$\mathrm{Ca}$ balance mg/day & $573+161$ & $908 \pm 110$ & $926 \pm 113$ \\
$\mathrm{GI}$ absorption $\mathrm{Pi}$ & $69.6 \%$ & $94.4 \%$ & $80.1 \%$ \\
$\mathrm{Alk}$ phosphatase mU/ml & $286 \pm 12$ & $262 \pm 6$ & $271 \pm 27$
\end{tabular}

At 6 mos. intervals, two new mineral balance studies were per formed. The data at the end of 19 mos. of therapy showed sustained biochemical improvement. The TRP which was $12.5 \pm 3.5 \%$ during the initial short-term study showed slight improvement to 21 $+5.3 \%$ at 19 mos. of therapy. iPTH remained within normal range throughout the study. She was however still in $3 \mathrm{rd}$ percentile for height, but radiologically, she achieved healing of the rachitic lesions and complete correction of her bowlegs and waddling gait.

THE LATE METABOLIC ACIDOSIS (LMA) OF LOW BIRTH 655 WEIGHT (LBW) INFANTS: FACT OR FICTION? George B. Haycock, George J. Schwartz, Chester M. Edelmann, Jr., Adrian Spitzer. Albert Einstein Coll. of Med., Bronx, New York. Some LBW infants with failure to thrive (FTT) have been found to have low $+\mathrm{CO}_{2}$; this condition was named LMA. More recently the same label has been applied to healthy infants with low $+\mathrm{CO}_{2}$. Well LBW infants, fed standard formula, were screened for blood $\mathrm{HCO}_{2}$ q 3-4 days. Values for $\mathrm{tCO}_{2}$ for the whole group were normally distributed $(19.1 \mathrm{mmol} / 1 \pm 2.6 \mathrm{SD})$. From $1-21$ days the means rose linearly from 18.2 to $19.7 \mathrm{mmol} / 1$ and then plateaued. 16/74 infants had 'acidosis', arbitrarily defined as $+\mathrm{CO}_{2}<18$ $\mathrm{mmol} / \mathrm{l}$. Urine $\mathrm{pH}$ at the age of detection of acidosis (7-31 days) was $5.18 \pm 0.32$. Eight infants were matched for maturity and weight and randomly allocated to treatment either with $5 \% \mathrm{NaHCO}_{3}$ p.o. to raise $\mathrm{HCO}_{2}$ to $>21 \mathrm{mmol} / \mathrm{l}$, or $0.9 \% \mathrm{NaCl}$ (I ml/feed). After 3 weeks of therapy the rate of growth was $38 \pm 9 \mathrm{~g} / \mathrm{wk}$ in the bicarbonate and $39 \pm 19 \mathrm{~g} / \mathrm{wk}$ in the saline treated group. There was no difference either between the two groups, or between acidotic and non-acidotic infants, in net acid excretion (NAE) in response to $\mathrm{NH}_{4} \mathrm{Cl}$ loading; minimum urine $\mathrm{pH}$ was slightly lower in non-acidotic $(4.9 \pm 0.11)$ than in acidotic $(5.16 \pm 0.35)$ infants. However, the $\mathrm{HCO}_{3}$ threshold was lower $(\mathrm{P}<0.01)$ in 'acidotic' $(18.7 \pm 2.5$ $\mathrm{mmol} / \mathrm{l})$ than in non-acidotic $(21.9 \pm 0.38 \mathrm{mmol} / \mathrm{l})$.

Thus, blood $\mathrm{HCO}_{2}$ levels in LBW infants reflect their $\mathrm{HCO}_{3}$ threshold and not limited NAE. Since no association was seen between FTT and $+\mathrm{CO}_{2}$, FTT in LBW infants with low $+\mathrm{CO}_{2}$ may be spuriously attributed to LMA.
658 25-HYDROXY-VITAMIN D DEFICIENCY IN OSTEOPENIA OF THE EXTREME PREMATURE. Nancy $\frac{\mathrm{W}}{\mathrm{S}}$. Hoff, Eileen $\frac{\mathrm{E}}{\text { Hillman (Intr. }}$. Tyrala, Hillman) Washington Univ. Sch. Med., St. Louis Children's Hosp. Dept. of Ped., Jewish Hosp. of St. Louis, Dept.of Med. St. Louis.

Four premature infants with birth weights of $780,880,930$ and $1010 \mathrm{gms}$ presented at $8-14$ weeks of age with bone disease varying from osteopenia and elevated alk. phos. $(650-1,800$ I.U.) to radiographic rickets, fractures, and flail chest. All infants had been fed orally a standard formula and supplements of .3 to $1 \mathrm{cc}$ Poly-vi-sol and $25 \mathrm{u}$ of vitamin $\mathrm{E}$ per day. In 3 cases studied, 25 -hydroxy-vitamin $D(25-O H D)$ serum conc. were $<2 \mathrm{ng} / \mathrm{ml}$ in two and $5 \mathrm{ng} / \mathrm{ml}$ in the third (rachetic range: $<6 \mathrm{ng} / \mathrm{ml}$ ). All 4 infants responded to $4,000 \mathrm{u}$ q.d. vitamin $D$, p.o. and repeat 25-OHD serum conc. were normal $(>15 \mathrm{ng} / \mathrm{ml})$. All 4 infants had biochemical evidence of liver dysfunction, elevated bilirubin (9-11mg\%) and liver enzymes, at 6-12 weeks of age, prior to the clinical onset of bone disease. The etiology of this self-limited liver disease is unknown. To assess the premature infants' ability to absorb fat soluable vitamins, especially with liver disease, vitamins $A$ and $E$ were measured in 3 of the cases. Vitamin A levels were 6,16 and 18 (normal 35-60) and vitamin $\mathrm{E}$ levels were .04, $0.2,0.4$ (normal $0.8-1.2$ ). These returned to normal on daily $3,000 \mathrm{u} A$ and $50-75 \mathrm{u} \mathrm{E} \mathrm{p.o.} \mathrm{The} \mathrm{relative} \mathrm{contribution} \mathrm{of} \mathrm{decreased} \mathrm{vitamin} D$ intake and absorption, liver immaturity, and superimposed liver disease is unclear. These cases resemble the "Dystrophie Osteomalacique du Prematuré" reported in the french literature and confirm by actual serum conc. that defficiency of 25-OHD contributes to some of the bone disease seen in extreme prematures. 
659

FAMILIAL PROTEIN-TOLERANT ORNITHINE TRANSCARBAMYLASE DEFICIENCY - A CAUSE OF REYE'S SYNDROME. Nicholas J. Hoogenraad and M. Michael Thaler. (Spon. by Charles J. Epstein). University of California, San Francisco, CA., and Stanford University, Palo Alto, CA.

A variant of ornithine transcarbamylase (OTC) deficiency with markedly decreased affinity for ornithine and greatly increased affinity for carbamylphosphate was discovered in a 4 year-old boy with repeated episodes of a prodromal illness, vomiting, coma and convulsions. Liver biopsy findings in this child and in ayounger sibling who died from a similar disorder were characteristic of Reye's syndrome. In both patients, SGOT was $>450$ I.U./L, prothrom. bin times $>16 \mathrm{sec}$., blood ammonia 220-250 $\mu \mathrm{g} / \mathrm{ml}$, blood glucose 60-72 mg\%. Urinary glutamic acid was strikingly elevated. Liver OTC activity was 0.71 umoles/g 1 iver $/ \mathrm{min}$ ( $6.2 \%$ of normal), with normal pH optimum, a 30 -fold decrease in apparent $K_{m}$ for carbamytphosphate, a 7-fold increase in apparent $k_{m}$ for ornithine, and substrate inhibition for both substrates. 'Liver carbamylphoshate synthetase activity was normal. Among 14 maternal siblings and first cousins, 6 died ( 5 males, 1 female) in infancy or early childhood with unexplained encephalopathy. Urinary orotic acid after a protein test load $(\mathrm{lg} / \mathrm{kg})$ was $297 \mu \mathrm{g} / \mathrm{ml}$ in the patient, $174 \mathrm{\mu g} / \mathrm{ml}$ in a maternal aunt, $40 \mathrm{\mu g} / \mathrm{ml}$ in the patient's mother, and within the control range $(3-23 \mu \mathrm{g} / \mathrm{ml})$ in 15 other family members. The patient was protein-tolerant and treatment with arginine p.०. appeared to allay 2 incipient episodes of coma. These findings indicate that in certain patients, recurrent Reye's syndrome may be due to inherited forms of OTC deficiency.

660 QUANTIFICATION OF GLUCOSE TURNOVER WITH GLUCOSE$1-13 \mathrm{C}$ TRACER: ATTENUATED GLUCOSE PRODUCTION IN NEWBORN INFANTS OF DIABETIC MOTHERS. Satish Kalhan, Samuel Savin, Naoki Uga and Peter Adam. Case Western Reserve Univ., Dept. Peds. at Cleve. Metro. Gen. Hosp., and Dept. Earth Sciences, Cleveland, Ohio.

The effect of maternal diabetes on systemic glucose production in the newborn was studied by measuring glucose turnover at 2 hrs of age in 4 infants of normal and 2 infants of insulin-dependent diabetic mothers (IDM). The infants of both groups were delivered at 38-39 wks gestation, were appropriate for gestational age, and were normoglycemic during the study. During the 4 weeks preceding delivery, maternal diabetes was controlled in hospital with 4 daily doses of crystalline insulin in order to maintain fasting and $2 \mathrm{hr} p, \mathrm{c}$. blood glucose levels between 50 and $150 \mathrm{mg} \%$. Glucose- $1-13 \mathrm{C}$ was administered according to the prime-constant infusion technique of Steele and the rate of glucose production estimated by tracer dilution. $\mathrm{CO}_{2}$ was evolved from f $^{-1}$ of glucose by enzymatic decarboxylation and its ${ }^{13} \mathrm{C} /{ }^{12} \mathrm{C}$ ratio measured with a magnetic deflection double collector mass spectrometer. Systemic glucose production in the normal infants was $4.03,4.21,4.51$ and 4.93 $\mathrm{mg} / \mathrm{kg} \cdot \min (4.42 \pm 0.39$, Mean $\pm \mathrm{S}$. D. $)$. Glucose turnovers in both IDMs $(2.76$ and $3.17 \mathrm{mg} / \mathrm{kg}$. $\mathrm{min})$ were below the $95 \%$ confidence limits for the normal infants. Thus, variable intrauterine hyperglycemia and hyperinsulinemia in the IDM inhibited hepatic glucose production after birth.

\section{1} CHLORPROPRAMIDE CONCENTRATIONS IN INFANTS OF DIA(Spon. by Alex F. Robertson) Med. Col. of Ga. Ped., Ann Arbor

Despite controversy, chlorpropramide is used by physicians in the control of diabetes mellitus in gravid patients. We have studied drug concentrations in 2 neonates whose mothers were treated during pregnancy with this drug. Measurement was by ultraviolet during pregnancy with this drug. Measurement was by ultraviolet

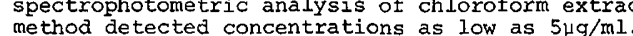

patient $A$, mother diagnosed as diabetic 11 years prior to delivery, was admitted at 17 hours of age. Matexnal chlorpropramide concentration on the day of delivery was $27.8 \mathrm{\mu g} / \mathrm{ml}$. Patient $B$, mother diagnosed diabetic $3 \frac{1}{2}$ years previously, was admitted a $70 \mathrm{hrs}$. Maternal medications included chlorpropramide $250 \mathrm{mgm}$. b.i.d. To prevent hypoglycemia the infants required intravenous
giucose until 156 and $144 \mathrm{hrs}$. of age at maximum rates of 14 and $7 \mathrm{mg} / \mathrm{kg} / \mathrm{min}$.

Age (hrs.)

Age (hrs.)

Glucose mgms

\begin{tabular}{rrrrrrrr}
\multicolumn{4}{c}{ A } & & & \multicolumn{3}{c}{ B } \\
\hline 53 & 59 & 68 & 77 & 103 & 88 & 91 & 108 \\
127 & 113 & 67 & 76 & 186 & 26 & 51 & 42 \\
30 & 67 & 76 & 67 & 112 & 35 & 75 & 70
\end{tabular}

A had chlorpropramide concentrations of $23.6,20.4,14.0$, and $10.5 \mu \mathrm{g} / \mathrm{ml}$ measured in sera obtained at $53,77,103$, and 128 hours of age. Sera from B at 91 and 108 hours had no measurable chlorpropramide.

These data show a half life of 60 houxs for chlorpropramide in the neonate and suggest that hypoglycemia may persist even though therapeutic concentrations of chlorpropramide are no longer present.
662 STUDIES ON THE EFFICACY OF CONTINUOUS LOW-DOSE I.V. ACIDOSIS IN CHILDREN. Michael S. Kappy and Elmer S. (Spon. by Grant Morrow, III) Univ. of Arizona College Lightner, trics, Tucson, Arizona.

A continuous insulin infusion using the standard pediatric I.V. drip set without an infusion pump was used to further simplify current methods of I.V. insulin therapy for ketoacidosis. Regular insulin was added without albumin to the patients' I.V. fluids. After an initial I.V, bolus of 2 units, a dose of 0.1 unit $/ \mathrm{kg} / \mathrm{hr}$ (maximum 3 units/hr) was sufficient to bring all patients out of ketoacidosis. The seven patients' average initial $\mathrm{pH}$ was 7.08. Using this regimen, the average time to reach $\mathrm{pH}$ 7.20 was 5.3 hours without the use of added bicarbonate. The majority of patients showed a prompt and steady increase in endogenous bicarbonate on the $I . V$. insulin regimen. Blood glucose fell smoothly to $50 \%$ of initial values in an average of $3 \frac{7}{2}$ hours. Glucose was added to the I.V. fluids when blood glucose fell to $250 \mathrm{mg} \%$ (in an average of $4 \frac{1}{2}$ hours), and no hypoglycemia was observed. Likewise, there was no hypokalemia or rapid fall in serum osmolarity associated with this regimen. The I.V. insulin was terminated (and subcutaneous insulin begun) when the patient had serum ketones positive on $1 y$ in a $1: 2$ dilution. This took an average of 11 hours. This modified regimen appears to be a safe and easy method of treating diabetic ketoacidosis in children and is suited for use in community hospitals. We are also measuring insulin, glucagon, amino acids, lactate, pyruvate, acetoacetate, and $\beta$-hydroxvbutyrate durino the rmirse of treatment.

\section{TREATMENT OF NEONATAL HYPOCALCEMIA WITH}

S.W. Kooh, D. Fraser, R.D. Toon*, H.F. Deluca*. Depts. of Paed. \& Physiol., Univ. of Toronto, and Hosp. for Sick Children, Toronto, Canada; and the Dept. of Biochem., Univ. of Wisconsin, Madison, WI.

$1,25(\mathrm{OH}){ }_{2} \mathrm{D}_{3}$, the hormonal metabolite of vitamin $\mathrm{D}$, has been shown to correct hypocalcemia in several conditions including hypo- and pseudohypoparathyroidism. Six infants with hypocalcemia and twiching (tetany of the newborn) were treated with $1,25(\mathrm{OH})_{2} \mathrm{D} 3$. They received $0.05 \mathrm{\mu g}$ metabolite/kg body weight/ day for 5 to 12 days except that two received $0.2 \mu \mathrm{g} / \mathrm{kg}$ body weight/day initial1y. $1,25(\mathrm{OH})_{2} \mathrm{D}_{3}$ was given intravenous1y in five patients and orally in one. Before treatment, plasma calcium values were less than $6.6 \mathrm{mg} / \mathrm{d} 1$, and four of the infants had had hypocalcemia for longer than two weeks. In all infants, the plasma calcium level started to rise within 24 hours of initiating $1,25(\mathrm{OH}){ }_{2} \mathrm{D}_{3}$ treatment, had increased above $7.5 \mathrm{mg} / \mathrm{dl}$ within three days, and reached the normal range within eight days. Two of the infants became hypocalcemic again when $1,25(\mathrm{OH})_{2} \mathrm{D}_{3}$ was discontinued. One infant became mildly hypercalcemic during treatment with $0.2 \mu \mathrm{g}$ metabolite $/ \mathrm{kg}$ body weight/ day. From our results, we believe that $1,25(\mathrm{OH})_{2} \mathrm{D}_{3}$ is a safe and dependable treatment of neonatal hypocalcemia.
664 HYPOMETABOLIC MITOCHONDRIAL DISEASE. Ingeborg Krie-

664 ger, Zwi Hart, and Chung-Ho Chang. Wayne State Univ. Sch. of Med. \& Chil dren's Hosp. of Mich., Detroit.

A 15-year old male with familial progressive CNS disease, minimal functional myopathy, and EKG evidence of cardiomyopathy had intermyofibrillar and subsarcolemmal aggregates of abnormal mitochondria and fat droplets in striated muscle and some smooth muscle involvement, elevated lactate $(24.9 \mathrm{mg} \%)$, pyruvate $(1.24$ $\mathrm{mg} \%$, urate, free fatty acids, and alanine (49.7 umoles/100 ml). Arterial, venous, and CSF lactate and pyruvate were in the same range and did not change in response to a 13 hour fast, a glucose or alanine load, glucagon, a ketogenic diet, or thiamine. Following muscular activity venous lactate was $29.5 \mathrm{mg} / 100 \mathrm{ml}$. Oral alanine $(100 \mathrm{mg} / \mathrm{kg}$ ) caused a rise in blood glucose. Basal $\mathrm{O}_{2}$-consumption was $0.123 \mathrm{1} / \mathrm{min}$ ( $\mathrm{RQ} 0.68$ ), corresponding to a $\mathrm{BMR}$ of $30.1 \mathrm{cal} / \mathrm{hr} / \mathrm{M}^{2}$ (normal $50.4 \pm 3.95$ ); it rose during an attack of agitation and hyperventilation to $43.6 \mathrm{cal} / \mathrm{hr} / \mathrm{M}^{2}$. While awake and restless $100 \mathrm{gm}$ glucose caused no rise in $\mathrm{O}_{2}$-consumption $(0.128+0.132 \rightarrow 0.1261 / \mathrm{min})$ although the $R Q$ increased from 0.71 to 0.90 ; at rest this load caused a drop from 0.112 to $0.0891 / \mathrm{min}$, corresponding to $23.6 \mathrm{cal} / \mathrm{hr} / \mathrm{M}^{2}$, while the $\mathrm{RQ}$ rose from 0.71 to 0.99 . These values can be explained by glucose conversion to fat. In vitro analysis (courtesy of S. DiMauro), using muscle homogenate, tagged substrate, ATP, ADP, and cytochrome $\mathrm{C}$, showed normal conversion of glucose and pyruvate to $\mathrm{CO}_{2}$, and a slightly increased rate for palmitate. This patient was morphologically similar to Luft's disease, but functionally different. Defective oxidation may be due to an error in cyto-
chrome structure or one of the enzymes in the respiratory chain. 
665 GLUCOSE UTILIZATION IN HYPERVISCOSITY. R.D. Leake, G.A. Chan, S. Zakauddin, M.E. Dodge, R.H. Fiser, and W. Oh, UCLA School of Medicine, Harbor General Hosp., Department of Pediatrics, Torrance, California.

The etiology of the hypoglycemia in neonatal hyperviscosity (HV) remains unclear. Nine HV infants (venous blood viscosity > 2 S.D. of the norm), 32-41 weeks gestation, had I.V. Glucose to $\overline{1}-$ erance tests ( $1 \mathrm{gm} / \mathrm{kg}$ of $508 \mathrm{glucose}$ ) prior to and after a partial exchange transfusion. Glucose disappearance rates (Kt) were significantly elevated initially $(M+$ S.E.M. $=3.5+1.0)$ and decreased following partial exchange transfusion $(2.4 \pm 0.6)$. Both rates remained elevated over that of 5 non-HV infants of similar gestation and age $(1.3 \pm 0.6 ; p=<.05)$. Basal and stimulated plasma insulin, growth hormone and alanine obtained before and 30 minutes after glucose tolerance, were similar between the two groups.

HV was experimentally produced in 7 lambs (5-19 day old) by a $500 \mathrm{ml}$ isovolemic exchange transfusion with heparinized maternal packed cells. Seven non-HV lambs underwent an identical exchange transfusion with maternal whole blood. Each lamb received a $2 \mathrm{hr}$ intravenous infusion of $6 \mathrm{mg} / \mathrm{kg} / \mathrm{min}$ glucose immediately before, and 1-24 hrs, after the exchange transfusion. Serial blood glucose (steady state) and serum insulin values were similar between the 2 groups before induction of $\mathrm{HV}$; but after induction, the HV lambs had significantly lower blood glucose levels. Insulin levels were similar in both groups. Our data suggest that hypoglycemia in the neonatal $\mathrm{HV}$ is due to increased glucose disposal unassociated with hyperinsulinism or substrate deficiency.

666 RESPONSE TO FASTING IN EXPERIMENTAL INTRAUTERINE GROWTH RETARDATION INDUCED BY MATERNAL STRESS. Lynne L. Levitsky, Anne Kimber, Jo Ann Marchichow and Jane Uehara (Spon. by Samuel P. Gotoff), Pritzker Sch. Med., Univ. of Chicago, Michael Reese Med. Ctr., Dept. Peds., Chicago, I11. Intrauterine growth retardation was produced in the rat neonate by maternal sham surgery at 17 days gestation $(n=13)$, or by the nonspecific stress of a maternal forced swim on days 17-20 of gestation $(n=20)$. Infants were delivered by cesarean section at term. After fasting for 4 hours, surgery and swim neonates had lower plasma glucose and higher plasma insulin levels than controls $(n=9)$.

$\begin{array}{lccc} & \text { Control } & \text { Surgery } & \text { Swim } \\ \text { Exsanguinated } & & & \\ \text { Birth Weight (g.) } & 5.4 \pm .1 & 4.7 \pm .1 \quad(p<.001) & 5.1 \pm .1(p<.05) \\ \text { Glucose mg/dI } & & & \\ \quad \text { Birth } & 92 \pm 7 & 92 \pm 5 \quad & 87 \pm 3 \\ \quad 4 \text { hrs } & 82 \pm 7 & 54 \pm 5 \quad(p<.005) & 58 \pm 6(p<.025) \\ \text { Insulin ng/m1 } & & & \\ \quad \text { Birth } & 3.45 \pm .33 & 3.12 \pm .33 & 3.48 \pm .25 \\ \quad 4 \text { hrs } & .45 \pm .16 & .62 \pm .05(p<.05) & .78 \pm .15 \text { (N.S.) }\end{array}$

These data further substantiate our postulate that hyperinsulinemia may accompany the low plasma glucose of experimental intrauterine growth retardation and parallel the clinical finding of hyperinsulinism in sore small for gestational. age infants.

667 GLUCONEOGENESIS IN THE FASTING BABOON NEONATE. Lynn L. Levitsky, John B. Paton, David E. Fisher, Clarence W. De Lannoy, Pritzker Sch. Med., Univ. Chicago at Michael Reese Med. Ctr., Dept. Peds., Chicago.

Fasting arterial blood levels of glucose and gluconeogenic substrates were evaluated in 7 baboon neonates after delivery by cesarean section at term and in 11 6-week old baboon infants. $\begin{array}{lllr}\text { Birth } & 2.2 \pm .2 & 3.3 \pm .4 & .327 \pm 023 \\ 6 \text { weeks } & 2.3 \pm .2 & 1.2 \pm 1 & .131 \pm 007\end{array}$

Substrate $(\mathrm{mM} / \mathrm{L}) \quad$ Glycerol Glutamine Glutamate $\begin{array}{lrrr}\text { Birth } & .36 \pm .04 & .38 \pm 04 & .57 \pm .06\end{array}$ 6 weeks $\quad .28 \pm .03 \quad .18 \pm 02 \quad .32 \pm .02$

Levels of gluconeogenic substrates were higher at birth than at 6 weeks ( $p<.001$ except for glycerol). In contrast, $\beta$-hydroxybutyrate was higher at 6 weeks $(.14 \pm .02$ vs. $3.30 \pm .44 \mathrm{mM} / \mathrm{L}$, $\mathrm{p}<.001)$. Net renal glucose production was estimated by measuring inferior vena cava blood glucose levels above and below the rena veins and calculating renal vein glucose and renal glucose production based upon blood flows determined by the radioactive microsphere method. 6 of 7 newborn and 9 of 11 6-week old infants demonstrated net renal release of glucose $(5.0 \pm 2.8$ and $3.3 \pm 1.1$ $\mu \mathrm{M} / \mathrm{min})$. Glucose release did not correlate with arterial Jactate or $\mathrm{pH}$. Lactate and glycerol were the only substrates consistently taken up by the kidney. We have previously estimated that neonatal splanchnic glucose production is $10.8 \pm 4.4 \mathrm{\mu M} / \mathrm{min}$. Therefore, renal glucose release may account for $1 / 3$ of total glucose production in these infants.
AN EVALUATION OF THE INTERRELATIONSHIP BETWEEN DIET \& PLASMA LIPIDS. A. Harold Lubin
George M. Owen, Ronald D. Pearson
(Spon. by Bruce D. Graham) Ohio State University, College of Medicine, Children's Hospital, Department of Pediatrics, Columbus, Ohio.

In a sample of 25 sibling pairs of closely-matched, isocioeconomic status, maternal education, \& close physician supervision) preschool \& school-age, healthy males the significant difference between triglyceride concentrations in black \& white, preschool children originally noted in the National Pre-School Nutrition Survey has been corroborated. This difference is not significant $n$ their school-age siblings. Total mean lipid levels are higher $n$ both white preschool and school-age children compared with the slack subgroup mean values, with a trend toward higher means in all lipid categories (cholesterol, triglycerides, phospholipids, \& total lipids) in the white males. Hematocrits in the black preschool children were significantly less than in the black school-age children, $\mathrm{p}<.005$, \& the same difference was observed between white preschool \& school-age children, $\mathrm{p}<.03$. Anthropometric measurements document in both age groups that black children tend to be taller \& heavier than their white counterparts and that mean head circumference \& mean triceps circumference increase more rapidly in black children \& are larger in the black school-age child. Although food energy intake \& the percent of total food energy provided from carbohydrate \& from fat suggest explanations for some of the observed biochemical differences, further evaluation following dietary alteration is required for Spon. vy Grant $\$ 74-118$, International Sugar Research Found., Inc.

66 IRON ABSORPTION FROM HUMAN MILK. JUIIa A. McMillan, Stephen A. Landaw and Frank A. Oski. Departments of Pediatrics and Medicine, State University of New York, Upstate Medical Center, Syracuse, N.Y. of 14 infants, ages 6 to 18 months who were exclusively fed human milk, 13 were found to have normal hemoglobin levels, MCV's, free erythrocyte porphyrins, serum iron, and transferrin saturations. In order to better understand this observation, iron absorption studies were performed in 5 healthy adults employing both human milk and whole cow milk. After the ingestion of milk containing added $\mathrm{Fe}^{59}$, initial plasma radioactivity and eventual red cell iron incorporation were determined. After correction for the relative specific activity of the administered iron, initial plasma radioactivity was 2.5 to 5.0 times higher following the consumption of human milk. Red cell iron incorporation was 1.3 to 8.0 times higher when the iron was administered with human milk. Minimum estimate of per cent iron absorption averaged $18.9 \%(2.2-31.9 \%)$ from human milk and $9.1 \%(1.7-19.0 \%)$ from cow milk.

The iron content of the human milk employed in the studies averaged $0.78 \mathrm{mg} / \mathrm{L}$ - the cow milk $0.87 \mathrm{mg} / \mathrm{L}$. These studies suggest that because of the superior iron absorption from human milk, even in iron replete normal adults, human milk is adequate to meet the iron requirements of the exclusively breast fed full-term infant during the first 6-12 months of life.

670 DIABETIC RETINOPATHY IN CHILDREN, Malone, J. I., Van Cader, T.C., and Edwards, W.C., Depts, of Pediatrics and Ophthalmology, University of South Florida, College of Medicine, Tampa, Florida The incidence of retinopathy in 153 children with diabetes was determined using indirect ophthalmoscopy (IO), retinal color photos (RCP), and retinal fluorescein angiography (FA). The grading system used: Stage 0 , no microaneurysms (MA); Stage 1, $<5$ MA; Stage $\frac{\text { Sta }}{5}$

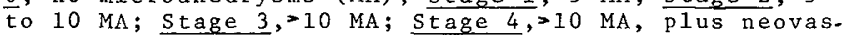
cularization. The results: $\%$ of

\begin{tabular}{lrrrrr} 
Diabetes Duration (Yrs.) & $=1$ & $2-5$ & $6-10$ & $11-16$ & Total \\
\hline (Stage 0) & 9 & 17 & 7 & 1 & 22 \\
\hline (Stages 1-4) & 20 & 58 & 32 & 9 & 78 \\
\hline$\%$ with Microaneurysms & 69 & 74 & 82 & 90 & -
\end{tabular}

MA were found by FA in $75 \%$ of the 133 children who had negative findings by $I O$ and $R C P$. The increase of MA with duration of diabetes suggests an acquired metabolic etiology. However, MA were found in children with diabetes of 1 month duration. $69 \%$ of children with diabetes of one year or less had vascular abnormalities demonstrated by FA while $10 \%$ with duration $>10$ years had no MA. These findings suggest that in addition to metabolic effects, genetic or other factors also are operative in causing vascular abnormalities in diabetes. 
671 PREVENTION OF PROLONGED NEONATAL HYPOGLYCEMIA WITH ANTENATAL GLUCOCORTICOIDS IN RATS WITH INTRAUTERINE GROWTH RETARDATION (IUGR). Robert L. Manniello,

Anthony J. Adams and Philip M. Farrell, NICHD, NIH, Bethesda, MD Neonatal patterns of hypoglycemia and hepatic glycogen content were studied in 200 IUGR progeny obtained by utilization of the Wiggelesworth surgical technique on carefully timed rat pregnancies. Hypoglycemia ( $<40 \mathrm{mg} / \mathrm{dl}$ ) was present at birth in $39 \%$ of these pups as compared to $13 \%$ in control littermates (mean plasma glucose $=44 \pm 2$ vs. $63 \pm 1.8$ in controls; $p<.001$ ). While controls did not change over the first $6 \mathrm{hrs}$ of life, the extent of hypoglycemia in IUGR pups was greater with a mean of $25 \pm 3.2 \mathrm{mg} / \mathrm{dl}$ at 2-4 hrs. The degree of hypoglycemia noted at birth and in early neonatal life correlated with both reduced liver glycogen content $(r=.64 ; p<.001)$ and liver weight $(r=.69 ; p<.001)$. In an attempt to influence hepatic glycogen and hypoglycemia patterns, we administered a potent cortisol analog to mothers 24 and $48 \mathrm{hrs}$ prior to delivery. This treatment decreased the IUGR neonate's trend toward hypoglycemia as evidenced by a significantly higher ( $p<.005$ ) plasma glucose of $4 \mathrm{l} \pm 4.5$ at $2-4 \mathrm{hrs}$ and $70 \pm 5 \mathrm{mg} / \mathrm{dl}$ at $6 \mathrm{hrs}$. Thus, the incidence of hypoglycemia during the 2-6 hr interval fell from $77 \%$ to $36 \%$ in the IUGR group after glucocorticoid therapy. Total liver glycogen was also increased $(p<.001)$ from $1.67 \pm .21$ in the non-treated IUGR progeny to $3.5 \pm .33 \mathrm{mg}$. These data indicate that prenatal corticoids, by promoting hepatic glycogenesis, are effective in preventing the prolonged hypoglycemia associated with IUGR. The utilization of glucocorticoids for the study of liver development in relation to enzyme induction is advocated.
674 PARADOXICAL EFFECT ON CYCLIC AMP (CAMP) OF ARGININE STIMULATED GLUCAGON SECRETION. R. Owen, A. Sadeghi-
Nejad and B. Senior. Tufts University School of Medicine, New England Medical Center, Pediatric Endocrine-Metabolic Service, Boston.

Glucagon activates liver adenylate cyclase to form cAMP. This is reflected by a striking rise in blood levels of cAMP following glucagon administration. The cAMP then activates the phosphorylase complex; glycogenolysis ensues, and glucose levels rise.

In order to study the response to endogenous glucagon, we administered arginine $(0.5 \mathrm{gm} / \mathrm{kg} \mathrm{I.V.)}$, a potent stimulus of glucagon secretion, to normal volunteers and to patients having either Type I glycogenosis or diabetes mellitus. Thirteen studies were done. As an index of the response we assayed cAMP (protein binding) as well as glucagon, insulin and glucose.

As expected the levels of glucagon rose as did the levels of insulin. The levels of glucose increased except in the Type I glycogenosis patients. Paradoxically, despite the rise in glucagon, cAMP not only failed to increase but fell profourdlymean decrement 54 per cent.

We conclude that the rise in glucose after arginine was mediated by a present1y unknown mechanism which did not involve cAMP and therefore was not caused by the increased secretion of glucagon.

Somatostatin suppresses levels of insulin, glucagon and glucose as well as growth hormone; effects which are the opposite of those produced by arginine. We speculate that arginine may cause inhibition of somatostatin.
672 REYE'S SYNDROME: EVALUATION OF 25 CASES FROM A WEST 672 COAST HOSPITAL: Wilbert H. Mason,* Frank R. Sinatra," ment of Pediatrics, University of Southern California, Childrens Hospital of Los Angeles and Stanford University, Palo Alto, Calif. 25 cases of Reye's Syndrome (RS) were admitted to Childrens Hospital of Los Angeles during a 7-year-period. The ages of the patients ranged from 9 weeks-12 years, and there was a 2:1 female to male ratio. 2 of the patients were black and one was an American Indian. Many patients were referred from suburban or rural settings. The clinical manifestations observed were similar to those formerly described. Stage of coma on admission and rapidity of progression seemed predictive of the outcome. All patients had $\uparrow$ SGOT levels, and 19 of 22 had $\uparrow$ levels of serum ammonia. Patients with maximum serum ammonia levels $>300 \mu / 100 \mathrm{ml}$ were more likely to have a fatal outcome, but the difference was not statistically significant. Salicylate levels were detected in the serum of all 20 patients tested, despite histories of no ingestion in 6 patients. Pathologic material was obtained from 15 patients either by autopsy or liver biopsy. Electron microscopy was performed on 2 liver specimens. Carbamyl phosphate synthetase (CPS) and ornithine transcarbamylase (OTC) enzyme activities were performed on 3 patients and were noted to be $\downarrow$ in 2 . In one patient with normal CPS and OTC activity, kinetic analysis showed a marked reduction in $\mathrm{V} \max$ and $\mathrm{Km}$ for carbamyl phosphate. 12 of the 25 patients were treated conservatively, 10 received exchange transfusions, and 3 had peritoneal dialysis. Numbers of patients in each group were too small to evaluate statistically. The need for collaborative studies to evaluate RS therapy is suggested.

673 CONTINUOUS INFUSION OF INSULIN IN THE TREATMENT OF JUVENILE DIABETTC KETOACIDOSIS, WiIliam J, Oetgen and Alan R. Figelman (Spon. by James W. Bass.) Departments of Internal Medicine and Pediatrics, Walter Reed Army Medical Center, Washington, D.C.

Traditional therapy of diabetic ketoacidosis (DKA) includes large bolus doses of insulin given every four to six hours. Several recent reports have described continuous infusion of low doses of insulin in adults with DKA, but there have been no controlled studies reporting the use of this method in juvenile DKA. This paper compares the clinical courses of four patients in seven episodes of JDKA treated with continuous infusion of low doses of insulin (CII) with the courses of a controll group of five patients in seven episodes of JDKA treated with traditional insulin high dose boluses (HDB.) CII was administered at the rate of ten units per hour following an initial IV bolus of ten units on admission. HDB was administered SC every four hours in doses of 1-3 units/kg depending on the severity of the DKA. The mean age on admission for the CII group was 12.7 years and did not differ significantly from the HDB group (13.4 yr.) The groups were also of comparable body weight, and there was no difference between the two groups in the length of time of insulin dependence. Chemical parameters on admission were 11 kewise comparable. Mean admission glucose: CII=508 and HDB=571 $\mathrm{mg} \%$. Mean serum bicarbonate: $\mathrm{CII} \Rightarrow$ ? and $\mathrm{HDB}=6 \mathrm{mEq} / \mathrm{L}$. Mean $\mathrm{pH}$ : $\mathrm{CII}=$ 7.08 and $\mathrm{HDB}=7.11$. The mean time to normal glucose (<150 mg\%) in CII was $3.6 \mathrm{hr}$ and in HDB was $9.4 \mathrm{hr}(\mathrm{p}<.05$.) The mean amount of insulin required was 42 units in CII and 163 in HDB $(p<.05$.$) CII is a rapid, simple and safe therapy for JDKA.$

\section{A AESSEIIGER ROLE FOR ALANIINE IN KETONE METABOLISM.}

675 Pinar T. Ozand, Jean Girard, Robert L. Hawkins, Roger M. Collins, Jr. W. Douglas Reed, J. Tyson Tildon and Marvin Cornblath. Dept. Of Pediatrics, Univ. of Md. Sch. Of Med., Baltimore, Maryland 21201 and Universite de Paris, Faculte des Sciences, Paris, France.

The effect of alanine on ketone metabolism was investigated in infused, 48 hour fasted, 35 day old rats. L-alanine was injected into the femoral vein and contralateral arterial blood was collected for analyses. D-alanine was injected in all control experiments. With fasting, alanine decreased from 653 to $132 \mu \mathrm{M}$ $\beta$-hydroxybutyrate $(B O H B)$ and acetoacetate $\left(A C_{2}\right)$ increased from 103 to $824 \mu \mathrm{M}$ and 16 to $236 \mu \mathrm{M}$, respectively. Injection of $100 \mathrm{mg} /$ $\mathrm{kg} \mathrm{L}$-alanine led to transient maximum levels not exceeding the postabsorbtive values with a return to fasting levels within 5-10 $\mathrm{min}$. Although glucose, lactate, pyruvate and $\mathrm{AC}_{2}$ levels did not change, $B O H B$ decreased significantly from $824 \pm 44(\bar{M} \pm$ S.E.M.) to $381 \pm\{13 \mu \mathrm{M}$ at 5 minutes with a concurrent decrease in [BOHB]/ $\left[\mathrm{AC}_{2}\right]$ ratio from $3.5 \pm 0.2$ to $1.5 \pm 0.6$. Levels of insulin and glucagon did not change. Neither anti-insulin serum (to bind $1440 \mathrm{~mL} / \mathrm{kg})$, nor dichloracetate $(300 \mathrm{mg} / \mathrm{kg})$ to block the peripheral utilization of ketones altered the hypoketonemic effect of L-alanine. Injected glucose, lactate or pyruvate $(100 \mathrm{mg} / \mathrm{kg})$ did not result in hypoketonemia. The hypoketonemic effect of alanine in fasted rats may be explained by a decreased redox state of liver mitochondria but not by insulin release. (Supported in part by NIH grants AM18127-01, HD06291-04, HD03959-05 and The John A. Hertford Foundation, inc. grant \$74266). 
677

CHRONIC EFFECTS OF ORAL GLUCOSE ALIMENTATION (GA) AND PORTACAVAL SHUNT (PCS) IN PATIENTS WITH GLYCOGEN STORAGE DISEASE (GSD), TYPE I. T.F. Roe and M.D. Kogut, USC School of Medicine, Childrens Hospital of Los Angeles, Depaitment of Pediatrics, Los Angeles.

Because patients with GSD, type I, have severe metabolic and growth disturbances which appear to be secondary to hypoglycemia $(\downarrow B G)$, attempts were made to restore $B G$ to normal by PCS or oral GA. Results following PCS in 2 children, aged $8 \frac{1}{2}$ and $13 \mathrm{yr}$, were compared with those of 4 patients receiving continuous overnight intragastric GA $(0.5 \mathrm{gm} / \mathrm{kg} / \mathrm{hr})$. After PCS, growth increased from 2 to $7 \mathrm{~cm} / \mathrm{yr}$ in one and from 2 to $10 \mathrm{~cm} / \mathrm{yr}$ in the older patient and puberty began;elevated ( $\uparrow$ ) lactic acid (LA), lipids (L) and uric acid (UA) levels and platelet (P) function returned to normal and hepatomegaly (H) diminished. In both symptomatic $\downarrow B G$ occurred following 3-6 hr fast. In one $\downarrow B G$ was not improved by diazoxide despite \& plasma insulin (IRI); plasma glucagon (IRG) levels were normal. In the other, IRG levels were markedly elevated with $\downarrow B G$. In 4 children before GA, plasma IRI levels were abnormally low and IRG levels were elevated. Post GA, plasma IRI levels increased and IRG levels fell. In 2 , aged $2 \frac{1}{2}$ and $4 \frac{1}{2} \mathrm{yr}$, post GA, growth was 12$14 \mathrm{~cm} / \mathrm{yr}, \mathrm{H}, \uparrow L A, \uparrow U A$, and $\uparrow \mathrm{L}$ diminished and $\mathrm{P}$ function was normal. In both, symptomatic $\downarrow B G$ occurred after fasting. In 2 others, aged $3 \frac{1}{2}$ and $19 \frac{1}{2}$ yr, recently started on GA, clinical effects were similar. Conclusions: 1) PCS and GA are both successful in reversing abnormalities; 2) the improvement may be mediated by the effects of increased insulin relative to glucagon on liver and other tissues; 3) symptomatic $\downarrow B G$ occurs post PCS and GA and does not appear to be due to IIRI or +IRG.

GLUCAGON SECRETION IN GROWTH HORMONE DEFICIENCY

678 PATIENTS BEFORE AND AFTER GROWTH HORMONE ADMINISTRATION: Carolyn A. Romshe and Juan F. Sotos, Dept. of Ped., Ohio State Univ. Coll. of Med., Children's Hosp., Columbus, Ohio.

The study was undertaken to obtain additional information on the pathogenesis of hypoglycemia in growth hormone deficient patients. Glucagon, glucose, and insulin levels were obtained during an oral glucose tolerance (GTT), arginine infusion, and Diazoxide-arginine infusion before and after 2 months of growth hormone ( $2 \mathrm{mg}$. three times a week). Diazoxide was given since it has been reported to stimulate glucagon secretion.

Time (min) GLUCAGON LEVELS - pg/ml

Oral GTT

$\begin{array}{ccc}\text { pre G.H. } & 143 \pm 26 & 117 \pm 17\end{array}$

60

90

$105 \pm 10 \quad 100 \pm 14$

$149 \pm 49 \quad 142 \pm 36$

$271 \pm 45 \quad 182 \pm 27$

$\begin{array}{lll}\text { pre G.H. } & 147 \pm 15 & 490 \pm 37\end{array}$

$\begin{array}{lllll}\text { post G.H. } & 218 \pm 67 & 760 \pm 234 & 440 \pm 94 & 300 \pm 106\end{array}$

$12 \pm 10$

Arginine

$\begin{array}{lrlllll} & \text { post G.H. } & 218 \pm 67 & 760 \pm 234 & 440 \pm 94 & 300 \pm 106 & 264 \pm 59 \\ \text { Arginine- } & \text { pre G.H. } & 168 \pm 34 & 518 \pm 132 & 228 \pm 45 & 163 \pm 23 & 173 \pm 25\end{array}$

$\begin{array}{lrlllll} & \text { post G.H. } & 218 \pm 67 & 760 \pm 234 & 440 \pm 94 & 300 \pm 106 & 264 \pm 59 \\ \text { Arginine- } & \text { pre G.H. } & 168 \pm 34 & 518 \pm 132 & 228 \pm 45 & 163 \pm 23 & 173 \pm 25\end{array}$

$105 \pm 23$

$172 \pm 19$

$\begin{array}{llllll}\text { Diazoxide post G.H. } & 128 \pm 18 & 725 \pm 107 & 258 \pm 34 & 194 \pm 29 & 170 \pm 31\end{array}$

Glucose and insulin levels with an oral GTT and arginine infusion were not significantly different before and after growth hormone. Glucagon secretion during an oral GTT was similar before and after growth hormone treatment. The arginine-induced glucagon response was not statistically different before and after growth hormone. Diazoxide pretreatment appears to enhance arginine induced glucagon secretion after growth hormone administration.
680

EXERCISE INDUCED GROWTH HORMONE RELEASE IN JUVENILE DIABETES MELLITUS. Chitra Sekaran, Fima Lifshitz and Pavel Fort, Dept. of Peds., North Shore Univ. Hosp., Manhasset, NY and Dept. of Peds., Cornell Univ. Med. Col., NY, NY. Growth hormone $(\mathrm{GH})$ release induced by exercise in juvenile diabetes mellitus (JDM) was assessed in 20 pts. in relation to the clinical and biochemical status of the disease. They were 6-6/12 to 19-6/12 yrs. of age and had JDM for 1 to 10 yrs. Fasting serum chemistries and $G H$ were measured prior to and after $30 \mathrm{~min}$. of vigorous exercise. Daily urine glucose (Glc) excretion was determined in relation to dietary intake. Responses were compared with 70 constitutional and primordial short stature controls (SSC) of comparable weights and ages. The JDM's had significantly lower basal GH levels than the SSC (means \pm SE): $2.0 \pm .3$ vs $6.5 \pm 1.1 \mathrm{ng} / \mathrm{ml}$, $p<.001$. The JDM's with a urine Glc excretion under $10 \%$ of daily carbohydrate $(\mathrm{CHO})$ intake had significantly increased $\mathrm{GH}$ responses after exercise $(18.1 \pm 4.0)$, whereas those with urinary Glc excretion of more than $20 \% \mathrm{CHO}$ intake had a blunted response $(2.7 \pm .7 p<001)$. SSC had no glycosuria and had a mean $G H$ response of $11.8 \pm 8$. 1 after exercise. Three of the 4 pts with subcapsular cataracts or with retinopathy had impaired GH release after exercise. The presence of motor neuropathy or lipodystrophy had no specific influence on $G H$ responses to exercise. The duration of JDM as well as the serum fasting levels of GIc, cholesterol, triglycerides, or thyroid antibodies had no correlation with $\mathrm{GH}$ responses. These studies suggest that well controlled JDM's with minimal glycosuria have higher GH release after exercise than poorly controlled JDM's.
681 GLUCOSE HOMEOSTASIS IN PREMATURE RHESUS MONKEYS W. Geoffrey Sherwood, Donald E. Hill, Graham W. Chance Children, Research inst. Richard Hamilton). The Hosp. for Sick Sixteen rhesus monkeys were delivered by cesarian section five near term (158 days) and eleven prematurely (six at $140+1$ and five at $143+1$ days). At $2-3$ hours of age a primed glucose infusion (PGI) was administered via the umbilical vein $(0.5 \mathrm{~g} / \mathrm{kg}$ and $8 \mathrm{mg} /$ $\mathrm{kg} / \mathrm{min}$ for $\left.180^{\prime}\right)$. Blood samples were obtained from the umbilical axtery. The five term and five of the premature newborn had similar basal glucose $(G), 55 \pm 6$ vs. $61 \pm 8 \mathrm{mg} \%$, and basal insulin (IRI) $27 \pm 12$ vs. $16+4 \mu \mathrm{U} / \mathrm{m} 1$. PGI induced a new steady state $(G)$ in both groups in the range $80-100 \mathrm{mg} \%$, (IRI) peaked at $60^{\prime}, 62+10 \mathrm{vs}$. $68+14 \mu \mathrm{U} / \mathrm{ml}$. Basal free fatty acid levels (FFA), $0.42 \mathrm{vs} .0 .31 \mathrm{mM}$ were minimally suppressed by PGI. Blood lactate (L) did not rise above $1.5 \mathrm{mM}$, and basal lactate/pyruvate ratios $(\mathrm{L} / \mathrm{P}), 25 \pm 6$ vs. $33+9$ were suppressed to $20 \pm 3$ in both groups. In the other six prematures, basal $\mathrm{G}$ was higher $80+11 \mathrm{mg} \%$ and basal IRI was lower $4+2$ $\mu \mathrm{U} / \mathrm{ml}$. The steady state (G) induced by PGI was higher $220+18 \mathrm{mg} \%$. IRI peaked at $60^{\prime}$ to $47+5 \mu U / \mathrm{ml}$. Basal L $1.8+0.9 \mathrm{mM}$ and $\mathrm{L} / \mathrm{P} 46+8$ were higher. However, $\bar{L} / \mathrm{P}$ was suppressed by $\overline{\mathrm{P}} \mathrm{GI}$ to $33 \pm 2$ at $60^{\circ}$ and $26 \pm 2$ at 120 . Basal (FFA) were higher $0.58 \mathrm{mM}$ and rose to $0.68 \mathrm{mM}$ during PGI. Thus, two separate responses to glycemic stimulus were seen in the premature groups. In retrospect, the six who developed hyperglycemia were the youngest of the prematures (140+1 day). Tissue hypoxia cannot be implicated as evidenced by a decreasing $\mathrm{L} / \mathrm{P}$ ratio. The ongoing lipolysis, low basal IRI, reduced IRI release relative to the glycemic stimulus, are suggestive of inappropriate autonomic function.
679

REGULATION OF INSULIN (IN) BINDING (B) TO CULTURED

FIBROBLASTS (F) IN PRECOCIOUS AGING (PA) AND LIPO-

ATROPHIC DIABETES (LD). Arlan L. Rosenbloom, University of Florida College of Med., Dept, of Pediatrics, Gainesville. $F$ were studied at confluence $6-10$ subcultures after harvesting from skin explants from 4 control children (CC) age 4 mo to $10 \mathrm{yr}$, 4 patients 2-9 yrs with PA ( 3 progeria, 1 Rothmund Syndrome), 4 non-diabetic adult controls (AC) $22-36$ yrs and 3 with LD age 15 19 and 30 yrs (very high levels of circulating In and resistance

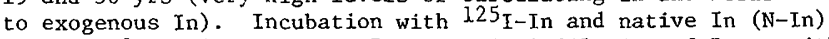
was at $24^{\circ}$ for 45 min at $\mathrm{pH} 7.0$. Maximal dilution of $B$ was with 25-50 fold concentrations of $\mathrm{N}-\mathrm{In}$, the counts remaining were "nonspecific" (NSB). PA differed significantly from CC in protein content per cel1 (PA $6.7 \mathrm{pg} \pm 1.6 \mathrm{SD}, \mathrm{CC} 3.8 \pm .6, \mathrm{AC} 6.1 \pm 2.2$ ) and concentration of $\mathrm{N}-\mathrm{In}$ required to produce $50 \%$ competition of $\mathrm{B}$ at $1 \mathrm{nM} 125 \mathrm{I}-\mathrm{In}$ (PA $5.63 \mathrm{nM}$ vs CC 1.15); insignificant differences were found in no. of In molecules SB and NSB per cell or per pg protein and in no. of cells at confluence. LD grew to greater $(p<.05)$ density $\left(3.9 \times 10^{6} \mathrm{~F} / 100 \mathrm{~mm}\right.$ dish $\left.\pm .9 \mathrm{SD}\right)$ than $\mathrm{AC}\left(1.79 \times 10^{6}\right.$ $\pm .3)$ and were more like $C C$ in: $50 \%$ competition concentration of $\mathrm{N}-\mathrm{In}(1.4 \mathrm{nM}$ vs 2.2 for $\mathrm{AC}, \mathrm{p}<.05)$; molecules $\mathrm{SB}$ at $1 \mathrm{nM}$ (AC 1301 \pm 217 SD, LD $975 \pm 291$, CC $961 \pm 422$, PA $976 \pm 188$ ); and molecules NSB at $1 \mathrm{nM}$ (AC $885 \pm 168$, LD $614 \pm 118$, CC $651 \pm 142$, PA $876 \pm 196, \triangle \mathrm{AC} / \mathrm{LD}$ $\mathrm{p}<.05)$. Marked insensitivity of putative In receptors of PA F to competition by $\mathrm{N}-\mathrm{In}$ implies decreased negative cooperativity as their genetic difference, consistent with in vivo resistance to insulin. LD F have exceptional multiplication and $B$ sensitivity for donor age; their In resistance in vivo cannot be explained by a genetic defect in cell surface binding.
GLUCOSE KINETICS IN PREMATURE RHESUS MONKEYS. W.GeOff682 rey Sherwood, Donald E. Hill, Graham W. Chance (Spon. ren, Research Inst., and Dept. of Paediatrics, Toronto, Canada. Eleven rhesus monkeys were delivered prematurely by cesarian section and formed two groups. They differed in terms of mean birth weight 369 vs. $341 \mathrm{~g}$, gestation $143+1$ vs. $140+1$ days and mortality $2 / 5$ vs. 6/6. Clinical hypoxia was not seen. At age 2-3 hours a primed infusion of $3 \mathrm{H}-2-\mathrm{g}$ lucose in $\mathrm{N}$. saline $(5 \mu \mathrm{Ci}$ and $0.2 \mu \mathrm{Ci} / \mathrm{min}$ ) was commenced. A basal steady state glucose specific activity was induced within 100'. This was perturbed by a primed glucose infusion PGI $(0.5 \mathrm{~g} / \mathrm{kg}$ and $8 \mathrm{mg} / \mathrm{kg} / \mathrm{min})$. A11 infusions were administered via the umbilical vein. All blood samples were obtained from the umbilical artery and assayed for glucose concentration (G) and glucose specific activity. PGI induced a new steady state $(G)$ in all eleven prematures. In five, this was in the range $80-100 \mathrm{mg} \%$, in the other six, it was in the range 190$210 \mathrm{mg} \%$. The basa1 (G) were $61+8$ vs. $80+11 \mathrm{mg} \%$. The corresponding glucose turnover rates were $6 . \overline{2}+0.7 \mathrm{vs} .7 .3+0.8 \mathrm{mg} / \mathrm{kg} / \mathrm{min}$. In the former group, PGI suppressed the endogenous hepatic glucose output (HGO) to $0.5 \mathrm{mg} / \mathrm{kg} / \mathrm{min}$. whereas in the latter group, HGO was minimally suppressed to a level of $5-6 \mathrm{mg} / \mathrm{kg} / \mathrm{min}$. Moderate increases in peripheral glucose utilization was seen in both groups. Thus, in those premature monkeys who develop hyperglycemia when challenged by glucose failure of suppression of glycogenolysis and or gluconeogenesis may be implicated. The premature rhesus monkey represents a model for the investigation of the hyperglycemia noted in certain human premature infants. 
INTERACTIONS OF LEAD, CALCIUM, VITAMTN D, AND NUTRITION IN LEAD-BURDENED CHILDREN. Michael Sore11, John F. Rosen and Martin Roginsky, The Albert Einstein College of Medicine, Montefiore Hosp. \& Med. Ctr., Department of Pediatrics, Bronx, New York; Nassau County Med. Ctr., Department of Medicine, New York.(Spon. by L. Finberg)

This study was undertaken to evaluate relationships between blood or serum levels of lead (Pb), calcium, and 25-hydroxyvitamin $D(25-\mathrm{OHD})$, within the framework of a formal nutritional survey in lead-burdened children. The results demonstrate: 1) Regardless of blood $\mathrm{Pb}$ leve1s and season of the year, serum 25-OHD concentration reflects vitamin $\mathrm{D}$ intake; 2) High blood $\mathrm{Pb}$ levels $(\geqq 60 \mathrm{\mu g} / \mathrm{dl})$ were associated with decreased levels of $25-0 \mathrm{HD}(18 \pm$ $1 \mathrm{ng} / \mathrm{m} 1$ vs $32 \pm 1$ in controls, $\mathrm{P}<.001)$; 3) Levels of $\mathrm{Pb}$ and calcium were inversely correlated in control and lead-burdened children, and 4) Children in the high blood $\mathrm{Pb}$ group ( $\geqq 60 \mu \mathrm{g} / \mathrm{d} 1)$ had lower mean daily intakes of both calcium $(610 \pm 20 \mathrm{mg}$ vs 770 \pm 20 in controls, $\mathrm{p}<.001)$ and vitamin $\mathrm{D}(210 \pm 17$ IU vs $325 \pm$ 20 in controls, $\mathrm{p}<.001$ ).

These data indicate that, in $\mathrm{Pb}$-burdened children, multiple factors, including metal-metal interactions, appear to modify the absorption and toxicity of $\mathrm{Pb}$ and relative vitamin $\mathrm{D}$ deficiency, not excess, was associated with high blood $\mathrm{Pb}$ levels. Assessment of nutrition, calcium metabolism, and vitamin D status is recommended in evaluating children known to have undue absorption of $\mathrm{Pb}$.

Effect of Maternally administered Dexamethasone and Galactose on Fetal Rat Liver G1ycogen. John W. Sparks, Almorris Lynch, and Walter Glinsmann

Kretchmer), Lab. Biomed. Sel. Nhly to the developing rat fetus inrease glycogen levels and total glycogen synthase activity (GS$\mathrm{T}$ ), while galactose (GAL) increases active or I-form synthase activity (GS-I). We questioned whether these agents given to the mother would also alter glycogen metabolism in the fetal liver. Dexamethasone (DEX) was given ( $1 \mathrm{mg} / \mathrm{kg}, \mathrm{i} . \mathrm{m}$.$) to pregnant rats$ at 48 and 24 hours before sacrifice at 17,19 and 21 days (term = 21.5 d). Fetal livers from DEX-treated mothers showed 2-3x higher glycogen levels at all three gestations compared with untreated controls, and this correlated with higher GS-T levels. Fetal liver GS-I was not affected by DEX. Fetal livers from pregnant rats receiving $5 \%$ GAL in drinking water for $48 \mathrm{~h}$ before sacrifice showed a 50\% increase in glycogen levels at 19 and 21 days compared with controls on normal diet. GS-T was unchanged by GAL, but GS-I was higher in the GAL group compared with normal controls and with controls receiving $5 \%$ glucose in drinking water. Animals receiving both maternal DEX and GAL showed the highest glycogen levels and the highest GS-T. Neither DEX nor GAL alone or in combination affected fetal liver phosphorylase, placental glycogen, or maternal liver glycogen, GS-T, or GS-I.

We conclude that maternally administered DEX and GAL selectively increase fetal liver glycogen. Since fetal liver glycogen is important for postnatal glucose homeotasis, prenatal steroids or diet may affect postnatal carbohydrate metabolism.

685 Galactose Enhancement of Hypoglycemic Glucose Release 685 In Perfused Perinatal Rhesus Liver. John W. Sparks, Almorr is Lynch, and Walter Glinsmann (Spon. by Norma

Kretchmer), Laboratory of Biomedical Sciences, NICHD, Bethesda.

Isolated neonatal Rhesus liver perfused with hypoglycemic medium rapidly releases glucose from glycogen. Since galactose (GAL) from milk both regulates glycogen metabolism and can be rapidly converted to glucose by liver, we questioned whether GAL might alter hypoglycemic glucose release. Livers from normal fetuses at 70 and $85 \%$ of gestation and from 6 hour old term newborns were perfused with aglycemic medium with and without GAL. In the presence of GAL, each Iiver released glucose at twice the rate observed in the same liver in the absence of GAL. GAL uptake was rapid ( $3 \mathrm{mg} / \mathrm{g}$ liver $/ 30 \mathrm{~min}$ in the fetuses, increasing to $15 \mathrm{mg} / \mathrm{g} / 30 \mathrm{~min}$ post-partum) and more than accounted for the increased glucose release. Livers perfused with aglycemic media containing GAL had higher glycogen synthase activity $(15 \pm 3$ vs. $8 \pm 3 \%$ Active form) and lower phosphorylase activity ( $18+6$ vs. $45 \mp 11 \mathrm{U} / \mathrm{g}$ protein). Measured glycogen change during perfusion could account for glucose release in the Control but not GAL group. A single infant of a streptozotocin-diabetic mother (STZ-IDM) showed normal GAL uptake and doubled glucose release in response to aglycemia in the presence of GAL, compared with perfusion of the same liver without GAL. Livers from 5 other STZ-IDM's showed normal GAL uptake under other perfusion conditions. We conclude that GAL increases hypoglycemic glucose release and acts by providing an efficient gluconeogenic substrate rather than by increasing glycogenolysis, and we suggest that GAL may be useful in the management of neonatal hypoglycemia.
686

METABOLIC EFFECTS OF SOMATOSTATIN (SRIF) IN NEWBORN LAMBS. Mark A. Sperling, Luiz A. Grajwer, Rosemary D. Hospital, Department of Pediatrics, Torrance, CA.

Immediately after birth, glucose $(G)$ falls, insulin (I) secretion is blunted, and a surge in glucagon (GLU) occurs. The contribution and relative significance of GLU and $I$ in $G$ homeostasis was explored in newborn lambs by means of SRIF, a known inhibitor of pancreatic hormone secretion. A pulse of cyclic SRIF $(50 \mu \mathrm{g})$ was followed by constant infusion of $400 \mu \mathrm{g}$ for 2 hours. Plasma $G, I$ and GLU were assayed in samples obtained sequentially during three time periods; $A(0-60 \mathrm{~min})$ only SRIF was infused, $B(60-120$ $\mathrm{min}) \mathrm{GLU} 5 \mathrm{ng} / \mathrm{kg} / \mathrm{min}$ and SRIF were simultaneously infused, and $c(120-150 \mathrm{~min})$ during recovery. During $A$, GLU fell abruptly (within $5 \mathrm{~min}$ ) by $43 \pm 17 \mathrm{pg} / \mathrm{ml}$ (mean $\pm S E ; p<0.02$ ) and remained suppressed throughout the first hour $(p<0.01)$. Similarly, plasma I fell to significantly lower values at 5,30 and $60 \mathrm{~min} \quad(p<0.05)$. With both I and GLU suppressed, G fell by $12 \pm 4 \mathrm{ma} / 100 \mathrm{mI}(p<0.05)$ Reinfusion of GLU during $B$ raised plasma concentration within the physiologic range by $208 \pm 71 \mathrm{pg} / \mathrm{ml} \quad(p<0.05)$ and this was associated with modest increments in $G$ and I. During recovery GLU fell by $83 \pm 40 \mathrm{pg} / \mathrm{ml}$; I rose by $12 \pm 5.6 \mathrm{uU} / \mathrm{ml} \quad(p<0.05)$. The new GLU/I ratio was associated with a fall in $G$ of $16 \pm 5 \mathrm{mg} / 100 \mathrm{ml}(p<0.05)$. These results suggest that: 1) Changes in GLU within the physiological range can significantly affect neonatal ig homeostasis, 2) GLU's effect predominates over I since $G$ falls as GLU secretion is suppressed despite concomitant insulinopenia; if I were predominant, hyperglycemia would be expected, 3) GLU or the fiLU/I ratio appears to be important for neonatal $G$ homeostasis.

BONE MINERAL CONTENT(BMC)AND 25 HYDROXY D IN PRETERM (AGA) AND SMALL FOR GESTATIONAL AGE (SGA) INFANTS. Jean Tsang, University of Cincinnati. Dept. of Pediatrics Reginald C. Tsang. University of Cincinnati. Dept. Of Pediatrics There is little information for in vivo bone mineral measure-
nts of newborn low birth weight infants and their relation to gestational and postnatal age. Ten preterm appropriate for gestational age infants (AGA) 30 to 36 wks, 10 SGA (32 to 41 wks, birth

weight 1000 to $1970 \mathrm{grams}$ ) and 42 term AGA infants were studied. 25 hydroxy vit $D$ (Belsey's, normal 13 to $8 \mathrm{Ing} / \mathrm{ml}$ ), parathyroid hormone (PTH, radioimmunoassay), and BMC were measured at birth and ages, $2 \mathrm{wks}, 4 \mathrm{wks}, 6 \mathrm{wks}, 8 \mathrm{wks}$ and $12 \mathrm{wks}$. BMC was measured (left $1 / 3$ distal radius)by direct photon absorptiometry(Norland-Cameron) modified by increased bone edge detector sensitivity, magnetic tape record of the $\log$ minute count rate and Hewlett Packard integration. BMC in preterm AGA infants at birth (mean $34 \mathrm{wks}$ ) was lower $(.056 \mathrm{gm} / \mathrm{cm} \pm$ .003 , mean $\pm S . E$.) than in term (mean $40 \mathrm{wks}$ ) infants $(.091 \pm .003$, t test, $\mathrm{p}<.001)$. In AGA infants, BMC correlated significantly with gestation $(r=.437, p<.001)$ and birth weight $(r=.739, p<.001)$. In SGA infants, BMC was within the $95 \%$ confidence limits for AGA infants. Postnatally, in preterm AGA and SGA infants, BMC did not increase from birth $(.063 \pm .004)$ to $6 \mathrm{wks}(.071 \pm .003)$ but had increased by $12 \mathrm{wks}$ $(.093 \pm .003$ paired $t, p<.02) .25$ hydroxy $D$ levels were $54.7 \pm 4.6$ at birth and $48.2+2$ at 4 wks, within normal limits. 25 hydroxy $D$ and PTH were not different between AGA and SGA infants. The rate of increase in bone mineral content of low birth weight infants appears to be less for extrauterine growth than that for intrauterine growth. 

INSULIN ADMINISTRATION TO DIABETIC RABBITS. GOrO Takada, Lyell J. Thomas, Jr.., Ennis C. Layne and Samuel P. Bessman. Univ. of So. California School of Medicine, Department of Pharmacology, Los Angeles.

A micropump approximately the size of a walnut has been constructed from piezoelectric crystals and a solenoid valve which delivers fluid at approximately l/loth microlitex per pulse controllable at any rate from zero to approximately 100 microliters per minute. Using this pump insulin has been administered continuously over 8 hour periods to 30 rabbits rendered diabetic by intxavenous administration of 200 milligrams of aloxan per kilogram. Insulin was administered at rates from 2 units to $.04 \mathrm{u} / \mathrm{Kg} / \mathrm{hr}$. Blood sugar was monitored with Technicon or glucose electrode. After a control period of 1-2 hrs. insulin is started at a preselected rate. At $.05 \mathrm{u} / \mathrm{Kg} / \mathrm{hr}$ the blood sugar remains at the starting level. At $0.1 \mathrm{u} / \mathrm{Kg} / \mathrm{hr}$ the diabetic rabbit shows a marked continuing fall. Rate of fall is independent of the initial glucose level. 10 rabbits were given $0.1 \mathrm{u} / \mathrm{Kg} / \mathrm{hr}$ till the blood glucose level was 150 to 200 milligrams percent. The infusion was changed to .05 units per $\mathrm{Kg} / \mathrm{hr}$ producing a plateau for 5 to 6 hours. During this plateau an intravenous glucose tolerance test was performed. The log rate of fall of glucose in diabetic rabbits treated with .05 units of insulin was similar to the log rate of fall seen in the glucose tolerance test in normal rabbits. There is a close similaxity between these experiments with rabbits and the recent experience in many laboratories with the treatment of diabetic ketoacidosis using very low intravenous insulin infusion rates.

690 FANTS OF DIABETIC MOTHERS, Robert Taylor, Philip Felig, Joseph B. Warshaw, Yale University School of Medicine, Departments of Pediatrics and Medicine, New Haven, $\mathrm{Ct}$. Hypoglycemia in the infant of the diabetic mother remains a group of these infants to show a glycemic response to an infused alanine load. Three infants of diabetic mothers less than $3 \mathrm{hrs}$. of age and five between 16 and 24 hours of age received a slow infusion of alanine ( $125 \mathrm{mg}$ per $\mathrm{kg}$ ). All infants showed a 1.5 $2.0 \mathrm{mM}$ increase in serum levels of alanine which declined to 0.8 $\mathrm{mM}$ by $60 \mathrm{~min}$. Infants 16 to 24 hours of age showed an increased glucose response following alanine infusion. In this group, mean sexum glucose increased from $48 \pm 12$ to $72 \pm 14 \mathrm{mg} \%$ with the peak level not being observed until $6 \overline{0} \mathrm{~min}$. A g 1 ycemic response to alanine infusion was not observed in the infants less than 3 hours of age. Baseline glucagon levels in the less than 3 hours of age group $(233 \pm 100 \mathrm{pg} / \mathrm{ml})$ were comparable to baseline levels in the 16-24 hour age group ( $155 \pm 33 \mathrm{pg} / \mathrm{ml}$ ). Mean elevations in serum glucagon levels $(200-400 \mathrm{pg} / \mathrm{ml})$ were observed in both groups during alanine infusion although the response of the 16 hour group was more variable. The late glycemic response to infused alanine by the 16-24 hour age group suggests that alanine is converted to glucose with development of gluconeogenic pathways. However, stimulation of glycogenolysis induced by alaninestimulated glucagon release in the presence of a more favorable glucagon:insulin milieu at 16 hours of age can not be excluded.

691 INFANT OF HYPOPARATHYROID (HP) MOTHER. Reginald C. Tsang, Jean J.Steichen, Hector Cabot, David R. Brown, J.Robert Johnson, I-Wen Chen, University of Cincinnati \& Good Sam. Hosp.

Fetal and neonatal hyperparathyroidism has been reported with maternal HP, but PTH function tests have not been evaluated; the fetal effect of large vit $D$ doses for maternal HP are uncertain.A $3200 \mathrm{gm}, 38$ week male was born of a woman with idiopathic HP. During pregnancy, serum Ca ranged $7.7 \mathrm{mg} / \mathrm{dl}$ to 9.3 , ionized $\mathrm{Ca} 3.3 \mathrm{mg} / \mathrm{d} 1$ to 3.5 , (normal 3.5 to 4.5 ) ; vit D 50,000 IUs/day was increased to 100,000 in the last 2 mos. Infant serum Ca(total and ionized) birth to 5 mos., were normal without signs of hypercalcemia.PTH levels ranged from undetectable to normal ( $N$-terminal radioimmunoassay, $89 \%$ adults detectable). Day 4 EDTA infusion $50 \mathrm{mg} / \mathrm{kg}$ showed normal $140 \%$ PTH response. Oral Ca tolerance $(50 \mathrm{mg} / \mathrm{kg})$ decreased PTH to $28 \%$. Urinary cyclic AMP(Gilman's) rose normally from day 2 ( $37 \mathrm{nmol} / \mathrm{day}, 0.75 \mathrm{pmol} / \mathrm{mg}$ creatinine) to day $4(95 \mathrm{nmol} / \mathrm{day}, 1.8 \mathrm{pmol} / \mathrm{mg}$ creatinine). Bone Mineral (photon absorptiometry, infant adapted, normal white male newborn 0.05 to.105), $0.074 \mathrm{gm} / \mathrm{cm}$ at $1 / 3$ distal radius at 10 days and rose to 0.12 at 4 mos. Skull and 1 imb Xrays were normal. Neonatal 25 hydroxy D levels (modified Belsey's, normal 13 to $81 \mathrm{ng} / \mathrm{ml}$ ) were high normal: at birth it was 77.1 (simultaneous maternal 64 ), rose to 86 at $37 \mathrm{hrs}$ and gradually fell to 63 in the first mo. With oral $\mathrm{Ca}$ test, $15 \%$ decrease in 25 hydroxy $\mathrm{D}$ occurred. Careful control of serum $\mathrm{Ca}$ in hypoparathyroid pregnancy was associated with normal neonatal PTH function (PTH stimulation and suppression, cyclic AMP and bone); large maternal vit D doses for HP resulted in high normal neonatal 25 hydroxy $D$ levels that gradually decreased.
INSULIN ADSORPTION TO INTRAVENOUS INFUSION SETS

692 DURING CONTINUOUS INSULIN INFUSION. Yvonne Vaucher Eileen McLanahan (Spon. by Corrigan, J. J., Univ. of Arizona, Coll. of Medicine, Dept. of Pediatrics, Tucson.

Continuous insulin infusion has been recommended for treatment of juvenile diabetic ketoacidosis. Insulin adsorption to glass and plastic surfaces of infusion sets may alter expected insulin delivery. Albumin reduces this loss, but data are not available on the effect of albumin after more than two hours of infusion. Regular Insulin (50U) was added to $250 \mathrm{cc}$ normal saline (with and without $0.3 \mathrm{gm} \%$ albumin) in nine $250 \mathrm{cc}$ glass bottles and allowed to run through standard length minidrip infusion tubing at $5 \mathrm{cc} /$ hr. Samples from bottles and distal IV tubing were collected at $\frac{1}{2}, 2,6$, and 24 hours. Insulin recovery was determined by radioimmunoassay. Albumin significantly $(\mathrm{p}<.05)$ increased insulin recovery $(\bar{x} \pm S E)$ from bottles at $\frac{1}{2}$ hour $(86 \pm 11$ vs $60 \% \pm 3), 2$ hours $\left(93 \pm 8\right.$ vs $53 \% \pm 4$ ) and from distal tubing at $\frac{1}{2}$ hour (110 \pm 8 vs $55 \% \pm 3), 2$ hours $(96 \pm 8$ vs $53 \% \pm 4)$ and 6 hours $(100 \pm 12$ vs $62 \% \pm 9$ ). Insulin recovery from bottles at 6 hours was not significantly different $(58 \pm 12$ vs $42 \% \pm 2)$. Recovery from all samples at 24 hours was less than $5 \%$ of total initial insulin activity. These results suggest that fresh insulin solution be prepared every 6 hours during continuous insulin infusion therapy of ketoacidosis to allow even insulin delivery. Distal insulin recovery may be increased approximately $40-50 \%$ by prior addition of $0.3 \%$ albumin to the insulin infusion solution.

693 y. Weisman, R. Sapir, A. Harel1 and S. Edelstein. (Spon. by A. Root). Municipal-Governmental Med Ctr., Depts. of Ped. and Endocr., Tel-Aviv, Israel.

The perinatal metabolism of $D$ was examined by maintaining pregnant rats $(M)$ on a $D$ deficient diet supplemented by $\left(1,2-{ }^{3} \mathrm{H}, 4-{ }^{14} \mathrm{C}\right) \mathrm{D}_{3}\left(\mathrm{D}^{*}\right)$. Distribution of $\mathrm{D}^{*}$ metabolites was analyzed in lipid extracts of whole 18 day fetuses $(F)$ and 2 day newborns (N) and in 18 day $M$ plasma (MP), kidney (MK), intestinal mucosa (MI) and bone (MB).

$\mathrm{D*}$ $(\mathrm{pM} / \mathrm{g})$

$25 \mathrm{OHD}_{2}$ $24,25(\mathrm{OH}){ }_{2} \mathrm{D}^{*}$ $1,25(\mathrm{OH}){ }_{2} \mathrm{D}^{*}$

\begin{tabular}{cccccc}
$\mathrm{F}$ & $\mathrm{N}$ & $\mathrm{MK}$ & $\mathrm{MI}$ & $\mathrm{MB}$ & $\mathrm{MP}(\mathrm{pM} / \mathrm{m} 1)$ \\
\hline 0.04 & 0.11 & 16 & 1.1 & 1.5 & 0.08 \\
2.31 & 3.29 & 10 & 0.25 & 0.9 & 1.15 \\
1.68 & 1.94 & 3.9 & 0.12 & 0.7 & 0.73
\end{tabular}
In non-pregnant rats much greater amounts of $1,25(\mathrm{OH})_{2} \mathrm{D}^{*}$ were found in D target organs and plasma. 7 days after administration of $\mathrm{D}^{*}$ to 1 day $\mathrm{N}, 25 \mathrm{OHD} *(2.0 \mathrm{pM} / \mathrm{g})$ and $24,25(\mathrm{OH})_{2} \mathrm{D} *(0.64 \mathrm{pM} / \mathrm{g})$, but not $1,25(\mathrm{OH})_{2} \mathrm{D}^{*}$, were detected in whole body extracts. 1-hydroxylase activity was low but demonstrable in renal homogenates of $F$ and $M$ but not in 1 day $N$. 24-hydroxylase activity was absent in $F$ and $M$ but was marked in $N$. Conclusions: 1 ) $1,25(\mathrm{OH})_{2} \mathrm{D}$ is not detected in fetuses and newborns and none or very little is found in maternal plasma, kidney, bone and intes tinal mucosa. 2) Newborns do not form detectable levels of $1,25(\mathrm{OH})_{2} \mathrm{D}$. 3) $24,25(\mathrm{OH})_{2} \mathrm{D}$ may serve an important perinatal function. 4) If the human newborn is also unable to form
$1,25(\mathrm{OH}){ }_{2} \mathrm{D}$, this may account in part for the occurrence of neonatal hypocalcemia.

EVIDENCE INDICATING GLUCAGON (GIU) SUPPRESSION IN INFANTS OF INSULIN DEPENDENT DIABETIC MOTHERS (IDM-I). Paul R. Williams, Mark A. Sperling, Zenaida Racasa. Departments of Pediatrics, William Beaumont Hospital, Royal Oak, Michigan, and UCLA - Harbor General Hospital, Torrance, CA.

Neonatal hypoglycemia in IDM-I is generally attributed to hyperinsul inemia consequent upon chronic in-utero stimulation by maternal hyperglycemia, but suppressed GLU secretion resulting from chronic hyperglycemia may also contribute. To evaluate this possibility, spontaneous (birth to 1 hour) and alanine stimulated (1st-2nd hr) GLU secretion was studied in 9 normal and 10 IDM-I infants. Cord blood glucose $(G)$ and GLU were similar in both groups but the fall in $G$ during the first hour was greater in IDMI (Table below). GLU rose significantly in control infants but not in IDM-I; the rise in GLU and fall in $G$ in controls was significantly correlated. I.V. alanine $150 \mathrm{mg} / \mathrm{Kg}$ given at $1 \mathrm{hr}$ of 7 ife elicited a significant rise in G $30 \& 60 \mathrm{~min}$ later, and a significant rise in GLU at $60 \mathrm{~min}$ in controls; GLU and G were positively correlated. However, in IDM-I, G rema ined unchanged, GLU response was blunted and did not rise significantly. The results suggest 1) GLU secretion is blunted in IDM-I, 2) This blunted GLU may contribute to neonatal hypogiycemia, 3) Infusion of GLU at physiologic concentrations may avert hypoglycemia in IDM-I.

\begin{tabular}{|c|c|c|c|c|}
\hline - & Cord & Baseline $(7 \mathrm{hr})$ & $30^{\prime}$ Post- & Alanine $60^{\circ}$ \\
\hline ;ontrol: $\mathrm{G}(\mathrm{mg} / \mathrm{dl} \pm \mathrm{SEM})$ & $94 \pm 73$ & $5 ? \pm 5^{*}$ & $63 \pm 6^{*}$ & $63 \pm 5^{\star}$ \\
\hline IDM- & $101 \pm 16$ & $? 3 \pm 8 *$ & $30 \pm 5$ & $33 \pm 6$ \\
\hline Control & $114 \pm 16$ & $16 ? \pm 28 *$ & $29 \pm 15 \%$ & $34 \pm 74 \% *$ \\
\hline IDM-I & $145 \pm 28$ & $179+21$ & $11 \pm 7 \%$ & $10 \pm 8 \%$ \\
\hline
\end{tabular}


695 TYROSINEMIA: ASSOCIATION WITH ACUTE INTERMITTEN PORPHYRIA AND EPISODIC HYPERTENSION. Edward L. Zuroweste, C. Frederic Strife, Edward A. Emmett, Vincent $\underline{N}$. Finelli and Helen $\underline{K}$. Berry, (Spon. by Clark D. West), University of Cincinnati College of Medicine and Children's Hospital, Depts. of Peds. and Environ. Health, Cincinnati.

A patient with hereditary tyrosinemia was observed during and following an illness resembling acute intermittent porphyria with associated hypertension. As is common in tyrosinemia, increased urinary excretion of $\Delta$ aminolevulinic acid (ALA) associated with normal or only slightly elevated ALA metabolites was observed. These results were possibly explained by markedly decreased red blood cell activity ( $172 \mathrm{nmol}$ porphobilinogen/hr/ml RBC) of the zinc dependent enzyme, $\triangle$ ALA dehydratase (ALA-D) $(\mathrm{N}=1083 \pm 178 \mathrm{nmo} 1 \mathrm{PBG} / \mathrm{hr} / \mathrm{ml} \mathrm{RBC})$. This enzyme deficiency suggests that the elevated ALA levels in tyrosinemia may result not only from increased ALA synthesis, as has been previously suggested, but also from inhibition of ALA degradation. In addition, the patient was found to be $\mathrm{Zn}$ deficient, suggesting an association between the diminished enzyme activity and the metal deficiency. However, addition of exogeneous $\mathrm{Zn}$ to the AIA-D assay system did not result in increased enzyme activity, suggesting decreased synthesis rather than competitive inhibition. The patient's hypertension appeared to be related to both increased urinary norepinephrine levels and elevated peripheral vein renin activity. Neither diminished ALA-D activity nor elevated renin activity have been described previously in association with hereditary tyrosinemia.

\section{MORPHOGENESIS}

696 A/JAX MOUSE IN THE PRESENCE OF EPIDERMAL GROWTH FACTOR (EGF). Alan Bedrick and Roger L. Ladda (Spon.
M. Nelson). Penn State Univ. Coll. Med., M. S. by Nicholas M. Nelson). Penn Sta

Injection of corticosteroid into pregnant A/JAX mice between days 10-15 of gestation has been reported to induce a high incidence of cleft palate (CP) in the offspring. The initial period of palatal shelf fusion occurs during days $10-15$ and presumably is inhibited by elevated levels of corticosteroids. We have studied the influence of EGF, a specific promotor of epithelial cell growth, on the incidence of CP or cleft lip and palate (CL/ $\mathrm{P})$ in the $\mathrm{A} / \mathrm{JAX}$ cortisone $\mathrm{CP}$ induction model. $\mathrm{CL} / \mathrm{P}$ was reported to occur spontaneously in $2-10 \%$ of of fspring and CP alone rarely occurred. In our experiment fondled uninjected controls produced $91 \%$ normal offspring and $9 \%$ with spontaneous $\mathrm{CL} / \mathrm{P}$ and none with CP alone. Saline-injected controls produced $80 \%$ normal animals and $20 \%$ with CL/P. The cortisone injected group showed $66 \%$ fetal resurption; $60 \%$ of formed embryos had CP on $1 y$; $7 \%$ had $\mathrm{CL} / \mathrm{P}$ and $33 \%$ were normal. EGF alone caused $21 \%$ fetal resorption; $91 \%$ of formed embryos had normal palates; none had CP alone while $5 \%$ had CL/P. Cortisone and EGF together produced $80 \%$ resorption; $100 \%$ of formed embryos had CP. EGF may have enhanced rather than decreased cortisone effect on palatal fusion. Stress also appeared to have a significant influence on fusion of palatal shelves, as saline injected controls had $6 \%$ fetal resorption and $20 \%$ of fspring affected with CL/P. (Supported by NIH Grant 非01 HD 09232 01).

69 ELECTROCARD IOGRAPHIC (EKG) ABNORMALITIES IN PATIENTS

697 WITH CLEFT LIP AND PALATE. N. Beligere, A. Hastreiter S. Pruzansky, Abraham Lincoln School of Medicine, U.
p., Dept. Pediatrics, Ctr. Craniofacial Anomalies, of 111. Hosp., Dept. Pediatrics, Ctr. Craniofacial Anomalies,

Association of abnormal EKG changes in patients with cleft lip and palate has been reported previously. In order to further study the relation of cleft $l i p$ and palate with abnormal EKG findings, we compared EKG's of 48 patients with facial clefts alone (study grp) with EKG's of age and sex-matched population (control grp) who had no congenital heart disease or facial clefts. The age ranged from $2 \frac{1}{2}$ mo. to $18 \mathrm{yrs}$.

Analys is of EKG findings showed the following. Heart rate was not significantly different between the two groups $(86.9 \pm 3.1$ vs $95.6 \pm 3.3 / \mathrm{mt})$. 15/48 patients in study grp had $r s R^{\prime}$ pattern, whereas only $3 / 48$ patients in control grp showed rsR' pattern. This was a significant difference $(p<.001)$ by $\chi^{2}$ test. Right axis deviation was seen in 13/48 of study grp whereas only $3 / 48$ of control grp had right axis deviation $(p<.001)$. QRS duration in study grp was significantly less than controls $(.058+.003$ vs $.070 \pm .002$ sec, $P<.01)$. The amplitude of $S$ wave in $V_{5}$ was significantly larger in study grp $(5.2+0.5$ vs $3.4+0.5 \mathrm{mv})$ than control grp ( $<<.025)$.

The data suggest that al though no increase in structural cardiac defects were demonstrable in patients with facial cleft, there was an increase in incidence of EKG abnormalities when compared with age and sex-matched controls. This study further suggests the possibility that the developmental process accounting for the cleft defect may have wider systemic effects than previously suspected.
RISK OF NEURAL TUBE DEFECTS TO IST COUSINS OF

698 AFFECTED INDIVIDUALS IN A UNITED STATES POPULATION Joann Bodurtha, Elaine H. Zackai, Mary Ames, William J. Mellman. Univ. of Pennsylvania Medical School, Children's Hospital of Philadelphia

Dept. of Pediatrics, Human Genetics. Philadelphia

Since the introduction of prenatal diagnosis it is particularly important to identify in the United States high risk populations for neural tube defects.

In England and wales . $7 \%$ of 1 st cousins of individuals affected with myelomeningocele or anencephaly have neural tube defects (maternal lst cousins - 1\%, paternal 1st cousins .4\%); Nance, W., Nature $224: 373,1969)$. The general population risk for these anomalies is approximate $1 \mathrm{y} 3 / 1000$.

To establish the risk to 1st cousins in a United States population family data was collected on 143 patients attending the Myelomeningocele Clinic at the Children's Hospital of Philadelphia. $7 / 663$ maternal lst cousins $(1.0 \%)$ and $2 / 763$ paternal 1st cousins (. $3 \%$ ) had neural tube defects ( 1 anencephalic, 8 myelomeningocele). Thus the rate for maternal lst cousins is 6.5 times and for paternal lst cousins 2 times the general population risk (based on the combined occurence rates of myelomeningocele, anencephaly and encephalocele determined by the Collaborative Perinatal Project). This result has prompted us to encourage prenatal screening of this cousin population.
SELECTIVE MUSCLE FIBER HYPOPLASIA AND EPIPHYSEAL

699 osteolysis: A NEW SYNDROME. S. Caste1ls, J.H. Sher, H.C. Anderson, S. Shafiq, S.E. Hashemi, Dept. of Ped. Pathol., Rad. and Neur., S.U.N.Y., Downstate Med. Ctr., Brooklyn A $10 \mathrm{yr}$. old female, presented with foreshortened, clawlike hands and feet, joint pain with stiffness and contractures, marked muscle atrophy, osteoporosis and epiphyseal osteolysis. $\mathrm{Ca}_{4} 7$ turnover was accelerated. Muscle biopsy revealed a population of hypoplastic muscle fibers, 10 to 15 microns in diameter, scattered singly or in small groups among a majority of larger morphologically normal fibers. Myofibillar ATPase stains showed that all Types I and II A fibers present were hypoplastic, and al1 large fibers present were Type II B. Electron microscopic study of the skeletal muscle showed normal myofibrils, but vacuolated mitochondria with disorganized cristae, in the hypoplastic fibers. Synthetic salmon calcitonin given at $2 \mathrm{MRC}$ units per $\mathrm{Kg}$ 3 days a week, produced a marked improvement in bone pain and reduced urinary excretion of hydroxyproline from 127 to $84 \mathrm{mg}$ per $24 \mathrm{hr}$, and calcium metabolic balance changed from an average of 2 weeks control of +124.4 to $+383.0 \mathrm{mg}$ per day. Iliac crest biopsy before calcitonin, revealed increased uncalcified matrix and increased osteoclastic activity. After 3 months of calcitonin there was a decrease in osteoclastic activity. Skin biopsy was normal. This new syndrome associated grotesque skeletal deformities, selective muscle hypoplasia of fibers Types $I$ and II A and increased skeletal resorption. Calcitonin, a hormone that reduces bone resorption, appears to improve the metabolic bone disease.

Supported by the NIH Grant RR-318

70 VENTRICULAR SEPTAL DEFECT AFTER REDUCTION OF BLOOD

700 FLOW TO SIXTH AORTIC ARCHES IN CHICK EMBRYOS. Edward B. Clark, D. Richard Martini, Glenn C. Rosenquist. The Johns Hopkins Hospita1, Baltimore, Maryland

We are currently exploring the effect of reduction of blood flow through the sixth aortic arches on cardiopulmonary morphogenesis. Fertile white leghorn eggs were preincubated at $37.8^{\circ} \mathrm{C}$ to Hamilton-Hamburger stages 23-25, when sixth aortic arches were electrocoagulated with a $0.01 \mathrm{~mm}$ unipolar platinum electrode connected to a Grass ML-4 Lesion maker. The 20ma radiofrequency current was applied directly to the vessel at a point equidistant between the heart and dorsal aorta until blood flow ceased for a 3 minute period. Eggs were sealed with parafilm and reincubated 2-5 days. Embryos were fixed in $10 \%$ formol in chick Ringer's saline and their thoracic contents micro-dissected. Lung and pulmonary artery dimensions were measured with a micrometer eyepiece.

Fourteen embryos survived to stage 36 or older ( 9 right, 3 left and 2 bilateral sixth aortic arch coagulations). Of these 10 had decrease in lung and pulmonary artery size on the operated side(s); 8 of these 10 had a large ventricular septal defect high in the septum. Three of 4 embryos with normal lung and pulmonary artery measurements had high ventricular septal defects, suggesting that transient variations in extracardiac blood flow may effect intracardiac morphogenesis. AI1 control embryos had intact ventricular septa. These data support the concept that both prolonged and transient reduction of blood flow through sixth aortic arches may effect intracardiac as well as lung morphogenesis. 
701

SIZE REGULATION DURING EAFLY FMIBRYOGENESIS: MACRO-

MOLECULAR SYNTHESES IN AGGREGATED MOUSE EMBRYOS. Donna $L_{0}$ Daentl (Intr. by Carolyn F. Piel). Univo of California, San Francisco, Depts。 of Growth and Development and Ped., San Francisco.

Understanding of some critical factors related to normal human development may be derived from biochemical study of the mouse embryo.

Despite vast alterations of cell number during the "labile" period of embryogenesis (morula stage), the ultimate size of a mouse developing in vivo to term is relatively constant. An initial investigation is proceeding on the precise embryonic size regulation which apparently takes place. Up to fifteen mouse morulae have been successfully aggregated, allowed to form a single giant blastocyst and develop to the egg cylinder stage in vitro. Giant embryos were then compared to normal-sized embryos cultured in parallel with respect to thymidine uptake, thymidine kinase activity, DNA polymerase activity and incorporation of precursors into DNA, RNA and protein.

The results obtained after development in vitro show a general correlation between these biochemical measures and the numbers of embryos initially aggregated, suggesting that control of embryo size may occur at a stage later than egg cylinder formation. Since differentiation in aggregated embryos generally coincided with that of single embryos of the same chronologic age, the mechanism of control appears more likely to be regulation of growth than acceleration of differentiation.

\section{2}

A FAMILY WITH MONILETHRIX. Irene M. Gray and

Annemarie Sommer (Spon. by Stella B. Kontras). Ohio State Univ. Col1. of Med., Dept. of Ped., Div. of Genetics and Children's Hospital Research Foundation, Columbus.

At least thirty members in seven generations of a family were affected with monilethrix, a hereditary disorder characterized by dry brittle scalp hair which is easily broken and perifollicular hyperkeratosis of the scalp. This disorder also shows sparse generalized body hair and decreased axillary and pubic hair. Dry flaking skin and defective or easily broken nails were observed in varying degrees by family members. Scalp hair from three members of this family was examined using various techniques including light microscopy, scanning electron microscopy and biochemical analyses. Most of the hair was darkly pigmented and averaged $10 \mathrm{~mm}$ or less in length and varied from 0.06 to $0.1 \mathrm{~mm}$ in width. Some hair exhibited regular beading with elliptical nodes $1 \mathrm{~mm}$ apart separated by narrower internodes. A11 hair showed various stages of defective cuticle, from nearly normal to almost absent and transverse fractures (including greensticktype) were frequently observed. The sulfur content of the hair was normal which differentiates this disorder from trichoschisis which has a low sulfur content. Trace element levels of the monilethrix hair were also normal. Serum and urine amino acids from 2 family members tested normal. Sweat pore counts of palms of three family members in 2 generations were normal. It is suggested that in this pedigree, monilethrix may be associated with a form of hidrotic ectodermal dysplasia and may be due to abnormal keratin production. This disorder is probably the expression of a single dose of an autosomal dominant gene.
704 CALVARIECTOMY IN THE MANAGEMENT OF SEVERE MULTIPLESUTURE CRANIOSYNOSTOSIS IN THE INEWBORN. JameS W. Hanson, Martin P. Sayers, Larry Knopp, Constance Macdonald, and David W. Smith. Departments of Pediatrics, University of Washington School of Medicine and Group Health Hospital Seattle; Department of Surgery. Ohio State University, Columbus. Synostosis of multiple cranial sutures in the newborn may result in blindness, mental retardation and/or severe craniofacial deformities. Past surgical treatment consisting of linear craniotomy of involved sutures with or without the interposition of non-absorbable synthetic materials has met with variable success and has often given unsatisfactory cosmetic results. This report presents the results of early calvariectomy in 3 infants treated in the neonatal period. All 3 had involvement of multiple sutures with early signs of impending neurologic complications. Regeneration of a new calvarium from the underlying dura occurred over a period of several months during which time the growing brain molded a more normal appearing skull in which sutures were present in a more normal fashion than in the synostotic calvarium. Neurologic and intellectual performance have been satisfactory and cosmetic results were preferable to those obtained by more limited surgery. This approach also favors better craniofacial growth by minimizing secondary deformities such as midfacial retrusion and shallow orbit, which can occur because of impaired forward growth of the cranial base secondary to coronal synostosis.

PATIVINS OF CELL POLALITY IN DEVELOPING LIMB. 705 Lewis B. llolmes and Robert L. Trelstad. Harvar fedical School, Massachusetts General Hospital, (i)ildren's Service, Boston.

More precise descriptions of the cellular events in normal morphogenesis are needed for the stuch of malformations. Changes in the spatial arrangement and orientation of celis occur repeatedly during 1 inib development. To quantitate this process, we have studied the polarity of $1 \mathrm{imb}$ bud mesenchyne and epithelium using a silver staining technique which identifies simultaneously two poles of the cell, the Golgi and nucleus. Studies of the developing cornea, tooth and endocrine glands have shown the end of the cell with the Colgi is the site of active secretion.

The following patterns have been found between the initial limb bud (day 10) and digit formation (day 14): 1) in the epithelium the Golgi poles remain alnost entirely in the apex of the cell--d. 10, $03 \%$ apjical (A), 35\% lateral (L) and $2 \%$ basal (B) ; d. $12,81 \% \mathrm{~A}, 18 \% \mathrm{~L}$ and $1 \% \mathrm{~B}$; d. $14,77 \% \mathrm{~A}, 21 \% \mathrm{~L}$ and $2 \% \mathrm{~B}$; during this time interaction between the ectodermal ridge and mesenchyme is alleged to ocaur; 2) the mesenchvrie cells adjacent to the epithelium initially have their folgi poles directed away from the epithelium (d. 10, 3\% toward, 48\% lateral and $44 \%$ away), but shift (a. 14, 65\% toward, 21\% lateral, 14\% away) during the tinle wher the extracellular matrix accumulates above these cells; 3) inesenchyme cells in the digit area aggregate in colums on d. 12 prior to chondrogenesis with the Golgi poles of the cells pointing toward the central axis; 4) the early cartilage aggregates have the ends of the Golgi apparatus pointing inward as they form concentric circles and matrix accumulates.

\section{3}

706 A NEW FORM OF CRANIOFACIAL DYSOSTOSIS WITH HAND AND FOO'T ANOMALIES. Roger L. Ladda (Spon. by Nicholas M. Nelson). Penn State Univ. Coll. Med., M. S. Hershey Med. Ctr., Dept of Ped., Hershey, Pa.

Six distinct forms of acrocephalosyndactylism and acrocephalopolysyndactylism are known. We now report a kindred of five generations with male and female members affected with a new type of craniofacial dysostosis associated with abnormalities of hand/ feet. Craniofacial abnormality is characterized by unilateral or bilateral fusion of the coronal sutures causing variable brachyoxycephaly. Hand malformations were variable, but characteristically consisted of short digits and large, broad thumbs without syndactyly. Dista1 interphalangeal joints of second and fifth digits were fused; middle phalanges of the other digits were consistently hypoplastic. Foot abnormalities consisted of fusion of the interphalangeal joint of the large toe; middle phalanges of the second thru fifth toes were often absent. All affected members had varying degrees of syndactyly of toes 2-3. Fusions of tarsal bones (first cuneiform-tarsal navicular and cuboidcalcaneous) were found in most affected individuals. Hand and foot anomalies varied as widely as the craniofacial anomaly, but, in each affected individual, both cranio facial and hand/foot anomalies were present. Several individuals had anomalies of the cervica1 spine consisting of abnormal articular facets. All affected individuals had normal mental function. Members of this family previously had been suspected of having Crouzon's Syndrome or Pfeiffer's Syndrome, but in fact are representative of a separate type of dominantly-inherited acrocephalosyndactylism based on the specific hand/foot defects. 
707 CORTISONE-INDUCED CLEFT PALATE STUDIES III TIWH $\leftrightarrow$ C57BI/6J ALLOPHENIC MICE. Kathleen K. Hiller (Spon. by R. L. Summitt), U. of Tenn. Ctr. for the Health Sci., Dept. of Anat., Memphis.

TlWh, an albino strain of mice, is homozygous for a $5 ; 19$ Robertsonian translocation which provides conspicuous chromosomal markers. Preliminary studies revealed that cleft palate (CP) is induced in all the offspring of TlWh females treated daily with $2.5 \mathrm{mg}$. of cortisone acetate on days $11-14$ of pregnancy and that palatal closure is accomplished at a later morphological age than in the C57Bl/6J strain mice. The latter strain had a CP frequency of $42 \%$ under similar experimental conditions in this lab.

Cleavage stage, 8-16 cell embryos, were fused in the following combinations, TlWh TlWh, C57Bi/6J↔C57BI/6J, and TiWh $\leftrightarrow$ $65781 / 6 \mathrm{~J}$. The resulting allophenic blastocysts were surgically transferred to pseudopregnant $[57 \mathrm{~B} 1 / 6 \mathrm{~J}$ recipients which were given the above cortisone treatment and sacrificed on their 18 th day of pregnancy. CP was found in all of $48 \mathrm{~T} 1 \mathrm{Wh} \leftrightarrow \mathrm{T}$ IWh fetuses, $4(20 \%)$ of $20 \mathrm{C} 57 \mathrm{Bl} / 6 \mathrm{~J} \leftrightarrow \mathrm{C} 57 \mathrm{BI} / 6 \mathrm{~J}$, and in $17(26.3 \%)$ of 65 TIWh $\rightarrow C 57 \mathrm{Bl} / 6 \mathrm{~J}$ fetuses. Analysis of eye pigmentation and palate and skin chromosomal preparations from the TIWh $\leftrightarrow$ C57BI/6J fetuses revealed that the simple presence of TlWh cells in the palate or other tissues does not predispose it to CP formation. However, in those TIWh $\rightarrow$ C57BI/6J mice with cleft palates, the percentages of $T i l / h$ cells were, on the average, greater in their palatal tissues than in mice without clefts. The data indicate a possible growth advantage in favor of $C 57 \mathrm{~B} 1 / 6 \mathrm{~J}$ cells in the palates of $T / W h \leftrightarrow 57 B I / 6 J$ allophenic mice.

708 NEURAL TUBE DEFECTS (NTD) AND FALSE POSITIVE/NEGATIVE 708 ALPHA-FETOPROTEIN (AFP) RESULTS. Aubrey Mi lunsky and Elliot Alpert, Harvard Medical School, E. Kennedy Shriver Center, Massachusetts General Hospital, Departments of Pediatrics and Medicine, Boston.

Assay for AFP in amniotic fluid (AF) has proved to be a valuable adjunct in the prenatal diagnosis of open NTD's. About $90 \%$ of such defects are diagnosable in utero by this method. A clear perception of the frequency of faise positive/negative AFP results is only now beginning to emerge. We have assayed 2,495 AF's made up of 637 cases at risk for NTD's and 1,858 cases whose amniocenteses were for other reasons. False positive elevations (AFP > 2 standard deviations (SD)) were observed in 122 cases - a rate of $4.9 \%$. Analysis of these 122 cases revealed that 85 resulted in a normal baby (or fetus) suggesting a true false positive rate of $3.5 \%$. AF fetal $\mathrm{Hb}$, IgM and total protein were measured in 40 of the 122 original false positive cases. Normal pregnancy outcomes were noted in 29 of these 40 cases, no clue to explain the elevated AFP level being apparent in 12 cases. Fetal or maternal blood contamination of AF served as the most likely explanation for most of the 17 other cases. The only false negative in all 2,495 cases was 1 closed NTD. A11 48 open NTD's had AFP values $>+3 S D$, al though the AFP level in one case was only $0.01 \mathrm{mg} \%>3 \mathrm{SD}$. False positive results with AFP values $>+3$ SD were found in 57 cases $(2.3 \%)$. No reasonable explanation could be offered for 30 of these cases $(1.2 \%)$ where a normal child was born (29) or a normal fetus aborted (1).

709

THE MECHANISM OF DIPHENYLHYDANTOIN TERATOGENESIS.

of Pediatrics, University of Florida, Gainesville. Use of diphenylhydantoin during pregnancy appears to result in an increased incidence of major congenital malformations and an expanded syndrome of minor anomalies. The teratogenic mechanism of diphenylhydantoin may involve folate antagonism. Women taking this compound have decreased folate levels. The folic acid antagonist, aminopterin, used as an unsuccessful abortifacient, resulted in congenital anomalies similar to those seen with diphenylhydantoin.

Treatment of pregnant rats with the folic acid antagonist, 9methyl pteroylglutamic acid (9-methy1 PGA) causes defects in 95\% of the litter. Significantly decreased rates of cellular respiration have been demonstrated in these maldeveloping embryos in our laboratory.

Treatment of mice with diphenylhydantoin produces embryopathy similar to the major anomalies in man following use of this compound or aminopterin. To study the possibility of altered oxidative metabolism in such animal models, primagravid Swiss albino mice were injected intraperitoneally on day 10 with $75 \mathrm{mg}$ dipheny1hydantoin per $\mathrm{kg}$ body weight. Oxygen consumption of embryonic cells on day 12 were determined by the direct Warburg technique. Embryos from treated mothers consumed oxygen at a rate which was decreased from controls $(\mathrm{P}<0.01)$. These results are comparable to our findings using 9-methyl PGA; thus, decreased oxidative metabolism may offer evidence that diphenylhydantoin affects normal embryogenesis through a mechanism similar to folic acid deficiency.

\section{0} BIRTH WEIGHT ANALYSIS OF CHILDREN WITH PHENYLKETONURIA

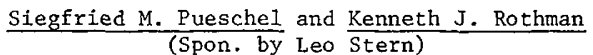

Brown University, Rhode Island Hospital, Department of Pediatrics, Providence, and Harvard School of Public Health, Department of Epidemiology, Boston.

The assumption that children with Phenylketonuria (PKU) deve1op normally until birth was brought into question by Saugstad who found that children with PKU weighed several hundred grams less at birth than their unaffected siblings.

We have examined the intra-family differences in birth weight in 40 sibships with at least one affected and one unaffected child. The mean birth weight for 52 children with PKU was 3300 grams; for their 79 unaffected siblings it was 3390 grams. In order to assess whether the intra-family differences in birth weight could be explained by corresponding differences of a variety of factors which might affect birth weight (gestational age, birth rank, maternal age, smoking, significant infections, toxemia, endocrine disturbances, emotional stress situations), and to control such confounding, a multivariate analysis was employed. The adjusted estimate of the birth weight difference between children with PKU and their siblings obtained from the fitted multiple aggression function is -51 grams, even less in absolute value than the unadjusted -69 grams.

The small difference in birth weight observed in children with PKU and their unafected siblings is statistically not significant and suggests that the intrauterine growth of children with classical PKU is not adversely affected.

\section{1}

712 THE ASSCCIATION CF SICKLE TRAIT AND LOWFR KFICHT. 12 Michael A. Ncirer and Sergio Piomelli, NYU School of Medicine, Department of Pediatrics, New Yor
This study evaluated ir. yourger children the recent suggestion that adolescents with hgt. AS have height (ht) within the normal limit, rui lower weight (w't). Routine screening for sickling is periormed cr: all clildren in the pediatric clinics at Bellevue Hospital. Search of the laboratory and clinical records yielded 52 Black children with positive sickle test, normal Higb and Hict values for age and normal peripheral smears (AS group). Non-Black children with positive sickle test were excluded from the study as was any child with a medical problem. Fifty-two Black children with negative sickle test, normal $\mathrm{Hgh}$ and Hct for age and normal peripheral smears were also selected (control group). Each control child had been tested and examined on the same day as one of the $A S$ children and the same nurse had ascertained measurements of ht and wt. From these 104 children it was possible to obtain 32 pairs, 15 male and 17 female, matched for age and sex. N11 subjects were between 6 months and 13 years of age. Percentiles for ht and wt were obtained using standard anthropometric charts to normalize with regard to age and sex. The data were analyzed by paired t-test. The mean percentiles for ht were $50.3^{ \pm} 12.2$ and $58.7 \pm 11.8$ for $\Lambda S$ and control group respectively $(p>.30)$. The mean percentiles for wt were $37.5 \pm 10.2$ and $59.5 \pm 11.1$ for AS and control group respectively $(p<.01)$. These findings confirm the hypothesis that children with AS have normal development for ht, but a statistically significant lower wt starting at a younger age. 
77 RENAL AGENESIS - A GENETIC DISORDER?-Jonathan Zonana, David L. Rimoin, David w. Hollistex, Ralph S. Lachman, Dennis A. Sarti \& Michael M. Kaback - UCLA General Hospital, Depts. Oi Peds. \& Rad., Torrance, Ca.

Bilateral renal agenesis (BRA) and unilateral renal agenesis (URA) have an approximate incidence of 1:3000 and 1:1000 births respectively. URA can also occur with an accompanying contralateral renal dysplasia. These entities have previously been considered to have separate etiologies, and since the majority of cases have appeared sporadic, a genetic etiology has generally been discounted.

We have examined clinically normal relatives of two "sporadic" cases by intravenous pyelography and nephrosonography. The first proband, a 16 month old male with renal failuxe, had URA with contralateral renal dysplasia. Two of his four siblings were found to have URA and the father had a duplication of the left collecting system. The second proband, a stillborn at 38 weeks gestation, had classical BRA. Subsequently, the father was discovered to have URA. There are no siblings, but we are currently following a subsequent pregnancy by serial ultrasonography.

It is apparent from ours and previously reported cases that familial cases of $B R A$ are clinically and pathologically indistinguishable from "sporadic" cases. Though an autosomal dominant pattern of inheritance is suggested by some pedigrees, the mode(s) of inheritance is unclear since most previous studies of familial and sporadic cases have failed to adequately examine clinically normal family members. Studies are now in progress to determine the true incidence, modes of inheritance and feasibil-

\section{NEONATOLOGY}

PROPHYLACTIC EXCHANGE TRANSFUSION IN THE VERY LOW

714 BIRTH WEIGHT TNEANT Gregor Alexander, Nadarassa Visveshwara and Charles R. Bauer, (Intro. by W. W. Cleveland) Univ. of Miami, School of Med. Dept. Of Ped., Miami, Fl. Sixty infants with birth weights less than 1250 grams were studied to determine the effects of exchange transfusion on the outcome of Hyaline Membrane Disease (HMD), Bleeding Disorders and Infection. Each infant was randomly assigned to one of four groups. Of the two groups that were not exchanged, one received no special therapy (Control) and the other was given fresh frozen plasma (F.F.P). Two groups were exchanged with ejther fresh walkin blood (F.W.I.B.) or with old red blood cells ( $>72$ hours) reconstituted with fresh frozen plasma (O.R.B.C.). This latter group received platelet infusions. Arterial Blood Gases, Hematocrit, clotting studies and immunoglobulins were monitored on admission, at 12 hours, or pre and post exchange. In spite of improving the above determinations post-exchange, the incidence of disease (Inc.) and the mortality rates (Mort.) were not significantly different.

Control (15)

F.F.P.

F.W.I.B.

O.R.B.C.

\begin{tabular}{|c|c|c|c|c|c|}
\hline \multicolumn{2}{|c|}{ HMD } & \multicolumn{2}{|c|}{ Bleeding } & \multicolumn{2}{|c|}{ Infection } \\
\hline Inc. & Mort. & Inc. & Mort. & Inc. & $\overline{\text { Mort. }}$ \\
\hline $33 \%$ & $40 \%$ & $20 \%$ & $66 \%$ & $33 \%$ & $40 \%$ \\
\hline $3 \%$ & $80 \%$ & $33 \%$ & $100 \%$ & $26 \%$ & $33 \%$ \\
\hline 33 & $100 \%$ & $53 \%$ & $87 \%$ & $20 \%$ & $33 \%$ \\
\hline $20 \%$ & $100 \%$ & $33 \%$ & $100 \%$ & $26 \%$ & $33 \%$ \\
\hline
\end{tabular}

These results suggest that exchange transfusion has no prophylac-

tic role in the care of the small infant. The increased mortality in the exchanged groups raises the possibility that exchange trans fusion itself may be detrimental.

715

EFFECTS OF RESPIRATORY DISTRESS ON CARDIAC OUTPUT AND ORGAN BLOOD FLOW IN THE PIGLET. Charles T. Alward, Michael D. Bailie, Jerry B. Hook and Thomas A.

Helmrath. Michigan State Univ., Col. of Human Med., East Lansing.

Respiratory distress and necrotizing enterocolitis occur frequently in ill premature infants. We have studied the possible relationship between these entities in unanesthetized piglets $6 \rightarrow$ 86 hours old. In 15 animals control values for cardiac output (CO), heart rate (HR), blood pressure (BP), organ blood flow and arterial $\mathrm{pO}_{2}, \mathrm{pH}$ and $\mathrm{pCO}_{2}$ were determined. Respiratory distress was produced for $90 \mathrm{~min}$ by increasing the respiratory dead space and determinations repeated. Arterial blood gases and $\mathrm{pH}$ changed as follows: $\mathrm{pO}_{2}(55 \rightarrow 39 \mathrm{mmHg}), \mathrm{pH}(7.26 \rightarrow 7.03)$, and $\mathrm{pCO}_{2}(44 \rightarrow$ $61 \mathrm{mmHg})$. CO $\left(\mathrm{L} / \mathrm{min} / \mathrm{m}^{2}\right)$, total peripheral resistance (TPR) in pressure resistance units, and upper and lower GI blood flow $(\mathrm{m} 1 / \mathrm{min} / \mathrm{g})$ are presented in the table.

$\begin{array}{lllllll} & \mathrm{CO} & \frac{\mathrm{HR}}{81} & \frac{\mathrm{BP}}{79} & \frac{\mathrm{TPR}}{.40} & \frac{\mathrm{UGI}}{1.05} & \frac{\text { LGI }}{1.04} \\ \text { Control } & 2.08 & 181 & 8^{*} & .61^{*} & .60^{*} & .67^{*} \\ \text { Resp. Dis. } & 1.82 & 216^{*} & 98^{*} & & \end{array}$

Resp. Dis.

$216 * 98 *$

Scattered areas of hemorrhage were seen in UGI and LGI. Total blood flow to kidneys, pancreas, and spleen decreased while flow measured. In the piglet $90 \mathrm{~min}$ of respiratory distress causes redistribution of blood flow away from intraabdominal organs presumably to heart and brain. This shift in blood flow may have significance in the pathogenesis of necrotizing enterocolitis.
$716 \frac{\mathrm{J} . \mathrm{V} . \text { Aranda, W. Gorman, H. Bergsteinsson, T. Gunn. }}{\text { (Intro.by E.Colle) Dept. Newborn Med.Montreal Child- }}$ dren's Hosp., Dept.Pharmacol.\& Therapeutics (Roche Dev.Pharmacol. Unit) McGill Univ. Montreal, Canada.

The efficacy of theophylline, a methylxanthine, in premature neonates with apnea has been demonstrated in recent studies. Caffeine (C), a related methylxanthine is thought to have a greater central respirogenic activity, therefore its potential efficacy was evaluated in 16 pre-term neonates, (gest,age: 25-32 wks, Bt.wt: 760-1985 gm) with apnea (cessation of breathing $>30$ secs.) and bradycardia $(<100 / \mathrm{min})$.C-citrate $10-20 \mathrm{mg} / \mathrm{kg} /$ dose was administered I.M.,I.V. or p.o. twice or thrice daily as per clinical response and was initiated from ages 1 to 44 days.All infants except one showed a significant decrease in the frequency of apneic episodes coincident with C-therapy $(14.75 \pm 3.65$ episodes/day before $\mathrm{C}$, and $1.74 \pm 0.69$ episodes/day during $C ; p<0.01)$. Physiologic variables obtained within $24 \mathrm{~h}$ before and after the first $\mathrm{C}$ administration showed a significant increase in respiratory rate (RR) without change in heart rate (HR). $(\mathrm{H})^{+}$ion concentration and $\mathrm{PCO}_{2}$ were

decreased and plasma bicarbonate was increased.
$(\mathrm{n}=8)$ $\begin{array}{llllllll}\text { PreC } & 58.0 \pm 3.2 & 155.0 \pm 4.0 & 50.1 \pm 2.4 & 52.9 \pm 3.5 & 25.1 \pm 1.2 & 1106.2 \pm 101.9\end{array}$ $\begin{array}{lllllll}\text { C } & 65.0 \pm 4.1 & 148.0 \pm 8.4 & 42.9 \pm 2.2 & 47.8 \pm 3.3 & 27.7 \pm 1.2 & 1116.2 \pm 101.1\end{array}$ $\begin{array}{lllllll}p & 0.01 & \text { NS } & 0.01 & 0.05 & 0.05 & \text { NS }\end{array}$ The data indicate that $C$ maybe an effective respirogenic agent in apnea of prematurity, but caution should be exercised in its use. Pending definition of pharmacokinetic disposition of $\mathrm{C}$ in neonates, a rational dose regimen has not been established.

77 EFFECT OF EXCHANGE TRANSFUSION ON UNBOUND BILIRUBIN

717 AND BINDING CAPACITY. Howard Arkans, George Cassady. Department of Pediatrics, Division of Perinatal Medicine, University of Alabama, Birmingham, Alabama.

Unbound (UB-nmol/1) and total bilirubin (TBR-mg/d1) were determined by the peroxidase method in 10 babies before and after exchange transfusion(ET)。 In 7 with preET UB of $0-3.3(\bar{x} 1.7)$, the post.ET UB was unchanged $(1,8-8,9 ; \bar{x} 3.8)$; in 3 with preET of $13.4-$ 13.7 , a significant decline was observed after ET $(\bar{x}$ prel3.5; $\overline{\mathrm{x}}$ post6.9).

Each sample was then titrated with crystalline bilirubin and the relation of UB to TBR ("binding capacity") was determined. This relation was unchanged by ET in 8 (groupI); an increase in "binding capacity" was observed in 2 (group II)。 In the group II babies, the "binding capacity" returned to preET values within 4-12 hours. Group I babies were lighter $(\bar{x}=1600 \mathrm{gm} ; 5 / 8 \leq 1100 \mathrm{gm})$ and more immature $(6 / 8 \leq 32 \mathrm{wks})$ than group II babies $(>3120 \mathrm{gm}, \geq$ 40wks). Seven of 8 group I babies had ET for coagulapathy; the group II babies were Coombs' positive.

TBR levels at which the UB was $\geq 30$ (possibly "dangerous" Wennberg) in group I wexe $14-29(\bar{x} 19.5)$ preET and $14.3-27.5$ ( $\bar{x} 19.2)$ postET ( $\mathrm{ns}$ ). In group II, these levels were increased $(20,5 \rightarrow 26,5 ; 28,8 \rightarrow 35.0)$ immediately postET, but returned to preET levels within 4-12 hours.

These data and our total experience with the peroxidase method (147 samples-55 infants) (1)confirm the relation between $\mathrm{JB} / \mathrm{TBR}$ and (2) suggest that ET only transiently effects "binding sapacity".

RELATIONSHIP OF PREMATURE RUPTURE OF THE MEMBRANES

718 TO HYALINE MEMBRANE DISEASE. Henrietta S. Bada, Leticia C. Alojipan, Billy F. Andrews, Univ. of Lou. Sch. of Med., Lou. Gen. Hosp., Dept. of Peds., Louisville.

To have a broader perspective and better understanding of neonatal problems related to birth after premature rupture of membranes (PROM), 963 deliveries with PROM were analyzed from a total of 19,420 deliveries in an eight-year period (1964-1972). Both maternal and neonatal histories were reviewed and incidence of neonatal complications, i.e., mortality, sepsis, asphyxia, and hyaline membrane disease (HMD), was correlated with hours following PROM and gestational age. Though the already high incidence of complications including HMD was not affected by $P R O M$ in infants less than 33 weeks gestation, the incidence of sepsis, asphyxia, and mortality increased with prolonged rupture of membranes in those over 33 weeks gestation. No infant over 37 weeks gestation developed HMD. Regarding preterm deliveries, it appears that the best time for delivery after PROM for infants 33 to 37 weeks is also 12 to 24 hours when incidence of HMD, sepsis, asphyxia, and mortality is low. Our data failed to show benefit from prolonged rupture of membranes in reduction of HMD. 

719 BLCK OF RELATTON BETWEEN ARTERTAL BLOOD PRESSURE AND Penrhyn E. Bailey, James E. Sumners, George Cassady. Department of Pediatrics, Division of Perinatal Medicine, University of Alabama, Birmingham, Alabama.

Aortic blood pressure(BP) and blood volume(BV)were measured in 42 preterm infants. Fourteen infants were hypotensive(mean BP $30 \mathrm{mmHg}) ; 28$ were normotensive. The hypotensive babies were significantly lighter(1370vs1870 gm), had lower Apgar scores $(8 / 12$ vs $7 / 25 ; \leqslant 6)$ and were younger at the time of study $(13 / 14 \mathrm{vs} 17 / 28 ;<12$ hrs). There were no significant differences in gestation( 31 vs 32 wk), frequency of hyaline membrane disease (HMD, 6/14vs19/28) or arterial $\mathrm{pH}$ and blood gases at the time of study.

The BV, plasma volume( $P V=10$ minute T1824 dilution space), and hematocrit(HCT) in the hypotensive babies $(88.9 \pm \mathrm{sd} 15.2 \mathrm{ml} / \mathrm{kg} ; 48.7 \pm$ $7.9 \mathrm{ml} / \mathrm{kg} ; 45.5 \pm 6.5 \%$ respectively) were not significantly different from those in the normotensive babies $(90.7 \pm 20.8 \mathrm{ml} / \mathrm{kg} ; 48.9 \pm 7.9 \mathrm{ml} /$ $\mathrm{kg} ; 47.5 \pm 8.7 \%)$. There was no significant correlation of mean $B P$ with BV, PV or HCT. There was a significant correlation of mean BP with both birth weight and gestation.

Eight hypotensive infants were then given $10 \%$ salt poor albumin, $1.0 \mathrm{gm} / \mathrm{kg}$, over 10 minutes. Mean $\mathrm{BP}$ and arterial $\mathrm{pH}$ and blood gases were measured 30,45 and 60 minutes after volume expansion. There was a small but significant increase in mean BP from $26.8 \pm$ $2.0 \mathrm{mmHg}$ before expansion to $29 . \mathrm{l} \pm 1.1 \mathrm{mmHg}$ at 60 minutes, but 5 of 8 were still hypotensive. There was no significant change in arterial $\mathrm{pH}$ and $\mathrm{PCO}_{2}$ with volume expansion. In 7 infants with HMD there was a trend toward a decrease in the arterial/alveolar $\mathrm{PO}_{2}$ ratio with volume expansion implying increased venous admixture.

72 TRANSCUTANEOUS $\mathrm{PCO}_{2}(t \mathrm{cPCO})$ MEASUREMENT. Anthony $\mathrm{V}$.

720 Beran, Robert F. Huxtable, $\frac{\text { Donald R. Sperling (Spon. }}{\text { by Thos. L. Nelson). University of California, College }}$ of Medicine, Department of Pediatrics, Irvine, CA.

Both $\mathrm{O}_{2}$ and $\mathrm{CO}_{2}$ diffuse through intact skin. Huch et al reported good correlation between $\mathrm{PaO}_{2}$ and $t c \mathrm{PCO}_{2}$ using a surface $\mathrm{O}_{2} \mathrm{e}$ lectrode, and Johns et al measured tissue $\mathrm{PCO}_{2}$ through the skin window. This report describes the construction and evaluation of a sensor for the measurement of $\mathrm{tcPCO}_{2}$. As $\mathrm{CO}_{2}$ diffuses from the heterogeneous tissue cylinder across the skin and the sensor's membrane, the $\mathrm{pH}$ of the electrolyte changes. Change in $\mathrm{pH}$ is measured by an $\mathrm{Sb}-\mathrm{SbO}$ electrode against an $\mathrm{Ag}-\mathrm{AgCl}$ reference. Standard sensor potential at $42^{\circ} \mathrm{C}$ was $-525 \pm 6.6 \mathrm{mV}$. Following 20$40 \mathrm{~min}$. stabilization, the sensor showed $5.2 \pm 2.2 \mathrm{mV}$ drift over 16 hours. Voltage output was a linear function of $\log \mathrm{PCO}_{2}$ in the range of $10-80 \mathrm{~mm} \mathrm{Hg} \mathrm{PCO}$, with a slope of $66.9 \mathrm{mV}$ per $1 \mathrm{log}^{2}$ unit change. The response time $(95 \%)$ was $2.7 \pm .3 \mathrm{~min}$. The effect of temperature was $5.5 \mathrm{mV} /{ }^{\circ} \mathrm{C}$. In vivo tests were performed on 5 adult human volunteers. Following initial calibration at $4 \%$ and $10 \% \mathrm{CO}_{2}$, the sensor was applied to the surface of the forearm. Methyl ${ }^{2}$ salicylate ointment was placed between the membrane and the skin to produce hyperemia and maintain seal and thermal conductivity. A heating unit maintained temperature at $42^{\circ} \mathrm{C}, 20 \mathrm{~min}$, were allowed for temperature stabilization, at which point the sensor's output stabilized. The $\mathrm{tcPCO}_{2}$ in these normal volunteers was 40 $44 \mathrm{~mm} \mathrm{Hg}$, increasing to $54-56 \mathrm{~mm} \mathrm{Hg}$ when breathing $4 \% \mathrm{CO}_{2}$. This indicates: 1) the usefulness of the $\mathrm{Sb}-\mathrm{SbO}$ electrode for measurement of $\mathrm{PCO}_{2}$; and 2) the usefulness of this new non-invasive technique for continuous transcutaneous measurement of arterial $\mathrm{PCO}_{2}$.

NEONATAL NECROTIZING ENTEROCOLITIS (NEC): GEOGRAPHTC

721 AND TEMPORAL CLUSTERING OF CASES 1972-1975. Linda S. Book, John J. Herbst, James C. Overall and Rex Spendlove. U. of Utah Medical School, Department of Ped Salt Lake City and Utah State University, Logan, Utah.
Recurrent epidemic of NEC mainly in summer or fall have not Reported. At the Intermountain Newborn Intensive Care Center $37 / 71(52 \%)$ cases of NEC occurred in only 6 of the 45 months between January 1972 and September 1975. Fourteen of 71 cases $(20 \%)$ occurred in september.

We attempted to isolate a transmissible agent in an outbreak of NEC involving 11 infants between May 18, 1975 and July 20, 1975. Stool cultures revealed no bacterial pathogens or predominant organisms and viral cultures were negative. A prevalence study on June 6,1975 showed no qualitative difference in fecal flora among 8 infants with NEC and 12 asymptomatic infants. Stool samples from $10 / 11$ wi.th NEC were examined by electron microscopy with complement fixation for orbivirus with no viral particles identified. The onset of the epidemic of NEC was associated with gastrointestinal symptoms among the nursery personne1. NEC developed only in infants cared for in 2 of the 4 nursery rooms. For each of the 4 rooms the number of cases/patient weeks exposure (weekly incidence) was $6 / 54(9.3 \%), 0 / 54$ $(0 \%), 6 / 36(16.6 \%), 0 / 54(0 \%)$. Although we were unable to identify an infectious etiology for NEC, the temporal clustering of cases, the geographic clustering of cases to 2 of the 4 nursery rooms, and the occurrence of the disease when nursery personne 1 had gastrointestinal symptoms, suggest that a transmissible agent is involved and isolation procedures are advisable.
722 VIDEO TAPE RECORDING (VTR) IN THE INTENSIVE CARE NURSERY (ICN) AS AN UNPARALLELED CLINICAL AND RESEARCH DEVICE, Gary G. Carpenter, Herbert C. Mansmann, II, Jef ferson Med. Coll., Pediat. Dept., Phila.

Use of a VTR CR-6300 separated from the ICN by a window offers increasing benefit, with function and experience. Recording respiratory movements in premature and SGA infants during oxygen \% changes by hood, CPAP and PEEP plus signs of withdrawal in infants of heroin, methadone and Darvon addicted mothers were the first of several previously unsuspected capacities. Infants of $1500 \mathrm{gm}$. and less who lived up to 24 hours as week-end itinerants had clinical signs assessed. Playback ability enhanced discussion of them when the assembled staff later reviewed their findings at postmortem pathology, genetic counselling, and teaching conferences about infants with sacral agenesis, multisystic kidneys immaturity, achondroplasia, Cornelia de lange syndrome, and hemifacial microstomia. Infant's small movements of eyes, digits and very brief respiratory changes superimposed with zoom on EEG recording, allowed a single view of both. The benefits of complete utilization of audio-visual equipment in the ICN may not yet be completely appreciated. 723 RELATIONSHIP OF SERUM TOTAL BILIRUBIN BINDING CAPAWilliam J. Cashore, Arthur Horwich, and William Oh. Brown University Program in Medicine, Women and Infants Hospital of Rhode Island, Department of Pediatrics, Providence, R.I.

The influence of maturity and of clinical status on the bilirubin binding capacity may be an important consideration in evaluating the risk of kernicterus in jaundiced newborns. Total bilirubin binding capacity (TBBC) was determined at 1-5 days of age by Sephadex G-25 gel filtration in 43 well newborn infants and in 45 with significant illness (acidosis, hypoxia, hypoglycemia, hypothermia, neurological complications, and sepsis). Gestational ages were between 26 and 41 weeks. TBBC was directly related to gestational age $(p<.01)$ and was significantly lower in sick than in well infants, throughout the range of gestation studied $(p<.05)$. Serum albumin concentration and the molar binding ratio of bilirubin to albumin $(B / A)$ were also lower in sick infants $(p<.05) ; B / A$ was directly related to gestation in well infants $(p<.01)$, but not in sick infants. Serial determinations of TBBC showed no change in TBBC from birth to 10 days in patients who were clinically well. In 16 ill patients, clinical improvement was associated with a significant rise in TBBC $(p<.01)$ and in $B / A(p<.025)$ during the first 10 days. The data indicate that TBBC and $B / A$ increase with maturation and that clinical illness results in significant reduction in $T B B C$ and $B / A$ in preterm as well as in term infants.

70 LONG-TERM UMBILICAL ARTERY CATHETERIZATION. Morton L.

724. Cohen, Alan E. Shumacher and Larry S. Johnsgard (Spon by Stanley A. Mendoza), Division of Neonatology, Children's Hospital, San Diego, California.

Umbilical artery catheters (UAC's) were maintained for more than 3 weeks (mean $46 \mathrm{~d}$., maximum 134 d.) in 48 neonates (mean birth weight 1285 gms) with chronic respiratory disease requiring long-term arterial blood gas monitoring. Most ( $92 \%$ ) required prolonged artificial ventilation, averaging 42 days on positive longed artificiai ventilation, averaging 42 days on positive
pressure. 38 infants ( $79 \%)$ survived, including 16 of the 18 under
1000 gms. Though there were no UAC-related deaths, 12 complica1000 gms. Though there were no UAC-related deaths, 12 complications occurred in 10 patients. Three non-survivors with end-stage
bronchopulmonary dysplasia developed renal or mesenteric artery
thrombi. In one survivor, partial renal artery occlusion caused thrombi. In one survivor, partial renal artery occlusion caused hypertension which resolved spontaneously; blood pressure and re-
nal function have since remained normal. In the last 36 long-term cases, since UAC's have been kept at L3-4, there have been no renal or mesenteric thromboses. Five survivors developed unilateral iliac artery occlusion without clinical circulatory insufficiency; in all cases femoral pulses returned and followup has shown no abnormality in growth or function of the affected extremity. The only UAC-related infection was a subclinical case of Candida fungemia (in an infant receiving parenteral nutrition via UAC) which resolved after removal of the UAC. In most patients prophylactic resolved after removal of the duc. In most patients prophylactic nificant blood loss from the UAC, occurring in 2 early cases, was nificant blood loss from the

Despite the risk of thromboses, the high survival rate and absence of UAC-related sequellae among survivors suggests that long-term UAC's may be useful in neonates who require prolonged blood gas monitoring. 
APNE'A IN NORMAI BABIES, P, J. Collipp, K. Sabarin-

725 athan, S. R. Ravi, Nassau County Medical Center, East Meadow, New York 11554.

Apnea (cessation of breathing recorded as 0 on an Air Shield's Apnea Monitor) duration and frequency was recorded for 102 normal fill-term infants during their first 72 hours of life. Data summary indicates the average baby had 90 episodes of 10 second apnea on the first day and 30 on the third day. Babies had 11 episodes lasting 20 seconds, 2 lasting 40 seconds, and 0.30 lasting 80 seconds ( 29 in 17 babies). While episodes lasting 10 seconds became less frequent during the 72 hours, the 20 second episodes were as common on the third day as the first. Ifore apnea (20 second) occurred between midnight and 6:00 AM than other time periods. Larger babies had slightly more apnea than smaller babies. Nurses' observations of the duration of apnea correlated well with data from 35 infants using an automatic striprecorder. 250 of these infants have been followed up for more than one year.

\section{8}

THE VARIABILITY OF PO IN NEWBORN INFANTS IN RESPONSE TO ROUTINE CARE. B.C. Dangman, $T$. Hegyi, M. Hiaft, L。 Indyk, and L。S. James, Coll. of

Phys。\& Surg。, Columbia Univ。, Dept of Pediatrics, New York.

Unexpected and clinically important variations in arterial oxygen tension $\left(\mathrm{PO}_{2}\right)$ have been noted in infants at rest and during routine clinical or diagnostic maneuvers not normally thought to have a significant effect on the infant's oxygenation. Using the Huch transcutaneous sensor to measure $\mathrm{PO}_{2}$ in sick newborns, we have monitored continuously for periods of several hours the $\mathrm{PO}_{2}$, heart rate, respiratory rate and wave form, and the peripheral perfusion. Our analysis of each event includes the time delay from start of the procedure to some effect seen in the cardiac, respiratory and oxygen signal, the maximum change produced in each parameter, the time course of these changes and the recovery time. The $\mathrm{PO}_{2}$ of an infant at rest with stable heart rate and respiratory pattern can vary by $+15 \mathrm{mmHg}$. During crying the $\mathrm{PO}_{2}$ usually decreases, often by as much as $50 \overline{\mathrm{mmHg}}$. Simple manipulations such as taking vital signs and physical examination produce falls of up to $30 \mathrm{mmHg}$ while endotracheal suctioning can produce a decrease in $\mathrm{PO}_{2}$ of up to $40 \mathrm{mmHg}$, with recovery time as long as $7 \mathrm{~min}$. The accompanying bradycardia is much more transient than the hypoxemia. Thus isolated blood gas samples do not give $\mathrm{PO}_{2}$ values which are representative of the infant's oxygenation. These observations have significant implications relating to present-day practices in the management of sick newborn infants.
726 THE RELIABILITY OF THE FOAM STABILITY TEST ON GASTRIC ASPIRATE STUDIED SEQUENTIALLY AFTER BIRTH. R.M. Cowett, B.D. Chandlex, A.C. Yao, P.E. Doyle, and W. Oh. Depts. of Pediatrics, Brown University Program In Medicine Women and Infants Hospital of R.I., Providence, and the Downstate Medical Center, SunY, New York.

The gastric aspirate foam stability test (GA FST) performed within $30 \mathrm{~min}$. after birth is a reliable method for the assessment of fetal lung maturity. (NEJM 293:413, 1975). The high gastric aspirate $\mathrm{pH}$ (mean $=6.47$ ) suggests that swallowed amniotic fluid is the predominant component of GA at birth. The subsequent fall in $\mathrm{GA}$ pH may reflect increasing concentration of gastric secretion which may influence the reliability of the FST. The GA FST and $\mathrm{pH}$ of 33 normal term infants were determined at half hourly intervals from birth through five hours. Twenty infants showed positive GA FST throughout in spite of decreasing $\mathrm{GA} \mathrm{pH}$; and in 8 the FST became intermediate or negative unassociated with a change in GA pH. In 5 the FST became intermediate or negative with decrease in $\mathrm{GA}$ pH. Similar studies were performed in 15 infants < 37 weeks of gestation from birth to 5 days. Eleven (4 with mild RDS) had positive GA FST throughout in spite of falling GA $\mathrm{pH}$. Of the remaining 4 , 1 had intermediate GA FST at bixth that turned positive without a fall in $\mathrm{GA}$ PH and in 3 who had RDS the negative GA FST became positive unrelated to GA $\mathrm{pH}$ changes as the clinical condition improved. The data suggests: 1) the GA FST is a reliable test for lung maturity in $65 \%$ of cases during first 5 hours of life, and 2) in those with RDS, the increasing positivity of GA FST reflects the improvement in the clinical course.

\section{7}

RESPIRATORY DISTRESS FOLLOWING ELECTIVE CESAREAN SECIION-A PREVENTABLE MORBIDITY. R.M. Cowett and W. Oh. Brown University Program in Medicine, Women and Infants Hosp. of R.I., Department of Pediatrics, Providence, R.I. Respiratory Distress (RD) may occur in infants delivered by elective cesarean section (ECS) as a result of either lung immaturity or CS or both. It may be argued that amniocentesis for assessment of fetal lung maturity should preceed ECS, but data for such recommendation is lacking. In 121 ECS transuterine needle aspiration for amniotic fluid (AF) was attempted (successfully in 75) prior to hysterotomy. In 19 unsuccessful cases, gastric aspirates (GA) were obtained from infants within 30 minutes of birth. The $A F$ and $G A$ were analysed by the foam stability test (FST) as an indicator of lung maturity. The timing and indications of ECS were independently determined by attending obstetricians who were unaware of the aims of the study. Objective scoring for transient respiratory distress (TRD) and respiratory distress syndrome (RDS) were done by nursery staff unaware of FST results. The mean birth weight was 3280 gms: mean gestation 38.6 wks. Of the 73 infants with a positive FST, 2 had TRD. In 13 infants with an intermediate FST 1 had TRD and 1 RDS. In 8 infants with a negative FST 4 de

\begin{tabular}{|l|r|r|r|}
\hline \multicolumn{4}{|c|}{ AF OF GA } \\
\hline GROUP & POS & INT & NEG \\
\hline TRD OF RDS & 2 & 2 & 6 \\
\hline NORMAL & 71 & 11 & 2 \\
\hline
\end{tabular}
veloped TRD and 2 had RDS. The incidence of respiratory difficulty due to inadequate lung maturity is $9 \%(8 / 94)$ which could be reduced to $3 \%(2 / 73)$ if there were appropriate delay for ECS when intermediate or negative FST was observed. The data suggest that assessment of fetal lung maturity seems indicated to minimize respiratory difficulty in infants delivered by ECS.
72 PHYSIOLOGICAL CHANGES INDUCED BY THEOPHYLLINE IN THE

729 TREATMENT OF APNEA IN PRETERM INFANTS. Maria Davi. Kor Sankaran, Keith Simons, Estelle Simons, Mary M. Seshia, Henrique Rigatto, Univ of Manitoba, Dept. of Ped., Winnipeg. Five preterm infants (b.w.0.9-1.3 kg) with periodic breathing or apnea $(>10 \mathrm{sec})$ were studied on 14 occasions, before and 48 hrs after the administration of theophylline $(3 \mathrm{mg} / \mathrm{kg}, q 6 \mathrm{~h})$. We determined the incidence of apnea,minute ventilation $\left(\dot{V}_{\mathrm{E}}\right)$, alveolar $\mathrm{PO}_{2}$ and $\mathrm{PCO}_{2}$, arterial $\mathrm{pH}, \mathrm{PCO}_{2}$ and $\mathrm{PO}_{2}$, respiratory responses to $\mathrm{CO}_{2}$ and specific dynamic compliance (Csp). We measured ventilation with a nosepiece and screen flowmeter, using a constant flowthrough to eliminate dead space and valves. Analyses were made from 3-5 min after the baby began breathing room aix or $\mathrm{O}_{2}$ enriched mixtures $\left(21 \%\right.$ or $\left.40 \% 0_{2}\right)$, alone or with $2 \% \mathrm{CO}_{2} 2$. Results: $\begin{array}{llllllll}\text { Apnea } & \dot{\mathrm{V}}_{\mathrm{E}} & \mathrm{PaCO}_{2} & \mathrm{P}_{\mathrm{A}} \mathrm{CO}_{2} & \mathrm{P}_{\mathrm{A}} \mathrm{O}_{2} & \mathrm{PaO}_{2} & \mathrm{CO}_{2} & \text { responset }\end{array}$ $\mathrm{No} / \mathrm{hr} \mathrm{L} / \mathrm{min} / \mathrm{kg} \mathrm{mm} \mathrm{Hg} \mathrm{mm} \mathrm{Hg} \mathrm{mm} \mathrm{Hg} \mathrm{mm} \mathrm{Hg} \mathrm{slope} \mathrm{posi-} \mathrm{cm} \mathrm{H} \mathrm{O}^{-1}$ \begin{tabular}{llllllllll}
\hline B* & 60 & $.249 \pm .024$ & $49 \pm 2$ & $49 \pm 2$ & $138 \pm 16$ & $71 \pm 12$ & $.015 \pm .006$ & $53 \pm 6$ & $.11 \pm .02$
\end{tabular}

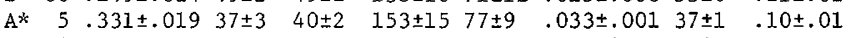
$\mathrm{P}<.05<.005<.01<.005<.01 \quad$ N.S. $<.05<.05 \quad$ N.S. All values: mean \pm S.E.; $B^{*}=$ before; $A^{*}=$ after theophylline † Response curve in $\mathrm{L} / \mathrm{min} / \mathrm{kg} / \mathrm{mm} \mathrm{Hg} ; \pi \mathrm{PACO}_{2}$ at $.300 \mathrm{~L} / \mathrm{min} / \mathrm{kg}$. These findings suggest: a) theophylline does decrease the incidence of apnea; b) this decrease is associated with an increase in alveolar ventilation, an increased sensitivity to $\mathrm{CO}_{2}$, and a pronounced shift of the $\mathrm{CO}_{2}$ response curve to the 1 eft; and c) lung mechanics are not affected by the use of this drug. The data are consistent with the idea that apnea is a reflection of a depressed respiratory control system.

THE EFFECT OF PREVIOUSLY ADMINISTERED

730 ANTIBIOTICS ON THE TIME OF ONSET AND CLINICA L COURSE OF NECROTIZING ENTEROCOLITIS (NEC). Robert A. de Lemos, Donald M. Null, Jr. , \& Lawrence C. Franklin (Spon. by M.J.Sweeney), Wilford Hall USAF Medical Center, Dept of Pediatrics, San Antonio, Tx.

To document the relationship between prior antibiotic administration and the development of $\mathrm{NEC}$, we analyzed records of all infants under 2000 grams admitted since a standard approach to NEC was established (Amer J Surg 126:758, 1973). Two groups of infants were identified;those receiving antibiotics within 48 bours of birth and those given none unless signs of NEC appeared. The no antibiotic infants had higher apgar scores and a lower incidence of IRDS and vascular catheterization. The incidence of NEC was significantly lower (table) in those babies who had NEC was significantly lower (tab) Age at Dx Surg Deaths

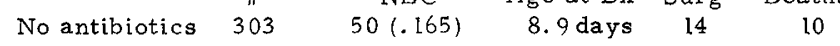
Antibiotics $\frac{634}{937} \quad \frac{51(.080)}{101(.108)} \quad \frac{19.9 \text { days }}{13.5} \quad \frac{3}{17} \quad \frac{1}{11}$ received antibiotics previously. When NEC occurred in these infants it appeared later, had fewer surgical complications and lower mortality. When NEC developed after 2 ! days pneumatos is of the caecum and small intestine was uncommon, occurring in only $9.5 \%$ of these babies as compared to $80 \%$ of those presenting in the first two weeks. Prior use of antibiotics in prematures prevents or lessens the severity of NEC. 


\section{1}

ALBUMIN DISAPPEARANCE IN NEWBORNS DURING EXCHANGE TRANSFUSIONS WITH ALBUMIN-ENRICHED ACD BLOOD. B. Doray ${ }^{+}, G$. Manlan ${ }^{+}$and $\mathrm{H}_{\text {. B Bard }}$ Un. of Montreal and Hôpital Ste-Justine, Montreal.

Blood for exchange transfusions (ET) of newborns is often enriched with human serum albumin for increased removal of bilirubin, the fate of albumin has not been investigated in these infants. In $28 \mathrm{ET}, \mathrm{ACD}$ blood was enriched by adding $50 \mathrm{cc}(12.5 \mathrm{gm})$ of salt-free serum albumin, after removal of $50 \mathrm{cc}$ of plasma, increasing the serum albumin level of the donor's blood to $7.150 \mathrm{gm} \%( \pm 0.882)$. The receiver's albumin was $3.735 \mathrm{gm} \%$ ( 1 0.414) at the beginning of the ET and only increased to $4.820 \mathrm{gm} \%( \pm 0.517)$ at the end of the procedure. This was $67.4 \%$ of the donor's level. Simultaneously, the donor's serum gammanglobulin level was $0.860 \mathrm{gm} \%$ ( \pm 0.272 ) while the receiver's was $0.722 \mathrm{gm} \%\left( \pm_{0.168)}\right)$ before and $0.573 \mathrm{gm} \%( \pm 0.118)$ at the end of the exchange. The final level of globulin being $66.6 \%$ of the donor's level. Since the changes in serum albumin and gamma-globulin were equal, it suggested either a rapid and marked increase in blood volume or a transcapillary escape of albumin and globulin, or both. The average disappearance rate of $33 \%$ found in this study was larger than the $13-20 \%$ reported for normal newborns. These results are in favor of both volume increase and a transcapillary escape of added albumin.

PERSISTENCE OF THE FETAL CIRCULATION (PFC): DEVELOP-

732 MENT OF AN ANIMAL, MODEL. Willa $H$. Drummond and John $M$ Bissonnette. Univ. Oregon Health Sciences Cente

Perinatal Medicine, Portland (Spon. by Robert C. Neerhout)

Clinical studies have shown a coorelation between PFC and pregnancy complications which may have produced intrauterine asphyxia. Chronic hypoxia was successfully induced in $31 \mathrm{amb}$ fetuses from

115 days gestation by micro-embolization of the maternal placenta (Creasy, R. et al. Am.J.Obs.Gyn. 112:566, 1972). Catheterization studies on the hypoxic fetuses delivered at term by Cesarean section showed an increase in pulmonary artery pressure (PAP) compared to control lambs. In two of the chronic hypoxic lambs PAP exceeded systemic arterial pressure (SAP)

\begin{tabular}{|c|c|c|c|c|c|c|c|}
\hline & tal $\mathrm{F}$ & to & eta & pH & m & Newborn & AP Newborm \\
\hline & high & on & high & low & $\mathrm{mm} \mathrm{Hg}$ & $\operatorname{men} \mathrm{Hg}$ & $\mathrm{pH}$ \\
\hline E1 & 24 & 16.8 & 7.38 & 7.33 & $64.5 \pm 6.6^{*}$ & $88.5 \pm 5$ & $50.0 \pm 4$. \\
\hline E2 & 27 & 18.2 & 7.41 & 7.29 & $83.0 \pm 9.9$ & $77.8 \div 2.8$ & $57.8 \pm 1$. \\
\hline E3 & 25.1 & 17.8 & 4.39 & 7.21 & $63.7 \pm 5.4$ & $61.7 \pm 3.7$ & $44.8 \div 3.27 .27 \div .01$ \\
\hline $\mathrm{C} 1$ & 27.6 & $\overline{24.4}$ & 7.4 & 7.38 & $44.1 \pm 12.4$ & $98.8 \pm 9.2$ & $55.2 \pm 5.97 .30 \pm .0$ \\
\hline $\mathrm{C} 2$ & - & - & - & - & $49.4 \pm 5.6$ & $116.3+10.6$ & $59.6 \pm 2.67 .33 \pm .02$ \\
\hline $\mathrm{C} 3$ & - & - & - & - & $20.3 \pm 2.2$ & $61.2 \pm 5.8$ & $67.8 \pm 5.97 .24 \pm .40$ \\
\hline
\end{tabular}

These data suggest that intrauterine hypoxic stress is important in the development of pathologic neonatal pulmonary hypertension. The successful production of a large animal model for PFC holds promise for detailed investigation of the pathophysiology and therapeutics of this important clinical entity.

Supported by the Oregon Heart Association.

A PROSPECTIVE CONTROLLED TRIAL OF ORAL KANAMYCIN PRO-

733 PHYLAXIS FOR NEONATAL NECROTIZING ENTEROCOLITIS. Edmund A. Egan, Gonzalo A. Mantilla, Robert M. Nelson, and Donald V. Eitzman, University of Florida College of Medicine, Department of Pediatrics, Gainesville.

In July, 1974, a prospective controlled study was initiated to determine if a regime of oral kanamycin, $15 \mathrm{mg} / \mathrm{kg} / \mathrm{day}$ in a t.i.d. dosage prevented neonatal necrotizing enterocolitis. The study was limited to infants under $1500 \mathrm{grams}$ birthweight who survived to feedings. Placement in treatment or control group depended only on whether the last digit of the hospital number was even or odd. Blood levels of kanamycin were determined in the first 12 treatment infants and were less than. $0.5 \mathrm{mg} / \mathrm{ml}$ the level of detectability of the test. Diagnosis of necrotizing enterocolitis required radiologic confirmation of pneumatosis or pneumoperitoneum. In the 13 months of the study, there were 5 cases in 40 control infants and 0 cases in 35 treatment infants $(p=0.038$, Fisher's Exact Test). Further analysis of 18 separate factors, possibly associated with enterocolitis, showed no difference between treatment and control groups. The infection rate was the same in both groups, although kanamycin resistance reappeared in coliform organism isolated from nursery patients during the study. The study demonstrated effectiveness of prophylactic oral kanamycin in preventing necrotizing enterocolitis in small premature infants.
EXPERIENCE IN A RURAL LEVEL II PERINATAL CENTER.

734 Robert N. Enberg, Robert H. Cox, S. Michaeleen Frahm, Hays, Ks. Sponsored by Herbert Wenner, Kansas City,Mo.

A Level II Perinatal Center was developed in Hays, Kansas to serve the 60,000 people in sparcely populated Northwestern Kansas. The center provides services in 4 areas: (1) obstetric-including capacity for evaluation of fetal status before and during labor, (2) neonata1-including a NICU staffed with trained nurses on all shifts, (3) transport - providing infants with supportive care while in transit by trained NICU nurses and (4) education-providing a series of weekly training sessions for physicians and nurses in 14 out1ying hospitals.

One hundred twelve patients have been admitted to the NICU from its beginning in January, 1974 through December 1975. Sixty-six infants were born in outlying hospitals, and 49 of these were transferred to Hays NICU with the center's transport system. Fortysix were inborn. Among the 112 infants, 73 were preterm, 66 had respiratory distress, 60 had umbilical artery catheterization, 11 received CPAP, 9underwent exchange transfusion. Twenty-three were transported to leve1 III centers. There were 21 deaths -6 locally, 3 in transport and 12 after reaching a tertiary center.

The Hays Perinatal Center is staffed solely by physicians in private practice and without a grants-in-aid or outside sources of funding. Ongoing consultation is obtained from one of three tertiary centers nearly equadistant from Hays.

This experience indicates that Level II Perinatal Centers can be developed and supported from services developed within an isolated community augmented by tertiary centers.

TRAUTERINE GROWTH RETARDATION (IUGR): THE SPONTANE-

735 OUS CONTRACTION STRESS TEST IN TIMING THE DELIVERY. A. Fanaroff, J.Goldfarb, M. Gyves, and 1. Merkatz. CWRU School of Medicine Depts of Ped \& Rep. Biol. Cleveland, Ohio.

Timing of delivery remains the most problematic aspect of antepartum management for severe IUGR. There has been no way to distinguish those babies for whom immediate delivery is indicated from those for whom continued suboptimal growth and further maturation may be beneficial. We report the significance of late fetal heart rate(FHR) decelerations associated with spontaneous uterine contractions in patients with suspected IUGR. Over an 8 month period, 12 patients with suspected IUGR prior to term were supervised with frequent monitoring by external cardiotocography. 62 non-stressed recordings of at least one hour's duration were obtained. Periodic contractions were noted on 43 occasions $(70 \%)$. All 12 patients evidenced spontaneous contractions on 1 or more recordings and 4 patients demonstrated FHR decelerations in response to the contractions. Delivery of these 4 infants was expedited by cesarean section at 29-37 weeks. They were all SGA(800$1420 \mathrm{gms})$. None developed RDS. 2 infants survived and are norma to date. One died of necrotizing enterocolitis and 1 has porencephaly. Both complications may be related to chronic intrauterine hypoxia reinforcing the significance of the abnormal FHR finding. Negative monitoring observations on the 8 remaining infants were utilized to extend expectant management until pulmonary maturity, labor or preeclampsia ensued. The spontaneous contraction stress test is a non-invasive physiologic test of placental reserve which provides important data in the pre-term management of these high risk pregnancies.

SCALP ABSCESS: A COMPLICATION OF FETAL MONITORING.

736 Henry M. Feder, Jr., William C. MacLean, Jx., E.

(Spon. by David H. Carver)

(FM) with the spiral electrode is widely accepted. Scalp abscess has been reported to be a rare $(0.33 \%$ ) and mostly non serious complication, usually requiring only local treatment. A review of the outcome of infants born after FM at Johns Hopkins (est. 508 of 2061 live births) during the past year suggests that scalp abscess related to FM may be more common and serious than previously recognized.

Nine cases of scalp abscesses were found. All were diagnosed within 4 days of birth. Abscesses ranged from 0.5 to $8 \mathrm{~cm}$. Organisms were recovered from the abscess in 6 of 9 cases. In 5, a single organism was isolated - 3 with $H$. influenzae $b$ (HIB), I Group A strep and 1 microaerophilic strep. The sixth case grew 4 different organisms. In 2 cases identical organisms were cultured from blood and abscess. In the first, HIB was isolated. In the second, Group A strep was grown from abscess, blood, and maternal cervix. This case was further complicated by a skin to bone slough over the abscess site requiring a graft.

The 0.878 incidence of scalp abscess in this patient population was higher than previously reported. Local and systemic complications of these infections emphasize the potential hazard of FM. 


\section{7}

NON-SIGNIFICANCE OF POSTIJATAL ASPHYXIA IN THE DEVELOPMENT OF INTRAVENTRICULAR HEMKRRHACE (IVH). JOSEph A Garcia-Prats, John D. Kenny, Anthony J.S. Corbet, James M. Adams, Arnold J. Rudolph. Der

Baylor College of Medicine, Houston.

To test the hypothesis that postnatal asphyxia causes IVH, 35 high risk premature infants (Apgar score 3 or less, GA 32 weeks or less, HMD or IPPV) were followed after the first hour of life with measurements of blood lactate, pyruvate and arterial blood gases every 8 hours for 3 days. The ratio of lactate to pyruvate (L/P) was calculated. IVH was diagnosed in 8 subjects by sudden clinical deterioration, hemorrhagic CSF and typical neurologic signs. Al1 8 died and autopsies were obtained on 6 which confirmed the diagnosis of IVII. Subjects with IVH were less mature (IVH-29.8 weeks + 0.6 SE, no IVH-32.5 weeks $+0.5 \mathrm{SE}, \mathrm{P}<0.01$ ) and had a higher incidence of HND ( IVH-8/8, no IVH-11/27, P<0.025), however prior to IVH no difference in any acid-base parameter was seen: no IVH $61.1 \mathrm{nM}+2.8 \quad 48$ torr+4 48 torr+3 $4.1 \mathrm{mM}+0.5 \quad 89+15$ IVH $\quad 72.3 \mathrm{nM}+7.2 \quad 47$ torr $+4 \quad 57$ torrt6 $5.4 \mathrm{mM}+1.1 \quad 185 \mp 88$ (Values are mean $\pm \mathrm{SE}$, all $\mathrm{p}>0.10 \overline{)}$

The large difference seen in $L / P$ between the two groups may be explained by the significant negative correlation found between $\mathrm{L} / \mathrm{P}$ and $\mathrm{GA}$ in the no IVH group ( $\mathrm{r}=-0.4, \mathrm{P}<0.05)$. After IVH and subsequent clinical deterioration the maximum $\mathrm{L} / \mathrm{P}$ and blood lactate were significantly higher than in the no IVH group (P<0.025) We conclude that prematurity is the most important factor in the etiology of IVH and postnatal asphyxia could not be implicated by this study.

SURVEY OF HUMAN MILK SAMPLES FOR INHIBITOR OF BILI-

738

Winkler and Ronald L. Polano, Wayne State Univ., Children's Hospital, Hutzel Hospital, Depts. of Peds. and Nurs., Detroit, Michigan

We studied $\varepsilon 1$ consecutive human milk samples obtained on the 2nd to 5th postpartum day from healthy lactating volunteers using the assay system of Strebel and Odell (Pediatr. Res. 5:548, 1971). with rat liver microsomes as the enzyme source and bilirubin and UDP-glucuronic acid as substrates. Only one of 81 samples had an inhibitor (0s of control activity) and no jaundice was detected in this mother's baby or the older sib (also breast-fed).

In contrast, when 11 other milk samples obtained from mothers of infants with unexplained prolonged unconjugated hyperbilirubinemia were similarly tested, $5 / 11$ had an innibitor.

In series of standard enzyme assays where increasing amounts of inhibitory milk were added, enzyme activity decreased asmilk concentration was increased up to $128(\mathrm{v} / \mathrm{v})$. Unknown milk samples were added to the enzyme assay to a final concentration of $13.5 \%$. "Normal" milks gave 47-135\% of control enzyme activity. Inhibitory milks gave 0-11\% of control activity. None of the samples fell between 12 and $46 \%$ of control.

In an unselected population of lactating women, the incidence of an in vitro inhibitor of bilirubin UDP-glucuronyl transferase is low. Enzyme inhibitor was more frequently detected when there was a prior history of prolonged "idiopathic" neonatal jaundice. In one instance, an inhibitor was detected in maternal milk unassociated with prolonged neonatal jaundice.

NEONATAL HYPOXEMIA:TOLAZOLINE RESPONSIVENESS. 739 B. Goetzman, P. Sunshine, J. Johnson, R. Wennberg, Pediatrics, University of California, Davis-Sacramento Medical Center and Stanford University Hospital.

Forty-six hypoxemic neonates were treated with tolazoline, a pulmonary vasodilator. Infants were selected to receive the drug when 1) hypoxemia(mean $\mathrm{Pa}_{2}-34$ torr) was refractory to mechanical ventilation or 2) severe pulmonary hypertension was documented by cardiac catheterization. Tolazoline was infused via scalp vein $1-2 \mathrm{mg} / \mathrm{kg}$ over 10 minutes followed by $1-2 \mathrm{mg} / \mathrm{kg} / \mathrm{hr}$. Ten infants without apparent lung disease were considered to have primary pulmonary hypertension of the newborn and $8(80 \%)$ responded with a mean increase in $\mathrm{PaO}_{2}$ of 116 torr within 1 hour of beginning tolazoline infusions. One responding infant and the two non-resp onders died. Thirty-six infants(including 26 prematures) with a variety of pulmonary disorders including hyaline membrane disease (21 infants), meconium aspiration(6) and intrauterine pneumonia(3) received tolazoline. Twenty-one (58\%) of the 36 responded with a mean increase in $\mathrm{Pa}_{2}$ of 130 torr within 1 hour of beginning tolazoline infusions and $13(62 \%)$ of these survived. Fifteen patients did not respond to tolazoline and only $3(20 \%)$ survived. Responders could not be distinguished from non-responders by $\mathrm{clin}$ ical or laboratory features prior to tolazoline administration. Complications possibly related to tolazoline occurred in 14 patients. These data suggest that pulmonary vasospasm is etiolog ically important in a significant number of hypoxemic neonates both with and without pulmonary parenchymal disease. Tolazoline may be a useful therapeutic agent in this selected group.
LIGHT INDUCED VITAMIN DEFICIENCY IN THE NEONATE 740 Donald S. Gromisch, Rafael Lopez, Harold S. Cole and Jack

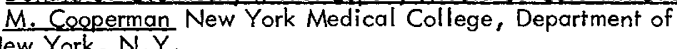
Pediatrics, New York, N.Y.

Phototherapy is widely used to reduce serum bilirubin levels in the moderately hyperbilirubinemic neonate. The light most effective for these purposes has an emission spectrum which encompasses the maximum absorption peak of riboflavin. From in vitro studies it has been postulated that photodynamically activated riboflavin generates singlet oxygen which decomposes bilirubin. During this process the vitamin is also decomposed. It was shown that such light can penetrate the skin layers, including vascular layers, permitting photochemical reactions to occur. This investigation was designed to determine whether riboflavin deficiency occurs in infants undergoing phototherapy. Twenty-one hyperbilirubinemic neonates were tested before and after exposure to light. Five infants with comparable bilirubin levels not requring phototherapy were the controls. Riboflavin deficiency was determined from the estimation of erythrocyte glutathione reductase activity, using a specific and sensitive method previously shown to reflect the riboflavin nutritional status of the neonate. Sixteen of the 21 neonates in the study group became vitamin deficient after light therapy. None of the controls developed deficiency during a comparable period of time. The deficiency was not correlated with race, sex, birth weight, dietary regimen nor serum bilirubin level. The vitamin deficiency was cor related with duration of time that the infants were exposed to light. All of those exposed for more than 48 hours became deficient; half of those for lesser periods of time became deficient. The vitamin deficiency may affect the wellbeing of the neonate and may contribute to the previously reported transient failure to thrive in infants undergoing phototherapy.

74. FANT-INCIDENCE AND RISK. n.n. Hakanson and $\mathrm{w}$. Oh. Brown University Program in Medicine, Women and $\overline{I n}-$ fants Hospital of R.I., Department of Pediatxics, Pxovidence, R.I. To determine the incidence and clinical morbidities of hyperviscosity syndrome in small-for-gestational age (SGA) infants, a one-year prospective study was conducted at a maternity hospital in 1975. Of the 4974 livebirths, 79 (1.6\%) were SGA (birth weight <loth percentile and signs of malnutrition). Whole blood viscosity measurement on these SGA infants revealed a $17.78(14 / 79)$ incidence of hyperviscosity (viscosity $\geq 2$ S.D. above the norm.) No significant differences were observed between the hyperviscous and normoviscous groups with respect to toxemia, placental separations, fetal anomalies, sepsis, hypoglycemia, and hypocalcemia. Morbidity as scored by symptoms in respiratory, cardiovascular, neurological, and gastrointestinal systems were significantly greater in the hyperviscous than in the normoviscous groups $150 \%$ vs. $8.3 \%$ with $>2$ of 4 morbidity score, $\mathrm{p}<0.005)$. Polycythemia (central venous hematocrit $((\mathrm{CVH}))>64 \%$ ) was more common in the hyperviscous group, and with increasing $\mathrm{CVH}$, blood viscosity increased. The CVH at which symptomatic hyperviscosity became manifest was 648 ; and no correlation could be shown for morbidity score and (hyper) viscosity value. Significantly, 4 of 14 hyperviscous infants (28.6\%) developed necrotizing enterocolitis (NEC) within three days of birth, while only 1 of 65 normoviscous infants (1.5\%) developed the condition ( $x^{2}$ analysis, $\left.p<0.005\right)$. These data indicate that in SGA infants hyperviscosity frequently occurs at $\mathrm{CVH} \geq 64 \%$ and carries significant risk of neonatal moxbidity, including NEC.

NECROTIZING ENTEROCOLITIS AND THE PATENT DUCTUS

742 ARTERIOSUS. Richard E. Heath, Frank W. Bowen, (Spon. by Gordon B. Avery), William Beaumont Army Medical Center, Dept. of Ped., Neonatal Svc., El Paso, Texas

The patent ductus arteriosus (PDA) can allow $L \rightarrow R$ shunting of blood which may cause bowel ischemia. Ischemia is a proposed factor in the pathogenesis of necrotizing enterocolitis (NEC). To evaluate the relationship of the PDA to NEC, serial echocardiograms were performed on 21 premature infants. A ratio of the left atrial to aortic root size (La/Ao) was determined for each tracing. The $L a / A_{0}$ determination and its reflection of ductal shunt have been described by Silverman. Based on La/Ao determinations, 2 groups of infants were identified. Group 1 had serial La/Ao ratios that were either persistently elevated ( $>1.0$ for 7 days or until PDA ligation) or were normal initially $(<1.0)$ but became elevated prior to the development of NEC. Group 2 had La/Ao ratios that were either consistantly normal or were elevated initially but showed a consistant downward trend. Each group was divided into a Subgroup A (had NEC) and a Subgroup B (no NEC). NEC was diagnosed on the basis of abdominal distention, blood in the stools, and pneumatosis intestinalis. 9 of $13(69 \%)$ of Group 1 developed NEC compared to 1 of $8(12.5 \%)$ of Group 2. 7 of 10 in Subgroups A developed congestive heart failure (CHF) whereas 3 of $11(27 \%)$ of Subgroups B had CHF. 5 of $10(50 \%)$ of Subgroups A required PDA 1igation; whereas 2 of $11(18 \%)$ of Subgroups $B$ required ligation. A persistent or increasing PDA shunt appears to be related to the subsequent development of NEC. 
743 CONTINUOUS MONITORING OF PO 2 IN SICK NEONATES DURING PERIODS OF RESPIRATORY INSTABILITY. T. Hegyi M. Hiatt, B.C. Dangman, L. Indyk \& L.S. James. Coll. of Phys. \& Surg., Columbia Univ., Div. of Perinatal Medicine, New York. We have been able to quantitate rapid changes in arterial oxygen tension $\left(\mathrm{PO}_{2}\right)$ in response to both spontaneous events (apnea, periodic breathing) and to alterations in ventilatory support, by means of the Huch transcutaneous sensor. Single and complex events have been recorded in 50 newborns and correlated with $\mathrm{PO}_{2}$, heart rate, respiratory rate and waveform and peripheral perfusion. During apnea the time from cessation of respiration to the onset of the $\mathrm{PO}_{2}$ change ranged from $0-10$ sec. with $\mathrm{PO}_{2}$ decreasing by as much as $50 \mathrm{~mm} \mathrm{Hg}$ (range 5-50). The time needed to regain a stable $\mathrm{PO}_{2}$ ranged from 30-300 sec. with more severe episodes requiring longer recovery periods. Greater instability of $\mathrm{PO}_{2}$ correlated with severity of illness. The effect of various forms of ventilatory support could be closely followed. Manual IPPB often resulted in very rapid elevation of the $\mathrm{PO}_{2}$ to the hyperoxic range with the $\mathrm{PO}_{2}$ remaining quite elevated for from $1-2$ minutes after bagging was discontinued. Institution of ventilatory support (CPAP, etc.) produced rapid changes in $\mathrm{PO}_{2}$ (onset of $10-50$ seconds with equilibrium attained in $1-6$ minutes).

Thus continuous monitoring of $\mathrm{PO}_{2}$ enables

1. earlier assessment of severity of illness,

2. more rapid stabilization of infants requiring ventilatory support,

3. minimization of exposure to hyperoxia.

Postnatal somatic Growth In Babies with abNormal FETAL 744 GROWTH PATTERNS. Grace E. Holmes, Herbert C. Miller, Dept. of Ped. and Biometry, Kansas City, Ks. This prospective study differentiates patterns of postnatal physical growth in 62 babies ( $61 \mathrm{FT}, 1 \mathrm{PM}$ ), who demonstrated either normal or abnormal somatic types of fetal growth (FG) at birth. The babies were divided into 4 groups: (1) 18 who were underweight for length (low ponderal index or PI), (2) 16 with short crown-heel (C-H) lengths for dates and with average PI, (3) 14 babies who were overweight for length (high PI) and (4) 14 babies in a control group with avg. weight (wgt) for avg. length (avg. PI). Analysis of variance and Duncan's test for multiple comparisons were used to determine distance and velocity growth of the 4 groups during the 1st year of life. There were significant and marked differences in postnatal growth between groups 1 and 2 . The latter group of short babies had consistently lower mean wgts., C-H lengths and head circumferences (HC) at birth and throughout the 1st year as compared with group 1. These differences were significant to either $p^{<.01}$ or $<.05$. Weight-gain velocity in group 1 was significantly higher during the 1st year of life than was that of group $2,(p<.05)$. The postnatal growth pattern of infants in group 2 appeared to be a continuum of their fetal growth pattern. The postnatal picture of infants in group 1 indicates an acceleration in wgt. gain and a reversal of their malnourished state (low-PI) at birth. The postnatal findings give additional evidence of two distinct types of abnormal fetal growth. Mean wgts., $\mathrm{C}-\mathrm{H}$ lengths and HC of the high PI and control groups were not significantly different.

745 INCIDENCE OF APNEA IN INFANTS AT HIGH AND LOW RISK FOR SUDDEN INFANT DEATH SYNDROME (SIDS). TOke T. Hoppenbrouwers, Joan E. Hodgman, Ronald M. Harper, Dennis J. McGinty, Maurice B. Sterman. Univ. Southern Cal Ped Sepulveda, Univ. of California, Dept. of Anatomy, Los Angeles. Epidemiological studies have shown that subsequent siblings of infants who die of SIDS are at a 4 to 5 times higher risk for SIDS. The objective of the present investigation was to document the incidence of apnea in subsequent siblings (SSIDS) and control infants during sleep states. Nine SSIDS and 9 control infants, matched for birth weight, gestation and parent education, were monitored during the first week of life and at monthly intervals thereafter to six months. A 12-hour polygraphic record of 4 sleep and 3 cardiopulmonary variables was obtained. Apnea was defined as cessation of breathing $\geq 6 \mathrm{sec}$. Apnea density was calculated from the number of minutes with apnea in each sleep state. The percentage of time spent in Active Sleep (AS) decreased and Quiet Sleep (QS) increased with age in both groups. Apnea density during AS decreased while remaining stable in QS $(p \leq 0.001)$. Apnea density was higher in AS than QS particularly in control infants. SSIDS differed from control infants in exhibiting less apnea $(p=0.002)$, fewer long apneas $(\geq 8 \mathrm{sec})$ and a higher respiratory rate at 3 and 6 months $(p=0.02)$. These findings suggest a difference in respiratory regulation in SSIDS, and are compatible with a response to chronic mild hypoxia.
746 EXTRACORPOREAL MEMBRANE OXYGENATION (ECMO) CARDIOPULMONARY SUPPORT IN THE NEWBORN Robin Jefferies, Robert Huxtable, Susie W. Fong, Alan B. Gazzaniga, Robert H. Bartlett, Spon. by Thomas L. Nelson from the Departments of Surgery and Pediatrics, Orange County Medical Center, University of California-Irvine, Irvine, Calif. Prolonged ECMO support has been used in adult cardiac and pulmonary insufficiency with moderate success. Many acute conditions of the newborn might benefit from this technique, but few trials have been attempted and no success has been reported. This report describes our experience with 5 critically ill newborn ( 1.8 to $3.3 \mathrm{~kg}$ ) babies supported with ECMO for 1 to 6 days. Indications and results were: two respiratory distress syndrome (Died, Survived), two meconium aspiration $(S, D)$ and one persistent fetal circulation (S). Superior vena cava to carotid access gave excellent flows ( $80 \%$ of cardiac output) in all cases. Stable hemodynamics, normal gas exchange, and normal organ function could be maintained without difficulty. Most cases had consumption coagulopathy prior to ECMO which improved during bypass. Progressive thrombocytopenia was uniformly present but bleeding did not occur. One infant successfully underwent ligation of a PDA while heparinized on ECMO. In addition to passive support of perfusion and gas exchange, the use of ECMO eliminates lung damage from barotrauma and high inspired oxygen concentrations. Indications for ECMO have not been defined in infancy. However, ECMO has been proven successful and should be considered in any newborn suffering from life threatening cardiac or pulmonary insufficiency before irreversible damage occurs.

747

THE USE OF AGAR FEEDINGS IN CONJUNCTION WITH PHOTOTHERAPY IN THE TREATMENT OF NEONATAL JAUNDICE Lois H. Johnson, David Rubinstein, Carolyn S. Crawford Thomas R. Boggs Univ. of Pa. Med. School, Pa. Hosp Dept. Ped Phi ia Phototherapy significantly increases excretion of unconjugated bilirubin into the duodenal bile and much of its effect in lowering serum bilirubin concentration resuits from this phenomenon. Therefore, use of a gut sequestering agent, in addition to phototherapy, should increase its efficiency and decrease the duration of light exposure necessary by decreasing the reabsorption of unconjugated bilirubin from the small intestine. As can be seen in the following table the opposite occurred. Superblue phototherapy (SB)was started when serum bilirubin in strong $>1300 \mathrm{~g}$ infants exceeded $12 \mathrm{mg} \%$ and continued until it fell to $10 \mathrm{mg} \%$. Treatment was supplemented, if necessary, with exchange transfusion (Ex). The average duration of phototherapy required per baby was calculated for three time periods, one before and one after a period when agar $(1500$ to $1600 \mathrm{mg} / \mathrm{d})$ was given at the end of each feeding. The agar used was from Amend $\mathrm{Co}$. as recommended by 0dell. The possible reasons for these unexpected results will be discussed.

$\begin{array}{lccc} & 1973 & \text { Spring 1975 } & \text { FaIl 1975 } \\ & \text { SB } 7 \text { ites } & \text { Agar + lites } & \text { SB 1ites } \\ \text { \# infants } & 49 & 39 & 49 \\ \%<25 C 0>1300 \mathrm{~g} & 38 \% & 28 \% & 30.5 \% \\ \text { \# requiring Ex. } & 4 & 3 & 4 \\ \text { Total \# of Ex. } & 6 & 4 & 4 \\ \% \text { Hemolytic Disease } & 43 \% & 46 \% & 29 \% \\ \text { Mean hrs photo Rx } & 44.5 & 55 & 45\end{array}$

748 Lois H. Johnson, David B. Schaffer, David Rubinstein Caroiyn S. Crawford and Thomas R. Boggs., Univ. of Pa. Med, School, Pa. Hosp. and Children's Hosp. Dept. Ped. PhiTa.

Retrolental Fibroplasia (RLF) still occurs in spite of careful $\mathrm{O}_{2}$ control. Therefore premature infants cared for at $\mathrm{Pa}$. Hosp. are examined regularly by indirect ophthalmoscopy until retinal vasculature is mature or RLF, if present, has regressed. The classification system used has already been detailed (Am. J. Clin. Nut. 27:1158, 1974). It agrees with the recent work of Foos and Flynn (Graefs Arch. klin. exp. Ophthal. 195:87 \& 101, 1975) and differs slightly from the Patz modification of the standard Reese \& Owens classification. It includes the findings visible by indirect ophthaimoscopy. Severity of RLF in our nursery does not usually exceed Stage 2. The effect of treatment with Vitamin $E$ acetate to maintain serum $E$ levels at $1.5 \mathrm{mg} \%$ has been evaluated. Infants with Birth Weights $\leq 2000 \mathrm{gm}$ Born Feb. 1972 to May 1974

Number of infants

Number of RLF

Incidence of RLF

vitamin
49
17
$22 \%$
0.55

Place

46
16
$35 \%$

$35 \%$
1.02

41

Mean Severity

0.55

$T(134)=-2.3$

Incidence/Severity
Treatment with the Vitamin $E$ in these infants was associated with

Analysis of Variance

a decrease in both incidence and severity of RLF. 
749 THE ROLE OF VITAMIN E IN RETROLENTAL FIBROPLASIA. II. Lois H. Johnson, David B. Schaffer, David Rubinstein, Pa. Med. School, Pa. Hosp. and Children's Hosp. Dept. Ped. Phila.

The relationship of low grade acute stage RLF in the nursery to subsequent eye pathology and the possible role of Vitamin $E$ on this long-term morbidity is being evaluated by indirect ophthalmoscopy in the nursery and again at age 1 year. The aim of treatment among $E$ supplemented infants was to raise the serum $E$ level to $1.5 \mathrm{mg} \%$ within 36 hours of birth and to maintain it in the range of $1.5 \mathrm{mg} \%$ until the eyes were mature or RLF had regressed. Control infants received placebo (Plac) or no injection (None). The $E$ content of the nursery formula was 5 IU/L before 1972 and 9 IU/L after 1972. Findings at age 1 year (corrected for gestational age) in 48 infants with birth weight $\leq 2000 \mathrm{gm}$ are presented below. Abnormalities included low-grade cicatricial RLF, myopia, anisometropia, strabismus, amblyopia and heteropia of the macula. Each deviation from normal at age 1 year was assigned a numerical score consistent with its severity.
Birth Year

Dx in the Nursery $\mathrm{Rx}$ in the Nursery \# Seen-age 1 year \# with normal eyes Deviations Score Mean Deviations/baby

\begin{tabular}{c|c}
$1968-71$ & $7972-74$ \\
RLF & RLF \\
None & Plac/None \\
12 & 18 \\
7 & 7 \\
84 & 35 \\
7 & 1.9
\end{tabular}

$$
\begin{gathered}
1972-\overline{74} \\
\text { RLF } \\
\text { Vit. E } \\
8 \\
6 \\
6 \\
0.75
\end{gathered}
$$

0.75
1972-74

No RLF E/Plac/None

10

2

0.2
MONITORING CRITICALLY ILL NEWBORNS WITH DIGITAL CAP750 ILLARY BLOOD SAMPLES: AN ALTERNATIVE. Padmani Karna Hospital of Michigan, Dept. of Peds.; Detroit, Michigan

In a series of 33 critically ill newborn infants, capillary blood samples obtained from a warmed distal phalanx of the right hand were compared with either temporal or right radial arterial blood samples for $\mathrm{pO}_{2}, \mathrm{pCO}_{2}$ and $\mathrm{pH}$. Results are listed:

\begin{tabular}{llllc} 
& $\mathrm{N}$ & \multicolumn{2}{c}{ Regression equation } & Correlation $(\mathrm{r})$ \\
\hline $\mathrm{pO}_{2}$ & 68 & $\mathrm{Y}(\mathrm{art})=-0.25+1.20 \times(\mathrm{cap})$ & .92 \\
$\mathrm{pCO}_{2}$ & 68 & $\mathrm{Y}(\mathrm{art})=2.17+0.86 \times(\mathrm{cap})$ & .84 \\
$\mathrm{pH}$ & 51 & $\mathrm{Y}(\mathrm{art})=0.31+0.96 \times(\mathrm{cap})$ & .94 \\
\hline
\end{tabular}

At the time of sampling the $\mathrm{FiO}_{2}$ and the infants' blood pres sures and skin temperatures were recorded. The presence of pallor. oliguria and type of respiratory assistance was also noted. The

$\mathrm{pO}_{2}$ differences found did not correlate with any of these factors.

Murdock and Swyer (Biol. Neonat. 13:194, 1968) compared right radial axtery to descending aorta; 65 paired samples were obtained for $\mathrm{pO}_{2}$ with like linear correlation $(x=0.83)$ and a mean $\mathrm{pO}_{2}$ difference of $23 \mathrm{mmHg}$. In that study, 3/65 pairs had a $\mathrm{pO}_{2}$ difference of $100 \mathrm{~mm} H \mathrm{Hg}$ or greater as compared with the $2 / 68$ samples in our study. Our two outlier values with 100 and $110 \mathrm{mmHg} \mathrm{pO}_{2}$ differences were the first two obtained from the same 28 wk. infant with grade IV hyaline membrane disease. Subsequent comparisons on the same infant fell within 2 S.D. The mean $\mathrm{pO}_{2}$ difference in our study was $13.8 \mathrm{mmHg}$.

Right digital capillary blood sampling presents a safe and reliable alternative for the monitoring of critically ill newborns.

EARLY NEONATAL CONTACT :EFFECT ON GROWTH,BREASTFFEEDING

751 AND INFECTION IN THE FIRST YEAR OF LIFE. John KennelI, Marshall Klaus, Roberto Sosa, Juan Urrutia. Case Western Reserve University, Dept. of Ped., Cleveland \& Institute of Nutrition of Central America and Panama, Guatemala.

To assess the physical benefits for the infant of early skin to skin contact, we studied 40 poor urban primiparous mothers and infants in Guatemala City. As is routine at the Social Security Hospital, 20 mothers (Controls) were separated from their infants from the time of delivery until the first feeding at 24 hours. 20 other mothers (Early Contact) spent 45 minutes after the episiotomy repair alone in a private room with their undressed infants under a heat panel, skin to skin. The mothers and infants in the two groups were similar in demographic characteristics and subsequent care. Free powdered milk was available upon request for all babies during the first year. Follow up visits to the homes were made at 7,21 and 35 days and at $3,6,9$ and 12 months to obtain data on the infants' growth, food intake and illnesses.

At six and twelve months of age, the babies in the early contact group had gained significantly more weight than the controls ( 6 mos. -4.5 vs $3.7 \mathrm{Kg} p<.05$ ) and more of their mothers were still breastfeeding $(p<.05)$. Also infants of early contact mothers had fewer episodes of infection in the first year (45 vs 68) in this study and in a similar study at another hospital (65 vs 144).

This is the first study to reveal differences in weight gain and health status as a consequence of early postpartum mother-infant contact. This simple maneuver, which appears to have far-reaching effects on infant health and growth during the first year, could be easily and inexpensively applied even in developing countries.
INT'RACRANIAL HEMORRHAGE SECONDARY TO YOLYCYTHEMIA IN

752 PUPPIES. Herbert Koffler (Spons. by Robert E. Greenberg). Univ. of New Mexico Sch. of Med. Dept. of Ped., Albuquerque.

Polycythemia, a venous hematocrit value (Hct) $\geq 70 \%$, was produced in four newborn Beagle puppies. The remaining three pups in the litter served as controls. All seven pups had daily hicts. During the first few hours after birth the pups had ficts ranging from $62 \%$ to $79 \%$. By 12 hours of age, six of the pups had Hcts of $55 \%$ to $59 \%$ and one pup had a Hct of $45 \%$. On the first and second postnatal day the experimental group was given packed RBCs from the bitch via an intraperitoneal route. Two of them were transfused again on the fifth postnatal day. Hets of $\geq 70 \%$ were produced in all four of the experimental group for a period of two to flve days. Hets in the three control nups continued to fall until the lith postnatal day when they stabllized in the range of $35 \%$ to $38 \%$.

On the 18th postnatal day, the Hcts of the experimental group were similar to those of the controls. At this point, two of the control pups and the four experimental pups were examined for intracranial lesions. Intracranial hemorrhage was noted in three of the four experimental puppies; no intracranial lesions were found in one of the experimental pups or in the control group.
753 S.Waldman, P.A.M.Auld. Cormell Univ. Med. Coll. Dept. of Pediatrics, New York, N. Y. 10021 .

Alterations of limb blood flow in response to postural changes and feeding are dependent on autonomic control of the vascular resistance. Head-up tilting and feeding both result in a fall in limb blood flow in association with a rise in vascular resistance. Limb blood flow was measured by occlusion plethysmography in 38 non-distressed infants and found to have a coefficient of variation of $12 \%$. Changes of over $25 \%$ from resting levels were juaged significant. Resistance was calculated from simultaneous intra-aortic blood pressure. Measurements of calf blood flow and resistance were made during tilting in 11 non-distressed premature infants and 9 infants with hyaline membrane disease. A fall in calf blood flow and a rise in resistance were found in $6 / 11$ non-distressed and $1 / 9$ HMD infants, while $2 / 11$ well and 5/9 HMD infants demonstrated a rise in limb blood flow and no change in resistance. Reduction in limb blood flow was found in $61 \%$ of well infants during the first week of life and $80 \%$ during the second week in response to feeding. HMD infants had $44 \%$ and $57 \%$ appropriate responses during weeks 1 and 2 of life. These studies demonstrate a higher rate of inappropriate reflex control of calf blood flow in infants with $M M D$, and suggest that lack of control of vascular resistance may play an important role in the response of the newborn infant to respiratory distress. 
ALBUMTN BINDING OF BIILIRUBIN-COMPARISON OF METHODS.

755 Kwang S. Lee and Lawrence M. Gartner. Dept. of Pediatrics, Albert Einstein Coli. Med., Bronx, N.Y

To compare four methods, albumin binding capacity for bilixubin (BICAP) was determined in artificially jaundiced fresh adul sera (A) and purified human serum albumin (HSA) preparations [Sigma(S), Pentax(P) and Hyland(H)]. The methods used simultaneously were fluorescent dye method (DY7), Sephadex G-25 column chromatography (G-25), HBABA and Saturation Index method (SI).

A decreasing order of BICAP for each HSA preparation (A>H>S $>P$ ) was observed with 3 of the methods (DY7, G-25, and HBABA). Setting BICAP of adult sera without bilirubin as $100 \%, D Y 7$ and HBABA method were compared utilizing BICAP of each preparation at the lowest bilirubin:albumin molar ratio (B:A MR) at which G-25 became positive (Fig. 1). Those samples which gave positive

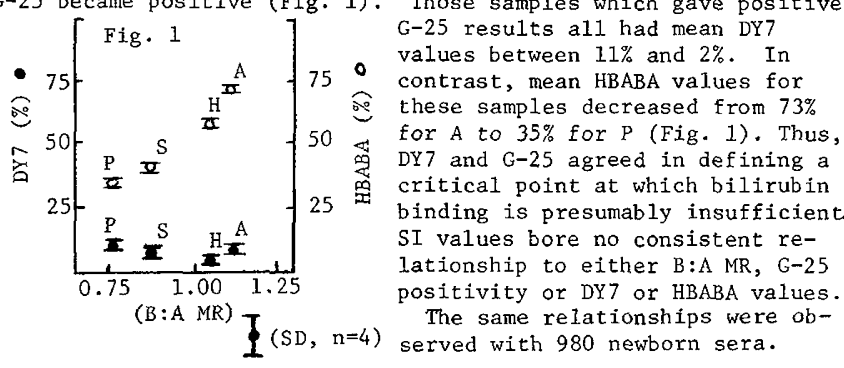

A NEW METHOD FOR DTRECT TITRATTON OF BILIRUBIN

756 BINDING CAPACITY BY FLUORESCENCE QUENCHING. Rodney L. Levine (Sponsored by Frederick $C$.

Battaglia) University of Colorado Medical Center, Division of Perinatal Medicine, Department of Pediatrics, and The Children's Hospital, Denver.

The method of fluorescence quenching has been adapted for titration of neonatal serum with bilirubin. Indirect techniques of assessing bilirubin saturation can identify groups of infants at statistically increased risk of damage, but they are difficult to apply in a specific clinical setting. The quenching method yields the binding capacity of the serum as well as the association constant of bilirubin for albumin $(K)$. The unbound bilirubin concentration (presumably the toxic fraction) can be calculated. A sample of $0.10 \mathrm{ml}$ suffices and can be analyzed in less than 1 hour in the clinical laboratory. This method showed the serum of term infants to bind bilirubin in a concentration equimolar to the albumin, in agreement with other techniques. It has also been utilized to investigate $\mathrm{pH}$ and drug effects on the binding of bilirubin to albumin. A drug can be screened for effect on binding in a few hours, and one can distinguish effects on capacity from effects on affinity (competitive versus noncompetitive effects).

757 INFANTS. Michael J. Light, Joan E. Hodgman, and Medicine, Department of Pediatrics, Honolulu, Hawail.

Nine infants had open lung biopsies, either at the time of patent ductus ligation (PDA), or because of worsening ventilatory status. As shown in the table, seven of the nine had an initial clinical and radiologic diagnosis of hyaline membrane disease (HMD). Three had biopsies consistent with WilsonMikity Syndrome (WMS), one showed a mixed picture (MIX) of WMS with changes of bronchopulmonary dysplasia (BPD), and one showed atelectasis (ATL), with no structural lung disease. The remaining four showed $B P D$.

\begin{tabular}{l|l|l|l|l|l|l|l|l|l|}
\multicolumn{10}{c}{ TABLE } \\
\hline Case No. & \multicolumn{1}{c}{1} & \multicolumn{1}{c}{3} & \multicolumn{1}{c|}{4} & 5 & 6 & 7 & 8 & 9 \\
\hline Birth Weight (kg) & 2.3 & 1.0 & 0.9 & 1.6 & 1.2 & 1.1 & 1.0 & 1.4 & 1.2 \\
FIO $2 \geq 60 \%$ (hrs) & 48 & 33 & 0 & 12 & 112 & 0 & 12 & 63 & 13 \\
PDA Ligation & NO & YES & NO & YES & NO & YES & YES & YES & YES \\
Age at Biopsy (days) & 33 & 15 & 35 & 13 & 38 & 35 & 16 & 8 & 24 \\
Clinical Diagnosis & HMD & HMD & WMS & HMD & WMS & HMD & HMD & HMD & HMD \\
Biopsy Diagnosis & WMS & WMS & WMS & MIX & BPD & BPD & BPD & BPD & ATL \\
\hline
\end{tabular}

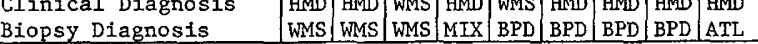
These findings suggest that the diagnosis of WMS cannot be made on clinical or radiologic grounds where the course has been altered by intermittent positive pressure ventilation. In addition, it is suggested that the incidence of WMS is more common than previously recognized in ventilated infants. SERIAL PLATELET COUNT AS A SCREENTNG PROCEDURE FOR

758 McCarthy, Vincent $J$. Fuselli and Charles $\frac{\text { Jeane A. }}{R \text {. Bauer }}$ (Intro. by w. W. Cleveland) University of Miami, School of Medicine, Department of Pediatrics, Miami, Florida

Previous reports indicate that, regardless of gestational age, platelet counts of less than $100,000 / \mathrm{mm}^{3}$ are abnormal. Thrombocytopenia in the newborn is most often induced by infection, phototherapy or drugs. This study was undertaken to determine if serial platelet counts could be used as an early indicator of infec tion. All Newborn infants admitted to the special care nurseries during $a$ one month period, regardless of diagnosis, were included. Platelet counts using direct microscopy were done on admission and then daily in all infants. Ninety three infants were studied. Three septic infants with low platelets were eliminated due to early exchange transfusicns. Results:

Culture Proven Sepsis Suspected Sepsis or Pneumonia No known infection Total

\begin{tabular}{c|c|c} 
Results: & \\
Number & $\leq 100,000$ Plat. & $>100,000$ p2at \\
\hline 8 & 6 & 2 \\
9 & 7 & 2 \\
73 & 19 & 54 \\
\hline
\end{tabular}

Four infants with normal platelets had evidence of infection (7\% false negative). Nineteen infants with low platelets did not have evidence of infection but were associated with circumstances known to cause lowered platelets. In summary, low platelets were associated with infection in $75 \%$ of infants with culture proven sepsis and in $85 \%$ of infants with radiologically demonstrated pneumonia $(p<.001)$. This procedure may be a useful adjunct to the standard sepsis work-up for the early detection of neonatal infection.

\section{9} PULMONARY VASCULAR RESISTANCE IN DIAPHRAGMATIC HERN IA: . Naeye. Penn State Univ. Coll. Med., M. S. Hershey Med. Ctr. Depts. of Ped., Surg, and Path., Hershey, $\mathrm{Pa}$.

An increased muscle mass has been observed in the pulmonary arteries of infants dying with congenital diaphragmatic hernias (Naeye et al. SPR 1976). This, together with the previous observation of marked right to left shunting in such infants, suggested that lowering the pulmonary vascular resistance might improve the outcome. Two infants who manifested respiratory distress irmediately after birth and who had $\mathrm{AaDO}_{2}$ (descending aorta) consistently $>500$ postoperatively (very poor prognostic category) received a continuous IV infusion of tolazoline hydrochloride $(2 \mathrm{mg} / \mathrm{kg} / \mathrm{hr})$ from 21 and 27 hours after operation. Arterial blood gases were measured in the temporal artery (TA) and aorta $(\mathrm{AO})$. Both infants responded with a dramatic increase in $\mathrm{TAPaO}_{2}(36 \rightarrow 238$ and $26 \rightarrow 312)$ but with less of an increase in $\mathrm{AOPaO}_{2}(36 \rightarrow 132$ and $20 \rightarrow 84)$. This response suggests a decrease in total $R \rightarrow L$ shunting but a marked increase in $R \rightarrow L$ shunting across the ductus arteriosus (DA). This may be explained by a fall in pulmonary vascular resistance but possibly a greater fall in peripheral resistance. In one infant the DA shunt decreased over 4 hours with subsequent rapid recovery. In the other a large DA shunt remained for about 30 hours with subsequent resolution. Both infants survived. Increased pulmonary vascular resistance, rather than abnormalities of ventilation, appears to be the important mechanism in the (often fatal) hypoxemia found in these infants.

760 THE WHITE BLOOD COUNT (WBC) IN THE DIAGNOSIS OF NEONATAL GROUP B STREPTOCOCCAL SEPSIS. B. Manroe, R. Browne, A.G. Weinberg, C.R. Rosenfeld, Dept. of Ped., Biometrics and Pathology, Univ, of Texas, Southwestern Medical School, Dallas, Texas. (Spon. by George H. McCracken).

Neonatal Group B streptococcal (GBS) sepsis has a high mortality. Early diagnosis is difficult; thus laboratory tests aiding in recognition are needed. Normal newborn values for the absolute neutrophil (AN) and absolute band ( $A B$ ) counts and the ratio of \% bands to \% total neutrophils $(B / N)$ were determined from 3000 counts and applied prospectively to 46 cases of blood culture proven GBS sepsis between Apri1,1974-December,1975. A11 but 7 of the organisms were $\beta$-hemolytic. 19 infants were preterm, 23 term, and 4 post-term. 39 cases were early onset $(<3$ days). None of these infants had a normal WBC: $20(51 \%)$ had $+A N$; $18(46 \%)$ had $+A B$; and $31(79 \%)$ had $\uparrow B / N$. Only 7 had the classically described $\uparrow$ in both $\mathrm{AN}$ and $\mathrm{AB}, 32(82 \%)$ had abnormalities in two parameters. 7 cases occurred after 3 days; 5 had $\uparrow A N$ and 2 a $\downarrow$ AN. Mortality was $26 \%$ $(12 / 46)$, all in infants with early onset disease. Early initiation of antibiotic therapy was undertaken in infants in whom an abnorma1 WBC was recognized. It was delayed or not initiated in 10 of the 12 deaths $(83 \%)$, all of whom were infants with severe hyaline membrane disease, and in whom there was a failure to appreciate an abnormal WBC. These data demonstrate the usefulness of a readily available laboratory test in the early recognition of GBS sepsis, allowing early initiation of therapy and a probable decrease in mortality. 
NORMAL LEUKOCYTE (WBC) VALUES IN NEONATES. B. Manroe, R. Browne, A.G. Weinberg, C.R. Rosenfeld, Dept. of Ped., Biometrics, and Path., U of Texas, Southwestern Medical School, Dallas. (Spon. by Chester W. Fink).

Several authors have described the normal neonatal WBC. None have evaluated noninfectious conditions other than surgery which might affect it. $3000 \mathrm{WBC}$ 's were performed on 920 infants from birth to 29 days of age. Gestational age was not influencial; therefore, infants were grouped by birth weight: $<2500$ gm (Group I) and $>2500 \mathrm{gm}$ (Group II). Many conditions were found to influence the WBC, including infants of mothers with diabetes, hypertension, or fever, those with meconium aspiration, 5 min Apgar $\leq 6$, hyaline membrane disease, hypoglycemia, and seizures. All such abnormal conditions were excluded. Only infants without clinical problems $\pm 48 \mathrm{hrs}$ from the time the WBC was drawn were considered normal. The following norms were determined. The absolute neutrophil count (ANC, $\times 10^{3} / \mathrm{mm}^{3}$ ) was maximum at 215 hrs of life, ranging from 8.0 to 12.0 in Group I and 8.0 to 14.0 in Group II. After 96 hrs ANC stabilized, ranging from 2.0 to 4.7 in Group I and 2.4 to $4.5 \times 10$ in II. The absolute band count $\left(\mathrm{ABC}, \mathrm{x} 10^{3} / \mathrm{mm}^{3}\right)$ was also maximum at $215 \mathrm{hrs}$, reaching $1.4 \times 10$. After $96 \mathrm{hrs} A B C$ did not exceed $500 / \mathrm{mm}^{3}$. The ratio of $\%$ bands to $\%$ total neutrophils $(B / N)$ was evaluated and found to be a sensitive method for detecting sepsis. In the first 15 hrs of life, it did not exceed 0.17 . After 96 hrs it was 0.14 . Nucleated red blood cells never exceeded $1200 / \mathrm{mm}^{3}$, and were not present after 3 days of life. By excluding numerous maternal and neonatal complications, the $A N C, A B C$ and $B / N$ had narrower ranges of normal than previously described and were found to be more sensitive in screening for neonatal infection.

762

A DEFLATION HERING-BREUER REFLEX IN THE PRETERM INFANI: A MECHANISM BY WHICH A LOW CONTINUOUS POSITIVE AIRWAY PRESSURE (CPAP) DECREASES. APNEA? R.J.Martin,H.Nearman, P.Katona, M. Klaus. CWRU, Dept. of Ped. \& Biomed. Engr., Cleveland. To explore the action of CPAP in reducing apnea of prematurity, we studied reflex control of respiration by the airway occlusion technique in 12 preterm AGA infants (mean birth wt. $1.3 \mathrm{Kg}$, GA 30 weeks) from 3-13 days of age. We measured inspiratory duration prior to occlusion $(T$,$) , duration of initial occluded inspiratory$ effort (To), \% change of inspiration $\left[\% T=100\left(T_{0}-T_{1}\right) / T_{1}\right.$ ] 0 on occlusion pressure generated at $100 \mathrm{msec} .(\mathrm{P} / 00)$ and peak inspiratory pressure $(\mathrm{Pm})$, at a CPAP of $\mathrm{O}$ and $3-5 \mathrm{~cm} \mathrm{H} \mathrm{H}_{2} \mathrm{O}$ using a nosepiece fitted with a thermistor. 5 infants (GPA) showed the expected positive prolongation of oT at $O$ CPAP with no change in mean $\% T$, $\mathrm{T}$, or Pm on CPAP, suggesting no effect of CPAP on the inflation reflex. In 7 infants (Gp B) mean \% was negative at 1 CPAP with one or more very short inspiratory efforts ( $\%$ T $<-30 \%$ ) on occlusion with higher PI00. These responses were eliminated by CPAP, occurring 32 times at 0 CPAP but only twice on CPAP. CPAP significantly increased T, To, \%T and Pm. 3 of these 7 infants Group B $(n=7)$ sec sec $\quad \mathrm{cm} \mathrm{H} O$ ly responded to CPAP. These short

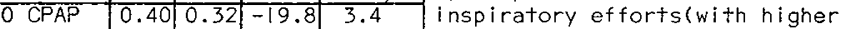
3-5 CPAP $0.490 .71+45.3 \quad 7.1 \quad$ PI00) and their disappearance at $p \quad k .025<.005 \mid<.005<.005$ increased lung volume appear the deflation reflex of Hering and Breuer. This reflex has not hitherto been implicated in normal respiration in man; its el imination with CPAP may stabilize respiratory control in the preterm infant.

CONTINUOUS IN VIVO MONITORING OF ARTERIAL PO AND $763 \mathrm{PCO}_{2}$ IN FOETAL IAMBS. Sadi V. Matalon, Patrick J. Hunt, Depts. of Pediatrics, Physiology and ob-Gyn, university of Minnesota, Minneapolis, Minnesota. Spon. by Henry L. Nadler.

An in vivo blood gas catheter-mass spectrometer system has been used to continuous $1 y$ and simultaneous $1 y$ measure foetal arterial $\mathrm{PO}_{2}$ and $\mathrm{PCO}_{2}$. In 7 pregnant sheep (128-135 days gestation) the maternal inspired mixture was varied and the following changes in foetal $\mathrm{PO}_{2}$ and $\mathrm{PCO}_{2}$ were observed: (1) $100 \% 0_{2}$ to room air: Foetal $\mathrm{PO}_{2}$ decreased from $21.5 \pm 0.8$ (Mean \pm S.E.M.) to $14 \pm$ $4.1 \mathrm{mmHg}$ at a rate of $1.47 \pm 0.3 \mathrm{mmHg} / \mathrm{min}$. Following the return to $100 \% \mathrm{O}_{2}$ the $\mathrm{PO}_{2}$ returned to $21 \pm 1.1 \mathrm{mmHg}$ at a rate of $2.1 \pm$ $0.35 \mathrm{mmHg} 7 \mathrm{~min}$. (2) $100 \% \mathrm{O}_{2}$ to $12 \% \overline{\mathrm{O}}_{2}$ and $10 \% \mathrm{CO}_{2}$ : After $6 \mathrm{~min}$. of ventilation, the $\mathrm{PO}_{2}$ fell to $6.3+0.3 \mathrm{mmHg}$ at a rate of 3.55 $\mathrm{mmHg} / \mathrm{min}$ and the $\mathrm{PCO}_{2}$ rose from $3 \overline{7} \pm 8$ to $70 \pm 5 \mathrm{mmHg}$. Following the return to $100 \% \mathrm{O}_{2}$, the foetal $\mathrm{PO}_{2}$ and $\mathrm{PCO}_{2}$ returned to their previous baseline values within 4 and $10 \mathrm{~min}$. respectively at a rate of $6.5 \mathrm{mmHg} / \mathrm{min}$. (3) $90 \% \mathrm{O}_{2}$ and $10 \% \mathrm{CO}_{2}$ : Within $8 \mathrm{~min}$. $\mathrm{PO}_{2}$ and $\mathrm{PCO}_{2}$ increased 4 and $20 \mathrm{mmHg}$ respectively. In all instances, the observed foetal changes were initiated within 1 min, after changing the maternal mixture, except in the case of recovery from $12 \% \mathrm{O}_{2}$ and $10 \% \mathrm{CO}_{2}$, when such changes began within $34 \pm 12$ sec. These results indicate that changes in maternal $\mathrm{PO}_{2}$ and $\mathrm{PCO}_{2}$ are rapidly transmitted to the foetus, and that foetal hypoxia secondary to maternal hypoxia can be rapidly alleviated by the administration of $100 \% \mathrm{O}_{2}$ to the mother.
EFFECT OF PHOTOTHERAPY ON ACID-BASE BALANCE

764 IN LOW BIRTH WEIGHT INFANTS. DaWn G. Mueller, Barry V. Kirkpatrick, Joan W. Stoerner Cheryl $R$. Hunter, and Harold M. Maurer, Medical College of Virginia, Department of Pediatrics, Richmond, Va. Tachypnea and water loss have been reported during the use of phototherapy. To monitor these effects on acid-base balance, serial blood gases were obtained from well low-birth weight ( $L B W$ ) infants ( $\leq 2 \mathrm{Kg})$ treated with Daylight bulb phototherapy (Group I) and from a non-treated control group (Group II) matched for birth weight and gestational age. Group I received continuous phototherapy for $96 \mathrm{hrs}$ by $\approx 80 \mathrm{Joules} / \mathrm{cm}^{2} / \mathrm{day}$ at $460 \mathrm{~nm}$ while Group II received $\approx 13$ Joules $/ \mathrm{cm}^{2} / \mathrm{day}$ at $460 \mathrm{~nm}$ from ambient light. $p H$, pCO2 and bicarbonate were compared at onset (24t $12 \mathrm{hrs}$ after birth) and 24 , 48 , and $96 \mathrm{hrs}$ later. At $24 \mathrm{hrs}$ a significant difference in bicarbonate was found between Groups $I$ and $I I$.

\begin{tabular}{|c|c|c|c|c|c|c|c|}
\hline$G p$ & $N$ & $p H$ & & $p C 02$ & $m m H g$ & $\mathrm{HCO} 3$ & $m E g / L$ \\
\hline I & $(16)$ & 7.36 & \pm .01 & 31.8 & \pm 2.1 & 17.3 & \pm 1.0 \\
\hline$I$ & (16) & 7.37 & \pm .01 & 34.9 & \pm 2.1 & 19.6 & \pm 0.8 \\
\hline & alue & $<$ & .10 & & .10 & & .025 \\
\hline
\end{tabular}

No significant differences were found at 48 and 96 hrs. Continuous phototherapy caused a transient fall of bicarbonate which returned to control levels by $48 \mathrm{hrs}$. Though of little clinical significance to the well LBW infant, bicarbonate loss may not be tolerated

UNSOLVED EPIDEMIOLOGY OF NECROTIZING ENTEROCOLITIS (NEC). J. C. Mulligan, A. C. Allen, H. M. MacDonald

765 (MWH), Department of Pediatrics, Pittsburgh, Pa.

NEC is reported to be associated with hypoxia, hypotension, compromised perfusion of the GI tract, various feeding practices, and infection yet none of the published series contains standardized controls. 27 of 36,000 infants born at MWH between '70-'75 developed NEC; each was matched with 4 inborn infants as to year of birth, birth weight, and gestational age.

Birth weight (mean) Asphyxia neonatorum Respixatory distress syndrome Umbilical arterial catheters Umbilical venous catheters

Exchange transfusion

Feeds: Median age of first Vo1. $(\mathrm{ml} / \mathrm{kg} / \mathrm{d})-$ mean for $3 d$ prior to NEC Caloric content: $20 \mathrm{cal}$

\begin{tabular}{|c|c|c|c|}
\hline & $\operatorname{NEC}(27)$ & Control(105) & P Value \\
\hline & $1680 \mathrm{gm}$ & $1675 \mathrm{gm}$ & \\
\hline & $2(7 \%)$ & $32(30 \%)$ & $<0.02$ \\
\hline me & $9(33 \%)$ & $23(21 \%)$ & $<0.5$ \\
\hline & $4(15 \%)$ & $20(19 \%)$ & $<0.9$ \\
\hline & $4(15 \%)$ & $9(8 \%)$ & $<0.6$ \\
\hline & $2(7 \%)$ & $4(4 \%)$ & $<0.8$ \\
\hline & $28 \mathrm{hr}$. & $15 \mathrm{hr}$. & \\
\hline EC & 93 & 112 & \\
\hline cal & $7(30 \%)$ & $28(26 \%)$ & $<0.9$ \\
\hline $\mathrm{cal}$ & $11(41 \%)$ & $38(35 \%)$ & $<0.8$ \\
\hline $27 \mathrm{cal}$ & $7(26 \%)$ & $36(33 \%)$ & $<0.7$ \\
\hline
\end{tabular}

Despite clustering of cases, bacteriologic studies in these 27 infants and in 20 outborn infants who developed NEC at MWH in '70-'75 did not indicate an infectious etiology.

Our experience thus fails to confirm associations between NEC and the clinical circumstances listed above. Control infants must be studied to unravel the epidemiology of NEC.

766 CAROTID BODY ABNORMALITIES IN THE SUDDEN INFANT DEATH SYNDROME. Richard L. Naeye, Penn State Univ. Col. Med. Dept. Pathology, Hershey, Pa. 17033

Prolonged apneic periods during sleep have been described in several SIDS victims prior to death. Such apneic episodes may be a common final pathway of death. The current study searched for carotid body abnormalities in SIDS victims. A defect in the chemoreceptor function of the organ might impair ventilatory control in SIDS. Using serial sections and point counting, the volume of glomic cells and the volume of nerve fibers were determined in the carotid bodies of 31 SIDS victims who had no evidences of infection, 25 who had mild infections and 26 non-hypoxic controls. The volume of glomic tissue/body weight did not change with age in the controls. Usirg this ratio, $19 \%$ of SIDS victims without infection had a volume of glomic tissue in the upper 10th percentile of control values and $75 \%$ in the lower 10 th percentile. The SIDS victims in the upper 10th percentile had marked evidences of chronic alveolar hypoxia and hypoxemia before death, i.e. increased mass of muscle in pulmonary arteries, hypertrophic cardiac right ventricles, abnormally retained brown fat and hepatic erythropoesis. SIDS victims in the lower 10th percentile (hypoplastic glomic tissue) also had anatomic markers of chronic alveolar hypoxia and hypoxemia but the abnormalities were not as severe as in those with hyperplastic glomic tissue.

Most of the SIDS victims with mild infections had a normal volume of carotid body glomic tissue. There were other differences between the infected and non-infected victims. Infected victims were older at death, had smaller thymus glands and milder tims were older at death, had smallex thymus glands and milde 
767

THE FORMATION OF BILIRUBIN GLUCURONIDE BY GUNN RAT LIVER FROM DI-METHYLESTER-BILIRUBIN G.B. Odell; J.O. Cukier; and A.C. Maglalang, Dept. of Pediatrics, Johns Hopkins Univ. Sch. Medicine,

Both Wistar (JJ) and homozygous jaundiced Gunn (jj) rats were infused with $20 \mathrm{mg}$ of synthetically prepared di-methylester bilirubin dissolved in $1 \mathrm{ml}$ of acetone over a period of $1 \mathrm{hr}$. Pre, inter and post-infusion bile was collected from the cannulated common bile duct. In both JJ and jj animals, pre-infusion bilirubin concentrations were between $1-2 \mathrm{mg} / \mathrm{dl}$ and rose to $17-25 \mathrm{mg} / \mathrm{dl}$ during the last $30 \mathrm{~min}$. of the methylester infusion. Thin layer chromatographic separation on silica G plates of the interinfusion bile showed diazo positive pigments with $\mathrm{R} f$ characteristics of both mono and diglucuronides of bilirubin and no free di-methylester bilirubin. The major bile pigments after chromatographic separation and elution had molar ratios of glucuronic acid: bilirubin of $1: 1$ and $2: 1$ respectively as chemically determined by the napthoresorcinol and VandenBerg reactions respectively. Invitro incubation of hepatic microsomal isolates from $j j$ animals with di-methylester bilirubin showed that they form free bilirubin in the absence of UDP glucuronic acid and in its presence both mono and diglucuronides of bilirubin are formed. These results indicate that the jj rat has a bilirubin glucuronyl transferase but it is packaged in the endoplasmic reticulum in a way that it is not available to bilirubin per se.
OXYGEN CONSUMPTION IN BILIRUBIN PHOTOXIDATION

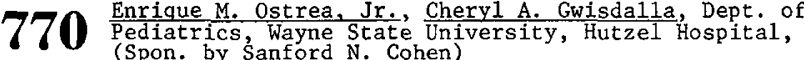

This study presents data on oxygen consumption during the phoodegradation of bilirubin \& the factors that affect it . Differ(2) 4.25 g\% human albumin (0.3-0.5 molar ratio) \& (3) cord blood serum. The solutions were exposed at varying times to daylight fluorescent bulbs energy $=1.2 \mu$ watts $/ \mathrm{cm}^{2} / \mathrm{nm}$ at $425-475 \mathrm{~nm}$ ) at ${ }^{37}$ Model 53 Biological Oxygen Monitor). Total bilirubin was measured by the Evelyn-Malloy method.

RESULTS: At $37^{\circ} \mathrm{C}$, the oxygen consumption during the photoxidation Bi1-Serum (BS)

Bil-Alb (BA) Bil-NaOH (BN) Units $19.00 \pm 3.11_{(\mathrm{p}<0.02)} \frac{14.53 \pm 3.62}{21.03 \pm 5.41} \quad \frac{\mathrm{n}<0.001)}{\mu 10_{2} / \mathrm{mg} \mathrm{Bil} / \mathrm{h}}$ $0.49 \pm 0.08^{(p<0.02)} 0.38 \pm 0.10^{(p<0.001)} 0.55 \pm 0.14 \quad \mu \mathrm{M} 02 / \mu \mathrm{M} \mathrm{Bil/h}$ The mean molar ratio of oxygen to bilirubin is $0.5: 1$ which indioms for oxidation. The most efficient breakdown of bilirubin occurs in the BA system--not only is less oxygen consumed per mg of bilirubin destroyed, but the rate of bilirubin breakdown is fastIt is likely that the binding of bilirubin to albumin results in a spatial configuration that renders the bilirubin molecule more susceptible to oxidation. The photoxidation of bilirubin in NaOH occurs in 2 phases: an initial reaction of rapid consumption of oxygen \& breakdown of bilirubin \& a subsequent lag phase characsumption of oxygen. This is not observed in the BA \& BS systems. At temperatures between $30-40^{\circ} \mathrm{C} \& \mathrm{pH}$ between $7.15-8.0$ there is during photoxidation in the BA system.
768 THE INFLUENCE OF FATTY ACIDS ON THE ALBUMIN ILIRUBIN LIGAND: G.B. Odell; J.O. Cukier; Pediatrics, Johns Hopkins Univ. Sch. Medicine, Balt.Md. When free fatty acids(FFA) are bound to albumin the lock the high affinity sites for bilirubin and force the latter to be transported at the secondary sites where it is more readily dissociated. Bilirubin carried at these secondary sites is easily displaced and is associated with a high incidence of bilirubin encephalopathy. Sixteen neonates with hyperbilirubinemia were identified whose clinical histories indicated caloric insufficiency and elevation of FFA was expected. Each was treated by caloric supplementation. Plasma concentrations of bilirubin, FFA, and saturation Index(SI) were determined before and after supplementation and the results analyzed by a paired $t-t \in s t$.

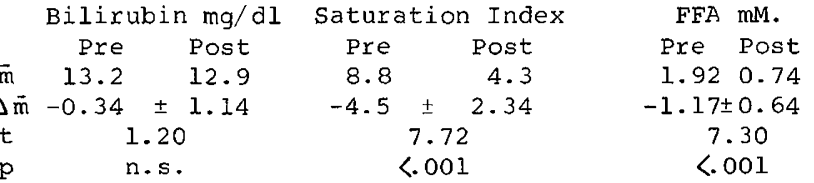

The results show a highly significant correlation between the FFA and the SI suggesting that fasting of jaundiced neonates may increase the possibility of bilirubin encephalopathy.

EFFECT OF SURFACTANT THERAPY ON RESPIRATORY DISTRESS 769 OF PREMATURE LAMBS. Alan Osher, Forrest Adams, Bernard of Medicine, Department of Pediatrics, Los Angeles.

This study was designed to see if the endotracheal delivery of surfactant (S) either in suspension or after nebulization produces a favorable effect on the lungs of prematurely delivered twin lambs with clinical respiratory distress. Thirty-two pregnant ewes with known breeding dates and twins or triplets were delivered of their offspring by cesarean section under spinal anesthesia. A11 deliveries were between 124-134 days of gestation, a tfme when lung surfactant is not normally present in sufficlent amounts and respiratory distress develops. The trachea of each fetus was cannulated with a tube and connected to a respirator. On an alternate basis, one twin recelved surfactant and the other saline. Four mafor treatment varlables were studied: natural surfactant and synthetic DPL; delivery of $S$ either by ultrasonic nebulization or by direct tracheal instillation. The lambs were ventilated for 2 hours during which time blood gases, blood pressure, heart rate and body temperature were monftored. No attempt was made to correct the lamb's metabolic ac1dosis when it occurred. After 2 hours, the lambs were sacrificed and the lungs were carefully removed for studies which included pressure-volume curves, water content, surface tension, histology and histochemistry.

Under the conditions of the study, nelther nebulization or instillation of natural or synthetic $S$ produced recognizable favorable effects on any of the parameters.
PHOTOXIDATION OF ESSENTIAL FATTY ACIDS: A POSSIBLE

771 COMPLICATION OF PHOTOTHERAPY Enrique M. Ostrea, Jr, Wayne State University, Hutzel Hospital, Detroit, Michigan (Spon.
by Sanford N. Cohen)

Fatty acids (FA) undergo spontaneous oxidation which can be measured by the amount of oxygen consumed $\&$ the analysis of pro-
ducts of oxidation by the thiobarbituric acid (TBA) test. Many factors accelerate the autoxidation of FA \& this report presents treatment of jaundice in the newborn, has a similar effect.

Solutions of linolenic $\left(\mathrm{C}_{18: 3)}\right.$, linoleic $\left(\mathrm{C}_{18: 2)}\right.$, oleic $\left(\mathrm{C}_{18: 1}\right)$ \&A palmitic (C16:0) acid were prepared. At $37 \mathrm{oC}^{2}$ a volume of each while an equal volume was protected with Al foil (dark control). thod (YSI Model 53 Biological Oxygen Monitor). The TBA test was done with a spectrophotometer using malonylaldehyde as a standard. RESULTS: Spontaneous oxidation of unsaturated FA is significantly

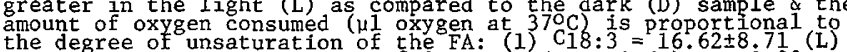

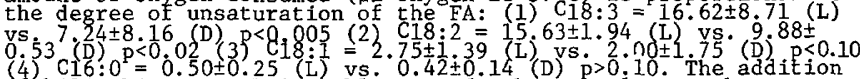
0.53 (D) $\mathrm{p}<0.020 \pm 0.25$ (L) vs. $0.42 \pm 0.14$ (D) $\mathrm{p}>0.10 \pm$ The addition
of bilirubin or methylene blue can further acceierate the oxygen consumption of $C_{18}: 1$ but not the saturated FA $C_{16}: 0$. The forma-
tion of TBA + products is also higher in the 1 ight exposed sam--
ples: TBA (C18:2) $=240 \pm 56.8 \mu \mathrm{M} / \mathrm{L}$ (L) vs. $174 \pm 25.9$ (D), $\mathrm{p}<0.05$. The use of the TBA test as a marker of in vivo essential FA
oxidation was studied in premature, term \& SGA infants undergoing phototherapy. As compared to matched controls not under photo-

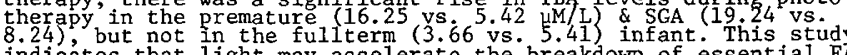
in the premature \& SGA infants.

EFFECT OF PHOTOTHERAPY AND NURSERY LIGHT ON NEONATAL

772 BIORHYTHMS: Tong SOo Park, Sonia Padget, Theres Temple University School of Medicine, Department of Pediatrics, Philadelphia, Penna.

The effect of differing light environments on blood glucose, serum calcium, and plasma HGH was studied in 40 normal newborn infants on the second day of 1 ife, 13 of whom (Group I) were treated by phototherapy for physiologic jaundice. A group of 14 infants (Group II) were in nursery light (100-120 ftcd.) from 0800 to $2000 \mathrm{hrs}$, and in the dark from 2000 to 0800 , and 13 infants (Group III) were in nursery light from 0700 to $2200 \mathrm{hrs}$., in dim light (5-8 ftcd.) from 2200 to $0700 \mathrm{hrs}$. Blood was drawn $9.4 \mathrm{~h}$. for $24 \mathrm{hrs}$, , before feedings, for measurement of $\mathrm{HGH}$, glucose and calcium.

There was no detectable biorhythm of blood glucose or calcium in any of the three light environments. However, a circadian rhythm of HGH was observed in Groups II and III under dark:light and dim:bright light cycling. In infants receiving phototherapy, with photoreception blocked, the normal circadian rhythm (whose peak is reached between 2400 and 0400 hrs.) was completely erased. It is not clear whether the constant exposure to intense light of phototherapy obliterates the biorhythm or if this is the result of the block of light perception by eyeshields applied to those infants. 
TREATMENT OF EARLY POSTNATAL CARDIOVASCULAR SHOCK

773 WITH AUTOLOGOUS FETAL BLOOD TRANSFUSIONS. Charles L. $\frac{\text { Paxson, Gary L. Heaton, Eugene W. Adcock, and Frank }}{\text { (Spon. by R.R. Howell). Univ. of Texas Med. School }}$ H. Morriss.

This prospective study of 4500 neonates was conducted to identify before their delivery those infants at risk for shock immediately after birth and to assess the effectiveness of treatment with transfusions of autologous blood collected from the placenta at delivery.

Shock occured in 27 neonates $(0.6 \%)$ and was associated $(p<.005)$ with maternal vaginal bleeding, prolapsed umbilical cord, twinning, and low birth weight (LBW), and maternal sepsis $(p<.025)$. Among $544 \mathrm{LBW}$ infants the incidence of early postnatal shock was $5 \%$.

Twelve of the neonates were treated with transfusions of fetal blood $(20 \mathrm{~m} 1 / \mathrm{kg})$ obtained from the placenta after delivery, and 15 similar neonates were treated with cross-matched banked adult blood. Seven of the latter also received immediate infusions of $1.0 \mathrm{~g} / \mathrm{kg}$ of $12.5 \mathrm{~g} / \mathrm{dl}$ salt-poor albumin.

\begin{tabular}{|c|c|c|c|c|c|}
\hline Treatment & Mortality & $\mathrm{p}$ & Age BP & restored, hrs. & P. \\
\hline Autologous blood & $8 \%$ & & & 0.8 & \\
\hline Banked blood (tota1) & $67 \%$ & $<.01$ & & 2.5 & $<.01$ \\
\hline+ I.V. albumin & $71 \%$ & $<.025$ & & 0.8 & $<.5$ \\
\hline No albumin & $63 \%$ & $<.05$ & & 4.1 & .00 \\
\hline
\end{tabular}

Conclusion: Mortality for LBW babies can be reduced by prospective identification of the fetus at risk for shock, collec tion of autologous fetal blood from the placenta, and rapid correction of low blood pressure with transfusions of fetal blood.

THE EFFECT OF TOLAZOLINE ON TRANSCUTANEOUS $\mathrm{PO}_{2}$ MUNI-

774 TORING. Joyce L. Peabody, George A. Gregory, Mary M. Willis and William H. Tooley. Cardiovascular Research Institute and the Depts. of Pediatrics and Anesthesia, University of California, San Francisco, Calif.

We have measured transcutaneous oxygen tension $\left(\mathrm{tcPO}_{2}\right)$ with the Huch electrode and correlated it with oxygen tension of arterial blood $\left(\mathrm{PaO}_{2}\right)$ on 105 occasions in 15 infants. The correlation coefficient was 0.987 , and the SD of regression of the line described by $y=0.952+2.65$ was \pm 3.75 .

We then measured the $\mathrm{PaO}_{2}-\mathrm{tc} \mathrm{PO}_{2}$ in 6 infants receiving tolazoline (Priscoline). The average dose for 5 of the infants was 29 (3-61) $\mathrm{mg} / \mathrm{kg}, 27$ paired measurements were made while the infants were receiving the drug. The mean difference of $\mathrm{PaO}_{2}-\mathrm{tcPO}_{2}$ was $11.6 \mathrm{~mm} \mathrm{Hg}$. This difference is significant at the $p<.001$ level by paired t-test. On 14 paired samples $8-18$ hours after withdrawing the drug, the mean difference of $\mathrm{PaO}_{2}-\mathrm{tcPO} 2$ was 0.29 (p) .200). The other infant received a total of $275 \mathrm{mg} / \mathrm{kg}$ of tolazoline over four days and had a mean difference of $\mathrm{PaO}_{2}-\mathrm{tcPO}$ of $38.8(\mathrm{p}<.001)$ on 27 occasions. His $\mathrm{PaO}_{2}$ and $\mathrm{tcPO}_{2}$ remained significantly different until 10 days after the drug was stopped.

We conclude that tolazoline adversely affects the accuracy of the Huch transcutaneous $\mathrm{PO}_{2}$ electrode and warn against relying on this method for $\mathrm{PO}_{2}$ monitoring during the administration of this drug. The inaccuracy of $\mathrm{tCPO}_{2}$ appears to vary directly with dose. The effect is reversible when the drug is withdrawn.

\section{5} THE HUCH TRANSCUTANEOUS $\mathrm{PO}_{2}$ ELECTRODE IN SICK INFANTS. 2 Joyce L. Peabody, George A. Gregory, Mary M. Willis tute and the Depts. Of Pediatrics and Anesthesia, University of California, San Francisco, Calif.

We tested the transcutaneous $\mathrm{PO}_{2}\left(t c \mathrm{PO}_{2}\right)$ electrode developed by Huch et a1 in 15 infants $(550-4480 \mathrm{~g}$.). All infants had arterial catheters. We obtained 105 arterial blood samples while recording the $\mathrm{tcPO}_{2}$, blood pressure, respiratory rate, and relative perfusion. In addition, we measured Hct, total protein, bilirubin, limb perfusion, skin thickness, and skin, axillary, and environmental temperature and estimated peripheral capillary filling. We noted the gestational age, present age; birth weight, present weight, major diagnoses, drugs and other therapies of each infant.

In the 105 pairs of $\mathrm{PaO}_{2}$ and $t c \mathrm{PO}_{2}, \mathrm{r}=0.987$, and the SD of regression of the line described by $y=.952 x+2.65$ was \pm 3.75 . $95 \%$ of paired values showed discrepancies of $\langle 10 \%$. The electrode's accuracy was unaffected by gestational age, skin type, edema, blood pressure, temperature, diagnosis, or perfusion routinely encountered. In addition, we observed changes in $\mathrm{tcPO}_{2}$ during apnea, suctioning, periodic breathing, positioning, heel sticks and changes in respirator settings. 4 infants with $\mathrm{BW}<1000$ grams had periodic breathing and fluctuations in tcPO from 30 to $100 \mathrm{~mm} \mathrm{Hg}$. tcPO $>90 \mathrm{~mm} \mathrm{Hg}$. was observed repeatedly in these infants which had not been suspected by intermittent sampling.

We conclude that the method is reliable and useful for monitoring sick infants and that continuous monitoring may allow more precise regulation of $\mathrm{PaO}_{2}$ and reduce the risk of retrolental fibroplasia and improve respiratory care.
776 SILENT ALARMS AND INCUBATOR CONTROL. Paul H. Per $1-$ stein, Harry D. Atherton, Neil K. Edwards and James Medicine, Department of Pediatrics, Cincinnati.

The thermal protection provided by incubators is easily disrupted when power cords become unplugged, skin temperature thermistors dislodged and when inadequately heated gases flow into an infant's microclimate via headhoods or respiratory support devices. To assess the frequency of these and other impediments to predictable care, incubators in an active intensive care unit were monitored continuously while used in caring for 89 high-risk infants. Incubators were declared out of control when they failed to change their heater "on" or "off" state for longer than 20 minutes, which is more than 2 standard deviations greater than the mean cycling time of a properly functioning incubator in the study nursery. During the first 3 months of a 6 -month study, 43 infants were housed in the study incubators a mean of $117.5 \pm$ (SE) 19.0 hrs during which time the mean of malfunctioning time was $10.4 \pm$ (SE) $2.0 \%$. Following the initial study period, a computer-driven non-a'scible TV alarm system was used to automatically notify nursery personnel when incubator malcontrol occurred. The high incubator malcontrol rate tabulated during the first 3 months was reduced significantly ( $p<.001$ ) to $2.3 \pm(\mathrm{SE}) 0.77 \%$ during the subsequent 3 months, when 46 infants were each provided a mean of $123.5 \pm$ (SE) 17.4 hrs of care in the monitored incubators. The study demonstrates some common hazarcis associated with incubator care and how computer-generated non-audible alarms can be used surcessfully to warn of impediments to consistent care without adding to the already excessive noise levels in intensive care areas. THE TELEPHONE IN REGIONAL NEWBORN CARE. PaUL H. P世: ? stein, Neil K. Edwards and James M. Sutherland. Univ. Newborn death rates were compared in three community "study" hospitals before and after their nurseries were connected to a regional newborn intensive care center by direct unswitched telephone "hot lines." Death rates were also calculated for infants born in 4 "control", non-phone-connected hospitals serving populations similar to the study hospitals. Deaths occurring after transfex to the regional care center were reassigned to the hospitals of birth. Of 5037 infants born in the 3 study hospitals one year before the "hot line" phone installations, 55 died. This $10.9 / 1000$ live birth death rate is significantly higher $(p<.005)$ than the $8.1 / 1000$ deaths that occurred one year after the installation of the "hot lines", when 40 of 4946 infants died. In the 4 control hospitals, 46 of 4052 infants died $(11.4 / 1000$ bixths) one year before, and a similar 44 out of 3850 infants died (11.4/1000 bixths) in the year after the "hot line" service was installed in the study hospitals. Falling moxtality rates in the study hospitals were not associated with increases in the percentage of infants transferred to the regional center following the phone installations. However, there were significant increases in 1) the number of infants in whom the decision to transfer was made prior to 2 hours of age $(p<.001), 2)$ the mean admission tempexature of infants transfexred $(p<.025)$, and 3$)$ post-transfer survival rates $(p<.05)$. Direct unswitched telephone service provides an effec. tive mechanism for improving ongoing communications between regional care centers and the community hospitals they serve.

\section{UNIQUE APPLICATION TO THE NEONATE.}

778 Jeffrey J. Pomerance, Ricardo L. Liberman and Dept. Ped. and UCLA Sch. Med., Los Angeles (Spon. by B. M. Kagan) Thermography is the recording of infrared radiant heat patterns from a surface. Recorded surface temperatures are a funcIn adults, information about deep lying structures is ordinarily made by inference only as intervening structures insulate to a large extent. The term, and especially the preterm newborr, offers a unique opportunity to examine deep structures more directly. The small premature infant has almost no subcutaneous fat, very little muscle, and extremely thin skin. Therefore, heat radiated by "deep" structures $(1-2 \mathrm{~cm}$. away) may be visualized relatively unaltered at the surface. In addition, thermography may be applied to the neonate as originally used to detect differences in blood flow to the skin.

Anterior and posterior thermographic patterns have been recorded in 37 neonates over a 3 month period. Studies were performed under a radiant heater servo-controlled to an abdominal skin temperature of $36.0^{\circ} \mathrm{C}$. Heart, liver, and kidneys, being highly vascular structures and therefore warmer than surrounding tissues, are detected as "hot" areas on the body surface. Photographs of representative normal thermographic patterns will be demonstrated. A longitudinal study of an infant with a patent ductus arteriosus and congestive heart failure demonstrating a generally cooler chest following ligation will be shown. Thermographic evaluation of the viability of a pedicle flap in a neonate will also be presented. 


\section{9}

A NEW TECHNIQUE FOR LONG TERM ARTERIAL ACCESS IN THE HIGH RISK NEWBORN.

Gregory W. Prian (Spon. by L. Joseph Butterfield) Department of Perinatology, The Children's Hospital, Denver

The hazards of umbilical artery catheterization necessitates the adoption of an alternate arterial cannulation site for monitoring arterial pressure and oxygen tension in the critically ill newborn. A newly developed method of catheter placement in the temporal artery not only provided arterial access for substantially longer than that reported for radial artery cannulation but over 50 percent of the infants in which it was performed weighed less than 1500 grams.

Close adherence to the technique of arterial cannulation and catheter fixation is essential for prolonged arterial access. The method involves: (1) Doppler location of the temporal artery, (2) a 3-4 mm transverse incision directly over the vessel, (3) bevel down insertion of a winged hub teflon catheter with inner needle stylet, (4) securing the catheter with suture and collodoin, and (5) maintenance of catheter patency with a constant infusion of heparinized solution.

Temporal artery cannulation using this technique has thus far been performed 50 times in 41 infants with severe respiratory distress. The patients weighed between 540 and 3900 grams. Catheters remained operational for an average of 6.5 days (24 to 396 hours) with 45 percent of all catheters providing arterial samples for more than 5 days. The cannulae worked well for arterial pressure monitoring and pulse contour matched that of the umbilical artery in infants with catheters in both vessels.

POVIDONE IODINE (PI), ETHYL ALCOHOL (AL) AND TRIPLE DYE

780 (TD)--CONTROL OF NEONATAL STAPHYLOCOCCAL (S. aUreuS) COLONIZATION. S. PYATI, R. S. RAMAMURTHY, T. KRAUSS AND R. S. PILDES, COOK COUNTY HOSP. DEPTS.PEDIAT., CHGO. MED. SCHOOL, AND UNIV. OF ILL., CHICAGO.

The need for maintainance of low $S$. aureus colonization in the neonate has led to the search for a topical agent which would achieve this without significant absorption. Over a two year period, four groups of infants were studied concurrently: TD (once), AL 70\% (q 8 hrs $\times 3$ days) and $\mathrm{Pl} 1 \%$ (q $8 \mathrm{hrs} \times 3$.days) were applied to the umbilical cord and an inch of surrounding skin after the initial bath. Anterior nasal and cord cultures were taken on day 3 of nursery stay. Nasal colonization using the three agents (TD 617 neonates, 8.1\% pos.; AL 357, 8.9\% pos.; PI $204,2.5 \%$ pos.) was significantly lower $(P<.01)$ than in the control group $(305,16 \%$ pos.). The nose and cord colonization using PI was lower $\left(P<<_{0} 01\right)$ than TD or AL; there was no difference between TD and $A L$. Alcohol levels in 6 infants on day three were not detectable. Total plasma lodine $\left(l_{2}\right.$-MeantS.D.) in cord blood of six controls (C) was similar to that of $\overrightarrow{12} \mathrm{PI}$ neonates $(15.9+7.7$ vs $16.6+9 \mu \mathrm{g} \%$ respectively). At 3 days of age, a higher $\mathrm{I}_{2}$ level $(\overline{\mathrm{P}}<001)$ was seen in the $\mathrm{Pl}$ group $(C-15.9 \pm 3.8$ vs PI $70.0+46.9 \mu \mathrm{g} \%$ ). Reducing $P I$ to a single application also resulted in a higher ${ }_{2}$ level at 3 days as compared to controls $(26.2+4.3$ vs $15.9+3.8 \mu \mathrm{g} \%)$. In addition, colonization increased $(P<O T)$ with a single PI application. Therefore, PI cannot be recommended for routine cord care. TD or AL may be equally effective in maintaining low colonization of $S$. aureus.

\section{MULTI-PATIENT ON-LINE COMPUTER BASED PERINATAL} IMPROVED PATIENT CARE. H. R. Rey, S. Caritis, R.I. Stark, R. Dwarka, E. T. Bowe, M. Finster, R. Vande Wiele, L. S. James Coll. of Phys. \& Surg., Columbia Univ., Div. of Perinatology, New York. A data mangement system has been designed to provide a reliable and dynamic on-line computer evaluation of risk factors associated with each patient's pregnancy, labor and delivery, by integrating information obtained from bedside monitors with biophysical and biochemical data and medical history. Using a mini-computer, the system automatically acquires stores and displays data for up to 32 patients, and allows for manual entry of other pertinent information. In addition to data acquisition and storage, the computer provides a multi-dimensional approach to data analysis includr ing 1) automatic pattern recognition of important events during labor, 2) instantaneous on-line analysis of the beat-fo-beat heart rate, 3) multiparametric analysis of uterine activity and the progress of labor, 4) mathematical predictive scoring system for neonatal outcome based on a set of cumulative risk indices generated by the computer from pregnancy, labor and delivery data. This system has been successfully implemented and is currently used both clinically and as a research tool. The system will lead: to a rapid and efficient build-up of a data base for statistical analysis, to improved communication and information flow between members of the staff and areas of the hospital, to a more precise prediction of neonatal outcome on the basis of antepartum and intrapartum risk factors, and to improved patient management.
THE EFFECTS OF A SINGLE INFUSION OF HYPERTONIC NaHCO

782 IN NEONATES WITH ACIDOSIS. Philip G. Rhodes, Robert T. Ha11, Stanley Hellerstein, The Children's Mercy Hospita1, Kansas City, Missouri.

The effects of infusion of hypertonic $\mathrm{NaHCO}_{3}$ on acid-base equilibrium, plasma water and solute, and red cell composition have been examined in 20 infants with IRDS and 4 with other forms of neonatal asphyxia. One molar $\mathrm{NaHCO}_{3}$ was infused at 0.3 $\mathrm{ml} / \mathrm{kg} / \mathrm{min}$ to provide a dose in $\mathrm{mEq}=(0.4)$ (body weight in $\mathrm{kg}$ ) (base excess). Most infants received ventilatory assistance during the infusion.

\begin{tabular}{|c|c|c|c|}
\hline Item & Baseline & $3^{\prime}$ Post Inf. & $30^{\prime}$ Post Inf. \\
\hline$\overline{\mathrm{PaO}_{2}}$ & $82 \pm 24$ & $78 \pm 48 \%$ & $97 \pm 50 *$ \\
\hline $\mathrm{PaCO}_{2}$ & $39 \pm 9$ & $44 \pm 10 * *$ & $41 \mp 11 *$ \\
\hline WB $\mathrm{pH}$ & $7.20 \pm .10$ & $7.30 \pm .06 * *$ & $7.31 \pm .08 * *$ \\
\hline $\mathrm{RBC} \mathrm{pH}$ & $7.09 \pm .10$ & $--\overline{-}$ & $7.19 \pm .08 * *$ \\
\hline $\mathrm{P} 1[\mathrm{Na}+]$ & $137.8 \pm 5.1$ & $\pm 5.0 * *$ & $142.9 \pm 4.8 * *$ \\
\hline $\mathrm{P} 1$ [ $\mathrm{mosm}]$ & $287.3 \pm 17.8$ & $296.7 \pm 16.6 * *$ & $294.3=$ \\
\hline $\mathrm{RBC} \mathrm{H}_{2} \mathrm{O}$ & $664.5 \mp 9.1$ & $-\overline{--}$ & $654.9 \mp 5.6 * *$ \\
\hline $\mathrm{RBC}[\mathrm{K}+]$ & $135.2 \pm 4.6$ & ---- & $140.7 \pm 5.8 * *$ \\
\hline $\mathrm{RBC}\left[\mathrm{Cl}^{-}\right]$ & $76.8 \pm 4.0$ & ---- & $71.4 \pm 4.6 * *$ \\
\hline
\end{tabular}
$*=\mathrm{N} . \mathrm{S} . * *=\mathrm{p}<0.001$ Paired $\mathrm{T}-\mathrm{Test}$, Compared with Baseline
The infused $\mathrm{NaHCO}_{3}$ provided sufficient base to buffer some of the protons initially present in $\mathrm{ECF}-\mathrm{H}_{2} \mathrm{O}$ as well as those produced during or subsequent to the infusions. There was a significant rise in Plasma[mosm], a decrease in $\mathrm{RBC}-\mathrm{H}_{2} \mathrm{O}$ and $\mathrm{RBC}\left[\mathrm{C1} 1^{-}\right]$and an increase in RBC [ cation]. The lack of significant change in $\mathrm{PaCO}_{2}$ may be due, in part, to the use of ventilatory assistance
during the infusion period.

783 CARE NURSERY RELATED TO INTRAVENOUS FLUID CONTAMINABrown University . Barbara S. Ross, Georges Peter and william Roger Williams Gen. Hosp., Dept. of Pediatrics, Providence, R.I.

Duxing a 6-day period in August 1975,9 infants $(2-60$ days old) in an intensive care nursery developed type 68 Klebsiella Pneumoniae (K.p.) sepsis, 3 of whom had meningitis and 5 had scalp abscesses at intravenous (IV) infusion sites. One additional non-septic infant developed a K.p. scalp abscess at an IV site. Nine of the 10 cases occurred in the intermediate care room (ICR). All infected infants received IV fluids by peripheral vein and 3 non-infected infants did not. One of 18 IV fluid recipients in the maximum care xoom (MCR) was infected. Cultures of IV fluid yielded type $68 \mathrm{~K} . \mathrm{p}$. in 3 cases from the tubing adjacent to the scalp vein needle. Just prior to the epidemic, a policy change resulted in the addition of electrolytes to IV fluids in the nursery. Cultures of 90 randomly obtained rectal swabs discinsed K.p. colonization rates of $58 \%$ in the entire nursery, $62 \%$ in ICR and $12.5 \%$ in the MCR. Typing of 14 isolates demonstrated multiple K.p. serotypes in adaition to the predominant type $68(8 / 14)$. The epidemic probably resulted from contamination of IV fluids in the nursery resulting from an inadvertent change in technique. This epidemic denotes another potential source for nosocomial infection in an intensive care nursery

\section{DISSIMILAR CARDIOVASCULAR RESPONSES TO APNEA IN THE}

784 PREMATURE. Jonelle C. Rowe, William J. Flanagan, David E. Woodrum, W. Alan Hodson, Dept. of Pediatrics Univ. of Washington, Seattle, washington.

The relationship of cardiovascular changes to apneic events in premature infants is inadequately described and the mechanisms poorly understood. Respiratory frequency, intra aortic blood pressure, heart rate and peripheral limb blood flow were measured during 32 apneic episodes (duration> $20 \mathrm{sec}$.) that occurred in the lst 2 weeks of life in 7 premature infants (27-33 wks gest.). The data indicated there were 2 recognizable pattexns of cardiovascular response to apnea. 5 infants demonstrated decrease in peripheral blood flow $(\bar{X}=47 \pm 98)$ during apneic periods. Blood pressure remained constant or slightly decreased during decrease in flow indicating that reduction in flow was due to an increase in peripheral resistance. Continuous recordings of heart rate and respixation in these infants during the lst 5-7 days of life demonstrated that bradycardia ( $<100$ BPM) occurred with 70-100\% of apneic episodes. 2 infants failed to manifest a decrease in peripheral blood flow during apnea. Continuous recordings of heart rate and respiratory frequency in these infants demonstrated that bradycardia failed to occur with apnea $80 \%$ of the time. These 2 distinct patterns of cardiovasculax response to apnea suggest diffexent physiologic mechanisms occur during apneic episodes. The lack of an "appropriate" cardiovascular response may indicate an abnormality in CNS integration of cardiorespiratory control. Delineation of the patterns of apneic response and their cardiovascular correlates is necessary before one can establish the indications for or evaluate the results of therapeutic intervention. 
THE INFLUENCE OF RUPTURED AMNTOTIC MEMBRANES (ROM) ON

785 FETAL OUTCOME. Elsa Sel1 and Thomas R. Harris, Dept. (Spons. by J. J. Corrigan).

Evidence suggests that infection risk and mortality increase with more time between ROM and delivery. The effect of ROM on the incidence of IRDS is unclear. We compared records on 694 infants with ROM $<12$ hrs. and 152 infants with ROM $>24$ hrs. before delivery. The incidence of IRDS was significantly lower at 28-33 wks. gestation when ROM was $>24 \mathrm{hrs}$.

\begin{tabular}{lcl} 
Wk. Gestation & $\begin{array}{c}\text { Incidence of IRDS } \\
\text { ROM }<12 \text { hrs. }\end{array}$ & ROM $>24 \mathrm{hrs}$. \\
\hline $28-29$ & $19 / 24 *(79 \%)$ & $3 / 8\left(37 \frac{1}{2} \%\right) * *$ \\
$30-33$ & $85 / 133(64 \%)$ & $15 / 41(37 \%) * *$ \\
$34-37$ & $106 / 327(32 \%)$ & $15 / 70(21 \%)$ \\
\hline$*=$ number of infants & $* * \%=\mathrm{p}<.05$
\end{tabular}

Furthermore, the incidence of IRDS with respiratory failure (RF) was lower in infants with ROM $>24 \mathrm{hrs}$.

\begin{tabular}{lll}
\hline \multicolumn{3}{c}{ Incidence of IRDS with RF } \\
Wk. Gestation & ROM $<12$ hrs. & ROM $>24$ hrs. \\
\hline $28-29$ & $13 / 24(55 \%)$ & $3 / 8\left(37 \frac{12}{2} \%\right)$ \\
$30-33$ & $48 / 133(40 \%)$ & $7 / 41(17 \%) * *$ \\
$34-37$ & $49 / 327(15 \%)$ & $5 / 70(7 \%)$ \\
\hline
\end{tabular}

There was no significant difference in the incidence of sepsis or morcality between ROM groups. We conclude that $>24$ hrs. between ROM and delivery will significantly reduce the incidence of IRDS in 28-33 wk. infants, and IRDS with RF at 30-33 wks. This benefit is not accompanied by a higher incidence of sepsis or mortality.

THE MULTISYSTEM INVOLVEMENT OF THE ASPHXXIATED NEWBORN. William R. Sexson, Sandra B. Sexson, John E. University of Mississippi Medical $\frac{\text { Rawson and Alfred }}{\text { Center, Department of Pedia- }}$ trics, Jackson

The central nervous system, with its involvement manifested by seizures and lethargy, has been the organ system most frequently recognized in association with perinatal asphyxia. The multiple organ system involvement in human neonates was implied from recent fetal monkey organ blood flow data following intrauterine asphyxia. A review of 6315 deliveries over the 21 months from 1 Jan. 1973 elucidates the potential spectrum of organ involvment. Out of 59 term infants found with 5 minute Apgar scores of 5 or less, 38 had symptoms within the first five days of life. These symptoms were sufficient to differentiate them from the normal infant and involved 5 organ systems. The frequency of involvement was as follows: pulmonary, $39 \%$; cardiovascular, $34 \%$; CNS, $24 \%$; GI, $20 \%$; and renal, $15 \%$. $53 \%$ of patients with symptoms had involvement of more than one system. When the combined $1+5$ minute Apgar score was used, $78 \%$ of infants with a value of 5 or less had symptomatic involvement, while only $25 \%$ with a combined Apgar score of 6 or more exhibited symptoms. If only the 5 minute Apgar score was used, $63 \%$ of the infants were identified. From the data presented, it is evident that intrauterine asphyxia affects multiple organ systems in the human full term infant. A combined $1+5$ minute Apgar score is more useful as a predictor of involvement than the 5 minute Apgar score alone. The specific organ involvement could not be predicted from the Apgar score. With multiple organ involvement, the CNS was not invariably included.

787 SOME EFFECTS OF THEOPHYLLINE IN INFANTS WITH APNEA Daniel C. Shannon, Felicita Gotay. Harvard Medical School

Service, Boston.

Theophylline, $11 \mathrm{mg} / \mathrm{kg} / \mathrm{day}$, was given for 5 days, 8 doses/d, to 10 infants $(\bar{x}$ B.W. $=1.75 \mathrm{Kg})$ with gestationally-related apnea and achieved a mean pre-dose serum concentration of 16.1 $\mathrm{ug} / \mathrm{ml}$ (range $11-21 \mathrm{ug} / \mathrm{ml}$ ). The frequency of apnea $\geq 20 \mathrm{sec}$ per 12 hour recording was reduced from 12.0-0.8 $(\mathrm{p}=<.01)$. They were compared to 10 infants ( $\bar{x}$ B.W. $2.25 \mathrm{Kg}$ ) without apnea. Lowsolute formula was given by continuous $N-G$ infusion. All repeat measurements wexe performed after 5 days of theophylline and compared to a similar time span in un-treated normal controls. Respiratory frequency ( $46 / \mathrm{min})$, systolic blood pressure (Doppler) and $\mathrm{PaO}_{2}, \mathrm{PaCO}_{2}$ and $\mathrm{pH}$ were unchanged. Heart rate increased 17/ min $(p=<.01)$. Blood glucose, pre-dose, increased from 74 to $102 \mathrm{mg} \%(p=<.02)$. Serum concentrations of $\mathrm{Na}, \mathrm{K}+, \mathrm{Ca}, \mathrm{Ca}+\mathrm{H}$, $\mathrm{PO} 4$ and $\mathrm{Mg}$ did not change. Urine volume $92 \mathrm{ml} / \mathrm{Kg} / \mathrm{day}$ and $\mathrm{Na}$ content $25 \mathrm{mEq} / \mathrm{L}$ did not change. Urine excretion of cAMP was related to post-conceptional age $(r=0.86)$ and weight $(r=0.75)$ in all 20 infants. Urine cAMP increased from 104 to $149 \mathrm{nN} / 24 \mathrm{Hr}$ $(p=0.02)$ with theophylline. Excretion of epinephrine $(r=0.72)$ and norepinephrine $(r=0.76)$ were related to weight but not age and did not increase with theophylline. Theophylline prevents apnea without altering fluid and electrolyte or acid-base balance. It may act through increased cAMP or aerobic substrate delivery at a key site.
OXYTOCIN AND NEONATAL JAUNDICE: Thomas R.C. Sisson,

788 Tonq S. Park, Edward Bartlett, Sudha Rao (Spon. by A. DiGeorqe), Temple University School of Medicine. Department of Pediatrics, Philadelphia.

A prospective study of the relation of maternal oxytocin administration and neonatal jaundice was done in 122 infants: of these 60 were controls, in 32 labor was augmented by oxytocin, and labor was so induced in 30. Infants SGA, pre-term, who had a potential for hyperbilirubinemia from sepsis, HDN or other cause, or whose mothers were at high risk were exciuded. Results are tabulated below. Bilirubin concentrations are in $\mathrm{mg} / \mathrm{dl}$ :

\begin{tabular}{|c|c|c|c|c|c|c|c|c|c|c|}
\hline \multirow{3}{*}{$\begin{array}{l}\text { Group } \\
\text { B.Wt. } \\
\text { Cont T. } \\
3230\end{array}$} & \multirow{3}{*}{$\frac{n}{60}$} & \multirow{3}{*}{$\begin{array}{l}\text { hrs. } \\
7 \mathrm{br} . \\
6.9\end{array}$} & \multirow{3}{*}{$\begin{array}{c}\text { hrs. } \\
\text { oxyt } \\
0\end{array}$} & \multirow{3}{*}{$\begin{array}{l}\text { ava. } \\
\text { bili } \\
3.5\end{array}$} & \multicolumn{2}{|c|}{$0-4.9$} & \multicolumn{2}{|c|}{$5-9.9$} & \multicolumn{2}{|c|}{$10-16$} \\
\hline & & & & & & $\%$ & & $\%$ & & $\%$ \\
\hline & & & & & 38 & 64 & 19 & 32 & 3 & 5 \\
\hline $\begin{array}{l}\text { Augm. } \\
3335\end{array}$ & 32 & 17.4 & 4.0 & 6.2. & 12 & 38 & 14 & 44 & 6 & 18 \\
\hline $\begin{array}{l}\text { Indct. } \\
32.6 ? \text { ? }\end{array}$ & 30 & 8.0 & 6.8 & 7.9 & 5 & 17 & 13 & 43 & 12 & 40 \\
\hline
\end{tabular}

In vitro study of the effect of oxytocin (to $1200 \mathrm{mU}$ ) on UDP gTucurony t transferase activity in liver microsomes showed no enzyme inhibition. We conclude that oxytocin qiven to mothers in labor is related to increase of jaundice in newborns, and that higher concentrations are more frequent when oxytocin in aiven in higher dose per unit time. This seems not to be an effect on bilirubin conjugation - but possibly is due to inhibition of ligand acceptance of bilirubin in the liver cell.

\section{9} EFYECTS OF PHOTOTLERAPY ON NEONATAL BEHAVIOR. David M. Snyder, Robert Telzrow, Edward Tronick, T. Children's hospital Medical Center, Boston, Mass.

This study documented changes in newborm behavior associated with phototinerapy using the Neonatal Behavioral Assessment Scale (Brazelton, 1973). 3 groups each with' 10 neonates were examined; a hyperbilimbinemia group treated with phototherapy, a control group with hyperbilirubinemia not treated with phototherapy, and a group of non-jaundiced controls. The phototherapy group was examined before, during and after phototherapy, the 2 control groups on postnatal days 3,6 , and 10 . All 30 subjects were full term AGA and had no perinatal complications other than hyperbilirubinemia. Medical management decisions were made exclusively by the infants' primary pediatricians. Both control groups showed improvement over time in alertness and orientation to visual and auditory stimuli as expected. The phototherapy group failed to show improvement in these areas, even at 2 weeks of age. In state organization and control, the normals showed progressive improvement. Jaundiced controls failed to improve through day 6 , but by day 10 were indistinguishable from normals. The phototherapy group failed to show this improvement as late as 2 weeks of age. We conclude that 1 ) neonates treated with phototherapy have altered functioning lasting well beyond the termination of phototherapy, 2) these alterations would produce a diminished ability to elicit optimal parental responsiveness, 3) the resulting disruption of normal parent-infant interactions may contribute to the observed persistence of apparent CNS disorganization associated with hyperbilirubinemia and phototherapy.

790 William $\bar{T}$. Speck, (Intr. by Richard $\mathrm{E}$. Behrnan). INTRACELLULAR UNA-MOD IFYING POTENTIAL OF PHOTOTHERAPY. Col. of Physicians and Surgeons, Columbia University, Babies llosp., Department of Pediatrics, New York, New York 10032

The effects of environmental agents on child health is a subject of considerable intersst. Of special concern are the long term effects of such agents and more specifically, their carcinogenicity, mutagenicity and/or teratogenicity which are believed to result in part from the ability of such agents to modify intracellular DNA. Little information is available on the intracellular consequences of high intensity illumination with visible light or phototherapy. The purpose of the present study is to determine whether DNA isolated from human cells growing in tissue zulture undergoes structural modification as a result of exposure to high intensity visible light in the absence of added photosensitizers. Analyses of such DNA on alkaline sucrose gradients reveals a diminution in molecular size following illumination. The structural lesion is repairable when the treated cells are subsequently incubated in the dark. These changes are observed at wavelengths of 1 ight $(450 \mathrm{~nm})$ identical to those utilized in phototherapy and with a total light dosage $\left(70.4 \mathrm{KJ} / \mathrm{m}^{2}\right)$ representing only $5 \%$ of that received by a newborn infant undergoin!s phototherapy in our nursery for a 24-hour period $\left(1.3 \times 10^{3}\right.$ $\mathrm{KJ} / \mathrm{m}^{2}$ ). In view of the known relationship between DiNA-modifying activity and mutagenic and/or carcinogenic potential, the present results suggest that phototherapy is a complex process with an inherent potential for serious long-term sequelae. 
791

EFFECT OF DECREASED OXYGEN AFFINITY FOR HEMOGLOBIN ON THE DISTRIBUTION OF REGIONAL BLOOD FLOW IN ANEMIC NEWBORN LAMBS. A.R. Stark, R. Hollister, R.E.Forster and M. Delivoria-Papadopoulos. Univ.of Pa., School of Med.,Phila., Pa Acute changes in the distribution of blood flow to different organs of newborn lambs following exchange transfusion which increased blood $P_{50}$ from a mean of $22.5 \pm 2.9$ to $32.3 \pm 3.1 \mathrm{~mm} \mathrm{Hg}$ but decreased Hct from $36.9 \pm 5.3$ to $26.5 \pm 1.4$ were studied by the microsphere method. Six lambs, 4 days old, were injected through a chronic catheter in the left ventricle with $15 \mu$ plastic spheres labelled with one of either $141 \mathrm{Ce}$ or $85 \mathrm{Sr}$ for baseline blood flows. An exchange transfusion was then performed with maternal blood and a second injection of microspheres labelled with the other isotope was given 3 hours later. Mean values for blood flow $\pm S D$ in $\mathrm{ml} / \mathrm{min} / 100 \mathrm{~g}$ tissue before and after exchange transfusion respectively were as follows: adrenal:245 \pm 77 and $198 \pm 58(p<0.05)$, kidney: $221 \pm 20$ and $132 \pm 55(p<0.01)$, heart: $111 \pm 22$ and $158 \pm 60(p<0.05$ brain: $77 \pm 19$ and $91 \pm 11(p<0.20)$, cardiac output: $241 \pm 84$ to $181 \pm 70$ $\mathrm{m} 1 / \mathrm{min} / \mathrm{kg}(\mathrm{p}<0.20)$. Five animals which were studied in a similar manner but not receiving an exchange transfusion demonstrated no significant changes in cardiac output or organ blood flow. Although the net effect of the increased blood $P_{50}$ and the lower Hct on $\mathrm{O}_{2}$ transport did not significantly change cardiac output, there was a redistribution of blood flow among the organs. Thus, anemia may counteract the effect of an increased $P_{50}$ in newborns undergoing exchange transfusion, particularly those at risk, and increase the stress on individual organs even though the effect o; the circulation as a whole is to reduce circulatory demands.

A NEW APPROACHTO PREDICTING THE HI-RISK NEONATE.

792 A SCORING SYSTEM FOR INTRAPARTUM MONITORING R. I. Stark, H. Rey, R. Dwarka and L. S. James, Coll. of Phys, \& Surg., Columbia Univ., Div, of Perinatology, New York.

A reliable scoring system for evaluating intrapartum fetal heart rate (FHR) and uterine pressure (UP) monitor records has been developed. The FHR-UP score is derived from a weighted assessment of the duration and severity of 12 different FHR-UP patterns and baseline characteristics defined by the Standard International Nomenclature. A retrospective analysis of $424 \mathrm{elec}-$ tronically monitored patients of gestational age $>32$ weeks revealed strong, positive and statistically significant relationships between FHR-UP score and Apgar scores $(p<.001)$, incidence of neonatal complications requiring intensive care $(p<0.001)$, and the incidence of suspicious or abnormal clinical assessment of outcome at discharge $(p<0.001)$. From information theory and analysis of R.O.C. curves, a cut-off point was derived to distinguish between "normal" and "abnormal" scores. Using the FHR-UP score alone, decision matrices, and Bayes' theorem of posterior probabilities, prospective identification of a fetus at risk for neonatal complication is increased 25fold. Conversely, for a fetus with a normal score the assurance of an uncomplicated neonatal course is improved 10-fold. The FHR-UP score together with other "scoring" systems can provide a cumulative index of fetal risk factors which will advance the understanding of the relationships between risk factors of pregnancy, perinatal events, and outcome. The score has been adapted to an on-line computerized system, enhancing its predictive value and permitting immediate clinical application.

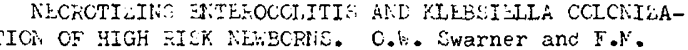

793 Barnes, (Spon. by Eoseph J. Guilligan), Departments of Pediatrics, Lome Iinda tiniversity School of liedicine and liniversity of California-ran Liego, Loma Linda and aan Diego, Calif.

A prosfective study of the bacterial colonization of neonates in two Neonatal Intensive Care Hurseres (IIICV) during the past four years has shown a striking: association between the occurance of Necrotizine. interocolitis (NEC) and Klebsiella colonization. A total of 52 cases of $\mathrm{NE} . \mathrm{C}$ have occured during 1972-75. 34 occured in the Ban Diego NICL and 18 occured in the Loma Linda VICT. The following table summarizes pertinent data.

Cases of $\mathrm{MiC}$

$\%$ with $K$ lebsiella

$$
\begin{gathered}
\operatorname{san} \\
34 \\
94 \%
\end{gathered}
$$

\% who had umbilical catheter

Hyperalimentation

Exchange transfusion

Fespiratory listress Syn.

vit. $<1500$ erams

Wit. $>150 \mathrm{C}$, erams

Patent Ductus Arteriosis Mortality

$\begin{array}{ccc}\text { an Liego } & \text { Ioma Iinda } & \text { Total } \\ 34 & 18 & 52 \\ 94 \% & 89 \% & 90 \% \\ 55 \% & 89 \% & 67 \% \\ 0 \% & 56 \% & 19 \% \\ 12 \% & 50 \% & 25 \% \\ 41 \% & 61 \% & 48 \% \\ 73 \% & 55 \% & 67 \% \\ 26 \% & 44 \% & 32 \% \\ 52 \% & 33 \% & 46 \% \\ 32 \% & 50 \% & 38 \% \\ \text { redominent organism found in the }\end{array}$

Klebsiella was by far the predominent stool, blood and peritoneum, although . coli was found in 9 pts. and $r$ seudomonas in 4. The occurance of most of the NEC was clustered durinf. periods when the colonization rate of Klebsiella was hi.gh. Nine cases of NEC occured during one month when a Rhamnose
neq. Kiebsiella colonized the NICU.
ANALYSIS OF NEWBORN INTENSIVE CARE BY TIME-LAPSE 793 A PHOTOGRAPHY. Jon E. Tyson, John E. Clarkson, Karyn Dept, of Pediatrics, Hamilton, Ontario, Canada.

The activities of nurses, residents and fellows providing newborn intensive care were studied by time-lapse photography and multivariant computer analysis. Six of 40 frames taken each hour during a 23 day period were evaluated (3312 total frames) excluding the first two weeks after camera placement. The time spent in various activities (bedside care, charting, handwashing, absent, or other) was estimated for persons caring for 4 infants. The amount of care needed by each infant was assessed independently by a clinical scoring system (infant care score). Physician activities were significantly diminished at night and on weekends. Only 3.28 of frames on weeknights vs 13.58 on weekdays showed a resident at any of the 4 beds. The average nurse spent 428 of each shift in bedside care and an additional $20 \%$ in the filming area. Nursing activities were unaffected by time-related variables (day vs night, weekday vs weekend, nursing rotations). Regression analysis showed a linear two to three fold increase in bedside nursing time with increasing infant care score. Individual nurses differed significantly in two ways: (1) the relation of mean bedside time to that expected from infant care scores (2) the gradient of bedside time on increasing infant care score. Differences between nurses accounted for as much of the total variation in bedside time $(30 \%)$ as did differences between infants in their care scores.

We relate these observations to our previously described diurnal variation in quality and outcome of newborn intensive care.
NOISE POLLUTION IN THE NEONATAL INTENSIVE CARE UNITS:

794 A POTENT IAL HAZARD. D. Vidyasagar, M. Joseph and L. Hamilton, Abraham Lincoln School of Medicine, University of Illinois Hospital, Department of Pediatrics, Chicago, Ill. Because of increasing concern over effects of high noise levels on neonates, we measured environmental and equipment noise in 9 different neonatal units using a Bruel \& Kjaer sound level meter. Measurements were made at the midpoint of the rooms of neonatal intensive care units (NNICU), inside the incubators and close to respiratory devices within the unit. Data, grouped into that obtained from (1) respiratory care area (RCA) and (2) nonrespiratory care area (NRCA) for analysis, is shown below. Numbers indicate sound pressure levels (SPL) in decibels (dB).

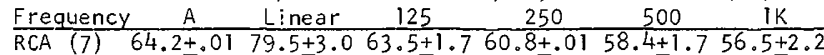
NRCA (9) $55.4+1.1 \quad 65.6+1.0 \quad 53.9+1.3 \quad 51.2+1.5 \quad 52.2+1.2 \quad 48.1 \mp 1.4$

The SPL's were significantly higher $(\bar{p}<.01)$ in the RCA than in NRCA on $A$ and linear scale and different octave band frequencies. The differences were related to number of equipment in the unit rather than to number of infants. SPL of different respirators ranged from 51-7I dB: CPAP devices had 64-7l dB; air compressor had $67 \mathrm{~dB}$; suction machines had SPL of $68 \mathrm{~dB}$ on A scale. Further it was shown. in our unit that high noise levels were similar both in $A M$ and $P M$ hours suggesting that high noise levels persisted throughout the day. Data from industrial standards indicate that 8 hour exposure to high noise level is damaging to hearing. Since many high risk infants spend prolonged length of time in this environment, there is need for further evaluation of noise levels in the NNICU and its effect on the outcome of infants.

795 RESPONSE OF INEUROTOXIC BILIRUBIN TO ALBUMIN INFUSION. $\frac{\text { Glenn W. Waterkotte, Jay P. Goldsmith, and Montgomery }}{\text { C. Hart. (Spon. by William J.R. Daily) Division of }}$ Reproductive Medicine, St. Joseph's liospital, Phoenix, Arizona.

Kernicterus is thought to result from a bilirubin ( $\mathrm{Br}$ ) fraction which is unconjugated and unbound to albumin (neurotoxic bilirubin $\mathrm{NBr}$ ). We have studied the effect of the infusion of adult saltpoor albumin $(1-2 \mathrm{gms} / \mathrm{kg})$ in 7 Coombs negative newborns with significant levels of $\mathrm{irr}\left(20.02 \mathrm{mgms}_{\%}^{\prime}\right)$. The measurement of $\mathrm{NBr}$ was made by solvent extraction, separating this fraction from the total unbound (conjugated and unconjugated) $\mathrm{Br}$ eluted by standard Sephadex column chromatography. Glucose, $\mathrm{pH}$, albumin, total Br, total unbound $B r$, and $i{ }^{\prime} B r$ were measured at fixed time intervals. Patient weights ranged from 1162 to 3376 gms (mean 1924 gms); total $\mathrm{Br}$ ranged from 6.5 to $24.8 \mathrm{mgms} \%$ (mean $13.4 \mathrm{mgms} \%$ ); and $\mathrm{NBr}$ ranged from 0.02 to 0.10 mgrms\% (mean 0.05 mgms\%).

In every study serum aloumin rose and $\mathrm{HBr}$ decreased to zero by 1-2 hours after albumin infusion. The effect on total $\mathrm{Br}$ was variable. In 2 patients ( 3 studies), no alternative therapy was required. Significant levels of $\mathrm{NBr}$ reappeared in 5 patients by 4 hours post-infusion, requiring exchange transfusion. No adverse effects of the infusion were noted. Two patients eventually died; in one of these ( $1200 \mathrm{gms}$, highest total $\mathrm{Br} .8 .3 \mathrm{mgms} \%$ ) both albumin infusion and exchange transfusion failed to prevent pathologic kernicterus. These data suggest that albumin infusion may represent a clinical alternative to, or interim therapy before, exchange transfusion for the management of neurotoxic bilirubin in selected patients. 
796 CLINICAL SIGNIFICANCE OF UNBOUND BILIRUBIN.

The serum unbound bilirubin concentration (UBC) reflects the toxic potential of bilirubin. Under usual clinical conditions the $\mathrm{UBC}$ is determined by (1) the total bilirubin concentration (TBC), (2) the albumin concentration (AC), (3) the binding affinity $\left(K_{1}\right)$, and $(4)$ the binding capacity $(\mathrm{N})$, i.e. the bilimbin/albumin molar ratio at saturation of the primary binding sites (normally $\mathrm{N}=1$ ). UBC and binding parameters were determined in 320 sera from 236 newborns using the peroxidase assay $\left(27^{\circ} \mathrm{C}\right)$

Clinical evidence of kernicterus occurred in 2 infants with estimated UBCs of 29 and $23 \mathrm{nmol} / 1$. No kernicterus occurred when UBC was maintained below $20 \mathrm{nmol} / 1$. Serum titrations with bilirubin helped to predict the TBC at which a given infant would reach 20nmol/1. This level was achieved at TBCs ranging 19-32mg\% (mean $25.9 \mathrm{mg} \%$ ) in 36 healthy term infants, and $8.5-28 \mathrm{mg} \%$ (mean $16.7 \mathrm{mg} \%$ ) in 101 sick premature infants. This variation can account for the difficulty in using TBC to predict the risk for kernicterus in sick infants.

The poor serum binding in sick premature infants was due to low $A C$ and binding affinity. Mean $K_{1}$ in 21 normal term infants was $20.4+4.6 \times 10$ compared to $9.0+3.2 \times 10$ in 41 premature infants with $\mathrm{HMD}^{-}(\mathrm{p}<.001)$. Decreased binding capacity $(\mathrm{N}<.80)$ was observed in only 13 infants, and was unrelated to gestational age.

Measurements of UBC and serum binding may be used as a guide for phototherapy, exchange transfusion, and hospital discharge planning of jaundiced but otherwise healthy newborns.

MEASUREMENT OF TOTAL, MUSCLE AND SKIN PERIPHERAL

797 BLOOD FLOW IN NEWBORN INFANTS. Paul Y.K. WU, Gonzalo Guerra, Woon H. Wong, Norman E. Levan. Univ. of So. Cal ifornia Sch. of Med., Los Angeles County-USC Medical Center. Depts. of Pediatrics and Internal Medicine (Dermatology).

Aithough data on total blood flow in the limbs of newborn infants are available, little is known of the distribution of blood flow between the skin and deeper tissues. Previously, we reported that the alteration in blood flow during phototherapy is due to change in skin blood flow rather than blood flow through deeper tissues (Ped. Res. 8:257, 1974). The electrocapacitance plethysmograph with local counter pressure was used to measure total, skin and muscle peripheral blood flow in 86 well newborn infants, ( 56 preterm and 30 term). Mean blood flow expressed as $\mathrm{ml} / 100 \mathrm{ml}$ tissue/min were:

\begin{tabular}{|l|r|c|c|}
\hline \multirow{4}{*}{ Preterm } & TOTAL & MUSCLE & SKIN \\
\cline { 2 - 4 } Term & 13.20 & 5.46 & 7.74 \\
& 8.80 & 3.50 & 5.30 \\
\hline
\end{tabular}

Daily measurement of blood flow in 12 preterm infants during the first week of life did not show any significant changes when infants were at "rest" with skin temperature maintained at $36.5^{\circ} \mathrm{C}$. Blood flow increased with activity and change in skin temperature. Effect of exposure to radiant heat from radiant heat warmers was studied in 12 infants. Blood flow was increased from 65 to $90 \%$, the greater increment being in skin rather than in deeper tissues.

798

HYDROCORTISONE THERAPY IN MECONIUM ASPIRATION SYNDROME (MAS): A CONTROLLED STUDY - T. Yeh, G. Srinivasan, V. Harris, and R. S. Pildes, Cook County Hosp. Dept. Pediat., Univ. of III. Coll. of Medicine, Chicago

To evaluate the efficacy of glucocorticoids for treatment of infants with MAS, a double blind study using hydrocortisone $(H)$ or a lactose placebo $(P)$ was undertaken. Thirty five infants, 17 $H$ and $18 \mathrm{P}$ were included in the study. Hydrocortisone $(20 \mathrm{mg} / \mathrm{kg})$ or placebo in equal amounts was given on admission (mean $5 \pm 0.3$ range $1-8 \mathrm{hrs}$ ), and $\mathrm{q} 12 \mathrm{hrs}$ for 4 doses via the umbilical catheter. The clinical features were similar in $H$ and $P$ groups: mean + S.E. birth weight in $H$ was $3.1+0.1$ and in $P, 3.2+0.1 \mathrm{~kg}$; gestaEional age, $40.7+0.4$ in $H$ and $40.8+0.3$ wks in $P$; Apgar score $5+0.6$ in $H$ and $4+0.6$ in $P$ groups. Initial severity based on respiratory distres's (RDS score), blood gases, $\mathrm{FiO}_{2}, \mathrm{~A}-\mathrm{aDO}_{2}$ and $\mathrm{X}-$ ray findings was similar in both groups. No significant differences were found either in mortality ( $I H$ and $2 P$ ) or in respiratory failure requiring IPPB ( $4 \mathrm{H}$ and $3 \mathrm{P}$ ). $\mathrm{H}$ group, however, needed $\mathrm{FiO}_{2}>60 \%$ for a significantly $(\mathrm{p}<.05)$ longer period than did $P$ group $(27 \pm 7$ vs. $6.8 \pm 3$ hrs $)$. Infants in $H$ group took longer $(\mathrm{p}<.01)$ to wean to room a ir $(68.9+9.6 \mathrm{hrs})$ than those in $\mathrm{p}$ group $(33.6+6.9 \mathrm{hrs})$. RDS score remained above normal for a significanti $\bar{y}(p<.05)$ longer period in $H$ than in $P$ group $(79.6+11.5$ vs. $43.5+8.9 \mathrm{hrs})$. Clearing of $X$-ray findings and duration of hospitaTization were not significantly different between $H$ and $P$ groups. Hydrocortisone, therefore, did not alter the outcome of MAS but prolonged the duration of respiratory distress and oxygen requirement. Thus, hydrocortisone can not be recommended
INCREASING INCIDENCE OF NECROTISING ENTEROCOLITIS (NEC) : FAILURE TO IDENTIFY PERINATAL RISK FACTORS. Victor Y.H. Yu, David I. Tudehope, Gerald J. Gill, Hamilton, Ontario, Canada.

A significant increase in incidence of NEC was observed in infants below 2500 gms over a 33 month period (Table). Diagnosis was based on tissue examination in 23 and on the clinical syndrome, which included pneumatosis, in 21 nonoperated infants who recovered. Previously suspected NEC risk factors(including maternal and delivery complications, low Apgar scores,

respiratory distress syndrome, umbilical axterial catheters, exchange transfusions, among $>20$ analysed) occurred in no greater frequency in infants with NEC than in over 400 infants under 2500 gms admitted in the same period who did not have NEC. A critical review of the radiological features at diagnosis was made: these included distended loops of bowel (24), foamy pattern (21), asymmetry of bowel pattern (14), pneumatosis (32), pneumoperitoneum (12), portal vein gas (6) and free peritoneal fluid (5). Mortality was $25 \%$ overall.

\begin{tabular}{|lllll|}
\hline Birthweight $(\mathrm{gm})$ & 1973 & 1974 & 1975 & Total \\
\hline$<1500$ & $3 / 51(68) *$ & $5 / 67(78)$ & $13 / 95(148)$ & $21 / 213(208)$ \\
$2500-2499$ & $0 / 55(08)$ & $5 / 107(58)$ & $10 / 130(88)$ & $15 / 292(58)$ \\
$\geqq 2500$ & $1 / 99(18)$ & $5 / 262(28)$ & $2 / 231(18)$ & $8 / 592(28)$ \\
\hline Ail cases & $4 / 205(28)$ & $15 / 436(38)$ & $25 / 464(58)$ & $44 / 697(48)$ \\
\hline
\end{tabular}

*No. with NEC/No. of Admissions

\section{NEPHROLOGY}

799 NEONATAL RENOVASCULAR HYPERTENSION (NRH) - A NONSURr ICAL APPROACH. R. AdeIman, B. Goetzman, J. Vogel $R$. Wennberg, and D. Merten. Univ, of Calif., Davis, School of Medicine., Depts. of Pediatrics \& Nuclear Medicine

Neonatal renovascular hypertension (NRH) is emerging as a significant neonatal problem, perhaps as a complication of indwelling umbilical artery catheters (UAC's). Prompt nephrectomy is the usual recommended therapy. This report describes the succesfuT non-surgical management of NRH.

7 newborns, 1970-5910 gms. at birth, developed NRH (blood pressures $140 / 100$ to $280 / 140$ ) at 2-35 days of age. 6/7 infants had UAC's prior to the onset of NRH. Unilateral renal artery narrowing was diagnosed in 6 infants by angiography and in 7 infant at autopsy. 5/7 infants had positive, lateralizing renal scans. 4/4 infants had positive renal scans using computerized compartmental analysis of data (ADAC). There was good correlation between renal scans, especially with ADAC, and angiography. $6 / 6$ infants had plasma renin activity (PRA)>3no $\mathrm{ng} / \mathrm{mi} / 3 \mathrm{hr}(\mathrm{N} 1<10 \mathrm{ng} / \mathrm{ml} / 3 \mathrm{hr})$. A1] patients responded to antihypertensive medications, usually diuretics and hydralazine, methyldopa and/or propanalol. 6 inf ants (1 lost to follow-up) followed for 3-11 months (mean, 5.5 months) became normotensive. 4/6 infants are off al1 medications. PRP decreased in 5/5 patients, reaching normal levels in three.

Immediate nephrectomy is not mandatory in NRH. Medical management may stabilize blood pressure until additional studies can be obtained or until the infant's condition is suitable for surgery. The natural history of NRH is unclear but our experience suggests that many infants may not require surgery.

OXYGen CONSUMPTiOn $\left(\mathrm{VO}_{2}\right)$ in UREMic Rats

800 R.D. Adelman and M.A. Holliday. University of California at Davis and San Francisco, School of Medicine, Department of Pediatrics.

It has been suggested that undernutrition seen in uremia results in part from excessive basal calorie expenditure. To test this hypothesis oxygen consumption $\left(\mathrm{V}_{2}\right)$ was determined in young male Sprague Dawley rats 10,16 and 3 I days after having been made moderately uremic by partial nephrectomy. Fasted, resting sham operated control rats and uremic rats were tested simultaneously and under identical conditions in a 6 chamber Haldi apparatus. Decreased $\mathrm{VO}_{2}$ seen in uremic rats was related to their decreased body weight. When $\mathrm{V}_{2} 2$ values were weight adjusted by regression of $\mathrm{VO}_{2}$ to weight. 75 , analysis of covariance demonstrated no difference between control and uremic anima $7 s$ at 10,16 and 31 days.

\begin{tabular}{|c|c|c|c|c|c|}
\hline $\begin{array}{l}\text { Anima 1 } \\
\text { Uremic } \\
\text { Control }\end{array}$ & $\begin{array}{l}(n) \\
(6) \\
(6)\end{array}$ & $\begin{array}{l}\frac{\text { Days }}{10} \\
10\end{array}$ & BUN, $\mathrm{mg} / 100 \mathrm{ml}$ & $\begin{array}{l}\text { Weight, g } \\
\text { oi } 126(5.8))^{4} \\
143(3.3)\end{array}$ & $\begin{array}{l}\frac{\mathrm{VO}_{2}, \mathrm{ml} / \mathrm{hr}}{\mathrm{q} 227(27.2) \dagger} \\
277(6.2)\end{array}$ \\
\hline $\begin{array}{l}\text { Uremic } \\
\text { Control }\end{array}$ & $\begin{array}{l}(6) \\
(6)\end{array}$ & $\begin{array}{l}16 \\
16\end{array}$ & $\begin{array}{l}\pi 97.2(6.0)++t \\
25.0(0.7)\end{array}$ & $\begin{array}{l}158(5.8)^{++} \\
188(5.6)\end{array}$ & $\begin{array}{l}226(10.8) \mp+ \\
308(4.5)\end{array}$ \\
\hline $\begin{array}{l}\text { Uremic } \\
\text { Control }\end{array}$ & $\begin{array}{l}(6) \\
(6)\end{array}$ & $\begin{array}{l}31 \\
31\end{array}$ & $\begin{array}{l}120.0(15.9)++f \\
25.7(1.1)\end{array}$ & $\begin{array}{l}224(17.2)+ \\
260(10.2)\end{array}$ & $\begin{array}{l}374(24.3) \\
370(27.3)\end{array}$ \\
\hline
\end{tabular}

IMean (SEM) $\quad+p<.05, \quad+p<<.01,++p<<.001$

Moderately uremic rats do not have abnormal basal calorie expenditure as determined by measurement of oxygen consumption. 
801 ACCELERATED CHRONIC RENAL FAILURE IN RATS: AN IMPROVED SURGICAL PROCEDURE. Alam, S., Wapnir, R. A., Mrozinska, K., Schneider, S., Lifshitz, F., McVicar M. Dept. of Peds. N. Shore Univ. Hosp. Manhasset, N.Y. and Comell Univ. Med. Col., N.Y., N.Y.

In an attempt to surgically induce more severe chronic uremia in a shorter time, the conventional 5/6th nephrectomy was modified to further reduce the renal cortical mass. In addition to the removal of the upper and lower poles of one kidney during the initial procedure, the lateral cortical tissue of the remaining mid-portion was also excised. One week later, contralateral total nephrectomy was performed leaving approximately $1 / 2$ th cortical mass vs. the conventional 1/6 th. Male Wistar rats, 100$170 \mathrm{~g}$, were made uremic by the conventional or by the modified procedure, Body weight and BUN were measured every 2 wks. Comparison of the 2 groups was as follows: Conventional Modified $($ Mean + SEM), $\mathrm{N}=20 \quad$ (Mean + SEM), $\mathrm{N}=20$

\begin{tabular}{|c|c|c|}
\hline Highest BUN & $85 \mathrm{mg} \%+12.9$ & $122 \mathrm{mg} \%+24.2$ \\
\hline Interval to highest BUN & 14 wks $\ddagger 0.4$ & 7.5 wks $\mp 0.4$ \\
\hline Interval to $\mathrm{BUN}>60 \mathrm{mg} \%$ & 8 wks $\mp 3.2$ & 3.5 wks $\mp 1.3$ \\
\hline leight gain & $\mathrm{tgm} / \mathrm{wk} \mp 0.7$ & $8.8 \mathrm{gm} / w \mathrm{k} \mp 1.1$ \\
\hline Mortality & $4 / 2 \overline{0}$ (Total) & $6 / 20$ (Total) \\
\hline
\end{tabular}

The more aggressive, modified technique produced a more rapid, severe chronic uremia with an acceptable mortality. The 3-4 weeks required to evolve a high BUN was still long enough to be consistent with an experimental model for chronic rather than acute uremic.

EFFECTS OF RESPIRATORY DISTRESS ON RENAL FUNCTION IN

802 THE PIGLET. Charles T. Alward, Michael D, Bailie and
Jerry B. Hook. Michigan State Univ., Col, of Human Med., East Lansing.

Utilizing the unanesthetized piglet we have examined the effects of respiratory distress (hypoxia, hypercarbia and acidosis) on renal function. Following a control period respiratory distress was induced in 15 animals 6 to 86 hours of age and maintained for 90 min by increasing the respiratory dead space. During control and experimental periods glomerular filtration rate (GFR), total renal blood flow (RBF), outer cortical (OC) and innex cortical (IC) blood flow, sodium excretion, plasma renin concentration (PRC) as well as other systemic hemodynamic variables were determined. During respiratory distress there was a significant decrease in arterial $\mathrm{pO}_{2}$ and $\mathrm{pH}$ while $\mathrm{pCO}_{2}$ increased. While GFR remained unchanged respiratory distress led to a fall in RBF from $3.9 \pm 0.4(\mathrm{sem})$ to $2.2 \pm 0.4 \mathrm{ml} / \mathrm{min} / \mathrm{g}$. OC/IC remained unchanged (control: $1.47 \pm 0.12$, experimental $1.31 \pm 0.14$ ). Sodium excretion increased from $2.0 \pm 0.8$ to $7.3 \pm 2.0 \mu \mathrm{Eq} / \mathrm{min}$ and plasma renin concentration increased from $67 \pm 10$ to $109 \pm 14 \mathrm{ng} / \mathrm{ml}$. These changes were associated with a $24 \%$ increase in blood pressure (BP) and a $52 \%$ increase in total peripheral resistance (TPR). The fall in RBF with no change in GFR is compatible with an increase in both afferent and efferent arteriolar resistance. There is no evidence of redistribution of intrarenal blood flow. The increase in sodium excretion may be secondary to the increased BP. The elevated $B P$ and $T P R$ may be in part maintained by the renin-angiotensin system.

RECOVERY FOLLONIRG COHGEMITAL NEPHROTIC SYNDROME(CIS)

803 Suchir $\mathrm{K}$. Anand, James $\mathrm{D}$. Northway, (Spon. by Ira K.

CIS is a procressive illness characterized by onset of nephrotic syndrome in early iniancy and death unjer $4 \mathrm{yrs}$; renal biopsy reveals large numbe: of dilated euboicial lined tubules, fetal gloneruIi projecting into dilated Bowman's capsule and arterioles with thickened media. A 4 -day-old white boy developed edema which prosressively worsened. At 5 whs $4+$ proteinuria was noted. SUN was $7 \mathrm{mr}$, creatinine $(\mathrm{Cr}) 0.8 \mathrm{mg}$ and albumin $\mathrm{l} . \mathrm{l} \mathrm{g} / \mathrm{d} l$. VDRJ, CID and toxo titers were negative. C'3 was normal. Renal biopsy revealed typical changes $0:$ CHS. Glomerular fluorescence was negative. Patient ras treated vith aiuretics.

At 14 mo urinary protein excretion was $300 \mathrm{mg} / 12 \mathrm{hr}$. Serum albumin was $2.3 \mathrm{~g} / \mathrm{a}$. . Repeat renal biopsy showed healing. Most glomemli were normal; $<10 \%$ showed complete sclerosis. Most tubules were normal; mild focal tubular atrophy was present.

At $2 \%$ mo urine became protein iree. Sun was 6 and $\mathrm{Cr} 0.3 \mathrm{mg} /$ dl. Patient's growth has been over joth cille in ht. and wt.

It is of interest that patient's mother during the rirst 4 wks of restation took steroids for allergy; othervise gestation was normal. In rojents gestational steroid intake causes renal cystic lesions (without nephrotic syndrome) in the offspring.

clinically and histologically this child at onset had classical features of CIS, however, unlike other children with this disorder he has recovered. Whether his CIS is a variant or etiologically related to maternal steroid intake remains to be determined.

(We would like to thank Dr. Robert L. Vernier, Prof. of Peds., Univ. of kinn. for reviewing the histopatholngic slides.)
TmPAH AND GT RELATIONSHIPS IN THE MATURING CANINE KID-

804 NEY. Billy S. Arant,Jr. (Spon. by James N. Etteldorf), Univ. Tenn. Center for Health Sci., Dept.Ped., Memphis.

TmPAH, inulin clearance(Cin) and dry kidney weight(DKW) were determined for 41 puppies frombirth-10 wks and 2 adults. TmPAH,Cin and DKW increased with age $(r=.82, .88, .83)$. TmPAH/Cin during the first week of $1 \mathrm{ife}(.143 \pm .075)\left(\bar{m}_{1}\right.$ S.D.) was not different from adults $(.147 \pm .014)$, however the ratio increased to $.171 \pm .011 *$ at 4 wks, decreased to .115 $\pm .027 *$ at 6 wks and increased thereafter to adult values. TmPAH/gDKW $(\mathrm{mg} / \mathrm{min} / \mathrm{g})$ was observed to increase in

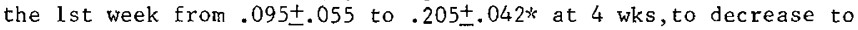
$.113+.041 \%$ at 6 wks, and to increase thereafter to adult values $(.58 \pm .067 *)$. Cin $/ g D K W(\mathrm{ml} / \mathrm{min} / \mathrm{g})$ increased slightly between weeks $1(.793 \pm .395)$ and $6(1.075 \pm .207)$, and increased thereafter to adult values $(3.95+.07 \%)$. DKW $(\mathrm{g})$ increased from $1.67+.534$ in the first week to $3.8 \overline{3} 3+1.527 \%$ at 4 wks, did not change from $4-6$ wks $(4.4 \pm$ $1.56)$ but was increased in the adult $(11 \pm 5.6 \%)$. Therefore the GT relationship for PAH during renal maturation in the dog is affected by differential rates of tubular and glomerular maturation after the first week of life before GT balance noted in mature kidneys is achieved. It is concluded that maturation of tubular transport of PAH from birth to 4 wks and between 6 wks and maturity results from both tubular growth and increased transport capacity of the tubular cell. The significant decrease in TmPAH/gDKW noted between 4 and 6 wks when DKW had not changed suggests a decreased transport capacity for the tubular cell, an interpretation supported by finding during this interval a small decrease in actual TmPAH (.693..133 to $.54 \pm .153 \mathrm{mg} / \mathrm{min})$.

$(* \mathrm{p}<0.05)$

\section{5}

THE EFFECT OF $\mathrm{FE}_{\mathrm{Na}}$ AND EXTRACELLULAR VOLUME(ECFV) EXBilly S. Arant, Jr. (Spon. by James N. Etteldorf),

Center for Health Sci, Dept. Ped., Memphis.

Early studies of GT balance in humans utilizing glucose as the marker of tubular function, indicated lower ratios of $\mathrm{TmG} / \mathrm{C}$ in in infants than in adults. More recently'Brodehl found a mature level of GT balance for glucose in infants and children. We have reported similar findings in human infants as early as 24 wks gestation and have reported matured GT balance for glucose at birth in the dog. It is known that glucose reabsorption is affected by ECFV expansion and by changes in $\mathrm{FE}_{\mathrm{Na}}$ and GFR, any of which may have accounted for the lower ratios of $\mathrm{Tm} / \mathrm{C}$ in in infants reported by Tudvad. A study was undertaken to examine TmG and GT balance in the developing canine kidney without producing changes in ECFV, FENa or GFR. TmG was reached during glucose titration experiments in 6 puppies from 1-5 days of age, in 6 others from 1351 days and in 1 adult without producing significant changes in $\mathrm{ECFV}, \mathrm{FE}_{\mathrm{Na}}$ or GFR. TmG/Cin in the younger puppies(3.24t.38) was not different from that of older puppies $(3.45+.28)$, was similar to that of the adult studied(2.88) and was not different from reported adult values $(3.03 \pm .39, p>0.1)$. ECFV expansion with $0.85 \%$ saline $(20 \mathrm{ml} / \mathrm{Kg})$ resulted in a decrease in $\mathrm{TmG}$ and in $\mathrm{TnG} / \mathrm{C}$ in $(2.375 \pm .78)(\mathrm{p}=.005)$ in every instance that $\mathrm{FE}_{\mathrm{Na}}$ increased significantly at all ages. It is concluded that low ratios of $\mathrm{TmG} / \mathrm{Cin}$ re ported in human infants were likely the results of ECFV expansion and/or increased $\mathrm{FE}_{\mathrm{Na}}$. The present study confirms mature GT balance for glucose from birth in the dog and is in agreement with studies in human infants reported from this laboratory.

\section{Q06 6 (une Harcrove, Jose}

$806 \frac{\text { Darla Erhard, Sarah Harding, June Hargrove, Jose A. }}{\text { Ramirez, Malcolm A. Holliday, Department of Pediat- }}$ rics, University of California, San Francisco.

Children with renal insufficiency do not grow normally. More than half of those who develop renal failure are growth retarded ( $>2$ SD below normal for height). Betts \& Magrath (BMJ 1974) reported severe growth failure and decreased growth velocities in children whose clearances fell to $<25$ \% of normal. We have followed 11 growth-retarded children with clearances of $<20 \%$ in a clinic where nutritional and social support were emphasized. Ages ranged from birth to 11 years. Patients were followed from 1 to 9 years (average 4.1 years). These children grew at a rate of 83\% of expected growth for their height age. For 6 months, 8 children have been followed in a special clinic where energy intakes were maintained at $80 \%$ or more of Recommended Dietary Allowance for height age and where educational and social intervention was possible. Patient population included children with congenital nephrosis, cystinosis, urologic disorders and severe renal hypoplasia. These children grew at $109 \%$ of expected for height age. This experience suggests that nutritional and social support can lead to accelerated growth. It is not yet clear whether this accelerated growth can be sustained over long periods. 
807

THE SIGNIFICANCE OF CHARACTERISTIC ULTRASTRUCTURAL ABORMALITIES (CUA) IN HEREDITARY PROGRESSIVE NEPHRITIS (HPN). Andrew J. Aronson, Ronald J. Kallen, Rosa T. Ong, and Benjamin $H$. Spargo (Spon. by Eddie S. Moore). University of Chicago Pritzker School of Medicine, La Rabida Children's Hospital, Departments of Pediatrics and Pathology, Chicago.

The CUA of HPN are glomerular basement membrane thickening, reticulation, and the presence of granular electron-dense particles They are associated with progressive renal disease, renal failure and positive family history. This report describes 6 children (5 boys, l girl) with HPN referred because of urinary abnormalities documented between 2.5 and 18 years of age. CUA was found in 4 and 5 year-old boys who are among the youngest thus far described with CUA. All 6 had been previously said to have acute glomerulonephritis, idiopathic hematuria or urinary tract infection. All have hematuria, 5 albuminuria, 2 nephrotic syndrome, 2 hypertension and 1 renal failure. All have normal audiograms. Family history is positive in 5 families available for study with apparent transmission of the deleterious gene from father-to-son (1), mother-to-son (3), and mother-to-daughter (1). Two patients have a brother and father respectively who have developed chronic renal failure. Two children have had multiple renal biopsies documenting significant progression and confirming the predictive importance of finding CUA of HPN early even in the absence of positive family history.
810

CHLORAMBUCIL THERAPY IN NEPHROTIC SYNDROME, (NS): FREQUENT RELAPSERS AND STEROID RESISTENT. H. JORGF BALUARTE, LINDA HINER, MARY L. COTE and ALAN GRUSKIN, Dept. Ped. Temple Univ. Sch. Med., St. Christopher's Hosp. for Child., Phila., Pa.

Chlorambucil (C) has been used in combination with Prednisone (P) to treat 21 children with frequent relapsing NS and 9 children with steroid-resistent NS. Renal biopsy in 10/21 frequent relapsers revealed minimal changes and their treatment consisted of a single course of $\mathrm{C}$ at two dosage levels: 10 children on stable dose $(0.2 \mathrm{mg} / \mathrm{kg} /$ day $)$ and 11 children on increasing dose $(0.2$ to $0.63 \mathrm{mg} / \mathrm{kg} /$ day $)$ in combination with $\mathrm{P}\left(60 \mathrm{mg} / \mathrm{m}^{2} / \mathrm{QOD}\right)$ for 8 weeks. Complete remissions were initially achieved in all. Follow up averaged 12.8 mos (range: 2-30 mos). One patient from each group subsequently relapsed at 7 and 17 mos. and responded to $P$ alone with sustained remissions. Nine children with steroid resistent, focal segmental glomerulosclerosis (FSCS) received 10 courses of increasing doses of $C(0.2$ to $0.83 \mathrm{mg} / \mathrm{kg} /$ day $)$ with alternate day $P$ for 8 weeks. Seven patients went into complete remission averaging 17.3 mos (range: $31 / 2-35$ mos). The other 2 patients remained chemically nephrotic, but more easily manageable. Conclusions: 1) $\mathrm{C}$ is effective in the treatment of frequent relapsing NS. 2) The stable dose regime was as effective as the increasing dose regime. 3) $\mathrm{C}$ also appears effective in the treatment of steroid-resistent NS associated with FSGS. Supported by NIH grants RR-75 and RR-5624.
MULTIPLE UNILATERAL RENAL ABSCESSES ANO SICKLE CELL

808 TRAIT (AS) IN A YOUNG CHILD. Andrew J. Aronson, Rosa Benjamin H. Sparqo (Spon. by Eddie s. Moore). University of Chicago Pritzker School of Medicine, La Rabida Children's Hospital, Departments of Pediatrics, Pathology and Radiology, Chicago.

A previously-well four-year-old black girl with AS was referred with fever, abdominal pain, myalgia, proteinuria $(4+)$ and hematuria following an URI. Urine cultures grew E.coli. Evaluation of renal function included BUN $(13 \mathrm{mg} / \mathrm{dl})$, serum creatinine $(0.7 \mathrm{mg} /$ di), endogenous creatinine clearance $\left(75 \mathrm{cc} / \mathrm{min} / 1.73 \mathrm{~m}^{2}\right)$ and urine specific gravity $(1.017)$. AS was confirmed. IVP revealed a large left kidney, incomplete filling of left lower pole and splaying of calyces. Renal uitrasound showed increased renal parenchyma? thickness and a probable intrarenal mass. The right kidney was normal. A left nephrectomy was performed, the kidney was found to be large with miltiple abscesses and the ureter, renal artery and vein were normal. Light microscopy illustrated ectasia of glomerular vessels with sludging of deformed RBCs, tactoids of fibrin between impacted sickled RBCs, and occasional leukocytes. Ultrastructurally RBCs showed characteristic rods of abnormal hemoglobin. Renal function is normal and urine has remained sterile during a six-month post-operative period. Bacteriuria, pyelonephritis and hematuria are associated with sickle-cell disease (SS). Bacteriuria, pyelonephritis, papillary necrosis and hematuria are now recognized as associated with AS. This child represents a striking example of the apparent predisposing role of AS in the development of renal disease in young children and emphasizes the need for careful evaluation of children with AS as well as SS.

ESSENTIAL HYPERTENSION IN CHILDHOOD. Lorenzo $C$.

809 Aschinberg, Robert A. Miller, Petros M. Zeis, Massoun University of lllinois Hospital and Cook County Hospital, Departments of Pediatrics, Chicago, 111 inois

Between 1974-75 eleven males and four females 8-17 years of age were found to have hypertension defined as supine 5 th phase diastolic pressure repeatedly above $90 \mathrm{~mm} \mathrm{Hg}$ (mean $97.3 \pm S . D$. $4.66 \mathrm{~mm} \mathrm{Hg}$ ). A family history of hypertension was obtained in 5 cases. Eleven patients were Black. Obesity was present in 3 cases. Evidence of hypertensive cardiovascular disease on ophthaImologic, roentgenographic or electrocardiographic examination was present in 6 patients. Diagnostic evaluation including physical examination, serum cholesterol, triglycerides, lipid electrophoresis, assays of peripheral plasma renin activity, aldosterone, cortisol, urinary $170 \mathrm{H}, 17 \mathrm{KS}$, VMA, creatinine clearance, and intravenous pyelography was normal in all cases except for one patient who was found to have Type IV hyperlipoproteinemia. Renal arteriograms and selective renal vein renins obtained in the first 9 patients were normal.

Seven patients were treated with Hydrochlorothiazide; 5 of them are currently normotensive (Diastolic $<80 \mathrm{~mm} \mathrm{Hg}$ ). Out of 8 untreated patients, 3 became normotens ive after one vear of observation. These results suggest that 1) essential hypertension is not uncommon in the young. 2) A significant number of patients become normotensive without therapy. And 3) extensive diagnostic work-up in these patients is unwarranted.
LONG-TERM TREATMENT WTTH 1-ALPHA HYDROXYVITAMIN $D_{3}$ IN

81 1 TWO PEDIATRIC HEMODIALYSIS PATIENTS. Mary G Beale, James CM Chan, Susan B Oldham and Hector F DeLuca (Intr by Gilbert August). George Wash Univ, Children's Hosp Nat Med Ctr, Wash DC; Univ So Calif, Dept Med, Los Angeles; Univ Wisc, Dept Biochem, Madison.

Two hemodialysis patients, ages 13 and 17 years, with renal osteodystrophy (RO) received oral 1-alpha hydroxyvitamin $\mathrm{D}_{3}(1-\alpha-$ $\mathrm{OH}-\mathrm{D}_{3}$ ) for 22 and 20 months, respectively. In the younger patient, plasma calcium rose to normal in the fifth month. Alkaline phosphatase returned to normal in the seventh month, coinciding with radiologic improvement.

During the early study period plasma iPTH was determined with both amino (N) specific and carboxy (C) specific antisera. In the seventh month, the $I$ PTH level was $28 \%$ of the pretreatment level using the $\mathrm{N}$-terminal antiserum and $62 \%$ of the initial value using the C-terminal antiserum. At 14 months when the dosage of $1-\alpha-O H-D_{3}$ was decreased to $0.5 \mathrm{\mu g} /$ day to avoid hypercalcemia, a1kaline phosphatase and iPTif showed a sharp increase.

Early $x$-ray improvement in the older patient could not be maintained because of poor medication compliance. Stmilar changes in $\mathrm{iPTH}$ were observed with the $\mathrm{N}$-terminal and $\mathrm{C}$-terminal antisera.

$1-\alpha-\mathrm{OH}-\mathrm{D}_{3}$ promotes healing of $\mathrm{RO}$. The dosage requirements change, necessitating close monitoring of $x$-ray and biochemical studies. Too low a dosage results in rapid deterioration.
87 GLOMERULAR LESIONS OF CHRONIC PYELONEPHRITIS (CPN)

812 AND REFLUX NEPHROPATHY. Dinyar Bhathena, H. Philip Stalker and Nancy H. Holland. Depts. of Path. and Ped., Univ. of Ky. Med. Sch., Lexington, Kentucky.

A variety of glomerular lesions, mostly focal and segmental hyalinosis, have been described in the group of tubulo-interstitial diseases associated with urinary reflux. Fluorescent antibody studies have shown deposits of immune globulin and complement in this glomerular lesion. Antibodies to Tamm-Horsfall (T-H) protein, a mucoprotein secreted by tubular cells directly into the urine were found by Hodson in the serum of refluxing pigs suggesting this normally sequestered protein may be exposed to immune competent cells by intrarenal reflux. We have examined renal tissue from patients with end-stage renal disease (ESRD) and reflux for evidence of glomerular deposits of $\mathrm{T}-\mathrm{H}$ protein or $\mathrm{T}-\mathrm{H}$ immune complexes.

Specimens were obtained at nephrectomy from 5 patients with ESRD and reflux. By light microscopy focal local or global glomerular lesions were seen. Tissue sections were stained with fluorescent antibodies to $\mathrm{T}-\mathrm{H}$ protein, IgG, IgA, IgM, $\mathrm{B}_{1} \mathrm{C}$ globulin and fibrin. In all patients focal granular deposits of IgM and BIC were seen in the mesangium and/or the periphery of lobules. $\mathrm{T}-\mathrm{H}$ protein was not demonstrated in any glomerulus not even those containing $\mathrm{IgM}$ and $\mathrm{B}_{1} \mathrm{C}$ but was present in tubular casts and cytoplasm of tubular cells. In one kidney an isolated interstitial focus of $\mathrm{T}-\mathrm{H}$ protein was noted. The results indicate no evidence for T-H immune complex deposition in the pathogenesis of the glomerular lesions associated with CPN and reflux. 
SERUM FREE POLYAMINES (SFP) IN HUMAN DISEASE BY RADIO

813 IMMUNOASSAX (RIA). Robert A. Campbell, Dagmar Bartos, Frantisek Bartos, Donald P. Grettie, Yeshawant B. Talwalkar, James E. Musgrave. (Spon. by Robert C. Neerhout), Department of Pediatrics, University of Oregon Health Sciences Center, Portland, Oregon.

Milligram amounts of free and conjugated polyamines are normally disposed of in urine each day. SFP levels in patients before and after hemodialysis grossly elevated. Patients prior to dialysis: n 17, range $90-260$ ng spermine equivalent/ml, $x 182$, SE 12.3. After dialysis values range $100-230, \bar{x} 125$, SE \pm 8.9 , ( $p-$ $<0.001$ ). Normal adult (N) $\bar{x}$ for SFP, n 26 , was $44 \mathrm{ng} \pm \mathrm{SE} 3.6$. Children with systemic lupus erythematosus (SLE), idiopathic nephrotic syndrome (INS) and kidney transplant were tested.

$\begin{array}{lrcrl} & \mathrm{n} & \text { Range } & \bar{x} & \text { SE } \\ \text { SLE } & 6 & 135-250 & 187 & +21 \\ \text { INS } & 24 & 58-305 & 161 & \mp 11.3 \\ \text { KT } & 9 & 18-132 & 55 & \ddagger 14.5 \\ \text { N (child) } & 9 & 13-40 & 25 & \pm 6.5\end{array}$

SFP levels in uremic patients may reflect accumulation due to inability to excrete these potent middle molecules. A $35 \%$ decrease In level was noted post-dhalysis. Elevated levels in SLE are attributed to immune-complex mediated cell damage with leakage of polyamines into the serum. Levels in INS may be due to multiple causes including the disease diathesis. Children with transplants have normal values despite drug treatment. Elevated values, where found, may or may not indicate occult rejection.

作

814 STRUCTURE BY SPERMINE (SPM). Robert A. Campbell, Thomas LaBerge, Robert E. Brooks, Mark C. Boswell, and Yeshawant B. Talwalkar, (Spon. by Robert C. Neerhout), Department of Pediatrics and Anatomical Pathology, University of Oregon Health Sciences Center, Portland, Oregon.

SPM, a small endogenous polycation, can be nephrotoxic. A small pharmacological dose is diuretic. A large dose produces proteinuria. Proximal tubular cell damage occurs. In man, a "nephritis-1ike" picture has been reported following SPM administration. Large polycations such as protamine and polylysine induce pseudopod changes similar to those seen in the idiopathic nephrotic syndrome of childhood.

Pre-adolescent Sprague-Dawley rats were injected I.P. with a single daily dose of $40 \mathrm{mg} / \mathrm{kg}$ of SPM hydrochloride for periods uf $i$ to 5 days. Animals were perfused by a modified Maunsbach technique. No EMC in the glomeruli were noted from $4 \mathrm{hrs}$. to 5 days. Zipper regions were intact. Progressive EMC developed in proximal tubule cells. First observed were luminal vesiculation (lollipops) and disarray (chinese figures) of microvillae. Pinocytotic vesicles increased in size and number. Rarifaction of cytoplasm, rounding up of mitochondria and increase in secondary lysosomal-like structures were then noted. Bizarre cytosegrasomes were seen. By day 5 sloughing of microvillae and cellular elements occurred. Tubular basement membranes were intact. Profound effects of SPM or its metabolites on the proximal tubule, morphologically first demonstrable about the luminal portion of the microvillae, suggest the possibility of glycocalyx envolvement as an early event in the cytolytic sequence.

FAMILIAL NEPHROTIC SYNDROME WITH FOCAL AND

815 SEGMENTAL GLOMERULOSCLEROSIS. Manju Chandra

Med. Col1, $\frac{\text { John R. Hoyer and John E. Lewy. Corne }}{- \text { N.Y. Hosp., Dept. of Ped., New York. }}$

The diagnosis of steroid resistant nephrotic syndrome (NS) was established in 3 of 5 siblings within a 3 mo. period. Renal biopsy of the 16 y.o. gir 1 with NS, at onset showed focal and segmental glomerulosclerosis (FSGS). Her disease progressed to severe renal insufficiency in 14 mo. despite therapy with prednisone and cytoxan. Renal biopsies of the 14 and 11 y.o. boys with NS showed minimal change by light and electron microscopy on initial biopsy and FSGS on subsequent biopsies 3 and 5 mo. later. Both boys retained normal creatinine clearances and showed a decrease of urinary protein excretion, normalization of serum albumin and loss of edema during and following treatment for 90 days with cytoxan and alternate day prednisone. The girl with NS, the 11 y.o. boy with NS and his male twin without NS have sickle cell disease (SS). The $14 \mathrm{y.o}$. boy with NS and his $9 y .0$. brother have thalassemia trait (Th). The twin with SS and without NS and 14 y.o. boy with $T h$ and NS are identical on HLA typing and mixed lymphocyte culture (MLC). The three children with NS and the twin without NS share HLA 23 and 41 . Thus, there is no apparent linkage of NS with FSGS to HLA type or specific hemoglobinopathy in this family.
816 SALT LOSING NEPHRITIS IN THE PRUNE BELLY SYNDROME.

Richard D. Zachman.) University of Wiscons in School of Medicine, University of Wisconsin Hospitals, Department of Pediatrics, Madison, Wisc.

A male infant with features of the prune belly syndrome (PBS) developed hyponatremia (to $116,118,122 \mathrm{mEq} / \mathrm{L}$ ) and weight loss several times while on I.V. solutions or milk formulas providing 3-6 mEq/ $\mathrm{kg} \mathrm{Na}$ daily. At a serum $\mathrm{Na}$ Tevel of $118 \mathrm{mEg} / \mathrm{L}$, the urine Na was $96-114 \mathrm{mEq} / \mathrm{L}$, an excretion of $18 \%$ of filtered load. He required up to $12 \mathrm{mEq} / \mathrm{kg} \mathrm{NaCl}$ daily to correct hyponatremia and maintain serum sodium at $>135 \mathrm{mEg} / \mathrm{L}$. He had signs of chronic renal failure with a serum creatinine $(\mathrm{Cr})$ of 2.1 to $5.0 \mathrm{mg} / \mathrm{dl}$, BUN $30-80 \mathrm{mg} / \mathrm{dl}$, and serum $\mathrm{HCO}_{3}<14 \mathrm{mEq} / \mathrm{L}$ requiring $3-6 \mathrm{mEg} / \mathrm{kg}$ $\mathrm{NaHCO}_{3}$ daily. Endogenous $\mathrm{Cr}$ clearance from both kidneys was $9 \mathrm{ml} /$ min $/ \mathrm{M}^{2}$ and $0.8-1.8 \mathrm{mg} / \mathrm{min} / \mathrm{M}^{2}$ from the left kidney thru a nephrostomy tube. This left kidney excreted $12-21 \%$ of filtered sodium load. Renal biopsy tissue revealed bilateral dysplastic kidneys with small cysts and marked hydronephrosis on the left.

To ascertain the prevalence of salt loss in PBS, the records of 7 other patients were reviewed. 5 of these were males. One male infant died at 18 days with a serum $\mathrm{Cr}$ of $10.5 \mathrm{mg} / \mathrm{dl}$; all the others had a serum $\mathrm{Cr}$ of $<1.8 \mathrm{mg} / \mathrm{dl}$. None of the 7 were hyponatremic on a normal diet or on $2-4 \mathrm{mEg} / \mathrm{kg} \mathrm{NaCl}$ given I.V.

Salt losing nephritis can mimic Addisons disease and has been reported in interstitial nephritis, polycystic disease and medul. lary cystic disease. As demonstrated in this patient, extensive renal salt loss may be seen with PBS and should be anticipated in this disorder, particularly in patients with severe dysplasia. ON RENAL FUNCTION IN UTERO. Ellis E. Chung, Eddie S. Moore, Eduardo E. Cevallos, Margarita Ocampo and Elizabeth Lyons. Dept. Peds. Univ. Chicago at Michael Reese Med. Ctr. Chicago.

The effect of mean arterial pressure (MAP) and gestational age on renal function was studied in 17 fetal lambs age 90-150 days. Initial opening and subsequent stable control arterial pressures were measured. Renal blood flow (RBF) and distribution of intrarenal flow (IRF) were measured by injecting 7-15u radioactive glass microspheres. Glomerular filtration rate (GFR) was measured with Il31 sodium iothalamate. MAP increased with gestational age $(\mathrm{p}<.001)$ from a low of $33 \mathrm{mmHg}$ at age 100 days to $80 \mathrm{mmHg}$ at age 150 days. MAP also correlated wi th fetal weight $(\mathrm{p}<.001)$. Total $\mathrm{RBF}(\mathrm{ml} / \mathrm{min} / \mathrm{gm})$ did not increase with gestational age and also did not correlate with MAP or fetal weight. Renal vascular resistance (RVR) rose from $18 \mathrm{mmHg} / \mathrm{mL} / \mathrm{min} / \mathrm{gm}$ in early gestation to $143 \mathrm{mmHg} /$ $\mathrm{ml} / \mathrm{min} / \mathrm{gm}$ near term. IRF $(\mathrm{ml} / \mathrm{min} / \mathrm{gm})$ in early gestation was greater in the outer $1 / 3$ of the cortex compared to the two inner cortical zones and did not change with gestational age. GFR did not correlate with RBF, MAP or gestational age. Urine flow (ml/ $\mathrm{min}$ ) did correlate with fetal age, RBF or GFR. Fractional urine flow (V/GFR) was constant throughout gestation. These studies demonstrate that although fetal MAP increases with gestational age, a simul taneous increase in RVR prevents an increase in RBF. From early gestation, IRF, GFR and urine flow are independent of arterial pressure and RBF. Glomerular-tubular balance for water is present throughout gestation as suggested by a constant fractional urine flow.

PROFILES OF A NEW NEPHROLOGY PROGRAM, Thomas $H$

$818 \frac{\text { Coleman, }}{\text { Beale and James CM Chan }}, \frac{\text { Demetrius Ellis }}{\text { Jntr by Robert H }} \frac{\text { Jose R Salcedo G }}{\text { Parrott) }}$ George Wash Univ-Children's Hosp Nat Med Ctr, Washington, DC To evaluate the referral and utilization of a teaching nephrology program and pediatric hemodialysis unit, analysis of patient statistics was undertaken. Since the program started, in the first 23 months (Jan 1974 - Nov 1975), 379 children with 546 renal disease categories were seen; kidney biopsies were done in 49 patients (13\%); and 13 end-stage kidney failure patients were started on hemodialysis; of which 3 received kidney transplantations. The more frequently occurring of the disease categories are presented:

urinary tract infection - 73 patients (19\%)

- hypertensive - 58 patients ( $15 \%$ )

- anatomic anomalies - 56 patients (15\%)

- hematuria - 49 patients $(13 \%)$

glomerulopathies - 47 patients (12\%) including 33 (9\%) 1diopathic nephrotic syndromes

- fluid and electrolyte disorders - 30 patients ( $8 \%$ ) includding SIADH (4), RTA (3), nephrogentc diabetes insipidus (3), Bartter's (2), hyperuricemia (2)

- enuresis - 20 patients (5\%)

- renal failure -7 acute $(2 \%), 13$ chronic (3\%)

hereditary conditions - 17 patients (4\%) including hypophosphatemia (4), hereditary nephritis (3), cystinosis (2)

- renal calculi - 4 patients $(1 \%)$

Wilm's Tumor - 3 patients $(0.7 \%)$

- renal trauma - 2 patients $(0.5 \%)$ 
THE ORIGIN OF CYSTS IN THE RENAL TUBULE. John F.

819 Crocker, Stephen Safe, and Otto Hutzinger. Dept. Ped., Da1. Univ., Halifax, N.S.; Dept. Chem., Univ. Guelph, Guelph, Ont., Canada; Dept. Chem., Univ. Amsterdam, Amsterdam, The Netherlands.

Polycystic kidneys induced by diphenylamine (DPA) in rats are manifested in two ways: (1) long term exposure in adult rats gives collecting duct cysts similar to the adult human disease, (2) in DPA exposed pregnant animals, cysts in the cortical or medullary areas of the kidney in the offspring. Crocker, et al, showed in 1972 that DPA was not the active chemical in the model but the impurity.

Four impurities were isolated from crude DPA and identified using TLC, GLC and mass spectra and the chemical structure of the four impurities confirmed by synthesis as $0-$ and p-cyclohexylaniline, and 2- and 4-amino biphenyl. A fifth impurity is a substituted aromatic amine $\left(\mathrm{T}_{1}\right)$. Timed pregnant rats were fed the chemicals or a control solution for the last six gestational days. Newborn rats were killed and one kidney fixed for histological evaluation and the other frozen for cytofluorescence by photon scanning. Cyst formation was graded on a negative to $4+$ basis. All kidneys except controls showed some degree of cyst formation, with 4-amino biphenyl and $T$ demonstrating the most marked cystic change. Natural cytofluorescence of the renal tubules occurred at $350 \lambda$. When animals were exposed to the purified teratogens, this fluorescence was absent.

Polycystic formation in this model may not be a degeneration of cells away from the tubular basement membrane (Darmady, et al) but a proliferation and alteration of tubular cell metabolism.

URINARY LDH ISOENZYME 5 EXCRETION IN EXPERIMENTAL

820 PYELONEPHRTTIS. Robert J. Cunningham, III, Hugo F. Carvajal, and Richard B. Passey, Spon. by Luther B. Travis, Univ. of Texas Med. Br. and Shriners Burns Insti., Depts. of Ped. and Path., Galveston, Texas.

Studies to determine the effect of kidney parenchymal infection upon urinary $\mathrm{LDH}$ isoenzyme composition, were performed in twelve female Sprague Dawley rats on day 0 and on days 2,5 and 10 after experimental inducement of $E$. coli pyelonephritis (dir ect inoculation). An additional group of 12 animals were subjected to similar experimental manipulations and served as sham controls. On day 10 all animals were sacrificed and the presence or absence of infection was confirmed by kidney homogenate cultures. Twenty-four hour urine collections (days 2,5 and 10) re vealed lower urine osmolalities, and significantly higher levels of urinary LDH isoenzyme 5 excretion in the experimental thail in the sham operated animals $(p<0.05)$. These differences persist ed for the length of the experiment. Leukocyte excretion rates were also higher in the experimental than in the sham group and a high correlation with ULDH 5 activity was demonstrated ( $R=$ $0.815)$. No other evidence that the latter two variables may be causually related was found. These studies support previous clinical investigations and corroborates the value of urinary LDH isoenzyme 5 determination in the diagnosis of pyelonephritis.

THE USE OF DIPYRIDAMOLE IN POST RENAL TRANSPLANT 821 PATIENTS: A PROSPECTIVE STUDY. Robert J. Cunningham [II, Luther B. Travis, Ben H. Brouhard and Michael Berger, Department of Pediatrics, Division of Nephrology, University of Texas Medical Branch, Galveston, Texas.

Anti-platelet agents have been used to prolong renal allograft survival in post transplant patients. The present prospective study evaluates the effect of Dipyridamole during the first 9 months post transplant on the number and severity of acute rejection episodes, a graft function and survival. Following random allocation, 4 patients received Dipyridamole $(6-7 \mathrm{mg} / \mathrm{Kg} /$ day $)$ and 4 patients did not. The diagnosis and treatment of acute rejection was done by a predetermined protocol as was other immunosuppressive therapy. Those patients receiving Dipyridamole underwent significantly $(p<.05)$

fewer acute rejection episodes $(1.75 \pm 0.25$ vs. $4.0 \pm 0.7)$; they also required less bolus methylprednisolone therapy $(15.0 \pm 3.1$ doses vs. $27.0 \pm$ 3.1 doses, $p<.05)$. One patient in the non-treated group has lost graft function and two others have serum creatinines of greater than $1.3 \mathrm{mg} \%$. All the Dipyridamole treated patients currently have serum creatinines of less than $1.2 \mathrm{mg} \%$. These results suggest that Dipyridamole is a useful adjunct to immunosuppressive therapy in post renal transplant patients. $\mathbf{8 2 2}$ RENAL RESPONSE IN THE FETAL LAMB TO COMPLETE OCCLUSION OF THE UMBILICAL CORD.S.S. Daniel, K.M.
Husain, R.I.Stark, J.Milliez, M-N Yeh, L.S.James. Coll. Phys. \& Surg. Columbia Univ., Div. of Perinatology, N.Y.C.

In order to study some of the causes underlying the abnormal plasma electrolyte composition and kidney function present in infants asphyxiated at birth, the renal response of the fetal lamb to repeated complete occlusion of the umbilical cord was studied in 6 animals chronically catheterized and intact in utero. In each experiment there were 5 episodes of cord occlusion of $2 \mathrm{~min}$ duration followed by $13 \mathrm{~min}$ recovery. Fetal arterial $\mathrm{pH}$ fell from a mean of 7.37 to 7.31 following the first episode; during the 5th episode $\mathrm{pH}$ fell from 7.31 to 7.24 and rose to 7.34 during the next hour of recovery. These intermittent periods of occlusion of the umbilical cord produced a rise in fetal urine osmolality from a mean control value of 186 to $243 \mathrm{mOsm} /$ $\mathrm{kg}$ following the first episode of occlusion to a maximum of $344 \mathrm{mOsm} / \mathrm{kg}$ at the end of the 5th episode; urinary output remained essentially unchanged. During the same period, urine sodium concentration rose from 46 to 98 $\mathrm{mEq} / \mathrm{L}$, chloride from 22 to $65 \mathrm{mEq} / \mathrm{L}$ and potassium from 5.0 of 12.8 $\mathrm{mEq} / \mathrm{L}$. Urinary electrolyte concentrations remained elevated for at least two hours after the termination of the occlusions. These studies indicate that complete interruption of the umbilical circulation even though of short duration can lead to a loss of electrolytes in the urine. These losses are even greater than have been reported previously with partial cord occlusion of longer duration and appear to be related to a sudden rise in serum levels of vasopressin following each episode of cord occlusion.
BRANOUS DEPOSITS : ANOTHER VARIANT OF MEMBRANOPROLIFERATIVE GLOMERULONEPIIRITIS (MPGN). Davis, A.E. Schneeberger, E.E., McCluskey, R.T. and Grupe, w.E., Children's Hospital Medical Center, Boston, Massachusetts.

MPGN has been divided into 2 major morpholoqic subqroups; nure MPGN and dense deposit disease (DDD) account almost exually for the vast majority of patients. A 3 rd intermediate variety, recently described, is characterized hy suhendothelial denosits and dunlication of hasement membranes (GBM) together with subenithelial deposits and deposits within GBM. A 4 th variant has heen seen in 2 qirls, followed 6 and 10 vrs., with low $C 3$ and sersistent nephritis but with a mild course, persistently nornal renal function and spontaneous morpholoqic imorovement. No family member had low $\mathrm{C} 3$. Levels of nroperdin Factor $\mathrm{B}, \mathrm{C} 4$ and $\mathrm{C} 2$ are normal; C3 nephritic factor was not detected. Mesangial proliferation is mild. Ultrastructurally, irreqular intramembranous denosits are present primarily in the lamina densa separated by lencths of normal appearing GBM. Intramembranous and subendothelial electron lucent areas, containing vesicles, are also seen. Different from either MPGN or DDD, neither subendothelial denosits, mesancial interposition, splitting of the GBM nor ribbon-like intramembranous deposits were identified. While morphologically distinct, this variant is clinically indistinquishahle at onset from other forms of MPGN. The possibility of a variant with mild clinical course, no proqression and spontaneous morphologic improvement is important in planning and assessine treatment programs in this disease group.

824 MERULONEPHRITIS. Charles A. Davis, Paul I. McEnery, A. James McAdams and Clark D. West. University of Cincinnati College of Medicine and Children's Hospital, Dept. of Peds., Cincinnati.

Rapidly progressive glomerulonephritis is a disease with a uniformly poor outcome, unless it is the result of a previous streptococcal infection. The clinical courses and glomerular morphology in nine children with idiopathic rapidly progressive nephritis are described. Al1 patients presented with hematuria and proteinuria. Four patients were febrile and three had rashes. A11 developed azotemia and five of the patients became oliguric. All had normal serum complement levels. Examination of renal biopsy sections showed glomerular crescents, interstitial round cell infiltrates, and focal necrotizing lesions, without cellular proliferation of the glomerular tuft. Fluorescein labeled antibody studies revealed deposits of complement and foci of fibrin, but revealed no immunoglobulin deposits. Electron microscopy revealed large subepithelial deposits at the junction of the capillary loops and the mesangial stalk. Eight of the patients were treated with corticosteroids. Four improved and on 12 to 40 month follow-up have stable renal function. Four patients progressed to end-stage renal disease. Follow-up biopsies were performed in seven and showed inactive lesions in all. Two patients developed recurrent disease. Re-institution, or increase in their dose, of corticosteroid again resulted in inactivation of their nephritis. 
ACID-BASE DYNAMICS DURING HEMODIALYSTS IN PEDTATRIC PATIENTS. Alfredo J. Elberg, Rex Baker, Helen Gorman, Vincent R. Finlay, A. Jose Wenger and Jose Strauss. University of Miami, School of Medicine, Department of Pediatrics, Miami, Florida 33152

Ten five hour dialyses were performed in four pediatric paUltraflo II ${ }^{R}$ dialyzer $\left(1.4 \mathrm{~m}^{2}\right)$. Blood flow through the dialyzer was $275 \pm 29 \mathrm{ml} / \mathrm{min}$ achieving a urea clearance of $4.7 \pm 0.5$ $\mathrm{ml} / \mathrm{min} / \mathrm{kg}$. Ultrafiltration was adjusted to remove excess body fluid. The dialysate (Diasol ${ }^{R}$ B-S-L-120) contained $37 \mathrm{mEq} / 100 \mathrm{ml}$ sodium acetate as its only buffer. During dialysis $\mathrm{pH}$ increased from $7.40 \pm 0.01$ to $7.51 \pm 0.02(\mathrm{P}<0.0005) ; \mathrm{PCO}_{2}$ decreased from $32.4 \pm 1.1$ to $22.5+1.4 \mathrm{~mm} \mathrm{Hg}(\mathrm{P}<0.0005)$; actual $\mathrm{HCO}_{3}$ decreased from $20.1+0.8$ to $17.7+1.0 \mathrm{mEq} / 1 \quad(\mathrm{P}<0.05)$. Base deficit was $3.9 \pm 2.7 \mathrm{mEq} / \mathrm{l}$; hemoglobin, $5.1 \pm 0.2 \mathrm{~g} \%$; lactic acid, $13.0 \pm$ $5.1 \mathrm{mg} \%$; pyruvic acid, $0.83 \pm 0.4 \overline{0} \mathrm{mg} \%$; and ketone bodies, absent these variables did not significantly change during dialysis. No significant changes in respiration or pulse rates were noted throughout dialysis. The presence of an elevated arterial blood $\mathrm{pH}$ with lowered $\mathrm{PCO}_{2}$ and $\mathrm{HCO}_{3}^{-}$does not agree with reports in the literature. The following explanations seem possible: 1) preferential loss of $\mathrm{CO}_{2}$ through a dialyzer of relatively large exchange area and at high blood flow rate; 2 ) decrease in $\mathrm{HCO}_{3}^{-}$limited by metabolism of acetate from the bath. Thus, reported results cannot be generally applied. When different dialyzers, dialysates and blood flow settings are used, specific acid-base changes must be identified and evaluated frequently to avoio potentially
dangerous situations.

826 SODIUM NITROPRUSSIDE IN PATIENTS UNDERGOING HEMODIALYSIS. Eduardo H. Garin, Robert S. Fennel1, III, Car1 D. Sorgen, Norman D. Pryor, and George A. Richard (Spon. by E1ia M. Ayoub), University of Florida College of Med., Department of Pediatrics, Gainesville.

Three girls with severe hypertension and end stage renal disease received nine intravenous infusions of sodium nitroprusside. The drug was administered for three hours during hemodialysis. In each instance, desired levels of blood pressure were reached at an average infusion rate of $1.94 \mathrm{\mu g} / \mathrm{kg} / \mathrm{min}$ (range $0.4-3.5$ ) within pressure increased slightly but did not attain pretreatment values. When each child served as her own control, the immediate post dialysis weight and blood pressure were not significantly different whether she did or did not receive an infusion of sodium nitroprusside during hemodialysis. Side effects were minimal (headaches, nausea, restlessness), inconstant, and remitted regularly when the infusion rate was reduced. In no instance did hypotension occur. Sodium nitroprusside appears to be effective and safe for the control of blood pressure in patients undertients (b.w. $37.0 \pm 4.2 \mathrm{~kg}$ (SE); s.a., $1.22 \pm 0.08 \mathrm{~m}^{2}$ ) using an 4 to 23 minutes. Upon discontinuance of the drug, the blood going hemodialysis.

828

COPPER AND ZINC DEPLETION IN END STAGE RENAL DISEASE (ESRD). Grupe, W.E., Kopito, L.E., Lazarus, J.M. and Brown, J.A., Children's Hospital Medical Center Boston, Massachusetts.

The role of trace element abnormalities in ESRO in children has not been previously investiqated. Analysis of 50 sera from 11 males and 13 females, ages 24 mos. -22 yrs. (mean 12.8 yrs.) with ESRD disclosed $62 \%$ of samples with low $\mathrm{Cu}$ and $50 \%$ with low $\mathrm{Zn}$, while levels of $\mathrm{Mg}, \mathrm{Mn}, \mathrm{Al}$ and $\mathrm{Cr}$ were normal. During stable dialysis, 768 of children had at least one samble with low $\mathrm{Cu}$ and 648 with low $\mathrm{zn}$. Sixty-two percent still had low $\mathrm{Cu}$ 3-5 mos. after successful transplantation while all had returned $\mathrm{Zn}$ levels to normal by 3 mos. Levels of $\mathrm{Cu}$ and $\mathrm{zn}$ were below normal in skin, kidney and fat; $\mathrm{Cu}$ was a third of nomal in muscle with $\mathrm{zn}$ levels only slightly decreased. $\mathrm{Cu}$ levels in hair were normal but $\mathrm{Zn}$ was elevated to levels similar to that seen in proteincalorie malnutrition. Serum $\mathrm{zn}$ levels are related to caloric intake; of those with intakes $>80 \mathrm{Cal} / \mathrm{kq}$, all had normal $\mathrm{zn}$, while 598 with intakes $<60 \mathrm{Cal} / \mathrm{kg}$ had low $\mathrm{zn}$. Cu levels vary independently of $\mathrm{Zn}$ and are not related to caloric intake. Neither $\mathrm{Cu}$ nor $\mathrm{Zn}$ correlated with protein intake. Cu levels were lower in non-nephrectomized patients while $\mathrm{zn}$ levels were lower in the nephrectomized children. Neither $\mathrm{Cu}$ nor $\mathrm{Zn}$ correlate with the duration of dialysis nor do serum levels vary pre- and post-dialysis.

The results are consistent with both $\mathrm{Cu}$ and $\mathrm{zn}$ depletion in ESRD. The reason for low $\mathrm{Cu}$ is not clear. Low $\mathrm{Zn}$ is related to insufficient caloric intake and may contribute to the poor appetite and poor growth characteristic of these children.

\section{PLASMA CREATININE (Pcr) AND ITS USE IN ESTIMATING 829 GLOMERULAR FILTRATION RATE (GFR) IN CHILDREN.} George B. Haycock, George J. Schwartz, Chester M. Edelmann, Jr., and Adrian Spitzer. Albert Einstein Col. of Med., Bronx, New York.

The range of values for Pcr in normal children is not well documented. Moreover, it is difficult to estimate the level of GFR corresponding to a given Pcr. Pcr was measured with a Technicon AutoAnalyzer in 1398 children 1-20 years of age with normal creatinine clearances $(\mathrm{Ccr})$. Pcr increased significantly with age, more rapidly in males $(M)$ than females $(F)$, $P<0.001$. The coefficient of variation was about $25 \%$ of the mean values in both sexes, at all ages. The relationship between age and Pcr was expressed by the regression equations:

$M: \operatorname{Pcr}(\mathrm{mg} / \mathrm{dl})=0.35+0.025$ age $(\mathrm{yr}) ; r=0.53$.

F: $\operatorname{Pcr}(\mathrm{mg} / \mathrm{dl})=0.37+0.018$ age $(\mathrm{yr}) ; \quad r=0.49$.

In the steady state creatinine $(\mathrm{Cr})$ production must equal $\mathrm{Cr}$ excretion. $\mathrm{Cr}$ production is a function of muscle mass, which can be approximated from body volume (V). Excretion is dependent on GFR, which, after 1 year of age, is proportional to body surface area $(S A)$. Since $V\left(\mathrm{~cm}^{3}\right) / S A\left(\mathrm{~cm}^{2}\right)=$ body length $(L)$, Pcr should be proportional to $L(\mathrm{~cm})$. Accordingly, an estimate of GFR was derived from analysis of these variables in 186 children with Ccr ranging from $5-220 \mathrm{ml} / \mathrm{min} / 1.73 \mathrm{~m}^{2}$. The resulting formula, GFR $\left(\mathrm{ml} / \mathrm{min} / 1.73 \mathrm{~m}^{2}\right)=0.55 \mathrm{~L}(\mathrm{~cm}) / \mathrm{Pcr}(\mathrm{mg} / \mathrm{dl})$, was tested against clearance data in a separate group of 223 children and gave excellent agreement with $\operatorname{Ccr}(r=0.94)$ and inulin clearance $(r=0.91)$. We conclude that Pcr, normalised for $L$, provides a good estimate of GFR. COID INSOLUBLE IMMUNE COMPLEXES AND GLOMERU-

827 LONEPHRITIS, William R. Griswold, Ray Brady, and Noel K. Dysart. Department of Pediatrics, University of California, San Diego and Clinical Investigative Center, Naval Regional Medical Center, San Diego.

The relationship between cold insoluble circulating immune complexes (CIC) and acute glomerulonephritis was studied. Acute nephritis was produced in rabbits by injecting $250 \mathrm{mg} / \mathrm{kg} 125 \mathrm{I}$ labelled bovine albumin. Serial determinations of urine volume, urine protein, serum creatinine, Farr complexes (FC) - done by ammonium sulfate precipitation -, and CIC were performed in 17 animals.

All animals developed FC. Fourteen of 17 animals developed CIC. FC appeared at an average of 6.8 days after injection. CIC appeared at 10.8 days. Nephritis, determined by oliguria, proteinuria or elevated serum creatinine occurred in $86 \%$ of animals, beginning at 11. 3 days. In each animal the appearance of CIC closeIy paralleled the onset of nephritis.

Animals were divided into two groups. Group I animals had cumulative CIC over $14 \mu \mathrm{g} / \mathrm{ml}$ of serum. Group II animals had CIC under $14 \mu \mathrm{g} / \mathrm{ml}$ of serum. The mean peak serum creatinine was $3.0 \mathrm{mg} \%$ in group I and 1.4 $\mathrm{mg}$ \% in group II $(\mathrm{p}<0.01)$.

In animals, as in man, CIC are a reliable sign of acute immune complex glomerulonephritis. CIC may be of immunopathologic significance in this model.
MATURATION OF RENAL ORGANIC ACID TRANSPORT IN VIVO:

830 SUBSTRATE STIMULATION BY PENICILLIN. Jenny T. Johnson, Univ., Col. of Human Med., East Lansing.

PAH accumulation by renal cortical slices from newborn rats, rabbits and pups was enhanced by pretreatment in vivo with substrates of the organic acid transport system (Science 165:909, 1969; Biochem. Pharm. 23:754, 1974). These experiments were designed to demonstrate that pretreatment of newborns with penicil1 in will enhance the maturation of the renal organic anion transport system in vivo. Littermate pups were injected with procaine penicillin G $(300,000 \mathrm{IU} / \mathrm{kg}$ B.I.D. for 3 days) or saline prior to 2 weeks of age. Animals were surgically prepared on day 16 or 17 . Transport of p-aminohippurate (PAH) as an indicator of renal organic anion transport, was quantified by the following procedures: (1) disappearance of a bolus injection from the plasma, (2) renal extraction, and (3) determination of the tubular maximum (Tm). The renal handling of inulin was measured simultaneous$1 \mathrm{y}$ in all procedures for comparison. Penicillin-treated animals excreted a single bolus injection of PAH more rapidly than control animals with the $T_{2}^{\frac{1}{2}}$ decreased and the Ke increased. The extraction of PAH by kidneys of penicillin-treated animals was $165 \%$ that of controls. The maximum transport capacity for PAH was handling of organic anions in vivo is enhanced by pretreatment with a substrate of the secretory system. also increased in treated pups. These data confirm that the renal 
831 HYPERKALEMIA AND RENAL TUBULAR UNRESPONSIVENESS TO Ronald J. Kallen, Rosa T. Ong, and Andrew J. Aronson (Spon. by Eddie S. Moore) University of Chicago Pritzker Schoo of Medicine, La $R$

A is year old girl with short stature, failure of sexual hyperkalemia was found to have glomerular basement membrane associated dense deposits. Creatinine clearance was $75 \%$ of 17-OHCS were normal. An oral potassium load suggested a limited capability to augment renal potassium excretion. Treatment with alpha-fluorohydrocortisone and DOCA, at pharmacologic dosage levels, depressed serum potassium to high-normal range. Buffer replacement treatment of metabolic acidosis failed to correct hyperkalemia. We conclude that renal tubular unresponsiveness to physiologic levels of endogenous aldosterone may be a manifestation of sickle cell disease nephropathy. MINERALOCORTICOID IN SICKLE CELL DISEASE NEPHROPATHY. maturation, sickle cell disease, renal tubular acidosis, and normal and maximal urinary osmolality was depressed $(264 \mathrm{~m} 0 \mathrm{sm} / \mathrm{kg})$. Plasma renin activity, urinary aldosterone excretion, and urinary

834

NONCOMPLIANCE IN FEMALE ADOLESCENTS WITH KIDNEY 834 and Vida Francis-Neqrete. Univ. of Southern Cal if. Systematic Dept. of Peds., Chi 83 pediatric patients with functioning allografts, from 1 to 8 years following kidney transplantation, was carried out to assess quality of life. Analyses focused on noncompliance with immunosuppressive regimen. Noncompliance was suspected when unexplained changes in allograft function or lessening of cushingoid features were noted, and con$f i r m e d$ by means of exploration \pm confrontation with appropriate psychosocial team members. Noncompliance led to partial or complete rejection of the allograft in 14 patients. Twelve of the 14 were female adolescents. In most of the noncompliant patients, there was evidence of family disorganization and/or lack of emotional support for the patient. The father's role in family functioning also was found to be significantly related to compliance. Personality test results indicated extremely low personal adaptation and low self-esteem in all but 2 of the noncompliant patients. Discriminant analys is yielded a function which selects compliant patients in the sample with $98.5 \%$ reliability and noncompliant patients with $92 \%$ reliability. The discriminant function is comprised primarily of demographic, family and personality test variables. Follow-up data suggests that female adolescents with depressed personality functions and insufficient family support are at the highest risk for noncompliance and need special attention from the treatment team. These risks need to be considered in formulating treatment plans. Also noncompliance needs more emphasis as a possible cause of allograft rejection.

835 CRITERIA FOR RADIOGRAPHIC EVALUATION OF CHILDREN IN VIVO ANALYSIS ON PAH TRANSPORT IN THE 832 MATURING RABBIT KIDNEY. Matthew R. Kaplan, Bama Rucker and John E. Lewy. Cornell Univ. Med. Coll. -N.Y. Hosp., Dept. of Ped., New York.

PAH transport in rabbits as estimated by extraction of PAH (EPAH) can be enhanced in vivo by injection with penicillin (PCN) on days 10-13 of life. This study was undertaken to determine if enhancement can be induced throughout the neonatal period, the duration of stimulation and the effect of preinjection on renal mass. 62 rabbits were injected with 6 doses of procaine PCN prior to study. 60 littermates served as saline injected controls. Maturation was shown in controls with EPAH increasing from $29.6 \pm 2.2 \%$ at 10 days to $35.5 \pm 2.0 \%$ at 14 days, $62.6 \pm 2.9 \%$ at 21 days and $74.5+1.5 \%$ at 28 days of life. PCN injection on the 3 days prior to study resulted in enhancement of EPAH on days 10 and 14 to $42.7 \pm 2.4 \%$ and $58.1 \pm 3.1 \%$ $(p<.005)$; however on days 21 and 28 less stimulation was noted $(69.1 \pm 2.2 \% ; 82.5 \pm 4.7 \%)$. Preinjection on days 6-9 with study delayed to day 14 also led to enhancement $(p<.05)$ but less than that achieved after injection on days 10-13. EPAH on day 21 was not augmented when pretreatment occurred on days 10-13. Kidneys of groups showing most stimulation were significantly heavier than controls. These data suggest that EPAH stimulation may he transient, decreases as maturation is neared, and may be related in part to substrate availability or increased renal mass.

IHE PROGRESSIVE REDUCTION IN BICARBONATE REABSORPTTON $\left(\mathrm{HCO}_{3} \mathrm{R}\right.$ ) IN A PATTENT WITH LOWE'S SYNDROME (L.S $\mathrm{S}_{\circ}$ ) Edward C. Kohaut, George A. Edwards, Arthur L. Beaudet, and L. Leighton Hill, Baylor College of Medicine, Dept. of Pediatrics, Houston.

This study was undertaken to better define the nature of the renal acidification defect seen in $L_{\circ} S$. The diagnosis of L.S. was made at two weeks of age. The patient presented with bilateral cataracts, congenital glaucoma, and marked hypotonia. A generalized aminoaciduria was noted. At three weeks of age, inulin clearance $\left(\mathrm{CL}_{\mathrm{I}}\right)$ and $\left(\mathrm{HCO}_{3} \mathrm{R}\right)$ were measured. Titratable Acidity (T.A.) and Ammonium Excretion $\left(\mathrm{NH}_{4}\right)$ were measured after an acute ammonium chloride load. These studies were repeated at ages 1 and $2 \frac{1}{2}$ years. The following data was obtained.

\begin{tabular}{|c|c|c|c|c|c|c|}
\hline Age & CLI avg. & $\begin{array}{c}\text { Max. } \\
\mathrm{HCO} 3 \text { fil.* }\end{array}$ & $\begin{array}{c}\text { Max. } \\
\text { HCO } 3 \underline{R}^{*}\end{array}$ & $\mathrm{pCO}_{2}^{\mathrm{u}}-\mathrm{pCO} 2$ & $\begin{array}{c}\text { Max. } \\
\mathrm{NH}_{4} \text { Exc.+ } \\
\end{array}$ & $\begin{array}{l}\text { Max. } \\
\text { T.A. }+\end{array}$ \\
\hline wks. & 5.8 & 3.1 & 2.7 & & 27.5 & 19.2 \\
\hline yr. & 20.0 & 2.3 & 1.8 & & 62.3 & 24.8 \\
\hline & 23.4 & 1.9 & 1.5 & 7.3 & 88.4 & 23.5 \\
\hline
\end{tabular}

$\mathrm{pCO} 2^{-} \mathrm{pCO}=$ difference between $\mathrm{pCO} 2$ in urine and plasma

*corrected to $\mathrm{mm} / 100 \mathrm{cc}$ GFR

tcorrected to $\mathrm{uEq} / 1.73 \mathrm{~m}^{2} /$ minute

The only defect demonstrated is bicarbonate wasting. Ammonia excretion was normal. A gradient between urine and plasma $\mathrm{pCO}_{2}$ was generated. Of interest, maximum $\mathrm{HCO}_{3} \mathrm{R}$ was normal at 3 weeks but decreased over $2 \frac{1}{2}$ years. This suggests that reduced $\mathrm{HCO}_{3} \mathrm{R}$ is not a primary defect in L.S. but may be secondary to a yet unknown defect which leads to a functional derangement of the proximal tubular cell.
835 WITH URINARY TRACT INFECTION (UTI). Levitt, S.B. Bekirov, H., Kogan, S., Spitzer, A., Greifer, I., Lutzker, L., Edelmann, C.M., Jr. Albert Einstein Col. of Med., Bronx, New York. Uncertainty persists regarding the criteria of selection for investigation and the extent of the work-up indicated in children with UTI. An 8-year experience with 218 children with documented UTI, studied at a primary care center, is reported. Radiographic evaluation of the urinary tract was performed several weeks after sterilization of the urine. IVU abnormalities, such as horseshoe kidney, pyelonephritic scarring, and hydronephrosis, were found in 3/99 girls with symptoms of lower UTI (Gp A), but in 23/57 with symptoms related to the upper urinary tract $(G p B)(p<.001)$. VCU, done in 77 of Gp A, was abnormal in 18, including all 3 with abnormal IVU. VCU in Gp B was abnormal in 10/20 with abnormal IVU and 18/24 with normal IVU. In males, abnormalities on IVU were found in 19/62, a significantly higher proportion than in females $(\alpha<.02)$. VCU was abnormal in $11 / 15$ with abnormal IVU and in $11 / 28$ with normal IVU. As with girls, a higher percentage of boys with upper UTI symptoms had abnormal ities on either IVP or VCU or both. Neither study alone, however, detected all abnormal boys in either the upper or the lower group. This experience suggests that $\mathrm{VCU}$ is the procedure of choice in the investigation of girls with symptoms of lower UTI. If normal, and response to therapy has been satisfactory, no further radiologic studies are indicated. In girls with upper urinary tract symptoms, and in all boys, both IVU and VCU should be performed to detect abnormalities of the upper and lower tracts.

936 THE TIME OF APPEARANCE OF GAMMA GLOBULIN COATED BAC836 TERIA IN THE URINE DURING EXPERIMENTAL HEMATOGENOUS PYELONEPHRITIS. Sherwood A. Libit, Barbara J. McMann, Ellis E. Chung and Karen J. Bogatz. Spon. by Eddie S. Moore

Univ. Clicago at Michael Reese Med. Ctr. Chicago.

Detection of antibody coated bacteria in the urinary tract of patients with clinical urinary tract infection (UTI) appears to be a useful clinical technique and correlates well with the site of UTI determined by bladder washout. This study was undertaken to determine the time of appearance of garma globulin coated bacteria (GGB) in the urine relative to the onset of bacteriuria and histopathologic lesions in a model of experimental pyelonephritis. Sprague-Dawley rats were given streptococcus faecalis ( $3 \times 10^{9}$ organisms/animal) intravenously and their urine was serially studied for the appearance of GGB and the onset of bacteriuria. Bacteriuria was regularly present in the animals sacrificed $24 \mathrm{hrs}$ after inoculation as has been previously clescribed. GGB were detected by fluorescent antibody in the urinary sediment of $10 / 10$ animals studied 72 hours after inoculation, $24 \mathrm{hrs}$. after the apthe onset of bacteriuria. The animals had no antecedent antibody to this organism by testing serum and urine with the indirect fluorescent antibody technique. The interval between the appearance of bacteriuria in this model of renal infection and that of GGB in the urine is $48 \mathrm{hrs}$. This study supports the clinical usefulness of this test in identifying patients with upper UTI. pearance of pyelitis and interstitial nephritis and $48 \mathrm{hrs}$. after 
837

ROLE OF TUBULAR BASEMENT MEMBRANE AND PERITUBULAR PROTEIN CONCENTRATION IN THE CONTROL OF RENAL TUBULE CELL VOLUME. Michael A. Linshaw, Mark Dellasega, and Jared J. Grantham, Depts. of Pediatrics and Medicine, Univ. of Kansas Medical Center, Kansas City, Kansas. (Spon. by Cheng T. Cho).

Renal tubule cell volume is thought to be regulated by active cation transport. Ouabain, by inhibiting ATPase, blocks sodium transport, allowing passive cellular uptake of saline with resultant cell swelling. However, the observed degree of swelling in ouabain is less than expected were active transport completely inhibited. This has led some investigators to postulate a ouabain insensitive cation pump. Alternatively, the elastic basement membrane might mechanically restrict swelling and thereby develop a hydrostatic pressure gradient between cells and interstitium. To test this hypothesis, 29 single proximal straight tubules with collapsed lumens were isolated from rabbit kidneys and both ends tightly crimped in pipets. In control serum (6 gm\% protein), cell volume as assessed from tubule diameter increased $40 \%$ upon addition of ouabain $\left(10^{-4 M}\right)$. Removing the basement membrane with collagenase $(500 \mathrm{u} / \mathrm{ml})$ caused more cell swelling. Furthermore, final cell volume was determined largely by bath protein concentration. Tubules treated with ouabain and collagenase swelled $111 \%$ above control volume in the absence of protein, but could be shrunk to normal size in hyperoncotic protein. Similar changes occurred using pure bovine albumin. On the basis of these findings we conclude that after treatment with ouabain, the ability of renal tubule cells to resist maximal swelling may be due to hydrostatic and colloid osmotic forces rather than from a unique ouabain insensitive cation pump.

THE EFFECT OF PTH ON RENAL TUBULAR PERMEABILTTY

838 William B. Lorentz, Jr., Department of Pediatrics Bowman Gray School of Medicine, Winston-Salem, N. C.

The effect of parathyroid hormone (PTH) on renal tubular perme. ability has been studied utilizing micropuncture techniques in the rat kidney, Nanoliter quantities of a solution of $3 \mathrm{H}$ inulin and ${ }^{14} \mathrm{C}$ mannitol in normal saline was microinjected into surface nephrons and urine from both kidneys analyzed for radioactivity. During control periods, inulin $(98.8 \pm 2.7 \%)$ and mannitol $(97.2+$ $2.4 \%$ ) recovery from the experimental kidney was essentially complete, Following intravenous infusion of PTH, reinjections were performed at the same puncture site. Inulin $(99.3 \pm 2.9 \%)$ recovery was not different from contro1. Mannitol recovery was signif. icantly decreased following both early proximal ( $84.7 \pm 5.8 \%$, $\mathrm{p}<0.001)$ and late proximal $(89.7 \pm 2.8 \%, \mathrm{p}<0.001)$ injection. There was no significant loss of either mannitol or inulin following distal tubular injection. Late proximal $\frac{T F}{P}$ inulin ratios during control conditions were $2.10 \pm 0.20$ and decreased to $1.99 \pm$ 0.21 during PTH. This difference was not significant $(0.10>\mathrm{p}>$ $0.05)$. Late proximal $\frac{\mathrm{TF}}{\mathrm{T}}$ manitol ratios were, during control periods, $2.09 \pm 0.21$. Puring PTH infusion this decreased significantly $(P<0.001)$ to $1.78 \pm 0.19$. These results indicate that PTH induces a change in proximal tubular permeability to a usually impermeable non-electrolyte, mannitol. Therefore, the effects of PTH on proximal tubular transport could be partially explained by this alteration of the permeability characteristics of the proximal tubular epithelium. There would be an increased passive backflux of actively transported species, thus decreasing net transport while having no effect on the active transport component.

THE RELATIONSHIP BETWEEN OBESITY, ANXIETY, AND OTHER

839 RISK FACTORS TO ONSET OF ESSENTIAL HYPERTENSION IN CHILDREN. Barbara J. McMann, Eddie S. Moore, Ellis E. Chung, Eduardo E. Cevallos and Sherwood A. Libit. Dept. Peds. Univ. of Chicago at Michael Reese Med. Ctr. Chicago, I1.

We have studied the possible influence of certain risk factors on the pathogenesis of essential hypertension in childhood. Essential hypertension was diagnosed in 25 children age 4 to 17 years with diastolic pressures greater than 2 S.D. above the normal for age. Other causes of hypertension were ruled out. The mean age at onset was 12 years and the follow-up period has been 3 to 42 months. A family history of hypertension was present in $80 \%$ of the cases. The hypertension was fixed in $33 \%$ of the patients, while $67 \%$ had episodic hypertension on two or more occasions. There are 9 females and 16 males. 3 of the patients were white and 22 were black. 3 patients were from social class 1 (lowest), 3 in class 2,2 in class 3,4 in class 4 , and 5 in class 5 (highest). $86 \%$ of the patients were in the 97 th percentile for weight at onset. A history of excessive salt intake was not present. $48 \%$ of the patients have a positive history of anxiety or emotional complaints. Control and furosimide stimulated renin levels were normal in 23 patients, low in one patient and high in one patient. This study identifies certain risk factors such as emotional state, sex, weight, race, social class, and family history which may precipitate onset of hypertension during childhood. A multi-clinic prospective study is necessary to confirm these results.
ACTIVE JEJUNAL TRANSPORT IN AMINONUCLEOSIDE
N4.0 NEPHROSIS. Mor, J., Wapnir, R.A., Lifshitz, F.,
Teichberg, S., Mrozinska, K., McVicar, M. Dept. of Peds
Peichere Univ. Hosp. Manhasset, N.Y., N.Y. and Cornell and Path. N. Shore Univ. Hosp.
Univ. Med. Col., N.Y., N.Y.

Male $180-200 \mathrm{~g}$, Wistar rats, were rendered nephrotic by $\mathrm{i} . v$. injection of $7.5 \mathrm{mg} / 100 \mathrm{gm}$ body weight aminonucleoside of puromycin (PAN). Fourteen days later, $20 \mathrm{~cm}$ of proximal jejunum was perfused in vivo with Krebs Henseleit bicarbonate buffers containing $1 \mathrm{mMole}$ of a single $3 \mathrm{H}$ amino acid (AA) and a $14 \mathrm{C}$ carbohydrate $(\mathrm{CHO})$. Polyethylene glycol was used as a non-absorbable marker. Rats were hypoalbuminemic and proteinuric at the time of study. The mean absorption expressed as mMole/ $\mathrm{min} / \mathrm{cm}$ of jejenum $+\mathrm{SEM}$. was:

$\begin{array}{llll} & \text { Nephrotic }(\mathrm{N}) & \text { Control }(\mathrm{C}) & \mathrm{p} \\ \text { phenylalanine } & 4.5 \pm .19 & 5.3 \pm .25 & <.01 \\ \text { histidine } & 4.6 \pm .14 & 6.3 \pm .16 & <.001 \\ \text { glycine } & 4.4 \mp .13 & 5.3 \pm .16 & <.001\end{array}$

The absorption of 3-0-methy $\bar{\Gamma}$ glucoside was also impaired in nephrotic rats; mean $10.10 \pm .56 \mathrm{mMoles} / \mathrm{min} / \mathrm{cm}$ vs. $17.3 \pm .74$ in controls. The rate of $\mathrm{Na}$ transport was decreased in nephrotic rats: Na $136 \mathrm{mMoles} / \mathrm{min} /$ $\mathrm{cm}+25.1(\mathrm{~N})$ vs. $225 \mathrm{mMoles} / \mathrm{min} / \mathrm{cm}+17.9$ (C), p<.001. Fructose absöption was normal. No jejunal edema was detectable by histology or comparison of wet and dry weights and ultrastructure was normal. These studies indicate an impairment of active jejunal transport in PAN induced nephrosis.

ANTTBODY-COATED BACTERTA IN URINE SEDIMENT FOR LOCALIZATION OF THE SITE OF INFECTION. Larry
Nussbaum, Eileen Kennedy, Stanley Hellerstein The Children's Mercy Hospital, Kansas City, Mo.

Studies in adults have shown that an immunofluorescent test for antibody-coated bacteria in urine sediment is highly reliable for localizing the level of a urinary tract infection. In pediatric patients we found antibody-coated bacteria in roughly $50 \%$ of children with urinary tract infections. However, there was poor correlation between the level of infection indicated by clinical findings and that defined by the test for antibody-coated bacteria in the urine sediment. For this reason we are comparing the latter test with bladder washout for localization of infections. We have now done combined tests during 19 urinary tract infections in 16 children. The results are shown in the table.

Bladder Washout* Antibody-Coated Bacteria

Upper Tract Lower Tract Total

$\begin{array}{ccc}\text { Present } & \frac{\text { Absent }}{7} & \frac{\text { Total }}{7} \\ 10 & \frac{3}{9} & \frac{12}{19}\end{array}$

Localization by ureteral catheterization in one patient.

A comparison of bladder washout with antibody-coated bacteria for localization of the site of infection in children does not show close correlation as reported for adults.
842 LINEAR GROWTH IN LONG TERM RENAL ALLOGRAFT RECIPIENTS. Alfred J.Pennisi, Mohammad H. Malekzadeh, Christel Uittenbogaart \& Richard N. Fine. USC School of Medicine, Childrens Hospital of Los Angeles, Dept. Pediat., Los Angeles.

Suppressed linear growth due to corticosteroid (C) administration is a frequent occurrence in pediatric renal transplant recipients (RTR). The mechanism of $C$ induced growth suppression has not been delineated. Linear growth was assessed in 39 RTR receiv ing daily $C$ (serum creatinine $\leq 2 \mathrm{mg} \%$ ) 3 to 8 yrs. post ransplant. In 17 of 39 RTR, bone age (BA) was $>12$ yrs. at transplant; 11 had no growth posttransplant and the remaining 6 grew $2-3 \mathrm{~cm}$. Twenty two of 39 RTR had BA $<12$ yrs. at transplant; growth rate was compared with expected rate for normal child of same sex and chronological, bone and height ages growing at the 50th percentile. Eight of 22 RTR grew at greater than $80 \%$ of expected for chronological age. In contrast when height and bone age were used for growth assessment, only 3 and 4 respectively grew at $>80 \%$ of expected. $C$ therapy was changed from daily to alternate day $(A D)$ in 10 of 18 patients with growth rate $<80 \%$ of expected for $B A$. All had $B A<12$ yrs, and stable renal function at the time of therapy change. Growth rate for the subsequent 8 to 18 months was $>80 \%$ of expected for $B A$ in 3, improved in 1, and unchanged in 6 . $A D$ was not associated with deterioration of allograft function.

The data demonstrate that normal growth occurs in $14-36 \%$ of pediatric $R T R$ with $B A<12$ yrs. receiving daily $C$; the incidence of normal growth varies with method of assessment. $A D$ appears to be associated with some improvement of linear growth without impairment of allograft function. 
843

TREATMENT OF HYPERRENINEMIC HYPERTENSION WITH PROPRANOLOL IN CHILDREN AFTER RENAL TRANSPLANTATION. DOnald E. Potter, Morris Schambelan, Oscar Salvatierra, Jr., Malcolm A. Holliday, Depts, of Pediatrics, Medicine \& Surgery, University of California, San Francisco.

Although hypertension is frequently associated with hyperreninemia in post-transplant patients, treatment with propranolol has not been evaluated in this group. Treatment of hypertension with propranolol also has not been evaluated in children. The use of propranolol was studied prospectively in eight children 6 to 14 years of age with hypertension and elevated peripheral plasma renin activity (PRA) 3 to 16 months after transplantation. Four presented with hypertensive encephalopathy, and 7 were treated with multiple antihypertensive agents with poor control of BP prior to starting propranolol. Three had acute rejection, 3 chronic rejection, 1 recurrent glomerulonephritis, and 1 normal renal function. Plasma volume was normal in 5 and low in 1 . Mean PRA in the recumbent and upright positions was 44.6 and $66.9 \mathrm{ng} / \mathrm{ml} / 3$ hours respectively (normal 2-10 and 5-32). When propranolol, $1.0-9.0 \mathrm{mg} / \mathrm{kg} / \mathrm{day}$ was added to the antihypertensive regimen, BP fell significantly in 6 children $(137 / 99+120 / 79)$ within $1-7$ weeks. Two childxen did not respond and were subsequently treated with minoxidil. Upright PRA, repeated in 5 of the 6 children who responded, decreased in all $(66.7 \rightarrow 2 l .2)$.

Propranolol was well tolerated and bradycardia and postural hypotension were insignificant. It is concluded that 1) propranolol is a valuable drug in the treatment of post-transplant hyperreninemic hypertension, and 2) propranolol can be recommended for hypertension in children.

LOCALIZATION STUDIES IN COLLEGE COEDS WITH BACTERI-

844. URIA. Norman D. Pryor, Carl D. Sorgen, Robert S. Fenne11, III, Eduardo H. Garin, R. Dixon Walker, and George A. Richard, (Spon. by Elia M. Ayoub), University of

Demonstration of antibody-coated bacteria in the urine by an immunofluorescent test (FA) and depressed concentrating capacity may be indicative of upper tract infection and have been shown to correlate well with direct methods of localization such as the bladder washout technique.

During a 1-year period, 209 college coeds presenting with dysuria were shown to have significant bacteriuria. Several therapeutic regimens were utilized for the eradication of the causative organism. Those patients with significant bacteriuria when initially evaluated were shown to be FA positive (FAt) in $95 / 190$ $(50.0 \%)$, had depressed concentrating capacity in $53 / 180(29.4 \%)$, and demonstrated both findings in $29 / 130$ (16.1\%).

The recurrence rate 1 month post $\mathrm{Rx}$ was $20 / 190(10.5 \%)$. Of these 20 recurrences, $13(13.7 \%)$ were in the group who were initially FAt (95) and $7(7.4 \%)$ were in the group initially FA(95). In the 13 initially FAt who experienced a recurrence within 1 month post Rx, 8 remained FAt and 5 became FA-; however, of the 7 initially FA-, 6 remained FA- and 1 became FAt.

of 196 dysuric and bacteriuric coeds evaluated, 60 had associated back pain. Of these, 30 were FAt and 30 were FA-; therefore, back pain was not an indicator of upper tract infection.

Localization of the site of urinary tract infection by the demonstration of antibody-coated bacteria in the urinary sediment appears to be a most sensitive and reliable method.

PRIMARY DISTAL RENAL TUBULAR ACIDOSIS (RTA) OF TRAN-

845 SITORY EVOLUTION DURING THE FIRST YEAR OF LIFE. David D Rao, Margaretia L Jackson, Gladys H Hirschman and Ctr, Dept of Nephrology, Washington, DC (Intr by Robert H Parrott) Transient types of primary proximal. RTA with onset within the first year of life and complete recovery have been reported previously. This study documents a case of transient type of primary distal RTA, bicarbonate wasting, diagnosed at 4 months of age which reverted to normal after 12 months of treatment and close followup.

Clinical features included slow weight gain, recurrent urinary tract infection and persisting enuresis. Lab data showed impaired $\mathrm{H}^{+}$secretory capacity with $\mathrm{UpH} 7.17$, blood $\mathrm{pH} 7.34, \mathrm{tCO}_{2}$ $14 \mathrm{mMol} / 1, \mathrm{Cl} 110 \mathrm{mEq} / 1$. Short-term $\mathrm{NH}_{4} \mathrm{Cl}$ loading tests (75-100 $\mathrm{mEq} / \mathrm{m}^{2}$ ) were performed: net acid excretion (NAE) data in $\mu \mathrm{Eq} /$ $\mathrm{min} / 1.73 \mathrm{~m}^{2}$ at 0,6 and 12 months of treatment are presented in the table below. The dosage of $\mathrm{HCO}_{3}^{-}$was $5-6 \mathrm{mEq} / \mathrm{kg} / \mathrm{day}$ and

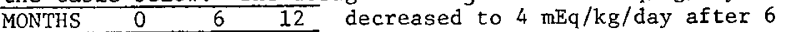
$\begin{array}{lcccc}\text { MONTHS } & 0 & \frac{6}{10} & 12 & \text { decreased } \\ \text { UpH } & 6.0 & 5.30 & 5.05 & \text { months of therapy. The fractional. }\end{array}$ NAE $\quad 35.5 \quad 50.9 \quad 94.4$ excretion of filtered $\mathrm{HCO}_{3}^{-}$at plasma TA $\quad 12.5 \quad 11.3 \quad 29.2 \quad \mathrm{tCO}_{2} 1$ eve 1 of $19 \mathrm{mMol} / 1$ was $5.3-8.7 \%$; $\mathrm{NH}_{4} \quad 22.9 \quad 39.7 \quad 65.2 \quad 1.4-5.7 \%$ and $1.3-1.4 \%$ for 0,6 and 12 months of treatment, respectively.

The acidification became normal after 12 months of treatment, permitting the alkali therapy to be discontinued. Laboratory data at 1 month intervals 4 months later showed sustained normal values for blood acid base and UpH.
846 LOCALIZATION OF SITE OF INFECTION IN INFANTS AND CHILDREN WITH BACTERIURIA. George A. Richard, Robert S. Fenne11, III, Eduardo H. Garin, Norman D. Pryor, Carl D. Sorgen, and R. Dixon Walker (Spon. by Elia M, Ayoub), 82 patients presenting with recurrent bacteriuria were evaluated with an antibody-coated bacteria test (FA+) and depressed maximal concentrating capacity $\left(<800 \mathrm{mOsmls} / \mathrm{kg} \mathrm{H}_{2} \mathrm{O}\right)$ to localize the site of infection.

\section{Groups}

A Rec. UTI

B Ureteral Reflux

C Abnormal IVP

D Post Ureteral Reimplantation
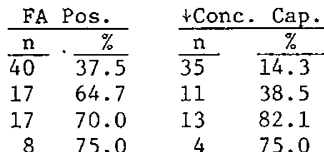

Each child in the recurrent urinary tract infection group $A$ had a normal IVP, VCU, and GFR. The reflux group $B$ includes only those with normal ureters and kidneys. The abnormal IVP group $\underline{C}$ includes those with hydrodynamic and irreversible changes in the ureters and kidneys. The ureteral reimplantation group D includes children who required reimplantation for reflux or renal transplantation.

FAt tests appeared to be more sensitive than + conc. cap. in children in groups A $(37.5 \%$ vs. $14.3 \%)$ and B $(64.7 \%$ vs. $38.5 \%)$. A1so, evidence of upper tract infection was more frequent with pre-existing complicated upper tract processes, $\underline{C}(70.0 \%)$ and $D$ $(75.0 \%)$. FA tests for antibody-coated bacteria appear to be an excellent and sensitive indicator of upper tract infection.

847 AND RENAL TRANSPORT OF SODIUM AND CHLORIDE DURING FETAL LIFE. J. Robillard, C. Sessions, L. HamelRobillard and F.G. Smith Jr. Univ. of Iowa, Dept. of Ped., lowa City, Iowa, and Univ. of Montreal, Dept. of Ped., Montreal, Que.

Renal transport of $\mathrm{Na}$ and $\mathrm{Cl}$, and GFR were studied serially in 16 chronic fetal lamb preparations (91-14l days). There was a significant ( $p<0.005$ ) positive correlation between GFR ml/min, gestational age (GA) $(r=+0.68)$ and fetal body weight (FW) $(r=+0.60)$. No increase in GFR $\mathrm{ml} / \mathrm{min} / \mathrm{kg}$ of FW (GFR - FW) (mean $1.15 \mathrm{ml} / \mathrm{min} / \mathrm{kg} \pm 0.39$ ) was found during gestation or when related to FW. There was a significant parallelism between the excretion of $\mathrm{Na}$ and $\mathrm{Cl}(\mathrm{p}<0.01)$, between the amount of filtered $\mathrm{Na}$ and $\mathrm{Cl}$ $(p<0.01)$ and between the fractional excretion of $\mathrm{Na}(\mathrm{FENa})$ and $\mathrm{Cl}(\mathrm{FECl})(\mathrm{p}<0.01)$. A significant decline in FENa expressed per $\mathrm{Kg}$ of fetal body weight (FENa-FW) was found when correlated to G.A. $(r=-0.71)(p<0.001)$ or to FW $(r=-0.65)(p<0.001)$ or to GFR $\mathrm{ml} / \mathrm{min}(\mathrm{r}=-0.58) \quad(\mathrm{p}<0.005)$. A similar significant decline in FEC1-FW was found when correlated to GA $(x=-0.71)$ $(p<0.001)$ or FW $(r=-0.69)(p<0.001)$ or GFR ml $/ \mathrm{min}(r=-0.62)$ $(p<0.005)$. However there was no correlation between FENa-FW or FEC1-FW and GFR-FW. Also no correlation was found between FENa or FECl and GA, FW, GFR ml/min or GFR-FW. The data thus indicate a) that the increase in GFR $\mathrm{ml} / \mathrm{min}$ is paralle1 to the increase in FW and explains the absence of variation in GFR-FW; b) that the fetal kidney reabsorbs more $\mathrm{Na}$ per $\mathrm{kg}$ of $\mathrm{FW}$ at the end of gestation even if there is no increase in GFR-FW. This study suggests that the transport capacity for $\mathrm{Na}$ and $\mathrm{Cl}$ by the fetal kidney develops at faster rate than the GFR.

848 CLEARANCE IN CHRONIC FETAL LAMB. J. Robillard, C. Sessions, R. Kennedey, L. Hame1-Robillard, F.G. Smith $\mathrm{Jr}$. Univ. of Iowa, Dept of Ped. Iowa City, Iowa and Univ. of Montreal, Dept of Ped. Montreal, Canada.

Osmolar clearance ( $\mathrm{Cosm})$, free water clearance $\left(\mathrm{CH}_{2} \mathrm{O}\right)$, urinary osmolarity (Uosm) and urinary flow $\mathrm{ml} / \mathrm{min}$ (V) were studied serially in 16 chronic fetal preparations (91-141 days). Glomerular filtration rate $\mathrm{ml} / \mathrm{min} / \mathrm{kg}$ of fetal body weight (GFR-FW) was $1.08 \pm 0.35(\mathrm{SD}), \mathrm{V}$ varied from 0.175 to 0.973 with a mean of $0.469 \pm 0.262$, Uosm varied from 97 to $284 \mathrm{mosm} / \mathrm{kg} \mathrm{H}_{2} \mathrm{O}$ with a mean of $148 \pm 50.7$. The Cosm and Cosm-FW varied respectively from 0.036 to $0.441 \mathrm{ml} / \mathrm{min}$ and from 0.036 to $0.204 \mathrm{ml} / \mathrm{min} / \mathrm{kg}$ with respective mean of $0.218 \pm 0.11$ and $0.123 \pm 0.045$. The $\mathrm{CH}_{2} \mathrm{O}$ and $\mathrm{CH}_{2} \mathrm{O}$ - FW varied respectively from 0.002 to $0.582 \mathrm{ml} / \mathrm{min}$ and from 0.001 to $0.270 \mathrm{ml} / \mathrm{min} / \mathrm{kg}$ with respective mean of $0.248 \pm 0.167$ and $0.137 \pm 0.079$. There was a positive and significant correlation $(\mathrm{p}<0.0 \mathrm{l}$ ) between GFR $\mathrm{ml} / \mathrm{min}$ (GFR) and $\mathrm{V}(\mathrm{r}=0.76)$, GFR and $\operatorname{Cosm}(r=0.77)$, and GFR and $\mathrm{CH}_{2} \mathrm{O}(\mathrm{r}=0.64)$. There was a positive correlation between fetal age (FA) and $V(r=0.58)$ and between FA and $\operatorname{Cosm}(r=0.62)$. These results suggest that the unstressed fetus does not produce a concentrated urine even though Cosm increases with FA. It also demonstrates that the increase in Cosm parallels the increase in GFR. 
DEFECTIVE PHAGOCYTE ADHERENCE AS AN INDICATOR OF COM-

849 ix P. Heald) Ruley, Judith Plaut and Shih-Wen Huang (SPON BY: FeDept. of Ped., Baltimore.

The ability of circulating phagocytes to adhere to glass was measured in 17 patients with acute post-streptococcal nephritis (P-SGN), 9 patients with lupus erythematosus nephritis (SLE-GN), 3 patients with membranoproliferative nephritis (MPGN), 48 patients with other renal diseases, 47 healthy adults and 16 healthy children. The phagocyte adherence of patients with P$S G N, S L E-G N$ and MPGN was significantly reduced compared to patients with other renal diseases and normal adults and children $(\mathrm{P}=<0.001)$. In patients with $\mathrm{P}-\mathrm{SGN}$ there was a positive correlation between phagocyte adherence and plasma $\mathrm{C}_{3}$ but not with plasma $\mathrm{C}_{4}, \mathrm{C}_{3}$ proactivator, severity of illness or drugs administered. Two patients with P-SGN had reduced adherence even though they were normocomplementemic. As the patients with PSGN recovered, their phagocyte adherence became normal while patients with SLE-GN and MPGN usually had reduced adherence regardless of their serum $\mathrm{C}_{3}$ or clinical status. The in vitro addition of functional $\mathrm{C}_{3}$ to whole blood produced the adherence defect in normals and failed to correct the defect in patients with P-SGN. These findings suggested that this phagocyte abnormality was related to an effect of complement activation on the phagocyte membrane and not a result of reduced serum complement. This test has been useful in the diagnosis of patients suspected to have complement activated renal disease.

MAINTENANCE DIALYSIS IN CH $\perp$ LREN WITH END STAGE REN-

850 STE (ESRD) SECONDARY TO SICKLE CELL ANEMIA (SSA) Shobha Sahney, Anil K. Bidani, Paula Roach and Larry E. Fleischmann (spon. by Flossie cohen) Wayne State U., Childrens Hosp. of Mich., Dept of Ped., Detroit

SSA is associated with hemodynamic structural, and functional abnormalities of the kidney. Hemodialys is for ESRD has rareiy been attempted in these patients for fear of thrombotic, hemolytic and cardiovascular complications. l'here have been no previous reports of dialysis therapy for ESRD in children with SSA. We wish to report two such patients on hemodialysis.

L.H. temale, presented to us at age 15 in Jan. 1972 in congestive heart failure (CHF) with nephrotic syndrome, hypertension, and renal insufficiency. Conservative therapy for her anemia and CHF with digitalis, diuretics, antihypertensives and transfusions was initiated. Renal function deteriorated; she was begun on peritoneal dialysis in Jan. 73. Repeated episodes of peritonitis Led us to begin hemodialysis in July of 75; despite her compromised cardiovascular state, the added demand of the access means has not led to decompensation. Her rehabilitation is exceilent. A.S., a 17 yr maie, presented with SSA, nephrotic syndrome and $\mathrm{CHF}$. A membranoproliferative glomerulonephritis was diagnosed on biopsy. His renal function deteriorated over nine months; hemodialysis was begun in Nov. of 75 . Complications have included a mycotic aneurism of the fistula site. Maintenance dialysis therapy is feasible in children with ESRD secondary to SSA, and no unusual complications are to be expected.

DISTAL RENAL TUBULAR ACIDOSIS (RTA) IN AN INFANT WITH

851 LEIGH'S SYNDROME (LS), Jose R Salcedo, Hooshan Hodjati, Gladys H Hirschman, James CM chan (Intr by Robert H Parrott). George Washington University, Children's Hospital National Medical Center, Dept of Nephrology, Washington DC.

In $L S$, or infantile subacute necrotizing encephalomyelopathy, an apparent deficiency of pyruvate carboxylase leads to accumula tion of pyruvate or lactate, inhibiting metabolic pathways for energy production and utilization; probably also contributing to the development of RTA as reported in two cases by Gruskin.

Our patient presented at age 7 months with failure to thrive, muscular hypotonia, nystagmus, strabismus and systemic metabolic acidosis. Blood $\mathrm{pH}$ was $7.26, \mathrm{pCO}_{2} 33 \mathrm{~mm} \mathrm{Hg}, \mathrm{tCO}_{2} 8 \mathrm{mMol} / 1, \mathrm{pO}_{2}$ $63 \mathrm{~mm} \mathrm{Hg}, \mathrm{Cl} 118 \mathrm{mEq} / 1$; other $1 \mathrm{ab}$ data were normal. The renal acidification capacity was grossly inadequate as documented by the net acid excretion of $33.35 \mu \mathrm{Eq} / \mathrm{min} / 1.73 \mathrm{~m}^{2} ; \mathrm{NH}_{4}+20.10 \mu \mathrm{Eq} /$ $\mathrm{min} / 1.73 \mathrm{~m}^{2}$, titratable acid $13.25 \mu \mathrm{Eq} / \mathrm{min} / 1.73 \mathrm{~m}^{2}$. During oral bicarbonate loading tests, fractional excretion of filtered $\mathrm{HCO}_{3}-$ increased from 0.7 to $14.9 \%$ with increasing doses of $\mathrm{HCO}_{3}$ - from 2 to $8 \mathrm{mEq} / \mathrm{kg} /$ day. Thereby the diagnosis of distal RTA, hybrid type, was established.

$\mathrm{HCO}_{3}{ }^{-}$therapy of $4-6 \mathrm{mEq} / \mathrm{kg} /$ day corrected the metabolic acidosis for the next 4 months. At age 12 months with development of coma, the patient died within a few hours. Post-mortem findings included symmetrical areas of scattered encephalomalacia, gliosis and capillary prolfferation in basal ganglia and spinal cord. This data implies that a third case of LS, but with distal RTA, is now documented.
ENDOGENOUS NET ACID PRODUCTION IN INFANTS, CHILDREN AND ADULTS. Jose R Salcedo, Margaretia L Jackson, Thomas H Coleman, David D Rao and James CM Chan(Intr by Gordon Avery) George Wash Univ-Children's Hosp Nat Med Ctr, Washington, DC

The rate of acid production in infants and young children was quoted to be 1-2 mEq/kg/day (Soriano: Ped CI N Am 18:529, 1971; Morris et a1: Kidney Int 1:322, 1972; Chan et al: JAMA 220:1700, 1972 ) but in fact, the sole reference (Albert et al: Pediatr 37: 728,1966 ) used in all these instances did not permit such quantitation. In order to evaluate the rate of endogenous acid production, we applied the quantitative technique of Lennon et al (JCI $45: 1601,1966$ ) in the following normal subjects, where $\mathrm{H}^{+}$sol is hydrogen ion released from metabolism of sulfur-containing amino acids; $\mathrm{H}^{+}$is hydrogen ion released from incomplete oxidation of organic acids; the sum of $\mathrm{H}^{+} \mathrm{so}_{4}+\mathrm{H}^{+} \mathrm{Oa}$ gives an estimation of endogenous net acid production (NAP):

\begin{tabular}{|c|c|c|c|c|c|}
\hline Age & $\mathrm{n}$ & $\mathrm{H}^{+} \mathrm{SO}_{4}$ & $\mathrm{H}^{+} \mathrm{Oa}$ & NAP & NAP \\
\hline & & & $\mathrm{Eq} / \mathrm{min} / 1.73$ & & $\overline{\mathrm{mEq} / \mathrm{kg} / \mathrm{da}}$ \\
\hline & 23 & 6.0 & $20.1+12.5$ & 26]$. & $1.81+0.9$ \\
\hline 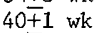 & 4 & 7 & 7.1 & 21 & $1.25 \pm 0$. \\
\hline $8+4$ & 11 & 22.7 & $\mp 16.3$ & 53. & $5+0$ \\
\hline $88+10 \mathrm{yr}$ & 10 & $23.8+5.2$ & $24.5+8.4$ & $48.3+11.3$ & $1.03+$ \\
\hline
\end{tabular}

This is the first study documenting the rate and the components of endogenous NAP in infants, children and adults. This will serve as a rational basis for investigations into a less acidogenic diet for treating end-stage kidney failures and the growth failure associated with renal acidosis.

853

THE ULTRASTRUCTURE OF THE GLOMERULAR SLIT DIAPHRAGM

3 (SD) DURING PROTEINURIA IN AUTOLOGOLS IMMUNE COMPLEX Children's Hospital Medical Center, Boston, Massachusetts

The mechanism of proteinuria is unknown. Farly observations suggest the SD to be either the final filter for macromolecules or the control of hydraulic flux across the qlomerulus. SD is a highly ordered, zipper-like substructure of the mammalian glomerular capillary wall, containina nores measurina $5 n \times 120 \AA$ in the human. To determine whether proteinuria is associated with subtle alterations in the SD and whether changes nrecede. or are the consequence of proteinuria, the ultrastructure was examined in AIC, an experimental rat model very similar to human membranous olomerulonenhropathy. Early chanaes include displacement of foot processes (FP) and associated SD toward the urinary snace by the imuune complex deposits. With the onset of proteinuria, FP spread with shortening of the interenithelial slits, SD becomes further disnlaced, then folded and nleated in the residual interenithelial snaces. With massive proteinuria, many slit pores are replaced by either close junctions or an intermediate narrowed structure. In some areas, FP become detached from the underlyina deposits. nccasionallv a slit pore lacking a SD.is seen. In no animal was a primary defect in the SD observed. It appears that changes in the SD follow the onset of proteinuria and are secondary to a decrease in nodocyte surface area. While the SD may control hydraulic flux, the areas of $\mathrm{FP}$ detachment, and/or the enithelial slits lacking a SD, are more likely to constitute the sites of massive protein leakace.

IDIOPATHIC BYPERTENSION FOLLONING NEONATAL RESPIRA-

854 TORY DISTRESS. Richard L. Siegler, Margaret W. Miller (Spon. by Lowe11 A. Glasgow). Dept. of Ped. Univ. of Utah Col. of Med., Univ. Hosp., Salt Lake City.

We have identified 11 infants during a 2 year period ( $1 \%$ of NBICU admissions) who developed unexplained hypertension while recovering from severe neonatal respiratory disease (10 cases of RDS, one case of meconium aspiration).

Their birth weights averaged $2192 \pm$ SD $461 \mathrm{gms}$, the average time from birth until the onset of the hypertension was 12 days (range 4-60 days), and the highest recorded systolic blood pressure for the group was $141+$ SD $22 \mathrm{~mm} \mathrm{Hg}$.

Almost al1 (10/11) had transiently abnormal urinalysis (proteinuria, hematuria, granular casts) that generally preceded the onset of the hypertension. Moreover, while the group mean plasma renin activity $(29.2 \pm$ SD $24.9 \mathrm{ng} / \mathrm{cc} / \mathrm{hr})$ was significantly elevated $(\mathrm{p}<.025)$ compared to weight-matched normotensive RDS infants $(8.72+5.85)$, on 1 y 7 of the 11 infants had values greater than one SD above the normotensive control mean.

While those with the more persistant or severe hypertension had thorough evaluations including intravenous pyelography, and renal arteriography, no explanation for the hypertension was ever found.

And except for 2 infants who died (ages 7 and 90 days) while still hypertensive, the blood pressure eventually returned to normal $(79+\mathrm{SD} 70$ days) in the remaining 9 infants. 
855 EFFECT OF UNILATERAL URETERAL OCCLUSION ON COMPENSATORY GROWTH AND FUNCTIONAL ADAPTATION. N.J. Siege1, M.H.Veeder, R.M.Weiss. (Spons. by J. Warshaw). Dept. of Ped. \& Urology, Yale Univ. Sch. of Med., New Haven, Conn.

The effect of unilateral ureteral occlusion on compensatory adaptation of the contralateral kidney is not well documented. Changes in renal mass, glomerular filtration rate (GFR), and renal blood flow (RBF) were examined 21 days after surgery in 3 groups of rats: controls (Grp A); uninephrectomy (Grp B); unilateral ureteral occlusion (Grp C). Since the animals were pair-fed, final body weight and changes in body weight were similar in all 3 groups $(p=N S)$. In Grp $B$ animals there was a $79 \pm 9 \%$ (mean $\pm S E M$ ) increase in kidney weight as compared to a $25 \pm 10 \%$ increase in Grp A animals $(p<0.01)$. However, in Grp C rats kidney weight increased only $65 \pm 8 \%$, significantly less than in Grp $B \quad(p<0.01)$. Thus, while compensatory growth occurred in both Grps B and C, the magnitude of the compensatory changes was significantly less in animals with unilateral ureteral occlusion (Grp C).

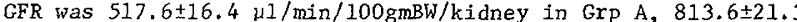
in Grp B, but only $608.2 \pm 25.2$ in Grp C. Likewise, RBF was $2.95 \pm$ $0.09 \mathrm{ml} / \mathrm{min} / 100 \mathrm{gmBW} / \mathrm{kidney}$ in Grp A, $4.32 \pm 0.32$ in Grp B, and 3.38 \pm 0.41 in Grp C. Thus, while changes in GFR and RBF in both Grps $B$ and $C$ were significantly greater than in Grp A $(p<0.01)$, the degree of functional and hemodynamic adaptation in Grp $C$ animals was consistent1y less than that in $\operatorname{Grp} B$ rats $(p<0.01$ ).

These data suggest that following unilateral ureteral occlusion either the rate of the compensatory changes or the magnitude of the adaptive response in both mass and function is altered compared to similar changes following unilateral nephrectomy.

\section{6}

STFUCTURAL RELATIONSHIPS BETWEEN METABOLISM AND TPANSPORT OF PROLINE IN TIE NEPIRON. Olli Simell, les Scriver \& Fazl Mohyuddin. MRC Genetics Group,

Two sets of treal Children's hosp. Res. Inst., Montreal, P.Q. duols (luminal, cellular \& peritubular) are traversed in series during net transepithelial reclamation of proline in the mamal. When net reabsorption occurs, movement "inward" from: the cellular pool exceeds backflux into the lumen. Metakolic "run cut" (chemical conversion) appears to regulate the reabsorption and $\mathrm{T}_{\mathrm{m}}$ of a solute when its intracellular conc. is high; asymetry of carrier activity at luminal and baso-lateral menbranes, to pemit enhanced baso-lateral efflux, may determine reclamation of solutes whose intracellular conc. is low. Intrarenal conc. of proline is higher than in plasma or ultrafiltrate; and symetrical transport characteristics exist for luminal \& baso-lateral proline carriers; (Mohyuddin \& Scriver, Amer. J. Phys. 219, 1,1970); accordingly chenical conversion of proline is likely to be a major deteminant of its tukular reclamation. We have now examined mitochondrial proline oxidase activity in nomal $(\mathrm{A} / \mathrm{J})$ mouse kidney using a modified_jsotopic assay. Specific activities (umoles F5C fomed. $\mathrm{g}^{-1}$ prot. ${ }^{-1} \mathrm{~min}$ ) at $25 \mathrm{mM}$ proline are $24.9 \mathrm{~g}$ in outer cortex; 16.7, inner cortex; 15.0, medulla; \& 11.8, papilla. anter cortex activity in PRO/Re mouse is <l\% of nomal. This hyperprolinemic strain has $4 \mathrm{x}$ normal kidney proline \& $50 \mathrm{x}$ normal urinary excretion (Scriver et al, PNAS 72, 1431, 1975). These findings confirm that proline oxidation in proximal tubule is a critical determinant of its reabsorption in vivo.

SEVERE HYPERTENSION AND CHRONIC PYELONEPHRITIS (CPN)

857 IN CHILDREN. Sorrenti, R.W. and Lewy, P.R. (Spon. by H.L. Nadler) Children's Memorial Hospital

Northwestern University Medical School, Chicago, Tllinois

Since 1966 fifteen children $(14+, 16)$, aged $7-15$ yr., presenting with hypertension (HT) have been found to have bilatera renal scarring and caliectasis (CPN) radiographically. The diagnosis of CPN is supported histologically in 10 patients. Initial serum creatinine was elevated in 14 patients (mean $2.2 \mathrm{mg} \%$, range 1.1-5.5). Though initial blood pressures (BP) ranged from 150/96 to $245 / 190 \mathrm{mmHg}$, detection of 6 cases was at routine examination. Two others presented with seizure, 6 with headache and 1 with renal insufficiency. Vesicoureteral reflux (VUR) was present in 11 patients, bilateral in 7 , but none was obstructed. Resection of abnormal renal tissue in 4 patients ( 3 with abnormal selective renins) did not improve BP. Six (non-operated) patients progressed to renal failure in $1-5$ (mean=3) years and 2 others have declining function. All 8 had initial creatinine clearance $<30 \mathrm{ml}$ $\min / 1.73 \mathrm{~m}^{2}$. Seven patients are stable after $1-4$ yrs. BP regulation appears more adequate in these 7 patients. VUR is not related to outcome: 3 of 8 patients with renal deterioration have no VUR while $4 / 7$ with stable function have VUR. Documented urin ary infection occurred in only 5 patients during follow-up, recurrent in none. The role of VUR or infection in the evolution of CPN is unclear but neither appears related to ultimate prognosis; surgical repair of VUR in such patients seems unnecessary. Careful BP control may forestall progression of disease, but surgical management of this form of hypertension is unrewarding even when selective renin studies appear lateralizing.
DISTRIBUTION OF SINGLE NEPHRON FILTRATION (SNF) IN

858

THE PUPPY. Nick J Tavani, Jr., Steve Zimmet,

Philip L. Calcagno, Gilbert M. Eisner, and Pedro A. Jose. Georgetown Univ. Sch. of Med., Georgetown Univ. Hospital Depts. of Peds., Physiol., Biophys., and Medicine, Washington, D. C.

The maturational pattern of glomerular blood flow distribution in the puppy is well established. However, the distribution of SNF has not been reported. This aspect of ontogeny was studied in 18 puppies 2 hrs to 41 days old using the $14 \mathrm{C}$ ferrocyanide method. 148 nephrons were dissected ( 43 outer cortical nephrons $[O C], 31$ middle cortical nephrons $[M C], 74$ juxtamedullary nephrons $[J M])$. The ratio $O C / J M$ was used as an index of SNF distribution.

In a 2 hr old puppy, filtration in OC could not be detected. By 2 wks $(n=5)$ OC/JM was 0.30 and 0.83 at $4-6$ wks $(n=4)$. This rise in $O C / J M$ was not linearly related with age; an accelerated increase occurred between $2-4$ wks of age. At one wk a clear distinction in SNF was seen among $O C, M C$ and $J M$ nephrons with $J M$ values highest and MC values intermediate between $O C$ and JM. Mean arterial pressure (MAP) increased linearly with age from $46 \mathrm{~mm} \mathrm{Hg}$ at one wh to $89 \mathrm{~mm} \mathrm{Hg}$ at 6 wks $(r=0.73 \mathrm{p}<.01)$.

These studies show that SNF distribution with maturation proceeds in a centripetal fashion similar to that described for cortical blood flow. This increase in OC/JM with age can only be partially accounted for by $r$ ise in MAP $(r=0.54 \mathrm{p}<.05)$, since the magnitude of change in $O C$ filtration was much greater than MAP.

Q59 HEMOLYTIC-UREMIC SYNDROME: FOLLOW-UP AND PROGNOSTIC INDICES。 John A. Tilelli and Richard L。 Siegler (Spon. by L.A. Glasgow). Dept. of Pediatrics, Unive of Utah College of Medicine, Salt Lake City, Utah.

We reviewed 29 occurrences of the Hemolytic-Uremic Syndrome (HUS) in 28 patients. All but 3 were treated supportively without heparin. Their neurologic and renal status has been followed for periods ranging from 2 to 43 months (mean 14.8 months). One of the 28 patients was lost to follow-up. Twenty-three $(85 \%)$ had a good outcome. (13/28 were entirely normal by 6 months; $10 / 23$ cleared within 14 months; $2 / 10$ with seizure disorders and $10 / 10$ with abnormal urinalysis). Four children (11\%) had poor outcomes. One died during the acute phase. One requixed dialysis and transplant, and later died in chronic renal failure. Another had a persistently abnormal U/A, and experienced a recurrence of the HUS complicated by minoxidil resistant chronic hypertension. A fourth child had mild azotemia and severe encephalopathy 2 months post onset. Late onset hypertension or renal failure has not been observed.

The data was subjected to chi-square tests and analysis of variance. The following indices observed during the acute phase were found to correlate with a poor outcome: 1. Anuria and/or oliguria greater than 7 days $(p<.001) ; 2$. Seizures $(p<.01)$; 3. Transient hypertension $(p<.05) ; 4$. Hematocrit less than $20 \%$ (p<.05). Thrombocytopenia had no prognostic significance. No variance observed during the acute phase correlated significantly These observations suggest that the outcome of the HUS may be predictable during the acute phase of the disease.
860 URINARY HYDROXYLYSINE GLYCOSIDES IN HEREDITARY NEPHRITIS. L.U. Tina, M. Lou, D. DiZio, and P.L. Washington, D.C., Dept. of Biochem., Alfred I. DuPont Inst., Washing ton, D. Delaware.

The urinary hydroxylysine glycosides (OHLG'S) - hydroxylysine galactose glucose (HGG) and hydroxylysine galactose (HG) come directly from collagen and basement membranes. These 2 hydroxylysine glycosides reflect endogenous collagen turnover more closely than hydroxyproline and their ratio represents that proportion of collagen degraded. No previous studies exist defining the collagen turnover via the utilization of urinary (OHLG'S) in hereditary nephritis (HN).

On the assumption that $\mathrm{HN}$ represents a defect in biosynthesis or metabolism of collagen, urinary OHLG'S were measured by ion exchange chromatography in 12 subjects (Females-7, Males-5, ages 4-49 yrs) which included 4 children with renal biopsies consistent with $\mathrm{HN}$ and 8 family members. As controls, 18 children ( $3-18$ yrs) and seven adults (22-60 yrs), without previous treatment were studied.

A significant inverse correlation was noted with urinary OHLG'S excretion with age. In all young children with microscopic

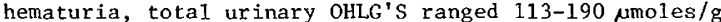

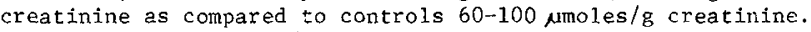
Since the ratio of HGG/HG in the controls was not significantly different to the HN suggest that collagen degradation and synthesis de novo are increased proportionately early in the natural history of $\mathrm{HN}$. 
861 J. Wassner. Sheldon IN NORMAL AND UREMIC RATS. Steven Pediatrics. $\frac{J \text {. Wassner }}{\text { versity of }}$ california, San $\frac{\text { Sheldon Orloff }}{\text { Fancisco, Department of }}$

Both normal and uremic growing rats undergo cyclic variation of muscle protein synthetic rates. Highest rates were in the postabsorptive period and the lowest just prior to feeding. The purpose of this study was to determine whether muscle protein catabolic rates (MPCR), like synthetic rates, have a diurnal pattern and whether moderate uremia alters the pattern or rate of catabolism. 3-Methylhistidine (3MH) is an amino acid found exclusively in skeletal muscle and its excretion reflects muscle protein catabolism. The 3MH/Creatinine $(\mathrm{Cr})$ excretion ratio defines MPCR.

Thirty-nine-day-old rats were made uremic by $5 / 6$ nephrectomy ( $B U N=80 \mathrm{mg} \%, G F R=25-30 \%$ ). Sham operated and uremic rats were allowed access to rat chow from $8 \mathrm{pm}$ to $8 \mathrm{am}$ daily. Weight gain was 8 $\mathrm{gm} /$ day. On the study day, urine was collected from 8 am to noon and from $4-8 \mathrm{pm}$. 3MH was determined by amino acid analyzer and $\mathrm{Cr}$ by autoanalyzer. The results are as follows:

\begin{tabular}{|c|c|c|c|c|}
\hline & $A M$ & $\frac{H / m g ~ C x}{P M}$ & $P M / A M$ & $n$ \\
\hline Sham (6) & $0.33 \pm .08$ & $0.54 \pm .14$ & $1.66 \pm .29$ & $<.005$ \\
\hline Uremic (12) & $0.28 \pm .07$ & $0.47 \pm .08$ & $1.79 \pm .75$ & $<.001$ \\
\hline
\end{tabular}

We have demonstrated by these results cyclic variation of MPCR in both normal and uremic growing rats which in contrast to synthetic rates is highest prior to feeding and lowest in the postabsorptive period. We have not demonstrated an effect of moderate uremia on MPCR in the unstxessed state.

THE EFFECT OF A PROSTAGLANDIN SYNTHETASE INHIBITOR ON 862 THE RENAL RESPONSE TO HYPOXIA. DOuglas N. Weismann, Daniel K. Day, Robert J. Roberts, Fred G. Smith and Harold E. Williamson, University of lowa, Col. of Medicine, Depts. of Pediatrics and Pharmacology, lowa City.

Prostaglandin $E_{2}\left(P_{G E}\right)$ is an endogenous vasoactive lipid synthesized in the renal medulla and produced in response to various stimuli. $\mathrm{PGE}_{2}$ acts as a vasodilator and apparently participates in modulation of renal hemodynamics. Hypoxia $(H)$ was induced in 16 adult mongrel dogs ( 11 experimental, 5 controls) by a $10 \mathrm{~min}$. exposure to $0.10 \mathrm{~F} \mathrm{O}_{0}$ before and after treatment with indomethacin (1), $5 \mathrm{mg} / \mathrm{kg}, \mid$.. ., a potent prostaglandin synthetase inhibitor. The animals were artificially ventilated and received a saline infusion which induced a mild diuresis. Renal blood flow (RBF) was measured simultaneously by means of the clearance of $p$ aminohippurate and an electromagnetic flow probe placed around the left renal artery. Glomerular filtration rate (GFR) was estimated by the clearance of inulin. $H$ induced an increase in RBF from a mean RBF of $253 \mathrm{ml} / \mathrm{min}$ to a mean RBF of $275 \mathrm{ml} / \mathrm{min}$. I administration resulted in a decline in baseline $R B F$ to a mean of $183 \mathrm{ml} / \mathrm{min}$ and the increase in mean RBF in response to $H$ was blocked $(p<.05)$ GFR also increased during $H$ from a mean of $34 \mathrm{ml} / \mathrm{min}$ to a mean of $36 \mathrm{ml} / \mathrm{min}$. GFR increased further (mean GFR $38 \mathrm{ml} / \mathrm{min}$ ) during the post-H recovery period. After $\mathrm{I}, \mathrm{H}$ induced a fall in GFR from a mean GFR of $37 \mathrm{ml} / \mathrm{min}$ to a mean GFR of $33 \mathrm{ml} / \mathrm{min}$. Thè mean change in GFR with $H$ was significantly different $(p<.05)$ from that induced by $H$ before 1 . This study indicates that PGE may play a protective role in the renal response to hypoxia. 'Supported by GMI 2675 and PMA Fellowship.)

URINARY BETA-GLUCURONIDASE IN NEWBORN INFANTS.

863 Paul Y.K. Wu, Reynaldo Miranda and Theodora EwusiMensah. University of Southern California School of Medicine, Los Angeles County-USC Medical Center. Department of Pediatrics.

Beta-glucuronidase is present in the proximal, distal and collecting tubules. It is normally present in the urine. Schapiro et al reported an increase in urinary $\beta$-glucuronidase activity following ischemic tubular necrosis (J. of Urology 100:146, 1968). Neonatal asphyxia or RDS has been known to be associated with increased sodium loss in the urine. One of the factors responsible may be due to tubular damage resulting from asphyxia and ischemia. B-glucuronidase activity was measured daily in the first seven days of life, according to the method described by Schapiro et al, from urine collections obtained from 78 newborn infants. A unit of enzyme activity was expressed as micrograms of phenolpthale in liberated per $0.3 \mathrm{ml}$ urine divided by creatinine concentration in $\mathrm{mg} / \mathrm{cc}$. In well infants the range of $\beta$-glucuronidase activity was found to be from 2 to 59 units. Infants with low Apgar scores or RDS had elevated activity as high as 450 units. The data demonstrated a close correlation between elevated levels of enzyme activity and severity of asphyxia and ROS. Urinary $\beta$-glucuronidase decreased with recovery and with increasing postnatal age.
864 HEMOLYTIC UREMIC SYNDROME (HUS): CHARACTERIZATION OF THE DISEASE AT A CENTER IN THE MIDWEST. Young, L.M. and Lewy, P.R. (Spon. by H.L. Nadler) Chi 1dren's

Memorial Hospital, Northwestern Univ. Medical School, Chicago,I11. HUS has been reported from relatively few centers in the U.S.A. our series consists of 18 cases from the Chicago area between 1968-75. Clustering of cases was evident in 1971-72 ( 7 cases)and 1974-75 (7 cases). A majority of cases occurred in April-June (11 cases). The age at onset was $0.5-16$ yrs. (mean 4.6 yrs.), with 10 patients over 3 years of age. Diarrhea and/or vomiting preceded HUS in all instances. Presenting signs and symptoms included lethargy in all, seizures in 6 ( 5 with hyponatremia, 1 with hypocalcemia), hypertension (HT) in 8 and edema in 5. Oligoanuria $\left(\leq 200 \mathrm{ml} / \mathrm{m}^{2} /\right.$ day) occurred in 12 . Mean presenting $\mathrm{Hb}$ was $8.3 \mathrm{gm} \%$ (range $3.7-13.5 \mathrm{gm} \%$ and mean platelet count $68,000 / \mathrm{mm}^{3}(10,000-$ $\left.233,000 / \mathrm{mm}^{3}\right)$. The mean initial BUN and creatinine were 93 (29$197)$ and $5.0 \mathrm{mg} \%(1.9-11.1)$ respectively. Transfusions were required in 16 patients and dialysis in 15 . Heparin was used only in 2 patients with clotting abnormalities and one other. Diuresis occurred after a mean of 9 days $(2-20)$ in the 12 oliguric patients who recovered, and BUN fell below $20 \mathrm{mg} \%$ by 20 days $(2-60)$. Hemoglobin began to rise in 9 days (4-15) while platelet counts exceeded $100,000 / \mathrm{mm}^{3}$ by 8 days $(1-23)$. Refractory HT (BP $>180 / 140$ $\mathrm{mmHg}$ ) occurred in 2 patients; both failed to recover renal function and one died at 8 weeks. A third patient also failed to recover. Thirteen patients recovered completely, 2 others have residual HT and proteinuria. HUS is not uncommon in the Midwest but occurrence clusters by season and year. Recovery rate is good $(83 \%)$ and the use of heparin appears unnecessary.

865 RENAL EFFECTS OF THEOPHYLLINE IN THE PRETERM INFANT.

865 Shereen Zakauddin, Rosemary D. Leake, and Carl W. Trygstad, UCLA \& Davis Schools of Medicine, Harbor
General Hospital \& Sacramento Medical Center, Departments of Pediatrics, Torrance and Davis, California.

Theophylline administered to human adults and animals produces an immediate diuresis by increasing glomerular filtration rate (GFR) and decreasing proximal tubular sodium reabsorption. Theophylline is being used for treatment of the apnea of prematurity. Therefore, 20 studies were performed on 10 preterm infants (mean $w t=1115$ gms; mean gestational age $=31$ wks) requiring theophylline for repeated apneic spells. GFR, fractional sodium reabsorption, urine volume (Uv), free water and sodium clearance, distal sodium reabsorption, and heart rate were measured immediately prior to and following a one hour intravenous infusion of $5 \mathrm{mg} / \mathrm{kg}$ of theophylline.

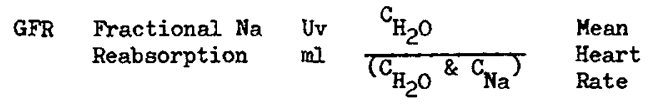

$\begin{array}{llllll}\text { Before } & & & & \\ \text { Theophylline } & 1.17 & 98.8 & 6.9 * & 57.6 * & 147 \\ \begin{array}{l}\text { Following } \\ \text { Theophylline } 1.41\end{array} & 97.8 & 8.4 * & 63.4^{*} & 165 \\ \text { P } & 0.01 & >0.05 & >0.05 & >0.05 & <0.01\end{array}$

In the preterm infant GFR increases with theophylline treatment, but we could not demonstrate a naturesis or diuresis.

ULTRASOUND LOCALIZATION FOR PERCUTANEOUS RENAL

866 BIOPSY IN CHILDREN. Petros M. Zeis, Demetrios Spigos, Conrado Samayoa, Vlastimil Capek, and Lorenzo C. Aschinberg. (Spon. by I.M. Rosenthal.) Abraham Lincoln School of Medicine, University of Illinois Hospital, Department of Pediatrics and Medical Radiology, Chicago, Ill.

B-scan ultrasound was used to localize the kidneys during renal biopsy in 27 children ranging in age between 1 and 15 years. Twenty-two patients had diffuse renal parenchymal disease; 5 patients were renal allotransplant recipients. Glomerular filtration rate (GFR) ranged between 7 and $157 \mathrm{ml} / \mathrm{min} / 1.73 \mathrm{~m}^{2}$. The lower pole was accurately localized in all patients. Adequate amounts of tissue yielding at least 10-20 glomeruli were obtained following a maximum of 3 attempts in all cases except one. Serious complications were not observed. Transient gross hematuria occurred in 2 patients. Ultrasonography obviates the need for iodinated contrast material, exposure to radiation and expense of image intensification. The method provides accurate information concerning kidney depth, size and delineation. In uremic patients with poor excretory capacity, ultrasonography was particularly valuable since the kidneys could be accurately localized despite marked impairment of renal function. We were able to obtain adequate specimens in 8 children with glomerular filtration rates of less than $50 \%$ of normal. In transplanted patients in whom relatively frequent samples of renal tissue were obtained, this technique was found to be fast, easy to teach, and totally without complications. 


\section{NEUROLOGY}

867

PHE BEKAVIOR OF THE FULLTELM YET UNDERWEIGHT NEWBORN INFANT. Heidelise Als, Edward C. Tronick, Lauren Adamson, T. Berry Brazelton, Harvard University

Medical School, The Children's riospital Medical Center, Departof Pediatrics, Boston.

Ten thin, otherwise healthy full-term newborns, weighing 6 lbs. (Ponderal Index $<2.3$ ) (less than 5th\%) were compared to ten healthy fullweight newborns (Ponderal Index72.3) on the Brazelton Neonatal Behavioral Assessment Scale administered on days $1,3,5$, and 10 。 The Brazelton Scale data were analyzed with an a priori established cluster and profile scheme. This behavioral exam differentiated the two groups clearly on the reflexes walking, crawling, passive movements of arms and legs, rooting and sucking. It furthermore, and more importantiy, differentiated the two groups on those behaviors which one must assume are of key value for the caretaker of the baby. These are attractiveness, need for stimulation, interactive processes, and motoric processes. The thin newborns did substantially more poorly than their fullweight peers. in responding to social interaction, alerting to voice and face, to being held in cuddling. Mothers were "turned of $f$ " by these infants" behaviors but felt it was their own inability to draw out satisfactory responses from the babies. The underweight infants were followed up at a later point during the first year. They showed temperamental organizational difficulties of a hyperkinetic hypersensitive overreaction to stimuli. It is speculated that the underweight newborn's fragile organization elicits anxiety in the caretaker which makes a snooth interaction and stabilization of organization difficult. The possibility of subtle cerebral dys-

96 BRAINSTEM MATURATION IN PRE-TERM INFANTS. Ragnar N

868 Amlie, Arnold Starr, Stephen Sanders, and Robert F. Co1. of Med., Dept. of Ped., Irvine, CA.

Auditory evoked responses(AER) were studied in pre-term infants soon after birth and then at intervals. AER are the far field reflections of electrical activity in the auditory pathway in its course through the brainstem. The potentials are recorded from scalp electrodes. Ten clicks per second at 65,45 and $25 \mathrm{dBSL}$ are transmitted through earphones to the infants while in incubators. The responses are amplified and averaged on line in a computer. Seven consistent waves can be defined in the first $15 \mathrm{msec}$ following the clicks. The latency of each was measured at each sound level. Forty-four infants with gestational ages(GA) of 30-44 weeks were tested. The table shows the mean latency in msec from the GA(wks) Wave I III VI-V fixst tests as a function of GA of waves I 30-32 $3.6 \quad 6.2 \quad 8.6$ III \& IV $\mathrm{IV}$ at $65 \mathrm{~dB}$. The data shows a de32-34 $2.9 \quad 6$. 8.2 crease in latency with decreased GA. The 34-36 2.2 5. 7.6 rate of maturation appears to decrease 36-38 $1.9 \quad 4.87$. after 36 weeks. Subsequent tests indicate 38-40 $1.9 \quad 4.97$. that extrauterine life before 40 weeks 40-42 $1.8 \quad 4.7 \quad 6.9$ may affect maturation. If the $\Delta$ between $\begin{array}{llll}42-44 & 1.5 & 4.5 & 6.6\end{array}$ waves $I$ and $I V-V$ in each infant is an indication of myelination of auditory pathways, it reflects the same maturation trend as the decreasing wave latency with GA. Significant deviations of latency and amplitude have proved to be an indicator of neurclogic abnormalities. AER can serve as an objective measure for hearing, reflect maturation and indicate the integrity of the brainstem as it affects the auditory pathway.

SEARCH FOR TRANSMISSIBLE AGENTS IN PEDIATRIC PATIENTS
WITH NEUROLOGICAL DISEASES OF UNKNOWN ETXOLOGY.

869 David M. Asher, Richard T. Yanagihara, D. Carleton

Gajdusek and Clarence J. Gibbs, Jr., NINCDS, NIH, Bethesda.

The demonstration of infectious agents in kuru, creutzfeldtJakob disease, PML and SSPE stimulated efforts to find viruses in other subacute and chronic diseases of the nervous system using both conventional virological techniques and inoculation of primates. We report our attempts over a 10-year period to demonstrate infectious agents in tissues of 43 selected pediatric patients with various neurological diseases of unknown etiology.

\begin{tabular}{|c|c|c|c|c|c|}
\hline \multirow{2}{*}{\multicolumn{2}{|c|}{ Disease Category }} & \multirow{2}{*}{$\begin{array}{l}\text { No. of } \\
\text { patients }\end{array}$} & \multirow{2}{*}{$\begin{array}{l}\text { Ages } \\
\text { (range) }\end{array}$} & \multicolumn{2}{|c|}{ Primates Inoculated } \\
\hline & & & & Species & Number \\
\hline $\bar{I}$. & Inflamatory & 6 & $\overline{2-12}$ & 10 & 18 \\
\hline \multicolumn{6}{|c|}{ II. Degenerative } \\
\hline & A. Neuronal & & & & \\
\hline & 1. Cerebral & 10 & $0.5-6$ & 6 & 27 \\
\hline & 2. Bulbospinal & 4 & $1.5-16$ & 4 & 10 \\
\hline & B. Myelin sheaths & 9 & $6-18$ & 5 & 28 \\
\hline III. $\mathrm{I}$ & Hereditary & 12 & $4-17$ & 9 & 31 \\
\hline IV. 1 & Neuropatiny, myopathy & 2 & 14,16 & 3 & 6 \\
\hline
\end{tabular}

These patients included 6 with seizure disorder and chronic encephalitis, 10 with Alpers' or unclassified degenerations of cerebral cortical gray matter, 4 with a vaxiety of motor neuron degenerations of medulla and/or spinal cord, 8 with Schilder's disease and $l$ with MS. Thus far none of these specimens has yielded an infectious agent by conventional techniques or in animals observed for as long as 7 years.
Q70 EFFECT OF PHOTOTHERAPY ON VISUAL FUNCTION MEASURED BY ELECTRORETINOGRAPHY - K. Bhupathy, R. Sethupathy, A. Constantaras, J. H. Fournier, and R. S. Pildes, Cook County Hosp., Depts. of Peds. and Ophtha1., Univ. of I11., Chicago

The short term effect of phototherapy (P) on visual function was studied in 50 full term neonates, 28 in $P$ and 22 in control (C) group. Infants were tested by electroretinography under dark and jight adaptation using Buriain-Allen corneal electrodes. Amplitudes of the ' $a$ ' and ' $b$ ' waves were measured. Infants in the $P$ group had a mean + S.D. birth weight of $3.2+0.4 \mathrm{kgm}$, gestational age of $39.6+1 . \overline{6}$ wks and Apgar Score $8+0 . \overline{9}$. Birth weight in the $C$ group was $3.3 \pm .4 \mathrm{kgm}$, gestational age $39.6 \pm 0.8$ and Apgar Score $9 \pm 0.6$. All infants were clinically well. Phototherapy was given for non-hemolytic idiopathic hyperbilirubinemia and administered intermittently, $6 \mathrm{hrs}$ on and $2 \mathrm{hrs}$ of $\mathrm{f}$. Mean duration of $P$ was $50.8+26 \mathrm{hrs}$. (range 9-121 hrs). At the time of testing the mean post-natal age of the infants in $P$ group were $16.8 \pm 2$ days and in the $C$ group, $16.4 \pm 3$ days. Under dark adaptation the mean amplitude of the ' $a$ ' wave in both eyes was $67.9+22.4$ uvolts in the $P$ and $69.3+21.1$ uvclis in the $C$ group. The ' $b$ ' wave measured $142.1 \pm 50.6$ in the $P$ and $133.8 \pm 39.1$ uvolts in the $C$ group. Under light adaptation ' $a$ ' wave was $40.8+16.9$ in $P$ and $37.3+17.4$ in $C$ groups, whereas ' $b$ ' amplitudes were $80.7 \pm 30.6$ in $P$ and $72.2+20.5$ in $C$ groups. There were no significant differences between $P$ and $C$ groups. These studies indicate that phototherapy in the neonatal period does not have any deleterious short-term effect on retinal function as measured by electroretinography.

THE NEONATAL ELECTROENCEPHALOGRAM IN INFANTS OF

871 ADDICTED MOTHER. Vivian V. Bosch, Joan B. Cracco, Intr.by Arthur . Shin, Jing J. Yoon, and Roger Q. Cracco. Med. Center, Brooklyn, N.Y.

Wake and sleep electroencephalograms were recordeo during the newborn period in 37 infants (gestational age-35 to 41 weeks) born to methadone and/or heroin addicted mothers. All these infants, except one, experienced withdrawal symptoms, including hyperirritability and tremors. Their neurological examinations were otherwise normal and none experienced focal or generalized seizures.

The EEGs were normal in 21 infants. In 16 , the EEGs revealed paroxysmal abnormalities; 4 had focal sharp waves and 12 had bilateral independent sharp waves. The EEG abnormalities did not correlate with the severity of withdrawal symptoms, maternal drug dosage, birth weight, gestational age, age at which the EEG was performed, or other antenatal or perinatal complications.

Follow-up EEGs were performed on 8 infants between 1-2 months of age, including 5 infants with abnormal neonatal EEGs. Two of these 5 infants had abnormal follow-up EEGs. The remainder (6) had normal EEGs. Follow-up neurological and developmental evaluations during the first year were normal in 18 patients and questionably abnormal in one.

The transient EEG abnormalities found in the newborn period in these infants may be associated with cerebral dysfunction related to the withdrawal syndrome rather than to irreversible structural changes. However, only further follow-up of these infants will confirm this.

872 DIHYDROPTERIDINE REDUCTASE DEFICIENCY. Thomas G. DIHYDROPTERIDINE REDUCTASE DEFICIENCY. Thomas G.
Brewster, Israel F. Abroms, Seymour Kaufman, Jan L. Breslow, Michael A. Moskowitz, Dorothy B. Villee, Robert S. Snodgrass. Depts Ped. and Neurol. Harvard Medical School, Boston, Lab Neurochem NIMH Bethesda Md.

Isolated dihydropteridine reductase deficiency has been found in an infant with mild hyperphenylalaninemia and neurological dysfunction. This male infant born to first cousins has had a mild phenylalanine intolexance since newborn screening with plasma phenylalanine levels between 5 and $10 \mathrm{mg} .8$ on Enfamil diet. Increased tone was noted at age 4 mos. with onset of seizures at 5 mos. An open liver biopsy was performed at age 9 mos. because of progressive seizures and developmental delay. Enzyme analyses revealed normal levels of phenylalanine hydroxylase and dihydrofolate reductase but less than $2:$ levels of dihydropteridine reductase (DHPR). The DHPR regenerates tetrahydrobiopterin a cofactor in the hydroxylation of phenylalanine, tyrosine, and tryptophan. The latter two reactions are necessary for the synthesis of the neurotransmitters dopamine and serotonin, respectively. The catabolites of these neurotransmitters, homovanillic acid and 5-hydroxyindoleacetic acid, were decreased in the CSF and urine. It is suggested that the neurological picture may be due to deficiency of these neurotransmitters. Treatment with 5hydroxytryptophan and "Carbicopa", a peripheral inhibitor of aromatic amino acid decarboxylase, has begun. Since commencement of treatment a decrease in seizure activity has occurred. L-dopa will be added. This variant form of hyperphenylalaninemia may be responsive to exogenous neurotransmitter precursors. 
873 A STUDY OF THE ACUTE CONVULSIVE EPISODE OF MAGNESIUM DEFTCIENT WEANLTNG RATS. JOan i. Caddell, Deborah Woodville, and Marie Sherman. (Spon. by Arthur E. McElfresh). Dept. Ped. and Path., St. Louis Univ. Sch. Med., St. Louis, Missouri 63104

The nonspecific biochemical changes associated with acute magne:sium $(\mathrm{Mg})$ deficiency were investigated in a series of studies using male weanling Sprague-Dawley rats. Controls were fed $20 \%$ casein and $100 \mathrm{mg} \% \mathrm{Mg}$, and deficient rats were fed $20 \%$ casein and no added $\mathrm{Mg}$. Rats were killed with nembutal and studied at three times: on day 6 , before known convulsions; on day 7 , during or immediately after audiogenic seizures; and on day 8 , in rats that survived the convulsive, shock-Iike episode. Investigations included whole blood $\mathrm{pH}$, microhematocrit, and a series of serum chemistries analyzed on the Technicon SMAC autoanalyzer. For the latter, some-biood was pooled. Mg deficient rats had lower serum protein, albumin, alkaline phosphatase, and higher SGOT, LDH, uric acid, and markedly higher serum triglyceride val ues than did controls. During the convulsions the blood $\mathrm{pH}$ decreased; the hematocrit, serum albumin, and protein increased; and some SGOT levels were remarkably high. Some rats died during these convulsions. 24 hours later, the blood pH, hematocrit, total serum protein, and serum albumin had all returned to preseizure levels, and serum glucose was now decreased. The study notes the transient as well as the lethal nature of the convulsive episode of acute $\mathrm{Mg}$ deficiency and the similarity of the nonspecific biochemical changes to those already reported in the sudden infant death syndrome (SIDS; crib death). This work was supported by the Missouri Heart Association.

FACTORS MODULATING THE OUTCOME OF ACUTE

874 IDIOPATHIC POLYNEURITIS. Lawrence T. Ch'ien, Hubert J. Chen, Sergio Facchini, and J. T. Jabbour. Univ. of Tenn., Sch. of Med., Dept. Ped., an Memphis state Univ., Dept. Math. Sci., Memphis, Tenn. Spon. by: J. N. Etteldorf, Univ. of Tenn., Memphis. From 1964 to 1975,69 cases of acute idiopathic polyneuritis (Guillain-Barré syndrome) were seen in 4 hospitals and $58 \%$ of the patients were in the pediatric age group. According to the extent, distribution and severity of the muscles involved, patients were classified into 3 main groups: mild, moderate, and severe. This classification correlates well with the outcome, particularly with the length of hospitalization and the total duration of the muscle weakness. Age, sex and race do not appear to affect the outcome. Twenty-five of 69 patients (36\%) received various forms of steroid therapy within 7 days of onset. There was no statistically significant difference between those treated and untreated in terms of mean peak CSF protein level, the interval time between the maximal weakness and the onset of improvement and of the length of hospital stay. However, there were complications attributed to steroid therapy. The incidence of neurological sequelae and the total length of weakness were increased in the steroidtreated groups. A double-blind controlled study is warranted to evaluate the risk vs benefit of steroids

\section{T75ERAPEUTIC TRIALS IN TWO PATIENTS WITH OCULOCRANIO-}

875 SOMATIC NEUROMUSCULAR DISEASE WITH RAGGED -RED FIBERS (OCSNMD-RR). J.D. Cook and W.K. Enge], Medical Neurology Branch, NIH. (Sponsor J. Sidbury, NIH, Bethesda, MD.) OCSNMD-RR is a rare, usually non-familial multisystem disorder consisting, as in our patients, of: progressive ophthalmoplegia, diffuse muscle weakness, ataxia, atypical retinal pigmentation, deafness and short stature; elevated blood basal pyruvate (Pyr) and lactate (LaC), low serum Mg, elevated CSF protein, impaired glucose tolerance, cardiac conduction defects and "ragged-red" muscle fibers (with excessive bizarre mitochondria and some increased lipid). No pathogenesis is known. Seeking biochemical and clinical evidence of reversal of a possible defect in Pyr metabolism, we have completed three therapeutic trials, 2-3 wks each, using high doses of the co-factors of pyruvate decarboxylase: thiamine (Th) and lipoic acid (Lp). Mg supplementation was evaluated since Th-deficiency states are unresponsive to Th if hypomagnesemia exists. Fasting non-ambulatory Pyr and Lac were determined qod. Treatment regimens were: Th $200 \mathrm{mg}$ tid, Lp 25mg I.V. qd, Mg 12 meq qid po (serum levels of Mg became normal). Means of 3-10 values given for each period; normal basal values Pyr $<0.9 \mathrm{mg} \% \mathrm{LaC}$ $<12 \mathrm{mg} \%$.

P.t.

R. 0
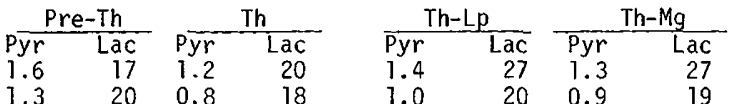

No clinical $1.3 \quad 20 \quad 0.8 \quad 18$ conclusion: this vigorous supplementation of co-factors of pyruvate decarboxylase did not significantly alter biochemical and clinical parameters of OCSNMD-RR.
SUCKING BEHAVIOR IN LOW BIRTH WEIGHT (LBW) INFANTS.

876 R.M. Cowett, w. Oh, and L.P. Lipsitt, Brown Univ. Program in Med., Women and Infants Hosp. of R.I. Depts. of Ped. and Psych., Providence, Rhode Island.

Psychophysiologic recording of sucking behavior (SB) can reflect neurological integrity of healthy term infants. In LBW infants, with an abnormal neonatal course, aberrant SB may be observed as a result of neurological deficit. SB studies were performed in $18 \mathrm{LBW}$ infants (bixthweight $=1.3 \mathrm{~kg}$., gestation ((GA)) $=31.4$ wks.). Nine infants had a severe neonatal course (SN): growth failure, sepsis, prolonged respirator use, and PDA with cardiac failure; and 9 had a mild neonatal course (MN): none of these complications. At 42.8 and 37.6 wks. chronological age for SN and MN respectively, polygraphic recording to calculate total responses (TR), inter-suck response time (IRT), and number of responses/burst (NRB) was performed while non-nutritive (NN) and $15 \%$ sucrose (S) tastants were presented over $2 \mathrm{~min}$. intervals.

\begin{tabular}{|c|c|c|c|c|c|c|c|c|c|}
\hline \multicolumn{4}{|c|}{$\mathrm{TR}(\# / 2 \mathrm{~min})$} & \multirow[t]{2}{*}{ IRT } & \multicolumn{2}{|c|}{ (millisec) } & \multicolumn{3}{|c|}{$\mathrm{NRB}(\#)$} \\
\hline & NN & $15 \% \mathrm{~S}$ & $\Delta$ & & $15 \% \mathrm{~S}$ & $\Delta$ & NN & $158 \mathrm{~S}$ & \\
\hline & $75.5^{*}$ & 125.4 * & 49.9 & 698.6 & 876.2 & 177.6 & 7.0 & 23.7 & 16.7 \\
\hline & 54.4 & 87.0 & 32.6 & 738.6 & 870.8 & 132.2 & 5.4 & 13.1 & 7.7 \\
\hline
\end{tabular}
values are $\widetilde{m}, \Delta=15 \% \mathrm{~S}-\mathrm{NN}, *$ = statistically significant SB of MN infants were similar to that of term infants. The SN infants had fewer TR and NRB than MN. The MN group had greater ability to discriminate tastants $(\triangle 15 \% \mathrm{~S}-\mathrm{NN})$ than $\mathrm{SN}$. One of 9 MN and 5/9 SN had abnormal neurological signs (seizures, abnormal tone, and head circum.). The data suggest that the SB of LBW infants with MN is comparable to that of term infants and that LBW infants neurologically impaired by SN have retardation of SB. $\mathbf{8 7 7}$ NEVELOPMENTAL PATTERNS OF INFANT MUSCLE OBTAINED BY (intr. by J.J. Corrigan, Jr.) Department Arizona. trics and Neurology, University of Arizona, Tucson, Arizona.
Needle muscle biopsies in infants and toddlers have shown that the small fibers in this age group allow a good cross section of a large number of fibers in $80 \%$ of biopsies. By this method we have investigated the patterns of histochemical development in infants with cerebral hypotonia. Particular emphasis was placed upon the ph lability of the myofibrillar ATPase reaction in order to divide the fibers into types I, IIA, IIB, \& IIC. IIC fibers are present in less than $1 \%$ in normal human muscle but have been found in large numbers $(20 \%)$ in the human fetus. In this study fiber type analysis was performed in 23 infants with cerebral hypotonia from 4 months to 5 years of age who have been followed for up to 24 months. None of the patients had histological evidence indicative of a classical neuromuscular entity. Seven patients demonstrated greater than $2 \%$ of Type IIC fibers and 8 also had an abnormal pattern of distribution of the remaining fiber types. One infant with benign congenital hypotonia had a normal distribution of fiber types with no IIC fibers. This data suggest that immature fiber types may provide an indicator of cerebral dysfunction as the cause of hypotonia in some infants. This could be a helpful diagnostic tool in the evaluation of "floppy babies" in which cerebral development is otherwise normal.

\section{(E) CHILDREN}

878 Jacqueline R. Farwell and George J. Dohrmann, (sponsored by Howard A. Pearson), Yale University School of Medicine, Dept. of Pediatrics and Sections of Neurosurgery and Neuropathology, New Haven.

Histologically verified cases of ependymoma and ependymoblastoma ("malignant ependymoma") occurring in children in Connecticut from 1935 to 1973 were analyzed. Of the 488 central nervous system tumors diagnosed in that period, $44(9 \%)$ of the 467 intracranial neoplasms and five (24\%) of the 21 intraspinal tumors were of ependymal origin. An increase in the incidence of ependymomas was noted since the mid-1950s. The mean age at diagnosis of ependymomas and ependymoblastomas was 5.6 and 5.0 years respectively. The male to female ratio was $0.6: 1$ for ependymomas and $1.7: 1$ for ependymoblastomas. Ependymomas were found above and below the tentorium with similar frequency; however, virtually all of the ependymoblastomas occurred supratentorially. Presenting symptoms and physical findings were reviewed. A significantly* increased survival was associated with supratentorial ependymal neoplasms relative to infratentorial. Contrary to the reports of no clinical difference between ependymomas and ependymoblastomas, children with supratentorial ependymomas were noted to have a significantly* longer survival than those with similarly situated ependymoblastomas. The children treated by operation and radiation had a significantly* greater survival than those treated by other methods. This difference became significant* at 27 months after diagnosis. The clinical course of intracranial ependymal neoplasms in adults and children was compared and appeared to be essentially the same. $*_{p}<0.05$ 
879 GLIOBLASTOMA MUITIFORME IN CHILDREN Jacqueline R. Farwe11 and George J. Dohrmann, (sponsored by Howard A. Pearson), Yale University School of Medicine, Dept. of Pediatrics and Sections of Neurosurgery and Neuropathology, New Haven.

of 490 children with central nervous system neoplasms, 43 (8.8\%) were glioblastomas: 22 occurred in the cerebral hemispheres, 16 in the brain stem, two in the cerebellum and three in the spinal cord. The male to female ratio was $3: 2$. Glioblastoma multiforme of the cerebral hemispheres occurred at a mean age of 12.7 years and the frontal lobe was the most commonly involved. Main presenting symptoms included headache $(77 \%)$, nausea/vomiting $(59 \%)$ and seizures $(32 \%)$. Papilledema $(45 \%)$ was the most common physical finding. The longest survivals were accomplished by a combination of operation and radiation $(22.0$ months). Brain stem glioblastomas occurred at a mean age of 6.7 (50\%) and headache $(36 \%)$ were the main presenting symptoms and the major physical findings were ataxia (43\%), cranial nerve palsies $(28 \%)$ and paresis $(28 \%)$. The length of survival was greatest with radiation alone (10.5 months). Survivals of children with glioblastoma multiforme were significantly increased with steroid therapy. Glioblastoma multiforme behaves similarly in children and adults. Intracranial glioblastomas have a more rapidly fatal course than that of other similarly

Sofia Franco, Billy F. Andrews. University of Louisville School of Medicine, Comprehensive Health Care Center for High Risk Infants, Department of Pediatrics.

Analysis of our data on comprehensive health care of high risk infants revealed maternal, fetal and neonatal factors associated with cerebral palsy. Of 31 infants 14 were preterm, 17 term. Maternal factors included 3 teen age pregnancies, 4 abnormal fetal presentation, 2 polyhydramnios, 1 alcoholism. Twenty-three infants were delivered spontaneous$1 y, 4$ by ceserean section 4 by forceps extraction. Fetal 10,5 meconium stained, 5 prolonged rupture of membranes, 3 cord prolapse 3 nuchal cord and 2 placental bleeding. Initial Apgar score was 6 or less in 20 of 31 infants with 9 requiring endotracheal intubation. Neonatal seizures were exhibited in 11 infants 6 of whom continued to have seizures on followup while 6 developed seizures subsequent to neonatal period. Other neonatal neurological signs were spasticity (2) hypotonia (2) cranial nerve palsy (2) nystagmus (1) microcephaly (1). Further reduction in incidence of cerebral palsy require earlier recognition and treatment of these maternal and perinatal factors. years and the pons was the most frequent site. Nausea/vomiting situated gliomas in childhood.

\section{$\mathbf{8 8 0}$ FACTORS RELATED TO LONG TERM FOLLOW-UP OF} distress was documented by external or internal monitors in

882

KE'RNICTERUS IN PREMATURES ASSOCIATED WITH NEUROTOXIC BILIRUBIN. Jay P. Goldsmith, Glenn W. Waterkotte, and Montgomery C. Hart. (Spon. by William J.R. Daily) Div. of Reproductive Medicine, St. Joseph's Hosp., Phoenix, Ariz. Pathologic kernicterus was observed in 8 Coombs negative infants among 33 infants of 1000-2000 gm birth weight who came to neurologic examination during the past 11 months. This represents an incidence of $4.4 \%$ of all infants in this weight category admitted to our institution during this period. Serial determinations of total bilirubin were obtained from birth to within 24 hours of death in 7 of 8 infants affected with kernicterus, with the highest value ranging from 4.1 to $11.4 \mathrm{mgms} \%$.

Neurotoxic (unconjugated, non-albumin bound) biliruoin was determined by solvent extraction, separating neurotoxic bilirubin from the total unbound bilirubin eluted by standard Sephadex column chromatography. Associated factors in patients who developed neurotoxic bilirubin were severe acidosis, hypoxia, and sepsis.

of the 33 patients autopsied, 19 had determinations of neurotoxic bilirubin including 6 of 8 kernicteric infants. Eight of the 33 patients had neurotoxic biliruvin values $\geq 0.04 \mathrm{mgms} \%$, 5 of whom had pathologic kernicterus. Eleven infants had values $\leqslant 0.03$ mgms\% of whom only one had kernicterus.

A high corelation between pathologic kernicterus and neurotoxic bilirubin values $\geq 0.04 \mathrm{mgms} \%$ ( 5 of 6 patients) strongly suggests that the determinations of neurotoxic bilirubin may significantly assist in the prediction of low birth weight infants at risk for kernicterus.

883 ( $\mathrm{Cu}$ ) METABOLISM. Warren D, Grover, Robert T Henkin, Temple U. School of Med, St Christopher's Hospital for Child., Dept. of Ped. and Neurol., Phila. and Georgetown U. Med. Center, Dept. of Molecular Nutrition \& Sensory Disorders, Wash., D.C. (Spon. H. W. Baird)

Previous reports concerning infants with TPD(Menkes Syndrome) have suggested that the sex-linked neurodegenerative disease is caused by a defect in absorption of oral $\mathrm{Cu}$ early in life. Studies in 2 infants $A$ ( 1 day), $B$ (3 days) have revealed biochemical findings indicating an intrauterine defect in $\mathrm{Cu}$ metabolism.Clinical features included a distinctive pattern of scalp and body hair composed of abnormal protein units. Cu data in infant A includes

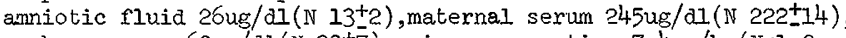
newborn serum 60:xg/dl (N 3347 ), lurinary excretion $7.4 \mathrm{ug} / \mathrm{kg}(\mathbb{N}<1$. oug/ $\mathrm{kg})$, liver $16 \mathrm{ug} / \mathrm{gm}$ dry wt. [N 215-498 (3)]. Cu values in infant $B$

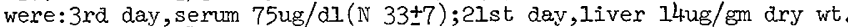
[N 107-383 (4)]. Ceruloplasm levels varied with serum $\mathrm{Cu}$ values. No activity of dopamine beta-hydroxylase (DBH) was detectable in the serum of either patient. Zinc levels were normal in all tissues and fluids studied. The administration of large amounts of $\mathrm{Cu}$ Salts by infusion ( 800 ug $\mathrm{Cu} \mathrm{SO} / \mathrm{kg} / \mathrm{wk}$ ), or subcutaneous injection or by mouth (Cu-trisodium nitrilotriacetate) was required to increase serum and hepatic $\mathrm{Cu}$ levels but DBH activity was not increased. These data suggest that maternal-fetal transport of $\mathrm{Cu}$ represents an attempt to force the ion into the fetus and that the binding of $\mathrm{Cu}$ by receptors in tissues is defective. This hypothesis accounts for the increased renal excretion of the unbound ion and the inability of several fetal tissues to retain $\mathrm{Cu}$. HOW EARLY IS EARLY CONTACT? DEFINING THE LIMITS OF THE SEQUEIAE OF NEONATAL HYPERVISCOSITY, Karen E.

881 Goldberg, Lula 0. Lubchenco, Mary A. Guggenheim, Univ. Colo. Med. Ctr., Div. Perinatal Med., Denve A randomized, controlled study of early partial plasma exchange transfusion for neonatal hyperviscosity was done in 22 UCMC newborns. 10 were exchanged within the first 8 hours after birth, 10 were not exchanged, \& 2 assigned to the non-exchanged group developed neurologic symptoms and were treated. These 22 infants, pius 10 controls with normal blood viscosity, were followed for hematologic, cardiac, pulmonary and neurologic status. At 8 months of age, a Bayley Motor and Mental Test, postural reflex assessment and neurologic exam were done (the latter by an examiner blind to the neonatal course). The Bayley scores were highest in the control group, 122 me atal \& 113 motor; next highest in the exchanged group, $112 \& 108$; and lowest in the nonexchanged infants, $109 \& 104$. The differences were not statistically significant. The neurologic exam was abnormal in 1 of 7 controls, 4 of 11 treated infants and 8 of 11 non-exchanged infants. 1 treated and 1 non-exchanged hyperviscous infant had developmental delay. 3 hyperviscous infants had spastic diplegia, 3 were tremulous and 4 had monoparesis of an upper extremity. The abnormal control infant was tremulous. The findings in nonexchanged infants vs controls were significant by the $x^{2}$ Test $(p<0.025)$. The difference between the exchanged group vs the untreated group did not quite reach significance $(p<0.08)$. Untreated neonatal hyperviscosity was associated with an increased incidence of neurologic abnormalities. Therapeutic benefit from early plasma exchange transfusions is suggested but not yet established.
884

SENSITIVE PERIOD. Deborah Hales, John Kennell, Roberto Sosa. CWRU Dept. of Ped.,Cleveland \& INCAP, Guatemala.

To explore the timing of the maternal sensitive period we studied the behavior of 60 primiparous Guatemalan mothers at Roosevelt Hospital. Using random assignment 20 mothers(Early Contact Group) lay skin to skin with their nude infants under a heat panel for 45 minutes immediately after they left the delivery room. A second group of 20 mothers (Delayed Contact) had the same 45 minutes of private skin to skin contact but at 12 hours after dellvery. A third group of 20 mothers(Control) was separated from their infants after delivery. These mothers did not have private skin to skin contact and first received their infants at 12 hours to begin breast feedings (the routine of this hospital).

Observations of these women in seven-bed rooms were made 36 hours after birth by an observer who did not know the previous experience of the mothers. Mothers who had contact with their neonates immediately after birth showed significantly more affectionate behavior("en face", looking at the baby, talking to the baby, fondling, kissing, smiling at the infant)when compared to the mothers in the delayed $(p<.01)$ and control $(p<.005)$ groups. There was a progressive decrease in affectionate behavior from the first to the third group. There were also significant differences in proximity-seeking behaviors $(p<.05)$ but not in measures of caretaking. No significant difference was noted between the delayed and control groups. This study indicates that the maternal sensitive period is less than twelve hours in length, suggests the importance of skin to skin coniact and compels reconsideration of hospital practices that even briefly separate mother and infant. 
885 A FOLLOW-UP STUDY OF TRANSIENT NEONATAI HYPERPHENYLALANINEMIA. Holger Hansen (Spon. by David Rush), Columbia University School

of Public Health, New York.

A search for children who had elevated PKU screening test results as newborns, and who were not referred for diagnostic evaluation, yielded 45 cases who could be

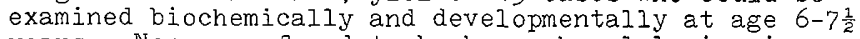
years. None was found to be hyperphenylalaninemic or mentally retarded. Degree and duration of neonatal hyperphenylalaninemia is known for 41 children. They were grouped according to highest phenylalanine level observed duxing neonatal retesting (means of values):

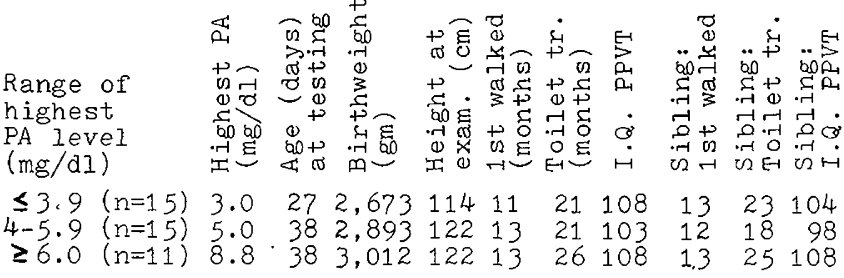

Children with more severe and longer lasting hyperphenylalaninemia were at no developmental disadvantage compared with their siblings as well as with childrer: with milder forms of neonatal hyperphenylalaninerila.

RISKS TO THE OFFSPRING OF WOMEN TREATED WITH

886 HYDANTOINS DURING PREGNANCY. James W. Hanson, Ntinos C. Myrianthopoulos, Mary Ann S. Harvey, and David W. Smith. Department of Pediatrics. University of Washington Scho Communicative Disorders and Stroke, Bethesda.

The fetal hydantoin syndrome is a variable pattern of altered growth and performance consisting of unusual facies, distal phalangeal hypoplasia and other defects occurring in some infants exposed in-utero to hydantoins. A prospective study of 35 infants exposed prenatally to this class of anticonvulsants showed that $11 \%$ had sufficient features to be classified as having the fetal hydantoin syndrome. An additional $31 \%$ displayed some features compatible with the prenatal effects of hydantoins. One hundred and four children whose mothers had a convulsive disorder and were treated with hydantoins throughout pregnancy were identified from the Collaborative Perinatal Project of the National Institute of Neurological and Communicative Disorders and Stroke. Abnormalities of growth, performance, and morphogenesis in this group of children were compared with those in a carefully matched control group. The results support the conclusion that about $11 \%$ of exposed infants have a recognizable pattern of abnormalities with serious clinical and developmental implications. Reduction of intellectual ability in infants with the fetal hydantoin syndrome is the area of greatest concern. Epileptic women being treated with bydantoin anticonvulsants should be told of the nature and magnitude of risks to the developing fetus before considering a pregnancy.

LEAD ENCEPHALOPATHY: A CONSEQUENCE OF CAPILLARY

887 DYSFUNCTION. Joseph A. Kochen and Yigal Greener Albert Einstein College of Medicine, Montefiore Hosp. \& Med. Ctr., Department of Pediatrics, Bronx, New York.

Lead-induced encephalopathy in the chick embryo is associated with capillary endothelial cell injury and focal hemorrhages. This study was undertaken to investigate the extent to which entry of $\mathrm{Pb}$ into the brain is due to extravasation of $\mathrm{Pb}$-containing red cells and to determine whether $\mathrm{Pb}$-induced endothelial cell injury increases capillary permeability to $\mathrm{Pb}$. Tracer doses of $59 \mathrm{Fe}$ and $210 \mathrm{~Pb}$, with and without added non-racioactive $\mathrm{Pb}$, were injected into the yolk sacs of 4 day embryos. Isotope uptake by red cells and brain was measured on day 18. As a result of exposure to adde $\mathrm{pb},{ }^{59} \mathrm{Fe}$ uptake by brain increased from $905 \pm 201 \mathrm{cpm} / \mathrm{gm}\{19\}$ to $1668 \pm 751 \mathrm{cpm} / \mathrm{gm}\{46\}(\mathrm{p}<.001)$. This corresponded to an increase in red cell volume from $0.023 \mathrm{~m} 1$ to $0.033 \mathrm{~m} 1 / \mathrm{gm}$ brain, presumably because of extravasation of red cells. $210_{\mathrm{Pb}}$ uptake by brain increased from $198 \pm 34\{19\}$ to $386 \pm 160 \mathrm{cpm} / \mathrm{gm}\{46\}$ (p<.001). Only $47 \%$ of this increase in $210 \mathrm{~Pb}$ uptake was accounted for by the increased retention of red cells in the brain. The remainder of the increase in brain $210 \mathrm{~Pb}$ uptake presumably represented increased capillary permeability to $\mathrm{Pb}$. This fraction of $210 \mathrm{~Pb}$ uptake remained linearly related to ${ }^{5}{ }^{9} \mathrm{Fe}$ uptake $(\mathrm{r}=0.95, \mathrm{p}<.001)$, suggesting that capillary damage is responsible for both types of $\mathrm{Pb}$ uptake. In conclusion, the movement of $\mathrm{Pb}$ into the immature CNS is facilitated during $\mathrm{Pb}$ exposure by increased capillary permeability to $\mathrm{Pb}$ and by the extravasation of red cells. These findings indicate that capillary dysfunction may be an important factor in the pathogenesis of lead encephalopathy.
888 CIRCUMVENTION OF BLOOD-BRAIN BARRIER BY INTRA-CEREBRAI LEAD IMPLANTATION. Joseph A. Kochen and Asao Hirano, Albert Einstein Coll. Med., Montefiore Hosp. \& Med. Ctr. Departments of Pediatrics and Pathology, Bronx, New York.

The resistance of the mature animal to the development of lead encephalopathy despite high blood $\mathrm{Pb}$ levels may be a function of a more effective blood-brain barrier. The intra-cerebral concentration of $\mathrm{Pb}$ in the mature brain of rats was increased by the direct intra-cerebral implantation by trochar of small pellets of $\mathrm{Pb}$ acetate into one cerebral hemisphere. W'ithin one week the pellet was surrounded by a large zone of inflammation, which by 4 weeks had largely subsided. In adjacent areas, small blood vessels, many unaccompanied by the usual astrocytic vascular feet, as well as disorganized neuronal and glial elements traversed edematous spaces. Some blood vessels showed striking endothelial cell changes. These included surface infolding, large numbers of pinocytotic vesicles, tubular bodies and the appearance of vessels with fenestrated endothelium in regions where such vessels are not known to be normat $1 y$ present. At varying distances from the implant, macrophages, reactive astrocytes and glial cells contained dense $\mathrm{Pb}-1$ ike granular and fibrillar cytoplasmic inclusions. In addition, a number of these cells showed dense fibrillar intranuclear inclusions identical to those found in renal tubular cells in $\mathrm{Pb}$ intoxication. Spongy changes due to axonal swelling in the hypothalamus similar to these previously found in the cerebral cortex of $\mathrm{Pb}$ intoxicated young monkeys were also observed. These results strengthen the mature CNS is due, at least in part, to the development of a bloodbrain barrier to $\mathrm{Pb}$.

ENTRICULAR AND SPINAL FLUID (CSF) GLYCINE IN NON-

889 KETOTIC HYPERGLYCINEMIA. CLINICAL CORRELATION AND TREATMENT BY USE OF A SHUNT. Ingeborg Krieger and Arthur B. Eisenbrey. Wayne State Univ., school of Med. \& Children's Hospital of Michigan, Detroit.

A major site of abnormal glycine metabolism appears to be in brain. Glycine is thus less elevated in plasma (4-fold in our cases) than in CSF (28-fold). By treating 3 patients with Nabenzoate we lowered pl.glycine from 85.5 to 33.9 umoles $/ 100 \mathrm{ml}$ through conjugation and excretion as hippurate. On 9 tests plasma/CSF ratios remained constant, 6:1 (normal 40:1). Normalization of CSF glycine was not accomplished apparently because conjugation with benzoate occurs in liver mitochondria and mixing of the two pools is limited. Mental retardation was not averted in $\mathrm{j}$. infant treated since the $3 \mathrm{ra}^{\mathrm{a}}$ day of $\mathrm{life}$ although pl.glycine was almost normal and CSF glycine was 6 umoles $/ 100 \mathrm{ml}$. Acute symptoms were a function of CSF glycine, with minimal brainstem depression at 9 umoles $/ 100 \mathrm{ml}$, increasing until respiratory assistance was required at $18 \mu$ moles $/ 100 \mathrm{ml}$. These observitions suggested that further reduction of CSF glycine, an inhibitory neurotransmitter, might improve mental function. This was attempted in 1 patient with a ventricular-peritoneal shunt which accelerates fluid production with time and was expected to remove glycine-rich fluid quicker than normal reabsorption mechanisms. ventricular glycine prior to shunting was twice as high as CSF glycine (15 vs 8 umoles/100 ml). One week later, slycine in fluid withorawn from the shunt was 38 umoles $/ 100 \mathrm{ml}$. Early follow-up shows that the patient no longer requires anticonvulsants.

EVALUATTON OF INFANTS WITH BLOODY SPINAL FLUID USING 890 COMPUTERIZED TOMOGRAPHIC (CT) BRAIN ȘCAN. Kalpathy S. Krishnamoorthy, Raymond A. Fernandez, K. Jack Momose, G. Robert De Long, Daniel C. Shannon. Harvard Medical School, Massachusetts General Hospital, Children's Service, Department of Neurology, Department of Radiology, Boston.

Twenty eight infants (mean birth weight: $1.7 \mathrm{Kg}$, mean gestational age: 32 weeks) receiving positive pressure mechanical ventilation (MV) for R.D.S. demonstrated unexplained neurologic deterioration and grossly bloody CSF. A CT scan using EMI scanner was performed during continued MV with the aid of muscle relaxation. Intracranial hemorrhage (ICH) was found in 23 , hydrocephalus secondary to old ICH in 2 and no evidence of ICH in 3 infants. $14 / 23$ had massive intra-ventricular hemorrhage (IVH) and died, 6 had lesser degrees of IVH and survived and $3 / 23$ had subarachnoid hemorrhage (SAH) and survived. Autopsy was performed on 6/14 infants with massive IVH and correlated well with CT scan. Among 6 survivors with IVH, 5 had dilated lateral ventricles at initial CT scan; $2 / 5$ developed rapidly enlarging head size confirmed as hydrocephalus by repeat CT scan. In follow-up, neurologic development is delayed. Among 3 with SAH, development is normal in two at 5-12 months of age. CT scan is useful in diagnosing the presence and extent of ICH in infants, the presence of hydrocephalus and following the natural history of infants who survive. 
891 EARLY HOME CARE; IMPACT CN VISITING SICK INFANTS, John Lampe, Mary Trause, John Kenne!1. Case Western Reserve To determine the effects of (1) the length of time an infant had been home prior to hospitalization, and 2) the presence of a visible congenital anomaly on parental attachment, the frequency of visiting was monitored for 44 infants on an infant division during two 2-week periods. When divided into groups for comparisonl of visiting rates, the groups were approximately matched for infant's sex and race, parents' socio-economic status and distance from hospital. During the second 2-week period a parental interaction score was computed based on whether or not the parent fed the child, held the child, or stayed for at least one hour during a visit. Parents of infants who had been home two weeks or more visited more often than parents of children who had not $(p<.05)$. Of the 12 infants who had been home two weeks or more, $7(58 \%)$ were visited every day. Of the 32 who had been home less than two weeks, only $5(16 \%)$ were visited every day. The parental interaction scores corroborated this result $(p<.05)$. Although the frequency of visiting for infants with visible congenital anomalies was less than for babies with no malformation or with a nonvisible congenital defect, the difference was not significant. The observation that parents of children who had been home for at least two weeks visited more often suggests the importance of full caretaking and intimate interaction with an infant in the early days at home for parent-to-infant bonding. These findings suggest the value of al lowing newborns to go home for a short time whenever possible and providing facilities for parents to "live in"and care for their infants in the hospital.

SEIZURE DISORDER, TPP-ATP PHOSPHORYL TRANSFERASE 892 INHIBITOR AND REYE'S SYNDROME: Derrick Lonsdale, Marta Steinberg and Samuel J. Horwitz, Cleveland Clinic Foundation, Depts. of Pediatrics and Pathology and Case Western Reserve University, Department of Pediatrics, Cleveland, Ohio. Spon. by Irwin A. Schafer

A 2-year old child with a seizure disorder failed to respond to all anti-convulsants used. Urine contained $3.0 \mathrm{mg} / 100 \mathrm{ml}$. of alpha keto glutarate $(\mathrm{N}<2.5 \mathrm{mg} / 700 \mathrm{ml}$.) Urinary pyruvate was normat. Empirical trial therapy with pyridoxine was followed 3 days later by acute acidosis and clinical and laboratory criteria indistinguishable from those of Reye's Syndrome. Gastrointestinal symptoms were associated with recovery of Shigella organisms from stool and may have been a predisposing factor. Gas chromatography of urine revealed an excess of lactate and paper chromatography an excess of pyruvate. Atthough urine contained TPP-ATP phosphoryl transferase inhibitor and smaller amounts were found in the urine of both parents, autopsy revealed changes of Reye's Syndrome rather than Leigh's Disease. Brain sections of cerebellum and cortex were histologically similar to those of a 2-month old lamb dying from polioencephalomalacia, known to be caused by the enzymatic formation of a thiamine analog inhibitor similar to amprolium. Fresh frozen sections of liver revealed normal activity of pyruvate carboxylase and dehydrogenase of both ketoglutarate and pyruvate. The animal model of polioencephalomalacia may be useful in further study of Reye's syndrome. Pyridoxine in this case may have had "anti thiamine" effect as reported in a case of Leigh's Disease.*

*Weil, M.L., et al, Clinical Research, 19:229; 1971.

SEIZURES IN A NEONATAL INTENSIVE CARE UNIT: A PROS-

893 PECTIVE STUDY. Richard Marshall, Michael Sheehan, Marilyn Escobedo, Pra-On Chavalit dhamrong and Joseph Volpe (Intr. by R.E. Hillman), Depts. of Ped. and Neurology, Washington Univ. Sch. Med, , St. Louis, Missouri.

Seventy patients with seizures were studied prospectively. Thirty three (47\%) patients weighed less than $2500 \mathrm{~g}$. (LBW) and 37 $(53 \%)$ more than $2500 \mathrm{~g}$. Half of the seizures occurred on the first day, and $75 \%$ by the third day. Tonic (27), subtle (20) and multifocal (19) seizures predominated. Multifocal (74\%) and subtle seizures $(65 \%)$ were common in term patients; tonic (70\%) seizures were found predominantly in LBW infants. Seventy-five percent of patients with subtle and multifocal seizures survived compared to $41 \%$ survival with tonic seizures. Etiological diagnoses were established for 64 patients $(91 \%)$. Only 12 patients (17\%) had a single etiological diagnosis. Perinatal hypoxia \pm intracranial hemorrhage was the etiology in $75 \%$ of cases. Hypocalcemia (13\%) and hypoglycemia (9\%) were never isolated causes without other associated factors. Bacterial and viral meningitis were responsible for seizures in $12 \%$ of patients. Overall survival was $69 \%-80 \%$ for patients weighing over $2500 \mathrm{~g}$. and $47 \%$ for LBW patients. Sixty-one percent of patients with perinatal hypoxia survived. Only $25 \%$ of patients with intracranial hemorrhage survived. Fifty percent of patients with infections survived. Thus, important prognostic information can be obtained from detailed neurological observations of seizure types and attempts at etiological diagnosis. 894. ENERGY METABOLISM OF BRAIN IN HUMAN PROTEIN-CALORIE
MALNUTRITION

Saroj Mehta, H.K. Kalsi, C.K. Nain and John H. Menkes Postgraduate Institute of Medical Education and Research, Department of Paediatrics, Chandigarh, India and UCLA School of Medicine, Brentwood V.A. Hospital, Departments of Psychiatry, Pediatrics and Neurology, Los Angeles, California.

Cerebral blood flow (CBF) and carbohydrate metabolism were studied in five normal children and 25 children, aged 40 months or less with varying degrees of protein-calorie malnutrition (PCM). CBF in normal children and those suffering from grade 1 $P C M$ was $90.3 \pm 5.7 \mathrm{ml} / 100 \mathrm{gm} / \mathrm{min}$, a value comparable to that obtained by other investigators, but the proportion of glucose taken up by the brain which combined with oxygen, the oxygen/ glucose index (OGI), was $65.8 \%$, suggesting significant conversion of glucose into brain proteins, lipids and cholesterol. With increasing severity of PCM there was an increased glucose utilization and a progressive reduction in the OGI. In grade IV PCM, CBF was $68.7 \pm 5.4 \mathrm{ml} / 100 \mathrm{gm} / \mathrm{min}$, and the OGI was $34.7 \%$. These findings suggest that in severe human PCM the proportion of glucose undergoing aerobic oxidation is reduced, possibly as a consequence of abnormally increased conversion into structural proteins or lipids. $\mathbf{8 0 5}$ A PROSPECTIVE STUDY OF THE FETAL ALCOHOL SYNDROME, Eileen M. Ouellette, Henry L. Rosett, N. Paul Rosman Boston City Hospital, Department of Pediatrics, of Medicine, Boston City Hospital, Department

A prospective study of the effects of excessive prenatal maternal alcohol ingestion on of fspring was carried out. A questionnaire administered to 450 pregnant women at the first prenatal visit sought demographic data, nutritional and smoking habits, alcohol and other drug use. Utilizing the VolumeVariability Index of Cahalan, mothers were rated as abstinent or rare, moderate, or heavy drinkers. At delivery, the questionnaire was re-administered and data compared. Detailed pediatric, neurologic and developmental examinations were carried out on newborns without prior knowledge of the mother's or child's history. $50 \%$ of mothers were abstinent or rare drinkers, $40 \%$ drank moderately and $10 \%$ drank heavily. No significant differences were found in general nutrition or vitamin intake among women in the 3 drinking groups. Heavy drinkers tended to smoke heavily. Of 193 infants born to date, $7 / 24$ born to heavy drinking women had congenital anomalies, $6 / 7$ were major. This compares with $8 / 88$ in the moderate group ( $1 / 8$ major) and $6 / 81$ in the rare and abstinent group ( $1 / 6$ major). Microcephaly was found in $4 / 24$ infants born to heavy drinking women. An increase in infants born small for gestational age, many with hypotonia and/or jitteriness, was also found. Excessive maternal alcohol ingestion during pregnancy is associated with increased congenital and growth abnormalities in offspring but a recognizable pattern of anomalies has not yet been found.
896 ONE YEAR OF AGE. M.J.Painter, P.0'Donoghue, Q.R. Depp, (Spon. by P.M.Taylor), Univ. of Pgh. \& Magee-Womens Hosp., Depts. Neurology, ob \& Pediatr., Pittsburgh.

We tested the hypothes is that FHRP would be a better predictor than Apgar scores of developmental outcome. Fifty high-risk, appropriate weight for gestational age, term infants were identified prospectively on the basis of their FHRP. Infants were grouped according to FHRP: 1) normal pattern, 2) moderate severe decelerations, 3) severe variable or late decelerations. The parity, socioeconomic status, race of the mothers, and sex of the infants were similar within each group. Infants were evaluated neurologically by detailed examinations and developmentally by Denver testing at 2, 4, 6, 9, and 12 months of age.

$\begin{array}{lccc}\text { Group } 1 & \text { No. Infants } & \frac{\text { Examinations }}{12} & \frac{\text { Abnormal Exams }}{68(9 \%)} \\ \text { Group 2 } & 17 & 100 & 29(29 \%) \\ \text { Group 3 } & 21 & 124 & 47(38 \%)\end{array}$

Thirty infants had abnormal neonatal examinations. Clinical neonatal asphyxia (Apgar $<4$ at $7 \mathrm{~min},<7$ at $5 \mathrm{~min}$ ) predicted 10 $(33 \%)$ of these abnormal neonates while abnormal FHRP's (Groups 2 and 3) predicted $27(90 \%)$ of the abnormal neonates. Eight infants were abnormal at 1 year of age; each had an abnormal FHRP while only 2 had neonatal asphyxia as determed by Apgar.

Proper interpretation of the prognostic significance of FHRP and the effect of intervention in response to abnormal FHRP during labor and delivery can be adequately evaluated only if the infant's neurological and developmental outcome is known. 
897 RELIABILITY OF THE NEUROLOGICAL ASSESSMENT AT 6 MONTHS POST-TERM IN THE INFANT $\leq 1500$ GMS. BIRTH WEIGHT. Karen E. Pape, Pamela M. Fitzhardinge (Spon. by Paul R. Swyer), Research Inst., Hosp. for Sick Children, Univ. of Toronto, Toronto, Canada.

In order to determine how early a definitive diagnosis of neurologic status can be made in the highest $r$ isk premature, examinations were done at 6 and 12 mos, post term on 82 babies (B.W. $\leq 1500$ g.) referred for neonatal care in 1974 . The CNS evaluations at 6 and 12 mos. post term (Table) were grouped as follows : Group A - normal; Group B - abnormal (hydrocephalus, microcephalus, cerebral palsy, D.Q. $\leq 50$ ); Group C - "soft signs" (dystonia, inappropriate righting reactions, D.Q.51-80). Infants in Group A at 6 mos. remained normal or showed only mild dystonia or developmental delay at 12 mos. Infants in Group B remained abnormal. Greatest change in status was seen in Group C. By 12 mos., 21 had improved to normal. However, 7 were shifted to the abnormal group because of a definitive diagnosis of microcephaly (3) or hemiplegia (4). The remaining 23 infants in Group $C$ retained their "soft" signs and thus could not be given a specific prognosis. These results suggest that there is a high predictive reliability for the 31 infants in Group $A$ and $B$ at 6 mos. but poor reliability for the $5 \mathrm{l}$ presenting with "soft" signs only.

\begin{tabular}{lr|c|c|c|c}
\hline \multirow{3}{*}{6 mos. examination } & \multicolumn{3}{|c}{12 mos. examination } \\
\cline { 2 - 6 } & No Change & Improved & \multicolumn{2}{c}{$\begin{array}{c}\text { Meteriorated } \\
\text { Mild Severe }\end{array}$} \\
\hline Group A & 25 & 21 & - & 4 & - \\
Group B & 6 & 6 & - & - & - \\
Group C & 51 & 23 & 21 & - & 7
\end{tabular}

\section{DEFICIENCY OF MUSCLE CARNITINE PRESENTING AS REYE'S}

898 ENCEPHALOPATHY IN A MALE INFANT. D. Parker, S. Di E. Reiter and A. Root. Univ. of So. Fla. College of Medicine, All Children's Hospital, Dept. Pediat., St. Petersburg, Florida.

Deficiency of muscle carnitine is usually associated with progressive muscular weakness. Our patient presented with an illness initially diagnosed as Reye's encephalopathy. At 12 months of age a previously well infant developed vomiting and lethargy two weeks after an upper respiratory infection, subsequently becoming flaccid and unresponsive. During that episode, peak serum $\mathrm{NH}_{3}$ was $343 \mu \mathrm{g} / \mathrm{dI}$, SGOT $6,760 \mathrm{IU}$, SGPT $2,310 \mathrm{IU}$, CPK 46,860 IU. Following treatment with dexamethasone he improved clinically and laboratory data returned to normal. Muscle strength became normal during the next 5 months. A similar episode occurred at 19 months of age when peak $\mathrm{NH}_{3}$ was only 133 , with SGOT 2783, SGPT 1111, CPK 151,500. He responded to glucocorticoid therapy, and muscle strength returned to normal over the ensuing 2 months. Biopsy of the gastrocnemius muscle 3 weeks after the 2nd episode revealed a carnitine concentration of $0.29 \mu \mathrm{M} / \mathrm{g}$ (normal: $\overline{\mathrm{X}}=2.52$; range 1.64-3.54). Serum carnitine concentrations were 2.94 and $2.44 \mathrm{\mu M} / \mathrm{dl}$ (normal 2.3-7.0). Iight and electron microscopic examinations of the muscle biopsy revealed findings similar to previously described cases of muscle carnitine deficiency. Large quantities of 6-carbon dicarboxylic fatty acids and myoglobin were identified in the urine during the 2 nd episode. It is concluded that a patient with deficiency of muscle carnitine may present with characteristics of Reye's encephalopathy before myopathy becomes apparent.

BRAIN ULTRASTRUCTURE IN REYE'S SYNDROME. John C.

899 Partin, Jacqueline S. Partin, William K. Schubert, A. James McAdams and Robert L. McLaurin, University of Cincinnati College of Medicine, Departments of Pediatrics and Neurosurgery, Cincinnati.

Brain biopsies were obtained from 3 children with severe Reye's Syndrome at cranial decompression approximately 50 hours after the onset of encephalopathy, and again at the time of cranial restoration, 43, 54, 75 and 586 days later. Al1 patients displayed the same ultrastructural changes in their first biopsy specimens: astrocyte swelling, a neuronal injury which included universal mitochondrial matrix rarification similar to that seen in the hepatocyte mitochondria, disaggregation of neuronal ribosomes and a striking blister-like lesion of the myelin sheaths resembling that seen in triethyltin intoxication. Blood vessels appeared normal and inflammation was absent. The extracellular space was not increased and the brain swelling was mainly due to watery myelin blebs and astrocyte swelling. In recovery: the least severely affected child's biopsy contained normal neurons, extracellular collections of dense myelin debris and occasional lipid-filled oligodendrocytes. The most severely injured child showed persistent vacuolation of neuropil with neuron loss and proliferation of fibrous astrocytes. Extracellular myelin debris was easily visible 586 days after the original illness. Conclusion: Neuronal mitochondria are universally altered as in the liver. This and the other findings are indicative of a specific cytotoxic brain injury in Reye's Syndrome which is not typical of anoxic change, ammonia intoxication or hypoglycemia.

\section{0}

PERCEPTUAL-MOTOR FUNCTION IN TREATED PHENYLKETONURIC CHILDREN WITH NORMAL INTELLIGENCE

Siegfried M. Puesche1, Elissa Koff, Patricia M. Boyle (Spon. by Leo Stern)

Brown University, Rhode Island Hospital, Department of Pediatrics, Providence, and Harvard Medical School, Children's Hospital Medical Center, Department of Medicine, Boston.

The objective of this study was to investigate whether there were significant perceptual-motor deficits in a group of schoolage treated PKU children currently on a liberal diet.

Two measures were used, an age equivalence score derived from the composite error score in the Bender Gestalt Test and the verbal IQ-performance IQ discrepancy on the Wechslex Intelligence Scale for Children. Sixty-one percent of the 32 PKU children had significantly lower performance IQ's than verbal IQ's $(p<0.05)$. Age equivalent scores on the Bender Gestalt Test ranged from 14 months above to 47 months below chronological age (p<0.001). There were no significant relationships between either the IQ or Bender Gestalt Test scores, and a set of variables associated with outcome in PKU and/or abnormalities in central nervous system development and function, except for dietary control. The regression coefficient for dietary control was weak1y suggestive $(p=.020)$ of a relationship between dietary control and perceptual-motor performance as measured by the Bender Gestalt. The question of significant perceptual-motor dysfunction is especially critical because of the high risk of learning disabilities and school problems associated with such an impairment.

901 GRADING CSF XANTHOCHROMIA, SexSOn, W. R., sippi School of Medicine, Department of Pediatrics, Jackson, Mississippi. Intr. by Stahlman, M. Traumatic LP's in neonates occur frequently enough that at times differentiation of the infant with a CNS bleed from the infant with a traumatic LP is difficult. Xanthochromia is not uncommon in the CSF of newborns. Increasing xanthochromia on the other hand is seen primarily with intracranial bleeding. A visual scale was used to evaluate degree of CSF xanthochromia from $0 / 6$ to $6 / 6$. CSF was examined within the first 4 days after birth in most cases. Repeat LP was done as indicated or within 48 to 96 hours after obtaining bloody CSF. "Normal" CSF was obtained from infants with suspected sepsis. All CSF was spun immediately and the supernatant was compared to the scale. No "normal" newborn including those with jaundice had xanthochromia of greater than $2 / 6$. Suspected intraventricular and subarachnoid bleeding was found to be clear or slightly xanthochromic immediately following the episode, with an increase to $3 / 6$ or more after several days. This method offers a simple clinical tool to help distinguish a traumatic tap from intracranial bleeding. It is suggested that xanthochromia of $3 / 6$ or more indicates that blood has been present chronically in the CSF, but gives no indication as to the locus of the bleed.

A SYNDROME RESEMBLING MINIMAL BRAIN DYSFUNCTION (MBD)

902 IN RAT PUPS BORN TO ALCOHOLIC MOTHERS. Bennett A. Shaywitz, Jeffrey H. Klopper, Judith W. Gordon, Dept. of Ped., Yale Univ. Sch. of Med., New Haven.

Evidence from both human (Jones et al) and mouse studies (Chernoff) suggest that maternal ingestion of ethanol produces a disorder (fetal alcohol syndrome) characterized by pre and postnatal growth retardation, craniofacial, cardiac and joint abnormalities and developmental delay. We now report that rat pups born to alcoholic mothers develop a syndrome similar to that of MBD found in children. Ethanol ( $35 \%$ of total calories) or isocaloric sucrose was added to liquid sustacal from the second day of gestation and continuing for the first month of postnatal life. Maternal alcohol averaged $126+55.7 \mathrm{mg} \%$ and pups were cross fostered between 1-3 days after bixth. Body weight was significantly reduced in rat pups born to alcoholic mothers from birth onward, and maturation of righting responses and eye opening was significantly delayed as well. Total activity was significantly greatex in pups born to alcoholic mothers at 12 days $(55.3 \%$ vs. $48.1 \%$, $p<0.001)$ and at 19 days $(75.8 \%$ vs. $57.5 \%, p<0.001)$, but by 26 days of age the hyperactivity of the prenatal alcohol groups had abated and was not different from controls (44.0\% vs. $40.0 \%$, p 0.05$)$. Rat pups born to alcoholic mothers were significantly impaired in T-maze learning at 21 days, and in shuttle box performance at 27 and 33 days. Our findings suggest that maternal ingestion of alcohol may produce subtle deficits in the offspring characterized by hyperactivity that abates with maturation and cognitive difficulties that persist - a developmental pattern
strikingly similar to the syndrome of MBD found in children. 
PROLONGED CONTINUOUS MUNITORING OF CEREBRAL VEN-

903 TRICULAR PRESSURE IN STAGE 4 REYE SYNDROME. Bennett A. Shaywitz, John M. Leventhal and Michae1 S. Kramer, Yale Univ. Sch. of Med., Dept. of.Ped., New Haven.

Evidence from both clinical and pathological investigations suggest that increased intracranial pressure(ICP) is a significant factor in the mortality of Reye syndrome. This, coupled with a critical review of our previous 22 cases which failed to document the efficacy of exchange blood transfusion led us to include continuous ventricular pressure monitoring in the supportive care of children with Reye syndrome. To date, three children ages 3-5 years have been managed with continuous ICP monitoring. All had stage 4/5 coma by both clinical and EEG criteria. Blood $\mathrm{NH}_{3}$ concentrations were 1086,316 and $586 \mathrm{ug} \%$ and all had elevations of SGOT, prothrombin time and CPK as well. Rapid unpredictable increases in ICP occurred for many days in all children, though difficulty controlling ICP was most pronounced during the first three days of illness coinciding with maximum blood $\mathrm{NH}_{3}$. Therapeutic measures most successful in controlling ICP were the intravenous administration of mannitol and hyperventilation. Elevations in ICP occurred despite serum osmolarity as high as 416 mosm。 and following oral glycerol. Rapid increases in ICP also ret sulted from routine manipulation of the patients (eg。 tracheal suction). Exchange blood transfusion increased ICP in one child and had no effect on ICP in another. All children are now completely normal 6 months after their illness. Our experience in these children suggests that vigorous supportive therapy including careful monitoring of ICP should be the basis on which any claims of specific treatment in Reye syndrome must be compared.

904 HYPERTENS IVE ENCEPHALOPATHY -THE DEFINITION AND REAPPRAISAL OF A COMMON COMPLICATION OF HXPERTENSION IN CHILDREN. John T. Sladky, Bennett A。 Shaywitz and Normal J. Siegel, Dept. of Ped., Yale Univ. Sch. of Med., Yale New Haven Hospital, New Haven.

Despite recent interest in hypertension in the pediatric age group, hypertensive encephalopathy remains a poorly defined entity. Over a ten year period 65 children and adolescents with documented hypertension (>95\% for age) were evaluated at YNHH. Eleven presented with neurologic involvement as the primary manifestation of hypertension and eight of these 11 demonstrated encephalopathy with decreased level of consciousness and convulsions. Papilledema was present in on 1 y $3 / 8$ at the time of their presentation. Of significance, was the focal nature of their neurological symptoms with focal motor seizures and/or localizing, neurological findings present in 5/8. In addition two patients developed cortical blindness and one presented with an acute personality change. Lumbar puncture revealed moderate elevations in the opening pressure $(5 / 6)$ and CSF protein (5/6). EEG evidence of focal slow wave activity was seen in $3 / 6$ patients shortly after their acute episode, one corresponding to a region of increased focal uptake on brain scan. The diverse CNS manifestations of hypertension in this series are consonant with theories that postulate local and multicentric baro injury to the blood brain barrier as the primary pathophysiologic mechanism of hypertensive encephalopathy. The evolution of this insult is complex with initial focal abnormalities that may progress to a more generalized brain involvement characterized by cerebral edema and increased intracranial pressure.

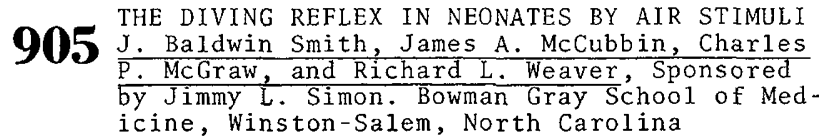

The diving reflex has been implicated in the etiology of the Sudden Infant Death Syndrome. In neonates, we elicited this reflex by delivering a $2-3 \mathrm{sec}$. burst of air at $25^{\circ} \mathrm{C}$ at a rate of $15 \mathrm{~L}$./min. to the face over the distribution of the ophthalmic or maxillary division of the trigeminal nerve. The air stimulus, electrocardiogram, instantaneous heart rate change, and respirations (by chest pneumograph) were recorded by a Grass Polygraph. The occurrence of immediate apnea and brady cardia were considered a positive diving reflex. Of 76 neonates between 38-44 weeks postconceptional age (ges tational age plus postnatal age), 21 normal and 50 abnormal infants had no diving reflex. However, 5 neonates with major neurological abnormalities had a positive diving reflex in at least $20 \%$ of the trials. Studies of 23 older normal and abnormal infants $(46-99$ weeks postconceptional age) revealed an increasing instance of diving reflex in both groups. Thus, while the diving reflex, as elicited by air stimulation, is not present in normal neonates at 38-44 weeks postconceptional age, it may be present in neurologically abnormal infants. Further, with increasing age this reflex is more easily evoked.
CHANGING PROGNOSIS FOR INFANTS WEIGHING $\leq 1000$ GMS.

906 AT BIRTH. Christinia T. Ukrainski, Jeffrey J. Pomerance, Diane H. Henderson, Andrea H. Nash, and Donald A. Lackey, Dept. Ped., Cedars-Sinal Medical Center and UCLA Sch. Med., Los Angeles. (Spon. by B. M. Kagan)

Improved neonatal outcome has been confined primarily to infants weighing $>1000$ gms, at birth. Recently published survival rates for infants weighing 51000 gms. at birth range between 8 and $31 \%$.

Between January 1973 and June 1975, 77 infants weighing s1000 gms. at birth were admitted to the Neonatal Intensive Care Unit. Thirty-three (43\%) infants were inborn, $44(57 \%)$ were transported from outlying hospitals. Thirty infants (39\%) survived with birth weights ranging between $620-1000$ gms. (avg. 875 gms). Gestational ages ranged between 25-32 wks. (avg. 28.5 wks). Six of $26(23 \%)$ infants weighing between 500-750 gms. survived. To date, 23 of 30 surviving infants have had neurological and Gesell developmental evaluations at post-natal ages varying from 6 months to $2 \frac{1}{2}$ years. The mean Developmental Quotient (D.Q.), corrected for prematurity, is 89 (range $36-114$ ). Sixteen of 23 (70\%) infants have D.Q.'s $>80$. One of these has grade I retrolental fibroplasia (RLF). Two infants (twins) have spastic quadriparesis with D.Q.'s in the $60^{\prime} s$. Four of 23 are severely retarded. One of these has grade V RLF. One infant could not be evaluated developmentally because of minor motor seizures.

Advances in perinatal care are benefiting even the smallest infants. Most importantly, intact survival is occurring with increasing frequency.

907 INTRACRANIAL PRESSURE MEASUREMENTS IN NEWBORNS US ING A NON INVASIVE TECHNIQUE. D. Vidyasagar and T.N.K. Raju, Abraham Lincoln School of Medicine, Univer. of 111. Hosp., Department of Pediatrics, Chicago, 11.

Because of technical difficulties intracranial pressure (ICP) in the sick neonate is not routinely monitored. We describe a simple noninvasive technique of measuring $1 C P$ in newborns. A fiber optic transducer is fixed over anterior fontanel and connected to ICP monitor (Ladd ICP Model \#1700, Hoffmann LaRoche, Inc) with a recorder; anterior fontanel pressure (AFP) was measured in 3 groups of infants. The mean+S.E. pressure in $\mathrm{cmH}_{2} \mathrm{O}$ in 3 positions of the head are shown below.

\begin{tabular}{lrrr} 
& Horizontal & \multicolumn{1}{c}{ Head Up } & Head Down \\
\hline Term (30) & $10.14 \pm 0.39$ & $5.64 \pm 0.33$ & $14.58+0.57$ \\
Preterm (6) & $9.56+0.83$ & $5.33 \pm 0.46$ & $17.14 \pm 2.30$ \\
Acutely i11 (15) & $13.58+0.85$ & $10.32 \pm 1.52$ & $16.68 \pm 1.19$
\end{tabular}

Acutely ill (15) $13.58+0.85 \quad 10.32 \pm 1.52 \quad 16.68+1.19$
The AFP in horizontal position was significantly higher in acutely ill infants than in healthy term or preterm infants $(p<05)$ The increased AFP in the sick infants may be related to relative hypoxia and brain edema. Change in AFP with different head positions indicates the sensitivity of transducer. Simultaneous measurement of spinal fluid pressure in 3 infants and ventricular pressure in 3 hydrocephalic infants correlated well with AFP ( $n=6$ * $r=0.95, p<.01)$. In 3 infants with clinical diagnos is of intracranial hemorrhage. AFP rose to $19.6,18.4,23.4 \mathrm{cmH}_{2}$. AFP did not increase significantly with varying pressures of constant distending pressure in 4 infants tested. The data suggests that this simple noninvasive technique of measuring ICP has a great potential in the management of sick neonates.

\section{ONCOLOGY}

908 DEMONSTRATION OF A PHOSPHATURIC SUBSTANCE IN EPIDERMAL NEVI SYNDROME (E.N.S.) ASSOCIATED WITH RESISTANT RICKETS.

Lorenzo C. Aschinberg, Lawrence M. Solomon, Petros M. Zeis, Parvin Justice and Ira M. Rosenthal. Abraham Lincoln School of Medicine, Univ. of Ill. Hosp., Depts. of Ped. and Derm., Chicago, I11.

Resolution of Vitamin $D$ resistant rickets following removal of several types of tumors has been previously reported. A 12 year old male was found to have severe progressive Vit.D. resistant rickets with maxked hypophosphatemia in association with hyperpigmented, linear, verrucous, epidermal tumors typical of E.N.S. Serum calcium was $9.4 \mathrm{mg} \%$, phosphorus (P) $2.2 \mathrm{mg} \%$, alkaline phosphatase 313 I.U. Fractional excretion of phosphorus (FEp) was $65 \%$ Serum parathyroid hormone (P.T.H.) was normal. Marked loss of bone density and signs of rickets were observed on roentgenograms. Following removal of several nevi, Vit.D. and phosphate supplements, previously ineffective, resulted in an increase in serum $\mathrm{P}$, a decrease in FEp to $28 \%$, improvement in symptoms and radiological evidence of healing. A portion of excised tissue was homogenized and the supernatant injected into a puppy. Two hours after injection a marked phosphaturia was observed with an increase in FEp from $43 \%$ to $100 \%$. No changes in hematocrit, glomerular filtration rate and fractional excretion of $\mathrm{Na}$ were noted. It is postulated that phosphaturic substances elaborated by some of the nevi were responsible for the rickets in this case and that secretion of related compounds are responsible for resistant rickets and osteomalacia associated with other tumors. 
HIGH-DOSE STEROIDS FOR INTERSTITIAL PHEULONITIS IN

909

PATIENTS RECEIVIUS CANCER CHEMOTHERAPY Jerry L. Barbosa, Nancy B. Mcllilli iams and Harold Il. Maurer.

Medical College of Virginia, Uepartment of Pediatrics, Richmond, Va High-dose steroids (methylprednisolone, $2 \mathrm{mg} / \mathrm{Kg} \mathrm{I.V.,} 9 \mathrm{~h}$ ) produced marked clinical improvement within 12 hours in 6 episodes of severe interstitial pneumonitis occurring in 5 children with leukemia or lymphoma.

Nonproductive cough and progressive dyspnea with temperatures ranging from $102^{\circ}$ to $106^{\circ} \mathrm{F}$ were present in all cases. Severe hypoxemia ( $\mathrm{pO}_{2}$ ranging from 28 to $51 \mathrm{mmll} / \mathrm{g}$ ) without hypercapnia was present. Absolute neutrophil counts ranged from 750 to $3,000 /$ $\mathrm{mm}^{3}$. Lymphopenia $\left(<1,000 / \mathrm{mm}^{3}\right)$ was present in 2 patients. Oniy 2 of the children were receiving methotrexate and another 2, prednisone in standard doses when symptoms developed

Radiologic exarnination disclosed a diffuse interstitial pattern bilaterally in all cases. Lung biopsy in 3 patients showed interstitial infiltration with mononuclear cells, intra-alveciar fibrin and increased interstitial fibrous tissue. Bacterial, viral, fungal and protozoan studies were negative and broad spectrum antibiotics were without effect.

High-dose steroid therapy was tapered after 3 to 5 days and discontinued at 7 to 10 days with no recurrence of symptoms. We conclude that in children with severe interstitial pneumonitis, where bacterial, fungal and protozoan causes have been excluded, high-dose steroids can produce a prompt and dramatic clinical response.

910 CHOLEDOCHAL CXST: REVIEW OF NINETEEN CASES. Barbara Barlow, Edward Tabor, William A. Blanc, Thomas V. Santulii, Ruth C. Harris. Columbia Univ. Col. of Physicians \& Surgeons, Depts. Pediatrics, Surgery and Pathology, New York, N.Y.

A 25 year experience with choledochal cyst identified 2 distinct groups of patients: 8 infants under one year initially diagnosed as biliary atresia, with associated atresias, severe cirrhosis, portal hypertension, and high mortality, and 11 older patients with mild cirrhosis and associated choledochocystlithiasis and pancreatitis. The review suggests that the infants had a congenital form and the children an acquired form of common duct dilatation, perhaps related to a common channel with reflux of pancreatic juice into the common bile duct.

The classic symptom triad occurred in $18 \%$ of the older patients and in none of the infants. Serial $I^{131}$ rose bengal scan was the most accurate diagnostic test. Follow up from 6 months to 25 years showed a high incidence of late post-op complications: $80 \%$ for choledochocyst-duodenostomy, $57 \%$ for choledochocyst-jejunostomy, $20 \%$ for choledochocyst-jejunostomy with cholecystectomy. The review encourages a high index of suspicion since the symptoms are intermittent, non specific and delayed diagnosis is common. The importance of long term follow up is emphasized because of the frequency of late complications such as cholangitis, stomal stenosis, pancreatitis, and choledochocystlithiasis.

CHANGING ERYTHROCYTE CLONES IN JUVENILE CHRONIC MYELOID LEUKEMIA. George J. Dover, Samuel H. Boyer, Dept. of Pediatrics, Baltimore.

Clones of erythrocytes in a 3 year old, Black female with juvenile chronic myeloid leukemia (JCML) were defined by membrane characteristics, $\mathrm{F}$-cell and A-cell reactions, Orskov-JacobsStewart hemolysis, and enzyme activities. The characteristics of the clones identified over 8 months are summarized below.

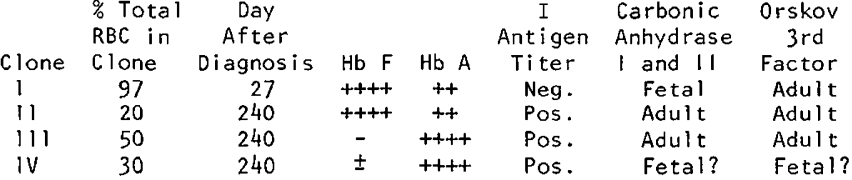
Whole blood erythrocyte G6PD, 6PGD, hexokinase, pyruvate kinase, and lactate dehydrogenase were fetal like on Day 27 and Day 240; Hb $F$ was $68.5 \%$ on Day $27,36.1 \%$ on Day 221 , and $16.7 \%$ on Day 240 ; $\mathrm{Hb} \mathrm{A}_{2}$ was always less than $1.5 \%$. These data indicate: (1) different clones of erythrocytes appear during the course of JCML, (2) individual clones may exhibit inappropriate fetal and adult characteristics, (3) progression to predominance of fetal erythropoesis as described in previous patients with JCML is not a characteristic of this patient. Reasons for the emergence of different clones of RBC may include effects of chemotheraputic agents and/or etiologic factors responsible for malignant transformation.
912 CENTRAL NERVOUS SYSTEM TUMORS IN CHILDREN Jacqueline R. Farwell and George J. Dohrmann (spons. by Howard A. Pearson), Yale University School of Mearcine, Dept. of Pediatrics and Sections of Neurosurgery and Neuropathology, New Haven, Connecticut.

of 488 central nervous system neoplasms occurring in children over a 39 year period, 467 were intracranial and 21 were intraspinal. The most common intracranial neoplasms were astrocytoma $(28 \%)$, medulloblastoma (25\%), craniopharyngioma $(9 \%)$, glioblastoma multiforme (9\%), and ependymoma (6\%). Of the intracranial tumors, $24 \%$ were located in the cerebral hemispheres, $15 \%$ in the brain stem, $47 \%$ in the cerebellum, and $14 \%$ in the sella-chiasm region. The average age at diagnosis was 7.7 years, with a peak in occurrence at age three. Male to female ratio was $1.3: 1$, the male predominance being found in nearly all histopathological types. Overall mean survival was 53.4 months and varied greatly relative to the type of tumor, ranging from 10.8 months in glioblastoma multiforme to 135.6 months in optic glioma. Survival also differed with location: mean survival was 45.3 months in the cerebral hemispheres, 13.7 months in the brain stem, 63.9 months in the cerebellum, and 71.3 months in the sella-chiasm region. of the intraspinal neoplasms, the most frequently noted were astrocytoma (47\%) and ependymoma (24\%). The mean age at diagnosis was 10.2 years with a male to female ratio of $1: 1$. The average survival from diagnosis ( 54.1 months) was comparable to that of intracranial neoplasms. Detailed analysis of each histopathological type of tumor relative to age at diagnosis, sex, anatomical location and survival from diagnosis are reported.
ECHOCARDIOGRAPHIC ASSESSMENT OF LEFT VENTRICULAR 913 FINCTION IN CHILDREN RECEIVING ADRIAMYCIN (ADR) OR DAUNORUBICIN (DNR). Angela C. Gilladoga, Charlotte T. C. Tan, M. Lois Murphy and Howard Thaler. Memorial SloanKettering Cancer Center. Department of Pediatrics. New York City.

Studies were done on 146 children, ages $2-17$, to determine whether cardiotoxicity can be detected early in patients (pts) receiving ADR or DNR, two effective antitumor antibiotics with total dose now 1 imited to $50 \mathrm{ng} / \mathrm{m}^{2}$ because of cardiomyopathy (CMY) which manifests late and often only when severe. The

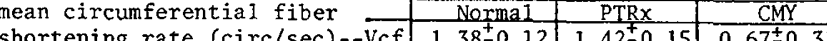
\begin{tabular}{l|r|r|r} 
shortening rate (circ/sec) $--V c f$ & $1.38-0.12$ & $1.42-0.15$ & $0.67 \pm 0.31$ \\
fractional shortening (\%) --FS & $36.5 \pm 3.2$ & $35.7 \pm 3.1$ & $16.8 \pm 5.8$
\end{tabular} \begin{tabular}{ll|l|l|l|l} 
fractional shortening $(\%)$ & $-\mathrm{FS}$ & 36.5 & \pm 3.2 & $35.7 \pm 3.1$ & $16.8 \pm 5.8$ \\
and ejection fraction $(\%)$ & $-\mathrm{EF}$ & $74.6 \pm 3.9$ & $73.4 \pm 3.5$ & $41.4 \pm 12.4$ \\
\hline
\end{tabular} from simultaneously recorded echocardiogram, electrocardiogram, phonocardiogram and carotid pulse showed no significant difference between 15 normal children and 20 DNR 615-1275 $12 / 22$ 55\% pts prior to treatment (PTRx) compared to decreased indices in 5 pts with heart failure (HF) from ADR or DNR CMY of 106 pts without $\mathrm{HF}, 16$ with incidental cardiac radiation (CRT), $39(37 \%)$ had 2-3 indices below 2SD. Results grouped according to total dose received $\left(\mathrm{mg} / \mathrm{m}^{2}\right)$ are shown. It appears that early cardiotoxicity can be detected by echocardiography and should be useful for rollow-up of pts to individualize dose limitation.

Supported by NCI Grant CA-05826 \& J.H. Selig Mem. Fund, Inc.

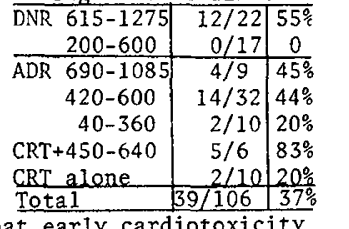


915 NEUROBLASTOMA IN INFANTS UNDER A YEAR OF AGE: THE VALUE OF THERAPY. Alexander A. Green, F. Ann Hayes, St. Jude Children's Research Hospital, Divisions of Hematology-Oncology and Immunology, Memphis, TN. Sponsored by Joseph Simone.

Thirty-seven infants with neuroblastoma diagnosed during the first year of life were treated according to discase stage, as follows: surgery only - 3 patients with stage I disease; surgery + irradiation - 5 stage llA patients: surgery + irradiation + cyclophosphamide (Cyclo) and vincristine (VCR) -6 stage IIB patients. All but one of these infants with localized disease survive for 1 to 12 years (median 5 years) with no evidence of disease.

Twenty-three patients had disseminated (or stage III) neuroblastoma, 18 with bone marrow involvement. In addition to limited surgery and occasional irradiation, therapy for this group consisted of one of the following drug combinations: (i) Cyclo + VCR, (ii) Cyclo + VCR + Adriamycin, or (iii) Cyclo + Adriamycin. Complete responses to treatment were obtained in 13 of these infants and a partial response in 1 . Nine patients had no response. Of the complete responders, 2 have developed recurrent disease, 1 of whom has died. Nine of the 10 patients without a complete response have died of progressive disease. Thus, 14 of 23 patients $(60 \%)$ are still alive for 4 months to 12.5 years (median 4.5 years). Only 1 infant in this group has evidence of disease. This analysis demonstrates that among infants with disseminated disease survival correlates with response to therapy. It was not possible to substantiate the popular notion that infants with IVS (Evans') neuroblastoma have a better prognosis than others. We conclude that extent of disease is the only criteria for selecting a subgroup of infants with good prognosis, and therefore that all infants with neuroblastoma should receive a course of therapy appropriate to their disease stage.

16 TERMINAL DEOXYNUCLEOTIDYL TRANSFERASE (TdT) IN CHILD-

916 HOOD MAI IGNANCY. Martha F. Greenwood, Mary S. Coleman, John J. Hutton, Beatrice C. Lampkin, Fred J. Bollum, and Phillip Holland, Univ. of Ky. Med. Sch., Depts. of Ped., Med., Biochem., Lexington and Children's Hosp., Cincinnati.

TdT, a DNA polymerase, is found in high concentration in thymus, low concentration in normal BM and is absent in all other normal tissues. TdT was examined in PB and BM cells in acute $1 \mathrm{ym}-$ phoblastic leukemia (ALL), non-Hodgkin's lymphoma with leukemic transformation, rhabdomyosarcoma and neuroblastoma with BM metastasis, ITP and aplastic anemia (AA). Results are:

\begin{tabular}{lccccc}
\hline Diagnosis & PB blasts $\%$ & PB TdT & BM blasts & BM TdT \\
\hline ALL-diagnosis(13) & $14-94$ & $47 \pm 26$ & $76-97$ & $57 \pm 44$ \\
ALL-remission(24) & 0 & $.60 \pm .42$ & $0-5$ & $2.90 \pm 2.19$ \\
ALL-relapse(7) & $0-32$ & $36 \pm 50$ & $67-98$ & $256 \pm 314$ \\
Non-Hodgkin's lymphoma (3) & $7-97$ & .76 & $76-97$ & 4.47 \\
Neuroblastoma(2) & 0 & 2.20 & present & .74 \\
Rhabdomyosarcoma (2) & 0 & .87 & present & 4.76 \\
ITP(7) & 0 & $.44 \pm .08$ & 0 & $5.17 \pm 3.13$ \\
Aplastic anemia (5) & 0 & $\mathrm{ND}$ & 0 & $2.36 \pm .91$ \\
Controls(12) & 0 & $.29 \pm .57$ & 0 & $3.26 \pm 1.14$ \\
\hline
\end{tabular}

TdT activity (units $/ 10^{8} \mathrm{cel1s}$ ) was markedly elevated in all ALI patients at diagnosis and relapse while remission TdT levels were within the control range. TdT assays in leukemic transformation of non-Hodgkin's Iymphoma, metastatic neuroblastoma and rhabdomyosarcoma, ITP and AA were within the control range. Elevated TdT levels appear diagnostic of disease activity in ALL and are of value in distinguishing ALL from other BM malignancies and conditions producing cytopenias and marrow hypoplasia.

\section{7} ELEVATED SERUM IMMUNOREACTIVE CALCITONIN (iCT) IN LEUKEMIA-LYMPHOMA. Nasrollah Hakami, Alan D. Mease, and Constantine S. Anast, Univ. of Mo. Schoot of Medicine, Dept. of Child Health, VA Hospital, Columbia, Mo. and Walter Reed Army Hospital, Washington, D.C.

Calcitonin (CT), a hypocalcemic polypeptide hormone produced by thyroid $\mathrm{C}$-cells is typically elevated in medullary carcinoma of the thyroid, a C-cell tumor. In addition, elevated levels of serum ICT have been reported in other malignancies, but not in acute lymphocytic leukemia (ALL) or lymphoma. We have studied three patients, two with ALL and one with Burkitt's lymphoma with elevated levels of serum iCT.

Patient (Age/Sex(yrs) Diagnosis $\frac{\mathrm{Ca}(\mathrm{mg} / \mathrm{d} \mathrm{l})}{7 \mathrm{P}(\mathrm{mg} / \mathrm{d} \mathrm{l})} \frac{\mathrm{iCT}(\mathrm{pg} / \mathrm{ml})}{3.0}$

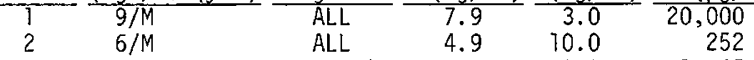

$\begin{array}{lllll}3 & 7 / M \quad \text { Burkitt's } 7.0 & 3.3 & 1,045\end{array}$

(iCT in normal and 6 children with ALL in remission $<150 \mathrm{pg} / \mathrm{ml}$ )

The chromatographic elution pattern of serum iCT in patient

(1) was undistinguishable from ICT of patients with medullary carcinoma of thyroid. There was no clinical evidence or family history of medullary carcinoma of thyroid in these patients. In addition, post mortem tissue examination by light and electron microscopy in patient (1) failed to show any C-cell tumor of thyroid. Although impaired metabolism of CT or increased secretion by thyroid C-cells (possibly induced by chemotherapy) need to be excluded as the cause of elevated serum iCT, these observations suggest that iCT can be ectopically produced by leukemia or lymphoma cells. For this reason serial assay of iCT may be potentially useful in detection of residual malignancies.
INCREASED NUMBERS OF MITOTIC CELLS IN NON-STIMULATED BLOOD CULTURES OF CHILDREN WITH ACUTE LEUKEMIA (AL). James R. Humbert, Helvise G. Morse, John J. Hutter, Arthur Robinson (Spon. by Charles s. August). Univ. Colo. Med. Ctr., Depts. of Biophysics/Genetics and Pediatrics, Denver Children's Hosp., Hematology/Oncology Ctr., and National Jewish Hosp., Denver.

High numbers of spontaneous mitoses (SM) are often present in blood cultures of leukemic patients. These dividing cells have been assumed to be leukemic in origin, as they often carry cytogenetic aberrations identical to those of simultaneous bone marrow (BM) cultures during relapse. We investigated SM in unstimulated blood cultures of 46 children with AL (39 lymphoblastic, 7 granulocytic). The number of SM was determined in 24hr. blood cultures without PHA, and reported as mitoses per $5 \times 10^{5}$ leukocytes. BM cell chromosomes were simultaneously evaluated. Of 21 remission (REM) patients, 14 long-term (LT) patients were off therapy from 4-20 months. Twenty-five children were in relapse (REL). Low numbers of SM were observed in 15 non-leukemic controls $(\overline{\mathrm{X}}+\mathrm{SEM}: 0.7+0.4$; range: $0-3)$. Results in REL and REM patients were respectively $44.9 \pm 10.9$ and $14.8 \pm 4.0$ and differed significantly $(p<0.01)$ from control values and from themselves. Three REL patients and 7 REM patients (including $6 \mathrm{LT}$ ) had normal numbers of SM. In 5 REL cases who displayed chromosomal abberations in $57 \pm 11.5 \%$ of their mitoses, the abnormality was present only in $22.6+9.8 \%$ of their blood SM $(p<0.05)$; most SM were thus of normal karyotype in these cases. Our data indicate that most dividing cells present in the blood of leukemic childxen during REI or REM may be non-leukemic in origin.

MULTIPLE CASES OF LYMPHOEPITHELIOMA AND BURKITT'S

919 LYMPHOMa in a CaNADIAN Family. I. HLA typing, ebV ANTIBODIES AND SERUM IMMUNOGLOBULINS

Jean H. Joncas, Ernest Rioux, Jean-Pierre Wastiaux, Michele Leyritz, Louise Robillard and José Menezes (Sponsored by R. Collu) Axmand Frappier Institute, Montreal; Ste Justine Hospital, Montrea1; University of Montreal, Montreal; Enfant Jesus Hospital, Québec, Canada.

Two nasopharyngeal carcinomas of the lymphoepithelioma type and two Burkitt's 1ymphomas with the characteristic histopathologic features have been observed in three young siblings and one first degree cousin from a large french Canadian family. The Epstein-Barr virus antibody titers available in the two lymphoepithelioma but not in the Burkitt lymphomas were, as expected, markedly elevated especially in one of the two. HL-A typing of the family members failed to disclose HL-A 2 and $\mathrm{HL}-\mathrm{A}$ Sin 2 previously associated with lymphoepithelioma in Asia. The occurrence, however, of a plasmacytoma in 1 other first degree cousin, and of low serum IgA in several siblings and cousins, suggest the possibility of a predisposing $B$ cell dysfunction in the development of these tumors in the family under study. Peripheral lymphocytes from all surviving members of this family have been put in culture and their throat washings put on cord blood lymphocytes (CBL) in an attempt to document defective intracellular control of the persistent EBV genome which could manifest itself by rapid transformation of their peripheral 1ymphocytes in vitro into permanent cell lines and excretion of the CBL transforming agent (presumable EBV) in their saliva.

NULL CELL LYMPHOBLASTIC LEUKEMTA IS A MALIGNANCY OF IMMATURE B LYMPHOCYTES. Joseph Kaplan and Ward D. Peterson, Jr. Wayne State University Medical School,Children's Hospital of Michigan, Department of Pediatrics, Detroit.

We have compared rabbit antisera to human $B$ and $T$ lymphoblast cell lines for reactivity to normal $T$ and $B$ cells and to leukemic lymphoblasts from patients with $T$ cell leukemia (those with Erosette positive lymphoblasts and/or thymic enlargement) and Nul1 cell leukemia (patients without thymic enlargement whose 1 ymphoblasts are E-rosette negative and lack surface immunoglobulin and complement receptors). By complement-dependent cytotoxicity and absorption of cytotoxicity antiserum to B cell line CCRF-SB absorbed with autocthonous $\mathrm{T}$ cell line $\mathrm{HSB}-2$ reacts with some normal B lymphocytes and with lymphoblasts from patients with Null cell leukemia but did not react with normal $\mathrm{T}$ cells or lymphoblasts from $T$ cell leukemia. Conversely, antisera to $T$ cell line HSB-2 absorbed with B cell line CCRF-SB reacted with some normal $T$ cells and with $T$ cell leukemia lymphoblasts but did not react with $B$ cells or 1ymphoblasts from Null cell leukemias. These findings indicate that Null cell lymphoblastic leukemia, the most common form of childhood leukemia, is a malignancy of immature B lymphocytes. 
921 RESPONSE OF CHILDHOOD GLIOMA TO METHOTREXATE-CITROVORUM RESCUE. Jung S。 Kim, Isaac Djerassi, Kenneth Shu1man ${ }^{*}$, and Soon JoChoi , Mexcy Catholic Medical Center, Department of Research Hematology, Darby, Pennsylvania,

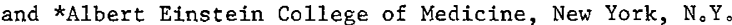

Four children with brain glioma were treated with High Dose Methotrexate and Citrovorum Factor. Neurological improvement was noted in all four. Methotrexate $(0.7$ to $3.25 \mathrm{umg} / \mathrm{ml})$ was found in their spinal fluid following $I . V$ o treatment. A patient with Astrocytoma Grade III, recurring following surgery, radiation and other chemotherapy was given intracarotid infusions of 5 to $70 \mathrm{mg} / \mathrm{kg}$ of MTX in 2 to 4 hours. Marked neurological improvement was observed. Following 1 year of periodic I.v. infusions of 100 $\mathrm{mg} / \mathrm{kg}$ of MTX almost complete neurological recovery was accompanied by normalization of the angiogram and brain scan. This patient is alive and well 8 years later without further treatment. Three other children with advanced pontine (2 patients) and third ventricle glioma (1 patient) who had relapsed after radiation and other chemotherapy were treated with 50 to $1000 \mathrm{mg} / \mathrm{kg}$ of MTX I.V. and Citrovorum Rescue at intervals of 3 to 4 weeks. All 3 responded initially with marked but temporary neurological improvement. Subsequent symptomatic responses were observed in 2 of the 3 patients with either higher doses of MTX or prolonged infusions of the same amount of drug.These 2 patients expired 4 to 5 months following discontinuation of treatment.

Supported in part by NIIl Grant-11630-05.

922 QUALITY OF SURVIVAL IN HISTIOCYTOSIS X. D. Komp, Charlottesville, A. El-Mahdi*, Charlottesville, K. Starling, Houston, J. Easley*, Houston, T. Vietti, St. Louis, D. Berry*, Little Rock and S. George*, Houston for the Southwest Oncology Group-Pediatric Division.

Forty-six patients with histiocytosis $\mathrm{X}$ survived for more than five years after diagnosis. Three of the $39 / 46$ with generalized disease subsequent1y died from pulmonary involvement. Two of 21 who received radiotherapy developed "secondary" malignancies in irradiated sites (1 thyroid CA; 1 osteosarcoma).

A single course of chemotherapy was adequate to arrest disease in $3 / 8$ long-term survivors with visceral involvement and $8 / 31$ with bone + visceral involvement. The length of active disease ranged from 3 months to $149 / 12+$ years with a median of $46 / 12+$ years. of the 29 survivors with bone + visceral involvement, $20(69 \%)$ survive with significant disabilities other than diabetes insipidus: growth retardation-8, neurologic disease-4, orthopedic disease-4, intellectual impairment-4, emotional disorders-4, chronic lung disease-5, hearing impairment-2, miscellaneous-4. None of the 7 long-term survivors with visceral disease had any residual disabilities recognized.

Al though bone + visceral involvement was fatal in only $11 / 63$ (16\%) of patients followed for longer than six months from diagnosis, single agent chemotherapy and radiotherapy have not impressively shortened the chronicity of the disease or prevented substantial disability in survivors. Future therapeutic trials should attempt more rapid disease control to prevent disability but weigh the possibility of therapy-related complications such as "secondary" malignancies.

RENAL TOXICITY OF HIGH-DOSE MFTHOTREXATE. Link, D.A.I,

923 Fosburq, M.T. Ingelfinger, J.R., Tobias, J.S., Jaffe, N., Sponsored by W.E. Grupe, Children's Hospital

Medical Center, Boston, Massachusetts

Methotrexate (MTX) is primarily excreted by the kidneys and is itself nephrotoxic. Compromise in renal function can prolong serum MTX half-life and contribute to systemic toxicity. We prospectively studied renal function and high-dose MTX elimination in 8 patients using pre- and post-treatment inulin (CIn) and creatinine (CCr) clearances, serial urine and serum MTX levels and daily serum creatinine determinations. None of the 8 patients developed clinical or laboratory evidence of systemic MTX toxicity. Three additional patients, with established systemic toxicity, were retrospectively studied.

In $7 / 8$ non-toxic patients, CIn fell an average of 518 (17-758) and $\mathrm{CCr} 428(4-808)$ from pre- to 40 hours post-treatment. Their serum creatinine did not rise more than $0.05 \mathrm{mgz}$. Serum MTX levels in the non-toxic group were comparable, with immediate post in-
fusion values of $1 \times 10^{-3}$ Molar and a mean decline of $1.3 \times 10^{-1} \mathrm{M}$ per $24 \mathrm{hrs}$. Two of 3 toxic patients exhibited markedly abnormal CIn $(10 \& 23 \mathrm{ml} / \mathrm{min})$ and $\mathrm{CCr}$, with sianificant elevations of serum creatinine. The third patient had minor changes in renal function The MTX levels in all 3 toxic patients remained elevated for 1-3 weeks with full recovery of renal function within 6 weeks. The renal defect in 8 non-toxic patients had no effect on the plasma disappearance of MTX. The data sugqest that high-dose MTX therapy regularly produces a reversible decline in GFR; the local renal toxicity, if sufficiently severe, may contribute to development of systemic toxicity.
CHILDHOOD CANCER (CC) IN SIBLINGS, Anna T. Meadows, 924 Frederick P. Li, Louise C. Strong, Odile Schweisguth, and Edward S. Baum (Spon. by Warren Nichols), Children's Hospital of Philadelphia; Sidney Farber Cancer Center, Boston; M.D. Anderson Hospital, Houston; Institut Gustave-Roussy, Villejuif, France; Children's Memorial Hospital, Chicago.

It has been suggested that some CC occur in hereditary and nonhereditary forms. The former is often associated with multiple primaries of the same or different sites among relatives and within individuals. Examination of familial aggregates provides opportunities to study tumors with common etiologies.

85 families (fams) with $190 \mathrm{CC}$ in 179 children $\mathbf{2} 16$ yrs were studied. In the total sample soft tissue sarcomas (STS), Wilms tumors, retinoblastoma (RB), ana adrenocortical carcinomas were excessive based on expected proportions in the CC population. Concordance for tumor type, observed in $7 / 8$ multiple case fams and $41 / 77$ pairs including 2 sets of monozygous and 1 set of dizygous twins, was excessive for all types except leukemia (AL) and Hodgkin disease (HD) compared with expected proportions. (5 children with HD were omitted because 1 or both sibs were $>16$ yrs.) Frequent combinations included AL with neuroblastoma and brain tumors (BT) with STS, bone tumors, AL/lymphoma, and RB. Predisposing genetic conditions included neurofibromatosis in 2 pairs with $B T$ and xeroderma pigmentosum in 1 pair with multiple primaries. Second malignant neoplasms (SMN) were noted in 11 individuals in 8 fams.

These data suggest a non-random distribution of $\mathrm{CC}$ among sibs which may be due to tissue specific predispositions. SMN may occur excessively in survivors of hereditary neoplasms.

\section{NEUROBlastoma: a CLUE FOR LABORATORY RESEARCH FROM}

925 GEOGRAPHIC VARIATIONS IN FREQUENCY. Robert W. Miller. Clinical Epidemiology Branch, NCl, Bethesda, Maryland. Under the auspices of the International Union Against Cancer (UICC), a worldwide comparison was made of childhood neoplasia according to cell type. More than 40 pediatric centers or tumor registries contributed data on about 40,000 childhood cancers. One result of particular interest is the near absence of neuroblastoma in parts of the African lymphoma belt, and a low frequency of neuroblastoma in other parts of central Africa, India, Pakistan, and in data from the excellent Puerto Rico Tumor Registry. The deficiencies of cases may have been due to some extent but not entirely to underascertainment. There were no corresponding deficiencies in the occurrence of Wilms' tumor. The areas with low frequencies of neuroblastoma have high frequencies of circulating parasites. This infestation markedly raises IgG levels, which when passed from mother to fetus may conceivably act as a transplacental anti-carcinogen and inhibit tumor development or induce maturation to normal tissue. Neuroblastoma is known for its capacity on occasion to regress spontaneously, and for its high frequency of in situ (microscopic) lesions soon after birth, which disappear spontaneously by 3 months of age. The results of the international comparison, admittedly crude, suggest that neuroblastoma cells or their products should be studied in vitro for their reactivity against high-titre antiparasitic sera.

\section{6} CHROMOSOME ABNORMALITIES IN ACUTE LEUKEMIA OF August), Universittex, Arthur Robinson (Spon. by Charles $\mathrm{S}$. Biophysics/Genetics of Colorado Medical Center, Departments of Hematology/Oncology Center, and National Jewish Hospital, Denver.

Chromosome abnormalities in bone marrow (BM) and blood cells of 46 children with acute leukemia (AL) were evaluated with a Giemsa banding technique. Twenty-five patients were in relapse at the time of study and 21 in remission, including 14 long-term (LT) patients off therapy from 4 to 20 months. Fourteen relapse patients (56\%) displayed chromosome abnormalities in leukemic cells. Hyperdiploidy was predominant in acute lymphoblastic leukemia (ALL) and consisted of (a) trisomies involving chromosomes 4 to 21 , (b) increased tetraploidy, and (c) abnormalities of lq. Hypodiploidy was observed only in acute granulocytic leukemia and involved a missing $Y$ in 1 case. Three of $20 \mathrm{com}-$ plete remission cases showed discrete aberrations in their cells (increased tetraploidy, isolated trisomic cells). Another patient studied during hematological remission showed a clone of hyperdiploid cells in his BM and developed relapse 6 weeks later. All 14 Lr patients had normal BM karyotypes except 1 who showed increased tetraploidy. Our data suggest that (1) hyperdiploidy in groups B to $G$ exists in leukemic cells of over $50 \%$ of children with ALL, (2) the significance of increased tetraploidy in AL is open to question, and (3) in patients with apparent hematological remission, the absence of BM cell chromosome aberrations may indicate adequately treated leukemia while their presence may signify the onset of relapse. 


\section{7}

CUTANEOUS ASPERGILLUS INFECTION IN COMPROMISED HOSTS - CLINICAL AND EPIDEMIOLOGIC FEATURES. E. Richard Moxon, Bert Vogelstein, Richard L. Riley, William G. Merz, Margaret L. Dobson, Mardel Knight. Johns Hopkins Univ. Sch. of Med., Departments of Pediatrics, Microbiology, Laboratory Medicine, and Environmental Medicine., Baltimore. (Spon. by William H. Zinkham).

In $1974-75,3$ children ( 2 with aplastic anemia, 1 with leukemia) developed localized cutaneous infections caused by Aspergillus flavus. Lesions began as erythematous papules at sites covprogressively invasive with tissue necrosis. All subsequently died. Disseminated aspergillosis was later confirmed in 2 (at autopsy) and clinically suspected in the other (autopsy denied).

The unusual nature of these infections resulted in studies to define its epidemiology. During $174-175$ a total of 204 Aspergillus isolates from 154 patients were A. flavus $24.6 \%$, niger $21.1 \%$, fumigatus 14.88, other species $40.5 \%$. Samples of air, using an Anderson air-sampler, revealed that $A$. flavus was the most common fungal species in $2 / 9$ patient caxe areas. The particle size in all areas was predominantly $<5 \mu$. A. flavus was cultured from adhesive tape in one of the treatment rooms. The outside air and the tape may be 2 unrelated but potential sources of A. flavus. Possible modes of infection with Aspergillus include: air to skin (with lung as portal of entry), air direct to skin, and adhesive tape to skin. The last possibility may be the major route of transmission in the cutaneous form of the disease.

\section{0} ECHOCARDIOGRAPHIC EVALUATION OF ADRIAMYCIN CARDIOMYOPATHY IN CHILDREN Agustin Ramos, Richard A. Meyer, Joan Korfhagen, Kwan Yuen Wong, Samuel Kaplan, University of Cincinnati, Chitdren's Hospital, Dept. of Pediatrics, Cincinnati, Ohio

A severe dose dependent cardiomyopathy is the major limiting factor in the prolonged use of Adriamycin. It may occur in 30\% of patients receiving a total cummulative dose of $>550 \mathrm{mg} / \mathrm{M}^{2}$. Once congestive heart failure appears, there is a mortality of $50 \%$. The drug is usually discontinued when this level is reached. Early changes in cardiac performance in the asymptomatic patient are generally unrecognized. We evaluated left ventricular function by serial echocardiography in 28 children receiving Adriamycin ( $50 \mathrm{mg} / \mathrm{M}^{2} /$ dose). Changes in the mean $\mathrm{VCF}$, systolic time intervals and ejection fraction showed some correlation with increasing dose. However, shortening fraction appeared to be the best parameter: Before the onset of therapy, only $10 \%(2 / 20)$ of the patients had an abnormally low shortening fraction (<28\%), whereas $75 \%(9 / 12)$ of those who received $300 \mathrm{mg} / \mathrm{M} 2$ had a SF $<28 \%$. The mean SF progressively decreased from a baseline value of $34.5 \%$ to $32.5 \%$ after $100 \mathrm{mg} / \mathrm{M}^{2}$, to $30.9 \%$ after $200 \mathrm{mg} / \mathrm{M}^{2}$ and to $27.0 \%$ after $300 \mathrm{mg} / \mathrm{M}^{2}$. Using the patients as their own controls, serial echocardiography appears to be a reliable method for detecting early alteration of cardiac function in the asymptomatic patient. By stopping the drug at an appropriate time, it may be possible to prevent cardiomyopathy. In addition, it may be possible to prolong therapy beyond the established limit in the presence of adequate cardiac function.
928 CHILDHOOD OSTEOSARCOMA, LIMB ANOMALIES, AND ERYTHROID MACROCYTOSIS IN AN AMERICAN INDIAN FAMILY. JOhn J. Mulvihill, Harvey R. Gralnick, Jacqueline Whanq-Peng, and Brigid 5. Leventhal. Clinical Epidemiology, Medicine, and Pediatric Oncology Branches, National Cancer Institute; and Hematology Service, Clinical Center, National institutes of Health, Bethesda, Maryland.

Three of 9 children born to possibly consanguineous American Indian parents developed typical osteosarcoma in a 2-year period. Etiology investigations detected, in the surviving tumor patient, several of her sibs, and her father (but not mother), limb anomalies (LA) and elevated mean corpuscular volumes $\left(98-109 \mu^{3}\right)$ LA ranged from simple clinodactyly with brachmesophalangy to absence of one digital ray of the foot and bilateral radioulnar synostosis. The red cell macrocytosis ( $M$ ) was not accompanied by anemia or explained by the usual causes of $M$. One sister with LA and $M$ had a breast fibroadenoma. No unusual environmental exposures were found and screening for possible oncogenic viruses by culture, electron microscopy and serology was negative. All family members had elevated antibody titers to Epstein-Barr viral antigens. Except for cutaneous anergy in one boy, immune function was intact. The father had excessive chromosomal breaks in the bone marrow and his leukocytes, like the proband's, failed to grow on 2 occasions. This unusual familial pattern of osseous malignancy and malformation and defective erythropoiesis, tentatively called OSLAM syndrome, may represent impaired regulation of bone development.

929 DETECTION OF SARCOMA VIRUS SPECIFIC RNA TRANSCRIPTION IN CELLS TRANSFORMED BY USUALLY NONTRANSFORMTNG RNA LEUKEMIA TYPE $C$ VIRUSES: A MODEL FOR LEUKEMOGENESIS by Paul T. Peebles, Edward M. Scolnick, Richard S. Howk (Spon. by Dr. Michael R. Blaese). National Cancer Institute, Bethesda, Md. An important unanswered question of tumor virology is how do leukemia type $C$ viruses induce leukemia in vivo while in infected tissue cultures they cause no transformation with loss of contact inhibition control of cell division. Mink lung cells nonproductively infected with a defective murine sarcoma virus (MSV) were isolated ( $\mathrm{S}+\mathrm{L}$ - mink cells). They were morphologically flat like uninfected cells and exhibited contact inhibition. Superinfection of S+L- mink cells with normally nontransforming feline leukemia type viruses caused morphological transformation and loss of contact inhibition. Using as a probe MSV specific DNA sequences obtained by reverse transcription of the MSV RNA genome, no sarcoma virus specific transcription was demonstrated in S+L- mink cell RNA. Leukemia type $C$ virus superinfection of $\mathrm{S}+\mathrm{L}-$ mink cells induced detectable sarcoma virus specific RNA transcription. The usually non-transforming leukemia type $C$ viruses may in like manner cause leukemia in animals by inducing detectable RNA transcription of normally repressed or nonfunctioning cell control or integrated viral transforming genes.
CENTRAL NERVOUS SYSTEM DISEASE IN CHILDREN WITH

Q31 HODGKIN'S DISEASE. Narayan R. Shah, James A. Wolff, Harold C. Neu, Babies Hospital, New York, N.Y.

Central nervous system symptoms occurring in children with Hodgkin's disease (HD) can be the result of CNS involvement or due to infection. In the past 5 years we have had 3 children with HD who developed CNS complications which illustrate the problems of diagnosis and treatment: A male diagnosed at age 11 as stage II, lymphocyte predominant $H D$ received radiotherapy and multiple agent chemotherapy. He was admitted 8 years later with fever, headache and emesis. An LP showed 131 WBC, 61\% 1ymphs, protein $91 \mathrm{mgll}$, glucose $42 \mathrm{mg} / \mathrm{dL}$. The patient died and autopsy showed extensive CNS toxoplasmosis. A second patient was admitted for severe headaches and emesis at age 13. Diagnosis of stage IVB, lymphocyte predominant HD had been made 19 months earlier. The patient's CSF grew cryptococci and treatment was initiated with amphotericin and $5 \mathrm{FC}$. Although the CSF cleared of organisms, cryptococcal antigen could be detected and 7 months later the patient developed increased intracranial pressure, blindness and died with cryptococci. The third patient a $41 / 2$ year old male, stage 11B, lymphocyte depletion HD developed fever emesis and lethargy. Physical exam revealed a VI cranial nerve palsy. The LP showed $57 \mathrm{WBC}, 96 \%$ lymphs, protein $390 \mathrm{mg} / \mathrm{dL}$ and glucose $5 \mathrm{mg} / \mathrm{di}$. Millipore examination revealed neoplastic cells and autopsy showed only HD of the CNS. Various techniques to establish a cause of the CNS involvement in children with HD twill be discussed.

IN VITRO CHARACTERISTICS OF CHILDHOOD LEUKEMIC MONO-

932 BLASTS. Robert M. Weetman, Robert R. ChIlcote, W. Joseph Rterden, and Robert $L$. Baehner, Department of Pediatrics, J.W. Riley Hospital for Children, Indiana University School of Medicine, Indianapolis, Indiana.

Plasma from aduits with acute monocytic (AMOL) and acute monomyelocytic (AMML) leukemia has been shown to contain colony stimulating activity (CSA), as has conditioned media prepared from adult AMML cells. Two children with AMoL (DB;OJ) and one with AMML (JD) were studied for evidence of CSA production by feeder layers (FL) prepared from $1 \times 10^{6}$ washed peripheral blood (PB) leukocytes containing monoblasts as documented by cell morphology, alpha naphthyl acetate esterase stain, glass adherence, and maturation to macrophages in vitro. FL prepared from DB and JD PB failed to support normal bone marrow (BM) colony growth $(0$ colonies), while control FL prepared from normal human PB leukocytes stimulated 4 different normal BMs to produce a mean of $21.8,24$, 99 , and 165 colonies $/ 2 \times 10^{5}$ cells/plate. Normal BMs stimulated by 0,6 , and 17 day old FL prepared from OJs PB monoblastsmacrophages produced $6+6,1 \pm 0$, and $0 \pm 0$ colonies compared to $25 \pm 8$,

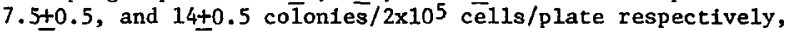
using control $\overline{\mathrm{FL}}$. Leukemic BM from each patient cultured over control FL grew small numbers of colonies (mean: DB 2.25 colonies, OJ 0.75 colonies, JD 0 colonies $/ 2 \times 10^{5}$ cells/plate), while none of the leukemic BMs grew colonies in the absence of an exogenous source of CSA. These studies suggest childhood leukemic monoblasts lack the ability to produce CSA in vitro, produce abnormal CSA, or produce an inhibitor to colony proliferation. 
IMPROVED RESULTS IN THE DIAGNOSIS AND TREATMENT OF INTERSTITIAL PNEUMONIA IN IMMUNOSUPPRESSED CHILDREN. Lawrence J. Wolff, Trent K. Ruebush, Robert A. Weinstein, Jay L. Grosfeld, Frank Gonzales-Crussi, James W. Smith, and Robert L. Baehner, Indiana University School of Medicine, Indianapolis and Center of Disease Control, Atlanta, Georgia. Modern cancer chemotherapy induces immunoincompetence of ten resembling primary immunodeficiency disorders. Both groups are at high risk for opportunistic infections. During a 17 month period 13 children with Acute Lymphocytic Leukemia, 1 with Hodgkin's D1sease, and 1 with combined immunodeficiency developed interstitial pneumonitis (IP). Every patient presented with tachypnea and/or fever. All patients had abnormal chest $x$-rays. Eight had diffuse interstitial infiltrates. Room a1r arterial $\mathrm{P}_{2}$ was less than $66 \mathrm{~mm} \mathrm{Hg}$ in 12 of 13 . Each patient was studied according to a prospective protocol with open lung biopsy (OLB) providing tissue for Giemsa, Gram-Weigert, Methenamine silver, and H\&E stains, and bacterial, fungal, and viral cultures. The interval between suspicion of IP and OLB was less than 24 hours in 12 of 15. Pneumocystis carinii (PC) was identified in 13, Candida albicans in 2 ( 2 with PC), Staphylococcus aureus in 1 , and Respiratory Syncitial virus in 1 . Pentamidine isethionate was begun in 12 of 13 within 12 hours. Twelve of 15 patients (10 of 13 with $P C$ ) survived. One patient died of accidental self-extubation. The excellent therapeutic results of this study with an $80 \%$ survival support the approach of early open lung biopsy in immunosuppressed children with arterial undersaturation and pulmonary infiltrates.

\section{PULMONOLOGY}

FLOW-VOLUME RELATIONSHIP AT LOW LUNG VOLUMES IN

934. HEALTHY TERM INFANTS. Saul M. Adler, Univ. of South Florida, Dept. of Pediatrics, Tampa, Fla, and Mary Ellen Wohl, Harvard Medical School, Children's Hospital Medical Center, Boston, Mass. (Introduced by L. A. Barness).

At low lung volumes the relationship of maximal expiratory flow ( $v \max$ ) to lung volume is determined by the characteristics of the airways and the elastic recoil of the lung. To study these mechanical properties we obtained maximal expiratory flow-volume (MEFV) curves in 9 healthy term infants by transiently applying positive pressure in a chamber around the infants' body. Flow was measured with a Fleisch pneumotachograph attached to an anesthesia mask. Volume was obtained by integrating flow and expressed as \% of the maximal expired volume (MEV) below FRC. With increasing chamber pressure expiratory flow increased above values for quiet breathing and expiration continued below FRC. We considered that at any given lung volume $\dot{V} \max$ was reached when further increases of chamber pressure produced no further increase of flow. At FRC this was achieved at chamber pressures ranging from $28-47 \mathrm{~cm} \mathrm{H} \mathrm{H}_{2} \mathrm{O}$.

The mean volume expired below $\mathrm{FRC}$ was $22 \pm 6 \mathrm{ml}(\mathrm{SD})$. Peak expiratory flow during tidal breathing averaged $78 \pm 15 \mathrm{ml} / \mathrm{s}$. In 7 infants $\dot{v} \max$ at FRC was $185 \pm 47 \mathrm{ml} / \mathrm{s}$ and at $50 \% \mathrm{MEV}$ was $108 \pm 38 \mathrm{ml} / \mathrm{s}$. Two infants failed to reach $\mathrm{V}$ max at FRC, and had higher flows at low lung volumes $(218-230 \mathrm{ml} / \mathrm{s}$ at $50 \% \mathrm{MEV})$. Seven of 9 curves were convex to the volume axis which is compatible with flow limitation in peripheral airways. These results demonstrate the feasibility of obtaining partial MEFV curves in infants and suggest that substantial increases in expiratory flow can occur in normal infants before the mechanical properties of the lung limit expiration.

COMPARATIVE ANALYSIS OF PURIFIED SURFACTANT FROM NEW-

935 BORN INFANTS. John U. Balis, Miroslav Kovacevic, Delivoria-Papadopoulos) Stritch Sch. Med., Loyola Univ. Med. Ctr. Depts. of Path. \& Ped., Maywood, II. \& V.A. Hosp., Hines, I1.

Based on studies reported previously (Shelley et al., J. Lipid Res. $16: 224,1975)$, a reliable density gradient centrifugation method was developed for purification of lung surfactant present in small samples contaminated with blood constituents. Using this method surfactant was isolated from serial tracheal aspirates (TA) of infants with respiratory distress syndrome (RDS) and from controls which included TA of infants without RDS, pharyngeal aspirates of term newborns (PA), amniotic fluids with mature L/S ratios and lung washings obtained post-mortem from normal lungs of term infants. Phosphatidyl glycerol was consistently found in PA surfactant but was frequently absent in samples from infants with RDS. Phosphatidyl choline (PC) of all control groups revealed essentially identical fatty acid compositions with an average of $72 \%$ palmitic $(16: 0)$ and $10 \%$ oleic $(18: 1)$ as the major fatty acids. Surfactant PC from newborns with RDS revealed significantly lower percentage of $16: 0$ and higher percentage of $18: 1$, irrespective of gestational age. Characteristic patterns of progressive alterations in the surfactant PC fatty acids were noted during prolonged survival of infants with RDS including those with bronchopulmonary dysplasia. Surfactant from infants recovering from RDS demonstrated gradual changes toward control values. The results indicate that evaluation of purified surfactant from TA effectively monitors severity and progression of RiS. (Supported by NIH Grant HD-04434).
936 GLUCOCORTICOID BINDING BY LUNG CYTOSOL AND ISOLATED TYPE 2 EPITHELIAL CELLS OF ADULT RAT. Philip L. Ballard, J. Patricia Granberg and Wilitiam H. Douglas. Spec. Center of Res. in Pulm. Dis., Cardiovas. Res. Inst. and Dept. of Ped., Univ. of Calif., San Francisco and W.A. Jones Cell Science Center, Lake Placid, New York.

Cytoplasmic receptors appear to mediate the effects of glucocorticoids in target tissues. These binding proteins have been detected previously in fetal lung of man and various animals, but their presence in adult lung other than rabbit has been uncertain. We measured $3 \mathrm{H}$-dexamethasone binding by cytosol preparations of adult lung at $2^{\circ}$ using the charcoal assay. In the presence of $1 \mathrm{mM}$ dithiothreitol we found specific binding in lung of mouse, dog, hamster, monkey and rat. In the absence of a sulfhydryl compound in vitro receptor activity of rat lung decreased after homogenization with a $t 1 / 2$ of $45 \mathrm{~min}$. In adrenalectomized rats (5-20 wk) lung contains an average of 19,400 sites/cell compared with 6000 / cell during late fetal life. Binding is specific for steroids with glucocorticoid activity and the $\mathrm{K}_{\mathrm{d}}$ for dexamethasone is $2.5 \mathrm{nM}$. At $37^{\circ}$ there is nuclear localization of steroid by intact cells isolated from rat lung. We also found nuclear binding by a freshly isolated population of type 2 cells ( $70-80 \%$ pure) and by a cell line (L-2) which apparently originated from a type 2 cell. Binding studies with cytosol of $\mathrm{L}-2$ cells indicated high affinity binding (dexamethasone $k_{d} 2.8 \mathrm{nM}$ ) and an equivalent number of sites $(14,500 /$ ce11) for dexamethasone, triamcinolone acetonide, cortisol and corticosterone. Thus, type 2 cells and apparently many other cell types of adult lung contain the receptor mechanism necessary for responsiveness to corticosteroids.

937 SYNDROME (W-M). Hordur Bergsteinsson, Allan L. Coates Katharine J. Desmond, Eugene W. Outerbridge and Pierre H. Beaudry. (Spon. by Keith N. Drummond) Depts. of Newborn Medicine and Respiratory Function, McGill University-Montreal Children's Hosp. Res. Inst., Montreal, P.Q.

Pulmonary function testing, including flow volume curves in air and in helium and oxygen $\left(\mathrm{HeO}_{2}\right)$ was performed on $5 \mathrm{~W}-\mathrm{M}$ survivors, aged 8-10 years, and compared to normal children, 5 born prematurely and 5 born at term. All had normal lung volumes. The W-M survivors had lower maximum mid-expiratory flow rates (MMEF) and flow rates at $25 \%$ vital capacity ( $25 \% \mathrm{vC}$ ) in air than the other 2 groups. This difference is statistically significant $(p<0.01)$. The volume of isoflow (VisoV) between the groups was not significantly different.

$\begin{array}{lccr} & \text { MMEF (\% pred.) } & \text { V } 25 \% \text { VC }(T L C / \text { sec.) } & \text { VisoV } \\ \text { W-M } & 55 \pm 22 & 0.20 \pm 0.08 & 25 \pm 22 \\ \text { Prems } & 100 \pm 3 & 0.45 \pm 0.09 & 10 \pm 11 \\ \text { Terms } & 102 \pm 13 & 0.57 \pm 0.12 & 8 \pm 8\end{array}$

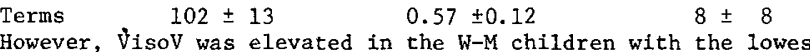
MMEF, suggesting that small airway changes may be a major contributor to this disease. This may be a sequel of the disease process, prolonged oxygen therapy, or of a deranged growth pattern. One could speculate that the abnormal flow rates are in keeping with persisting abnormal airway compliance from the neonatal period. This could support the concept of high airway compliance as an etiologic factor in this syndrome.

FATE OF BIOGENIC AMINES IN RABBIT LUNG: 938 DEMONSTRATION OF AGE DEPENDENCE. Michael A. Berman, K. Baker, C.N. Gillis. Yale Univ. Sch. Med., Depts. Ped, \& Anesth., New Haven. (Intr. by N.S. Talner) Norepinephrine and 5-hydroxytryptamine (NE \& 5HT) uptake and metabolism were measured in isolated rabbit lung preparations at ages $1,2,3,10,17,24,31,38,50,140$ days. Lungs were perfused through individual right and left pulmonary artery cannulae with $20 \mathrm{ng} / \mathrm{ml}$ of $\mathrm{NE}$ and $5 \mathrm{HT}$ at $2 \mathrm{ml} / \mathrm{min}$. Effluents were assayed by column chromatography. The \% uptake of NE increased from newborn $(18.4+2.5)$ to adult levels at 50 days $(51.0+$ 2.5). Uptake of $\mathrm{NE}$ per $\mathrm{ml}$ of inulin space (net removal corrëcted for perfusable space), however, showed highest values in the newborn $(3.9+.7)$ declining to a low at $2-3$ weeks and then increasing to apparent adult levels by 50 days. Similarly, $5 \mathrm{HT}$ uptake increased from $36.9+2.5$ ( 1 day) to $50.8+1.1$ ( 140 days). Uptake of $5 \mathrm{HT}$ per $\mathrm{ml}$ of inulin space ranged from $5+.2$ (1 day) to $1.6+.1$ (140 days). The \% of catechol-O-methylated product (COMT) and monoamine oxidase (MAO) values for NE were lowest at 1 day, rising steadily to adult levels (MAO 10-20\%, COMT 2-5\%). 5HT MAO ranged from 2-17\%. In conclusion, newborn $x$ abbit lung has the capacity for uptake and metabolism of $N E$ and $5 \mathrm{HT}$. Values of $\mathrm{NE}$ and $5 \mathrm{HT}$ removal obtained in the newborn, when corrected for perfusable space, are greater than adult levels and cannot be explained by an increasing number of lung units with age. 
CORRELATIONS BETHEEN GIYCOGEN AND LECITHTN LEVELS IN

939 DOPING FETAL LUNG FOLLOWING VARIOUS HORMONE INJECTIoNS. Will R. Blackburn, Phylis A. Logsdon, John A. Alexander and Jan Delli-Bovi. University of South Alabama College of Medicine, Mobile. Fetal lung accumulates glycogen prior to the period of active surfactant synthesis, however, by the time of birth little glycogen is present. Evidence is accumulating which suggests that fetal lung glycogen is a major contributor to the glycerol moiety of the surfactant molecule. In order to evaluate the relationships between glycogen and lecithin (L) levels in developing lung, fetal rats were injected (day 18.5 in utero) with thyroxine $(\mathrm{T}-4)$, dexamethasone $(\mathrm{C})$, and testosterone $(\mathrm{T})$ and the lungs harvested for analysis at 24-hour intervals. Peritoneal punctured and vehicle $(5 \mathrm{ul})$ injected littermates served as controls.

Dexamethasone $(1,2,4,6,20 \mathrm{ug} /$ fetus $)$ resulted in lower glycogen levels at each sampling period (P 0.01) and significant (P.001) elevations of both phospholipid (PL) and lecithin. Thyroxine injections $(2,4,6,8 \mathrm{ug} / \mathrm{fetus})$ leci to reduced lung glycogen at each of the sample periods: this effect was not dose related. T-4 injections did not influence PL levels although lecithin levels were increased only at $72 \mathrm{hrs}$ post injection. Testosterone $(5,20,40$, $60 \mathrm{ug} / \mathrm{fetus})$ did not influence either glycogen, PL or Lecithin levels in developing fetal rat lung.

These studies further support the concept that the initial synthesis of lung surfactant is closely tied to glycogen utilization and may offer a key to the fact that hyperinsulinemia in utero (infants of diabetic mothers) increases the risk for surfactant deficiency at birth and ultimately hyaline membrane disease.

Supported by USPHS NIH Grant HL 17328-02.

LUNG FLUID AND PROTEIN TRANSPORT IN AWAKE

940 NEWBORN LAMBS AND ADULT SHEEP. Richard D. Bland and Douglas D. McMillan (Spon. by Phillip L. Balliard). Cardiovascular Research Institute, Department of Pediatrics, University of California, San Francisco, CÁ.

To compare pulmonary microvascular permeability to plasma proteins and lung fluid filtration in newborn lambs and adult sheep, we measured steady-state lung lymph flow (Q/ym) and protein flow (Qpr) in 7 oneweek-old unanesthetized lambs and 9 sheep. We surgically prepared the animals to isolate and collect lung lymph and measure mean pulmonary arterial ( $\mathrm{Ppa}$ ) and left atrial ( $\overline{\mathrm{Pla}}$ ) pressures, allowing 2 days for postoperative recovery and 2-4 hr for each study. Summary of results:

$$
\text { No. Exper. Pिpa P̄la Q்lym Q̀pr Lymph Plasma L:P }
$$

\begin{tabular}{lllllllll} 
& \multicolumn{4}{c}{ torr } & $\mathrm{ml} / \mathrm{hr} *$ & $\mathrm{mg} / \mathrm{hr}^{*}$ & $\mathrm{~g} / 100 \mathrm{ml}$ & \\
Lambs & 16 & 19 & 2 & .11 & 3.1 & 3.02 & 5.01 & .60 \\
Sheep & 22 & 14 & 1 & .07 & 2.8 & 4.11 & 6.09 & .68
\end{tabular}

*Calculated per $g$ of dry, bloodless lung (post-mortem measurements) We also found that intravenously-injected 125 -albumin entered lymph from plasma more rapidly in lambs than in sheep, and extravascular lung water per $\mathrm{g}$ of dry, bloodless lung was greater in the younger animals $(4.90 \pm .11$ vs. $4.45 \pm .08)$. Higher lung vascular pressures, Q lym, and $Q \mathrm{pr}$ in lambs, coupled with the lower lymph:plasma total protein ratio, suggest that pulmonary transvascular fluid filtration and associated bulk protein flow are greater in lambs than sheep, but that microvascular membrare permeability to plasma proteins is not significantly different.

CONTROL OF SEVERE CHILDHOOD ASTHMA WITH TRIAMCINOLONE

941 ACETONIDE AEROSOL. DOuglas P. Boldon, F. Stanford Massie, Keith N. Megathlin, Gilberto E. Rodriguez, (Spon. by Harold M. Maurer) Dept. of Pediatrics, Medical ColTege of Virginia, Richmond.

Thirty-one children with severe asthma (mean age 11.4 years) were treated with triamcinolone acetonide aerosol (TA) for 8 weeks. There were fourteen steroid-dependent jatients (SD) on oral steroid therapy for at least 6 months, and 17 patients on no steroid (SI) for at least 30 days. The mean duration of $i 17 n$ ness was 9.6 years.

Clinical evaluations and pulmonary functions (FEV , MMEF) were followed weekly. Cortisol and laboratory studies were done at 0 , 4 , and 8 weeks. Oral steroids were tapered after 2 weeks on TA and were discontinued in 9 of 14 SD patients. Daily symptoms and nights awakened by wheezing were greatly reduced.

Results of spirometry showed improvement for both SD and SI groups of patients. Cortisol values were not effected by TA.
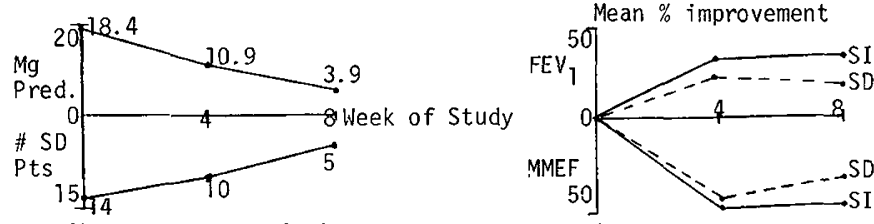

Short term use of TA is safe and efficacious in the treatment of severe childhood as thma.
942

MONITORING OF ESOPHAGEAL PRESSURE FOR THE DETERMINATION OF OPTIMAL CPAP, B. W. Bonta, L. Weksler, J. B. Warshaw, and E. K. Motoyama, Yale Univ. School of Medicine, Depts. of Pediatrics and Anesthesiology, New Haven, Ct. Continuous positive airway pressure (CPAP) is an effective treatment for IRDS. However, there is no simple and reliable method to determine the optimal level of airway pressure (Pao) for each infant. At optimal $\mathrm{Pao}, \mathrm{PaO}_{2}$ increases markedly, due to reopening of collapsed alveoli with an increase in resting lung volume (FRC) toward normal. Such changes in FRC should be reflected by an increase in esophageal pressure (Pes). To test this hypothesis, we monitored Pes with a water-filled \#5 French feeding tube placedin the lower third of the esophagus with the proximal end connected to a pressure transducer. Pao was measured by inserting a needle into either a nasal prong or endotracheal tube adaptor. $P a o$ and Pes were continuously monitored on a two channel recorder. In 5 infants with IRDS breathing spontaneously, $\mathrm{Pao}, \mathrm{Pes}, \mathrm{PaO}_{2}$, and $\mathrm{PaCO}_{2}$ were measured before and after successive $2 \mathrm{~cm}_{\circ} \mathrm{H}_{2} \mathrm{O}$ increments in $\mathrm{PaO}$ with a constant $\mathrm{FiO}_{2}$. At Pao below optimal CPAP, Pes increased only mildly but when $\mathrm{Pao}$ reached the optimal level, $\mathrm{PaO}_{2}$ increased $\geqslant 20$ torr and Pes increased $\geq 3 \mathrm{~cm}_{0} \mathrm{H}_{2} \mathrm{O}$. Above this level, $\mathrm{PaO}_{2}$ and Pes changed little while $\mathrm{PaCO}_{2}$ increased markedly (8-13 torr). Chest radiographs below and at optimal CPAP did not differ appreciab1y. Thus, continuous monitoring of Pes provides an accurate and safe method to determine the optimal CPAP in infants with IRDS.

COMPARISON OF THE EFFECTS OF VENTILATION AT I:E

943 RATIOS OF $1: 1$ vS 1:4 ON RESPIRATORY PARAMETERS OF PREMATURE LAMBS WITH SEVERE RDS. Stephen J. Boros, Sadi V. Matalon, Randall Ewald, Arnold S. Leonard and Car1 E. Hunt. Depts. of Pediatrics, Physiology and Surgery. University of Minnesota, Minneapolis, Minnesota. Spon. by Henry I. Nadler.

In 5 premature lambs with severe RDS ventilated with $100 \% 0_{2}$ at a constant $V_{T}$, PEEP levels and $I: E$ ratios were varied as indicated. $\mathrm{PAO}_{2}$ and $\mathrm{PACO}_{2}$ were obtained from continuous analysis of the expired air. The measured parameters and their observed changes were as follows: (Mean \pm 1 S.E.M.) $*_{p} \leq 0.05$

PEEP $=0 \quad$ PEEP $=5 \quad$ PEEP $=10$

\begin{tabular}{|c|c|c|c|c|c|c|}
\hline $\begin{array}{l}\mathrm{PaO}_{2} \\
(\mathrm{mmH})\end{array}$ & $88 \pm 15$ & $106 \pm 31$ & $132 \pm 31$ & $87 \pm 15$ & $147 \pm 67$ & $127 \pm 82$ \\
\hline $\begin{array}{l}\mathrm{PaCO}_{2} \\
(\mathrm{mmHg})\end{array}$ & $40 \pm 7$ & $35 \pm 5$ & $28 \pm 4$ & $31 \pm 7$ & $27 \pm 5 *$ & $-38 \pm 6$ \\
\hline$(\mathrm{a}-\mathrm{A}) \mathrm{DCO}_{2}$ & $19 \pm 7$ & $16 \pm 5$ & $3 \pm 1.5$ & $10 \pm 5$ & $6 \pm 5 *$ & $-19 \pm 8$ \\
\hline$(\mathrm{A}-\mathrm{a}) \mathrm{DO}_{2}$ & $417 \pm 49$ & $430 \pm 54$ & $386 \pm 82$ & $406 \pm 81$ & $355 \pm 69$ & $383 \pm 83$ \\
\hline $\begin{array}{l}\text { Mean Air- } \\
\text { way Pres. } \\
\left(\mathrm{cmH}_{2} \mathrm{O}\right)\end{array}$ & $22 \pm 3$ & $18 \pm 3$ & $23 \pm 4$ & $22 \pm 3$ & $24 \pm 4$ & $24 \pm 6$ \\
\hline $\begin{array}{l}\text { Peak Air- } \\
\text { way Pres. } \\
\left(\mathrm{CmH}_{2} \mathrm{O}\right)\end{array}$ & $43 \pm 5$ & \pm 5 & $40 \pm 9$ & $33 \pm 7$ & $34 \pm 9$ & $32 \pm 11$ \\
\hline
\end{tabular}

$\left(\mathrm{cmH}_{2} \mathrm{O}\right)$

VA $/ Q$ did not significantly improve at higher $\mathrm{L}: \mathrm{E}$ ratios and appeared to worsen with higher $I: E$ ratios plus PEEP.

\section{SELECTIVE BRONCHIAL INTUBATION FOR THE TREATMENT OF}

944. SEVERE LOCALIZED INTERSTITIAL EMPHYSEMA. John $G$. Brooks, Beverly L. Koops, Saskia Hilton, Richard L. Wesenberg, Michael A. Simmons, (Spon. by Ernest K. Cotton), Dept. of Pediatrics, University of Colorado School of Medicine, and Denver Children's Hospital, Denver.

Pulmonary interstitial emphysema (PIE) is a serious and frequent complication of severe hyaline membrane disease (HMD). Although lobectomy has produced improvement in some patients with localized PIE, a procedure which preserves potentially functiona lung tissue is desirable. We have employed selective bronchial intubation (SBI) at 20-25 days of age in 4 prematurely born neonates mechanically ventilated for severe HMD who developed progressive, localized PIE, unresponsive to usual therapy. The localized hyperinflation disappeared in all patients after 5-48 hours of contralateral SBI. We withdrew the endotracheal tube to above the carina after 36-48 hours of SBI in two twins (gestational age $(G A)=28$ weeks) and after 5 days in two patients ( $G A=32$ and 35 weeks) PIE did not reappear after 5 days of SBI, and both patients improved progressively after the procedure. Followinj the shorter SBI, both patients redeveloped PIE requiring further SBI. One subsequently died of nonpulmonary causes. SBI is a simple procedure for the treatment of severe localized PIE, if conservative management fails. No significant complications have been recognized and the procedure is well tolerated. Minimal $\mathrm{CO}_{2}$ retention and no increased $R \rightarrow L$ shunt have occurred. SBI should not be done for localized hyperinflation due to anatomic abnormalities such as bronchial obstruction. 
FACTORS CONTRIBUTING TO DEVELOPMENT' OF YELLOW PUL945 MONARY MEMBRANES IN THE NEONATE. Audrey K. Brown, Medical Center. Depts. of Pediatrics \& Pathology, Brooklyn. Only recentiy have pathologists reported yellow membranes in the lungs of infants dying with the respiratory distress syndrome (RDS). The pigment has been identified as bilirubin, but the mechanism remains obscure. In a recent review of our neonata. autopsy data (1971-75), we identified seven infants with yellow pulmonary membranes. Findings in these infants were compared with data from other infants also dying with RDS. Since the average age at death was significantly greater in the yellow membrane group ( 14.3 days) than in the total group of infants with RDS (5.2 days) and since all yeliow membrane infants had been on ventilators, the control RDS group was limited to infants who had lived more than 5 days (average 12.5 days) and had also been on ventilamore than 5 days (average 12.5 days) and had also been on ventilators. The following features were found: 1) peak serum bilirubin leveis were significantly higher in infants with yellow membranes than in the controls $(15.9 \mathrm{mg} \%$ vs. $10.5 \mathrm{mg} \%, \mathrm{p}<.05), 2)$ all infarts have bronchopulmonary dysplasia, while $6 / 9$ control infants had severe RDS and only one infant developed bronchopuimonary dysplasevere RDS and only one infant developed bronchopulmonary dysplasia, 3) $/ 7$ infants with yellow merbranes showed evidence of cholestasis (including one infant with hepatitis) compared with $4 / 9$ control infants. It is postulated that the prolonged survival of infants with severe RDS in the presence of hyperbilirubinemia may result in the observed yellow pulmonary membranes; since the infants with yellow membranes seemed to have more severe RDS, this may be a contributing factor. Furthermore, all of the infants given 100\% oxygen, treated with positive end expiratory pressure and mechanically ventilated, so that this therapy may also have contributed to the formation of the yellow membranes.

\section{6} COMBINED OBSTETRICAL AND PEDIATRIC MANAGEMENT OF MECONIUM STAINING. Bonita S. Carson, Rosalind W. Losey, Watson A. Bowes, Jr., and Michael A. Simmons (Spon. by Frederick C. Battaglia). University of Colorado Medical Center, Department of Pediatrics, Denver.

Meconium aspiration syndrome (MAS) continues to cause significant neonatal mortality and morbidity. In May, 1974, a regimen for obstetrical and pediatric management of meconium staining was introduced which included: (1) deep suctioning of the oral nasopharynx by the obstetrician while the infant's head was still on the perineum, prior to the delivery of the shoulders, and usually prior to the first breath; (2) routine inspection of the larynx by the pediatrician; and (3) direct tracheal suction if meconium was present.

Number of births

Meconium staining

Respiratory distress $>5$ days

MAS deaths

The 23 cases of MAS occurring during the study period were mild, with no infant requiring assisted ventilation. In a prospective study from $4 / 75$ to $10 / 75$, one mild case of MAS occurred among 273 infants with meconium staining. We have not found routine tracheal suctioning necessary unless meconium is visible at the cords. The addition of saline lavage to the suctioning procedure led to increased morbidity. Severe MAAS has been effectively prevented by the suctioning alone.

\section{7} DEVELOPMENTAL STUDIES OF PULMONARY ALVEOLAR SEPTAL EL.ASTIN IN FETAL LAMBS. J. M. Cerreta, R. 1. Stark, S. Keller, L. S. James, I. Mandl and J. A. Fierer, Coll. of Phys. \& Surg., Columbia Univ., Div. of Perinatology, New York. In view of the importance of elastin in maintaining the structural stability of the lung and the known relationship between RDS and immaturity, a study of the ultrastructural and biochemical maturation of pulmonary septal elastin has been undertaken. Eighteen fetal lambs have been studied at 6 different gestational ages ranging from 90 days to term. Mature elastin fibers consist of two morphologically and biochemically distinct components: a large amorphous component, rich in neutral amino acids and containing the unique cross-linking amino acids desmosine and isodesmosine and a smaller microfibrillar portion having the chemical composition of structural glycoprotein. These components can also be differentiated morphologically. At 90 days gestation only the microfibrillar component is present. In the 110 day lamb small amounts of amorphous elastin appear with gradual increase in this component. At 130 days gestation the microfibrils and amorphous component are present in approximately equal amounts. Increase in amorphous elastin accelerates at this stage to reach the mature proportion of approximately $90 \%$ amorphous component and $10 \%$ microfibrils. At term the composition of the pulmonary al veolar septal elastin resembles that in the adult ewe. The mature form of elastin is susceptible to digestion by elastolytic enzymes which reduce the proportion of the amorphous component to that found at the more immature phases of development. The immature form of elastin found in RDS could be the result of impaired develop
damage by elastolytic enzymes released as a result of hypoxia.
948 ENHANCED FETAL ERYTHROCYTE CARBONIC ANHYDRASE ACTIVITY BY STEROIDS. Chernick, Victor,
Loesebrink, Brigitte, and Russell, Brian Department of Pediatrics, University of Manitoba and Health Sciences Centre, Winnipeg, Canada.

Infants with hyaline membrane disease (HMD) have been shown to have lower erythrocyte carbonic anhydrase activity than normal infants of comparable gestational age. Previous studies have shown that steroids accelerate lung enzyme systems during fetal life. The present experiments were undertaken to study the effect of exogenous corticosteroids on carbonic anhydrase activity in fetal erythrocytes (CA). Rabbit fetuses from 24 to 30 days of gestation were injected intraperitoneally with either $0.2 \mathrm{ml}$ of $0.9 \%$ saline or $2.5 \mathrm{mg}$ hydrocortisone succinate (St). Non-operated, non-injected animals served as controls. CA measured at 24 hours after injection was increased in the saline injected group on days $26-28$ some 1.3 to 3 fold but there was no effect on days 29 or 30 . This response was likely due to fetal stress induced by the saline injection. A marked increase (2 to 7 fold) in enzyme activity was demonstrated after steroid injection at 24 hours after treatment and $\mathrm{CA}$ activity remained elevated at 48 hours except on day 30 . An increase in CA activity was also demonstrated after incubating fetal erythrocytes for 2, 4, and 8 hours in the presence of St. It is suggested that low CA in infants with HMD may reflect lack of enhancement by steroid of a number of enzyme systems, including those necessary for surfactant synthesis, in preparation for extrauterine life. (Supported by the M.R.C. and Children's Hospital of Winnipeg Research Foundation, Inc.)

\section{MECHANISM FOR PILOCARPINE-INDUCED SECRETION OF}

949 PULMONARY SURFACTANT (S) IN FETAL RABBITS. Anthony $\frac{\text { J. Corbet, Phyllis K. Flax, and Arnold J. Rudolph }}{\text { urdina M. Desmond). Department of Pediatrics, Baylor }}$ (Spon. by Murdina M. Desmond)
College of Medicine, Houston.

Pilocarpine (PC) induces secretion of $\mathrm{S}$ (Goldenberg et. al. Lab. Invest. 20, 147, 1969), but the mechanism is not known. Static pressure-volume curves were used to assess release in fetal rabbits sacrificed 3 hours after injection with Pc (5mg) or Saline ( $\mathrm{Sa}$ ). Airspace $S$ was indicated by lung volume at $5 \mathrm{~cm}$ water on deflation from total lung capacity (\% TLC-5). There was increased $S$ with increasing maturity, and with $\mathrm{PC}$ injection at $25.5,26.5,27.5$ days gestation.
G.A
\% TLC-5
1) $\mathrm{Sa} \quad \begin{array}{lll}12 & 25.5 & 26.5 \\ \text { 2) } \mathrm{Pc} & 20 * & 25\end{array}$
$26.5 \quad 27.5$
28.5
2) $\mathrm{PC} \quad 20 * \quad 25 * \quad 43 * \quad 56 \quad(* \mathrm{p}<0.001)$

No improvement beyond 27.5 days may mean that lining of airspace with $S$ was optimal by then.

The effect of Pc at 27.5 days was blocked by Atropine (Ap), Propranolol $(\mathrm{Pp})$ and Phenoxybenzamine $(\mathrm{Pb})$.

$\begin{array}{lllllll} & \mathrm{Sa} & \mathrm{Pc} & \mathrm{Pc}_{\mathrm{Ap}} & \mathrm{P}_{\mathrm{C}+\mathrm{Pp}} & \mathrm{Pc}_{\mathrm{Pb}} & \\ \% & \text { TLC-5 } & 25 & 43 & 25 * & 30 * & 25 *\end{array}(* \mathrm{p}<0.01)$ Blocking agents given alone had no effect, nor did Muscarine alone improve lung stability. We conclude that Pc released $S$ by stimulation of sympathetic ganglia with release of adrenergic agonists from the adrenal medulla or nerve endings adjacent to the granular pneumocyte.
950 Department of Pediatrics, Denver.

Ventilatory response to $13 \% \mathrm{O}_{2}$ has been measured in 25 full term infants born at Leadville, Colorado, altitude 3,100 meters. Infants that were studied were conceived and gestated at that altitude. Measurements were made using an infant body plethysmograph with the face exposed. $13 \%, 30 \%$ and $100 \%$ oxygen mixtures were given and tidal volume and respiratory rate recorded. Alveolar $\mathrm{O}_{2}, \mathrm{CO}_{2}$ and $\mathrm{N}_{2}$ gas tensions were measured using a mass spectrometer. Results showed that 14 babies increased minute volume by $18 \%$ after 5 seconds of hypoxia and maintained a $10 \%$ increase until 90 seconds when a depressed response occurred. Eleven babies had no response to hypoxia at 5 seconds and by 10 seconds showed a depressed response of 118 which persisted during the 2 minutes of hypoxia. Repeat studies were carried out at 15 and 20 days on 6 infants with the absent response. All had the "sea level" response. We conclude that the ventilatory response to hypoxia is lacking on a large number of infants born at altitude. Either hypoxia is not an important ventilatory stimulus in the immediate newborn pexiod or is over ridden by the central depressive effect of hypoxia. 
951 EFFECTS OF ISOPROTERENOL AND CROMOLYN PRE-TREATMENTS ON EXERCISE-INDUCED BRONCHOSPASM (EIB) IN ASTHMATIC CHILDREN. G.J.A. Cropp and S.S. Fisher. Nat. Asth. Ctr., Dept. Clin. Physiol., Denver, Colorado.

Strenuous physical activity triggers acute EIB in many asthmatic children. We evaluated the effectiveness of pre-treatments with $250 \mu \mathrm{g}$ iscproterenol (I) or $20 \mathrm{mg}$ cromolyn sodium (C) aerosols in 22 asthmatics (age $7-16$ yrs.) with known EIB. Pulmonary functions (PF: Forced vital capacity-FVC; functional residual capacity-FRC; airway resistance-R) were measured before and after pre-treatments and repeatedly after exercise. Exercise was performed at incrementally increasing loads on a cycle ergometer until heart rates of at least $180 / \mathrm{min}$ were reached. Baseline PF's were no different on the 3 test days, were not altered by $C$, but were improved by $I$. The table summarizes the benefits derived from pre-treatment. Without pre-treatment there was a highly significant deterioration in all $\mathrm{PF}^{\prime} \mathrm{s}$ which lasted for 30-40 minutes. Both $I$ and $C$ lessened and shortened EIB significantly, but $I$ was more effective than $C$ in reducing functional and clinical abnormalities.

\begin{tabular}{|c|c|c|c|c|c|c|}
\hline & \multicolumn{2}{|c|}{ No Pre-Treat. } & \multicolumn{2}{|c|}{ I Pre-Treat. } & \multicolumn{2}{|c|}{ C Pre-Treat. } \\
\hline & Basel. & Worst $p$ Ex. & Basel & Worst $p$ Ex. & Basel. & Worst $p$ Ex. \\
\hline FVC & $104 t$ & $73^{*}$ & 105 & $96 *$ & 105 & $87 *$ \\
\hline $\mathrm{FEV}_{1}$ & 84 & $54 *$ & 86 & $76 *$ & 84 & $65 *$ \\
\hline & 101 & $239^{*}$ & 99 & 133 & 115 & $189^{*}$ \\
\hline FRC & 145 & $242^{*}$ & 158 & $173^{*}$ & 148 & $202^{*}$ \\
\hline
\end{tabular}

tMeans of predicted values; *statist. sig. change from baseline

952 OL (PG) IN INFANTS OF DIABETIC MOTHERS (IDM) WITH RESPIRATORY DISTRESS (RD). M. Douglas Cunningham, J. Michael Greene, Sue A. Thompson, and Nirmala S. Desai. (Spon. by C. Charlton Mabry). Albert B. Chandler Med. Ctr., Univ. of Kentucky, Dept. of Ped., Lexington.

Despite mature antenatal $\mathrm{L} / \mathrm{S}$ ratios, $\mathrm{RD}$ continues to occur in some IDM. Amniotic fluid (AF) PG, an important surface active component of pulmonary phospholipids (PPL), was studied in relation to other PPL, gestational age, and infant outcome. PPL were identified using DEAE cellulose microcolumns, and thin layer chromatography. Integrated scanning densitometry was used to derive percent of PPL fractions. 19 non-diabetic (17 pt) and 38 IDM (27 pt) AF specimens were assayed. Gestations were $\leqslant 33 \mathrm{wk}$, $34-37 \mathrm{wk}$, and $\geqslant 38 \mathrm{wk}$. Lecithin (Lec) was 25 and $28 \%$ of total PPL from 34 wk to term in non-diabetic and IDM, respectively. PG levels fell during 34-37 wh period in all cases, concomitant with Lec rise. PG was absent in $45 \%$ of all IDM. Class A and B IDM had $54 \%$ absence of PG in the $34-37$ wk period. Lowest mean $P G$ values were seen in IDM with $R D$. PG values \pm 1 SD were:

\begin{tabular}{|c|c|c|c|}
\hline & $\leqslant 33 \mathrm{wk}$ & $34-37$ wk & $\geqslant 38 \mathrm{wk}$ \\
\hline non-diabetic & $22.0 \% \pm$ & $7.0 \% \pm 6.41$ & $12.7 \% \pm 8.47$ \\
\hline IDM & $7.9 \% \pm 8.06$ & $3.8 \% \pm 5.31$ & $11.6 \% \pm 6.07$ \\
\hline IDM with & $11.1 \% \pm 8.73$ & $2.3 \% \pm 3.89$ & \\
\hline
\end{tabular}
IDM with RD $\quad 11.1 \% \pm 8.73 \quad 2.3 \% \pm 3.89$

$6 / 27(22.2 \%)$ IDM had mild to moderate RD; 5 of the 6 had no AF $P G$ and were delivered (34-37 wk) with a mean $\mathrm{L} / \mathrm{S}$ ratio of 2.4 . The absence of PG in AF PPL assays may be an adjunctive index to fetal lung immaturity in diabetic pregnancies.

TRANSIENT HYPOXEMIA IN THE CRYING NEONATE RECOVERYNG 953 FROM THE RESPIRATORY DISTRESS SYNDROME. RObert Dinwiddie, Savitri P. Kumar, Bakulesh D. $\overline{\text { Patel, }}$, and William W. Fox (Spon. by Jean A. Cortner) The Children's Hosp. of Philadelphia, Philadelphia, Pa.

Blood gas management of infants recovering from RDS is complicated by the fact that peripheral arterial puncture induces crying. Little is known about the effects of crying on blood gases in RDS. To quantitate the physiologic effects of crying, arterial blood gases (indwelling arterial lines), inspired oxygen concentration $\left(\mathrm{FiO}_{2}\right)$, intraesophageal pressure (Pes), heart rate, respiratory rate and blood pressure (B.P.) were measured in the control state, after 90 seconds of crying and at 10 minute followup in ten infants $(0.09-2.94 \mathrm{~kg})$ recovering from RDS. Mean $\mathrm{FiO}_{2}$ for all studies was $0.38 \pm 0.12 \mathrm{~S} . \mathrm{D}$. There was a significant decrease between control and crying values for $\mathrm{PaO}_{2}$ mean $\pm S$.D. $(89 \pm 15$ vs $70 \pm 17, \mathrm{P}<0.01)$. Follow-up mean $\mathrm{PaO}_{2}$ was $79 \pm 23 \mathrm{~mm}$ $\mathrm{Hg}$, not significantly different from the control value. During crying there was a significant increase in Pes $(7.3 \pm 3.3$ vs $\left.31.5 \pm 13.1 \mathrm{~cm} \mathrm{H} \mathrm{C}_{2} \mathrm{O}, \mathrm{P}<0.01\right)$ heart rate $(143 \pm 15$ vs $159 \pm \overline{19}$, $\mathrm{P} 0.05)$ and diastolic B.P. ( $39 \pm 10 \mathrm{vs} 44 \pm 10 \mathrm{~mm} \mathrm{Hg}, \mathrm{P}<0.05)$, these values returned to control levels at 10 minute follow-up. It is possible that the rise in Pes causes an increase in right to left shunting and results in the drop in $\mathrm{PaO}_{2}$. This study demonstrates a significant fall in $\mathrm{PaO}_{2}$ during crying in infants recovering from RDS and this should be considered before altering $\mathrm{FiO}_{2}$.
EFICACY OF TRACHEAL EXTUBATION IN NEONATES AT 2-3 CM $\mathrm{H}_{2} \mathrm{O}$ CPAP. William W. Fox, Lawrence S. Berman, and Thomas H. Shaffer (Spon. by Jean A. Cortner) Univ. of Penna. Sch. of Med., Depts. of Ped. and Physiol., and Phila. Naval Hosp., Phila., Pa.

The criteria for tracheal extubation of infants on CPAP therapy have not been clearly established. Previous recommendations suggest extubation at ZEEP whereas recent studies indicate that extubation at higher than $\mathrm{O} \mathrm{cm} \mathrm{H}_{2} \mathrm{O}$ CPAP would be more physiologic and would reduce total intubation time. To investigate the efficacy of extubation at higher levels of CPAP, 48 newborns $(0.95$ to $4.0 \mathrm{~kg}$ ) were extubated at 2 to $3 \mathrm{~cm} \mathrm{H} O$ following 1 to 50 days of CPAP therapy. Pre and post extubation measurements of $\mathrm{PaO}_{2}$, $\mathrm{PaCO}, \mathrm{pH}, \mathrm{FiO}$, and CPAP were made in all infants. No significant ${ }^{2}$ differences ( $P>0.05$ ) were found between pre and post extubation arterial blood gas values for all patients (mean \pm S.E.) $\mathrm{PaO}_{2}: 73.9 \pm 3.58$ vs $76.8 \pm 3.24 \mathrm{~mm} \mathrm{Hg}, \mathrm{PaCO}_{2}: 39.1 \pm 1.01$ vs $39.9 \pm 1.29 \mathrm{~mm} \mathrm{Hg}$, and $\mathrm{pH} ; 7.37 \pm 0.01$ vs $7.36 \pm 0.01$. Comparisons of pre and post extubation blood gas data with respect to birth weight $(<2.0 \mathrm{~kg}$ vs $>2.0 \mathrm{~kg}$ ) and intubation time ( $<8$ days vs $>8$ days) also indicated no statistical differences. Mean A - a oxygen differences for 43 infants showed a progressive decrease at 24,48 , and 72 hours post extubation. Six of the 48 infants required re-intubation within 48 hours following extubation. The results of this study indicate that newborns with respiratory disease requiring CPAP may be extubated at 2-3 $\mathrm{cm} \mathrm{H} \mathrm{H}_{2} \mathrm{O}$ with no significant changes in arterial blood gas values thus preventing prolonged intubation associated with weaning to zero $\mathrm{cm} \mathrm{H}_{2} \mathrm{O}$ CPAP. EFFECT OF HYPEROXIA ON SUPEROXIDE DISMUTASE (SOD) ACTAPY OF HYALINE MEMBRANE DISEASE (HMD) AND BRONCHOPULMONARY DYSPLASIA (BPD). Lee Frank, Anne P. Autor, Robert J.
Roberts, Univ. of lowa, Depts. of Ped. and Pharmacol. lowa City SOD is an enzyme thought to provide protection against superoxide anion, believed to be a prime agent of $\mathrm{O}_{2}$ pulmonary toxicity. A previous report (Ped. Res., In press) showed that exposure of normal young animals to hyperoxia resulted in a rapid increase in lung SOD activity. The lung SOD activity of infants with HMD, however, was no greater than that of their normal peers despite exposure to hyperoxic therapy. Because examination of hyperoxia in normal premature infants is not possible, more detailed animal studies were undertaken. Normal premature, term, and young rats ( $<3$ weeks of age) showed marked increases in lung 500 activity $(30 \%$ to $70 \%$ ) after a 24 -hour exposure in vivo to $85 \% \mathrm{O}_{2}$. Young hamsters and mice showed a similar response. Increased SOD activity was also observed when excised lung tissue was exposed in vitro to hyperoxia. Plasma or serum in the incubation medium was necessary for the increase in SOD activity. This observation led to a study designed to compare the ability of plasma from normal prematures vs. HMD infants to support this effect in rat lung tissue. Whereas plasma from 15 of 17 normal prematures supported this effect, plasma from 7 of 10 HMD infants proved ineffective. A plasma factor, therefore, may be important for the normal response of lung SOD to hyperoxia. Inability to generate increased lung SOD activity upon exposure to hyperoxic therapy may seriously compromise the lungs of infants with HMD (and BPD). Supported by GM 12675 and GM 07013 .

956 CONTINUOUS POSITIVE AIRWAY PRESSURE (CPAP) IN NEWBORN LAMBS. Giorgio Gabriele, David E。 Fixler, and Charles R. Rosenfeld, Dept. of Ped., Univ. of Texas, Southwestern Medical School, Dallas, Texas.

The headbox has been proposed as a noninvasive method of applying CPAP in the treatment of hyaline membrane disease. Its effects on cardiovascular hemodynamics are unknown. The purpose of this study was to evaluate tissue blood flows after application of headbox CPAP. Eight healthy, spontaneously breathing, newborn lambs breathing room aix were studied. Flows were measured with differently labeled $25 \mu$ radioactive microspheres infused into the left ventricle at pressures of $0,8,15$ and $0 \mathrm{~cm}$ of $\mathrm{H}_{2} \mathrm{O}$, applying the pressure in a random sequence. Application of CPAP did not significantly alter heart rate, left ventricular pressure or arterial gases. However, at 8 and $15 \mathrm{~cm} \mathrm{H}_{2} 0$, cardiac output. $(\mathrm{m} 1 / \mathrm{min} \cdot \mathrm{kg})$ fell from control of $215 \pm 16(\mathrm{SE})$ to $172 \pm 13^{*}$ and $179 \pm 14^{*}$ respectively; while jugular pressure $(\mathrm{mm} \mathrm{Hg})$ rose from $6 \pm 2$ to $19 \pm 2 *$ and $24 \pm 2 *$, and central venous pressure $(\mathrm{mm} \mathrm{Hg})$ from $1.3 \pm 0.4$ to $4.3 \pm 0.4^{*}$ and $6.6 \pm 0.4^{*}$, respectively. Blood flow to spleen, liver, GI tract, and heart did not change when CPAP was applied, but rose significantly after its removal. Cerebral blood flow (ml/min.gm) was unaltered, averaging $1.1 \pm 0.1,1.1 \pm 0.1$, and $1.0 \pm 0.1$ at control, 8 , and $15 \mathrm{~cm} \mathrm{H} \mathrm{H}_{2} \mathrm{O}$, respectively. Retinal blood flow (m1/min. gm) fell from $1.5 \pm 0.3$ to $1.0 \pm 0.2^{*}$ and $0.6 \pm 0.1^{*}$ at 8 and $15 \mathrm{~cm} \mathrm{H}_{2} \mathrm{O}$, respectively. These data demonstrate significant hemodynamic alterations when headbox CPAP is applied to newborn lambs with normal lungs, in particular, as much as a $60 \%$ fall in retinal blood flow while cerebral flow is unchanged. $(* \mathrm{P}<0.05)$. 
RELATION BETWEEN LUNG ETASTANCE AND EFFECTIVE ELAS-

957 IN PREMATURE AND FULL TERM NEWBORNS. Tilo Gerhardt, Eduardo Bancalari, (Intro. by W.W. Cleveland) Univ. of Miami, School of Med., Dept. of Pediatrics, Miami, Fl.

The purpose of this study was to determine in what proportion the reported higher effective elastance (E'rs) in prematures compared to full term infants is due to differences in lung elastance $\left(E_{\mathrm{L}}\right)$ or to differences in reflex activity and intrinsic properties of the muscles. E'rs was measured in 7 premature infants (mean gest. age 30 wks) on 19 occasions and in 10 full term newborns on 2l occasions. Each determination consisted of 5-10 air way occlusions at end expiration, measuring continuously esophageal pressure, (Pesoph), tidal volume and mouth pressure ( $\mathrm{P}_{\mathrm{MOP}}$ ). E'rs $\mathrm{cmH}_{2} \mathrm{O} / \mathrm{ml} \quad \mathrm{cmH}_{2} \mathrm{O} / \mathrm{ml} \quad \mathrm{cmH}_{2} \mathrm{O} \quad \mathrm{cmH}_{2} \mathrm{O}$

$\begin{array}{llllrl}\text { F. Term } & 0.44 \pm 0.02 & 0.22 \pm 0.01 & 5.1 \pm 0.3 & 11.0+0.7 & 2.2 \pm 0.1 \\ \text { Premature } & 0.80 \pm 0.05 & 0.43+0.02 & 5.1 \pm 0.3 & 9.4 \pm 0.6 & 1.9 \pm 0.1\end{array}$ $\begin{array}{llllll}\text { Premature } & 0.80 \pm 0.05 & 0.43+0.02 & 5.1+0.3 & 9.4+0.6 & 1.9+0.1 \\ \mathrm{P} & <0 . \overline{0} 01 & <\overline{0.001} & \mathrm{NS} & \overline{\mathrm{NSS}} & \mathrm{NS}\end{array}$ In both groups the resoiratory effort was the same during quiet respiration (Pesoph) and doubled during airway occlusion ( $P_{M O P}$ ) resulting in an E'rs which was near twice the $\mathrm{E}_{\mathrm{L}}$. This relation could be predicted by transforming the equation $\mathrm{E}^{\prime} \mathrm{rs}=\mathrm{P}_{\mathrm{MOP}} / \mathrm{V}_{\mathrm{T}}$ to $E^{\prime} r S=P M O P / C_{I} \times$ Pesoph to $E^{\prime} r S=E_{L} \times F_{M O P} /$ Pesoph. The results and the last equation indicate that E'rs is proportional to a passive component, $E_{\mathrm{L}}$, and to an active one, the increase in inspiratory effort during elastic loads (PMop/Pesoph). Since the increase in inspiratory effort was similar in full term and premature infants, the higher E'rs observed in prematures was due only to their higher lung elastance.

EFFECTS OF NEONATAL GASTRIC ASPIRATES ON THE RABBIT

958 LUNG. Edwin L. Gresham and William C. Kirby (Spon. by Robert L. Baehner). Indiana Univ. Sch. of Med. James W. Riley Hospital for Children, Dept. of Ped., Indianapolis Although it has become a common practice to feed normal newbor infants within a few hours of birth, controversy exists concerning the composition of the first feeding. Previous studies indicated that sterile water was less irritating than a $5 \%$ glucose solution $\left(D_{5} \mathrm{~W}\right)$ when instilled into the rabbit's lung; however, these early experiments did not evaluate the effects of regurgitated diets which were contaminated by gastric contents.

Gastric aspirates were obtained from newborn infants fed either sterile water or glucose solutions at $6 \pm 0.5$ hours after birth. A total of 40 rabbits were divided into four groups and sacrifice at $24 \mathrm{hrs}$. after tracheal instillation of $4 \mathrm{cc} / \mathrm{kg}$ of sterile water, $D_{5} \mathrm{~W}$ or gastric aspirates of the same solutions. $\mathrm{P}_{8} \mathrm{O}_{2}$ fell in all groups immediately after treatment but remained significantly below a control value of $81.7 \pm 0.9$ ( $\mathrm{mmHg} \pm \mathrm{SEM}$ ) at $24 \mathrm{hrs}$. only in those animals treated with the $D_{5} \mathrm{~W}$ gastric aspirate $(64.1 \pm 3.7)$ and aspirated sterile water $(65.2 \pm 3.1)$. Instillation of pure $\mathrm{D}_{5} \mathrm{~W}$ or sterile water resulted in a $\mathrm{P}_{2} \mathrm{O}_{2}$ at $24 \mathrm{hrs}$. of $83.0 \pm 2.0$ and $81.9 \pm 0.9$ respectively. Corrected lung volumes were smaller and lung weights greater in the gastric aspirate groups than in either the controls or those animals treated with unadulterated fluids. Histologic evidence of atelectasis and interstitial pneu monia was also more prominent in animals treated with aspirates.

These data indicate that sterile water contaminated with gastric contents is highly irritating to the rabbit lung and may have no advantage over $\mathrm{D} 5 \mathrm{~W}$ as a test meal for the normal newborn.

THE EFFECTS OF STABILIZATION OF THE RIB CAGE ON

959 RESPIRATION IN PRE-TERM INFAIITS. Ronald Hagan, A. Charles Bryan, M. Heather Bryan, EGordon Gel iston. Research Institute, Hospital for Sick Children, Toronto, Canada.

During REM sleep in infants there is irregularity of respiratory rate and tidal volume. This is associated with marked distortion of the rib cage, which significantly increases the work of breathing. We have studied the effect of stabilizing the chest wall by either continuous negative pressure (CNEG) or continuous positive airway pressure (CPAP). Motion of the rib cage (RC) and abdomen were monitored with AP magnetometers. The RC pair was used to assess the rate of RC distortion (RCD). This was taken as the slope of any inward RC movement during inspiration. The abdominal pair were used to assess diaphragmatic shortening. Tidal volume $V_{T}$ and inspiratory time Ti were measured with a pneumotachograph. Six normal pre-term infants were studied. There was a highly significant correlation between inspiratory time $\mathrm{Ti}$ and the rate of distortion $\mathrm{Ti}=0.572-0.003$ RCD ( $p<0.001)$. With both CNEG and CPAP there was a marked reduction in rib cage distortion $(p<0.001)$. This had two effects: 1. there was a significant increase in Ti $(p 0.001)$ and. hence a fall in respiratory rate and, 2 , for a given tidal volume the diaphragm had to shorten less. We conclude that chest wall distortion decreases the efficiency of the diaphragm and generates afferent information that disrupts the normal timing mechanisms. Part of the therapeutic value of CNEG and CPAP are therefore related to stabilization of the rib cage.
The Effect of Maternal Isoxsuprine on Fetal Rabbit

960 Lung Biochemical Maturation. William R. Hayden, Earl B: 0lson, Jr., and Richard D. Zachman, Univ. of Wis. Dept. Peds., Madison, $\overline{W I}$.

Clinical observations have suggested improved neonatal respir atory adaptation if the mothers had received isoxsuprine. Fetal rabbit lungs have improved pressure-volume curves $3 \mathrm{hr}$ after receiving isoxsuprine at 28 da gestation (Wyszogrodski et.al. Am. J. Ob. Gyn. 119:1107, 1974). Since maternal isoxsuprine is used frequently to stop labor, we investigated its effect on fetal lung lecithin synthesis indirectly by measuring lecithin content, the $\mathrm{L} / \mathrm{S}$ ratio of tracheal washes, and the activity of phosphorylcholine glyceride transferase (PCGT).

Fourteen pregnant rabbits of $26 \frac{2}{2}$ da gestation were injected with isoxsuprine, ( $3.33 \mathrm{mg} / \mathrm{kg}$ IM q8h for 3 doses) (7) or a saline placebo (7). Twenty six $\mathrm{hr}$. after the last injection does were sacrificed. The right fetal lung was assayed for lecithin and PCGT, and the left was lavaged for the $L / S$ ratio.

Maternal isoxsuprine had no effect on fetal birth wt. (31.6 vs $33.3 \mathrm{~g})$. Lecithin in fetal lungs of treated mothers was higher than controls $(6.67$ vs 5.35 यmoles/g wet wt, $p<0.001)$. The $\mathrm{L} / \mathrm{S}$ ratio of pulmonary lavage fluid of fetuses born of isoxsuprine injected mothers was 5.85 vs 4.08 for controls $(p<0.001)$. The controls $(2.57$ vs 3.56 nmoles lecithin $/ \mathrm{mg}$ protein $/ 30$ min., $p<0.001)$ The exact role of maternal isoxsuprine in fetal lung maturation is not known. However, the results of antenatal enhancement of pulmonary maturation must be evaluated carefully since several drugs can affect fetal lung biochemistry.

OXYGEN PROFILES IN RESPIRATORY DISTRESS. W.L.HOLman,

961 A. N. Krauss, P.A.M.Auld. Cornell Univ. Med. Coll., Dept. of Pediatrics, New York, N. Y. 10021.

Sequential determinations of arterial oxygen tension ( $\mathrm{PaO} 2)$ at increasing levels of inspired oxygen (FiO2) yield the oxygen profile of a patient. These values may be used to estimate the amount of venous admixture present at any FiO2. Venous admixture remains constant at all levels of $\mathrm{FiO} 2$ in pure venoarterial shunting. A rising (positive) profile may be obtained if venous admixture is due to a loss of diffusion or underventilation since these factors are overcome by progressively increasing fio2. Twenty-one infants with hyaline membrane disease (HMD) were studied on 47 occasions, 19 with CPAP/PEEP, and 28 on spontaneous breathing. Infants receiving continuous alveolar distending pressures showed a $33 \%$ rate of negative profiles (increasing venous admixture with increasing $\mathrm{FiO2}$ ) while spontaneously breathing infants had a 53\% rate. A negative profile suggests that resorption atelectasis with increased venoarterial shunting takes place as FiO2 is increased in patients with HMD. This suggests that the role of distending alveolar pressures is to stabilize alveoli which lack surfactant. It also suggests a need to reassess the 100\% oxygen breathing test as this study shows that shunt in $100 \%$ oxygen may be increased by performance of this test. Resorption atelectasis may also explain the "flip-flop" phenomenon--lower than expected $\mathrm{PaO} 2$ when attempts are made to lower FiO2. Loss of trapped gas by resorption is associated with fall in $\mathrm{PaO} 2$. Maintenance of alveolar volume is of prime importance in the therapy of HMD.

Lecithin-Cholesterol Acy1transferase Activity in Cord

962 Blood. Richard Inwood, Francis H.C. Tsao and Richard D. Zachman, Univ. of Wis., Dept. Peds., Madison, WI.

Lecithin-cholesterol acyltransferase (LCAT) is the enzyme that catalyzes the transfer of fatty acids from lecithin to cholesterol to form cholesterol esters and lysolecithin. Deficiency in LCAT has been implicated as a possible cause of pulmonary hyaline membrane disease.

We evaluated LCAT activity in the cord blood of premature an term neonates and compared it with adult values. LCAT activity was defined as the incorporation of $(14 \mathrm{C})$ cholesterol, equilibrated with plasma cholesterol, into $(14 \mathrm{C})$ cholesterol ester and was expressed as counts per minute of formed cholesterol ester/mg protein $/ \mathrm{hr}$. Product formation was linear with time (up to $6 \mathrm{hrs}$.) plasma protein concentration up to $8 \mathrm{~g} \%$, and nearly independent of total cholesterol concentration.

LCAT activity (cpm/mg protein $/ \mathrm{hr}$ ) averaged 104.6 for adult plasma. Cord plasma activity for 19 term infants ( $>37$ weeks) was $54.55+16.55$ vs $31.78+18.07$ for 12 premature AGA infants $\leqslant 37$ weeks). The difference was significant to $p<0.001$. There was no statistically significant sex difference for activity in term babies. One set of 38 week female twins had comparable activities of 67.61 and 56.64 , respectively. One mother and premature infant had values of 94.77 and 28.41 , respectively.

LCAT activity is probably dependent on fetal gestational development. Its role in recycling lecithin and maintaining the lipoprotein pool in neonates needs to be studied. We are currently evaluating its activity and postnatal levels in infants with RDS. 
PREDICTION OF PULMONARY SURFACTANT FROM GASTRIC

963 ASPIRATE. Pura Elor D. Isleta and Jeffrey $B$ coule (Boston City Hospital, Boston, Massachusetts)

Samples of amniotic fluid obtained at the time of cesarian section and gastric aspirate obtained within one hour of birth were evaluated for L/S ratio by TLC (Borer Method) and the bubble stability screening test (Shake test). Although a high correlation was obtained between TLC $\mathrm{L} / \mathrm{S}$ ratios determined from amniot ic fluid and gastric aspirate $(P<0.01)$, the shake test gave variable results.

The determination of gastric aspirate $L / S$ ratios by cold acetone extraction and TLC may prove helpful in assessing the functional maturity of the pulmonary surfactant sytem at birth. This information could be used as a criteria for the early initiation of continuous positive airway pressure.
966 EVIDENCE FOR LOWER AIRWAY CHEMO RECEPTORS IN NEWBORN LAMBS. Paul Johnson*, Andrew Wilkinson*, Richard Harding* and Mary McClelland*. Nuffield Institute for Medical Research, Oxford. (Spon. by George A. Gregory)

The ventilatory response to decreases in inspired oxygen (10 min. at $12 \%, 8 \%, 5 \% \mathrm{O}_{2}$ ) with and without added $\mathrm{CO}_{2}$ were studied in 14 unanesthetized lambs less than 24 hours old. All breathed through a tracheotomy and pneumotachograph and had duplicate measurements of $\mathrm{PaO}_{2}, \mathrm{PaCO}_{2}$ and $\mathrm{pH}$ at each gas mixture. Respiration, blood pressure and heart rate were measured continuously. Five lambs showed no significant ventilatory response to hypoxia. They hypoventilated and became apneic at an average $\mathrm{PaO}_{2}$ of 22 torr. The other 9 increased minute ventilation significantly $(+31$ to $+120 \%)$ at a $\mathrm{PaO}_{2}$ below 40 torr. Below 20 torr they also became apneic. $\mathrm{PaCO}_{2}$ fell from 33.5 to 21.5 torr. When $1.5 \% \mathrm{CO}_{2}$ was added to air, resting minute ventilation increased significantly to $196 \%$ of control with a rise in $\mathrm{PaO}_{2}$ from 72 to 91 torr but no significant change in $\mathrm{PaCO}_{2}$ in 10 of the 14 . When $\mathrm{FIO}_{2}$ was reduced in these 10 , minute ventilation further increased to $332 \%$ of control. $\mathrm{PaCO}_{2}$ fell to 23.5 and $\mathrm{PaO}_{2}$ to 25 torr. The remaining 4 required $5 \% \mathrm{CO}_{2}$ in order to increase the ventilatory response to $\mathrm{CO}_{2}$ above that observed with hypoxia alone. This suggests a lower airway receptor sensitive to airway $\mathrm{CO}_{2}$ with a direct or indirect interaction with alvelar oxygen.
USE OF NEBULIZED SURFACTANTS IN TREATMENT OF THE RESPIRATORY DISTRESS SYNDROME (RDS) OF INFANCY. Hallam H. Ivey, Stephen Roth, and John Kattwinkel, (Spon. by Robert M. Blizzard), Univ. of Va., Dept. of Ped., Charlottesville, and The Johns Hopkins Univ., Dept. of Biol., Baltimore. RDS of infancy is associated with a lack of the pulmonary surfactant complex. In a pilot study, we have nebulized a mixture of two phospholipids, dipalmitoyl phosphatidyl choline (DPPC) and dipalmitoyl phosphatidy1 glycerol (DPPG), to 6 babies with RDS. DPPC is the major component of the pulmonary surfactant system. DPPG is also a surfactant component, has the same surface tension lowering properties as DPPC, and when mixed with DPPC will increase interaction of DPPC with biologic membranes by a factor of 25 . It has also been shown that formation of vesicles by sonication of a DPPC emulsion will enhance fusion with biologic membranes. Previous studies utilizing surfactant aerosols in the treatment of RDS may have been unsuccessful because of failure to add another phospholipid to DPPC, need for vesicle formation by sonication, or failure to utilize CPAP to enhance delivery to atelectatic areas.

On 18 occasions, 6 infants (mean wt. $1.2 \mathrm{~kg}$ ) with severe RDS received a $0.8 \%$ solution of $90 \%$ DPPC $+10 \%$ DPPG which was sonicated in saline and administered by a $1.35 \mathrm{MHz}$ ultrasonic nebulizer in series with a Bournes respirator. AIl infants were on PEEP and had stable arterial blood gases in constant $\mathrm{F}_{\mathrm{I}} \mathrm{O}_{2}$ prior to nebulization. In the time period from onset to $1 \mathrm{hr}$. postnebulization (mean $3.7 \mathrm{hrs}$. ), the $\mathrm{F}_{\mathrm{I}^{\circ}} \mathrm{O}_{2}$ was decreased by $6 \%$ and the $\mathrm{PaO}_{2}$ increased by $17 \%$.

These preliminary results suggest that, following determination of optimum dose and timing of administration, aerosolization of a
DPPC-DPPG mixture may be a valuable tool in the treatment of RDS.

965

THE BIOLOGICAL HALF-LIFE OF LUNG LECITHIN AT BIRTH. Alan Jobe and Louis Gluck. University of California San Diego, Department of Pediatrics, La Jolla.

Lung lecithin of newborn rabbits was labeled with ${ }^{4} \mathrm{C}-$ or $3 \mathrm{H}-$ palmitic acid or $3 \mathrm{H}$-choline. The mother was injected with two isotopes: one 18-24 hrs. before delivery and the second $10 \mathrm{~min}$. before c-section. The isotope injected initially measures lecithin synthesized before birth; the second isotope is a pulse label for lecithin synthesized at C-section and term birth (late 30 - early 31 days). At various times the newborn lungs were lavaged with saline (alveolar wash, AW). A fraction of total lung (TL) was extracted for lecithin. Residual lung tissue was processed for microsomal (M) and lamellar body (LB) fractions by density centrifugation in sucrose. Lecithin was isolated by two dimensional chromatography and radioactivity expressed as CPM/ $/ M$ lecithin $\mathrm{PO}_{4}$. Biological half-life $(t / / 2)$ of each fraction was determined from a semi-log plot of the data. The $t / / 2$ of TL lecithin was about $60 \mathrm{hrs}$ for lecithin synthesized before birth and about 40 hrs for lecithin appearing at birth. Similar values for $t 1 / 2$ were found in the $M$ fraction. The $t 1 / 2$ for AW lecithin was about $40 \mathrm{hrs}$; however, no lecithin labeled at birth appeared in AW for $3 \mathrm{hrs}$ and release of this lecithin was not complete for about $20 \mathrm{hrs}$. These $T 1 / 2$ values are significantly longer than those published previously for adult animals and may be relevant to RDS. No $t \quad 1 / 2$ values have been presented previously in $M$ or LB fractions in either adult or newborn animals. work supported by NIH HD-04380, SCOR HL-14169 and HL-18375.
PULMONARY FUNCTION DURING RECOVERY FROM NEAR-DROWNING

967 Karl H. Karlson, Howard Eigen, Richard J. Lemen, William W. Waring Departments of Pediatrics and Physiology, Tulane University School of Medicine, New Orleans. The acute phases of near-drowning have been well documented, but little is known of the recovery process. We studied pulmonary function during recovery of an $11 \mathrm{y} .0$. boy who nearly drowned in brackish water and who required mechanical ventilation. He had no history of previous lung disease or allergy. He was evaluated 7 times in the period from 2 to 25 weeks after the accident. Each evaluation included history and physical examination, chest roentgenograms, and detailed pulmonary function studies. Physical examination of the chest was normal at two weeks and the chest roentgenogram was normal at four weeks after the near-drowning. Pulmonary function studies were consistent with moderate restrictive pulmonary disease that gradually resolved. Total lung capacity (by plethysmography and helium dilution) increased from $63 \%$ of predicted at 2 weeks to normal $(86 \%$ of predicted) by 14 weeks post near-drowning. Changes in vital capacity followed a similar pattern. The single breath oxygen test and the MMEFR were still abnormal at 25 weeks, the MMEFR being $65 \%$ of predicted. Diffusing capacity was normal throughout the recovery period. No significant response to inhaled bronchodilators was observed on five occasions.

We conclude that 1) radiological and clinical signs may not reflect the prolonged pulmonary injury resulting from neardrowning and 2) pulmonary function testing of victims of neardrowning is needed to assess accurately the patient's condition.

PULMONARY FUNCTION ABNORMALITIES IN SYMPTOM FREE 968 CHILDREN 10 YEARS AFTER BRONCHIOLITIS. Meyer Kattan Tom Keens, Jean-Guy Lapierre, Henry Levison and B.J. Reilly. Research Institute, Hospital for Sick Children, Toronto, Canada.

Fifteen children who had clinical and radiological evidence of bronchiolitis requiring hospitalization under 18 months of age and remained symptom free thereafter were studied to determine pulmonary function 10 years later. Lung volumes, forced expiratory volume in the first second (FEV 1 ), maximal midexpiratory flow rates (MMEF) and peak expiratory flow rates (PEFR) were measured before and after exercise. The maximal expiratory flow $\left(\dot{V}_{\max }\right)$ at $25 \%$ and $50 \%$ of vital capacity $\left(\dot{V}_{25}\right.$, $\left.\dot{V}_{50}\right)$ and the volume at which $\dot{V}_{\max }$ becomes density independent $\left(V_{i s o v}\right)$ were also measured. Chest $x$-rays were also taken. Ten children had abnormal arterial oxygen tensions $\left(\mathrm{PaO}_{2}\right)$. Five of the children with abnormal $\mathrm{PaO}_{2}$ had $V_{\text {isov }}$ measured and all five were abnormal. One child had exercise induced bronchospasm. We conclude that: 1. bronchiolitis may result in residual abnormalities in asymptomatic children which are unrelated to bronchospasm. $2 . \mathrm{PaO}_{2}$ and $V_{\text {isov }}$ are sensitive unrelated to bronchospasm. $2 . \mathrm{PaO}_{2}$ and $\mathrm{isov}$ are sensitives to detect these abnormalities and 3. there are residual lesions in small airways with or without changes in elastic recoil after bronchiolitis. 
ASYMMETRIC ACOUISITION OF RHESUS MONKEY PULMONARY STABILITY, DISTENSIBILITY AND IECITHIN PRODUCTION OVER GESTATION. Robert V. Kotas, Rodney E. Ulane, and Phillip M. Farrell, W. K. Warren Medjcal Res. Ctr., Tulsa Ok, and NICHD, NIH, Bethesda, Md.

Sixteen fetal Rhesus monkeys were studied between 111 and 150 days gestation (DG). An abrupt increase in deflation stability (DS) and pulmonary distensibility (PD) occurred between 140 and 150 DG ( $\overline{\mathrm{m}}$ Term $=165 \mathrm{DG}$ ). Cephalad lobes (UL) were more distensible and held more air during deflation than caudad lobes ( $L L$ ). c14 lecithin production per $\mathrm{mg}$. lung per hour from $\mathrm{C}^{14}$ choline was 60 to $80 \%$ higher in UL than in LI after 130 DG. Lung choline kinase, cytidyl transferase and choline phosphotransferase activities did not change significantly. Increase in PD lagged approximately 10 days behind the increase in DS. Morphologic changes (MC) consisting of thinning of air space walls and proliferation of capillaxies accompanied the increase in PD. Anatomic and biochemical maturation of the fetal Rhesus monkey lung seemed to advance at different speeds. The discordance between the development of DS and the acquisition of PD and MC in the normal animals and differences between lobes suggest that specific pulmonary tissue responsiveness to various stimuli may appear in different anatomical locations at different times over gestation. These findings may account for differential lung fluid clearance during the first breaths and may help to explain why hyaline membrane disease has been sometimes noted to clear in the UL before the LI.

970 . Kravath, Charles P.Pollak, Albert Einstein College of Medicine, Montefiore Hosp. \& Med.Ctr., Departments of Pediatrics and Neurology, Bronx, New York. (Spon. by L. Finberg).

Two children with sleep apnea, alveolar hypoventilation, apparent mental retardation, and poor growth associated with chronically en larged tonsils and adenoids were treated effectively with a nasopharyngeal tube (NPT) followed by tonsillectomy and adenoidectomy (T\&A). The effectiveness of this therapy and the relationship of the apneic spells to sleep was documented by polygraphic recording of sleep stage and respirations in one of the patients, and in both by correlation with serial arterial blood gases and $\mathrm{ph}$. The table shows the effect of the NPT and T\&A on arterial pH,PCO2, and $\mathrm{PO}_{2}$ of both patients and on the frequency per hour of apnea of greater than 10 seconds during REM sleep in one patient.

$\begin{array}{lcccc} & \mathrm{pH} & \mathrm{PCO}_{2} & \mathrm{PO}_{2} & \text { Apneas/Hr. (REM) } \\ \text { Without NPT } & 7.42,7.30 & 69,58 & 44,46 & 43 \\ \text { With NPT } & 7.48,7.45 & 39,39 & 65,66 & 0 \\ \text { After T\&A } & 7.40,7.38 & 37,37 & 95,82 & 0\end{array}$

$\begin{array}{ccccc}\text { After T\&A } & 7.40,7.38 & 37,37 & 95,82 & 0 \\ \text { Criteria for } T \& A \text { should be revised to include such patients. }\end{array}$ They fit the category of patients who hypoventilate during sleep, which includes those with Ondine's Curse,the Pickwickian Syndrome, idiopathic sleep apneas and perhaps some with the sudden infant death syndrome (SIDS). The NPT was so well tolerated by the patients, so easy to use, and so effective in diagnosis and treatment that we suggest that its use be evaluated in some of the other patients within this category, in particular those who might be at greater risk for SIDS.

\section{CHANGES IN AIRWAYS DYNAMICS DURING ANTIGEN INDUCED}

971 "ASTHMA' IN DOGS. Jean-Guy Lapierre, Milton Gold, Hospital for Sick Children, Toronto, Canada.

We induced "asthma" in anaesthetized, spontaneously breathing dogs by aerosols of Ascaris Suum extracts to which the animals were sensitive. The Ascaris produced a bronchoconstriction which was maximal within the first minute after challenge. Pulmonary resistance (RL) increased by $322 \% \pm 100 \%$ (mean \pm s.d.) while dynamic compliance (Cdyn) decreased by $73 \% \pm 4 \%$. By five minutes, static compliance showed a $31 \%$ decrease and there was a significant shift to the right of the pressure-volume curve. Thoracic gas volumes were slightly lower (18\%) during the bronchoconstriction. Static compliance and RL returned to normal within 15 minutes. However, there was a persistent tachypnea and Cdyn was still only $50 \%$ of control at 15 minutes. Intravenous atropine $(0.2 \mathrm{mg} / \mathrm{kg})$ given 30 minutes before the challenge significantly reduced the increase in RL. However, it had only a small influence on the observed change in Cdyn. In addition intravenous administration of atropine $(0.2 \mathrm{mg} / \mathrm{kg})$ during the period of bronchoconstriction showed no effect on Cdyn whereas it rapidly reduced RL. We conclude that there are two lesions in this model: 1. a vagally mediated large airways constriction that can be blocked by atropine and 2 . a nonvagally mediated lesion presumably in the small airways that may account for the persistent tachypnea and reduced Cdyn.
972 TORY FAILURE IN NEWBORN RABBITS. EdWard E. LaWSOn and Bradley T. Thach. (Spon. by Mary Ellen Avery) Dept. of Pediatrics, Harvard Medical School, Boston, Massachusetts. Newborn animals exhibit prolonged respiratory activity in response to asphyxia. However, functional respiratory failure (apnea followed by intermittent gasps) occurs after a brief period of hypexpnea. To study the transitional period from hyperpnea to gasping we measured inspiratory $\left(\mathrm{T}_{j}\right)$ and expiratory $\left(\mathrm{T}_{e}\right)$ duration and maximal inspiratory tracheal pressure $\left(\mathrm{P}_{\mathrm{m}}\right)$ following airway occlusion at FRC in rabbit pups 1-5 days old. EMG activity was recorded from multiple muscle groups of several additional animals.

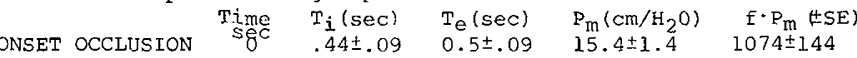

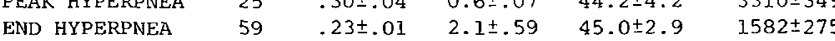

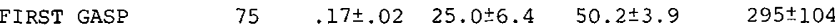

The decline in respiratory output $\left(\mathrm{f} \cdot \mathrm{P}_{\mathrm{m}}\right)$ during the transitional period preceding sasping is due to prolonged $\mathrm{T}_{e}$. In all animals a brief seizure (extensor rigidity and maximal EMG activity in limb and trunk muscles) occured at onset of apnea. The onset of gasping coincides with the loss of EMG activity in all muscle groups except inspiratory muscles. That $P_{\mathrm{m}}$ is preserved after onset of respiratory failure is evidence that central and peripheral inspiratory motor pathways remain fully functional. We conclude that asphyxial respiratory failure in rabbit pups results from depression of the central frequency regulators. Seizure activity appears to precipitate asphyxial apnea and may be directly involved in the central transition to respiratory failure.

\section{INCIDENCE OF RESPIRATORY DISTRESS SYNDROME AND}

973 COMPLICATIONS OF PREGNANCY. Kwang 5 . Lee, Arthur 1. Eidelman, $P_{0-1}$ Tseng, Stephen R. Kandall, and Lawrence M. Gartner. Dept. of Pediatrics, Albert Einstein Coll. Med., Bronx, N. Y. (Spon. by C. M. Edelmann, Jr.)

Four hundred and twelve infants with birth weights (BW) 501 to $2,500 \mathrm{gm}$ and gestational age (GA) of 36 wks or less were studied for the influence of both ruptured amniotic membranes greater than 24 hours (PROM) and maternal hypertension (MH) on the incidence of idiopathic respiratory distress syndrome (IRDS)

The occurrence of PROM and/or MH was associated with a significant decrease in the incidence of IROS only in infants with BW 1,501 to $2,500 \mathrm{gm}(37.4 \%$ in the no-complication (NC) group versus $12.8 \%$ in the complication (C) group; $p<0.01$ ) or GA of 33 to 36 weeks (35.2\% in the NC group versus $13.1 \%$ in the $C$ group; $p(0.01)$. PROM and $M H$ did not alter the incidence of IRDS in infants with BW $1,500 \mathrm{gm}$ or less $(54.0 \%$ in the NC group versus $56.9 \%$ in the C group; $p>0.05$ ) or with $G A$ of 32 wks or less $(53.1 \%$ in the NC group versus $62.2 \%$ in the C group; $p>0.05)$. Rupture of the membranes for greater than 72 hours had no greater effect on the incidence of IRDS than did PROM between 24 and 72 hours in either of the $B W$ or GA groups.

974 ENDOTHELIAL FILTRATION COEFFICIENT IN ISOLATED, PERFUSED PIGLET LUNGS, o. Robert Levine, Arthur B. Ritter, John Fahey and Eric Rall. New Jersey Medical Schoo1, Dept. of Ped. and Med., Newark, and Stevens Inst. of Tech., Hoboken, N.J.

In previous studies lung fluid accumulation rate during hemodynamic pulmonary edema was greater in intact puppies than in adult dogs; the estimated filtration coefficient $\left(K_{f}\right)$ was 10 20 times higher in puppies. This could be due to a difference in the endothelial membrane or in perivascular forces. In the present study endothelial filtration was assessed in the isolated perfused lungs of 6 piglets weighing $1.9-9 \mathrm{~kg}$ (av. $4.2 \mathrm{~kg}$ ). The animals were pentobarbitalized and the blood exchanged with a buffered, balanced $10 \%$ bovine serum albumin solution to obtain the perfusate. The heart and lungs were removed, the pulmonary artery (inflow) and left atrium (outflow) cannulated and connected to pressure gauges and to a gravity-feed perfusion system. The preparation was mechanically ventilated and suspended from a load-cell for continuous measurement of lung weight. The response of filtration rate (weight slope) to step-change in outflow pressure was recorded. $\mathrm{K}_{\mathrm{f}}$ was calculated assuming endothelial surface arealiung weight $\times 500 \mathrm{~cm}^{2} \mathrm{~g}^{-1}$. In 47 weight slope responses, $K_{f}$ averaged $0.117 \pm 0.018$ (2 SE) $\mathrm{cm} \mathrm{sec}^{-1}$, as compared to a comparable value in adult dogs of $0.050 \pm 0.015$ (Perl et al). The pulmonary endothelial $\mathrm{k}_{\mathrm{f}}$ in the immature lung may contribute to the greater rate of lung edema formation in intact immature animals. 


\section{5}

FIRST REPORTED CHILD WITH GOODPASTURE'S SYNDROME (GS) AND ATYPICAL RENAL IMMUNOFLUORESCENT FINDINGS. Gerald M. Loughlin and Lynn M. Taussig (Sponsored by Vincent Fulginiti), Univ. of Arizona, Dept. Pediatrics, Tucson.

GS (pulmonary hemorrhage and glomerulonephritis) has been reported in very few children. Linear depositions of anti-glomerulax basement membrane (GBM) antibody have been found in typical GS. The entity of atypical Goodpasture's Syndrome (AGS) has been reported rarely in adults but never in children. AGS is associated with granular deposition of immunological material along the GBM indicating circulating antigen-antibody ( $\mathrm{Ag}-\mathrm{Ab}$ ) complexes. An 8 year old female presented with hemoptysis, glomerulonephritis and renal failure. She had a 5 month history of cough and migratory lung infiltrates. The initial uremia was insufficient to account for lung hemorrhage. Renal biopsy showed widespread glomerular hyalinization, crescent formation and thickening of GBM. Immunofluorescent studies revealed granular deposition of IgG, IgM and C3 along the GBM suggesting circulating $\mathrm{Ag}-\mathrm{Ab}$ complexes. Lung biopsy revealed hemorrhage and fibrosis; immunofluorescent studies were negative. Rheumatoid factor was present early in her disease but reverted to negative. Serum was negative for circulating anti-GBM antibody, and serum $\mathrm{CH}_{50}$ and $\mathrm{C} 4$ were normal. ANA and LE preps have consistently been negative. Her renal function rapidly deteriorated and she is on dialysis. She was started on steroids and azathioprine and has had no further pulmonary manifestations. There has been no other organ involvement. This is the first pediatric patient reported with $A G S$ and the youngest reported with any form of GS.

EARLY MECHANICAL VENTILATION IN RDS WITH A PROLONGED

976 Inspiration. F.L. Mannino; B.H. Feldman; G.P. Heldt; N.A. DeLue; J.E. Wimmer; M.A. Fletcher; L. Gluck

Univ, of Calif. San Diego, Dept. of Ped., La Jolla, Calif.

72 infants with RDS were treated according to the following protocol when $\mathrm{PaO}_{2}<50$ torr, $\mathrm{FiO}_{2} 60 \%$ a) $<1500 \mathrm{gm}$ : intubated and intermittent mandatory ventilation (IMV) with pressure cycled ventilator (Baby Bird); b) $>1500 \mathrm{gm:}$ trial of mask or prongs CPAP until $\mathrm{FiO}_{2} 80 \%$, then treated as (a); c) initial ventilator rate 20-30 $\mathrm{CPM}$, pressure 25-35 $\mathrm{cmH}_{2} \mathrm{O}$, inspiratory time (IT) $1 \mathrm{sec}$. As infants stabilized over first few hours of treatment IT was gradually prolonged to $1.5-2$ sec. with corresponding diminution of rate to 10-15 CPM. PEEP was 2-4 $\mathrm{cmH}_{2} \mathrm{O} .29$ conventionally treated non-protocol infants were used for comparison.

Results of Groups:

b) <1500 gm

$\%$ of infants ventilated

Ventilator hrs., first 5 days, $\mathrm{FiO}_{2}>60 \%$

\%ventilator time at pressure $>35 \mathrm{cmH}_{2} \mathrm{O}$

\%ventilator time at pressure $21-31 \mathrm{cmH}_{2} \mathrm{O}$

Air leaks (overall)

Bronchopulmonary dysplasia

*Statistically sionificant differences

Results indicate that early IMV, without prior CPAP, improves the survival of infants $<1500 \mathrm{gm}$. IMV with prolonged inspiratory time allows use of lower pressures, lower cycling frequencies and lower $\mathrm{FiO}_{2}$ and results in decreased ventilatory complications. (Partly supported by NIH Grants HL-0111 and SCOR HL 14169)

\section{ASPHYXIA AS A CAUSE OF HYALINE MEMBRANE DISEASE.}

977 Gonzalo Mantilla, Barry V. Kirkpatrick, William H. Donnelly, Hugh W. Calderwood, Jack R. Hessler, and Donald V. Eitzman, Univ. of Fla. Coll. of Med., Depts. of Ped., Comp. Med., Path., Anesth., Gainesville.

To evaluate asphyxia as a significant determinant in hyaline membrane disease, premature monkeys were delivered by $\mathrm{C}$-section at a gestational age of 139-150 days, which is before the surge of surfactant as measured by the $\mathrm{L} / \mathrm{S}$ ratio. An experimental group was asphyxiated for five minutes at the time the infant was delivered. The control group was matched for age and both groups were managed the same way with oxygen as needed and ventilation. There was a significantly greater incidence of hyaline membrane disease on the basis of histopathology in the asphyxiated group. The other data analyzed includes right-to-left shunts and at the termination of the experiment ( 3 hours), when the animals were killed, the lungs were excised and several lung stability indices and the area of hysteresis was plotted during the generation of pressure volume curves, as described by Avery et al.

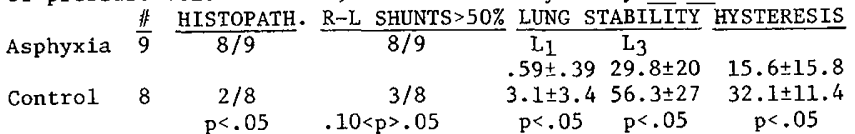

Asphyxia is a significant determinant of hyaline membrane disease in the premature primate and modifies the animal's ability to stabilize its lung. Management of the perinatal period in the high-risk premature infant is critical to the development of pulmonary disease and to the survival of this group of babies.
THE RESPIRATORY EFFECTS OF PEEP IN PREMATURE LAMBS

978 WITH SEVERE RDS. Sadi V. Matalon, Stephen J. Boros, Randall Ewald, Arnold S. Leonard and Carl E. Hunt. Depts. of Pediatrics, Phys Minnesota, Minneapolis, Minnesota. Spon. by Henry L. Nadler.

Nine premature lambs with severe RDS were ventilated with $100 \% \mathrm{O}_{2}$ at a constant $\mathrm{V}_{\mathrm{T}}$. The level of PEEP was varied from 0 to 5 or to $10 \mathrm{cmH}_{2} \mathrm{O}$ in an arbitrary order. $\mathrm{PAO}_{2}$ and $\mathrm{PACO}_{2}$ were obtained from continuous analys is of the expired air. Changes from baseline $(P E E P=0)$ were calculated using each animal as its own control.

\begin{tabular}{|c|c|c|c|c|}
\hline \multirow[b]{2}{*}{$\mathrm{mmihg}$} & \multicolumn{2}{|l|}{ Baseline } & \multicolumn{2}{|c|}{$\%$ Change from Baseline } \\
\hline & $\mathrm{PEEP}=0$ & $!$ & PEEP $=5 \mathrm{CmH}_{2} 0$ & PEEP $=10 \mathrm{cmH} 20$ \\
\hline $\mathrm{PaO}_{2}$ & $87 \pm 16$ & ! & $58 \pm 12.5 *$ & $-22.5 \pm 14 *$ \\
\hline $\mathrm{PaCO}_{2}$ & $51 \pm 10$ & & $-8 \pm 15$ & $7.2 \pm 11$ \\
\hline$(\mathrm{a}-\mathrm{A}) \mathrm{DCO}_{2}$ & $34 \pm 13$ & & $-56 \pm 19 *$ & $25 \pm 12 *$ \\
\hline$(A-a) \mathrm{DO}_{2}$ & $477 \pm 53$ & & $-6.5 \pm 5$ & $-5 \pm 12$ \\
\hline $\begin{array}{l}\text { Mean Pulm } \\
\text { Art. Pres }\end{array}$ & $21 \pm 2$ & & $-7 \pm 15$ & $4.5 \pm 11$ \\
\hline
\end{tabular}

\section{Art. Pres}

These results indicate that although there was an improvement of $V A / Q$ and shunting at a PEEP of $5 \mathrm{cmH}_{2} \mathrm{O}$ as shown by the decrease of $(\mathrm{a}-\mathrm{A}) \mathrm{DCO}_{2}$ and increase of $\mathrm{PaO}_{2}$, increasing the PEEP to $10 \mathrm{cmH}_{2} 0$ led to the worsening of the above parameters.

HEMODYNAMIC EFFECTS OF CONTINUOUS PRESSURE BREATHING

979 IN RABBITS. Pierre J. P. Monin, (Spon. by William oh). Faculte de Medecine, Universite de Nancy, Maternite Regionale "A Pinard", Laboratoire de Physiologie, Nancy, France.

Continuous pressure breathing is currently an accepted form of treatment for respiratory failure and could conceivably influof the neonates. Twenty-two rabbits weighing 2115 to 3890 grams were subjected to continuous bits weighing 2115 tive airway pressure (CPAP) and continuous negative pressure positive airway pressure (CPAP) and continuous negative press atrial pressure (RAP) increased with CPAP and decreased with CNP from its base line in correlation with the level of both CNP from its base line in cardiac output (CO) types of continuous pressure by thermodilution technique decreased significantly with measured by thermodilution technique decreased significantly with CPAP and CNP. Furthermore, the reduction in CO is directly proportional to the level of positive or negative pressures applied $12.5 \%$ and $16.1 \%$ of $\mathrm{CO}$ for $\mathrm{CNP}$ at $4,6,8 \mathrm{~cm} \mathrm{H} \mathrm{H}_{2} \mathrm{O}$ pressure, re$12.5 \%$ and $16.1 \%$ spectively). No significant difference in the reduction of CO was observed between CPAP and CNP at various levels of pressure. These data indicate that both CPAP and CNP result in
Q ASSISTEd VEnTtLATION IN THE NEONATE - COMPARISON BE-

980 TWEEN POSITIVE AND NEGATIVE RESPIRATORS. pierre $\underline{\mathrm{J}}$ p. Monin, William J. Cashore, David O. Hakanson Richard M. Cowett, and William oh. Brown University Program in Medicine, Women and Infants Hospital of Rhode Island, Department of Pediatrics, Providence, Rhode Island.

From October 1973 through October, 1975, 115 infants with severe respiratory distress syndrome and/or apnea were treated by assisted ventilation, using either intermittent positive pressure (IPP) or intermittent negative pressure (INP) respirators. Personnel were equally trained in the use of both types of respirators. Severity of disease between those treated with IPP and INP was comparable, as indicated by similarity in birth weight, gestational age, and criteria for assisted ventilation. Forty-four percent of the 58 infants treated by IPP and $46 \%$ of the 57 treated by INP survived. The incidence of patent ductus arteriosus (298 in IPP and $22 \%$ in INP) was not significantly different. In those who died the incidence of intracranial hemorxhage was also similar (17\% in each group). The IPP treated infants had a significantly higher incidence of pneumothorax ( $36 \%$ vs. $17 \%$, p. <.05) and broncho pulmonary dysplasia (BPD) diagnosed radiologically $(24 \%$ vs. $5 \%, p<.01)$. The severity of $B P D$ in the IPP infants was significantly greater than in the INP group. The concentration and duration of oxygen exposure between the two groups were similar, but the duration of ventilation was greater with IPP. These data indicate that assisted ventilation for respiratory failure in infants using INP has a lower incidence than IPP of two major complications, pneumothorax and broncho pulmonary dysplasia. 
981

BRONCHOPULMONARY DYSPLASIA AND MECHANICAL VENT ILATION OF R.D.S. Fergus M.B.Moylan, Susan K, Kramer, I David Todres, Danie1 C. Shannon. Harvard Medical School, Massachusetts General Hospital, Chíldren's Service, Department of Radiology, Boston.

The chest radiographs and charts of 99 surviving neonates, treated in a four year period with positive pressure mechanical ventilation (MV) for R.D.S. were reviewed. Alveolar rupture during MV occurred in $40 / 99(40 \%)$ of the patients. By radiographic diagnosis, bronchopulmonary dysplasia (BPD) developed in $27 / 40(68 \%)$ of the infants with alveolar rupture and in $3 / 59$ $(5 \%)$ of those without $(p<0.001)$. The incidence of alveolar rupture in the first three years reviewed was $31 / 61$ (51\%) compared to $9 / 38(24 \%)$ the fourth year $(p<0.01)$ while the incidence of BPD in the respective periods was $26 / 61(43 \%)$ and $4 / 38(11 \%)(p<0.001)$. The incidence of BPD in those patients with alveolar rupture in the two time periods was not significantly different: $23 / 31(74 \%)$ versus $4 / 9(44 \%)$. The decrease in incidence of $B P D$ was unrelated to duration of intubation, MV, $\mathrm{FIO}_{2} \geq 0.6$, inspiratory pressure $\geq 40, \geq 50 \mathrm{~cm} \mathrm{H} \mathrm{H}_{2} \mathrm{O}$ or positive end expiratory pressure $\geq 5 \mathrm{~cm} \mathrm{H} \mathrm{H}_{2} \mathrm{O}$. $\mathrm{BPD}$ in these infants with R.D.S. was related to alveolar rupture occurring during $M V$ and the fall in incidence of BPD paralleled the decrease in alveolar rupture.
984 ING RDS. Michael Obladen, T.Allen Merritt, Louis Gluck Univ. of Calif., San Diego, Dept. Ped., La Jolla.

Serial measurements of the following tracheal phospholipids were made in 22 infants during the first 5 days of life: Sphingomyel in (SPH), Phosphatidyl chol ine(PC), Phosphatidyl serine(PS), Phosphatidyl glycerol(PG). Hethods: 2-dimensional TLC, reflectance densitometry, phospholipid phosphorus. Tracheal L/S ratios at birth of 10 normal term newborns was greater than 3.0 and $P G$ was present. In 12 prematures with RDS $(1915+503 \mathrm{~g}, 33+3$ wks $) P G$ was not detected, initial tracheal L/S ratios were less than 2.3 in all cases and rose after $24 \mathrm{hrs} . \mathrm{Pl}$, the $2 \mathrm{~d}$ major phospholipid of prematures, showed a marked increase during recovery from RDS by day 5 (initially $16.7 \%$ by dens itometry or $7.4 \% \mathrm{P}$ to $38.3 \%$ or $19.7 \%$ respectively). PI/PS ratio increased from 1.02 to 3.84 .

\begin{tabular}{|ll|llllll|ll|}
\hline NEWBORNS & Age & SPH & PC & PS & PI & PE & $P G$ & $L / S$ & $P I / P S$
\end{tabular}
\begin{tabular}{llllllll|ll} 
Heal thy term I d & 9.7 & 41.6 & 11.6 & 15.7 & 6.7 & 15.0 & 5.20 & 1.43
\end{tabular}

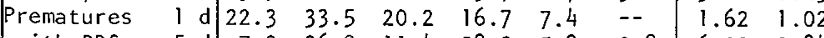
\begin{tabular}{ll|lllllllll} 
with RDS & $5 \mathrm{~d}$ & 7.3 & 36.9 & 11.4 & 38.3 & 5.8 & 0.8 & 6.39 & 3.84 \\
\hline
\end{tabular}

The table shows the distribution of the phospholipids as per cent density. In addition to PC, an acidic phospholipid, PG or $\mathrm{PI}$, seems necessary to maintain normal surfactant function. The increased PI at the 3rd to 5th day of RDS was associated with clinical improvement and might represent an adaptive mechanism, restoring alveolar stability in prematures. Work supported by NIH HD-04380, SCOR HL-14169 and Deutsche Forschungsgemeinschaft.

UNILATERAL DIAPHRAGMATIC HERNIA, MECHANISMS OF NEO-

982 NATAL DEATH. Richard Naeye, Stephen Shochat, Victor Whitman, M Jeffrey Maisel Penn State Univ Col. Med, Depts. Pathology \& Pediatrics, Hershey, Pa. 17033

Unilateral diaphragmatic hernia with displacement of abdominal viscera into the thoracic cavity is a common anomaly with a $12 \%$ immediate neonatal mortality and a $30-47 \%$ mortality after corrective surgery. In the current study, four infants with the defect developed severe ventilatory insufficiency immediately after birth. It was not relieved by oxygen and they died. Their lungs were also airless due to antemortem compression by mediastinal shift.

Surgical correction of the hernia in 6 infants permitted Iung expansion and adequate ventilation for a few hours. This honeymoon period was followed by cardiac failure, the reappearance of ventilatory insufficiency and death. Blood gases sampled proximal and distal to the ductus arteriosus revealed a fetal-type circulatory pattern with large right-to-left ductal shunting. Using histologic quantitation, 9 out of 10 infants with hernias had nearly twice as much muscle in pulmonary arteries as did matched controls. This abnormality increases pulmonary vascular resistance and apparently was responsible for pulmonary arterial hypertension, the abnormal ductal shunts and terminal cardiac failure. Hemorrhagic pulmonary edema contributed to the terminal ventilatory insufficiency.

983 ING IN ALLOXAN AND HEMODYNAMIC PULMONARY EDEMA. Robert M. Nelson, Edmund A. Egan, Bruce R. McIntyre, and Ira H. Gessner, Univ. of Fla. Coll. Med., Dept. of Ped., Gainesville. Alveolar epithelial permeability and fluid volume flux in the alveolus was studied in dogs with acute pulmonary edema, 10 produced by aortic constriction and saline infusion and 5 produced with alloxan (5,6 dioxyuracil). Alveolar permeability was determined from measurements of efflux of Blue Dextran, 125I-albumin and $57 \mathrm{Co}-\mathrm{cyanocobalamin}$ from a saline filled isolated lung segment and influx of $131_{I-a l b u m i n}$ from blood. Prior to edema the alveolar epithelium was impermeable to either albumin tracer or Blue Dextran and slightly permeable to cyanocobalamin. Slow absorption of saline occurred. Induction of edema increased fluid volume in the lung segment an average of $218 \%$ in hemodynamic and $291 \%$ in alloxan edema. In both forms of edema, ${ }^{131}$ I-albumin entered lung saline from the vascular system. In alloxan edema, ${ }^{125}$ Ialbumin diffused out of lung saline and appeared in blood, but in hemodynamic edema, 125 I-albumin did not efflux across alveolar epithelium and remained confined to the alveolus. Thus, two different mechanisms of alveolar flooding were observed. Alloxan edema results from an epithelial injury allowing free diffusion of water and all solutes between alveolus and interstitium. Hemodynamic edema results from discrete leaks which allow bulk flow of interstitial fluid into the airspace, but does not permit general transfer of protein solutes across the epithelium. Definition of two different patterns of pulmonary edema suggests that effective therapy may depend on the mechanism of production of the edema.
RESTORATION OF LUNG PRESSURE-VOLUME CHARACTERISTICS

985 WITH SURFACTANT. llachiko Ohi, Tom llesterberg, Masahiko Medicine, Department of Pediatrics, Los Angeles.

This study was designed to see if nebulization or instillation of surfactant (S) can restore the pressure-volume (PV) characteristic of adult rat lungs in which $S$ has been removed by washing with saline. Twenty-one adult rats were anesthetized, then sacrificed by exsanguination following which the lungs were removed, degassed, and a PV curve was obtained. After removal of $\mathrm{S}$, another PV curve was done. Then either natural or synthetic (dipalmitoyl lecithin, DPL) surfactant was administered by either an ullungs were ventilated at a rate of $40 / \mathrm{min}$. at $20 \mathrm{~cm} \mathrm{H}_{2} 0$ peak inspiratory pressure and with $7 \mathrm{~cm} \mathrm{H} O$ PEEP and then a final PV curve was obtained. The duration of ventilation was 2 hours in those receiving $S$ by nebulization and $5 \mathrm{~min}$. in those receiving $S$ by instillation. Results were analyzed from the deflation limb of the PV curve at 5 and $10 \mathrm{~cm} \mathrm{H}{ }^{0}$ and expressed as \% of original total lung capacity (TLC). Nebulization or instillation of DPL produced no significant increase in \% TLC. Nebulization of natural $S$ produced only a slight increase in \% TLC whereas instillation of natural $S$ produced a very striking increase of \% TLC: from 13.2 to 55.6 at $5 \mathrm{~cm} \mathrm{H}{ }^{0}$ and from $47: 9$ to 78.6 at $10 \mathrm{cr} \mathrm{H} O$. From these studies as well as others, it appears that nebulization is not an effective method for depositing material into the alveoli, on the other hand direct instillation of the material into the tracheal bronchial tron seems to be a promising method worthy further study.

ACCELERATED FETAL LUNG MATURATION AND INCOMPETENT

986 CERVIX. Donald M. Okada and Donald W. Thibeault, UCLA Schoot of Medicine, Harbor General Hospita?, Departments of Pediatrics and Obstetrics, Torrance, California.

The purpose of this study was to observe the growth and development of 40 fetuses of pregnancies with incompetent cervix weeks gestation (21) and those $<34$ weeks (19). The infants $>37$ weeks from pregnancies with IC were compared with infants of elective cesarean section of the same gestation. The infants < 34 weeks gestation were compared with infants $<34$ weeks randomly selected. The mean age at cerclage in infants born $>37$ weeks was 18 weeks. There was no difference in birth weight or body of the infants had either tibial torsion or metatarsus adductus (MA) whereas only one of the "control" infants had an MA. Nineteen infants born at $<34$ weeks gestation (mean 27.5) had a mater nal history of IC. Five of the mothers with IC did not have cerclages. In five pregnancies, Foam Stability Tests (FST) were performed on amniotic fluid and they were intermediate or positive. One of the fetuses was 24 weeks gestation with intact membranes and a lecithin/sphingomyel in ratio of 2.1 . The 19 infants had minimal or no Respiratory Distress Syndrome. "Nine infants died. The weights and lengths of the study and "control" infants were not different. Ten of the "control" infants had RDS and 12 died. Except for a possible increase of 1 imb abnormalities, IC and/or cerclages do not appear to effect somatic growth of the fetus, however, there does appear to be accelerated lung surfactant as early as 24 weeks gestation. trasonic nebulizer or instilled directly into the trachea. All (IC). The infants could be separated into two groups, those $>37$ length between the study and "control" infants $>37$ weeks. Five 
EFFECTS OF CONTINUOUS POSITIVE ATRWAY PRESSURE ON

987 PHYSIOLOGIC RESPONSES TO OLEIC ACDD-INDUCED IUNG INJURY IN DOGS: Alan B. Osher, Barbara Gothe, Robert L. Waymost, Daniel H. Simmons, (Spon. by Forrest H. Adams). UCLA School of Medicine, Departments of Pediatrics and Medicine, Los Angeles.

Spontaneous ventilation with continuous positive airway pressure (CPAP) has been widely used in the management of idiopathic neonatal respiratory distress syndrome (IRDS) because of its effect in raising arterial oxygen tension $\left(\mathrm{PaO}_{2}\right)$. Other potentialIy important physiologic consequences of $\mathrm{CPAP}$, e.g., effect on cardiac output and tissue oxygenation have not been systematically studied. The physiologic effects of CPAP of 5, 10, 15 and 20 $\mathrm{cm} \mathrm{H}_{2} \mathrm{O}$ during spontaneous ventilation was studied in 6 mongrel dogs with a simulated respiratory distress syndrome induced by intravenous infusion of oleic acid. Initially, hypoxemia was severe, with a mean $\mathrm{PaO}_{2}$ of $64 \mathrm{~mm}$ Hg \pm 3 while breathing 100 percent oxygen. $\mathrm{PaO}_{2}$ improved significantly at the two highest levels of CPAP (e.g., $\mathrm{PaO}_{2} 271 \mathrm{~mm} \mathrm{Hg} \pm 41$ at $20 \mathrm{~cm} \mathrm{H} \mathrm{H}_{2} \mathrm{CPAP}$ ). Mean Pvo2 rose from $37 \mathrm{~mm} \mathrm{Hg} \pm 1.5$ with no CPAP to $61 \mathrm{~mm} \mathrm{Hg} \pm 3.1$ at $20 \mathrm{~cm} \mathrm{H} 2 \mathrm{O}$ CPAP. Tissue oxygenation appeared to remain constant or improve with CPAP, since cardiac output, oxygen delivery and serum lactate were not significantly affected and $\mathrm{P}_{\mathrm{V}}^{-} \mathrm{O}_{2}$ rose significantly at the two higher levels of CPAP. However, significant hypoventilation occurred at all but the lowest level of CPAP. Mean $\mathrm{PaCO}_{2}$ rose from $44.1 \mathrm{~mm} \mathrm{Hg} \pm 1.8$ with no CPAP to $77.6 \mathrm{~mm}$ $\mathrm{Hg} \pm 6.8$ at $20 \mathrm{~cm} \mathrm{H}$ O CPAP. The explanation for the hypoventilation response to CPAP is consistent with increased work of breathing.

ERF EFECT OF TOLAZOLINE ON PERSISTENT HYPOXEMIA IN NEO-

988 NATAL RESPIRATORY DISTRESS. Sharada Pai, Dilip M. Purohit, Candace C. Caldwell and Abner H. Levkoff, (Spon. by Mitchell I. Rubin). Department of Pediatrics, Medical University of South Carolina, Charleston, South Carolina.

Tolazoline can produce pulmonary vasodilatation by its alpha adrenergic blocking effect and thereby improve ventilation/perfusion imbalance in the presence of pulmonary vasoconstriction. In the present study, this drug was used in 15 newborn infants with respiratory distress and persistent hypoxemia $\left(\mathrm{PaO}_{2}<50\right.$ torr in $100 \%$ oxygen) with or without assisted ventilation. The respiratory distress was due to hyaline membrane disease in 8 infants, persistent fetal circulation in 5, meconium aspiration in 1 and lobar atelectasis in 1 .

Acidosis was corrected with sodium bicarbonate and plasminate infusions. Tolazoline, $2 \mathrm{mg} . / \mathrm{kg}$. was infused by push via scalp vein. Arterial blood gases, blood pressure and heart rate were monitored. An immediate response, defined as rise in $\mathrm{PaO}_{2}>15$ torr was seen in all but 4 infants. In one infant, the $\mathrm{PaO}_{2}$ increased after 2 hours and in three others after 4 hours. In 15 infants, the mean rise in $\mathrm{PaO}_{2}$ within 10 minutes after infusion was 42.7 . Continuous infusion of tolazoline $(2 \mathrm{mg} . / \mathrm{kg} . / \mathrm{hr})$ was continued in 14 out of 15 infants. Side effects encountered were systolic hypertension in one, hypoglycemia in one and blotchiness of skin in all infants. Since 11 out of the 15 infants showed an immediate response and 10 of the 15 survived, it appears that a trial of tolazoline is indicated if $100 \%$ oxygen and assisted ventilation fail to maintain a $\mathrm{PaO}_{2}$ above 50 torr.

989

SYNDROME OF INAPPROPRIATE ANTIDIURETIC HORMONE

SECRETION IN NEONATES WITH LUNG DISEASE. Charles L. Paxson, Joan W. Stoerner, Susan E. Denson, Eugene W. Adcock, and Frank H. Morriss. (Spon. by R. R. Howell). Univ. of Texas Med. School at Houston, Dept. of Pediatrics, Houston.

The syndrome of inappropriate ADH secretion (SIADH) rarely has been reported in neonates. Since the advent of positive end expiratory pressure therapy, we have documented 9 episodes of SIADH in 5 patients among 657 high-risk neonates. Diagnostic criteria were: hyponatremic, hypo-osmolal serum; serum osmolality < urine; continued sodium excretion; absence of volume depletion; and apparently normal renal and adrenal function. This study was performed to identify the neonates at risk for development of SIADH.

The mean birth weight, gestational age, and postnatal age of the 5 patients were $1730 \mathrm{~g}, 32 \mathrm{wk}$, and 16 days, respectively. AlI 5 required positive pressure therapy for IRDS or pneumonia. SIADH was associated with pneumothorax, atelectasis, and pneumonia $(p<0.025)$, and occurred $3-40 \mathrm{hr}$ (mean $=13 \mathrm{hr}$ ) following radiographic evidence of acute loss of lung volume. SIADH occurred after $10 \%(3 / 31)$ of pneumothoraces, $14 \%(5 / 36)$ of episodes of atelectasis and/or pneumonia, and in $14 \%(1 / 7)$ of patients with CNS disease. However, SIADH was not significantly related to ventilator therapy, pneumomediastinum, interstitial emphysema, IRDS, ligation of patent ductus arteriosus, post-operative status, or airway pressures. Evidence of SIADH resolved in $\simeq 2$ days with treatment of atelectasis or pneumothoraces and fluid restriction. Conclusions: 1) Neonates as pre-term as 31 wh may experience SIADH, and 2) SIADH should be anticipated in neonates with atelectasis, pneumothorax, or pneumonia.
Q LUNG MATURITY, INTRAUTERINE GROWTH, NEONATAL ASPHYXIA AND SHOCK AND THE RISK OF HYALTNE MEMBRANE DISEASE. Roderic H. Phibbs, John A. Clements*, Robert K. Creasy*, George A. Gregory, Joseph A. Kitterman*, Mureen A. Schleuter ${ }^{*}$ and William H. Tooley. Department of Pediatrics and Cardiovascular Research Institute, University of California, San Francisco, San Francisco, CA 94143.

We measured maturity of the pulmonary surfactant system by the amniotic fluid foam test within 24 hours before delivery of 305 infants. Forty-one infants developed typical hyaline membrane disease (HMD) as diagnosed by a fixed set of criteria designed to exclude mild or questionable cases. Comparison of the foam test to outcome showed that it can be used to predict a graded risk of HMD, depending upon the degree of amniotic fluid reactivity. On this basis, we defined four risk categories, IV, III, II and I, with incidences of $71 \%, 33 \%, 12 \%$ and $<1 \%$ of HMD, respectively.

We studied cardiorespiratory function immediately after birth in 92 infants, including all in groups IV and III and half in group II. Half of those studied were asphyxiated and 30\% were in shock as determined by measurements of aortic and central venous pressures. Within each amniotic fluid risk group there is a close association between neonatal shock and occurance of HMD apparent$1 y$ independent of prenatal lung maturity. There is a similar association for birth weight or gestation and $\mathrm{HMD}$, but not for sex or mode of delivery.

EFFECT OF VITAMIN E ON OXYGEN TOXICITY TN THE MOUSE.

991 Fonald L. Poland and Padmani Kaxna, Wayne State Univ., Detroit, Michigan.

Thirty-two adult female white mice were divided into two groups. E- mice were fed a diet deficient only in vitamin E. E+ mice were given the identical mixture with $D-\alpha$-tocopherol added (100 $\mathrm{mg} / \mathrm{kg}$ of chow). D.fter 5 weeks of the special diets, both groups wexe maintained in $100 \%$ oxygen. The mice were reexposed to room air for $30 \mathrm{~min}$. daily. The mean survival time in oxygen of $\mathrm{F}^{+}$ mice was 4.9 days and of $E-m i c e$ was 3.3 days $(t=3.20 ; P \leq .005)$.

In the second group of experiments, 17 male mice were similarly pretreated with E+ (8) and E- (9) diets for 5 weeks and placed in $100 \%$ oxygen. Pairs of mice were sacrificed at 0 time and then daily until $96 \mathrm{hrs}$. Body weight, lung weight (wet and after dxying in $65^{\circ}$ oven), lung sodium content were measured. The body weight did not change significantly with time in oxygen. Both groups had an increase in lung wet weight with time from $5.5 \pm 0.5$ $\mathrm{mg} / \mathrm{Gm}$ mouse to $8.0 \pm 2.0 \mathrm{mg} / \mathrm{Gm}$ with overlap between groups. There was also an increase in lung sodium content and water content in both groups.

Survival times of mice exposed to 1008 oxygen were significantly related to the $\alpha$-tocopherol content of theix diets. Both $\mathrm{E}+$ and $\mathrm{E}-\mathrm{mice}$ developed pulmonary edema to a similar degree. Further studies will be needed to assay the cause of the greater susceptibility of vitamin $\mathrm{E}$ deficient mice to oxygen.

PULMONARY HYPERTENSION (PULHY) COMPLICATING, LUNG DIS-

992 EASE IN THE NEONATE: ROLE OF CHLORPROMAZINE (C) IN MANAGEMENT. Jeffrey J. Pomerance, Gray $\triangle$. B. Choto, and Christinia T. Ukrainski, Cedars-Sinai Medical Center, Dept. Ped, and UCLA Sch. Med., Los Angeles. (Spon. by B. M. Kagan)

C lowers pulmonary vascular resistance through inhibition of centrally mediated pressor reflexes, $\alpha$-adrenergic blockade, and direct action on blood vessels. Pulmonary vasculature is more sensitive to these actions than systemic vasculature. C has been used with temporary success in the treatment of PulHy complicating congenital diaphragmatic hernia.

Twenty-six infants received $\frac{1}{4} \mathrm{mg} / \mathrm{kg}$ of $\mathrm{C}$ via an umbilical artery catheter through which all blood gases were drawn. $C$ had no apparent depressant affect on respiration nor was systemic hypotension observed. Sixteen infants had either an equivocal or no response. Ten infants ( 3 with meconium aspiration, 1 with pneumonia and 6 with RDS) with birth weights between 0.81 and 4.0 $\mathrm{kg}$. and gestational ages between 26 and 41.5 wks. had an average $\mathrm{PaO}_{2}$ rise of $80 \mathrm{~mm}$. Hg $(27-202)$, measured between $\frac{3}{2}$ and 2 hours post $\mathrm{C}$ administration. No changes were made in ventilatory support during this interval. A rise in $\mathrm{PaO}_{2}$ of $\geq 25 \mathrm{~mm}$. Hg was interpreted as indicative of previously existing PulHy and a subsequent decrease in right to left shunting across the ductus arteriosus and/or foramen ovale. Cardiac catheterization to obtain confirmatory pressure data could not be justified.

PulHy may unexpectedly complicate neonatal lung disease. In infants whose clinical course is unsatisfactory, the use of $\mathrm{C}$ may be both diagnostic as well as therapeutic. 
993 EFFECTS OF CONTINUOUS POSITIVE AIRWAY PRESSURE (CPAP) ON PULMONARY FUNCTTON IN EARLY STAGES OF RESPIRATORY DISTRESS SYNDROME (RDS). C. Peter Richardson, Stephen O. Atherton, and August L. Jung. (Spon. by L. Glasgow). Dept. of Pediatrics, University of Utah, Salt Lake City, Utah.

Ten infants with clinically diagnosed RDS were studied using nitrogen washout and blood gas analyses while breathing at 5,10 , and $15 \mathrm{~cm} \mathrm{H} \mathrm{H}_{2} \mathrm{O} \mathrm{CPAP}$. All infants were under 12 hours of age and were intubated after demonstrating the inability to maintain $\mathrm{PaO}_{2} \geq 60$ torr on $\mathrm{FIO}_{2} \geq 0.6$.

As applied airway pressures were raised large increases in $\mathrm{PaO}_{2}$ were observed but changes in $\mathrm{PaCO}_{2}$ were not statistically significant. Results from the nitrogen washout tests indicate that the increases in $\mathrm{PaO}_{2}$ were consistent with 1 ) increases in functional residual capacity (FRC) and 2) alterations in the distribution of ventilation. Increases in FRC probably caused decreases in intrapulmonary shunting since most of the volume increases were measured as part of the slowly ventilated lung space whereas the rapidly ventilated lung space remained relatively constant. Also, as airway pressures were increased a greater portion of the cases, at the higher airway pressures, alveolar ventilation of the rapidly ventilated space was reduced to levels indicating lveolar overdistention.

The results indicate that determining optimal CPAP levels must be made on an individual basis and may require pulmonary function measurements in addition to blood gas analyses. EFFECTS OF CONTINUOUS DISTENDING AIRWAY PRESSURE INFANTS WITH AND WITHOUT PATENT DUCTUS ARTERIOSUS (PDA). Kor Sankaran, Maria Davi, Carey Lee, Victor Chernick, Henrique Rigatto, Univ. of Manitoba, Dept. of Pediatrics, Winnipeg, Canada.

We studied the effects of CDP on ventilation and lung mechanics in 5 preterm infants $(n=10)$ with and $5 \quad(n=10)$ without PDA. After a control period, we subjected the infants to -4 and $-8 \mathrm{~cm} \mathrm{H}_{2} \mathrm{O}$ of continuous negative pressure (Isolette incubator) for 10 minutes each. Ventilation was measured using a nosepiece and a screen flowmeter, and intraesophageal pressure, using an air filled balloon. We determined minute ventilation $\left(\hat{V}_{E}\right)$, alveolar $\mathrm{PO}_{2}$ and $\mathrm{PCO}_{2}$, functional residual capacity (FRC), and specific dynamic compliance (Csp). During control period, $\mathrm{P}_{\mathrm{CO}}$ was higher in PDA infants than in those without PDA $(\mathrm{p}<.025)$. $\dot{\mathrm{V}}_{\mathrm{E}}$ and $\mathrm{Csp}$ were lower in the PDA group $(\mathrm{p}<.05)$. With pressures of -4 and $-8 \mathrm{~cm} \mathrm{H}_{2} \mathrm{O}, \dot{\mathrm{V}}_{\mathrm{E}}$ and $\mathrm{P}_{\mathrm{A}} \mathrm{CO}_{2}$ increased and $\mathrm{Csp}$ decreased by more than $50 \%$ in both groups $(p<.05)$. Csp decreased more in infants without PDA $(p<.05)$ due to greater increase in FRC $(p<.05)$. We suggest: 1$)$ preterm infants with PDA hypoventilate and have lower Csp than those without PDA; 2) CDP induces similar deleterious effects on ventilation and lung mechanics in both groups of infants; and 3) benefits, if any, of using CDP in the treatment of pulmonary edema refractory to medical therapy in infants with PDA are probably due to changes other than in lung mechanics.

\section{A PROSPECTIVE LONGITUDINAL STUDY OF PULMONARY} 995 FUNCTION STATUS IN CHILDREN SURVIVING PNEUMOCYSTIS CARINII PNEUMONITIS (PCP). Shyamal K. Sanyal, William I. Mariencheck, Walter T. Hughes, and Scott Harris. St. Jude Children's Research Hospital, Cardiopulmonary and Infectious Diseases Services, Memphis, TN.

It has bcen reported that PCP, a diffuse form of bilateral alveolar disease, may cause significant interstitial fibrosis with permanent sequelae. Clinical, radiographic and pulmonary function data were studicd prospectively in 19 children with PCP (proven by lung aspirate) at hospital discharge, and at 1- and 3-mo intervals for 1 year. Within 1 month of hospital discharge, all patients werc ambulatory without respirat ory symptoms, the chest roentgenograms were free of pulmonary infiltratcs, and blood-gas profiles returned to normal in all but 3 pulmonary infiltrates, and blood-gas profiles returned to normal in all but 3
patients in whom mild hypoxemia $\left(\mathrm{PaO}_{2}, 75-89 \mathrm{~mm} \mathrm{Hg}\right)$ and intrapulmonary patients in whom mild hypoxemia $\left(\mathrm{PaO}_{2}, 75-89 \mathrm{~mm} \mathrm{Hg}\right)$ and intrapulmonary
right-to-left shunt $(11.8$ to $14.4 \%)$ persisted. By contrast, in 16 of 19 children, the vital capacity (VC) and total lung capacity (TLC) were below 2 S.D. of predicted normal values (based on height) and bclow 1 S.D. in 2 others. Values for MVV and FEV 1 generally paralleled the decreases in VC and TLC. In 15 patients, the diffusion capacity showed reduction below 1 S.D. of predicted normal. These diffusion capacity showed reduction below 1 S.D. of predicted normal. These
findings suggested restrictive lung disease as well as impairment of gas transfer. Five patients died within 3 months of recovery from PCP; in each patient the lung functions had been abnormal. In 13 survivors, the pulmonary functions returned to normal by the end of 3 months in 9,6 th month in 3 , and 12 th month in 1 . The expiratory flow values were normal in all the 19 patients excluding obstructive lung disease. We conclude that (i) PCP produces a restrictive type of lung disease and impairment of diffusion capacity that persist during convalescence, (ii) in survivors, the abnormal pulmonary functions return to normal within 1 year from acute stage of the disease.
996 THE EFFECTIVENESS OF ASSISTED LIOUID VENTILATION IN PREMATURE LAMBS. Thomas $\mathrm{H}$. Shaffer S. David Rubenstein, Gordon D. Moskowitz, and Maria Delivoria-papadopoulos. Univ. of Penna., Sch. of Med., and Drexel Univ. Philadelphia, Pa. 19174.

The clinical and physiological significance of liquid ventilation as a therapeutic modality for treating respiratory distress was assessed in 9 premature lambs of 135 days gestation. The lambs were delivered by cesarean section following spinal anesthesia. Arterial and venous catheters and a tracheal cannula were placed before clamping the cord. Following assisted gas ventilation $\left(\mathrm{FiO}_{2}=1.0\right)$ for 20 minutes, the lambs were ventilated with $\mathrm{FC}-80$ liquid $\left(\mathrm{PO}_{2}=500 \mathrm{mmHg}, \mathrm{PCO}_{2} \mathrm{r}\right.$ $5 \mathrm{mmHg}$ ) for periods of 1 to 3 hours. During normothermic liquid ventilation arterial blood gases for all lambs were stable in the range of $\mathrm{PO}_{2} 175-200 \mathrm{~mm} H, \mathrm{PCO}_{2} 38-41$ mmHg and $\mathrm{pH}$ 7.31-7.35. During post-1iquid assisted gas ventilation $\left(\mathrm{FiO}_{2}=1.0\right)$, peak intratracheal pressure sig-
nificantly decreased from $36.2-3.5 \mathrm{SE} \mathrm{cm}$. $\mathrm{H}_{2} \mathrm{O}$ pre-1iquid to $25.0-2.6 \mathrm{SE} \mathrm{cm}$. $\mathrm{H}_{2} \mathrm{O}$ post-liquid period ( $\mathrm{p}<0.001$ ) with identical tidal volume and respiratory rates. These results indicate that liquid ventilation provides an effective means for reducing inflation pressures in addition to maintaining stable blood gases in premature lambs. Future development of this method may become applicable to the human neonate with respiratory failure after current techniques fail to sustain ventilation. (USPHS Grants\#HL17154\#HD07135).

DIPALMITOYL LECITHIN AEROSOL IN R.D.S. Daniel C.

997 Shannon, J. Bert Bunne11. Harvard Medical School, Massachus

Twenty infants, mean age $22 \mathrm{Hr}$, B.w. $2.03 \mathrm{Kg}$, G.A. 34 Wks, with severe R.D.S. were admitted to a blind, randomized trial of $1 \%$ dipalmitoyl lecithin in water aerosol (DPL) compared to a water aerosol (W) to determine whether lung function improved with DPL. We estimated that deposition of $3 \mathrm{mg} / \mathrm{Kg}$ DPL (4l $\AA^{2}$ ) would provide an alveolar monolayer. Aerosol delivery rate was designed to maintain this level of deposition by infusing $20 \mathrm{ml} /$ $2 \mathrm{Hr}$, every $8 \mathrm{Hr}$ for 3 doses into a MHZ ultrasonic generator, inline with relaxants. We omitted six infants (3 DPL) from final analysis due to complications e.g. PDA. The initial alveolararterial $\mathrm{PO}_{2}$ gradient $\left(\mathrm{A}-\mathrm{a} \Delta \mathrm{PO}_{2}\right)$ at $\mathrm{FIO}_{2} 1.0$ was $466 \mathrm{~mm} \mathrm{Hg}$ (DPL) and $461 \mathrm{~mm} \mathrm{Hg}(\mathrm{h}) ; \mathrm{P}_{\mathrm{CO}}$ was $34 \mathrm{~mm} \mathrm{Hg}$ (DPL), 32 (W). After dose $\# 1$, the $\mathrm{A}-\mathrm{a} \Delta \mathrm{P}_{2}$ fell $8 \mathrm{~mm} \mathrm{Hg}$ (DPL) and rose $21 \mathrm{~mm} \mathrm{Hg}(W)$; ( $\mathrm{p}$ of difference $=0.2)$. After dose $\$ 2$, the A-a $\Delta$ PO2 fell $30 \mathrm{~mm} \mathrm{Hg}$ $(p=<.05)$ in DPL treated and rose $23 \mathrm{~mm} \mathrm{Hg}(\mathrm{p}=.1)$ in $\mathrm{k}$ treated ( $p$ of difference $=\left\langle .02\right.$ ). After dose $" 3$, the A-a $\triangle \mathrm{PO}_{2}$ fell 44 $\mathrm{mm} \mathrm{Hg}(p=\langle .05)$ in DPL treated and rose $9 \mathrm{~mm} \mathrm{Hg}(p=.2)$ in $\mathrm{W}$ treated ( $p$ of difference $=<.02$ ). $\mathrm{PCO}_{2}$, static $\mathrm{CL}_{\mathrm{L}}$ and passive $V C$ (volume to inflate from FRC to static $P_{t p}$ of $30 \mathrm{~cm} \mathrm{H}_{2} 0$ ) did not change. Lower $A-a \triangle P_{0} 2$ values after $1 \%$ DPL aerosol were not maintained for the 8 hours between doses.

98 NEONATAL LUNG DEFENSE MECHANISMS. M. Sherman, E. Goldstein, W. Lippert and R. Wennberg. Departments of Pediatrics and Medicine, School of Medicine, Univ. of California, Davis.

Enhanced susceptibility to bacterial pulmonary infection is a major complication of the neonatal period. In adult animals the pulmonary alveolar macrophage(PAM) has a major role in preventing bacterial infection. We investigated the function of the PAM in newborns by expgsing rabbits of varying postnatal age to high concentrations (. $10^{8}$ bacterial $\left./ \mathrm{ft}^{3}\right)$ of a Staphylococcus aureus aerosol for a 30 minute period. The aerosol chamber was designed to produce uniform infection and deposited $>10^{\circ}$ bacteria per lapine lung. At 0 and 4 hours after infection, the number of viable bacteria was determined in homogenates of the left lung by pour plate technique. The bacterial clearance was calculated comparing the numbers of viable bacteria. The right lung was perfused and fixed in situ, and bacterial ingestion rates were determined and compared at 0 and 4 hours by histologically ascertaining the intracellular or extracellular location of 100 bacteria. The results are summarized below:

\begin{tabular}{lllll}
\hline Post Natal Age (Days) & I & 2 & 4 & 14 \\
\hline Clearance (\%) & -0.6 & 14.4 & 52.4 & 70.3 \\
Phagocytized 0 hr (\%) & 29 & 54 & 32 & 49 \\
Phagocytized 4 hr (\%) & 63 & 77 & 61 & 86 \\
\hline
\end{tabular}

The results demonstrate a severe impairment in killing of ingested bacteria by the PAM. The enhanced susceptibility to neonatal pulmonary infection can be explained, at least in part, by this severe impairment. 
999

DISCORDANT LUNG MATURATION AND CORTICOSTEROID LEVELS IN TWINS. Barry T.Smith and Dennis $W$. Werthington. (Spon by M.W. Partington)

Queen's University, Departments of Paediatrics and $\mathrm{Ob}-$ stetrics, Kingston, Ontario, Canada.

Endogenous fetal glucocorticoids may play a role in lung maturation as evidenced by accelerated lung development in conditions with increased fetal steroid levels. We describe twins, one of whom demonstrates this relationship in reverse: delayed lung maturation with subnormal steroid levels.Born at $33 \mathrm{wk}$, twin $\mathrm{A}$ weighed $1960 \mathrm{~g}$ and experienced no pulmonary or other problems; twin $\mathrm{B}$ weighed $720 \mathrm{~g}$ and died at $3 \mathrm{hr}$ with symptoms of IRDS. Twin B was anencephalic and at autopsy the adrenals were markedly hypoplastic without identifiable provisional cortex.The lungs were small, hemorrhagic with immature alveolar epithelium. Amniotic fluid and cord blood were studied from each twin:

\begin{tabular}{lccc} 
CORD BLOOD: & $\begin{array}{c}\text { TWIN A } \\
\text { (normal) }\end{array}$ & $\begin{array}{c}\text { TWIN B } \\
\text { (anencephalic) }\end{array}$ & $\begin{array}{c}\text { NORMAL RANGE } \\
( \pm S . D .)\end{array}$ \\
Cortisol(ng/ml) & 34.7 & 4.6 & $31.8 \pm 4.5$ \\
cortisone" & 83.2 & 26.6 & $79.4 \pm 5.0$ \\
AMNIOTIC FLUID: & & & \\
\hline L/S ratio & 4.0 & 0.9 & $>2.0$ \\
cortisol(ng/ml) & 19.6 & 3.9 & $20.7 \pm 8.9$ \\
cortisone" & 16.5 & 16.6 & $14.5 \pm 6.7$
\end{tabular}

These findings provide further support for a role of

fetal glucocorticoids in normal pulmonary development.

\section{0}

NEONATAL APNEA J. Baldwin Smith, Charles P. by Jimmy L. Simon, Bowman Gray School of Med-

The significance of apnea in the neonatal period has not been determined. Seventy-three infants with clinically noted apnea were studied by means of continuously recorded (minimum- $4 \mathrm{hr}$., maximum- $24 \mathrm{hr}$.) EEG, EKG, instantaneous heart rate change, respirations (by chest pneumograph), expired $\mathrm{CO}_{2}$ (by mass spectometer analysis) and extraocular movements. Forty-one neonates had apneic episodes $20 \mathrm{sec}$. Or longer. Four types of apnea patterns were detected. The most common type $(\mathrm{N}=22)$ was an exag geration of normal periodic respirations. This type of apneic episode, which was seen most often in premature infants may be related to an immaturity of respiratory centers. A second type $(\mathrm{N}=4)$ was associated with clusters of rapid respirations rather than a gradual change in depth. The breathing pattern did not improve during the neonatal period. A third type $(\mathrm{N}=3)$ was associated with a single fixed pattern similar to a central neurogenic respiratory pattern. A fourth type $(\mathrm{N}=12)$ was associated with an electroencephalographic seizure. Infants with type 2 pattern all developed signs of severe neurological dysfunction. Infants with type 4 pattern had a prognosis related to the cause of their seizures. EEG polygraph evaluations of apneic episodes can be of value in the determination of the pathogenesis and prognosis of neonatal apnea.

1001 THE FOAM STABILITY TEST (FST) ON GASTRIC ASPIRATE IN THE PREDICTION OF RESPIRATORY DISTRESS SYNDROME (RDS) Michael E. Speer, Anthony J. S. Corbet, and Arnold J. Rudoloh. Baylor College of Medicine, Dept. of Ped., Houston.

The amniotic fluid L/S ratio and FST have been shown to correlate with fetal lung maturity. Studies have also demonstrated cor relation between the L/S ratio of neonatal gastric aspirate and the development of RDS. Samples of gastric aspirate were collected at delivery or within 30 mins. thereafter from 194 infants $\leq 36$ weeks gestation. The samples were frozen at $-20{ }^{\circ} \mathrm{C}$ until the FST was performed. Samples containing gross blood or meconium were excluded. An inadequate sample volume $(<0.5 \mathrm{ml})$ was obtained in 45/194 (23.2\%). A further 27 samples had negative FST at a $1: 2$ dilution but were of inadequate volume to be tested at lower dilutions. These were also excluded from analysis. Of the 122 adequate samples 44 were + at $1: 2,43$ were + at a dilution $<1: 2$, and 35 were negative at all dilutions. RDS was found in $2.3 \%, 23.3 \%$ and $22.8 \%$ of each group respectively. The predictability of the FST was found to be significant only if the test was $+1: 2 \quad(p<0.01)$ There was no significant difference in the incidence of RDS from that expected if a negative FST was found or if + dilutions at $<1: 2$ were present. Our data indicate that the usefulness of the gastric aspirate FST is limited by the difficulty in obtaining an adequate sample volume and the lack of correlation between positive or negative results to the development of RDS at lower dilutions than $1: 2$. The test appears to be of value only if the $1: 2$ dilution is + , which correlates with previous FST amniotic fluid studies although not with other studies utilizing neonatal gastric aspirate FST.
1002

THE ADEQUACY OF RESPIRATORY COMPENSATION TO INCREASED LUNG VOLUME IN THE INFANT. Ann R. Stark, Elizabeth R. Brown, and IVan D. Frantz, ITI. (Spon. by H.W.

Taeusch, Jr.) Dept. of Pediatrics, Harvard Medical School, Boston

We have examined the effect of increasing lung volume on respiratory center output and vagal activity in ten normal, sleeping, full-term infants during the first three days of life. Inspiratory time $(t i)$, tidal volume, minute ventilation, static compliance, and maximum inspiratory pressure $\left(\mathrm{P}_{M}\right)$ after occlusion at FRC were measured before and after application of $5 \mathrm{~cm} \mathrm{H}_{2} \mathrm{O}$ negative pressure (CNEG) to the thorax. The mean increment in lung volume with CNEG was $8.5 \pm 2.0$ (SD) $\mathrm{ml}$. Respiratory rate $(55 \pm 8$

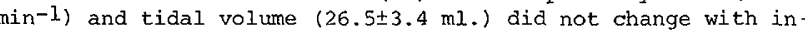
creased lung volume. Ventilation decreased in six infants on CNEG and increased in two, with no significant change in the mean $(396 \pm 23$ (SE) to $366 \pm 28 \mathrm{ml} / \mathrm{min} / \mathrm{kg}$ ). $\mathrm{P}$, indicating respiratory centex output, was also unaffected by ${ }^{\mathrm{M}} \mathrm{CNEG}$. The relationship of $t i$ during airway occlusion at FRC to ti of the preceding unoccluded breath did not change, suggesting that vagal influence does not change with the alteration of lung volume. Total thoracic compliance, determined from mask pressure in passive expiration following occlusion at known volumes, was unchanged $14.05 \pm$ $1.85 \mathrm{ml} / \mathrm{cm} \mathrm{H}_{2} \mathrm{O}$ ) with CNEG. We conclude that lung volume increases of $30 \%$ of tidal volume do not influence respiratory center output or vagal tone.

The lung volume increment with CNEG was 28-768 of that expected from compliance measurements. This smaller than expected lung volume change with CNEG may represent an active compensatory response of the respiratory muscles or glottis to distending pressure,

THE EFFECT OF OXYGEN ON TRACHEOBRONCHIAL SECRETION OF 1003 LYSOZYME IN NEWBORN INFANTS. Osvaldo Stoliar, Avroy Fanaroff. Thomas Boat.Case Western Reserve University School of Medicine, Dept.of Ped. \& Rep. Biology, Cleveland.

Secretion of lysozyme by explanted human neonatal tracheal epithelium is depressed by exposure to $80 \%$ oxygen for more than 72 hours (Boat, Pediatr. Res. 7:607,1973). To determine whether this effect occurs in vivo we measured lysozyme in tracheobronchial secretions collected from 9 term and 16 preterm infants at the time of routine suctioning through endotracheal tubes. Infants were on mechanical ventilators for RDS, apnea, asphyxia or post operative care. Infants with infection were considered separately. Lysozyme levels, expressed as $\mathrm{ug} / \mathrm{mg}$ secretion, were significantly depressed by high oxygen ( $\mathrm{FiO}_{2}>0.8>48$ hours).

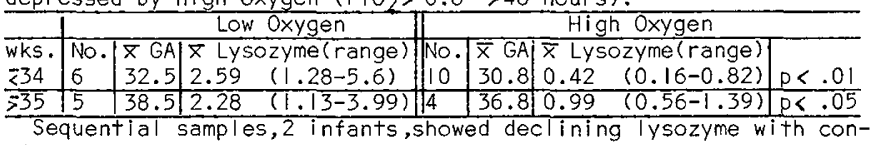
tinued exposure to high oxygen. An effect of gestational age on lysozyme secretion could not be del ineated due to the small number of infants $<32$ weeks gestation. Low levels of lysozyme ( 1.26 and 0.68 ) were obtained from tracheobronchial secretions of 2 term infants with group B streptococcal pneumonia but not infants with streptococcal or other septicemias. These data confirm that tracheobronchial lysozyme secretion is depressed in vivo by prolonged intensive oxygen therapy which together with disrupted mucociliary transport may impair antibacterial defense mechanisms.
104 HYPOXIC DEPRESSION OF RESPIRATION IN THE NEWBORN AND ADULT RABBIT. Bradley T. Thach and Edward E. Lawson. (Spon. by H.W.

Medical Scnool, Boston, MA. The newborn rabbit like the human newborn hypoventilates after several minutes in a low oxygen environment, supporting the hypohypoxic depression than the adult's (Dawes, J.Physiol., 1959). We measured arterial blood gases and respiratory center output (RCO) in newborn and adult rabbits made acutely and progressively asphyxic by prolonged airway occlusion at FRC. After tracheal and arterial cannula placement, the animals (47 pups $1-5$ days old and 5 adults) were maintained under light ether anesthesia. Instantaneous RCO (frequency $x$ tracheal pressure) was computed serially from onset of the occlusion. Blood gases corresponding to time from occlusion onset were interpolated from curves based on 1 or 2 samples per pup and 5 serial samples per adult. When severe hy $\rightarrow$ poxemia is reversed independently of $\mathrm{PaCO}_{2}$ and $\mathrm{pH}$, by giving the occluded pup a single breath of $100 \% \mathrm{O}_{2}$, RCO rapidly increases indicating that respiratory failure results largely from hypoxia. RCO peaked and began to decline at about the same time in the pups and adults ( $23 \pm 2$ vs $20 \pm 5$ secs from occlusion, mean $\pm S E M$ ) however this occurred when the pups were more hypoxemic (18 2 vs $66 \pm 10$ torr). In contrast, respiratory failure (apnea $>5 \mathrm{sec}$ ) occurred earlier in the pups $(57 \pm 5$ vs $124 \pm 7 \mathrm{sec})$ but again at a greater degree of hypoxemia ( $9 \pm 1$ vs $20 \pm 1$ torr). A consideration of the $\mathrm{PaO}_{2}$ at which depression occurs suggests that the newborn's respiratory. center is actually somewhat more resistant to hypoxic depression than the adult's. Earliex respiratory failure in the pups may be due to reduced $\mathrm{O}_{2}$ stores and increased $\mathrm{O}_{2}$ consumption. 
1005

Enzyme Hydrolysis and Acylation of Lysolecithin in Human Neonatal Lung. Francis H.C. Tsao and Richard D. Zachman. Univ. Wis., Dept. Peds., Madison, WI

The metabolism of lysolecithin $(L L)$, a breakdown product of lecithin which could act as a precursor for surface active pulmon ary lecithin synthesis, is not yet well studied in lung. We found that lysolecithin was a common substrate of three enzymatic reactions in the newborn human lung homogenate. They were (1) hydrolys is of the acyl-group of 1ysolecithin by lysophospholipase (LPL); (2) acylation at the hydroxyl group with acyl-CoA to form lecithin by acy 7 COA: lysophosphoglyceride acyltransferase (COA-LAT); and (3) exchange of the acyl-groups of two molecules of lysolecithin by lysophosphoglyceride-lysophosphoglyceride acyltransferase

(LAT) to produce a new molecule of lecithin.

The enzyme assays were carried out on human newborn lung autopsy homogenate. Activities were measured at $\mathrm{pH}$ optimum, at linear dependence with protein, and at saturating substrate. LL was stochio-metrically converted to free fatty acid and lecithin by LPL and LAT. The hydrolys is and acylation of 1-palmitoyl lysolecithin $\left(\mathrm{C}_{16}\right)$ and 1 -oleyl lysolecithin $\left(\mathrm{C}_{18.1}\right)$ had the same reaction rates. The $\mathrm{pH}$ optimum was 8.0 for $L P L, 7.4-8.0$ for $L A T$, and was 7.4 for COA-LAT. AT1 three enzymes were inhibited by detergents. Cat+ stimulated LPL and LAT, but inhibited CoA-LAT. Serine blocking agents inhibited all three enzyme activities, suggesting that they are probably serine-histidine type enzymes. Iodoacetic acid had no effect on LPL or LAT.

The enzymes for lysolecithin breakdown and recycling to lecithin are present in human neonatal lungs. Their quantitative role

\section{6}

Marked Differences Between the Lecithin-Lysolecithin Cycle Enzymes and the CDP-Choline Lecithin Synthesis Enzymes in Developing Feta] Rabbit Lungs. Francis H.C. Tsao and Richard D. Zachman, Univ. Wis. Dept. Peds. Madison, WI Lysolecithin $(\overline{L L})$, a breakdown product of lecithin, might be important in pulmonary lecithin synthesis through fatty acid exchange reactions. This study defines the levels of the enzymes of the lecithin-lysolecithin cycle; lysophospholipase (LPL) and lyso phosphoglyceride acyl transferase (LAT), in the developing fetal rabbit lung and compares them with the enzymes of the CDP-choline synthetic pathway:choline kinase (CK), cytidyl transferase (CyT), and phosphorylcholine glyceride transferase (PCGT)

Fetal lung homogenates from rabbits of known gestation were used for the enzyme, protein, and phospholipid analyses. Total lung phospholipid, lecithin and protein increased with gestational age. 30 days gestation, newborn, and maternal lung activities of CK, CYT, and PCGT decreased to only $50 \%$ of their activities at 22-26 days gestation. In contrast, LPL and LAT activities increased $3-$ fold from $22-26$ days to 30 days gestation, 2.0 vs 6.0 nmole FFA/mg protein/min. for LPL and 0.5 vs 1.6 nmole lecithin/ $\mathrm{mg}$ protein/min. for LAT), and increased further in the newborn lung to a Tevel matching maternal lung (about 8-10 fold higher than the 22-26 days gestation activities).

In fetal rabbit lung, enzyme activities for the apparent major lecithin synthetic pathway decreased. In contrast, the marked increases in LPL and LAT activities with increasing gestational age and at birth suggests importance of the lecithin-lysolecithin cycle in regulating synthesis and turnover with maturation.

1007 ISOLATION AND KINETIC STUDY OF PULMONARY ENZYMES FOR LECITHIN BIOSYNTHESIS. Rodney E. Ulane, Laura L. Stephenson and Philip M. Farreli, NICHD, NIH, Bethesda, Md.

Lecithin, a primary component of pulmonary surfactant, is synthesized de novo in lung via the choline pathway. Choline kinase (CK), being the pathway's first enzyme, could be a key regulatory site in the synthesis of surface active lecithins. In these studies, CK has been isolated in a highly purified form for the first time, enabling us to make a meaningful assessment of the enzyme in terms of its regulatory properties. The purification of the enzyme was achieved following development of a new system for measuring CK activity. The new assay which employs radioisotopic substrate and paper chromatography, is sensitive, rapid, accurate, reproducible and yields extremely low blanks. CK was found entirely in the supernatant fraction of monkey lung homogenates centrifuged at $100,000 \mathrm{~g}$. This fraction was then subjected to $\left(\mathrm{NH}_{4}\right)_{2} \mathrm{SO}_{4}$ precipitation, DEAE-and $\mathrm{CM}-c e l l u l o s e$ ion exchange chromatography, G-200 gel filtration, and iso-electric focusing. On kinetic analysis, the purified enzyme exhibited two distinct $\mathrm{km}$ values for choline, one at high and the other at low substrate concentrations, suggesting the possibility of isozymic forms. The activity is inhibited $40 \%$ at glycerol concentrations of $25 \%$ and by p-hydroxymercuribenzoate. CK is also inhibited by ADP, and phosphorylcholine. It is activated by $\mathrm{Mg}^{++}, \mathrm{Na}^{+}, \mathrm{K}^{+}, \mathrm{Ca}^{++}$and inactivated by $\mathrm{Zn}^{++}$. These results suggest that the enzyme is sensitive to regulation by physiological levels of metabolites and may explain the increase in choline pathway rates in fetal primate lung during late gestation.
1008

RELATIONSHIP OF INITIAL CHEST ROENTGENOGRAMS TO SUBSEQUENT PULMONARY FUNCTION AND SURVIVAL IN CYSTIC FIBROSIS by Pierre A. Vauthy, Robert C. Stern, Thomas F. Boat, Arthur S. Tucker, Frank P. Primiano, LeRoy W. Matthews, Carl F. Doershuk, Case Western Reserve University, CTeveland, Ohio.

Ninety-five patients with cystic fibrosis were divided into two groups after one year of therapy using chest roentgenogram scores: Group I, 19 points or greater(maximum 25); Group II, less than 19 points. Scores were assigned by a pediatric radiologist without knowledge of the clinical status. During 18 years of therapy 28 patients died; one $(2.5 \%$ ) in Group I, and $27(47 \%)$ in Group II (mean age at death 14 years 7 months). The remaining patients have been followed for a minimum of 13 years.

Progression of putmonary disease by roentgenogram scoring and pulmonary function was significantiy slower $(p<0.01)$ in Group I patients than in Group II patients. The males in Group I, mean age 18.1 years, maintained remarkabiy good pulmonary function with mean vital capacity $96+24$ (SD)\% of predicted, mean conductance $84+37$ (SD)\% of predicted and mean RV/TLC ratio of $0.35+0.12$ (SD). Mean chest roentgenogram and pulmonary function in Group I females showed a greater deterioration than Group I males but the difference is not yet statistically significant. The surviving Group II patients showed greater deterioration of mean pulmonary function values than patients in Group I but with considerable indivicual variation. Cumulative survival rate in the 95 patients was 0.64 after 18 treatment years, 0.98 in the Group I patients and 0.40 for Group II. Chest roentgenogram scoring after one year of intensive therapy can provide a useful prognostic index of subsequent pulmonary function and survival.

\section{DISTRIBUTION OF VENTILATION IN BRONCHO-}

PULMONARY DYSPLASIA. John L. Watts, Ronald L. Cardiovascular Research Institute, University of California, San Francisco

The histologic and radiologic findings in bronchopulmonary dysplasia (BPD) suggest that maldistribution of ventilation is a major abnormality. Nitrogen wash-out studies and calculated pulmonary clearance delay (PCD) were performed in 3 groups of 10 low birth-weight infants. Group A (mean birth-weight of $1370 \mathrm{gm}$ ) had mild-moderate respiratory disease and received no ventilatory assistance; group B (mean birth-weight 1290 $\mathrm{gm}$ ) were ventilated for severe respiratory disease; group $C$ (mean birth weight $1410 \mathrm{gm}$ ) were also ventilated for severe respinatory disease but all developed BPD. Sex ratio, 1 and 5 minute Apgar scores and gestational age were similar.

Mean PCD values at 1 month of age in groups $A$ and $B$ were similar ( $71 \%$ and $72 \%$ ). However, group $C$ (infants with BPD) had severe maldistribution of ventilation, with a mean $P C D$ value of $231 \%$, significantly greater than both $A$ and $B(P<0.005)$. Severity of disease, as represented by duration of oxygen dependence, was significantly related both to maximum recorded PCD, and PCD at one month $(r=0.55, p=0.01$ and $r=0.57, p<0.01$ respectively). Our findings suggest that measurement of PCD may be useful in assessment of progress and prognosis in $B P D$, and that maldistribution of ventilation is the major cause of respiratory insufficiency.

\section{PULMONARY INTERSTITIAL EMPHYSEMA AND THE}

1010 ETIOLOGY OF BRONCHOPULMONARY DYSPLASIA (BPD).

John L. Watts and Robert S. Arkoff. (Sponsored by Samuel T. Giammona) Children's Hospital and Cardiovascular Research Institute, University of California, San Francisco.

The etiology of BPD is unknown. Previous studies have incriminated high inspired oxygen, prolonged positive pressure ventilation and severity of initial disease. We assessed two groups of ventilated, low birth-weight infants. Group I developed the classical clinical and $x$-ray findings of BPD; group II did not (clinical data are listed in table).

$\begin{array}{llllllll} & N & \text { Male } & \begin{array}{c}\text { Apgar } \\ 11 / 5^{\prime}\end{array} & \begin{array}{l}\text { B. Wt. } \\ \text { gm }\end{array} & \begin{array}{l}\text { Gest. Age } \\ \text { wks }\end{array} & \begin{array}{c}\text { IPPV } \\ \text { days }\end{array} & \begin{array}{c}O_{2}>0.6 \\ \text { hrs }\end{array} \\ \text { Gp I } & 10 & 8 & 5.4 / 6.9 & 1413 & 30.9 & 23.9 & 62 \\ \text { Gp II } & 10 & 8 & 5.0 / 7.1 & 1292 & 30.0 & 20.5 & 21\end{array}$

The mean times in $\mathrm{FIO}_{2}>0.60$ and $>0.40$ were greater for group I but this was not statistically significant. The major difference between the two groups was the presence, on the first day of life, of pulmonary interstitial emphysema (PIE) in the initial chest $x-r a y$, in $8 / 10$ of group 1 and only $2 / 10$ in group $11\left(\mathrm{Chi}^{2}=5.0, \mathrm{p}=0.025\right)$. Four of the 8 in group 1 had PIE present prior to the onset of mechanical ventilation. The 2 infants who developed BPD, but did not have early PIE, both had focal emphysema prior to artificial ventilation. These findings suggest that the presence of early PIE may significantly predispose to the development of BPD, especially if high inspired oxygen and mechanical ventilation are used. 
NEBULIZED RACEMIC EPINEPHRINE ADMINISTERED BY INTER1011 MITTENT POSITIVE PRESSURE BREATHING (IPPB) MORE EFFECTIVE THAN NEBULIZED SALINE BY IPPB IN THE TREATMEN' OF CROUP: A DOUBLE BLIND CONTROLLED STUDY. C. ROSS Westley, John G. Brooks, and Exnest K. Cotton, University of Colorado Sch. of Medicine, Department of Pediatrics, Denver.

Racemic epinephrine ( $R E$ ) has been advocated for the treatment of croup, but controlled studies have failed to prove it more effective then saline. Twenty children (age 4 mo - 5 yrs) with stridor at rest were randomly assigned to one of two treatment groups: normal saline or 2.25\% RE each diluted $1: 8$ with sterile water. Each patient was assigned a clinical score based on the state of consciousness, the adequacy of air entry, the severity of stridor and chest wall retractions, and the presence or $a b-$ sence of cyanosis, before and 10, 30 and 120 minutes after each treatment. A total of 40 treatments were evaluated. All treatments were administered for 10-15 minutes with a Mark $V$ Bird IPPB machine. All children received continuous cool mist therapy; none required intubation; and no supplemental oxygen was administered except with the treatment. The clinical score was significantly improved $(p<0.01)$ following treatment with $R E$, but there was no significant improvement after saline. There was a significant difference $(p<0.01)$ between the effect of $R E$ and saline at 10 , and 30 minutes but not at 120 minutes after the treatment. We conclude that nebulized RE by IPPB but not saline is an effective treatment for croup. Because of the relatively short duration of clinical benefits in some patients, nebulized RE by IPPB may have to be administered repeatedly and therefore should not be administered to outpatients.

107 EFFECT OF FEEDING ON PERIPHERAL BLOOD FLOW IN HYALINE 1012 membrane diseAse (HMD). Victor Y.H. Yu, Richard W.I. Cooke and Peter Rolfe (Intr. by John C. Sinclaix)., Univ. of Oxford, John Radcliffe Hosp., Department of Pediatrics, Oxford, England.

Lower limb blood flow was measured before and after gavage feeding in 12 neonates with HMD and 12 healthy controls by means of venous occlusion plethysmography. Expressed human breast milk was given in volumes between $5-8 \mathrm{mls} / \mathrm{kg}$. Results for peripheral blood flow (PBF $\mathrm{ml} / \mathrm{min} / 100 \mathrm{ml}$ ) and peripheral resistance (PR $\mathrm{mmHg} / \mathrm{ml} / \mathrm{min} / 100 \mathrm{ml}$ ) are shown in the Table. PBF in infants with HMD tended to be lower than that in the controls. This was due mainly to a higher PR. A significant rise in PBF associated with a decrease in PR was observed $60 \mathrm{~min}$ postfeed in both groups. Abdominal aortic BP, central (rectal) and peripheral (foot) temperatures were unchanged. The data indicates a similar peripheral circulatory response to feeding in neonates with HMD and healthy controls. The physiological significance of this in HMD deserves investigation.

\begin{tabular}{|c|c|c|c|c|c|}
\hline & & Prefeed & $15-30 \mathrm{~min}$ & $60 \mathrm{~min}$ & $90 \mathrm{~min}$ \\
\hline Controls & $\begin{array}{l}\text { PBF } \\
\text { PR }\end{array}$ & $\begin{array}{r}6.7+0.9 \\
10.1 \pm 2.3\end{array}$ & $\begin{array}{r}6.5+0.9 \\
10.4 \pm 1.2\end{array}$ & $\begin{array}{l}8.3+0.9 * \\
8.3 \pm 1.0 *\end{array}$ & $\begin{array}{l}8.1+0.9 \\
8.4 \pm 1.0^{*}\end{array}$ \\
\hline HMD & $\begin{array}{l}\text { PBF } \\
\text { PR }\end{array}$ & $\begin{array}{r}4.9+0.7 \\
13.2 \pm 1.4\end{array}$ & $\begin{array}{r}4.1+0.6 \\
14.8+1.3\end{array}$ & $\begin{array}{l}6.6+0.9 * \\
9.4+1.0 *\end{array}$ & $\begin{array}{r}5.9 \pm 0.7 \\
10.6 \pm 1.1\end{array}$ \\
\hline
\end{tabular}

(Values in Mean \pm SEM, * $=$ significant)
1013 CARDIORESPIRATORY EFFECTS OF CONTINUOUS POSITIVE AIRWAY PRESSURE BREATHING (CPAP) IN HYALINE MEMBRANE DISEASE (HMD). Victor Y.H. Yu and Peter Rolfe (Spon. by John C. Sinclair). Univ. of Oxford, John Radcliffe Hosp., Dept. of Pediatrics, Oxford, England.

In normal animals and humans, CPAP significantly impairs cardiorespiratory function. Six human neonates with HMD were studied on ten occasions, before and during CPAP $(5-15 \mathrm{~cm} \mathrm{H} 20)$ by endotracheal tube. Ventilation was measured by pneumotachography. (Results in Mean + SEM, * $=$ significant) without CPAP with CPAP $\mathrm{pH}$ $\mathrm{PaCO} 2$ ( $\mathrm{mmHg}$ ) $\mathrm{PaO2}$ (mmHg)

A-aDO2 (mmHg)

Heart rate (bpm)

Mean art. blood pressure (mmHig) End-expiratory CVP (mmHg)

Respiratory rate (bpm)

Tidal volume (ml)

Minute volume (ml) Dynamic compliance (ml/cmH20)
$7.22+0.2$

$50+3.4$

$52 \pm 5.7$

$424+48$

$157 \overline{ \pm 2} .7$

$49+2.2$

$5.5 \pm 0.9$

$94+3.8$

$8+1.2$

$749+110$

$1.0 \pm 0.1$
$7.24+0.1$ $50 \pm 2.9$ $75 \pm 9.7 *$ $395+39$ *

$156+2.5$

$48+1.9$

$7.4 \pm 1 \cdot 2 *$

$77 \pm 3.9 *$

$9+1.0$

$706+95$ $0.8 \pm 0.1$ *
The average increases in central venous and intraesophageal

pressures were $20 \%$ and $34 \%$ respectively of the applied CPAP. All infants showed improvement in oxygenation. None had significant bradycardia or hypotension. The effect of CPAP on the breathing pattern was evaluated by breath-by-breath analysis of tidal volume and instantaneous respiratory rate. Significantly less variation in both parameters was observed during CPAP.

\section{4} CARDIOVASCULAR RESONSES TO APNEA: A TOOL FOR E. Hodgman, Bijan Siassi. Univ. of So. Calif. School of Medicine, Los Angeles County/Univ. of S. Calif. Med. Center, Depts. of Ob/Gyn and Peds., Los Angeles. Continuous monitoring of instantaneous heart rate(HR), its variability (HRV), respirations (expired $\mathrm{CO}_{2}$ ), aortic blood pressure(ABP), temperature, instantaneous electromechanical interval(EMI) of the cardiac cycle measured from the $Q$ wave of the EKG to the onset of the aortic pulse pressure upstroke were explored as predictive parameters for the significance of apnea. 47 spontaneous apneic episodes $(A E) \geqslant 15$ seconds, occurring in 10 prematures(GA:28-36 wks,BW:1210-2480 gms) in the first day of life were studied. 5 had no $\operatorname{HMD}(I)(A E=21) ; 5$ had $H M D(I I)$ $(A E=26)$, of whom 3 went on to assisted ventilation $(\mid \mathrm{la})(A E=17)$ and 2 did not (IIb) $(A E=9)$. Temperature, total calcium, blood sugar and arterial blood gases were normal except for increased $0_{2}$ alveolar-arterial gradient in II. HR, ABP, and EMI changes were timed from the onset of apnea(OA). Duration of $A E$ was different only between I vs $11(p<.01)$. HRV decreased in II vs I $(p<.01)$ and in IIa vs IIb (p<.01). OA was followed by HR decelerations and prolongation of EM! in all groups. The peak drop in HR preceded the maximum change in EMI. EMI recovery appeared later in II than I, and in II a than IIb(p<.0I). The ABP changes after OA were: Hypertension and pulse pressure(PP) widening in lib and initial hypotension without PP change in Ila(p<.0I)

Two types of responses to apnea were noted: One with decreased HRV, initial hypotension and later EMI recovery as in severely ill infants(IIa); the other, with greater HRV, hypertension, widened PP and earlier EMI recovery as in normal(I) and moderately ill(llb) infants. A classification of apnea based on these variables allows better understanding of hemodynamic changes and offers prognostic value. 